A DICTIONARY OF VURËS, VANUATU 



\section{A DICTIONARY OF VURËS, VANUATU}

\section{CATRIONA MALAU}

WITH CONTRIBUTIONS FROM

PHILEMON ALA, KATHERINE E. HOLMES,

ARMSTRONG MALAU AND ELI FIELD MALAU

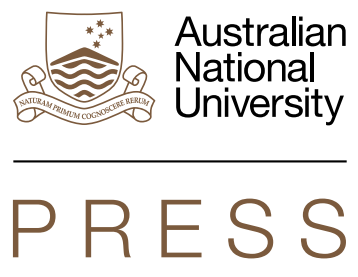

ASIA-PACIFIC LINGUISTICS 


\section{ANU \\ PRESS}

Published by ANU Press

The Australian National University

Acton ACT 2601, Australia

Email: anupress@anu.edu.au

Available to download for free at press.anu.edu.au

A catalogue record for this book is available from the National Library of Australia

ISBN (print): 9781760464608

ISBN (online): 9781760464615

WorldCat (print): 1268007302

WorldCat (online): 1268007983

DOI: 10.22459/DVV.2021

This title is published under a Creative Commons Attribution-NonCommercial-NoDerivatives 4.0 International (CC BY-NC-ND 4.0).

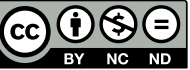

The full licence terms are available at

creativecommons.org/licenses/by-nc-nd/4.0/legalcode

Cover design and layout by ANU Press. Cover photograph (c) Katherine E. Holmes.

This edition @ 2021 ANU Press 


\section{Contents}

Acknowledgements vii

About this dictionary $\quad 1$

1 Who this dictionary is for and how to use it 1

2 The Vurës language $\quad 2$

2.1 Vurës sounds and spelling 4

2.2 Vurës punctuation notes $\quad 8$

3 Bislama in the dictionary 8

4 The Vurës corpus 9

5 Structure of the dictionary and entries $\quad 13$

$\begin{array}{ll}5.1 \text { Alphabetical ordering } & 13\end{array}$

$\begin{array}{ll}5.2 \text { Structure of the entries } & 14\end{array}$

$\begin{array}{ll}5.3 \text { Part of speech labels used } & 17\end{array}$

$\begin{array}{ll}5.4 \text { Variants, variation and complex forms } & 19\end{array}$

6 Word origins 21

7 References $\quad 22$

8 Abbreviations used in the dictionary 24

Vurës - English - Bislama Dictionary 25

English - Vurës Finderlist $\quad 261$

Bislama - Vurës Faendalis $\quad 291$

Organisation of the thesaurus $\quad 315$

$\begin{array}{ll}\text { A Landscape, country and water } & 320\end{array}$

B Weather and cyclic time $\quad 324$

$\begin{array}{ll}\text { C Spatial relations } & 326\end{array}$

$\begin{array}{ll}\text { D Plants } & 328\end{array}$

E Fauna 363

F Physical properties $\quad 392$

$\begin{array}{ll}\text { G Quantification } & 394\end{array}$ 
H Human classification $\quad 396$

I Human qualities and emotions 399

$\begin{array}{ll}\text { J Body parts and products } & 400\end{array}$

$\begin{array}{ll}\mathrm{K} \text { Body states and functions } & 405\end{array}$

L Stance and motion $\quad 408$

M Artefacts, material culture and manufactured things 411

N Kinship 426

O Marriage, sex, nurturing $\quad 428$

$\begin{array}{ll}P \text { Speaking, communicaton and sounds } & 430\end{array}$

Q Social interaction, relationships and behaviour 434

R Religion, ceremony, law and the supernatural 436

S Hunting, fishing, farming 443

$\begin{array}{ll}\mathrm{T} \text { Fire and cooking } & 447\end{array}$

U Human uses of natural resources $\quad 454$

V Thinking, perception and attention $\quad 457$

W Handling and physical transfer 458

$\begin{array}{ll}X \text { Impact and surface contact } & 460\end{array}$

Y Speed, manner and relative time 463

$\begin{array}{ll}\text { Appendix } & 465\end{array}$ 


\section{Acknowledgements}

This dictionary has been in gestation for close to 22 years, so naturally there are very many people who have contributed to its production in different ways. The two people who have made the most substantial contributions are the two people who were there right from the start, who met me when I landed at Sola airport on Vanua Lava in March 1999. That is, Eli Field Malau, a fieldworker with the Vanuatu Cultural Centre, who had requested that I come to help him write his language, and his son, Armstrong Malau, who was to become my husband. My father-in-law was the single most significant contributor in the early days of my research. He worked with me, discussed language issues with me, and sought out all the Vurës speakers who ended up working with me as language consultants. Armstrong has had an impact throughout, as he was my companion from the beginning of my time on Vanua Lava, so had a great influence on me learning to speak Vurës. Throughout the years he has worked on text transcription and translation, and answered countless questions about the words in this dictionary.

All other members of the Malau family have also contributed greatly to this work. They have assisted with all aspects of data collection and analysis, and they have looked after me and supported me with love. Most importantly, they have cared for my young children so that I could manage to get enough work done. In particular, my children's grandmother, Joana Leo, has always worked hard to make sure that my and my children's every need was attended to each time we visited the village. I would also like to single out Kali Malau and his wife, Nesta, who have worked with me and cared for us with boundless energy and enthusiasm.

I can't thank individually every other Vurës speaker who has contributed to this dictionary, as there are too many who have been involved. All those who provided a recording of them speaking in their language are listed in the appendix, but there are also many others in the community who have contributed. Some who deserve special mention are my Vurës parents, Doran Rörösōq and Noris Menegqiat, and my grandmother, Emely Rēlin̄veg Qiat. As with the Malau family, they and their children have consistently supported me, both on a personal level and with contributions to my work. 
I thank the chiefs of Vurës for granting me permission to carry out research with the community. Thanks to those who helped out from the beginning and took part in discussions about the spelling, in particular Father Gregory Manliwōs, Owen Qalgislē, Hosea Waras, Roy Kipe and John Elizabeth Kökör. Others who devoted long hours to working with me on transcription and translation are Kathy Doris and Kate Ruth Elman.

During the time that I have been engaged in research on Vurës, I have been employed at several institutions that have supported my work: The Vanuatu Cultural Centre (1998-2002), The Research Centre for Linguistic Typology at La Trobe University (2002-2006), The University of the South Pacific (2006-2008), and The University of Newcastle (2009-present). I thank these institutions for providing the personal, financial and institutional support that has enabled me to work on my research. I also thank the Vanuatu Cultural Council and Vanuatu Cultural Centre (VKS) for granting me permission to carry out research on Vurës. The VKS staff have always been supportive, and in particular I would like to thank Henlyn Mala for her friendship and assistance over the years.

My fieldwork expenses have been supported for the most part through grants from the Endangered Languages Documentation Programme (ELDP) and the DOBES Documentation of Endangered Languages Programme. Aside from the vital financial support, these programs and the staff who work with them have provided excellent technical training and advice over the years.

The DOBES-funded project was a collaborative project, and I am appreciative of my fellow researchers who contributed to this dictionary: the late Philemon Ala, Raymond Ammann, Sabine Hess, Kate Holmes, Chanel Sam and Stefan Schnell. In particular, many thanks to Kate Holmes, who provided scientific identifications for many fish and other marine species, contributed a large portion of the photos in the dictionary, and shared fun times with me during fieldwork. Thanks also to Sophie Caillon for fieldwork support and for contributions relating to taro and coconut farming and names.

Thanks to Nick Thieberger for technical advice over the years. I appreciate the input of Alex François on a number of matters, particularly his early contributions regarding phonology and spelling. Thanks to Daniel Krausse who provided some additional data and revisions. I'm grateful to Garry Innes for allowing me to use his bird photos in the dictionary.

I'm incredibly grateful to Beth Evans for the amount of time she has put into helping me with dictionary formatting issues. Thank you to the three anonymous reviewers who provided valuable feedback to help me improve the final work. I would like to thank Tonya Stebbins and Brenda Boerger for their input regarding compilation of the thesaurus. I also acknowledge Tonya's advice and input regarding the structure of the introduction to the dictionary. Thanks to Angela Terrill for copyediting and in particular for advice regarding inclusions in the introduction. 


\section{About this dictionary}

\section{Who this dictionary is for and how to use it}

This dictionary is intended for two main audiences, who may want to find in it different types of information. The first intended audience is the Vurës language community. I hope they will be able to use this dictionary to help them write their language, and to promote and maintain the use of traditional words and meanings. It was for this audience, particularly the younger members, that I decided to include many colour photos, especially to illustrate plant and animal names, and a thesaurus, that teachers will be able to refer to when working with school children. The second main audience is linguists and other scientists, for whom I have included more extensive fields that may not be relevant for community members, such as etymologies and literal meanings.

There is a lot of information packed into some of the entries in the dictionary, so Vurës speakers who are not very familiar with using a dictionary should read the explanations in the following sections. These show how to use the dictionary and how different parts of an entry for a word are separated. After a brief introduction to the Vurës language in Section 2, the Vurës spelling system is explained in Section 2.1. Section 2.2 provides a brief note about punctuation. This is a trilingual dictionary, with definitions of Vurës words presented in both English and Bislama, the national language of Vanuatu. Some information about the Bislama used in the dictionary is provided in Section 3. Section 4 presents some background information about the Vurës corpus and how the dictionary was compiled. Section 5 explains how the dictionary, and entries within the dictionary, are structured, and how to find information. Section 5.1 details the alphabetical ordering that is used. In section 5.2 some sample entries are presented to illustrate and explain how entries are set out. Some details about the part of speech labels that are used in the dictionary are given in Section 5.3. Section 5.4 discusses variants and complex word forms. The final section comments on word origins. 


\section{The Vurës language}

Vurës is a language spoken by about 2,000 people who live mostly in the southern part of the island of Vōnō Lav - called Vanua Lava by outsiders - in Vanuatu, a small island nation in the South Pacific (Malau 2016). Map 1 shows the location of Vanua Lava, in the Banks group of islands, part of Torba, the most northerly province of Vanuatu. Map 2 shows just Vanua Lava, the shaded part indicating the area where Vurës is spoken, with labels for the main Vurës-speaking villages. Around half of the people who speak Vurës live in the large village of Vētuboso. The remainder live mostly in villages up to an hour or two walk away from Vètuboso, with small communities of speakers also in the major Vanuatu towns of Port Vila, the capital, and Luganville, on the island of Espiritu Santo.

The most recent count puts the number of vernacular languages of Vanuatu at 138, with 17 of these spoken in the islands of Torba province (François et al. 2015). Vurës, along with all other indigenous languages of Vanuatu, belongs to the Oceanic subgroup of languages, which in turn are all part of the larger language family called Austronesian. Within the Oceanic subgroup, languages of Vanuatu are all part of the Southern Oceanic linkage, which is divided into several groups, and Vurës, like all languages spoken in Torba province, is part of the Northern Vanuatu linkage (Lynch 2000; Lynch, Ross and Crowley 2002).

Vurës speakers are multilingual, and are all fluent in Bislama, which is an English-lexifier creole (Crowley 2003, 2004). Many speakers also have some level of proficiency in the other main language of Vanua Lava, Vera'a and/or one of the languages of neighbouring islands, such as Mwotlap or Mota. Many Vurës people also have a working knowledge of English, and some have knowledge of French. For most, literacy was taught in English, and people have varying literacy levels in English, French and Bislama. Most people have not been taught how to read and write in their own language and thus find it difficult to transfer their English literacy skills to Vurës. This dictionary aims to help speakers overcome these difficulties. 


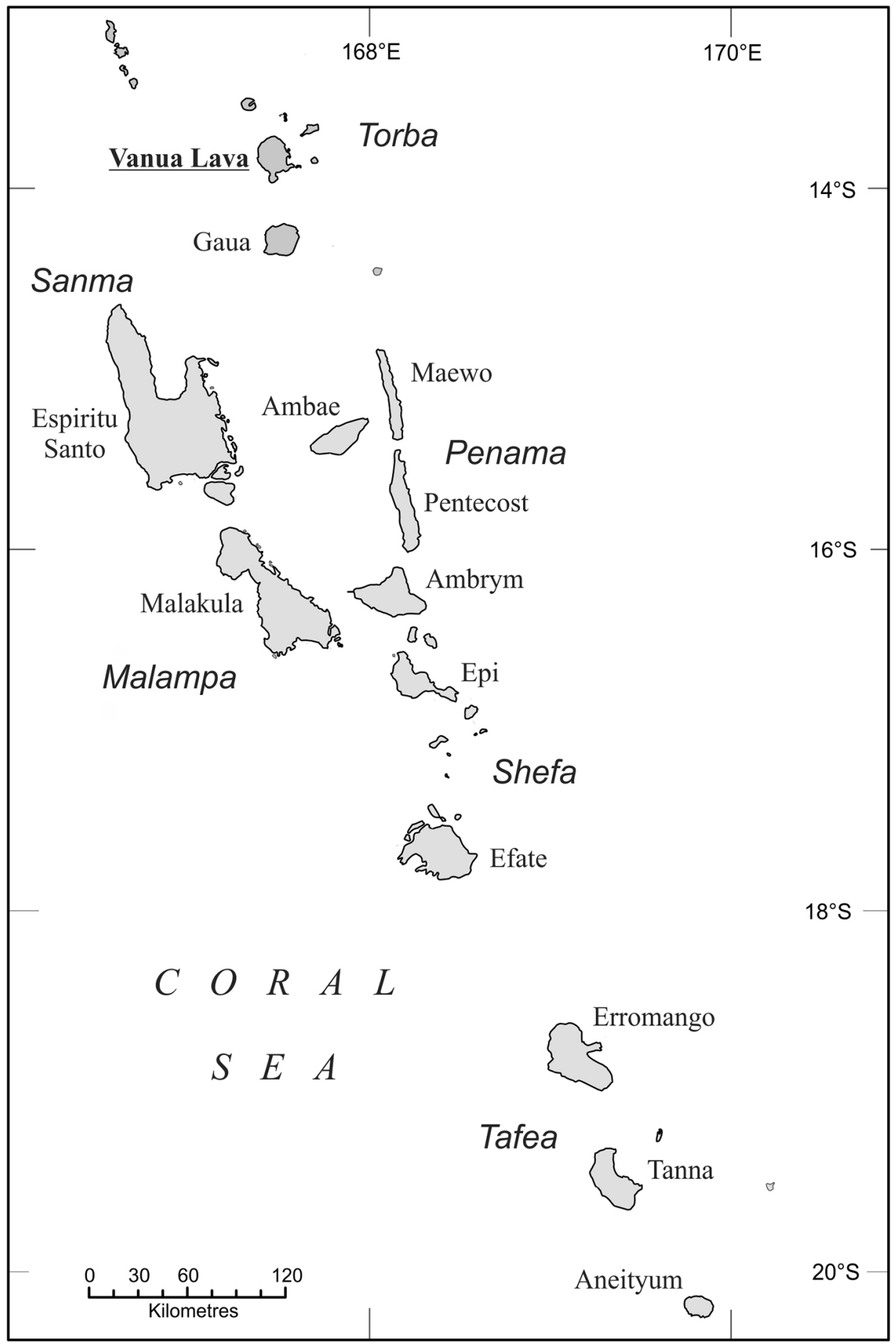

Map 1: Area map of Vanuatu, showing Vanua Lava in the north

Source: () Malau (2016) 


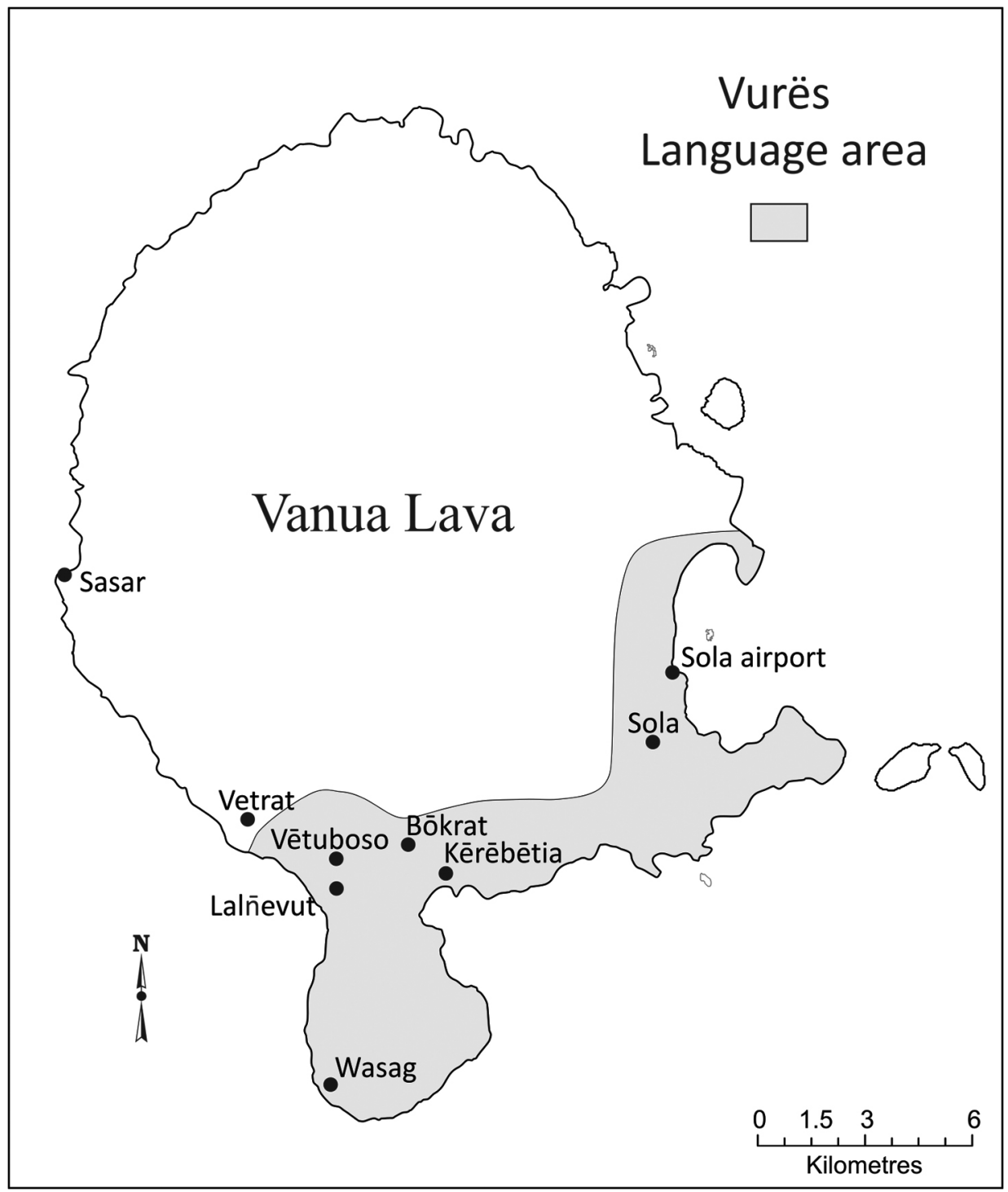

Map 2: Vanua Lava, showing area where Vurës is spoken

Source: (c) Malau (2016)

\subsection{Vurës sounds and spelling}

There is no established tradition of writing in the Vurës language. Most speakers of Vurës have been taught how to read and write in English during their schooling, and have never been taught how to read and write their own language. This has been the norm in schools throughout Vanuatu, with only English and French being used as languages of education. The situation is changing, however, the 2012 Vanuatu National Language Policy (Ministry of Education 2012) stipulates 
that all children should be taught in their mother tongue for the first three years of primary school, with English or French only introduced in Year 3. As of 2016, this policy has begun to be implemented at the Sanlañ primary school, the main school within the Vurës-speaking area. In 2016, as part of the Vanuatu Education Support Program, I assisted with the translation of 51 readers and story books, along with other literacy materials such as posters, for classroom use for Years 1-3 of primary school. The literacy materials that have been produced for Vurës follow the spelling system outlined and used in this dictionary.

Table 1 presents the letters of the Vurës alphabet matched with symbols for the sounds that they represent. Each letter of the alphabet is presented in the first column of the table. In the next column is an example of a Vurës word that has that letter in it. The example words can be used by Vurës speakers as keywords to serve as a reminder of the sound that some of the more unfamiliar letters represent, especially those with marks on top of them, such as $\overline{\mathbf{e}}$ and $\ddot{\mathbf{e}}$. In the last column is a symbol from the International Phonetic Alphabet (IPA), which specifies the sound that the alphabet letter represents. The IPA symbols are to help those who do not already know how to speak Vurës, to show them how words are pronounced. More information about the sounds and phonology of the language can be found in Malau (2016: 19-48), which is a grammar of Vurës.

Table 1: Spelling conventions for Vurës

\begin{tabular}{|c|c|c|}
\hline Letter & Example & Sound \\
\hline $\mathbf{A} \mathbf{a}$ & añañ 'yellow' & [a] \\
\hline $\mathbf{B} \mathbf{b}$ & bibis 'roll up' & {$\left[\mathrm{b},{ }^{\mathrm{m}} \mathrm{b}\right]$} \\
\hline D d & dödöm 'think' & {$\left[\mathrm{d},{ }^{\mathrm{n}} \mathrm{d}\right]$} \\
\hline Ee & es 'spear' (vt.) & {$[\varepsilon]$} \\
\hline$\overline{\mathbf{E}} \overline{\mathbf{e}}$ & ès 'alive' & [e] \\
\hline$\ddot{\mathbf{E}} \ddot{\mathbf{e}}$ & ës 'smoke' & {$[œ]$} \\
\hline $\mathbf{G} \mathbf{g}$ & gegen 'sharp' & {$[\mathrm{\gamma}]$} \\
\hline $\mathbf{I} \mathbf{i}$ & ilsi 'end' & [i] \\
\hline $\mathbf{K} \mathbf{k}$ & kakaka 'story' & {$\left[\mathrm{k}, \mathrm{k}^{\mathrm{h}}\right]$} \\
\hline $\mathbf{L} 1$ & lelev 'fast' & {$[1]$} \\
\hline M m & mamas 'dry' & {$[\mathrm{m}]$} \\
\hline$\overline{\mathbf{M}} \overline{\mathbf{m}}$ & '̄̄ō̄o 'tame' & {$\left[\tilde{y m}^{\mathrm{w}}\right]$} \\
\hline $\mathbf{N} \mathbf{n}$ & nonor 'yesterday' & {$[\mathrm{n}]$} \\
\hline$\overline{\mathbf{N}} \overline{\mathbf{n}}$ & n̄ōōōorr 'snore' & {$[\mathrm{y}]$} \\
\hline 0 o & on 'large octopus' & [0] \\
\hline$\overline{\mathbf{o}} \overline{\mathbf{o}}$ & ōn 'sand' & [o] \\
\hline$\ddot{0 ̈ ~ o ̈ ~}$ & götö 'hermit crab’ & [ø] \\
\hline
\end{tabular}




\begin{tabular}{|l|l|l|}
\hline Letter & Example & Sound \\
\hline P p & Pōpō 'Grandma, Grandpa' & {$[\mathrm{p}]^{1}$} \\
\hline Q q & qaqaq 'talk' & {$\left[\widehat{k p}^{\mathrm{w}}\right]$} \\
\hline R r & rörö 'news' & {$[\mathrm{r}, \mathrm{c}]$} \\
\hline S s & sasar 'island cabbage' & {$[\mathrm{s}]$} \\
\hline T t & Tatat 'Uncle' & {$\left[\mathrm{t}, \mathrm{t}^{\mathrm{h}}\right]$} \\
\hline U u & usuk 'my life' & {$[\mathrm{y}, \mathrm{v}]^{2}$} \\
\hline V v & vivi 'umbrella' & {$[\beta, \mathrm{p}]$} \\
\hline W w & wiwieg 'work' & {$[\mathrm{w}]$} \\
\hline
\end{tabular}

There are some difficult aspects of the spelling system. When I first started documenting Vurës in 1999, there were no books or other materials published in the Vurës language and few people ever tried to write in the language. When people needed to write anything, they would mainly write in English or Bislama. Eli Field Malau, one of the main people who has helped me to document the language, was one of a very few people who had tried to write in Vurës. He had faced difficulties with how to write the sounds that do not occur in English or Bislama, such as the vowels that we now write as $\ddot{\mathbf{e}}$ and $\ddot{\mathbf{o}}$. We were assisted in the development of the spelling system by linguist Alexandre François.

As people are familiar with writing in English, if a Vurës sound is fairly similar to an English sound, then it is easy to use the same letter that is used for English. This is the case for a lot of the sounds of Vurës, even if the sound is a little different. For example, a Vurës $\mathbf{v}$ is made by putting both lips together, whereas an English $\mathbf{v}$ is made by moving the bottom lip up to the top teeth. These sounds are similar enough that it makes sense to use $\mathbf{v}$ for both. Several other sounds, mostly some of the vowels, are more difficult. There are also a few consonants that present some difficulties. I'll explain each of the issues here.

To start with the consonants, Table 1 shows that there are two letters that have a line on top, $\overline{\mathbf{m}}$ and $\overline{\mathbf{n}}$. The letter $\overline{\mathbf{m}}$ sounds a bit like an English $\mathbf{m}$ followed by $\mathbf{w}$ and $\overline{\mathbf{n}}$ sounds like English $\mathbf{n g}$. Therefore, we could have decided to write these sounds with $\mathbf{m w}$ instead of $\overline{\mathbf{m}}$ and $\mathbf{n g}$ instead of $\overline{\mathbf{n}}$. Eli Malau and I decided, with input from some interested members of the community, on $\overline{\mathbf{m}}$ and $\overline{\mathbf{n}}$ because quite a lot of older people are familiar with these two symbols being used for Mota language, which has been used as a common language by the Anglican church in the northern islands of Vanuatu for over a hundred years. There was also a sense within the group who participated in discussions that using the

\footnotetext{
1 In Vurës /p/ only occurs in words borrowed from Bislama or English, like pomken 'pumpkin'. It is included here as there are some entries in the dictionary of words beginning with $\mathbf{p}$ that have been fully incorporated into the language.

2 The letter $\mathbf{u}$ represents two different sounds. The sound [v] only occurs in a very few words that come from Mota, like bulsal 'friend'. The decision was therefore made not to introduce a new letter for this sound.
} 
$\overline{\mathbf{m}}$ and $\overline{\mathbf{n}}$ instead of $\mathbf{m w}$ and $\mathbf{n g}$ gives the written language a distinctive Torba character, in contrast with the spelling used for some of the languages spoken on islands to the south.

Over the past 20 years, Eli Malau and I have held various workshops aimed at teaching the proposed spelling system to community members. Armstrong Malau, Eli's son, has continued these literacy teaching efforts through a Facebook group. There has been a reasonable level of interest in our efforts, with perhaps 20 per cent of the community engaging in one way or another. There has been general acceptance of the alphabet that we have proposed by the people who have engaged with us in our literacy teaching efforts. When we have explained the need for symbols that differ slightly from those used for English, the reasoning has been understood and accepted. Today, many speakers of Vurës have become familiar to some extent with using this spelling system, but many other speakers have not. This means that in some cases, when a Vurës speaker tries to write in Vurës without having been taught, they might make different decisions about how to write a sound, based on the language(s) that they already know how to write. The most common difference is choosing $\mathbf{m w}$ instead of $\overline{\mathbf{m}}$ and $\mathbf{n g}$ instead of $\overline{\mathbf{n}}$. People do this to some extent because these are easier to type on computers and smart phones, without needing to put a line on top of the letters. This would be an argument in favour of using $\mathbf{m w}$ and $\mathbf{n g}$. However, this dictionary reflects the earlier community decision. The main reason for this is that the members of the community who I have worked with most closely feel strongly that the orthography has become established, through the literacy materials that we have produced and through community teaching.

The other difficult letter in Vurës is q. This represents a sound which is not close to any sound of English, French or Bislama. It sounds a little bit like English $\mathbf{k}$ and $\mathbf{p}$ said at the same time, then followed by $\mathbf{w}$, all in one sound. So in some ways it is similar to English qu which is pronounced as $\mathbf{k}$ followed by $\mathbf{w}$. For that reason, we use $\mathbf{q}$ for this sound, and also because the letter $\mathbf{q}$ isn't needed for any other sound in Vurës.

As for the vowels, the letters $\mathbf{a}$ and $\mathbf{i}$ are fairly straightforward and close to English and Bislama sounds. The sound represented by letter $\mathbf{u}$ is like a French $\mathbf{u}$ rather than an English one. That is, the letter represents the high front rounded vowel [y]. The symbols based on letters e and $\mathbf{o}$, each with a line on top or two dots on top, are the difficult ones for people to learn which symbol goes with which sound. What is important to realise is that it is always best for a spelling system to have a different letter for each sound, and in Vurës each of these letters does represent a different sound. If you want to write that your grandmother is ēs 'alive' but you write instead that she is ës 'smoke', then obviously that could be a bit confusing!

There are nine vowel sounds in Vurës, but only five vowel letters in the English alphabet, so somehow the English alphabet needed to be adapted so that the Vurës alphabet has a different symbol for each vowel sound. An alternative option would be to use diagraphs, which is when two letters together represent 
one sound, such as in English 'bee'. Many literacy experts believe that it is easier for people learning how to read if each sound has just a single symbol to represent it, rather than two letters together. So this thinking influenced the choice of the symbols.

\subsection{Vurës punctuation notes}

As speakers of Vurës are literate mostly in English, it makes sense to generally follow principles of English punctuation where relevant, like capital letters at the beginning of a sentence and full stops at the end. One punctuation issue of relevance to the format of the dictionary is capitalisation. Headwords for nouns referring to place names, or people's names and kinterms used to address people, belong respectively to the subclasses of absolute location nouns and proper nouns. These nouns are listed capitalised in the dictionary, as they would be written.

There are several proclitics in the language. Proclitics are words that in some ways sound like separate words, but in other ways sound like they are joined to the word that follows. In the dictionary, as in other literacy materials that have been printed since we started using this spelling system with the community, the proclitics are written separately. This is done to try and keep words as short as possible, which makes it easier for people learning to read and write. An entry in the dictionary that is preceded or followed by '-' means that the form is a prefix or a suffix. Prefixes join on to the beginning of words. For example, to qiat 'taro' we can add di- to form diqiat 'taro leaf'. Suffixes join onto the ends of words. So matan with $-\bar{n}$ on the end means 'your eye', whereas matan with $-n$ on the end means 'her eye' or 'his eye'.

\section{Bislama in the dictionary}

This is a trilingual dictionary, with definitions of Vurës words given in both English and Bislama. All Vurës people are fluent in Bislama. Some use Bislama on a daily basis, but many use it less regularly, only when they come into contact with people who do not speak Vurës. While Vurës people are fluent speakers of Bislama, there is variation in the extent to which people are used to writing it. Most people have been taught literacy skills in English, but have not been systematically taught how to write Bislama. For this reason, not just on Vanua Lava, but throughout Vanuatu, it is quite common for people to be reasonably unsystematic in the way that they spell Bislama. Bislama is an English-lexifier creole. This means that the majority of words have an English origin. Due to this, many people who are more used to writing in English than in Bislama will transfer their knowledge of English spelling, even if the pronunciation and meaning of the word in Bislama is quite different. When writing Bislama, many people use a mix of English spelling combined with some knowledge of Bislama spelling conventions. 
Bislama spelling conventions do exist. There has been somelevel of standarisation of Bislama spelling, which started in the 1970s when the first Bislama translations of the gospels were produced (Crowley 2004). Since Independence in 1980, as part of the promotion of Bislama as the national language of Vanuatu, many groups with an interest in literacy advocacy, particularly the Literacy Association of Vanuatu, have further developed the standarisation of the written form of Bislama. For the most part, the spelling of Bislama in this dictionary follows the standardised spelling used in A New Bislama Dictionary (Crowley 2003). The only deviations from the spelling presented in Crowley (2003) are in rare cases where experience tells me that the spelling does not reflect the most widespread current pronunciation. Pronunciation does vary within Vanuatu and I decided to consistently use the spelling that is seen as being the most common on a national level.

There is variation, not just in pronunciation and spelling, but also in the words of Bislama. The majority of words are commonly used across the country, but others, especially those derived from local languages, can be limited in use to a particular area. Again, where this is the case, I have tried to represent as much as possible a standard national form of Bislama. For example, with plant names, if Crowley (2003) gives a word that is clearly derived from a word in Vanuatu languages, then I use only that word as the definition if I am sure that knowledge of that word is completely widespread. If there is any doubt regarding knowledge of any Bislama words, then the definition will consist of an explanation of the meaning in simple Bislama.

\section{The Vurës corpus}

This dictionary was compiled from data collected over a 20-year period, from March 1999 to the end of 2018. The majority of headwords have been taken from and are represented multiple times in a corpus totalling approximately 32.5 hours of recorded speech of a variety of genres. Approximately half of these recordings are audio only and the remainder are video and audio. The majority are monologues. The majority of the texts do not include any form of elicitation, but rather are people speaking in response to a request to explain a procedure, present a narrative, describe a situation, etc. The texts were recorded in two phases as part of separately funded projects, and thus are archived in different digital archives. The data collected in the period from 2002-06 was part of a project funded by the Endangered Languages Documentation Programme $(\mathrm{ELDP})^{3}$ and this data is archived both with the Endangered Languages Archive (ELAR), hosted by the School of Oriental and African Studies (SOAS), and with the Pacific and Regional Archive for Digital Sources in Endangered Cultures (PARADISEC). The second phase was funded by the Documentation of Endangered Languages project (DOBES), ${ }^{4}$ with data collected from 2007-13. 
The corpora in these digital archives are publicly available, and the access details and list of texts are presented in the appendix. For some entries, an example sentence illustrating the headword is presented. The majority of those example sentences has been extracted from recorded texts, and, if this is the case, a reference code for the relevant text is given after the example, to enable dictionary users to verify the use of the headword by accessing the text in one of the digital archives. The corpus was compiled with the primary goal of producing a grammatical description, published as Malau (2016). Compilation of a dictionary was a secondary aim. Thus, the definitions provided for some words are not as complete and thoroughly verified as for others.

The texts in the corpus represent the language of over 100 different speakers, ranging in age from young children to the oldest members of the community. There is no significant dialectal variation in Vurës and each of the main communities in different villages are represented in the corpus. The recordings for the ELDP project were all made by me. The recordings archived with the DOBES project were recorded by me and by two other members of that project team, Armstrong Malau and Katherine Holmes. Transcriptions and translations of the texts were produced by me in consultation with a number of speakers, the main language assistants being Armstrong Malau, Eli Field Malau, Kali Malau, Samson Blondel, Kathy Doris, and Welsam Segir Malau Qiat. For the most part, translations made with the help of language assistants were from Vurës into Bislama. At a later stage, I then translated the texts into English. As my competence in Vurës developed, I was able to refine both the Bislama and English translations. While working on translations, the language assistants helped with refining the definitions of many of the words in the dictionary. Definitions of many of the more common, everyday words are based less on direct consultation, and more on observations of the language in use. Over the past 20 years, I have spent a combined total of a little more than a year with the Vurës community and am married to a speaker, Armstrong Malau, thus I have daily exposure to the language. Although not fluent, I am a competent speaker of the language. I am a fluent Bislama speaker.

The majority of words, mostly nouns, in a number of semantic domains was directly elicited rather than documented through textual use. Marine biologist Katherine Holmes was a member of the DOBES project team and was responsible for documenting most of the marine terms, including names of fish, shellfish, and other marine species, and some fishing terminology. She has provided scientific identification for many species documented in the dictionary, mostly through spending time with speakers engaged in fishing and reef gleaning. Holmes checked identifications with multiple speakers, and scientific names for marine species are only given where the name has been checked and she was confident that the identification is correct. She also took almost all the photos of marine species that are presented to illustrate these nouns. 
Plants were identified by two botanists who work with the Vanuatu Department of Forestry, Sam Chanel and Philemon Ala. In the early stages of my documentation of the language, in 2002, I asked Sam Chanel for identifications of approximately 100 plants, and he identified these from specimens that I took and delivered to him at the Vanuatu Herbarium. Later, in 2007, Philemon Ala visited Vanua Lava and made further identifications, working with several Vurës speakers. Again, I have tried to give the scientific identification only when I am relatively confident that the scientific name is correct. Some of the better-known species that are distributed throughout Vanuatu, particularly food-bearing trees and plants, I was able to identify on my own, and by consulting Wheatley et al. (1992). The names and descriptions of some varieties of coconuts and taro cultivars have been recorded based on the research of agronomist Sophie Caillon.

The scientific identifications provided for bird names should be taken as less accurate, and are based solely on identifications from pictures in Bregulla (1992) and from my own experience in Vanuatu. As the bird life of Vanuatu is not incredibly diverse, these identifications can still be considered to be reasonably accurate, as there are not many instances of similar species that might easily be confused when identifying through images.

Anthropologist Sabine Hess provided some assistance with the definitions of kin terms. Linguist Daniel Krausse carried out fieldwork on Vurës as part of his $\mathrm{PhD}$ research in 2018 and contributed a small number of lexical items that were not yet included in the database and also provided modifications for some definitions.

In terms of technical aspects of the production of this dictionary, the lexical data was entered and stored in a database, using the FieldWorks Language Explorer (FLEx) software. ${ }^{5}$ All data was entered by me, regardless of whether it was produced as part of the process of text annotation, or as direct entry of elicited lexical data. A configured dictionary, finderlists and the thesaurus were exported using the Pathway program. This is a program that is linked to the FLEx program, which enables styles to be applied consistently to the dictionary data that is stored in separate fields in the database, so that the output is a somewhat formatted dictionary. The output files were then further formatted in Microsoft Word to improve the aesthetics and make the final product more user-friendly. There are just over 3,500 headword entries in the dictionary.

In order to produce the thesaurus, I added the semantic category of individual words as a field within the FLEx database. This is an inbuilt part of the program, with a very detailed ready-made set of semantic domains. For the most part, the divisions into semantic domains that are specified within FLEx matched the categories that I wanted to distinguish for Vurës, but in some cases I adjusted

$5 \quad$ FieldWorks is a set of software tools that have been created by the organisation SIL for storing and anlaysing language data. See: software.sil.org/fieldworks/. 
the domains to suit. I also added some new categories that were not included, such as having a specific category for taros and coconuts, as these are such a pervasive part of Vurës culture.

Due to my collaborations with Katherine Holmes, Chanel Sam and Philemon Ala, and the input of Sophie Caillon, this dictionary is quite heavily weighted towards names for flora and fauna, with close to one third of the entries belonging to these semantic domains. The dictionary includes 431 names for various fauna, of which 295 are marine species, including 158 fish species and 50 shellfish species. There are also 62 bird species included. There are 608 entries for plant names, including 228 trees and palms, 109 taro varieties, and a range of other plant and animal species.

Any words that occurred in a text as part of the corpus have been included. The emphasis on flora and fauna was intentional to the extent that I actively sought collaboration with a marine biologist and botanists, knowing that engagement with the marine and bush environments are important sociocultural emphases for the community. Most omissions are unintentional, in that this dictionary is simply the result of my best efforts at documenting the words of Vurës with the time and resources that I have had available. I have chosen to publish the dictionary as it stands so that it can be used by the community now, but with the hope that it will be added to in future.

One category of omissions that some users might observe is swear words. The inclusion of such words is an issue that can often be contentious when compiling a dictionary, particularly for small language communities. I have included words referring to body parts and functions that might be thought of as 'rude' by some, but I have not included words that are solely used for swearing. There were differing opinions as to whether these words should be included or not, and I decided that it was appropriate to respect the more conservative members of the community.

Almost all of the images used to illustrate headwords are photographs that have been taken by members of the DOBES team, specifically for the Vurës documentation project. Copyright belongs to the photographer and this is indicated by a watermark on each photo. Researchers who contributed photos are Katherine Holmes, Catriona Malau, Armstrong Malau, Philemon Ala, Raymond Ammann, Stefan Schnell and Sabine Hess. The only photos included that were not taken as part of the DOBES project are those depicting bird species. Permission to include these to illustrate bird names was kindly given by the photographer, Garry Innes, whose name is also watermarked on the photos that he contributed. 


\section{Structure of the dictionary and entries}

There are four sections to the dictionary itself. First comes the main part, which lists Vurës words in alphabetical order and gives meanings in English and Bislama and other kinds of information. This is a translation dictionary, which means that the meanings of the Vurës words are given in both English and Bislama, but the definitions or meanings of words are not given in Vurës itself. The different types of information that are provided about each word and how to find that information in an entry are explained below in Section 5.2. After the main part of the dictionary, there are two finderlists, one from English to Vurës and the other from Bislama to Vurës. A finderlist lets the user look up a word in English or Bislama and find the equivalent word or words in Vurës. There is not much information in the finderlist, compared to in the main part of the dictionary, so when the user finds the word that they are looking for, they can then go to the main entry for that word if they want more information. There is a short introduction at the beginning of each finderlist, which covers some important points about how the finderlists are set out. Finally, the last section is a thesaurus. This is where words are grouped according to a meaning-based category. For example, there are lists of all of the fish names in the language, and names for different food crops, like taros, yams or bananas, and many other things. Further explanation of the structure of the thesaurus, and the different categories that have been included, is provided at the beginning of the thesaurus.

\subsection{Alphabetical ordering}

In Section 2.1 an explanation was given about the letters used in Vurës spelling. In terms of alphabetical order used in the dictionary, as Vurës speakers know how to write in English, Bislama or French and the letters are based on letters for those languages, it was decided that the Vurës letters should be put together to form an alphabet in an order similar to that which is used for English. I thought that this was the most sensible decision and asked the opinion of a range of Vurës people over the years, who have all expressed agreement with the proposed order. Therefore, where the letters are exactly the same, they occur in that order. The only letters that are different are still based on English letters, so they follow that basic letter in the Vurës alphabet. For example, $\mathbf{e}$ is followed by $\overline{\mathbf{e}}$ and then $\ddot{\mathbf{e}}$. The alphabetical order of Vurës is as follows:

\section{a b d e è ëgi k l m m n ñ oōö pqrst u vw}

Looking at this alphabet, some people might wonder why some of the letters of the English alphabet are missing; that is $\mathbf{c}, \mathbf{f}, \mathbf{h}, \mathbf{j}, \mathbf{x}, \mathbf{y}$ and $\mathbf{z}$. There are two reasons for this. Mostly the reason is that the sounds that these letters represent for English do not occur in the Vurës language. This is the case for $\mathbf{f}, \mathbf{h}, \mathbf{j}$ and $\mathbf{z}$. In other cases, this is due to the fact that the English spelling system is actually 
not very regular. For example, in English c can either represent the same sound as $\mathbf{k}$, or $\mathbf{c}$ can represent the same sound as $\mathbf{s}$. Vurës spelling simplifies spelling by consistently using either the letter $\mathbf{k}$ or $\mathbf{s}$, and not using $\mathbf{c}$ at all.

\subsection{Structure of the entries}

The entries in the dictionary vary in terms of how many different types of information are given about each word. At the very least, for each word, the part of speech is given, followed by the meaning in English, then the meaning in Bislama, as in the following:

butöliaw n.com. rainbow $\sim$ renbo

Other entries contain much more information. Here are five sample entries, to explain. Each part of each of these entries has a raised red number after it, and below there is an explanation for each number about what category of information that is. Note that different styles of typing fonts and different colours are used to try to distinguish one kind of information from the information next to it, in order to make it easier to find different types of information.

$\operatorname{ar}^{12}{ }^{2}$ (fr.var. waar) $)^{3}$ n.com. ${ }^{4}$ kind of tree ${ }^{6}$

$\sim$ wan kaen tri ${ }^{7}$ Premna corymbosa ${ }^{8}$

[Used for making bows. Ol i yusum blong mekem bonaro. $]^{13}$ [POc * qarop 'Premna spp.'] ${ }^{16}$

$\mathbf{l e n}_{1}^{1}{ }_{1}^{2}$ n.com. ${ }^{4} \mathbf{1} \cdot{ }^{5}$ wind $^{6} \sim$ win $^{7} \mathbf{O}$ leñ nē ge meneg, o len mö uv me, ni tek nē in̄kē, ni tek nē in̄kè. ${ }^{9}$ The wind was strong, the wind blew this way and it took it (bird) this way, it took it this way. ${ }^{10}$ Win $i$ strong, win i blu i kam, $i$ tekem hem (piin) olsem ia, i tekem hem olsem ia. ${ }^{11}(\mathrm{DHN})^{12} \mathbf{2} \cdot{ }^{5}$ air $^{6} \sim$ win $^{7}$ La masawre nē me le o leñ timiak in̄kē, ni rōñteg o bunë. ${ }^{9}$ When he took in the wind like that, he sensed a smell. ${ }^{10}$ Taem hem i tekem win olsem ia, hem i harem wan smel. ${ }^{11}(\mathrm{RTF}){ }^{12}\left[\mathrm{POC}\right.$ *lanit 'sky'] ${ }^{16}$

len̄limerlav ${ }^{18} \quad$ (comp. $)^{19} \quad$ n.com. $^{4}$ southeast tradewind $^{6} \sim$ tokelau, saotis win we $\mathrm{i}$ kam long Mere Lava ${ }^{7}$ \{Lit. wind from Mere Lava win i kam long Mere Lava\} ${ }^{15}$

$\mathbf{l o}^{1}{ }_{1}^{2}$ n.loc. ${ }^{4}$ seashore, seaside, area by sea, but not including sea itself $^{6}$ solwota, eria klosap long solwota ${ }^{7}$ See: naw ${ }_{1}^{14}\left[\right.$ POC *laur 'sea, seawards'] ${ }^{16}$ $\mathbf{l o}_{2}{ }_{2}^{2}$ art. ${ }^{4}$ locative preposition, to, at, on, marking common nouns ${ }^{6} \sim$ long $^{7}$ No mēèl nēk lo sto. ${ }^{9}$ I sawyou at the shop. ${ }^{10}$ Mi lukim yu long stoa.11 (Allomorphs: la, le, lē, lë, li, lō, lö) ${ }^{17}$ See: $\mathrm{a}_{1}^{14}$

Entries within the dictionary are organised as follows:

1. Headword: The Vurës word is the first part of any entry in the dictionary. The Vurës headword is presented the way that it is most commonly pronounced. Note that in Vurës, words mostly don't change their shape (i.e. there is little allomorphy). A common exception is with nouns that take endings that show who owns the thing expressed by the noun (possessive suffixes). See the discussion of nouns in 5.3 for more explanation. 
2. Homonym number: Sometimes there are two words in a language that sound exactly the same, and therefore are spelt the same, but have completely different meanings. These are called homonyms. If words are homonyms, they are listed separately, with their separate meanings, as they are different words. For example, Vurës lo is both an absolute location noun meaning 'seashore, seaside' and an article that is used before common nouns. These are totally different words and it is just a coincidence that they sound the same. In cases like these, the headword is followed by a number, indicating which homonym entry it is. This is particularly important for when a different entry makes a reference to the entry for a word that is a homonym.

3. Variant: For some words, there is more than one way in which the word can be pronounced, and therefore spelt. This can be for different reasons. Types of variants are explained in Section 5.4. For all words that have variants, the headword that is given is the most common or easily identifiable for speakers of the language, and the variant is in brackets after the headword. Within the brackets is a label for the type of variant, and then the word itself, like this: (fr.var. waar).

4. Part of speech: After the Vurës word is an abbreviation label in small font for the part of speech of the word. That is, whether it is a noun, a verb or some other type of word. Some words belong to more than one category. The part of speech is given in italics: n.com. There is a full list of the abbreviations used, with some further explanation, in Section 5.3.

5. Sense number: Some words have more than one meaning or slight variations in their meaning. We call these different meanings the senses of the word. For example, bē can refer to fresh water or to a river. These meanings are obviously related to each other, but they are not the same, so they need to be listed separately under the entry, with distinct meanings and other information. The start of a sense is indicated with a number, like this: $2 \cdot$. Words can have up to five senses.

6. English meaning: The English meaning is given next. In some cases this can be just one word, but other Vurës words require a much longer English definition.

7. Bislama meaning: The Bislama translation or meaning comes after the English. It is separated by and is in a different colour to make it easy to distinguish the Bislama definition from the English.

8. Scientific name: For many plants, animals, fish and birds, a scientific name has been given. See Section 4 for more on this.

9. Example sentence: An example sentence in Vurës has been included for as many words as possible, to help illustrate the meaning and use of the word. This is not necessary for things like names of plants and fishes, which usually have other types of information like a description of their appearance, or a photo.

10. English translation: An English translation of the example sentence follows the Vurës example. 
11. Bislama translation: A Bislama translation of the example sentence then follows the English translation.

12. Reference: Where an example sentence has been provided, in some cases it is followed by a reference abbreviation in brackets: (DDL.006). If a reference is given, the example sentence was taken from a recording of a Vurës speaker. The reference is a label for the particular recording. The recordings and where they can be found on the internet are listed in the Appendix at the back of the dictionary, so anyone interested can listen to these examples.

13. Encyclopedic information: For some entries, additional encyclopedic information has been provided in square brackets like this: [English. Bislama.]. This information is not an essential part of the meaning of the word, but it aids in understanding the meaning and use of the word.

14. Lexical relations: In some cases there are two words that have the same or very similar meanings. These are called synonyms and are indicated with the label Syn. For example, Syn: gösuv occurs as part of the entry for as. For some words, there is a word that is almost exactly the opposite in meaning. These are called antonyms and are indicated with the label Ant. For example, Ant: mamas occurs as part of the entry for döw. Other words are related in meaning in some way, which is worth drawing to the attention of users of the dictionary. For example, See: naw occurs as part of the entry for lo 'seaside', because the two words have similar meanings and the range of use of the words lo and naw is a bit different to the way the words 'sea' and 'seaside' are used in English, and 'solwota' in Bislama.

15. Literal meaning: For some words, especially compounds formed from two or more other words, the meaning of the compound is not obvious from the words that make up the compound. In cases like these it is of interest to know what the individual words mean. For example, the expression gen mōvōt means 'close one's eyes', but this is an idiom formed from the words meaning 'eat' and 'sleep'. Literal meanings are indicated towards the end of the entry, like this: \{Lit. eat sleep kakae slip\}.

16. Etymology: Two different but related types of information are listed in square brackets at the very end of a small number of entries. This information is about the historical origin of the word. More detail about this is given in Section 6. The etymological information is presented in square brackets like this: [POC *qarop 'Premna spp.'] with a label indicating the source language.

17. Allomorphs: As mentioned at note 1, there is little allomorphy in Vurës. A significant exception is with some proclitics and prefixes that are important to the grammar of the language. The vowels in these forms match the vowel of the word they occur before. So one says in Vurës, for example, lo sto 'at the shop', but le ev 'in the fire' and lö gövur 'at the house'. See Malau (2016: 44-47) for a detailed explanation of this vowel variation. 
18. Subentries: Some entries include a subentry. A subentry is a complex form that contains the main entry headword. Subentries are indented after the main entry and can themselves include all the same information as a main entry.

19. Complex form type: For subentries, the subentry headword is always made up of more than a single morpheme - word or unit of meaning - like a compound or a reduplicated form. This is indicated with an abbreviation label in brackets after the headword, like this: (comp.). There are six different types of complex forms and explanations and abbreviations for these are given in Section 5.4.

Some of the entries have pictures to help illustrate the meaning. The picture is always located at the end, directly below the entry that it relates to.

\subsection{Part of speech labels used}

The part of speech or word class labels used in the dictionary reflect the analysis of word classes presented in Malau (2016). The abbreviations used for each word class and subclass are presented in Table 2. Readers who would like to know more about the properties of particular word classes are directed to the main section of the grammar in which that word class is discussed. The relevant page numbers are provided in the last column of Table 2. A few points that are particularly relevant are discussed briefly here.

Table 2: Parts of speech in Vurës

\begin{tabular}{|l|l|l|}
\hline Abbrev. & Part of speech label & Reference \\
\hline adj. & Adjective & $117-120$ \\
\hline adv. & Adverb & $122-124$ \\
\hline art. & Article & $230-241$ \\
\hline cl. & Relational classifier & $290-308$ \\
\hline conj. & Conjunction & $700-728$ \\
\hline dem. & Demonstrative & $423-460$ \\
\hline dir. & Directional particle & 132 \\
\hline inter. & Interjection & 138 \\
\hline loc. & Locational & 131 \\
\hline loc.adj. & Locational adjunct & 131 \\
\hline mod. & General modifier & 124 \\
\hline n.com. & Non-suffixing common noun & $107-108$ \\
\hline n.com.suff. & Suffixing common noun & $108-109$ \\
\hline n.loc. & Absolute location noun & $105-106$ \\
\hline n.p.bound & Bound proper noun & $104-105$ \\
\hline n.p.fr. & Free proper noun & $103-104$ \\
\hline & & \\
\hline
\end{tabular}


A DICTIONARY OF VURËS, VANUATU

\begin{tabular}{|l|l|l|}
\hline Abbrev. & Part of speech label & Reference \\
\hline num. & Numeral & $124-127$ \\
\hline part. & Particle & 134 \\
\hline pre. & Prefix & 135 \\
\hline prep. & Preposition & $313-370$ \\
\hline pron. & Pronoun & $127-128$ \\
\hline quant. & Quantifier & $120-121$ \\
\hline subord. & Subordinator & $136-137$ \\
\hline suff. & Suffix & 128,135 \\
\hline temp. & Temporal & $121-122$ \\
\hline v. & Ambitransitive verb & $116-117$ \\
\hline vi. & Intransitive verb & $110-114$ \\
\hline vt. & Transitive verb & $114-116$ \\
\hline v.part. & Verbal particle & $134-135$ \\
\hline
\end{tabular}

In Vurës there is a considerable degree of word class flexibility (Malau 2016: 139-145). This means that, as in English, there are many words that can be used as different parts of speech, in particular both as a noun and as a verb. For words like this, the meanings of the word as different parts of speech are presented as different senses of the word. The following entry for tañsar provides an example of how this looks in the dictionary:

tan̄sar $\mathbf{1} \cdot n . c o m$. person $\sim \operatorname{man} \mathbf{2} \cdot v i$. become a person, feel like you have become human again $\sim$ kam man

Following on from this example, note that if all senses of a word belong to the same part of speech category, the part of speech is indicated directly after the headword, before the sense numbers and their associated definitions. If, as with tañsar, the parts of speech are different, then it is indicated after the sense number for each sense.

As stated, this word class flexibility occurs particularly with words that can be both nouns and verbs. To a lesser extent, there is also some overlap for words in other categories. For example, very much like in English, timiak 'like' is a preposition, a transitive verb, a subordinator and a discourse particle. Again, this will be indicated through the different senses.

There are five noun subclasses in Vurës. It is important when speaking Vurës to know which subclass a noun belongs to for two main reasons. One is that the subclass of noun determines which article the noun will occur with. The other reason is that some nouns - bound proper nouns and suffixing common nouns - have a suffix attached to indicate the possessor of the item referred to by the noun. When it comes to flexibility, some nouns can occur as a member of more than one subclass. A helpful example to illustrate both the different classes and subclasses, and also the flexibility is with the words that mean 'dad, 
father'. Mam 'Dad', when used as an address term or to refer to one's own father is a free proper noun, and as such can be preceded by the personal article $i$. When used to refer to a member of the generic class 'father, dad', mam is a nonsuffixing common noun and will be preceded by the common article $o$. The word mam also occurs as a transitive verb meaning 'to call someone father'. In order to say whose father someone is, you need to use a different word, which is a bound proper noun and takes a suffix to show the possessor. So tëmëk means 'my father', taman is 'her/his father' and temen $i$ Kali means 'Kali's father'. These examples show how the nouns show variation in the vowels of the root, depending on the possessor. The rules about how the vowels change are quite complicated and readers who wish to know more detail should refer to Malau (2016: 276-281). Due to this complexity, for each bound proper noun, the form listed in the dictionary is that which occurs when the possessor is a noun. Then, to illustrate the allomorphy (the variation in the vowels within the word), first person singular ('my') and third person singular ('her/his/its') possessed forms are presented as subentries. Suffixing common nouns behave in the same way, so they are also presented in this way.

The main division for the class of verbs in Vurës is into intransitive, transitive and ambitransitive subclasses. Intransitive verbs are labelled in the dictionary as $v i$. and transitive verbs as $v t$. For ambitransitive verbs, if the meaning difference between the intransitive and transitive uses of the verb is significant, then these are listed as separate senses of the word, with the meaning and label given for each sense. If, however, there is no significant meaning and distribution differences for a verb that can be used both intransitively and transitively, then the part of speech label given is simply $v$. For example, garmöo means 'spit' as an intransitive verb and 'spit on' as a transitive verb. It is thus just marked as $v$. with the definition 'spit, spit on'.

There are a number of entries for which the occurrence of the verb is limited in the corpus, and thus it has not been possible to determine conclusively the range of transitivity for the verb. In these cases, the word class is also indicated simply as $v$.

\subsection{Variants, variation and complex forms}

In Vurës, there is almost no dialectal variation. It is less than two hours walk between each of the villages within the original Vurës area and people from these villages are in regular contact. Instead, most variation that occurs is free variation; that is, alternate forms can be used freely by speakers. This is particularly the case with the pronunciation of some vowels, especially the two vowels $\ddot{\mathbf{e}}$ and $\overline{\mathbf{e}}$. There are some words that contain the vowel $\ddot{\mathbf{e}}$, for which some speakers, particularly younger people, replace the ë with e. For example, dëm 'yam' is pronounced as dem by most people today. Older speakers note that the original pronunciation was dëm. Similar variation occurs with the vowel $\overline{\mathbf{e}}$, which some speakers pronounce as $\mathbf{i}$ in some words. For example, the first person plural inclusive pronoun, 'we, us' is nēn, but is sometimes 
pronounced as nin. In the dictionary, the pronunciation that has been observed most commonly is given as the headword, with the other pronunciation given afterwards in brackets: (fr.var. dëm).

There are two other types of variants that are identified in brackets after the headword. One is common abbreviated forms of a word, such as timiak 'like', which is frequently reduced, and pronounced as tiak and so this is given as a variant after the headword preceded by the label abbrev. The other variant type indicated is for a small number of words for which the root of the word does not occur very often on its own, but is generally fused with a word that comes before it, which is usually a separate word. For example, the article that goes before place names, $\mathbf{a}$ is usually a separate word and would be written as such. There are a few exceptions to this, such as the word for 'where', for which a joins with vē to form a single word avē. As speakers think of avē 'where' as the word for 'where', with the a attached, it makes more sense to list this as the headword. The root, which is generally not thought of by speakers as occurring on its own, is listed after as a variant: (root vē).

There are several different types of complex word forms that are listed as subentries in the dictionary. By complex form, I mean a word that is made up of more than one word or a word with another morpheme attached. This means, a single word with two different elements contributing to the meaning. For each subentry, the specific type of complex form is indicated with an abbreviation in brackets after the headword. The different types are explained here:

- Compound (comp.): A compound is a word that is formed from two or more other words put together. In Vurës these words are mostly nouns that are formed from one noun plus another noun or verb following it. Compounds are quite common as a way of making new nouns for different plants and animals that are related or similar. For example, under mōto 'coconut' in the dictionary are many different types of mōtō. Most of the names of different types of coconut are compounds, with the name being the word mōtō followed by another word, such as mōtō dēdērēs, which is the name of a type of coconut that has sweet flesh, with dēdērēs meaning 'sweet'. Compounds can be made up of more than one other word. For example, wotag dere timiat is a kind of nut that has dark red skin, and so it is named for the dere 'blood' of the timiat 'devil'.

- Possessed noun (n.poss): When some nouns are indicated for their possessor, or owner, especially parts of the body and names for family, the form of the root of the word changes. For example, the word for 'father' is teme, but 'my father' is tëmëk and 'his father' is taman. For each noun that changes its form in this way, the root noun is listed as a headword, and the forms for 'my X' and 'her/his/its X' are listed as subentries to show the variation.

- Reduplicated form (redup.): Reduplication, the repetition of a word or part of a word to form a new word, is a very common process in Vurës. In some cases, the reduplication is a regular process, and adds to, but does not really change, the meaning, such as indicating that an action continues for 
an extended period of time. (See Malau 2016: 172-197 for details.) In other cases, reduplication can form a new word that has a meaning that is not entirely predictable from its parts, and belongs to a different part of speech category. For example, the verb gen 'eat', when reduplicated, can be a noun, gengen 'food'. As this meaning is not predictable - and this is particularly the case when verbs are reduplicated to form nouns - then the form gengen and others like it must be listed separately in the dictionary. They are listed with (redup.) following the headword.

- Serial verb construction (SVC): Serial verb constructions, when two or more verbs are used in combination, are common in Vurës. (See Malau 2016: 557620 for details.) In some cases, the meaning resulting from the combination of verbs is not predictable. In these cases, the serial verb construction is included as an entry in the dictionary, followed by (SVC).

- Derived form: A headword is listed as (der.) if it has been formed by adding a derivational prefix or suffix to an original word to form a new word. For example, there is a prefix, w0-, that is used to form new nouns, from other verbs, nouns, or combinations of nouns and verbs. The meaning and form of the words produced using the prefix is not predictable. An example of this is the noun wōtōtōw 'measuring stick', which is derived from the verb tōw 'to measure' reduplicated with the w0- prefix attached.

- Idiom (id.): An idiom is an expression, formed from a combination of words, the meaning of which is not at all predictable from the meaning of the individual words. An example is, qaq tala lam, which means 'Bislama'. The literal meaning of this expression is 'the language (qaq) of the deep sea (lam)'.

\section{Word origins}

The Vurës language belongs to the grouping called Oceanic within the Austronesian language family. Oceanic languages include most of the indigenous languages spoken in the Pacific region nations. They all share a common ancestor language, which means that they have a common source (Lynch et al. 2002). The ancestor of all Oceanic languages is known as Proto Oceanic. Proto Oceanic can be thought of as the great-great-granddaughter of Proto Austronesian, which sits at the top of the family tree. The languages of the islands of Torba are all very closely related to each other, so they can be thought of as sisters, whereas languages of central and southern Vanuatu are like cousins to Vurës, and Polynesian languages are more distant cousins. Most of the words in Vurës, and thus most of the words in this dictionary, are indigenous to the language and this means that they have been inherited from Proto Oceanic. The group of Oceanic languages, which has about 450 members, has been well studied, as has the history of the development of the languages from Proto Oceanic. As such, we can often tell from the form of a Vurës word, comparing it to words in other Oceanic languages, roughly what that word must have sounded like in Proto Oceanic. It is useful for those who study relationships 
between languages, to know which words can be traced back to the ancestor language. Some reconstructions are thus included in the dictionary, where they are known. These occur at the end of an entry in square brackets, with the format $\left[\mathrm{POC}{ }^{*}\right.$ word 'meaning'], POc being the abbreviation for Proto Oceanic. All of the Proto Oceanic reconstructions presented come from four volumes on The Lexicon of Proto Oceanic (Ross, Pawley and Osmond 1998, 2003, 2008, 2011).

In the majority of cases where I have provided a reconstruction of a word, this is for the word in Proto Oceanic. In some cases, a confident reconstruction for Proto Oceanic has not been produced, but it has been possible to trace back to a more immediate ancestor, Proto North Central Vanuatu. These reconstructions are from Clark (2009) and are preceded by the label PNCV.

The national language of Vanuatu, Bislama, is a creolised pidgin language most of whose words have come from English. All speakers of Vurës are also fluent speakers of Bislama and use it quite regularly to communicate with outsiders - people who are from Vanuatu but do not know Vurës. As a result of regular contact and use, today some of the words that are used in Vurës are not words that have been handed down into the language from the ancestor language, but which instead have come from Bislama. In some cases, a Bislama word might have almost completely replaced the original Vurës word, such that almost no one today uses the original word. An example of that is taosen replacing tar 'thousand'. The word tar is still known by many, but rarely used. Alternatively, a word might be used quite regularly, although the original word is still known and used by most people. An example of such is wok, which is used by many in place of wiwieg 'work'. In other cases, the word derived from Bislama is the only word used, because it refers to an item or concept that has recently been introduced, since Europeans settled in Vanuatu. These are words like trak to refer to cars and other vehicles, derived from English 'truck'. Words like these, although not of Oceanic origin, are used on a daily basis when speaking Vurës and have been included in the dictionary. Some speakers of the language may not approve of this, because they feel that it is not 'real' language, but nevertheless as they are well incorporated into the language, it is an accurate reflection to include them. Words that have come into the language from Bislama are indicated with [Bis Bislama word 'English meaning'] at the end of the entry. If the word borrowed from Bislama has the same meaning as a word that already exists in Vurës, then this is indicated with See: word [Bis].

\section{References}

Bregulla, Heinrich L. 1992. Birds of Vanuatu. Shropshire, England: Anthony Nelson.

Clark, Ross. 2009. *Leo Tuai: A Comparative Lexical Study of North and Central Vanuatu Languages. Pacific Linguistics 603. Canberra: The Australian National University.

Crowley, Terry. 2003. A New Bislama Dictionary. (2nd Edition) Vanuatu: Institute of Pacific Studies, University of the South Pacific. 
Crowley, Terry. 2004. Bislama Reference Grammar. Oceanic Linguistics Special Publication No. 31. Honolulu: University of Hawai'i Press.

François, Alexandre, Michael Franjieh, Sébastien Lacrampe, and Stefan Schnell. 2015. The exceptional linguistic density of Vanuatu: Introduction to the volume. In Alexandre François, Sébastien Lacrampe, Michael Franjieh, Stefan Schnell. The Languages of Vanuatu: Unity and Diversity, 5, Asia-Pacific Linguistics Open Access, pp.1-21, 2015, Studies in the Languages of Island Melanesia.

Lynch, John. 2000. Linguistic subgrouping in Vanuatu and New Caledonia. In Proceedings of the Second International Conference on Oceanic Linguistics (SICOL), vol. 2 Historical and descriptive studies, ed. by Bill Palmer and Paul Geraghty, 155-184 Canberra: Pacific Linguistics.

Lynch, John, Malcolm Ross, and Terry Crowley. 2002. The Oceanic Languages. Richmond, Surrey: Curzon.

Malau, Catriona. 2016. A Grammar of Vurës, Vanuatu. Berlin, Boston: De Gruyter Mouton.

Ministry of Education. 2012. Vanuatu National Language Policy. Republic of Vanuatu. moet.gov.vu/docs/policies/Vanuatu $\% 20$ National $\% 20$ Language $\% 20$ Policy $\% 20$ (English)_2012.pdf

Ross, Malcolm, Andrew Pawley, and Meredith Osmond (eds). 1998. The Lexicon of Proto Oceanic. Volume 1: Material culture. Pacific Linguistics C-152. Canberra: The Australian National University.

Ross, Malcolm, Andrew Pawley, and Meredith Osmond (eds). 2003. The Lexicon of Proto Oceanic. Volume 2: The physical environment. Pacific Linguistics 545. Canberra: The Australian National University.

Ross, Malcolm, Andrew Pawley, and Meredith Osmond (eds). 2008. The Lexicon of Proto Oceanic. Volume 3: Plants. Pacific Linguistics 599. Canberra: The Australian National University.

Ross, Malcolm, Andrew Pawley, and Meredith Osmond (eds). 2011. The Lexicon of Proto Oceanic. Volume 4: Animals. Pacific Linguistics 621. Canberra: The Australian National University.

Stebbins, Tonya N. 2012. Mali (Baining) Dictionary. Studies in the Languages of Island Melanesia. Canberra: Asia-Pacific Linguistics.

Wheatley, J.I., M. Tebbs, S. Seoule, and Sheila Gowers. 1992. A Guide to the Common Trees of Vanuatu: With Lists of Their Traditional Uses \& Ni-Vanuatu Names. Port Vila, Vanuatu: Department of Forestry. 


\section{Abbreviations used in the dictionary}

\section{Grammatical categories}

adj.
adv.
art.
cl.
conj.
dem.
dir.
disc.
inter.
loc.
loc.adj.
mod.
n.loc.
n.p.bound.
n.com.
n.p.fr.
n.com.suff.

num.

part.

pre.

prep.

pron.

quant.

subord.

suff.

temp.

v.

$v i$.

$v t$.

v.part.
Adjective

Adverb

Article

Relational classifier

Conjunction

Demonstrative

Directional particle

Discourse particle

Interjection

Locational

Locational adjunct

General modifier

Absolute location noun

Bound proper noun

Common noun

Free proper noun

Suffixing common noun

Numeral

Particle

Prefix

Preposition

Pronoun

Quantifier

Subordinator

Suffix

Temporal

Verb

Intransitive verb

Transitive verb

Verbal particle

\section{Other abbreviations}

abbrev.

Ant

Bis

comp.

der.

dl.

Eng

excl.

fr.var.

Gen

id.

incl.

Lit.

n.poss

pl.

PNCV

POC

redup.

Spec

SVC

Syn
Abbreviation

Antonym

Bislama

Compound

Derived form

Dual

English

Exclusive

Free variant

Generic

Idiom

Inclusive

Literally

Possessed noun

Plural

Proto North-

Central Vanuatu

Proto Oceanic

Reduplicated form

Specific

Serial verb construction

Synonym 


\section{Vurës - English - Bislama Dictionary}

\begin{tabular}{|c|c|}
\hline \multicolumn{2}{|c|}{$\mathbf{A} \sim \mathbf{a}$} \\
\hline 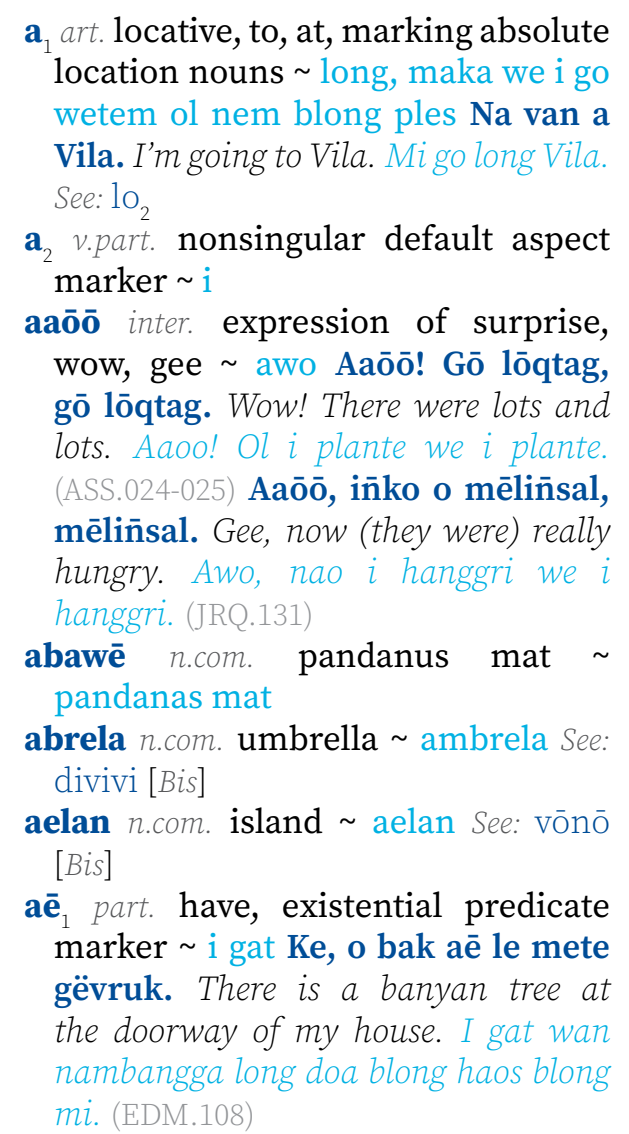 & $\begin{array}{l}\text { aēe part. anaphoric marker, referring } \\
\text { to previously mentioned place or } \\
\text { topic; there, about (it) long hem, } \\
\text { long ples ia (wan samting o ples } \\
\text { we i bin tokbaot finis) Nē mē gil o } \\
\text { qäörö, gil gil giigil, ni van, ni taq } \\
\text { lin̄ereg aē. He dug a hole, dug and } \\
\text { dug, and he went and hid in it. Hem } \\
\text { i digim hol, digim, digim, i go i haed } \\
\text { long hem. (AGG.019) Qēt kemem a } \\
\text { dēn me o masawre nitiwial, kemem } \\
\text { a gengen aē. Then we arrived at a } \\
\text { place and we ate there. Finis mifala } \\
\text { i kam long wan ples, mifala i kakae } \\
\text { long ples ia. (AAD.273) Kemek döl mö } \\
\text { vugvug aē. We all had a meeting about } \\
\text { it. Mifala i miting long hem. (CAA.061) } \\
\text { [POc * (q)i-ai 'previously mentioned } \\
\text { place; 'there, thereat"] } \\
\text { ak n.com. canoe, boat, ship kenu, sip } \\
\text { Si kēmi gö mörös o ak talo kopra } \\
\text { na mögun kēmi, nēk i wöl. If you } \\
\text { all want to own a copra boat, you buy } \\
\text { (one). Sapos yufala i wantem wan sip } \\
\text { blong kopra blong yufala, yu pem. } \\
\text { (EDM.160) See: bakalav } \text { [POc *waga } \\
\text { 'canoe (generic)'] }\end{array}$ \\
\hline
\end{tabular}


ak gavgav (comp.) n.com. plane plen \{Lit. flying canoe kenu we i stap flae\}

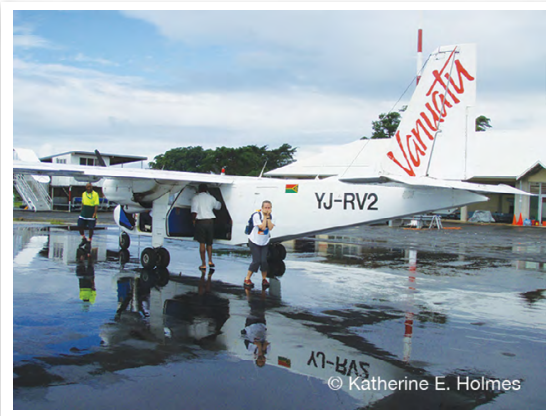

ak gavgav plane

ak geben (comp.) n.com. sailing canoe $\sim$ sel kenu

ak sōsō (comp.) n.com. canoe kenu E rōrō a siōl sar me, rēv kal sar o ak sōsō. Then the two of them floated ashore and pulled ashore the canoe. Ale tufala i flot $i$ kam antap, pulum i kam antap kenu. (ATB.048) Syn: wēlwēl

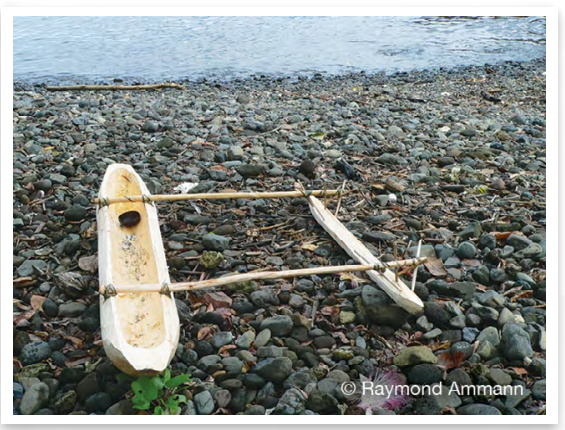

ak sōsō canoe

ake inter. ow! expression used to indicate that one is hurt awo! toktok we i talemaot se yu kasem kil Akē, akē, akē! Gë mëmës, gë mëmës! Ow, ow, ow! It really hurts! Awo, awo, awo! I so we i so! (KMTM)

akrikalja n.com. variety of taro, 'male', which occurs in a variety of colours and shapes $~$ kaen man taro we $\mathrm{i}$ gat defren saes mo sep blong hem [Bis agrikalja 'agriculture'] ale $\overline{\mathbf{e}}_{1}$ conj. so, then, conjunction used to link coordinate clauses ale Mem sur na mōgōñ o tere savsav, alē van me, dōrōk a van sar la gamelik. Put down your things, then come, and we'll go up to my men's house. Putum ol ting blong yu, ale kam, yumitu go antap long nakamal blong mi. (BQD) See: e [Bis]

ale $\bar{e}_{2}$ inter. ok, alright $\sim$ ale See: söwlē [Bis]

a⿳亠⿴囗十 (root $\overline{\mathrm{m}} \mathrm{)}$ ) n.loc. $\mathbf{1}$ ・ before, time before now bifo, fastaem $\mathbf{B a}$ a⿳亠̄ō min nēn a Vōnō Lav, o tō odiañ aē. But before with us on Vanua Lava there were no chickens. Be bifo wetem mifala long Vanua Lava i nogat faol. (RTF.065) 2 • first, in front $\sim$ fastaem Kōmōrōk a var kal siag li tōw, no a⿳亠̄⿲ō, nē töwur. The two of us walked up the hill, me in front and him behind. Mitufala $i$ wokbaot $i$ go antap long hil, mifastaem, hem bihaen. (ASS.002)

a⿳亠̄̄o den subord. before, introduces temporal adverbial clause $\sim$ bifo long $\mathbf{O}$ bē, nēk i liaq vagvet a⿳亠̄̄o den o qiat ni mian. The water, you irrigate four times before the taro ripens. Wota, yu putum wota fo taem bifo taro hem i raep. (RPT.014)

an $v i$. be uncooked, not properly cooked $\sim$ no dan gud

anm̄eg vt. bother, disturb spolem Mōlio ni sōsō ti, ni sō sōsō, rōrō a sōsōsō, wo sar e i timiat mō rōw lō me, ni anmèeg o tō. Mōlio paddled, he paddled and paddled, the two of them paddled, and on the shore the devil came along and disturbed the chickens. Mōtio i parel, i parel go go, tufala i parel, parel, mo antap ia, devil i kamtru, hem i spolem faol. (DMM.038)

añan̄ 1 $v i$. yellow, be yellow yelo Dödöu ga añan̄ ine, o gē ine na sian o wistaban. That one with yellow leaves, that kava is called wistaban. Lif i yelo ia, kava ia, nem blong hem wistaban. 
(OSK.039) 2 • n.com. yellowness, yellow (one) yelowan $[P O C$ *yayoyajo 'yellow']

aqa inter. expression of surprise, disbelief, horror; gee, gosh awo, toktok blong singaot blong talem se yu sapraes long wan samting Qēt no wo, 'Aqa, in̄ko timiak avē Rëwël?' Then I said, 'Gee, now what are we going to do, brother-in-law?' Afta mi se, 'Awo, nao ia olsem wanem, tawian?' (ASS.010) aqit (root qit) n.loc. far longwe, farawe So nēk aqit ren vēti e, nēk i rōw. When you are still far away, you jump. Taem yu stap longwe yet, yu jam. (ADT.041) Syn: arës

$\mathbf{a r}_{1}$ (fr.var. waar) n.com. kind of tree $\sim$ wan kaen tri Premna corymbosa [Used for making bows. Ol i yusum blong mekem bonaro.] [POc *qarop 'Premna spp.']

ar $\mathbf{o l}$ (comp.) n.com. kind of ar tree which grows in the bush $\sim$ wan kaen ar tri blong bus

ar tur (comp.) n.com. kind of ar tree which grows near the sea and is strong and does not bend wan kaen ar tri we i stap long solwota mo i strong mo i no save benben

$\mathbf{a r}_{2}$ n.com. fenced in area, pen, compound, enclosure fenis Nēk ma tatag ere buluk ine van van van van vavavan, nēk mē tēqēl sōw le qereñ bē bilēñ nitiwial. You follow the cattle fence on and on and on until you go down to another creek. Iu folem fenis blong buluk ia go go go go go, yu go daon long wan wota bakegen. (RRS.031) Ni van la ar, ni lēg o tere qō ine, ni van me ni lēg lē wērēntenge. He went to the enclosure, he tied up the pigs, and he came and tied them to the posts. Hem igo long fenis, ifasem ol pig ia, i kam i fasem long wud. (ESP.098) [POc * $q a R a(r)$ 'fence'] $\mathbf{a r}_{3} v t$. bore, have enough of, tire of les long No me geegen o vetel, ma ar no. I have eaten bananas so much and I am tired of it. Mikakae banana go go, miles long hem. Syn: dir

arës (root rës ${ }_{2}$ ) n.loc. distant, far, far away longwe, farawe Nèr ga vanvan arës. They go far away. Ol $i$ stap go farawe. (ESP) Syn: aqit

as vt. $\mathbf{1} \cdot$ rub $\sim$ rabem Jessica nē ni as na matan, o dödömia taē vita nē gö mörös mōvōt. When Jessica rubs her eyes it means that she wants to go to sleep. Taem Jessica i rabem ae blong hem, mining blong hem se hem i wantem slip. Nēk i le me o tenge, nēk i as, nēk i vun la panken, e ni sum. Bring the medicine leaf, rub it, squeeze it into a cup, then she'll drink it. Karem lif meresin i kam, yu rabem, skwisim $i$ go long kap, ale bae hem $i$ dring. 2 - wash, scrub (plates) wasem Lö lölö gövur, na wur o lölö gövur, na as o plet. Inside the house, I sweep inside the house and I wash the plates. Insaed long haos, mi brum insaed long haos, mi wasem plet. (DDL.006) Syn: gösuv $[P O c$ *asa(q) 'grate, sharpen by grating or rubbing']

asaw $a d v$. separate, apart $\sim$ stap hem wan Nē go tog asaw den o tan̄sar, nē go tog asaw den o men, wo nē go tog asaw den o animol. It (flying fox) is apart from people, it is apart from birds, and it is apart from animals. Hem (flaengfokis) i stap defren long ol man, hem i stap defren long ol pijin, mo hem i stap defren long ol animol. (DFF.062) Mem asaw, den nana qal ni mës. Put it separate, in case I touch it and it falls. Putum i stap hem wan, nogud mi kasem i foldaon.

at $v$. tie thatch slabs onto bamboo rafters fasem lif natanggura i go antap long bambu Nēn mo so o doot, nēn a tek me, a vidir, a vēn, qēt, in̄ko nēn a at. We remove the sago 
palm leaves, we bring them, we remove the midribs, we sew them and then we tie the thatch to the rafters. Yumi tekem natanggura, yumi karem i kam, karemaot bun, somap, finis, nao ia yumifasem i go long bambu. (RBH.016) at rēv (comp.) v. tie sago roof thatching loosely, not close together $\sim$ fasem lif natanggura we i no fasfas tumas

at tit (comp.) v. tie sago roof thatching with lots of leaves close together fasem lif natanggura we i fasfas gud wetem plante lif klosap

atale $a d v$. all over the place $\sim$ olbaot $\mathbf{R i}$ kere vanvan avē, ba ri togtog gem le mete gëvrun nēn ine, ri oror atalē. Don't go anywhere, but just stay by the door to our house and play around. Yufala i no go samples, be stap nomo long doa blong haos blong yumi, yufala i pleplei olbaot. (OQQ.015) Nē gata vanvan atalē, nē gē sigsiag gem. She didn't go all about, she just sat down. Hem i no stap go olbaot, hem i sidaon nomo. (JRQ.007)

atan n.com.suff. her/his/its soul sol blong hem See: ete

atelen̄ vi. face upwards, for items that have division into top side and underside, such as person lying, bowl, plate, cup fes o luk i go antap, olsem man i leidaon, kap o plet Ōn rōw ateleñ. Lie down facing upwards (i.e. on your back). Leidaon fes igo antap. Ant: vateqev

atik adj. small, be small smol Darag, nēk i èl na wian, na wian, la masawre nē atik, nē o wewese. The wild nutmeg, you see its fruit, when it is small, it is round. Nandae, yu luk frut blong hem, frutblong hem, taem i smol, hem i raon. Syn: nötu, 1 [POc *qitik 'small'] atiktik (redup.) n.com. small smolsmol Wōl nē atiktik e, nē mita van le. Because he is small, he won't be able to go. From hem i smolia, bae hem ino save go. (GMM.074)

atina n.com. cassava variety $\sim$ kaen maniok

atm̄ēn n.com. 1 • man man Qön̄ nitiwial i rege min ginon, atm̄ēn wo reqe, rōrō me leg. One day there was a couple, a man and a woman, the two of them were married. Wan dei $i$ gat man wetem woman blong hem, man mo woman, tufala $i$ mared. (HWM.005) 2 • male man I nōtōn ine o atm̄ēn, ma qal wot o atmēn. Her child was a boy, the first-born was a boy. Pikinini blong hem ia boe, fasbon hem i boe. (ESS.014) Nēk gö mörös o tō atm̄ēn si o tō reqe? Do you want a rooster or a hen? Yu wantem man faol o woman faol? [POc *qata-m(w)aqane 'man, male'] auw inter. gee! awo!

av $v$. make mistake, be wrong, incorrect mestem Oo mamarseg, na lölön kōmōrōk mō qōr, e no ma qaq av vita nēk ma van a Wētika. Oh sorry, the two of us forgot, so I made a mistake and said that you had gone to Wètika. O sore, mitufala i foget, ale mi talem rong se yu bin go long Wëtika. (AWW.041)

avē $\left(\right.$ root $\left.\mathrm{ve}_{2}\right)$ n.loc. where wea Qèt kōmōrōk a van lö gövur avē i Sabine go togtog aē. Then the two of us went to the house where Sabine was staying. Afta mitufala i go long haos we Sabine stap long hem. (AAD) [POc *vai 'where'] aw vi. shout, cry out $\sim$ singaot Ma var ten̄ten̄ nē ine, var gōr na mōgōn o mōnōg, nē ni awaw tek. He stepped on him and made him cry, stepped on his sore, and he cried out because of it. Hem i stanap long hem mekem i krae, stanap antap long soa blong hem, hem i singaot wetem. (JTF.074) 
awi inter. argh! awe! Awi, no gëtë kër! Argh, I don't want it! Awe, mi no wantem! (FRN.034)

awo inter. $\mathbf{1}$-general interjection, used to express positive emotion, wow! woah! awo! Awo, gōwē idian.
Wow, it's really good. Awo, i gud tumas. $\mathbf{2} \cdot$ general interjection, used to express negative emotion, hey! ow! awo! Awo, Die, Mam, na miat e! Ow, Mum, Dad, I'm dying! Awo, Mama, Papa, bae mi ded ia! (JRQ)

\section{$\mathbf{B} \sim \mathbf{b}$}

ba conj. but be Nēn gö dödöm ko vita nē miti kur le o tan̄sar, ba nē mo kur o tan̄sar. We thought that he wouldn't be able to eat a person, but he ate a person. Yumi ting se bae hem i no save kakae man, be hem i bin kakae man. (SSW.070)

babēris $1 \cdot n$.com. wall of house $\sim$ wol blong haos Nēk ga sar gem o qiat so lö kulë babēris ine. You will just plant the taro behind the wall of the house. Bae yu jas planem taro bihaen long wol blong haosia. (ESP) $\mathbf{2} \cdot v$. make wall of house $\sim$ mekem wol blong haos Syn: butun̄

baēr $v t$. ask (someone) for something, ask (something) from someone askem wan samting long wan man No go baēr nēk vita nēk i van me. I will ask you to come. Bae mi askem yu se bae yu kam. (VSG.032) No ma baēr o söm den i Eli. I asked Eli for money. Mi askem mane long Eli. See: vörus

bagbaglō n.com. swiftlet (bird) wan kaen pijin Aerodramus spp.

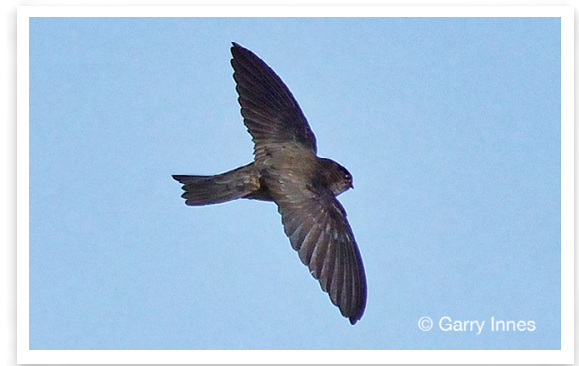

bagbaglō swiftlet $\mathbf{b a k}_{1}$ n.com. generic name for banyan trees, kind of banyan with fruit that are round and light red when ripe wan kaen nambangga we frut blong hem i raon mo i grin mo taem i raep i red waet Ficus spp. O bak aē le mete gëvrōñ? Is there a banyan tree at the entrance to your house? I gat nambangga long doa blong haos blong yu? (EDM) La laln̄e bak, o gersal liwō mè dēñ aē gēn. Under the banyan tree, the main road reaches up to there. Andanit long nambangga, bigfala rod $i$ kasem long ples ia nao. (RRS) $[P O c * b a(k, g) a$ 'banyan tree, mediumsized Ficus spp., not stranglers']

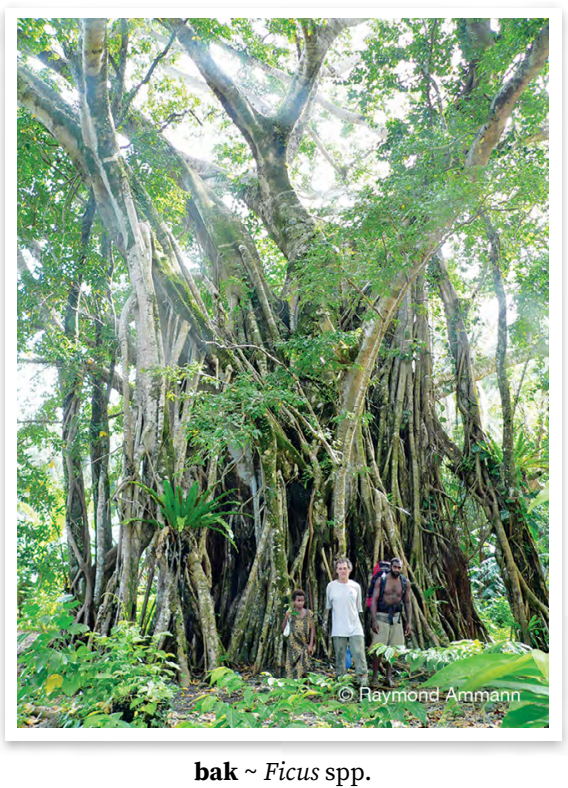


bak matqō (comp.) n.com. kind of banyan with long fruit wan kaen nambangga we frut blong hem i longwan

bak $_{2} v$. join together (two sides of prawn trap) joenem tugeta (tufala basket naora) Armstrong, le o tere waga nirō ine, nēk i bak tiwial rōrō. Armstrong, take those two prawn trap baskets, you join the two of them together. Armstrong, tekem tufala basket naora ia, yu joenem tugeta tufala.

bakalav $_{1}$ n.com. ship sip See: ak

bakalav $_{2}$ n.com. variety of yam that is between grouping of 'female' and 'male' yams and is hard to distinguish due to leaf. It is very thorny and is red and white wan kaen yam we hem i no man mo hem i no woman. Hem i had blong luk save from lif blong hem. I gat tumas nil blong hem mo insaed hem i waet mo red

bakan $n$ married lover of man or woman $\sim$ fren blong man o woman we hem i mared finis

bakasē n.com. abdomen, under side of crabs and other crustaceans andanit, bel blong krab o naora Si no gē gial, nēk i le o sursal, o tere giav döl, nēk i tar wor nē, nēk i wiak sōw o bakasē tabaē, nēk i ēl i Qaslaklakatēg tö tur ti lö lölö giav. If I am lying, then you get a sursal crab, all the crabs, smash it open, open up its abdomen, and you will see Qaslaklakatèg there inside the crab. Sapos mi giaman, yu tekem sursal krab, evri kaen krab, yu smasem brekem, yu openem bel blong hem, bae yu luk Qaslaklakatēg $i$ stanap insaed long krab. (OQQ.180)

bakō n.com. skin condition like ringworm where the skin becomes scaly and isn't strong, peeling off easily wan sik blong skin we i olsem ringwom mo skin i luk olsem skel blong fis mo i save kamkamaot bakrës n.com. variety of banana wan kaen banana

bal $v t$. $\mathbf{1} \cdot$ use wooden tongs to pick up hot stones or charcoal from stone oven $\sim$ tekemaot ston o jakol long oven wetem wud Nana le o gabal, na bal o vet. I get the wooden tongs and I remove the stones. Mi karem wud, mi karemaot ston. (NBQ.008) See: gabal $2 \cdot$ cut (with scissors) katem (wetem sisis) Nēk ma bal na vōlōñ? Did you cut your hair? Yu bin katem hea blong yu?

balbal (der.) n.com. scissors $\sim$ sisis

$\mathbf{b a l}_{2}$ n.com. stack hip Ni lēg nē la bal ralèt. He tied it to the stack of firewood. Hem $i$ fasem long hip faeawud. (WRN.078)

balak n.com. variety of fig tree with edible fruit and leaves wan kaen tri we hem $i$ famle blong nambangga mo yu save kakae frut mo lif blong hem Ficus wassa

dabalak (der.) n.com. leaf of balak which is eaten as wild greens lif blong balak we ol man ol i save kakae olsem bus kabis

balsian n.com.suff. her/his/its chin, jaw jin, jo blong hem See: balsiē

balsiēe n.com.suff. chin, jaw jin, jo [PNCV *balase 'jaw bone (of pig), chin, cheek']

balsian (n.poss) n.com.suff. her/his/its chin, jaw jin, jo blong hem

balsiēk (n.poss) n.com.suff. my chin, jaw jin, jo blong mi

balsiēk n.com.suff. my chin, jaw jin, jo blong mi See: balsiē

ban $c l$. her/his/its (things for bedding) $\sim$ ting blong slip blong hem See: be

banēn n.com.suff. her/his/its hand han blong hem See: bēni 
baon n.com. 100 vatu, monetary unit used to refer to multiples of 100 vatu 100 vatu [This term is now considered archaic, only used by the oldest generation of speakers. Nao ia ol i nomo stap yusum wod ia tumas, ol olfala nomo ol i yusum yet.] See: mil, selen [Eng 'pound']

bar $_{1} \mathbf{1} \cdot$ n.com. mature coconut that has very thin flesh and so is not good for making copra and spongy growth inside is not good for eating kokonas we mit blong hem $\mathrm{i}$ tintin tumas mo yu no save mekem kopra long hem [The stage of coconut growth after sōl. Kokonas i kam sōl fastaem, afta hem $\mathrm{i}$ bar.] 2 - vi. (of coconut) has reached stage where the flesh has become thin and spongy growth inside is no longer good for eating (kokonas) i nomo gudfala navara blong kakae mo i nomo gud blong mekem kopra long hem Taklē ma barbar. Some (copra) has thin flesh and is not good for making copra. Samfala (kopra) i tintin tumas mo yumi no save mekem kopra long hem.

$\mathbf{b a r}_{2}$ n.com. variety of coconut that has very small fruit that do not have much liquid in them but the liquid is very sweet $\sim$ kaen kokonas we hem i smol tumas be wota blong hem i swit

$\mathbf{b a r}_{3}$ n.com. kind of palm $\sim$ wan kaen pamtri Pelagodoxa henryana

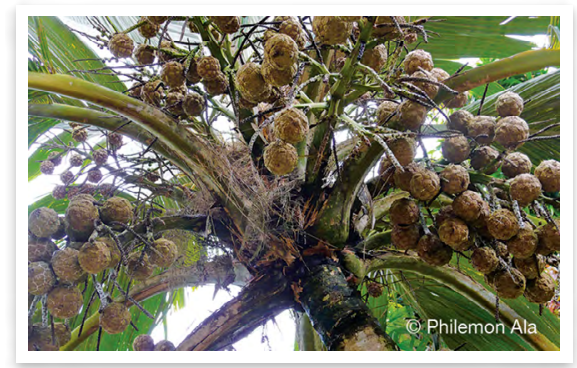

bar Pelagodoxa henryana barbar $v i$. across krosem Nēk i mem ni mönö ōn barbar, ni ōn barbar kēl sōw wo ni ōn barbar kēl wōl. Put it so that it lies across a little, it lies crossing towards the right and it lies crossing towards the left. Putum i leidaon krosem smol, i leidaon krosem olsem ia, mo i leidaon krosem olsem ia. (TT2.005) $\mathbf{O}$ rērēi rēntenge më mës barbar gōr o gersal. A tree branch fell across the road, blocking it. Brans blong wud ifoldaon i krosem blokem rod.

barbar $_{2}$ n.com. axe $\sim$ akis Syn: tele bargōr ${ }_{1}$ n.com. kind of flying fish that is found close to shore $\sim$ wan kaen smol flaengfis we i stap klosap long so

bargōr $\mathbf{r}_{2}$ n.com. yam that is unable to produce large tubers because it is planted with other large crops taem yu planem yam wetem bigfala kakae, yam i no save mekem bigfala kakae

barnaw n.com. doublespotted queenfish $\sim$ kaen fis Scomberoides lysan

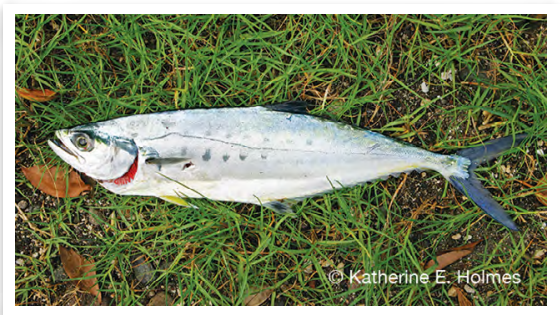

barnaw Scomberoides lysan

basbasne num. last laswan Nēn töl a big basbasne na tönö banēn. The three of us will eat from his hands for the last time. Yumitri kakae laswan long han blong hem. (JMT.287) Syn: qētqētne

bat $v i$. go secretly in search of sexual partner krip from fren Nē ma bat sur nē. He secretly went to try and have sex with her. Hem i krip from hem.

batbat n.com. red-tailed leatherjacket (fish) kaen strongskin (fis) 
baw n.com. onespot snapper (fish) $\sim$ kaen pulefis we i gat yelo tel mo wan blak spot long hem Lutjanus monostigma $\left[P O c{ }^{*} b^{w} a[p, w] a\right.$ 'Lutjanus spp., snapper']

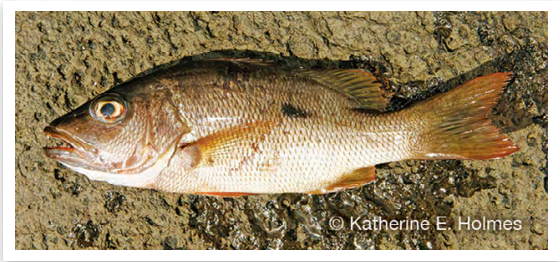

baw Lutjanus monostigma

be $c l$. relational classifier used to mark possession of items that are used for bedding by the possessor $\sim$ blong (yusum blong tokbaot ona blong bed o samting blong slip) Nē mō mōvōt lë bëk. He slept on my bed. Hem i slip long bed blong mi.

ban (n.poss) cl. her/his/its (things for bedding) ting blong slip blong hem ben i nötuk (n.poss) cl. my child's (bedding) ting blong slip blong pikinini blong $\mathrm{mi}$

bëk (n.poss) cl. my (things for bedding) ting blong slip blong $\mathrm{mi}$

be tētē n.com. 1 • placenta samting we i kamaot long mama, taem bebe i bon, we i bin fidim pikinini $\mathbf{2}$ uterus $\sim$ basket blong pikinini [There is some confusion over current usage of this term. It appears to be used to refer both to the placenta and uterus, but there is uncertainty about whether this is 'correct'. Sam man ol i talem se wod ia i gat tufala mining ia, be i no rili klia gud se hem i stret o no.] Syn: ben rōrō \{Lit. baby's bed bed blong bebe\}

Bek n.loc. place name $\sim$ nem blong ples

bele n.com. handle, hard handle as of knife, cup, kettle handel, olsem blong naef, kap, ketel syn: siri $_{2}$

bemrin $_{1} v t .1 \cdot$ help $\sim$ halpem syn: biriñ $\mathbf{2}$ - be with $\sim$ stap wetem $\mathbf{N e ̄}$ gete bemriñ o men, nē gete bemrin̄ o seleqō. Nē to tog gëm ti lē vētitne.
It (flying fox) isn't with the birds, and it isn't with the animals. It just stays in the middle. Hem (flaengfokis) i no stap wetem ol pijin, i no stap wetem ol animol. I stap nomo long medel. (DFF)

bemrin̄ 2 prep. Comitative preposition, with wetem Garqe nēk gō mōvōt bemrin no. Today you are going to sleep with me. Tede bae yu slip wetem mi. Nēk i le o bōk, nēk i mem bemrin̄ o wooror, nēk i nës lē tibiar. You take the book, put it with the toys, and put (them) in the basket. Yu tekem buk, yu putum wetem pleplei, yu fulumap long basket. Syn: birin̄

ben i nötuk $c l$. my child's (bedding) ting blong slip blong pikinini blong mi See: be

ben rōrō $n$.com. placenta samting we i kamaot long mama, taem bebe i bon, we i bin fidim pikinini Syn: be tētē $\{$ Lit. their (two) bed bed blong tufala\}

benmē $n . c o m$. kind of large flying fish with reddish pectoral fins wan kaen flaengfis we hem i bigwan mo wing blong hem i haf red

benm̄omolo n.com. black sweetlips (fish) tiklips (fis) Plectorhinchus gibbosus

ber $_{1} \mathbf{1} \cdot$ n.com. grass skirt, skirt made from beach pandanus $\sim$ grasket $\mathbf{2} \cdot v i$. wear grass skirt werem grasket No ge ber. I am going to wear a grass skirt. Bae mi werem grasket.

ber $_{2} v$. hollow out the inside of a canoe $\sim$ klinim insaed long kenu

beraw n.com. striped surgeonfish renbofis, blumak piko Acanthurus lineatus $[P O c$ *bi[r,R]apa 'Acanthurus lineatus, blue-lined surgeonfish']

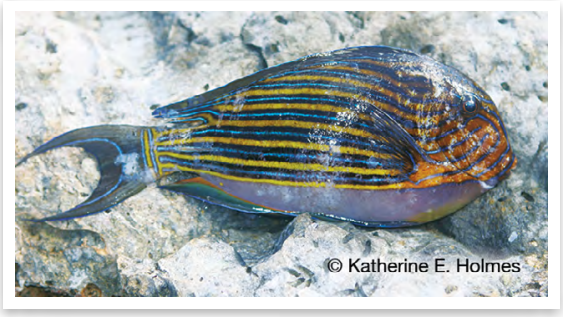

beraw Acanthurus lineatus 
beret $v_{1}$. try to shoot something (e.g. fish with bow and arrow, bird with shanghai or person with stone) but be unsure if the target will be hit as it is far away or can't be seen clearly traem sut (olsem fis wetem bonaro, pijin wetem lastik o stonem man we i stap long bus) be yu no sua se yu save kasem from maet i stap longwe tumas o yu no save luk gud beberet (redup.) v. training to shoot a target with a bow and arrow trening blong sut wetem bonaro No gē èl o men mō rōw siag ba no ma daēl talō sōk sösögöt, ba odian̄, qēt ine na beret gem. I saw a bird land and I tried to find it but couldn't, so then I just shot with the hope of catching it. Mi luk pijin i sidaon be mi traem blong faenem, bo nogat, finis mi sut olbaot nomo.

beret $_{2}$ n.com. surface of sea $\sim$ antap blong solwota

beretnaw n.com. common dart (fish) kaen fis Trachinotus botla

beriri $n$ side saed Syn: bersi

bersi $n$ side, edge saed Nēk i le o rōr ine, mem rōw me le bersi qēl. Take that garden rubbish and put it down at the edge of the taro paddy. Yu tekem doti ia, putum daon ia long saed garen taro. Isē mö tur ti le bersin John? Who was standing beside John? Hu ia i stanap long saed blong John? Syn: beriri, kikse

bertan̄tañ $v$. feel body of canoe to test whether it is thick or not, to see if it needs to be hollowed out more $\sim$ holem bodi blong kenu blong testem se i tik o no In̄ko na këk o ak na berbertan̄tañ. Now I am testing the body of my canoe to see if it needs to be hollowed out more. (This means that it is nearly finished.) Nao ia mi stap holem bodi blong kenu blong mi blong luk se mi mas katem moa o no. (Sapos i talem olsem i minim se klosap i finis.) bes $1 \cdot n$.com. coconut oil, oily substance lining the flesh of germinated coconuts $\sim$ krim we $i$ stap wetem navara, oel blong kokonas $\mathbf{2} \cdot v t$. put coconut oil or grease on something, as to heal a sore or grease something rusty putum oel o krim blong kokonas long wan samting olsem long soa o long wan rosta aean Nē ge bes o mōnōg na mögun i Jessica. She is going to put some coconut grease on Jessica's sore. Bae hem i putum oel blong kokonas long soa blong Jessica.

bet $v t$. pick up pikimap No me bet o ñe, na nës lē tibiar. I picked up the canarium nuts and put them in the basket. Mi pikimap nangae, mi fulumap long basket.

$\mathbf{b} \overline{\mathbf{e}}_{1}$ n.com. 1 fresh water $\sim$ freswota Nēr mö suvsuv lē bē. They swam in the water. Ol $i$ bin swim long wota. $\mathbf{2} \cdot$ river $\sim$ wota

bē mamas (comp.) n.com. dry creek bed $\sim$ drae wota, rod blong wota Van wōl me o qeren̄ bē mamas nitiwial, na sian o Bē Qiqiar. Go round that way a bit further and there is a dry creek bed called Qiqiar Creek. Go olsem smol, i gat wan drae wota, nem blong hem Qiqiar Reva. (RRS)

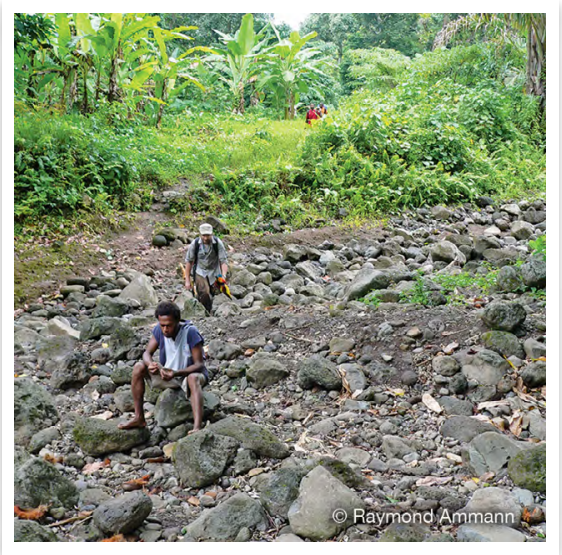

bē mamas $\sim$ dry creek 
kērē bēe (comp.) n.com. mouth of river, place where river meets sea as blong wota, ples we wota $\mathrm{i}$ joenem solwota \{Lit. bottom of water as blong wota\}

mete bē (comp.) n.com. source of river, creek ae blong wota, ples we wota i stat $\{$ Lit. eye of water ae blong wota\}

nögö bē (comp.) n.com. place where the irrigation channel is directed into a taro paddy ples we ol i joenem wota $i$ go insaed long garen taro \{Lit. face of water fes blong wota\}

nötu bē (comp.) n.com. stream smol reva $\{$ Lit. small water smol wota\}

$\mathbf{b} \overline{\mathbf{e}}_{2} v t$. bait hook putum bet long huk See: wēbē $[P O c$ *bayan 'fish bait, trolling lure, trolling hook']

$\mathbf{b} \overline{\mathbf{e}}_{3}$ n.com. variety of banana with very long, large fruit, and the fruit must be squeezed to enable peeling kaen banana we kakae blong hem i longlongfala mo i bigwan, mo yu mas skwisim bifo yu pilim

Bē Daragrō n.com. river name $\sim$ nem blong wan wota

Bē Gavteg n.com. name of a river nem blong wan wota

Bē Luwō n.loc. name commonly used to refer to Bē Taqren, the largest river in the west Vanua Lava area nem we ol i stap yusum blong singaotem Bē Taqren, bigfala wota long eria blong wes Vanua Lava Syn: Bē Taqren \{Lit. large river bigfala wota\}

Bē Matwetegem n.com. name of a river $\sim$ nem blong wota

Bē Metentuv n.com. name of a river nem blong wan wota

Bē Nunur n.com. name of a river $\sim$ nem blong wan wota

Bē Qiqiar n.com. name of a river $\sim$ nem blong wan wota

Bē Ran̄ran̄kör n.com. name of a river nem blong wan wota
Bē Sōn̄sōñtēr n.com. name of a river nem blong wan wota

Bē Taqren n.com. name of a river nem blong wan wota Syn: Bē Luwō

Bē Tultulvet $n . c o m$. name of a river nem blong wan wota

Bē Veqog n.com. name of a river $\sim$ nem blong wan wota

bē- prep. purposive preposition, for blong

bēbètiqū n.com. kind of tree that has two varieties, one with white leaves, the other with red leaves $\sim$ wan kaen tri we $i$ gat tu kaen blong hem, wan lif $\mathrm{i}$ waet, narawan lif $\mathrm{i}$ red [The wood is used for making arrows. The leaves are used as a local medicine and the young shoots are cooked as baby food. Ol i yusum wud blong katem aro blong sutum pijin o faol. $\mathrm{Ol}$ i yusum blong lif meresin mo ol i melekem top blong hem blong smol pikinini.]

bēkil n.com. kind of shrub wan kaen plant Polyscias samoensis [Used traditionally in taro farming to produce large taro corms. An infusion made from the leaves can be used as an antidote for poisoning. Kastom yus blong hem blong putum long garen wota taro blong mekem se taro i bigwan. Mo tu, taem man i posenem yu, bae yu dring lif o insaed long andanit blong skin blong hem.]

bēl $v$. have sexual intercourse $\sim$ fak Rōrō mē bēl. The two of them had sex. Tufala i bin fak. Rōrō a bēlbēl. The two of them are having sex Tufala ifak. Nēk mē bēl nē? Did you fuck her? Yu bin fakem hem?

bēm 1 n.com. butterflyfish $\sim$ bataflae fis Family Chaetodontidae [POc *bebek 'generic for Chaetodontidae, coralfish and butterflyfish'] 


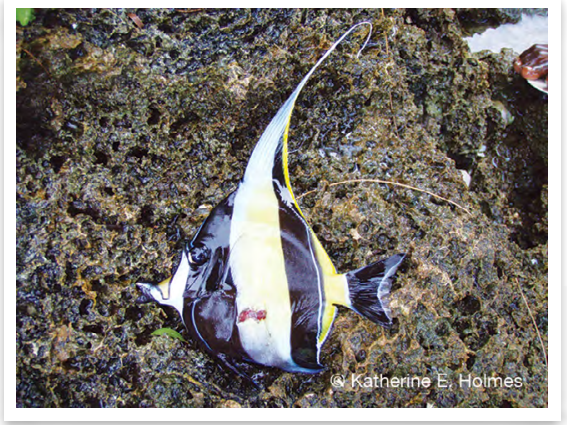

bēm Family Chaetodontidae

bèm $_{2} v$. carry (baby) with piece of cloth, carry person or thing on back $\sim$ karem pikinini o man long baksaed Dōrōk a bēm. I'll carry you on my back. Mi karem yu (long baksaed). Na bēm nēk. I'll carry you on my back. Mikarem yu (long baksaed).

bēm naw $v$. surf, go surfing $\sim$ karem wef long solwota

bēmn̄eg (fr.var. bimn̄eg) vt. carry on shoulder, hang over shoulder, as carrying basket with strap over shoulder and basket hanging down $\sim$ karem long solda, hangem long solda, olsem basket Na bēmn̄eg na non̄ o tibiar. I'll carry your basket. Bae mi karem basket blong yu.

bēmtawal n.com. piece of sago palm thatch which covers over the top of the roof $\sim$ natanggura we ol $i$ somap blong blokem top blong haos Hilton, nēk i le kal me o bēmtawal ine, nana gëv o qötu gövur. Hilton, pass up that piece of sago thatching, I'll cover over the top of the house. Hilton, pasem $i$ kam antap natanggura $i a, m i$ blokem top blong haos.

bēn lo n.com. brackish water, spring water that comes up by the sea and thus is slightly salty but drinkable wota we i spring aot long solwota, yu save dring be i sol smol

bēni $n$.com.suff. $\mathbf{1}$ hand, arm han Nēn ge mem lē bēnin Samson. We will put it in Samson's hands. Bae yumi putum long han blong Samson. (VSG) Nē gē gis o rēntenge la banēn lō mōtō? Is he holding the stick in his hand on the right side? Hem i holem wud long han blong hem long raet saed? (M2E) 2 • wing of bird wing blong pijin $[P O C$ *banic 'arm, hand, wing, fin (probably pectoral)']

banēn gala (comp.) n.com.suff. her/ his/its left hand lef han blong hem banēn mōtō (comp.) n.com.suff. her/ his/its right hand $\sim$ raet han blong hem

banēn (n.poss) n.com.suff. her/his/its hand han blong hem

bēnik (n.poss) n.com.suff. my hand han blong $\mathrm{mi}$

bēnik n.com.suff. my hand han blong mi See: bēni

bēr $v$. tie bamboo rafters to beam of roof before tying thatch onto rafters $\sim$ fasem bambu long ol wud (bifo somap taj i go long hem) See: wēbēr

bērn̄ē n.com.suff. partner, friend, person one associates with patna, fren Na bērn̄ēn̄ aē, gètē nēk masgiañ. You have a partner, it is not you by yourself. Iu gat patna blong yu, ino yu wan. (JNN.022)

bērn̄ēn (n.poss) n.com.suff. her/his/its partner patna blong hem

bērn̄ik (n.poss) n.com.suff. my partner $\sim$ patna blong $\mathrm{mi}$

bērn̄ēn n.com.suff. her/his/its partner patna blong hem See: bērn̄ē

bērn̄ik n.com.suff. my partner patna blong mi See: bērn̄ē

bērseg $v t$. wring skwisim Nēk i bērseg o gogov. You wring out the (wet) clothes. Yu skwisim wota i kamaot long ol klos.

bès ${ }_{1} v t$. prepare cane or vine for weaving by stripping woody insides from outer bark karemaot insaed blong loeaken o rop, redi blong wivim Nēk i nin wor o gawol, e nēk i bēs. You break open the lawyer 
cane, then you remove the fibre inside. Yu brekem loeaken, ale yu karemaot samting we i stap insaed.

bēs ${ }_{2} v t$. add water to (grated coconut, kava, traditional leaf medicine) when there is not enough liquid, in order to dilute or make liquid enough kapsaedem wota i go (long kokonas, kava, lif meresin) taem wota i no naf Brenden, nēk i ut o bē, nēk i bēs me ti min no o mōtō, nana wōr o sasar. Brenden, get some water and add it to the grated coconut for me and I will squeeze the coconut milk onto the island cabbage. Brenden, yu kasem wota, kapsaedem i go long kokonas blong mi, mi melekem kabis.

bēsbēs vi. come with a purpose, reason, interest $~$ kam giaman, kam from i wantem wan samting Nē mē sigsiag bēsbēs sur o gengen. He sat waiting expectantly for some food. Hem i sidaon giaman from kakae. Nē ni vanvan bēsbēs me sur o reqe ko. He comes just to see this woman. Hem i stap kam giaman from woman ia.

bētul $v$. lie down leidaon

Bēut $n . l o c .1 \cdot$ place name $\sim$ name blong wan ples $\mathbf{2}$-tribe name, members of this tribe are thought to be talkative, proud and often making trouble nem blong traeb, mo olgeta long traeb ia ol i man blong toktok mo ol i save praod

bēw ${ }_{1}$ n.com. 1 • kind of slug that is brown slag, samting olsem snel be i nogat sel blong hem $\mathbf{2}$. kind of sea slug samting olsem besdemea we i stap long solwota

bēw $\mathbf{w}_{2}$ n.com. variety of breadfruit kaen bredfrut

bëk $c l$. my (things for bedding) ting blong slip blong mi see: be

bël $v$ t. $\mathbf{1} \cdot$ steal $\sim$ stilim Rōrō a van vël tiqē talë bëlbël gengen. The two of them went to every garden to steal food. Tufala $i$ go long evri garen blong stilim kakae. (WRN.003) 2 - record, with audio or video recorder $\sim$ rikodem voes o video

bëlbëlvōlōn $n$.com. small Vanuatu fruit dove (bird) grinpijin \{Lit. steals its feathers stilim feta blong hem\}

bëtutu adj. big, large bigfala Rōrō gētē ēl sur o bëtutu naw nitiwial ma van kal me. The two of them didn't see a big wave that came up. Tufala $i$ no luk wan bigfala wef we i kam antap. (ATB.120) Syn: luwō 1

bibiag vi. stage of coconut growth when the flesh is dry but the skin is still green $\sim$ mit blong kokonas $\mathrm{i}$ drae be skin blong hem i grin yet

bibian $n$.com.suff. her/his/its neck $\sim$ nek blong hem See: bibiē

bibiē $n$.com.suff. neck $\sim$ nek Na bibian gē mie. It has a long neck. Nek blong hem i longfala. (SBB)

bibian (n.poss) n.com.suff. her/his/its neck nek blong hem

bibiēk (n.poss) n.com.suff. my neck nek blong mi

bibiēk $n$.com.suff. my neck nek blong mi See: bibiē

bibis $\mathbf{1} \cdot v t$. roll up, as pandanus, hair, mat $\sim$ rolemap wan longfala samting Na mōgōñ o ga e gē mie nimal, bibis qëtutu. That rope of yours is too long, roll it up shorter. Rop blong yu ia i longwan tumas, rolemap ikam sotwan.

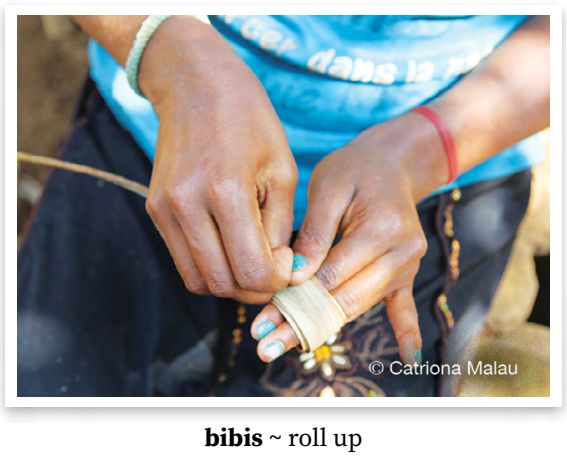


2 - n.com. roll (of something) rol (blong wan samting) Isē mö wöl o bibis ga ko? Who bought this coil of rope? Hu i bin pem rop ia we i stap long rol?

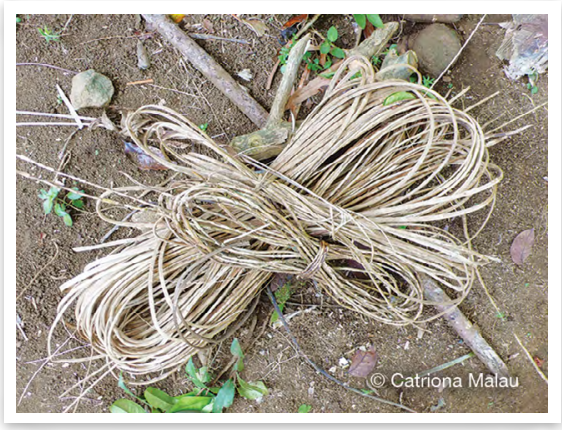

bibis roll

$3 \cdot v$. roll together, as group without edges (in dancing) rol tugeta olsem we yu no save luk kona (olsem taem wan grup i danis)

bien $v t . \mathbf{1}$ - feed $\sim$ fidim Ni le o vin garat, ni van me ni bien i tasēn aē. He took some food scraps and he came and fed his younger brother with them. Hem i karem doti blong kakae, i kam fidim smol brata blong hem long hem. (GMM.024) Syn: ven̄en $\mathbf{2} \cdot$ put bait in trap putum bet long basket naora Na mon o mōtō, na bien o waga. I wrap up the coconut, and I bait the prawn trap. Mi paselem kokonas, mi putum long basket naora. (KBN.020)

biēg n.com. breadfruit bredfrut Artocarpus altilis See: döwis [POc *baReko 'breadfruit']

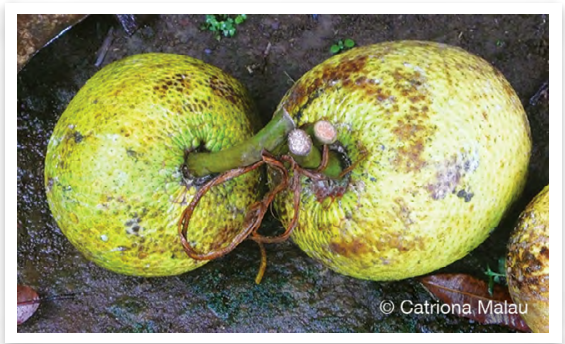

biēg breadfruit biēg ge mirmiar n.com. variety of breadfruit that has small fruit kaen bredfrut we kakae blong hem i smolwan

biēg löt n.com. variety of breadfruit that is good for making löt as it is neither too hard nor too soft $~$ kaen bredfrut we hem i gud blong nalot from hem i no sofsof tumas mo i no had tumas

biēg ta motlav n.com. variety of breadfruit that is good for boiling kaen bredfrut we hem i gud blong kuk

biēn n.com. young unmarried male, in early stages of puberty, no facial growth as yet (younger than lomegev) $\sim$ yangfala boe, olsem se hem i nomo pikinini be i nogat mustas yet

big $v t$. eat (meat, greens, protein with main meal) kakae (mit o kabis wetem kakae) Meter ma da o löt, big tek o tō. Meter made a nalot, and they ate it with chicken. Meter hem i mekem nalot, kakae wetem faol. (HWM.082)

bigbig (redup.) n.com. meat, greens, protein component of meal. Generally in Vanuatu, a meal is conceived of as consisting of the starchy, staple part (gengen), such as root vegetables, unripe bananas, or rice. This will be accompanied by the bigbig, which ideally will be meat, or otherwise greens, other non-staple vegetables, or simply coconut cream $\sim$ mit, pat blong kakae we i no men kakae, olsem mit, kabis o kokonas Ke, nēn töl ta wanwan ko, ba na gen nēn o bigbig odiañ aē. Hey, the three of us are drinking kava now, but we don't have any meat to eat. Ei, yumitri stap dring kava be mit blong yumi i nogat. (ADT.035) See: gen 
big töw $v$. eat grated coconut flesh, scraped straight from the shell, either on its own or with a staple food kakae kokonas, taem yu skrasem kokonas, yu kakae hem wan o tugeta wetem wan kakae $\mathrm{Na}$ èl nē ni bigbig töw. I saw that he was eating coconut flesh. Mi luk hem i stap skrasem drae kokonas mo kakae.

bilēn (fr.var. of bilēen)

bilēñ (fr.var. bilēn) mod. 1 • again, modifies verb in the verb complex bakegen $\mathbf{E}$ gunön Tëqël ni tel kēl bilēn. Then Tëqël's wife was pregnant again. Ale woman blong Tëqël $i$ gat bel bakegen. (KM̄T) 2 - as well, too, modifies noun in the noun phrase $\sim$ tu $\mathrm{O}$ tërëw bilēñ nē gö mörös $\mathrm{o}$ darag. The Vanuatu mountain pigeon too likes wild nutmeg. Nawimba blong hil tu hem i laekem nandae. (SBB.092) 3 - more, other, another, modifier in the noun phrase $~$ moa, bakegen Qēt lē vētitne o qarörö nitiwial bilēñ nē go tog. Then in the middle there is another hole. Afta long medel wan hol bakegen i stap. (TT3.006)

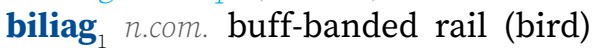
$\sim$ nambilak (pijin) Gallirallus philippensis

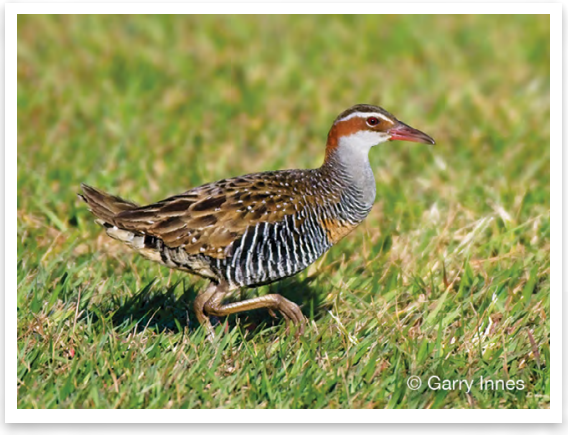

biliag Gallirallus philippensis

biliag mamē (comp.) n.com. whitebrowed crake (bird) wan kaen pijin Poliolimnas cinereus \{Lit. red buff-banded rail red nambilak\} biliag 2 n.com. darkfin hind (fish) kaen los (fis) Cephalopholis urodeta

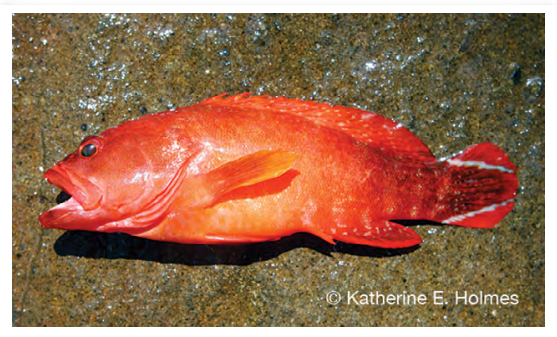

biliag Cephalopholis urodeta

biliag lam (comp.) n.com. leopard grouper (fish) kaen los (fis) Cephalopholis leopardus \{Lit. grouper from deep sea los blong dipsi\}

biliag $_{3}$ n.com. variety of taro, 'female', a type of mare, with a dark red stem $\sim$ kaen mare taro we hem i woman we han blong hem i dak red

biliag $_{4}$ n.com. speckled, modifier derived from name for buff-banded rail, to indicate that the referent has speckled colouring dotdot, olsem kala blong nambilak

biliagtat n.com. kind of laplap food dish that has two layers of laplap with a layer of grated canarium nuts between, either roasted or raw wan kaen laplap we i gat tu laen wetem grin o drae nangae long medel

bimn̄eg (fr.var. of bēmn̄eg)

bin $v$ t. peg pin [Bis pin 'peg']

bioñ n.com. proud praod

birbir n.com. sea hearse, lantern tree nambirimbiri Hernandia nymphaeifolia $\quad[\mathrm{POc} \quad *$ *biRi-biRi 'Hernandia nymphaefolia']

biriat n.com. garden where taro is planted in dry ground on the hillside $\sim$ drae garen taro antap long hil Rōrō gata vas kēl o biriat. The two of them didn't replant the dry taro garden. Tufala i no planem bakegen garen taro long drae graon. (HWM.040) 
birin̄ $v t$. help givhan long, halpem No gö mörös na biriñ nēk ti nēk i van me o tan̄sar gö luwō. I want to help you so that you become an important person. Mi wantem givhan long yu blong yu kam wan bigman. (ESP.019) Syn: bemrin̄ 1 See: tek

biriñ $_{2}$ prep. with, marks a concomitant role wetem No gö lölö kakaka birin̄ nēk. I want to speak with you. Mi wantem storian wetem yu. Nēn ge gen o qiat, gē big birin̄ o buluk. We will eat taro, and will eat it with beef. Iumi kakae taro wetem buluk. (EDM.091) Syn: bemriñ $_{2}$, tek $\mathbf{1}$

bis $v t$. pinch and twist to break off, as in picking (fruit) from tree or removing prawn legs $\sim$ aksen blong karem (frut) long tri, karemaot leg blong naora Nēk i rem, nēk i bis na gen nēn o miak. You climb and you pick some Tahitian chestnuts for us to eat. Yu klaem, yu karem sam namambe blong yumi. (JRQ.056)
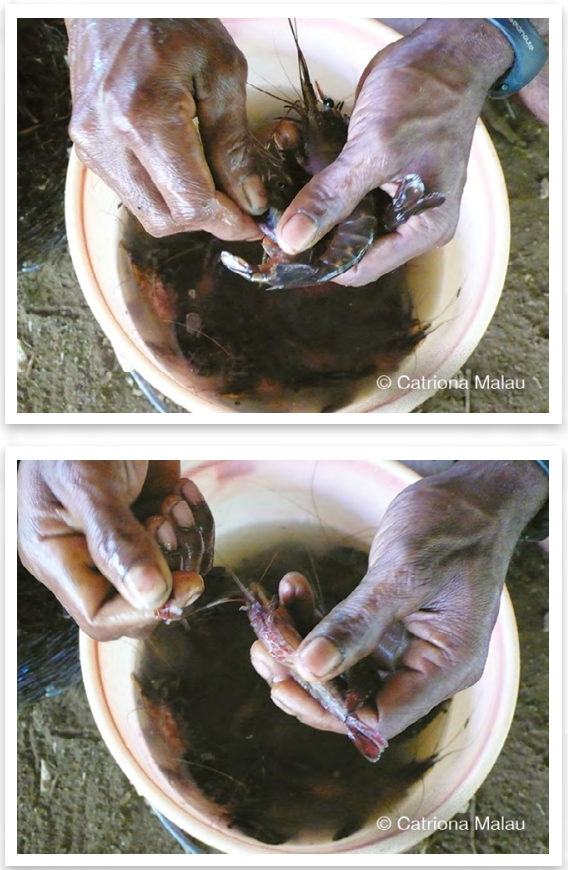

bis break off bit $v t$. break off length of shell money $\sim$ karemaot pis sel mane Nēr döl nēr mē bit o söm min nē. All of them broke off some shell money for him. Olgeta evriwan ol i karemaot pis sel mane blong hem. (ESP)

bitbitiq̄̄ n.com. kind of small tree with small red fruit and yellow flowers wan kaen tri we i smol mo frut blong hem i smol mo i red mo flaoa blong hem i yelo Psychotria trichostoma

bo prep. towards long Nē ni gis rak nē wo ni wöt bo Tores, ni wöt bō Ōrbarbar, ni wöt bō Rō, ni wöt bē Rēvian̄, nē ni wöt kal rōw bo Motlav. He picked her (parts of her body) up, and he threw her towards Torres, he threw her towards Ureparapara, he threw her towards Rowa, he threw her towards Revian, he threw her towards Motlav. Hem i pikimap hem, i sakem $i$ go from Torres, $i$ sakem $i$ go from Ureparapara, $i$ sakem $i$ go from Rowa, i sakem $i$ go from Rēviañ, $i$ sakem $i$ go from Motalava. (JRQ)

boboros n.com. kind of tree related to balak, with small fruit that are white or pinkish when ripe $\sim$ wan kaen tri we hemi famle wetem balak. Kakae blong hem i smol mo taem i raep $i$ waet o laet red [It has soft wood and is only used for firewood. Wud blong hem i sofsof mo i gud blong faeawud nomo.]

boboto n.com. cheese tree; kind of tree with small yellow flowers and small fruit that are black when ripe wael namamao, wan kaen tri we flaoa blong hem i smol mo i yelo mo frut blong hem i blak Glochidion sp.

boko $v$. tie fasem See: lēg $[$ [POc *buku 'tie (a knot); fasten']

bor 1 vi. laugh laf Angelina ni bor tek nè. Angelina was laughing with him. Angelina i stap laf wetem hem. 
borbor ${ }_{1}$ (redup.) n.com. laughter laf Ni miat ren lo borbor. He died of laughter. Hem i ded long laf. (DNS.116)

bor 2 n.com. $\mathbf{1} \cdot$ kind of large coconut leaf basket that is used for transporting food wan kaen basket blong lif kokonas we ol i yusum blong paselem plante kakae insaed olsem blong sendem Nēr më nës o qiat lo bor talo vetreñ a Santo. They put the taro in a large coconut leaf basket to send it to Santo. Ol i fulumap taro $i$ go long basket kokonas blong sendem i go long Santo. $\mathbf{2} \cdot$ small basket that is filled with food and hung up outside someone's house to indicate that another person has failed to meet an appointment, such as for a meal or drinking kava, with that person. The community will then know that this person has been let down by another $\sim$ basket we man i fulumap wetem kakae mo hangem aotsaed long haos blong ol man ol i save se wan narafala man i mestem mo i no kam taem hem i bin mekem promes wetem man we i hangem basket ia

borbor $_{2}$ n.com. kind of fish kaen fis bormiak n.com. evodia, shrub with distinctive, pleasant-smelling leaves, variety with smaller leaves wan kaen lif we i gat naes smel, lif blong hem i bigwan Evodia hortensis [The leaves are used as decoration on one's body, valued for the scent. $\mathrm{Ol}$ i yusum blong flasem bodi from smel blong hem i naes.] See: seg

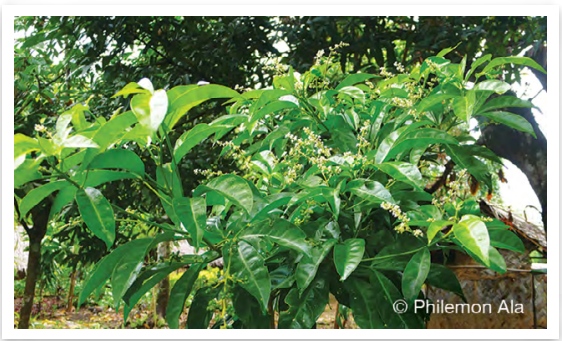

borm̄iak Evodia hortensis borseg $v t$. laugh at laf long Nēr a borseg nē, qaq titisē nē van vaavan. They laughed at him, and said bad things about him on and on. Ol i laf long hem, toktok nogud long hem go go go. (ESP)

boso $a d v$. why, interrogative marker occurring clause initially from wanem Boso nēk to borbor sur? Why are you laughing? From wanem yu stap laf? (WRN)

bō n.com. 1 - length of bamboo used for carrying water $\sim$ bambu blong fulumap wota 2 - length of bamboo used for making beat in dance bambu blong kilim long wud long taem blong danis [POC *bue '(made of) bamboo']

bōak n.com. 1 • mud sofmad Nē mo ror ti a Daragrō lō bōak. She had become stuck in the mud at Daragrō. Hem i bin draon long sofmad long Daragrō. (AAD) $\mathbf{2}$ - taro garden planted in mud $\sim$ garen taro we ol i planem long sofmad $\mathbf{O}$ qiat ta vasvas vaga li tōw, rōrō gata vas kēl o bōak. They were always planting taro on the hillside, the two of them hadn't planted the taro garden in the mud again. Ol i stap planem taro long hil nomo, tufala i no plantem bakegen long sofmad. (HWM)

bōbōn $v$. kiss kis, kisim Bōbōn i Jessica vagvet na mögun kemem döl. Kiss Jessica four times for all of us. Kisim Jessica fo taem blong mifala evriwan.

bōgereg $v t$. feed and look after (domesticated animals and plants) $\sim$ fidim mo lukaotem, olsem animol O qō ine, nēn gō bōgereg. We will feed and look after that pig. Pig ia bae yumifidim. (ESP) Syn: veñen

bōgēt $v i$. feel proud, act proudly, show off flas, mekem flas Nēn a bōgēt sur o plan na mögun nēn. We are feeling proud because of our plan. Mifala i stap mekem flas from plan blong mifala. 
bōgō ${ }_{1}$ n.com. shark sak $[P O c$ *bakiwa 'shark (generic)']

bōgō dēm̄ēl (comp.) n.com. blue shark wan kaen sak \{Lit. cycad leaf shark namele sak\}

bōgō mes (comp.) n.com. sandbar shark wan kaen sak \{Lit. fish shark fis sak\}

bōgō n̄ōrn̄ōr (comp.) n.com. leopard shark wan kaen sak \{Lit. snoring shark sak we i stap pulum win\}

bōgō $\overline{\mathbf{o}}_{2}$ n.com. man who has not learned the traditional ways in the men's house to gain rank $\sim$ man we hem $\mathrm{i}$ no bin go long nakamal blong lanem stret rod blong kastom

bōgōn n.com.suff. her/his/its fault fol blong hem See: bugë

bōgōr n.com. ballnut, Alexandrian laurel, beach mahogany, tamanu (tree) nambakura, tamanu blong solwota Calophyllum inophyllum [Oil from the seeds has medicinal uses. Oel we i kamaot long sid blong hem ol i yusum olsem meresin.]

bōgōrēmēs (comp.) n.com. kind of tree tamanu blong bus Calophyllum neo-ebudicum

bōk n.com. book buk [Bis]

Bōkrat n.loc. place name $\sim$ nem blong ples

bōl $1_{1} v t$. $\mathbf{1} \cdot$ throw (something roundish and short) at stonem (wetem raon, sotfala samting) Nē mō bōl no tek o vin mōtō. He threw a coconut shell at me. Hem i stonem mi wetem sel kokonas. See: gatötöqulqërët $\mathbf{2} \cdot$ fall down onto (of something heavy) (wan samting hevi) foldaon i go antap long Nēk i ēlgōr gem vita o rēntenge mitō bōlbōl nēk. You just have to watch out that the branches don't fall down onto you. Bae yu mas lukaot nomo se ol wud i no foldaon mo kasem yu. (VVCMVDP21Nov0701AS.012) bōl ${ }_{2} v i$. unripe, ripening but not yet ripe $\sim$ klosap i raep be i no raep gud yet $\mathbf{O}$ tere $\overline{\text { möl }}$ ko gō bōl vēti. These oranges are still not quite ripe. Ol aranis ia ol ino raep gud yet.

bōlan $c l$. her/his/its (plant or animal) blong hem (plant o animol) See: bulë bōn vi. smell bad, as rotten food smel nogud $\mathrm{O}$ n̄e ine gō bōn, gō bōn. Those canarium nuts smell really rotten. Ol nangae ia i smel nogud we. See: bunë

bōnan n.com.suff. her/his/its smell smel blong hem See: bunë

bōnësnës $v t$. fill length of bamboo with food (e.g. taro, yam, meat, prawns) and roast over fire $\sim$ fulumap kakae insaed long bambu mo rusum long faea Nēr mō bōnësnës o ōr nonor. Yesterday they cooked prawns in bamboo. Yestedei ol i bin rusum naora insaed long bambu.

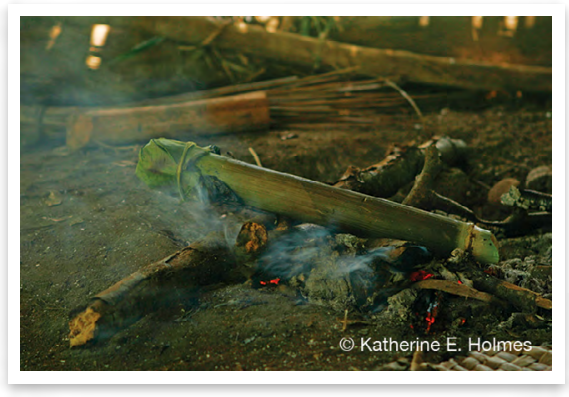

bōnësnës cook in bamboo

bō̄n n.com. baby sling, cloth used for carrying baby kaliko blong karem pikinini

bō̄n $\overline{\mathbf{n}}_{2} v t$. tie up fasem No mō bōñ o go. I tied the hook onto the fishing line. Mi fasem huk long string.

bō̄n $\overline{\mathbf{n}}_{3}$ n.com. cicada wan kaen bebet we hem $\mathrm{i}$ singaot long naet [There is a belief that if one hears a cicada in the night, it means the spirits will come. Long kastom ol i talem se sapos i kam long naet, i minim se ol devel ol i kam.] Syn: wōsōr 
bōrō̄n n.com. kind of beetle shaped like a turtle with hard wings and a strong smell wan kaen bebet we wing blong hem i strong mo sep blong hem i olsem totel. Hem i sting

bōrōt n.com. shore, sea shore, strip of sand or land close to the sea so, sanbij o eria we i klosap long solwota Mō sō sō, sō dēn̄ sar lō bōrōt. He paddled and paddled until he reached the shore. Hem i pul, pul kasem long so. (OHS.024)

bōsōn n.com.suff. her/his/its finger, toe fingga blong hem See: busu

böl n.com. variety of taro, an old variety with a whitish stem that is no longer planted kaen taro blong bifo we ol i nomo planem we han blong hem i waet

brisben n.com. variety of banana kaen banana [Bis Brisben 'Brisbane']

bugë n.com.suff. fault; error or mistake caused by a particular person $\sim$ fol, problem we wan i kosem

bōgōn (n.poss) n.com.suff. her/his/its fault fol blong hem

bugën i tëmëk (n.poss) n.com.suff. my father's fault fol blong papa blong mi

bugëk (n.poss) n.com.suff. my fault fol blong mi

bugëk n.com.suff. my fault fol blong mi See: bugë

bugën i tëmëk n.com.suff. my father's fault fol blong papa blong mi See: bugë

bul n.com. suicide tree, kind of tree with red or black fruit that has sticky sap wan kaen tri we frut blong hem i red o blak mo wota blong hem i olsem glu Cerbera manghas, C. odollam \{Lit. join, sticky, so called because sap and juice from the fruit is sticky joen, fas, from yu save yusum wota blong frut olsem glu\} [POc *bulit 'gum; resin'] bul ${ }_{2}$ n.com. tattoo tatu La masawre nềr a ēl o bul odian̄ aē nēk, nēr a èl ta nēk o sarsar. When they see that you don't have any tattoos, then they will know that you are not a woman of rank. Taem ol iluk se yu nogat tatu, bae ol $i$ save se yu woman we yu no bin go long nakamal.

bul- num. > num. prefix added to numbers to indicate a group of people of that number who are together smol pat blong wod ia i go long bigining blong ol namba, taem namba i talemaot wan grup blong pipol we ol i stap tugeta Qèt i Gavin, Sabine, Catriona wo i no kemem bulvet a van a lo vita a suvsuv la naw. Then Gavin, Sabine, Catriona and I, the four of us went to the seaside to swim in the sea. Afta Gavin, Sabine, Catriona mo mi, mifala fo i go long solwota blong swim long solwota. (AAD.288)

bulalèv n.com. variety of taro, 'male', with a black stem kaen taro we hem i man we han blong hem i blak bulbul 1 - n.com. cooperation, togetherness, friendship kopereisen $\mathbf{O}$ bulbul odian̄ ren. There is no cooperation whatsoever. I nogat kopereisen nating. Hilton o mēsi bulbul birin tek ira Kelly. Hilton is always hanging out with Kelly and his friends. Hilton $i$ stap hangraon nomo wetem Kelly olgeta. 2 - vi. cooperate kopereit Iutikas, nēk götö bulbul wareg min kemek. Iutikas, you don't cooperate well with us. Iutikas, yu no stap kopereit gud wetem mifala.

bulë $c l$. relational classifier used to mark possession of items that are looked after by the possessor, such as crops, domestic animals blong (yusum blong tokbaot ona blong animol o plant) Nēk ge ven̄en na bōlan̄ o tok. You will feed your dog. Bae yu fidim dog blong yu. (DDP.002) 
bōlan (n.poss) $c l$. her/his/its (plant or animal) blong hem (plant o animol)

bulën i tëmëk (n.poss) cl. belonging to my father (plant or animal) blong papa blong mi (plant o animol) bulëk (n.poss) cl. my (plant or animal) blong mi (plant o animol)

bulëk $c l$. my (plant or animal) blong mi (plant o animol) See: bulë

bulën i tëmëk $c l$. belonging to my father (plant or animal) blong papa blong mi (plant o animol) See: bulë

bulës $v t$. place stone or something heavy on top of something else to hold it firm putum ston o wud o samting we $i$ hevi antap long wan samting blong holem taet o blokem No ma tar lēt o rēntenge ko, ba mö bulës na bulëk o qōōr. I cut down this tree, but it fell on top of my wild yam plant. Mi jopem daon wud ia, be $i$ foldaon antap long waelyam blong mi. Ri van me, ri bulës no. You all come and lie on top of me. Yufala $i$ kam, leidaon antap long mi. Le o vet ine, bulës gōr siag o bōk. Take that stone and weigh down the book with it.

Tekem ston ia, putum $i$ go antap long buk.

bullevetē num. six together, group of six (of people) sikisfala (man)

bullōvōrō num. seven together, group of seven (of people) sevenfala (man)

bullövötöl num. eight together, group of eight (of people) eitfala (man)

bullövövet num. nine together, group of nine (of people) naenfala (man)

bulrō num. two together, group of two (of people) tufala (man) Qön̄ nitiwial i rege bulrō min tasēn a oror ti. One day, two people together, two brothers were playing. Wan dei tufala wetem brata blong hem i stap pleple. (ADT) bulsal $1 \cdot n . c o m$. friend, mate, can be sexual partner or platonic friend $\sim$ fren 2 - n.p.fr. friend, used as an address term fren Bulsal, nēk gē biriñ le no? Friend, can you help me? Fren, bae yu save givhan long mi? (ATB) $\mathbf{3} \cdot v i$. be friends, become friends fren Rōrō mo bulsal, rōrō mo togtog, rōrō a lugög vita, 'Dōrōk, dōrōk gē vēnvēn.' The two of them became friends, and they stayed, and they made a plan that, 'The two of us will go shooting.' Tufala i kam fren, tufala $i$ stap, tufala $i$ plan se, 'Yumitu, bae yumitu go sut.' (JTF.005) 4 $v i$. become sexual partners $\sim$ fren (olsem gelfren mo boefren)

bulsamul num. ten together, group of ten (of people) tenfala (man)

bultarō n.com. yellowmask angelfish, regal angelfish $\sim$ papiong fis Pomacanthus xanthometopon, Pygoplites diacanthus

bultevelēm num. five together, group of five (of people) faefala Qēt ine ma da vita in̄ko kemem bultevelēm. Then it meant that now we were a group of five. Afta $i$ mekem se nao ia mifala faeflong grup. (AAD)

bultiwial n.com. unity yuniti Lö bultiwial nēn a tōtōw. In unity we progress. Long yuniti yumi progres.

bultöl num. three together, group of three (of people) trifala (man) Nē mè qilian̄ den nēr bultöl. He passed away, leaving the three of them. Hem $i$ lus long trifala. (ATW)

buluk n.com. cow, cattle buluk [Bis]

bulvet num. four together, group of four (of people) fofala (man) I Mem ni vanvan me sur kemem bulvet. Mem was coming for the four of us. Mem i stap kam from mifala fofala. (AAD)

Bum n.p.fr. Grandma, Grandpa, Grandson/daughter; reciprocal address term used by grandparent 
to grandchild or grandchild to grandparent $\sim$ Bubu [Mostly used by grandparent to grandchild, but able to be used as reciprocal term. Mos taem bubu i yusum blong singaot long smol bubu.]

Bum tal n.p.fr. Great-Grandma, Great-Grandpa mama o papa blong bubu

Bum vövörus n.p.fr. Great-greatgrandparent $\sim$ Bubu blong bubu

bum n.com. triggerfish $\sim$ strongskin (fis) Family Balistidae $[\mathrm{POC}$ *bubu 'Balistes taxon, triggerfish']

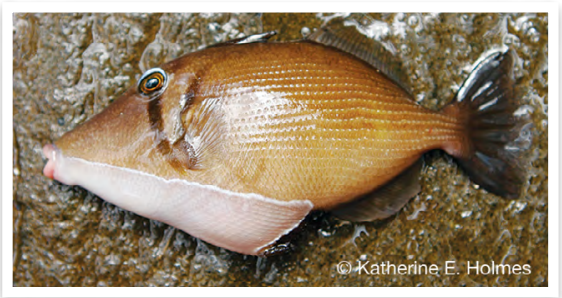

bum $\sim$ Family Balistidae

bum gōlōw (comp.) n.com. black triggerfish $~$ kaen strongskin (fis) $\{$ Lit. orphan triggerfish strongskin we i nogat mama mo papa\}

bum lam (comp.) n.com. starry triggerfish kaen strongskin (fis) Abalistes stellaris \{Lit. deep sea triggerfish strongskin blong dipsi\}

bum lo (comp.) n.com. kind of triggerfish kaen strongskin (fis)

bum qërët (comp.) n.com. redtooth triggerfish $\sim$ kaen strongskin (fis) Odonus niger [Lit. flying fox triggerfish flaengfokis strongskin\}

bum timiat (comp.) n.com. orangelined triggerfish kaen strongskin (fis) Balistapus undulatus $\{$ Lit. headdress triggerfish hed dres strongskin\}

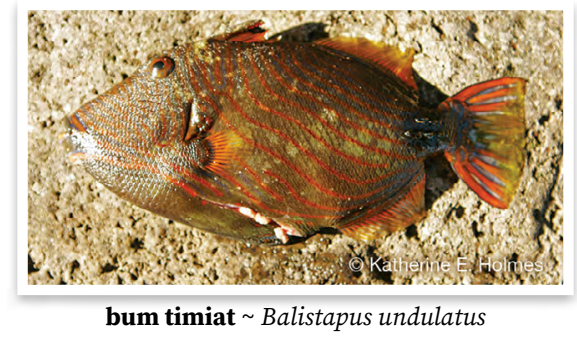

bun $v$. place stones on fire of oven putum ston antap long faea No mö tuwegev tuwegev kal qēt, nana bun kēl o vet ni van lē mēèkē qañris. I make the fire, then I place the stones on top of the oven. Mi mekem faea finis, mi putum ston $i$ go antap long oven. (NBQ.005)

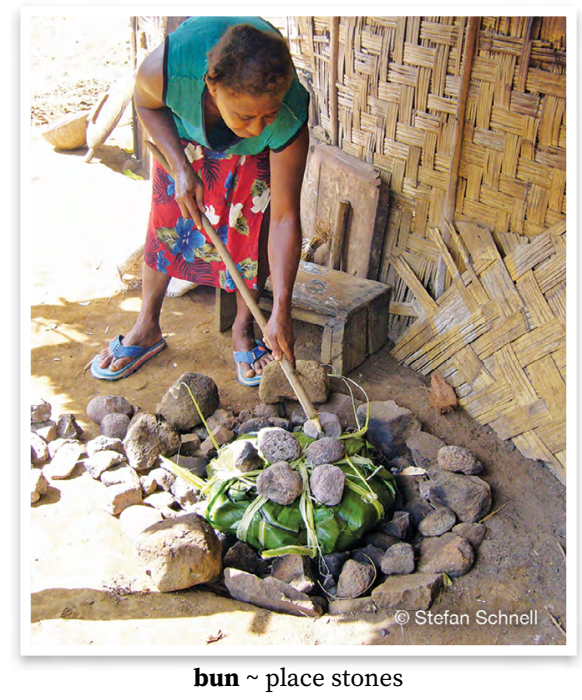

bunë qërët $n$.com.suff. flying fox's smell $\sim$ smel blong flaengfokis See: bunë bunë n.com.suff. smell smel $\mathrm{O}$ bunë gengen gōwē idian. The smell of the food is really good. Smel blong kakae i gud we. See: bōn

bōnan (n.poss) n.com.suff. her/his/its smell smel blong hem

bunë qërët (n.poss) n.com.suff. flying fox's smell smel blong flaengfokis bunëk (n.poss) n.com.suff. my smell smel blong mi

bunëk n.com.suff. my smell smel blong mi see: bunë 
bunia $n . c o m$. food dish, where different kinds of root vegetables are baked in leaves in stone oven with coconut milk bunia [Bis]

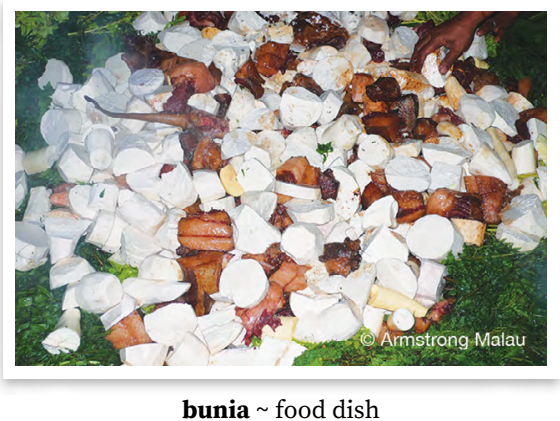

bur 1 n.com. light laet E nēr a ti o bur, van sar lö lölö gövur. Then they lit the light and went into the house. Ale ol i laetem laet, go insaed long haos. (RPP.104)

bur $_{2} \mathbf{1} \cdot v t$. close, shut $\sim$ sarem Na bur na mëtëk. I close my eyes. Mi sarem ae blong mi. I Wöwut mö bur gōr o mete gövur. Wöwut shut the door of the house. Wöwut $i$ sarem insaed long haos. (WWM̄) 2 - vi. be closed klos $\mathrm{O}$ mete gövur gö bur. The door is closed. Doa i klos.

bur ñe n.com. canarium sap wota blong nangae

burmat vi. flutter eyelashes, make seductive looks to someone mekem ae, lukluk long wan man o woman we yu laekem hem Nē mö burburmat min gunök. She was making eyes at my husband. Hem i mekem ae long man blong mi.

burmatan n.com. variety of taro, 'male', with a dark green bottom, dark purple top and black petiole $\sim$ kaen taro we hem i man we han blong hem i dak grin daon mo antap i dak popel

burus mete $n$.com. eyelashes, eyebrows $\sim$ hea blong ae

bus lav n.com. thumb nambawan fingga bus titivōnō n.com. index finger, pointer finger nambatu fingga long han Lit. finger pointing to village fingga i poen long vile]\}

buskat n.com. cat $\sim$ puskat Syn: tivi [Bis puskat 'cat']

busmaraw n.com. nail, wooden stake $\sim$ nil \{Lit. spider's finger fingga blong spaeda\}

bustavēl n.com. kind of colourful, shiny beetle wan kaen bebet we wing blong hem i strong mo i kalakala mo i saen

bustutun $n . c o m$. kind of hardwood tree $\sim$ wan kaen wud Guettarda speciosa

bustuwur n.com. pinky, baby finger las fingga long han

busu n.com.suff. 1 ・ digit, finger, toe fingga $2 \cdot$ claw $\sim$ fingga blong pijin, fingga blong krab, naora

bōsōn (n.poss) n.com.suff. her/his/its finger, toe $\sim$ fingga blong hem busuk (n.poss) n.com.suff. my finger, toe $\sim$ fingga blong $\mathrm{mi}$

busu ōr (n.poss) n.com.suff. prawn or lobster claw fingga blong naora

busuk n.com.suff. my finger, toe fingga blong mi see: busu

busumat n.com. kind of insect $~$ kaen bebet

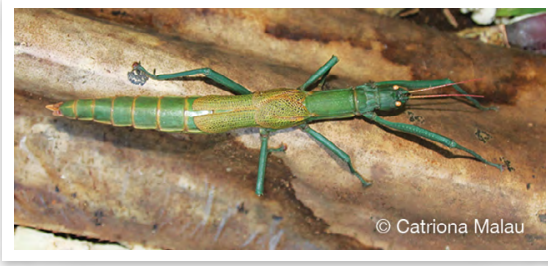

busumat kind of insect

busuōr n.com. variety of taro, 'female', with light red stem and top with light green stripes $\sim$ kaen taro we hem i woman we han blong hem i laet red wetem grin straep long hem Syn: wēlēbēōr \{Lit. prawn claws fingga blong naora\} 
busuqaēn n.com. kind of small flowering shrub with white flowers and small fruit that are red when ripe wan kaen wud we i gat waet flaoa, frut blong hem i smolsmol mo i grin be taem i raep i red. Lif blong hem i smolsmol mo stamba i no save gru bigwan Psychotria aneityensis

busuqërët $n$.com. kind of shrub kaen smol wud Ascarina lanceolata

buswōtōt n.com. wooden stakes hammered into float to join float to cross booms of outrigger $\sim \mathrm{smol}$ wud we i holem taet rel i joen long nasama blong kenu

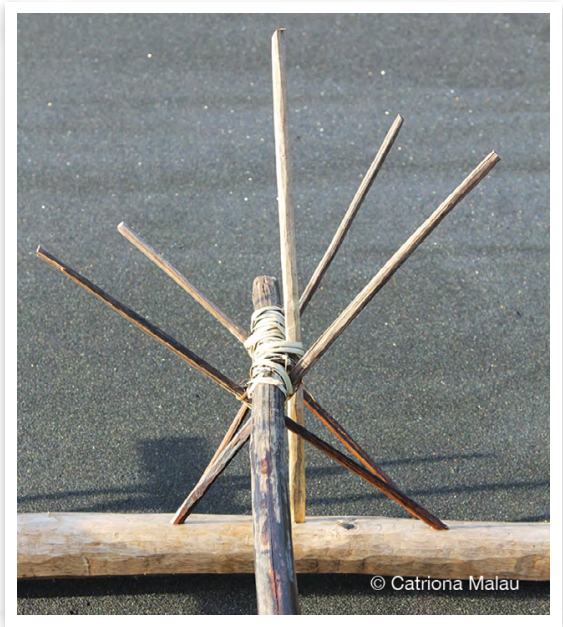

buswōtōt outrigger stakes

buton n.com.suff. her/his/its navel nambuton blong hem See: butö

butö n.com.suff. navel nambuton

buton (n.poss) n.com.suff. her/his/its navel nambuton blong hem butök (n.poss) n.com.suff. my navel nambuton blong mi

butök n.com.suff. my navel nambuton blong mi see: butö

butöliaw n.com. rainbow $\sim$ renbo

butu $n$ thorn (of citrus fruits) nil (blong aranis) 0 butu möl me er na mölök. The orange thorn tore my clothes. Nil blong aranis i terem klos blong mi.

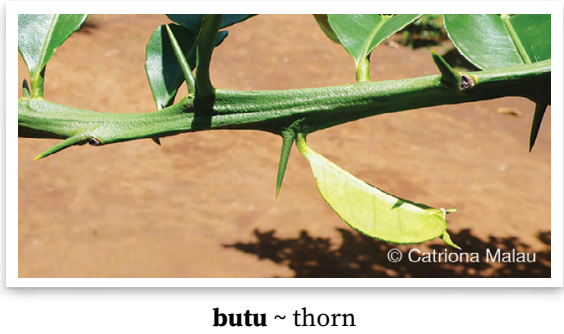

butuñ $1 \cdot n . c o m$. wall of house $\sim$ wol blong haos $2 \cdot v$. make wall of house mekem wol blong haos Iñko nēn a tōt o tere woo ta nēn a butuñ o gövur. Now we cut some bamboo to make the front walls of the house. Nao ia yumi katem ol bambu blong yumi wivim wol blong fran blong haos (RBH.022) Syn: babēris

butuñ lav (comp.) n.com. walling of house made by tying together lengths of large diameter bamboo wol blong haos we ol i fasem tugeta ol bigfala bambu

butū̄ rēg (comp.) n.com. walling of house made by tying together lengths of small diameter bamboo wol blong haos we ol i fasem tugeta ol smolsmol bambu

\section{$\mathbf{D} \sim \mathbf{d}$}

da $v t .1 \cdot$ make, do $\sim$ mekem Nēk ma da oso? What did you do? Yu bin mekem wanem? 2 - cause; occurs as initial verb in causative serial verb construction mekem Ke, nēk ma da mamarseg no. Yes, you caused me to feel bad. Yes, yu bin mekem mi mi sori. (GMM) $\mathbf{3} \cdot$ do something mean or bad to someone; mistreat, tease, hassle mekem Nēk ma da nē sur oso? Why were you mean to her? Yu mekem hem from wanem? 
da ēs $v$. resuscitate, revive, make somebody come back to life mekem laef bakegen (sapos wan man klosap i ded) Nē mi miat ti, nēr ma da ēsēs nē, mö vuv o bē lō qōtōn. She was unconscious but they revived her, they poured water over her head. Hem i bin ded be ol i mekem hem i laef bakegen from ol i kapsaedem wota long hed blong hem.

da gese $v$. do what $\sim$ olsem wanem, mekem wanem Ei, nēk i da gese tek o vo gēn? Hey, what are you doing with that thing? Ei, yu mekem wanem wetem samting ia? (RPP.030) No gētē gilal ta dōrōk a da gese. I don't know what we are going to do. Mino save se bae yumitu mekem wanem. (ADT.082)

da lagē $v$. pay bride price $\sim$ pem woman See: legleg (leg)

da lèt $v t$. break (something brittle like wood or glass) brekem (samting we i strong, olsem brans blong wud o glas) Nē ma da lēt o gasel. He broke the knife. Hem ibrekem naef. See: mēlēt da titisē $v t$. damage, affect something in a negative way $\sim$ spolem

daēl $v$ t. try traem Ni daēl talē rēv, nē mitē rēv le. She tried to pull it, but she wasn't able to pull it. Hem i traem blong pulum, hem ino save pulum. (JRQ.169) \{Lit. make see mekem luk\}

dagarē (root garē) n.com. victory leaf, ti plant $\sim$ nanggaria Cordyline terminalis, C. fruticosa $\left[\mathrm{POC}{ }^{*} k a R i(q) a\right.$ 'taxon of decorative plants']

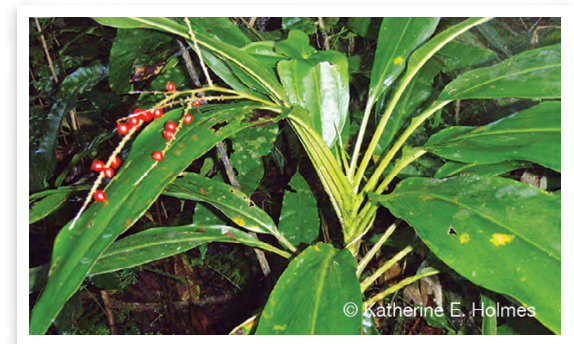

dagarē $~$ Cordyline terminalis dagawis (fr.var. diwis) n.com. young leaf of breadfruit, which is cooked and eaten as greens top blong bredfrut, blong kakae olsem kabis

dalagē n.com. payment of bride wealth $\sim$ braed praes

damalalal n.com. kind of shrub whose leaves are eaten as greens $\sim$ kaen bus kabis Syn: dimililial

damarēn̄it n.com. pandanus leaves that have dried on the branches lif pandanas we i drae nomo long stamba See: dēmērir

damat n.com. heliconia, plant with large leaves that are used to wrap laplap for cooking in stone oven $\sim$ lif laplap Heliconia indica

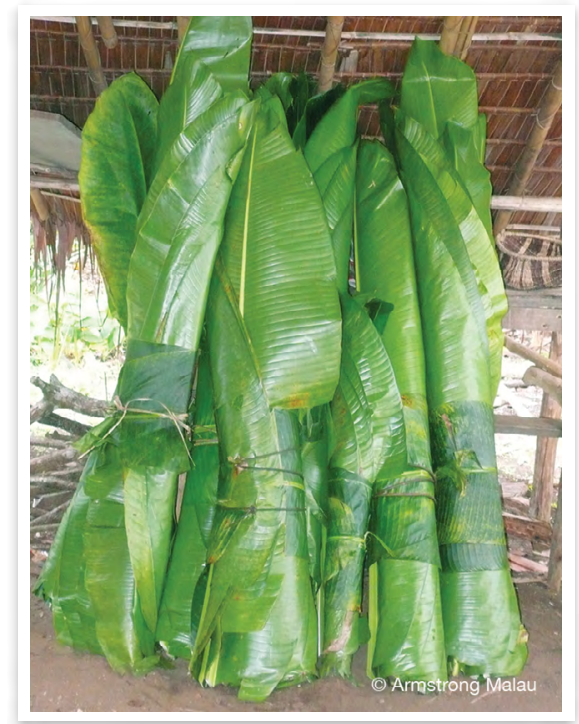

damat $\sim$ Heliconia indica

damat dēnin (comp.) n.com. variety of heliconia that has small leaves $\sim$ kaen lif laplap we lif blong hem i smolsmol

damat möt (comp.) n.com. variety of heliconia that grows wild in the bush and is not cultivated $\sim$ kaen lif laplap we i gru long bus nomo

damatmot n.com. canna lily wan kaen flaoa Canna indica 
damösur $v$. comfort, try to make someone feel better when they are upset or worried kamfotem, mekem tingting $i$ go daon sapos i sore o wari tumas La masawre $\mathbf{i}$ Sophie mō mōl, no mō lōlōlwōn nimal, Catriona ma damösur no. When Sophie went home I was really sad, and Catriona comforted me. Taem Sophie i go hom, mi sad tumas, Catriona i kamfotem mi.

damalak $n$.com. crinum lily, spider lily kaen waet flaoa Crinum asiaticum [POC * $m^{w}$ alak 'spider lily, Crinum asiaticum']

damamas n.com. kind of tree, one traditional use of which is to help crawling babies to be able to walk strongly wan kaen wud we kastom yus blong hem i blong ol pikinini we ol i wokabaot fo leg blong mekem se ol i save wokabaot strong See: wā̄amas

damataq n.com. kind of small flowering plant with tiny mauve flowers $\sim$ wan kaen plant we i gat smolsmol popel flaoa Veronia cinnerea [Liquid from leaves can be used to stop a cut from bleeding Taem wan man i katem hem, bae yu skwisim wota blong hem i go, bae i stopem blad.]

dananar n.com. Indo-Pacific sergeant, scissortail sergeant (fish) wan kaen fis Abudefduf vaigiensis, Abudefduf sexfasciatus

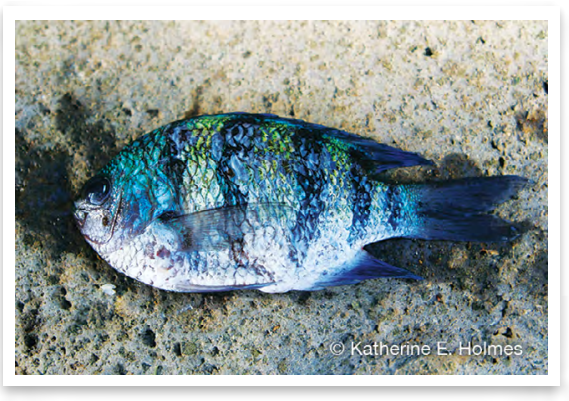

dananar $\sim A b u d e f d u f s p p$. dan̄dan̄ n.com. sugarcane variety kaen sugaken

dar $1 \cdot$ n.com. blood blad Nē mē ēl gem ke o veglal tala matien rōrō, o dar. He saw only the mark of the death of the two of them, blood. Hem i luk nomo mak blong ded blong tufala, blad. (GMM) 2 vi. bleed (blad i) ron [POc *draRa (q) 'blood']

daran (n.poss) n.com.suff. her/his/its blood blad blong hem

dere qō (n.poss) n.com.suff. pig's blood blad blong pig

dërëk (n.poss) n.com.suff. my blood blad blong mi

darag n.com. wild nutmeg nandae Myristica fatua $[P O c *(d r, d) a R a(q, k) a$ 'wild nutmeg, Myristica sp.']

darag tōwlo (comp.) n.com. kind of tree like wild nutmeg but which has larger fruit which are round wan kaen tri olsem nandae be kakae blong hem i bigwan mo i raon Myristica inutilis

daran n.com.suff. her/his/its blood blad blong hem See: dar

dasagēl n.com. plants used as decorative boundary of land $\sim$ plant we ol i yusum blong makem baondri mo blong flasem ples

dasas $n . c o m$. fern (generic) fens

dasas qötu riaw $n$.com. kind of fern wan kaen fens Angiospermum evecta [Young shoots can be taken to cure fish poisoning. Taem fis i posenem yu, bae yu kakae yangfala lif blong hem.]

dasas tie tō n.com. kind of fern wan kaen fens Spherostephanos invisus [Used to make sus tawlen where bush nuts are threaded onto the branch of the fern.] \{Lit. chicken faeces fern sitsit blong faol fens\}

dasimreg $v t$. hate, reject $\sim$ heitem, no wantem nating Jon ma dasimreg $\mathrm{i}$ ginon tasga. John rejected his wife all the time. John hem i heitem woman 
blong hem oltaem. O nötu m̄irmiar ine, nēr ga dasimreg nē tasga. That small child, they reject him all the time. smol pikinini ia, ol i stap heitem hem oltaem.

datalès n.com. flametail snapper (fish) $\sim$ kaen pulefis Lutjanus fulvus

datā̄al n.com. caricature plant, kind of shrub with edible leaves wan kaen lif we yumi save kukum mo kakae Graptophyllum pictum [There are threekinds, one has yellow leaves and two have green leaves, one of which is long, the other more oval shaped. The plant grows to about 2 metres and has white flowers. I gat tri kaen blong hem. Wan i gat yelo lif, tufala narawan, lif i grin. Wan i longfala, wan i raon. Flaoa blong hem i waet.] Spec: dömöwön

datā̄al añ (comp.) n.com. kind of shrub with edible leaves eaten as greens $\sim$ kaen bus kabis

datā̄alq̄o $n$.com. kind of tree $\sim$ wan kaen tri Pisonia grandis

datamiar n.com. small shrub with succulent stem and pale purple flowers wan kaen plant we flaoa blong hem i laet popel Coleus scutellarioides [There are two kinds, one is red and one green. It can be used to prevent pests from attacking taro. Also used to treat cracked, irritated skin between toes which is caused by excessive moisture. I gat tu kaen blong hem, wan i red, wan i grin. Hem i lif blong stopem gōsōs, wan kaen bebet we i stap spolem taro. Tu ol i yusum blong gaglētlēt, skin we i brok long leg we sofmad i mekem.]

datartarawöw n.com. bandicoot berry, kind of shrub with small round black fruit $\sim$ wan kaen plant we smol frut blong hem i raon mo i blak Leea indica [Used as a local medicine. Hem I tenge tala dar, sapos i nogat blad, dring lif blong hem.]

datawalwal n.com. kind of small tree, grows to about 3 metres, with small yellow flowers and small fruit that are red when ripe $\sim$ wan kaen tri we frut blong hem i smolsmol mo i grin be taem i raep i red, mo flaoa blong hem i yelo Phyllanthus ciccoides [Used as a local medicine for babies with breast feeding problems, and also as a treatment when children have eaten too many nuts. Hem i wan tenge sus. Givim long pikinini sapos titi i mekem hem, mo tu givim long pikinini taem ol i kakae tumas navele o nangae.]

datek $v t$. 1 • look after lukaotem Söw rōrō ma datek o mirmiar ine. Then the two of them looked after the child. Ale tufala i lukaotem pikinini ia. (KM̄T.022) 2 - have knowledge of, possess and use a certain skill or knowledge gat save long wan samting, holem wan save blong yusum Nē mo togtog so ine, ni datek o dödörug nitiwial. He stayed like that and he had a trick that he was using. Hem i stap olsem ia, hem i holem wan giaman blong mekem long man. (BQD) davaqal $n . \mathrm{com}$. kind of edible fern that grows in the water or swamp wan kaen bus kabis we i olsem fens be i gru long wota o swam Pteris sp. Syn: detewel

davarteker n.com. kind of shrub, grows to about 2 metres with tiny white flowers wan kaen plant we i gat smolsmol waet flaoa Maesa sp. [Used in taro farming to encourage the corms to grow big. Tenge tale qiat, blong mekem se taro i gru bigwan.]

deben tenēnēg n.com. kind of small plant wan kaen smol plant Melicytus ramiflorus [If one rubs the leaves on one's arm it will improve 
one's aim at shooting. Blong mekem se yu save sut stret, rabem lif long han blong yu.]

dege n.com. leaf lif Syn: dödöu

degetget n.com. kind of shrub with leaves that are eaten as greens kaen bus kabis

delē $v$. flick, as in to shake something off one's hands sekemaot, olsem yu sekem han blong mekem wota i lus

deli $n$.com. very heavy rain $\sim$ ren we i strong tumas

delti n.com. buttress root, kind of tree root that starts high up on the trunk and is wide and long, reaching down to the ground $\sim$ kaen rus blong wud we hem i stat antap long stamba mo i kasem daon long graon, olsem blong nakatambol, bluwota, siok, natavoa o namambe [Used as a carving wood, especially for wooden platters, and for flat piece of wood used to drum beat for dances. Ol i stap yusum rus ia blong mekem kaving, olsem natambia o blong kilim long danis]

dem (fr.var. dëm) n.com. yam yam Dioscorea spp. Ba lö lölö ñeñere ine wo o vetel, o qiat, o dëm, o vël sele gengen. But inside the garden there were bananas, taro, yam, every different kind of food crop. Be insaed long garen ia i gat banana, taro, yam, evri kaen kakae. (KM̄TT) [PROc *damu 'Dioscorea sp.']

dem atatīèn (comp.) n.com. primary grouping of varieties of yam, yams that bear long tubers ol kaen yam we kakae blong olgeta ol i longlongfala \{Lit. male yam man yam\} dem bōnōbōn (comp.) n.com. variety of yam belonging to 'male' grouping, that has a distinctive smell $\sim$ wan kaen yam we i longfala mo i waet mo i gat smel blong hem dem getō (comp.) n.com. variety of yam that has red tuber, with varieties belonging to both 'male' and 'female' groupings wan kaen yam we insaed i red evriwan, i gat woman kaen mo man kaen tu \{Lit. chicken's yam yam blong faol\}

dem kēlēglēg (comp.) n.com. primary grouping of varieties of yam, yams that bear crooked tubers ol kaen yam we kakae blong olgeta ol i kruked \{Lit. crooked yam kruked yam\} dem rereqe (comp.) n.com. primary grouping of varieties of yam, yams that bear short tubers ol kaen yam we kakae blong olgeta ol i sotsotfala \{Lit. female yam woman yam\}

dem ga n.com. variety of yam that is not planted but grows wild in the bush kaen wael yam blong bus Syn: wēlir

demeles n.com. general name for immature parrotfish species ol smol blufis we ol i no kam bigwan yet Chlorurus sordidus

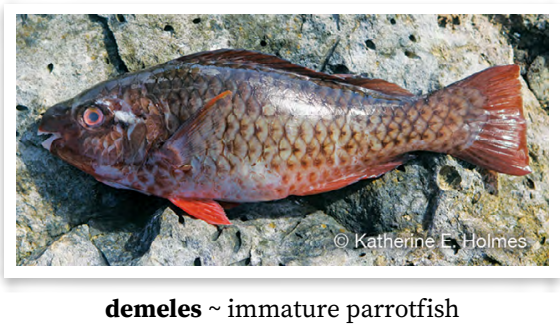

demen mav n.com. kind of shrub wan kaen plant Ficus septica [Custom stipulates that if the leaves of this plant are placed behind one's ear, they will protect anyone who goes to a place that has negative spiritual associations Putum bihaen long sora blong yu sapos yu go long wan rabis ples.] 
demete n.com. leaves fastened together and used to cover opening of stone oven ol lif we ol i fasem tugeta blong blokem ae blong oven Syn: dōrōq

den $_{1} \mathbf{1}$ - prep. ablative; from, motion away long Kemem döl a kalōlō den o plen. All of us got out from the plane. Mifala evriwan $i$ kamaot long plen. (AAD.030) 2 - prep. marking transfer or movement from a source $\sim$ aot long, kam long Nēr mē girē den nē o tere q⿳亠丷厂. They bought pigs from him. Ol i pemaot long hem ol pig. (ESP.100) 3 - prep. than, marker of standard in comparative construction aot long $\mathbf{O}$ gōsōw gö luwō den o tere taklē gōsōw gē sisia go tog lö lölö ar. The rat was bigger than some of the different rats that stayed inside the enclosure. Rat hem i bigwan bitim samfala narafala rat we $i$ stap insaed long fanis. (ESP.023) 4 • prep. of, expressing partitive relationship, of a group aot long Gö tutuqë den nēn o sul, nēn ma qaq vita nēn tē wiwieg ko. Many of us people say that we are working here. Fulap aot long yumi of pipol, yumi talem se yumi stap wok ia. (VSG.047) 5 • vt. originate, come from, be from kam long 0 kakaka ine me den o tamarge nitiwial, na sian i Wēmolo. This story comes from an old man, his name is Wēmolo. Storian ia i kamaot long wan olfala man, nem blong hem Wèrinolo. (SSW.003)

den $_{2}$ subord. lest, in case, introduces adversative clause $\sim$ nogud $\mathbf{R i}$ van, ba kōmōrōñ ri ēlgōr, den kōmōrōn̄ a mës. You two go, but watch out, in case you two fall down. Yutufala i go, be yutufala i lukaot, nogud yutufala i foldaon. (OQQ.038)

den $v$. beat to call attention, as beating on bamboo to call people to meeting kilim (tamtam, dram) blong singaotem man i kam Mam Tomas ge deñ o wōkōr lō vōrōg gēn. Papa Tomas will beat the bamboo gong this morning (to call people to a meeting). Papa Tomas bae i kilim tamtam long moning ia (blong singaot man i kam long miting). (VSJ) See: lam

dere q̄̄ n.com.suff. pig's blood blad blong pig See: dar

deteqeñtōw n.com. kind of edible fern that grows on hill bus kabis we hem i gru long saed blong hil Pteris sp. \{Lit. leaf of top of hill lif blong top blonghil\}

detewel n.com. kind of edible fern that grows in the water or swamp kaen bus kabis we i olsem fens be i gru long wota o swam Pteris sp. Syn: davaqal

devga n.com. sago palm leaf which is dry and has brown mark on it lif natanggura we $i$ gat braon mak olsem i drae

dēbit n.com. good spirit (can be of dead or live person) who gives people good things gudfala spirit, blong man we i ded o laef yet, we i save givim gudfala samting long yu

dēdērēs vi. taste good $\sim$ swit $\mathbf{O}$ tin̄i tewen gē dēdērēs. The juice from the native lychee is sweet. Wota blong nandao $i$ swit. (OQQ)

dēm n.com. childless woman, woman who has never given birth $~$ woman we i neva karem pikinini Iro vo ko nē o dēm, ba tōar wē, nē gētē dēm, na nōtōn nirō. That woman is childless, but the other one yes, she isn't childless, she has two children. Woman ia i neva karem pikinini, be narawan yes, hem $i$ karem pikinini, hem i gat tu pikinini.

dēmē conj. plus, used to conjoin numerals, thousands and hundreds and tens and units wod we i joenem tugeta tu pat blong hae namba, olsem tu handred mo twenti Qet na tasēn sā̄ul tiwial dēmē nirō. 
Qet had twelve (i.e. ten plus two) younger brothers. Qet hem i gat twelef (olsem ten mo tu) smol brata. (JRQ.008) dèmērir n.com. pandanus that has dried on the plant $\sim$ pandanas we $\mathrm{i}$ drae long stamba See: damarēnit

dēmērmēer n.com. tree fern blakpam Dicksonia spp. [Comparing dèmērmèr and qatē, démérmèr does not grow as tall; the hands grow closer together; the leaves start further from the base; and the trunk is harder to cut and not soft inside. qatē is used as a grater for tubers, but dèmérmèr is not.]

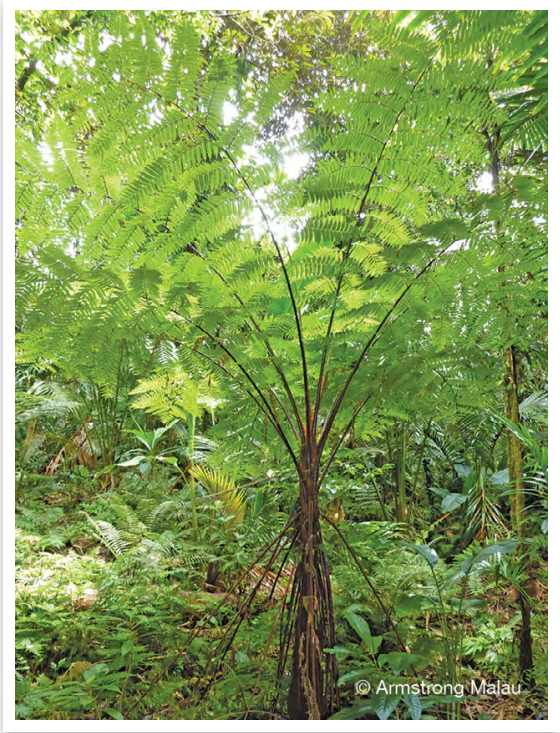

dēmērmēr Dicksonia spp.

dēmēè n.com. variety of breadfruit that has elongated fruit kaen bredfrut we kakae blong hem i longwan

dēn $v$. beat, tap lightly, bang, beating just to make a noise, rather than to make music or signal to people kilim (bambu, wud) blong mekem noes nomo Nēk i rōñteg ni dēn bilēn̄ o ak vagōrō. You hear her bang again on the canoe a second time. Yu harem hem $i$ kilim kenu bakegen nambatu taem. (ATB.033) Isē tē dēn o delti rēntenge avē? Who is banging on a tree root some place? Hu i stap kilim wud sam ples?

dēnin (fr.var. dinin) n.com. leaf like heliconia but smaller lif olsem lif laplap be i smolsmol Cominsia gigantea [Used for wrapping up food for baking. I olsem lif laplap be i smolsmol, be stil ol i yusum blong pasolem kakae.]

dēñ $\mathbf{1} \cdot v i$. arrive $\sim$ kasem Nē mē dēn̄ vitia. She arrived already. Hem i kasem finis. 2 - vt. reach, arrive at $\sim$ kasem Nè gè dēñ o ilsi. It reaches the end. Hem i go kasem en.

dēqēt $n$.com. bird's nest fern wan kaen lif we i stap gru long stamba blong wud Asplenium nidus [POc * $p^{w}$ ete 'bird's nest fern, Asplenium nidus']

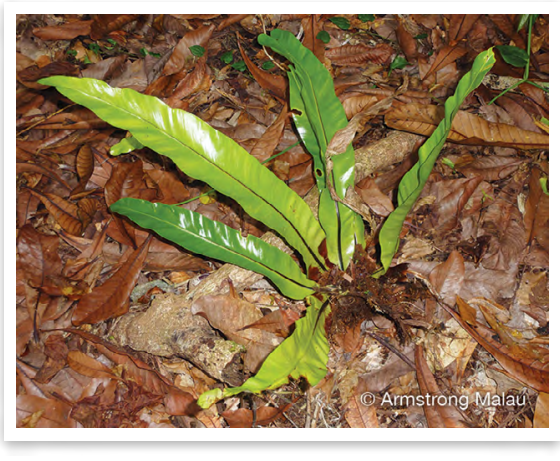

dēqēt Asplenium nidus

dēr n.com. coconut crab krab kokonas Birgus latro

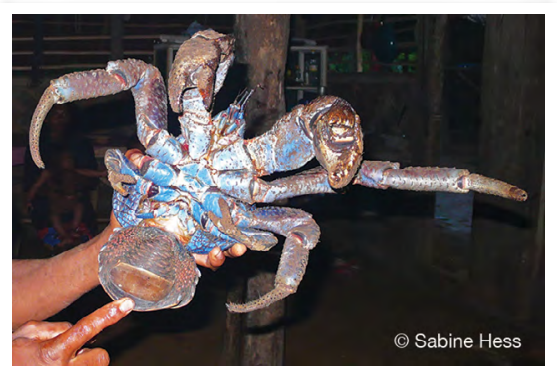

dēr $\sim$ coconut crab 
dērē 1 • vt. wait for wet long Qēt nēk kara dērē tōar masawre o ak min van me. Then you just wait for another time when the ship will come. Afta bae yu jas wet long narafala taem we sip bae i kam. (VSB.057) Syn: $\operatorname{diar} \mathbf{1} \mid \mathbf{2}$ • vi. wait wet Qet nē mē dērē dērē, oo mö möul. Then he waited and waited, oh it was a long time. Afta hem $i$ wet, $i$ wet, oo, i longtaem. (JRQ.087) Syn: diar $\mathbf{2}$

dērēn n.com.suff. her/his/its leg leg blong hem See: diri

dērēs $v t$. tear strip from edge of coconut or pandanus leaf to make narrower for use in weaving teremaot pat blong lif kokonas o pandanas sapos i bigwan tumas taem yu wantem wiv No mē dērēs o dōmōtō ko sur gö luluwō idian. I removed a strip off the edge of the coconut leaf because it was too wide. Mi teremaot pat blong lif kokonas from we i bigwan tumas. Na mōgōn o dōmōtō e gē dēdērēs si gètē dēdērēs? Have you neatened the edges of your coconut leaves or not? Yu bin teremaot ol saed blong lif kokonas blong yu blong stretem o nogat?
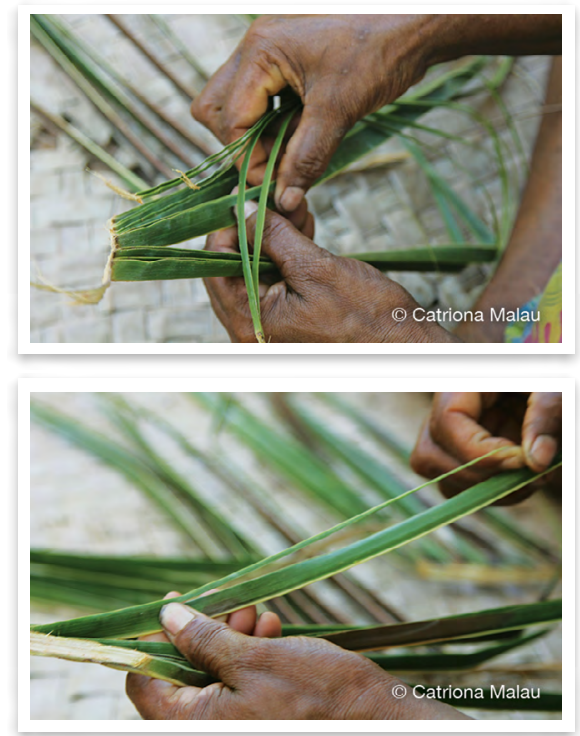

dērēs tear off edge dērivriv (fr.var. dirivriv) n.com. fan fan See: riv $_{2}$

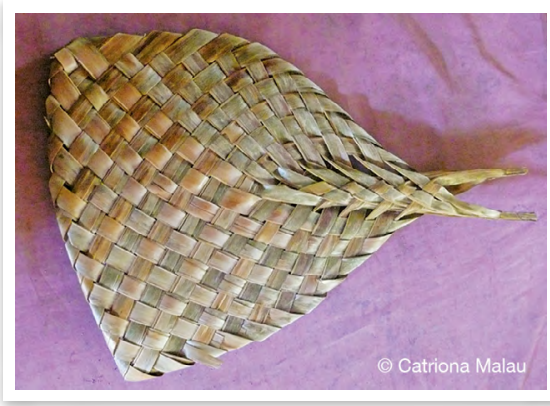

dērivriv $\sim$ fan

dērvag n.com. beach pandanus waelpandanas, wan kaen pandanas we i gru long solwota mo i save gru bigwan mo i gat longfala rus. [The fruit can be eaten. The leaves are used for traditional clothing and also for covering the roofline of houses. Leaves together with canarium sap used to make a local light. Man i save kakae nat blong hem. Ol i yusum blong wivim kastom dresing mo tu ol i save yusum blong blokem top blong haos. Ol i save yusum drae lif wetem wota blong nangae blong mekem kastom laet.] Pandanus sp. [POc *pakum 'Pandanus dubius']

dēsēn n.com. kind of tree $\sim$ wan kaen tri we i save gru eni ples [The plant has many uses in local medicine. Also used as a firewood. I gat plante yus long kastom meresin mo ol i yusum top blong lif we i red blong lif meresin. Ol i save yusum blong faeawud tu.]

dētēriv n.com. leaves of cottonwood tree that have been pinned together to produce a large leaf mat that is used to cover over food when it is baking in stone oven stikim tugeta lif blong burao o navenue blong mekem i bigwan blong kavremap kakae taem i bek long oven 
DĒVĒLAVĒL

dēvēlavēl n.com. steephead parrotfish kaen blufis Chlorus microrhinus

dēwērēt n.com. aibika variety with reddish stems and veins of leaf kaen aelan kabis we $\mathrm{i}$ gat red han blong hem Abelmoschus manihot variety $\{$ Lit. octopus leaf lif blong nawita\}

dëm (fr.var. of dem)

dëmēl n.com. ming aralia, kind of plant with edible leaves, that are yellowish green $~$ nalaslas, wan kaen tri we lif blong hem i gat ol poenpoen long saed blong hem Polyscias fruticosa, Polyscias guilfoylei [There are two species of the same genus that are given this name. Ol i save kakae yang lif blong hem olsem kabis mo hem i gud speseli blong bekem wetem mit.] Spec: mölöl kör

dër vi. rot, be rotten, as in food deteriorating or other items disintegrating roten, olsem kakae, klos, haos I rege siag ko li tōw, na gen nēr o gengen ni dërdër viteg gem. The people living up there on the hill, their food was just rotting away. Olgeta antap long hil, kakae blong olgeta $i$ stap roten nomo. (RPP) $\mathbf{O}$ qatē nē më dër den, ba o tere siri tabaē nē ge meneg. The tree fern has rotted away, but only the hard bits inside it are still strong. Blakpam i roten aot long hem, be bun blong hem nomo i strong i stap. (SBB)

dërëk n.com.suff. my blood blad blong mi See: dar

dëvër n.com. orbicular batfish wan kaen fis Platax orbicularis

diam $v i$. hang hang $\mathbf{O}$ tere gogov gē diam la ga. The clothes are hanging on the line. Ol klos ol $i$ hang long rop. No mi dimdiam ti sar me la gakōkō. I was hanging up there on the swing. Mi bin stap hang antap ia long swing.
O tibiar siag me ti diam ti. That basket is hanging up there. Basket antap ia i stap hang. See: simēdēg dimdiam (redup.) n.com. hammock, something one can hang in $\sim$ hamak dian̄ (fr.var. of odian̄)

diar $\mathbf{1} \cdot v t$. wait for $\sim$ wet long Nē go tog diar in̄ko ripot na mögun nēn. He will wait now for our report. Bae hem $i$ stap wet nao long ripot blong yumi. (VSJ.091) Syn: dērē $\mathbf{1} \mid \mathbf{2} \cdot$ vi. wait $\sim$ wet Nēk ma da qēt, in̄ko nēk i siag diar. When who have finished doing that, then you sit and wait. Taem yu mekem finis, nao ia yu sidaon wet. (SBB.055) Syn: dērē $\mathbf{2}$

diat $v i$. mushy, soft (of food that has been overcooked) sofsof kakae we $\mathrm{i}$ dan tumas, olsem raes $\mathrm{i}$ kam olsem laplap, o yam i tumas wota Kemem mo kuk o kōmar, ba kōmar mi diat. We cooked the sweet potato, but the sweet potato was overcooked and mushy. Mifala i kukum kumara, be kumara i dan tumas mo i sofsof. Sas sur gamlöt o qiat, den nē mi ditdiat. Remove the taro from the fire quickly, in case it gets overcooked. Karemaot taro long faea hariap, nogud hem i dan tumas.

diat $_{2}$ vi. make a mistake and not reach the end when performing a dance mestem mo no kasem stret en blong danis Nonorēs lē qēn̄ē vōnō i rege rereqe mi diat tek na mögun nēr o laklak. The other day on the village's Saint's Day the women didn't finish their dance properly. Bifo yestedei long dei blong vilej ol woman ol i mestem mo no kasem stret en blong danis blong olgeta.

Die n.p.fr. Mum Mama Mam tan̄ Die, no gö mörös na gunök. Dad and Mum, I want a wife. Papa mo Mama, mi wantem woman blong mi. (ATB) 
Diebum (comp.) n.p.fr. Grandma Bubu woman See: Mambum (comp. of Mam), Pōpō

die $\mathbf{1} \cdot n$.com. mother (generic) mama Maranag luwō ta Vōnō Lav, i rege maranag, i rege döl go tog oko, o m̄irmiar, o die, o mam, tan̄sar döl, i rege, kēmi ma van me den taval lam. Big chief of Vanua Lava, chiefs, all the people who are here, children, mothers, fathers, everybody, you who have come from across the sea. Bigfala jifblong Vanua Lava, ol jif, ol man we i stap long ples ia, ol pikinini, ol mama, ol papa, olgeta everiwan, yufala we $i$ kam long narafala ples. (EKK.001) 2 v t. call (someone) mother $\sim$ singaotem mama long

$\operatorname{dim} v t$. suck $\sim$ titi long No ma van ti lē dim waga. I have been to suck wild passionfruit. Mi bin go blong kakae wael pasenfrut.

dimililial n.com. kind of shrub whose leaves are eaten as greens $\sim$ kaen bus kabis Syn: damalalal

dimia n.com. kind of shrub, native mulberry wan kaen plant Pipturus argenteus [Rub the leaf on fishing line before going fishing in order to catch lots of fish. Sapos yu rabem lif $\mathrm{i}$ go long string blong yu taem yu go huk, bae yu hukum fulap fis.] [POc * qaram ${ }^{w}$ aqi 'Pipturus argenteus']

dimiarēsul n.com. aibika variety with lots of branches, eaten as greens $\sim$ kaen aelan kabis we i gat fulap han long hem Abelmoschus manihot variety $\{$ Lit. leaf many children, so called as it produces enough leaves to feed many lifblong fulap pikinini, from se i save fidim fulap pikinini\}

dinin (fr.var. of dēnin)

dinir n.com. strainer for kava sef blong kava Nēk ga taōrmat na mōgōñ o dinir, nēk ga taōrmat na mōgōñ o tabē. You prepare your strainer, and you prepare your platter (for making kava). Iu mekem redi sef blong yu, yu mekem redi natambia blong yu. (OSK) See: sēnsēn (sēn) [POc ${ }^{*}$ Runut/nuRut 'sheath around base of coconut frond, used as strainer']

dinsöw $n . c o m . \mathbf{1} \cdot$ saliva, dribble coming out of mouth, especially as with baby spet we i kamaot long maot (olsem blong bebe) Na dinsan ni ōlōl. Her saliva was dribbling out. Spet long maot blong hem i kamkamaot. 2 - slime, something that is slimy samting we $i$ glis, wota we i olsem spet $\mathbf{O}$ qiat ko o ke dinse. This taro is very slimy. Taro ia iglis.

din̄venqiat n.com. variety of taro, 'female', with a stem that is whitish at the bottom and light green at the top kaen taro we hem i woman we han blong hem i waet daon mo i grin antap

dir $_{1}$ vi. hatch (eg i) brok Na bulëk o tō mē dir. My chicken hatched. Faolblong mi ikamaot long eg.

$\mathbf{d i r}_{2} v t$. bore, tire of les long Mē dir no talo togtog oko. It bores me to stay here. Mi les blong stap long ples ia. Syn: ar

$\operatorname{dir}_{3} v$. break off $\sim$ brekem

diri n.com.suff. leg, foot $\sim$ leg

dērēn (n.poss) n.com.suff. her/his/its leg leg blong hem

dirik (n.poss) n.com.suff. my leg leg blong mi

dirik n.com.suff. my leg leg blong mi See: diri

dirivriv (fr.var. of dērivriv)

ditial $_{1}$ n.com. kind of shrub $\sim$ wan kaen plant Selaginella durvillei [The leaves are tied together to make a local broom. Also rub one's legs with the leaves when walking in the bush and it is believed that this will prevent one's legs from getting tired. Also used as a sösölö ‘taboo'; if it is put on a tree then this means that people are not allowed to take the fruit or 
nuts. Men can put the leaves in their hair when performing a traditional dance. $\mathrm{Ol}$ i fasem lif blong mekem brum. Sapos yu rabem long leg blong yu taem yu go wokbaot, bae leg blong yu i no save taed. Ol man ol i putum long hea blong olgeta taem ol i danis.]

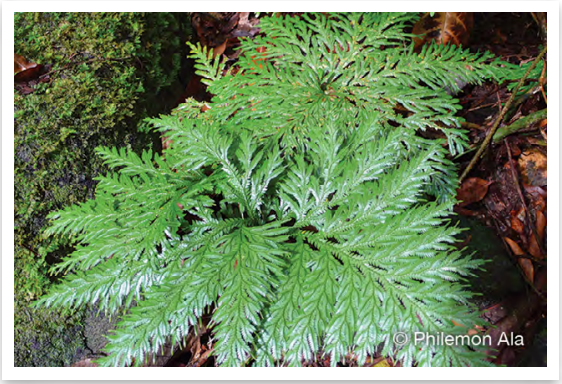

ditial Selaginella durvillei

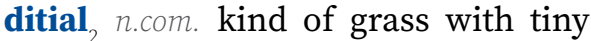
white flowers wan kaen gras we $i$ gat smolsmol waet flaoa

divivi n.com. umbrella leaf ambrela lif Licuala grandis

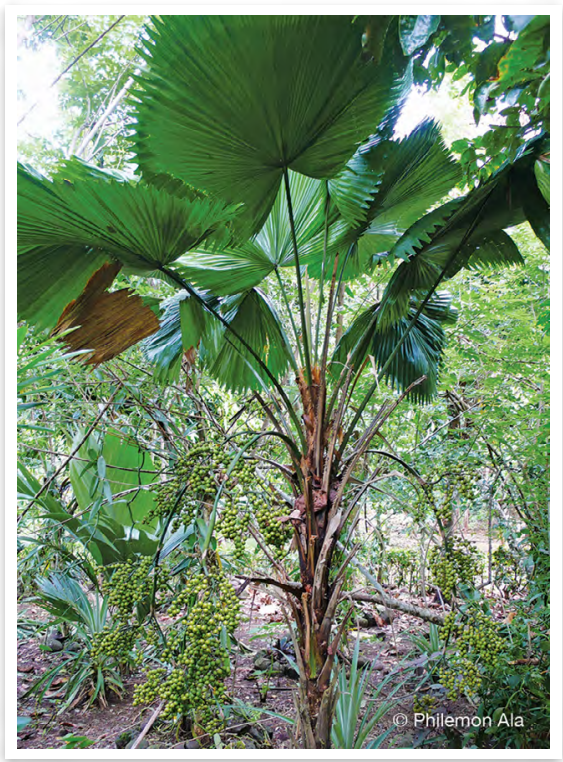

divivi $~$ Licuala grandis diwinwian n.com. kind of plant $\sim$ kaen lif Freycinetia tannaensis $\{$ Lit. rain leaf, so called because it is believed that burning the leaves will cause it to rain lif blong ren, from se ol man i talem se sapos yu bonem, bae i mekem ren\}

diwis (fr.var. of dagawis)

do n.com. kind of vine $\sim$ wan kaen rop do man (comp.) n.com. kind of leaf from a vine that is used to poison fish wan kaen kastom lif blong wan rop we ol i yusum blong posenem fish Derris elegans

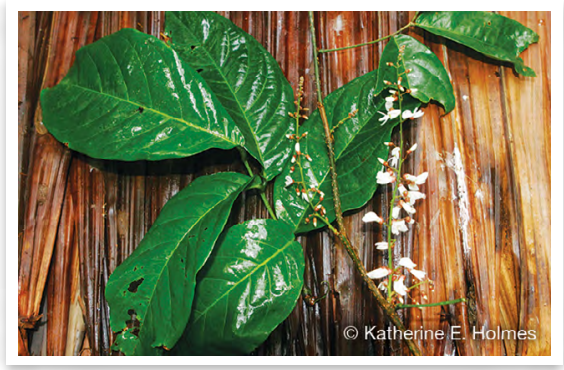

do man Derris elegans

do qōrgavgav (comp.) n.com. leaf which looks like do man that is used to poison fish, but is not poisonous wan lif we i luk olsem do man be i nogat paoa blong posenem fis

do- pre. leaf of; derivational prefix which occurs both on nouns referring to plants, to specify a leaf of that plant, and also with a limited use to produce nouns referring to useful products made with leaves, such as a fan (dérivriv) or broom (döwurwur) lif blong (wan samting), wan maka we i talemaot se lif blong wan tri o plant o wan samting we i mekem long lif, olsem fan (dérivriv) o brum (döwurwur) (Allomorphs: da-, de-, dē-, dë-, dō-, dö-) dobormiak n.com. plant with strong scented yellowish, green leaves $\sim$ lif we i gat smel blong hem we i yelo, grin Kopsia flavida [Used in dancing 
for the scent. Ol i yusum blong danis wetem from naes smel blong hem.] See: wesev

dogon n.com. variety of taro kaen taro dolē n.com. doll doli [Bis doli 'doll']

dom inter. great; interjection used by and with deaf people to express a positive reaction to something top, long lanwis blong ol def [Bis top 'great']

doot n.com. 1 - Vanuatu sago palm natanggura Metroxylon warburgii

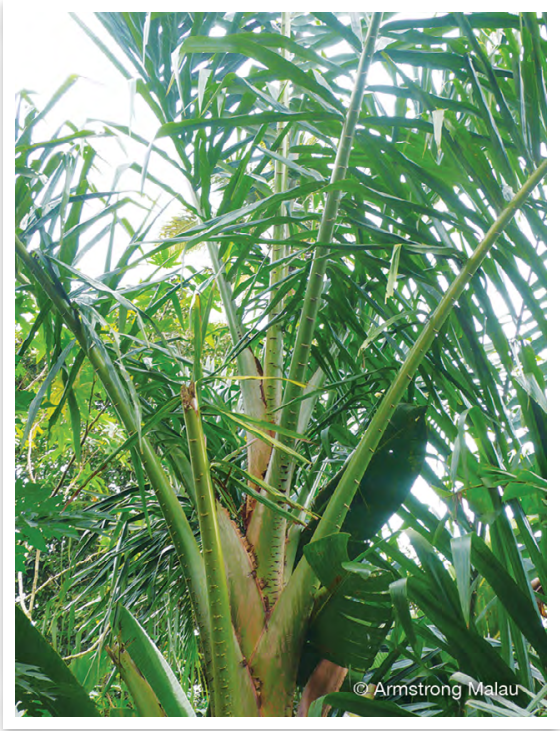

doot $\sim$ Metroxylon warburgii

2. sago palm thatch lif natanggura, taj

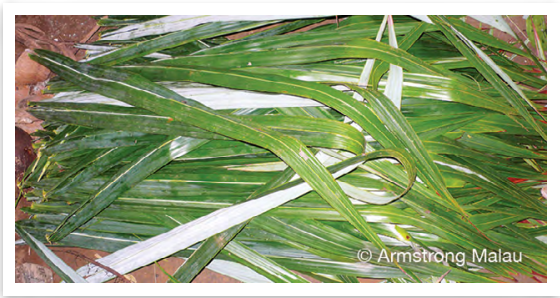

doot $\sim$ sago palm thatch

dootnönqet n.com. wild bush pandanus that grows in moist environments. Leaves have very sharp edges. waelpandanas blong bus we i laekem ples we i gat wota. Lif blong hem i sap mo i save katem yu. [There are no current uses but traditionally the leaves were used for roofing. I nogat yus tede be bifo ol i mekem ruf wetem olsem natanggura.] Freycinetia sp. \{Lit. Qet's sago palm natanggura blong Qet\}

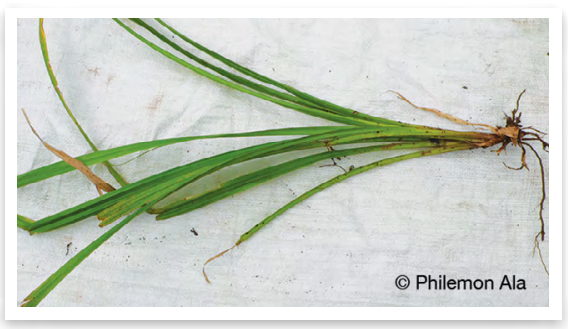

dootnönqet $\sim$ Freycinetia sp.

dordor $v$. make continuous rattling, rippling, shaking or reverberating noise $\sim$ mekem noes we i stap gohed semak $\mathbf{O}$ bē rōw me to solsol dordor wo. The river down there really makes a rippling noise as it flows. Wota daon ia $i$ stap mekem plante noes taem wota $i$ ron. Da dordor ten na mōgōñ o wēviriak e. Try and rattle your dancing rattles. Traem mekem navangge blong yu i mekem noes. Na möguk o gasel ko mo dordor. This knife of mine rattles (handle). Naef blong mi ia i stap seksek. See: dorveg

dorveg $v t$. bang together $\sim$ bangem tugeta See: dordor

dō n.com. cliff $\sim$ klif Ba o masawre ine, nē o bëtutu dō. But that place, it was a big cliff. Be ples ia hem i wan bigfala klif. (AAD)

dōdōr vi. be slow in movement or performing activity slo blong wokbaot o blong mekem wan samting Nēk o dōdōr, nēk o dōdōr. You are really really slow. Yu slo we yu slo tumas.

dōkōlōkōl n.com. kind of tree wan kaen tri Dillenia biflora $[P O c$ *kulapu(R) 'Dillenia schlechteri'] 
DŌL

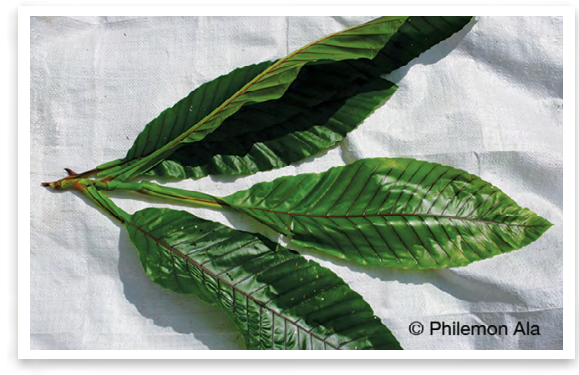

dōkōlōkōl Dillenia biflora

Dōl n.p.fr. name of mythical creation figure, enemy of the main creation figure Qet nem blong man long kastom storian

dōl vt. swallow swolem (daon) In̄ko rōrō mō dōl o garm̄ēs vita, 'Dōrōk a gengen soko.' Then the two of them salivated (swallowed down their spit) saying, 'We're going to eat now.' Nao ia tufala i swolem daon spet blong tufala, $i$ se, 'Yumitukakaenao.' (HWM) Na rem kal lö wön̄sial, o naw ni dada nim̄al, na m̄ës tēqēl, na dōl o naw, no mitë gër le. If I climb on some driftwood, and the sea is too rough, I'll fall down and swallow some seawater, and I won't be able to swim. Sapos mi klaem long wan wud we i stap flot, solwota $i$ mekem tumas, bae mi foldaon, mi solem solwota, bae mi no save swim. (WRN)

dōleg $v$. mix löt food dish after it has been pounded $\sim$ miksim nalot wetem wud afta yu bin kilim finis Mō dōleg so ine, a èl o qiat, ga qag mē qilian̄. Vilēs ga mamē. They mixed it together, and they saw that the taro, all of the white had disappeared. It was all red. Ol $i$ miksim olsem ia, ol i luk se taro, waetwan $i$ lus. I red evriwan. (ATW)

dōlgia $1 \cdot v i$. choke $\sim$ no solem daon gud kakae o wota mo i fas long trot Gen mölumlum, den nēk i dōlgia. Eat slowly, in case you choke. Kakae slosto, nogud kakae i fas long trot blong

$y u .2 \cdot v t$. choke on jok long Ëlgōr den nēk i dōlgia o siri mes. Careful, in case you choke on a fish bone. Lukaot, nogud bun blong fis i fas long trotblong yu.

dōmōtō n.com. blackspot emperor (fish) blakspot redmaot (fis) Lethrinus harak \{Lit. coconut leaf lif kokonas\}

dōmōtōtak n.com. plant related to kava but which cannot be made into kava drink waelkava Macropiper latifolium

dōmōtōtak mamē (comp.) n.com. plant related to kava with dark red leaves and branches waelkava we lif mo han blong hem i red \{Lit. red wild kava waelkava we ired\}

dōmōvōtrev n.com. kind of small tree whose leaves close in the late afternoon wan kaen tri we lif blong hem i klos long aftenun Albizzia lebbeck \{Lit. leaf sleeps afternoon lif we $i$ slip long aftenun\}

dōn n.com. earwax doti blong sora No gōtō rōn̄rōñteg wareg sur na qörök ko o dōn mö tur gōr. I can't hear properly because earwax is blocking my ear. Mi no save harem gud from doti blong sora i blokem sora blong mi.

dōn ${ }_{2}$ n.com. small dip or ditch in path that is not easily visible as the path appears flat hol we yu no luk save se hol from graon i flat nomo, be yu prumbut long hem mo foldaon insaed No ma van qal o dōn sar me le gersal. I stumbled across a small dip in the road up there. Mi wokbaot kasem wan smol hol antap ia long rod.

dōōt n.com. blind your eye, milky mangrove (tree) wan kaen tri Excoecaria agallocha [It is believed that if one burns the wood, then it will result in deafness. Sapos yu bonem olsem faeawud, bae sora blong yu i fas.] [POc *dotoq 'sticky 
liquids including the sap of (some?) trees; a mangrove tree, probably Excoecaria agallocha']

dōqōmur See: qōmur

dōr n.com. variety of wild yam strong wael yam

dōrdōrse naw n.com. tideline ples we solwota i finis long hem

dōrōk pron. we two, us two; first person dual inclusive pronoun $\sim$ yumitu

dōrōq n.com. cottonwood leaves fastened together to form a lid for covering the opening of a steam oven lid blong blokem ae blong oven we ol i mekem long lif blong burao o navenue No me mem gōr siag o mete qan̄ris, nana tër tek o dōrōq, o dege susus. When I have put it on top of the opening of the stone steam oven, I cover it up with the leaf cover, the leaves that have been sewn together. Mi putum finis antap long ae blong oven, mi kavremap wetem lif kava, ol lif we ol i bin fasem tugeta. Syn: demete See: rōq

dōrōt n.com. type of edible fern bus kabis, kaen fens we yumi save kakae Diplazium harpeoides

dōsdōs vi. 1 • coo, make noise like dove (grinpijin o sotleg) i singaot, mekem noes blong hem Vël qön̄ no gō rōñteg o malqon ni dōsdōs. Every day I hear the fruit dove cooing. Evri dei mi harem grinpijin i stap singaot. [If one hears a dove cooing like this at night, it is believed to be a sign of something, such as someone being sick or dying. Sapos yu harem noes blong pijin i singaot long naet, hem i saen blong wan samting, maet man i ded o sik] 2 - groans and sighs of sick man noes man i mekem taem hem i sik La masawre i Gavin ni los, nē o mēsi dōsdōs. When Gavin is sick he groans a lot. Taem Gavin hem i sik, $i$ stap mekem plante noes. dōsōw n.com. kind of tree wan kaen tree Geniostoma rupestre

dōsōwtöl n.com. kind of vine, ground creeper with purple flowers wan kaen rop we flaoa blong hem i popel Canavalia rosea

dōvōr nem n.com. kind of small tree $\sim$ wan kaen smol tri Vitex negundo [Useful for discouraging mosquitoes. Blong ronemaot moskito.]

dōw $v i$. (of garden) become overgrown after having been prepared for planting bus i kavremap garen bakegen taem we yu bin klinim finis No mō ōm qèt na tiqēk, no mö möul talē gē riv, e na tiqēk mō dōw kēl. I finished preparing the place for my garden, then I was slow to do the planting, then my garden became overgrown again. Miklinim finis ples blong garen blong mi, ale mi slo blong planem, ale garen blong mi i kam bus bakegen.

dōwōn n.com. variety of breadfruit that has small fruit kaen bredfrut we kakae blong hem i smolwan

döbumsër n.com. variety of breadfruit that has small fruit $\sim$ kaen bredfrut we kakae blong hem i smolwan

dödöm vt. think, think that, think about ting, ting se Nēk gö dödöm vita no gētē gilal aē. You think that I don't know about it. Yu ting se mi no save long hem. (JRQ)

dödöm tutun $v i$. think strongly, be determined $\sim$ tingting strong blong mekem wan samting $\mathrm{Na}$ gëvrun Hilton rōrō ine, si rōrō mö dödöm tutun aē, timiak ine nē mē qēt ren ti vitia. Hilton's house, if the two of them had thought strongly about it, in that case it would have been finished ages ago. Haos blong Hilton tufala, sapos tufala $i$ bin tingting strong long hem, olsem ia hem i finis longtaem finis. 
dödömia n.com. 1 - thought, thinking, idea tingting Na non o dödömia gete tenēnēg. His thinking isn't straight. Tingting blong hem ino stret. 2 • meaning mining $\mathbf{O}$ dödömia taē vita la masawre o miar ni wot me, ni miat. Its meaning is that when a child is born, it dies. Mining blong hem se taem pikinini ibon i ded.

dödöu n.com. leaf, leaf of (a particular plant) lif, lif blong (wan tri) E ni rōñteg o bē tē tirtir tēqēl lö dödöu dērvag. Then he heard the water dripping down onto the leaf of the beach pandanus. Ale hem i harem wota i drop i go daon long lifblong wael pandanas. Syn: dege

dökurut n.com. kind of wild pandanus, with yellow flower, fruit is red when ripe $\sim$ wan kaen wael pandanas we $i$ gat nil long lif blong hem Freycinetia tannaensis [Leaves are used traditionally when laying a prawn trap in the water to ensure that one catches lots of prawns. Ol i yusum taem ol i putum basket naora, blong mekem se ol i karem fulap naora long wota.]

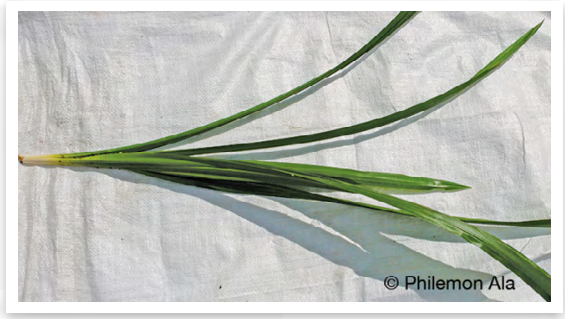

dökurut Freycinetia tannaensis

döl quant. all evriwan Kemek döl mö vugvug aē. All of us had a meeting about it. Mifala evriwan i miting long hem. (CAA) $\mathbf{O}$ gōsōw gö luwō sel den o tere gōsōw döl. The rat was bigger than all the other rats. Rat $i$ bigwan bitim evri rat. (ESP)

döm $v t$. think ting döm dērē (comp.) $\mathbf{1}$ • vt. expect ekspektem No mö döm dērē vita $\mathbf{i}$ Weten̄ nē ge tek me na gëk o qiat, ba nē gete tek me. I expected that Weteng was going to bring me some taro, but he didn't bring it. Mi bin ting se Weteng bae hem i karem sam taro blong mi, be hem ino karem i kam. No mö döm dērē nēk ta nēk ga van me, ba odiañ. I expected you to come, but you didn't. Mi bin ekspekt se bae yu kam, be nogat. 2 n.com. expectation $\sim$ tingting se bae wan samting i olsem Na möguk o döm dērē vita nē go kuk timiak gēn, in̄ko nē ma da gē sisia. My expectation was that he would cook like this, then he did it differently. Tingting blong mi se bae hem i kuk olsem ia, nao ia hem imekemi defren.

döm kēl (comp.) vt. think about, remember tingbaot Nēk gö döm kēl i vo so nēn ma kakaka ti e, nēk gö döm kēl vēti na sian? You remember that guy who we spoke with, do you still remember his name? Yu tingbaot man ia we yumi bin storian wetem hem, yu tingbaot nem blong hem yet? No ga da döm kēl nē tale le o magal. I will remind her to get the ants. Bae mi rimaenem hem blong karem anis. No götö döm kēl nēk ma qaq ti min no. I don't remember that you told me. Mino tingbaot se yu bin talem long mi. döm mav (comp.) $\mathbf{1} \cdot v t$. respect $\sim$ ting hevi, respek Nē götö döm mav ira marmarōōn. She doesn't respect her uncles. Hem i no respektem ol angkel blong hem. Gōwē nēn a döm mav i rege maranag. It is good for us to respect the chiefs I gud yumi ting hevi long ol jif. 2 - n.com. respect $\sim$ respek Kemem ma kakaka ba i Tony ta da ren ti o qōrōtōt, o döm mav odian̄ ren min nē. We were talking but Tony kept making noise, he lacks any respect at all. Mifala i bin storian be Tony $i$ stap mekem noes, inogat respeknating. 
döm siër (comp.) vt. think about, think of tingbaot ōō, ke rege burō, kōmōrōn a döm siër i maröun kōmōrōn, kōmōrōñ a van me. Oh, gee, you two, you think about your uncle, and you come. Awe, yutufala, yutufala i tingbaot angkel blong yutufala, yutufala i kam.

döm viteg (comp.) 1 • $v$. forgive fogivim Na lölök me en nēk ti am̄ō ba no mö döm viteg, in̄ko no mē qèt lōl enen nēk. I was angry with you before but I have forgiven and now I have finished being angry with you. Bifo mi kros long yu be nao ia mi fogivim yu, mi nomo kros long yu nao. I Andrew Bō më bël na bulëk o gè ba no mö döm viteg min nē. Andrew Bō stole my kava but I have forgiven him. Andrew Bō i stilim kava blong mi be mi fogivim hem. 2 - n.com. forgiveness fogivnes I Mam o döm viteg odian̄ ren min nē. Dad doesn't have any forgiveness in him. Papa $i$ nogat fogivnes long hem nating.

dömöwön n.com. variety of caricature plant, kind of shrub with edible leaves kaen bus kabis Gen: datam̄al dön̄ n.com. coconut leaf mat $\sim$ lif kokonas mat Syn: tönöam

dörug $v t$. trick, play a trick on, deceive trikim, giaman long Vita rōrō a dörug nēr, vita a tek viteg nēr lö wömötmöt. Such that the two of them were going to trick them, by taking them and losing them in the bush. Se bae tufala i trikim olgeta, se ol i tekem olgeta i lus long bus. (RPP)

dötöl n.com. kind of tree with red flowers. Birds and flying fox eat the fruit wan kaen tri we i save gru bigwan mo i gat smel. Ol flaoa blong hem i red mo ol pijin mo flaengfokis i stap kakae [Used as a local medicine, for firewood, and also for timber, as pests don't destroy the wood. Ol i yusum blong kastom meresin, faea mo timba tu from ol bebet ol i no wantem kakae.]

dötöqötun n.com. plant with stinging nettle like leaves with a serrated edge and tiny flowers wan kaen plant we hem i save kakae skin blong yu

dötösqar n.com. green (undried) pandanus leaf $\sim$ grin lif blong pandanas

dötöv n.com. bluefin trevally (fish) blu karong (fis) Caranx melampygus \{Lit. sugarcane leaflifblong sugaken\}

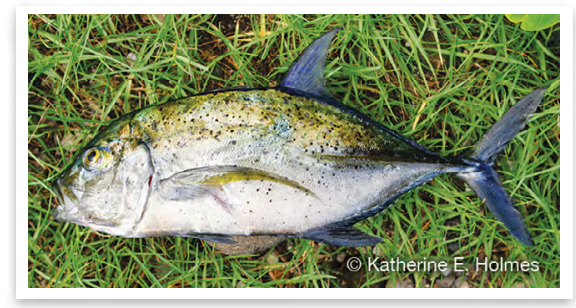

dötöv Caranx melampygus

döturkulo (fr.var. of duturkulo)

dövuln̄ö n.com. dinner plate aralia, kind of plant with edible leaves nalaslas, kaen wud we yumi save kakae lif blong hem Polyscias scutellaria

döw vi. wet, be wet wetwet Ant: mamas

döwis n.com. soft edible tips of breadfruit leaves $\sim$ top blong bredfrut See: biēg

döwurwur n.com. broom brum Nēk i le o döwurwur, nēk i wur waliög den o kikse um. You get a broom, and you sweep around the edge of the stone oven. Yu karem brum, yu brum raon long saed blong oven. (JNN) Syn: wōtōgsiar See: wur \{Lit. sweeping leaves lifblong brum\} 


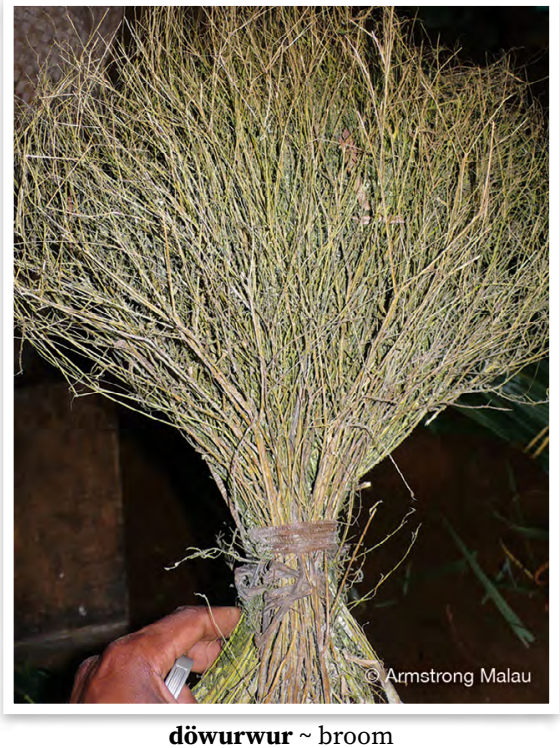

dun $1 \cdot v i$. true, be true, real $\sim$ tru $\overline{0} \bar{~}$ odian̄, gö dun, no no tek o döbit. No, it is true, I have a spirit with me. Nogat, hem i tru, mi stap wetem wan spirit. (EDM) Ōō, ika, o timiat dun soko. Oh, my dear, it is a real devil. O, mae dia, hem i wan tru devel ia. (OQQ) 2 vi. sure, tell truth $\sim$ sua, tru Nēk gö dun vita o ak gē dēñ me tōlōw? Are you sure that the ship is coming tomorrow? Yu sua se sip bae i kasem tumora? $\mathbf{3} \cdot n$.com. truth tru Talē nēn a da o dun ni rōw tur, ni ès. So that we make the truth come out and come back to life. Blong yumi mekem wanem i tru hem i stanap, i laef. (EKK.102)

dun̄ög $v$. leave something with someone, leave something behind putum wan samting, livim i stap (olsem se bae yu jas kambak from o man i karem i kambak o wan i kam from) Wölus, nonor i Kali mö dun̄ög viteg ti min no o bibis ga wo ni van den a Sanleñ. Sister-in-law, yesterday Kali left a roll of string with me and went to Sanlang. Tawian, yestedei Kali i bin livim wan rol string $i$ stap wetem mi mo i go long Sanlang. O tōar kartō̄ no mö dun̄ög viteg ti a Mēèsēn, tōlōw no kara kèl sur. I left the other carton at Mosina, tomorrow I will go back for it. Narafala Katon mi livim i stap long Mosina, tumoro mi jas gobak from.

dunregōsōw n.com. kind of tree wan kaen tri [Used as a local medicine to mend a broken tooth. Wan kaen tri we hemi meresin we yu save yusum blong fiksim wan tut sapos i brok.]

dunremēēèg n.com. kind of plant, shrub wan kaen wud Leucosyke australis

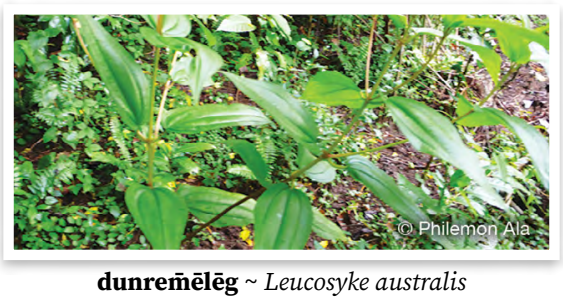

dun̄ vt. bang, beat, bang to keep rhythm, bash kilim, bangem

dun̄bönö n.com. Java cedar, bishop wood (tree) nakoka Bischofia javanica

dur $v t$. $\mathbf{1} \cdot$ stab, poke hole in something stikim Nē mö dur mi miat o qō tek o gasel. He stabbed the pig to death with a knife. Hem i stikim pig $i$ ded wetem naef. $\mathbf{2} \cdot \operatorname{dig}$ hole $\sim$ digim hol Nēk tö durdur o qarörö sur o so? What are you digging a hole for? Yu stap digim holblong wanem? $\mathbf{3} \cdot$ hollow out inside of canoe digimaot insaed blong kenu Nēn a dur o ak tek o vidëm. We will hollow out the canoe with an adze. Bae yumi digimaot insaed blong kenu wetem aj.

dutgōr $v$. tie knot mekem not, fasem joen long rop o fasem taet long en blong hem

duturkulo (fr.var. döturkulo) n.com. variety of aibika, shrub with leaves which are commonly eaten as vegetable greens kaen aelan kabis Abelmoschus manihot variety 


\section{$\mathbf{E} \sim \mathbf{e}$}

$\mathbf{e}_{1}$ conj. then, so; conjunction used to join coordinate clauses ale Nē ni vus mi miat i ginon, e ni tog masgian ti. He killed his wife, and then he lived alone. Hem i kilim woman blong hem i ded, ale hem i stap hem wan.

$\mathbf{e}_{2}$ dem. 1 - that, distal modifier; as a modifier of locationals, indicates a greater distance $\sim$ ia longwe $\mathbf{~ N i}$ rem kal, reerem ni dēñ ren siag e. He climbed up, climbed way up until he reached right up high. Hem $i$ klaem antap, klaem go go i kasem antap olgeta. (ATW) 2 - emphatic demonstrative, when modifying pronouns, specifies emphasis $\sim$ ia nao Nēk so mö vus sër nēr e, nēk tö vusvus nēr e. It was you who killed them, you are killing them. Yu nao yu bin kilim olgeta ia, yu stap kilim olgeta ia.

e'e inter. yes yes Syn: ke

ei inter. hey ei

el $v i$. dive down $\sim$ daeva i go daon, stap antap long sefes mo go daon Nēk i el tēqēl sōw e, nēk i vēn o terit. Dive down and shoot the porcupinefish. Yu daeva i go daon, yu sutum nilfis. See: qōt

elel vi. 1 • high, high up, tall hae, antap $\mathbf{O}$ tōw ine nē ge elel, $\mathbf{o}$ malēg gō rōw siag aē. The hill was high, and there were clouds sitting on top of it. Hil ia hem $i$ antap, mo i gat klaod i sidaon long hem. (DHN.005) $\mathbf{O}$ mal nē ma gav elel kal siag e la maē. A hawk flew high up in the sky. Namala i flae hae antap long skae. (DHN.023) 2 • important impoten $\mathrm{Na}$ sian mö luwō, me elel. His name became big and important. Nem blong hem i kam bigwan mo ikam hae. (ESP)

elñan n.com.suff. her/his/its voice voes blong hem See: eln̄e elñe n.com.suff. $\mathbf{1} \cdot$ voice $\sim$ voes Iñko $\mathbf{i}$ Mōlgon ni töqul kal na eln̄an, ni teñ. Then Mōlgon lifted up his voice, and cried. Nao ia Mōlgon i sakem voes blong hem $i$ go antap, hem i krae. (GMM) $\mathbf{2} \cdot$ words, opinion presented, what someone has said $\sim$ toktok folem tingting blong wan man Ba nē gōtō rōñteg na eln̄en i tëmëk. But he didn't hear my father's words. Be hem i no harem toktok blong Papa blong mi. (KM̄T.180)

eln̄an (n.poss) n.com.suff. her/his/its voice voes blong hem

eln̄ëk (n.poss) n.com.suff. my voice voes blong mi

elnetiti n.com. style of dance that is performed at the ceremony marking one thousand days since someone's death wan danis we ol man ol i danis long wan taosen dei blong wan ded man. Ol man ol i danis blong soemaot se ol i glad mo ol i stat blong fogetem

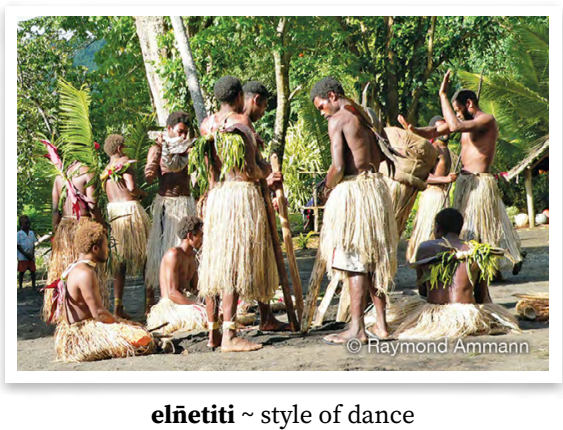

eln̄eul $n$.com. explanation $\sim$ toktok we i mekem i klia $\mathbf{O}$ elñeul nē ga mas taoseg. The explanation must be clear. Toktok we $i$ talemaot gud hem $i$ mas klia.

eln̄evu n.com. traditional story kastom storian Iñko nana gagneg o eln̄evu tale me. Now I am going to tell a traditional story about a sea snake. 
Nao ia bae mi talem kastom storian blong sisnek. (ESS.001) \{Lit. voice of the spirits voes blong ol spirit\}

eln̄ëk n.com.suff. my voice voes blong mi See: eln̄e

elvet $v i$. long, be long long, longfala $\mathrm{Ba}$ o gersal ine ge elvet idian. But the road was very long. Be rod ia $i$ longwan tumas. (AAD) Ant: qëtutu $\mathbf{1}$

en $v$. flatten out löt $\bar{n} e$ mereñ food dish

flatem kaen nalot ia löt ñe mereñ

en $_{2} v$. be angry, cross (with) kros (long) Na lolon me en no. She was cross with me. Hem i kros long mi.

er 1 t. peel off, remove pandanus leaves from trunk, cut around and peel off layer of wood from tree trunk karemaot pandanas long stamba Nesta, garqe in̄kē o lō gōwē, nēk i er na mōgōñ o videge. Nesta, today it is sunny, you peel off your pandanus leaves. Nesta, tede i gat gudfala san, yu tekem lif pandanas blong yu. [Used to describe way of removing pandanus leaves and Tahitian chestnuts from the plant. Blong talem se tekem pandanas o namambe long stamba.] $\mathbf{e r}_{2} v$. bite $~$ kakae

es $v t .1 \cdot$ spear $\sim$ sperem $2 \cdot$ pierce, poke, use sharp pointed object to pierce something stikim, yusum wan samting we i sap long en blong hem blong stikim wan samting Akē, bulsal, nēk me es na kērēk sur oso? Ow, friend, what did you pierce my bottom for? Awei, fren, yu stikim as blong mi from wanem?

es do (comp.) v. pound leaves for making fish poison $\sim$ mekem posen lif, sperem lif wetem wud blong mekem plante

es mōtō (comp.) v. remove husk from coconut sperem kokonas blong karemaot skin
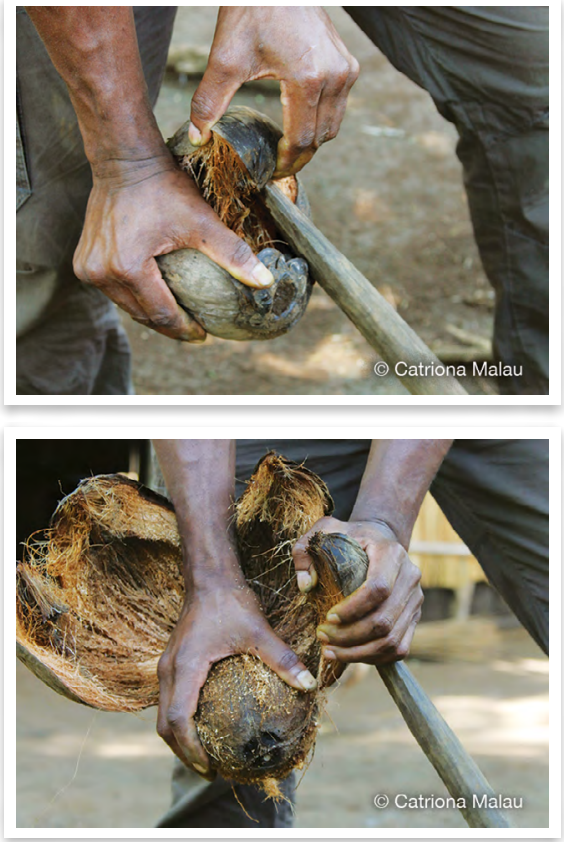

es mōtōo husk coconut

ete n.com.suff. soul sol Nēk gōtō mōlmōl la tatar, in̄ko na atan̄ mi miat. You don't go to prayer, now your soul is dead. Iu no stap go long prea, nao ia sol blong yu i ded. [POc *qata(r) 'image, reflection, soul, spirit']

atan (n.poss) n.com.suff. her/his/its soul sol blong hem

ëtëk (n.poss) n.com.suff. my soul sol blong mi

$\mathbf{e v}_{1}$ n.com. $\mathbf{1}$ fire $\sim$ faea Nēk mē ēl vita in̄ko rivteg o ev ni nōn. You see that now the fire has nearly died down to coals. Yu luk se nao ia klosap faea i go daon. (JNN) $\mathbf{2} \cdot$ hell hel Kēmi gō mōl le ev. You are all going to go to hell. Bae yufala igo long hel. [POc *api 'fire']

$\mathbf{e v}_{2} v t$. use digging stick to loosen taro, after pull out of ground $\sim$ yusum wud blong mekem graon i sofsof, mo pulumaot taro long graon Nēn a van a ev o sinag. Let's go and remove the taro from the ground with a digging stick. Yumi go karemaot taro wetem wan wud. 


\section{$\overline{\mathbf{E}} \sim \overline{\mathbf{e}}$}

èl 1 • vt. see luk Kōmōrōk mē èl o wuw mes. The two of us saw lots of fish. Mitufala i bin luk fulap fis. (AAD) 2 • vi. look luk Rōrō gē ēl kēl vē? In which direction are the two of them looking? Tufala i stap luk long wanem daerekson? (M3E) Nēk i ēl ris. You look behind. Yuluk bihaen. (ATB)

èlēl (redup.) 1 • n.com. view, vision, insight, opinion lukluk lē èlēl na mōgōn timiak avē nēn gō tōtōw in his view about how we are going to progress long lukluk blong hem se olsem wanem bae yumi kam antap (VSG) $\mathbf{2} \cdot v$. light $\sim$ laet

$\overline{\mathbf{e}} \mathbf{l}$ rak $v t$. recognise, realise (by seeing) $\sim$ luk save Nē mē ēl rak o masawre kōmōrōk to tog ti aē. She recognised the place where we were. Hem i luk save ples we mitufala i stap long hem. (AAD) ēlgōr 1 - vt. look after, care for lukaotem No mē ēlgōr nē gaidēn̄ o to nitiwial. I looked after her for a year. Mi lukaotem hem kasem wan yia. (CAA) 2 - vi. watch out, be careful lukaot Nēk i ēlgōr, den ta nitiwial ni m̄ës sōw, ni bōl lö kulë gevrōn, nē ni rōn̄teg nēk. You be careful, in case one falls down and bangs the roof of the house, and he hears you. Yu lukaot, nogud se wan i foldaon, i bangem top blong haos, hem i harem yu. (JRQ)

ès $1 \cdot v i$. alive, be alive laef No gō rōñteg vita no miti ēs. No gē miat. I can feel that I am not going to live. I am going to die. Mi harem se bae mi no laef. Bae mi ded. (GMM) 2 - n.com. life laef (blong) Nēn a èlēl vita o ès nē ni mavmav. We can see that life is difficult. Yumi luk se laef hem i had. (VSV)

ōsōn (n.poss) n.com.suff. her/his/its life laef blong hem

usuk (n.poss) n.com.suff. my life laef blong mi

ēwatē n.com. wooden sticks used to join crossboom to outrigger boom of canoe and hold it in place $\sim$ smol wud we i nilim wud i go long nasama blong kenu blong holem taet

\section{$\ddot{\mathbf{E}} \sim \ddot{\mathbf{e}}$}

ër n.com. casuarina, she-oak, beefwood, ironwood, whistling pine oktri, siok Casuarina equisetifolia [POc *aRu 'a shore tree, Casuarina equisetifolia']

ërlam n.com. sea fan samting blong solwota we i luk olsem wan fan Plexauridae spp. \{Lit. casuarina from the deep sea oktriblong dipsi\}

ërmamē n.com. variety of taro, with a light red stem with white stripes kaen taro we han blong hem i laet red mo i gat waet straep \{Lit. red casuarina red oktri\} $\ddot{e ̈ s}_{1} \mathbf{1} \cdot n . c o m$. smoke $\sim$ smok $\mathbf{2} \cdot v i$. smoke $\sim$ smok Na ukuëg o ev ni ës ti. I leave the fire to smoke. Mi lego faea i stap smok. (NBQ.005) [POC *qasu 'smoke']

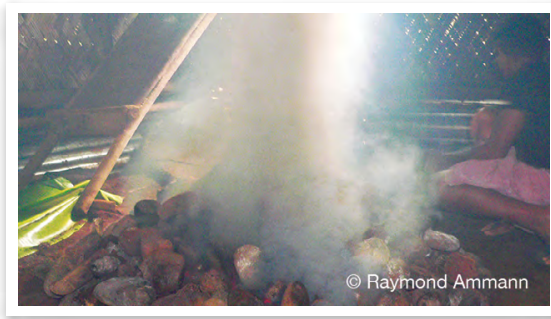

ës smoke 
ës n.com. song singsing Nēk gō rōnteg i rege tö sursur o ës e? Can you hear the people singing that song? Yu harem ol man ol $i$ stap singim singsing ia? (BTB.052)

$\ddot{e ̈ s}_{3} v$. thick (of bush) fasfas No ma van lö möt ba o masawre gë ës idian, no mita van veselo le. I went to the bush but the bush was too thick and I couldn't get through. Mi go long bus be ples ifasfas tumas, mi no save gotru. ët $n$.com. tuna (fish) tuna (fis)

ëtëk n.com.suff. my soul sol blong mi See: ete

ëtëtlam n.com. variety of banana that has a very tall trunk with small fruit growing in a long bunch kaen banana we stamba blong hem i gru hae bitim ol banana, kakae blong hem i smolsmol mo bandel i longfala

\section{$\mathbf{G} \sim \mathbf{g}$}

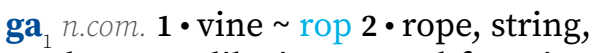
other rope-like items used for tying $\sim$ rop Nēk i le o ga, nēk i viris. You get a rope, and tie it up. Yu karem rop, yu fasem. (EDM) [PNCV *kaRo 'vine, rope; vein']

ga $_{2}$ vi. burn up, burn out of control bon nogud $\mathbf{O}$ ev ma ga lö möt. The fire burnt out of control in the bush. Faea ibon nogud long bus.

ga atat (fr.var. ga qir) n.com. climbing cane-like vine wan rop we ol i yusum blong fasem haos Flagellaria indica [Used to tie thatch to bamboo in making roof of house. Ol i yusum blong fasem natanggura.] \{Lit. vine for tying thatch rop blong fasem natanggura\}

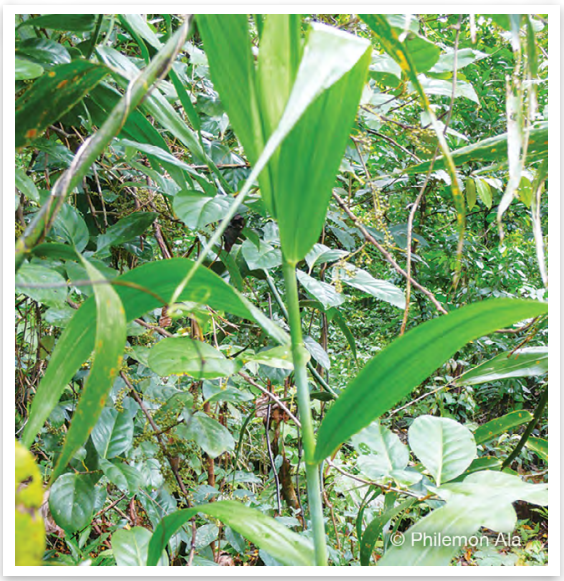

ga del n.com. kind of large leafed vine that grows up and wraps around trees wan kaen rop we i gru antap long wud we lif blong hem i bigwan Epipremnum pinnatum [Used to stop blood flow from a wound and to heal skin sores. Ol i yusum kastom lif blong blokem blad sapos i ron tumas mo blong soa (tenge talō mōnōg).]

ga dōvōr $r_{1}$ n.com. vine, creeper that grows on beach, seashore wan kaen rop we i stap gru long sanbij mo klosap long solwota Ipomoea pescaprae [It is believed that if someone wants to make the sea rough, they should uproot the plant and throw it into the sea. Sapos yu sakem lif blong hem long solwota, bae i mekem solwota i raf.]

ga dōvōr ${ }_{2}$ n.com. variety of sweet potato which is named for plant of same name, as it has large leaves like that vine $~$ kaen kumala we i gat bigfala lif

ga iaw n.com. kind of vine wan kaen rop Anodendron paniculata [It is used for making fishing nets as it is very strong. Ol i yusum insaed blong rop blong mekem net from i strong we i strong olsem naelon.]

ga matawasē n.com. kind of vine that grows straight and is white when immature and light brown when 
mature wan kaen rop we hem $\mathrm{i}$ smol nomo mo i save gru stret. Taem i yang i waet mo hem i kam laet braon Stephania forsteri [It is used as a local medicine and it is very bitter. $\mathrm{O} 1 \mathrm{i}$ yusum blong lif meresin mo i konkon we.] \{Lit. shrikebill vine rop blong wan kaen pijin\}

ga mien n.com. kind of vine wan kaen rop Epipremnum sp.

ga $\mathbf{o} \mathbf{l}$ n.com. kind of vine $\sim$ wan kaen rop

ga qir (fr.var. of ga atat)

ga sasin̄irnir n.com. kind of vine wan kaen rop Cayratia trifolia

ga susul n.com. morning glory; kind of vine with blue flowers wan kaen smolsmol rop we i gru long garen mo long haos be no long bus Ipomoea acuminata [Used as local medicine for problems with breastfeeding babies. Hem i kastom meresin blong pikinini (tenge talö sus).]

ga taqav n.com. kind of vine with large leaves wan kaen rop we lif blong hem i bigwan Merremia peltata [Used as medicine for fish poisoning. Main use for tying up firewood bundle. Ol i yusum blong fasem faeawud, mo tu olsem lif meresin blong fis i posenem yu.]

ga töus n.com. kind of vine that bears tubers similar to cassava wan kaen rop we i gat kakae blong hem olsem maniok [It is bitter and so only eaten in times of drought and famine. The leaves are also used as local medicine for coughs. Hem i konkon, mekem se ol i kakae nomo sapos san i strong mo kakae i nogat. Lif hem i wan gudfala kastom meresin blong kof.]

ga vörur n.com. kind of vine $\sim$ snek rop [The vine is used as a local medicine. The seeds are used as a decoration and percussion when dancing. The vine is used for making children's swings. Wan kaen rop we ol i yusum blong kastom meresin. Ol i yusum sel blong sid blong danis mo ol i yusum rop blong mekem swing blong pikinini.]

ga wöböw (abbrev. of wöböw)

gabal n.com. long wooden tongs used for picking up hot stones or charcoal from stone oven wud blong karemaot ston long oven See: bal 1

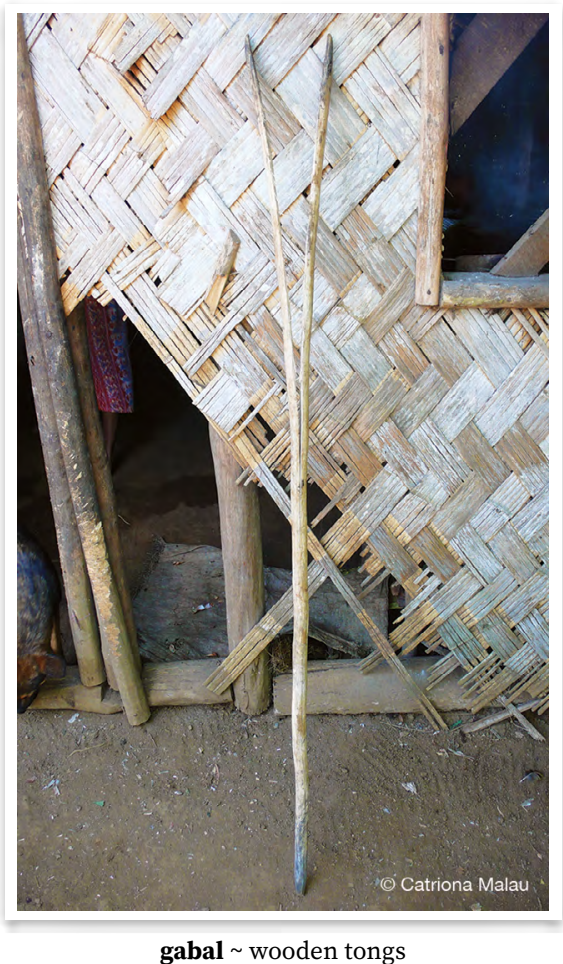

gabala mōgōn n.com. her/his/its vein string blong blad blong hem

gabelte $n . c o m$. belt $\sim$ strap

gabōōn n.com.suff. its wing wing blong hem See: gaböu

gaböu n.com.suff. wing wing gabōōn (n.poss) n.com.suff. its wing wing blong hem

gaböuk (n.poss) n.com.suff. my wing wing blong mi

gaböu tō (n.poss) n.com.suff. chicken wing jikinwing, wing blong faol 
gaböuk n.com.suff. my wing wing blong mi see: gaböu

gadödö qaqaq n.com. original, oldfashioned way of speaking olfala fasin blong toktok

gag vt. rip, peel off karemaot, teremaot Gag rieg o plasta e. Rip off that bandaid. Karemaot plasta ia.

gagarēn n.com. aerial roots $\sim$ rus we $\mathrm{i}$ gru antap

gagneg $v t$. tell, say to $\sim$ talem $\mathbf{O}$ gēnē nitiwial no mō rōñteg nēr ma gagneg min no. One thing that I heard them tell me. Wan samting we mi harem se ol i talem long mi.

gagö n.com. $\mathbf{1} \cdot$ stem or runner of vine longfala gru blong rop $2 \cdot$ string $\sim$ rop $\mathbf{3} \cdot$ handle $\sim$ handel $\mathbf{4} \cdot$ strap $\sim$ strap

gagö q⿳亠丷厂 (comp.) n.com. rope for tying up pig rop blong fasem pig No mē vir o gagö qō. I plaited the rope for tying up the pig. Mi tanem rop blong fasem pig.

gagö vus (comp.) n.com. string of bow rop blong bonaro

gagö butö n.com.suff. umbilical cord rop we i joenem nambuton i go long mama

gagönus n.com. taste very good i swit we i swit No me gen qal o kek ko, o gagönus. I tasted this cake, it's very tasty. Mikakae testem kekia, i swit we. gaidē̄n subord. until kasem

gakalkalbōgō n.com. jackbean; kind of flowering vine wan kaen rop Canavalia cathartica

gakarēs n.com. kind of climbing fern wan kaen rope Lygodium reticulatum [Used in weaving baskets to give colour to the basket. Blong wivim basket, blong givim kala long hem.]

gakōkō n.com. swing made from rope swing See: kōkō

gal $v t$. make rope by twisting strands together twistim tugeta gala n.com. left lefsaed $\mathbf{O}$ diri reqe e, o mōtō lö lölö gövur, wo o gala la varē. The woman's legs, the right one was inside the house, and the left one was outside. Leg blong woman ia, raetsaed $i$ stap insaed long haos, mo lefsaed i stap aotsaed. (LLF) Ant: mōtō $_{2}$ galatentur n.com. variety of taro that has white tuber, skin and stem kaen taro we hem i waet evriwan, kakae wetem han mo skin tu i haf waet

galēm n.com. long wooden pole used for evenly spreading charcoal and heat of stones in stone oven $\sim$ wud blong miksimap jakol mo ston long oven

galō n.com. silver croton wan kaen tri Croton insularis [The branches are used for making spears as they are very straight. Also used for firewood. Ol i yusum blong mekem spia wetem yangwan blong hem from we i stret gud. $\mathrm{Ol}$ i save yusum blong faeawud tu.]

gamalwuw n.com. kind of black vine $\sim$ wan kaen rop we i stap long bus mo hem i blak Smilax vitiensis [One of the main vines that is used for weaving food baskets, as it is strong. Ol i yusum blong mekem basket blong kakae from hem i strong.]

gamar n.com. mottled lightfoot, rocky shore crab wan kaen krab we i stap long so Grapsus albolineatus Syn: giav tamal

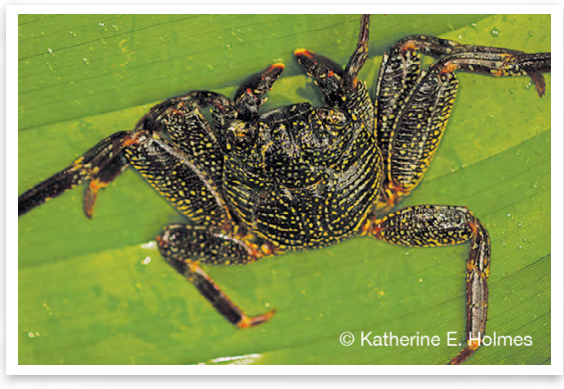

gamar Grapsus albolineatus 
gamērēr n.com. kind of vine wan kaen rop Freycinetia spp. [There are two varieties. Both varieties have common uses in weaving and house building. I gat tu kaen blong hem we ol i yusum blong wiv mo blong mekem haos. Red wan ol i yusum blong fasem top blong haos.]

gamērēr dēmadēm (comp.) n.com. kind of vine $\sim$ wan kaen rop Freycinetia flavida

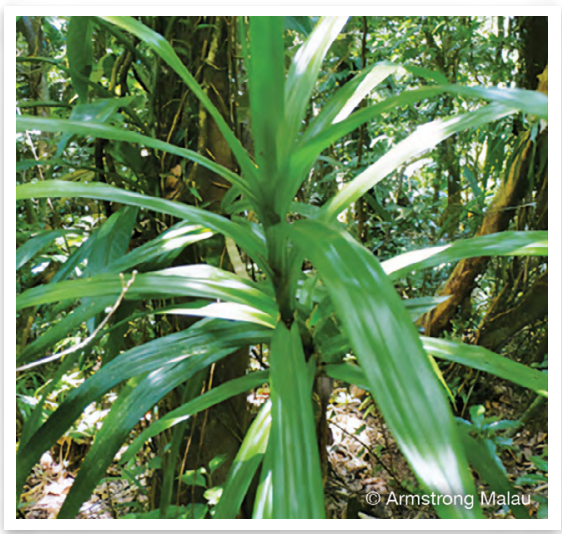

gamērēr dēmadēm Freycinetia flavida

gamērēr qō (comp.) n.com. kind of vine wan kaen rop Freycinetia impavida [Used to weave prawn traps. Ol i yusum blong wivim basket blong naora.]

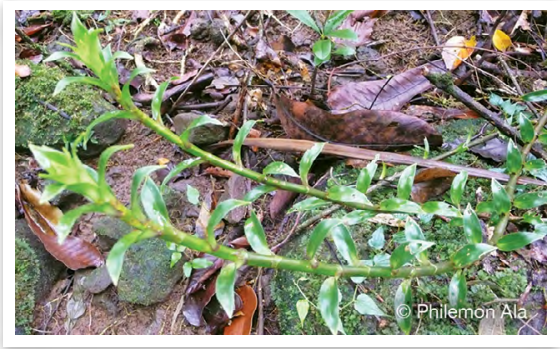

gamērēr qōo $~$ Freycinetia impavida

gamlöt $\mathbf{1} \cdot a d v$. quickly hariap, kwiktaem Nē gö mörös ta o lō ni tul gamlöt. He wanted the sun to set quickly. Hem $i$ wantem se san i draon kwiktaem. (LLF) 2 • vi. be quick, hurry up go hariap Nēk ma gamlöt sur oso? Why were you hurrying? Yu hariap from wanem?

gā̄esel n.com. midrib, midvein of Heliconia indica leaf, when used for tying up food parcel, etc. bun blong lif laplap we ol i yusum blong fasem kakae La masawre nēk i nin o damat, nēk i mem wurveg ti o gam̄esel. When you remove the midribs from the heliconia leaves, make sure that you put aside the midribs. Taem yu karemaot bun blong lif laplap, yu putum gud bun blong hem. (VVAMVDP04Jun0801-JN.027) gan $\mathrm{cl}$. her/his/its (food) blong hem (kakae) See: ge

gan men n.com. species of fig tree which has fruit that is orange when ripe, growing close to the branch wan kaen tri we hem i famle blong nambangga, taem i raep, frut blong hem $i$ aranis Ficus tinctoria [Fruit is commonly eaten by birds. $\mathrm{Ol}$ pijin ol i stap kakae frut blong hem.]

ganar n.com. rope made from cottonwood which one hangs around the neck as a sign that one has given up a particular type of food as a sign of respect when a close relative has died rop we man i hangem long nek blong hem blong soemaot se hem i givap wan kaen kakae taem mama o papa blong hem i ded See: nar, siganar

gon ganar (comp.) $v$. tie a rope around ones neck as a symbol to indicate that one is mourning the death of ones father or mother, the rope also indicates that one is giving up a particular food for the mourning period taem we man i fasem rop long nek blong hem blong soemaot se hem i givap wan kaen kakae taem mama o papa blong hem i ded

ganasēg n.com. freckled porcupinefish, black-blotched porcupinefish nilfis Diodon spp. 
gan̄veg $v$ t. lead lidim Nē ga gan̄veg nēn lö vugvug gōwē. He will lead us so the meeting is good. Bae hem ilidim yumi long miting i gud.

gaqō thick tik

gar $v$ t. bite kakae wetem tut No mē viris o genqirēg ine tala ga da vita mita gar le o tan̄sar. I tied up the dog so that it couldn't bite people. Mifasem dog ia blong mekem se i no save kakae man. [POc *kaRat 'bite']

gar bētin (comp.) $v$. clench teeth satem taet tut mo no openem maot

garaq $v i$. new niu Nēn a da qētqēt o gövur gōrōn̄ na mögun nēn gagaraq. We will finish making our new church. Bae yumi finisim niufala jos haos blong yumi. (VSG) [POc *ka(l,r)abwa 'new']

garat n.com. food scraps (e.g. taro skin, fish bones) doti blong kakae (olsem bun blong fis, skin blong taro) Rōrō a goro den o qō, rōrō a gen o garat. The two of them chased away the pigs and they ate the food scraps. Tufala $i$ ronemaot pig, tufala i kakae doti blong kakae. (HWM.044)

gargarke n.com. type of fatty looking substance, part of animal samting olsem gris we $i$ stap wetem mit blong animol

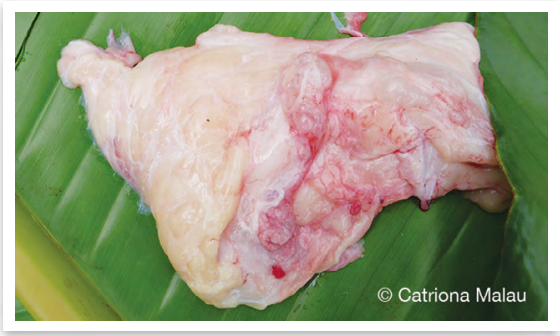

gargarke $\sim$ fatty substance

gargarmamas $v i$. fast, refrain from eating certain foods for a particular reason lego kakae mo dring No gö mörös o lō ti na sul na tiqēk, ba no go gargarmamas sur. I want it to be sunny so I can burn my garden, but
I must fast for it (according to tradition). Mi wantem san blong mi bonem garen blong mi, be mi mas lego kakae from.

garmian n.com.suff. her/his/its tongue tang blong hem See: garmiē

garmiē n.com.suff. tongue $\sim$ tang $\mathbf{R i}$ èl o bëtutu matriaw ko wo birin tek na garmian gō tōtōrōg. Look at this big lizard with its blue tongue. Yufala i iuk bigfala lised ia wetem blu tang blong hem. (AAD) [POc *kara-maya- 'tongue']

garmian (n.poss) n.com.suff. her/his/ its tongue tang blong hem

garmiēk (n.poss) n.com.suff. my tongue $\sim$ tang blong mi

garmiēbuluk n.com. variety of aibika, shrub with leaves which are commonly eaten as vegetable greens kaen aelan kabis Abelmoschus manihot variety $\{$ Lit. cow's tongue tang blong buluk\}

garmiēk n.com.suff. my tongue $\sim$ tang blong mi See: garmiē

garmēès (fr.var. of garmös)

garmös (fr.var. garmēs) $\mathbf{1} \cdot$ n.com. spit $\sim$ spet $\mathbf{2} \cdot v$. spit, spit on $\sim$ spet, spet long Nē ma garmöös no. She spat on me. Hem i spet long mi.

garqe temp. $\mathbf{1}$ today tede Na tetetel, sur garqe ko o weta nē gōwē, o masawre döl o lō. I'm just walking about because today the weather is good, and it is sunny everywhere. Mi stap wokbaot from tede ia weta hem $i$ gud, evri ples $i$ gat san. (DNS) $\mathbf{2}$ - later on today, today in the afternoon or evening; earlier today, earlier the same day tede long aftenun, tede long moning finis No gö tuwegev garqe lö qön. I'm going to bake on the fire later on today. Bae mi mekem faea tede long ivning. (DPD) No ma gagneg min kēmi garqe, kēmi gōtō nōmtuv no. I told you all earlier on today, but you didn't believe me. Mi talem long yufala tede finis, be yufala ino bilivim mi. (SSW) $\mathbf{3} \cdot$ at the present time or 
age; these days long taem tede O vu ine nē gō rōw lō le la tan̄sar garqe. That spirit can appear to people these days. Spirit ia i save kamtru long ol man tede. (ESP) $\mathbf{O}$ qō ta garqe, nē o qō gē sisia. The pigs of today are a different type of pig. Pig blong tede hem i defren kaen pig.

gartan n.com.suff. her/his/its leftovers hafkakae blong hem See: garte

garte n.com.suff. $\mathbf{1}$ - leftovers, part remaining from something $\sim$ pat we i stap yet Nēn ko lo garte to. We are in the middle of the year. Yumi stap long medel blong yia. 2 - leftovers, food that someone has left uneaten hafkakae No ge gen na garten i Ella. I'll eat Ella's leftovers. Bae mi kakae hafkakae blong Ella.

gartan (n.poss) n.com.suff. her/his/its leftovers hafkakae blong hem garten nōtōn (n.poss) n.com.suff. her child's leftovers hafkakae blong pikinini blong hem

gartëk (n.poss) n.com.suff. my leftovers hafkakae blong $\mathrm{mi}$

garte wōl n.com. kind of basket, with curved base $\sim$ wan kaen basket we hem i sep olsem hafmun \{Lit. half moon haf mun\}

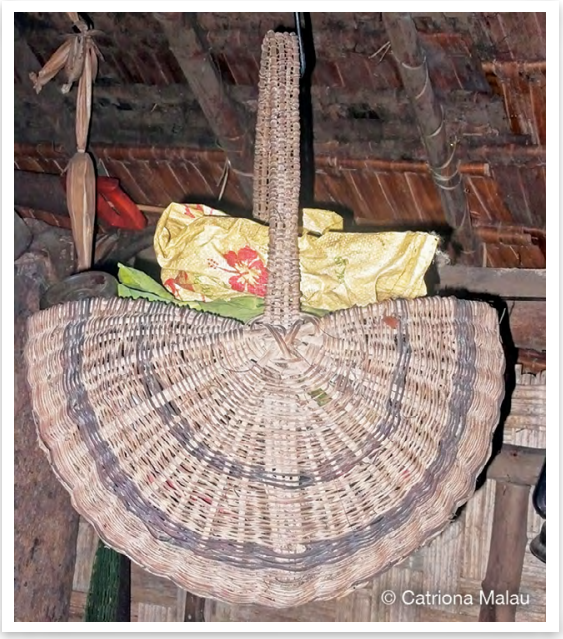

garte wōl kind of basket garten nōtōn n.com.suff. her child's leftovers hafkakae blong pikinini blong hem See: garte

gartëk n.com.suff. my leftovers hafkakae blong mi see: garte

garveg $v t$. cuddle, hold in arms holem taet long tufala han Nö gö mörös ta na garveg nē. I want to cuddle her. Mi wantem holem taet hem. gas $v$. make roof of house by making two lengths of bamboo join to make cross $\sim$ krosem tufala bambu i mit long antap blong haos $\mathbf{O}$ gövur ko, nēk gō rōq si nēk ga gas? This house, are you going to bend the bamboo over the top in making the roof, or are you going to cross it? Haos ia, bae yu putum bambu i go ova long top, o bae yu krosem? (RBH)

gasalē n.com. right size place for setting net where the fish won't be able to get away stret ples blong putum net we ol fis bae ol i no save lus

gasel n.com. knife naef Nēk i le o gasel, nēk i tes metestes o siri qatē ine. You take a knife, and you whittle the wood of the tree fern until it is sharp. Yu karem naef, yu katem wud blong blakpam ia se $i$ sap. (SBB) [POc* sele 'knife; cut with a knife']

gasel luwō (comp.) n.com. machete, large knife, used mostly for garden work and chopping wood busnaef, big naef Na sēk na möguk o gasel luwō tala ga van tek lē tiqēe. I'm looking for my machete to take it to the gardens. Milukaotem big naefblong mi blong karem i go long garen.

gasel nötu (comp.) n.com. small knife, kitchen knife used mainly for cooking, simple daily use smolnaef gasēg $v t$. share out, separate, divide seraotem Dōrōk a gasēg o n̄e. Let's share out the canarium nuts. Yumitu seraotem ol nangae. 
gasēsēdignönqet n.com. kind of tree $\sim$ wan kaen tri Ficus aspera \{Lit. rope for Qet's bird trap rop blong trap blong pijin blong Qet\}

gasim n.com. length of bamboo used for removing breadfruit from the tree bambu we ol i yusum blong karemaot bredfrut long stamba O gasim, nē o wo talē lis biēg. A 'gasim' is a length of bamboo used for removing breadfruit from the tree. 'Gasim' hem i wan bambu we ol iyusum blong karemaot bredfrut long stamba.

gasmeg $v$. insert something so that it hangs out, as with a piece of cloth pusum wan samting, olsem kaliko, i go samples, ale haf i stap hang Nēk i le o an̄kejiv, nēk i gasm̄eg lē qēgēn. You take the handkerchief and push it in the waist of your trousers so it hangs out. Yu tekem angkejif, yu pusum long traosis blong yu blong i hangdaon.

gasōg n.com. tree with small red fruit wud we frut blong hem i smolsmol mo i red Gnetum gnemon [POc *kusaq 'k.o. edible greens']

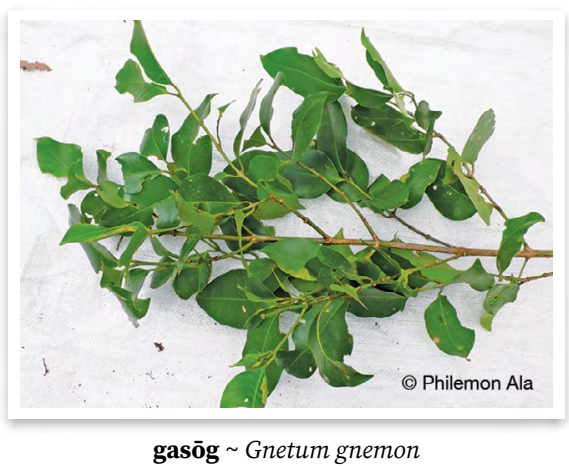

gasreg $v$. push something into tight space to hold it tight, as between slabs of thatch, walling, or in hair pusum samting i go long smol ples, olsem long natanggura, o long hea blong yu Gasreg kal o meteges siag me lo doot. Put the wooden knife up in the space between slabs of thatch. Pusum naefigo antap long natanggura.

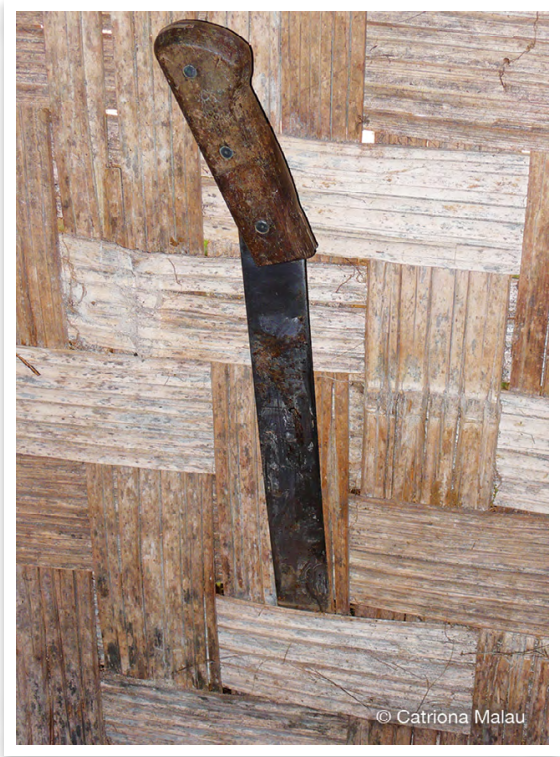

gasreg push into tight space

gatala n.com. web bed blong spaeda gateli maraw (comp.) n.com. spider web bed blong spaeda

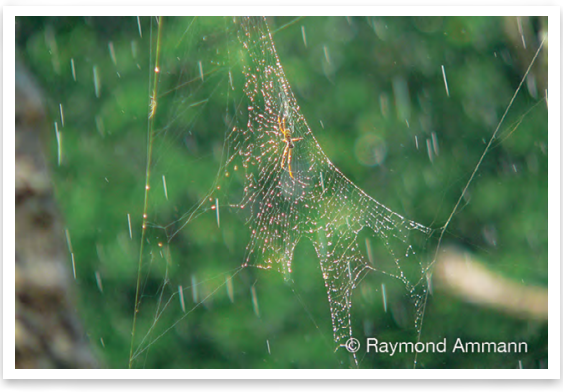

gateli maraw $\sim$ spider web

gatavdor n.com. type of ginger plant kaen flaoa Alpinia sp. [Leaves used as a local medicine. Lif hem i wan kastom meresin.]

gatege $n . c o m$. variety of breadfruit that has small fruit kaen bredfrut we kakae blong hem i smolwan 
gateteg n.com. kind of basket made from coconut leaves and used for carrying firewood wan kaen basket blong faeawud we ol i wivim long lif kokonas

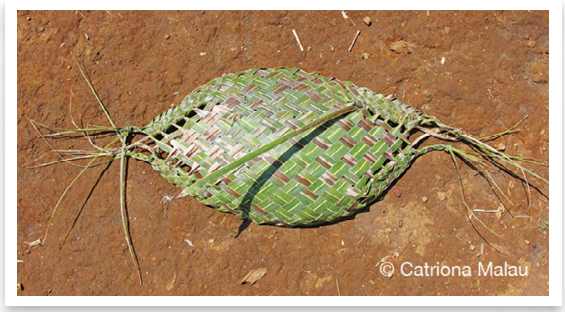

gateteg kind of basket

gatitieg n.com. bird or pig trap made from rope $\sim$ trap blong pijin o pig Si na da o gatitieg, no gē gis qal le o savger gēn, nē to kurkur na bulën nēn o gengen gēn If I make a trap, I will be able to catch that thing that is eating our crops. Sapos we mi mekem wan trap, bae mi save kasem samting ia we i stap kakae ol kakae blong yumi. (WRN) See: tieg

gatitimeles $n$.com. piece of wood with hooks on it for hanging food in the kitchen wud we i gat huk long hem we ol i hangem antap blong hangem kakae long hem \{Lit. rope for hanging leftovers rop blong hangem namalas\}

gatōw n.com. kind of strong vine that grows on hills and by the sea but not in the bush. It is hard but can be easily broken when immature wan kaen rop we i stap long hil mo long solwota be no long bus. I strong be yangwan i save brok isi Derris trifoliata [Has a wide, common use as a rope and as local medicine. $\mathrm{Ol}$ i yusum blong fasem ol samting olbaot mo blong kastom meresin.]

gatöqul $v$. throw (something long) at $\sim$ stonem (wetem longfala samting) Nē ma gatöqul o qërët tek o mie rēntenge. He threw a long stick at the flying fox. Hem i stonem flaengfokis wetem longfala wud.

gatötöqulqërët n.com. kind of dragon tree, shrubby plant wan kaen plant Dracaena sp. \{Lit. thing for throwing at flying fox samting blong stonem flaengfokis wetem\}

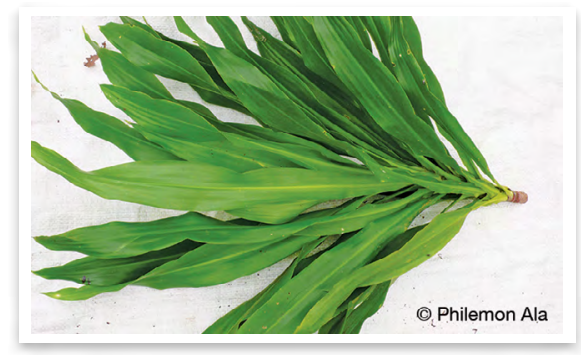

gatötöqulqërët $\sim$ Dracaena $\mathrm{sp}$.

gav vi. fly flae No ga gav timiak o men. I fly like a bird. Mi stap flae olsem pijin. (DFF.017)

gavan̄ n.com. kind of food basket woven with a black vine, gamalwuw wan kaen basket blong kakae we ol i wivim wetem blak rop [Only men make and use these baskets in the men's house. A tibiar gamalwuw is the same basket style, but it is made by women at home. $\mathrm{Ol}$ man nomo ol i wivim mo yusum kaen basket ia long nakamal. Tibiar gamalwuw hem i semak basket, be ol woman ol i wivim long haos.] See: tibiar gamalwuw (comp. of tibiar)

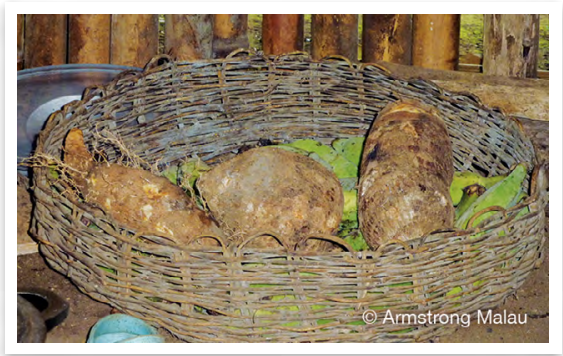

gavan̄ kind of food basket 
gavēg n.com. mountain apple, Malay apple, rose apple nakavika Syzygium malaccense Gōsōw mē dēn̄ me lē qētēgi gavēg, nē me rem. The rat reached the base of the Malay apple tree and it climbed it. Rat i kam kasem long stamba blong nakavika, hem i klaem. (FRN) [POC*kapika 'Malay apple, rose apple, Syzygium malaccense']

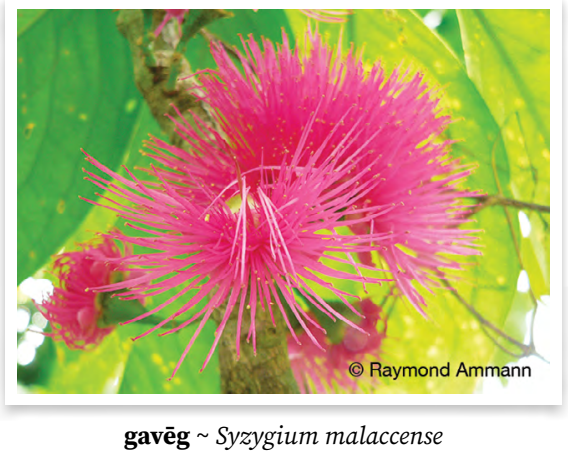

gavēgulul (comp.) n.com. kind of tree like mountain apple but with white flowers wan kaen tri olsem nakavika be flaoa blong hem i waet

Gavēgbalas n.loc. tribe name, named because they came from Malay apple tree nem blong traeb, ol i tekem nem blong olgeta aot long nakavika [People of this tribe are said to have lots of children without getting married. Olgeta long traeb ia ol i no mared be ol i karem fulap pikinini.]

gavivnan n.com.suff. her/his/its armpit $\sim$ andanit han blong hem See: gavivne gavivne n.com.suff. armpit $\sim$ andanit han

gavivnan (n.poss) n.com.suff. her/his/ its armpit andanit han blong hem gavivnëk (n.poss) n.com.suff. my armpit andanit han blong mi gavivnëk n.com.suff. my armpit andanit han blong mi See: gavivne gavō n.com. whitewood (tree) waetwud Endospermum medullosum gawol n.com. lawyer cane, rattan loeaken Calamus vanuatuensis [Used as a rope for house and canoe construction and for making prawn traps. Ol i yusum blong fasem haos o kenu mo blong wokem basket blong naora.]

gawōlō n.com. kind of tree that is used to make a strong rope $~$ wan kaen tri we $\mathrm{i}$ gat rop blong hem insaed we i strong tumas [Originally used for making barkcloth for clothes. It grows on hillsides. Bifo ol i yusum blong mekem klos olsem tapa. Hem i gru long hil.]

gawuw n.com. valley vali

ge $c l$. relational classifier used to mark possession of items that are eaten by the possessor $\sim$ blong (yusum blong tokbaot kakae) $\mathbf{O}$ mes ine na gëk. That fish is mine to eat. Fis ia blong mi blong kakae. (DNS) [POc *ka- 'possessive classifier: alimentary, subordinate'] gan (n.poss) cl. her/his/its (food) blong hem (kakae) ge tō (n.poss) $c l$. (food) for chickens (kakae) blong faol gëk (n.poss) cl. my (food) blong mi (kakae)

ge tō $c l$. (food) for chickens (kakae) blong faol

geben n.com. sail sel

gegen vi. sharp (edge, as in knife) sap (long saed, olsem naef) No gö mörös ta na tar o ralēt ba na möguk o tele gete gegen. I want to chop firewood, but my axe isn't sharp. Mi wantem katem faeawud be akis blong mi ino sap. Ant: löl See: metestes

gem (fr.var. gëm) mod. $\mathbf{1} \cdot$ just $~$ nomo Lō vōrōg kemem mo tog gem ti lö gövur. In the morning we just stayed in the house Long moning mifala i jas stap nomo long haos. (AAD) $\mathbf{2} \cdot$ only, just nomo Tan̄sar nitiwial gem nē mi tiveg na bōlan o tok nirō. Only one 
person has paid the fee for his two dogs. Wan man nomo $i$ bin pem fi blong tufala dog blong hem. (VSB)

gemel $n$.com. traditional building used only by men of rank for ceremonial purposes and meetings nakamal Ga sasarēt o tarn̄i sere ev go tog le gemel, si o tin̄tin̄ le gemel. It was enough for the different areas of the men of different rank inside the men's house. I stret long ol kaen tambu faea i stap insaed long nakamal, o ol eria blong nakamal. (ESP) [The gemel is strictly only for men, and women are not allowed to enter. Nakamal hem i haos blong ol man nomo mo ol woman ol i no save go insaed, be haos blong ol woman, ol man tu ol i no save go insaed.] [POc *kamali( $R$ ) 'men's meeting house']

gen $_{1} v t$. eat, eat staple part of meal kakae, kakae men kakae Dōrōk a gen o gavēg. Let's eat Malay apples. Yumitukakae nakavika. (FRN) [Cannot be used to refer to eating of meat, only eating staple or snack foods. Yu no save yusum wod ia blong talem se yu kakae mit, be ol kakae o frut nomo.] [POc *kani 'eat'] See: big

gengen (redup.) n.com. $\mathbf{1}$ food, meal, staple part of meal, either cooked or uncooked kakae, men kakae, we i dan o i no dan Nēk i da o gengen min kemem sur oso? Why are you making a meal for us? Yu stap mekem kakae blong mifala from wanem? (ESP) Syn: sinag $2 \mid \mathbf{2} \cdot$ food crops, staple food plants kakae long garen Riv wareg kēl na bulën nēn o tere gengen. Replant our staple food crops properly. Planem gud bakegen ol kakae blong yumi. (HWM)

gengen luwō (comp.) n.com. feast bigfala kakae, lafet

gengen luwömeren (comp.) vi. eat lunch, meal in middle of day $\sim$ kakae dina gengen revrev (comp.) vi. eat dinner, evening meal kakae long sapa gengen vōrōg (comp.) vi. eat breakfast dring ti long moning

gen $_{2}$ n.com. fire ant $\sim$ blakanis Solenopsis geminata [There are two different kinds, one black and one red I gat tu kaen blong hem, wan i blak mo wan i red]

genqirēg n.com. dog dog Syn: tok gengenur n.com. $\sin \sim \sin$ Metwu bëlbël nē o gengenur. The habit of stealing is a sin. Fasin blong stil hem i wan sin.

genqininimatē $v$. last feast that is made to celebrate someone's death, one thousand days after the death kakae las dei long wan taosen dei blong ded man. Hem i kakae blong finisim tingbaot man we i ded mo blong stat blong fogetem. Hem i no dei blong sore, i dei blong glad. Hem $i$ talem se i naf blong tingbaot, nao ol i save fogetem

genvēl n.com. someone who chooses not to eat some foods, usually due to illness or other dietary concerns $\sim$ man we i no stap kakae sam kaen kakae from wan sik blong hem

genwiwiris n.com. fussy eater, someone who is choosy about what they want to eat and won't eat if they are given any food other than what they want $\sim$ man we hem $i$ jusum kakae we hem i wantem nomo mo sapos yu kukum nara kaen kakae bae hem i no wantem kakae Nē o genwiwiris. She is a fussy eater. Hem i kakae nomo kakae we hem i wantem. ger $v t$. chop, cut using chopping action with considerable effort required for effect, as object is solid, hard katem, olsem yu katem wan samting we i strong mo yu mas hadwok long hem Nēn a ger o kopra. Let's chop the copra. Yumi katem kopra. Syn: $\operatorname{tar}_{1}$ 
geres vt. grate coconut $\sim$ skrasem kokonas Kemem a geres o mōtō, a wōr. We grate the coconut and we squeeze the milk from it. Mifala i skrasem kokonas, ale melekem. (VVKHVDP21Jul0702-CS) Syn: gör

geret n.com. mountain, high hill maonten, tolfala hil

gerger n.com. scabies sik blong skras gersal (abbrev. $\mathrm{sal}_{2}$ ) $\mathbf{1} \cdot$ n.com. path, road $\sim \operatorname{rod}$ Ba nē ma qal tēqēl tatag o gersal ta 1o. But he first went down following the path to the seaside. Be fastaem hem $i$ go daon folem rod blong go long solwota. (ADT) $\mathbf{2}$ - n.com. way, method or means for doing something wei Nē ga van me, nē gē birin̄ le gersal gö tutuqë. It will come, and it will help in many ways. Bae hem i kam, hem i givhan long fulap wei. 3 - n.com.suff. way (of doing something), path (of a person) rod blong (wan man o blong mekem wan samting) [POC *jalan, *salan 'path']

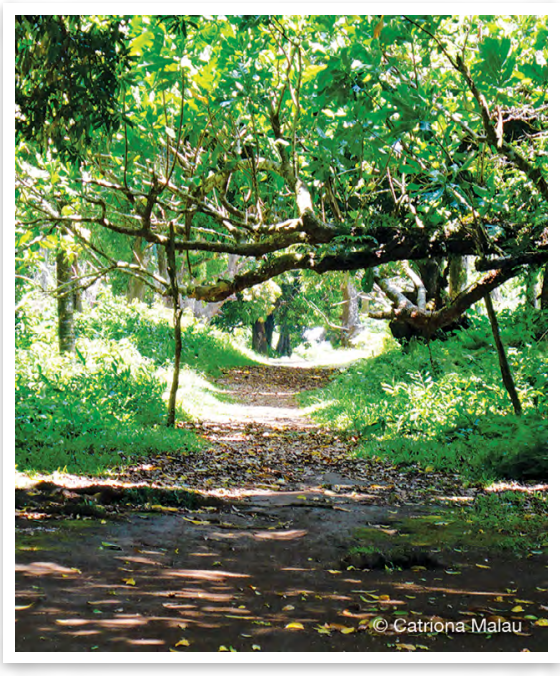

gersal path

gersal liwō (comp.) n.com. road, main road suitable for vehicles $\sim$ rod, men rod La laln̄e bak, o gersal liwō mē dēñ aē gēn. Underneath the banyan tree, the main road reaches up to there. Andanit long nambangga, men rod $i$ kasem long ples ia nao. (RRS)

gersalan (n.poss) n.com.suff. her/his/ its path rod blong hem

gersele legleg (n.poss) n.com.suff. way of marriage rod blong mared gersëlëk (n.poss) n.com.suff. my path rod blong $\mathrm{mi}$

gersalan n.com.suff. her/his/its path rod blong hem See: gersal

gersele legleg n.com.suff. way of marriage rod blong mared see: gersal

gersëlëk n.com.suff. my path rod blong mi See: gersal

gese $a d v$. how olsem wanem No gō mōl kēl gese? How am I going to get back home? Bae mi gobak long hom olsem wanem? (WRN)

gē n.com. 1 • kava plant kava Piper methysticum $\mathbf{O}$ mie gē nē mo wot lō me den o reqe. The first kava plant grew from a woman (from where a dead woman was buried). Fes kava hem ibon aot long woman. (OSK) $2 \cdot$ drink prepared from the roots of the kava plant kava we ol i mekem dring aot long rus blong hem I rege atatmēn, kemem gö sum o gē tastas. Us men, we drink kava all the time. Olgeta man, mifala i stap dring kava oltaem. (OSK.001)

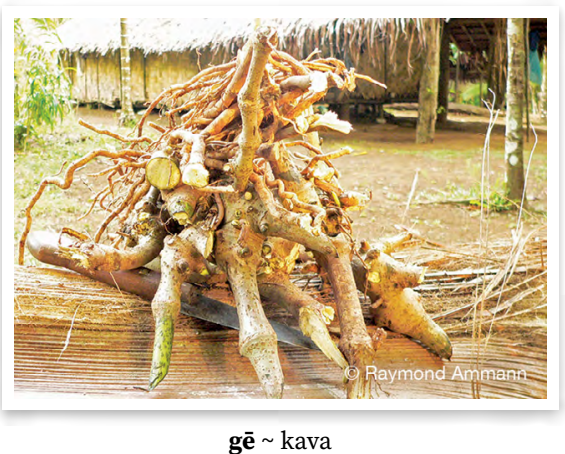


gēgēt $v i$. be on ball of feet or tippy toes, not full, flat feet stanap long en o fingga blong leg Nēk i tur gēgēt. Stand on your tippy toes. Stanap long fingga blong leg blong yu.

gēn (fr.var. gēnak, igēn) dem. this, this one, proximal demonstrative $\sim$ ia, hemia O kakaka talo qō nē ga van timiak gèn. The story of the pig goes like this. Storian blong pig i go olsem. (DDP.001) Nē gē kēl me sur o gengen gēn. It will return for this food. Bae i kambak from kakae ia. (DFF) Syn: in̄kē gēnak (fr.var. of gēn)

gēnē n.com. thing samting Ba i rege ta ine ma da o gēnē nitiwial, na sian vita Scenic Railway. But the people from there had made this thing, it is called the Scenic Railway. Be olgeta blong ples ia ol i mekem wan samting we nem blong hem se Scenic Railway. (AAD)

gēri n.com. root $\sim$ rus $[P O C *$ wakaRi'root']

gēri mōtō (comp.) n.com. stretch marks on belly mak long bel blong woman, olsem trak blong pikinini \{Lit. coconut roots rus blong kokonas\}

gēvir $v t$. squeeze skwisim Nē mēe gēvir na dirin Jessica. She squeezed Jessica's leg. Hem i skwisim leg blong Jessica.

gëk $c$ l. my (food) blong mi (kakae) See: ge

gëm (fr.var. of gem)

gër $\mathbf{1} \cdot v i$. swim $\sim \operatorname{swim~} \mathbf{2} \cdot v t$. wade through water wokbaot krosem wota Nēk më gër levga o Bē Taqren. You wade across Taqren creek. Iu wokbaot krosem wota blong Taqren. (RRS.021) See: Suvsuv

gëv $v t$. cover the ridge of a roof, where the two sides meet, make ridge flashing blokem top blong haos Nēn a so o doot talë gëv o qötu. We make the thatch from sago palm leaves in order to make the ridge flashing. Yumi mekem natanggura blong blokem top blong haos. (RBH)

gëv bulës (comp.) $v$. make the ridge flashing for house roof using method of bending sago palm thatch over join wei blong blokem top blong haos we yu benem wan natanggura $\mathrm{i}$ go antap blong blokem top blong haos

gëv gonsōgōrō (comp.) v. make the ridge flashing for house roof by sewing together two slabs of sago palm thatch and placing on join wei blong blokem top blong haos wetem tu natanggura we yu somap tugeta mo benem antap long haos gëv nag (comp.) v. cover up one's face at time of marriage, in case the man has chosen an unsuitable wife $\sim$ blokem fes taem blong mared, sapos man i mekem rong mared gëvgëv $v i$. perform ceremony when someone has killed another person, to pay the person's family for their life pem laef blong wan man we yu bin kilim

gëvtun $v i$. make meal using method where the food is wrapped in leaves, then place the parcel of food on top of the coals of the fire mekem kakae folem fasin we yu paselem kakae fastaem, afta yu putum long faea. Samtaem yu save rusum kakae fastaem Rōrō a gëvtun, rōrō a monon kal. The two of them made the meal, they wrapped it (the food) up. Tufala i mekem kakae, tufala i pasolem. (JMT)

gëwtu $n$.com. stomach $\sim$ bel

gial $1 \cdot n$.com. lie, tell untruth $\sim$ giaman Nēk o gial, na ginon̄ aē. You are lying, you have a wife. Yu giaman, woman blong yu i stap. (DSR) Syn: sen $\mathbf{2} \cdot v t$. lie, tell untruth to giaman long Nē ni gial kōmōrōñ vita a van a sigsig e, ni van e, ni sosodēmēl. He lied to the two of you that he had gone to harvest taro, 
and he went to gather together people. Hem i giaman se hem i go karem taro long garen ia, hem i go ia, ifaenem ol man. $3 \cdot v t$. trick, pretend to giaman long Dōrōk a gial nēr, dōrōk a tek viteg nēr. Let's trick them, let's take them away. Bae yumitu giaman long olgeta, yumitu karem olgeta i go. (RPP) gilgial (redup.) vi. lie giaman Nēk i gilgial, nēk mè èl nē. You are lying, you saw him. Iu giaman, yu bin luk hem. (OQQ)

giam n.com. $\mathbf{1} \cdot$ net $\sim$ net Nēr töl ma van me a Rō talē ti giam, ti o giam sur o mes. The three of them came to Rowa Island to place a net, place a net for fish. Trifala i kam long aelan blong Rowa blong putum net, putum net blong fis. (WDR) $\mathbf{2} \cdot$ net covering stomach $\sim$ net we i kavremap gat

giam vus (comp.) n.com. net with a handle $\sim$ kaen net wetem handel blong hem

ti giam (comp.) v. place net $\sim$ putum net [Catching fish with a net in this manner involves one person holding the net and another person chasing the fish into the net. Taem ol i yusum net ia wan man i ronem fis, narawan i holem net.]

giav n.com. crab krab

giav kukut (comp.) n.com. sargassum crab wan kaen krab blong solwota Family Grapsidae

giav matrōrōw (comp.) n.com. kind of crab with big eyes, blue back and white underside. Found in the bush or shore, close to the sea wan kaen krab we ae blong hem i bigbigwan mo baksaed blong hem i blu, front i waet. Hem i stap long bus o so be klosap long solwota

giav mërës (comp.) n.com. mottled lightfoot crab wan kaen krab we i stap long so Grapsus albolineatus giav ōwōw (comp.) n.com. spanner crab wan kaen krab blong solwota Ranina ranina giav semetöv (comp.) n.com. kind of crab that dwells in holes in rotten trees in the water. It is white and light brown wan kaen krab we $\mathrm{i}$ stap insaed long wota insaed long hol blong wud. Hemi lelebet waet mo laet braon

giav sēvsēv (comp.) n.com. kind of crab wan kaen krab

giav sōsō (comp.) n.com. kind of freshwater crab that lives in swamps krab Kaledoni

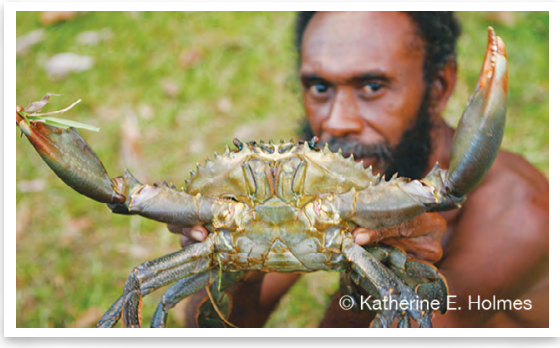

giav sōsō kind of freshwater crab

giav tamal (comp.) n.com. mottled lightfoot crab wan kaen krab we i stap long so Grapsus albolineatus Syn: gamar

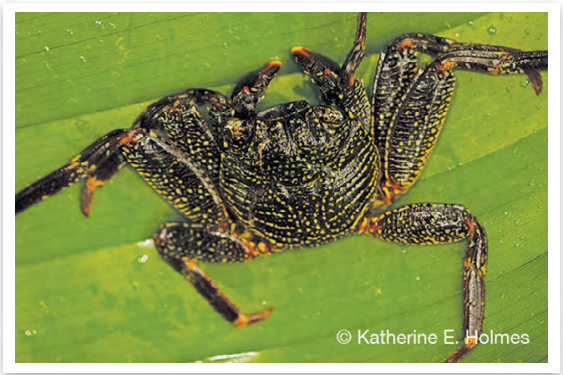

giav tā̄al Grapsus albolineatus

giav vaw (comp.) n.com. kind of reef krab with red spots on shell wan kaen krab we i stap long rif we i gat red dot long baksaed Carpilius maculatus

giav vet (comp.) n.com. kind of shore crab wan kaen krab we i stap long so Plagusia dentipes 
giavèdun n.com. land crab (with hairy legs) wan kaen krab we hem i blak mo hem i gat tumas hea blong hem. Hem i stap long drae ples be hem i go long solwota taem i gat eg Syn: sursal

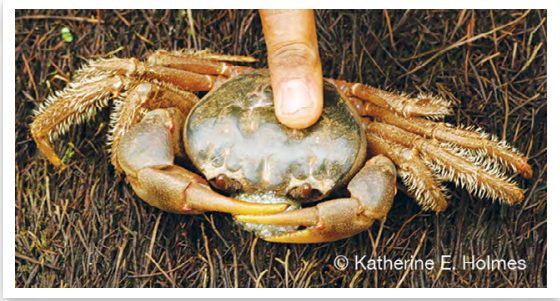

giavēdun $\sim$ land crab

gie n.com. whitespotted surgeonfish wan kaen fis Acanthurus guttatus

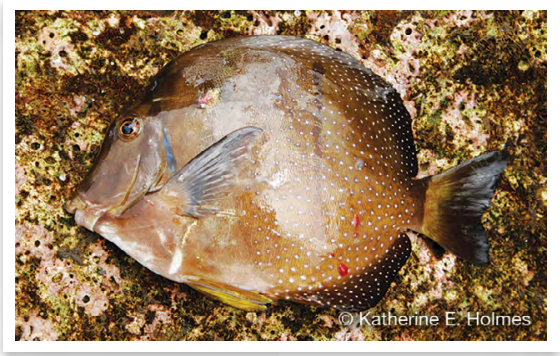

gie Acanthurus guttatus

giebē n.com. kind of freshwater fish that is black with white stripes. It is flat, can be large, and is always found in pairs wan kaen fis blong wota we hem i blak wetem dak waet laen. Hem i flat mo hem i save bigwan. Ol taem ol i stap tu tugeta

gil ${ }_{1} \mathbf{1} \cdot v t$. dig, dig up food plants, etc. $\sim$ digim Kemek a van a gil na gen kemek o walalas. We went to dig our wild yams. Mifala igo blong digim wael yam blong mifala. $\mathbf{2} \cdot$ n.com. digging stick stik blong digim graon Na gil o tan tek o gil. I dig the earth with a digging stick Mi digim graon wetem stik blong digim graon. 3 - n.com. Stick used for removing husk from coconut $\sim$ wud blong karemaot skin long kokonas $[\mathrm{POc} * k e l i$ 'dig, harvest (tubers)']

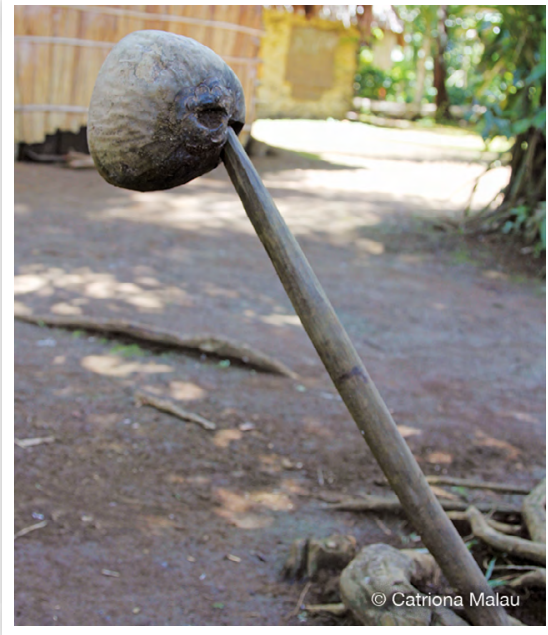

gil coconut husking stick

gil $_{2} v i$. get out of breath, feel unable to breathe sotwin, harem se yu no save pulum win Na memsan mē gil. She got out of breath. Hem $i$ sotwin. No me ten̄teñ vaavan, e na memsëk mi gil. I cried so much that I felt that I couldn't breathe. Mi krae krae go go, win blong mi isot.

gilal $\mathbf{1} \cdot v$ t. know $\sim$ save $\mathbf{2} \cdot$ n.com. knowledge, wisdom save, waes O tan̄sar ine, o gigilal gö luwō min nē. That man possesses great wisdom. Man ia igat bigfala waes. $\mathbf{3} \cdot v$. jealous, express jealousy jalus 0 reqe ine ni gigilal i ginon sur i bulsalan ta $\bar{m} \bar{o}$. That woman expresses jealousy to her husband about his previous girlfriend. Woman ia $i$ stap jalus long man blong hem from gelfren blong hem bifo.

gilgeg $v t$. stand up post stanemap pos

gin $v t$. $\mathbf{1} \cdot$ pinch, pinch off with fingers $\sim$ pinsim 2 - pick greens using pinching motion to break off leaves pinsim, karem Nēk i gin na gen nēn o sasar. You pick our aibika greens. Yu tekem aelan kabis blong yumi. [POC *kinit, *kinit-i- 'pinch off with fingers, nip with fingernails'] 
ginias n.com. sixfinger threadfin (fish) $\sim$ kaen fis Polydactylus sexfilis

ginon n.p.bound her/his spouse $\sim$ man/ woman blong hem See: gunö girē $v$. buy pem Nēr mē girē o qō gö tutuqë idian. They bought lots of pigs. Ol i pem fulap pig. (ESP)

girē qalēg (comp.) v. (at marriage ceremony) give money to parents of spouse, to confirm their relationship status as in-laws long taem blong mared man mo woman i givim mane long ol papa mo mama blong patna girē wël (comp.) v. (at marriage ceremony) give money to sisters of husband (for woman) to confirm their relationship status as in-laws long taem blong mared woman wetem ol famle blong hem ol $i$ givim smol mane long brata mo sista blong boe

girir vt. smooth out pandanus leaves using limpet shell, in preparation for weaving smutum pandanas wetem sel, redi blong wivim Ni girir o vudege tek o weger. She is making the pandanus smooth with a shell. Hem i smutum pandanas wetem sel.
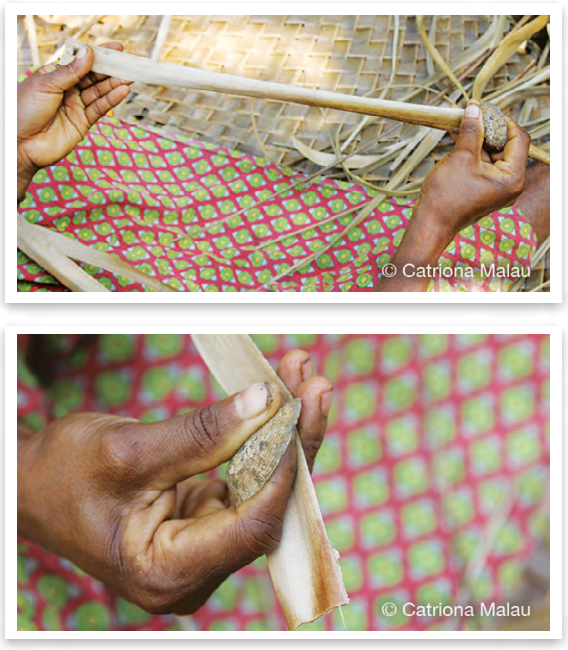

girir $\sim$ smooth pandanus gis $v t .1 \cdot$ hold, carry, hold onto holem, karem Gis wareg den ni mës. hold it tightly so it doesn't fall. Holem gud, lukaot bae $i$ foldaon. 2 - touch $\sim$ tajem $3 \cdot$ host, hold celebration, ceremony hostem lafet, seremoni Rege rōw e a lo nēr o gis o kōlkōl. The people down at the seaside were hosting a pig killing ceremony Olgeta daon long solwota ol i stap holem wan pig kiling. (KM̄MT)

gis qal (SVC) vt. catch, achieve aim kasem Nēr a vērēg tatag nē. Nēr gētē gis qal nē. They ran after him. They didn't catch him. Ol i resis folem hem. Ol ino kasem hem. (JTF)

gis tës (SVC) vt. accidentally drop, let go of something that one has been holding $\sim$ mestem wan samting we yu holem, i foldaon Ni gis tës nitiwial, ni mës lō den o tan̄. He dropped one, it fell out of the basket. Hem i lego wan ifoldaon, ikamaot long basket. (JRQ)

gis $_{2} v t$. build (house) bildim (haos) (RBH.001)

gis rak $v t$. pick up and carry pikimap mo karem Ni van me, ni gis rak nē, ni van tek. He came, picked him up, and took him away. Hem i kam, pikimap, i go wetem. (HWM)

gis salmiat $v$. make preparations for a feast $~$ priper blong mekem bigfala kakae, speseli long wan taosen dei blong wan ded man Nēn a gis salmiat sur o legleg. Let's make the preparations for the marriage. Yumi mekem redikakae blong mared.

gisine $a d v$. once upon a time, formulaic introduction to traditional stories wantaem (toktok we yu talemaot long stat blong kastom storian) Gisine i rege min ginon. Once upon a time there was a man and his wife. Wantaem $i$ gat man wetem woman blong hem. (ESS) 
gitiag $v$. be careful lukaot Kēmi wun suvsuv ba gitiag gōr den kēmi a tul. When you swim, watch out in case you drown. Yufala i swim, be lukaot, nogud yufala idraon.

go 1 n.com. hook, fish hook huk blong fis [POC *kawil 'hook; fishhook']

go $_{2}$ v.part. imperfective aspect marker, used to mark future and habitual events, and to describe states wan maka we $i$ go wetem veb we $i$ talemaot se aksen bae i tek ples long fiuja. Tu i refe long wan situesen we i stap olsem nomo o wan samting we man i stap mekem oltaem (Allomorphs: ga, ge, gē, gë, gi, go, gō, gö)

gogoreg $v$. sweep away $\sim$ brumum

gogov n.com. $\mathbf{1} \cdot$ clothes $\sim$ klos $\mathbf{2} \cdot$ cloth $\sim$ kaliko

golē n.com. variety of breadfruit that has elongated fruit $\sim$ kaen bredfrut we kakae blong hem i longwan

gomot n.com. striped mullet $\sim$ malet Mugil cephalus

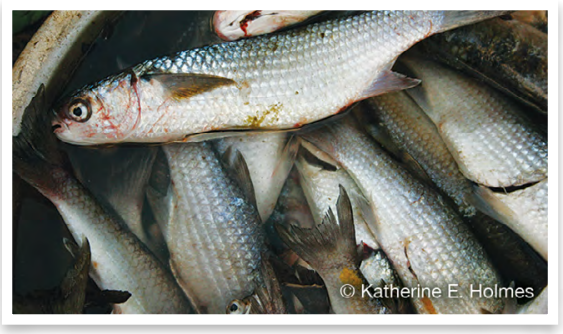

gōmot Mugil cephalus

gon wōmōōto $v$. make an agreement that two small children will marry when they come of age. The boy's family gives money and coconuts to the girl's father. Two germinating coconuts are tied together and must be planted together as a symbol of the bond. mekem agrimen se bae tufala pikinini i mared taem tufala pikinini i smol nomo. Famle blong boe ol i fasem tu kokonas tugeta mo givim long papa blong gel wetem mane. $\mathrm{Ol}$ i mas planem tu kokonas tugeta mo hem i wan saen se bae tufala i mared. Hem i wan strong agrimen we bae ol i no save brekem I Vikta nē mo gon wōmōōō gōr i Jessica. Vikta's family and Jessica's family have made an agreement that the two will marry when they are grown up. Vikta hem i blokem Jessica (blong tufala i mared taem tufala ibigwan).

gonsōgōrō n.com. two pieces of thatch joined together to make one piece to cover over top of roof $\sim$ tu natanggura we ol i somap tugeta blong kavremap top blong haos

goqtul $v$. fall down, over (of tree, banana) (wud i) foldaon $\mathbf{O}$ leñ ge meneg ma da o qētēgi man̄ko mo goqtul. The strong wind made the mango tree come out of the ground. Strong win $i$ mekem stamba blong mango i kamaot long graon.

goro $v t$. chase $\sim$ ronem

got vt. (of hook) catch on something $\sim$ (huk i) hukum wan samting $\mathbf{O}$ go mo got na busuk. The hook caught my finger. Huki kasem fingga blong mi.

goto (abbrev. $\left.\mathrm{to}_{2}\right)$ v.part. negative particle, not no (Allomorphs: gata, gete, gētē, gëtë, giti, gōtō, götö)

Gō n.loc. 1 • name of island, Gaua nem blong aelan, Gaua Syn: Lokon $\mathbf{2}$ tribe name, named after the island that they came from, Gaua nem blong traeb we i kamaot long Gaua

gōdōw n.com. red-eyed crab wan kaen krab blong solwota Eriphia sebana

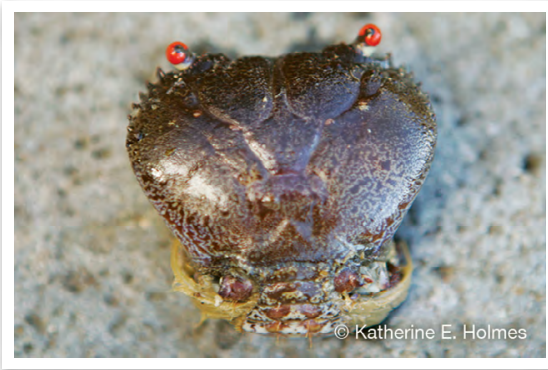

gōdōw Eriphia sebana 
gōg n.com. reef heron (bird) longnek; naova (pijin) Ardea sacra, Egretta sacra

gōg gabēt (comp.) n.com. white heron $\sim$ waet longnek \{Lit. white heron waet longnek\}

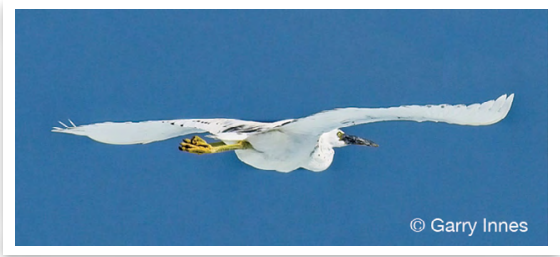

gōg gabēt white heron

gōg kör (comp.) n.com. black heron blak longnek \{Lit. black heron blak longnek\}

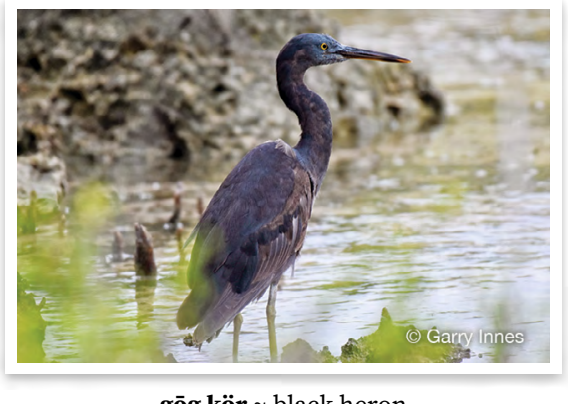

gōg kör black heron

gōglo n.com. kind of shrub, small tree kaen navenue Macaranga tanarius [POc *koka 'Macaranga spp.']

gōgōn vi. $\mathbf{1}$ • bitter, be bitter konkon Nēk gō rōn̄teg o lulumne gē, nē gō gōgōn timiak nēk gö sum o meresin nē gō gōgōn. You sense the taste of the kava, it is bitter like you drink medicine and it is bitter. Yu harem tes blong kava, hem i konkon olsem yu drink meresin hem i konkon. (OSK) $\mathbf{2}$ - salty, be salty $\sim$ konkon, sol Nē me mem idian o sol, o gengen gō gōgōn idian. He put too much salt and the food is too salty. Hem i putum tumas sol, kakae i sol tumas. $\mathbf{3} \cdot$ sour, be sour $\sim$ konkon $4 \cdot$ poisonous, be poisonous $\sim$ posen gōgōn $v i$. holy tabu

gōl ${ }_{1} v i$. slack, limp (of leaves, branches) slak, olsem ol lif o brans blong wud No tē rērē o damat sur ti a mon o laplap aē, ni da o damat ni gōl wurveg. I am turning the heliconia leaves over the fire so that I can wrap up the laplap with them, as it makes the heliconia leaves nice and flexible. Mi stap putum lif laplap long faea blong mi paselem laplap long hem, i mekem se lif laplap i sofsof gud. (VVAMVDP04Jun0801-JN.023)

gōl ${ }_{2}$ vi. shake, shudder $\sim$ seksek $\mathbf{O}$ malas ma da nē, na tarbian ni gōlgōl. The cold affected him so that his body was shaking. Kolkol i mekem hem, bodiblong hem i seksek.

gōlōw n.com. orphan, child whose parents have died pikinini we $\mathrm{i}$ nogat mama mo papa (GMM)

gōq $v t$. make hole with digging stick to plant taro digim graon wetem wud blong mekem hol blong taro Nē ni gōqgōq o lie sinag. She made a hole to plant the taro with a digging stick. Hem i digim hol blong planem taro.

gōqleg $v$. dig out earth with stick digimaot wetem wud La masawre nēk i dada na non̄ o um, nēk i èl vita götö luwō, gata sasarēt, nēk i gōqleg bilēn When you are digging out your oven, and you see that it is not big enough yet, then you dig it out with a digging stick too. Taem we yu stap mekem hol blong oven blong yu, yu luk se hem i no bigwan, i no naf, yu digimaot bakegen.

gōr $\mathbf{r}_{1} v$. hold together in bunch (as greens) and cut into strips katem (olsem bandel kabis) Nēk i gōr o sasar. Cut up the island cabbage. Iu katem aelan kabis. 


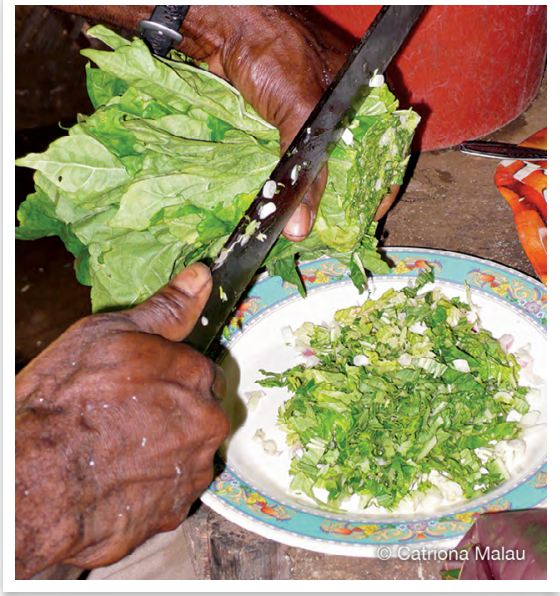

gōr slice while holding tightly

gōr ${ }_{2}$ vt. block blokem $[\mathrm{POc}$ *koro 'prepositional verb specifying motion against']

gōrga vi. cut the mourning rope from the neck of a man who has fasted during period of mourning for father or mother. The ceremony is performed in the morning of the 999th day after person has died. The same family who tied the rope around the person's neck must cut the rope, then they give the person the food that he has not eaten during the mourning period, and he must pay them. seremoni we ol i mekem long eli moning long naen handred naenti naen dei blong wan ded man. Ol man we ol i bin hangem rop long man we hem i fas long kakae, ol i kam katem rop. Afta ol i givim kakae long man we hem i no kakae kakae ia mo man ia i mas pem hem.

gōrgōrbiliag n.com. oriental sweetlips (fish) yelo tiklips (fis) Plectorhynchus orientalis

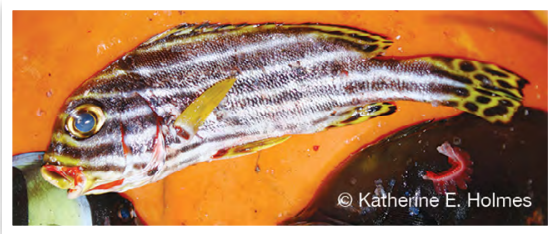

gōrgōrbiliag Plectorhynchus orientalis

gōrō̄n vi. holy, sacred, spiritually meaningful tabu Nēn ma da o gövur ko la masawre gōrōn. We made this house in a sacred place. Yumibin mekem haos ia long wan tabu ples. (EDM) Iñko o masawre ko mō gōrōn ren. This place has now become very holy. Nao ia ples ia $i$ kam tabu tumas.

gōsōs n.com. insect that damages yam and taro and Hong Kong taro bebet we i stap kakae yam mo taro mo Fiji taro Papuana uninodis

gōsōw n.com. rat rat Nēr ge ven̄en o gōsōw, o gōsōw na bulën nēr. They fed pigs, their pigs. Ol i fidim pig, pig blong olgeta. (ESP) [POc *kusupeq 'rat']

gōwē vi. good gud See: wē

gögörö $v t$. refuse to give something to someone when they ask for it; keep from no letem, no givim wan samting long hu we i wantem E i götö mö gögörö den nē o talēs. So the hermit crab wouldn't let it have the Indian almonds. Ale nakato $i$ no letem natavoa long hem. (BNR.018)

gök vi. 1 • boil boel $\mathbf{O}$ bē mö gök. The water has boiled. Wota $i$ boel. 2 bubble, dissolve boel (meresin) O panadol tö gökgök, nē tö gökgök, wo nēk i sum. The paracetamol dissolves and bubbles and you drink it. Panadol i mekem babol go go, afta yu dring:

göl vt. speak angrily to (someone) tok kros (long) Nē ma qaq sur no, na lölök me en, ba na göl nē. She talked 
about me and I felt cross, so I spoke angrily to her. Hem i tokbaot mi, mi kros long hem, ale mi tok kros long hem. gölö n.com.suff. tail tel

golon (n.poss) n.com.suff. her/his/its tail tel blong hem

gölök (n.poss) n.com.suff. my tail tel blong mi

gölörēki 1 • n.com. sprouting coconut that is just starting to germinate and has not yet formed spongy growth inside $\sim$ kokonas we i stat blong kam navara be wota i stap insaed yet $\mathbf{2} \cdot v i$. shoot of coconut begins to grow gru blong kokonas i stap kamaot be i no mekem gud navara yet $\mathbf{O}$ mōtō ko, in̄ko mö gölörēki. This coconut, now its shoot is beginning to grow. Kokonas ia, nao ia lif i kamaot long navara. [At this stage the spongy growth inside the coconut has only just started to develop and inside is still watery. Hem i taem we i stat blong kam navara be i nogat navara insaed long hem yet.] \{Lit. tail of topsail drummer tel blong bigbel fis\}

gör $v t$. grate (coconut), scrape out insides of something firm skrasem kokonas, skrasemaot insaed long wan samting we i strong Nēn a gör o mōtō talo nēn a wōr le gengen. We grate the coconut so that we can squeeze its milk onto the food. Yumi skrasem kokonas blong yumi melekem i go long kakae. (VVAMVDP04Jun0801-JN.033) Na gör den o lölö dem ti nana riv. I'm scraping out the inside of the yam so that I can plant it. Mi stap skrasemaot insaed long yam blong mi planem. Syn: geres
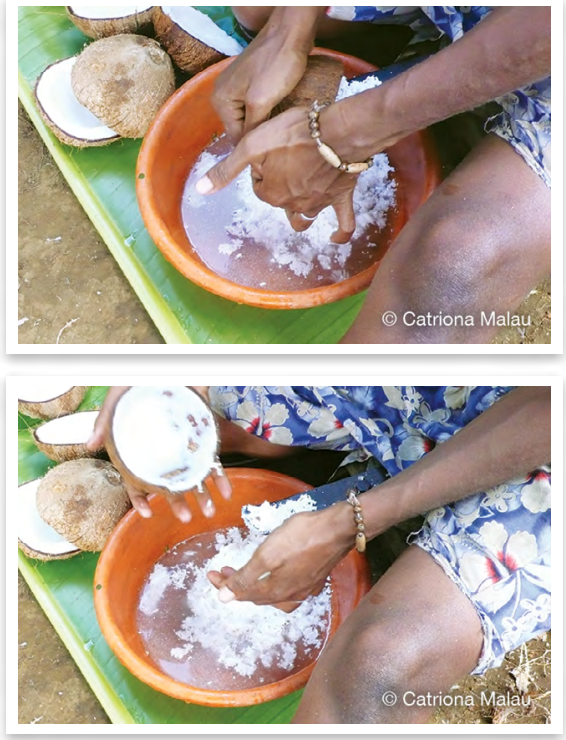

gör $\sim$ grate coconut

görgör n.com. kind of skin condition in which skin has lots of tiny, rough bumps, like sandpaper, but is not too itchy kaen sik blong skin we i gat ol smolsmol lam long hem, be i no skras tumas

görum n.com. black land crab that is red when cooked, lives in holes in trees or in the ground wan blak krab we i stap long bus insaed long wud o hol long graon. Taem yu kukum hem i red Gecarcinus quadratus

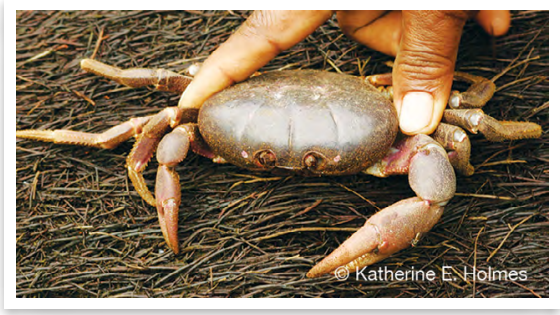

görum Gecarcinus quadratus

gösuv vt. wash (body, plates) wasem (bodi o plet) Nēk i gösuv na nogon. Wash your face. Iu wasem fes blong yu. Syn: as , See: suv 
gösuv miar $v$. bathe baby in a traditional ceremony that occurs 5-10 days after the baby is born, the aunts of the child bathe the child and the father pays the aunts for this contribution $\sim$ faef o ten dei afta fes pikinini i bon, ol sista blong papa ol i kam wasem pikinini mo flasem hem mo afta papa i mas pem olgeta gösuv rōñ $v t$. baptise baptaes Nēr mö gösuv rōn o nötu mirmiar nitiwial. They baptised a child. Ol i baptaesem wan pikinini.

götö ${ }_{1}$ n.com. hermit crab nakato

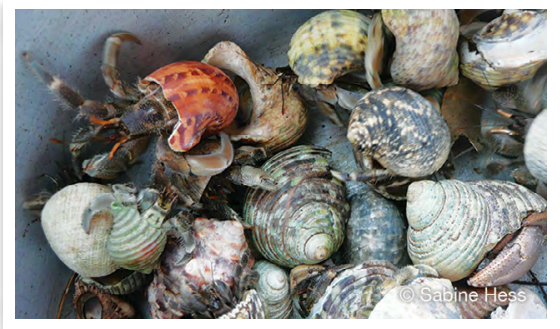

götö hermit crab

götö mamē (comp.) n.com. kind of land hermit crab wan kaen nakato we i stap nomo long drae ples \{Lit. red hermit crab red nakato\}

götö riam takak (comp.) n.com. kind of black hermit crab found in the bush wan kaen blak nakato blong bus

götö sërsër (comp.) n.com. kind of small hermit crab that lives in the sea wan kaen smol nakato we $i$ stap insaed long solwota

götö ${ }_{2}$ n.com. variety of taro, 'male', with black stem, and white corm with red spots $\sim$ kaen taro we hem i man we han blong hem i blak mo kakae blong hem i waet wetem red spot

götuë $n$ before, long time ago bifo, long taem finis

gövruq̄ō $n$.com. thin length of bamboo tied onto edge of thatching on roof to hold the thatching tight longfala bambu we i tintin we ol i fasem long en blong natanggura blong i holem taet $\mathbf{O}$ gövruqō nē ge seben gōr o doot. The gövruqō holds up the thatching. Gövruqo hem i holem taet natanggura \{Lit. pig house haos blong pig\}

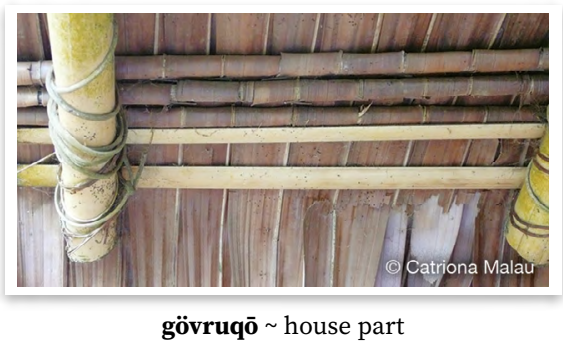

gövur n.com. house, generic name for building haos

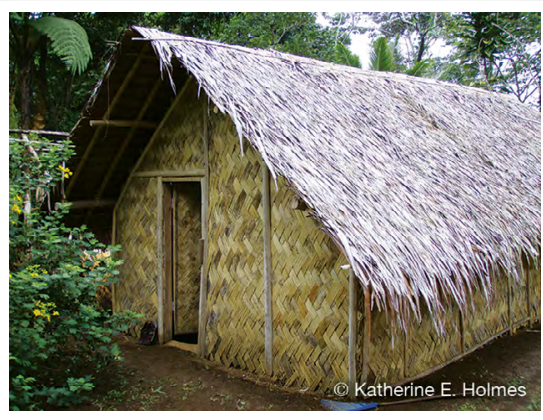

gövur house

gövur gōrōn̄ (comp.) n.com. church jos Syn: gövur tatar \{Lit. holy house tabu haos\}

gövur lisia (comp.) n.com. hospital, clinic klinik \{Lit. sickness house haos sik\}

gövur tatar (comp.) n.com. church jos Syn: gövur gōrōn \{Lit. prayer house haos blong pray\}

kulë gövur (comp.) n.com. back, sides and roof of house, sections of house behind the front $\sim$ baksaed blong haos, we i kavremap saed mo ruf \{Lit. back of house baksaed blong haos\} 
GÖVUT

nögö gövur (comp.) n.com. front of house $\sim$ fored blong haos $\{$ Lit. face of house fes blong haos\}

gövut $v t$. pull out something that is tightly held within something, as in ground, uproot pulumaot wan samting we i fas gud bitwin o insaed long wan samting, olsem pulumaot wud long graon Nēk i gövut rieg o tere mete qatē ine. You pull out completely all those spikes from the tree fern. Iu pulumaot gud ol nil blong blakpamia. (JRQ)

göwur n.com. white-margined moray wan kaen namarae gunö n.p.bound spouse man blong woman; woman blong man

ginon (n.poss) n.p.bound her/his spouse $\sim$ man/woman blong hem gunök (n.poss) n.p.bound my spouse woman/man blong mi

gunögōsōw n.com. kind of large freshwater shellfish wan bigfala sel blong wota we i stap long saed blong wota [People don't eat these because they have a strong smell and taste. Man i no kakae from hem i gat smel.] \{Lit. rat's spouse woman blong rat\}

gunök n.p.bound my spouse woman/ man blong mi see: gunö

\section{$\mathbf{I} \sim \mathbf{i}$}

i inter. ow! awo! Tëqël mō rōw lö lölö ev ine, i rege ine a tërtër, nē ta, 'Akē, akē, akē! Gë mëmës, gë mëmës.' Tëqel jumped into the fire, the people covered him up, and he said, 'Ow, ow, ow! It hurts, it hurts.' Tëgèl i jam i go insaed long faea, olgeta $i$ kavremap hem, i se, 'Awo, awo, awo! I soa, i soa.' (KM̄T.165)

$\mathbf{i}_{2}$ art. personal article denoting nouns in personal noun class $\sim$ maka we $\mathrm{i}$ go wetem naon we $\mathrm{i}$ talemaot nem blong man o famle $\left[P \mathrm{PC}^{*} i\right.$ 'personal article']

$\mathbf{i}_{3}$ v.part. second person singular default aspect marker wod we i folem nēk 'yu' bifo long veb long sentens

ia dem. here ia [Bis ia 'here']

iamarge n.com. shame sem I nē o iamarge gö luwō no vēti sur nē mē gial no nonorēs. He is still really ashamed with me because he lied to me the other day. Hem i sem bigwan long mi yet from hem i giaman long mi lastaem. iamron vi. tired taed Syn: $\bar{n} a l_{1}$

idian $a d v$. very, really, too, too much, degree adverb tumas Nē mē èl mē qiliañ, nē mō lōlwon idian aē. He saw that it was lost and he was very sad about it. Hem iluk se ilus, hem iharem nogud tumas long hem. (VSB) Nēk mite ven̄en idian nē. You shouldn't feed it too much. Bae yu no fidim hem tumas. (ESP) Syn: nimal

igēn (fr.var. of gēn)

ii inter. hey! ei!

Ika n.p.fr. 1 - wife, used as an address term waef, olsem yu singaot long hem 2 - dear; address term used to someone who has a close relationship with the speaker $\sim$ dia

iken (fr.var. of ken)

ikene (fr.var. of ken)

ilan̄ n.com. nothing, absence of something that one expected to be present $\sim$ nating, nogat wan samting long ples we yu ting se bae wan samting i stap 
ilēkē (fr.var. lēkē) dem. that one over there, distal presentative $\sim$ hemia longwe Dōrōk a gav ten sur o tōw ilēkē a Vōnō Lav? Shall we two try and fly to that hill over there on Vanua lava? Bae yumitu traem blong flae kasem hil ia longwe long Vanua Lava? (DHN)

iloko (fr.var. loko) dem. this here, proximal presentative hemia, hemia ia Nēr gö wöt na mögun nēr o vi min iloko, i vo ti siag ti gēn, John Vira. They are going to toss their fee to this one here, the guy sitting here, John Vira. Bae ol i sakem fi blong olgeta $i$ go long hemia nao, man ia i sidaon ia, John Vira. (VSS)

ilsi $n . c o m$. end en Wun o ilsi kakaka nē mē dēn gem ti aē gēn. I think that's the end of the story, it just reaches up to here. Ating en blong storian i kasem long ples ia nao. (KM̄T) Ba nēk i kōñ rak siag, rivteg o ilsi. But you snap it (plant) up here a bit, close to the end. Be yu brekem antap smol, klosap long en. (SBB)

ine (abbrev. ne) dem. that, anaphoric demonstrative, referring back to a previously mentioned participant or event $\sim$ ia, wan samting we yu bin tokbaot finish Rōrō a èlēl me sur o tōw a Vōnō Lav. O tōw ine nē ge elel. The two of them were looking this way towards the hill on Vanua Lava. That hill is very high. Tufala i lukluk i kam long hil long Vanua Lava. Hil ia hem i hae tumas. (DHN) E ni siag ti aē lē rērēi rēntenge nitiwial, ni sigsiag ine, ni teñten. And it sat there on the branch of a tree, it sat there and it cried. Ale hem i sidaon long brans blong wan wud, hemisidaonia, istap krae. (DHN)

in̄kè dem. this, this one, proximal demonstrative $\sim$ ia, hemia Lō tōtgial in̄kē, na èl o tōtgial in̄kē timiak mō tōtgial lö lölö naw. In this photo, I see this photo as if it were taken inside the sea. Long foto ia, mi luk long foto ia olsem se ol i karem foto insaed long solwota. (DPR.001) Syn: gēn

in̄ko temp. now nao

in̄ko ${ }_{2}$ conj. then $\sim$ nao Nēr me gengen qēt, in̄ko ni qön̄. They finished eating, and then night fell. Ol i kakae finis, nao naetikam. (ATB)

In̄lan n.loc. England Inglan

io inter. yes yes

ira $a r t$. plural personal article ol; wod we i go bifo long naon long sentens we i soemaot se i refe long tu o moa blong wan samting

irir vt. reject spouse because one has reason to believe that they have been unfaithful (man o woman) i no wantem stap gud wetem woman o man blong hem from hem i ting se hem i gat narafala fren Nē ti irir viteg na ginon sur na bakan aē lö möt. She is rejecting her husband because he has a lover who he meets in secret outside the village. Hem i nomo stap wantem man blong hem from hem igat narafala gelfren long bus.

is n.com. homebrew made from yeast dring we ol i mekem long yis

isē $\left(\right.$ root $\left.\mathrm{se}_{2}\right)$ n.p.fr. who hu Isē ma van, me le o bek nivet gēn? Who went and took those four bags? Hu $i$ bin go, i bin tekem fofala bag ia? (VST) [POc *sai 'who']

iv (fr.var. of uv) 


\section{$\mathbf{K} \sim \mathbf{k}$}

-k suff. first person plural possessive suffix, my blong mi Na qötuk gë mëmës. My head hurts. Hed blong mi isoa.

kaka $v t$. tell story, narrate $\sim$ stori No gö mörös na kaka o kakaka nitiwial, kakaka na mögun Mes Tuvun. I want to narrate a story, the story of Mes Tuvun. Mi wantem stori long wan storian, storian blong Mes Tuvun. (JMT.002)

kakaka $\mathbf{1}$ - vi. chat, speak storian, toktok $\mathrm{O}$ tölne tan̄sar tala kakaka lō vōrōg ko, Tomas, maranag talō tōtōtow. The third person to speak this morning is Tomas, the chief of progress. Nambatri man blong toktok long moning ia hem i Tomas, jif blong progres. (VSG) 2 - vi. talk about, tell story about $\sim$ storian (long) No gö mörös kakaka sur i rege min tasēn. I want to talk about a boy and his younger brother. Mi wantem storian long saed blong wan boe wetem smol brata blong hem. (OQQ.002) 3 $n$ com. story, tale, speech $\sim$ storian O kakaka na mōgōn e nē ga van lō bōk, nē go tog lav. His story will go in a book, and it will endure forever. Storian blong hem bae i go long buk, bae i stap oltaem. (VSE)

kakal (fr.var. of kalkal ${ }_{2}$ )

kakēlis n.com. brown striped lizard braon lised wetem laen

$\mathbf{k a l}_{1} v t$. pull in fishing line, pull up fish $\sim$ pulum string Nēk ma kal o mes nives? How many fish did you catch? Iu pulum hamas fis?

kal vi. $^{1}$ - go up, motion or event directed upwards go antap Alē kōmōrōk a var kal siag li tōw. Then the two of us went up the hill. Ale mitufala $i$ go antap long hil. Ant: tēqēl
1 | 2 go landward, ashore, inland go antap long so, long bus Ni vēn basbasne o mes, ni wöt kal siag. He shot the last fish and he threw it ashore. Hem i sutum laswan fis, hem i sakem i go antap long so. (JMT) Ant: tēqēl 2 | $\mathbf{3}$ - go upstream go antap (folem wota) Rōrō a sō kal tatag o bē e. The two of them paddled upstream. Tufala $i$ parel folem wota $i$ go antap. (GMM) Ant: tēqēl $\mathbf{3} \mid \mathbf{4} \cdot$ travel in a southeasterly direction (of travel beyond island to other islands) go long saot long narafala aelan Ant: tēqēl $\mathbf{4} \mid \mathbf{5} \cdot$ go inside, enter go insaed Qēt ine kōmōrōk a kal lin̄ereg sar lö lölö gövur. Then the two of us went inside the house. Afta mitufala $i$ go insaed long haos. (AAD) Ant: kalō

kalkal n.com. hill, slope of hill hil

kalkal (fr.var. kakal) vi. crawl wokbaot fo leg Ma datek vaavan o tētē ine ni kalkal. They looked after him until the baby could crawl. I lukaotem go go bebe hem i wokbaot fo leg. (ESS)

kalmōs n.com. kind of lizard which is black with green-yellow belly wan kaen lised we hem i grin mo blak

kalō $v i$. go outside go aotsaed Nē mi wiak o mete gövur tala kalō. She opened the door in order to go outside. Hem i openem doa blong $i$ go aotsaed. (DWB.008) Ant: $\mathrm{kal}_{2} \mathbf{5}$

kalqar n.com. green tree lizard grinlised Emoia sanfordii

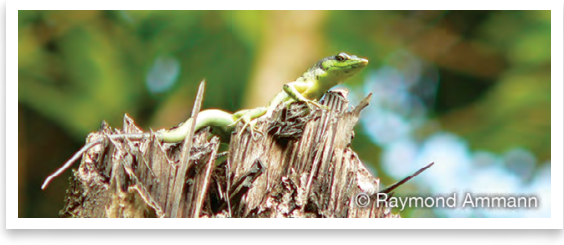

kalqar Emoia sanfordii 
Kalrōw n.loc. tribe name $\sim$ nem blong traeb

kan $c l$. her/his/its (canoe) blong hem (kenu) See: $\mathrm{ke}_{3}$

kar n.com. spear with four prongs and rubber spia we hem i gat raba blong hem

kara v.part. just, of an event that has happened very recently or will happen very shortly after an upcoming event $\sim$ jas No kara miat me ti, o bunë maram vēti min no. I have only just died, the smell of the world is still on me. Mijas ded ia nomo, smel blong wol i stap yet long mi. (JTF)

kartō̄ n.com. cardboard box katon [Bis katon 'cardboard box']

kasivi n.com. fork (of river, tree) fok (blong wota, wud) o kasivi bē fork of a river fok blong wota See: n̄iria

kastom n.com. tradition $\sim$ kastom

kat $v$. stick to $\sim$ fas long

kav n.com. corrugated iron $\sim$ kapa

$\mathbf{k e}_{1}$ inter. yes yes Syn: e'e

$\mathbf{k e}_{2}$ part. emphatic particle $\sim$ we, tumas Nē o ke mōnōg. She was completely covered in sores. Hem i gat fulap soa. (ATB)

$\mathbf{k e}_{3} \quad c$. relational classifier used to mark ownership of forms of transport, such as a canoe blong (yusum blong tokbaot ona blong kenu o trak) Ni rēv sur na kan o ak, ni sō. He pulled down his canoe, and he paddled. Hem i pulum kenu blong hem i go daon, hem i parel.

kan (n.poss) cl. her/his/its (canoe) blong hem (kenu)

ken tëmëk (n.poss) $c$. belonging to my father (canoe) blong papa blong mi (kenu)

këk (n.poss) cl. my (canoe) blong mi (kenu)

kel $v t$. stir $\sim$ tanem (wetem spun) $\mathbf{O}$ so nēk te kelkel? What are you stiring? Yu stap tanem wanem?

kemek (fr.var. of kemem) kemem (fr.var. kemek) pron. we, us; first person plural exclusive pronoun mifala

ken (fr.var. iken, ikene, kene) dem. that one, that one there, distal demonstrative hemia I rege iken. Those are the people there. Hemia olgeta ia. (RPP.098)

ken tëmëk $c l$. belonging to my father (canoe) blong papa blong mi (kenu) See: ke 3

kene (fr.var. of ken)

ker $v t$. comb komem Iutikas, nēk i van me, na ker na vōlōñ. Iutikas, come here and I'll comb your hair. Iutikas, yu kam, mi komem hea blong yu.

kere part. dehortative marker; don't no; wod we i go bifo long veb long sentens, taem yu talemaot long wan man se bae i no mekem wan samting Vita, 'Ōōō, ri kere mōlmōl kēti.' He said, 'No, don't go home yet.' I se, 'Nogat, yufala i no go hom yet.' (DPD.066)

keres $v t$. scratch, use scratching motion to dig skrasem, digim

keresi n.com. thorn (of wild yam, Chinese yam, umbrella leaf) $\sim$ nil blong wael yam, swityam, ambrela lif

kes $v$ t. argue, row with rao Kōmōrōn a rēs, nēk i kes nē, ni mōl kēl. You two fight, you argue with him, then he'll go home. Yutufala $i$ rao, yu rao wetem hem, bae hem i go hom. (JMT)

kev $v t$. dig earth digim graon

kewō $n$.com. large canoe $\sim$ bigfala kenu $\mathbf{k} \overline{\mathbf{e}}_{1}$ n.com. dolphin dolfin

$\mathbf{k} \overline{\mathbf{e}}_{2}$ disc. emphatic particle $\sim$ tumas Nē ma van ren kē a Worwor, e ni ēl i taman e ni los. He went all the way to Worwor, and he saw his father who was sick. Hem i go olwe long Worwor ia, ale hem iluk papa blong hem we hem i sik. (EDM.005) 
kēl 1 • vi. return kambak No mēe èl vita o masawre in̄kē no mi ti kēl bilēn̄ aē. I can see that I won't come back to this place again. Mi luk se ples ia, bae mi no gobak long ples ia bakegen. $\mathbf{2}$ - vi. face towards, be directed towards $\sim$ fes i go long Na kōlan gē kēl me sur no wo na nogon gē kēl sōw. His back is towards me and his front is facing to the right (when facing sea). Baksaed blong hem ifes i go long mi mo fran blong hem i fes $i$ go long raetsaed. (M2E) 3 • $a d v$. again bakegen Qēt i rètnan ni tel kēl. Then his mother got pregnant again. Afta mama blong hem $i$ gat bel bakegen. (ATB) $\mathbf{4}$ - prep. towards go long Ni qaq kēl rōw min i Mes. It spoke out down towards the sea to Fish. Hem i toktok i go daon long solwota i go long Fis. (DNS)

kēlēglēg $v i$. crooked, bent, not straight kruked Gōtō ōn tenēnēg, gō ōn kēlēglēg. It doesn't lie straight, it lies crooked. Hem i no lei stret, i lei kruked. (M4D.025) Ant: tenēnēg,

kēmi (fr.var. kimi) pron. you, you all; second person plural pronoun yufala

kērē 1 •n.com.suff. arse, bottom as O met ni lum vaavan ni lum dēn̄ na kirian. The tide came in until it came up to his bottom. Solwota i kamso gogo $i$ kamso kasem as blong hem. (JMT) Syn: wērē 2 - $n$ base, underside as, andanit $\mathbf{3} \cdot$ n.com.suff. rear, end, back, as rear of canoe or car, stern of ship bihaen, baksaed blong trak o kenu No mo qal tes ilēkē o kērē, ba in̄kē o qötu tabaē. I first carved that bit, the back, but this is the front of it. Fastaem mi katem hemia, baksaed, be hemia fored blong hem. (VVKHVDP180ct0801.030)

kērē vōnō (comp.) n.com. area of village that is on the seawards edge or section eria blong vilej we i stap long saed i go daon long solwota Ant: qötu vōnō (comp. of qötu) \{Lit. bottom of village as blong vilit\}

kērēk (n.poss) n.com.suff. my arse as blong mi

kirian (n.poss) n.com.suff. her/his/its arse as blong hem

kērēk n.com.suff. my arse as blong mi See: kērē

kērēt inter. yeah right! Interjection used teasingly to indicate that you know that what you or another interlocutor said is an exaggeration or inaccurate ei no! No gö sum gem o vinlas nitiwial. Kērēt! I'll just drink one cup. Yeah right! Bae mi dring wan sel nomo. Giaman!

kērēt ${ }_{2}$ n.com. 1 • poo, excrement $\sim$ sitsit $\mathbf{2} \cdot$ toilet $\sim$ toelet, smolhaos Ni vērēg lē kērēt. He raced to the toilet. Hem i resis $i$ go long toelet. Syn: nötu gövur, tes

kēt $v$. wild wael No ma van vita na vil bulëk o tō ba o tō e mē kēt. Mi go blong holem taet faol blong mi be faol blong mi i wael.

kēti (fr.var. kiti) $\mathbf{1}$ inter. wait, hold on wet, wet smol $\mathbf{2} \cdot a d v$. short time $\sim$ sot taem No ga mamar kōmōrōñ vita, dōrōk ge mem viteg kēti o kastom. I am going to plead with the two of you (polite) that we two put aside our traditions first. Mi plis long yu se yumitu livim kastom fastaem.

kētkēt n.com. 1 - in air, up high above ground antap, long ea, i no long graon $\mathbf{O}$ vetel ga da $\mathbf{o}$ wie lē kētkēt. Bananas produce their fruit up in the air. Banana i mekem kakae blong hem antap. Syn: m̄aē $\mathbf{2} \cdot$ heaven heven I God, na vanañ kal li kētkēt. God, your place is up in heaven. God, ples blong yu antap long heven.

këk $c l$. my (canoe) blong mi (kenu) See: $\mathrm{ke}_{3}$

kër $r_{1} v t$. scratch with fingernails $\sim$ skrasem, wetem sel fingga $[P O c * k a r u(t)$ 'scratch with fingernails or claws'] 
kër $\mathbf{r}_{2} v$ t. $\mathbf{1}$ - want $\sim$ wantem No gëtë kër nēk go kuk o raes, nēk ge le min nēr. I don't want you to cook rice and give it to them. Mi no wantem se bae yu kukum raes, yu givim long olgeta. 2•like laekem

kikbol $v i$. play soccer $\sim$ plei futbol kikse n.com. side (of) saed (blong) Syn: bersi

kilmat vi. wink, blink one's eyes or somehow make a funny face, as a sign to someone that one doesn't want others to see. This could be because one is wanting to keep a secret, is making a joke or teasing someone $\sim$ mekem ae o fani fes long wan man we yu stap toktok wetem hem olsem wan saen blong sikret blong yutufala we yu no wantem ol narafala man we i stap blong save

kilo vi. turn around tanem No mēe èl kilo. I looked behind. Miluklukbihaen. kimi (fr.var. of kēmi)

kir vi. fart brekwin

kirian n.com.suff. her/his/its arse $\sim$ as blong hem See: kērē

kirkiar n.com. croton $\sim$ kala lif Codiaeum variegatum

kirkiar mamēgin (comp.) n.com. variety of croton that grows in the bush and is not planted near houses $\sim$ wan kaen kala lif we i stap long bus nomo [If one has an eye infection, the sap from the leaves of this croton should be dripped into the eye. Sapos yu gat wan soa long ae blong yu, bae yu karemaot wan lif, ale mekem wota blong hem i go long ae blong yu.] \{Lit. cold croton kala lif blong kolkol\}

kirkiar meter (comp.) n.com. variety of croton wan kaen kala lif [Traditionally only used by women. Blong ol woman nomo.] \{Lit. Meter's croton kala lifblong M̄eter\}

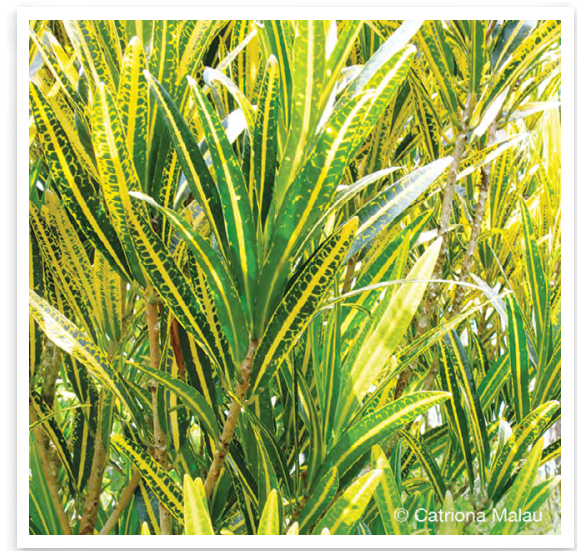

kirkiar $\overline{\mathbf{m}}$ eter $\sim$ croton variety

kirkiar qere qō (comp.) n.com. variety of croton with curly leaves wan kaen kala lif we lif blong hem i tantanem $\{$ Lit. pig's penis croton kala lifkok blong pig\}

kirkiar qet (comp.) n.com. variety of croton that is red and green wan kaen kala lif we i red mo grin [Traditionally only used by men. Blong ol man nomo.] \{Lit. Qet's croton kala lifblong Qet\}

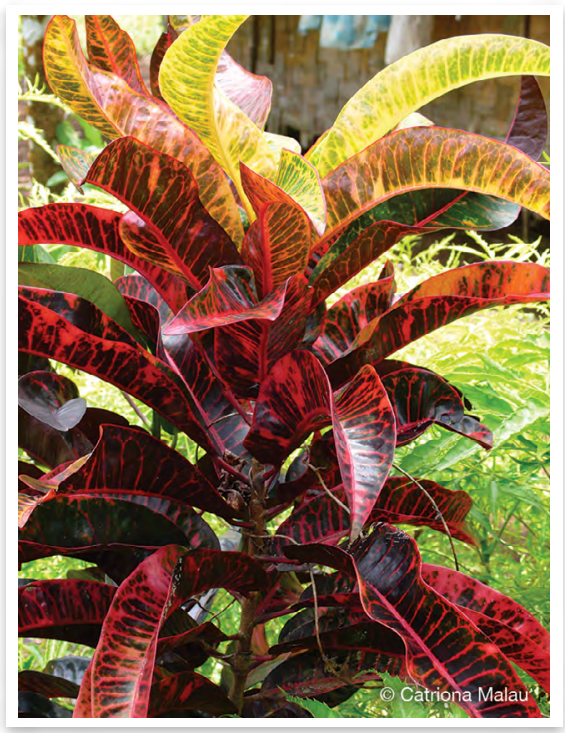

kirkiar qet $\sim$ croton variety 
kirkiar sas qȫn (comp.) n.com. variety of croton $\sim$ wan kaen kala lif \{Lit. night croton kala lif blong naet\}

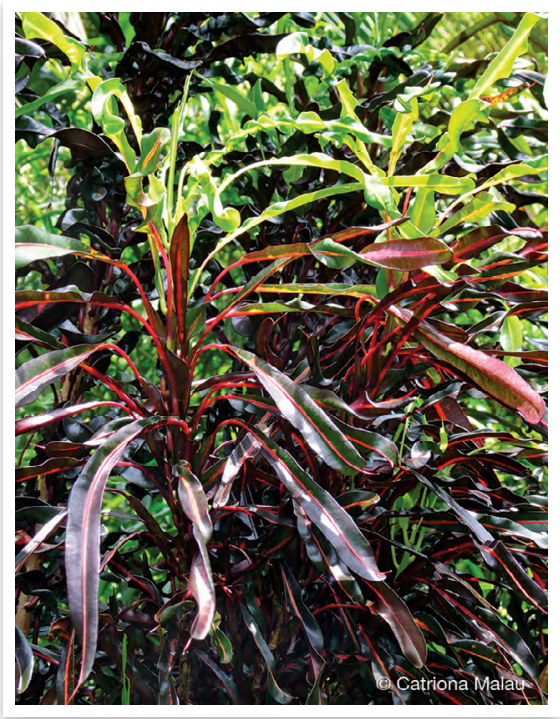

kirkiar sas qön̄ $~$ croton variety

kirkir $v i$. have diarrhoea $\sim$ sitsit wota

Nē o kirkir. She has diarrhoea. Hem i sitsit wota.

kiti (fr.var. of kēti)

klas n.com. glass, mirror glas [Bis glas 'glass, mirror']

ko dem. this, proximal modifier $\sim$ ia Nēn ma da o gövur ko la masawre gōrōn. We have made this house in a holy place. Yumi bin bildim haos ia long tabu ples. (EDM)

ko (fr.var. of oko)

Koimaram n.loc. place name $\sim$ nem blong ples

kokos vi. 1 - make narrow, tight mekem i smol $\mathbf{O}$ gersal e go kokos gem. That road is only narrow. Rod ia i smol nomo. Nēk i gis kokos na molon. Bring your clothes in tightly round your legs. Holem klos blong yu $i$ taetem leg blong yu. $\mathbf{2}$ - close together, hold together $\sim$ klosap 3 - tightly woven $\sim$ wivim taet kor $v$. make kindling; cut trees and branches into smaller pieces so that they will dry and can be used as firewood katkatem ol brans mo wud we yu bin katem blong livim i drae redi blong bonem Rēntenge, nēk ma tar tul vitia, e nēk i kor. The tree, you've already chopped it down, now cut it up. Wud, yu katem daon finis, ale yu katem pisisim.

korel n.com. coral korel See: weles [Bis] kosēv vi. break off (of branch) kamaot (brans blong wud) $\mathbf{O}$ rērēi mo kosēv den o rēntenge. The branch broke off from the tree. Brans $i$ kamaot long stamba.

kōk vt. surround with open arms when trying to catch something (e.g. chicken) raonem wetem open han Nē gö mörös ta ni gis varge o tō, e ni kōk gōr. He wants to catch a chicken, so he approaches it with open arms. Hem i wantem kasem faol, ale hem $i$ openem han blong hem iblokem.

kōkm̄eg vt. look after, guide lukaotem, kea long Si nēk i kōkm̄eg wareg gōr na ōsōn̄ nēk mito los le. If you look after your life well, you won't get sick. Sapos yu lukaotem gud laef blong yu bae yu no sik. No ta vanvan ba no ga van kōkm̄eg ra nötuk. As I go I look out for my children. Mi stap go, be mi lukaotem gud ol pikinini blong mi.

kōkō vi. swing swing I Jessica ni kōkō la gakōkō. Jessica is swinging on the swing. Jessica $i$ swing long swing. See: gakōkō

kōl vi. perform grade-taking ceremony, that a man performs in order to increase in rank as a chief, which involves killing of pigs $\sim$ kilim pig long seremoni blong leftemap rang

kōlkōl (redup.) n.com. grade-taking ceremony, ceremony that a man performs in order to increase in 
rank as a chief $\sim$ seremoni we man o woman i save mekem blong tekem rang. Woman i mekem long haos, man i mekem long nakamal Nēr te veveñen sur wo la masawre nēr a da o kōlkōl, nēr a vus. They were feeding them because when they performed a grade-taking ceremony, they would kill them. Ol $i$ stap fidim olgeta from taem we ol i tekem rang, bae ol i kilim olgeta. kōlan n.com.suff. her/his/its back baksaed blong hem See: kulë

kōmar n.com. sweet potato kumala Ipomoea batatas

kōmōrōk pron. we two, us two; first person dual exclusive pronoun mitufala

kōmōrō̄n pron. you two; second person dual pronoun $\sim$ yutufala

$\mathbf{k} \overline{\mathbf{n}} \overline{\mathbf{n}} v t$. snap, bend and break brekem Nēk i kōñ lèt o rēntenge. You snap the wood. Yu bendem brekem wud. Sasar, kēmi min ēl ni taw, ri kōn̄ o rērēi, ri riv kēl. When you see the aibika flowering, snap off a branch and replant it. Taem yu luk se aelan kabis $i$ gat flaoa, yu brekem brans, yu planem bakegen.

kōñqarqar n.com. someone who died unexpectedly while still young and healthy man we hem i no sik mo hem i no olfala be hem i jas ded nomo taem yumi no ekspektem \{Lit. break unripe brekem ino raep\}

kör n.com. dried breadfruit biscuit, used as famine food after hurricane $\sim$ bredfrut biskit [This is made by roasting the whole breadfruit first in the fire, then removing the soft part in the centre. It is then baked in a stone oven, covered with sago palm leaves. Finally it is wrapped in cottonwood leaves and hung over the fire, such that it can last for up to three years. Blong mekem bredfrut olsem, ol i rusum bredfrut finis, katemaot top wetem sofsof pat insaed, mekem faea long oven, putum bredfrut insaed mo putum tivtiv (lif natanggura we ol $\mathrm{i}$ bin somap) antap. Taem i dan i drae olsem biskit mo ol i paselem long lif burao, hangem ova long faea mo i save stap tri yia.]

körkör 1 • vi. black, be black blak Nē ge le min rege gogov gö körkör. He will pass it to the people in black clothes (Brotherhood). Bae hem i givim long olgeta long blak klos (Bratahud). 2 - n.com. blackness, black (one) blakwan 3 - vi. dark (colour) blak, tudak

kuëg $v t$. shake, move, push on swing sekem, muvum, pusum Kuëg mamamat nē. Shake him so he wakes up. Sekem hem i wekap.

kuk $v$. cook, used occasionally as generic cooking term, but more commonly with specific reference to cooking food in a saucepan, rather than over fire or in stone oven kuk, kuk long sospen Nēr a tuntun, nēr goto kukuk, nēr a tuntun. They roasted, they didn't cook in a saucepan, they roasted. Ol i rus, ol i no kuk long sospen, ol i rus. (JMT) [Bis]

kukuög n.com. variety of banana that is only eaten ripe, not cooked when unripe; the fruit will fall off when the trunk is shaken kaen banana we ol i kakae raepwan nomo mo taem yu sekem stamba ol raepwan i foldaon, mo hem i sotfala See: ruruñög

kulë n.com.suff. $\mathbf{1}$ - back (body part) baksaed Nēk i siag lö kulëk, e na gav tek dōrōk. You sit on my back and I will fly the two of us. Iu sidaon long baksaed blong mi, mi flae wetem yumitu. (WRN) $2 \cdot$ behind, at the back, after (place or event) bihaen, afta Tok nitiwial nē o mas tur lö kulë vaga. One dog must always stand at the back. Wan dog bae i mas stanap bihaen oltaem. (DDP) Tōlōw lö kulë tatar 
na mögun nēn. Tomorrow after our prayer. Tumoro after long prea blong yumi. (VSG)

kōlan (n.poss) n.com.suff. her/his/its back baksaed blong hem

kulëk (n.poss) n.com.suff. my back baksaed blong mi

kulëk n.com.suff. my back baksaed blong mi See: kulë

kulum $_{1} v t$. bend at joint, as legs benem

kulum $v t$. clench fist $\sim$ taetem han Nēk i kulum na banēn̄. You make a fist. Yu mekem taet han blong yu.

kulum $_{3} v t$. gather together in bundle and tie up mekem bandel mo fasem taet Ri kulum kalkal o tere doot gēn. Bundle together those sago palm leaves and tie them up. Putum ol lif natanggura long wan bandel mo fasem taet.

kur $v t$. $\mathbf{1}$ - eat, bite on something that is hard (e.g. nut, biscuit, bone) kakae wan samting we hem i strong (olsem nat, biskit, bun) Dōrōk a van a kur talēs? Shall we two go and eat Indian almonds? Bae yumitu go kakae natavoa? (BNR) 2 e eat person, term used in traditional stories to refer to devils or bad spirits eating people, as they eat them totally including bones kakae man, olsem long kastom storian ol devel i kakae man wetem bun blong hem Ba nēk wun mōl, nēk kara gagneg min i Die wo i Mam vita Qaslaklakatēg mo kur no. But when you go home, just tell Mum and Dad that Qaslaklakatēg ate mi. Be bae yu go hom, yu talem long Mama mo Papa se Qaslaklakatēg i bin kakae mi. (OQQ)

kurkurqōl n.com. blue-finned triggerfish kaen strongskin (fis)

kurqar not cooked properly hafdan, kakae we i no dan gud

\section{$\mathbf{L} \sim \mathbf{1}$}

la masawre subord. when, introduces temporal adverbial clause $\sim$ taem La masawre me meren, e ni ukuëg kēl o masawre ine. When it became daylight, then he left that place. Taem hem $i$ deilaet, ale hem i lego ples ia. (DFF)

labēvi n.com. stem or petiole and leaf of palm or non-branching plant, as taro, tree fern, coconut, sago palm; rachis, principal midrib han blong wud olsem kokonas o blakpam o taro

lag $v$. walk on narrow edge at height, as over narrow bridge or on branch $\sim$ wokbaot long smol ples, olsem long smol brij o brans blong wud O Bē Daragrō, nēn ga lag kal lē rēntenge. Daragrō Creek, we cross over it on a narrow log bridge. Wota blong Daragrō, yumi krosem antap long wan smol wud. (RRS.004)

lagē n.com. marriage mared See: leg lagē gē tisē (comp.) n.com. bad marriage, in that it is not correct according to the rules of tradition dictating whom one should marry mared we i no stret long kastom, olsem we wan i mared wetem famle \{Lit. bad marriage mared nogud\}

lagē gōwē (comp.) n.com. good marriage, in that it is correct according to the rules of tradition dictating whom one should marry $\sim$ mared we $\mathrm{i}$ folem stret rod blong kastom \{Lit. good marriage mared gud\} 
lak vi. dance $\sim$ danis

laklak (redup.) n.com. dance $\sim$ danis E söw, rōrō a kal lin̄ereg la laklak. And so the two of them joined the dance. Ale tufala i go insaed long danis.

lakakēris ${ }_{1}$ n.p.fr. garden spirit, good spirit that is positive force in garden $\sim$ gudfala spirit blong garen

lakakēris, n.com. kava variety that is very tall, is not generally used to make kava for drinking kaen kava we i bigwan, ol man ol i no yusum tumas blong mekem kava long hem

lakakēris ${ }_{3}$ n.com. variety of taro, 'male', with light green stem $\sim$ kaen taro we hem i man we han blong hem i laet grin

lakēr n.com. active, able to do things aktiv, i save mekem samting O mirmiar ine o lakēr ren. That child is very active and able. Pikinini ia hem i aktiv mo i save mekem samting.

lala $v i$. strong strong

lalakēt $v i$. win $\sim$ win I rege taklē nēr ma van lo kot, ba i Bill gem nē ma lalakēt. Some people went to court, but only Bill won. Samfala man ol i bin go long kot, be Bill nomo hem i win.

lalèbur n.com. thick slab of wood that is placed over hole in ground and used to make the sound and rhythm, especially for elñetiti dance $\sim$ bigfala pis wud we ol i putum ova long wan hol long graon mo ol i kilim wetem wud blong mekem gudfala saon blong danis, speseli blong elñetiti

laln̄e n.com. underneath $\sim$ andanit Syn: tawle

Laln̄etak n.loc. place name $\sim$ nem blong ples

lam n.com. deep water, deep freshwater pool, deep sea dip wota, dip solwota, dip ples In̄ko i Mōlwor ni tōrak den o ak ine, ni rōw lö lölö lam, lö lölö bē, ni tul qilian̄. Then Mōlwor got up from the canoe, and jumped into the deep pool, into the water, and he drowned. Ale Mōlwor i girap long kenu ia, hem i jam insaed long dip pul, insaed long wota, hem $i$ draon i lus. (ATB) I Dōl nē tōwō sōsō vēti lē vētitne lam. Dōl was still paddling around out in the deep sea. Döl i stap parel yet long dip solwota. (WDR) Ant: raras [POc *laman 'deep sea beyond the reef']

butölam (comp.) n.com. very deep sea solwota i dip olgeta

vētitnē lam (comp.) n.com. deep sea between islands medel si bitwin tu aelan Nēr töl mō sō sō sō sōōsō lē vètitne lam. The three of them paddled right out into the deep sea. Trifala $i$ parel parel go go long medel blong solwota. (WDR)

lā̄ $v t$. bash, hit, beat strongly kilim strong Nēr a gis qal nē ine, a lā̄. They caught him, and beat him. Ol i kasem hem, kilim hem. (ATW.124) See: den̄

lam̄lam $v$. flap wings (bird) (pijin) i bangem tufala wing blong hem

lamseg $v t$. bash against something hard, like a stone $\sim$ kilim long ston o long wan samting we i strong Ni lā̄seg nē, lā̄seg nē vaavan o wērēt mi miat. She bashed it and bashed it until the octopus was dead. Hem i kilim hem, i kilim hem go go nawita i ded. (WNW)

lan n.com. high rank chief $\sim$ hae jif

lantar n.com. variety of taro, 'female', with two types kaen taro we hem i woman mo hem i gat tu kaen blong hem

lantar lamkör (comp.) n.com. variety of lantar taro, 'female', with black stem $\sim$ kaen taro we hem i wan kaen lantar. Han blong hem i dak mo kakae blong hem i waet

lantar malgias (comp.) n.com. variety of lantar taro, 'female', one of the largest tubers with a soft corm, stem is purple with light green at top 
kaen taro we hem i wan kaen blong lantar. Han blong hem i laet gre mo kakae blong hem i waet

lān n.com. fly flae

lā̄ talōbō (comp.) n.com. blowfly bluflae

lan̄ $\tan \overline{\mathbf{o}}$ (comp.) n.com. kind of small fly that is found by freshly dug ground, they cause damage to fruits like oranges $\sim$ wan kaen smol flae we i kamaot taem yu digim niufala graon; ol i spolem ol frut olsem aranis

lan̄ vivis (comp.) n.com. small black fly smol blak flae

laplap n.com. laplap; food dish that is made from starchy vegetables such as taro, yam, sweet potato, cassava or bananas. The vegetables are first grated, then usually wrapped up in a large parcel using heliconia leaves and baked in a stone oven. The grated vegetable could also be wrapped in aibika leaves or simply cooked in coconut milk. There are many variations of the dish, with inclusion of coconut milk, leafy vegetables and/or meat laplap [Bis]

laqēsi $v i$. wide $\sim$ bigwan Tōt o damat laqēsi. Cut some wide heliconia leaves. Katem lif laplap we i bigwan. Si o dōmōtō o laqlaqēsi idian, nana dērēs. If the coconut leaves are too wide, then I strip off the edges. Sapos lif kokonas i bigwan tumas, mi karemaot sam. (JWM)

Lataqarqar n.loc. place name $\sim$ nem blong wan ples

$\mathbf{l a v}_{1} a d v$. forever, without ceasing oltaem, wantaem Nē go tog lav ren ti min nēk. She is going to stay with you forever and ever. Bae hem i stap wetem yu oltaem. (CAA.050)

$\mathbf{l a v}_{2}$ vi. big $\sim \mathrm{big}$

le 1 vt. $\mathbf{1}$ - take $\sim$ tekem Qet ni le o tibiar, e ni le na rērēn̄ēn, nē ni mōl kēl. Qet took his basket, and he took his things, and went home again. Qet $i$ tekem basket blong hem, ale hem i tekem of ting blong hem, hem i go hom bakegen. (BQD) 2 • get, bring karem, karem i kam Rètnan ni le me o ralēt, rōrō a tuwegev. Her mother brought the firewood, and the two of them made the fire. Mama blong hem ikarem faeawud i kam, tufala $i$ mekem faea. (WNW) $3 \cdot$ give $\sim$ givim $\mathbf{O}$ tamarge ni le min nē na gan o gengen. The old man gave him some food. Olfala i givim kakae blong hem long hem. (ATB)

le $e_{2} a d v$. be able to perform action indicated by verb that it postmodifies save mekem Ba nēk gē biriñ le kōmōrōk? But are you able to help the two of us? Be yu save givhan long mitufala? (WDR)

le den $v$. remove, take away, take out $\sim$ karemaot Na sēr wor nirō aē, na le den o vētitne siri. I split it (coconut leaf) in two, and I remove the midrib. Mi splitim brekem long tu pis, mi karemaot medel bun blong lif. (JWM)

le ris $v t$. turn, turn over $\sim$ tanem

leg vi. marry (in church), be married mared (long jos) Rōrō ge leg garqe. They are getting married today. Bae tufala i mared tede. See: lagē

legleg (redup.) n.com. marriage ceremony (in church) mared (long jos) Nēn ga van le legleg garqe. We are going to the church wedding today. Bae yumi go long wan mared tede. See: da lagē

leg verser $v$. a son marries daughter of an uncle and daughter marries son of the same uncle, so daughter is exchanged for son and there is no need to pay a bride price $\sim$ pikinini gel blong angkel i maredem boe blong nara angkel, mo narafala boe i maredem gel blong sem angkel bakegen, mekem se ol i jenjenisim pikinini mo i nid blong pem woman, jas mekem kakae 
legser n.com. man who promises to marry but then changes his mind $\sim$ no save mared gud $\mathbf{O}$ atm̄ēn e nē o legser. That man is one who keeps changing his mind about getting married. Man ia hem i stap mekem promes se bae hem $i$ mared, be afta $i$ no wantem. [Ol pikinini ol i no mas kakae kakae we i go tugeta wetem braed praes from sapos ol i kakae bae tufala i no save mared gud. I no long taem bae tufala i divos mo sapos we wan long tufala $i$ mared bakegen bae i divos tu] \{Lit. marry repay mared pembak\}

lele n.com.suff. rib(s) reb

lalan (n.poss) n.com.suff. his/her/its rib(s) reb blong hem

lëlëk (n.poss) n.com.suff. my rib(s) reb blong mi

lelev $v i$. fast, be fast $\sim$ hariap $\mathrm{O}$ vanvan na mōgōn ge lelev si vaga nēk ta vanvan o vanvan gö möul? Is your going along fast or do you always go at a slow speed? Wokbaot blong yu $i$ hariap o oltaem yu stap wokbaot, wokbaot i slo? (DNS) Ant: möul 2

leleverser ceremony in which the family of a man who has died give money to other members of the family wan seremoni we ol famle blong wan man we hem i ded ol i givim mane long olgeta insaed long famle

le $\overline{\mathbf{n}}_{1}$ n.com. 1 • wind $\sim$ win $\mathbf{O}$ leñ nē ge meneg, o leñ mö uv me, ni tek nē in̄kē, ni tek nē in̄kē. The wind was strong, the wind blew this way and it took it (the bird) this way, it took it this way. Win istrong, win iblu i kam, i tekem hem (pijin) olsem ia, i tekem hem olsem ia. (DHN) 2 • air win La masawre nē me le o leñ timiak in̄kē, ni rōnteg o bunë. When he took in the air like that, he sensed a smell. Taem hem i tekem win olsem ia, hem i harem wan smel. (RTF) [POC *layit 'sky'] len̄limerlav (comp.) n.com. southeast tradewind tokelau, saotis win we $i$ kam long Mere Lava \{Lit. wind from Mere Lava win i kam long Mere Lava\}

len̄limot (comp.) n.com. northeasterly wind win i kam long notis long Mota [A wind that brings rain. Win ia i karem ren.] \{Lit. wind from Mota win i kam long Mota\}

len̄lirōō (comp.) n.com. northerly wind $\sim$ win $\mathrm{i}$ kam long not long Reef Aelan [A wind that can bring hurricanes. Win ia hem i wan rabis win we i save karem hariken i kam.] \{Lit. wind from Reef Island win i kam long Rif Aelan\}

len̄vus (comp.) n.com. hurricane, cyclone $\sim$ hariken \{Lit. killing wind win we ikil\}

le $\overline{\mathbf{n}}_{2} v t$. cut and break off taro petiole from the corm katem brekem top blong taro Leñ o n̄èli sinag. Cut the petiole from the taro. Katemaot top blong taro.

leñas n.com. beauty, possessing beauty, nice looking naes tumas Plasta nē so ga da o len̄as talö gövur gōrōñ e. It is the plastering that will make the church look beautiful. Plasta ia nao bae i mekem se jos hem i luk naes tumas. (VSM)

len̄man n.com. variety of taro, 'female', with green stem with white or yellow stripes $~$ kaen taro we hem i woman we $i$ gat grin han wetem waet o yelo straep long hem

leqer $v$. spring up, shoot up $\sim$ jam antap, sut antap No mö tutgëg o gil la tan, no mē lēm, o tan me leqer lë mëtëk. I stuck the digging stick into the ground and levered it and dirt shot up into my eye. Mi stikim stik long graon, mi leftemap, graon $i$ jam antap long ae blong mi. Me leqer o bes. Oil spits when heating coconut milk to make oil. Oel blong kokonas i kamaot. Sër o ōr la baket, ba ēlgōr den nēr a leqer den o baket. Pour the prawns into the 
bucket, but watch out in case they jump up out of the bucket. Kapsaedem naora $i$ go long baket, be lukaot, nogud ol $i$ jamaot long baket.

les n.com. poisonwood (tree) posenwud Semecarpus neoebudica $[P O C$ *walasi 'tree sp. with poisonous sap, Semecarpus forstenii']

Lesa n.loc. place name nem blong ples

levetē num. six siks

levetēne num. sixth, sixth one nambasikis

levga $v$. go past, cross over (something) ovarem, bitim Nē mē vērēg levga me. It crossed over towards this way. Hem i resis i kam ia. (DNS) Diar nēr a levga o bē. Wait for them to cross over the water. Wet long ol i krosem wota. Nēk i gër levga o bē ine, in̄ko nēk i van kēl sōw timiak gēn. You wade through the creek, then you go round clockwise like that. Yu krosem wota ia, nao ia yu go raon olsem ia. (DRV.020)

$\mathbf{l} \overline{\mathbf{e}}_{1}$ n.com. cave $\sim$ kef

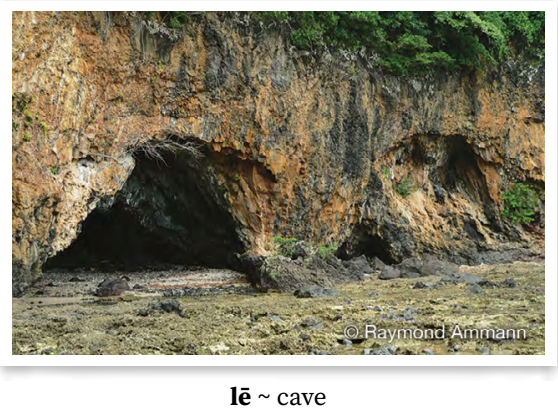

$\mathbf{l} \overline{\mathbf{e}}_{2}$ n.com. law $\sim$ loa

$\mathbf{l e ̄ g}_{1} v t$. tie up fasem Ni lēg o tere qō ine, ni van me, ni lēg lē wērēntenge. He tied up those pigs, he came and tied them up to trees. Hem i fasem ol pig ia, hem i kam, ifasem long ol wud. (ESP) See: boko

lēg $v$. hang $\sim$ hangem Lēg kal o vetel ni diam ti gèn. Hang the bananas up there. Fasem olbanana ihang antap ia. lēkē (abbrev. of ilēkē) lēl n.com. flounder, flatfish flatfis, wansaed fis Family Bothidae, Family Samaridae $[P O C *$ [l]alali 'flatfish incl. flounders, soles']

lēlè $v i$. nearly ripe, of bananas that are ripe enough to eat, but still slightly unripe klosap raep, olsem banana we $\mathrm{i}$ gud blong kakae be i no raep gud yet

lèm $v t$. stir up and spread charcoal and hot stones in stone oven with long stick (galèm); use lever action yusum wud (galèm) blong tekemaot bigfala pis wud o jakol long bigfala oven blong vësëw bifo i redi blong putum kakae Rōrō mo goro nē, ni kal lin̄ereg le vet, rōrō a lēm rak o vet. The two of them chased him and he hid under a rock and the two of them lifted up the rock using a stick as a lever. Tufala i ronem hem, hem i haed andanit long ston, tufala $i$ leftemap ston wetem wan wud. (OQQ.160)

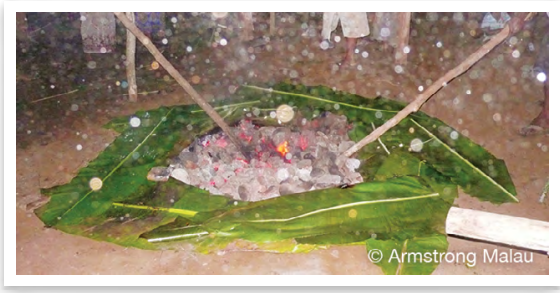

lēm lever with stick

lēn $v$. break off top of taro with knife karemaot top blong taro wetem naef lēnötō $\overline{1}_{1}$ n.com. rooster's comb red samting we $i$ stap antap long hed blong man faol

lēnötō ${ }_{2}$ n.com. red firespike, kind of flowering plant with red flowers that look like rooster's comb wan kaen plant we i gat red flaoa blong hem we i luk olsem samting antap long hed blong man faol Odontonema stricta \{Lit. rooster's comb kom blong man faol\}

lès n.com. nit, louse egg eg blong laos [POC *lisaq 'nit, louse egg'] 
Lēsē n.loc. place name nem blong ples

lēt $v t$. break brekem See: mēlēt

lēw (fr.var. löw) n.com. generic name for larger wrasses (fish) nem we i kavremap ol defren kaen bigfala napoleong (fis) Cheilinus spp.

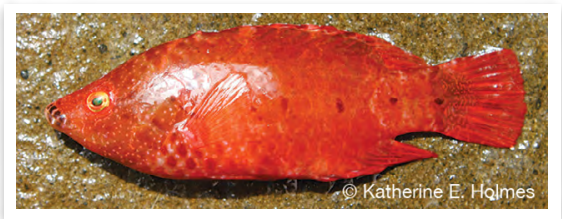

lēw wrasse

lēw biliag (comp.) n.com. kind of large wrasse (fish) kaen bigfala napoleong (fis)

lēw mal (comp.) n.com. humphead wrasse (fish) kaen napoleong (fis) Cheilinus undulatus \{Lit. hawk wrasse hoknapoleong\}

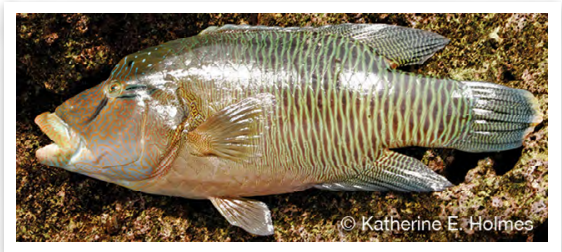

lēw mal Cheilinus undulatus

lëk $v$. nail piece of timber onto outside of bamboo walling in order to hold it in place $~$ nilim strap long haos See: wëlëklëk

lër $v t$. mix miksim

liañ n.com. style of traditional dance kaen kastom danis

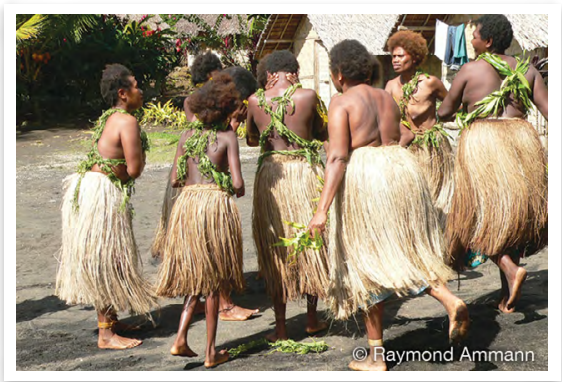

liañ $~$ style of dance lian manu n.com. variety of banana kaen banana

liaq $v t$. irrigate putum wota $\mathrm{i}$ go insaed long garen taro

liar $v i$. $\mathbf{1} \cdot$ lost, be lost $\sim$ lus $\mathbf{2} \cdot$ confused, be confused $\sim$ konfius

liaw n.com. $1 \cdot$ kind of freshwater fish $\sim$ wan kaen fis blong wota we i no save bigwan $\mathbf{2}$ - blenny, rockskipper species $\sim$ wan kaen smol fis we i stap jam long ston

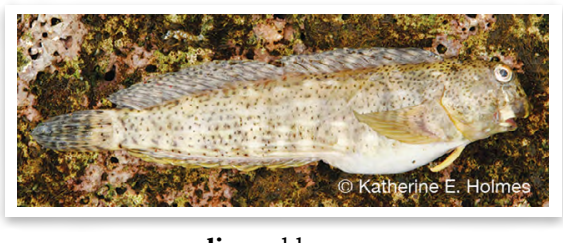

liaw blenny

lie n.com. hole hol Kōmōrōk a lēg o terē mōtō ine le terē lie dēr gēn. The two of us tied the coconuts by the coconut crabs' holes. Mitufala ifasem ol kokonas long ol hol blong krab kokonas. (VVKHVDP23Ju10701-CC)

lie giav (comp.) n.com. crab hole hol blong krab

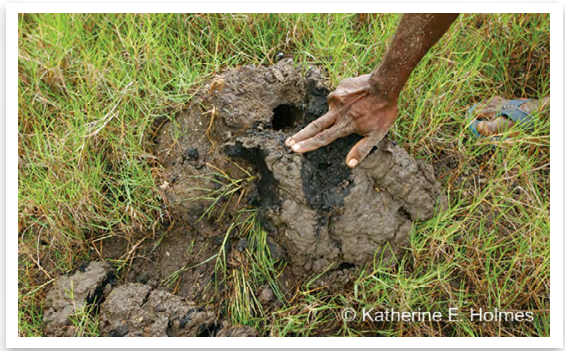

lie giav crab hole

lies $v t .1$ - change, exhange, replace one thing with another $\sim$ jenisim Kōmōrōk a van la Vanair a lies o tiket talō mōl kēl a Vōnō Lav. The two of us went to Vanair to change the ticket to go back home to Vanua Lava. Mitufala i go long Vanair mo jenisim tiket blong gobak hom long Vanua Lava. (AAD) 2 - take someone's place, do something in place of another $\sim$ tekem ples blong narafala man 
O tan̄sar ga qaq lies nē. Someone will speak in his place. Wan man bae $i$ toktok long ples blong hem. (VSJ.040)

lilies (redup.) vi. change (clothes) jenis, jenisim klos

lik $v$. serve food, remove food from oven sevem kakae, karemaot kakae long oven Ri kal me, nana lik o gengen ko, nēn töl a gengen. Come inside, I will serve the food, and the three of us can eat. Kam insaed, bae mi sevem kakae, yumitri kakae. (HWM)

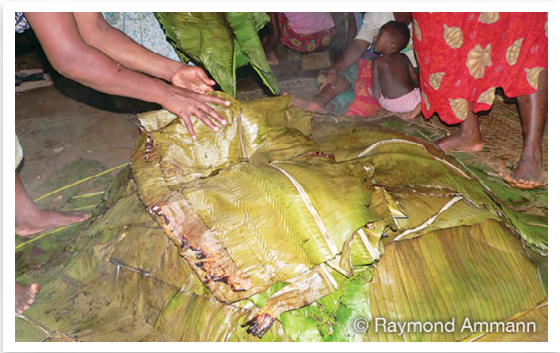

lik serve food

lil vi. run, move very quickly resis, ron kwiktaem tumas Oso mē lil levga ine? What just raced past there? Wanem i jas resis i go pas ia?

lin̄ vt. pour into something kapsaedem i go long wan samting Nēk i lin̄ o bē lö lölö baket. Pour the water into the bucket. Kapsaedem wota i go long baket. See: sër

$\operatorname{lin̄ereg}_{1}$ vi. hide $\sim$ haed I no ga taq lin̄ereg lē qētēgi rēntenge nitiwial gēn. I am going to sit and hide at the base of this tree here. Bae mi sidaon haed long stamba blong wan wud ia. (DSR)

$\operatorname{lin̄ereg}_{2}$ loc.adj. inside $\sim$ insaed $\mathbf{O}$ gōsōw ni van me, ni kal lin̄ereg kēl lē lian. The rat came, and it went back inside its hole. Ratikam, igobakinsaed long holblong hem. (WRN)

Lion n.loc. name of place on west coast of Vanua Lava nem blong ples long wes blong Vanua Lava liqet $v t$. cover food with large leaves for cooking in stone oven $\sim$ kavremap kakae wetem lif insaed long oven

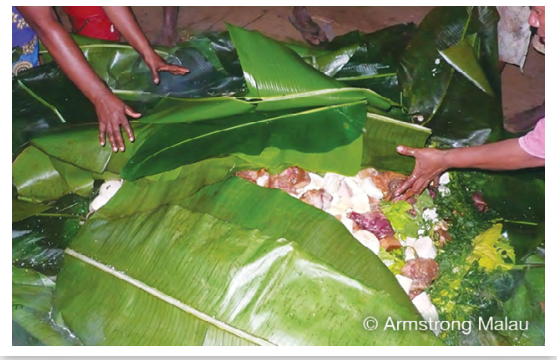

liqet cover food

lis $v$. remove breadfruit from the tree with length of bamboo karemaot bredfrut long stamba wetem bambu O gasim, nē o wo talē lis o biēg. A 'gasim' is a length of bamboo used for removing breadfruit from the tree. 'Gasim' hem i wan bambu we ol i yusum blong karemaot bredfrut long stamba.

liseg $v t$. disobey, argue with disobei, no folem tingting blong No gëtë kër nēk gē liseg no. I don't want you to diobey me. Mi no wantem yu disobei mi. O mirmiar ine o mēsē liliseg. That child is someone who disobeys all the time. Pikinini ia hem i save disobei tumas.

lisia n.com. sickness, illness, disease sik, siknes La masawre o lisia mö luwō, i rētnen rōrō ine wo nē mi miat. When the sickness got worse, then their mother died. Taem sik i kam bigwan, mama blong tufala hem i ded. (ATW)

liwō (fr.var. of luwō)

Liwōn n.loc. place name $\sim$ nem blong ples

Lo n.loc. tribe name, named because they came from the seaside $\sim$ nem blong wan traeb, we nem blong olgeta i talemaot se ol i kamaot long eria blong solwota 
lo n.loc. seashore, seaside, area by sea, but not including sea itself solwota, eria klosap long solwota E rōrō ma van rōw a lo, ni rēv sur na kan o ak. So the two of them went down to the seaside, and he pulled down his canoe. Ale tufala i go daon long solwota, hem i pulum kenu blong hem i go daon. (ATB) See: naw $_{1}$ [POc *laur 'sea, seawards']

$\mathbf{l o}_{2}$ art. locative preposition, to, at, on, marking common nouns $\sim$ long No mē èl nēk lo sto. I saw you at the shop. Mi lukim yu long stoa. (Allomorphs: la, le, lē, lë, li, lō, lö) See: $a_{1}$

lok vi. be locked lok [Bis lok 'be locked'] loko (fr.var. of iloko)

Lokon n.loc. Gaua, next island south of Vanua Lava Gaua Syn: Gō 1

lolon n.com.suff. her/his/its insides insaed blong hem See: lölö

lolov n.com. tsunami, tidal wave sunami, bigfala wef

lomonwer n.com. variety of taro with light green stem and yellow corm kaen taro we han blong hem i laet grin mo kakae blong hem i yelo

los vi. sick, be sick sik Nē mo los ine, nē mi miat ren. She got sick then, and she just died. Hem i sik ia, hem i jas ded. (KM̄MT)

loson n.com.suff. her/his genitals praevet pat blong hem See: lösö

$\mathbf{1} \overline{\mathbf{o}}_{1}$ n.com. $\mathbf{1} \cdot \operatorname{sun} \sim \operatorname{san} \mathbf{O} \mathbf{1} \mathbf{o}$ mö tul vitia. The sun has already set. San $i$ draon finis. (DPD) 2 - sunshine, sunny weather, sunny place $\sim$ ples 0 taem we i gat san Van kēti me den o lō. Come out of the sun for a while. Kamaot long san smol taem. (WWM) 3 - hour, time; measurement used in indicating the time $\sim$ taem, aoa O lō nivēs in̄ko? What time is it now? Wanem taem nao ia? [POc *qaco 'sun, daytime']

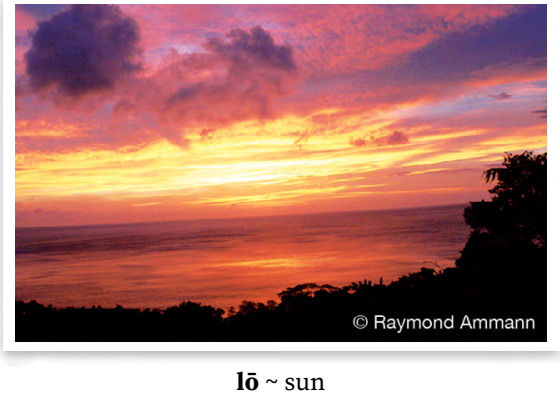

$\mathbf{1} \overline{\mathbf{o}}_{2}$ dir. out, come out, move out aot, kamaot No ma vasōgō lō ti na sien kēmi. I have read out your names. Mi ridimaot nem blong yufala. (VSB) I Dōl nē ni le lō o meles, ni le lō o mon qō. Dōl took out some leftovers, and he took out a parcel of pork. Dōl i karemaot hafkakae, hem i karemaot pasel pig. (WDR) [POc *lua 'outside']

$\mathbf{l} \overline{\mathbf{o}}_{3} v i$. vomit $\sim$ traot

$\mathbf{l o ̄} \mathbf{l}$ (redup.) n.com. vomit $\sim$ traot

lōat n.com. kind of fish wan kaen fis

lōk n.com. laplap, food dish made from grated tubers or banana, generally cooked in stone oven laplap

wōr lōk (comp.) n.com. simboro, food dish made from grated banana or tubers, wrapped in leaves of Abelmoschus manihot and cooked in coconut milk simboro

lōkreg n.com. variety of taro, 'female', with brown stem with light green stripes, and white corm $\sim$ kaen taro we han blong hem i laet braon mo kakae i waet. Hem i strong lelebet mo hem i save karem fok

lōkreg mamē (comp.) n.com. variety of taro, 'female', with red stem kaen taro we hem i woman we han blong hem i red

lōlenen $v t$. angry at kros long Nē gō lōlenen nēr. He was angry at them. Hem i kros long olgeta.

lōlgagar $v t$. very angry kros bigwan, kros tumas Nē mō lōlgagar i ginon, ma da vita nē ma tar lèt na dērēn 
tek o gasel. He was really angry with his wife, which meant that he chopped her leg with a knife. Hem i kros tumas long woman blong hem, mekem se hem $i$ katem brekem leg blong hem wetem naef.

lōlmusugsug n.com. nausea, feeling that one is going to vomit sik olsem yu harem se bae yu traot La masawre no ma van tatag o ak, o lōlmusugsug ma da no. When I went on the ship, I was affected by nausea. Taem we mi bin go long sip, sik blong traotimekem mi.

lōlōmeren 1 • vi. wise, intelligent waes Nē gē gilal o savsav o wuw sur nē gō lōlōmeren. She knows lots of things because she is wise. Hem i save fulap samting from hem $i$ waes. 2 • n.com. wisdom, knowledge waes, save Gōtō lōlōmeren na mögun i Jill. It wasn't Jill's knowledge. I no save blong Jill.

lōlōq $v t$. count how many rows of thatching are needed to make roofing faenemaot hamas ro blong lif (masa) inidim, sapos yu no makem fastaem No mō lōlōq o gövur tabō sōk siër vita o doot nives ga van kal la masa nitiwial. I measured the roof of the house to find out how many slabs of thatching are needed for the roofing. Mi meserem haos blong faenemaot hamas pis natanggura $i$ nidim blong go antap long wan ro blong ruf blong haos. (RBH)

lōlōqōr n.com. $\mathbf{1}$ - forgetful save fogetem tumas samting $\mathbf{2}$ - heathen, lacking Christian enlightenment taem o man blong tudak Mo tog me a⿳亠̄ō, la masawre o maram sönötu vēti, nē o maram lōlōqōr. In the past, when the world was still small, it was a heathen world. I stap bifo, long taem we wol $i$ smol yet, hem $i$ wan wol we $i$ stap long tudak. lōlōs n.com. food dish that is made from grated canarium nuts and datamal leaves, wrapped up and cooked on hot stones wan kaen kakae we ol i mekem smolsmol pasel blong datamal lif wetem nangae we ol i bin rasras mo ol i paselem mo putum antap long hot ston

lōlwōn vi. sad harem nogud, filing insaed long hat i no stret No gō lōlwōn sur i gunök mi miat. I am sad because my spouse died. Mi sad from (wo)man blong mi $i$ ded. O nötu m̄irmiar mō lōlwōn sur ira bērbērn̄ēn mō tō den nē a lo. The child is sad because her friends ran away from her to the seaside. Smol pikinini hem i stap sore from ol fren blong hem ol $i$ ronwe long hem ol $i$ go long solwota.

lōm $v$. fold foldem Nana lōm o demete, nana mem gōr o mete qan̄ris. I fold the leaf cover, and I place it over the hole at the top of the oven. Mi foldem lif, mi putum blokem hol blong oven. (NBQ.021)

lōmegev $\mathbf{1} \cdot$ n.com. youth, young unmarried man yangfala $\mathbf{E} \mathbf{O}$ lōmegev ni qaq vita, 'Na van me ko, no gö mörös legleg.' Then the young man said, 'I have come here, I want to get married.' Ale yangfala i talem se, 'Mi kam ia, mi wantem mared.' (ATB) $\mathbf{2} \cdot v$. be youth, become youth $\sim$ kam yangfala In̄ko nēr mo tog, tog vanvan rōrō mō lōmlōmegev. Then they stayed until the two of them had become young men. Oli stap, stap go go tufala i kam yangfala nao. (ATB)

lōqtag vi. plenty, many, be many plante, fulap I rege gō lōqtag nēr ma van bē rērēv mëkër. Many people went to catch flying fish. Fulap man ol i bin go blong pulum flaengfis. (OHS) Syn: m̄ōrag, tutuqë, wuw $\mathbf{2}$

lōt n.com. posset, baby milk vomit traot blong smol bebe 
lōv n.com. grated coconut that has had the milk squeezed out of it makas blong kokonas

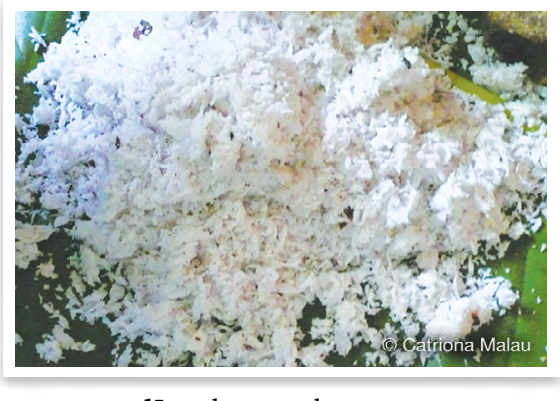

$\mathbf{l o ̄ v} \sim$ dry grated coconut

lōvōrō num. seven seven

lōvōrōne num. seventh, seventh one nambaseven $\mathbf{O}$ lōvōrōne nē ma van. The seventh one went. Nambaseven hem igo. (JRQ)

lōw n.com. kind of edible seaweed, which has two types, one red, one yellow kaen nalumlum blong solwota we yu save kakae

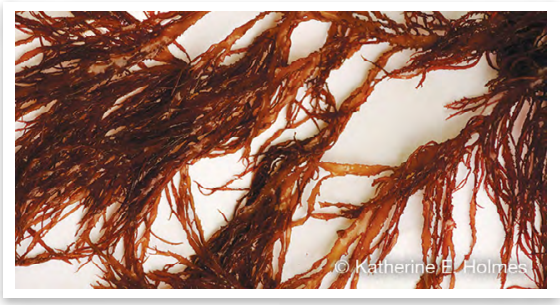

lōw kind of seaweed

löglē n.com. religion, church jos, skul $\mathbf{l o ̈ l}$ vi. blunt no sap $\mathbf{O}$ gasel gö löl. The knife is blunt. Naefino sap. Ant: gegen lölö $v t$. $\mathbf{1} \cdot$ want wantem No gö lölö idian vita na big o mes. I really want to eat fish. Mi wantem tumas blong kakae fis. 2 • like laekem No gö lölö o mirmiar ine. I like that child. Mi laekem pikinini ia.

lölö $\ddot{2}_{2}$.com.suff. inside $\sim$ insaed Matriaw nirō rōrō gō ōn lö lölö wötöv ine. Two lizards were lying inside the bottle. Tufala lised i leidaon insaed long botel ia. (EDM) [POc *lalo- 'inside'] lolon (n.poss) n.com.suff. her/his/its insides insaed blong hem

lölök (n.poss) n.com.suff. my insides insaed blong mi

lölök n.com.suff. my insides insaed blong mi see: $1 \mathrm{löl}_{2}$

lölön̄ö vōrōg n.com. dawn, early morning eli moning Mē dēñ lō vōrōg, ni mamat, lö lölön̄ö vōrōg vēti. When it became morning, he woke up, when it was still early morning. I kam long moning, hem i wekap, long eli moning yet. (ESP)

lölös $\mathbf{1}$ • vt. wrap up pasolem Dōrōk a lölös o qōōr ko. Let's wrap up this wild yam. Yumitu pasolem waelyam ia. 2 n.com. parcel pasol Syn: m̄on, wöwös

lön $1 \cdot v$. place leaf over fire in order to block and reduce the heat putum lif long faea fastaem blong blokem sapos i bigwan tumas 2 - vt. block off water to change watercourse so it flows into taro paddy blokem wota blong mekem i go long garen taro No mö lön gōr o bē lö nögö qēl. I blocked off the water at the entrance to the taro paddy. Miblokem wota long stat blong garen taro.

lör n.com. kind of laplap that has grated canarium nuts mixed in before being spread out and wrapped laplap we yu miksim wetem nangae, afta jas paselem

lösö n.com.suff. genitals, covers full genitals for male or female $\sim$ praevet pat blong bodi [POC *lasoR 'testicles']

loson (n.poss) n.com.suff. her/his genitals praevet pat blong hem lösök (n.poss) n.com.suff. my genitals $\sim$ praevet pat blong mi

lösök n.com.suff. my genitals praevet pat blong mi See: lösö

lösönq̄ō n.com. plant belonging to ginger family plant we hem i famle blong jinja Alpinia pacifica \{Lit. pig's genitals bolblong pig\} 
löt 1 - n.com. kind of food dish made from breadfruit, taro or cassava, which is boiled or roasted, pounded to make a thick paste, then mixed or covered with coconut cream or nuts nalot $\mathbf{2} \cdot v$. make löt food dish $\sim$ mekem nalot Nēn gö löt o biēg. We will make löt from breadfruit. Bae yumi mekem nalot long bredfrut.

löt bulmarwe (comp.) n.com. kind of food dish made from pounded root vegetables or breadfruit, that has grated roasted cutnuts on top $\sim$ wan kaen nalot we i gat drae navele antap long hem we ol i bin rasras

löt kel (comp.) n.com. kind of food dish made from pounded root vegetables or breadfruit, that has roasted cutnuts mixed with sugarcane juice on top wan kaen nalot we $i$ gat drae navele antap we ol i bin miksim wetem wota blong sugaken

löt matwōnwōn (comp.) n.com. kind of food dish made from pounded breadfruit, with a hole in the centre which is filled with grated cutnuts, cooked with hot volcanic stones wan kaen bredfrut nalot we ol i kilim finis mo afta rasras grin navele, putum insaed long hol long medel wetem hot ston, kavremap mo livim i dan

löt n̄e meren (comp.) n.com. kind of food dish made from pounded root vegetables or breadfruit, with grated roasted canarium nuts on top wan kaen nalot we $\mathrm{i}$ gat drae nangae antap long hem

löt n̄e qar (comp.) n.com. kind of food dish made from pounded root vegetables or breadfruit, with grated raw canarium nuts on top wan kaen nalot we i gat grin nangae antap löt salsim (comp.) n.com. kind of food dish made from pounded root vegetables or breadfruit, topped with coconut milk that has been cooked until the oil separates from the caramelised coconut $\sim$ wan kaen nalot we ol i putum melek kokonas antap we ol $\mathrm{i}$ bin kukum go go $\mathrm{i}$ braon mo oel blong hem i kamaot

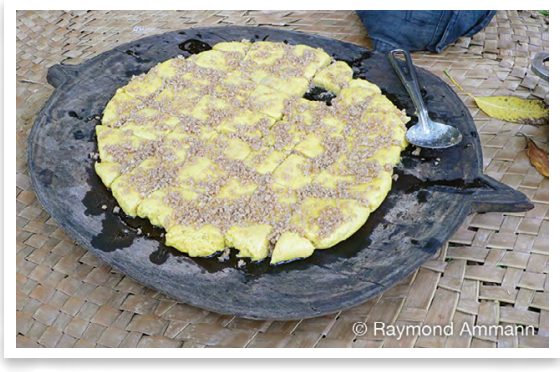

löt salsim food dish

löt teqesēg (comp.) n.com. kind of food dish made from pounded taro, with coconut milk poured in a hole in the centre, then the whole dish is wrapped in heliconia leaves and cooked over hot coals wan kaen taro nalot we ol i mekem hol long medel, melekem kokonas insaed, paselem mo putum antap long faea \{Lit. kingfisher belly löt, due to the fact that it is white like a kingfisher's stomach nalot bel blong nasiko, from i waet olsem bel blong nasiko\}

löt vasgēr (comp.) n.com. kind of food dish made from pounded root vegetables or breadfruit, topped with caramelised coconut cream and oil that has been cooked in a coconut shell over the fire wan kaen nalot wetem kokonas antap. $\mathrm{Ol}$ i melekem kokonas long sel kokonas mo putum antap long faea blong mekem se oel i kamaot mo kokonas i braon

löt wirsal (comp.) n.com. kind of food dish made from pounded root vegetables or breadfruit, topped 
with coconut cream that has been cooked by the heat of a hot stone inside a coconut shell wan kaen nalot we ol i putum melek kokonas antap. Ol i melekem insaed long sel kokonas we i gat hot ston insaed

löt wotag (comp.) n.com. kind of food dish made from pounded root vegetables or breadfruit, topped with grated roasted cutnuts $\sim$ wan kaen nalot we i gat drae navele antap löt wötön̄ö (comp.) n.com. kind of food dish made from pounded root vegetables or breadfruit, with a hole in the centre filled with grated green coconut flesh, which then has hot volcanic stones added, and is wrapped in heliconia leaves so that the coconut cooks wan kaen nalot we ol i rasras frut blong kokonas we i no grin be i no drae yet. $\mathrm{Ol}$ i kilim nalot finis, afta ol i mekem hol long medel mo putum kokonas wetem hot ston insaed. Afta ol i kavremap wetem lif laplap mo livim i dan.

löv n.com. orchard, plantation, area where lots of useful plants and fruit and nut trees are planted for future generations $\sim$ ples we ol i planem fulap stamba blong frut o nat mo ol yusful plant long hem blong ol man long fiuja i save karem $\mathbf{O}$ tere lövlöv ine, ira tubunēn ma taōrmat ti sur nēn. That area of fruit and nut trees, our ancestors prepared it for us. Olgeta stamba blong frut o nat, ol bubu blong yumi ol i bin putum redi blong yumi.

lövötöl num. eight eit

lövötölne num. eighth, eighth one nambaeit Iñko ni vetreñ o lövötölne. Then he sent the eighth one. Nao ia hem i sendem nambaeit. (JRQ)

lövövet num. nine naen

lövövetne num. ninth, ninth one nambanaen Ni le na gan nivet, ni le na gen tasēn nivet, lövövetne rōrō a tus wor. He gave himself four, he gave four to his brother, and the ninth, the two of them split it in half. Hem i givim fo blong hem, fo blong brata blong hem, nambanaen, tufala $i$ brekem long haf. (GMM)

löw (fr.var. of lēw)

luëglō $v$. spit out food $\sim$ spetemaot kakae

lugög $v$. plan plan Rōrō mö lugög ti vita rōrō a vēnvēn. The two of them planned that they would go shooting. Tufala i bin plan se bae tufala $i$ go sut. (JTF.009)

lukul $v t$. bundle up sago leaves that have already had midribs removed $\sim$ tekem ol lif nataggura we yu bin karemaot bun blong hem finis, fasem long wan bandel Nēn a lukul kalkal o doot. We bundled up all the sago palm leaves. Yumi fasem tugeta ol lif natanggura.

lul $v$. lift up, turn leftemap, tanem $\mathbf{N e ̄}$ mö lul kal na molon. She lifted up her clothes. Hem i leftemap klos blong hem.

lulu $v t$. repeat $\sim$ ripitim

lulum $v i$. $\mathbf{1}$. have a good taste (of food) $\sim$ (kakae) i swit 2 - have a nice appearance $\sim$ luk naes

lulumne n.com. taste (of something) tes blong wan samting

lum vi. (tide) come in (solwota i) kamso $\mathbf{O}$ met ni lum dēñ na varan. The tide came in and reached up to her chest. Solwota i kamso kasem jes blong hem. (JMT)

met mö lum (id.) high tide, tide coming in solwota i kam so

lumlum n.com. $\mathbf{1}$ - seaweed $\sim$ nalumlum (blong solwota) $2 \cdot$ moss, algae nalumlum (long wud, long bus) [POC *lumut 'moss, algae']

luw $v t$. count (something) kaontem O mes ine, no mö luw nēr, nēr samul tiwial dēmē nitöl. The fish, I counted them, and there are 13. Ol fis ia mi kaontem olgeta, olgeta 13. (DPR) Syn: vēvēg 
luwon n.com.suff. her/his/its tooth, teeth tut blong hem See: luwö

luwō (fr.var. liwō) vi. 1 • big, be big, sizable, become big, expand bigfala, kam bigwan $\mathbf{O}$ wērèt ine, nē gö luwō ren, nē o on, wērēt luwō, nēr gō ōl vita nē o on. That octopus was really big, it was an on, a big octopus that they call an on. Nawita ia hem i bigwan tumas, hem i wan on, bigfala nawita we ol i kolem se on. (WNW) Nēr mē ēlgōr nē vaavan nē mö luwō. They looked after him until he had become big. Ol i lukaotem hem go go hem i kam bigwan. (ATB) Syn: bëtutu 2 - important, high ranking $\sim$ hae, impoten No o maranag luwō. I am an important chief. Mi wan hae jif. (VVCMVDP21Nov0701-GM) luwö n.com.suff. $\mathbf{1}$ • tooth tut $\mathbf{2} \cdot$ tusk tut (blong pig) [POC *lipo- 'tooth']

luwö bas (comp.) n.com. toothless person, person who has lost all or most of their teeth man we i nogat tut

luwö qō (comp.) n.com. pig's tusk tut blong pig

luwon (n.poss) n.com.suff. her/his/its tooth, teeth $\sim$ tut blong hem

luwök (n.poss) n.com.suff. my tooth, teeth tut blong mi

luwömeren n.com. midday, middle of day medel dei

luwök n.com.suff. my tooth, teeth $\sim$ tut blong mi See: luwö

\section{$\mathbf{M} \sim \mathbf{m}$}

madagese (abbrev. medese) inter. what's happening? what's going on? olsem wanem? Bulsal, ti nēk ma van me ko, ma da gese? Friend, but you have come here, what's happening? Fren, be yu kam long ples ia, olsem wanem? (ATB)

Made n.com. Monday Mande

magal n.com. ant $\sim$ anis

magal tutun (comp.) n.com. kind of large, reddish, biting ant, produces painful bite wan kaen bigfala anis we hem i hafred. Taem i kakae man i soa mo i hot wantaem \{Lit. hot ant hot anis\}

magalmevesoqötu n.com. bigeye (fish) $\sim$ wan kaen fis Family Priacanthidae $\{$ Lit. ants eat clean head anis i kakae klinim hed\}

magte ${ }_{1}$ n.com. stonefish $\sim$ stonfis Family Scorpaenidae

magte $_{2}$ n.com. old woman olfala woman mako n.com. variety of taro, 'male', with stem that is red at bottom and green at top $\sim$ kaen taro we hem i man we han blong hem i red daon mo grin antap

mal n.com. common hawk, swamp harrier (bird) namala, igel, hok (pijin) Circus approximans

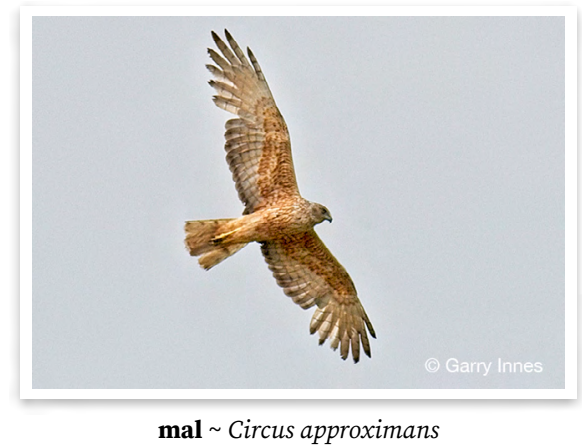

mal biliag (comp.) n.com. peregrine falcon (bird) kaen pijin olsem hok be wing blong hem i longwan, 
bodi blong hem i moa smol Falco peregrinus $\{$ Lit. buff-banded rail hawk nambilak hok\}

mala n.com. kind of tree with fruit that is red on the outside and black inside wan kaen tri we frut blong hem i red long aotsaed, be insaed i blak Sterculia banksiana

malabōs $a d v$. luckily, it's a good thing laki Malabōs ren nēk i van me. It's a good thing that you came. Laki se yu bin kam. (WRN)

malaēn $n$ ex-wife, ex-husband olfala woman o man blong yu blong bifo we yu nomo stap wetem hem

malagavēg n.com. kind of tree that is related to Malay apple, with pink flowers and small dark red to black fruit wan kaen tri we hem i famle blong nakavika, flaoa blong hem i pink mo frut i smolsmol mo i dak red o blak Syzygium buettnerianum

malaklak $\mathbf{1} \cdot v i$. happy $\sim \operatorname{glad}$ No ga malaklak sur nēn mē qētēg rēv o diksonari. I am happy because we have started to write the dictionary. Mi glad from yumi statem blong raetem diksonari. 2 n.com. happiness $\sim \mathrm{glad}$ No gē ēl rak o malaklak na mōgōn. I can recognise her happiness. Mi luk save glad tingting blong hem.

malala vi. tingle, have pins and needles $\sim$ ded, filing long bodi taem yu sidaon long leg o han blong yu Na bēnik ma malala. My arm has got pins and needles. Han blong mi $i$ ded

malaqōōr n.com. kind of tree with very long leaves that grows on the side of hills wan kaen tri we hem i gru long saed blong hil. Lif blong hem i longlongwan [Hem i gudfala timba mo ol i yusum blong faeawud tu]

malas n.com. cold kolkol No o malas. I am cold. Mi kolkol. Syn: mamēgin 1; Ant: tutun $\mathbf{1}$ [POC *malaso 'be cold']

malaw $v i$. grow gru Na bulëk o qiat ma malaw. My taro has grown. Taro blong mi i gru. malawsöm n.com. middle finger medel fingga \{Lit. grow money gru mane\}

male $\overline{\mathbf{e}}_{1}$ n.com. bird species $\sim$ kaen pijin

$\mathbf{m a l e}_{2}$ n.com. variety of banana that has roundish fruit, white flesh and does not have a strong flavour. The banana bundle grows upwards rather than hanging down $\sim$ kaen banana we kakae blong hem i raon mo i waet insaed be i nogat tumas tes. I sakem kakae blong hem i go antap

malēg n.com. cloud klaod $\mathbf{O}$ tōw ine nē ge elel, o malēg gō rōw siag aē. The hill was really high, and clouds gathered around it. Hil i antap tumas, klaod i sidaon raon long hem. (DHN)

malēw $v i$. sick with hunger $\sim$ sik blong hanggri

malkan n.com.suff. her/his/its sole andanit leg blong hem see: malke

malke n.com.suff. sole (of foot), footstep andanit fut, futstep I nēk wo i no, dōrōk ga tatag na malkan. You and $I$, the two of us will follow in his footsteps. Yu mo mi, bae yumitu folem futstep blong hem. (EKK.200)

malkan (n.poss) n.com.suff. her/his/its sole andanit leg blong hem

malkëk (n.poss) n.com.suff. my sole andanit leg blong mi

malkëk n.com.suff. my sole andanit leg blong mi see: malke

malmalasē n.com. kind of tree $\sim$ wan kaen tri [Used for making house posts. It is not good as firewood as it produces too much smoke. $\mathrm{Ol}$ i yusum blong mekem pos blong haos. Hem i nogud blong faea from $\mathrm{i}$ mekem smok nomo]

malmalei lantar n.com. variety of taro, 'male', family of lantar, with many stems that are dark green at bottom and light green at top, and small corms that are covered in roots and rot easily kaen taro we hem i man 
mo hem i famle lantar, we kakae blong hem i gat fulap rus blong hem mo i save roten isi. Han blong hem i dak grin daon mo i laet grin antap Syn: mensēkē

malmalē n.com. pretend giaman

malnët n.com. kind of tree like red silkwood but the fruit are smaller and not eaten $\sim$ wan kaen tri olsem naduledule be kakae blong hem i smol mo man i no stap kakae See: nët malqon n.com. fruit dove (bird) grinpijin Ptilinopus spp. Syn: men dōsdōs

malwērēt n.com. brittle star; kind of echinoderm closely related to starfish samting olsem stafis we bodi blong hem i smol mo ol leg i longwan, mo i gat ol smolsmol nil long hem \{Lit. bit like octopus lelebet olsem nawita\}

Mam n.p.fr. 1 • Dad Papa $2 \cdot \operatorname{God} \sim$ Papa God

Mambum (comp.) n.p.fr. Grandad, Grandpa Bubu man See: Diebum, Pōpō

mam $1 \cdot$ n.com. father (generic) papa Maranag luwō ta Vōnō Lav, i rege maranag, i rege döl go tog oko, o mirm̄iar, o die, o mam, tan̄sar döl, i rege, kēmi ma van me den taval lam. Big chief of Vanua Lava, chiefs, all the people who are here, children, mothers, fathers, everybody, you who have come from across the sea. Bigfala jifblong Vanua Lava, ol jif, ol man we i stap long ples ia, ol pikinini, ol mama, ol papa, olgeta evriwan, yufala we $i$ kam long narafala ples. (EKK.001) 2•vt. call someone father $\sim$ singaotem papa long man No ga mam nēr. I will call them Dad. Bae mi singaotem Papa long olgeta.

mamake $v$. be surprised at, about sapraes long Kemem ga mamakē aē nēk sur nēk mē gilal na mögun kemem o qaqaq gamlöt idiañ. We are surprised by you because you have learnt our language very quickly. Mifala i sapraes long yu from yu save gud lanwis blong mifala.

mamar $v t$. ask of, plead, implore askem bigwan No go mamar vita si kēmi ga van me le, gōwē. I implore you that if you can come, then that would be good. Mi askem bigwan long yufala se sapos yufala i kam, bae i gud. (VSB)

mamarōvōg n.com. small sign or indication of something, such as seeing smoke that indicates there is a fire wan smol samting, saen i kamaot, olsem luk smok o faea longwe

mamarseg (abbrev. marseg) 1 • v pity, feel sorry (for) sori (long) No ga mamarseg nēk sur i ginon̄ mi miat. I feel sorry for you because your husband/wife has died. Mi sori long yu from hasban/waef blong yu $i$ ded. 2 - vi. unfortunate, pitiful sori I Wōmōdō nē gō rōw lō le la tan̄sar ga mamarseg. Wōmōdō can appear to people who are unfortunate. Wömōdō $i$ save kamaot long ol sori pipol. (ESP) 3 - inter. sorry sori Ke, mamarseg, rege bulrō mîrmiar, no mo los in̄kē, no mō rōñteg vita no mitē wē le. Oh, sorry, you two children, I am sick now, and I feel that I will not get better. Oo, sori, tufala pikinini, mi sikia, miharem se bae mi no save kam gud. (HWM)

mamas vi. dry drae Ant: döw

mamat n.com. kind of freshwater fish that has large head and eyes. It is light grey and hides in holes in rocks. wan kaen fis blong wota we hem i save bigwan mo ae mo hed blong hem i bigwan. Hem i laet grei mo hem i stap long hol oltaem

mamat sërsërdiqiat (comp.) n.com. kind of small freshwater fish that is silver on its side with a black spot, and light grey on its back wan kaen 
smol fis blong wota we hem i silva long saed wetem blak spot. Hem i laet grei long baksaed blong hem

mamat $_{2} v i$. 1 • wake up wekap Lö qön̄ nitöl lō vōrōg, kōmōrōk a mamat, kōmōrōk a van lö suvsuv. On Wednesday in the morning, the two of us woke up and went for a swim. Long Wenesde long moning mitufala i wekap mo go swim. (AAD) $\mathbf{2}$-open eyes, have one's eyes open openem ae, ae i open E nē ta, 'Gen mōvōt.' Nēr a gen mōvōt, a mamat me, nēr a èl na gëvrun nēr o gövur kav wo o simen. Then he said, 'Close your eyes.' They closed their eyes, then opened them again, and they saw that their house was a cement house with corrugated iron roof. Ale hem i se, 'Satem ae.' Ol i satem ae, i openem bakegen, ol i luk se haos blong olgeta hem i haos kapa wetem simen. (RPP) Ant: gen mōvōt

mamē $\mathbf{1} \cdot v i$. red, be red $\sim \operatorname{red} \mathbf{2} \cdot n$.com. redness, red (one) $\sim$ redwan $\mathbf{O}$ mamē mōgōn gete meneg. Its redness is not strong. Red blong hem ino strongwan.

mamēgin $\mathbf{1} \cdot v i$. be cold, cold $\sim$ kolkol O naw ga mamēgin idian. The sea was really cold. Solwota i kolkol tumas. (AAD.034) 2 - n.com. cold kolkol o mamēgin ma qal no. The cold has got to me. Kolkol i kasem mi. Syn: malas; Ant: tutun $\mathbf{1}$

$\operatorname{man}_{1} \mathbf{1} \cdot v t$. intoxicate, stun, poison, as in kava, alcohol or poison making one feel drunk or delirious kilim, mekem i drong, olsem man i posenem fis o kava i kilim man $\mathbf{O}$ gē ma man no. The kava made me drunk. Kava i kilim mi. 2 - n.com. magic, words used when performing magic or to support use of traditional healing and other purposes $\sim$ majik, toktok blong talemaot long taem blong yusum kastom meresin mo sam narafala samting $\operatorname{man}_{2}$ n.com. right $\sim$ raet No ge le o man min nēk tala qaq. I will give you the right to speak. Bae mi givim raet long yu blong toktok.

$\operatorname{man}_{3}$ cl. her/his/its (drink) $\sim($ dring) blong hem See: $\mathrm{me}_{2}$

manene $n$.com. reason $\sim$ risen

maniok n.com. cassava, manioc maniok Manihot esculenta

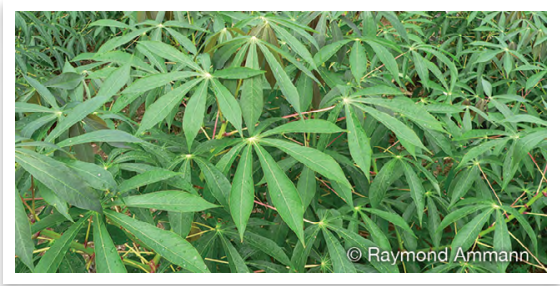

maniok cassava

maniok añañ (comp.) n.com. variety of cassava that has yellow tubers kaen maniok we kakae blong hem i yelo

maniok atatīēn (comp.) n.com. primary grouping of varieties of cassava, cassava that bear long, large tubers ol kaen maniok we kakae blong olgeta ol i longlongfala mo i bigwan

maniok rereqe (comp.) n.com. primary grouping of varieties of cassava that bear round, short tubers ol kaen maniok we kakae blong olgeta ol i raon mo i sotfala

manreva n.com. variety of taro kaen taro Syn: matēkmëgërsurletes

manrē n.com. piece pis

mañ n.com. serious cough, with wheezing, asthma kof nogud

mañde n.com. sabre squirrelfish kaen redfis Sargocentron spiniferum

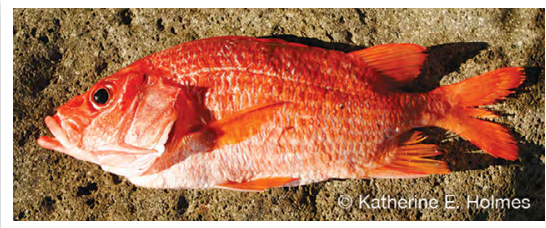

man̄de Sargocentron spiniferum 
man̄ko n.com. mango manggo [Bis]

mar n.com. famine $\sim$ taem we i nogat kakae I Qet birin̄ ra tatasēn, nēr, o gengen odiañ aē. Mar ma qal nēr lē Sēbēr. Qet and his brothers, they didn't have any food. Famine had affected them at Sëbèr. Qet mo ol brata blong hem, olgeta, kakae i nogat. Taem we i nogat kakae i kasem olgeta long Sébèr.

marak n.com. fat (of animal) gris (blong animol)

maram n.com. world $\sim$ wol $\mathbf{O}$ tere men döl waliög o maram, o mie na mögun nēr o mal. Of all the birds all around the world, their leader is the hawk. Olgeta pijin we ol i stap raon long wol, lida blong olgeta hem i hok. (DFF.004)

maranag n.com. chief $\sim$ jif See: tövusm̄ēl maraw n.com. spider $\sim$ spaeda

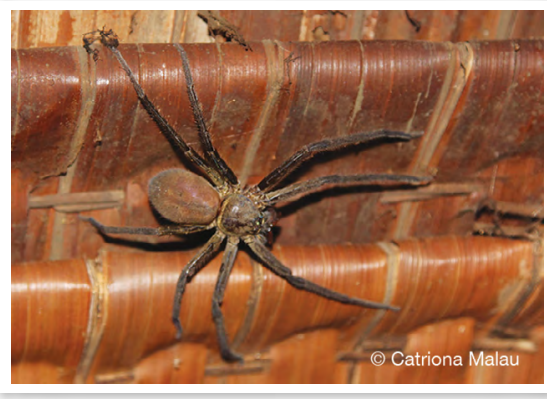

maraw spider

marē $\overline{\mathbf{e}}_{1}$ n.com. eel namarae, ilfis See: vusö [PROC *maraya 'sea eel']

marē bēluwō (comp.) n.com. kind of freshwater eel that lives in rivers, with a large head, light grey with small spots wan kaen namarae blong wota we hed blong hem i bigwan mo i laet grei wetem ol smolsmol spot. Hem i liv long bigfala wota $\{$ Lit. big water eel namarae blong big wota\}

marē genrō (comp.) n.com. kind of eel which dwells where water meets the sea. It is flat and yellow with black spots $\sim$ wan kaen namarae we i stap klosap long solwota long ples we solwota mo wota i miks. Hem i kakae long tu ples, long wota mo solwota. Hem i flat we i flat mo i yelo wetem blak spot long hem

marē mat (comp.) n.com. kind of freshwater eel, light grey with small spots wan kaen namarae blong wota we hem i laet grei wetem ol smolsmol spot. \{Lit. taro paddy eel namarae blong garen taro\}

marē mes (comp.) n.com. mottled moray wan kaen namarae blong solwota \{Lit. fish eel fis namarae\}

marē q⿳亠丷厂 (comp.) n.com. giant moray wan kaen namarae blong solwota \{Lit. pig eel pig namarae\}

marē teweswotag (comp.) n.com. starry eel, moray wan kaen namarae blong solwota \{Lit. cutnut flower eel namarae we flaoa blong navele\}

marē wosgötö (comp.) n.com. girdled reef eel wan kaen namarae blong solwota \{Lit. pound hermit crab eel kilim nakato namarae\}

marē wot (comp.) n.com. kind of freshwater eel, black with white belly, has large eyes. It dwells in swamps and muddy waters wan kaen namarae we baksaed blong hem i blak gud mo bel blong hem i waet. Ae blong hem i bigwan. I stap oltaem long swam mo ples we i gat sofmad \{Lit. born eel namarae we ibon\}

marē wōl (comp.) n.com. kind of freshwater eel, short and silver wan kaen namarae we kala blong hem i silva mo hem i sotwan \{Lit. moon eel mun namarae\}

mare $\overline{\mathbf{e}}_{2}$ n.com. grouping of kinds of taro which don't fork but which grow tall and crooked grup blong ol kaen taro we ol i no save karem fok be ol i save longfala mo benben (olsem namarae) [Taro in this group ripen 
more quickly than others. They are good for roasting. Taro long grup ia ol i redi kwiktaem bitim ol narawan. Ol i taro blong rus.] \{Lit. eel namarae\} marē wasalav (comp.) n.com. variety of taro, 'male' with stem that is white at bottom and light brown at top, with light black petiole kaen taro we hem i no save gru bigwan be i save longfala. Hem i sofsof nomo mo kakae i waet

marē wasalav mamē (comp.) n.com. variety of taro, 'male', with red stem and white corm kaen taro we hem i man we han blong hem i red mo kakae blong hem i waet

markō̄n n.com. kind of tree wan kaen tri Metrosideros collina

marōōn n.p.bound her/his uncle angkel blong hem See: maröu

maröu n.p.bound maternal uncle; brother of mother, husband of sister of father angkel

marōōn (n.poss) n.p.bound her/his uncle angkel blong hem

maröuk (n.poss) n.p.bound my uncle angkel blong mi

maröuk n.p.bound my uncle angkel blong mi See: maröu

marsam n.com. kind of tree, brown kurrajong wan kaen tri Commersonia bartramia $[\mathrm{POC} *$ jamaR 'Commersonia bartramia']

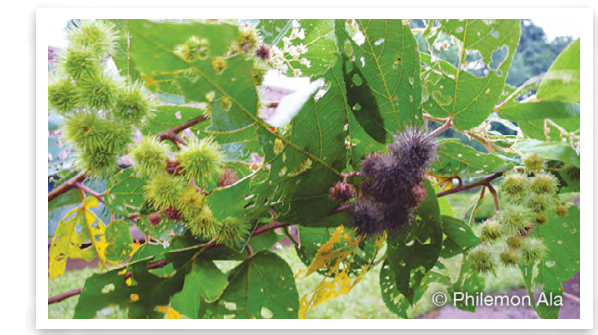

marsam $\sim$ Commersonia bartramia

marseg (abbrev. of mamarseg) martat n.com. yellowstripe snapper, bluelined snapper (fish) kaen pulefis we hem i yelo wetem fo blu laen long hem Lutjanus kasmira

mas v.part. must mas [Bis mas 'must']

masa n.com. row of slabs of thatch that make up roofing ro blong pis natanggura we ol i bin fasem blong mekem rufing blong haos Nēn mō tōw qēt o masa, in̄ko nēn a sōk sösögöt ta o masa nives. We measure the rows of thatch needed, then we find out how many rows of thatch. Yumi mesarem finis ol ro blong natanggura, afta yumi faenem se hamas ro. (RBI)

masa dirdir (comp.) n.com. short slab of thatch that is made if there is a short row left at the end when thatching a roof $\sim$ pis natanggura blong ruf we ol i mekem i sotwan blong filimap ples long en masa taq (comp.) n.com. side of roof that has more slabs saed blong ruf blong haos we hem i gat moa natanggura long hem

masa tur (comp.) n.com. side of roof with less slabs saed blong ruf blong haos we natanggura i no fulap Tavalgi o masa gō lōqtag, tavalgi o masa gōtō lōqtag. One side has lots of rows of slabs, the other side doesn't have lots of rows of slabs Wansaed i gat fulap natanggura, narasaed $i$ nogat fulap natanggura.

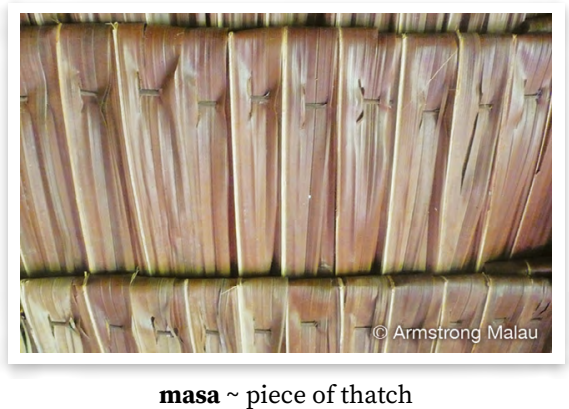


masalav n.com. southerly wind $\sim$ win we $\mathrm{i}$ kam long saot [This wind does not bring any rain but brings very high temperatures. Win ia i no karem ren be i mekem ples bae i hot we i hot.]

masansan $v i$. individually $\sim$ wanwan

masawre $n . c o m .1 \cdot$ time $\sim$ taem Nē mē èlgōr o tere qō ine vaavan mē dēn̄ o masawre tabaē. He looked after all of those pigs until it was time for it. Hem i lukaotem ol pig ia go go i kasem taem blong samting ia. (ESP) $\mathbf{2} \cdot$ place $\sim$ ples La masawre me meren, e ni ukuëg kēl o masawre ine, ni van. When it was daylight, then it left that place and went. Taem i deilaet, ale hem i lego ples ia, hem igo. (DFF)

masgian n.com.suff. by himself, herself, itself hem wan See: masgiē

masgiē n.com.suff. alone, by oneself hem wan Nē ni vus mi miat i ginon, e ni tog masgian ti. He killed his wife, then he lived alone. Hem i kilim woman blong hem i ded, ale hem i stap hem wan. (DSR)

masgian (n.poss) n.com.suff. by himself, herself, itself $\sim$ hem wan masgiēk (n.poss) n.com.suff. by myself $\sim$ mi wan

masgiēk $n$.com.suff. by myself $\sim$ mi wan See: masgiē

masrē $n$ piece pis No me gen o masrē qiat nivet. I ate four pieces of taro. Mi kakae fo pis taro.

masta n.com. variety of taro, with white stem that is red at bottom kaen taro we han blong hem i waet mo i red daon

mat n.com. taro garden planted in running water or mud, where the water is never drained off, taro paddy garen taro long wota $\mathrm{O}$ sofmad we wota i stap long hem oltaem Qèt, nēk i èl o tere mat, la masawre nēr mō sōwereg o qiat. Then you can see the taro paddies, at the place where they plant taro. Afta bae yu luk garen wota taro, long ples we ol i planem taro. (RRS) See: qēl

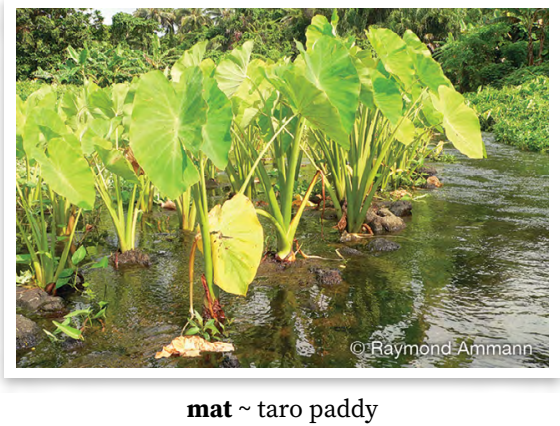

mat bōak (comp.) n.com. taro garden planted in mud with no stones, where water wells up when the ground is dug garen taro we ol i planem long sofmad, we i nogat ston mo wota $\mathrm{i}$ kam antap taem ol i digim graon

mat vetvet (comp.) n.com. taro garden planted only in stones with running water garen taro we ol i planem long ples we wota i ron long ol ston mat wöwörös (comp.) n.com. taro garden planted in small stones and thin soil with running water $\sim$ garen taro we ol i planem long ples we i gat ol smolsmol ston mo wota i ron long hem

matabōbōt n.com. mature coconut drae kokonas

matagraq n.com. firstborn, eldest child fasbon Na nötuk matagraq $\mathbf{i}$ roJokobet. My eldest child is Jokobet. Fasbon pikininiblong mi hem i Jokobet. matal n.com. puzzle tree $\sim$ namatal Kleinhovia hospita [PNCV *matala 'Kleinhovia hospita']

matan n.com.suff. his/her/its eye $\sim$ ae blong hem See: mete

mataq n.com. fresh wound, cut gat kil mataqē n.com. stone or clamshell axe $\sim$ ston akis o akis wetem sel blong natalae 
matawōsōl n.com. kind of lizard that is light brown with spots on its back and yellow belly. It sheds its skin when touched wan kaen lised we hem i laet braon wetem spot long baksaed mo yelo andanit. Taem yu holem, skin blong hem i kamaot

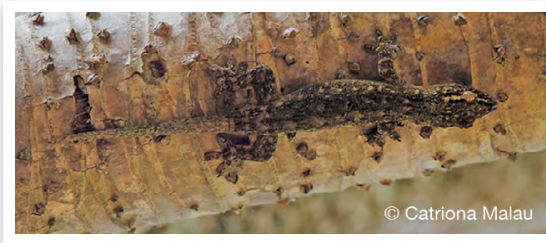

matawōsōl $~$ kind of lizard

matē n.com. 1 • death ded Nēr mē ès kēl qēt den o matē. They all came back to life from being dead. Ol i kam laef bakegen, aot long ded. (JRQ) Nē ni kēl me lō qōrqōr, qön̄ nives gōr na mōgōn o matē. He returned in a dream, several days after his death. Hem i kambak long drim, hamas dei afta long ded blong hem. (OSK) $\mathbf{2}$ • funeral, death ceremony seremoni blong ded

matian (n.poss) n.com.suff. her/his/its death ded blong hem

matiēk (n.poss) n.com.suff. my death ded blong mi

matēl $a d v$. unsuccessfully, unable to complete or do properly no finisim gud Si kēmi ge gengen matēl wo ri nës kal taklē siag me lē tibiar. If you eat it but you can't finish it then put some of it in the basket. Sapos yufala $i$ kakae be no save finisim, yufala $i$ fulumap samfala insaed long basket. (KM̄T.145)

matētatultulvet n.com. situation in which someone dies, then comes back to life, then dies again several times until they are finally dead kaen ded we man hem i ded, afta hem i laef bakegen, afta hem i ded, hem $i$ laef bakegen go go hem i jes ded matēwot $n$.com. accident $\sim$ aksiden

matgagar n.com. anger $\sim$ ae i kros O matgagar nē o qitigi gigilal. Seeing things that irritate you is the start of jealousy. Ae i kros hem i stamba blong jalusi. [Taem yu luk wan samting we yu no wantem luk, yu luk nogud fastaem, afta yu kros, hem i stamba blong jalusi]

matian n.com.suff. her/his/its death ded blong hem See: matē

matiēk n.com.suff. my death ded blong mi see: matē

matmamē n.com. rock shell wan kaen sel blong solwota Thais sp. \{Lit. red eye red ae\}

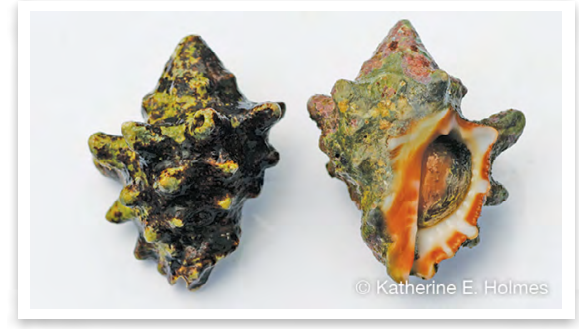

matmamē $\sim$ Thais sp.

matmat malgias n.com. variety of banana that has whitish fruit, leaves and trunk, with long, large fruit kaen banana we skin mo stamba mo lif blong hem i waet mo hem i bigwan

matmat mamē n.com. variety of banana that has reddish fruit, leaves and trunk, with long, large fruit kaen banana we skin mo stamba mo lif blong hem i red

matmuturtur n.com. sleepiness, drowsiness, desire to sleep $\sim$ harem se i wantem slip, ae i hevi

matnōn n.com. hot coals of fire asis wud

matqēt n.com. flat coconut leaf basket which is used especially in cooking, as a scraps basket and also for placing parcels of hot cooked food inside $\sim$ basket blong lif kokonas we 
hem i flat mo ol i yusum long saed blong kuk blong putum kakae o doti blong kakae insaed

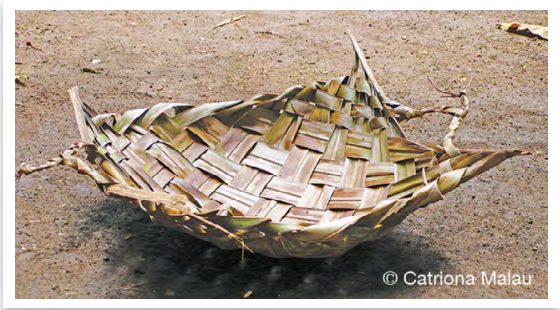

matqēt flat coconut leaf basket

matriaw n.com. lizard lised

matubu n.p.bound self $\sim$ (hem) wan matōbōn (n.poss) n.p.bound herself/ himself/itself $\sim$ hem wan matubuk (n.poss) n.p.bound myself mi wan

matwiwi n.com. cardinalfish $\sim$ wan kaen fis Family Apogonidae

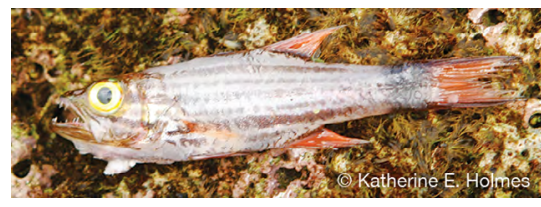

matwiwi Family Apogonidae

matwōn n.com. blind blaen

mav vi. $\mathbf{1} \cdot$ heavy $\sim$ hevi, no laet Nē ni kal o mes in̄kē, ni ron̄teg ga mav idian. He pulled in this fish, and he felt that it was very heavy. Hem i pulum fis ia, $i$ harem se $i$ hevi tumas. Ant: men̄meñ $\mathbf{1} \mid \mathbf{2}$ • difficult, hard, not easy had, no isi Qēt talo nēk i tar o sam, nē o wiwieg ga mav bilēn. Then for you to carve the outrigger, it is also a difficult job. Afta, blong yu katem nasama, hemi wan bigfala wok tu. (VVKHVDP180ct0801) Ant: men̄meñ 2 [POc *mapat 'heavy']

maw $_{1}$ vi. heal (soa i) drae, kam gud maw $_{2}$ n.com. secret $\sim$ wan save we bae yu yusum be yu no save lego na gëk o maw my secret knowledge save blong mi we bae mi no save lego

mawi part. dehortative, don't no, yusum blong talemaot se no mekem wan samting

me 1 - dir. towards speaker, in a direction towards the deictic centre $\sim$ kam Ri wöt tèqēl me min no o ga. Throw the rope down here to me. Sakem rop $i$ kam daon ia long mi. (AWW) Rōrō a èl o men nitiwial ta gav me. The two of them saw a bird flying this way. Tufala iluk wan pijin iflae i kam. (WDR) 2 - dem. close by, proximal location, close to deictic centre ia, ples klosap long man we i toktok Bet o söm ine, nēk i mem siag me le tibiar. Pick up that money, and put it just up there in the basket. Pikimap mane ia, yu putum antap ia nomo long basket. (EDM) [POc *ma[i] 'come from']

$\mathbf{m e}_{2} \mathrm{cl}$. relational classifier used to mark possession of items that are drunk by the possessor blong (yusum blong tokbaot ona blong dring) Ni le lō na man o bē, e nē ni sum. He took out his water, then he drank it. Hem i karemaot wota blong hem, ale hem $i$ dring. (WDR) [POc *ma'possessive classifier: 'drinkable"] man (n.poss) cl. her/his/its (drink) (dring) blong hem

mëk (n.poss) cl. my (drink) (dring) blong mi

medese (abbrev. of madagese)

megelgel $v i$. ticklish, tickle $\sim$ tajem yu sofsof, mekem se yu laf

meles n.com. leftover food, food from previous day, which may need to be reheated $\sim$ namalas, kol kakae, hafkakae Ni mem lin̄ereg kēl o gengen ine, o meles, lē tibiar na non. He put away the food again, the 
leftovers, in his basket. Hem i putum bakegen kakae ia, hafkakae ia, long basket blong hem (WDR)

mem $v$ t. put $\sim$ putum Ni mem o vet lēe mēkē damat. She put the stones on top of the heliconia leaves. Hem i putum ston $i$ go antap long liflaplap. (WNW)

memesgēt $n$.com. sweat swet

memsan n.com.suff. her/his/its breath win blong hem See: memse

memse n.com.suff. breath win (blong hem) Na memsan më qëtqëtutu. He was really short of breath. Win blong hem i sotsotfala. (JTF)

memsan (n.poss) n.com.suff. her/his/ its breath win blong hem

memse timiat (n.poss) n.com.suff. spirit's breath win blong devel memsëk (n.poss) my breath win blong mi

memse timiat $n$.com.suff. spirit's breath win blong devel See: memse

memseg vi. $\mathbf{1}$ breathe $\sim$ pulum win Ni èl i tasēn gete mememseg bilēñ. Rivteg vitia ni miat He saw that his younger brother wasn't breathing either. He was already nearly dead. Hem i luk se smol brata blong hem i nomo pulum win. Klosap hem $i$ ded. (HWM) 2 • rest spel Nēr mö wöwör vaavan ine, o malmal ni memseg. They weeded for a while, and the young woman rested. Ol $i$ widim garen go go $i a$, yangfala gel $i$ spel. (WWMi)

memsëk n.com.suff. my breath win blong mi See: memse

men n.com. bird $\sim$ pijin $\mathbf{O}$ men nē ma gav me. The bird flew this way Pijin $i$ flae $i$ kam. [According to folk taxonomy, this is a category that includes all animals that fly, thus includes flying fox, although modern schooling has caused this point to be debatable. Flaengfokis tu hem i wan men, iven tho yumi no talem se hem i wan pijin.] [POc *manuk 'bird']

men $_{2}$ n.com. thunder $\sim$ tanda

men dōsdōs n.com. fruit dove (bird) grinpijin Ptilinopus spp. Syn: malqon menar n.com. consideration, forethought waes No gö mörös ri qaq tek o menar. I want you to speak with consideration. Mi wantem se yufala i tok wetem waes.

meneg vi. 1 hard, be hard strong, olsem i no sofsof $\mathbf{O}$ tere siri tabaē ge meneg. All of its bones were hard. Ol bun blong hem ol $i$ strong. (SBB) 2 - be strong, powerful strong, olsem i gat paoa $\mathbf{O}$ wēgisgis nö wērēt me meneg so ine. Mē gis vavarge o reqe. The octopus's tentacles were strong. It held the woman tightly. Olhan blong nawita ol i strong. Hem i holem taet woman ia. (WNW) $\mathbf{3} \cdot$ intense strong, olsem i gat paoa blong hem Na bōnan bilēñ ge meneg. It has an intense smell too. Smel blong hem tu $i$ strong. (DFF) $4 \cdot$ be determined strong, olsem fasin i strong Rōrō a liseg, i rege burō mirmìiar e, rōrō a meneg. The two of them argued, the two children, and the two of them were determined. Tufala $i$ rao, tufala pikinini ia, tufala i strong. (OQQ)

menmav n.com. kind of tree, probably type of fig, with fruit that is green when unripe and white when ripe wan kaen tri we i olsem balak. Kakae blong hem $i$ grin mo taem i raep $i$ waet

menmenlul n.com. golden whistler (bird) wan kaen pijin

menn̄ērēs n.com. dried mucus, snot wota blong nus we i drae

mensēkē n.com. variety of taro, 'male', family of lantar, with many stems that are dark green at bottom and light green at top, and small corms 
that are covered in roots and rot easily kaen taro we hem i man, we hem i famle lantar, we han blong hem i dak grin daon mo laet grin antap. Kakae blong hem i smol mo i gat fulap rus mo i save roten isi Syn: malmalei lantar

mensirsirqōōr n.com. Vanuatu buffbellied flycatcher (bird) wan kaen pijin Neolalage banksiana

meñ $v t$. wipe $\sim$ waepem

men̄meñ vi. $\mathbf{1}$ • light (weight), not heavy laet, no hevi Ant: mav $\mathbf{1} \mid \mathbf{2}$ - easy isi, no had $\mathbf{O}$ ak sōsō, talo nēk i sōsōg aē nē ge men̄meñ, ba o wiwieg tabaē nē ga mav nē ga mav. For you to go fishing with a canoe is easy, but the work to make it is really hard. Wan kenu, blong yu go fising long hem i isi, be wok blong mekem hem i had we $i$ had. (VVKHVDP180ct0801) Ant: mav 2

Mere n.loc. place name $\sim$ nem blong ples

meren $1 \cdot n$.com. day, daytime dei In̄ko o sul ma datek o sum gē le meren. Now people have a tendency to drink kava in the daytime. Nao ia ol pipol ol i stap dring kava long dei. 2 - n.com. daylight $\sim$ deilaet Oko o meren vēti. Now it is still daylight. Nao ia hem i dei yet. $(\mathrm{QQ}) \mathbf{3} \cdot v i$. be daylight, become day kam deilaet Söw nēr töl mō mōvōt ine, me meren me, nēr töl ma van lē tiqē. So the three of them slept, and when it became daylight, the three of them went to the garden. Ale trifala $i$ slip ia, mo deilaet i kam, trifala $i$ go long garen. (AWW) [POc * rani 'daytime'] mere $_{1} v i$. lazy les

$\operatorname{mere}_{2}$ vi. dry, dry up, dry out (wood, leaf, fruit, etc.), become withered (vegetation) drae (olsem wud o lif) $\mathrm{O}$ vēlislis me mereñ qēt. The grass was all dry. Ol gras $i$ drae evriwan. Syn: $\operatorname{ran}_{1}\left[P O c{ }^{*}[m a-] r a j o\right.$ 'become withered (of vegetation)'] mes n.com. fish $\sim$ fis

mesbule n.com. kind of fish that lives in fresh water but moves to sea when mature. It is a dull silver and can be found in pairs wan kaen fis we i bon long wota be taem i bigwan i go stap long solwota. Hem i silva be i no braet mo i save bigwan. $\mathrm{Ol}$ i wokabaot tutu

mesgeben n.com. Indo-Pacific sailfish wan kaen fis Istiophorus platypterus \{Lit. sail fish sel fis\}

mesmame $\overline{\mathbf{e}}_{1}$ n.com. pink squirrelfish redfis Sargocentron spp. \{Lit. red fish red fis\}

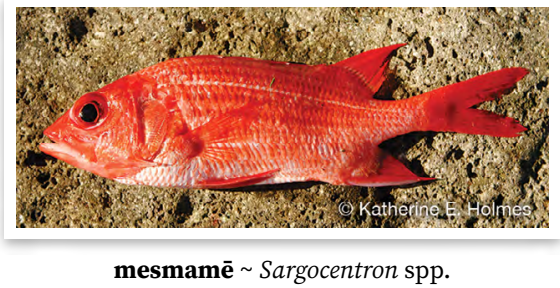

mesmame $\overline{\mathbf{e}}_{2}$ n.com. variety of taro, 'female', with red stem kaen taro we hem i woman mo stem blong hem i red \{Lit. red fish red fis\}

mestalisōr $n . c o m$. spotted unicornfish $\sim$ kaen strongskin (fis) Naso brevirostris

mesteveltiwial n.com. copper sweeper (fish) wan kaen fis Pempheris oualensis Syn: wetevel \{Lit. one sided fish wan saed fis\}

mestēv n.com. hogfish kaen fis Bodianus axillaris

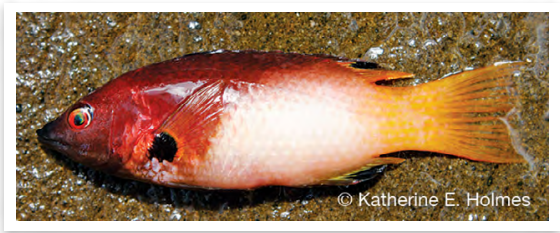

mestēv $~$ Bodianus axillaris 
mestiagōsōw n.com. black and white snapper (fish) kaen pulefis we hem i waet mo grei Macolor niger \{Lit. fish shit of rat fis sitsit blong rat\}

mesvōlōvōl n.com. variety of taro, 'female', with green stem kaen taro we hem i woman, we han blong hem i grin \{Lit. wrasse fish kaen fis\}

meswelwel $n$.com. fusilier (fish) wan kaen fis Family Caesionidae

met $n . c 0 m$. area of reef close to shore which is covered during high tide but exposed during low tide rif, ples we solwota mo rif i stap tugeta mo man i save wokabaot long hem mo yusum blong fising Rōrō ma van lo vovon̄on, met mō ōr, nēr gō lōqtag nēr ma van a lo, li met talo vovonon. The two of them went reef gleaning, the tide was low, and many of them went to the seashore, to the reef in order to collect seafood. Tufala i go blong karem mit, solwota i drae, olgeta fulap ol $i$ go long solwota, long rifblong karem mit. (WNW)

met siag (comp.) n.com. tide is neither high nor low $\sim$ solwota i stap semak nomo, i no save drae mo i no save kam so. [This happens when the moon is waning. This is not a good time for fishing. I hapen olsem taem mun i go blong tudak, bitwin ful mun mo niu mun. I no wan gud taem blong fising.]

met sörlav (comp.) n.com. time when there is a very high high tide $\sim$ taem we solwota i kam so we i bitim mak blong evri dei long manis [I hapen olsem wan dei bifo niu mun mo wan dei bifo ful mun]

mësu met ōrōr (comp.) n.com. extreme low tide, which enables reef gleaning of species that would normally not be accessible $\sim$ taem solwota i drae bigwan, mo i minim se yu save karem sam mit we mos taem i stap long dip ples met mō ōr (id.) low tide, tide going out solwota i drae

mete n.com.suff. eye $\sim$ ae Mes Tuvun me teeteñ vaavan na matan ma mamē qēt. Mes Tuvun cried on and on until his eyes were completely red. Mes Tuvun i krae krae gogo ae blong hem i red evriwan. (JMT) [POc *mata- 'eye, face']

mat möturtur (comp.) n.com. be sleepy taed

matan (n.poss) n.com.suff. his/her/its eye $\sim$ ae blong hem

mëtëk (n.poss) n.com.suff. my eye ae blong mi

mete gövur n.com. door doa In̄ko ni tōrak, ni wiak o mete gövur, ni kalō. Then he got up, he opened the door, and he went outside. Nao ia hem i girap, i openem doa, hem i go aotsaed. (WWM) \{Lit. eye of house ae blong haos\}

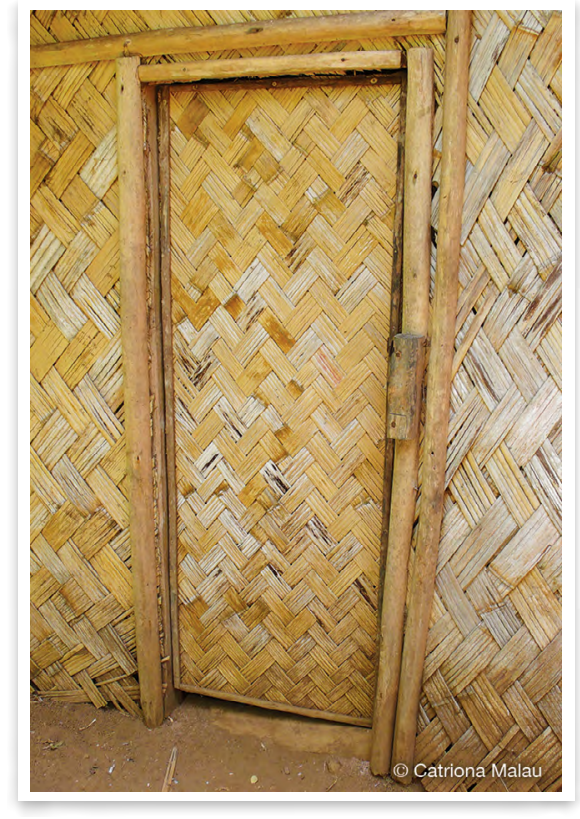

mete gövur door

mete lō $n$.com. $\mathbf{1}$ • east, place where sun rises $\sim$ ples we san i kam antap syn: nögö lō $\mathbf{2}$ • expression used to refer to Sola by people in Vurës, as Sola is 
towards the east, where sun rises wan wei blong talemaot Sola, from i stap long is, long ples we san i kam antap Tōlōw kemem a van le mete 10̄. Tomorrow we are going to Sola. Tumoro mifala i go long Sola. \{Lit. eye of sun ae blong san\}

mete mōtō (fr.var. wemete mōtō) n.com. pimple spot long skin, espesli we i stap long fes blong ol yangfala, mo i gat wota blong hem \{Lit. eye of coconut ae blong kokonas\}

mete qōsqōs n.com. source of spring water $~$ ples we spring wota i stat long hem Syn: mete vuvrës

mete vuvrës n.com. source of spring water ples we spring wota i stat long hem Syn: mete qōsqōs

meteër n.com. arrow made from wood of casuarina, with sharp point, used for shooting fish aro we ol i mekem long wud blong oktri, we i gat sap en blong hem mo ol i yusum blong sutum fis \{Lit. eye of casuarina ae blong oktri\}

meteërqōtōn n.com. bluespine unicornfish kaen strongskin (fis) Naso unicornis \{Lit. eye casuarina its head ae blong oktri hed blong hem\}

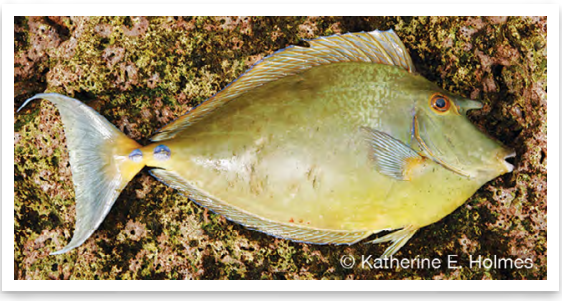

meteërqōtōn Naso unicornis

meteges n.com. wooden knife for cutting löt food dish wuden naef blong katem nalot

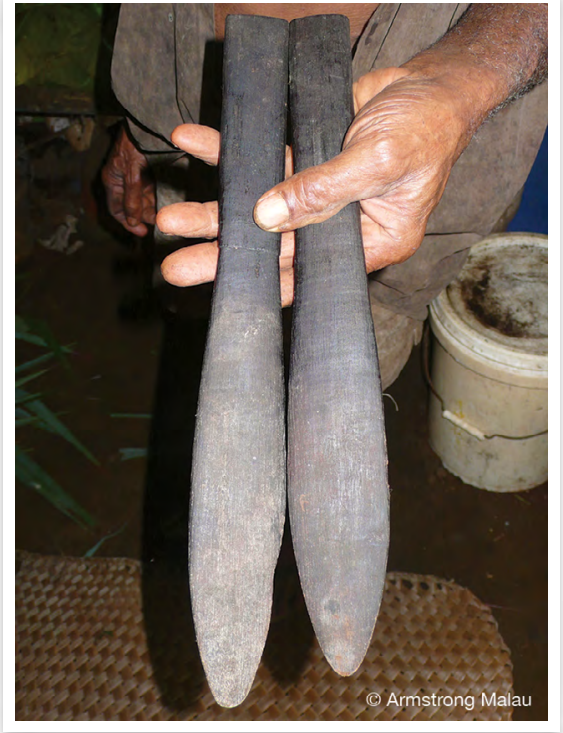

meteges $\sim$ wooden knife

metegongon n.com. zip, opening of trousers zipa, samting blong blokem fran blong traosis

metegrōw $v i$. afraid, frightened $\sim$ fraet Syn: mëtëwtëw, qeneg [POC *[ma]takut 'fear']

metekerker n.com. comb kom

meteraramat $n$. com. kind of tree $\sim$ kaen wud [This tree was traditionally used to start a fire, by rubbing sticks together. Bifo ol i yusum wud ia blong statem faea.]

metesa n.com. anchorage, harbour, landing place pasis Ni kal sar, mem ti o ak le metesa ren ine. He went ashore and put the canoe right at the landing. Hem $i$ go antap long soa, putum kenu stret long pasis ia. (OHS) [POC *mata-saway 'landing place, channel in fringing reef giving passage to boats']

metestes vi. sharp (point, as in end of spear, knife) sap (poen, olsem en blong spia o naef) No ma daēl vita nana lēn o qiat, ba na möguk o gasel gete metestes. I tried to break the top of the taro, but (the point of) my knife 
wasn't sharp. Mi traem blong karemaot top blong taro, be naef blong mi i no sap. See: gegen

metewōs $n$.com. water source $\sim$ ples we wota i kamaot long hem

metwōn n.com.suff. her/his/its personality fasin blong hem See: metwu

metwu $n$ personality, behaviour, manner, habit fasin No gē gilal wareg o metwu sul. I know well the behaviour of the people. Mi save gud fasin blong ol man. (VSB)

metwōn (n.poss) n.com.suff. her/his/ its personality $\sim$ fasin blong hem metwuk (n.poss) n.com.suff. my personality fasin blong mi metwuk n.com.suff. my personality fasin blong mi See: metwu

mewērwēr n.com. black-tipped grouper (fish) kaen los (fis) Epinephelus fasciatus

$\mathbf{m} \overline{\mathbf{e}}$ n.com. achiote, annatto; kind of shrub or small tree with bright red spiny fruits and red seeds wan kaen wud we $i$ gat red frut blong hem wetem red sid insaed Bixa orellana [The red seeds are used as a traditional face paint for men's dance performance. Ol i yusum red sid blong insaed blong frut blong penem fes blong man long kastom danis.]

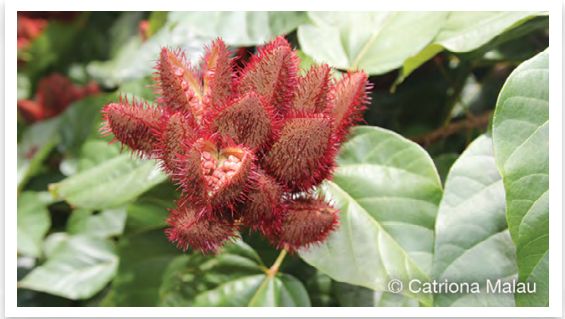

$\mathbf{m e \overline { e }} \sim$ Bixa orellana

mēl n.com. kind of tree $\sim$ wan kaen wud Elaeocarpus floridanus

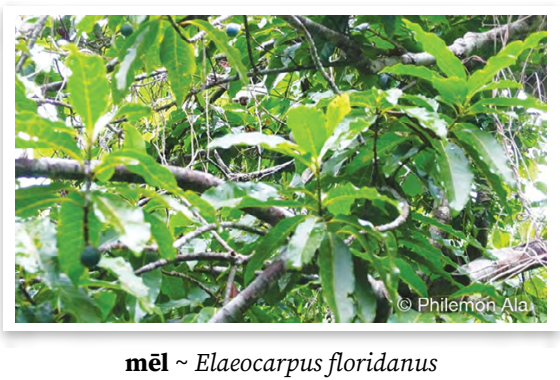

mēlēs n.com. worm wom

mēlēslēs $s_{1}$ vi. weak, slack, floppy slak $\mathbf{O}$ mēlin̄sal ma da nē na tarbian mē mēlēslēs aē. Hunger affected her such that her body was weak and slack. Hanggri i wokem hem, bodi blong hem i slak wetem. Syn: mölöslös

mēlēslēs $s_{2}$ n.com. kind of flowering plant in the nettle family kaen plant Elatostema macrophyllum mēlēslēs lav (comp.) n.com. kind of flowering plant in the nettle family $\sim$ kaen plant Elatostema beccadi

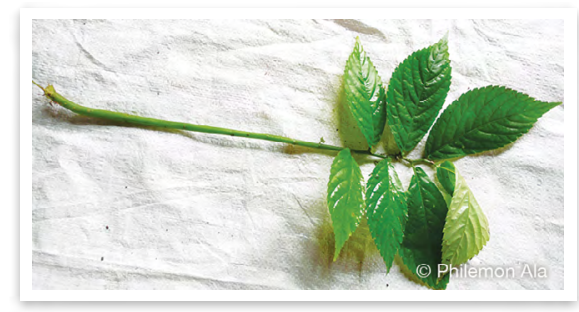

mēlēslēs lav Elatostema beccadi

mēlēsten̄teñ n.com. kind of insect which is similar to a cicada, both in the way it looks and noise that it makes wan kaen bebet we hem i wokabaot long graon mo hem i save jam. Hem i singaot oltaem

mēèēt $v i . \mathbf{1}$ • break, be broken brok o gasel mē mēlēt. The knife broke. Naef $i$ brok. 2 break (of waves) (solwota i) brok La masawre no mē èl rōw e, o naw mē mēlētlēt kal me. When I looked seaward, the waves were coming in. Taem mi luk i go daon long solwota, solwota i stap brok $i$ kamso. (DNS) See: da lēt, lèt 
mēlin n.com. variety of yam that is grouped with the 'male' yams and is white inside wan kaen yam we $\mathrm{i}$ longlongfala mo insaed $i$ waet

mēlin̄sal $n$.com. be hungry, hunger hanggri $O$ mēlin̄sal ni qal nēr töl. Hunger had affected the three of them. Hanggri i kasem trifala. (WDR) $\mathbf{O}$, nēn o mēlin̄sal wo. Oh, we are really hungry. Awo, yumi hanggri we. (JRQ)

mēr $\mathbf{1} \cdot v i$. angry, be angry $\sim \operatorname{kros} 2 \cdot$ n.com. anger $\sim$ kros $\mathbf{O}$ mēr ge meneg ren. The anger is really strong. Kros i strong tumas.

mērēn n.com. large stone, can be sharp used for cutting, or used to block and direct water for irrigation bigfala ston, i sap blong katem samting, o ol i yusum blong blokem wota long garen taro

mēs n.com. rainbow lorikeet (bird) nasiviru (pijin) Trichoglossus haematodus

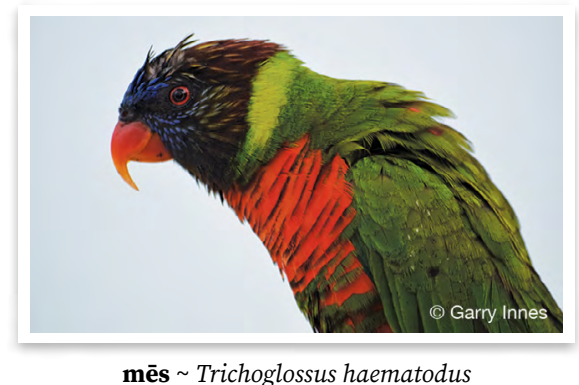

mēsēr vi. tear, be torn (kaliko o pepa) i brok Na non o gogov mē mēsēr. Her clothes tore. Kaliko blong hem ibrok. See: sēr

mēsi part. emphatic marker, premodifying nouns, indicating that the subject has the characteristic indicated to a large degree $\sim$ tumas Nē o mēsi qaqaq. He is one who talks a lot (can be angrily). Hem i mak blong toktok, save tok kros long ol man. Nē o mēsi borbor. He is one who laughs all the time. Hem i laf tumas. mētigtisē n.com. selfish, not kind hearted selfis, fasin i nogud Nē gōtō sōgsōg sur nē o mētigtisē. He is not generous with his possessions because he is a selfish person Hem i no stap givim ol ting from hem $i$ selfis (fasin blong hem inogud) Ant: mētigwē mētigwē n.com. generous, kind hearted, well mannered gladhat, gudfala fasin Ant: mètigtisē

Mētir n.loc. place name nem blong ples

mēvin n.com. shard of glass, mostly used to refer to piece of glass used as tool for scraping root vegetables when cooking $\sim$ pis glas, olsem ol i yusum blong skrasem kakae O mèvin mè tēv no. The shard of glass cut me. Pis glas i katem mi.

mēvinvin $v i$. thin tintin Ant: mötöltöl mëk $c l$. my (drink) (dring) blong mi See: $\mathrm{me}_{2}$

mëkbē n.com. broad-billed flycatcher (bird) wan kaen pijin Myiagra caledonica [The bird's name is derived from its call, which sounds like mëkbē, meaning 'my water'. People hearing the call consider this to be a sign that it is going to rain. Saon we pijin ia i stap mekem i saon olsem hem i stap talem mëkbè, we i minim se 'wota blong mi'. Mekem se ol i man i talem se hem i stap singaotem ren.] \{Lit. my water (to drink) wota blong mi (blong dring)\}

mëlmël 1 • n.com. shade, cool place sed, ples i kolkol Tek nē den o lō, lë mëlmël. Take it out of the sun, into the shade. Karemaot long san, long kolkol ples. 2 $v$ vi. become cool kam kolkol 0 gengen ni mëlmël. The food is cooling down. Kakae i stap kam kolkol. mëmës $v i$. hurt, be sore, ache soa Na qōtōn gë mëmës. She has a headache. Hed blong hem i soa. 
mërëv n.com. mist, fog sno, samting olsem smok we i kavremap ples taem i kolkol Nonor o mërëv. Yesterday it was foggy. Yestedei i gat sno. Syn: miaw mëtëk n.com.suff. my eye ae blong mi See: mete

mëtëwtëw $1 \cdot v i$. afraid, frightened fraet $\mathbf{2} \cdot v t$. afraid of, frightened of fraet long Lo sem sëkër ine vēti, no më mëtëwtëw o masawre ine. At that same reef yet, I was afraid of that place. Long sem rifia yet, mi fraet long ples ia. (TSR) Syn: metegrōw, qeneg

mëtëwtëw ev n.com. variety of breadfruit that cooks quickly kaen bredfrut we i dan kwiktaem $\{$ Lit. scared of fire fraet long faea\}

mëvtia $n . c o m$. wound gat kil $\mathbf{O}$ mëvtia ko gē liwō wo. This wound is really big. Gat kil ia hem ibigwan tumas.

$\mathbf{m e ̈ w}_{1}$ vi. open and close $\sim$ i open, i klos $\mathbf{m e ̈ w}_{2}$ n.com. dew drop wota long gras long eli moning

mi (abbrev. of $\min _{2}$ )

-mi suff. second person plural possessive suffix, your $(\mathrm{pl}) \sim \mathrm{blong}$ yufala

mian vi. ripe $\sim$ raep $\mathbf{O}$ wotag gi mian si gō bōl? Are the bush nuts ripe or not quite ripe? Ol navele ol i raep o ino raep gud? Syn: qōtō

mianisi $\mathbf{i}_{1}$ n.com. variety of banana with small, thin, bent fruit and short trunk kaen banana we hem i save gru hae. [This banana tastes similar to a samo. Tes blong kakae i defren lelebet be i olsem samo.]

mianisi $_{2}$ n.com. hardwood $\sim$ natora Malabōs o biēg ko nē o mianisi. Luckily this breadfruit is a hardwood. Laki se bredfrut ia hem inatora.

miarbē n.com. kind of freshwater fish that is red, and black on its back. It can live in the sea and grows quite large wan kaen fis blong wota we i red mo antap long hem i blak. Hem i save liv long solwota. Hem i save bigbigwan

miat vi. die, be dead ded Nēk i miat ti, nē ga da ēsēs kēl le nēk. If you die, he can bring you back to life. Sapos yu ded, hem i save mekem yu laef bakegen. [POC *mate 'die']

mie adj. long longfala Manene o qon, na bibian gē mie. Because a pigeon has a long neck. From nawimba, nek blong hem i longwan.

mil n.com. 1,000 vatu, monetary unit used to refer to multiples of 1,000 vatu 1,000 vatu Nēk ge le min no o mil nirō. You give me 2,000 vatu. Bae yu givim 2,000 vatu long mi. Nē ni le kēl den bilēñ o mil nitevelēm e, e sur oso? He took another 5,000 from it, for what? Hem i tekem 5,000 bakegen long hem, from wanem? (CAA) [From the French word for thousand, 'mille'. Note that monetary units referring to multiples of 10 and 100 vatu are derived from the imperial monetary system and only this term comes from French, introduced after the introduction of Vanuatu vatu currency. Wod ia i kamaot long Franis wod 'mille' we i minim se wan taosen. Tufala narafala wod blong koen i kamaot long sistem blong bifo long taem blong kondominium.] See: baon, selen

$\operatorname{mim} \mathbf{1} \cdot v i$. urinate $\sim$ pispis Nana van lè mim, na dada matēl. I went to urinate, but I was unable to do it. Mi go blong pispis, be mi no save mekem. (ADT) $2 \cdot$ n.com. urine $\sim$ pispis $\mathbf{E}$ ni rōn̄teg o mim e ni tōk rak kēl bilēñ. Then he felt the urine (the need to urinate) coming back again. Ale hem i harem pipis i kambak bakegen. (ADT) See: mimiē [POC *mimiR 'urinate']

mimian n.com.suff. her/his/its urine pispis blong hem See: mimiē 
mimiē n.com.suff. urine, wee $\sim$ pispis Na mimian e mē vērēg vēvēvērēg. His urine ran out a lot. Pispis blong hem iron we iron. See: mim 2

mimian (n.poss) n.com.suff. her/his/its urine pispis blong hem

mimiēk (n.poss) n.com.suff. my urine $\sim$ pispis blong mi

mimiēk n.com.suff. my urine pispis blong mi see: mimiē

$\min _{1}$ prep. $\mathbf{1} \cdot$ to, marks dative object, role of recipient of an action long I nēk ge vetreñ na salañ min marōōn. You will send your news to your uncle. Bae yu sendem nius blong yu long angkel blong yu. (RKM.010) 2 - for, on behalf, marks benefactive, role of beneficiary of an action long No gö mörös na da o nötu gengen min kēmi. I want to make a small meal for you all. Mi wantem mekem wan smol kakae long yufala. (ESP.061) 3 • have, for, prepositional marker of possessor in existential possessive construction $\sim$ wetem $\mathbf{S i}$ o söm aē min nēk, nēk i le bilēñ na mōgōn̄ o vi. If you have any money, then give your fee too. Sapos we yu gat mane wetem yu, yu givim mane blong yu tu. (VSS.019) $4 \cdot$ onto, on, to, motion onto, position on go long Nē mö ququs lō o gengen min no. She spat food out onto me. Hem i spetemaot kakae i go long mi. 5 - with, marks a Comitative role $\sim$ wetem Ne mite leg min Manbus si isē gēn. She will not get married with Manbus or whoever it is. Bae hem ino mared wetem Manbus o huia. (CAA.072)

$\min _{2}$ (abbrev. mi) v.part. high probability modality bae Syn: wun $_{2}$

mina disc. Syn: wana

miöw n.com. mist, fog samting olsem smok we i kavremap ples taem i kolkol syn: mërëv

misonari n.com. missionary misonari mito v.part. future negative proclitic bae i no; wod we i go bifo long veb we i talemaot se aksen bae i no tekples (Allomorphs: mita, mite, mitē, mitë, miti, mitō, mitö)

mo v.part. perfect aspect marker, used to mark events that are completed bin (Allomorphs: ma, me, mē, më, mi, mō, mö)

molon n.com.suff. her/his clothes $\sim$ klos blong hem See: mölö

monog $v i$. cooked dan La masawre o ōr mo monog, in̄ko a mem lö lölö aesboks. When the prawns were cooked, then we put them in the fridge. Taem naora $i$ dan, nao ia mifala $i$ putum insaed long aesboks. (AAD) $[P O C *[m a] n o k a$ 'be in good condition for eating: nicely ripe, well-cooked, soft']

mowor $v i$. be broken, break brok Ni mowor, lö lölö, o wie tabaē ga mamē. It breaks open, and inside, its fruit is red. I brok, insaed long hem, frut blong hem i red. (SBB) See: wor

mō n.com. long feather of a rooster's tail longfala feta long tel blong man faol

mōdō n.com. unfortunate person, who people feel sorry for because they have no family, partner, or some other bad things are affecting their life man sori we laef blong hem i nogud tumas from i nogat famle, nogat man o woman blong hem, mo sam samting nogud long laef blong hem i mekem se ol man i sori long hem Manene o mōdō, na taman odian̄, na rētnan odiañ. Because an unfortunate one, he doesn't have a father, he doesn't have a mother. From man sori, i nogat papa blong hem, i nogat mama blong hem. (ESP)

mōdōn n.com.suff. her/his/its nose nus blong hem See: mödu

mōgōn cl. hers/his/its blong hem See: mögu 
mōl $v i$. 1 . go home, go to a place to stay overnight go hom Rōrō a mōl kēl me lö gövur. The two of them returned to the house. Tufala $i$ kambak long haos. (ATB) Nēk gō mōl si nēk gē kēl me? Are you going to go and sleep or will you come back? Bae yu go, yu slip o bae yu kambak? 2 - go to bed, go in order to sleep go blong slip Söwlē nēr me gengen qēt, nēr a mōl kal lö gövur. So they finished eating, and they went into the house to sleep. Ale ol $i$ kakae finis, ol $i$ go long haos blong slip. $\mathbf{3} \cdot$ go to church, prayer $\sim$ go long jos Nēk gōtō mōl la tatar sur? Why didn't you go to church? Yu no go long jos from?

mōlkēl n.com. variety of taro, 'female', stem with dark green bottom and dark purple top kaen taro we hem i woman mo stem blong hem i dak grin daon, popel antap \{Lit. go home again go hom bakegen\}

$\mathbf{m o ̄} \mathbf{m} \overline{\mathbf{o}}_{1}$ n.com. squaretail grouper (fish) $\sim$ kaen los (fis) Plectropomus areolatus $\mathbf{m o ̄} \mathbf{m} \overline{\mathbf{o}}_{2}$ n.com. poumuli, tree species with small, black, inedible fruit namamao, namemiwa, wan kaen tri we i gat frut we i smol mo blak be man i no save kakae Flueggea flexuosa [POc *mapuqan 'Flueggea flexuosa']

mōnōg n.com. sore on body soa long bodi na mōgōn o mōnōg her/his/its sore soa blong hem

mōr $n$.com. lazy les Nēk o mōr, nēk o mōr. You are really lazy. Yu les tumas. mōs n.com. black lizard blak lised

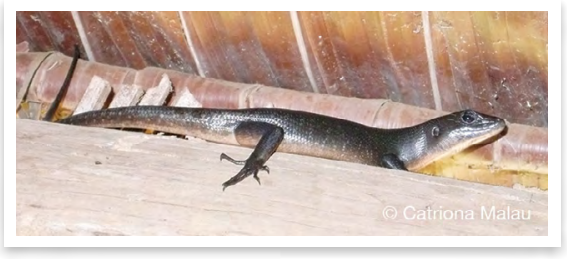

mōs black lizard mōotō ${ }_{1}$ n.com. $\mathbf{1} \cdot$ coconut, generic term for coconut palm, and for fruit when ripe kokonas Cocos nucifera Nana van lo qētēgi mōtō nitiwial. I go to a coconut palm. Mi go long stamba blong kokonas. (JWM.002) $2 \cdot$ coconut milk $\sim$ melek blong kokonas [POC *matuqu 'coconut growth stage 9: ripe, flesh hardened']

mōtō atm̄ēn (comp.) n.com. variety of coconut that is characterised by rapid growth but poor productivity kaen kokonas we i gru kwiktaem be i no karem tumas kakae \{Lit. male coconut man kokonas\}

mōtōo bal (comp.) n.com. variety of coconut where two palms grow from one coconut or two are planted side by side $\sim$ kaen kokonas we tu kokonas i gru i kamaot long wan kokonas o we ol i planem tu kokonas i stap klosap tumas \{Lit. coconut tongs kokonas tong blong karemaot hot ston\} mōtō dēdērēs (comp.) n.com. variety of coconut that has very sweet flesh and water kaen kokonas we kakae mo wota blong hem i swit tumas \{Lit. sweet coconut swit kokonas\}

mōtō elvet (comp.) n.com. variety of coconut that has long fruit $\sim$ kaen kokonas we frut blong hem i longfala Syn: mōtō ge metestes (comp. of mōtō ${ }_{1}$ ), mōtō sam̄sam̄ (comp. of mōtō̄ \{Lit. long coconut longfala kokonas\}

mōtō ga an̄añ (comp.) n.com. variety of coconut that has yellow fruit and yellowish leaves $\sim$ kaen kokonas we frut blong hem i yelo mo lif i lelebet yelo \{Lit. yellow coconut yelo kokonas\} mōtō ga garaq (comp.) n.com. variety of coconut that is a new hybrid kaen kokonas we i niuwan \{Lit. new coconut ninfala kokonas\}

mōtō ge metestes (comp.) n.com. variety of coconut that has long fruit $\sim$ kaen kokonas we frut blong hem i longfala syn: mōtō elvet (comp. 
of mōtō ${ }_{1}$, mōtō sam̄sam (comp. of mōtō $\left._{1}\right) \quad$ Lit. sharp-pointed coconut kokonas we i sap\}

mōtō gō tōtōrōg (comp.) n.com. variety of coconut that has green fruit kaen kokonas we frut blong hem $\mathrm{i}$ grin $\{\mathrm{Lit}$. green coconut grin kokonas\}

mōtō gö luwō (comp.) n.com. large variety of coconut with thick meat kaen kokonas we i bigwan mo i gat bigfala kakae \{Lit. big coconut bigfala kokonas\}

mōtō malgias (comp.) n.com. variety of coconut that has reddish greenish coloured fruit kaen kokonas we frut blong hem i grin red

mōtō mamē (comp.) n.com. variety of coconut that has red fruit kaen kokonas we frut blong hem i red $\{$ Lit. red coconut red kokonas\}

mōtō mete röwö (comp.) n.com. variety of coconut so called because the eyes on the shell stick out like those of the fish röwö kaen kokonas we ol i kolem olsem from ae blong hem i stik aot olsem ae blong wan fis, röwö

mōtō mölumlum (comp.) n.com. variety of coconut that has soft flesh that is thus easy to remove from the shell kaen kokonas we kakae i sofsof mo i isi blong selaot \{ Lit. soft coconut sofsofkokonas\}

mōtō mat (comp.) n.com. variety of coconut that has stripes encircling the fruit kaen kokonas we i gat ol straep i raonem frut \{Lit. snake coconut snek kokonas\}

mōtō reqe (comp.) n.com. variety of coconut that is characterised by slow growth yet high productivity kaen kokonas we i gru sloslo be i karem plante kakae \{Lit. female coconut woman kokonas\}

mōtō sā̄sā̄ (comp.) n.com. variety of coconut that has long fruit kaen kokonas we frut blong hem i longfala Syn: mōtō elvet (comp. of mōtō ${ }_{1}$ ) mōtō ge metestes (comp. of mōtō $_{1}$ ) \{Lit. chewing coconut juim kokonas\}

mōtō seseser (comp.) n.com. variety of coconut that is easily husked $\sim$ kaen kokonas we $i$ isi blong karemaot skin blong hem

mōtō sial me (comp.) n.com. variety of coconut that was introduced from another island $\sim$ kaen kokonas we i kam long narafala aelan $\{$ Lit. coconut that floated here kokonas we $i$ flotikam\}

mōtō silat (comp.) n.com. variety of coconut that has fragile leaves and inflorescences and is not highly productive $\sim$ kaen kokonas we lif mo flaoa blong hem i no strong mo hem i no karem tumas kakae \{Lit. nettle tree coconut nanggalat kokonas\}

mōtō taktak (comp.) n.com. variety of coconut kaen kokonas

mōtō us (comp.) n.com. variety of coconut with flesh that is sweet and soft with lots of liquid kaen kokonas we i swit, i sofsof mo i gat plante wota

mōtō vanvan (comp.) n.com. variety of coconut $\sim$ kaen kokonas \{Lit. walking coconut kokonas we i wokbaot\} mōtō vet (comp.) n.com. variety of coconut that has very hard flesh which is thus difficult to remove from shell kaen kokonas we i strong tumas mo i had blong selaot \{Lit. stone coconut ston kokonas\}

mōtō vin gaqō (comp.) n.com. variety of coconut with a thick husk kaen kokonas we skin blong hem i tik mōtō wulmēe (comp.) n.com. variety of coconut that has a reddish coloured ring surrounding where the peduncle is attached to the fruit $\sim$ kaen kokonas we i gat red ring long frut long ples we i joen long han 
mōtō ${ }_{2}$ n.com. right $\sim$ raetsaed Nē gē gis o rềntenge la banēn lō mōtō? Is he holding a stick in his right hand? Hem i holem wud long han blong hem long raetsaed? (M2E) Ant: gala

mōtōvōl n.com. areca palm belonging to same family as betel nut kaen pamtri we i famle blong bitelnat Areca macrocalyx

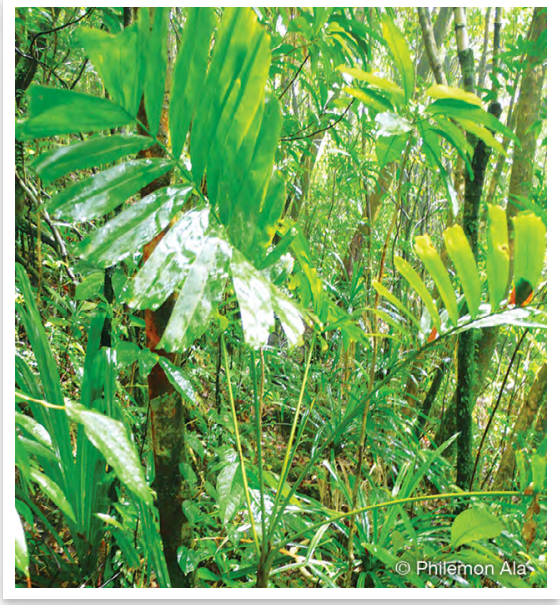

mōtōvōl Areca macrocalyx

mōvōt $v i$. sleep slip Kemek a gengen qēt, kemek a ōn sursur, a mōvōt. We finish eating, and we lay down and sleep. Mifala i kakae finis, mifala i leidaon, $i$ slip. (DDL) $[P O c *$ [ma]puta 'sleep']

gen mōvōt (id.) vi. close eyes satem ae Nēk i gen mōvōt wo nana rōw net. Close your eyes and I'll jump down to you. Satem ae mo bae mi jam i kam daon long yu. (AWW) Ant: mamat $_{2} 2$ \{Lit. eat sleep kakae slip\}

mōvōt tul (SVC) vi. be in a deep sleep slip blong ded E wana in̄ko mō vōrōg, e wana i vo e ni mōvōt tul. Then morning came, but the guy was in a deep sleep. Ale nao ia i kam moning, be man ia i slip blong ded. (RPP)

mödu n.com.suff. nose nus Ni rōn̄teg na mōdōn ni wëswës, timiak vita mō mōvōt tul ren. He heard his nose whistling, as if he was in a very deep sleep. Hem $i$ harem nus blong hem i stap wisel, olsem se hem i slip blong ded nomo. (ADT)

mōdōn (n.poss) n.com.suff. her/his/its nose $\sim$ nus blong hem

möduk (n.poss) n.com.suff. my nose nus blong mi

möduk n.com.suff. my nose nus blong mi see: mödu

mögu $c l$. relational classifier used to mark possession of general items that are not included in any of the other categories; the default classifier blong (yusum blong tokbaot ona blong wan samting) Nē ni le na mōgōn o tibiar. She got her basket. Hem i karem basket blong hem. (WWM̄)

mōgōn (n.poss) cl. hers/his/its blong hem

möguk (n.poss) cl. my blong mi möguk $\mathrm{cl}$. my blong mi See: mögu

mölö n.com.suff. clothes klos In̄ko kemem a sër na mölön kemem ga mamas. Then we put on our dry clothes. Nao ia mifala i werem drae klos blong mifala. (AAD)

molon (n.poss) n.com.suff. her/his clothes klos blong hem

mölök (n.poss) n.com.suff. my clothes klos blong mi

mölök n.com.suff. my clothes klos blong mi see: mölö

mölöl kör n.com. variety of Polyscias $\mathrm{sp}$. whose leaves are eaten as greens kaen kabis Gen: dëmël

mölumlum vi. 1 • soft $~$ sofsof $\mathbf{O}$ dëm gö mölumlum sel den o qiat. Yams are softer than taro. Yam i sofsof bitim taro. Na vōlōn gö mölumlum. Her hair is soft. Hea blong hem i sofsof. $\mathbf{2} \cdot$ slow, slowly slo, sloslo $\mathbf{O}$ qon nē ma gav mölumlum. The pigeon flew slowly. Nawimba i flae sloslo. (DHN) [POC * [ma]lumu 'soft, gentle, easy'] 
mönö (fr.var. mēnē) part. a bit smol No mö mönö gis o rörö timiak ine. I have caught a bit of news like that. Mi kasem smolnius olsem ia. (VSJ)

mörö n.com. thirst, be thirsty tosta o mörö ni qalqal rōrō. The two of them were struck by thirst. Tosta $i$ kasem tufala. (WDR) Na nötuk o mörö. My child is thirsty. Pikinini blong mi i tosta.

mörönërër n.com. kind of eel that is thin but long $(150 \mathrm{~cm})$ and speckled brown wan kaen namarae we hemi tintin be longfala mo kala blong hem i braon

mörös $1 \cdot v t$. want, need $\sim$ wantem No gö mörös idian vita na big o mes. I really want to eat fish. Mi wantem tumas blong kakae fis. 2 - vt. like laekem No gö mörös o mirmiar ine. I like that child. Mi laekem pikinini ia. 3 n.com. desire $\sim$ nid Ni damösur na non o mörös döl. It fulfils all of his desires. Hem i fulfilim evri nid blong hem. (OSK)

mörötröt vi. weak, faint, as from hunger slak olbaot, nogat wan samting i pulum tugeta paoa blong olgeta Ira nōtōn a tutuag aē o mēlin̄sal. Ra nōtōn a mömörötröt kēlkēl gem gēn. His children were suffering from hunger. His children were just weak all over. Ol pikinini blong hem ol i safa from hanggri. Ol pikinini blong hem ol i les olbaot nomo. (KM̄T) Syn: m̄ölöslös

mösön n.com. hiccups hikap $\mathbf{O}$ mösön ma da no. I have the hiccups. Hikap $i$ mekem mi.

mösövsöv vi. beautiful, in perfect shape (of person) naes tumas (bodi blong man)

mötöltöl $v i$. thick tik Ant: mēvinvin

möul vi. 1 • for a long time, take a long time tekem longtaem Nēr mo tog gö möul min rōrō. They stayed with the two of them for a long time. Olgeta ol $i$ bin stap longtaem wetem tufala. (JMT) Rōrō ma kal lin̄ereg sar ine wo götö möul wana o timiat e ni vērēg dēn. The two of them went inside and before long the devil ran up. Tufala $i g o$ insaed ia mo i no longtaem devel $i$ resis kam kasem. (DPD) $\mathbf{2} \cdot$ slow, be slow sloslo Nēk o götö, nēk gö möul, nēk gö möul. You are a hermit crab and you are really slow. Yu nakato mo yu slo we yu slo. (DNS) Ant: lelev

mövut vi. uproot kamaot long graon Nē ma da o gatitieg le vetel ine, ba o vetel ine gö mövut. Ba gë mës tēqēl, e gō ōn. He made a bird trap on the banana tree, but the banana tree uprooted. But it fell down, and it lay down. Hem i mekem trap blong pijin long banana ia, be banana ia i kamaot long graon. Be banana i foldaon, ale $i$ leidaon. (WRN)

mu n.com. striped large-eye bream (fish) wan kaen fis Gnathodentex aurolineatus

mulög $v$. replace stones from oven fire if they fall off while the fire is alight putum bak ston sapos ol $\mathrm{i}$ folaot long oven taem faea i laet

mulög $v$. use tongue to try and remove something from inside the mouth yusum tang blong traem tekemaot samting long maot

mun $v$. mend roof of house by changing individual pieces of thatch jenesim wanwan lif natanggura insaed long haos

murmur n.com. epaulet soldierfish wan kaen redfis wetem bigfala ae Myripristis kuntee

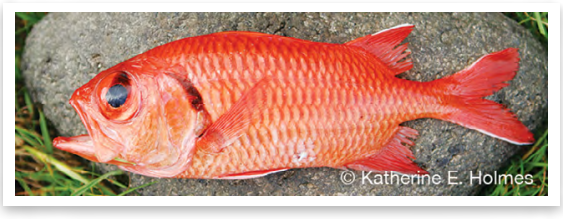

murmur Myripristis kuntee 
murmurbē n.com. kind of freshwater fish that is silver with a sharp mouth. It grunts like a pig. It lives in holes in rocks and can also move between fresh and salt water $\sim$ wan kaen fis blong wota we hem i silva mo fes blong hem i sap. Hem i mekem noes olsem pig. Hem i stap long hol mo hem i save stap long solwota

musmus $v$. get disoriented after going to a place that has traditional significance in order to get something good, but the power or the spirits of that place affect you go long wan tabu ples mo ples ia o spirit blong ples ia i spolem nogud yu, mekem se yu no save faenem rod blong yu Ēlgōr den i rege tala masawre ine ni musmus nēk. Watch out in case the spirits from that place affect you and make you disoriented. Lukaot, nogud olgeta blong ples ia $i$ spolem nogud yu.

\section{$\overline{\mathbf{M}} \sim \overline{\mathbf{m}}$}

$\overline{\mathbf{m a}} \overline{\mathbf{e}}$ n.com. sky, open sky, high in air skae, antap long ea $\mathbf{O}$ leñ gö luwō mö uvuv kēlkēl nē siag la maē. A strong wind blew it back and forth up in the open sky. Wan bigfala win ibloem hem i go i kam, antap long skae. (DHN) Syn: kētkēt 1 See: tōkmeren̄ (comp. of tōk)

mal n.com. sow pig woman See: $\mathrm{qo}_{1}$ malaglag $v i$. wobbly, be wobbly muvmuv Na luwök ko mō malaglag. My tooth is wobbly. Tut blong mi $i$ muvmuv. Syn: m̄ōgōlgōl

malan n.com. suprise, shock sapraes Na mëlëk mō rōw aē. I was shocked by it. Mi sapraes long hem.

malmal 1 - n.com. young unmarried woman, teenager yangfala gel o malmal e, in̄ko na nōtōn nivet vitia. That young unmarried woman, now she already has four children. Yangfala gel ia, nao ia hem i gat fofala pikinini finis. (CAA) 2 - vi. be young woman, become young woman kam yangfala gel Mo tog ine vaavan i Wōwut mō lōmegev, i Meter ma malmal. It stayed like that until Wöwut had become a young man, and Meter had become a young woman. I stap olsem ia go go Wöwut i kam yangfala man mo Meter i kam yangfala gel. (WWM- $\left[\mathrm{POC}{ }^{*} m^{w}\right.$ ala 'unmarried young woman']

malmalbak n.com. variety of banana that has long, large fruit. It bears fruit up high and is not very productive. The cooked unripe fruit are very dry to eat $~$ kaen banana we stamba blong hem i bigfala mo hem i gru hae be hem i no karem fulap kakae. Taem ol i kukum i drae we i drae

malrōw $v i$. be surprised sapraes No mē ēl malrōw. I was surprised. Mi sapraes

'̄amā̄ $v i$. clear, be open, not enclosed (of area) klia, open ples Rōrō a èl timiak o masawre ta mamaē. The two of them saw that the place was becoming more open. Tufala iluk olsem se ples i kam open. (KM̄T)

mā̄as n.com. grated canarium nuts wrapped in datamal leaves and baked in a stone oven $\sim$ nangae we ol i pasolem long lif wetem lif blong datainal mo bekem long oven

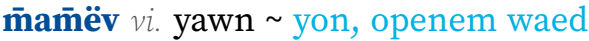
maot, olsem taem yu taed No ma mā̄ëv. I yawned. Mi openem waed maot from mi taed. 
marö adj. empty emti In̄kē o marö wötöv gem. This is just an empty bottle. Hemia wan emti botel nomo. Ant: wōr

mas n.com. goatfish mustasfis Family Mullidae [POc * $m^{w}$ acasi 'goatfish']

mas añan (comp.) n.com. goldsaddle goatfish wan kaen mustasfis Parupeneus cyclostomus \{Lit. yellow goatfish yelo mustasfis\}

mas sarsar (comp.) n.com. yellowfin goatfish, yellowstripe goatfish wan kaen mustasfis we ol i stap wokbaot long grup. $\mathrm{Ol}$ i longlongfala mo ol i waet wetem smol dak red spot long saed blong olgeta Mulloidichthys sp.

mas teñten̄ (comp.) n.com. dashdot goatfish wan kaen mustasfis Parupeneus barberinus \{Lit. crying goatfish mustasfis we i stap krae\}

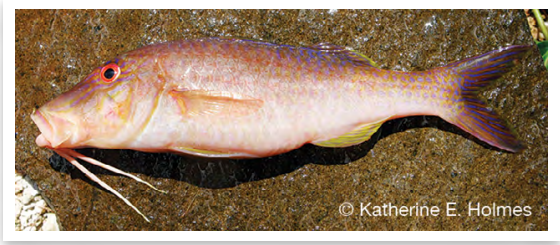

mas ten̄teñ $\sim$ Parupeneus barberinus

masar n.com. poor person, who has no valuables, such as pigs and other valuable goods, money and land pua man we hem i nogat ol samting olsem pig mo narafala samting we $\mathrm{i}$ gat valiu blong hem

masawsaw vi. loose, slack slak No mē lēg ti o ga in̄ko ma masawsaw vitiag. I tied up this rope, but now it is already loose. Mi bin fasem taet wan rop be nao ia i slak finis.

masēlasēl n.com. Job's tears; grainbearing plant wan kaen gras we $i$ longfala mo i gat sid blong hem we i waet mo i strong mo ol i yusum olsem bid Coix lacryma $[\mathrm{POC}$ *sila 'Job's tears, Coix lacryma-jobi']

masērig $v i$. cheap no sas mat n.com. snake $\sim$ snek $\left[P O c{ }^{*}\right.$ mwata 'snake']

mat añ (comp.) n.com. kind of snake which is yellow kaen snek we i yelo mat biliag (comp.) n.com. kind of snake which is spotted kaen snek we i gat dot long hem

mat tan tes (comp.) n.com. sea snake which is short and yellow and is found on surface $\sim$ wan snek blong solwota we i yelo mo i sotfala mo i stap swim antap long ol wef

mat $n$.com. variety of yam that belongs to the grouping of 'crooked' yams kaen yam we i stap long grup blong ol kruked yam

mataklav $v i$. very happy glad tumas No go mataklav sur o rörö vita no go van a Santo. I am really happy about the news that I will go to Santo. Mi glad tumas from nius ia se bae mi go long Santo.

mataktak $v i$. active, lively, feel fit smat; bodi i no hevi, bodi i laet Na tarbēk go mataktak talo vanvan alo sur no gö mörös vanvan alo. I am feeling fit to go to the seaside, because I want to go to the seaside. Mi smat blong go long solwota from mi wantem go long solwota.

matēk n.com. purple swamphen (bird) napiru, longnek (pijin) Porphyrio porphyrio

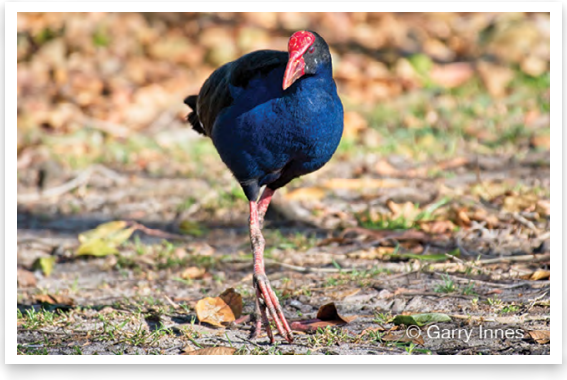

'̄atēk Porphyrio porphyrio 
matēkatēk $v i$. trouble trabol I vo ine go tog matēkatēk vaga. Man ia i stap mekem trabol oltaem.

matēkmëgërsurletes n.com. variety of taro, 'female', with black stem and yellow corm kaen taro we hem i woman, we han blong hem i dak lelebet mo kakae blong hem i ful yelo Syn: manreva \{Lit. swamphen wades towards it in the lake nambiru $i$ swim from long lek\}

matētē n.com. kind of small spider. wan kaen smol spaeda. [If the web of this spider is touched, then the spider shakes. Taem yu tajem web blong spaeda ia, i stap seksek.]

me n.com. $\mathbf{1}$. banded sea snake, banded sea krait sisnek we hem i blak mo waet Laticauda colubrina $\mathbf{2} \cdot$ name of a traditional dance $\sim$ nem blong wan kaen kastom danis

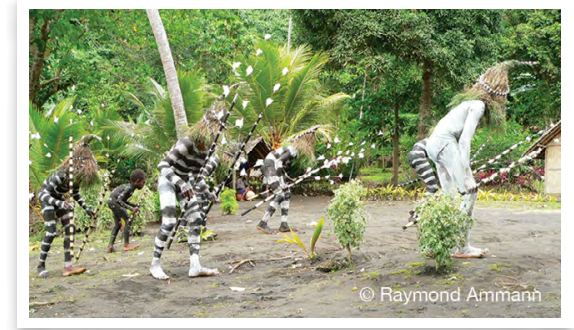

$\overline{\mathbf{m}} \mathbf{e} \sim$ snake dance

menek n.com. $\mathbf{1}$ - squid $\sim$ nawita 2 • cuttlefish $\sim$ nawita, katelfis

mereg n.com. Pacific emerald dove (bird) sotleg (pijin) Chalcophaps longirostris

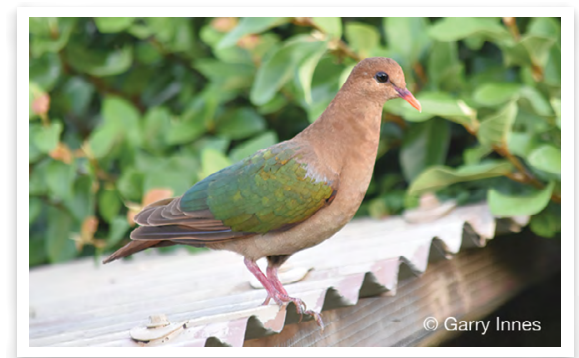

mereg Chalcophaps longirostris mereg n.com. variety of breadfruit that has small fruit kaen bredfrut we kakae blong hem i smolwan

Merlav n.loc. 1 • name of island, Mere Lava nem blong aelan, Mere Lava 2 - tribe name, named because they came from the island of Mere Lava $\sim$ nem blong traeb we olgeta ol i kamaot long aelan blong Mere Lava merlav n.com. variety of taro, with tall dark red stem, and large light red corm kaen taro we stem blong hem i longfala mo i dak red, kakae blong hem i laet red

meter n.com. woman of high rank who has studied to learn the true traditional way of life $\sim$ woman we hem $\mathrm{i}$ bin go long nakamal blong lanem stret rod blong kastom

$\overline{\mathbf{m}} \overline{\mathbf{e}} \mathbf{e}$ tele $n$.com. weaving pattern, for which two strands are woven over, two strands under, alternating the smooth side facing out, then the rough side facing out, can refer to bamboo walling, coconut, pandanus stael blong wiv we tu pis i go ova, tu pis i go andanit, mo yu jenisim, wan antap blong lif o bambu, narawan andanit blong hem

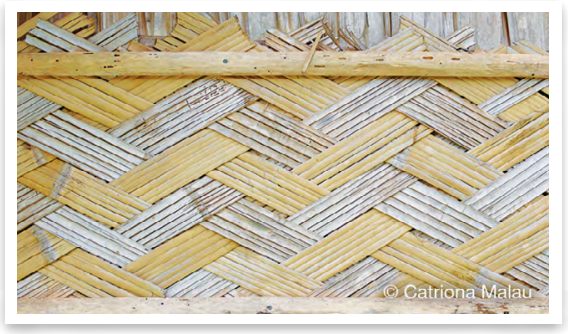

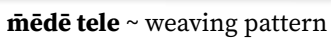

$\overline{\mathbf{m}} \mathbf{e} \mathbf{k} \overline{\mathbf{e}}$ n.com. top of, above, over antap Nēk i le o vet, nēk i mem kal siag lē mēkē damat. You get the stones, and you put them up on top of the heliconia leaves. Iu karem ston, yu putum i go antap long top blong lif laplap. $\mathbf{O}$ tere mes döl ko nēr go tog lē mēēkē sëkër 
ko. All of the fish were over the top of the reef. Evri fis ia ol i stap antap long rifia. (TSR)

mēel n.com. cycad namele Cycas seemannii

m̄èl añañ (comp.) n.com. kind of cycad kaen namele

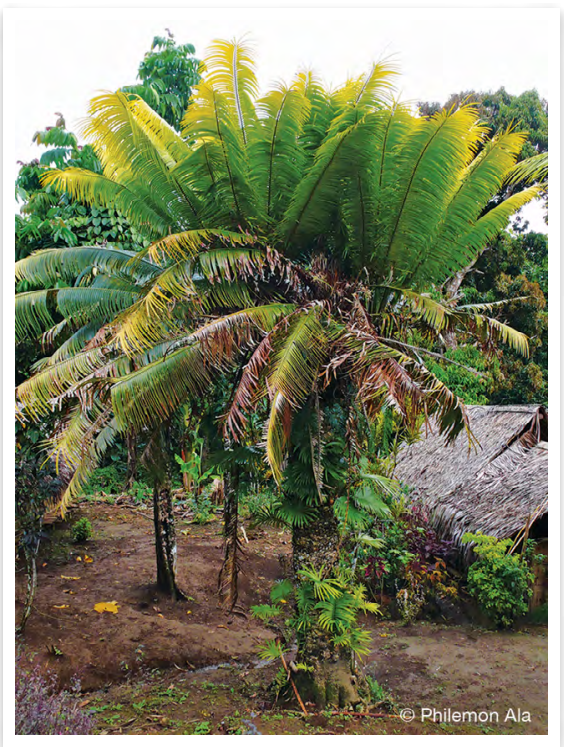

$\overline{\mathbf{m}} \mathbf{e} \mathbf{1} \sim \operatorname{cycad}$

mèèlèg n.com. Vanuatu white-eye (bird) nalaklak wetem yelo bel (pijin) Zosterops flavifrons

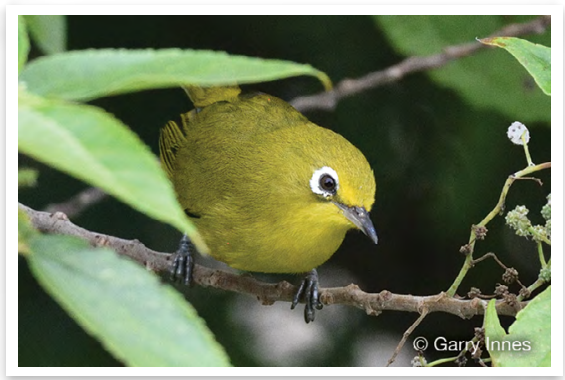

m̄èlēg Zosterops flavifrons

m̄ēlēg wö (comp.) n.com. silvereye (bird) nalaklak (pijin) Zosterops lateralis

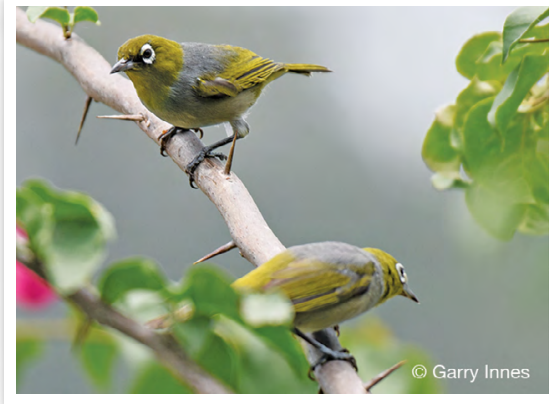

mēèlēg wö Zosterops lateralis

mēēmēri n.com. tentacle, arm of octopus han blong nawita

m̄ēninin $v i$. slippery glis

mēeriri n.com. kind of perennial climbing tree brata blong namariu, namariu blong solwota Acacia simplex

mēeririnek (comp.) n.com. kind of tree namariu blong solwota Acacia spirorbis

M̄èsēn n.loc. place name nem blong ples

mēesir $v$. $\mathbf{\bullet}$ warm $\sim$ wom, hot smol $\mathbf{2}$ • warm, make warm womem

m̄ēvinvian (fr.var. mivian, mivinvian) n.com. kind of plant $\sim$ wan plant Coprosma persicaefolia

mēvinvian 2 (fr.var. mivian, mivinvian) n.com. variety of taro, 'female', with light green stem with light red stripes and light red corm kaen taro we hem i woman, we han blong hem i laet grin wetem laet red straep mo kakae blong hem i laet red

'̄ēw n.com. variety of taro, 'female', with purple stem and wavy leaves with black veins on underside $\sim$ kaen taro we hem i woman we han blong hem i popel mo lif i gat blak laen long andanit blong hem \{Lit. Maewo (island) Maewo (aelan)\} 
mëg n.com. carver, carpenter, handyman, man who has skill for making things $\sim$ man we hem i save katem wud, o gat skil blong mekem samting

mëg simsim (comp.) n.com. carver who is very slow and careful about making good work man we hem i katem wud sloslo be hem i mekem gudwan

mëg sōbōr (comp.) n.com. person who carves randomly with no skill $\sim$ man we i katem wud olbaot nomo mëkër n.com. flying fish flaengfis [POc * $m^{w}$ agaRut 'Exocoetidae, flying fish']

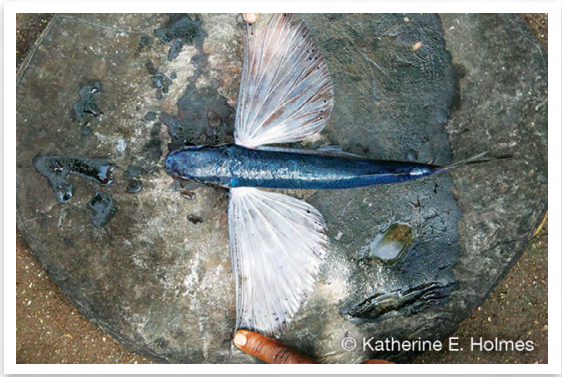

mëkër flying fish

mël n.com. green coconut that has no flesh on the shell, but has water inside, although the liquid is not sweet yet grin kokonas we hem i gat wota blong hem be i nogat mit mo yumi no stap dring from i no swit tumas

mës vi. fall foldaon $\mathbf{O}$ götö me rem kal la talēs, më mës sōw. The hermit crab climbed up the Indian almond tree, and it fell down. Nakato i klaem i go antap long natavoa, hem i foldaon. (GNR)

miak n.com. Tahitian chestnut namambe Inocarpus fagiferus

miar veser $v$. call someone father when they also call you father (of man) singaotem papa long man we hem i singaotem papa long yu

miarmōvōl n.com. stillbirth pikinini we hem i ded taem hem i bon miarōbōl n.com. new mother, mother who has recently given birth mama blong smol bebe

miarten $n . c o m$. noises that are made with bamboo flutes and other things when people are making a vōnōmōto ceremony ol noes we ol man ol i mekem wetem flut o wanem, taem ol i mekem vōnōm̄ōto See: vōnōmōlō

miartiam n.p.fr. darling daling Awo miartiam, no gö mörös idian nēk. Oh darling, I really like you. Awo daling, mi laekem yu tumas.

mie $\mathbf{1} \cdot$ adj. first $\sim$ fas Nēn a gis o mie vugvug na mögun nēn. We will hold our first meeting. Bae yumi holem fas miting blong yumi. (VSS) $\mathbf{2}$ - n.com. first-born child, first thing, first one fasbon, fas samting Mö tulëg o mie ma van. He sent the first one. Hem i sendem faswan $i$ go. (JRQ.009) O mie nēn ga tar o terē bōs. The first thing to do is we cut the posts. Fas samting yumi katem ol pos. (RBH) $\mathbf{3}$ • n.com. leader lida Varian Gilbert, mie talo sosol moning lō vōrōg. Thank you Gilbert, leader of the social morning this morning. Tangkiu tumas Gilbert, lida blong sosol moning long moning ia. (VSE.001)

miedar (abbrev. of wēmiedaran)

mirmiar n.com. child pikinini Ginon ni telsiër o nötu mirmiar nitiwial, ni wot rōw o atm̄ēn. His wife was pregnant with a child, and when it was born, it was a boy. Woman blong hem i gat bel wetem wan smol pikinini, hem i bon, i boe. (HWM) [POc *meRa-meRa 'baby, very young child']

misiak n.com. coconut leaf mat with only one side $\sim$ lif kokonas wetem wan saed nomo

mivian (fr.var. of m̄ēvinvian)

mivinvian (fr.var. of m̄ēvinvian)

molo n.com. incubator bird, scrub fowl, megapode skrabdak, namalao (pijin) Megapodius freycinet 
momo vi. tame, used to refer to animals that are domesticated or comfortable being handled by people no wael, olsem animol we hem i get yus blong stap wetem man $\mathbf{O}$ tere mes ine nēr gōwē idian wo nēr gō mō̄mo bilēñ. All those fish were really nice and they were tame too. ol fis ia ol i gud tumas mo ol $i$ no wael tu. (AAD)

mon $1 \cdot v t$. wrap up pasolem Na mon o mōtō, na bien o waga. I wrap up the coconut, and I bait the prawn trap. Mi pasolem kokonas, mi putum i go long pasket naora. (KBN) Syn: lölös 1, wöwös 2

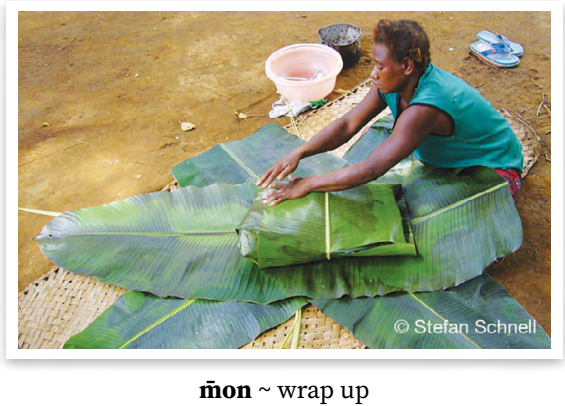

2 • n.com. parcel pasol I Dōl nē ni le lō o meles, ni le lō o m̄on qō, e nē ni ul o mon qō. Dōl took out the leftovers, he took out a parcel of pig, and he unwrapped the parcel of pork. Dōl hem i karemaot ol hafkakae, hem i karemaot pasol blong pig, ale hem i tekemaot rop blong pasol blong pig. (BQD)

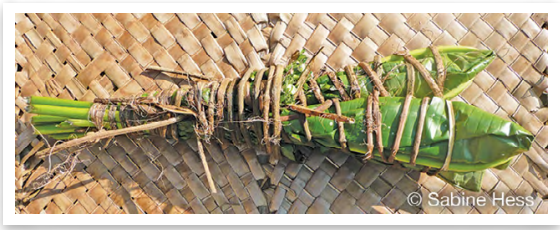

mon parcel

Mot n.loc. $\mathbf{1} \cdot$ neighbouring island in Banks group Mota, nem blong aelan long Bangkis $2 \cdot$ tribe name, named because they came from the island of Mota, in the daytime $\sim$ nem blong traeb we olgeta ol i kamaot long aelan blong Mota, long dei nomo

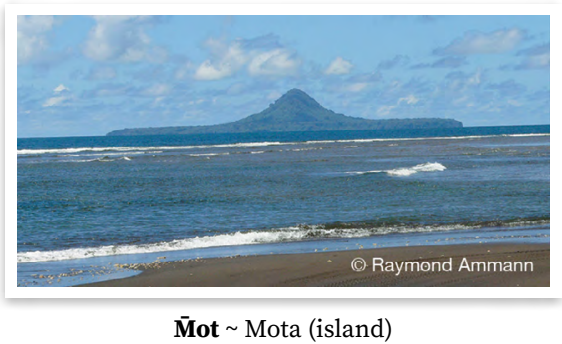

Motlav n.loc. Mota Lava, island to the east of Vanua Lava Mota Lava, nem blong aelan long Bangkis

$\overline{\mathbf{m}} \mathbf{o}$ (root of am̄ō)

'mōgōlgōl vi. wobbly, be wobbly muvmuv Na luwök ko mō mōgōlgōl. My tooth is wobbly. Tut blong mi i muvmuv. Syn: malaglag

$\overline{\mathbf{m}} \mathbf{o g o ̄ t}{ }_{1}$ n.com. kind of grass $\sim$ wan kaen gras Oplismenus hirtellus [People know that when it flowers, it is almost the season of rar, dry season. Taem i karem flaoa i minim se klosap taem blong rar, drae sisen.] '⿳亠⿴囗十⿱日一gōt ${ }_{2}$ n.com. name of a season, when $\bar{m} \overline{o g} \bar{o} t$ flowers, just before dry season of rar, roughly February, March nem blong wan sisen blong yia, taem blong imōgōt i gat flaoa, bifo long drae sisen

$\overline{\mathbf{m}} \mathbf{o} \mathbf{l} \overline{\mathbf{o}} v i$. form hole, have hole, cavity brok, gat hol Kemek go mörös talō wōn o ak ni m̄ōlō timiak in̄kē, kemek go yusum o burñe. If we want to seal up a canoe that has got a hole like this, we will use canarium sap. Sapos mifala i wantem fiksim kenu we i gat hol olsem ia, bae mifala i yusum wota blong nangae. (VVKHVDP180ct0801.117)

mōörag $n$.com. many, be many plante Syn: lōqtag, tutuqë, wuw $\mathbf{1}$

mōōmōōse n.com. bubbles, foam babol 
'̄ōrmōōrsenaw (comp.) n.com. sea foam spet blong solwota

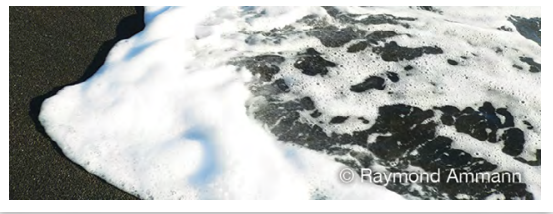

$\overline{\mathbf{m}} \mathbf{o} \mathbf{r m} \bar{o}$ rsenaw $~$ sea foam

mōōō vi. stuck in a hole or tight space

$\sim$ fas long hol o wan smol ples Nē mō mōrō le tes. He was stuck in the toilet hole. Hem i draon ifas long toelet.

'̄ōōōl n.com. variety of taro, 'female', with light purple stem, round leaves kaen taro, we hem i woman, stem i laet popel mo lif i raon [Grouped with rōvōl taro. Famle wetem taro we ol i kolem se rōvōl.]

mögus $v i$. move muvmuv $\mathbf{O}$ tere wut nēr mö möömögus lō qōtōn. The lice are moving around on her head. Ol laos ol i stap muv long hed blong hem.

möökölagē n.com. food and presents for the house, given with bride price $\sim \mathrm{ol}$ samting we ol i givim wetem braed praes olsem kakae mo ol samting blong haos

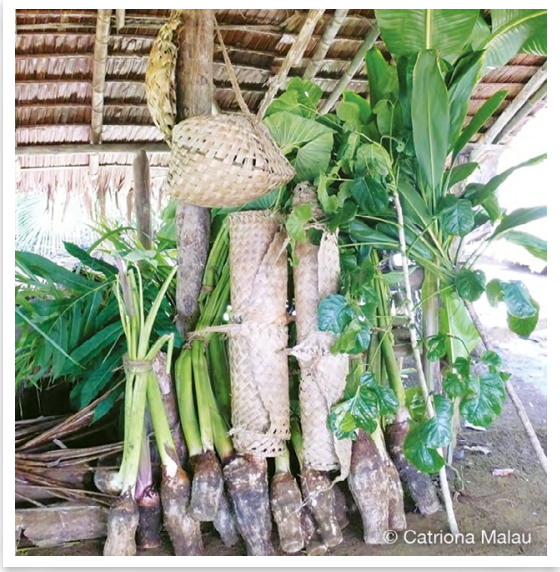

mökölagē wedding items möl n.com. orange; generic term for citrus fruit, but mostly referring to orange aranis Citrus spp. [POc ${ }^{*}$ molis 'citrus fruit or citrus-like fruit, perhaps Clymenia polyandra']

möldöl num. hundred, become a hundred handred, kasem handred $\mathrm{O}$ tiveg taē möldöl vagtöl. The price of it is three hundred. Praes blong hem $i$ tri handred. (VSB)

mööldöl vagōrō (comp.) num. two hundred $\sim$ tu handred

mööldöl vagtöl (comp.) num. three hundred tri handred

möölöslös $v$. weak slak Syn: mēlēslēs ${ }_{1}$, mörötröt

mönug n.com. joint, where two parts of skeleton are joined together $\sim$ joen blong bodi See: sö

mörögötö n.com. pallid triggerfish kaen strongskin (fis) Sufflamen bursa

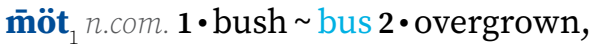
overgrown bushy area of land bus, eria we ol i no klinim gud O masawre ko o möt ren. This place is really overgrown. Ples ia i bus tumas. See: wömötmöt

mööt luwō (comp.) n.com. forest fores, bigfala bus

möt vandā̄eg (comp.) n.com. isolated location, interior of island medel bus

möt ${ }_{2}$ adv. quickly hariap, kwik Ale, qaqaq möt min no! OK, tell me quickly! Ale, talemaot hariap long mi! (EDM)

möt ${ }_{3} v i$. be broken, become broken (of something long and flexible, as rope) brok (wan samting olsem rop) $\mathrm{O}$ gōsōw ni n̄ōr mööt o ga den o diri matēk e. The rat chewed apart the rope from around the swamphen's legs. Rat ikakae brekem rop aot long leg blong nambiru ia. (WRN) [POc *ma-utus 'become, be broken off, severed']

musu adj. old olfala, wan samting (olsem naef, haos) we hem i olfala 
$-\mathbf{n}_{1}$ suff. third person singular possessive suffix, hers, his, its blong hem Na vōlōn ge elvet. Her hair is long. Hea blong hem i longfala.

-n $\mathbf{n}_{2}$ suff. construct suffix on directly possessed nouns, indicating nominal possessor maka long en blong noun we i talemaot se blong o dödömia na mögun i Hosea. Hosea's idea. Tingting blong Hosea.

na $_{1}$ art. the, a; article denoting noun that is in possessive construction wan, maka blong naon

$\mathbf{n a}_{2}$ part. first person singular default aspect marker $\sim \mathrm{mi}$

nagnagsamul $n$.com. kind of tree with flowers that are red, white and yellow wan kaen tri we flaoa blong hem $i$ gat ten flaoa insaed we ol $\mathrm{i}$ red, waet mo yelo \{Lit. ten faces ten fes\}

nak $v$. take notice, pay attention to $\sim$ tek not long, wari long Rōrō gō rōñteg, ba rōrō gata nak aē. The two of them could hear, but they didn't pay attention to it. Tufala iharem, be tufala ino tek notis long hem. (ESS)

nalumlum n.com. variety of taro, 'male', with black stem with stripes kaen taro, we hem i man, wetem blak stem we i gat straep long hem \{Lit. moss nalumlum\}

Namnog n.loc. place name $\sim$ nem blong ples

Namal n.loc. tribe name, this tribe originated from the tribe of Sêbeer nem blong traeb we i kamaot long Sēbèr

Nam̄asmas n.loc. place name $\sim$ nem blong ples

nan n.com. $\mathbf{1}$. pus $\sim$ doti wota blong soa o boela $\mathbf{2} \cdot$ rheum, gound, mucus discharge from eyes during sleep or from conjunctivitis doti wota blong ae o blong sik red ae nanar n.com. New Guinea rosewood (tree) nananara, bluwota Pterocarpus indicus [POc *naRa 'Pterocarpus indicus']

nar $1 \cdot n$.com. widow, widower $\sim$ wido, woman we man blong hem i ded, man we woman blong hem i ded $2 \cdot v$ give up one specific favourite food for 1,000 days out of respect after a close relative has died $\sim$ taem mama o papa i ded, ol pikinini ol i mas givap wan kaen kakae we papa o mama $\mathrm{i}$ bin fidim olgeta wetem taem ol i smol. Ol i no save kakae kasem wan taosen dei. Tu ol i mas givim smol mane long hem we i ded mo hangem rop (blong burao) long nek blong hem blong givim saen se hem i lego wan kakae See: ganar [POC *ñaro 'widower']

naw $n$.com. 1 • sea $\sim$ solwota Qēt kemem a van alo tabo suvsuv la naw. Then we went to the seaside to swim in the sea. Ale mifala $i$ go long solwota blong swim long solwota. (AAD) See: $\mathrm{lo}_{1} \mathbf{2} \cdot$ sea water, salty water $\sim$ solwota Nēk i le o naw, nēk mē bēsbēs qēt o ñe. You get some sea water, and you put it in with the (grated) canarium nuts. Yu karem solwota, yu kapsaedem i go wetem nangae. (JNN) $\mathbf{3} \cdot$ wave (of sea) $\sim$ wef (blong solwota) Nēk i luw o naw nivet. You count four waves. Yu kaontem fofala wef. (WRN) Rōrō gētē èl sur o bëtutu naw nitiwial ma van kal me, vus mi miat qēt ren rōrō. The two of them didn't notice that a big wave came ashore, and it killed the two of them. Tufala $i$ no luk se wan bigfala wefi kamso, i kilim tufala $i$ ded wantaem. (ATB) $4 \cdot$ salt $\sim$ sol [POC *napo(k) 'breaking wave; surf'] dōrdōrsenaw (comp.) n.com. tide mark mak we solwota i kam so, we yu harem noes blong solwota long ples ia 
naw gö ut (id.) rough sea solwota i raf

nawōn $a d v$. without purpose $\sim$ nating ne (abbrev. of ine)

-ne num. > num. ordinal suffix ending long namba we i talemaot oda blong namba, olsem namba tu, namba tri, etc No gē bēs kēl orōne. I'll thin it down a second time. Bae mi katem i tintin nambatu taem. (VVCMVDP22Nov0701-JR.004)

nem $_{1} v t$. lick likim Ni nem qal ko, ni rōñteg gē dēdērēs. He licked it, and he sensed that it tasted good. Hem $i$ likim kasem ia, i harem se i swit. (OQQ) nem $_{2}$ n.com. mosquito $~$ moskito [POC *namuk 'mosquito']

nem biliag (comp.) n.com. kind of mosquito that is black with grey spots wan kaen moskito we hem i blak wetem grei spot \{Lit. buff-banded rail mosquito nambilak moskito\}

nem talöqön̄ (comp.) n.com. kind of mosquito that is brown and comes out at night wan kaen moskito long naet we hem i braon

nen $v$. swell up a lot, as in a dead body or one's stomach if one has eaten too much solap bigwan olsem wan ded bodi o bel blong yu sapos yu bin kakae tumas

net $d i r$. towards addressee, in direction or facing towards person being spoken to $\sim \mathrm{i}$ go long man we yu toktok long hem [POc*(w)atu 'away, hence, 'motion away from speaker']

nē pron. he, she, it, him, her; third person singular pronoun $\sim$ hem

nēk pron. you; second person singular pronoun $\sim \mathrm{yu}$

nēn (fr.var. $\operatorname{nin}_{2}$ ) pron. we, us; first person plural inclusive pronoun yumi

nēr pron. they, them; third person plural pronoun $\sim$ olgeta nës vt. put into (container) fulumap insaed wan samting I nēn gë nës o tere gengen lo so? What shall we put the food in? Bae yumi fulumap ol kakae long wanem? Syn: sön̄ön

nët n.com. red silkwood, large tree, growing up to 30 metres with large edible green fruit naduledule Burckella obovata See: malnët [POc *ñatuq 'Burckella obovata']

ni (fr.var. nini) v.part. third person singular default aspect marker $\sim$ i

ni- num. numeral marker $\sim$ maka we i go long fored blong ol namba

nian $n . c o m$. shock $\sim$ sapraes

nimal $a d v$. very, really, too, too much, degree adverb tumas $\mathbf{O}$ naw ni dada nimal. The sea is too rough. Solwota $i$ mekem tumas. (WRN) Syn: idian

nin $v t$. split leaf or leaflet in two along midrib, split length of vine or cane into strips splitim lif, karemaot bun blong lif Kemem gē bēs o gawol, in̄ko kemem a nin wor vagōō le. We separate the inside layer from the outside of the lawyer cane, then we are able to split it into two. Mifala i karemaot insaed blong loeaken, nao ia mifala i save splitim tu taem. (VVKHVDP18Oct0801.056-7) [Used in reference to removing midrib from heliconia leaf used in food preparation or removing midrib from coconut leaf to divide it into two pieces to weave. Olsem tekemaot bun blong lif laplap o blong lif kokonas blong mekem tu pis blong wivim mat.]

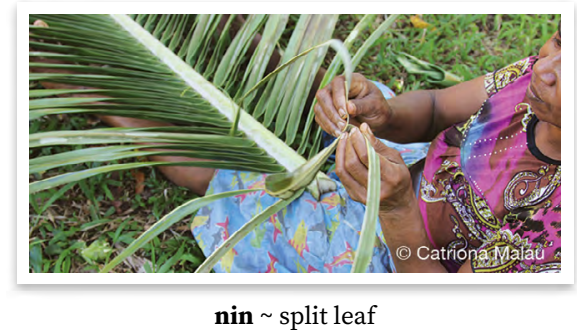




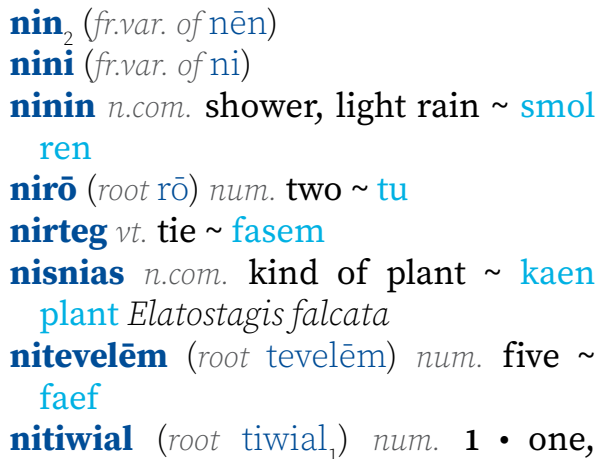
marker indicating a nonspecific referent, a wan Nē mē gis o mar ta lo to nitiwial. He made there be a famine for one year. Hem i holemtaet taem blong nogat kakae blong wan yia. (KM̄T) No gö mörös talo vet o waga nitiwial. I want to weave a prawn trap. Mi wantem wivim wan basket blong naora. (KBN.001) 2 • alone, just, one only from a set of those possible (following a pronoun) wan nomo aot long hamas we i stap No go representem nēn talö lölö vōnō ko, no nitiwial no go tog lo woksop. I will represent us from this village, I alone will be at the workshop. Bae mi representem yumi blong insaed long vilij ia, mi wan bae mi sidaon long woksop.

nitog v.part. prohibitive mood particle, dehortative marker (don't) no; wod we i go bifo long veb long sentens, taem yu talemaot long wan man se bae i no mekem wan samting

nitöl (root töl) num. three tri

nives (fr.var. of nivēs)

nivet $\left(\right.$ root vet $\left._{3}\right)$ num. four $\sim$ fo $\left[P O c^{*}\right.$ pat $[i]$ 'four']

nivēs (fr.var. nives) num. $\mathbf{1}$ how many $\sim$ hamas $\mathbf{2} \cdot$ few, several $\sim$ fiu, hamas nomo [POC *pican 'how many?']

no pron. I, me; first person singular pronoun $\sim \mathrm{mi}$

$\mathbf{n o}_{2}$ cl. relational classifier used to mark possession of items that are worn by the possessor, such as clothes $\sim$ blong (yusum blong tokbaot ona blong klos o wan samting we yumi werem) I Anita ni wöl na non o gogov bilēñ. Anita bought her clothes too. Anita $i$ pem klos blong hem tu. (AAD) non (n.poss) cl. her/his (clothes) klos blong hem

nök (n.poss) cl. my (clothes) klos blong $\mathrm{mi}$

nogon n.com.suff. her/his/its face $\sim$ fes blong hem See: nögö

non 1 n.com. blackfin barracuda (fish) $\sim$ sofis Sphyraena genie $[P O C$ * qonos 'mature Sphyraena spp., possibly generic for all barracuda']

non $_{2} \mathrm{cl}$. her/his (clothes) klos blong hem See: $\mathrm{no}_{2}$

nonor temp. yesterday $\sim$ yestedei [POc *na-ñoRap 'yesterday']

nonorēs temp. recent time period before yesterday bifo yestedei So i no ma van ti lo ofis nonorēs kēl la Made. I went to the office the other day on Monday. (Spoken 5 days later.) Mi bin pas long ofis bifo yestedei long Mande. (I talemaot 5 dei afta.)

nōmtuv $1 \cdot v$. believe $\sim$ biliv I rege le SDA nēr goto nōmtuv vita i Jisas nē mō tōrak kēl den o matē. Seventh Day Adventists don't believe that Jesus rose from the dead. OL SDA ol i no biliv se Jisas hem i girap long ded. $\mathbf{2}$-n.com. belief bilif $\mathrm{O}$ nōmtuv na mōgōr gètē timiak o nōmtuv na mögun nēn. Their beliefs are not like our beliefs. Bilifblong olgeta ino olsem bilif blong yumi.

nōn vi. (of fire) burn down to coals, ready for cooking go daon (faea) redi blong kuk Wo ni ēl o ev mō nōn, ga taōrmat tala bal. And he saw that the fire had died down, and it was ready to remove the hot stones. Mo hem i iuk se faea igo daon, i redi blong karemaot ston. (KMT)

nōnō̄n n.com. kind of small tree wan kaen smol tri Ficus adenosperma 
nōtōn n.p.bound her/his/its child pikinini blong hem See: nötu

nōvōk n.com. variety of taro, 'male', with tall brown stem kaen taro, we hem i man, we stem blong hem i longfala mo i braon \{Lit. Norfolk Island Nofok Aelan\}

nögö n.com.suff. $\mathbf{1}$ • face $\sim$ fes Na gösuv na nögök. I washed my face. Mi wasem fes blong mi. (AAD) $\mathbf{2}$ - front, at the front, before fran Nēk gē ēl vaga tok nirō lö nögö. You always see two dogs at the front. Yu luk oltaem tufala dog long fored. (DDP) [POc *nako- 'face, front']

nogon (n.poss) n.com.suff. her/his/its face $\sim$ fes blong hem

nögök (n.poss) n.com.suff. my face fes blong mi

nögö $1 \overline{0}$ n.com. east, place where sun rises ples we san i kam antap Syn: mete lō 1, rōwnölō

nögök n.com.suff. my face $~$ fes blong mi see: nögö

nök $\mathrm{cl}$. my (clothes) klos blong mi See: $\mathrm{no}_{2}$

nötu (fr.var. sönötu, sunötu) adj. $\mathbf{1}$ • small, be small, become small smol Alē in̄kē, la qaqaq na mögun kemem, o nötu gēnē in̄kē, kemem gō ōl nē vita nē o wamasē. So this one, in our language, this small shell is called a wam̃asé. Ale hemia, long lanwis blong mifala, smol selia, mifala i kolem se hem i wam̃asè. (VVKHVDP21Jul0702-
SW) Syn: atik 2 narrow, be narrow, become narrow smol, naro Rēvōrō ti a rēv, a da na bibian ni nötu. Weave two strands together, so that it will pull (the basket) in and make its neck narrower. Wivim tutu blong i pulum taet, $i$ mekem nek blong hem i smol. (VVCMVDP22Nov0702-KD)

nötu 2 n.p.bound child (of), offspring $\sim$ pikinini (blong hem) $\left[P O C *^{*}\right.$ natu 'child']

nōtōn (n.poss) n.p.bound her/his/its child pikinini blong hem

nötuk (n.poss) n.p.bound my child pikinini blong mi

nötu gövur n.com. toilet, out house smolhaos syn: kērēt 2 2, tes ${ }_{3}$

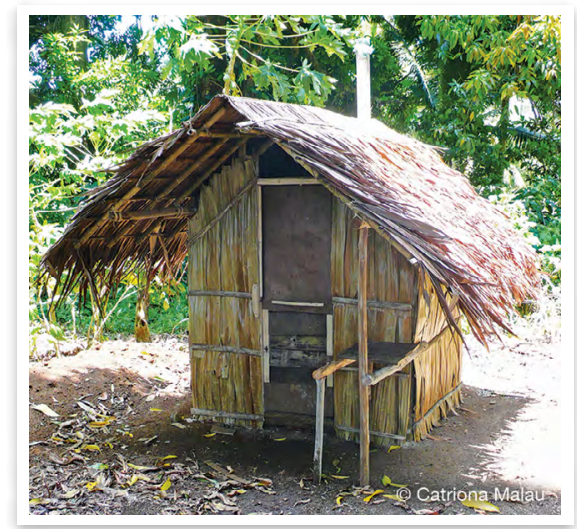

nötu gövur toilet house

nötuk n.p.bound my child pikinini blong mi See: nötu

\section{$\overline{\mathbf{N}} \sim \overline{\mathbf{n}}$}

- $\overline{\mathbf{n}}$ suff. second person singular possessive suffix, your blong yu Nēk wun tur masgiañ. If you stand alone. Sapos yu stanap yu wan. (CH1-200301-EKK.201)

naēs temp. when wataem $\overline{\mathbf{n}} \mathbf{l}_{1} v i$. tired, slack taed, slak No mē wiwiēg qön na tarbiēk ma nal aē. I worked until night and my body is tired. Mi bin wok tudak, bodi blong mi i taed long hem. Syn: iamron 
nal $2 v t$. eat only meat without the staple part of a meal kakae mit witaot kakae Nē ma ñal na gan o mes. He just ate his fish (without any main food). Hem i kakae fis blong hem nomo (ino kakae men kakae).

$\bar{n} \mathbf{n l}_{3} v t$. chew, as something chewy like toffee, bubble gum juim, kakae samting we i taf Nēk ta ñalñal o so? What are you chewing? Yu stap juim wanem?

nalte $n . c o m$. sap wota (blong wud)

n̄añ $v$. feed baby by chewing their food first to make it smooth, then feeding them fidim bebe, olsem yu juim kakae blong hem fastaem, afta fidim long hem taem i sofsosf $\mathbf{M a}$ ñañ o nötu mirmiar. She chewed the food then fed it to the little baby. Hem i juim kakae, afta ifidim long smol bebe. (DPD.061)

n̄añaēr $v$. happen $\sim$ hapen Oso ma nañaēr? What happened? Wanem i bin hapen?

nar $v$. want (only used in negative form, to indicate something that is not wanted) wantem (yusum blong talem nomo wan samting we yu no wantem)

n̄aran n.com.suff. her/his/its lips skin blong maot blong hem See: ñere

ne $n . c o m$. canarium nut, native almond $\sim$ nangae Canarium spp. [POc *[ka] yaRi 'canarium almond, Canarium indicum']

ne dawel (comp.) n.com. kind of wild native almond that grows in the bush and is not eaten. It has white bark and can grow tall like a whitewood wan kaen wael nangae blong bus we ol man ol i no kakae. Skin blong hem i waet mo hem i save gru longwan olsem waetwud

ne dun (comp.) n.com. kind of native almond that has flat nuts kaen nangae we i gat frut we i flat Canarium harveyi ne mereñ (comp.) n.com. dried, roasted canarium nuts $\sim$ drae nangae

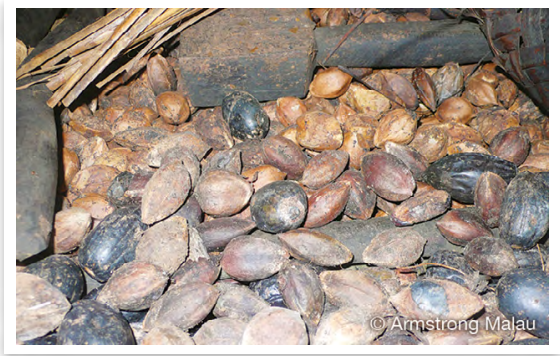

$\bar{n} \mathbf{e}$ mereñ $\sim$ dried canarium nuts

ne ta (comp.) n.com. kind of native almond that has round nuts kaen nangae we i gat frut we i raon Canarium indicum

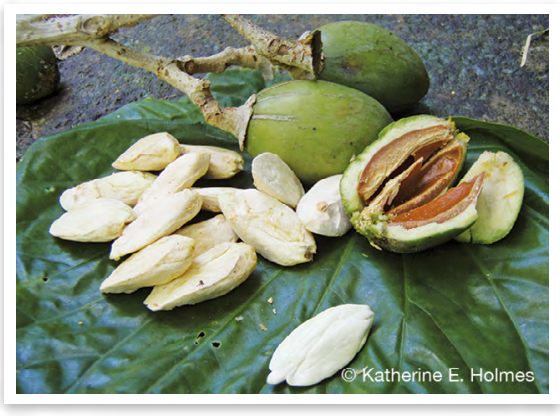

$\overline{\mathbf{n e}} \mathbf{t a ̄} \sim$ Canarium indicum

n̄eñere n.com. garden garen Lö lölö ñeñere ine wo o vetel, o qiat, o dëm, $o$ vël sele gengen ni dagdagese gëm döl lö lölö ñeñere gēn. Inside that garden there were bananas, taro, yams, every different kind of crop was there inside that garden. Insaed long garen ia we banana, taro, yam, everikaen kakae i mekem olsem wanem nomo insaed long garen ia. (KM̄MT)

nere $_{1} n$ edge $\sim$ ej blong wan samting nere $\mathbf{1} \cdot$ n.com.suff. lips $\sim$ lip, skin blong maot Kemem gètē wiak neren kemem ren aē. We haven't opened our lips about it at all. (i.e. We haven't spoken about it.) Mifala i no bin openem maot blong blong mifala 
nating long saed ia. (CAA) Syn: n̄usu $\mathbf{1}$ | 2 ・ n.com.suff. beak maot blong pijin Na naran ge gegen, ge gegen. Its beak is really sharp. Maot blong hem i sap we. (WRN) $\mathbf{3} \cdot$ n.com. point of land poen

naran (n.poss) n.com.suff. her/his/its lips skin blong maot blong hem n̄ërëk (n.poss) n.com.suff. my lips skin blong maot blong mi

nere ak n.com. variety of breadfruit kaen bredfrut

n̄ere ōn n.com. stretch of beach where the sand meets the sea $\sim$ ples we sanbij i kasem solwota

n̄ereg $v$ t. sell salem

Nereqe $n . l o c$. place name $\sim$ nem blong ples

N̄eretan $n$.loc. place name $\sim$ nem blong ples

n̄ēli (fr.var. of ñili)

n̄ēlida n.com. surface top (blong) nēlida naw surface of the sea top blong solwota (DHN.020)

n̄ēnēn $1 \cdot v$. (shoot) start to emerge from coconut gru i stat blong kamaot long kokonas 2 • n.com. newly emerging coconut shoot gru blong kokonas [Once a coconut has reached this stage in germination it is able to be planted. The stage of growth before sōl. Klosap i kam navara mo i redi blong planem.]

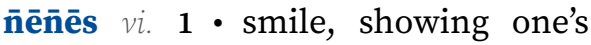
teeth smael, soem tut blong yu O mirmiar ni nēnēs. The child is smiling, showing her teeth. Pikinini hem $i$ smael. 2 - show teeth, bare teeth soemaot tut

n̄ërëk n.com.suff. my lips skin blong maot blong mi see: $\bar{n} e r e_{2}$

n̄ili (fr.var. n̄ēli) n.com. shoot, sprout, new growth of plant $\sim$ top blong wud, gro blong plant [POC * qili 'sprout, shoot (esp. of banana or taro)'] nili pomken (comp.) n.com. new growth of pumpkin plant, eaten as greens $\sim$ top blong pomken we hem i wan kabis

nili qiat (comp.) n.com. taro stems top blong taro In̄ko nēn a vas o ñili qiat. Now we plant the stem of the taro Nao ia yumi planem top blong taro (RPT.003)

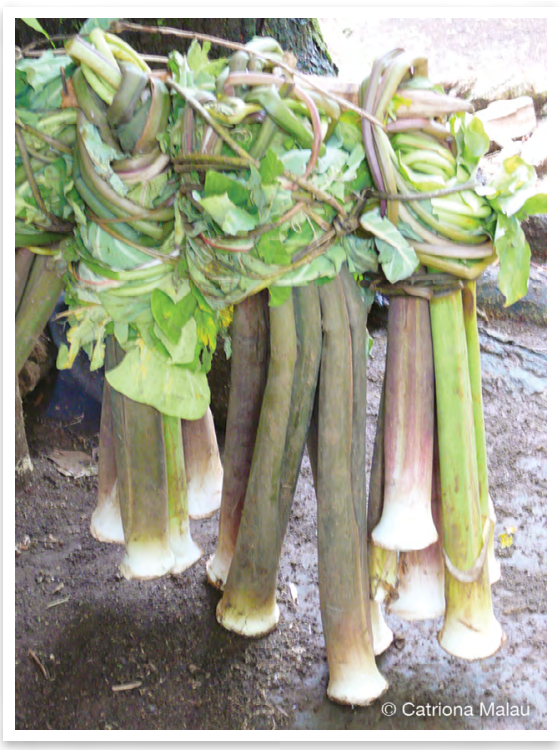

n̄ili qiāt $\sim$ taro stem

nilinaw $n . c o m$. wave $\sim$ wef $\{$ Lit. top of the sea top blong solwota\}

niria $v i$. fork (of road, tree) karem fok (rod, wud) O bē mē n̄iria. The river forks. Wota i karem fok. See: kasivi nōlseg $v$. chew with gums $\sim$ trae had blong kakae (olsem smolsmol pikinini o olfala we $i$ nogat tut) O mirmiar mō n̄ōlseg o masrē qiat. The baby is chewing the piece of taro with its gums. Pikinini i stap kakae pis taro.

n̄ōn̄ōr $v i$. snore pulum win, sno No gōtō mōvōt wareg lö qön̄ sur i birn̄ik mō n̄ōnōnōor idian. I didn't sleep well in the night because my partner was snoring too much. Mi no bin slip gud long naet from patna blong mi i pulum win tumas. [POc * yorok 'snore'] 
nōr $v$. gnaw, chew on something hard with teeth, as dog chewing bone kakae wetem tut (olsem rat i kakae o dog i kakae bun sloslo) $\mathbf{O}$ gōsōw mō n̄ôr lō o wo. The rat chewed through the bamboo. Ratikakae brekem bambu.

n̄ōsōn n.com.suff. her/his/its lips, beak maot blong hem See: n̄usu

$\overline{\mathbf{n o ̈ r}}$ n.com. snot wota blong nus $\mathbf{O}$ n̄ör lö möduk mē vērēg. Snot is running from my nose. Wota blong nus blong mi ron. [POC *yori 'channel above upper lip']

ñusu $1 \cdot$ •n.com.suff. lips, snout (of animal such as pig) lip, skin blong maot Na qötun nēr ge meneg, na nusun nēr ge elelvet. Their (pigs') heads are hard, and their snouts are very long.
Hed blong olgeta (pig) i strong, maot blong olgeta i longlongwan. (ESP) Syn: $\bar{n}^{2} e_{2} \mathbf{1} \mid \mathbf{2} \cdot$ n.com.suff. beak maot blong pijin $\mathbf{3} \cdot$ n.com. point of land poen Ni rēv kal sar o ak na kan taval nusu. He pulled ashore his canoe on the other side of the point. Hem i pulum kenu blong hem i kamso long narasaed poen. (WDR) [POc *yuju- 'external mouth, lips, snout, beak']

n̄ōsōn (n.poss) n.com.suff. her/his/its lips, beak maot blong hem

nusuk (n.poss) n.com.suff. my lips skin maot blong mi

nusuk n.com.suff. my lips skin maot blong mi See: n̄usu

\section{$\mathbf{0} \sim \mathbf{0}$}

o art. the, a, article denoting noun in class of common nouns wan, maka blong ol naon $\mathbf{O}$ atmēn mē ēl o reqe. The man saw the woman. Man ibin luk woman.

odian̄ (abbrev. dian̄) $\mathbf{1}$ • inter. no nogat $\mathbf{2}$ - pred. negative nonverbal predicate, be not $\sim$ nogat

oko (abbrev. $\mathrm{kO}_{2}$ ) dem. here, proximal location ples ia Bulsal, nēk go togtog oko. Friend, you are going to stay here. Fren, bae yu stap long ples ia.

(WRN) Na van me ko, no gö mörös legleg. I have come here, and I want to get married. Mi kam long ples ia, mi wantem mared. (ATB)

on n.com. kind of large octopus, can be taller than man, with a big head $\sim$ bigfala nawita we i gat bigfala hed O wērēt ine, nē gö luwō ren, nēr gō ōl vita nē o on. That octopus, it is really big, they call it an on. Nawita ia hem i bigwan tumas, ol i kolem se on. (WNW) [This octopus is white with red marks when cooked. Nawita ia $i$ waet mo i gat red mak long hem taem hem i dan.] oo inter. oh oo

or vi. play plei $\mathrm{O}$ tere mirmiar te ver or ti. The children were playing together. Ol pikinini $i$ stap pleplei tugeta. (OQQ)

oror (redup.) $\mathbf{1}$ ・ vi. play pleplei Ei, na këk o ak, o tan̄sar goto oror aē. Hey, my canoe, people don't play with it. Ei, kenu blong mi, ol man ino stap pleplei long hem. (WDR) 2 - n.com. game pleplei Qēt kemem a èl o taklē oror. Then we saw some games. Afta mifala i luk samfala pleplei. See: wooror

orès temp. time period in the near future, after tomorrow afta tumoro [POC *[i] waRisa 'two days from today'] oso $\left(\right.$ root $\left.\mathrm{SO}_{4}\right)$ n.com. what $\sim$ wanem Ei, nēk gö mörös nēk i da oso min no soko? Hey, what is it that you want to do with me? Ei, yu wantem mekem wanem long mi ia? (WDR) Bulsal, nēk to borbor sur oso? Friend, why are you laughing? Fren, yu stap laf from wanem? (WRN) 


\section{$\overline{\mathbf{0}} \sim \overline{\mathbf{0}}$}

$\overline{\mathbf{o}} \mathbf{l}_{1} v t . \mathbf{1} \cdot$ call, call out to $\sim$ singaotem $\mathbf{2}$ - call, give name to place or person singaotem (nem) O kakaka in̄kē me den lō vōnō siag e nēr gō ōl vita Böut. This story comes from a village round the coast that they call Böut. Storian ia $i$ kamaot long wan vilij antap ia we ol i kolem se Böut. (WNW) ōlōl (redup.) vi. shout, call out singaot

$\mathbf{o} \mathbf{l}_{2}$ n.com. maggot $\sim$ maget

ōligi $v t$. invite invaetem Nē mō ōligi o tere seleqō döl. He invited all of the animals. Hem i invaetem evri animol. (DFF)

$\overline{\mathbf{o}} \mathbf{l o} \mathbf{l}_{2}$ n.com. traditional secret prayer made at a sacred place to ask for something desired kastom prea long tabu ples blong askem wan samting

$\overline{\mathbf{o}} \mathbf{m}$ n.com. humpnose unicornfish kaen strongskin (fis) Naso tuberosus [POc *qume 'Naso unicornis, long-snouted unicornfish']

ōn $v i$. lie, lie down leidaon Nēk i ōn sur, nēk i mōvōt. You lie down, and you sleep. Yu leidaon, yu slip. (OSK) [POC *qeno 'lie down']

$\overline{\mathbf{o n}} \mathbf{n}_{2}$ n.com. $\mathbf{1} \cdot$ sand $\sim$ sanbij Nēn a tek na mögun nēn o wöwörös wo o ōn. We'll carry our small pebbles and sand (for making cement floor). Yumi karem smol ston mo sanbij blong yumi (blong mekem simen). (VSG) $\mathbf{2} \cdot$ beach sanbij Kōmōrōk a van sur i Sabine lō ōn a Bondi. The two of us went to see Sabine at the beach at Bondi. Mitufala $i$ go from Sabine long sanbij long Bondi. (AAD) [POc *qone 'sand, sandy beach'] ōn wöt (comp.) n.com. place where there is sand and surfable waves ples we i gat wef

ōnveg $v t$. lie beside, lie next to leidaon klosap long Nē ni ōnveg o ev. He lay next to the fire. Hem ileidaon klosap long faea. (DPD. 029) ōnvegtëm $v$. lay in the same house with the body of someone who has died after sunset, until the next day when the body can be removed in daylight sapos wan man hem i ded long sapa, ol i mas slip wetem hem kasem neks dei

$\overline{\mathbf{o}} \overline{\mathbf{o}}$ inter. no; interjection used as negative response to a question, or to indicate a negative attitude to a proposition nogat 'Ba nana mōl.' 'Ōōō, nēk i van me.' 'But I am going home.' 'No, you come.' 'Be mi mi go hom.' 'Nogat, yu kam.' (DSR) 'Ba kēmi gē gilal vita i Rēwilbam avē in̄ko?’ Nēr vita, 'Ōōō'. 'But do you know where Réwilbam is now?' They said, 'No.' 'Be yufala i save se Rèwilbam wea nao ia?' Ol i se, 'Nogat.' (JRQ)

$\overline{\mathbf{o r}} \mathbf{r}_{1}$ n.com. $\mathbf{1}$ - freshwater prawn $\sim$ naora Macrobrachium sp. Kemem a van vita a tut ōr lē lēk. We went to catch prawns at night in the lake. Mifala $i$ go blong faenem naora long naet long lek. (AAD) $\mathbf{2}$ - lobster naora blong solwota Panulirus spp.

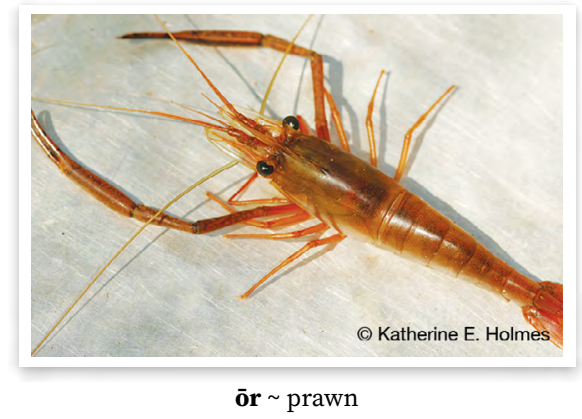

ōr bëlbël (comp.) n.com. kind of freshwater prawn, medium-sized that does not grow large, whitish with spots wan kaen naora we hem i medel saes mo i no save gru bigwan. Hem i dak waet wetem ol spot long hem 
or taban (comp.) n.com. painted rock lobster wan kaen naora blong solwota Panulirus versicolor

$\overline{\mathbf{o}} \mathbf{r}_{2}$ vi. low tide, tide go out $\sim$ solwota i drae, stap drae Rōrō ma van lo vovon̄on, met mō ōr. The two of them went looking for shellfish, the tide was low. Tufala i go lukaotem mit, solwota idrae.

Ōrbarbar n.loc. Ureparapara, island to the northwest of Vanua Lava Ureparapara

örbarbar n.com. variety of taro, 'female' $\sim$ kaen taro we hem i woman

ōsōn n.com.suff. her/his/its life laef blong hem See: ès
Ōt n.loc. name of a place in hinterland, where taro is planted $\sim$ nem blong wan ples antap we ol i planem taro long hem $[P O c$ *qutan 'bushland, hinterland']

ov n.com. canoe tree $\sim$ kenutri Gyrocarpus americanus $[\mathrm{POC}$ *qope 'Gyrocarpus americanus' PNCV *(q)ove 'Gyrocarpus americanus']

$\overline{\mathbf{o}} \mathbf{w}_{1}$ n.com. turtle $\sim$ totel

$\overline{\mathbf{o w}}_{2}$ vi. bear fruit $~$ karem kakae $\mathbf{0}$ miak lö kulë gëvrun Rēwilbam nē mō ōw. The Tahitian chestnut behind Réwilbam's house was bearing fruit. Namambe bihaen long haos blong Rēwilbam i karem kakae. (JRQ)

\section{$\mathbf{P} \sim \mathbf{p}$}

pawa n.com. variety of sweet potato that has green vine $\sim$ kaen kumala we rop blong hem i grin

Periōt n.loc. place name $\sim$ nem blong ples

pispol n.com. variety of breadfruit that has large fruit kaen bredfrut we kakae blong hem i bigwan plantesen n.com. plantation plantesen [Bis plantesen 'plantation'] plen n.com. plane plen [Bis plen 'plane'] pomken n.com. pumpkin pamken [Bis pamken 'pumpkin']

Pōpō n.p.fr. Grandma, Grandpa Bubu See: Diebum, Mambum [Bis Bubu 'Grandma, Grandpa']

\section{$\mathbf{Q} \sim \mathbf{q}$}

qaēl inter. hey! awo!

qaēn n.com. kind of small freshwater fish. Lays its eggs in the sea in a nest like a spider's nest. When the eggs hatch, they move upstream. They have suckers so they can cling to the rocks to move upstream. A popular eating fish. wan kaen smolsmol fis blong wota we hem i gud blong kakae. Hem i putum eg long solwota wan taem long wan yia mo basket blong eg i olsem blong spaeda. Taem ol pikinini i kamaot ol i klaem folem wota $\mathrm{i}$ go antap. $\mathrm{Ol} \mathrm{i}$ gat samting olsem nawita blong ol i save fas long ston blong klaem i go antap long wota

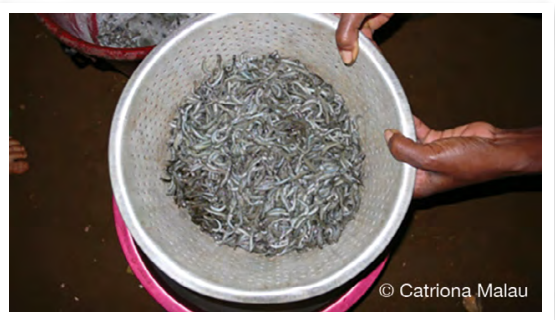

qaēn kind of fish 
qag $\mathbf{1} \cdot v i$. white $\sim$ waet $\mathbf{2} \cdot$ n.com. whiteness, white (one) waetwan 3 vi. light (in colour), pale $\sim$ laet kala Van tek min rege ga qag. Take it to the white people. Karem i go long ol waetman. (VVKHVDP21Jul0701MC.020)

qagar n.com. garfish wan kaen fis Family Hemiramphidae [POc * mwakoR 'Hemiramphus, garfish']

Qakē n.loc. 1 - place name nem blong wan ples $\mathbf{2} \cdot$ tribe name, came from the island of Mota and named because they landed at Qakē nem blong traeb blong ol man we i kamaot long Mota mo i kamso long Qakē

qal 1 • vt. affect, attain, reach $\sim$ kasem O mamēgin ma qal no. The cold got to me. Kolkol i kasem mi. 2 - $v$. be first kam faswan Nē ma qal vösus. She gave birth first. Hem i bonem fastaem. Nē ma qal vatvat min nēk. He made the arrangement with you first. Hem i promesem long yu fastaem.

Qalēg n.p.fr. reciprocal term used to address parents of spouse or spouse of child, father-in-law, mother-inlaw, daughter-in-law, son-in-law palika

qalēg gōr $v$. make an agreement between the parents that a particular girl or woman will be married to a man blokem, putum tabu long wan gel mo givim mane se bae tufala i mared

qalmiat n.com. variety of breadfruit kaen bredfrut

qan̄ris (fr.var. qarn̄is) $\mathbf{1} \cdot$ n.com. method of baking in stone oven $\sim$ fasin blong bek long oven See: um, vësëw $21 \mid \mathbf{2}$ - $v$. bake food in stone oven $\sim$ bek, bekem kakae long oven Tōlōw kōmōrōñ ri qan̄ris na gëk o gengen, ri vus na gëk o qō. Tomorrow you two bake my food, and kill a pig for me to eat. Tumoro yutufala i bekem kakae blong mi, yufala i kilim pig blong mi. (WDR) [This is the everyday way of baking, in contrast to baking in an oven called vësëw. Hem i oven blong evri dei yus be i gat wan kaen spesol oven tu, vësëw.]

qaq $\mathbf{1} \cdot v t$. say talem se Ni èl nēe, ni qaq vita, 'Bulsal, van me.' He saw him and he said, 'Friend, come'. Hem i iuk hem, italem se, 'Fren, kam.' (ATB) 2 • vi. speak, talk toktok Nēk i ēl o tamarge nitiwial, nēk i qaq min nē. When you see an old man, speak to him. Taem yu luk wan olfala, yu toktok long hem. 3 - n.com. language of a place lanwis $\mathbf{O}$ qaq ta Iñlan nēr a ōl ta histri. In English they call it history. Long Ingglis ol i kolem se histri. (EKK) qaq ta minēn (id.) n.com. language of Vurës lanwis blong Vurës \{Lit. language from us lanwis blong yumi\} qaq tala lam (id.) n.com. Bislama Bislama \{Lit. language from the deep sea lanwis blong dip si\}

qaq totono n.com. advice advaes \{Lit. speak teach tok lanem\}

qaqa 1 - n.com. silly, ridiculous behaviour, ridiculous behaving person krangke, krangke fasin 2 $n$.com. crazy person, behave in a ridiculous way $\sim$ mekem ol samting we i no stret olsem i hafmad; man we i krangke $\mathbf{O}$ qaqa gētē gilal oso nē ta qaqaq. A crazy person doesn't know what they are saying. Krangke man hem i no save wanem hem i stap talem. $\mathbf{3} \cdot \mathrm{mod}$. stupidly, not properly, randomly olbaot, long krangke fasin Mē rēv qaqaqa. She didn't write properly. Hem i raetem olbaot.

qaqet n.com. kind of small freshwater prawn $\sim$ wan kaen smolsmol naora qar vi. $\mathbf{1}$ - unripe $\sim$ no raep $\mathbf{O}$ vetel ga qar si gō bōl nē o gengen. Banana that is unripe or under ripe is a staple food. Banana we ino raep o no raep gud hem $i$ wan kakae. 2 - raw, uncooked 
no dan La masawre nē ga qar, nē gö körkör. When it is not cooked, it is black. Taem ino dan, hem i blak. (VVKHVDP01Aug0701-IK) 3 • undried, untreated, as of green leaves, rather than dried or heat treated leaves $\sim$ no drae, no dan, olsem lif we i grin nomo, we ol i no putum long faea blong mekem i strong. Si ilēkē nana vet nē, nē ga qar, gētē rē nē, a da ine, a vet nē, o dōmōtō tabaē gē mēsērsēr. If I weave that one, when it is undried and hasn't been heat treated, then I do it, weave it, and the coconut leaves will tear. Sapos hemia mi wivim, hem we i no dan, we i no bin putum long faea, i mekem ia, i wivim, bae lif kokonas blong hem bae i brokbrok. (VVCMVDP22Nov0701-MC)

qaran n.com.suff. his/its penis kok blong hem See: qere ${ }_{2}$

qarān n.com. hole, hole for grave hol, hol blong gref See: qarörö

qarn̄is (fr.var. of qan̄ris)

qarörö n.com. hole hol See: qaran̄

qas n.com. be bald, bald person bolhed Qaslaklakatēg na wēn̄ēn tö tur ti ko, na qōtōn o qas. Qaslaklakatēg had a beard, and his head was bald. Qaslaklakatèg, mustas blong hem i stanap, hem ibolhed. (OQQ) [POC* * $\left.p^{w} a\right)$ $p^{w}$ ata 'bald']

qate $\overline{\mathbf{e}}_{1}$ n.com. tree fern blakpam Cyathea spp. [Comparing dèmèrmèr and qatè, dèmérmèr does not grow as tall; the hands grow closer together; the leaves start further from the base; and the trunk is harder to cut and not soft inside. qate is used as a grater for tubers, but dēmērmèr is not. I gat tu kaen blakpam mo qatē i no bigwan olsem dēmèrmèr. Qatē nao ol i yusum blong rasras ol kakae.]

daqatē (der.) n.com. leaf of tree fern, eaten as greens $\sim$ lif blong blakpam, blong kakae olsem wan kabis qate $\overline{\mathbf{e}}_{2}$ n.com. arrow $\sim$ aro See: vus ${ }_{1}$ qatē $_{3}$ inter. peekaboo! Interjection used in playing game of surprise with small children

qeleg vi. plait together two strips of coconut leaflets or pandanus leaves as first stage in making basket or mat joenem tugeta tu saed blong lif kokonas o pandanas blong wivim (JWM)
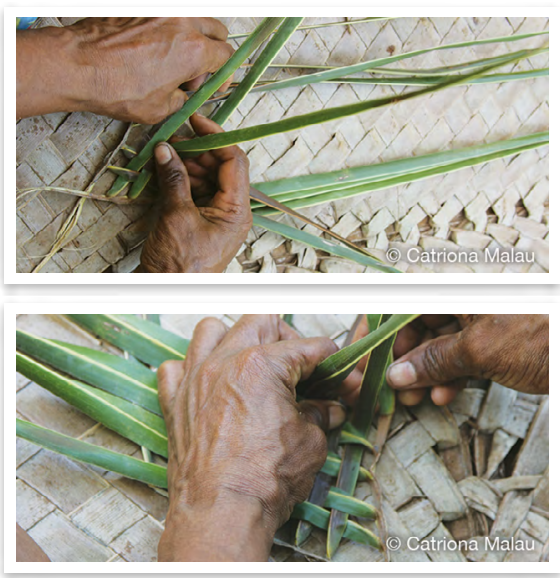

qeleg weaving term

qelgan n.p.bound her/his parent-in-law palika blong hem See: qelge

qelge n.com.suff. reciprocal term used to refer to parents of spouse or spouse of child, father-in-law, mother-in-law, daughter-in-law, son-in-law palika, papa o mama blong man o woman blong yu, man o woman blong pikinini blong yu

qelgan (n.poss) n.p.bound her/his parent-in-law palika blong hem qëlgëk (n.poss) n.p.bound my parentin-law palika blong mi

qeneg $\mathbf{1} \cdot v i$. afraid, frightened $\sim$ fraet 2 - vt. fear, be afraid of $\sim$ fraet long I rege ta Ambrym me qeneg no timiak no o timiat e. The people from Ambrym were afraid of me as if I were a devil. Ol man Ambrym ol $i$ fraet long mi olsem we mi mi devel. (VVCMAA03Apr1306.019) Syn: metegrōw, mëtëwtëw 
qere $_{1} n$ petiole $\sim$ stamba

qere ${ }_{2}$ n.com.suff. penis $\sim$ kok

qaran (n.poss) n.com.suff. his/its penis kok blong hem

qërëk (n.poss) n.com.suff. my penis kok blong mi

qereg $v$. flatten $\sim$ mekem i flat [This word is used specifically for the process of making löt flat. Toktok ia ol i speseli yusum blong talem se ol i mekem nalot i flat.]

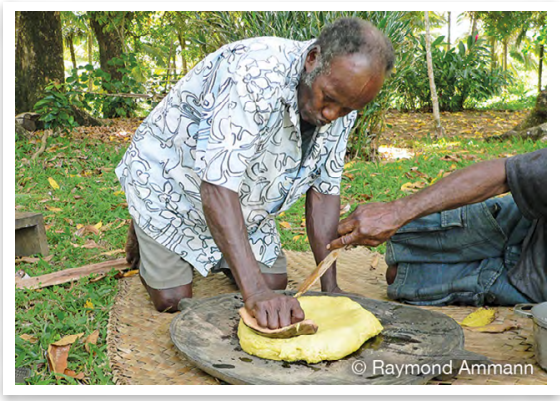

qereg flatten $l o ̈ t$

qereñ bē n.com. watercourse, creek gully, place where there is a creek, including creek and edge around it hol blong wota Ni tēqēl le qereñ bē, ni ut o bē. He went down to the creek, and collected some water. Hem $i$ go daon long wota, i kasem wota.

qeren̄ ōlōl n.com. cave, hole in reef or pool that has special significance, where people go to pray, give money and ask the spirits for something that they want, or to appease and ask forgiveness from the spirits if they have done something wrong $~$ tabu hol long kef, long rif o long wota we ol man ol i go long hem blong prea long ol spirit from wan samting o talem sori from wan samting we yu bin mekem

qese n.com.suff. top (of something tall, as person's head or hill) top (blong wan samting we i tolfala, olsem man o hil) qese tōw (comp.) n.com. top, peak of hill top blong hil Ni gav dēñ ren siag e, le qese tōw gere qon. It flew right up there, to the top of the hill of the pigeon. Hem iflae kasem antap olgeta, long top blong hil blong nawimba. (DHN.027)

qesekēr n.com. club, length of wood used as weapon $\sim$ nalnal

Qet n.p.fr. name of mythical creation figure, God God blong kastom

qet mëmës n.com. headache, have headache $\sim$ hed i soa

qet möwu vi. nod head, show agreement $\sim$ sakem hed se i oraet $\mathbf{N e}$ me qet möwu. She nodded her head. Hem i sekem hed blong hem blong talem se yes.

qetegak n.com. captain, leader kapten, lida

qetge n.com. kind of tree wan kaen tri [Popular for use as firewood as it gives strong heat. Taem i drae $i$ mekem gudfala strong faea.]

qetgengen n.com. feast, planned celebratory feast $\sim$ lafet, wan kakae we yumi ekspektem blong holem, plan blong mekem kakae

qetmëv n.com. surge wrasse (fish) wan kaen fis Thalassoma purpureum

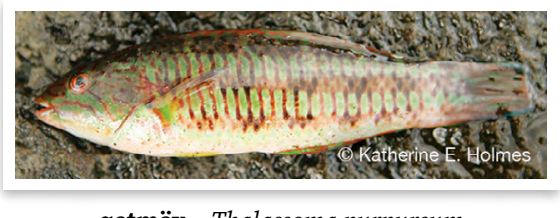

qetōlō̄n n.com. pillow pilo [POc *quluךan 'rest the head on; wooden headrest'] qetsōs n.com. kind of insect like a termite but larger wan kaen bebet we hem i olsem waetanis be i bigwan lelebet [They die after they have infested the wood. They are eaten by chickens. Taem hem i kakae wud hem i mas ded. Hem i kakae blong faol.] 
qetvet n.com. stubborn, obstinate stronghed \{Lit. stone head ston hed\} qev n.com. humpback snapper (fish) kaen pulefis Lutjanus gibbus

qēdëm n.com. yam platform, for drying yams after harvest $\sim$ bed blong yam qēel n.com. taro paddy; garden for planting taro that is sometimes irrigated and sometimes not during the period of growth. The taro are planted in dry ground, then irrigated, then drained for weeding, then irrigated again kaen garen taro we ol i planem long drae graon, afta ol i putum wota, afta lego wota long taem blong widim See: mat qēlaqēl ( $p l$. of qēel)

qēqēl n.com. mangrove natongtong Rhizophora spp.

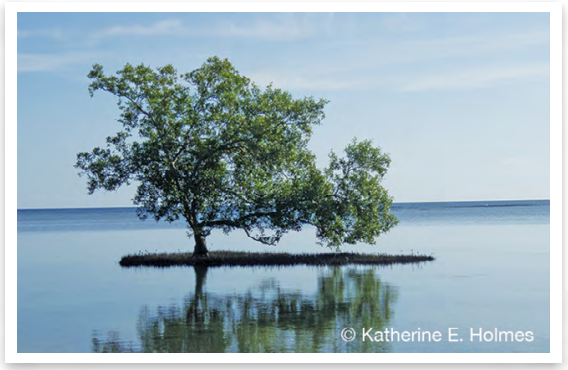

qēqēe $~ R h i z o p h o r a$ spp.

$\mathbf{q} \overline{\mathbf{e}} \mathbf{r}_{1}$ n.com. kind of tree $\sim$ wan kaen tri Osmoxylon orientale

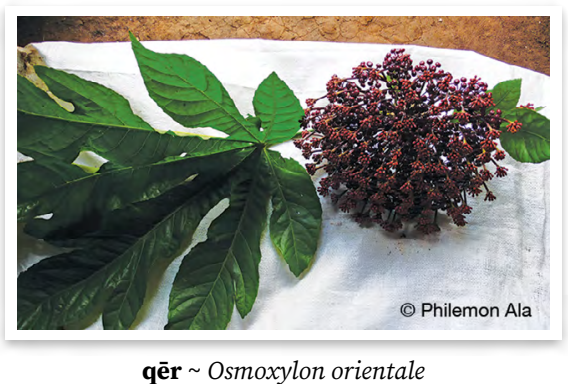

$\mathbf{q e} \mathbf{r}_{2} v$. bend over (of tree) $\sim$ wud $\mathrm{i}$ benben $\mathbf{O}$ vetel mē qēr tek na wian. The banana tree bent so much that it broke because of its fruit. Banana i ben ibrok wetem ol frut blong hem.

qērēt $v t$. push, push in button, push something down, press pusum $O$ reqe ni qērēt sōw o wērèt, o wērèt ni rèv tēqēl nē sōw. The woman pushed the octopus down, and the octopus pulled her down. Woman i pusum nawita $i$ go daon, nawita $i$ pulum woman i go daon. (WNW)

qēs $v$. bang against something bangem $\mathbf{O}$ mōtō mē qēs wor. The coconut fell and broke open. Kokonas $i$ foldaon ibrok. Fiona tē qēsqēs o mōtō le vet. Fiona is banging a coconut on a rock. Fiona i stap bangem kokonas long ston.

qēs mōnmōn $\mathbf{1} \cdot v i$. smash smas $\mathrm{O}$ wötöv më mës mē qēs mōnmōn le vet. The bottle fell and smashed on a rock. Botel $i$ foldaon $i$ smas long ston. 2 - vt. smash smasem Nē mē qēs mōnmōn o wötöv. He smashed a bottle. Hem i smasem botel.

qēsis n.com. sore, similar to a boil, caused by a small scratch which then becomes infected, swells up and fills with pus kaen soa we hem $i$ gat doti wota insaed olsem boela

qēsqēs $v$. fishing technique where one pounds hermit crabs so that they smell and then throws them in the sea to attract fish wan fasin blong kasem fis we yu kilim hed blong nakato blong mekem se i smel, mo afta yu sakem long solwota blong atraktem fis

qēt 1 - $v i$. finish, be finished, end, cease $\sim$ finis $\mathbf{O}$ ilsi kakaka, nē mē qēt gem gèn. The end of the story, it is just finished there. En blong storian, hem i finis nomo long ples ia. 2 vt. finish, complete, stop, cease finisim Tōrak ti, mē qēt o wiwieg, 
rōn̄teg ti. Get up, stop working and listen a minute. Girap, finisim wok, lesen fastaem. (ESS) $\mathbf{3} \cdot v$. discontinue, cease to engage in, minor verb in serial verb construction, indicating discontinuative aspect in relation to the following verb nomo mekem, finis No mè qēt sumsum o gè. I'm finished with drinking kava. Mi nomo stap dring kava. 4 - mod. completed action, indicates that the action described by the verb that it follows is completed, before moving on to another action finis Rōrō me gengen qēt, e rōrō a van. The two of them finished eating, then they went. Tufala $i$ kakae finis, ale tufala $i$ go. (ATB) La masawre no me vetvet qēt, no me vet qēt tavalgi, in̄ko na na vir. When I have finished weaving, I have finished weaving one side, then I plait. Taem mi wivim finis, mi wivim finisim wansaed, nao ia mi tanem. (JWM)

qèt ${ }_{2}$ conj. then, clause initial consecutive marker finis, afta Rōrō mē èlgōr nē van van ni kalkal. Qèt ni vanvan. Qèt i rētnan ni tel kēl. The two of them looked after him until he crawled. Then he walked. Then his mother got pregnant again. Tufala i iukaotem hem go go hem i wokbaot fo leg. Finis hem $i$ wokbaot. Finis mama blong hem igatbel bakegen. (ATB.005-7) qēt ${ }_{3} \mathbf{1} \cdot$ mod. all, every, postmodifier of a noun or pronoun, indicating that all referents are affected or that the referents are completely affected evriwan Na meten nēr ni mëës tēqēl, nēr qēt ren ga sasarēt. Their eyes fell down, absolutely all of them were the same. Ol ae blong olgeta $i$ foldaon, olgeta evriwan i semaknomo. (JTF) Nēr qēt, i rege mirmiar qēt mo wotwot vilēs a Vurës. All of them, all of the children were all born at Vurës. Olgeta evriwan, olgeta pikinini evriwan ol i bon evriwan long Vurës. (NRL) $\mathbf{2} \cdot v$. all, completely affected, minor verb following major verb in serial verb construction, indicating that the participants who engage in the event are all affected or are completely affected by the action evriwan Nēr a van qēt lo rot. They have all gone to the taro garden. Olgeta evriwan ol $i$ go long garen taro. $\mathbf{O}$ wedeldel ma van waliög qēt o gövur. The red ginger plants went completely around the house. Ol red flaoa ol $i$ raonem haos evriwan. (LLK)

qētēg $v . \mathbf{1} \cdot$ start $\sim$ stat, statem $\mathbf{R a}$ tubun kemem mē qētēg o sēsēdig. Our ancestors started the way of trapping birds. Ol bubu blong mifala ol $i$ statem rod blong trapem pijin. (SBB.004) $\mathbf{2}$ - inceptive, first verb in serial verb construction, indicating the inception of the action of the verb that follows statem Nēr mē qētēg mian. They started to ripen. Oli stat blong raep. (SBB)

qētēgi (fr.var. qētigi, qitēgi, qitigi) n.com. $\mathbf{1} \cdot$ trunk (of tree), body of tree stamba Ni vērēg dēñ me lē qētēgi miak, nē ni rem kal. He ran up to the Tahitian chestnut tree, and he climbed up. Hem i resis kasem stamba blong namambe, hem $i$ klaem $i$ go antap. $\mathbf{2}$ - basis, root of something $\sim$ stamba qētēt n.com. termite, white ant waetanis

qētigi (fr.var. of qētēgi)

qētqētne num. last laswan Syn: basbasne

qël $v$. grow and spread out (of vine or yam) rop i gru mo spred aot $\mathbf{O}$ dem gë qël kal lē rēntenge. Yams spread and climb up trees. Yam ol i gru antap long wud.

qëlgëk n.p.bound my parent-in-law palika blong mi see: qelge 
qër n.com. grave, graveyard $\sim$ gref, ples blong gref

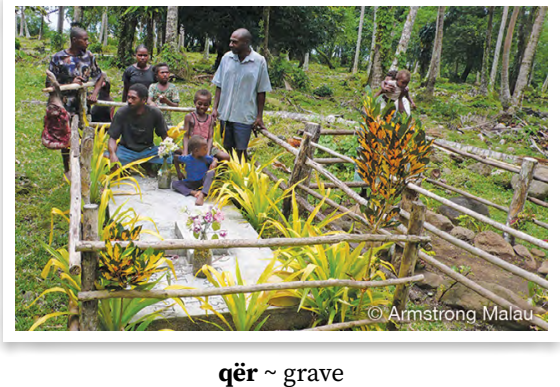

qërëk n.com.suff. my penis kok blong mi See: qere

qërët n.com. flying fox $\sim$ flaengfokis In̄ko, qërët nē to tog gëm ti lē vētitne, timiak vita in̄ko wo, nē gete bemrin o men, nē gete bemriñ o seleqō. Now the flying fox just stays in the middle, like now it is not with the birds, and it is not with the animals. Nao ia flaengfokis i stap nomo long medel, olsem se nao ia hem i no go wetem pijin, hem i no go wetem animol. (DFF)

qëtutu (fr.var. sëqëtutu) $\mathbf{1}$ •adj. short, be short sotfala No gö lölö gagneg o qëtutu kakaka. I want to tell a short story. Mi wantem talem wan sotfala storian. (TSR) Ant: elvet $\mathbf{2} \cdot v i$. be short, become short sotfala, kam sotwan Ba la masawre nēk mē ēl na mōgōñ o ga më qëtutu, nēk gö sö kēl le na mōgōn o ga. But when you see that your rope has become too short, you can add more onto your rope. Be taem yu luk se rop blong yu hem i kam sotfala, yu save joenem rop blong yu bakegen. (VVKHVDP180ct0801.141)

qëtutu masawre (comp.) n.com. moment, short time sot taem Nēr mo tog o qëtutu masawre gem. They just stayed for a short time. Ol i stap sot taem nomo.

qiag vi. run dry (of water source), dry out ron drae $\mathbf{O}$ bē mi qiag la paep. The water in the pipe has run dry. Wota long paep i ron drae. qias $v t$. spread apart something like hair, dense bush or grass, typically in order to look for lice or other small things openem hea blong lukaotem laos, lukaotem wan smol samting insaed long gras o bus Nër o qias wut. They are looking for lice.

Ol i stap lukaotem laos. See: wial

qiat n.com. taro $\sim$ taro Araceae spp.

diqiat (der.) n.com. taro leaf, cooked and eaten as greens $\sim$ lif blong taro qiat atm̄ēn (comp.) n.com. taro grouping, varieties of taro that are 'male' and don't fork grup blong ol taro, man taro we hem i no save karem fok \{Lit. male taro man taro\} qiat gōll (comp.) n.com. variety of marē taro, 'male', with light green stem and white corm kaen marē taro we hem i man mo i laet bitim evri taro. Hem i save bigwan smol mo hem i save longfala. Han blong hem i laet grin mo kakae i waet

qiat lēlē (comp.) n.com. variety of taro, 'female', that is considered to be the king of taros as it grows very tall, has a light green stem and white corm kaen taro we han blong hem i laet grin mo kakae i waet. Hem i save bigwan mo longlongfala, hem i king taro Syn: qiat minwog

qiat mingala (comp.) n.com. variety of taro, 'female', with light green stem kaen taro we hem i woman, we han blong hem i laet grin Syn: ten̄tur qiat minwog (comp.) n.com. variety of taro, 'female', that is considered to be the king of taros as it grows very tall, has a light green stem and white corm $\sim$ kaen taro we hem $\mathrm{i}$ woman, han blong hem i laet grin mo kakae i waet. Hem i save bigwan mo longlongfala, hem i king taro Syn: qiat lēlē 
qiat qet (comp.) n.com. variety of taro, 'female', with black stem and red corm kaen taro we hem i woman, han blong hem i blak mo kakae blong hem i red syn: qiat qön

qiat qȫn (comp.) n.com. variety of taro, 'female', with black stem and red corm kaen taro we hem i woman, han blong hem i blak mo kakae blong hem i red syn: qiat qet

qiat reqe (comp.) n.com. taro grouping, varieties of taro which can fork grup blong ol woman taro we ol i save karem fok \{Lit. female taro woman taro\}

qiat rev (comp.) n.com. variety of taro, a grouping that includes 'male' and 'female' varieties, all have many runners, there is variation in colour and shape grup taro we i gat plante kaen long hem, we sam i man, sam i woman, i gat defren kala mo sep be evriwan $i$ gat fulap saka \{Lit. pulling taro taro we i pul\}

qil $v$. order, tell someone that they must do something talem long wan man se i mas mekem wan samting See: tē

qilewer n.com. spinster, bachelor, unmarried man or woman man o woman we i nogat woman o man blong hem

qiliañ vi. $\mathbf{1}$ • be lost, go missing lus O ki ni qiliañ qēt, wo ni wöl. The key was lost and then he bought (it). Ki $i$ lus finis, mo hem i pem. I Mam ma da qiliañ o ki. Dad lost the key. Papa i lusum ki. 2 - die, pass away ded, lus long Nē mē qiliañ den kemem. He died leaving us. Hem i lus long mifala.

qin̄imatē n.com. measurement of days since someone's death $\sim$ dei blong ded we yu kaontem kasem wan taosen dei qiriag vt. confuse $\sim$ konfiusem o qaqaq nirō mē qiriag nē. The two languages confused her. Tufala lanwis i konfiusem hem.

qirir vt. grind kava or local plant medicines with a rough piece of dead coral graonem kava o lif meresin wetem pis korel Syn: qisis

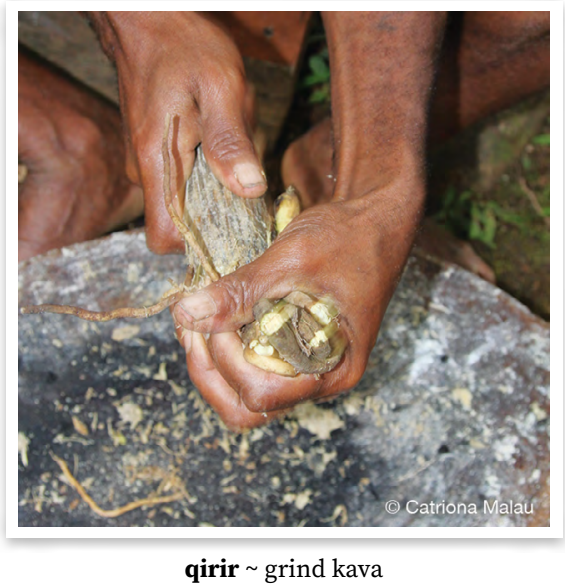

qisis vt. grind kava or local plant medicines with a rough piece of dead coral graonem kava o lif meresin wetem pis korel Syn: qirir qit (root of aqit)

qitēgi (fr.var. of qētēgi)

qitigi (fr.var. of qētēgi)

qog n.com. kind of tree $\sim$ wan kaen tri Neuburgia corynocarpa

qon n.com. pigeon (bird) nawimba (pijin) Ducula spp.

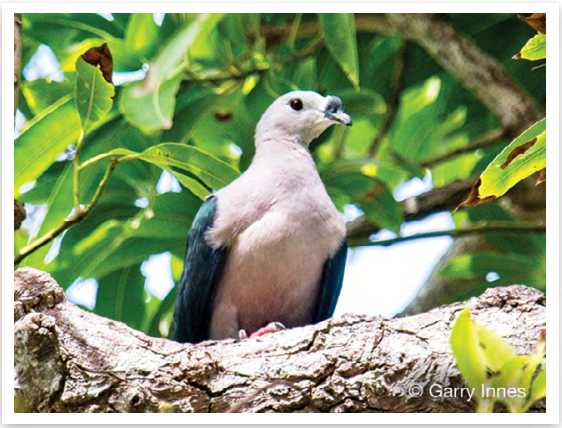

qon $\sim$ Ducula pacifica 
QOROGROG

qon bulënqet (comp.) n.com. kind of pigeon that has a ring on its leg kaen nawimba we i gat ring long leg blong hem \{Lit. Qet's pigeon nawimba blong Qet\}

qon bulënrēlē (comp.) n.com. kind of pigeon that has a lump on its beak (male) kaen nawimba wetem lam long maot blong hem (man) \{Lit. Rēlē's pigeon nawimba blong Rèlê\} qon tan̄sar (comp.) n.com. kind of pigeon that has a straight beak (female) kaen nawimba we i gat stret maot (woman) \{Lit. person pigeon nawimba man\}

qon wēbèt (comp.) n.com. kind of pigeon that is white $\sim$ waet nawimba \{Lit. white pigeon waet nawimba\}

qorogrog vi. have tiny lumps, spots, as on baby's skin gat spot long bodi (blong bebe) Na tarbian mo qorogrog. Her body is spotty. Hem i gat ol spot long bodi blong hem.

qoron n.com.suff. her/his/its ear sora blong hem See: qörö

qosmötöl $v$. blunt no sap

qoson n.com.suff. her/his groin $\sim$ tabu eria blong hem See: qösö

$\mathbf{q} \overline{\mathbf{o}}_{1}$ n.com. pig pig See: $\bar{m} a l[\mathrm{POC} *$ boRok 'domestic pig']

$\mathbf{q} \overline{\mathbf{o}}_{2}$ n.com. type of insect, large and black, that bites people and fights with cockroaches bebet we i save kakae man. Hem i blak mo bigfala mo hem i save faetem kakros

$\mathbf{q} \overline{\mathbf{o}}_{3}$ n.com. kind of plant with thick succulent leaves, white flowers and red fruit with black seeds kaen plant we i gat waet flaoa, red frut mo blak sid Tapeinochilus sp.

$\mathbf{q} \overline{\mathbf{o}}_{4} \quad v t$. swear swea Nē tō qōqō i Mackenzie. He is swearing at Mackenzie. Hem iswea long Mackenzie. qōbetge n.com. common Pacific vase (gastropod shellfish) wan kaen sel blong solwota Vasum turbinellus

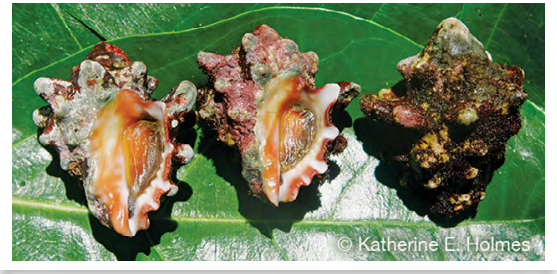

qōbetge Vasum turbinellus

$\mathbf{q} \mathbf{0} \mathbf{1}$ n.com. surgeonfish, name covers all the smaller species of surgeonfish fis we i gat naef, ol smolsmol wan blong hem Acanthurus spp. [This name covers all the smaller species of surgeonfish, compared with vag which covers the larger Acanthurus spp.] See: vag [POc *bolo 'small dark surgeonfish, possibly Ctenochaetus']

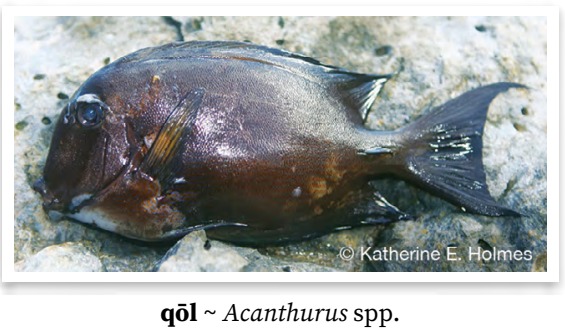

qōlmo n.com. variety of yam that belongs to the grouping of 'crooked' yams $\sim$ kaen yam we i stap long grup blong ol kruked yam

qōlo n.com. dugong kaofis Dugong dugon \{Lit. sea pig pig blong solwota\}

qōlō $1 \cdot n . c o m$. dirty, be dirty doti Na nogon o qōlō. His face is dirty. Fes blong hem $i$ doti. 2 - vi. become dirty, be dirty kam doti Na non o gogov mō qōlō kēl. His clothes got dirty again. Klos blong hem i kam doti bakegen.

qōmiat n.com. stinkwood; kind of tree with yellow fruit with red seeds inside stingwud; wan kaen tri we frut blong hem i yelo mo i gat red sid insaed Dysoxylum gaudichaudianum \{Lit. dead pig ded pig\} See: walag; wosvet 


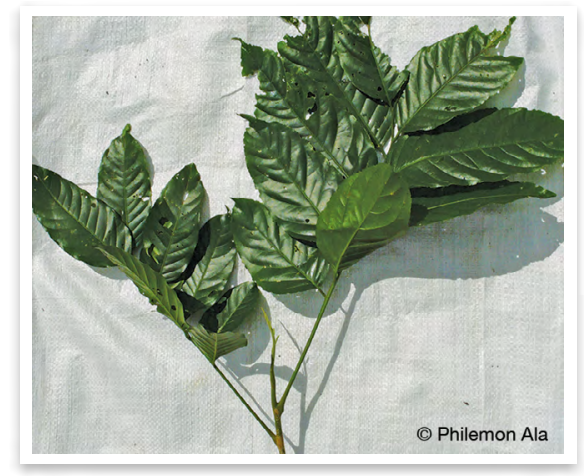

qōmiat Dysoxylum gaudichaudianum

qōmur n.com. kind of shrub kaen plant [Used as a local medicine to help with anaemia. Wan kastom meresin taem blad blong yu i finis.] See: dōqōmur

qōn̄ov vi. dull, be dull no braet

qōōn n.com.suff. her/his/its knee(s) ni

blong hem See: qöu

qōōr n.com. wild yam wael yam Dioscorea nummularia Syn: weag

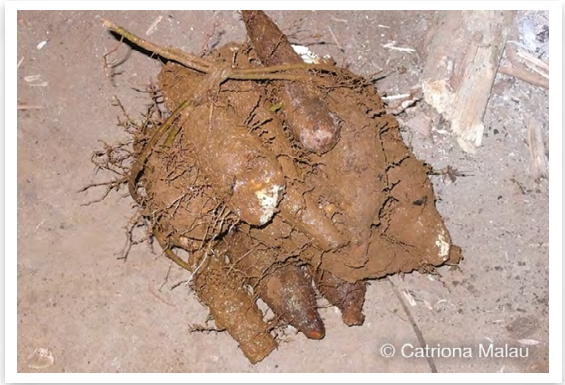

qōōor Dioscorea nummularia

q⿳亠े冖q⿳亠丷厂 n.com. mouldy food kakae we i gat nalumlum i gru long hem from i stap longtaem tumas

qōr $v$. forget foget, fogetem Na lölök miti qōr le o masawre ine. I will not be able to forget this place. Bae mi no save fogetem ples ia. (AAD) Ba na lölök gōtō qōr ten kēmi. But I haven't forgotten you all yet. Be mi no fogetem yufala yet. (AAD) qōrōtōt 1 1 vi. make a lot of noise, be noisy mekem tumas noes Ira sē tō qōrōtōt ko? Who are they making noise here? Hu ia olgeta i stap mekem noes ia? (DSR) 2 - n.com. loud sounds, too much noise $\sim$ tumas noes See: wölul qōrqōr $\mathbf{1} \cdot v i$. dream drim No mō qōrqōr vita no mo van a lo. I dreamed that I went to the seaside. Mi drim se mi bin go long solwota. 2 - vt. dream about drim long No mō qōrqōr nē. I dreamed about him. Mi drim long hem. 3 n.com. dream $\sim$ drim I ginon ni kēl me lō qōrqōr. His wife came back in a dream. Woman blong hem $i$ kambak long drim. (OSK.027)

qōrser n.com. kind of spear with several prongs on the end wan kaen spia we i gat plante poen long en blong hem [Originally the points were made from the inside of the trunk of a tree fern but they are now made from wire. Bifo ol i mekem poen long stamba blong blakpam be nao ia ol i mekem long waea.]

qōrwōn n.com. deaf person, be deaf sora $\mathrm{i}$ fas See: wōn , qörö $_{1}$

$\mathbf{q} \overline{\mathbf{o s}} \mathbf{s}_{1} \mathbf{1} \cdot v t$. pound, as in pounding löt with pestle, pounding clothes against rock $\sim$ kilim, olsem blong mekem nalot Mō qōs o wērēt e, ni da nē vita ni mölumlum. She pounded the octopus, she made it soft. Hem i kilim nawita ia, $i$ mekem se $i$ sofsof. 2・ $v$. wash (clothes) wasem (klos)

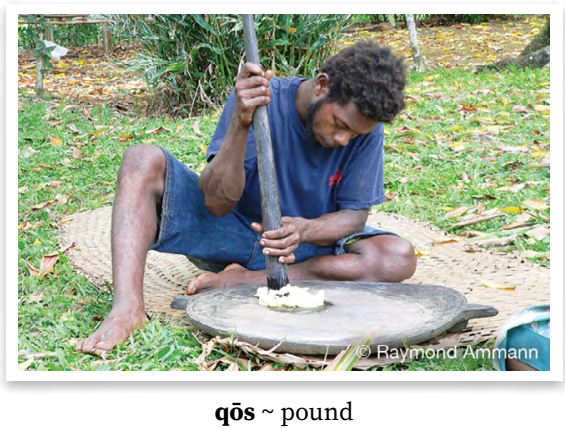


qōs do (comp.) v. pound leaf used for poisoning fish and mix with sand $\sim$ stonem lif blong posenem fis mo miksim wetem sanbij

$\mathbf{q} \overline{\mathbf{o}} \mathbf{s}_{2}$ vi. run strongly, rapidly (of water) $\sim$ (wota i) ron strong $\mathbf{O}$ bē ni qōs lō me. The river is running rapidly (due to heavy rain). Wota $i$ ron bigwan (from ren).

qōsqōs (redup.) vi. large movement of water, as in wake of boat, water stirred up by propellers, etc. wota i muv bigwan, olsem from enjin blong bot

qōt $v$ t. dive (for) daeva (long) See: el qōtqōt (redup.) vi. go diving daeva qōtarō n.com. angelfish papiong fis Family Pomacanthidae

qōtō n.com. semi-mature coconut, which has fizzy liquid inside $\sim$ haf drae kokonas we wota insaed long hem i gat gas long hem Syn: mian $\mathbf{q} \overline{\mathbf{o}} \mathbf{t} \overline{\mathbf{o}}_{2}$ n.com. cupboard that is built over the fire in kitchen to store canarium nuts or breadfruit, so that they are dried and kept safe from rats $\sim$ haos blong putum drae nangae o bredfrut insaed long hem. Hem i go ova long faea mo ol i somap doa blong rat i no save kakae ol nangae o bredfrut

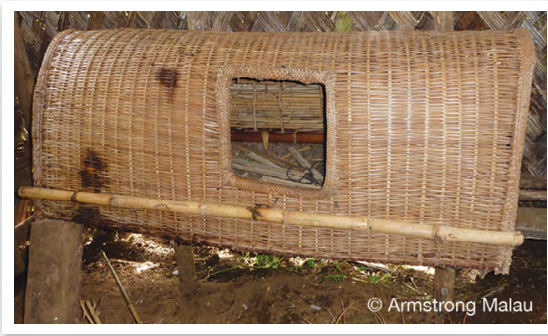

q⿳亠̄冖tō nut cupboard

qōtōn n.com.suff. her/his/its head hed blong hem See: qötu

qōtōnögōsōw n.com. empty complete shell of mature coconut that has not germinated and the husk and albumen inside have decomposed $\sim$ drae kokonas we hem i no kam navara mo skin mo kakae blong hem insaed i lus \{Lit. storage cupboard for rats kabod blong rat\}

qöl $v$. bear fruit karem kakae $\mathbf{O}$ möl mö qöl idian, o röröu mo kosēv. The orange tree bore lots of fruit and its branch broke. Aranis i karem fulap kakae, brans blong hem ibrok.

Qön̄ n.loc. tribe name, named because they arrived in the dark (qö $\bar{n})$ from Mota and also because they are seen as not wise $\sim$ nem blong traeb we olgeta ol i kamaot long aelan blong Mota long naet $(q \ddot{o} \bar{n})$ mo ol i no waes tumas

qön̄ $\mathbf{1} \cdot n . c o m$. night $\sim$ naet $\mathbf{2} \cdot n . c o m$. day (as measurement of time) dei (olsem blong tokbaot hamas dei) $3 \cdot v i$. become day $\sim$ kam dei $n$. anniversary of her/his/its death dei blong ded blong hem [POC *boyi 'night']

Qȫ̄n nirō n.com. Tuesday Tusde \{Lit. second day nambatu dei\}

Qön̄ nitöl n.com. Wednesday Wenesde \{Lit. third day nambatri dei\}

Qön̄ nivet n.com. Thursday Tosde \{Lit. fourth day nambafo dei\}

Qöñ tevelēm n.com. Friday Fraede \{Lit. fifth day nambafaef dei\}

qöñöön n.com. purple popel

qöqö n.com. young shoot yang lif See: qöqörö

qöqörö n.com. small leaf smol leaf (JWM) See: qöqö

qörö ${ }_{1}$ n.com.suff. ear sora See: qōrwōn qoron (n.poss) n.com.suff. her/his/its ear sora blong hem qörök (n.poss) n.com.suff. my ear sora blong mi

qörö ${ }_{2}$ n.com. strip of coconut leaflets incorporated into basket to form corner pis lif kokonas we ol i ademap long basket blong mekem kona blong hem 
qörö gōsōw n.com. kind of vine that grows along the ground wan kaen rop i gru long graon nomo Geophyla repens Lit. rat's ear, named for the shape of the leaf sora blong rat, from lif blong hem\}

qörök n.com.suff. my ear sora blong mi See: qörö

qösö n.com.suff. groin, area of body around genitals $\sim$ pat blong bodi raon long ol tabu pat

qoson (n.poss) n.com.suff. her/his groin tabu eria blong hem qösök (n.poss) n.com.suff. my groin tabu eria blong mi

qösök n.com.suff. my groin tabu eria blong mi See: qösö

qötqötumen n.com. cardinal honeyeater (bird, male) redhed (pijin, man) Myzomela cardinalis See: wētēsisis

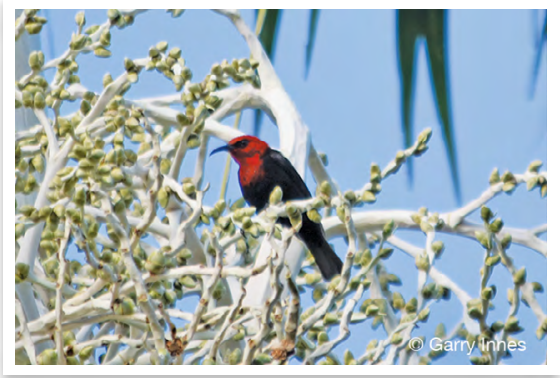

qötqötumen Myzomela cardinalis

qötu n.com.suff. $\mathbf{1}$ • head $\sim$ hed $\mathbf{O}$ reqe ni wēlēs ris o qötu wērēt. The woman turned the octopus's head inside out. Woman i tanem hed blong nawita $i$ kam aotsaed. (WNW) $2 \cdot$ top $\sim$ top $3 \cdot$ front, as in bow of ship, front of canoe, car fored, olsem blong kenu o trak No mo qal tes ilēkē o kērē, ba in̄kē o qötu tabaē. I first carved that bit, the back, but this is the front of it. Fastaem mi katem hemia, baksaed, be hemia fored blong hem. (VVKHVDP180ct0801.030) [POc * $b^{w} a t u(k)$ 'head; top of (something)'] qötu gövur (comp.) n.com. roof beam of house top blong haos

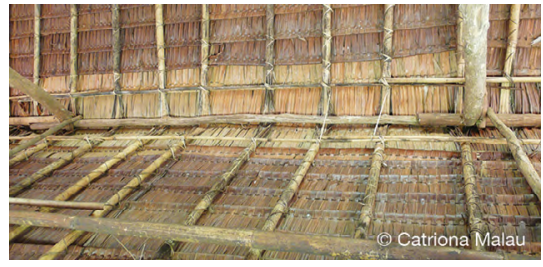

qötu gövur roof beam

qötu vōnō (comp.) n.com. area of village that is the section or edge towards the inland $\sim$ pat blong vilej we i stat long saed i go antap long bus Ant: kērē vōnō (comp. of kērē)

wöqötu (der.) n.com. part of house part blong haos

qōtōn (n.poss) n.com.suff. her/his/its head hed blong hem

qötuk (n.poss) n.com.suff. my head hed blong mi

qötubanēn n.com.suff. her/his/its shoulder $\sim$ solda blong hem

qötuk n.com.suff. my head hed blong mi See: qötu

qötuōw n.com. kind of basket made from coconut leaves and hung in the kitchen, used especially for putting bivalve shells in, which are used for scraping food and coconuts wan kaen basket we ol i mekem long lif kokonas mo yusum blong putum plasta sel insaed long kijin \{Lit. head of turtle hed blong totel\}

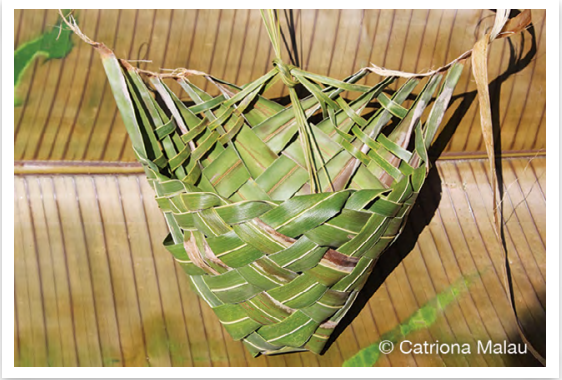

qötuōw kind of basket 
QÖTUQËRËT

qötuqërët $n . c o m$. big-eye bream (fish) $\sim$ wan kaen fis Monotaxis grandoculis \{Lit. flying fox head hed blong flaengfokis\}

qötuq $\overline{\mathbf{o}}_{1}$ n.com. variety of breadfruit that has large fruit $\sim$ kaen bredfrut we kakae blong hem i bigwan

qötuq $\overline{\mathbf{o}}_{2}$ n.com. variety of taro, 'male', with black stem and white stripes $\sim$ kaen taro we hem i man, we han blong hem i blak wetem waet straep \{Lit. pig's head hed blong pig\}

Qötusa n.loc. tribe name nem blong traeb

Qötuwis n.loc. place name nem blong ples \{Lit. owl head hed blong hoknaet\} qöu n.com.suff. knee ni $\mathbf{O}$ met mö lum dēn̄ na qōōn. The tide came in reaching up to his knees. Solwota i kamso kasem ni blong hem. (JMT)

qōōon (n.poss) n.com.suff. her/his/its knee(s) ni blong hem qöuk (n.poss) n.com.suff. my knee $\sim$ ni blong mi

qöuk n.com.suff. my knee ni blong mi See: qöu

qul n.com. bunch bandel Rōrō mē vên o qul mes nirō. The two of them shot two bundles of fish. Tufala i sutum tu bandel fis. (JMT 150)

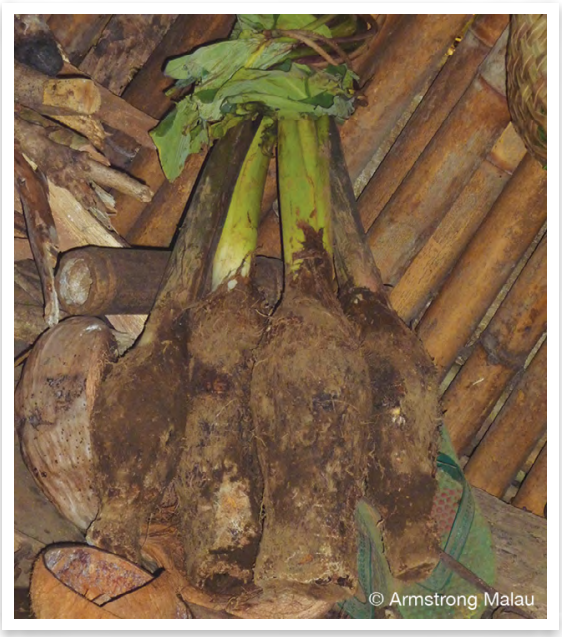

qul bunch qulön̄ $n$.com. ring-like mark around wood or plant stem, such as node of bamboo or sheath mark of sugarcane mak olsem ring we $\mathrm{i}$ ronem samting olsem wud, bambu o sugaken

qulös n.com. pool pul

qultian n.com.suff. her/his/its intestines, guts gat blong hem qultie n.com. intestine $\sim$ gat \{Lit. bundle offaeces bandel blong sitsit\}

quq $v$. leave something (food or drink) in the mouth without swallowing it $\sim \operatorname{livim}$ (kakae o wota) long maot be no solem daon

quqseg (der.) v. spit out liquid spetem aot wota

qus $v$. put a lot of food in the mouth fulumap gud maot wetem kakae

qusmamas $v i$. eat a meal without any meat $~$ kakae drae, kakae kakae we i no gat mit blong hem $\mathrm{Na}$ gen nēn o bigbig odiañ, nēn a qusmamas gem. We don't have any meat, we'll just have to eat the staple part of the meal. Yumi nogat mit, bae yumi kakae draenomo. 


\section{$\mathbf{R} \sim \mathbf{r}$}

-r suff. third person plural possessive suffix, their blong olgeta Nēr a èlgōr nor o sul. They look after their people. Ol i lukaotem ol pipol blong olgeta. (CH1-200301-EKK.367)

ra art. plural marker denoting personal nouns ol, maka we i go wetem nem blong man we i soemaot se i gat plante man long grup No ga mamarseg idian ra maranag ta Vōnō Lav. I really feel pity for the chiefs of Vanua Lava. Mi sori tumas long ol jif blong Vanua Lava. (CH1-200301EKK.176)

raba $n$.com. slingshot $\sim$ raba

rabiēg n.com. bluespotted cornetfish, smooth flutemouth wan kaen longfala fis Fistularia commersonii

raēs ga qag n.com. cassava variety, white $\sim$ kaen maniok we hem i waet raēs mamē n.com. cassava variety, reddish kaen maniok we hem i red $\mathbf{r a k}_{1} n$.com. long-finned drummer (fish) $\sim$ wan kaen fis Kyphosus vaigiensis

$\mathbf{r a k}_{2} v$. lift, lift up leftemap Nēk i rak kal o dön. You lift up the mat. Yu leftemap mat.

$\mathbf{r a k}_{3} v$. recognise save Nēk gētē èl rak no. You didn't recognise me. Yu no luk save mi.

$\mathbf{r a k}_{4} v i$. move a bit, a little bit in a certain direction $\sim$ muv smol long wan daereksen Nitog nēk o kōn̄kōñ rak sōw, ba nēk i kōn̄ rak siag, rivteg o ilsi. Don't snap it down there a bit, but you snap it up there a bit, close to the end. Yu no brekem daon smol ia, be yu brekem antap smol, klosap long en. (SBB)

raksiag $a d v$. especially, emphasising one or a certain number of things or people in particular out of a group speseli No gö mörös qaq vasösöv gem min rege sul, raksiag i rege timiak nēr me le me ti na sien nèr. I want to advise all the people, especially those who have given their names. Mi wantem advaesem olgeta pipol, speseli olgeta olsem ol i bin givim nem blong olgeta.

ralēt $n$.com. firewood $\sim$ faeawud Nēr a van a tar o ralēt. They went and chopped firewood. Ol $i$ go katem faeawud. (ESP) [POC *alito(n) 'firebrand, piece of burning wood']

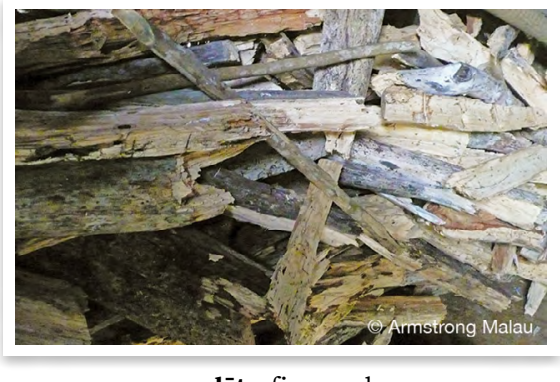

ralēt $\sim$ firewood

ram $v i$. give payback peibak Nana ram kēl ti min nē. I'll pay him back. Bae mi givim peibak long hem.

raminwōlōnmmögörviaomōtōsur n.com. bok choy, Chinese greens waetbun, jaenis kabis \{Lit. the woman and her sister-in-law grated lots of coconut for it woman wetem tawi blong hem $i$ skrasem plante kokonas from\}

$\boldsymbol{r a n}_{1} v i$. dry (yam, wild yam, taro, etc.) $\sim$ drae (olsem yam, wael yam, taro) Syn: $\operatorname{meren}_{2}[P O C *$ [ma-Jrano "become withered (of vegetation)']

$\boldsymbol{r a n}_{2} v t$. pull, pull strongly pulum (strong) $\mathrm{Na}$ ran̄ o tere gamērēr qōo na möguk talo vet o waga. I pull my vines in order to weave a prawn trap. Mi pulum rop blong mi blong wivim basket naora. (KBN) 
ran̄ōrō $v$. plait, weave twill pattern; plait strips over two crossing elements wivim tutu, wivim pis pandanas o bambu i go ova long tu narafala pis, i no wan nomo

ran̄ran̄ $v$. clean garden $\sim$ klinim, katem garen

$\operatorname{rar}_{1} v i$. arrive $\sim$ kamtru

$\mathbf{r a r}_{2}$ n.com. Indian coral tree $\sim$ narara Erythrina indica, E. variegata

$\operatorname{rar}_{3}$ n.com. season when rar flowers, time of dry, cold weather, April to August sisen we narara hem i gat flaoa, hem i taem blong kolkol, stat long Eprel kasem long Ogis

raras n.com. shallow, shallow place salo, ples we wota i no dip Ant: lam

rarö $n$.com. noise $\sim$ noes

rartan n.com. wild cane $\sim$ wael ken Miscanthus sp.

ras $1 \cdot v t$. grate nuts or tubers for nalot or laplap rasras, salem kakae Nēk i le o via, nēk i ras o ñe. You get the grater and you grate the canarium nuts. Yu karem samting blong rasras, ale yu rasras ol nangae. $\mathbf{2} \cdot v$. sharpen sapenem Nē o rasras gem na nök o gasel. He is just sharpening my knife. Hem $i$ jas sapenem naef blong mi. [POC rasi 'grate, scrape (tubers, coconuts); scratch']

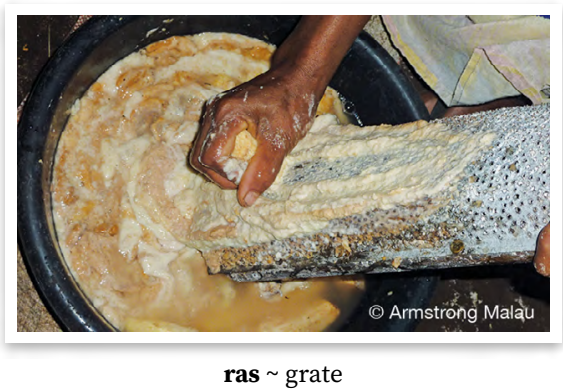

rat $n$.com. flat place, area of land $\sim$ flat ples Nēn gē gis o gövur la rat. We'll build a house on a flat place. Yumi bildim haos long flat ples. $\mathbf{O}$ tan ko o rat. This land is flat. Graon ia iflat. rege n.p.fr. people, group of two or more people ol man, olgeta I rege gō lōqtag nēr ma van bē rērēv mëkër. Lots of people went to fish for flying fish. Fulap man ol $i$ go blong pulum flaengfis. (OHS)

regeltëv $n . c o m$. variety of taro $\sim$ kaen taro

Rekel n.loc. place name $\sim$ nem blong ples

rem $v$ t. climb klaem Nēk i rem o miak. You climb the Tahitian chestnut tree. Yu klaem namambe. (JRQ)

ren part. emphatic marker; right, absolutely, totally, definitely tumas, tru, stret; i toktok we yu talemaot i moa strong Nēk gō sōw tek wareg dēn̄ ren la tan. You are going to take it down, reaching right down to the ground. Bae yu go daon wetem kasem daon long graon wantaem. (ATB) O bigbig odiañ ren. There was absolutely no meat. Mit i nogat nating. (BQD)

reqe n.com. $\mathbf{1}$ - woman $\sim$ woman 0 reqe e o magte. That woman was an old woman. Woman ia hem i olfala woman. (GMM) $2 \cdot$ female $~$ woman Nēr a gis varge o tō reqe. They caught the female chicken (hen). Ol $i$ kasem woman faol. (RTF)

rerev vi. slither, creep, movement low to the ground and generally as something that does not have legs kalkal, wokbaot, muv olsem wan samting we i nogat leg, olsem snek, snel, nawita, slug $\mathbf{O}$ wērēt ni rerev tēqēl kēl a lo. The octopus crept back down to the seaside. Nawita $i$ kalkal i gobak daon long solwota. (WNW)

retekraw n.com. variety of banana kaen banana [This variety of banana is no longer grown on Vanua Lava. Nao ia i nomo gat kaen banana ia long Vanua Lava.] 
Rev n.loc. place name, Arep, where secondary school is located, near Sola nem blong ples, Arep, ples we skul i stap long hem, klosap long Sola

revrev n.com. late afternoon, evening; period preceding and following sunset $\sim$ aftenun, ivining $\mathbf{O}$ qërët ge gengen le revrev. The flying foxes eat in the evening. Flaengfokis ol $i$ kakae long ivining. (EDM) $[P O C *(p a)$ Rapi 'evening']

re $\overline{\mathbf{e}}_{1} v t$. draw leaf, such as coconut, pandanus, heliconia, over fire to make more hardy and supple for use in weaving, cooking bonem lif long faea, olsem kokonas, pandanas, lif laplap, blong mekem se i sofsof mo i save stap longtaem Nēk i rē o damat talo qañqañris. You soften the heliconia leaves over the fire for baking in the stone oven. Yu bonem lif laplap long faea blong bekem kakae. (JNN)

$\mathbf{r} \overline{\mathbf{e}}_{2}$ n.com. rain $\sim$ ren

rēg vi. 1 • swell, become swollen solap Na dirik mē rēg. My leg has swollen. Leg blong mi i solap. $\mathbf{2}$ - fat, be fat fatfat Nē gētē rēgrēg. She isn't getting fat. Hem ino stap kam fatfat.

rēgèt $n . c o m$. variety of taro, 'male', with light green stem and small red top $\sim$ kaen taro we hem i man we han blong hem i laet grin mo i red antap rēki n.com. topsail drummer, Pacific chub, drummer (fish) bigbel (fis) Kyphosus cinerascens, K. pacificus

rèlēgtēl $n . c o m$. variety of taro, 'female', with stem that is red at the bottom and purple at the top $\sim$ kaen taro we hem i woman, han blong hem i dak lelebet mo kakae i waet. Skin blong hem i laet grin mo hem i save bigbigfala

rēntenge n.com. $\mathbf{1}$ - tree, plant with woody trunk and branches, also covering palms, but not smaller shrubs tri, wud Ni siag ti aē lē rērēi rēntenge nitiwial. It sat there on the branch of a tree. Hem i sidaon long ples ia long brans blong wan wud. (DHN) 2 - wood, length of wood, stick, the wood of a tree $\sim$ wud Nēk ge vetrug o rēntenge ga mamē nē gö tur aē. You are going to stand up the red stick there. Bae yu stanemap pis wud we $i$ redwan long ples ia. (TT3) Ni le o vötu rēntenge nē tö vusvus o tan̄sar aē. He took the big club of wood that he bashes people with. Hem i karem bigfala wud we hem i stap Kilim man long hem. (WDR)

rēn̄ē $v t$. roast meat over fire or charcoal rusum mit long faea Garqe nēn a rēn̄ē o qō. Today we are going to roast a pig. Tede bae yumi rusum pig. See: tun

rēn̄ēs $n$.com. green palm lorikeet (bird) wan kaen pijin olsem nasiviru Charmosyna palmarum

rēnēg $v$. wear around neck $\sim$ hangem long nek

rēneēen n.com. variety of banana that appears to be ripe when it is not completely ripe, and will be ripe approximately two days after first appearing to be ripe $\sim$ kaen banana we i save raep tu taem, hem i luk olsem i raep be yu mas wet, afta tumora bae i raep

rēq $v t$. pay fine for saying the name of one's parent-in-law or child-in-law, or otherwise not showing respect to them $\sim$ pem smol faen sapos yu no bin respektem gud palika blong yu, olsem yu singaotem nem blong palika o yu ovarem hem Nèk mō ōl qelgan̄, nēk gē rēq nē. You called your in-law's name, you'll have to pay a fine to him. Iu bin singaotem nem blong palika blong yu, bae yu mas faen long hem.

rēqrēq (comp.) n.com. fine paid for saying one's parent or child-in-law's name or otherwise not showing 
respect to them faen blong pem sapos yu no bin respektem gud palika blong yu, olsem yu singaotem nem blong palika o yu ovarem hem rēr $v i$. tide $\sim$ taed $\mathbf{O}$ naw ni rēr kēl. The wave drew back again. Solwota $i$ go daon bakegen. (BQD)

rērēg n.com. whale welfis

rērēi $n$ branch of tree or other plant han blong wud Nē me rem kal lē rērēi miak. He climbed up in the branches of the Tahitian chestnut. Hem i klaem antap long brans blong namambe. (JRQ.009) [POC *raqan "branch of tree or other plant' $P N C V$ *ra-ra- 'branch']

rērēi ${ }_{2} v i$. thin $\sim$ bunbun

rērēt $v$. peep, peek, look secretively or with particular intent $\sim$ pusum hed blong luk haed $\mathbf{O}$ wērēt nē ni rērēt kal den o tabē. The octopus peeped up out of the wooden platter. Nawita hem i pusum hed $i$ go antap, aot long natambia. (WNW) Ni van mölumlum, van mölumlum van vaavan, van dēn̄ me o qētēgi rēntenge nitiwial, ni rērēt. He went along quietly until he reached a tree, and he peeked out. Hem i go sloslo, go sloslo go go, i kam kasem stamba blong wan hed, ale i pusum hed ilukluk. (WDR)

rērēv n.com. towline tolaen [Towline used for fishing, such that the line is pulled along behind the canoe. Tolaen we yu yusum blong string i go bihaen long kenu.]

rēs vi. 1 • fight, wrestle, struggle faet Ni gis varge ine, rōrō mē rēs gö möul ren. She held onto it tightly, and the two of them wrestled for quite a long time. Hem i holem taet ia, tufala i faet longtaem lelebet. (WNW) 2 - struggle, fight an urge $\sim$ traem blong winim Nē gö mörös ta ni van ni mim, ni van me, nē mē rēs aē. He wanted to go and urinate, and he came and fought against the urge. Hem i wantem sebae hemigo pispis, hemikam, hemi traem blong winim. (ADT) rēsim n.com. variety of taro, with red stem and light red corm. It is not strong and cannot fork $~$ kaen taro we han blong hem i red mo kakae i miks red wetem waet. Hem i no strong mo hem i no save karem fok rētnan n.p.bound her/his/its mother mama blong hem See: rētne

rētne n.p.bound mother (of someone) mama (blong) I rētnan ni wotoqtoq kēl. His mother became pregnant again. Mama blong hem $i$ gat bel bakegen. (ESS)

rētnan (n.poss) n.p.bound her/his/its mother mama blong hem

rētnëk (n.poss) n.p.bound my mother $\sim$ mama blong mi

rētnëk n.p.bound my mother mama blong mi See: rētne

rētrēt $v t$. tie up fasem Nitog o rētrēt taet na dirik. Don't tie up my legs tightly. Yu no fasem taet leg blong mi. (DPD.096)

rēv vt. pull pulum Qèt rōrō a siōl, rèv kal o ak. Then the two of them came ashore, and they pulled up the canoe. Afta tufala $i$ kamso, i pulum kenu i kam antap. (GMM)

rēv vasese (comp.) v. pull canoe out of the water to the tideline, so it is half in and half out, in case one needs to make a quick getaway pulum kenu i kam antap smol nomo, haf long solwota, haf aotsaed, blong mekem se yu save pulum i go daon hariap sapos yu wantem go kwiktaem

$\mathbf{r e ̄ v _ { 2 }} v t$. write $\sim$ raetem

Rēvi $n$.loc. $\mathbf{1}$ eplace name $\sim$ nem blong ples $\mathbf{2}$ tribe name, named because they are known for their skills of fishing with a net $\sim$ nem blong traeb blong ol man we ol i waes blong pulum net

rēvōrō $v i$. weave two strands over and two strands under $\sim$ wivim tu i go antap, tu $i$ go andanit 
rēw n.com. kind of fig that does not have aerial roots. It has smaller fruits than a banyan, the fruits are red and yellow and are eaten by birds wan kaen nambangga we rus blong hem i no kamaot antap. Frut blong hem i no bigbigwan tumas olsem nambangga. Ol frut ol i red mo yelo mo ol pijin ol i laek blong kakae

rēwēsil n.com. variety of aibika, shrub with leaves which are commonly eaten as vegetable greens $\sim$ kaen aelan kabis Abelmoschus manihot variety

rēwurveg n.com. variety of taro, 'female', with light brown stem and many roots on the corm $\sim$ kaen taro we hem i woman we han blong hem i laet braon mo kakae blong hem i gat fulap rus long hem

rërës $v$. scratch skrasem (RTF.026)

rës vt. bail (water) belaot Gamlöt!

Rës den o bē. Quickly! Bail out the water. Hariap! Belaot wota. [POc *asu 'scoop or ladle out']

rës $_{2}$ (root of arës)

rësrës dene n.com. style of dance that is made at a ceremony held five days after someone's death, when their spirit leaves the earth to go to the place of the spirits (lisorr) wan kaen danis we ol i mekem long faef dei blong ded blong wan man, taem spirit blong hem i go long ples blong ol ded (lisōr)

rëw vt. sing singim, singsing Pōpō ni rëwrëw o ës min Tètē. Grandma is singing a song to Baby. Bubu i singim singsing long Bebi. Syn: sur

Rëwël n.p.fr. brother-in-law; wife's brother, sister's husband (for man), reciprocal same generation in-law term for men tawian, brata blong woman blong man, man blong sista blong man ri v.part. second person non-singular imperative marker yusum taem yu talem wan grup blong mekem wan samting

riam $_{1} v t$. rub rabem

riam $_{2} v i$. have eggs underneath (of crabs and prawns) krab o naora i gat eg (i stap andanit long hem)

riaw $n$.com. $\mathbf{1}$ hermaphrodite $\sim$ animol we hem i man mo woman wantaem 2• intersex pig pig we hem i man mo woman wan taem Isē na bōlan aē, o riaw? Who has one, a hermaphrodite pig? Hu i gat blong hem, wan pig we i man mo woman wantaem?

rid vi. read $\sim \operatorname{rid}$ [Bis rid 'read']

rieg $a d v$. away from, out, out of the way aot long Le rieg o tönö sigsiag den o gersal. Move the chair out of the way. Seftem jea ia aot long rod.

rir n.com. earthquake etkwek

ririn̄i n.com. belongings, possessions, things ting (blong wan man) wēririn̄i (der.) n.com. materials needed for making a particular item, such as house, canoe, basket ol samting we yu nidim blong mekem wan samting In̄ko nēn a tar o wēririn̄i gövur. Now we will chop the materials needed for making the house. Nao ia bae yumi katem ol ting blong mekem haos. (RBH)

rirve $v$. if one only has a small amount of meat, soak small pieces of it in salty water, then when it is eaten with a meal, one will feel that it is sufficient $\sim$ sapos yu gat smol mit nomo blong kakae wetem kakae, sapos yu tekem smolsmol pis mit ia mo yu putum long wota wetem sol, bae i mekem se yu fulap long smol mit

ris $1 \cdot v$. turn tanem I Wöwut ni rōn̄teg so ine, ni tal ris, ni èl nē odiañ. Wöw theard it, and he turned around, and saw that she wasn't there. Wowut i harem, hem i tanem hem, hem i luk 
se hem i nogat. (WWM̄) 2 v vt. change into tanem i kam E ni qērēt, nē ni ris o dem aē. Then he squeezed it and turned it into a yam. Ale hem i skwisim, itanem ikam yam. (DPD)

riv $_{1} v t$. plant planem Ri kōñ o rērēi, ri riv kēl. Break off the branches and replant them. Brekem brans blong hem, planem bakegen. (HWM)

rivriv (redup.) n.com. plant samting we yu planem Nē ga datek o rivriv. It looks after the plants. Hem ilukaotem samting we ol i planem. (DFF)

$\operatorname{riv}_{2} v t$. fan, use fan to fan something fanem See: dērivriv

rivteg $1 \cdot a d v$. near, close by $\sim$ klosap 2 - prep. near to, close to $\sim$ klosap long $\mathrm{O}$ qon nē ma gav tēqēl ren sōw e, rivteg o nēlida naw. The pigeon flew right down low, close to the surface of the sea. Nawimba i flae daon olgeta, klosap long top blong solwota. (DHN) Wöt ren me gēn, rivteg no. Throw it right here, close to me. Sakem i kam ia, klosap long mi. (DSR) 3 - adv. nearly, soon klosap Rivteg o ev ni nōn. The fire has nearly died down to coals. Klosap faea i go daon. (JNN)

ro $v$. soak, immerse in water draonem long wota Nē ni ro nēr e, ti nēr a manman kēl. He soaked them in water, so that they became soft again. Hem i draonem olgeta long wota, blong ol ikam sofsof bakegen. (JRQ)

ro- n.p.fr. >n.p.fr. female categorisation prefix that occurs on women's names maka blong nem blong wan woman (Allomorphs: ra-, re-, rē-, rë-, rō-, rö-)

ronog n.com. rank rang $\mathbf{O}$ ronog no to tog ti garqe nē ge elel, nē ge elel. The rank that I am at today is very high. Rang we mi stap long hem tedei hem i hae we ihae. (VVCMVDP21Nov0701-GM) ron̄ron̄oqar n.com. clown triggerfish $\sim$ kaen strongskin we i gat bigfala waet spot long hem Balistoides conspicillum rot n.com. taro garden $\sim$ garen taro $\mathrm{Na}$ vanvan tek lo rot, a suñun o qiat aē. I take it to the taro garden, and put taro in it. Mi go wetem long garen taro, fulumap taro long hem. (EWB)

rov $_{1}$ n.com. food dish made by mixing various leafy greens with grated raw canarium nuts, wrapping them and baking in stone oven wan kaen kakae we ol i rasras grin nangae mo paselem wetem defren kaen kabis, afta ol i bekem

$\mathbf{r o v}_{2}$ n.com. variety of taro, 'female', with white stem and corm and red veins under the leaf. kaen taro we han blong hem i waet mo kakae blong hem i waet mo hem i save karem fok [A popular variety of taro which is commonly used for making löt. Hem i wan strong kaen taro we mos taem ol i yusum blong nalot.]

$\operatorname{rov}_{3} v t$. pull back bow, ready to shoot pulum bonaro, redi blong sut E rōrō a rov o vus. Then the two of them pulled back their bows, ready to shoot. Ale tufala i pulum bonaro, redi blong sut. (DMM.112)

$\mathbf{r o ̄}$ (root of nirō)

rōm $\mathbf{1}$ - n.com. butterfly $\sim$ bataflae $\mathbf{2}$ • vi. turn into butterfly jenis i kam bataflae $\mathbf{3} \cdot$ n.com. butterfly fish bataflae fis Chaetodon spp.

rōm timiat (comp.) n.com. kind of big, black butterfly with white dots wan kaen bataflae we hem i bigbigfala mo hem i blak wetem waet dot \{Lit. devil butterfly devel bataflae\}

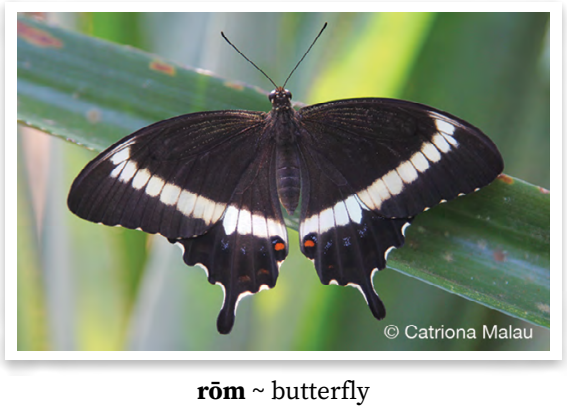


rōne num. second, second one nambatu In̄ko ni vetreñ o rōne. Then he sent the second one. Nao ia hem i sendem nambatu. (JRQ)

rōnōobōn $v t$. smell, passive sense of smell harem smel Nēr gō rōn̄ōbōn vita o bunë tan̄sar. They could smell that it was the smell of a person. Ol $i$ harem smel se smel blong man. (JTF)

rōn̄teg $v$ t. 1 • hear harem Matēk ni rōn̄teg o mēs ni sar. Matēk heard the lorikeet chirping. Matēk $i$ harem nasiviru i stap mekem noes. (KM̄MT) 2 - feel, sense $\sim$ harem, filim $\mathbf{N i}$ kalkal, ni rōn̄teg ga mav, ga mav. He pulled it up and he could feel that it was very heavy. Hem i pulum i kam antap, $i$ harem se $i$ hevi we. (OHS) [POC *rojoR 'hear']

rōñteg gē tisēe (der.) v. feel bad harem nogud No gō rōñteg gē tisē sur o rörö vita i Walter Lini mi miat. I feel bad because of the news that Walter Lini died. Mi harem nogud from nius se Walter Lini i ded.

rōn̄teg gōwē (der.) v. feel good harem gud Nonor no go los, garqe no mō rōn̄teg gōwē. Yesterday I was sick, today I feel good. Yestedei mi sik, tede mi harem gud.

rōn̄teg rak vt. understand, sense, comprehend, realise $\sim$ harem save No gō rōñteg rak vitia lē bēk vita no miti wē le. I can already sense in my body that I will not become well again. Mi harem save finis long bodi blong mi se bae mino kam gud. (KM̄T)

$\mathbf{r o ̄ q} \mathbf{q}_{1}$ n.com. kind of freshwater fish $\sim$ malet blong wota we $i$ save bigbigwan See: varvar

$\mathbf{r o ̄ q _ { 2 }} v t$. bend length of bamboo over the top of the house in making roof putum bambu i go ova long top blong mekem haos $\mathbf{O}$ gövur ko, nēk gō rōq si nēk ga gas? This house, are you going to bend the bamboo over the top in making the roof, or are you going to cross it? Haos ia, bae yu putum bambu $i$ go ova long top, o bae yu krosem? See: wōrōqrōq

$\mathbf{r o ̄ q}$ n.com. kind of tree like a varvar which grows in the bush and has a thick double layer of bark wan kaen tri we i olsem varvar be i blong bus mo skin blong hem i strong dabol skin [Used for making traditional wooden knives (meteges) and hooks for hanging food baskets (gatitimeles). $\mathrm{Ol}$ i katem wuden naef (meteges) mo wud blong hangem kakae long hem (gatitimeles) long hem.]

$\mathbf{r o ̄ q}{ }_{4} v$. hold tight $\sim$ holem taet, prestem i go daon See: dōrōq

rōr n.com. 1 - rubbish, scraps $\sim$ doti Tek o rōr. Take the rubbish. Tekem of doti. (HWM) 2 - rubbish heap, area for rubbish ples o hip blong doti Wöt o vēnti gengen lō rōr. Throw the vegetable peelings on the rubbish heap. sakem skin blong kakae $i$ go long ples blong doti.

$\mathbf{r o ̄}_{2}$ vi. go deep inside, either down or lengthwise, fall into hole, sink into something go dip insaed long wan samting, i go daon o go longwe, draon insaed long hol Nē mō rōr ti a Daragrō lō bōak. She sank into the mud at Daragrō. Hem $i$ draon long sofmad long Daragrō. (AAD)

rōrō pron. they two, them two; third person dual pronoun $\sim$ tufala

rōrōñ $a d v$. $\mathbf{1} \cdot$ slowly $\sim$ sloslo $\mathbf{2} \cdot$ quietly kwaet In̄ko ni siag rōrōn, ni siag sur, ni teñ. Then she sat quietly, she sat down, and she cried. Nao ia hem i sidaon kwaet, hem $i$ sidaon, hem $i$ krae. (DSR) Ri tur rōrōn̄, ri rōn̄teg o ës. Stand quietly, and listen to the song. Yufala i stanap kwaet, lesen long singsing. $\mathbf{3} \cdot$ calmly, still $\sim$ kwaet

rōt $v t$. cut with shell or small knife, placing finger on edge of knife or shell to guide and support the 
RŌVōL

cutting action katem long naef o sel mo yu yusum fingga blong gaedem Nèr a van lō rōt damat. They have gone to cut heliconia leaves. Oli go blong katem liflaplap.

rōvōl n.com. variety of taro, 'female' with stem that is white at bottom and red at top, with red veins under leaves kaen taro we hem i woman we han blong hem $\mathrm{i}$ waet daon mo red antap, mo i gat red mak long lif blong hem [Grouped with $\bar{m} \bar{o} v o \bar{l}$ taro. I famle wetem inōvōl.]

rōw $w_{1}$ vi. jump jam Na ēl o mat ni rerev me, e söw nana rōw, i Rëwël ni rōw. I saw a snake slithering this way, so then I jumped, and Brother-in-law jumped. Miluk snek i krolikam, ale mi jam, Tawi ijam. (ASS)

$\mathbf{r o ̄} \mathbf{w}_{2} \mathbf{1} \cdot$ loc. seaward, in a direction out to sea daon long solwota $\mathbf{E}$ rōrō ma van rōw a lo, ni rēv sur na kan o ak, ni sō. Then the two of them went down to the seaside, and he pulled down his canoe, and he paddled. Ale tufala $i$ go daon long solwota, hem i pulum kenu blong hem, i pul i go. (ATB) Nē ni èl o ak ma van kal rōw la maē vitia. $\mathrm{He}$ saw that the canoe had floated right out to sea already. Hem i huk se kenu $i$ go daon kasem en blong solwota finis. (WDR) $\mathbf{2}$ - loc. outside $\sim$ aotsaed Ba la masawre no mo kalō rōw, nana ēl so ine i Fiona. But when I went outside, I saw Fiona. Be taem mi go aotsaed, mi luk ia Fiona. (AAD.341) 3 - vi. go out, go outside $\sim$ go aotsaed, kamaot Kōmōrōk mō rōw lō den o bas. The two of us got out of the bus. Mitufala $i$ kamaot long bas. (AAD) 4 - vi. come out and show oneself, appear to kamaot long $\mathbf{O}$ döbit e mō rōw lō min no e, na tutuëk kēl. That spirit who showed itself to me was my own sister. Spirit we hem i kamaot long mi ia, hem i sista blong mi bakegen. (EDM) $\mathbf{O}$ gēnē ga garaq mo wot min nēr si mō rōw lō min nēr timiak o gōsōw qō. A new thing was born to them or appeared to them like a big rat. Wan niufala samting i bon long olgeta o kamaot long olgeta olsem wan bigfala rat. (ESP)

rōweg $v t$. tie rope or something long and flexible between two distant points $\sim$ fasem rop bitwin tufala ples we i longwe lelebet Nēk i rōweg den lö nögö gövur ni van lö kulë gövur. Tie it from the front of the house to the back of the house. Fasem long fored blong haos i go long baksaed blong haos.

rōwlav n.com. kind of flying fish, larger than other kinds, white with black spots, from deep sea wan kaen flaengfis we hem i bigwan olgeta. Hem i waet be i gat blak spot blong hem. Hem i blong big si blong aotsaed olgeta \{Lit. jump forever jam oltaem\}

rōwnölō n.com. east, place where sun rises $\sim$ is, ples we san i kam antap Syn: nögö lō; Ant: tulnö lō

rōwō n.com. kind of tree that has bark that is red on the underside wan kaen tri we insaed skin blong hem $i$ red [The wood is used for canoe paddles and also for firewood. $\mathrm{Ol}$ i yusum blong katem parel blong kenu long hem. Ol i yusum blong faeawud tu]

röböul payback for someone having killed a family member givim peibak taem ol i kilim wan famle blong yu

römöwuler n.com. variety of taro, 'female', with very dark red stem kaen taro we hem i woman we han blong hem i dak red [Said to be the first taro in Vētuboso. Ol i talem se hem i fes taro long Vètuboso.]

rönö $v$. plant stake in ground for yam to grow up planem wud long graon blong yam i save gru long hem See: wörönö 
rörö n.com. news nius

röröut vi. 1 - be narrow, become narrow smol, kam smol 2 - be skinny, become skinny smol, bunbun Na tarbian mö röröut. Her body has got skinny. Bodi blong hem i kam bunbun.

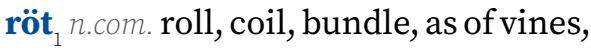
rope, leaves, mats bandel Ni dēn̄ tek me o röt damat. She arrived with a bundle of rolled up heliconia leaves. Hem i kam kasem wetem bandel blong liflaplap. (WNW)

röt $_{2} v t$. wrap around, encircle, hug, squeeze skwisim, fasem raon Na röt kirkir nēk! I'll hug you so hard that you fart! Bae mi skwisim yu go go yu brekwin! Kōmōrōk a ver röt. We hugged each other. Mitufala i hagem mitufala. rötö $v$. heat leftover food, reheat next to fire or on coals, to prevent from going off $\sim$ hitim namalas blong mekem se i no sting

röv n.com. vine used in weaving bird trap rop we ol i yusum blong wivim trap blong pijin

röwö n.com. bigeye scad (fish) kaen bigfala fis blong dipsi Selar crumenophthalmus

rurun̄ög n.com. variety of banana which is only eaten ripe, not cooked and eaten when unripe. The fruit will fall from bundle when the trunk is shaken kaen banana we ol i kakae raepwan nomo mo taem yu sekem stamba ol raepwan i foldaon. Hem i sotfala See: kukuög

ruruse (der.) n.com. payment pemen ruse $v$. pay, buy pem

\section{$\mathbf{S} \sim \mathbf{S}$}

Sade n.com. Sunday Sande

saemon n.com. cassava variety $\sim$ kaen maniok

sagar $v$. remove damaged piece of thatch from roof $\sim$ tekemaot olfala lif natanggura bifo yu mekem niu wan bakegen

sagēl vt. decorate, as with flowers and colourful leaves, or planting colourful plants on boundary dekoreitem, olsem yu putum flaoa long haos o planem kalakala flaoa long eria Nēn ga sagēl o yad aē. We decorate our yards with them. Yumi dekoreitem yad wetem.

sak vi. swell solap Wo o terit ni sak. And the porcupinefish swelled up. Mo nilfis i solap. (AWW)

sakreg $v$. take off, leave lego Wo rōrō a sakreg viteg, rōrō a gav. And the two of them took off, the two of them flew. Mo tufala ilego, tufala iflae. (DHN) sal 1 1 $n$.com. message $\sim$ mesej $\mathbf{O}$ sal ma van me ine. The news came. Nius $i$ kamia. (ADT) $2 \cdot v i$. send message sendem mesej Kēmi ta sal tultulëg me sur oso? Why do you keep sending a message for me to come? Yufala i stap sendem mesej from wanem?

$\mathbf{s a l}_{2} \mathbf{1} \cdot n$.com. working strip of plaiting or weaving element $\sim$ pis lif kokonas, pandanas o wanem we i stap lidim wiving 2 - vi. make trip with certain aim $\sim$ mekem rod blong wan samting Nē ma sal vagtevelēm vitia. He made five trips already. Hem i mekem faef trip finis.

$\mathbf{s a l}_{3}$ n.com. kind of tree $\sim$ wan kaen tri Homalanthus nutans

sal mamēgin style of making meal by which staple foods are wrapped with meat and greens in heliconia leaves with hot stones inside to help the food cook stael blong mekem 
kakae we yu pasolem ol hot ston insaed long lif laplap wetem kakae, mit mo kabis

salgōr n.com. sacred and restricted act or thing samting we i tabu Nēk tö sumsum o gē lö qön̄ manene o gē nē o salgōr. You drink kava in the evening, because kava is a sacred thing. Yu stap dring kava long naet, from we kava hem i wan tabu samting. (OSK)

salmiat $v i$. be ready, be prepared $\sim$ redi O damat mè qèt, na mem salmiat ti, na diar o ev ni nōn. The heliconia leaves are finished, I put it aside ready, and I wait for the fire to die down. Lif laplap $i$ finish, mi putum $i$ redi $i$ stap, mi wet long faea i go daon. (NBQ)

salōqōs n.com. kind of food dish (löt) made by pounding cooked taro, with coconut cream on top. The coconut cream should be thick but not separated wan kaen nalot wetem melek blong kokonas antap. Ol i kukum kokonas blong mekem se i tik be i no braon

salsal $v$. make meal by wrapping staple food with meat and greens in heliconia leaves with hot stones inside to help the food cook, the food is then placed on hot stones or coals so that it is cooking from inside and outside the parcel mekem kaen kakae we yu paselem kakae wetem mit, putum hot ston insaed bitwin lif laplap, mo afta yu putum long faea

salsim vi. separate oil from coconut cream kukum melek blong kokonas blong oel i kamaot

sam n.com. outrigger float nasama E nē o sam, sam lo qaq na mun kemem, kemek gō ōl ta nē o nutu ak talo ak sōsō. So it is an outrigger, the outrigger, in our language, we say that it is the child canoe of the canoe. Ale hem i nasama, nasama, long lanwis blong mifala, mifala i talem se hem i pikinini blong kenu. (VVKHVDP180ct0801.006) [POC *saman 'outrigger float']

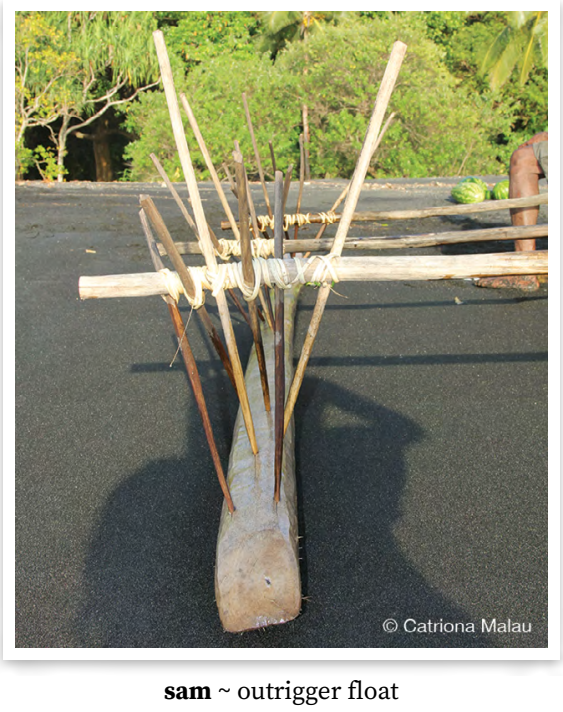

samo n.com. variety of banana with a short trunk and small, thin, bent fruit kaen banana we stamba blong hem i sotfala mo kakae blong hem i smol mo i benben \{Lit. Samoa Samoa\}

sam $v$ t. chew something and swallow the liquid but spit out the residue (e.g. sugarcane, pineapple or kava) kakae wan samting (olsem sugaken, paenapol o kava) we yu juim, yu solem daon wota blong hem mo yu spetem aot makas

sam̄ul num. ten ten

sā̄ul rō (der.) num. twenty twenti samul tiwial dēmē nilevetē (der.) num. sixteen $\sim$ sikstin

sam̄ul tiwial dēmē nilövövet (der.) num. nineteen $\sim$ naentin sam̄ul tiwial dēmēe nilōvōrō (der.) num. seventeen $\sim$ seventin

samul tiwial dēmē nilövötöl (der.) num. eighteen $\sim$ eitin

sā̄ul tiwial dēmē nirō (der.) num. twelve twelf 
sam̄ul tiwial dēmē nitevelēm (der.) num. fifteen $\sim$ fiftin

sam̄ul tiwial dēmē nitiwial (der.) num. eleven $\sim$ leven

sam̄ul tiwial dēmē nitöl (der.) num. thirteen $\sim$ totin

samul tiwial dēmē nivet (der.) num. fourteen $\sim$ fotin

samulne num. tenth, tenth one nambaten Samulne ni le na mōgōn o tañ, ni vērēg. The tenth one got his basket and ran. Nambaten hem i tekem basket blong hem, hem i resis. (JRQ)

san $1 \cdot v$. pull with quick movement, as in pulling in a fish $\sim$ pulum, jokem $\mathbf{2} \cdot v t$. kind of trap for catching chickens and birds on the ground. A rope is tied to a piece of wood and a loop made in the rope, then food is scattered around on the ground. Then the rope is pulled sharply when a bird walks inside the loop wan kaen trap blong kasem faol o pijin long graon we yu fasem rop long wud long graon, putum kakae raon long rop mo jokem kwiktaem taem pijin i stanap insaed long rop san via (comp.) v. try to pull in a fish but it gets away pulum fis be yu mestem

san qal (SVC) $v$. pull fish successfully $\sim$ pulum fis

Sanleñ n.loc. place name $\sim$ nem blong ples

Santo n.loc. Santo Santo

$\mathbf{s a n} \overline{\mathbf{n}}_{1}$ n.com. house post $\sim$ pos blong haos sañ tine (comp.) n.com. central post of house $\sim$ medel pos blong haos

$\mathbf{s a n} \overline{\mathbf{n}}_{2} v t$. touch, hold just with fingertips $\sim$ holem, tajem be wetem fingga nomo, i no wetem hol hand No ma san̄ qal o wut lō qōtōn. I caught a louse on her head. Mi holem kasem laos long hed blong hem.

san̄möt n.com. part of house pat blong haos saq $v t$. burn, as in person touching fire or something hot and getting burnt bonem, olsem faea i bonem yu, o wan samting we $\mathrm{i}$ hot $\mathbf{O}$ ev ma saq nēk. The fire burnt you. Faea $i$ bonem yu.

$\mathbf{s a r}_{1}$ n.com. ceremonial ground $\sim$ nasara

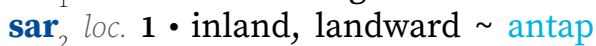
(long bus) E rōrō a siōl sar me, rēv kal sar o ak sōsō. Then the two of them came ashore, and pulled up the canoe. Ale tufala i kamso, pulum kenu i kam antap. (ATB) $2 \cdot$ inside $\sim$ insaed Qēt kemem a kal lin̄ereg sar la trak na ken i Gavin. Then we got into Gavin's car. Afta mifala i go insaed long trak blong Gavin. (AAD)

$\mathbf{s a r}_{3} v i$. noise made by lorikeet $\sim$ noes we nasiviru i mekem

$\mathbf{s a r}_{4} v t$. pull out, referring to pulling taro from ground pulumaot, olsem pulumaot taro long graon Nēk ga sar gem o qiat so lö kulë babēris ine. You are just going to pull out the taro which is behind the wall of the house. Bae yu pulumaot nomo taro we i stap bihaen long wol blong haosia. (ESP)

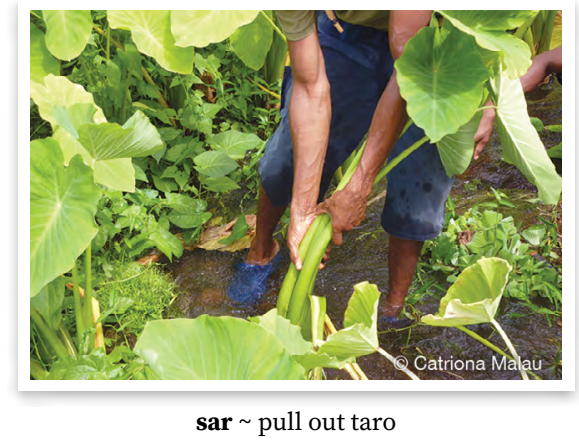

sara n.com. place ples

sara tiwial $a d v$. together $\sim$ wan ples No gö mörös ta na mem sara tiwial rōrō. I want to put the two of them together. Mi wantem putum tufala wan ples.

Sarere n.com. Saturday Sarede 
sarē n.com. variety of taro, 'male', with stem that is white with a small amount of red $\sim$ kaen taro we han blong hem i waet wetem smol red, kakae blong hem i waet mo hem i no save karem fok [Grouped with qiat rev as it has many runners. I grup wetem qiat rev from taem yu planem wan hem i save karem plante kakae.] sarōqer hol blong rop blong bodi blong kenu i brok mo wud i kamaot wetem rop mo yu nomo save fasem be mas mekem niu hol

sarsar n.com. woman who has not gained rank and learned the true traditional ways $\sim$ woman we hem $\mathrm{i}$ no bin go long nakamal La masawre nēr a èl o bul odiañ aē nēk, nēr a ēl ta nēk o sarsar. When they see that you don't have any tattoos, then they will know that you are not a woman of rank. Taem oliluk seyunogat tatu, bae ol i luk save se yu woman we yu no bin go long nakamal.

sarsarale $n . c o m$. reef lizardfish $\sim$ wan kaen fis Synodus variegatus

sartan first sign that yam is starting to dry out $~$ fes saen blong yam i stap go drae (lif i yelo o rop i drae)

sas $v$. carry something heavy, holding with one hand on either side, or two people carrying, one on each side $\sim$ karem, wan i holem wan saed, narawan i holem narasaed, o wan man i yusum tu han blong leftemap Syn: sesen̄

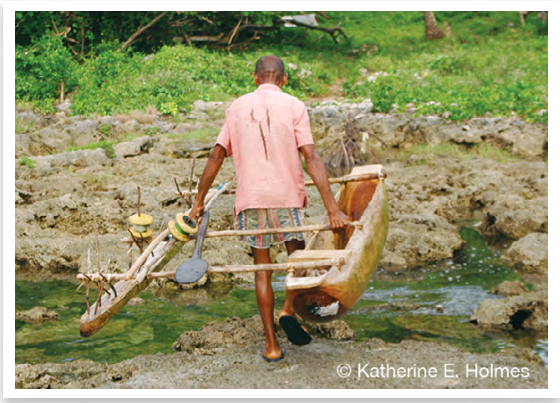

sas carry sasal vi. tie lengths of unsplit bamboo together to make walling for house wei blong mekem wol blong haos we yu dabolem ol pis bambu Nēn ga sasal lo woo ge mereñ. We make the walling with dried bamboo. Yumi fasem wol wetem drae bambu. (RBH) Syn: teren̄

Sasar n.loc. place name $\sim$ nem blong ples

sasar $_{1}$ n.com. $\mathbf{1}$ - aibika, kind of shrub which is planted for leaves that are eaten as greens aelan kabis Abelmoschus manihot $\mathbf{2} \cdot$ greens, generic term for leaves that are eaten as greens, whether planted as a crop or gathered from wild plants kabis, nem i kavremap ol defren kaen kabis [POC *wasa 'Abelmoschus manihot']

sasar $_{2}$ n.com. wrasse (fish) sop (fis) Family Labridae [This fish takes its name from sasar 'island cabbage'. It is unclear whether that is because the fish is slimy to touch or because it is a good eating fish. Fis ia i tekem nem blong aelan kabis. Maet hem $i$ from hem $i$ glis olsem aelan kabis o from hem i wan gud mo helti kakae.] sasarēt $v i$. 1 enough $\sim$ inaf $\mathbf{2}$ - same, equal $\sim$ semak, stret

sasargenravarnrōv $n$.com. bluespotted wrasse (fish) wan kaen fis Anampses caeruleopunctatus

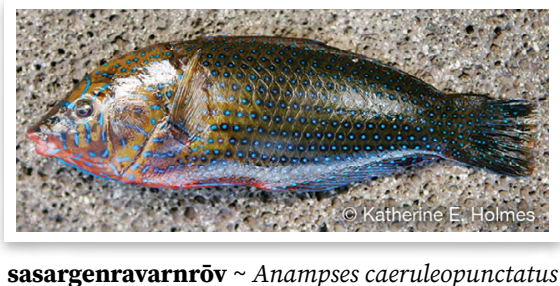

sav adj. which wanem

savan $n$ which relative of him/her, or which part of his/her body? wanem famle blong hem, o wanem 
pat blong bodi blong hem? Na savan i Nixon? What is he to Nixon? Hem i wanem famle blong Nixon?

savger n.com. thing samting

savsav n.com. thing, belongings samting, ol ting

sawa n.com. mountain lantern tree bluwud Hernandia moerenhoutiana

sawadañ $v i$. come out kamaot

seben $v$. hold up, prop up holem i stanap $\mathbf{O}$ rēntenge ine vita nē ni mès, ba no me seben gōr kēl nē. The tree was about to fall down but I propped it up again. Klosap wud bae i foldaon be mi mekem i stanap bakegen seg n.com. evodia, shrub with distinctive, pleasant-smelling leaves, variety with wider, larger leaves wan kaen lif we i gat naes smel, lif blong hem i bigwan Evodia hortensis [Leaves used as decoration on one's body, valued for the scent. Ol i yusum blong flasem bodi from smel blong hem i naes.] See: bormiak

sel $v t$. cut, using slicing or sawing motion, especially food, requiring minimal force katem (kakae o wan samting we i no strong) No me sel worwor o qiat. I cut (split) the taro (into pieces when raw). Mi katem pisisim taro. No me sel na bēnik. I cut my finger. Mi katem fingga blong mi. [POC *sele 'knife; cut with a knife']

sel $_{2} v t$. surpass, exceed, beat $\sim$ bitim $\mathrm{Ge}$ tisē sel. It is worse. I nogud bitim.

seleg $v t$. lay down flat, crosing over, as in strips as first step in working lattice basket, and laying heliconia leaves for parcelling food putum ol pis ken blong statem basket

selemëg n.com. different styles of carving defren kaen fasin blong katem wud

selen n.com. 1 10 vatu, 10 vatu coin, monetary unit used to refer to multiples of $10 \mathrm{vatu}$, up to 100 vatu 10 vatu, koen we i gat valiu blong
10 vatu mo yu save ademap kasem long 100 vatu $\mathbf{O}$ selen nitiwial nē mitē gis kēl le me. He wouldn't even bring back 10 vatu. Bae hem ino iven save karembak 10 vatu. (EDM) [This term derives from the English word 'shilling' and was introduced when imperial currency was in use, then applied to a 10 vatu coin due to the similarity in size and value. Nem ia hem i kamaot long 'shilling' we hem i mane blong Ingglis long taem blong Kondominium, mo nao ia ol i singaot nem ia long 10 vatu from saes mo valiu blong koen i klosap semak.] 2 - money, general term for money mane Mamarseg, no o selen odian̄. Sorry, I don't have any money. Sori tumas, minogat eni mane. See: baon, mil

seleqō n.com. animal animal [This class includes mammals, e.g. pig, dog, rat, etc., but not fish, birds, reptiles, etc. Wod ia i kavremap ol animol be i no fis o pijin o lised.]

selge n.com. variety of breadfruit that is good for making löt as it is neither too hard nor too soft kaen bredfrut we hem i gud blong nalot from hem i no sofsof tumas mo i no had tumas Selwō n.loc. place name $\sim$ nem blong ples

se⿳亠丷厂 n.com. white-barred triggerfish, wedge-tail triggerfish kaen strongskin (fis) Rhinecanthus aculeatus, $R$. rectangulus

seme n.com. residue remaining when the liquid has been extracted from such things as kava and sugarcane, also sawdust makas blong wan samting olsem kava, sugarcane, wud (sodas)

sen vi. lie, tell untruth giaman Syn: gial 1

sentawud n.com. sandalwood sentawud Santalum austrocaledonicum [Bis] 
ser ${ }_{1}$ n.com. spear spia

$\operatorname{ser}_{2}$ n.com. horn $\sim$ hon

$\mathbf{s e r}_{3} v t$. pay back, return money in exchange for something given pembak Nēr gete ser ten na möguk o söm. They haven't paid me back my money yet. Ol i no pembak mane blong mi yet.

sere plen n.com. airfield epot \{Lit. plane field nasara blong plen\}

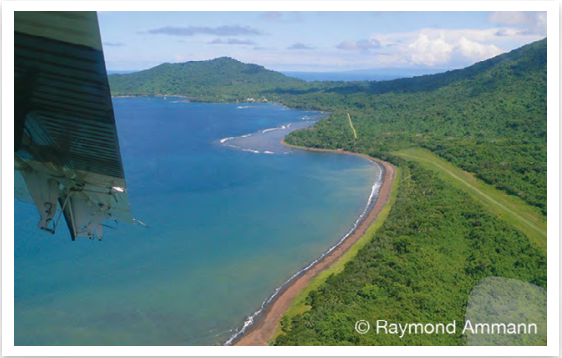

sere plen airfield

seretimiat $n . c 0 m$. area associated with the spirits of the dead, on the top of a hill nasara blong ol devel. Hem i wan ples antap long hil, wan ples we ol spirit ol i go stap long hem

$\operatorname{serev} v t$. pat $\sim$ rabem Na serev na kōlan̄ wo nēk i mōvōt. I'll pat your back and you sleep. Bae mi rabem baksaed blong. yu mo yu slip.

serevugvug n.com. meeting place for spirits of the dead, underneath a banyan tree ples we ol devel ol i mit long hem. Hem i wan ples andanit long wan nambangga we i klin we i klin

Serewan n.loc. place name $\sim$ nem blong ples

sergeg $v$. (after plaiting together sections composed of two pandanus half leaves) lay sections down, overlapping, ready to plait together $\sim$ tekem ol pis pandanas we yu bin wivim tu lif tugeta, joenem plante tugeta blong statem mat

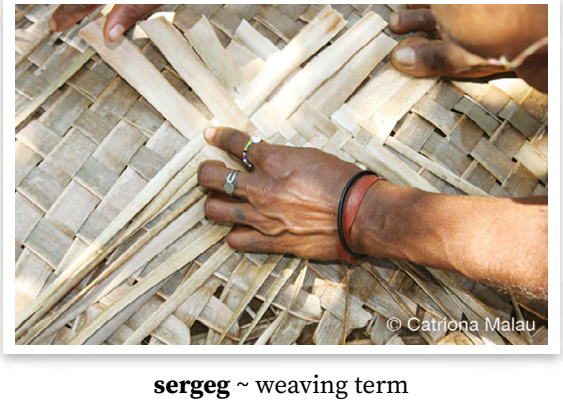

sernönqet n.com. kind of sea urchin with sharp spines samting blong solwota we i olsem sel mo i gat ol sap nil long hem Family Diaematidae See: wadas $\{$ Lit. Qet's spear spia blong Qet\}

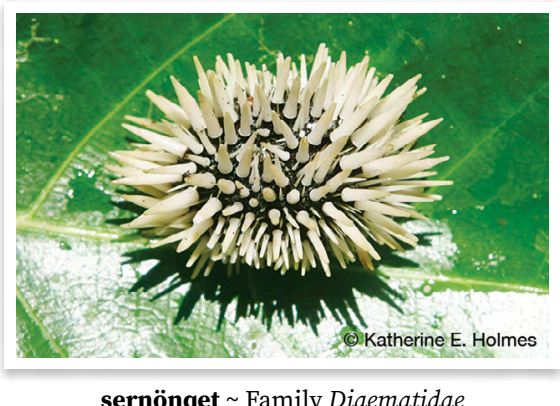

sersöm $v$. return some of bride price if a couple divorce, the woman's family must return money to the man's family who paid the bride price $\sim$ givim bak mane sapos mared $i$ finis mo man mo woman $i$ divos, famle blong woman i mas mekem long famle blong man we ol i bin pem woman ia

sesen $v t$. carry something heavy, holding with one hand on either side, or two people carrying, one on each side karem, wan i holem wan saed, narawan i holem narasaed, o wan man i yusum tu han blong leftemap Dōrōk a seseñ me o tabē ti a mem kal o wērēt le ev. Let's lift the wooden platter over here so that we can put the octopus on the fire. Yumitu 
leftemap natambia i kam blong yumitu putum nawita $i$ go antap long faea. (WNW) Syn: sas

sesev $v t$. shave wood, shave off bark or outside of wood sevem, karemaot pat blong wud wetem naef 0 rēntenge ko no te sesev in̄kē, na sian o wëër. This wood that I am shaving down is called casuarina. Wud ia we mi stap sevem ia, nem blong hem oktri. (VVKHVDP180ct0801-071)

$\mathbf{s e v} v$ t. $\mathbf{1} \cdot$ pluck $\sim$ tekemaot feda $\mathbf{2} \cdot$ strip, remove leaves from branch, remove branches from trunk tekemaot lif long wud Sev o doot den o labevi. Pull the sago palm leaves off the palm. Karemaot natanggura long han blong hem. [POc *sapi 'strip (leaves); pluck (fuit, nuts)']

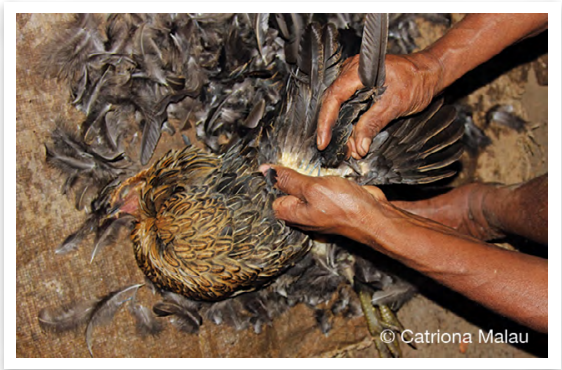

$\mathbf{s e v} \sim$ pluck

seve n.com. piece, crumb, scrap pisis, pat $\mathrm{Na}$ gëk o seseve gengen ni mësmës sōw lo qarörö. Bits of my food were falling down into a hole. Ol smol pis kakae blong mi i stap foldaon insaed long hol.

sewerles n.com. several species of hawkfish wan kaen fis Neocirrhites sp., Paracirrhites spp. See: weteltelvet

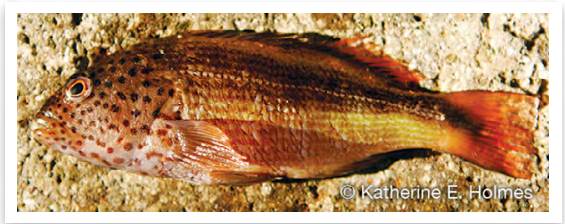

sewerles $\sim$ hawkfish $\mathbf{s} \overline{\mathbf{e}}_{1} v t$. peel bark from wood karemaot skin long wud

$\mathbf{s} \overline{\mathbf{e}}_{2}$ (root of isē)

Sēbēr $n$.loc. $\mathbf{1} \cdot$ place name $\sim$ nem blong wan ples $\mathbf{2} \cdot$ tribe name, members of tribe are considered to be wise, inventors nem blong traeb we olgeta ol i waes tumas

sēbēr $v t$. throw something out of the way when it is in the way or not wanted sakem wan samting we $i$ stap long rod o i blokem rod

sēg n.com. kingfisher (bird) nasiko, longmaot (pijin) Todiramphus spp.

sēg malgias (comp.) n.com. Pacific kingfisher nasiko Todiramphus sacer

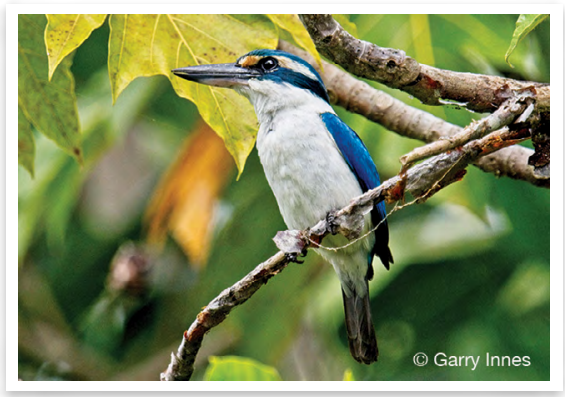

sēg malgias Todiramphus sacer

sēg mamēe (comp.) n.com. sacred kingfisher red nasiko Todiramphus sanctus \{Lit. red kingfisher red nasiko\}

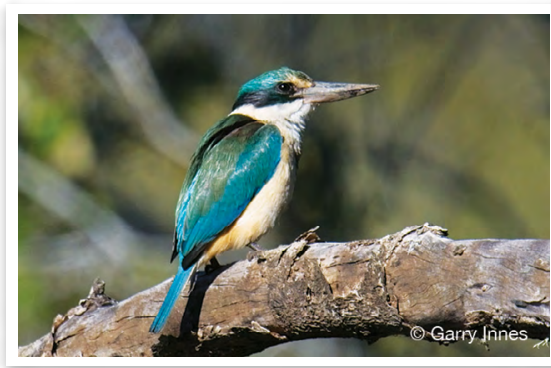

sēg mamē Todiramphus sanctus 
sēk $v t$. look for lukaotem Na van ti a lo, na sēk na möguk o barbar. I'll go down to the sea and look for my axe. Mi go daon long solwota, mi lukaotem akis blong mi.

sēlēg n.com. dark and cloudy, rain clouds tudak klaod, saen blong ren sēlēg vi. go to stay or sleep in another house because one is unable to sleep in one's own house due to damage as result of cyclone, rain, etc. go slip long narafala haos from haos blong yu i nogud from hariken o wan samting i spolem Ri sēlēg kēti me gēn, la masware o leñ ni qēt, kēmi kara mōl kēl. Come and stay here for a while, and when the wind is over, you can just go home again. Iufala $i$ kam stap long ples ia fastaem, ale taem win i finis, bae yufala i jas go hom bakegen. sēlēgtel $n . c o m$. variety of taro $\sim$ kaen taro

sēlsēl vi. shine, emit light laet, saen $\mathbf{O}$ tere wamasē nēr gē sēlsēl. The stars are shining. Ol sta ol $i$ saen. Ant: sil

sēm 1 t. 1 - peel, remove skin from (something hard, as uncooked vegetables, copra) tekemaot skin (blong samting we i strong olsem taro o kopra) Na sēm o kuk, na vasgēr kal. I peel the vegetables for cooking, and I put it up (on the fire). Mi karemaot skin blong kuk, mi putum igo antap (longfaea). (DDL) 2 • remove coconut flesh from shell and husk, to produce copra selaotem Nēr mē sēm o bek kopra nives? How many bags of copra did they produce? Ol $i$ selaotem hamas bag kopra? See: wul

sēm $_{2}$ vi. lead off main path, as small path or minor road kamaot long narafala rod, olsem smol rod Nēk ma var kal siag me, nēk i èl o gersal gē sēm kal sar. You go up a bit, and you see a path leads off inland. Iu go antap smol, yu luk wan rod i kamaot $i$ go antap. (RRS)

sēn vt. strain, pour through cloth or coconut sheath to remove residue (from kava, coconut, etc.) sevem (olsem kava, kokonas) Nē mē qēt, nēk i sēn, nēk i sēnsēn qēt, nēk i sum. When that is done, you strain it (kava), and when you have finished straining it, you drink it. Taem ifinis, yu sevem (kava), mo yu sevem finis, yu dring. (OSK)

sēnsēn (redup.) n.com. strainer for kava made of sheath of coconut frond sef blong kava we ol i mekem long blangket blong kokonas See: dinir

sēnē n.com. variety of banana kaen banana \{Lit. Sydney Sidni\}

sēqēg n.com. kind of tree wan kaen tri Elaeocarpus floridanus

sēr $v$ t. tear, rip terem $\mathbf{O}$ leñ mē sēr na mōgōr o geben. The wind tore their sail. Win i terem sel blong olgeta. See: mēsēr

sēriv n.com. waterfall wotafol

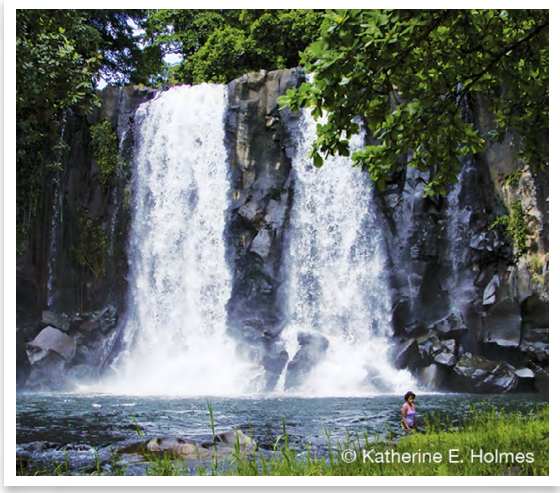

sēriv waterfall

sērsēr n.com. kind of tree like stinkwood but bigger and harder wood wan kaen tri olsem stingwud be i bigwan mo i strong 
sēs $v t$. remove stones from oven, take something out of basket $\sim$ karemaot ston long oven, karemaot samting long basket Na sēs qēt o um, nana le o ralèt, na tuwegev. I take all the stones out of the stone oven, I get the firewood, and I prepare and light the fire. Mi karemaot ol ston long oven, mi karem faeawud, mi mekem faea. (NBQ.004)

sēs um (comp.) $v$. remove stones from oven hole ready to make fire in oven tekemaot ston long hol blong oven redi blong mekem faea

sēsēdig n.com. method of trapping birds wei blong kasem pijin wetem trap we yu fasem rop long brans blong tri mo yusum frut blong atraktem pijin

ga sēsēdig (comp.) n.com. rope for trapping birds rop blong trap blong pijin

sēsēlēg vi. dark, of sky, place (ples i) tudak $\mathbf{O}$ masawre mē sēsēlēg. It is dark. Ples i tudak.

sēsēlit n.com. water music wota miusik

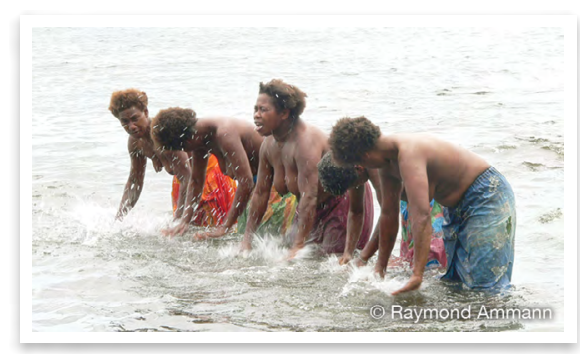

sēsēlit water music

sēsēvēl $v i$. slanted, not flat no stret, no lei flat $\mathbf{O}$ tan gō ōn sēsēvēl. The land is slanting. Graon i lei i no stret.

sēstan̄ n.com. variety of taro, 'male', with a tall light green stem with red stripes $~$ kaen taro we hem i man, we han blong hem $\mathrm{i}$ laet grin mo $\mathrm{i}$ gat red straep long hem sëkër n.com. reef, coral, refers both to an area of reef and to coral heads that comprise the reef $\sim$ rif

sëqëtutu (fr.var. of qëtutu)

$\mathbf{s e ̈ r}_{1} \mathbf{1} \cdot v i$. spill, be spilt $\sim$ kapsaed $\mathbf{O}$ gē më sër la tan. The kava spilt on the ground. Kava $i$ kapsaed long graon. 2 - vt. spill, pour, tip out (anything, either liquid or solid) kapsaedem Nē më sër o gē la tan. He spilt the kava on the ground. Hem i kapsaedem kava long graon. Taktaklē tan̄sar nēr a sër o gengen lō qō bulën nēr. Some people tip the food out for their pigs. Samfala man ol $i$ kapsaedem kakae blong givim long pig blong olgeta. (HWM) See: lin̄

sër $_{2} v t$. shine on (of sun) (san i) saen O lō ni sër mamas, alē nana vir. The sun shines on it (cottonwood) and makes it dry, then I plait it. San i saen long hem, draemap hem (burao), ale mi tanem.

sër $_{3} v t$. wear, put on (clothes, accessories) werem, havem (klos, savat) In̄ko kemem a sër na mölön kemem ga mamas. Then we put on our dry clothes. Nao ia mifala i werem drae klos blong mifala. (AAD) See: wësërsër

sër lō (comp.) vt. take off, remove (clothes, accessories) karemaot (klos) E i Peter Posa ni sër lō na mōgōn o sefti den o diri timiat, e nē ni sër. Then Peter Posa removed his work boots from the devil's feet, and he put them on. Ale Peter Posa i karemaot sefti blong hem long leg blong devel, ale hem i werem.

sërëv $v$. pat brasem (wetem han) sërëvtës $v i$. slip glis No më sërëvtës. I slipped. Mi glis.

sësëwu n.com. heat (of something) hit (blong wan samting we i hot) sësëwu bē (comp.) n.com. steam stim, smok blong wota we i boel 
sësëwu ev (comp.) n.com. heat of fire hot blong faea La masawre o bē ni gök o sësëwu ni sëw lō den. When water boils, steam rises from it. Taem wota iboel, stim i kamaot long hem.

sëwëw $v t$. grab, catch something with quick movement kasem kwiktaem nomo $\mathbf{O}$ genqirēg më sëwëw $\mathbf{o}$ gengen. The dog caught the food with a quick movement. Dog i kasem kakae hariap nomo.

si subord. if, introduces conditional clause sapos Ba si kōmōrōn̄ gö mörös, no ga gagneg le min kōmōrōn. But if the two of you want, I could tell you. Be sapos yutufala $i$ wantem, bae mi save talemaot long yutufala.

si ${ }_{2}$ conj. or o Nē gē riv o ur si o man̄ko. It will plant a hog plum or a mango. Bae hem i planem naos o manggo. (DFF)

$\operatorname{siag}_{1} v i$. sit $\sim$ sidaon

$\operatorname{siag}_{2}$ loc. 1 - upward antap Vël qön̄ rōrō a togtog siag e li geret. Every day the two of them stayed up there on the high hill. Evri dei tufala i stap antap ia long bigfala hil. (DHN) $2 \cdot$ to the left when facing the sea, of places that are some distance away, not close by $\sim$ fes i go daon long solwota, go long lefsaed, be longwe lelebet Nē mē qētēg siag a Revi, o sie masawre nēr gō ōl a Revi. It started up there at Revi, the name of that place, they call it Revi. I stat antap long Revi, nem blong ples ia ol i singaotem se Revi. (RTF.002) See: wōl $1_{2}$ [POc *sake 'up above, go up']

siag gēgēt ${ }_{1} v i$. squat $\sim$ sidaon, olsem yu bendem leg mo as blong yu i no tajem graon

siag gēgēt ${ }_{2}$ n.com. variety of taro, 'female', with light red stem with white stripes $\sim$ kaen taro we hem i woman we han blong hem i laet red wetem waet straep sial vi. float $\sim$ flot Rōrō a ēl o wabalak ni sial me. The two of them saw the fruit of the Ficus wassa floating towards them. Tufala i luk frut blong nambalanggo iflotikam. (GMM)

sial mōōtōtō vi. level, even level Nēn a sēk o masawre avē gē sial mōōtōtō tabo nēn a wos o gövur aē. Let's look for a place that is level so that we can build a house there. Yumi lukaotem wan ples wea hem i level blong yumi bildim wan haos long hem.

sialtislan̄ $v i$. slip glis Nēk i sialtislañ. You slipped. Yu glis.

sian n.com.suff. her/his/its name $\sim$ nem blong hem See: sie

siaw $_{1}$ n.com. food dish in which sliced ripe breadfruit is layered with canarium nuts, wrapped and baked in stone oven wan kaen kakae we ol i slaesem raep bredfrut mo lerem wetem nangae we ol i bin rasras

siaw $_{2}$ throw something disgusting, like rotten fruit or shit at someone sakem wan rabis samting long wan man Nēk mē siaw no tek na tian̄. You threw your shit at me. Yu stonem mi wetem sitsit blong yu.

sie n.com.suff. name $\sim$ nem Gisine i rege min tasēn, na sien rōrō, tōar na sian i Mōlwor, wo tōar i Mōlgon. Once upon a time there were two brothers, and the names of the two of them, one, his name was Molwor, and the other, his name was Mōlgon. Wantaem i gat tufala brata, nem blong tufala, wan, nem blong hem Mōlwor, mo narawan nem blong hem Mōtgon. (GMM.005)

sian (n.poss) n.com.suff. her/his/its name nem blong hem

sian gōgōn n.p.fr. name used to refer to or address someone who has the same name as an in-law whose name one is not permitted to say, due to cultural restrictions of the relationship nem we yu yusum 
blong talemaot wan man o woman we hem i gat semak nem olsem wan tawian o palika blong yu, from we nem i semak, i tabu blong singaotem nem Lit. her/his name is taboo nem blong hem i tabu\}

siëk (n.poss) n.com.suff. my name nem blong mi

siëk n.com.suff. my name nem blong mi See: sie

siër (fr.var. suër) vt. find faenem

siganar $v$. remove rope from the neck of a man who has not met the goal of giving up a certain food for 1,000 days during the mourning period after the death of a close relation tekemaot rop (ganar) long wan man we hem i no save lego kakae we hem i bin talem se bae hem i no kakae kasem wan taosen dei blong wan man long famle blong hem i ded See: ganar

sigsig $v$. harvest taro from paddy gardens $\sim$ karem taro long garen

sil n.com. darkness, pitch black tudak Ant: sēlsēl

silat n.com. $1 \cdot$ nettle tree, fever nettle, devil nettle $\sim$ nanggalat Dendrocnide spp. 2 - jellyfish $\sim$ nanggalat blong solwota

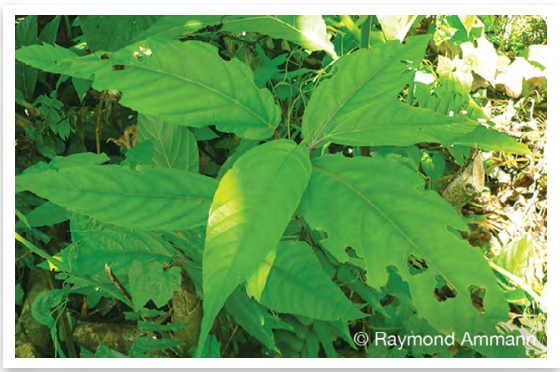

silat Dendrocnide spp.

silat gegeretō (comp.) n.com. kind of nettle tree that has a powerful sting wan kaen nanggalat we hem i kakae man i strong sili n.com. sucker of banana, shoots from taro or other tubers, used to propagate new plants saka, olsem blong banana o narafala plant, blong planem $\left[P O c *{ }^{*}[s, j] u l i(q)\right.$ 'banana or taro sucker, slip, cutting, shoot (i.e. propagation material)']

simēdēg (fr.var. simidēg) vt. hang up object, as in basket from strap, or objects hanging on length of rope $\sim$ hangem wan samting, olsem we i hang long rop $\mathbf{O}$ mes ine, ni simideg ti. The fish, he hung it up. Fis ia hem $i$ hangem. (JMT) See: diam

simidēg (fr.var. of simēdēg)

sinag n.com. 1 - taro $\sim$ taro Nēn a van a ev o sinag. Let's go and remove the taro from the ground with a digging stick. Yumi go karemaot taro wetem digging stick. 2 - food kakae Syn: gengen 1

sinav n.com. kind of insect that is like a cockroach but small wan kaen bebet we hem i olsem kakros be i smolsmol. Ol i laek blong stap antap long faea from we ol i laekem hit mo smok blong faea

sinsa n.com. ginger $\sim$ jinja [Bis jinja 'ginger']

siōl vi. go ashore kamso Ni sō dēn̄ lö nötu vōnō nitiwial, e ni siōl sar. He paddled until he reached a little island, then he went ashore. Hem i parel kasem long wan smol aelan, ale hem $i$ kamso $i$ go antap. (ATB.013) Syn: tiabasas

sir vt. 1 - scrape, scrape dirt from the outside of taro and other root vegetables $\sim$ skrasem doti (blong taro) $2 \cdot$ remove skin, bark $\sim$ tekemaot skin $\mathbf{3} \cdot$ shave $\sim$ sevem Nē mē sir qēt na wēwēn̄ēn, qēt nēr a van lē sir wērēntenge. He finished shaving his beard, then they went to 
remove the bark from some trees. Hem i sevem mustas blong hem finis, afta ol i tekemaot skin blong wud.

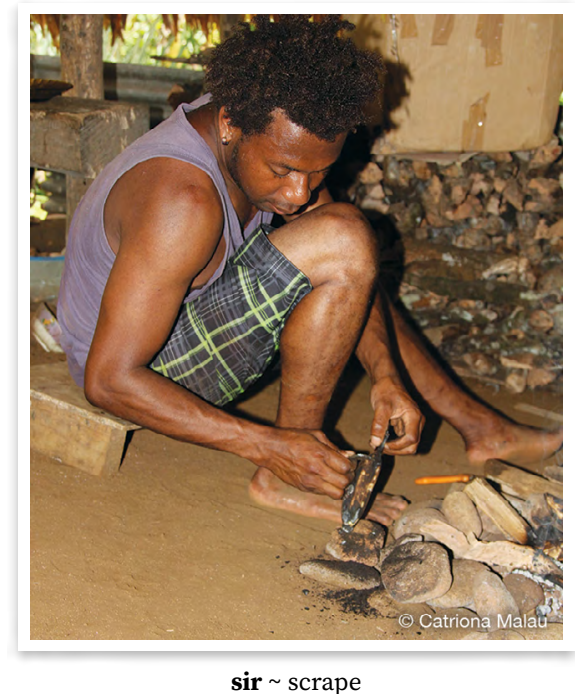

siri ${ }_{1}$ n.com. $\mathbf{1}$ bone $\sim$ bun $\mathbf{O}$ mes ine, siri na mōgōn gem mō ōn ti. That fish, just its bones were left lying there. Fis ia, bun blong hem nomo i leidaon $i$ stap. (DNS) 2 - midrib, midvein of leaf, leaflet $\sim$ bun blong lif $\left[P O{ }^{*}{ }^{*} s u R i\right.$ 'bone']

sērēn (n.poss) n.com.suff. her/his/its bone(s) bun blong hem

sirik (n.poss) n.com.suff. my bone(s) bun blong mi

siri ${ }_{2}$ n.com. handle, hard handle as of cup, saucepan handel, olsem blong kap, sospen Syn: bele

siri qatē n.com. pieces from inside of trunk of tree fern, which is composed of layers of old branches that are brittle ol sap pisis blong insaed blong blakpam

siri wawalag n.com. shin bone $\sim$ bun blong leg daon

siritimiat n.com. variety of taro, 'male', in mare grouping with dark stem, black petiole and red and white corm kaen marē taro we hem i no save bigwan be i save longfala mo i save benben. Han blong hem i dak popel mo kakae blong hem i miks laet red wetem waet. Hem i sofsof mo hem i taro blong rus we i save dan kwik \{Lit. devil's bones bun blong devel\}

siriv vt. peel dried pandanus leaves from the plant $\sim$ karemaot lif pandanas we i drae finis long stamba sis $v i$. be full, sated fulap Na tëqëk mē sis. I am full. Mi fulap.

sis $_{2}$ vi. melt, dissolve, liquify, flow $\sim$ melt, ron Kemek a le o bur ne, a mem le ev ni sis. We get the canarium sap and put it on the fire so that it liquifies. Mifala i tekem wota blong nangae, putum long faea hem i melt. (VVKHVDP180ct0801.122)

sisia $v i$. different $\sim$ defren

Sisiōl n.loc. place name $\sim$ nem blong ples

sislaqlaq n.com. chicken pox $\sim$ jikinpok siwia n.com. kind of public dance kaen pablik danis

siwit n.com. variety of banana $\sim$ kaen banana [Bis swit 'sweet']

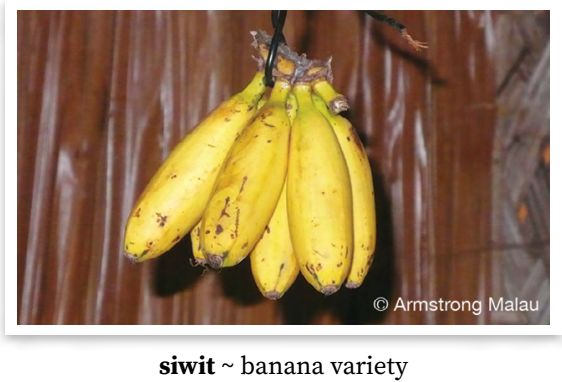

skul 1 •n.com. school $\sim$ skul $\mathbf{2} \cdot v i$. go to school, carry out schooling skul, go long skul

so 1 subord. relativiser, that $\sim$ ia Nēn a gengen lo Chinese Restaurant so $\mathrm{i}$ nēn me gengen ti aē birin̄ i Sabine. Yumi kakae long Chinese Restron we yumi bin kakaelong hem wetem Sabine. 
so ${ }_{2} v t$. cut sago leaves, bring and prepare thatch $\sim$ mekem natanggura Nēn mo so o doot, nēn a tek me, a vidir, a vēn, qēt, in̄ko nēn a at. We remove the sago palm leaves, we bring them, we remove the midribs, we sew them and then we tie the thatch to the rafters. Yumi tekem natanggura, yumi karem i kam, karemaot bun, somap, finis, nao ia yumi fasem i go long bambu. (RBH.016)

$\mathbf{s o}_{3} v t$. gather, harvest palolo worm karem palolo, wom blong solwota

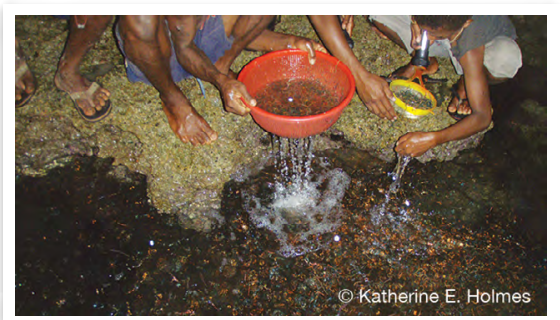

so gather palolo

$\mathbf{s o}_{4}$ (root of OsO)

so gamēlēs remove midrib from sago palm but fail to remove it properly, such that the leaf will snap when one tries to bend it to make thatch karemaot bun blong natanggura be yu no karemaot gud

sodēmēèl $v i$. arrange for people to come together, gather together people for

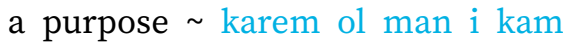
wan ples from wan samting

sogolo $v$. cross over, as in watercourse, piece of land, log krosem, olsem krosem wota o pis graon Nēk i èl o qereñ bē nitiwial, nēk i sogolo o qereñ bē ine, o bē ine na sian o Bē Nunur. You see a creek, and you cross over that creek, the creek is called Nunur Creek. Yu luk wan wota, yu krosem wota ia, wota ia, nem blong hem Nunur Reva.

sogon n.com.suff. her/his descent group laen blong hem See: sögö soko dem. emphatic marker, expression of surprise or emphasis regarding an item or event $\sim$ hemia ia $\mathbf{O}$ gēnē nitiwial in̄kē nē gē sisia soko. This thing here is really different. Wan samting ia hem i defren olgeta. (RTF) Soko oso? What on earth is that? Hemia wanem ia? (ESP.043)

soko $_{2} v t$. catch $\sim$ kasem

Sol n.loc. place name, Sola, on east coast, where province headquarters is located nem blong wan ples, Sola, ples we provins i stap long hem sol $v$. flow heavily (wota i) ron bigwan sosov n.com. skin condition caused by getting too wet and cold in rain, resulting in swollen, itchy, spotty skin skin i skras, i solap, i tik, from yu stap long taem tumas long ren mo kolkol No mö turtur gōr idian o wian, e ma da vita o sosov ma da no. I spent too long in the rain, and it affected my skin and made it itchy. Mi stanap tumas long ren, ale i mekem se skin blong mi inogud long hem.

sospen n.com. saucepan sospen

sot n.com. shirt sot [Bis sot 'shirt']

sō vi. paddle parel, pul long kenu See: wōs

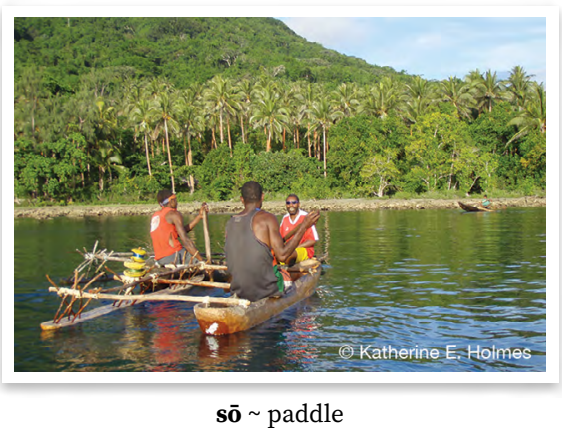

sōbōn $v t$. smell, sniff scent, active sensing of smell smelem In̄ko ni sōbōn tatag o requ mē vērēg. Then he smelt the trail of the woman who was running. Nao ia hem i smelem folem woman we i resis. (LLF.053) 
sōbōt n.com. variety of banana with small, thin, bent fruit and short trunk kaen banana we kakae blong hem i olsem samo be stamba i sotsotfala

sōg $v$. give generously giv wetem gladhat Nē gō sōg na gan vaga sur na metwōn o mētigwē. He often gives away his food because he has a generous character. Hem i givim gudfala kakae blong hem oltaem long ol man from fasin blong hemi gud.

sōgsōg $n$ n.com. variety of coconut that bears a large number of small fruit which have sweet liquid inside kaen kokonas we i karem fulap frut we ol i smolsmol mo wota blong hem i swit

sōgsōg $v$ vi. be generous $\sim$ gat gladhat sōgsōggenqet n.com. kind of tree kaen wud Phaleria pentecostalis

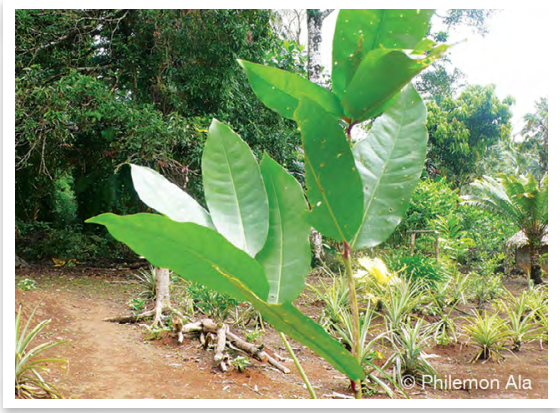

sōgsōggenqet $\sim$ Phaleria pentecostalis

sōk $v$. find $\sim$ faenem

sōksōk $v$. hunt hant

sōl n.com. young coconut seedling, general term for germinated coconut navara

sōlvet (comp.) n.com. young coconut seedling that is firm and sweet navara we $\mathrm{i}$ strong mo $\mathrm{i}$ swit \{Lit. coconut seedling stone navara ston\}

sōl kētkēt n.com. coconut which has germinated while still hanging to the tree, which has very sweet flesh $\sim$ kokonas we i kam navara taem i stap hang yet long stamba mo i no foldaon hem wan, navara blong hem i swit tumas \{Lit. germinated coconut up in the air navara long antap\}

sōltek $v$. carry on one shoulder, especially heavy items $\sim$ karem (wan hevi samting) long wan solda

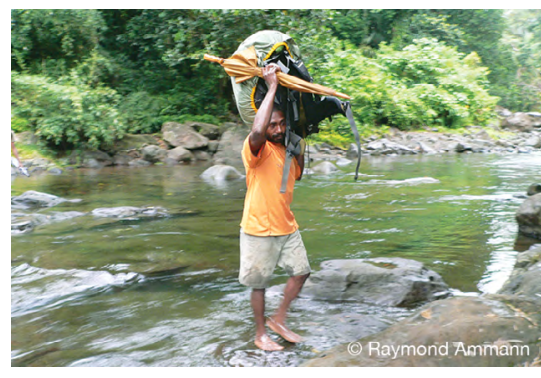

sōltek carry on shoulder

sōm n.com. parrotfish, refers to all large species, when they are blue blufis, ol bigwan blong hem Family Scaridae

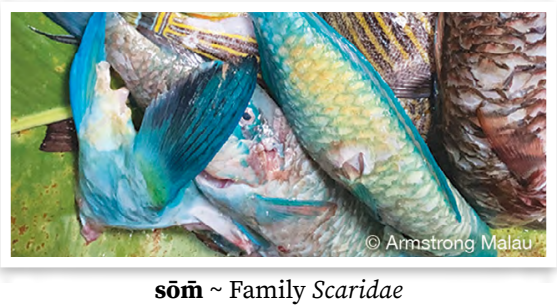

$\mathbf{s o ̄} \mathbf{q} v$. perform grade-taking ceremony, by killing of pig kilim pig long seremoni blong tekem rang sōravar n.com. Vanuatu fruit dove (bird) bigfala grinpijin Ptilinopus tannensis

sōrsōr $v i$. noise made by cicada noes we bebet we i kolem se bōn i mekem See: bōñ; wōsōr

sōs $v t$. start fire by rubbing stick on wood rabem wud blong statem faea Nēr gō sōs o ev aē. They would start a fire with it. Bae ol i statem faea long hem. (VVAMVDP05Jun0801-SF.011) sōsōg vi. fish, go fishing go huk 


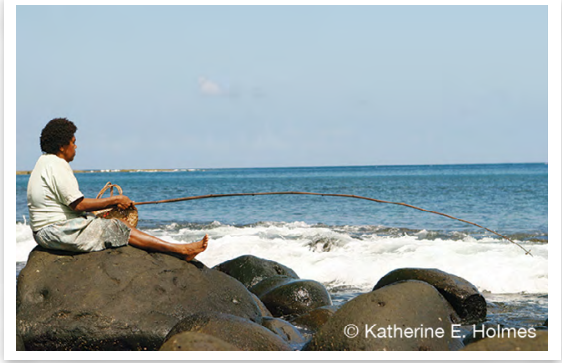

sōsōg go fishing

sōsōn n.com.suff. her breasts titi blong hem See: sus $_{4}$

sōsōrvël $v$. carry using length of wood over shoulder, with half the load in front and half behind karem long wud long solda No go sōsōrvël, wo sā̄ul tavalgi, sam̄ul tavalgi. I am going to carry them on my shoulder with a length of wood, with ten on one side, ten on the other side. Bae mi karem long wud long solda, ten wan saed, ten nara saed. (KM̄T)

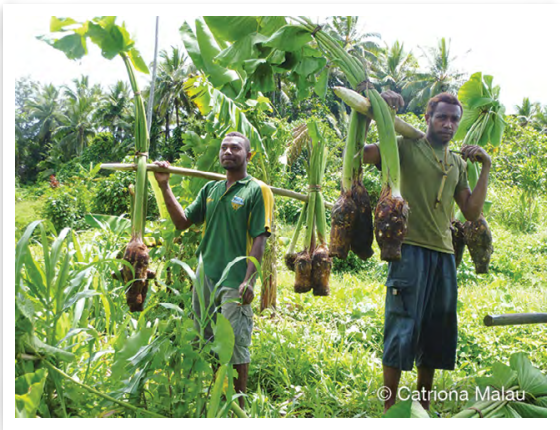

sōsōrvël carry on stick

sōw 1 - loc. towards the right when facing the sea, in a clockwise direction around the island $\sim$ sapos yu fes i go daon long solwota, go long raetsaed Na nogon gē kēl sōw sur o tönö tulnö lō. He is facing in a rightwards direction, towards the place where the sun goes down. Hem i fes $i$ go long saed ia, long ples we san i draon. (M2E) 2 - loc. down, downwards, down low $\sim$ daon, stap daon Ba o qon nē ma gav tēqēl ren

sōw e rivteg o n̄êlida naw. But the pigeon was flying right down low close to the surface of the sea. Be nawimba $i$ stap flae daon olgeta, klosap long sefes blong solwota. (DHN) $\mathbf{3} \cdot$ vi. go down $\sim$ go daon E ni sōw, sōw dēñ la tan. Then he came down, came down to the ground. Ale hem i kamdaon, kamdaon kasem long graon. (JRQ) 4 • vi. reduce in size, value, deteriorate $\sim$ go daon (saes, namba o kwaliti) Na tarbian mō sōw. You have lost weight. Bodi blong yu i go daon. [POc *sipo 'down below, go down']

sōwereg $v t$. plant yam or water taro in the same place where it was dug up planem yam o wota taro i gobak long sem ples we yu bin tekemaot Kēmi min le o gengen, sōwereg kēl. If you take any staple foods, then replant them. Sapos yufala i karem eni kakae, yu planem bakegen. (HWM) [Term is relevant because the usual practice is for yam to be dug up or taro pulled out of the ground and replanted in a different place.]

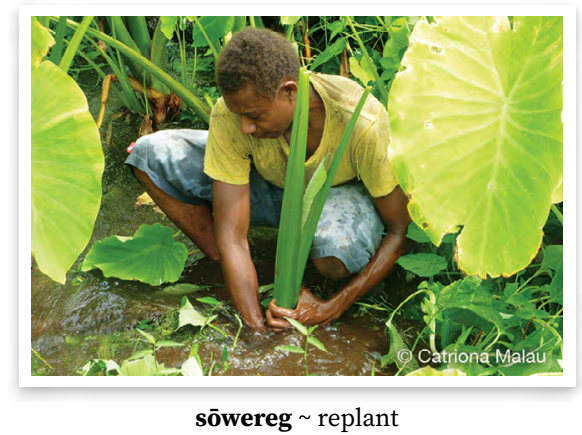

sö $\mathbf{1} \cdot v t$. join joenem No mö sö o vötu rēntenge nirō. I joined two pieces of wood. Mi joenem tufala pis wud. 2 • n.com. joint, as in joint where two bones meet, joint in crafted wooden item joen, olsem joen blong tufala bun o joen long wud See: m̄önug 


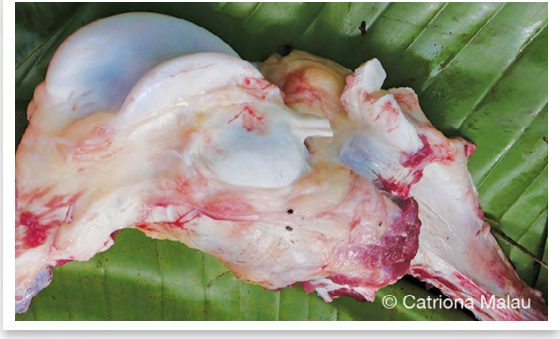

sö $\sim$ joint

sögö n.com.suff. moiety, descent group laen Nē gē ēl na ginon̄, na sogon.

Nē te lele kēl na sogon sur o tan. He finds your spouse, someone in his descent group. He takes someone who is in his descent group because of land ownership. Hem i luk woman o man blong yu, hem i laen blong hem. Hem i stap tekem laen blong hem bakegen from graon. (RKM)

sogon (n.poss) n.com.suff. her/his descent group laen blong hem sögök (n.poss) n.com.suff. my descent group laen blong mi

sögök n.com.suff. my descent group laen blong mi see: sögö

sökö $v$. throw in one place $\sim$ sakem long wan ples

sölö vt. put taboo, place marker to indicate restriction, usually a cycad leaf is placed to indicate, for example, that people should not fish or should not take the fruit from a tree putum namele lif, olsem tabu blong soemaot se ol man ol i no sud mekem wan samting, olsem tekem kakae long wan wud No mö sölö gōr o wotag. I placed a marker to indicate that people should not take the cutnuts. Mi putum namele blong blokem navele. sösölö (redup.) n.com. a cycad leaf placed to indicate a restriction on use of plant or area lif namele we yu putum blong makem tabu

söm n.com. 1 • money mane No tö wölwöl aē so o tere söm ine. I was buying things with that money. Mi stap pem ol samting wetem mane ia. (EDM) Kemek töl a mem o söm ine la ban̄. The three of us are going to put the money in the bank. Bae mitrifala $i$ putum mane ia long bang. (VSS) Syn: tiveg $\mathbf{1} \mid \mathbf{2}$ - shell money, traditional money, used as a value item for trading and payments in cultural exchanges sel mane we ol i bin yusum bifo blong pem ol samting long kastom Nēr töl nēr mē bit o söm min nē. The three of them broke off some shell money and gave it to him. Trifala i brekem sel mane i givim long hem. (ESP)

sömodiañ n.com. ring finger namba fo fingga long han \{Lit. no money nogat mane\}

sönötu (fr.var. of nötu)

sön $v t$. comfort, calm someone who is cross or sad kamfotem, traem mekem wan we hem i kros o i sad i harem gud bakegen Vita, 'Oo, ni ten̄teñ qaqaqa gem.' Rōrō a van me, rōrō a sön̄ nē. Vita, 'Taron̄, tarō̄, tarō̄, me dese?' He said, 'Oh, he's just crying unnecessarily.' The two of them came and comforted him. They said, 'Calm down, calm down, what's wrong?' I se, 'O, hem i stap krae krangke nomo.' Tufala i kam kamfotem hem. I se, 'Stap kwaet, stap kwaet. Wanem i rong?' (JMT.188-190)

sön̄ön vt. put inside (container) fulumap insaed Söw rōrō a bis o tere tewen ine, rōrō a sönön lē tibiar. So the two of them picked the native lychees and they put them in the basket. Ale tufala i tekem ol nandao ia, tufala ifulumap long basket. (OQQ.009) Syn: nës

sön̄sȫn $v$. make joke mekem fani sör n.com. nicolaia; plant from the ginger family that has nice-smelling leaves, used as decoration for dancing and in the house kaen lif we hem i famle blong jinja, i gat 
naesfala smel blong hem mo ol i yusum long danis olsem dekoresen Hornstedtia sp.

sösögöt $v$. find $\sim$ faenem

sötel n.com. proud, pride praod, wan blong mekem flas blong hem No gata tan̄sar talo sötel. I am not a proud man. Mi no man blong mekem flas blong mi.

söw (abbrev. of söwlē)

söw malgias n.com. variety of breadfruit $\sim$ kaen bredfrut

söw taban n.com. variety of breadfruit kaen bredfrut

söwlē (abbrev. söw) conj. then, so ale spidbōt n.com. variety of sweet potato that is named for the fact that it grows very quickly $\sim$ kaen kumala we ol i putum nem blong hem from hem i gru kwiktaem tumas

stori n.com. storey house stori haos

suër $v$. find $\sim$ faenem See: siër

sugsugte n.com. marks of pigs from where they have been digging around in the ground with their noses mak blong pig long ples we $\mathrm{i}$ stap digim graon

sul 1 vt. $\mathbf{1} \cdot$ light (fire) laetem faea $\mathbf{N a}$ van me lē kijin na mun kemek, na sul o ev. I come into our kitchen and I light the fire. Mi kam long kijin blong mifala, mi laetem faea. (DDL.001) 2 - burn (something) bonem (samting) [POC *sulu 'dry coconut leaf torch']

susul (redup.) v. $\mathbf{1}$ burn a bird after plucking bonem pijin taem yu tekemaot feta finis $\mathbf{2} \cdot$ irritate mouth (said of taro and wild yam) taro o wael yam i kakae man

sul $_{2}$ n.com. people, general population, unified group of people $~$ pipol, ol man In̄ko o sul ma datek o sum gē le meren. Now people generally are drinking kava in the daytime. Nao ia ol man ol i stap dring kava long dei. (OSK) sulör $v$. poke stikim Sulör na men nēn o vōs. Poke a drinking coconut for us (so it falls from the tree). Stikim (grin) kokonas blong yumi.

sum $v t$. drink dring Kemem gö sum o gē tastas. We drink kava all the time. Mifala i stap dring kava oltaem. (OSK.001)

sumsum (redup.) n.com. 1 • drink dring Rōrō a wöl o tere sumsum wo o taklē gengen wo o bred. The two of them bought drinks and some food and bread. Tufala i pem ol dring, mo samfala kakae mo bred. (AAD) 2 - alcohol, alcoholic beverage dring, alkohol

sunötu (fr.var. of nötu $)$

sur $_{1}$ prep. $\mathbf{1} \cdot$ towards, marks allative role, motion towards a goal long, from Kōmōrōk gö lölö vita a vērēg kal kēl sur o tōw. The two of us wanted to run up again towards the hill. Mitufala $i$ wantem resis $i$ go antap bakegen from hil. (ASS.008) $\mathbf{2}$ - for, marks purposive role, action towards a goal blong, from Nēr töl ma van me a Rō talē ti o giam sur o mes. The three of them came to Rowa to place a net for fish. Trifala $i$ bin kam long Rowa blong putum net blong karem fis. (WDR.051) $\mathbf{3}$ • because of, reason for, marks stimulus or cause of an action from Ei nōtōn te ten̄teñ sur oso? Hey, why is your child crying? Ei, pikinini blong yu i stap krae from wanem? (DPD.043) Rōrō a malaklak sur rōrō. The two of them were happy because of them (other two). Tufala i glad from tufala. (OQQ.018) $\mathbf{4}$ • about long saed blong In̄kē o kakaka sur i Matēk wo i Tëqël This is a story about Matēk and Tëqël. Hemia hem $i$ wan storian long saed blong Matēk mo Tëqël. (KM̄MT. 001) [POc ${ }^{*} s u[l, d, R] i$ 'prepositional verb, motion to/after a person'] 
sur subord. because, introduces adverbial clause of reason $\sim$ from Ba la masawre ine i no mo mamakē idian sur o meren mē dēñ o 9pm. But at that time I was very surprised, because it was daylight until 9pm. Be long taem ia mi sapraes tumas from $i$ deilaet kasem 9 klok. (AAD.115) Syn: wōl ${ }_{3}$

sur $_{3}$ loc.adj. down, in a downwards direction daon Ri van sar, ri ōn sur, ri mōvōt. You go inside, lie down and sleep. Yufala i go insaed, leidaon, slip. (RPP)

sur $_{4} v t$. sing singim Ale, in̄ko na sur o ës vagtöl. OK, now I'm going to sing the song three times. Ale nao ia mi singim singsing tri taem. (BQD) Syn: rëw

sur $_{5} v$. remove hook from fish tekemaot huk taem yu hukum fis

sursal n.com. land crab (with hairy legs) wan kaen krab we hem i blak mo hem i gat tumas hea blong hem. Hem i stap long drae ples be hem i go long solwota taem i gat eg Gecarcinidae sp. Syn: giavēdun

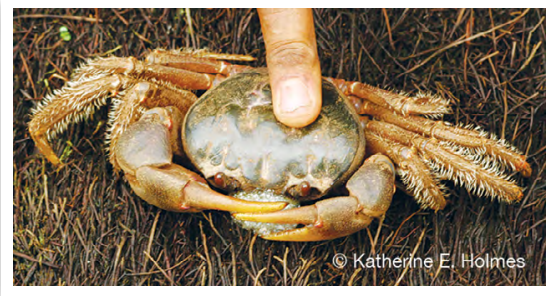

sursal $\sim$ Gecarcinidae sp.

sursur loc.adj. down low daon Nē ma gav sursur ren sōw e, rivteg o n̄ēlida naw. It flew right down low, close to the surface of the sea. Hem i flae daon olgeta, klosap long top blong solwota. (DHN)

survun n.com. greedy, putting oneself first before others, thinking highly of oneself $\sim$ kriti, putum yu wan mo daonem narawan Gunök o survun o survun. My wife is always critical of me, saying she does more than me. Woman blong mi i putum se hem yes be mino.

sus $v$ t. $\mathbf{1} \cdot$ poke, prod, pierce $\sim$ stikim Sus ten o gengen, mo monog si odian̄? Stick something in the food, is it ready or not? Traem stikim kakae, i dan o nogat? 2 - point (with finger) poen (wetem fingga) $\mathbf{3} \cdot$ inject, give injection to $\sim$ stikim (wetem stik meresin) Nē gö sus na kiriañ. She's going to give you an injection in your bum. Bae hem i stikim as blong yu. See: susgeg $[P O c *(s u) s u(k)$ 'anything used to pierce, prick']

sus $_{2} v$. take piece of löt (food dish) from dish that has already been cut leftemap mo karemaot nalot long natambia we yu bin katem finis

sus $_{3} v$. place marker to indicate position of house posts stikim mak, wud folem mak blong haos

sus $_{4} \mathbf{1} \cdot n$.com. breast $\sim$ titi Ba sur no ge ven̄en na nötuk tek o sus. But because I feed my offspring with the breast. Be from mi fidim pikinini blong mi wetem titi. (DFF) 2 - vt. suckle, suck on $\sim$ titi, titi long [POc *susu 'breast']

sōsōn (n.poss) n.com.suff. her breasts titi blong hem

susuk (n.poss) n.com.suff. my breasts $\sim$ titi blong $\mathrm{mi}$

sus atatīēn n.com. variety of cassava belonging to 'male' grouping, with long, large tubers kaen maniok we kakae blong hem i longfala mo i bigwan

sus rereqe n.com. variety of cassava that has round, short tubers $\sim$ kaen maniok we kakae blong hem i raon mo i sotfala

susdenlē n.com. variety of taro with red stem with white stripes kaen taro we han blong hem i red wetem waet straep 
susgeg $v t$. push (stake or length of other material) into tight narrow space pusum (stik o samting olsem) i go fas long wan hol See: sus susn̄eg $v$. push pusum

susuk n.com.suff. my breasts $\sim$ titi blong mi See: sus $_{4}$

susuki n.com. motorbike moto, susuki [Bis susuki 'motorbike']

susul $v t$. irritate, make itchy skrasem, olsem wan samting i mekem man i harem nogud Den o qōōr ni susul nēk. The wild yam might itch you. Nogud waelyam i skrasem yu.

susumalmà (fr.var. of wösusum̄almal) susuñu $v$. make fringe (on mat or cloth) katem pisisim en (blong mat o kaliko)

susur $v t$. sew somap (klos) O leñ mē sēr na mōgōr o geben, e nēr mö susur kèl. The wind tore their sail, so they sewed it up again. Win i terem sel blong olgeta, ale ol $i$ somap bakegen. [POC *(su)suRi 'bone (needle); sew'] susus $a d v$. close klosap

suv $v i$. bathe (in something), get washed or become wet from something swim (long wan samting) Nē mō rōw e, nē mö suv ren lè tian. He jumped, and he bathed right in its shit. Hem i jam ia, hem i swim stret long sitsit blong hem. (ADT) See: gösuv

suv wian $v i$. get rained on, not worry about getting wet in rain $\sim$ swim long ren Syn: löt wian

Suvren n.loc. place name $\sim$ nem blong ples

suvsuv vi. bathe, swim swim See: gër

suware $v$. invite $\sim$ invaetem

suwbē n.com. variety of taro, 'female', with white stem and corm. They are big and long and can be crooked like marē taro kaen taro we hem i woman mo han, skin mo kakae blong hem i waet. Hem i longfala mo bigfala mo hem i save ben olsem marē

\section{$\mathbf{T} \sim \mathbf{t}$}

$\mathbf{t a}_{1}$ (abbrev. of vita)

$\mathbf{t a}_{2}$ prep. from, denizen of $\sim$ blong No ko, no ta lo, no ta la naw. Me, I'm from the seaside, I'm from the sea. Mi ia, mi blong ples blong solwota, mi blong solwota. (JMT.045) No, no o vēnēm ta Sēbēr. Me, I am from the tribe of Sēbèr. Mi, mi blong traeb blong Sēbēr. (ESP.114)

tabaē (abbrev. taē) part. of it, for it, anaphoric form $\sim$ blong hem, toktok we i gobak long narafala toktok we yu talem finis Le revrev tabaē. That afternoon. Long aftenun blong hem.

tabē n.com. carved wooden platter, used for pounding nalot and making kava natambia, wuden plet Nēki le sur me o tabē löt, nēk i mem sōw lē kikse um na mōgōn. You get down the wooden platter for the löt pudding, and you put it by the side of your earth oven. Yu tekem daon natambia blong nalot, yu putum long saed blong oven blong yu. (JNN) [POc *tabiRa 'wooden bowl']

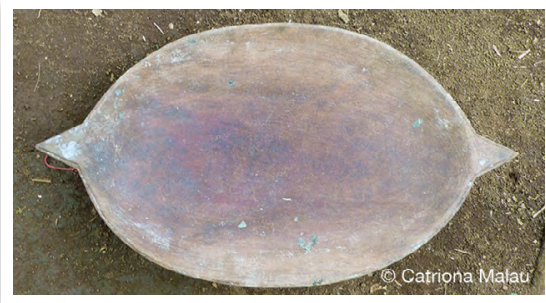

tabē $\sim$ wooden platter 
tabēva n.com. present $\sim$ presen Tabēva ine, nē ga van la ambasada talo Japan. The present is going to be given to the Japanese ambassador. Presen ia bae ol $i$ givim long ambasada blong Japan. (VSJ)

tabo subord. in order to, so that, introduces adverbial purposive clause blong Iñko na sir bilēn̄ o ga, tabo nana qētēg talo vet o waga. Now I am scraping more vines, so that I can start to weave the prawn trap. Nao ia mi stap skrasem moa rop, blong mi stat blong wivim basket naora. (KBN) Syn: talo $_{2}$

taē (abbrev. of tabaē)

tagar $_{1}$ n.com. 1 luck laki 2•behaviour, way of acting fasin mo tingting O tagar gōwē nēn gö mörös, o tagar tisē nēn gëtë kër. We like good behaviour, we don't like bad behaviour. Gud fasin yumi wantem, rabis fasin yumi no wantem. [Sapos tingting i gud, fasin i gud. Yu ekspresem tingting, mas se i gud o nogat. Sapos yu gat wan gudfala tingting, evri samting bae i oraet, sapos yu gat rabis tingting, bae i gat rabis aksen] tagar gōwē (comp.) good luck, good manner gud lak, gud fasin

tagar tisē (comp.) bad luck, bad manner bad lak, bad fasin

$\operatorname{tagar}_{2}$ n.com. kind of tree $\sim$ wan kaen tri Melochia odorata

tagrës $n$.com. piece of wood with hot embers stik faea

takak $v i$. have legs spread apart, either when standing, sitting or lying $\sim$ leg i open i stap, nomata se i stanap, sidaon o leidaon $\mathbf{O}$ giav e ta takak ti. The crab had its legs spread apart. Leg blong krab i open i stap. (OQQ 168)

takavkav $v i$. wide $\sim$ open $\mathbf{O}$ masawre ga takavkav idian. The space is too open. Ples is open tumas. taklē adj. 1 • some, part of samfala, pat Taklē weles ine nē ga qag, taklē gö mönö añañ, taklē nē gō tōtōrōg. Some of the coral is white, some of it is a bityellow, and some is green. Samfala korel ia hem i waet, samfala i haf yelo, samfala hem $i$ grin. (DPR) 2 - part, partial haf, pat Timiak o reqe e nē o taklē timiat, nē o taklē tañsar. So that woman she was part spirit and part person. Olsem woman ia hem i pat devel, hem i pat man. (GMM)

talēs n.com. sea almond, Indian almond natavoa Terminalia catappa [POC *talise 'Terminalia catappa']

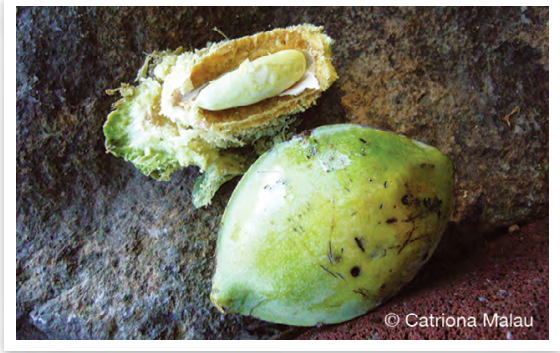

talēs Terminalia catappa

talēs toko (comp.) n.com. kind of tree related to an Indian almond but which has small nuts wael natavoa Terminalia sepicana $[\mathrm{POC}]$

taliwög $v i$. turn round $\sim$ tanem raon talo prep. of, for, marks an associative relationship blong Nēr ta van me ine, nēr a le o ripot talo projek na mögun nēn. They are coming to get the report for our project. Ol i stap kam ia blong ol i karem ripot blong projek blong yumi. (VSJ.080) Iñko na da o qarörö talō tōar waga. Now I am making the hole for the other prawn trap. Nao ia mi stap mekem hol blong narafala basket naora. (KBN.017) (Allomorphs: tala, tale, talē, talë, tali, talō, talö)

talo ${ }_{2}$ subord. in order to, so that, introduces adverbial purposive clause blong Na ran̄ o tere gamērēr qō na möguk talo vet o waga. I pull 
some Freycinetia vine for me in order to weave a prawn trap. Mi pulum rop blong mi blong wivim basket naora. (KBN) (Allomorphs: tala, tale, talē, talë, tali, talō, talö) Syn: tabo

taltal n.com. variety of taro, 'female', with black stem and light spots on leaves kaen taro we hem i woman, we han blong hem i haf dak mo han mo lif i gat waet spot. Kakae blong hem i waet mo hem i no stap karem fok

tamag n.com. lesser yam, Chinese yam $\sim$ wovile, swityam Dioscorea esculenta taman n.p.bound her/his father papa blong hem See: teme

tamarge $1 \cdot n$.com. old man olfala man $\mathbf{2} \cdot n$.com. old person $\sim$ olfala $\mathbf{3} \cdot$ $v i$. grow old, become old $\sim$ kam olfala tamn̄o n.com. coconut leaf mat $\sim$ lif kokonas mat

tamōt n.com. variety of breadfruit that is good for boiling kaen bredfrut we hem i gud blong kuk

tamtian n.com.suff. her/his/its shadow, soul sado, spirit blong hem See: tamtiē

tamtiē $n$.com.suff. $\mathbf{1} \cdot$ shadow, reflection $\sim$ sado $\mathbf{2} \cdot$ spirit, soul of a person spirit blong wan man See: vu

tamtian (n.poss) n.com.suff. her/his/ its shadow, soul sado, spirit blong hem

tamtiēk (n.poss) n.com.suff. my shadow, soul sado, spirit blong mi tamtiēk n.com.suff. my shadow, soul sado, spirit blong mi see: tamtiē

tam̄at n.com. peace pis In̄ko nēn a tog kēl la tamat. Now we live in peace again. Nao ia yumi stap long pis bakegen.

$\tan _{1}$ n.com. 1 earth, soil graon 2 ground $\sim$ graon $\mathbf{3} \cdot$ land $\sim$ graon [POC *tanoq 'earth']

$\tan _{2}$ prep. with, marks a comitative role $\sim$ wetem Die tan̄ Mam, èl siag me. Mum and Dad, look up here. Mama mo
Papa, lukluk i kam antap ia. Kōmōrōk tek na nötun kōmōrōk e, i Jacobeth wo i Joana. The two of us with our children, Jacobeth and Joana. Mitufala wetem pikinini blong mitufala, Jacobeth mo Joana. (EDM)

tan vōvō n.com. dust das Syn: usu tan tana n.com. variety of taro, 'female', with dark red stem $\sim$ kaen taro we hem i woman, we han blong hem i dak red \{Lit. Tanna Tanna\}

tanlēn̄lēn n.com. kind of small black lizard which is found underneath logs and rocks wan kaen smol blak lised we hem i stap andanit long wud o ston

$\tan$ n.com. pandanus basket, usually small, especially one used for carrying personal effects, also for collecting nuts or small amounts of food basket pandanas, blong yusum blong ol smolsmol ting blong yu Ni bis o miak, ni nësnës lö lölö tan̄. He picked the Tahitian chestnuts, and he put them into the basket. Hem $i$ tekem namambe, hem i fulumap insaed long basket. (JRQ) See: tibiar [POc *taya 'basket or bag, small, used for personal effects']

tan̄evsōs n.com. variety of taro, 'male' kaen taro we hem i man \{Lit. light fire by rubbing sticks together rabem wud blong mekem faea\}

tan̄ēs n.com. tears wota blong ae $\mathbf{E} 1$ ti o tan̄ēs ni vēvērēg den na matan. Look at the tears coming out of his eyes. Yu luk wota blong ae i stap ron long ae blong hem. Syn: tin̄is matan

tañqal $v$. touch (with hand) tajem (wetem han) Si nēk i tañqal o ev, nē gö tum nēk. If you touch the fire, it will burn you. Sapos yu tajem faea bae ibonem yu.

tan̄sar $\mathbf{1} \cdot n . c o m$. person $\sim \operatorname{man} \mathbf{2} \cdot v i$. become a person, feel like one has become human again kam man 
taōrmat $\mathbf{1} \cdot v i$. be ready, be prepared redi $\mathbf{O}$ gē ma taōrmat talö gö sum. The kava is ready to be drunk. Kava $i$ redi blong dring. (OSK.042) $\mathbf{2}$ - vt. prepare, make something ready mekem redi

dataōrmat (SVC) vt. prepare mekem redi Kōmōrōk a van a da taōrmat na mögun kōmōrōk o tere savsav. The two of us went and prepared our things. Mitufala i go mekem redi ol ting blong mitufala. (AAD.098)

taq vi. sit sidaon

taqan $n$.com.suff. her/his/its belly bel blong hem See: teqe

taqeqe $v i$. flat $\sim$ flat

taqēñi $n$.com. surface $\sim$ top blong Nana rēv na siëk la taqēn̄i vet. I wrote my name on the surface of the stone. Mi raetem nem blong mi long top blong ston. (AAD.353)

taqgilgil $v$. lie down motionless, with no energy, as when someone is very sick taem we siknes i kasem wan man mo hemi no save muv, i leidaon nomo

taqtaq $v t$. overcast, cloudy gat klaod, i no san be i no ren Garqe o taqtaq. Today it is overcast. Tede i no san be i no ren

taqtutun $v$. 1 sit on egg (of bird) pijin i slip long eg kasem taem eg i brok $\operatorname{tar}_{1} v t$. chop, cut using chopping action with considerable effort required for effect, as object is solid, hard katem, olsem long akis we i hadwok blong mekem Iñko nēn a van, a tar o wēririn̄i gövur. $O$ mie, nēn ga tar o tere bōs. Then we go and chop the parts for the house. The first thing is, we will chop the posts. Nao ia yumi go, katem ol pat blong haos. Fas samting, yumi katem ol pos. Syn: ger See: tēv, tōt [POC *taRaQ-i- 'chop with adze']

$\operatorname{tar}_{2} \mathbf{1} \cdot$ n.com. calm sea $\sim$ solwota i kwaet 2 $v i$. (sea) become calm solwota i kam kwaet

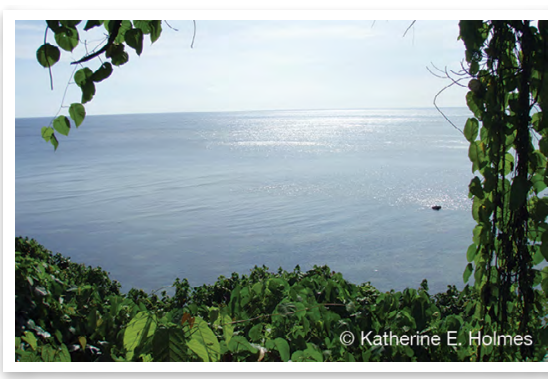

tar calm sea

$\operatorname{tar}_{3}$ num. become, reach a thousand kasem taosen

tar vagōrō (der.) two thousand tu taosen

tar vatiwial (der.) one thousand wan taosen

tarak n.com. kind of tree $\sim$ wan kaen tri Ervatamia orientalis, E. pandacaqui, E. obtusciuscula [Branches used for making slingshots, because they are straight. Leaf used as a traditional form of contraception. Wan kaen tri we ol i yusum blong kastom meresin mo ol i yusum wud blong fasem lastik blong sutum pijin.]

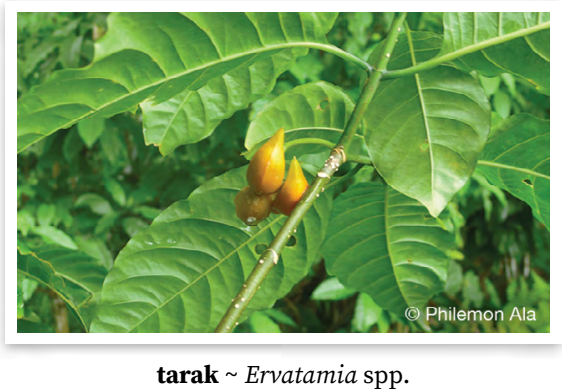

tarbian n.com.suff. her/his/its body bodi blong hem See: tarbiē

tarbiē n.com.suff. body bodi $\mathrm{Na}$ tarbian döl gö körkör, ba na qōtōn ga mamē. Its body was black all over, but its head was red. Bodi blong hem i blak evriwan, be hed blong hem i red. (WRN) 
tarbian (n.poss) n.com.suff. her/his/its body bodi blong hem

tarbiēk (n.poss) n.com.suff. my body bodi blong mi

tarbiēk n.com.suff. my body bodi blong mi See: tarbiē

tarere n.com. young chicken which is not a chick but not yet fully mature $\sim$ yang faol

tarēn̄ vi. sneeze $\sim$ snis

tarin̄i $n$.com. row laen

tarn̄i n.com. kind, variety kaen Qēt a van lo restron ta Chinatown tabe gen o tarn̄i gengen ine nēr gō ōl vita 'yum cha'. Then we went to a restaurant in Chinatown to eat a kind of food that they call 'yum cha'. Afta i go long restron blong Chinatown blong kakae kaen kakae we ol i kolem se 'yum cha'. (AAD)

$\operatorname{tar} \overline{\mathbf{n}} v i$. be quiet, be calm, be peaceful stap kwaet

tarōviti n.com. Fijian taro taro fiji

tartarawöw n.com. kind of plant kaen plant Leea indica

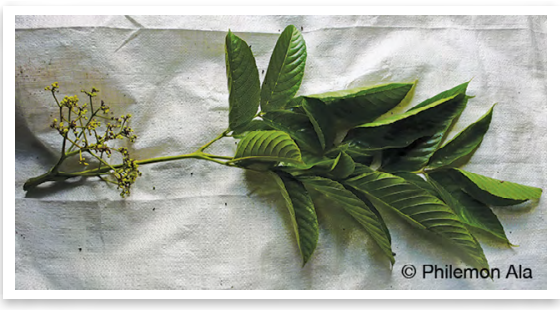

tartarawöw Leea indica

tas $\mathbf{1} \cdot a d v$. all the way olwei $\mathbf{O}$ gersal ni ñiria. Nitiwial ni kēl sōw sur o Sol, wo nitiwial ni van tas le sere plen. The road forks. One goes right towards Sola and the other goes all the way to the airport. Rod i fok. Wan i go raon from Sola, mo wan $i$ go olwei long epot. (RRS) $\mathbf{2} \cdot v i$. go all the way $\sim$ go olwei Nè ma tas. He went all the way. Hem igo olwei. tasēn n.p.bound her/his/its younger sibling smol brata o sista blong hem See: tēsi

tasga $a d v$. always oltaem

tastas $a d v$. always evri taem Syn: vaga Tat (abbrev. of Tatat)

tatag $1 \cdot v t$. follow folem Kōmōrōn ri tatag o gersal gēn. You two follow this path. Bae yutufala i folem rod ia. (DPD) Rōrō ga tatag o savger so no go qaq. The two of them are going to follow whatever I say. Bae tufala ifolem samting we mi talem. (CAA) $\mathbf{2} \cdot v t$. do according to, in accordance with mekem folem Qēt o tere m̄irm̄iar nēr a da tatag oso i Matēk ma qaq min nēr. Then the children did it according to what Matēk had told them. Afta ol pikinini ol $i$ mekem folem wanem we Matēek ibin talem long olgeta. (KMTT) $\mathbf{3}$ • prep. along folem Nē me mem o vet tatag o gersal vaavan me mem qēt. He put the stones along the road until he had put them all out. Hem i putum ol ston folem rod go go hem i putum evriwan. (RPP)

tatalēv $v$. carry load hanging on a stick held over shoulder $\sim$ karem wetem stik ova long wan solda

tatar $1 \cdot v i$. pray prea Söwlē in̄ko kōmōrōk a tatar sur timiak avē kōmōrōk gē gis qal o reqe. So then the two of us prayed for how we could get a girl. Ale nao ia mitufala i prea from olsem wanem bae mitufala i karem wan gel. (NRL) 2・n.com. prayer prea

Tatat (abbrev. Tat) n.p.fr. Uncle Angkel tatörur vi. noise made by pigeon noes blong nawimba

tavalalē n.com. enemy badfren, enemi No gëtë kër o tavalalē möguk. I don't want my enemy. Mi no laekem enemiblong mi.

tavalgi $n \mathbf{1} \cdot$ side $\sim \operatorname{saed} \mathbf{2} \cdot$ end $\sim$ en 
taw n.com. dark-brown honeyeater, grey-eared honeyeater (bird) kaen pijin wetem longmaot, famle blong redhed we hem i braon Lichmera incana

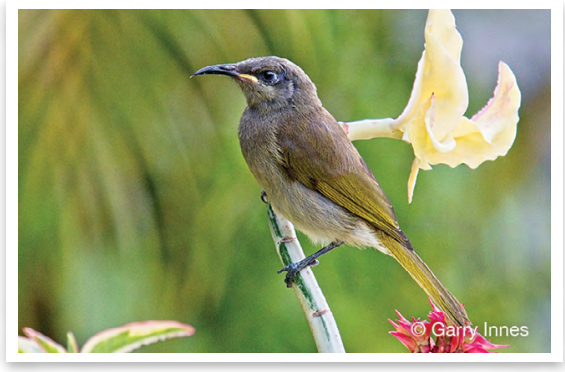

taw Lichmera incana

$\operatorname{taw}_{2}$ n.com. variety of yam that belongs to the grouping of 'female yams', which is white and soft wan kaen yam we i sofsof mo i waet. Hem i save gru raon be samtaem i flat

taw $_{3}$ vi. produce flower (plant) mekem flaoa

taw $_{4}$ have fissure which indicates that it will break (e.g. egg) gat laen we i soemaot se bae i brok (olsem eg)

taw $_{5}$ n.com. watercourse that starts after rain in a place where there was no water before $~$ wan wota we i stat niu long ples we i drae o i nogat wota bifo, be afta i ren mo i gat wota

tawagas n.com. flower $\sim$ flaoa See: tewes

tawian n.com. brother-in-law, sister-inlaw tawi, tawian

tawle n.com. under, underneath andanit Syn: laln̄e

tawseg vi. clear klia Nēk i qaq ulul wareg, nē gata tawseg min no. Try and explain well, it is not clear to me. Yu traem eksplenem gud, hem ino klia long mi.

tebe n.com. frond, leaf of palm tree or palm-like leaf lif blong pamtri $\mathrm{O}$ tebe doot nives? How many sago palm leaves? Hamas lif natanggura? tebedēmēēl n.com. centipede $\sim$ milpat Order Chilopoda \{Lit. frond of cycad han blong namwele\}

tebol n.com. table tebol

tegvōnō n.com. local person $\sim$ man ples tek 1 prep. with, marks an instrumental role wetem Nē mö dur mi miat o qō tek o gasel. He stabbed the pig to death with a knife. Hem i stikim pig $i$ ded wetem naef. Syn: bemrin $_{2}$, birin 2. prep. with, marks a comitative role, accompanied with wetem In̄ko nēk ge leg tek i gunön tōgan̄. Now you are going to get married with your older brother's wife. Nao ia bae yu mared wetem woman blong bigfala brata blong yu. (ATB) Nēn ge gen o qiat gē big tek o buluk. We will eat taro with beef. Yumi kakae taro wetem buluk. $\mathbf{3}$ • prep. with, marks a confective role wetem Nēk i turtur tek gem na vōsōn. You just stood there with your bow. Yu stanap nomo wetem bonaro blong yu. (JMT) 4 • vt. take, carry, be with tekem, karem Kemek ge tek tēqēl nē la naw. We will take it down to the sea. Bae mifala i karem igo daon long solwota. See: birin̄

tek na taqan (id.) $v$. be pregnant gat bel Syn: wotoqtoq 1

tektam $v$ unexpectedly come across misfortune wan samting we $\mathrm{i}$ nogud $\mathrm{i}$ hapen be yu no bin ekspektem

tel (abbrev. of telsiër) vi. be pregnant, become pregnant gat bel Rōrō mo tog vaavan ginon ni tel. The two of them stayed together until his wife got pregnant. Tufala $i$ stap go go woman blong hem i gat bel. (ATB.002)

tel $_{2} v t$. turn $\sim$ tanem

tele $e_{1}$ n.com. axe $\sim$ akis syn: barbar $_{2}$ See: wetele

tele ${ }_{2}$ n.com. clam $\sim$ natalae

tele tur n.com. giant clam $\sim$ bigfala natalae, klamsel Tridacna sp. \{Lit. standing clam klamsel i stanap\} 


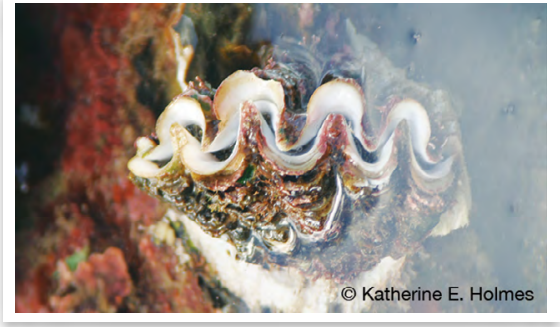

tele tur Tridacna sp.

teln̄an (root teln̄e) n.com.suff. as (X) says $\sim$ olsem toktok blong \{ Lit. from the voice of long voes blong\}

telne (root of teln̄an)

telsiër (abbrev. tel $)_{1}$

teme n.p.bound father, father's brother papa, brata blong papa Ba na temen rōrō mi miat den rōrō. But their father died leaving the two of them. Be papa blong tufala $i$ ded aot long tufala. (WNW) [POC *tama 'father'] taman (n.poss) n.p.bound her/his father papa blong hem

tëmëk (n.poss) n.p.bound my father papa blong mi

ten v.part. yet $\sim$ yet Temen rōrō gōtō mōl ten me. Their father hadn't come home yet. Papa blong tufala i no kam hom yet. (JMT)

ten $_{2}$ v.part. connative particle, try traem Dōrōk a sēksēk ten i rētnen dōrōk. Let's try and look for our mother. Yumitu traem lukaotem mama blong yumitu. (ATW)

ten $_{3}$ n.com. rain $\sim$ ren

tenēnēg vi. $\mathbf{1}$ • straight, not bent or curved stret, no kruked Ant: kēlēglēg $\mathbf{2}$ • correct, right, correctly $\sim$ stret, gud

tenge n.com. leaf remedy, plant used as a local medicine, for fertilising crops, aiding fishing and other traditional remedies and purposes lif meresin o lif we ol i yusum long saed blong kastom olsem blong fis o long garen Nēk i le min nēr töl o tenge talō sōksōk. You give the three of them (dogs) a remedy for hunting. Iu givim long trifala (dog) lif meresin blong hanting. (DDP)

tengeg n.com. example eksampol

tenmē n.com. red bass (fish) wan kaen snapa, pulefis Lutjanus bohar ten $v i$. cry krae ten̄seg (der.) vt. cry for a dead person $\sim$ krae long wan man we i ded ten̄ten̄ (redup.) n.com. cry krae [POc *tajis 'weep']

ten̄tur n.com. variety of taro, 'female', with light green stem $\sim$ kaen taro we hem i woman, we han blong hem i laet grin Syn: qiat mingala

teqe $n$.com.suff. belly bel Nana gengen wo nēk i turtur so ine wo na taqan̄ ni sisis. I'll eat and you just stand like that and your belly will get full. Mi kakae mo yu stanap ia nao mo bel blong yu bae i fulap. (JTF) $\left[P O c{ }^{*} t^{\prime} b^{w} a\right.$ 'bay, harbour; belly, stomach'] taqan (n.poss) n.com.suff. her/his/its belly bel blong hem

tëqëk (n.poss) n.com.suff. my belly bel blong mi

teqeliaw n.com. calf (of leg) mit we i stap bihaen long leg daon

teqelsan n.com.suff. back of head and back of neck bihaen hed, bitwin solda, stat antap long hed

teqeñ $n$.com. top top

teqesqē n.com. spiny squirrelfish kaen redfis Sargocentron spiniferum

tere (fr.var. of terē)

terē̄ $v$. make walling of house by lining up and tying together lengths of bamboo laenemap mo fasem ol bambu blong mekem wol blong haos Syn: sasal

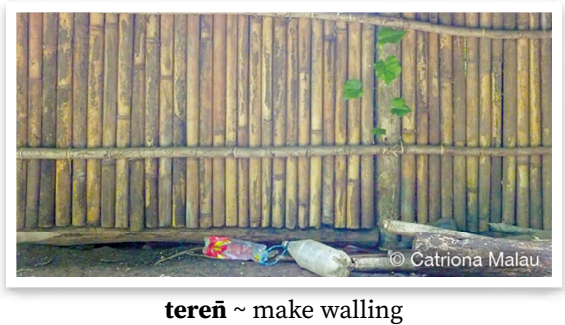


terē (fr.var. tere) part. plural marker, occuring before common nouns ol, olgeta

terit n.com. porcupinefish $\sim$ nilfis Family Diodontidae $\left[P O c{ }^{*}\right.$ taRutu $(m, \eta)$ 'Diodon spp., porcupinefish']

terit lam (comp.) n.com. spotted porcupinefish $\sim$ wan kaen nilfis Diodon hystrix \{Lit. deep sea porcupinefish nilfis blong dipsi\}

tes ${ }_{1}$ n.com. lake, large body of water bigfala wota, lek Rōrō go togtog li geret, le tes a Gō. The two of them were staying up on the high hill, by the lake on Gaua. Tufala i stap long bigfala hil, long lek long Gaua (DHN)

tes $_{2} v t$. carve, use knife or chisel to cut and shape something from wood katem wud Nēk i le o gasel, nēk i tes metestes o siri qatē ine. You get a knife and you cut the insides of the tree fern trunk so that they are sharp. Iu karem naef, yu katem sapenem insaed blong blakpam ia. (SBB)

tes ak (comp.) v. carve canoe katem kenu

tes $_{3}$ n.com. toilet, outhouse $\sim$ toelet, smolhaos Syn: kērēt 2 2, nötu gövur

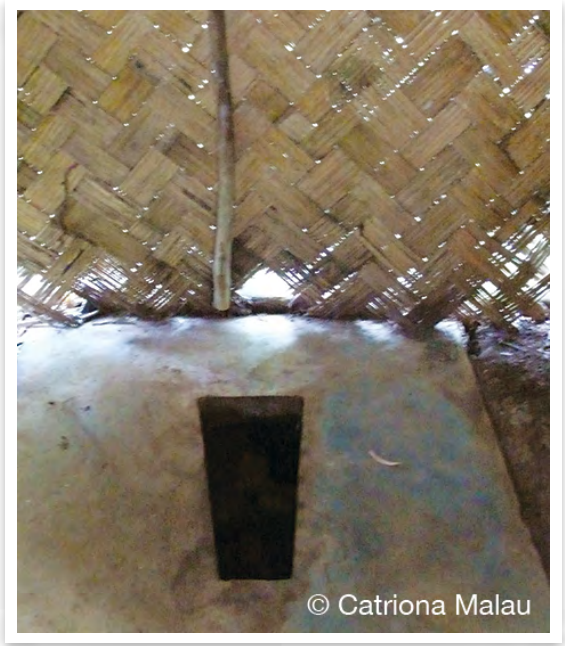

tes $\sim$ outhouse tes ${ }_{4}$ n.com. taste $\sim$ tes [Bis tes 'taste']

tesmērēre n.com. kind of freshwater eel, very long, grey with yellow belly and light red tail. It is quite aggressive wan kaen namarae blong wota we hem i save kakae man mo hem i save kamaot hanting long drae ples. Hem i longlongfala mo kala blong hem i grei, andanit i yelo mo fin mo tel i laet red

teteg $_{1} v t$. lift leftemap (KBH.012)

teteg $_{2} v$. tie up bundle of firewood fasem

teteg ralēt (comp.) n.com. tied up bundle of firewood bandel faeawud

tetel $v i$. walk around, go somewhere just for enjoyment, with no particular purpose wokbaot, wokbaot blong enjoem nomo Na tetetel, sur garqe ko o weta nē gōwē, o masawre döl o lō. I am just going for a walk because today the weather is good and it is sunny everywhere. Mi stap wokbaot from tede weta $i$ gud, $i$ gat san evri ples. (DNS)

Tetgen n.loc. place name $\sim$ nem blong ples

tevelēm (root of nitevelēm) num. five tevelēmne num. fifth nambafaef Nēk gö luw qȫn nitiwial, qȫn nirō, qȫ̄ nitöl, qön̄ nivet, o tevelēmne nēk i kēl me. You count one day, two days, three days, four days, and on the fifth day you return. Yu kaontem wan dei, tu dei, tri dei, fo dei, nambafaef dei yu kambak.

tewen n.com. native lychee (tree) nandao Pometia pinnata $[P O c$ *tawan 'Pometia pinnata']

tewes n.com. flower $\sim$ flaoa blong See: tawagas

tewesgavēg n.com. tailspot squirrelfish $\sim$ kaen redfis Sargocentron caudimaculatum \{Lit. flower of Malay apple flaoa blong nakavika\} 
tewesqēqēe n.com. variety of taro, 'female' with stem that is white at the bottom and red at top kaen taro we hem i woman, we han blong hem, daon i waet mo antap i red \{Lit. flower of mangrove flaoa blong natongtong

tewestamal n.com. variety of taro, with green stem and black petiole $\sim$ kaen taro we han blong hem $\mathrm{i}$ grin mo $\mathrm{i}$ joen long lif hem i blak [No longer cultivated. Hem i lus.] \{Lit. flower of caricature plant flaoa blong wan kaen kabis\}

tewesvër n.com. variety of taro, with red stem and light black petiole kaen taro we kala blong han mo kakae blong hem i miks red wetem waet, skin blong hem i red \{ Lit. flower of cottonwood flaoa blong burao\}

tē $v$. make payment for work that is done in addition to what was required $\sim$ givim smol mane taem yu askem man blong mekem samting we hem i no pat blong pemen See: qil tēbar n.com. kind of crab, white, which dwells by the sea where there is sand and mud wan kaen waet krab we i stap long solwota long ples we i gat sanbij mo sofmad

tēbēg $v t$. express love for $\sim$ fasin blong soemaot lav long No gē tēbēg i Brenden sur no götö tötöwös nē. I express love for Brenden by not hitting him. Mi soemaot lav long Brenden taem mino stap wivim hem.

tēg $v$. walk with walking sticking wokbaot wetem wud, holem stik Nē mē tēg tek o tēgatēg. He walks with a walking stick. Hem $i$ wokbaot wetem stik blong wokbaot.

tēgēr n.com. fantail (bird) pijin blong trik Rhipidura spp.

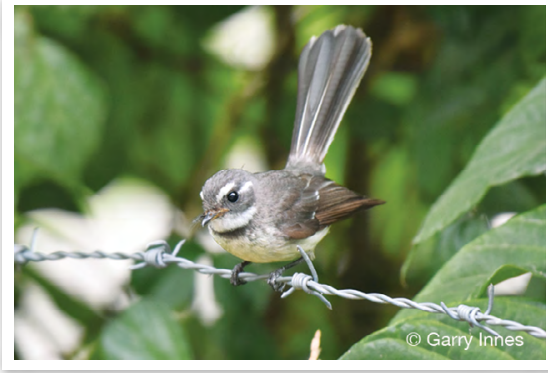

tēgēr Rhipidura spp.

tēk $v$. touch gently tajem sloslo, tajem smol Kemem gi siag lē sil, nē mē tēk qal no, na mëëlëk mō rōw. We were sitting in the dark, he touched me gently and I was taken by surprise. Mifala i sidaon long tudak hem i tajem mi mi sek we.

tēeq $\mathbf{q}_{1}$ n.com. kind of tree with small black fruit wan kaen tri we kakae blong hem i smol mo taem i raep $i$ blak Rhus taitensis

tēq $\mathbf{q}_{2} v$. shoot (with gun) $\sim$ sutum (wetem masket) Nēk i tēq o men tek o timiatēq gōwē sel den nēk i vēn o men tek o vus. Shooting a bird with a gun is better than shooting a bird with a bow and arrow. Yu sutum pijin wetem masket i mo gud bitim yu sutum pijin wetem bonaro. See: vēn, wētēq [POc tib( $\left.{ }^{w}\right) a \eta$ - $i$ - 'shoot with dart, arrow']

tēqatēq $1 \cdot v$. hunt with bow and arrow in the bush (birds and flying fox) hant wetem bonaro long bus (blong sutum pijin o flaengfokis) $2 \cdot$ n.com. gun masket See: vēn,

tēqēl vi. 1 • go down, motion or event directed downwards go daon Ri wöt tēqēl me o ga. You guys throw a rope down to me. Yufala i sakem rop i kamdaon. (AWW) Nē ni bisbis qēt, nē mō sōw tek tēqēl, dēn̄ la tan. He picked them all, and he took them down to the ground. Hem i pikim evriwan, hem i go daon wetem kasem long graon. (ATB) Ant: $\mathrm{kal}_{2} \mathbf{1} \mid \mathbf{2} \cdot$ go seaward, go 
further out to sea go long solwota Nēr töl a tēqēl rōw a lo. The three of them went down to the sea. Trifala $i$ go daon long solwota. (ATB) Ant: kal 2 3 3 go downstream go daon (folem wota) Ant: $\mathrm{kal}_{2} \mathbf{3} \mid \mathbf{4} \cdot$ travel in a northwesterly direction (of travel beyond island to other islands) go long not long narafala aelan $\mathbf{O}$ tō nē mō tō tēqēl bo Tores. The chicken ran away to Torres islands. Faol i ronwe igo long Torres. (RTF) Ant: $\mathrm{kal}_{2} \mathbf{4}$

tēqēs n.com. tool for cutting wood to make it flat $\sim$ tul blong katem wud i flat Nēk i le o tēqēs, nēk i tes na mōgōñ o tönö sigsiag tek. Take an adze and carve your stool with it. Tekem aj, yu katem ples blong sidaon blong yu wetem. See: vidëm

tēr vt. peck, action that bird makes with beak aksen we pijin i mekem wetem maot blong hem $\mathbf{0}$ kōmar, o maniok, o tere savsav ine, o gōsōw mo kur lōlō, o matēk mē tēr lōlō qēt. The sweet potatoes, the cassava, all those things, the rat chewed them all up, and the swamphen pecked them all to bits. Kumala, maniok, ol samting ia, rat i kakae tru long hem, nambiru $i$ kakae tru long evriwan. (WRN)

tēsi n.p.bound younger same sex sibling $\sim$ smol brata blong wan boe o smol sista blong wan gel [POC *taci 'younger sibling']

tēsi rësrës (comp.) n.p.bound youngest sibling lasbon brata, sista

tasēn (n.poss) n.p.bound her/his/its younger sibling smol brata o sista blong hem

tēsik (n.poss) n.p.bound my younger sibling smol brata o sista blong mi tēsik n.p.bound my younger sibling smol brata o sista blong mi See: tēsi

tètē 1 - n.com. baby bebe, smol pikinini 2 - n.p.fr. My child, term of endearment used by parent to child Bebe, nem we mama o papa i yusum blong singaotem smol pikinini blong hem

tētēr $1 v t$. lay flat, lay out something that is flat putum i leidaon Le sōw o tönöam, tētēr sōw. Get down the mat and lay it down. Karem mat, putum ileidaon. (DPD)

tētēr ${ }_{2}$ n.com. mirror glas, miro

tētērē $v$. rigor mortis $\sim$ bodi i kam strong, olsem taem man i ded

tētēw vt. smoke, dry over smoke smokem I nēk mē tētēw o ñe. You dried the canarium nuts. Yu smokem nangae.

wētētēw (der.) n.com. wood for smoking wud blong smokem

tēv $v$ t. $\mathbf{1} \cdot$ cut, slice $\sim$ katem, slaesem $\mathrm{Na}$ tēv möt o string ine. I'll cut the string. Bae mi katem brekem string ia, (TSR) Iñko nēn a tēv o löt ine. Then we'll slice the löt pudding. Nao ia bae yumi katem nalot ia. (JNN) 2 - operate on katem (long hospitel) No me telsiër bilēñ i Sophian, e nēr mē tēv no. I became pregnant again with Sophian, then they operated on me. Mi gat bel bakegen wetem Sophian, ale ol i katem mi. (NRL) See: $\operatorname{tar}_{1}$, tōt

tēwē n.com. shore birds pijin blong solwota

tēwē biliag (comp.) n.com. wandering tattler (bird) nasivi (pijin) Heteroscelus incanus \{Lit. shore bird like a buff-banded rail nambilak nasivi\} tēwē ōlqön (comp.) n.com. kind of shore bird smolsmol nasivi we hem i stap singaot oltaem (pijin) \{Lit. shore bird that calls night nasivi we i singaot long naet\}

tēwē rōwelteg (comp.) n.com. beach thick-knee (bird) kaen nasivi (pijin) Esacus magnirostris [This bird stays near the sea and then comes and visits the garden. Pijin ia hem i blong solwota, be bae i kam visit long garen.] 
tëmëk n.p.bound my father papa blong mi See: teme

tëqëk n.com.suff. my belly bel blong mi See: teqe

tëqël n.com. bat bat

tër $v t$. cover, as in covering food with leaves, ready to bake in oven, or covering blanket over person kavremap, olsem kakae long oven blong bekem, o kavremap bodi Tër gōr nēk! Cover yourself up! Kavremap yu!

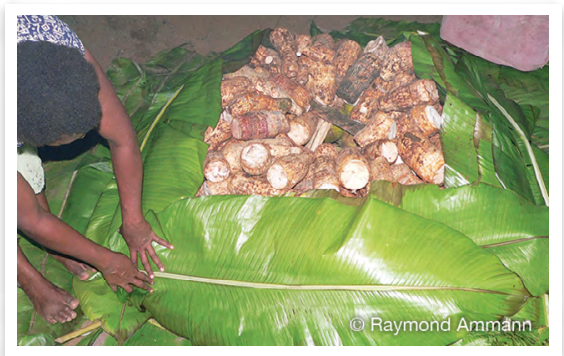

tër cover food

tërtër qō (der.) n.com. pork that has been baked overnight pig we hem i bek ovanaet

tërëkrëk $v$. dry up drae

tërëw n.com. Vanuatu mountain pigeon (bird) nawimba blong hil (pijin) Ducula bakeri

tërtërgōr n.com. fascia, covering over meat waet samting we i stap bitwin long skin mo mit

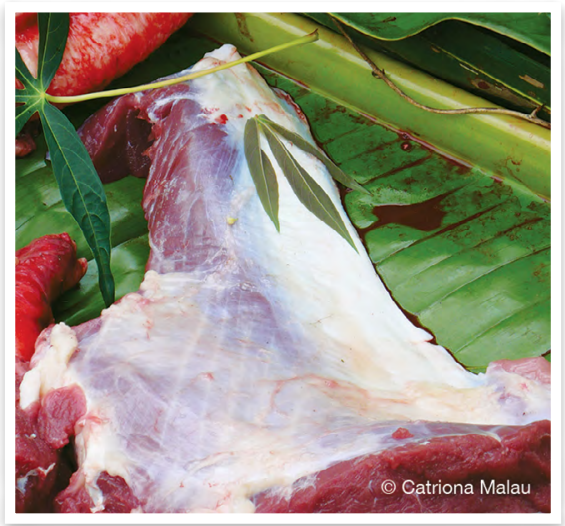

tërtërgōr fascia tës $a d v$. in error, wrongly, mistakenly mestem Ni gis tës o gasel, gasel më mès, e ni es na dērēn. He dropped the knife and the knife fell down and cut his leg. Hemi i holem mestem naef, naef $i$ foldaon, ale hem i sperem hem.

tëtës $v t$. massage holem (bodi)

tëw n.com. stranger $\sim$ strenja, man afsaed

tëwru n.com. back bihaen tëwru gövur back of the house bihaen blong haos

ti $v$.part. eventive aspect marker

$\mathbf{t i}_{2} v$ t. $\mathbf{1} \cdot$ start $\sim$ statem $\mathbf{2} \cdot$ light $\sim$ laetem

Na ti o bur. I'll light the lamp. Bae mi laetem laet.

tia n.com. shit, excrement $\sim$ sitsit [POC *taqe 'faeces']

tian (n.poss) n.com.suff. her/his/its faeces sitsit blong hem

tie tō (n.poss) n.com.suff. chicken faeces sitsit blong faol

tiēk (n.poss) n.com.suff. my faeces sitsit blong mi

tiabasas vi. go ashore $\sim$ kamso Ni tiabasas kal sar lō ōn. He went ashore, up onto the beach. Hem ikamso antap long sanbij. Syn: siōl

tiak (abbrev. of timiak)

tiam $_{1} v t$. love lavem Nē gē tiam nēr. He loves them. Hem i lavem olgeta. $(A A D)$ Ba nēn töl ga mas tiam nē timiak na turgi taman i no, na turgi rētnan i no. But the three of us must love him as if I am his real father, I am his real mother. Be yumitri mas lavem hem olsem se tru papa blong hem mi ia, tru mama blong hem mi ia. (JMT)

timtiam (redup.) n.com. love laf

tiam $_{2} v$. upsett (basket), i.e. to transition from weaving base to sides (of basket) leftemap basket, redi blong wivim saed blong hem (KBN)

tian n.com.suff. her/his/its faeces sitsit blong hem See: tia 
tiargōr vt. prevent, stop someone from doing something stopem Nē gö mörös van tas, ba kemem mē tiargōr nē. He wanted to go all the way, but we stopped him. Hem i wantem go olwe be mifala i stopem hem.

tibiar n.com. basket, generic term used to refer to coconut leaf, pandanus and cane or reed baskets basket, nem ia i kavremap ol kaen basket blong kokonas, pandanas mo narafala kaen Söw rōrō a bis o tere tewen ine, rōrō a sönön lē tibiar. So the two of them picked the native lychees, and they put them in the basket. Ale tufala i tekem ol nandao ia, tufala $i$ fulumap long basket. (OQQ) See: tan̄

tibiar gamalwuw (comp.) n.com. kind of food basket woven with a black vine wan kaen basket blong kakae we ol i wivim wetem blak rop [These baskets are made by women. They are the same style as the gavan basket, which is only made and used by men. Ol woman nomo i wivim kaen basket ia. Hem i semak long gavañ basket we ol man nomo ol i mekem mo yusum long nakamal.] See: gavan̄

tie menek n.com. meconium; baby's first faeces that is almost black in colour fes sitsit blong bebe after hem i bon \{Lit. squid faeces sitsit blong nawita\}

tie tō n.com.suff. chicken faeces $~$ sitsit blong faol See: tia

tieg vt. lay trap putum trap See: gatitieg

tiēk n.com.suff. my faeces $\sim$ sitsit blong mi See: tia

tig $v$. finish (basket or mat) by plaiting or threading in ends to prevent from coming undone $\sim$ finisim ol sotsot pis blong pandanas o rop blong mat o basket blong mekem se ol i no save kamkamaot

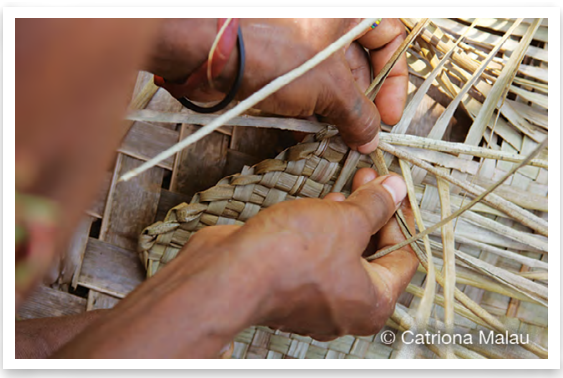

tig weaving term

tigian n.com. kind of sea crab that is black with claws that are white underneath $\sim$ krab blong solwota we hem i laet blak mo ol fingga blong hem ol i waet andanit

tikiar $v$. sound made by hen after laying an egg saon olsem woman faol i singaot afta hem i putum eg

til n.com. reef needlefish, keeltail needlefish, juvenile needlefish longmaot (fis), ol pikinini o smolsmol kaen blong hem Strongylura incisa, Platybelone argalus See: ut

tilē $\tan$ n.com. kind of snake $\sim$ kaen snek

timiak (abbrev. tiak) $\mathbf{1}$ - prep. like, marks manner or similative role $\sim$ olsem No ga gav timiak o men. I fly like a bird. Mi stap flae olsem pijin. (DFF.017) 2 v vt. be like $\sim$ i olsem $\mathbf{3}$ - subord. like, introduces adverbial clause of manner $\sim$ olsem $\mathbf{4} \cdot$ disc. like $\sim$ olsem Nè o tarn̄i men nitiwial, timiak vita nē gē rivriv o savsav timiak nēn o tan̄sar nēn gē rivriv e. It is a kind of bird, like, it plants things like us people plant. Hem i wan kaen pijin, olsem se hem i planem samting olsem yumi ol pipol yumi planem.

timiak avē $\mathbf{1} \cdot a d v$. how olsem wanem Nē gètē gilal vita nē ga da timiak avē. He didn't know how he would do it. Hem i no save se bae hem i mekem olsem wanem. (LLF) 2 - inter. what's going on? olsem wanem? Timiak 
avē? Nēk gata van ten? What's going on? Haven't you gone yet? Olsem wanem? Yu no go yet?

timiat n.com. ghost, spirit of dead person devel Rōrō a turtur ti, a ēl olwoman nitiwial e, ba o timiat. The two of them stood there, and they saw an old woman, but it was a spirit. Tufala i stanap ia, i luk wan olfala woman, be hem i devel. (DPD) [POc *taumate 'dead person']

timiat $_{2}$ n.com. headdress worn by men for particular dances $~$ hedres we ol man i werem long taem blong danis

timiatēq $n$.com. gun $\sim$ masket

timiatwos n.com. traditional wooden upright drum tamtam See: wōkōr

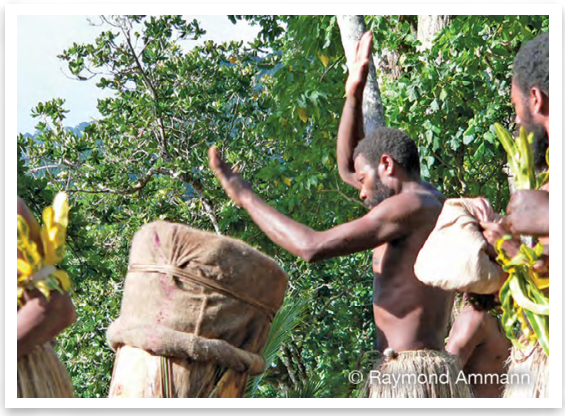

timiatwos $\sim$ wooden drum

tina n.com. lunch dina [Bis dina 'lunch, middle of day']

tine n.com. middle sibling brata $\mathrm{o}$ sista long medel

tinësqön̄ n.com. middle of night medel naet

$\operatorname{tin}_{1} n$ taro paddy unit within a qēl, mat or bōak separated by mud or stone walls step insaed long garen taro

tīn $2 v t$. create $\sim$ mekem I temen nēn mē tin̄ nēn. Our Father created us. Papa blong yumi i krietem yumi.

tin̄i n.com. 1 - liquid, juice from something, as from fruit or sauce, liquid from cooked food wota (blong frut, kakae) O tin̄i tabaē nē gè dēdērēs. The liquid from it tastes really good. Wota blong hem $i$ swit tumas. (VVKHVDP21Jul0702-SW) 2 - sap of plant wota blong wud Kemek go yusum o tin̄i wōbōgōr. We use the sap from the tamanu tree. Mifala i yusum wota blong tamanu. (VVKHVDP180ct0801-116)

tin̄i mōtō (comp.) n.com. liquid from coconut (green or dry) wota blong kokonas (grin o drae)

tin̄is matan n.com. his/her tears $\sim$ wota blong ae blong hem Na tin̄is matan ni mës sōw le veren i Mōlwor. His tears fell fown onto Mōlwor's chest. Wota blong ae blong hem i foldaon long jes blong Mōtwor. (GMM) Syn: tan̄ēs

tin̄qōr $v t$. listen lesen Varian gö luwō min nēk wo i rege sul talē tin̄qōr. Thank you very much to you and to all the people for listening. Tangkiu tumas long yu mo long ol pipol blong lesen. (VST)

tiq̄ē n.com. garden $\sim$ garen

tiqër $v t$. grab with two hands, tackle, wrap arms around $~$ kasem, holem taet

tiqialdaran̄rañ $n$.com. peacock grouper (fish) kaen los (fis) Cephalopholis argus

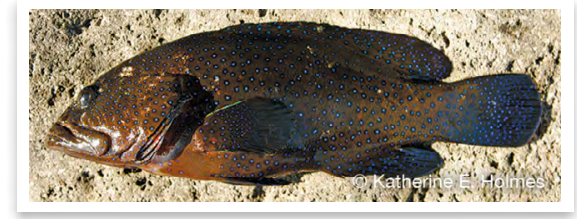

tiqialdaran̄ran̄ $\sim$ Cephalopholis argus

tiqialq̄o n.com. potato grouper (fish) kaen los (fis) Epinephelus tukula \{Lit. pig grouper pig los\}

tir vi. drip drop $\mathbf{O}$ bē mē tir lō den o wötöv. The water dripped out of the bottle. Wota $i$ drop $i$ kamaot long botel. (OQQ 105) O tin̄i taē mē tir qal no in̄kē. The juice from it dripped on me. Wota blong hem i drop i foldaon long mi. 
tiria n.com. lyretail grouper (fish) red los (fis) Variola louti

tiriakör n.com. redmouth grouper (fish) kaen los (fis) Aethaloperca rogaa \{Lit. black grouper blak los\}

tis vi. skim, skip on surface of water $\sim$ glis antap long wota $\mathbf{O}$ vet ni tis lē bē. The stone skimmed across the water. Ston $i$ glis antap long wota.

tisē $v i$. bad nogud

tit $v . \mathbf{1} \cdot$ split $\sim$ splitim $\mathbf{2} \cdot$ pull rope down quickly and sharply when trying to catch bird with a sēsédig bird trap jokem string blong kasem pijin long sēsēdig

titiav vi. shit, defecate $\sim$ sitsit

titigi n.com. bundle (of bananas) bandel blong banana

titiq̄o n.com. kind of tree kaen wud

titiritowwetam n.com. variety of taro with green stem with brown stripes at base $\sim$ kaen taro we han blong hem i grin wetem braon straep daon \{Lit. Dripping from Vetam Hill I drop long Hil blong Vetam\}

tiveg 1 - n.com. money, payment for something mane, amaon blong pem from wan samting Avē o tiveg taē? Where is the money for it? Wehem mane blong hem? Syn: söm $\mathbf{1} \mid \mathbf{2} \cdot v t$. pay (someone, something) pem Nēk i tiveg o tan̄sar. You pay someone. Yupem man.

tivi n.com. cat puskat Syn: buskat

tivtiv $_{1}$ n.com. kind of fish $\sim$ wan kaen fish

tivtiv , n.com. cover made from sago palm leaves, used to cover breadfruit when baking in stone oven in order to keep heat in wan samting we ol i somap lif natanggura mo yusum blong kavremap bredfrut long oven blong kipim hit i stap insaed

tivtivnönqet $n$.com. sailfin tang (fish)

$\sim$ kaen kalakala fis we i gat naef Zebrasoma desjardinii tiwial (root of nitiwial)

tiwial ${ }_{2}$ mod. together $\sim$ tugeta

tiwil vt. roll, toss around $\sim$ rolem O naw ni titiwil nē. The sea tossed her about. Solwota i rolem hem.

to n.com. $\mathbf{1} \cdot$ year $\sim$ yia $\mathbf{2} \cdot$ season $\sim$ taem töu leñ (comp.) n.com. hurricane season $\sim$ taem blong win, taem blong saeklon A vōnō lav o töu len vus gē qētēg lō wōl Noveba gaidēn̄ o ilsi Eprel. On Vanua Lava the season for hurricanes starts in November and goes until the end of April. Long Vanua Lava taem blong hariken $i$ stat long Novemba kasem Eprit.

to ${ }_{2}$ v.part. progressive aspect marker, indicates an event that is in progress stap, go go, yusum taem yu tokbaot wan aksen we i stap gohed (Allomorphs: ta, te, tē, të, ti, tō, tö)

tog vi. $\mathbf{1}$ - stay, be in a place, remain in a place or way, live in a place $\sim$ stap La masawre kemem me gengen qēt, in̄ko kemem a tog gem ti lö gövur. When we had finished eating, then we just stayed at the house. Taem mifala i kakae finis, mifala $i$ stap nomo long haos. (AAD) 2 - remain, be left, still be, still exist $~$ stap yet $\mathbf{O}$ wik nitöl gem so go tog vēti. Only three weeks still remain. Tri wik nomo i stap yet. (VSM)

tok n.com. dog dog Syn: genqirēg tokorës $v$. miss mestem (RTF.009)

tono $v t$. $\mathbf{1}$ • learn lanem No go mörös tono o qaqaq mōgōn. I want to learn your language. Mi wantem lanem lanwis blong yu. 2 - teach, instruct lanem, tijim Mis na mögun kōmōrōñ mo tono kōmōrōñ lo skul sur oso? What did your teacher teach you two about at school? Mis blong yutufala i lanem yutufala long wanem long skul? (DDL) 
toq n.com. bottle shaped basket which is used for storing dry canarium nuts basket blong fulumap drae nangae insaed we hem i sep olsem wan bigfala botel

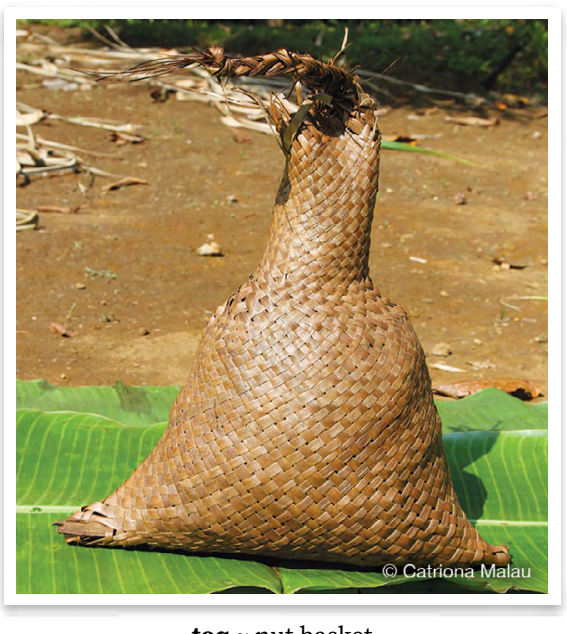

toq nut basket

toqlōn n.com. kind of flying fish wan kaen flaengfis we hed mo tel blong hem i smol be bel blong hem i bigwan. Hem i stap aotsaed be no tumas

toqsis n.com. full (stomach) (bel i) fulap

tor n.com. island teak, ironwood (tree) $\sim$ natora Intsia bijuga $[P O c$ *toRas 'a taxon of hardwood trees including Intsia bijuga']

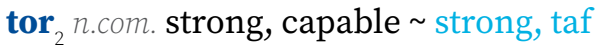
Nēk o tor talē rēvrēv. You are good at writing. Yu winim ol man blong raet.

Torba n.loc. Torba province $\sim$ Torba provins

Tores n.loc. place name $\sim$ nem blong aelan grup

tores n.com. variety of banana $\sim$ kaen banana

tortor n.com. variety of taro, 'female', with stem that is red at the bottom and green at the top kaen taro we hem i woman, we han blong hem i red daon mo grin antap [This taro is significant in grade taking ceremonies as it must be eaten for 100 days by a man who wants to pass the first step. Long saed blong kastom, man i mas kakae blong 100 dei bifo hem i pas long fes step blong nakamal.]

totenanar n.com. kind of tree that has long white flowers with a strong perfume wan kaen tri we flaoa blong hem i waet mo i longlongfala mo i gat smel [Used only for firewood. Ol i yusum blong faeawud nomo.]

towo v.part. immediate progressive aspect marker, marks an event which is currently in progress go go, yusum blong tokbaot wan samting we i stap hapen nao ia nomo (Allomorphs: tawa, tewe, tēwē, tëwë, tōwō, töwö)

$\mathbf{t o ̄}_{1}$ n.com. chicken $\sim$ faol Gallus gallus

nötu tō (comp.) n.com. chick pikinini blong faol

tō armiag (comp.) n.com. chicken with light reddish brown feathers faol we kala blong hem i laet braon

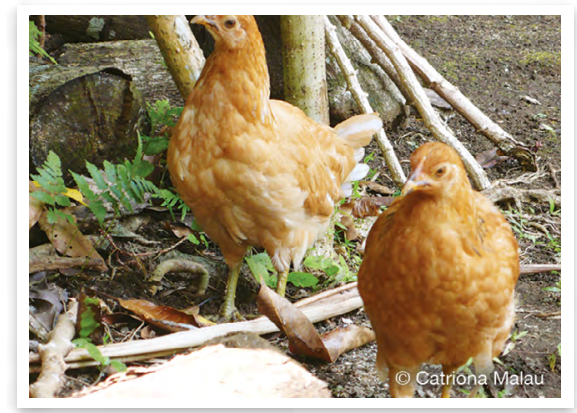

tō armiag brown chicken

tō atm̄ēn (comp.) n.com. rooster man faol \{Lit. male chicken man faol\} tō bēt (comp.) n.com. white chicken waet faol \{Lit. white chicken waet faol\} 
tō biliag (comp.) n.com. speckled chicken with colouring like buffbanded rail faol we kala blong hem i olsem nambilak \{Lit. buff-banded rail chicken nambilak faol\}

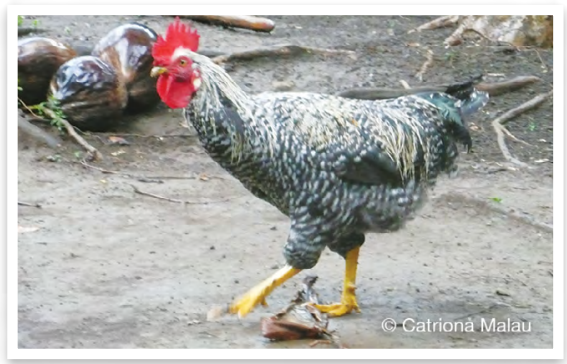

tō biliag speckled chicken

tō kör (comp.) n.com. black chicken blak faol \{Lit. black chicken blak faol\} tō mamē (comp.) n.com. red chicken red faol \{Lit. red chicken red faol\}

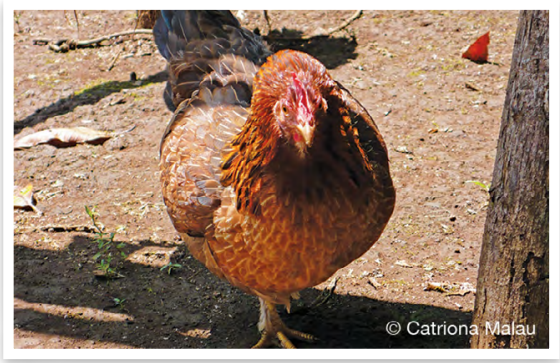

tō mamē $~$ red chicken

tō menmenlul (comp.) n.com. red, white and black chicken $\sim$ faol we i gat tri kala long hem, red, waet mo blak

tō reqe (comp.) n.com. hen woman faol $\{$ Lit. female chicken woman faol\}

$\mathbf{t} \overline{\mathbf{o}}_{2}$ vi. run away $\sim$ ronwe

tōar (fr.var. tuar) adj. other, another, the other, one of (a group) narawan

tōbōn n.p.bound her/his grandparent/ grandchild bubu blong hem See: tubu

tōbulënqet $n$.com. kind of insect like a dragonfly wan kaen bebet olsem dragonflae be i smolsmol mo i waet. Taem yu tajem i save jam longwe \{Lit. Qet's chicken faol blong Qet\} tōge n.p.bound older same sex sibling $\sim$ bigfala brata blong wan boe o bigfala sista blong wan gel

tōgan (n.poss) n.p.bound her/his/its older sibling bigfala brata o sista blong hem

tōgen Ella (n.poss) n.p.bound Ella's older sister bigfala sista blong Ella tōgëk (n.poss) n.p.bound my older sibling bigfala brata o sista blong mi

tōgan n.p.bound her/his/its older same sex sibling bigfala brata blong wan boe o bigfala sista blong wan gel

tōk n.com. blue sky blu skae

tōkmeren̄ (comp.) n.com. clear blue sky with no clouds skae we i blu nomo mo nogat klaod see: $\bar{m} a \bar{e}$

tōlōw temp. tomorrow tumora

tōm vt. dab, mark something with finger mekem mak long wan samting wetem fingga I sē mō tōm o bes lo bōs ko? Who marked this post with coconut oil? Hu i makem pos ia wetem oel blong kokonas?

tōmtōm $v i$. be spotted gat spot

tōqagat n.com. blackspot sergeant (fish) wan kaen fis Abudefduf sordidus

tōr $\mathbf{r}_{1}$ vi. bark singaot (dog)

tōr ${ }_{2}$ n.com. Bleeker's parrotfish wan kaen blufis Chlorus bleekeri

tōrak vi. stand up, get up stanap, girap Vël vōrōg no to mamat, na na tōrak den o am, na van me lē kijin na mun kemek. Every day I wake up, I get up from the bed, and I go to our kitchen. Evri dei mi wekap, mi girap long bed, mi go long kijin blong mifala. (DDL.001)

tōrō n.com. white-throated pigeon nataroa Columba vitiensis

tōrō butbut (der.) n.com. male whitethroated pigeon $\sim$ man nataroa 
tōrtōr 1 - vi. preach, give important speech, as in at wedding givim hevi toktok $2 \cdot n$.com. speech $\sim$ strong toktok

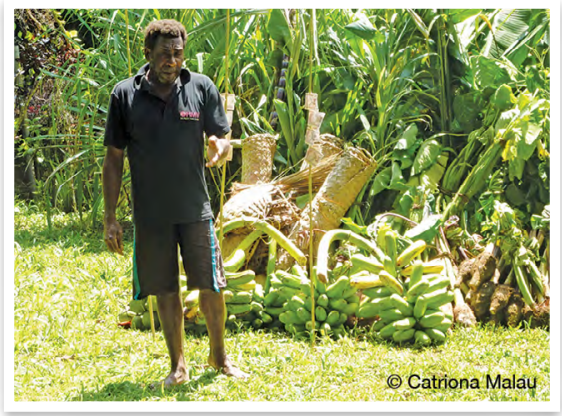

tōrtōr $\sim$ give speech

tōt $v t$. cut using chopping action, with minimal effort required for effect katem, jopem, be i no kadwok tumas Lö kulë luwë meren kōmōrōk a tōt o vēlis. After lunch the two of us cut the grass. Afta long medel dei mitufala $i$ katem gras. (AAD) (JWM) See: tar ${ }_{1}$, tēv

tōt $\mathbf{t}_{2}$.com. poison arrow $\sim$ posen aro buru tōt (comp.) n.com. lid for covering up tip of poison arrow $\sim$ lid blong posen aro

tōt vaslē n.com. variety of breadfruit kaen bredfrut

tōtag vi. producing furry feeling in mouth, as with unripe banana kaen filing yu harem long maot blong yu taem yu kakae banana we i no raep

tōtgial $1 \cdot$ n.com. image, photo, video foto, pikja Lō tōtgial in̄kē, na ēl o tōtgial in̄kē timiak mō tōtgial lö lölö naw. In this photo, I see this photo as if it has been taken inside the sea. Long foto ia, mi luk foto ia olsem ol i tekem foto insaed long solwota. (DPR.001) 2 $v t$. take photo, shoot video $\sim$ tekem foto, mekem video \{Lit. cut lie katem giaman\}

tōtōrōg $\mathbf{1} \cdot v i$. green, be green $\sim$ grin $2 \cdot n . c o m$. greenness, green (one) grinwan $\mathbf{3}$ •vi. blue, be blue $\sim$ blu $\mathbf{4}$ • n.com. blueness, blue (one) bluwan tōtōtōw n.com. progress, growth progres Nē o maranag talō tōtōtōw. He is the chief for progress. Hem $i$ jif blong progres.

tōtōw vi. grow gru In̄ko o qiat nēr mō tōtōw vitia in̄ko. Now the taro are growing already. Nao ia ol taro ol i stap gru nao. (RPT)

tōv n.com. $\mathbf{1} \cdot$ cough $\sim \operatorname{kof} \mathbf{2} \cdot$ have a cold $\sim$ kof, kasem kof

tōw ${ }_{1}$ n.com. hill, mountain hil, maonten Kōmōrōk ma var kal siag li tōw. The two of us went up the hill. Mitufala i klaem antap long hil. (ASS.003)

tōw , vi. grow gru

tōw ${ }_{3} v t$. measure, mark out makem, mesarem In̄ko nēn a tōw o gövur vitia inkko. Then we measure out (the area for) the house. Nao ia yumi mesarem eria blong haos nao. (RBH)

wōtōwtōw (der.) n.com. length of wood used to measure something out wud blong makem $\mathbf{O}$ wōtōwtōw taē ge elelvet, ge elelvet. The measuring stick for it is really really long. Wud blong makem hem i longfala we ilongfala.

tōw 4 (fr.var. tōw mölum) n.com. triton trumpet, conch (gastropod shellfish) pupu Charonia tritonis Rōrō a rōñteg o tōw, nē tö uvuv o tōw. The two of them heard the conch shell, he was blowing the conch. Tufala i karem pupu, hem i stap blo long pupu. (WDR.047) [POc *tapuRi(q) 'triton shell; Charonia tritonis, used as trumpet']

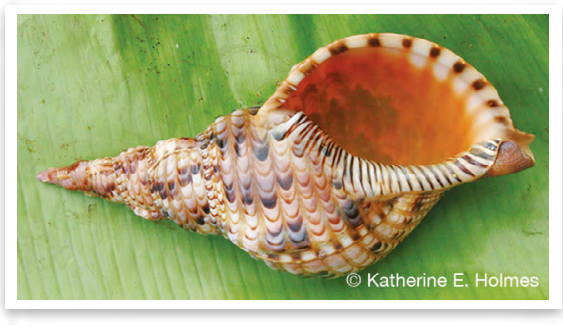

tōw $\sim$ Charonia tritonis 
tōw $\mathbf{w}_{5} v t$. compose (song) komposem (singsing) I Hosea mō tōw na sien i Kerely. Hosea composed a song about Kerely. Hosea $i$ komposem singsing blong Kerely.

Tōw Gereqon n.com. name of hill nem blong wan hil

tōw mölum (fr.var. of tōw ${ }_{4}$ )

Tōw Vetam n.loc. name of hill hil blong wivim mat [So named because it was traditionally a place where they wove mats Ples we ol i wivim mat fastaem.]

tōwav ${ }_{1}$ n.com. scribbled leatherjacket (fish) kaen strongskin (fis) Aluterus scriptus

tōwav $n$.com. rufous-brown pheasantdove (bird) longtel (pijin) Macropygia mackinlayi

tōwereg $v t$. give name to $~$ nemem, singaotem Nēn tō tōwtōwereg o gölörēki, mes nēn tē bigbig e, so lō sōl e. We gave the name of the 'gölörêki' fish, that we eat, to that stage of germinating coconut (because the shoot looks like the fish's tail). Yumi putum nem blong tel blong 'rëki' fis, hemia we yumi kakae, long navara ia (from lif blong hem i luk olsem tel blong fis ia).

tōwlo $n$.com. westerly wind $\sim$ win we i blo long wes [This wind causes rough seas. Win ia i mekem solwota i raf.]

tödun mian n.com. middle-aged man medel man

tödun reqe $n$.com. middle-aged woman $\sim$ medel woman

töl (root of nitöl) num. three tri (namba)

tölne num. third, third one nambatri O tölne tan̄sar tala kakaka lō vōrōg ko Tomas. The third person to speak this morning is Tomas. Nambatri man blong toktok long moning ia hem i Tomas. (VSG)

tömus n.com. rabbitfish piko (fis) Family Siganidae tömus añ (comp.) n.com. kind of rabbitfish kaen piko (fis) Siganus sp.

tömus bul kör (comp.) n.com. kind of rabbitfish kaen piko (fis) Siganus puellus

tömus kör (comp.) n.com. kind of rabbitfish kaen piko (fis) Siganus stellatus

tönö n.com. place ples I Gavin ni van kēl lö tönö wiwieg na non. Gavin went back to his work place. Gavin hem i gobak long ples blong wok blong hem. (AAD)

tönö sigsiag n.com. seat, chair, stool, bench ples blong sidaon, jea

tönöam n.com. bed or sleeping mat bed o mat we yu leidaon long hem Syn: dön̄

tönödem n.com. variety of breadfruit that has large fruit $\sim$ kaen bredfrut we kakae blong hem i bigwan

töqöl vi. boom, bang, make a loud booming noise faerap, mekem bigfala noes olsem saon blong tanda No mō rōnteg o rarö timiak o men gö töqöl. I heard a noise like thunder booming. Mi harem wan noes olsem tanda ifaerap.

töqul $v t$. throw away sakem Nēr wun töqul ti o vin garat lō rōr. They would throw the food scraps in the rubbish. Bae ol i sakem doti blong kakae long ples blong doti. (GMM.023)

törö $1 \cdot v$. make marking, draw, paint, dye $\sim$ kalarem, putum kala long wan samting Nē mö törö la tan tek na boson. He drew on the ground with his finger. Hem i dro long graon wetem fingga blong hem. Na qal törö o mak. I first make a mark. Fastaem mi mekem mak. (VVKHVDP180ct0801.083) $\mathbf{2}$ • n.com. colour, markings kala $\mathbf{O}$ törö na non ni van me, ni tōtōrōg, ni qag, ni mamē, ni añañ. Its colours became green and white and red and yellow. Kala blong hem i kam i grin, i waet, i red, i yelo. (WRN.123) 
törö ${ }_{2}$ n.com. orangeband surgeonfish kaen fis we $i$ gat naef, wetem orenj spot long hem Acanthurus olivaceus \{Lit. painted pentem\}

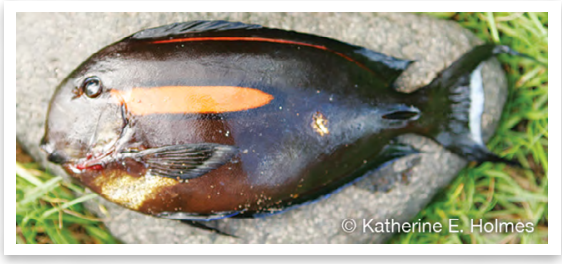

törö Acanthurus olivaceus

törtörök vi. crow, sound made by rooster man faol i singaot

tös $v t$. strip thorny edges (of pandanus) with needle-like instrument and split into strips ready for weaving $\sim$ splitim pandanas smolsmol mo tekemaot bun o nil

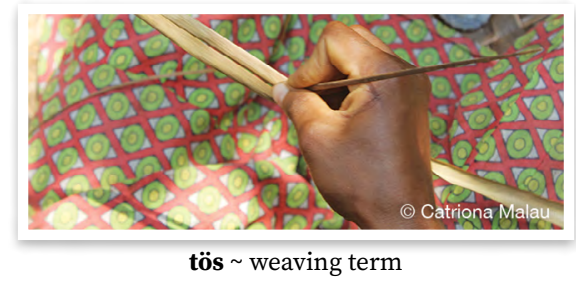

tötöu n.com. nest nes

tötöu men (der.) n.com. bird's nest nes blong pijin

töv n.com. sugarcane $\sim$ sugaken

Saccharum officinarum [POc *topu 'sugarcane']

tövun (fr.var. tuvun) vt. $\mathbf{1}$ • bury berem

Nēr mē ēlgōr nē vaavan götö möul ni miat. Qèt nēr a tövun nē. They looked after him until before long he was dead. Then they buried him. Ol $i$ lukaotem hem go go ino longtaem hem $i$ ded. Afta ol i berem hem. (ATB) 2 - fill in (hole), cover over something in hole berem Nēn mē gil qēt o qarqarörö, nēn a tövun o tere wasañ. We dig all the holes, then we fill in the holes with the posts. Yumi digim ol hol finis, yumi berem ol pos. (RBH) tövusm̄ēl n.com. chief who has gained rank in traditional way by kiling pig $\sim$ stret kastom jif we hem i bin kilim pig See: maranag

tövutwen n.com. variety of sweet potato that has a greenish vine kaen kumala we rop blong hem i grin smol

töwör (fr.var. of tuwur)

töwös $v t$. hit kilim Nēk mitö töwös nē. You shouldn't hit her. Bae yu no mas kilim hem. (ESP)

trak n.com. vehicle, car, utility vehicle $\sim$ trak [Bis trak 'vehicle']

traosis n.com. $\mathbf{1}$ - trousers $\sim$ traosis 2 - shorts $\sim$ sot traosis [Bis traosis 'trousers']

tri manis $n$.com. variety of sweet potato that is valued as it bears tubers only three months after planting kaen kumala we i stap tri manis nomo, afta i karem kakae [Bis tri manis 'three months']

tuar (fr.var. of tōar)

tubu n.p.bound $\mathbf{1}$ - grandparent, grandchild, reciprocal term used between grandparent and grandchild bubu, smol bubu No go gis o kakaka ine, me den ra tubuk. I have this story, and it comes from my ancestors. Mi holem storian ia, hem i kam long ol bubu blong mi. (ESP) 2 • ancestor $\sim$ bubu blong bifo

tōbōn (n.poss) n.p.bound her/his grandparent/grandchild bubu blong hem

tubuk (n.poss) n.p.bound my grandparent/grandchild bubu blong mi

tubuk n.p.bound my grandparent/ grandchild bubu blong mi See: tubu tuë vi. long ago longtaem

tul 1 1 vi. drown, fall down from height $\sim$ draon Na bulën nēn o genqirēg mö tul. Our dog drowned. Dog blong yumi $i$ draon. 2 - vt. submerge $\sim$ draonem $O$ ak ni van me ine, nēr a tul o vet, 
a van sar. The boat came, and they threw down a stone (as anchor) and went ashore. Bot i kam ia, ol i sakem ston, ol i go antap long soa. $\mathbf{3}$ - vi. set (of sun) go daon, draon (san) 0 lō mö tul vitia. The sun has already gone down. San idraon finis.

tul viteg (comp.) vt. drown irretrievably (i.e. resulting in death) $\sim$ draonem i go mo yu no save karem bakegen Nē mö tul viteg o genqirēg. He drowned the dog. Hem $i$ bin draonem dog $i$ go.

tuleg $v$. act of children of a dead man making payment to their father's family to claim their right to his land and property taem wan man i ded, ol pikinini blong ded man ol i mas givim mane long famle blong papa blong pemaot graon mo ol propeti long ol famle blong mekem se $\mathrm{i}$ graon blong olgeta nao

tulëg $v$. send sanem Syn: vetren

tulëg sal (der.) $v$. send word sanem toktok i go Wun i rege bulrō den wun mö tulëg nē me. Ating tufala ia ating i sendem hem i kam.

tulnö lō n.com. west, place where sun sets $\sim$ wes, ples we san i draon Ant: rōwnölō \{Lit. drowning of sun draon blong san\}

tulug n.com. variety of banana, considered to be one of the original endemic varieties on Vanua Lava, with very long fruit, eaten only when ripe, rather than cooked when unripe kaen banana we kakae blong hem i longlongfala. Hem i blong kakae taem i raep mo yu mas skwisim fastaem

tum $\mathbf{1} \cdot v t$. burn bonem $\mathbf{O}$ ev mö tum o gövur. The fire burnt the house. Faea i bonem haos. 2 - vi. burn, be burnt bon $\mathbf{O}$ laplap go tum. The laplap is burnt. Laplap ibon. tumsölönev food is burnt on the outside but raw in the middle after roasting in a fire that is too hot kakae $\mathrm{i}$ bon long afsaed be i no dan long insaed, taem yu rus be faea $i$ hot tumas

tun $_{1} v t$. roast staple foods, as taro, banana, cassava, etc. (not meat) over fire or embers fasin blong rusum kakae we yu putum ova long faea o jakol Ni tun o miak ine. He roasted the Tahitian chestnuts. Hem i rusum namambe ia. (JMT) [Refers to roasting of staple foods only, not meat. Yusum blong talem rusum kakae nomo, i no blong rusum mit.] See: rēn̄ē [POc *tunu 'roast on embers or in fire']

tuntun (redup.) vi. roast food $\sim$ rus

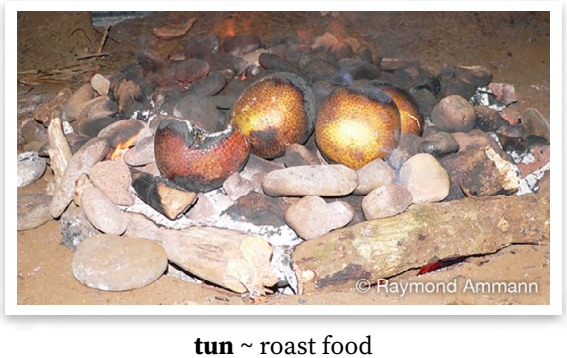

$\operatorname{tun}_{2} v t$. buy land from another tribe pemaot graon long narafala traeb long kastom Nēk gö tun o tan lō qō wo o riaw wo o tiveg. You buy land from another tribe with pigs, hermaphrodite pigs and money. Iu pemaot graon long pig, pig we hem i man mo woman wantaem mo mane. [When land is bought in this way it will then always remain property of the tribe who bought it and will not go back to the other tribe. Yu save askem blong pemaot graon sapos yu nidim. Sapos yu pemaot graon olsem ia bae i graon blong yu oltaem nao mo i no save gobak long narafala traeb.] See: ununseg 
tun tenēnēg $v t$. straighten, sort out stretem Nēr mö tuntenēnēg o qaqaq tale legleg nonor. They sorted out the business relating to the wedding yesterday. Ol i stretem toktok blong mared yestedei.

tuq̄ē (fr.var. of tiqē)

tur vi. $\mathbf{1} \cdot$ stand, stand up stanap Nēk i van me in̄kē talö tur in̄kē tale le o kakaka. Come here to stand here and give the speech. Yu kam ia blong stanap ia blong givim toktok. (VSG) 2 - have a stance, maintain a position on a particular thinking or argument stanap long wan tingting No gö tur so la qaqaq mögun Doran. I will make a stance according to the words of Doran. Bae mi stanap long tingting folem toktok blong Doran. (CAA)

tur mōtō n.com. coconut palm stamba blong kokonas

turgēn 1 1 n.com.suff. body bodi No mē èl o turgi tan̄sar gi miat. I saw the body of a dead man. Mi luk ded bodi blong man. 2 n.com. trunk of tree stamba blong wud

turgi adj. real, proper, true stret, barava, tru Kōmōrōn̄ me le na turgi tutuëk wo no me le na turgi tutuën kōmōrōn. You took (in marriage) my real sister and I took your real sister. Yu tekem stret sista blong mi mo mi tekem stret sista blong yu. (ADT)

turm̄̄o $v$. go first, be in front, lead stanap fastaem, go fastaem, lidim Tōar gö turm̄̄ō, tōar gö tur töwur. One is standing in front, and one is standing behind. Wan istanap fastaem, narawan istanap bihaen. (M2D)

tut 1 t. punch faetem

tut $_{2} v t$. hunt at night, using light to shine on prey, such as flying fox, coconut crab, crab, prawns mekem laet, olsem blong kasem krab, flaengfokis, naora long naet Kemem a van vita a tut ōr lē lēk.
We went to catch prawns in the lake at night. Mifala i go mekem laet blong karem naora long lek. (AAD)

tut $_{3} v t$. pull using quick sharp motion $\sim$ pulum taet hariap La masawre nitiwial nē min rōw me, nē mē siag tenēnēg na mōgōñ o ga, nēk i tut. When one (bird) arrives and sits straight at the place where your rope is, then you pull sharply. Taem wan $i$ kamtru, i sidaon stret long rop blong yu, bae yu pulum strong. (SBB)

tutgëg $v t$. stick, poke something into something stikim wan samting Nēk mö tutgëg o ñili wëër lē kērēk, na möguk o veglal. You stuck the end of a casuarina leafinto my bottom, and that is my mark. Yu stikim en blong oktri $i$ go long as blong mi, hem i mak blong mi. (FRN)

tutgö n.com. fin wing blong fis

tutrevak n.com. kind of bird like a Vanuatu mountain pigeon but bigger wan kaen pijin olsem nawimba blong hil be bigwan

Tutu n.p.fr. address term for opposite sex sibling, brother (of a girl), sister (of a boy) nem blong singaot long brata blong wan gel o sista blong wan boe See: tutue

tutuag vi. worry, suffer $\sim$ wari Nē go tutuag sur $\mathbf{i}$ taman go los. He is worrying because his father is sick. Hem i wari from papa blong hem i sik. (KMT)

tutue n.p.bound opposite sex sibling, brother (of a girl), sister (of a boy) brata blong wan gel o sista blong wan boe

tōtōan (n.poss) n.p.bound her/his/its opposite sex sibling brata blong hem (blong gel) o sista blong hem (blong man)

tutuen Hilton (n.poss) n.p.bound Hilton's sister sista blong Hilton 
tutuëk (n.poss) n.p.bound my brother (for girl), my sister (for boy) brata blong mi (gel i toktok) o sista blong mi (boe i toktok)

tutugēn tamarge n.com. kind of small shrub with purple flowers wan kaen plant we i gat flaoa we i popel Blumea lacinuata \{Lit. old man's walking stick wokingstik blong olfala man\}

tutugon n.com.suff. walking stick wokingstik

tutum $v i$. be greasy, fatty gat gris

tutun vi. $\mathbf{1}$ - hot $\sim$ hot $\mathbf{O}$ masawre gö tutun idian. It is too hot. Ples $i$ hot tumas. Ant: malas, mamēgin $\mathbf{1} \mid \mathbf{2}$. have fever $\sim$ gat fiva

tutuqë vi. many, plenty, be many fulap, plante Syn: lōqtag, m̄ōrag, wuw 2

tutwō $n$.com. kind of bird, like a Vanuatu mountain pigeon but smaller $\sim$ wan kaen pijin olsem nawimba blong hil be i smol lelebet

tuvun (fr.var. of tövun) tuw $1 \cdot v i$. come undone, unfastened kamaot (olsem samting we ol i bin fasem) Syn: ul $\mathbf{1} \mid \mathbf{2} \cdot v$ t. undo, untie karemaot Na tuw na vuluk. I undid my hair. Mi karemaot hea blong mi. No mö tuw o qō. I untied the pig. Mi karemaot pig (we mi bin fasem). Syn: ul 2

tuwegev (fr.var. tuwëgev) vi. make fire in stone oven $\sim$ mekem faea insaed long oven Nōtōn mē sēs qēt o um, rētnan ni le me o ralēt, rōrō a tuwegev. Her child removed the stones from the stone oven, her mother brought the firewood, and the two of them made the fire. Pikinini blong hem i karemaot ston long oven, mama blong hem $i$ karem faeawud $i$ kam, tufala $i$ mekem faea. (WNW)

tuwëgev (fr.var. of tuwegev)

tuwur (fr.var. töwör) loc.adj. behind bihaen

tutuwur (redup.) loc.adj. after afta, bihaen Nēk ga qaq tutuwur. You will speak afterwards. Bae yu toktok bihaen.

\section{$\mathbf{U} \sim \mathbf{u}$}

ukuëg $\mathbf{1}$ • vi. depart, leave go, lego Tawian, dōrōk a ukuëg. Brotherin-law, let's go. Tawian, yumitu go. 2 - vt. leave (place, person) lego I Papa John ni ukuëg kōmōrōk, ni van a Fiji tabo ēl i Rohan nēr. Papa John left us, and he went to Fiji to see Rohan and family. Papa John hem i lego mitufala hem i go long Fiji blong luk Rohan olgeta. (AAD) $\mathbf{3} \cdot v t$. release, let go of, allow to happen, let $\sim$ lego wan samting we yu holem I no mitö ukuëg le bilēn̄ o malmâl ko vita nē ni mōl tatag i Leven. I won't be able to let this young woman go to live with Leven. Bae mi no save lego yangfala gel ia blong hem i go stap wetem
Leven. (CAA) $\mathbf{4} \cdot v t$. leave something to continue, especially continue unaided for a time lego i stap Mē qēt na ukuëg o ev ni ës ti. Then I left the fire to smoke. Afta mi lego faea $i$ stap smok. (NBQ)

ul $\mathbf{1} \cdot v i$. come undone, unfastened kamaot (olsem samting we ol i bin fasem) Syn: tuw $\mathbf{1} \mid \mathbf{2} \cdot v t$. undo, untie, unwrap, unfasten $\sim$ slakem, openemaot, karemaot (rop) Qēt kemem a ul o tere tabēva. Then we unwrapped the presents. Afta mifala $i$ openem ol presen. (AAD.239) Nē ni ul o mon qō. He unwrapped the parcel of pork. Hem i openem pasel blong pig. (WDR.033) Syn: tuw 2 
ulēulēi $n . c o m$. variety of banana kaen banana

um n.com. oven made by digging hole in the ground and baking food with hot stones graon oven Na sēs qēt o um, nana le o ralèt, na tuwegev. I take the stones out of the oven, then I get the firewood, and I light the fire. Mi karemaot ston long oven, mi tekem faeawud, mi laetem faea. (NBQ) See: qan̄ris $\mathbf{1}$, vësëw $\mathbf{1}[P O c$ *qumun 'oven made with hot stones; cook in a stone or earth oven']

um val (comp.) n.com. kind of stone oven for which a big hole is dug in the ground which is then filled with big stones kaen oven we ol i digim hol, afta fulumap wetem bigfala ston

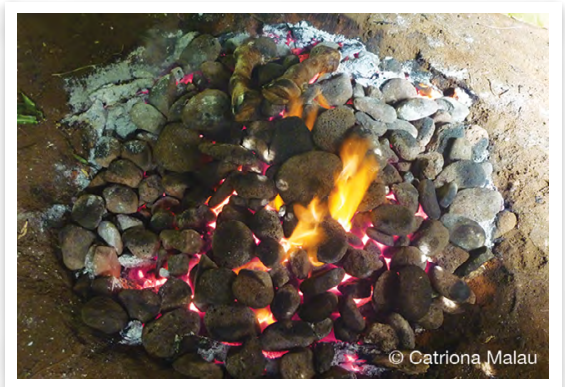

um stone oven

un n.com. palolo, sea worm, marine annelid palolo, siwom Palola viridis

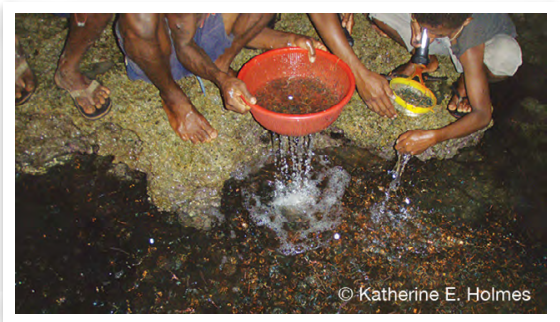

un $\sim$ Palola viridis

un gal (comp.) n.com. male of the species Palola viridis, distinguished by its light brown colouring palolo wom we hem i man wan, we hem i laet braon

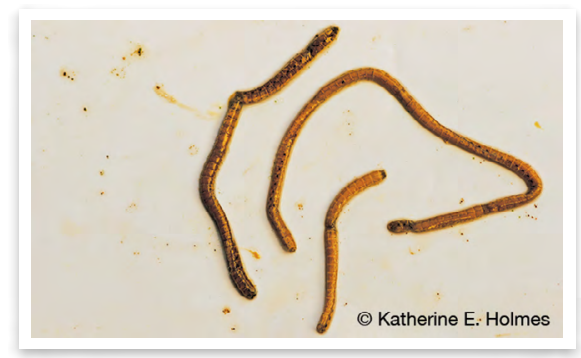

un gal $\sim$ male Palola viridis

un lam (comp.) n.com. female of the species Palola viridis, distinguished by its deep blue-green colouring palolo wom we hem i woman wan, hem i dak mo i blu o grin

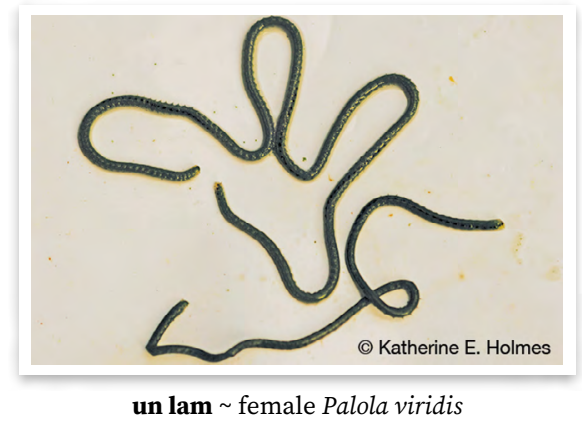

ununseg $v$. make ceremony to release land from your father's ownership to you, when father has died karemaot graon long Papa taem i ded [The land can go to someone else on the basis of marriage. Graon i save go long narafala, long saed mared] See: tun 2

ur n.com. great hog plum $\sim$ naos Spondias dulcis $[P O C$ *quRis 'Spondias cytherea']

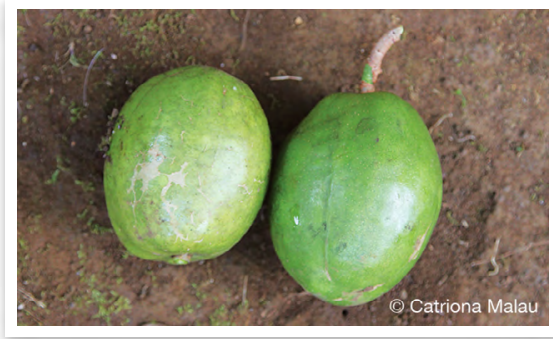

ur $\sim$ Spondias dulcis 
usu tan n.com. dust das Syn: tan vōvō usugiav n.com. kind of tree $\sim$ wan kaen tri we natora blong hem i blak Annona squamosa? [Used for making house posts. Blong mekem pos blong haos.]

usuk n.com.suff. my life laef blong mi See: ēs

ut ${ }_{1}$ n.com. reef needlefish, keeltail needlefish, mature needlefish longmaot (fis), ol bigwan blong hem Strongylura incisa, Platybelone argalus See: til $[P O C$ *qutur 'Sphyraena sp., barracuda'] ut lam (comp.) n.com. kind of needlefish $\sim$ kaen longmaot $\{$ Lit. deep sea needlefish dipsi longmaot\}

ut timiat (comp.) n.com. kind of needlefish kaen longmaot \{Lit. devil needlefish devel longmaot\}

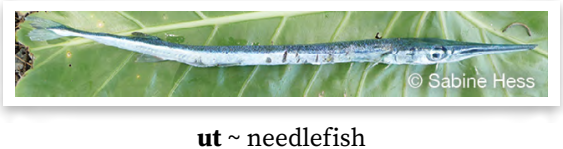

$\mathbf{u t}_{2} v t$. collect water $\sim$ kasem wota

uv (fr.var. iv) v. blow blo O len̄ ni uv me. The wind is blowing this way. Win iblo i kam. [POC *upi 'blow; native flute']

\section{$\mathbf{V} \sim \mathbf{v}$}

va- pre. causative prefix maka we $i$ go long fored blong veb we i minim se mekem Nē ma vaēs no. She resuscitated me. Hem i mekem mi mi laef bakegen.

vaēs $v$ t. resuscitate $\sim$ mekem i laef vag $n$ surgeonfish, name covers larger species of surgeonfish $\sim$ fis we $i$ gat naef, ol bigwan blong hem Family Acanthuridae, Acanthurus maculiceps, A. albipectoralis See: qōl

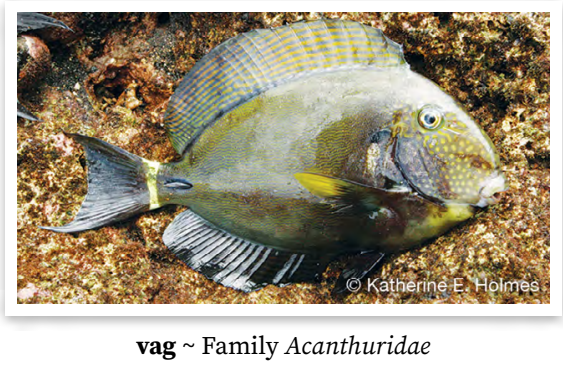

vag $\overline{\mathbf{m e}} \mathbf{l}$ (comp.) n.com. yellowfin surgeonfish $\sim$ wan kaen fis we $\mathrm{i}$ gat naef Acanthurus xanthopterus \{Lit. cycad surgeonfish namele fis we $i$ gat naef\} vag mël (comp.) n.com. eyestripe surgeonfish wan kaen fis we i gat naef Acanthurus dussumieri \{Lit. immature coconut surgeonfish grin kokonas fis we i gat naef\}

vag- num. > num. multiplicative prefix, added to numeral, indicates that the action is carried out specified number of times maka we $\mathrm{i}$ go long ol namba mo i talem se mekem hamas taem we namba i talemaot Nēr ma da vagvet. They did it four times. Olimekem fo taem.

vaga $a d v .1$ - always, all the time oltaem, evritaem Vaga nēk ta vanvan o vanvan gö möul. You always go at a slow speed. Evritaem yu stap go sloslo. (DNS.021) Syn: tastas $\mathbf{2} \cdot$ often, regularly fulap taem Nē ga van vaga a Sol. He goes to Sola often. Hem i go fulap taem long Sola.

vagal n.com. war, large scale fighting wo, bigfala faet

vaglevetē 1 - num. six times sikis taem $2 \cdot v i$. do six times $\sim$ mekem sikis taem 
vaglōqtag $1 \cdot$ num. many times $\sim$ fulap taem Nē më rëw o ës ine vaglōqtag. It sang the song many times. Hem $i$ singim singsing ia fulap taem. (WNW) 2 vi. do many times $\sim$ mekem fulap taem

vaglōvōrō $1 \cdot$ num. seven times seven taem $\mathbf{2} \cdot v i$. do seven times $\sim$ mekem seven taem

vaglövötöl 1 - num. eight times $\sim$ eit taem $\mathbf{2} \cdot v i$. do eight times $\sim$ mekem eit taem

vaglövövet $\mathbf{1} \cdot$ num. nine times $\sim$ naen taem $2 \cdot v i$. do nine times $\sim$ mekem naen taem

vagmööldöl 1 - num. hundred times handred taem $2 \cdot v i$. do a hundred times $\sim$ mekem handred taem

vagrō 1 - num. twice $\sim$ tu taem $\mathbf{O}$ to nitiwial, i ginon ge le o miar vagrō. In one year his wife would give birth twice. Long wan yia, woman blong hem bae i karem pikinini tu taem. $2 \cdot v i$. do twice $\sim$ mekem tu taem

vagrōne num. second time nambatu taem Nēk i rōñteg wun dēndēn o ak vatiwial, nitog ēlēl ris, vagrōne, nitog nitog èlēl ris ... When you hear banging on the canoe once, don't turn round, the second time, don't turn round... Y Iu harem i stap kilim kenu nambawan taem, yu no tanem raon, nambatu taem, yu no tanem raon ... (ATB)

vagsamul $1 \cdot$ num. ten times $\sim$ ten taem $\mathbf{2} \cdot v i$. do ten times $\sim$ mekem ten taem vagtar 1 - num. thousand times taosen taem $\mathbf{2} \cdot$ vi. do thousand times $\sim$ mekem taosen taem

vagtevelēm $\mathbf{1}$ - num. five times $\sim$ faef taem Si mi er nēr vagtevelēm, wo matien nēr töl ine. If it bites them five times, then that will mean their death. Sapos i kakae olgeta faef taem, be hemia ded blong trifala. (DDP) $\mathbf{2}$-vi. do five times $\sim$ mekem faef taem vagtevelēmne num. fifth time nambafaef taem vagtöl 1 - num. three times $\sim$ tri taem 2 - vi. do three times $\sim$ mekem tri taem

vagtölne num. third time $\sim$ nambatri taem

vagvag $n . c o m$. variety of banana $\sim$ kaen banana

vagvet 1 - num. four times $\sim$ fo taem 2 - vi. do four times $\sim$ mekem fo taem vagvetne num. fourth time $\sim$ nambafo taem

vagwuw $1 \cdot$ num. many times $\sim$ fulap taem $\mathbf{2} \cdot v i$. do many times $\sim$ mekem fulap taem

val n.com. hookjaw moray kaen namarae

valakreg $a d v$. randomly, not in ordered way olbaot Nēr ma da valakreg. They did it in a disorderly manner. Olimekem olbaot.

$\operatorname{van}_{1} \mathbf{1} \cdot v i$. go (unmarked for direction) go Kōmōrōk a van ti lē tiqē, kōmōrōk a kēl me. The two of us went to the garden, and we came back. Mitufala i go long garen, afta mitufala i kambak. (OQQ) See: kal $\mathbf{1}$, tēqēl $\mathbf{1} \mid \mathbf{2}$ • v.part. on and on, postverbal particle indicating continuous aspect, continuation of an activity $\sim$ go go

$\operatorname{van}_{2}$ n.com. kind of pandanus that grows by the sea and has thorny leaves. The fruit is eaten by people and flying foxes wan kaen pandanas we i stap long solwota we i gat nil. Man mo flaengfokis ol i save kakae frut blong hem mo ol i juim mo afta spetemaot makas

van $_{3}$ n.com. pineapple $\sim$ paenapol Ananas comosus

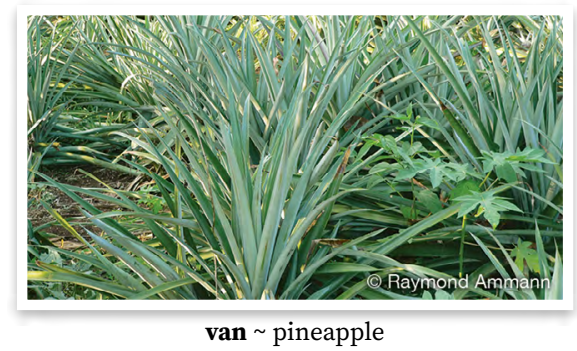


van me vi. 1 • come kam Nēk i van me! You come! Yu kam! 2 become kam Nēk ga van me o tan̄sar luwō. You will become an important person. Bae yu kam wan big man.

vanan n.com.suff. her/his/its place ples blong hem See: vene

vandam̄eg $a d v$. unused, wasted, without use, of no use nating, nogat yus blong hem Qaqaq na mōgōn ma vanvan dā̄eg gem, nēr gete le. His words were just wasted, they didn't take it in. Toktok blong hem i go nating nomo, olgeta ol i no tekem. Nē o vōnō vandam̄eg. It is a wilderness, place where there is nothing there. Hem i ples we inogat man.

Vanlis n.loc. place name $\sim$ nem blong ples

vann̄on n.p.bound niece, nephew, child of sister (of a man) smol angkel, pikinini blong sista blong wan man

Vann̄ö n.p.fr. niece, nephew, child of sister (of a man) smol angkel, pikinini blong sista (blong wan man)

Vanötur n.loc. tribe name, named because they go (van) and stand around (turtur) nem blong traeb we nem blong olgeta i minim se ol i stap stanap (turtur) tumas

Vanuatu n.loc. Vanuatu Vanuatu

vanvan n.com. going, movement wokbaot I rege ko, o sav vanvan soko? Those people, what kind of walking is that? Olgeta ia, wanem kaen wokbaot olsem ia?

van̄ $v t$. revive $\sim$ mekem i kam laef bakegen Wun nēr ma van̄ nē, van̄ nē tek o tere tenge. I reckon they revived him, revived him with local medicines. Ating ol i bin mekem hem i kam laef bakegen, mekem hem i laef wetem ol lif meresin. (JTF)

$\operatorname{var}_{1} \mathbf{1} \cdot v i$. stomp, tread heavily on $\sim$ prumbut $2 \cdot v i$. go in upwards direction $\sim$ go antap $\mathbf{3} \cdot v t$. stand on, kick something with $\sim$ stanap long, kik samting wetem See: varseg $\operatorname{var}_{2} v$. plant (island cabbage) planem (aelan kabis) No ma var o sasar. I planted island cabbage. Mi planem aetan kabis. [Only used in reference to planting island cabbage. Wod ia ol i yusum nomo blong planem aelan kabis.]

varam n.com. twin twin Rōrō a vösus nötu mirmiar nirō. Rōrō o varam. The two of them gave birth to two children. The two of them were twins. Tufala i bonem tufala pikinini. Tufala itwin. (DMM)

varan n.com.suff. $\mathbf{1} \cdot$ her/his/its chest jes blong hem $\mathbf{2} \cdot$ her/his/its liver leva blong hem; See: vere ${ }_{2}$

varar n.com. weaving pattern where two strips are woven under, two over; term used for weaving pandanus, coconut leaves, bamboo walling stael blong wiv we tu pis i go ova, tu pis i go anda; stael ia hem i blong pandanas, kokonas o bambu blong haos

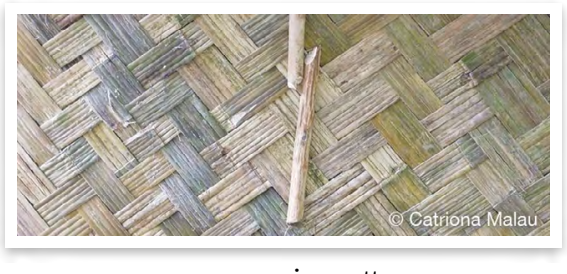

varar $\sim$ weaving pattern

varar tur (comp.) n.com. two over, two under weaving pattern, straight up and down $\sim$ stael blong wiv we tu pis i go ova, tu pis i go anda, i stanap stret

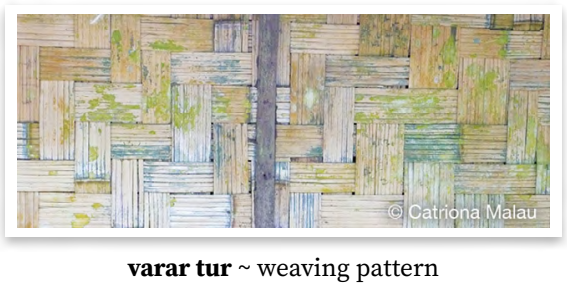

varē n.com. outside, open space outside of an enclosed space, as outside a house, in the open area of a village, on an open vehicle aotsaed Ni kal 
lin̄ereg me lö gövur, taval dērēn la varē, taval dērēn lö gövur. He was going into the house, and one of his legs was outside, and the other leg was inside the house. Hem i go insaed long haos, wansaed leg blong hem i stap aotsaed, narasaed leg blong hem i stap insaed long haos. (SSW) [POc *pera '(?) settlement, open space associated with a house or settlement']

varēg $v$. indicate, show $\sim$ soemaot

varge $a d v$. tightly $\sim$ taet

varian inter. thanks $\sim$ tangkyu

varseg $v$. stand firm stanap strong See: $\operatorname{var}_{1}$

varvar n.com. kind of tree wan kaen wud Neisosperma oppositifolia See: rōq

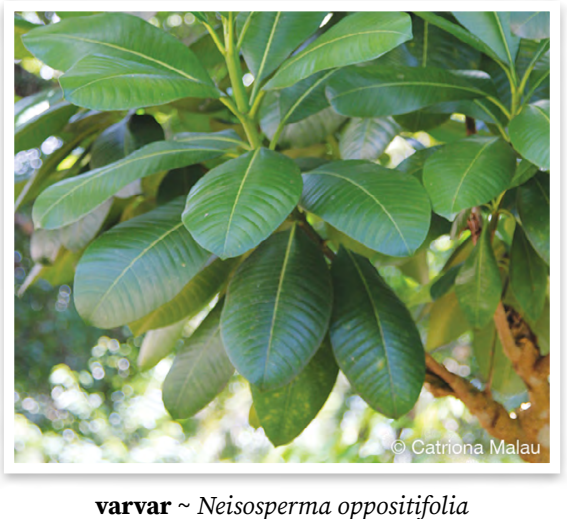

varvarsōm n.com. variety of taro with green stem and small black petiole, with white corm $~$ kaen taro we han blong hem i laet grin mo kakae i waet $\{$ Lit. stomp on parrotfish stanap long blufis\}

varwëgsērèt $n$ first flooding in process of irrigating of taro paddy. In this step the water remains in the paddy for five days before it is drained fas taem we yu putum wota i go long garen taro blong i stap 5 dei

varwëw $v$. troll (fishing line) $\sim$ fasin blong fising long bot we yu lego string we i stap long rol In̄ko nē ni varwëw viteg kēl o ga na non. Then he released his fishing line again. Nao ia hem i lego string blong hem bakegen. (OHS)

varwōs mōgōn n.com. her/his/its lungs lang blong hem

varwöt inter. serves you right $\sim$ i gud ia (talemaot long wan man taem wan nogud samting i hapen long hem be yu ting se i stret from wanem we hem i bin mekem)

varwöw $v$. troll (fishing) fasin blong fising long bot we yu lego string we i stap long rol

vas $v t$. plant taro planem garen taro

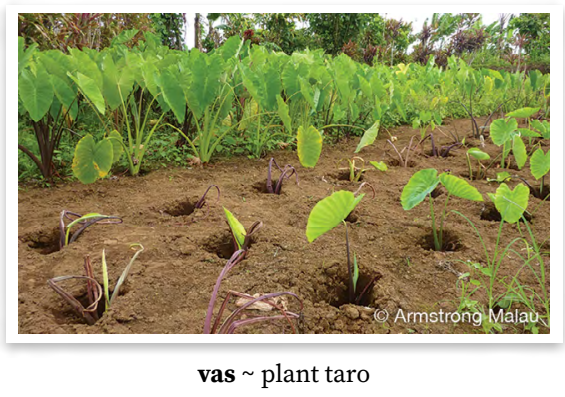

vasas n.com. something surprising or unexpected wan sapraesing samting Kōmōrōn ma da o vasas ine. The two of you did something unexpected and surprising. Yutufala $i$ mekem wan sapraesing samting.

vasēlē $v t$. launch (canoe) lonjem, putum (kenu) i go long solwota Kemem a vasēlē o ak la naw. We launch the canoe into the sea. Mifala i putum kenu $i$ go long solwota. (VVKHVDP180ct0801.171)

vasēr n.com. cassava variety $\sim$ kaen maniok

vasērēg $1 \cdot v$. think about, consider $\sim$ tingbaot Ba si tatag nēn o sul ga vasērēg vita nēn ge tek me, alē nēn a tek me. But if, following us people we think about it, that we will bring it, then we will bring it. Be sapos folem yumi ol pipol yumi tingbaot se bae yumi karem i kam, ale yumi karem i kam. (VSM) 2 - n.com. idea, thought 
tingting Kemem gē rēv tatag o vasvasērēg gē sisia. We would write following different ideas. Bae mifala $i$ raetem folem defren tingting.

vasgēr $v t$. prepare, have ready mekem redi i stap Nē mö tun vasgēr ti na gen nēr o miak. I have roasted their Tahitian chestnuts, ready for them. Mirusum namambe blong olgeta $i$ redi $i$ stap. (JRQ) Nē ni sar o qiat, nē ni sir, qēt nē ni da vasgēr o um, ni tuwegev. He harvested the taro, he scraped it clean, then he prepared the stone oven, and he lit the fire. Hem i pulumaot taro, hem i skrasem, afta hem $i$ mekem redi oven, hem i laetem faea. (ESP)

vaslē n.com. area of taro paddies (qēl) all planted together $\sim$ ples blong ol garen wota taro

vasōgō $v$. read ridim No go mamar i rege timiak na sien nēr no go vasōgō lō oko. I am going to ask those people whose names I read out now. Bae mi askem olgeta, we olsem nem blong olgeta mi ridimaot nao ia. (VSB)

vasösöv $v t$. advise, instruct givim advaes long Ni ōlōl me i Mōlgon sur nē o mie tan̄sar, ni vasösöv nē sur i tasēn. He called Mōlgon to come because he was the oldest child, and he instructed him about his younger brother. Hem i singaotem Mölgon ikam from hem i fasbon, hem i givim advaes long hem long saed blong smol brata blong hem. (GMM)

vateber $v t$. cause to wake up wekemap Boso nēk ma vateber nē? Why did you wake him up? From wanem yu wekemap hem?

vateqev $v i$. face downwards, for items that have division into top side and underside, such as person lying, bowl, plate, cup fes o lukluk i go daon, olsem man i leidaon o kap o plet Vateqev ti siag me le tebol. Put it face down, up there on the table. Putum fes $i$ go daon, antap long tebol. Ant: atelen

vatēv $v$. visit $\sim$ visitim Kemem ma vatēv

o tan̄sar go los nonor. We visited a sick man yesterday. Mifala i visitim wan sik man yestedei.

vatiwial $a d v$. $\mathbf{1}$ once $\sim$ wantaem No go qaq min nēk vatiwial gëm. I am only going to tell you once. Bae mi tok long yu wantaem nomo. 2 - at once, straight away $\sim$ wantaem

Vatov n.loc. place name $\sim$ nem blong ples

vatōgō n.com. teacher $\sim$ tija

vatvat $1 \cdot v i$. promise, arrange, make agreement $\sim$ promes Nēk i vatvat min nēr vita nēr gö wöl i Roan lo sav wōl. You make an agreement with them that they will pay the bride wealth for Roan in which month. Yu mekem promes wetem olgeta se bae ol $i$ pem Roan long wanem manis. (CAA) 2 - n.com. promise, agreement, arrangement $\sim$ promes Qēt in̄ko nēr me mem o vatvat nitiwial talō sōk sösögöt vita oso ren to kurkur ren na bulën nēr o tere gengen. Then they put in place an agreement to find out what exactly it was that was eating their crops. Nao ia ol i putum wan promes blong faenemaot se wanem stret i stap kakae ol kakae blong olgeta. (WRN) $\mathbf{O}$ götö ni le min nē o vatvat. The hermit crab made him a promise. Nakato i givim promes long hem. (DNS) vavam n.com. slipper lobster flat naora Parribacus caledonicus

vawē $\mathbf{1} \cdot v t$. bless $\sim$ blesem $\mathbf{2} \cdot n$ blessing blesing

vawnët $a d v$. all evriwan

vawot $v t$. give birth bonem Nēr ga vawot o mirmiar. They will give birth to a child. Bae ol $i$ bonem pikinini. Syn: vösus

vawseg $v$. clean around $\sim$ klinim 
veglal $1 \cdot n . c o m$. mark of something, sign saen, mak Nē mē èl gem ke o veglal tala matien rōrō, o dar. He just saw a sign of their death, blood. Hem $i$ luk saen nomo blong ded blong tufala, blad. (GMM.173) 2 - vt. make a mark, sign makem, mekem saen Van, nēk i bis o widiar nilōvōrō, nēk i van, nēk i veglal ra nötun dōrōk. Go, pick seven phosphorescent mushrooms, you go, you mark our children. Go, tekem seven masrum we $i$ saen long naet, yu go, yu makem pikinini blong yumitu. (RPP.108)

vegsañ $v$ give more information in support of another person's argument $\sim$ ademap tingting blong yu blong sapotem narafala man

vele $n$.com.suff. mouth maot

valan (n.poss) n.com.suff. her/his/its mouth maot blong hem

vëlëk (n.poss) n.com.suff. my mouth maot blong mi

veleg $v t$. beat (usually bamboo or wood) with one stick in each hand to make music or dance beat kilim tamtam, bambu blong mekem miusik, o man i danis long hem See: weveveleg

veleklek n.com. brown and crispy, crunchy (of baked laplap and vegetables) i dan gud mo i braon mo i strong (laplap o skin blong kakae we ol i bekem) o laplap o veleklek. The laplap is brown and crunchy. Laplap i dan gud. Kōmōrōk ma qan̄ris o laplap ba nē götö tum, nē ge veleklek gem The two of us baked a laplap but it didn't burn, it was just brown and crunchy. Mitufala i bekem laplap be hem i no bon, hem i dan gud mo ibraon.

velesgon n.com. plants growing closely together so one can't get through bus i fas veltiwial n.com. time when the family of a couple who are to be married come together in order to settle the couple together long taem blong mared ol famle blong man mo woman i kam tugeta blong putum tugeta tufala

Vemölö n.loc. tribe name, members of tribe are said not to marry well and marry own brothers and sisters $\sim$ nem blong traeb we ol i talem se ol i no stap mared gud from ol i stap maredem stret brata o sista blong olgeta

vene n.com.suff. place, owned area of land, place belonging to person or community ples, olsem ples blong man, ples we ol pipol ol i stap long hem Na vënëk ren la naw, no ren ta la naw. My place is really the sea, I am really from the sea. Ples blong mi stret hem i long solwota, mi rili blong solwota. (DSR) See: vōnō 2 [POc *panua 'settlement']

vanan (n.poss) n.com.suff. her/his/its place $~$ ples blong hem

vënëk (n.poss) n.com.suff. my place ples blong mi

ven̄en $v t$. feed, of humans and animals $\sim$ fidim No ge veñen na nötuk tek o sus. I feed my child with the breast. Mi fidim pikinini blong mi long titi. (DFF.013) Syn: bien 1, bōgereg

ver $v$.part. collective subject, preverbal marker that indicates either that the subjects are performing the action of the verb collectively or that the action is reciprocal long hem bakegen, tugeta In̄ko rōrō a ver liliseg aē o gisgis mes. Then the two of them argued with each other about carrying the fish. Nao ia tufala i rao long tufala long saed blong karem fis. (JMT.109)

Veran n.loc. tribe name $\sim$ nem blong traeb 
verdēñ $v i$. meet, join mit $\mathbf{O}$ vötu rēntenge nirō me verdēn. The two pieces of wood joined. Tufala pis wud ijoen

vere $_{1}$ n.com. edge $\sim$ saed

vere ${ }_{2}$ n.com.suff. $\mathbf{1} \cdot$ chest (of body) jes $\mathrm{O}$ met mē dēn vitia na varañ. The tide has come in reaching up to your chest already. Solwota $i$ kamso kasem jes blong yu finis. (JMT) $\mathbf{2} \cdot$ liver leva varan (n.poss) n.com.suff. $\mathbf{1} \cdot$ her/his/ its chest $\sim$ jes blong hem $\mathbf{2} \cdot$ her/his/ its liver leva blong hem;

vërëk (n.poss) n.com.suff. $\mathbf{1} \cdot$ my chest $\sim$ jes blong mi $\mathbf{2} \cdot$ my liver $\sim$ leva blong mi

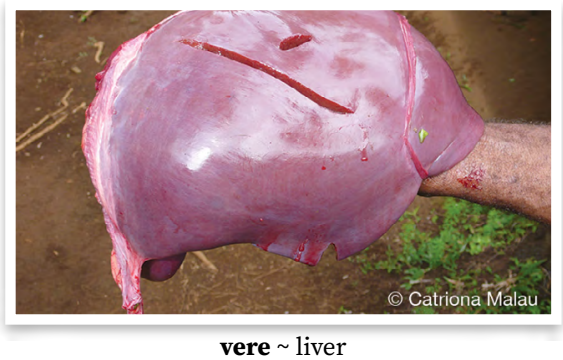

vere wutwut $n$. com. mound, pile, rise of land from where one can see over lower land hip, hip blong graon o sanbij o smol top blong hil

vergöl vi. argue, row rao Rōrō a vergöl vaga. The two of them argue with each other all the time. Tufala i stap rao oltaem.

verlē (fr.var. of vërlē)

verliseg $v$. argue $\sim$ rao Nonor nēr me verliliseg sur o tan. Yesterday they had an argument about land. Yestedei ol i rao from graon.

vernonog $v$. advise $\sim$ advaesem

verqirin $v i$. be too close together, so close that they almost touch fasfas, i klosap tumas Nēr mē riv o tere gē ine gē rivrivteg idian, nēr ge verqirin. They planted the kava too close together, they are touching each other. Ol i planem ol kava i klosap tumas, ol ifasfas.

vesel $v$. go through, poke through, thread through $\sim$ pusum i gotru vet n.com. stone, rock, either small individual stones or large standing stones or rock ston No ma bal o vet ni van lē mēēe qiat. I use the tongs to place the stones on top of the taro. Mi tekem ston, putum i go antap long taro. (NBQ) [POC *patu 'stone, rock']

vet bunbun (comp.) n.com. black stones used for making oven ston blong bek

vet gag (comp.) n.com. rock bigfala ston

vet lèg (comp.) n.com. anchor angka \{Lit. tying stone ston blong fasem\}

vet mërës (comp.) n.com. hard black rock strong blak ston

vet qöñ (comp.) n.com. limestone $\sim$ waet ston \{Lit. night stone ston blong naet\}

vet siag (comp.) n.com. stone for grinding kava on, that has a hollow like a platter $\sim$ ston we i sidaon $\mathrm{i}$ stap, yu no save muvum \{Lit. sitting stone ston $i$ sidaon?

vet ${ }_{2} v t$. plait, weave [refers both generally to interlacing of sets of elements (as plaiting of coconut leaves, pandanus) and specifically to interlacing of a set of weft elements across fixed warp elements] wiv, wivim Nē ga sasarēt talo no go vet o tönöam aē. It is suitable for me to weave a coconut leaf mat with it. Hem i stret blong mi wivim mat blong kokonas long hem. (JWM) [POc *patu(R) 'tie, plait, weave (mats, baskets +)'] vetvet (redup.) n.com. weaving, in process or completed items $\sim$ wiving we i stap mekem o i mekem finis

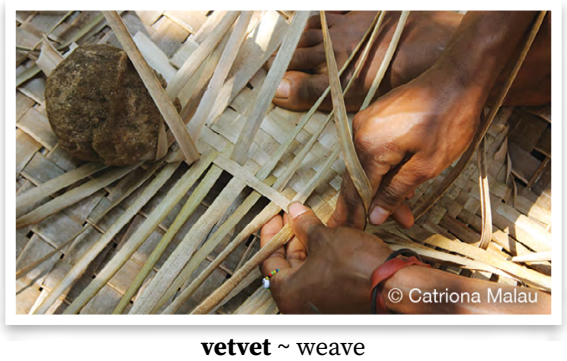


vet $_{3}$ (root of nivet)

vetbōn n.com. rope used for picking up a $w \bar{w} w$ fish trap from the bottom of the sea. It has a stone on the end which has a hole in it for tying the rope to it, and a special hook rop wetem ston mo huk long en blong hem blong pulum wōw net we i stap long solwota

vetel n.com. banana banana Musa spp.

vetel mal (comp.) n.com. variety of banana that has a large trunk but small fruit. The bundle can be quite long wan kaen banana we stamba blong hem i bigbigwan be kakae blong hem i smol. Samtaem bandel blong hem i longfala \{Lit. hawk banana banana blong igel\}

vetel talisōr (comp.) n.com. variety of banana, traditionally considered one of the original endemic varieties, with red fruit, leaves and trunk wan kaen banana we hem $\mathrm{i}$ red, lif i red mo stamba blong hem i smol mo red [Said to be the devil's food. Ol i talem se hem i kakae blong devel.]

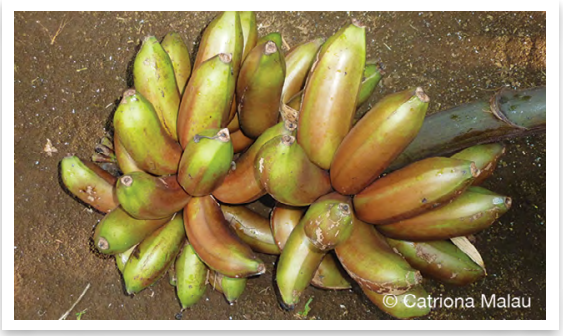

vetel talisōr $\sim$ banana variety

Vetmōl n.loc. place name $\sim$ nem blong ples

vetne num. fourth, fourth one nambafo o vetne dödömia no gö mörös tala gagneg lō min nēn the fourth thing that I want to say to us nambafo samting we mi wantem talem long yumi (VSB)

vetnia $v$ gut (fish, animal); strip off excess woody material from coconut leaf, cane, etc. in preparation for weaving, plaiting tekemaot gat; karemaot doti blong lif kokonas o rop blong wivim

Vetrat n.loc. Vatrata, name of a village $\sim$ nem blong wan vilej

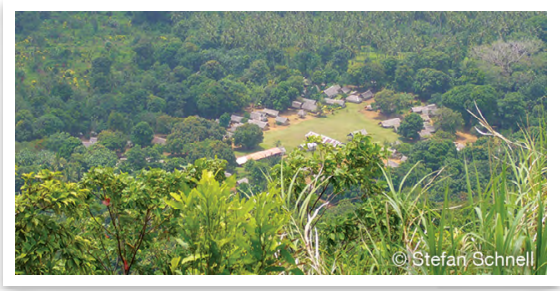

Vetrat $\sim$ Vatrata village

vetren $v t$. send (an item or person to a place) sendem Kōmōrōk a van a mem o katon le sere plen tabo nēr a vetreñ tēqēl a Vōnō Lav. The two of us went and left the box at the airport for them to send down to Vanua Lava. Mitufala i go putum katon long epot blong ol i sendem i go daon long Vanua Lava. (AAD) Syn: tulëg

vetrug $v t$. stand up, erect $\sim$ stanemap No me vetrug kal o tere wasañ. I erected the posts. Mi stanemap ol pos. Nē ni vetrug ti lē qētēgi man̄ko. He lay it against the mango trunk. Hem i stanemap long stamba blong manggo.

vetsur $v$. tell $\sim$ talem $\mathrm{Na}$ vetsur gem min nēk vita nēk gö wöl i Raangelina lō wōl gēn. I'm just telling you that you are going to pay the bride wealth for Angelina in this month. Mi talem nomo long yu se bae yu pem Angelina long manis ia. (CAA) 
$\mathbf{v} \overline{\mathbf{e}}_{1}$ n.com. giant taro, elephant ear taro waeltaro Alocasia macrorrhiza $\left[\mathrm{POC}{ }^{*}\right.$ piRaq 'giant taro, elephant ear taro, Alocasia macrorrhizos']

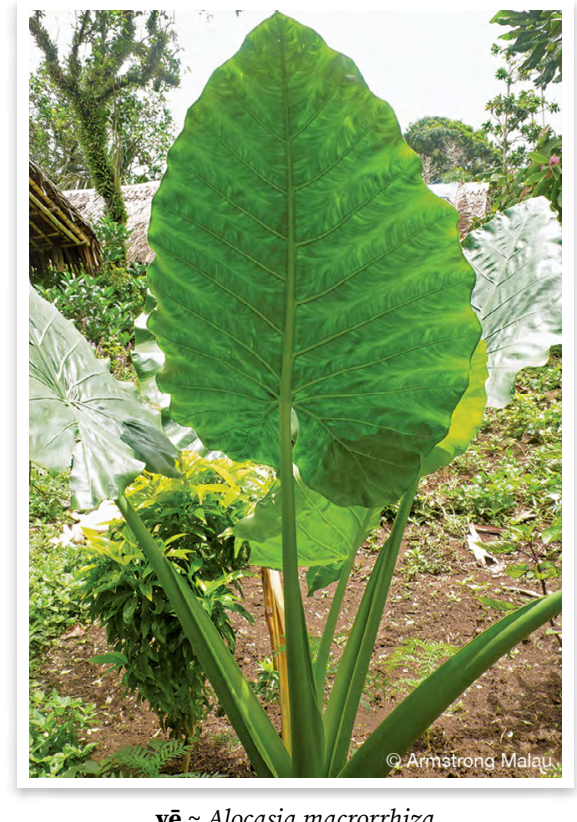

$\mathbf{v} \overline{\mathbf{e}}_{2}$ (root of avē)

vēdir (fr.var. of vidir)

vēkēmolo n.com. kind of tree that grows to about 4 metres, with purple flower and brown fruit $~$ kaen tri we i gat popel flaoa mo braon frut Psychotria aneityensis [O1 i yusum blong faeawud nomo; tenge tala vas qiat]

vēl n.com. lightning laetning [POC * pilak 'lightning']

vēl ${ }_{2} v t$. use magic to kill a person yusum majik blong kilim wan man i ded Isē mē vēl nē? Who killed him with black magic? Hu i kilim hem wetem majik?

vēe $_{3} v t$. divide, distribute, separate seraotem Nēk mē vēl wor rōrō. You separated the two of them. Yu seraotem tufala. vēlavēl n.com. toi (tree) huremi, navasvas (kaen tri) Alphitonia zizyphoides

vēlavēl ${ }_{2}$ n.com. person who knows how to poison people $\sim$ man we hem i save posenem man Tan̄sar ine, nē o tan̄sar vēlavēl. That person is someone who knows how to poison people. Man ia hem $i$ wan we hem i save hao blong posenem man.

vēlereg $v$. divide and put things of same kind together putum ol sem kaen samting long wan ples

vēlēt $v t$. divide, separate, share out seraotem $\mathbf{E}$ rōrō a vēlēt rōrō, i Marinō fesbon, Mōlio sekon bon, ni qaq vita, 'Nēk sur nēk sekon bon, van tavalgi mete gövur kēl wōl e, no fasbon, na van tavalgi mete gövur kēl me sōw e.' Then the two of them separated each other out, Marino the first-born, Mötio the second born, and he said, 'You, because you are the second born, you go on that side of the doorway, and me the first-born, I'll go on this side of the doorway.' Ale tufala $i$ seraotem tufala, Marinō fasbon, Mōlio sekon bon, hem i se, 'Yu from yu sekon bon, bae yu go long narasaed doa blong haos, mi fasbon, mi go long doa blong haos saed i kam.' (DMM.101)

vēlig $v$. prohibit, prevent from doing something stopem blong i no mekem sam samting

vēlis n.com. grass, weeds gras Lö kulë luwë meren kōmōrōk a tōt o vēlis. After lunch the two of us cut the grass. Afta long medel dei mitufala i katem gras. (AAD) [POC * pali[s,j]i 'grass']

vēlis ōlōl n.com. false dodder, love vine, kind of slender rambling vine that grows on ground $\sim$ wan kaen rop we i gru long graon Cassytha filiformis [The leaves can be steeped in coconut oil and rubbed in the hair 
to make it grow long. Kukum long oel blong kokonas mo rabem long hem blong bae i longfala.]

vēlwor $v t$. share out, divide $\sim$ seraotem, divaedem Iñko i Mōlgon ni le rak o wobalak nisam̄ul ine, ni vēlwor wo na gen tasēn nitevelēm, na gan nitevelēm. Then Mōlgon picked up the ten fig fruit and he shared them out and there were five for his little brother, and five for him. Nao ia Mōlgon i pikimap tenfala frut ia, hem i seraotem mo i gat faef blong smol brata blong hem, faef blong hem. (GMM)

vēn 1 t. shoot (with bow and arrow or spear) sutum (wetem bonaro o spia) Nēk gētē vēn qal o mes aē. You didn't manage to shoot any fish. Yu no sutum kasem eni fis. (JMT) See: tēq, tēqatēq

vēnvēn 1 (redup.) vi. go shooting go blong sut Qaq min Die tan Mam vita rōrō a tes na vusun dōrōk, dōrōk a vēnvēn. Tell Mum and Dad to carve our bows, and we'll go shooting. Talem long Mama mo Papa se tufala i kavem bonaro blong yumitu, yumitu go sut.

vēn ${ }_{2} v t$. make thatch slabs for roofing by pinning sago palm leaves over a length of reed $\sim$ pinim lif natanggura Nēn mo so o doot, nēn a tek me, a vidir, a vēn, qēt, in̄ko nēn a at. We remove the sago palm leaves, we bring them, we remove the midribs, we pin them and then we tie the thatch to the rafters. Yumi tekem natanggura, yumi karem i kam, karemaot bun, somap, finis, nao ia yumi fasem $i$ go long bambu. (RBH.016)

vēnēm n.com. tribe traeb Rōrō o vēnēm ta Rēvi. The two of them are from the Revi tribe. Tufala i traeb blong Rèvi.

vēntēn n.com.suff. her/his/its skin skin blong hem See: vinti

vēnti (fr.var. of vinti) vēnvēn $n_{2}$.com. kind of tree with small, pale yellow flowers and small round fruit wan kaen tri we flaoa blong hem i smol mo i yelo mo kakae blong hem i raon Pavetta opulina [Used as a local medicine. $\mathrm{Ol}$ i yusum olsem wan kastom meresin.] \{Lit. shoot, so called because the fruit are used in children's play to shoot people through a blowpipe made of bamboo sutum, from ol pikinini ol i yusum long wan plepleiblong sutum aot long bambu\}

vērē n.com. oyena mojarra, common mojarra (fish) silva fis Family Gerreidae

vērēg $\mathbf{1} \cdot v i$. run ron Nēr a vērēg tatag nē. Nēr gētē gis qal nē. They ran after him. They didn't catch him. Ol i ron folem hem. Ol i no kasem hem 2 - vi. flow (of water, liquid, etc.) (wota i) ron $\mathbf{O}$ tin̄i taē mē vērēg so ine. The liquid from it was flowing. Wota blong hem i ron ia. (OQQ) Nēk mē ēl o bē mamē tē vērēg ti ko. You have seen the red water flowing there. Yu luk red wota i ron long ples ia. (RRS) $\mathbf{3} \boldsymbol{\bullet}$ t. run (tap), cause to flow $\sim$ mekem i ron Na vērēg o bē. I run the water. Mi mekem wota i ron.

na tëqëk gēe vērēg (id.) I have diarrhoea. Bel blong mi i ron.

vēsēn n.com.suff. her vagina kan blong hem

vēti (fr.var. viti) v.part. still yet No mē dēñ me oko lō vōrōg ren vēti e. I reached here when it was still early morning. Mi kasem long ples ia long eli moning yet ia. (DNS) Nē go tog vēti lē vētitne möt. It is still there in the middle of the bush. Hem i stap yet long medel bus. (ESP)

vētitne $n$.com. middle, between $\sim$ medel Nitiwial ni siag lö qötu wo nitiwial ni siag le kere ak, wo i Dōl ni siag lē vētitne. One sat at the front and one sat at the back of the canoe, and Dōl sat 
in the middle. Wan i sidaon long fored mo wan $i$ sidaon long as blong kenu, mo Dōl i sidaon long medel.

Vètuboso n.loc. place name $\sim$ nem blong ples

vētuboso n.com. kind of tree that has thick bark and roots similar to a mangrove wan kaen tri we skin blong hem i tik mo rus blong hem i olsem natongtong Crossostylis cominsii [People use the wood as firewood and as timber for house building. Ol man ol i yusum blong faea o timba blong mekem haos.]

vēvēg ${ }_{1} \quad v t$. count (something) kaontem Ni vēvēg o qöñ, na qin̄in rētnen rōrō, ni gengen. He counted the days, the days since the death of their mother, then they ate (in her memory). Hem i kaontem ol dei, dei blong ded blong mama blong tufala, i kakae (ded blong hem). (KM̄T) Syn: luw vēvēg ${ }_{2} v$. charge (someone) with doing something rong $\sim$ jajem (man) from hem i mekem wan samting i rong vēvēr (fr.var. of vivir)

vëkrës $v t$. scale, remove scales from fish tekemaot skel blong fis

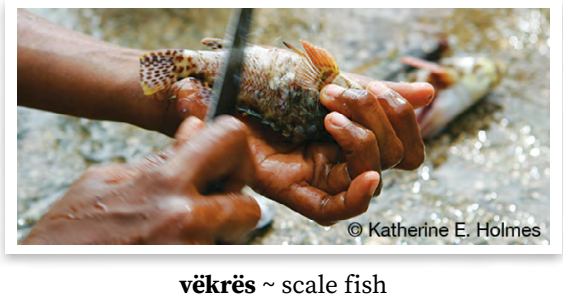

vël quant. every evri I no gē ēl TV vël qön. I watch TV every day. Mi luk TV evridei.

vël ${ }_{2} v t$. answer, reply to ansarem, ríplae long Sar e ni vël nē ta, 'Bulsal, no mē dēn ren ti e!' On the shore he replied to him, 'Friend, I arrived ages ago!' Antap long soa hem $i$ ansarem hem se, 'Fren, mi kasem long taem finis!' (DNS) vëlvël (redup.) n.com. answer ansa Nē ni le o vëlvël kal. He took the answer up. Hem $i$ tekem ansa $i$ go antap. (KMMT)

vëmlösal $v$. reply ansa

vënëk $n$.com.suff. my place ples blong mi See: vene

vër ${ }_{1}$ n.com. stingray stingre $\left[P O C{ }^{*} p a R i\right.$ 'stingray, ray (generic)']

vër dudut (comp.) n.com. blackblotched stingray wan kaen stingre Taeniura melanospila

vër mal (comp.) n.com. spotted eagle ray wan kaen stingre Aetobatus narinari \{Lit. eagle stingray hok stingray\}

vër matawasē (comp.) n.com. Kuhl's stingray wan kaen stingre Neotrygon kuhlii

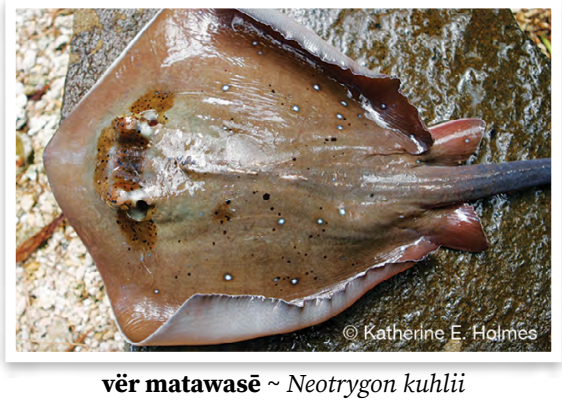

vër timiat (comp.) n.com. manta ray wan kaen stingre Manta birostris \{Lit. devil stingray devel stingre\}

vër ${ }_{2}$ (fr.var. wëvër) n.com. cottonwood, beach hibiscus burao Hibiscus tiliaceus [POC * ${ }^{*} a R u$ 'Hibiscus tiliaceus'] gavër (der.) n.com. $\mathbf{1}$ - inner bark of cottonwood $\sim$ insaed long skin blong burao 2 - cord, braid made from twisted or intertwined inner bark of cottonwood rop we ol i bin tanem long insaed skin blong burao $\mathrm{Na}$ vet qēt, nana van, nana tōt o gavër taē. I finish weaving it, and I go and cut some cottonwood bark for it (for the strap). Mi wivim finis, mi go, mi katem skin blong burao blong handel blong hem. (EWB) 
vërëk n.com.suff. $\mathbf{1} \cdot$ my chest $\sim$ jes blong mi $\mathbf{2} \cdot$ my liver $~$ leva blong $\mathrm{mi}$ See: vere ${ }_{2}$

vërlē (fr.var. verlē) v. compete, race kompit, resis Dōrōk a vërlē sur talē dēñ o tōw gēn. Let's race to see who can reach that hill. Yumitu resis blong kasem hil ia. (DHN)

vësëw $v t$. treat illness by taking leaves of certain medicinal plants, steeping them in boiling water, then covering the person with the treatment so that they can breath in the steam $\sim$ tritim sik man wetem lif meresin we yu kukum ol lif long wota, afta yu mekem man we i sik i smelem stim Nē go los idian, nēk i vësëw nē. He is really sick so you should treat him with a herbal steam remedy. Hem i sik tumas, yu sud stimim hem wetem lif meresin.

vësëw 2 $1 \cdot$ n.com. stone oven $\sim$ hol blong oven [A special kind of oven which is unique to the Banks Islands. A pit is dug, then stones are placed inside and a fire is lit on top. After the fire has died down the stones are not removed (as with most stone ovens in Vanuatu) but are spread flat to evenly distribute the heat, and then the midribs from heliconia leaves are placed on top of the stones before placing the taro or meat on top, so that the food does not burn. The taro or meat is then piled up into a mound, leaving a hole in the middle. Several thousand taro can be cooked in the one oven and when the taro have all been piled up the mound is wrapped with heliconia leaves and then tied up with rope. Water is then poured into the hole left in the middle of the mound and the top is sealed. The food is thus cooked with steam rather than directly from the heat of the rocks. Kaen oven ia hem i wan spesol wan blong Banks nomo.] See: qan̄ris 1, um $\mathbf{2} \cdot v$. bake food in stone oven bekem kakae long oven

vësrët $v$. force $\sim$ fosem

vëtës $v$. discuss, express one's opinion tokbaot, talemaot tingting Vëtës ten sōw, dōrōk ga van la sav masawre? Tell me what you think, when shall we go? Talemaot tingting, bae yumitu go long wanem taem? (WRN)

vëtur vt. place in piles, pile up hipimap

vëwvëwlös $v$. stare intensely lukluk strong

via n.com. grater for grating nuts and tubers to make laplap and nalot samting blong salem nangae o ol kakae Nēk i le o via, nēk i ras. You get the grater and you grate (the food). Iu karem samting blong salem laplap, ale yu salem. (HNN)

via qatē (comp.) n.com. grater made from stem of tree fern samting blong salem laplap, nat o samting olsem we ol i mekem long blakpam via tin (comp.) n.com. grater made from old tin that has holes punched into it samting blong salem laplap, nat o samting olsem we ol i mekem long tin

viabobo n.com. kind of dark brown lizard with large eyes. Its skin is rough and can scratch one's skin wan kaen lised we hem i dak braon mo hem i bigwan wetem bigbig ae. Skin blong hem i raf mo strong mo hem i save skrasem skin blong yu

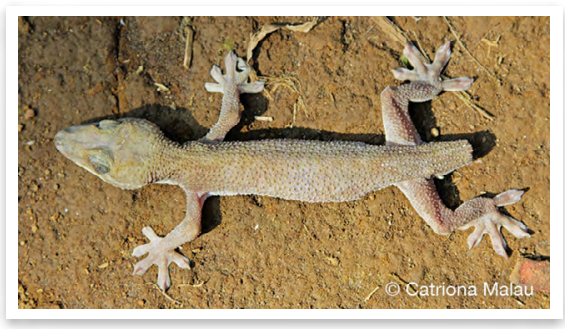

viabobo kind of lizard 
viaw vt. thread, put something on a string putum samting long rop, olsem fasem ol fis long rop o mekem nekles Nēk mē viaw o bitbit. You threaded some beads. Iu putum sam bid igo long string.

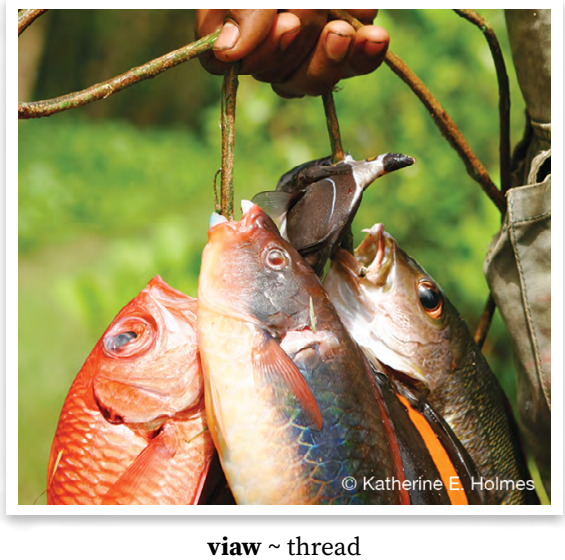

viaw mes n.com. sea is neither calm nor rough $\sim$ solwota i no raf be i no kwaet [This expression is derived from the idea that when it is unclear whether the sea will become rough or not, one should thread one's catch of fish on a rope so that one can be sure that they won't be lost. Ekspresen ia i kamaot long viaw 'putum samting long rop' mo i minim se maet solwota i kam raf, ale i gud yu putum ol fis long rop o maet ol i lus sapos solwota i kam raf.] \{Lit. string fish fasem fis long string\}

vidëm n.com. adze, tool for hollowing out canoe aj, tul blong katem kenu $O$ vidëm, nē talo dur o ak. A vidëm, it is for hollowing out a canoe. Vidëm hem iblong digim kenu. See: tēqēs

vidir (fr.var. vēdir) v. $\mathbf{1}$. remove midrib from sago palm leaf $\sim$ tekemaot bun blong lif natanggura Nēn mo so o doot, nēn a tek me, a vidir, a vēn, qèt, in̄ko nēn a at. We remove the sago palm leaves, we bring them, we remove the midribs, we sew them and then we tie the thatch to the rafters. Yumi tekem natanggura, yumi karem i kam, karemaot bun, somap, finis, nao ia yumi fasem i go long bambu. (RBH.016) 2 - shoot liaw fish sutum liaw fis vietnam n.com. variety of banana kaen banana

viër $v t$. open out flat openem i kam flat Viër na banēn̄! Open your hand! Openem han blong yu!

vikas $v$. communicate in sign language $\sim$ toktok wetem han $\mathbf{0}$ tan̄sar gètē gilal qaqaq, nē gē vikas gëm. A person who can't talk, communicates in sign language. Man we hem ino save toktok, i toktok wetem han blong hem.

vikian n.com.suff. her/his/its thigh top blong leg blong hem

vil $v$ t. pick up holem taet

vil 2 t. tie, tie up fasem

vila n.com. variety of banana with long, large fruit kaen banana we kakae blong hem i longfala mo i bigwan

vilēs mod. all, all over evriwan Nēr qēt, i rege mirmiar qēt mo wotwot vilēs a Vurës. All of them, all of the children were all born at vurës. Olgeta evriwan, ol pikinini evriwan, ol i bon evriwan long Vurës. (NRL) Na babanēn ine, na turturgēn ine, nē o tia vilēs. All over his hands, all his body, he was all covered in shit. Ol han blong hem, evri bodiblong hem, hem i sitsit evriwan. (ADT)

vinbaw n.com. variety of breadfruit kaen bredfrut

vinit $v$. plait in additional pandanus leaf strips to create second side of pandanus mat joenem lif pandanas blong statem narafala saed blong mat

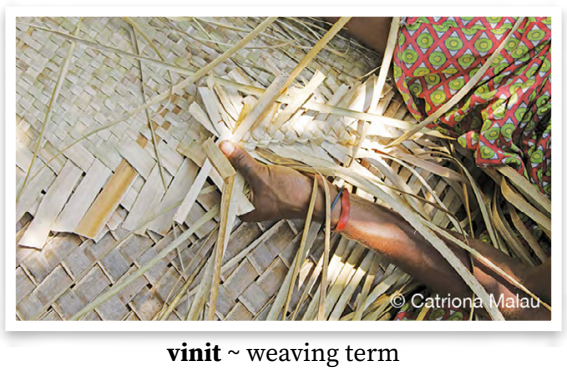


vinkörögör n.com. coconut shell that has had flesh removed sel kokonas Ni da o ev lē vin körögör. He made a fire with the coconut shells. Hem i mekem faea long sel kokonas. Syn: vinlas

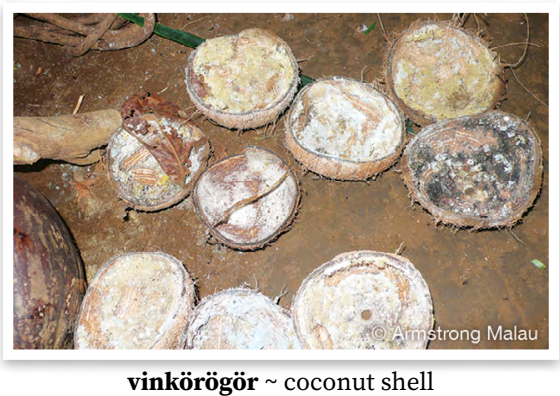

vinlas n.com. coconut shell with flesh removed, especially when used as a cup for drinking kava sel kokonas, olsem ol i dring kava long hem Syn: vinkörögör [POc *lasa 'coconut half-shell cup']

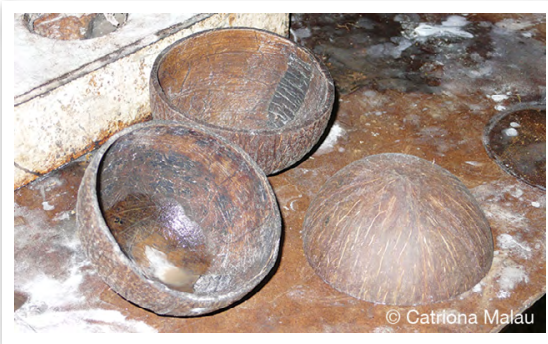

vinlas $\sim$ coconut shell

vinmötöl n.com. variety of taro, 'female', with black stem and white corm kaen taro we hem i woman, we han blong hem i dak lelebet mo kakae i waet [Good for roasting as it has a thick skin. Hem i gud blong rus from skin blong hem i tik.] \{Lit. thick skin skin itik\}

vinraq $n$.com. dead coral $\sim$ rif we i ded finis

vinti (fr.var. vēnti) $\mathbf{1} \cdot$ n.com.suff. skin skin $\mathbf{2} \cdot$ n.com. bark $\sim$ skin blong wud 3 n.com. husk (of nut, coconut), skin (of fruit, vegetable) skin blong nat o kokonas Oo, nana gengen gōwē wo nēk i gengen o vēnti tabaē. Oh, I'll eat the good bit and you eat the skin of it. Ale, mi mi kakae gudwan blong hem mo yu kakae skin blong hem. (OQQ) [POC *pinut 'skin, bark']

vēntēn (n.poss) n.com.suff. her/his/its skin skin blong hem

vintik (n.poss) n.com.suff. my skin skin blong mi

vintik n.com.suff. my skin skin blong mi See: vinti

vinwōn n.com. variety of banana that is long and a flat shape kaen banana we hem i olsem asis banana be hem i longfala lelebet mo hem i save flat vinwōn ga qag (comp.) n.com. variety of banana kaen banana

vinwōn gō tōtōrōg (comp.) n.com. variety of banana kaen banana

vir vt. plait, braid, French braid, plait a cord $\sim$ tanem (hea o rop o saed blong mat) Nē ni vir vasgēr vitia o gagö qō sam̄ul tiwial dēmē nirō. He had already plaited twelve pig tying ropes, ready to use. Hem i tanem redi finis twelf rop blong pig. (ESP) [POc* * piri 'plait a cord, twist, wrap around']

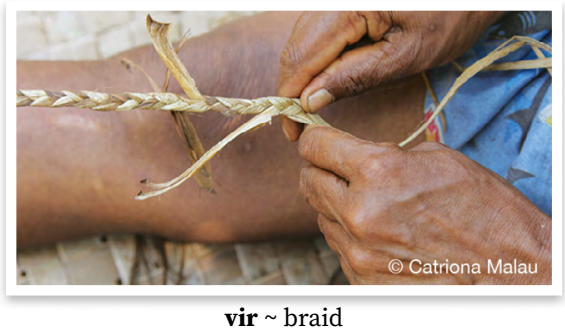

gavirvir (der.) n.com. braid, French braid rop we ol i tanem

viris $v$ t. tie, tie up, tie together $\sim$ fasem Ni viris tiwial o tere qiat. He tied together the taros. Hem i fasem tugeta ol taro. (BQD)

vis $v i$. wrap around $\sim$ fas $\mathbf{O}$ ga vërur, nē mē vis le teqen kōmōrōk. The vines wrapped around our bellies. Rop ia ifas long bel blong mitufala. (ASS.016) 
visō n.com. pitpit, duruka, vegetable cane naviso Saccharum edule $[P O C$ * pijo 'cane or reed taxon, including Saccharum spontaneum']

vita $_{1}$ (abbrev. $\left.\mathrm{ta}_{1}\right)$ subord. that, complementiser introducing complement clause se No gö mörös vita nē ni van kal me in̄ko. I want him to come up now. Mi wantem se bae hem i kam antap nao ia. (KMMT) Gagneg min i tēsik vita nē ni van kal me. Tell my younger brother that he should come up. Talem long smol brata blong mi se bae hem ikam antap. (KMTT) vita $_{2}\left(\right.$ abbrev. ta $\left.{ }_{1}\right) v t$. say talem $\mathbf{O}$ qon vita, 'Gōwē wo.' The pigeon said, 'That's really good.' Nawimba i talem se, 'I gud we.' (DHN)

viteg $a d v$. away, completely i go van viteg (id.) inter. leave it, don't worry about it, it doesn't matter lego, no wari Van viteg! Ine nē mi miat, nē ma van. Don't worry about it! He's dead, he's gone. Lego! Hem i ded, hem igo. (HWM)

viti (fr.var. of vēti)

vitia (fr.var. vitiag) v.part. already $\sim$ finis $\operatorname{vitiar} v$. mention $\sim$ mensonem

vivi n.com. umbrella ambrela

vivir (fr.var. vēvēr) vt. throw at, throw something at someone or something stonem, sakem i go long wan man o wan samting $\mathbf{O}$ gōsōw mē vivir nē vitia tek o tagrës e. The rat had already thrown the wood with burning embers at him. Rat i stonem hem finis wetem pis faeawud we i gat jakol long hem. (WRN)

vivis $v i$. guess $~$ gesem

vivivis (redup.) n.com. guess $\sim$ ges

vlawa n.com. flower $\sim$ flaoa [Bis flaoa 'flower']

vo 1 - $n$ thingummy, thingamabob, indefinite name used for something or someone the speaker chooses not to name or has forgotten the name for samting ia, man ia $\mathbf{E}$ kemek a èl ine wana i vo, i vo ... i Sivlag ni var kal me. Then we saw thingummy, um ... Sivlag coming up. Ale mifala i luk man ia, hu ia ... Sivlag i kam antap. (CAA) $\mathbf{O}$ vo ine nē gö kur nēk garqe. That thing is going to eat you today. Samting ia bae i kakae yu tede. (OQQ) $2 \cdot v$. do something unspecified, like thingummy, is used to refer to an event that the speaker cannot name because they can't think of the word $\sim$ mekem wan samting

$\operatorname{vorog}$ n.com. $\mathbf{1} \cdot$ sweet potato variety kaen kumala $\mathbf{2} \cdot$ frog $\sim$ frog [Bis frog 'frog']

vovoñon vi. go fishing, looking for shellfish or other sea or freshwater creatures lukaotem mit long wota o solwota [POC *panoda 'gather seafood on the reef']

vōl $v$. break off main new shoot, new growth from top of tree, shrub to make it branch

vōlō $n$.com. wart kaen soa

vōlōn n.com.suff. her/his/its hair hea blong hem See: vul

vōlōvōl n.com. Klunzinger's wrasse (fish) wan kaen smolsmol fis Thalassoma klunzingeri

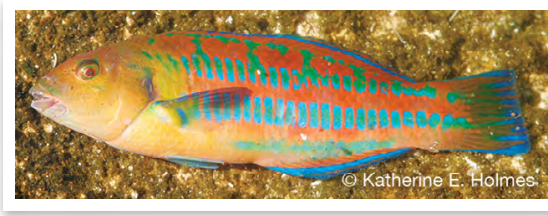

vōlōvōl Thalassoma klunzingeri

vōlōvōl sōōm (comp.) n.com. Christmas wrasse (fish) wan kaen smolsmol fis Thalassoma trilobatum

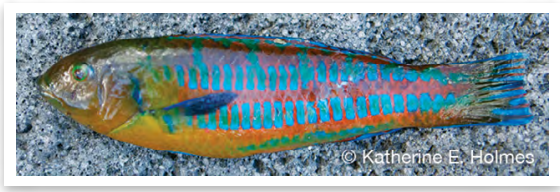

vōlōvōl sō̄̄̄ $~$ Thalassoma trilobatum 
vōn n.com. sore on scalp, caused by lice $\sim$ soa long hed we laos i mekem vōnō n.com. $\mathbf{1}$ • island aelan Dōrōk a qētēg vērēg den oko a vērēg waliög o vōnō. We'll start running from here, and we will run around the island. Yumitu resis stat long ples ia, resis raonem aelan. [The correct name for Vanua Lava island is Vōnō Lav. Stret nem blong Vanua Lava hem i Vōnō Lav.] 2 • village $\sim$ vilej $\mathbf{O}$ sie vōnō ine nēr gō ōl ta Nam̄asmas. The name of that village, they call it Namasmas. Nem blong vilej ia, ol $i$ kolem se Nam̃asinas. (RRS) See: vene [POc *panua 'settlement']

nögö vōnō (comp.) n.com. whole village, look or appearance of village eria blong vilej \{Lit. face of village fes blong vile)\}

vōnōdöwö n.com. kind of shrub, with furry leaves and stem and small mauve flowers wan kaen smol plant Ageratum conyzioides [Used as a local fertiliser to make taro grow big. I gat kastom yus blong mekem se taro i gru bigwan.]

vōnōmōōlō n.com. tradition that is performed in the afternoon of the fourth day after someone has died, in which the heads of the families of the dead person do such things as remove the dead person's house or cut wood in their garden. The children of the dead person must then pay the people who have done this long aftenun long namba fo dei blong wan man i ded, ol man (lida blong olgeta hem i famle blong man we hem i ded) ol i kam katem wud long garen o tekemaot haos blong ded man mo ol samting olsem. Afta pikinini blong ded man i mas givim mane long ol man we ol i mekem See: miarten̄ vōname n.com. kind of tree that has red flowers similar to a Malay apple. Flying foxes drink the nectar from the flowers wan kaen tri we flaoa blong hem i red mo i olsem nakavika be i strong. Flaengfokis i dring flaoa blong hem be ol i mas satem ae taem i dring o sapos no flaoa bae i stikim vōr n.com. volcano volkeno [POc * puro 'bubble up, boil, as hot spring']

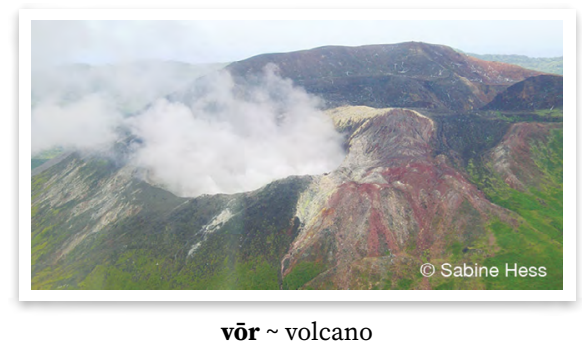

vōrōg $\mathbf{1}$ •n.com. morning moning $\mathbf{2}$ • $v i$. be morning kam moning

vōs n.com. green coconut $\sim$ grin kokonas

vōs gargarteqërët (comp.) n.com. green coconut at beginning of stage appropriate for drinking, with soft shell, thin flesh and liquid inside $\sim$ grin kokonas we sel blong hem i sofsof, kakae blong hem i tintin mo $i$ gat wota blong hem

vōsōsōs vi. (of strands for plaiting) lying overly close together (lif blong wivim) i fasfas tumas (JWM)

vōt n.com. kind of freshwater fish wan kaen fis blong wota we hem i no save agensem karen

vōvo $n$ thing samting Le me o vōvo gēn. Give me that thing. Givim samting ia.

vōvōrō̄n $v$ t. soothe, speak gently (to) toktok sloslo blong mekem i harem gud bakegen No mō vōvōrōñ gōr nē. I spoke gently to him. Mi toktok slosto long hem.

völöt $n$ traditional practice in which when the first child of a couple is born, the family of the mother come and try to throw stones at the father 
of the child and his family taem fes bon pikinini i bon, famle blong mama ol i traem stonem papa blong pikinini wetem ol famle

vörus $v$ t. ask (someone); ask (about) askem (man) Kemem töl a vörus nē vita 'I nēk timiak avēe' The three of us asked her, 'How are you?' Mitrifala i askem hem se, 'Yu olsem wanem?' (AAD) See: baēr

vörus völu $n$.com. question kwestin vösus $v$ t. give birth, lay egg bonem, karem pikinini, putum eg Ginon ine, nē ni vösus o m̄irmiar gēn. His wife gave birth to this child. Woman blong hem ia, hem ibonem pikinini ia. (KMT) Syn: vawot

vötu 1 - n.com. (of tree), trunk, above bole stamba, pat blong stamba daon $2 \cdot n$ very large $\sim$ bigwan tumas Jessica nē o vötu miar. Jessica is a very large child. Jessica hem $i$ wan bigfala pikinini.

vötu wōqōlqōol $n$.com. neck bone bun blong nek

vötubanēn n.com.suff. her/his/its arm han $(\mathrm{am})$ blong hem

vötudōldōl n.com. oesophagus rod blong kakae \{Lit. stem for swallowing stamba blong solemdaon\}

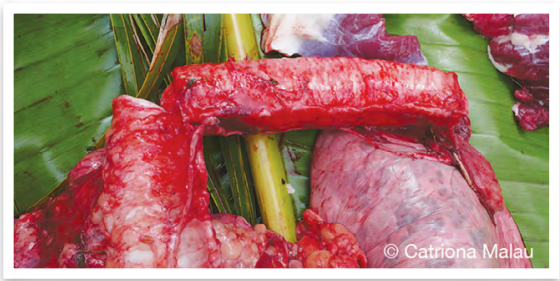

vötudōldōl oesophagus

vötuga n.com. reel, fishing reel $\sim$ wud blong rolem rop i stap long hem vötuga sēsēdig (comp.) n.com. reel for rope for catching birds wud blong rolem rop i stap long hem blong kasem ol pijin

vötuga sōsōsōg (comp.) n.com. fishing reel wud blong rolem string blong fising i stap long hem vötulöt (fr.var. of vutulöt)

vövötös $v$. discuss diskas Tala da o savger gōwē o mie nēn gö vövötös aè. In order to do something good, the first thing is we must discuss about it. Blong mekem wan gud samting yumi mas diskas fastaem.

vu n.com. magical spirit, a supernatural being that appears to people wan spirit we i save kam helpem man No, no o vu nitiwial, ma van me talo no gē birin̄ nēk. I am a spirit, I have come in order to help you. Mi wan spirit, mi kam blong givhan long yu. (ESP) See: tamtiē

vudege (fr.var. vudëgë) n.com. pandanus, screw pine $\sim$ pandanas Pandanus spp.

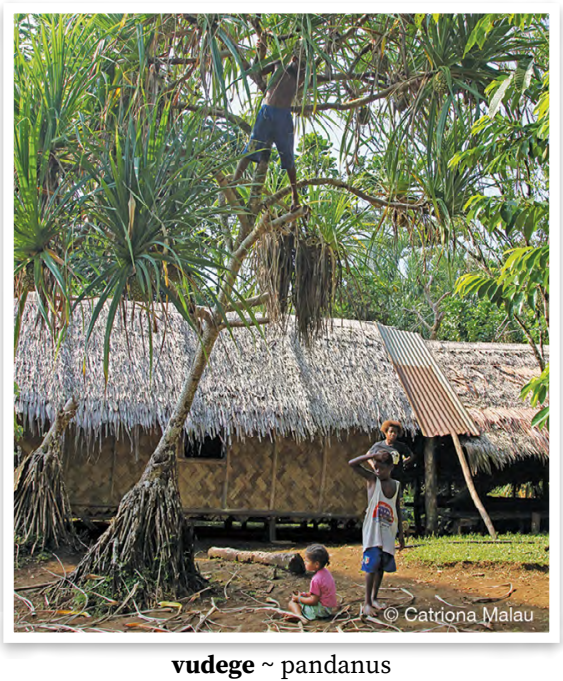

vudëgë (fr.var. of vudege)

$\operatorname{vug}_{1}$ n.com. row of yams in garden ro blong yam long garen Ri èlgōr wareg na bulën nēn o tere vugvug tale dem. Look after our yam plantings well. Lukaotem gud ol yam blong yumi long garen. (HWM)

$\operatorname{vug}_{2} v i$. have meeting $\sim$ miting vugvug (redup.) n.com. meeting miting Nēn a gis o mie vugvug na mögun nēn. We will hold our first meeting. Bae yumi holem fes miting blong yumi. (VSS) 
vukrē n.com. scale skel blong fis

vul n.com. $\mathbf{1}$ • hair hea Nē ga qag, na vōlōn më mèsmēës dēñ na teqelsan. She was white, and her hair fell down covering the back of her neck. Hem i waet, hea blong hem i folfoldaon kasem bihaen long nek blong hem. (ATB) 2 feather $\sim$ feta $\left[P O c{ }^{*}\right.$ pulu- '(body) hair']

vōlōn (n.poss) n.com.suff. her/his/its hair hea blong hem

vulu tō (n.poss) n.com. chicken feather feta blong faol

vuluk (n.poss) n.com.suff. my hair hea blong mi

vulëg $v t$. open (eye) openem (ae) No mö vulëg na mëtëk. I opened my eyes. Mi openem ae blong mi.

vulu tō n.com. chicken feather $\sim$ feta blong faol See: vul

vuluk n.com.suff. my hair hea blong hem See: vul

vun $v$. pour water into oven to produce steam by which food will cook kapsaedem wota antap long kakae long oven
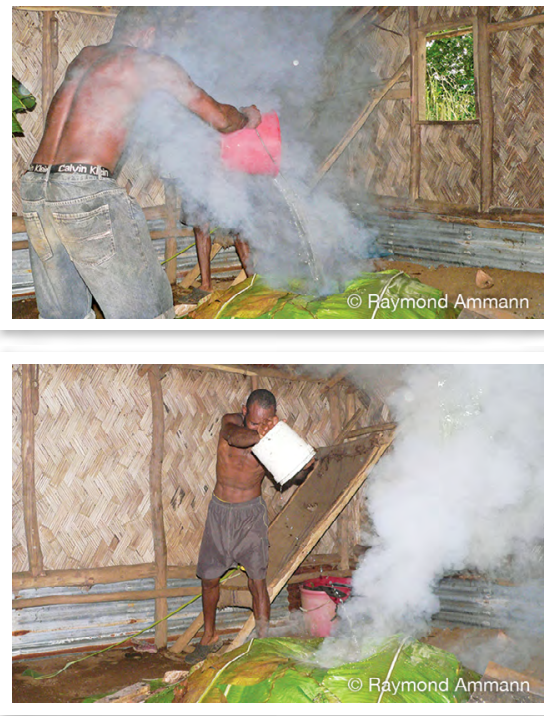

vun steam oven vun $_{2}$ n.com. kind of insect that destroys yam kaen bebet we i kakae yam

$\operatorname{vun}_{3} v$. squeeze liquid out of a leaf that is to be used for local medicine, using one hand melekem (lif) wetem wan han

$\operatorname{vun}_{4} v$. fart inaudibly $\sim$ man $\mathrm{i}$ fat we i kwaet nomo

vun $_{5}$ n.com. ringworm $\sim$ ringwom vun meren (comp.) n.com. type of ringworm where the skin is dry with no liquid coming out unless it is scratched kaen ringwom we wota $i$ no stap kamaot long skin oltaem

vun qar (comp.) n.com. 1 - type of ringworm where the liquid is coming out all the time kaen ringwom we wota blong hem i stap ron oltaem 2 - type of disease that affects trees and other plants, where liquid comes out of the affected part $\sim$ kaen sik blong wud we wota i stap kamaot long skin blong hem oltaem vunvun $v$. poison fish $\sim$ posenem fis [This method is especially used near the shore. Ol i mekem olsem speseli klosap long so.]

vun viteg (der.) $v$. method of poisoning fish in deep sea $\sim$ fasin blong posenem fis long dip si

vun n.com. section of yam garden eria blong garen yam Kēmi mē riv o vuñ nivēs? How many sections did you plant in the yam garden? Yu planem hamas eria blong garen yam?

Vurës n.loc. place name $\sim$ nem blong eria

vurōmōvōt n.com. pearly monocle bream (fish) wan kaen fis Scolopsis margaritifer 
vus n.com. bow $\sim$ bonaro vusun Nikson Nixon's bow bo blong Nixon See: qatē

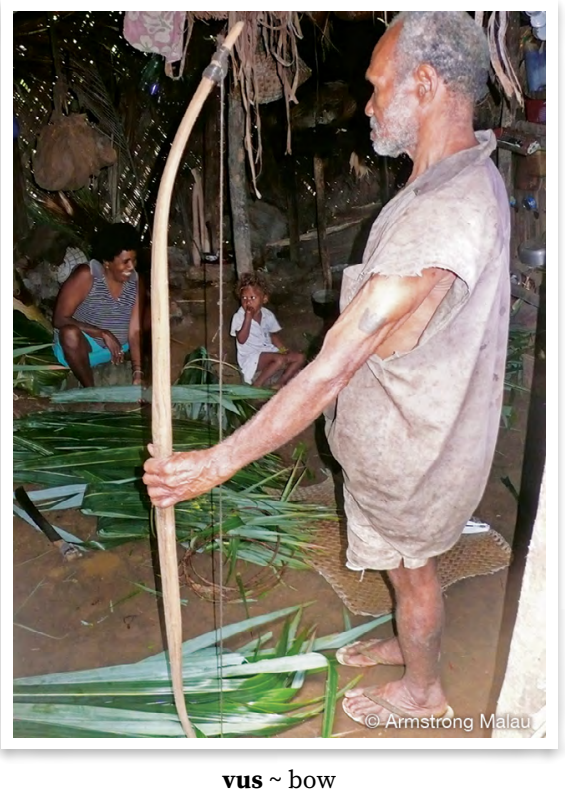

vus 2 t. kill kilim Nēr a vus o tatu na mögun Catriona. Olikilim tatu blong Catriona. (AAD)

vusarōr vi. kill pig in order to release the spirit of someone who has died. The dead person is quiet but the pig squeals to represent the person crying as their spirit is released. kilim pig blong lego spirit blong man we i ded. Ded man i kwaet be pig i singaot blong representem ded man i singaot mo hem i lego spirit.

vusën̄sën n.com. good spirit gudfala spirit

vusgon n.com.suff. her/his/its meat mit blong hem See: vusgö

vusgö n.com. meat, flesh mit $\mathbf{O}$ tere vusvusgēn nēr ni tōtōw tetenēnēg kēl. All their flesh grew back properly again. Mit blong olgeta $i$ gro stret bakegen. (JRQ) [POc * *isiko 'flesh, muscle, meat']

vusgon (n.poss) n.com.suff. her/his/its meat mit blong hem vusö n.com. black-blotched moray wan kaen namarae Gymnothorax sp. See: marēe $_{1}$

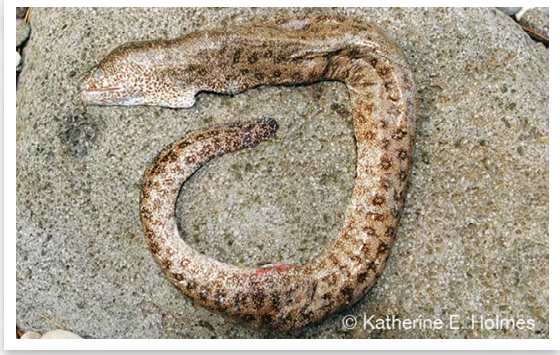

vusö Gymnothorax sp.

vusö ōn (comp.) n.com. painted moray kaen namarae \{Lit. sand moray namarae blong sanbij\}

vusveslivmat $v i$. kill a pig or chicken to close the eyes of someone who has died, a ceremony performed by one of the children of person who has died kilim pig o faol blong klosem ae blong man we i ded, wan pikinini blong man we i ded i mekem

vut $n$.com. fish poison tree, sea poison tree fisposentri Barringtonia asiatica $[P O C$ *putun 'Barringtonia asiatica']

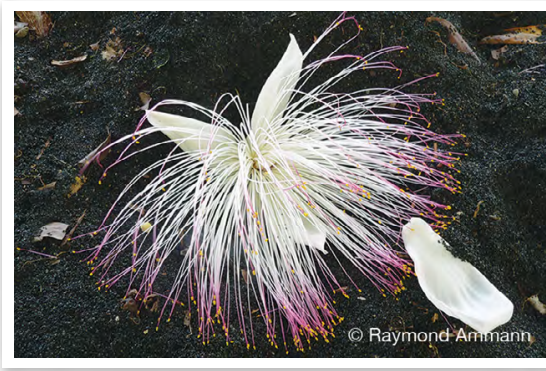

vut $\sim$ Barringtonia asiatic

vuteg $v t$. plant (yam) planem (yam) Nēr a van a vuteg o dëm. They went to plant yams. Ol i go planem yam.

vutrë $n$ piece $\sim$ pis

vutulöt (fr.var. vötulöt) n.com. large wooden pestle used for pounding löt food dish wud blong kilim nalot 


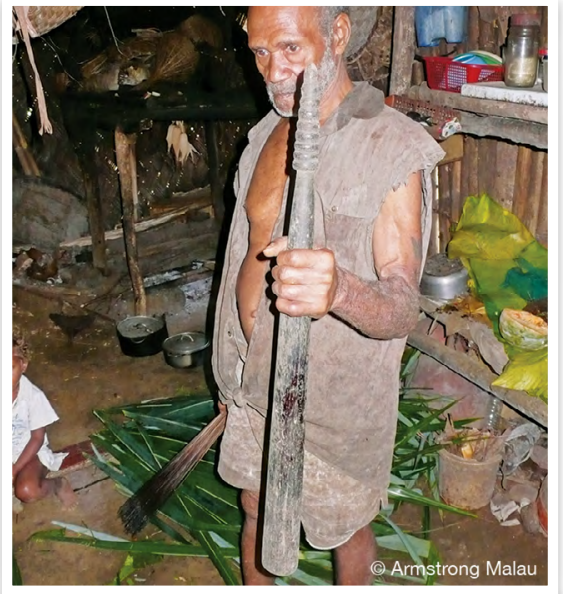

vutulöt $\sim$ wooden pestle vuv vt. pour water on $\sim$ kapsaedem wota long Nē mi miat ti, nēr ma da èsēs nē, mö vuv o bē lō qōtōn. He had been unconscious, but they revived him by pouring water on his head. Hem i bin ded be ol i mekem hem i laef bakegen from ol $i$ kapsaedem wota long hed blong hem.

\section{$\mathbf{W} \sim \mathbf{W}$}

waakak n.com. kind of freshwater mollusc that can be found in places where the water runs fast $\sim$ wan kaen flat sel blong wota we i stap long ples we wota i save ron strong

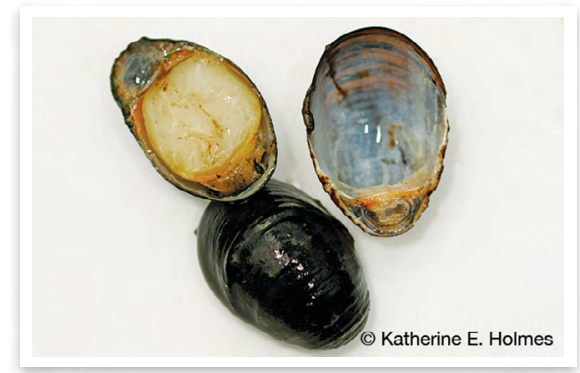

waakak freshwater shellfish

waan̄ n.com. turmeric kari jinja Curcuma longa \{Lit. yellow thing samting we i yelo\} [ $\mathrm{POC}$ *yajo 'turmeric, Curcuma longa']

waar (fr.var. of $\mathrm{ar}_{1}$ )

waatlëk n.com. part of house pat blong haos

wadaln̄an n.com.suff. her/his/its cheek saed blong fes blong hem See: wadaln̄e wadaln̄e n.com.suff. cheek saed blong fes

wadaln̄an (n.poss) n.com.suff. her/his/ its cheek saed blong fes blong hem wadaln̄ëk (n.poss) n.com.suff. my cheek saed blong fes blong mi

wadalnëk n.com.suff. my cheek saed blong fes blong mi See: wadaln̄e

wadas n.com. kind of sea urchin with thick blunt spines kaen sel blong solwota we i gat nil long hem be hem i no sap Family Cidaridae See: sernönqet

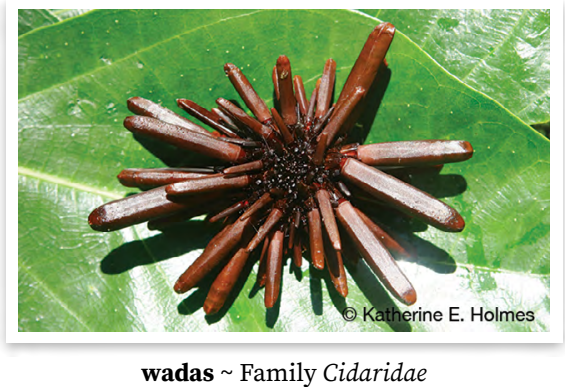

wadē n.com. money mane 
wag 1 n.com. ylang-ylang, perfume tree $\sim$ nandingori Cananga odorata

wag $_{2} v t$. squeeze with hand, like massaging skwisim long han, olsem yu holem man Wag ten na bēnik. Can you massage my arm. Traem holem skwisim han blong mi.

waga $_{1}$ n.com. basket trap for catching freshwater prawns basket blong kasem naora Na bien qèt o waga, na tek le qereñ bē, na wödöñ. I finish baiting the trap, I take it to the river, and I set it with stones around. Mi fidim trap finis, mi karem i go long wota, mi draonem mo putum ston long hem. (KBN)

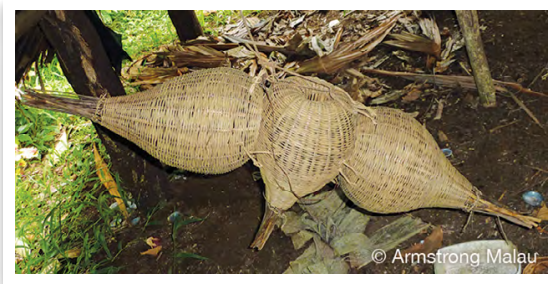

waga $\sim$ prawn trap

waga ${ }_{2}$.com. 1 1 passionfruit $\sim$ pasenfrut Passiflora edulis

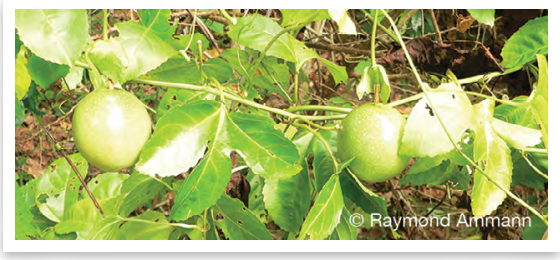

waga $\sim$ Passiflora edulis

2 - stinking passionflower $\sim$ wael pasenfrut Passiflora foetida

wakam n.com. kind of tree with yellow flowers. It has nuts similar to native almonds that are yellow when ripe $\sim$ wan kaen tri we flaoa blong hem i yelo. Kakae blong hem i olsem nangae, i grin be taem i raep i yelo Finchia chloroxantha [Children like to eat the nuts but they are very small. Ol pikinini i kakae be kakae i smol nomo.]

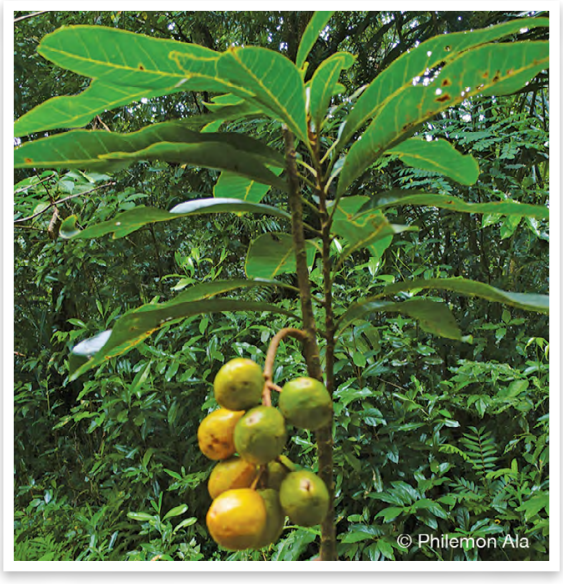

wakam Finchia chloroxantha

wakata n.com. variety of taro $\sim$ kaen taro

wakata gatgat (comp.) n.com. variety of taro, with white stem and white corm with red stripes $\sim$ kaen taro we han blong hem i waet mo kakae blong hem i waet wetem red straep wakata mamē (comp.) n.com. variety of taro, 'female', with tall red stem that has white stripes at the bottom, and white corm kaen taro we hem i woman, we han blong hem i longfala mo i red wetem waet straep daon, kakae blong hem i waet

wakata qagqag (comp.) n.com. variety of taro, 'male', with white stem and corm kaen taro we hem i man, we han mo kakae blong hem i waet

walag n.com. kind of tree with large fruit with yellow skin and red seeds wan kaen tri we wud blong hem i strong mo frut blong hem i yelo mo insaed ol sid i red Dysoxylum aneityensis [Used for making house posts. It is very common. Ol $\mathrm{i}$ yusum blong mekem pos mo evri pat blong haos. Hem i gru olbaot.] See: qōmiat; wosvet 


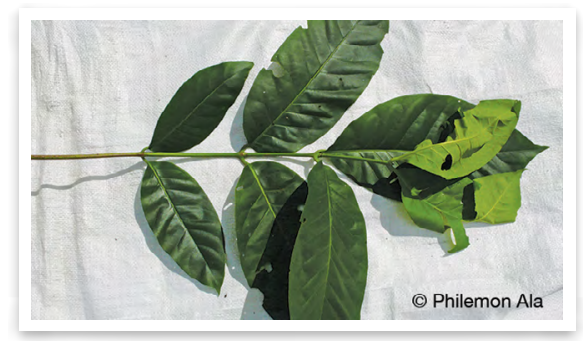

walag Dysoxylum aneityensis

walag n.com. lower leg, between knee and ankle pat blong leg daon, bitwin ni mo angkol

\section{walaglē n.com. orangespine} unicornfish $\sim$ yelo tel strongskin (fis) Naso lituratus

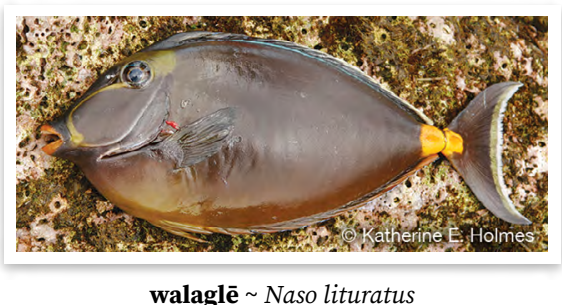

walal $_{1}$ n.com. top shell $\sim$ troka Trochus niloticus [POc *[la]lak[o] 'trochus shell; bracelet made from it']

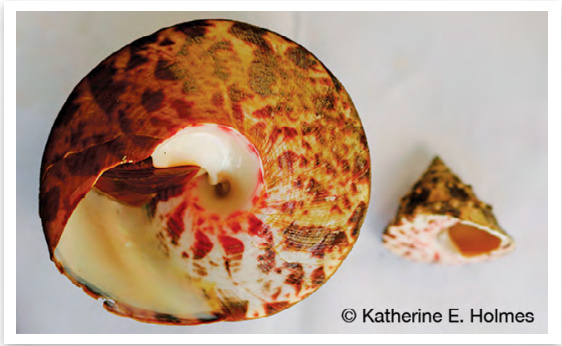

walal $\sim$ Trochus niloticus

walal $_{2}$ n.com. bracelet $\sim$ breslet [POC *[la]lak[o] 'trochus shell; bracelet made from it']

walalas n.com. kind of wild yam wan kaen yam we hem i gru wael Dioscorea pentaphylla

walalnelan $n$.com. kind of yam that has a round shape, is white and can grow large wan kaen yam we hem i raon gud mo hem i save bigbigwan mo hem i waet insaed \{Lit. underneath high chief andanit long hae jif\}

walaqlaq gōsōw n.com. rat trap $\sim$ trap blong rat

walas vi. peel back foreskin skinim kok

waliög $\mathbf{1}$ - vi. round, be round, spherical $\sim$ raon $\mathbf{2} \cdot v t$. go around something, cover all areas $\sim$ raonem wan samting, no mata wanem sep Dōrōk a qētēg vērēg den oko, a vērēg waliög o vōnō. Let's start running from here, and run all the way round the island. Bae yumitu stat blong ron aot long ples ia, ron raonem aelan. (DNS) See: wewese (der. of wese)

wamal n.com. variety of taro that has both 'male' and 'female' varieties, the 'male' with stem that is white at bottom and red at top, the 'female' with purple stem and black petiole kaen taro we i gat woman mo man wan blong hem, we woman, han blong hem i waet daon mo red antap, mo man, han blong hem i popel mo i blak long ples we lif i joen

wamamēgin n.com. type of croton with green leaves $\sim$ famle blong kala lif be lif blong hem i grin nomo Codiaeum variegatum

wamasal n.com. kind of unidentified lump on skin that becomes a sore kaen soa long skin

wamatwōn n.com. monodont (gastropod shellfish) wan kaen sel blong solwota Monodonta sp.

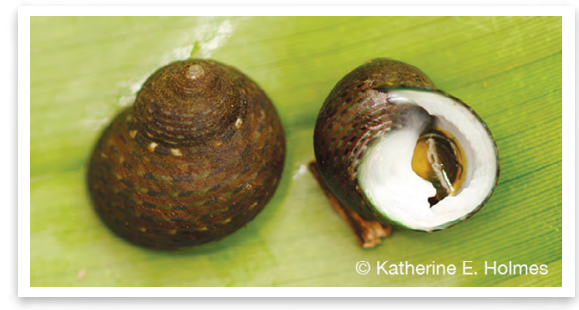

wamatwōn Monodonta sp. 
wamalak vi. white with black splotches, referring to colour of pig or sometimes dog kala blong pig (o dog) we i waet mo blak smol Tere nötu qō, ga qag, gö körkör, ga wamalak, tere qō nē gēn. The little pigs, white, black, all of those pigs. Ol smol pig, ol waet wan, ol blakwan, ol blak mo waet tugeta, ol pig ia nao. (VST) wā̄amas $_{1}$ n.com. kind of plant wan kaen plant Evodia latifolia See: dā̄am̄as

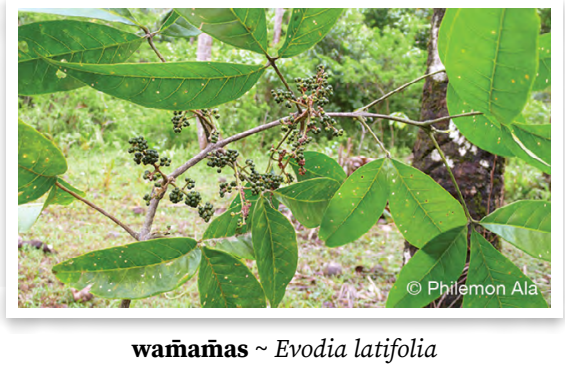

wam̄amas $_{2}$ n.com. food dish in which grated canarium nuts are rolled up in datamal leaves, then wrapped in heliconia leaves and baked in stone oven kaen kakae we ol i rasras nangae, rolem long lif datainal, pasolem long lif laplap, afta bekem

wamas $\overline{\mathbf{e}}_{1}$ n.com. star sta I rege ta $\overline{\mathbf{m}} \overline{\mathbf{o}}$ nēr ga tatag o tere wam̄asē. People from before followed the stars. Ol man blong bifo ol i folem ol sta. (AAD)

wamas $\overline{\mathbf{e}}_{2}$ n.com. kind of basket, shaped like a star wan kaen basket we i gat sep olsem sta

wamas̄e $_{3}$ n.com. abalone (gastropod shellfish) kaen sel Haliotis asinina

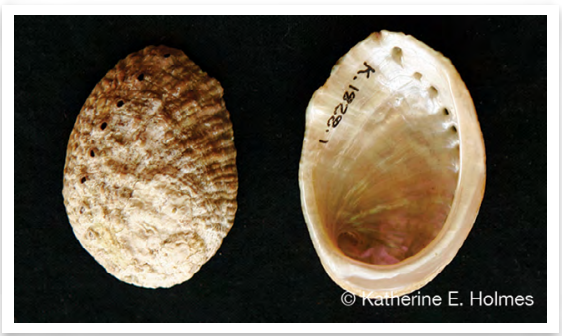

wam̄asē $\sim$ Haliotis asinina wan 1 - $v i$. have kava drinking session $\sim$ stap dring kava Nēn töl ta wanwan ko, ba na gen nēn o bigbig odiañ aē. We're having a kava session now, but we don't have any meat to eat. Yumi stap dring kava ia, be mit blong yumi nogat. (ADT) 2 - n.com. kava session taem blong kava

wan $_{2} v$. make holes, destroying crops mekem hol Na bulën nēn o gengen, ma wan sër. Ma wan lōlō gëm. All of our crops, it (a bird) pecked holes in them. It just made holes in them. Ol kakae blong yumi, hem i mekem hol long hem. I mekem hol gotru long hem nomo. (WRN)

wana disc discourse particle $\sim$ we Syn: mina

wan̄arñar n.com. kind of tree that has fruit similar to a mango, which are red when ripe wan kaen tri we kakae blong hem i olsem manggo we i laet grin, mo taem i raep i red Corynocarpus similis [The fruit needs to be bashed to make it soft before it can be eaten $\mathrm{Ol}$ i mas kilim kilim kakae i sofsof blong kakae.]

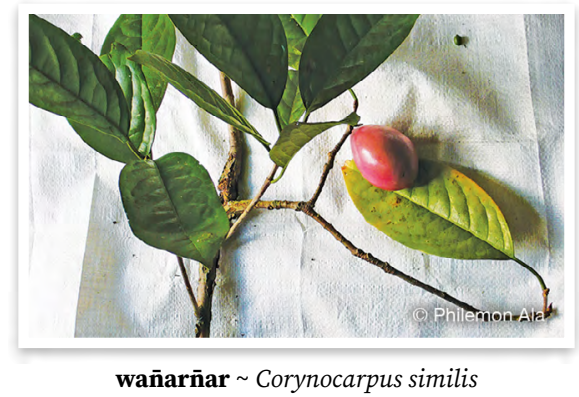

waqagal n.com. hibiscus $\sim$ haebiskas Hibiscus rosa-sinensis $\left[P N C V{ }^{*} b^{w}\right.$ akala 'Hibiscus sp.']

waraēl n.com. fishing bamboo pole bambu blong hukum fis

waran̄rañ $n . c o m$. kind of shrub wan kaen tri Acalypha spp. [It grows in overgrown gardens. Used as a local medicine and for firewood. I gru 
long ples blong olfala garen. $\mathrm{Ol} \mathrm{i}$ yusum blong kastom meresin mo faeawud.]

waran̄ran̄ ga qag (comp.) n.com. plant with pale leaves $~$ kaen plant we lif blong hem i waet Acalypha forsteriana

waran̄rañ mamēe (comp.) n.com. plant with red leaves kaen plant we lif blong hem i red Acalypha hispida

waran̄rañ marge n.com. kind of tree, fruit are red when ripe and similar to that of poumuli wan kaen tri we frut blong hem i red taem i raep mo hem i olsem kakae blong namamao Flacourtia rukam [Birds and children eat the small fruits. Ol nawimba ol i kakae frut blong hem mo hem i kakae blong pikinini tu.]

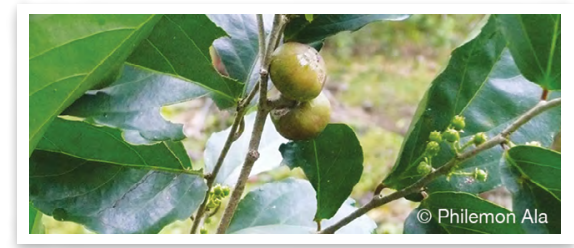

waran̄rañ marge $\sim$ Flacourtia rukam

waras n.com. variety of taro, 'male', with white corm with red points kaen taro we hem i man, we kakae blong hem i waet wetem red long hem

wareg $a d v$. 1 - well, completely, totally $\sim$ gud $\mathbf{O}$ tañ mo wor wareg ren. The basket was totally full. Basket $i$ fulap gud. (JRQ) 0 tewen e mi mian wareg. The native lychees were completely ripe. Nandao ia $i$ raep gud. (OQQ) Syn: wurveg 2 2 properly, correctly $\sim$ stret, gud Si tamañ nē mo tono wareg nēk, nēk gē gilal aē. If your father has taught you properly, then you will know about it. Sapos papa blong yu i bin lanem gud yu, bae yu savelong hem. (SBB)

warian n.com.suff. her/his/its forehead $\sim$ fored blong hem See: wariē wariē n.com.suff. forehead $\sim$ fored Nē ga gasreg o waqagal mamē la warian. He had put a red hibiscus flower in his hair at his forehead. Hem i pusum wan red haebiskas long fored blong hem. (KM̄T)

warian (n.poss) n.com.suff. her/his/its forehead fored blong hem

wariēk (n.poss) n.com.suff. my forehead $\sim$ fored blong mi

wariēk n.com.suff. my forehead $\sim$ fored blong mi See: wariē

warwar n.com. person with prominent forehead man we fored blong hem i bigwan Nē o warwar sur na warian gö wutwut lō. He is a person with a prominent forehead because his forehead bulges out. Hem i wan warwar from fored blong hem ibigwan.

Wasag n.loc. place name $\sim$ nem blong ples

wasalav n.com. variety of taro $\sim$ kaen taro

wasan n.com.suff. her egg eg blong hem See: wese

wasanto n.com. variety of taro that originated from Santo $\sim$ kaen taro we i kam long Santo

wasanto mamē (comp.) n.com. variety of taro originating in Santo that has red corm kaen taro we i kam long Santo we kakae blong hem i red

wasanto qagqag (comp.) n.com. variety of taro originating in Santo that has white corm kaen taro we $i$ kam long Santo we kakae blong hem i waet

wase $_{1}$ n.com. southern shrikebill (bird) wan kaen pijin we hem i braon mo blak Clytorhynchus pachycephaloides

$\mathbf{w a s} \overline{\mathbf{e}}_{2} n$.com. variety of taro that has two types, one with light grey stem, the other with light green stem. They can have lots of forks, but are not good for eating in that case $~$ kaen taro we i gat tu kaen blong hem, wan han blong hem i laet grei, wan han 
blong hem i laet grin. Hem i strong lelebet. Hem i save karem plante fok be sapos olsem kakae i no gud

\section{wasren straight $\sim$ stret}

watagōgōn n.com. kind of gastropod shellfish wan kaen nasese we i stap long ston [hem i konkon so yumi no kukum be yumi rusum]

watamarge n.com. kind of large tree with fruits that are similar to a Malay apple, but they are not commonly eaten as they are very sour. The fruit are red when ripe and long and oval shaped wan kaen tri we hem i bigwan lelebet. Kakae blong hem i olsem nakavika be man i no kakae tumas from i saoa. Taem i no raep i grin mo taem i raep i braet red. Hem i longfala lelebet Aceratium oppositifolium

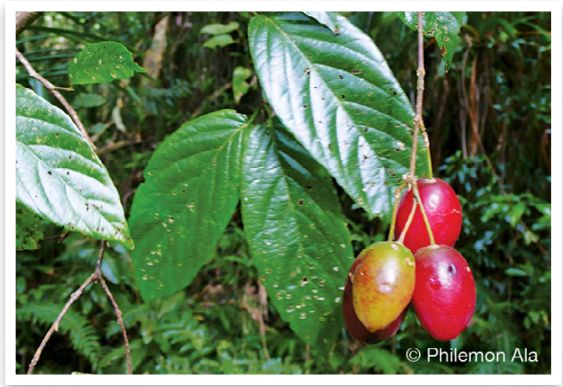

watamarge $\sim$ Aceratium oppositifolium

watavrēn n.com. charcoal jakol watawlētlēt $n$.com. Pacific star shell wan kaen sel blong solwota Astraea haematraga

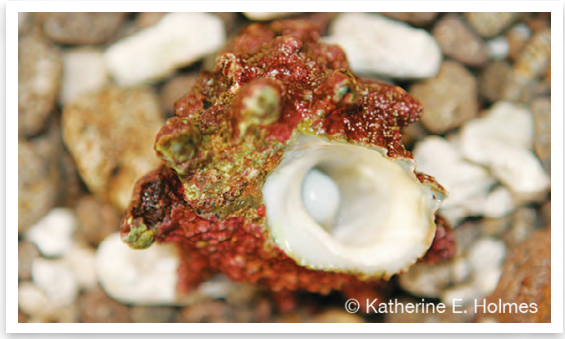

watawlētlēt Astraea haematraga wavagvag n.com. variety of banana that has a bundle that grows upwards instead of down. The fruit are roundish and yellow inside with red skin kaen banana we bandel blong hem i gru antap, i no hang daon. Kakae blong hem i raon mo taem i raep i red be insaed i yelo. Taem man i kakae i pispis yelo

wawan̄ $v i$. open mouth openem maot Bulsal, nana wawan̄, nēk i rōw tēqēl me, na kur nēk. Friend, I'll open my mouth, you jump down to me, and I'll eat you. Fren, mi openem maot, yu jam i kam daon, mi kakae yu. (AWW) [POc *paya 'be open, gape']

wawarēs n.com. lump on skin (unknown skin condition, like pimple but with no pus inside) spot long skin

wawasē n.com. sweetlip emperor (fish) kaen redmaot (fis) Lethrinus miniatus $[P O C$ *kasika 'large emperor, Lethrinus sp. ']

wawawn̄e n.com. gill sora blong fis wawlen̄ n.com. wing tip en blong wing

weag n.com. wild yam $\sim$ wael yam Dioscorea nummularia Syn: qōōr

webeges n.com. coconut spathe (leafy sheath that encloses spadix (cluster of flowers)) samting olsem lif we i kavremap bandel blong flaoa blong kokonas

wedeldel n.com. red ginger, kind of flowering plant $~$ red flaoa we hem i famle blong jinja Alpinia purpurata

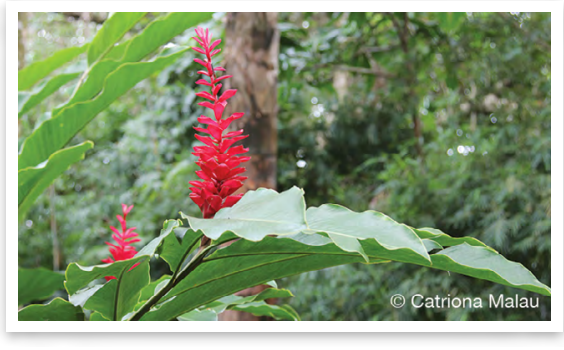

wedeldel Alpinia purpurata 
wederebiliag n.com. variety of marē taro that is not large but grows long and crooked, with light red stem with red stripes. The corm is white and is not strong but is good for roasting kaen marē taro we hem i no save bigwan be i save longfala mo i save benben. Han blong hem i laet red wetem ful red laen i go daon. Kakae blong hem i waet mo i no strong. Hem i spesel taro blong rusum \{Lit. blood of buff-banded rail blad blong nambilak\}

wegemgem n.com. large scar $\sim$ bigfala trak blong soa

weger n.com. kind of gastropod shellfish, generic term that covers a number of bivalve and limpet species grup blong sel blong solwota [Used in food production and for smoothing out pandanus leaves. Ol i yusum sel ia blong skrasem kakae mo smutum pandanas.]

weger dun (comp.) n.com. sunset shell wan kaen sel blong solwota \{Lit. true shell stret sel\}

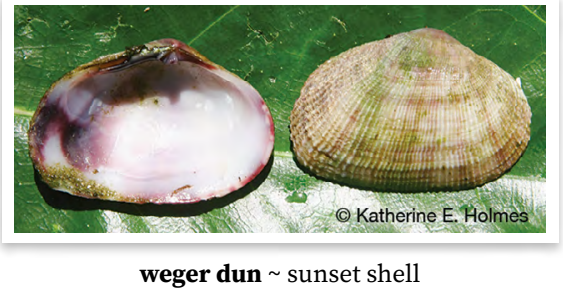

weger mel (comp.) n.com. common turtle limpet (gastropod shellfish) paua Cellana testudinaria [This shell is used for grating coconut. Sel blong skrasem kokonas.]

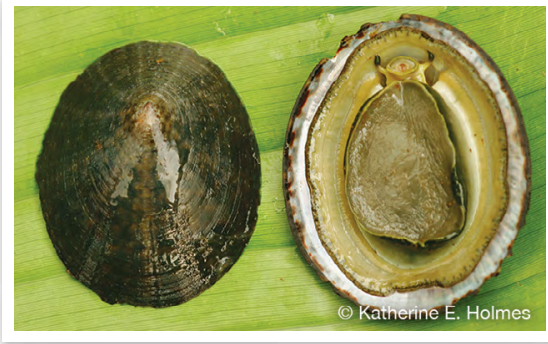

weger mel $\sim$ Cellana testudinaria

weger sōl (comp.) n.com. kind of small gastropod shellfish that is found on the sand $\sim$ kaen smolsmol selfis we i stap long sanbij

weger ta (comp.) n.com. Pacific sugar limpet (gastropod shellfish) wan kaen sel blong solwota Patelloida saccharina

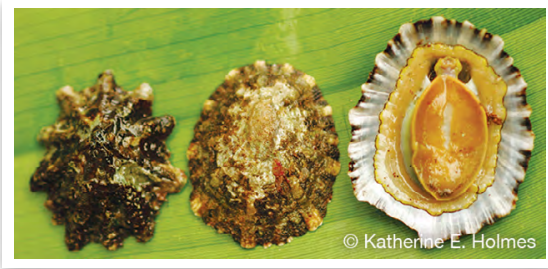

weger ta $\sim$ Patelloida saccharina

weger tur ${ }_{1}$ (comp.) n.com. pen shell wan kaen sel blong solwota Pinna bicolor $\{$ Lit. standing shell sel we $i$ stanap\}

weger tur (comp.) n.com. Pacific asaphis, sunset shell wan kaen sel blong solwota Asaphis violascens \{Lit. standing shell sel we i stanap\}

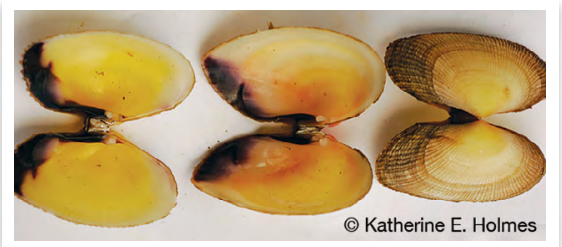

weger tur $\sim$ Asaphis violascens 
weger tele n.com. metal coconut grater aean blong skrasem kokonas \{Lit. metal shell aean sel\}

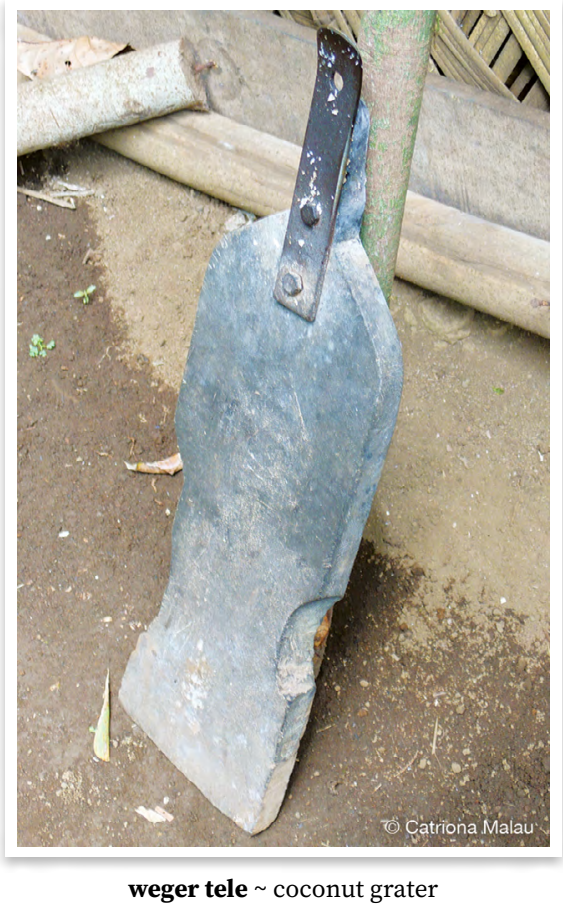

wegerbak n.com. kind of tree wan kaen tri [Used in house construction as the trunk grows very straight. They are plentiful on the hillsides. Ol i yusum blong wokem haos from hem i gru stret mo strong. Hem i gru plante long hil.]

wegeretqon n.com. variety of taro kaen taro \{Lit. pigeon hill hil blong nawimba\}

wel vi. pool, come together in clump $\sim$ hip, wan samting olsem wota $i$ fomem wan pul $\mathbf{O}$ mōtō me wel aē. The coconut milk pooled on top of it. Melek kokonas i hip long hem.

weles n.com. coral, both live and dead rubble korel, olsem pis rif o we $\mathrm{i}$ ded finis [The term covers corals, sponges and possibly also algaes.] weles miar n.com. eyeball aebol, raon pat blong ae

wemete mōtō (fr.var. of mete mōtō) wen̄emërës n.com. damselfish species wan kaen fis Family Pomacentridae $\{$ Lit. canarium ripens black nangae i raep iblak\}

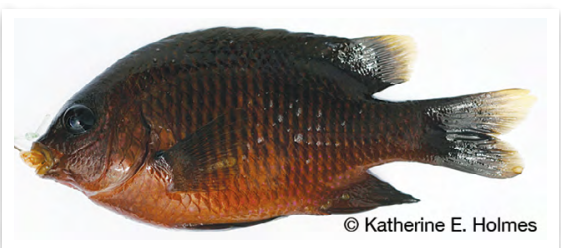

wen̄emërës $\sim$ Family Pomacentridae

weqet n.com. kind of freshwater shellfish that has spines that can stick into one's feet and break off if trodden on $\sim$ wan kaen nasese we $i$ stap long wota mo i save stikim man werir $v$. drill hole in coconut $\sim$ drilim hol long kokonas

wes $v t$. drill, chisel hole in something drilim hol long wan samting Iñko nana wes o terē qaqarörö la ak. Now I am chiselling holes in the canoe. Nao ia mi stap mekem ol hol long kenu wetem jisel. (VVKHVDP180ct0801.090)

wese n.com. egg eg

wewese (der.) vi. round $\sim$ raon wese molo (comp.) n.com. egg of incubator bird eg blong skrabdak

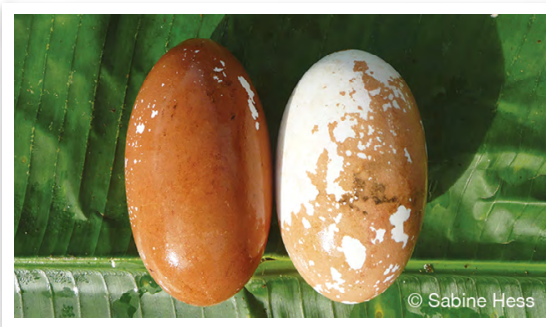

wese $\overline{\text { molo } ~}$ incubator egg

wese tō ${ }_{1}$ (comp.) n.com. chicken egg eg blong faol

wasan (n.poss) n.com.suff. her egg eg blong hem 
wësëk (n.poss) n.com.suff. my egg eg blong mi

wese $\mathbf{t}_{\mathbf{0}}$ n.com. variety of sweet potato that is so named as it is shaped like a chicken's egg kaen kumala we i tekem nem blong hem from i sep olsem eg blong faol \{Lit. chicken egg eg blong faol\}

weselegōsōw n.com. part of house pat blong haos \{Lit. path of rat rod blong rat\}

weseñebēr n.com. part of house pat blong haos

wesev n.com. plant with strong scented yellowish, green leaves lif we $\mathrm{i}$ gat smel blong hem we $\mathrm{i}$ yelo, grin Evodia hortensis [Used as body decoration during traditional dances for the scent. Ol i yusum blong danis wetem from naes smel blong hem.] See: dobormiak

wesrē n.com. honeycomb grouper (fish) smol braon los (fis) Epinephelus merra

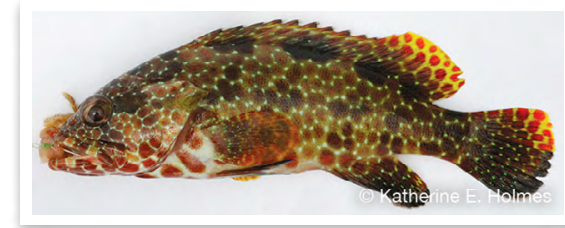

wesrē Epinephelus merra

weswes n.com. piles of fecal pellets, probably from a type of termite, appearance is like dried yeast, but slightly larger, pest unidentified and not seen, only the deposit $\sim$ hip blong ol smolsmol raon samting we i kamaot long wud taem wan kaen bebet i kakae

weswesak n.com. filamentous horse conch (gastropod shellfish) wan kaen sel blong solwota Pleuroplaca filamentosa [Used in the past to drill holes in canoe for binding canoe.
Ol i yusum sel ia olsem wan dril, speseli bifo blong mekem kenu.] \{Lit. drilling canoe drilim kenu\}

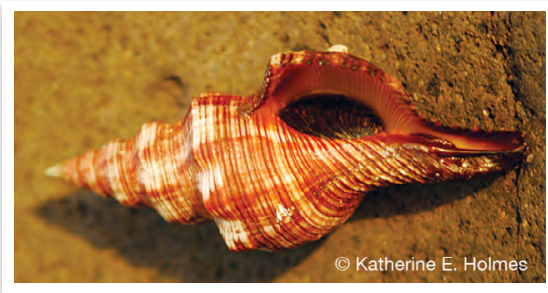

weswesak Pleuroplaca filamentosa

weteg $v t$. crack, break open, split splitim No me weteg wor o mōtō. I cracked open the coconut. Mi splitim brekem kokonas.

wetele n.com. iron aean See: tele

wetele lan̄lan̄ n.com. metal rods used as support for saucepan when cooking over fire aean blong ples blong kuk

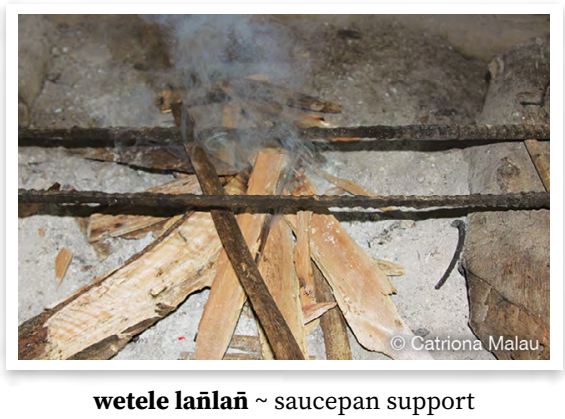

weteltelvet $n . c o m$. blackside, freckled hawkfish wan kaen fis Paracirrhites forsteri See: sewerles

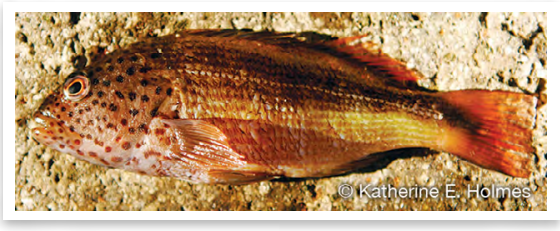

weteltelvet $\sim$ Paracirrhites forsteri 
wetelwa n.com. kind of vine, similar to a pumpkin with round fruit, the leaves are eaten as greens $\sim$ kaen rop we yumi save kakae top blong hem

wetenge n.com. necklace $\sim$ nekles Syn: wērērēn̄ëg, wevet

weteñe tō n.com. basket traditionally used for keeping chicks in to feed them, which now has other uses, made from Donax canniformis and woven in lattice style basket blong kipim smol faol insaed blong fidim See: wēnēn bulëvu

weterōw $n$.com. smooth moon turban (gastropod shellfish) wan kaen sel blong solwota Lunella cinerea

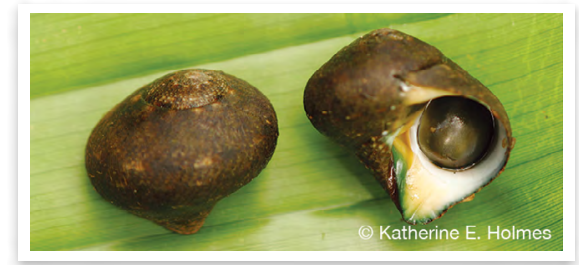

weterōw Lunella cinerea

wetevel n.com. copper sweeper (fish)

wan kaen fis Pempheris oualensis Syn: mesteveltiwial \{Lit. one-sided thing ting wetem wan saed\}

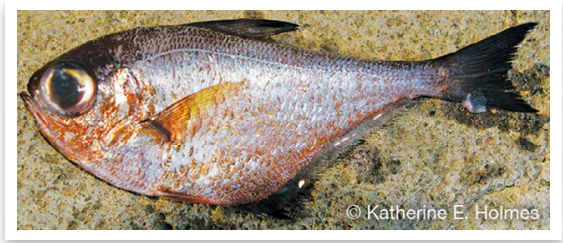

wetevel $\sim$ Pempheris oualensis

weverbak n.com. kind of tree wan kaen tri Olea paniculata [Used to make roof beam. Ol i yusum blong wöqötu gövur.]

weverbōn n.com. kind of moth that comes out in the early evening, at dusk, and makes a loud buzzing noise with the beating of its wings $~$ kaen bataflae blong naet we hem i kamaot taem tudak i kam, mo i mekem noes wetem wing blong hem

wevet n.com. necklace $\sim$ nekles Syn: wetenge

weveveleg n.com. sticks used for beating slitgong wud we ol i yusum blong kilim tamtam See: veleg

weviat n.com. type of insect that lives in wood, edible $\sim$ bebet we $i$ stap long wud we man i save kakae

wewe n.com. kind of laplap which is rolled in island cabbage and then cooked in saucepan rather than baked laplap simboro

wewestel n.com. area which used to be a vegetable garden, which is not currently being tended, but still produces crops, so people can go there to harvest crops ples blong olfala garen we ol man ol i nomo planem kakae long hem, be ol kakae i stap gru yet, mekem se ol man ol i save go karem kakae long ples ia

wewete n.com. elongated, long and narrow longfala mo bunbun O taklē dödöu maniok o wewete, ge elelvet. Some manioc leaves are narrow and really long. Samfala lif maniok i naro mo i longlongfala. (VVCMAA03Apr1306.073)

wē $v i . \mathbf{1} \cdot \operatorname{good}$, be good $\sim \operatorname{gud} \mathbf{2} \cdot$ be well, healthy $\sim$ stap gud, kam gud See: gōwē

wēbē n.com. bait bet Kōmōrōk ma van tala sōsōsōg ba no ma da ta na bē na möguk o wēbē, ba o go mo got na busuk. The two of us went to go fishing, but I went to put my bait on the hook, but the hook caught my finger. Mitufala i go blong huk be mi mekem blong putum bet long huk, be huk $i$ kasem fingga blong mi. See: bēe

wēbēbēs n.com. barnacle kokias we i fas long ston o botom blong sip wēbēlēt n.com. scar trak blong soa wēbēr n.com. beam rel See: bēr 
wēbiēg n.com. variety of yam with a double layer of skin wan kaen yam we i gat dabol skin \{Lit. breadfruit bredfrut\}

wēbiēg atatīēn (comp.) n.com. variety of yam with a double layer of skin and white flesh wan kaen man yam we i gat dabol skin mo insaed i waet \{Lit. male breadfruit man bredfrut\}

wēbiēg rereqe (comp.) n.com. variety of yam that can be red or white wan kaen woman yam we insaed i red o waet \{Lit. female breadfruit woman bredfrut\}

wēbigqō n.com. variety of taro kaen taro \{Lit. eat pig kakae pig\}

wēbigqō mamē $n$.com. variety of taro kaen taro $\{$ Lit. eat pig red kakae pig we ired\}

wēduduw (fr.var. wududuw) n.com. damselfish $\sim$ wan kaen fis Amblyglyphidodon spp.

wēēwatē n.com. blackfin squirrelfish, spotfin squirrelfish kaen redfis Neoniphon spp.

wēgēbil n.com. false tamanu; kind of tree with edible fruit that are dark red, black when ripe, red inside wan kaen tri we frut blong hem i dak red mo taem i raep i blak mo $i$ red long insaed blong hem Garcinia pseudoguttifera

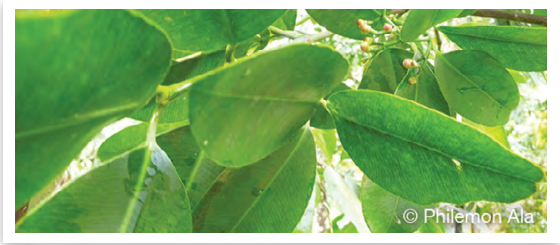

wēgēbil Garcinia pseudoguttifera

wēgēbil n.com. kind of small coconut leaf basket that is used especially when reef gleaning, for putting shellfish in $\sim$ kaen basket blong lif kokonas we ol i yusum taem ol i go karem mit long solwota, blong fulumap ol sel insaed long hem

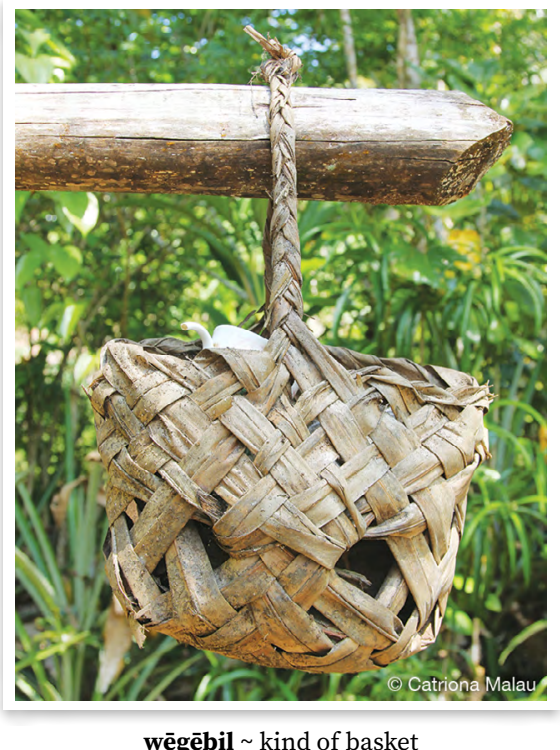

wēgēngēndilo n.com. kind of insect like a grasshopper but smaller, found around stones by the sea kaen bebet we hem i olsem grashopa be $i$ smol mo i stap long ol ston long saed blong solwota long ples we solwota i finis long hem

wēgēriv n.com. ghost crab wan kaen krab blong solwota Ocypode ceratophthalma

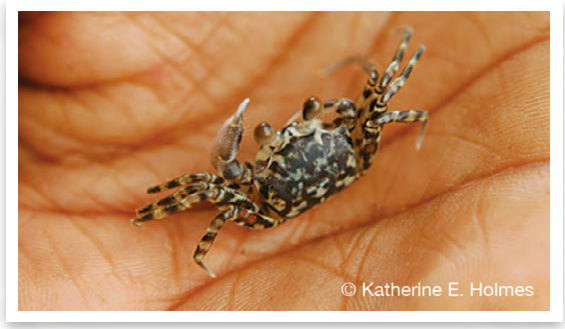

wēgēriv Ocypode ceratophthalma

wēgēt n.com. kind of coconutleaf basket used for holding and transporting food wan kaen basket blong lif 
kokonas we ol i yusum blong karem kakae long garen o hangem kakae long hem

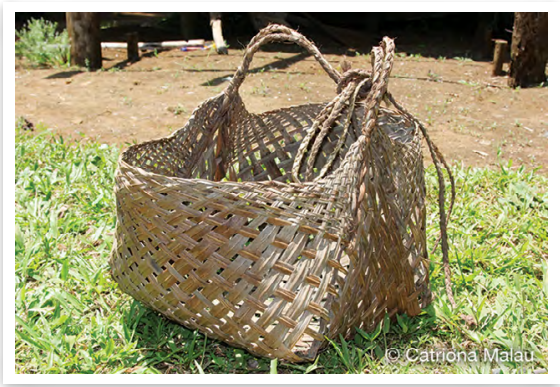

wēgēt kind of basket

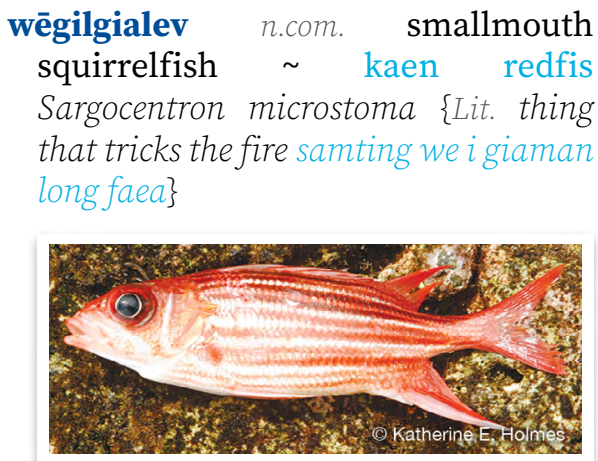

wēgilgialev $\sim$ Sargocentron microstoma

wēgilgialnaw n.com. fiddler crab kaen krab Uca sp. \{Lit. thing that tricks the sea samting we $i$ giaman long solwota\}

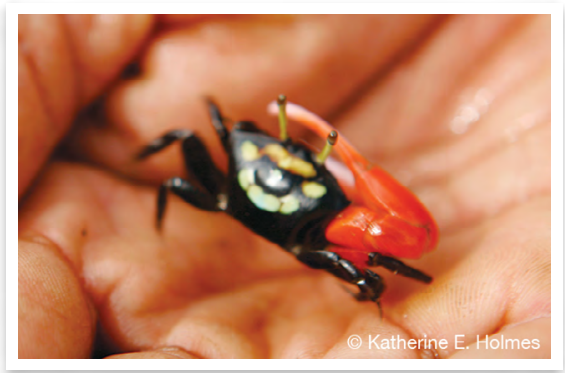

wēgilgialnaw $\sim U c a$ sp. wēgim n.com. giant clam that can move $\sim$ natalae we i save wokabaot Tridacna sp.

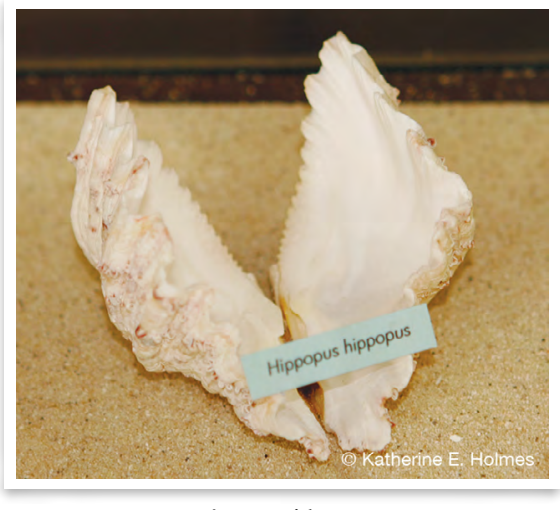

wēgim Tridacna sp.

wēkēr wōnwōn $n$.com. variety of yam belonging to the 'female' grouping which has short, white tubers wan kaen yam we hem i woman, we i no save gru longfala tumas be i save gru bigfala. Insaed blong hem i waet

wēlēbēōr n.com. variety of taro, 'female', with light red stem and top with light green stripes $\sim$ kaen woman taro we han blong hem i laet red wetem laet grin straep Syn: busuōr

wēlēgian $n$.com.suff. $\mathbf{1} \cdot$ her/his/its heel $\sim$ bihaen blong leg blong hem $\mathbf{2}$ • hoof (of animal) bihaen long leg blong animol

wēlēm n.com. immature trevally, jacks and kingfishes smolwan blong karong (fis), taem i bigwan i gat defren nem

wēlēs $v$. turn $\sim$ tanem $\mathbf{O}$ reqe ni wēlēs ris o qötu wērēt. The woman turned the head of the octopus inside out. Woman i tanem hed blong nawita.

wēlēwlēw $n$.com. coconut spadix (plant spike bearing flowers) samting blong kokonas we ol flaoa i stap gru long hem 
wêlir n.com. variety of yam that is not planted but grows wild in the bush $\sim$ wan kaen wael yam we i save longlongfala (olsem man) be i smol nomo antap we i mekem se i had blong digim Syn: dem ga

wēlwēl n.com. canoe kenu Syn: ak sōsō

wēmētigtig n.com. betel nut, areca palm bitelnat, rabis kokonas, waelkokonas Areca catechu

wēmēlēglèg $n$.com. variety of taro in mare grouping that has light red stem and white corm. It is harder than other mare but not that strong $\sim$ kaen mare taro we han blong hem i laet red mo stamba klosap i waet. Kakae blong hem i waet mo hem i strong bitim ol narafala marē be i no strong tumas

wēmiedaran (abbrev. miedar) n.com.suff. her/his/its heart hat blong hem

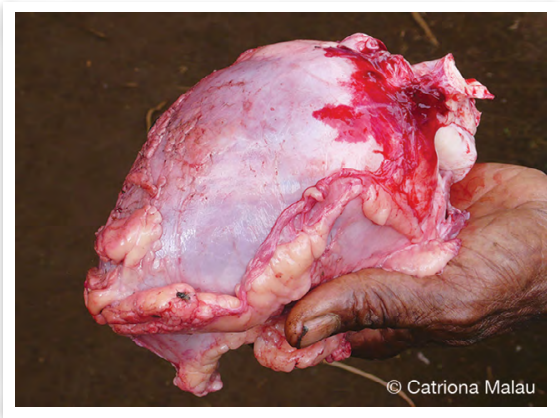

wēm̄iedaran heart

wēmikmiak n.com. kidney kidni

wēnēn bulëvu (fr.var. winin bulëvu) n.com. perennial herb with branched cane-like stems kaen flaoa Donax cannaeformis [Bark of stems are cut into strips and plaited to make lattice baskets, such as weteñe tō, used for keeping chicks. Ol i yusum skin blong hem blong wivim basket blong fidim faol.] See: weteñe tō [POC *nini(q) 'shrub, Donax cannaeformis']

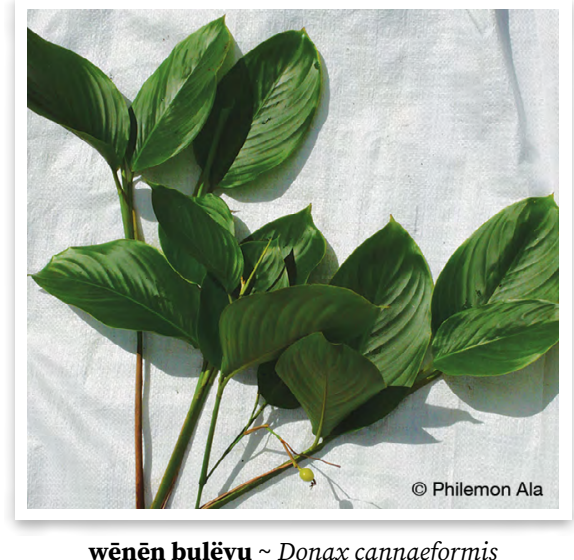

wēnir (fr.var. of winir)

wēnēn n.com.suff. his facial hair mustas blong hem See: win̄i

wēqē n.com. shelf, bed, storage platform bed Ira rētnar a tek o gengen, o wēqē ni wor, o wēqē qiat, o wēqē tamag, o wēqē dëm, o wēqē wēbēw, mo wor. The mothers brought the food, and the storage shelves were filled up, the shelf of taro, the shelf of Chinese yams, the shelf of yams, the shelf of aerial yams, they were filled. Ol mama blong olgeta ol $i$ karem kakae, ol bed ol i fulap, bed blong taro, bed blong swityam, bed blong yam, bed blong nabew, ol i fulap. (ATW)

wēqē n̄e (comp.) n.com. shelf built over fireplace for smoking canarium nuts bed we ol i bildim ova long faea blong smokem nangae

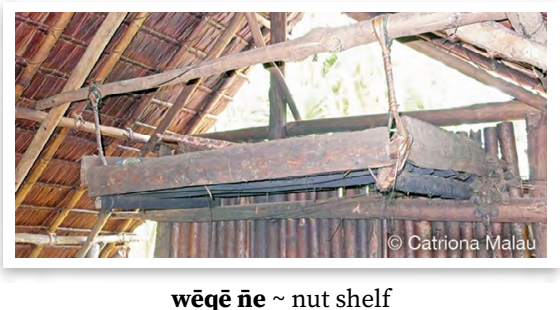


wēqiar ${ }_{1}$ n.com. convict surgeonfish, tang wan kaen fis Acanthurus triostegus

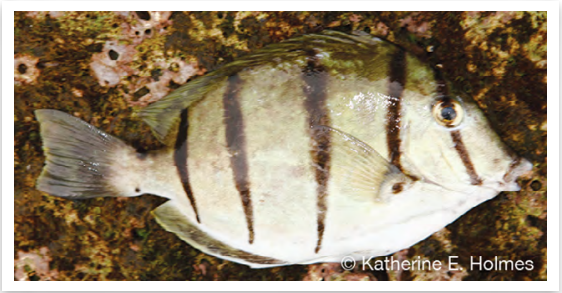

wēqiar $\sim$ Acanthurus triostegus

wēqiar ${ }_{2}$ n.com. kind of small tree $\sim$ wan kaen smol tri Desmodium umbellatum See: wēqiqiar

wēqig $n$.com. swelling, lump on person or tree that swells but does not hurt $\sim$ ples long bodi o wud we i solap be i no soa

wēqiqiar $n$.com. kind of tree that grows by the sea and has fruit that look like small beans wan kaen tri we i gru long solwota we kakae blong hem i olsem smolsmol bin [Fruit are not edible. Yumi no save kakae.] See: wēqiar

wēqitin̄ev $n$.com. samurai squirrelfish $\sim$ kaen redfis Sargocentron ittodai

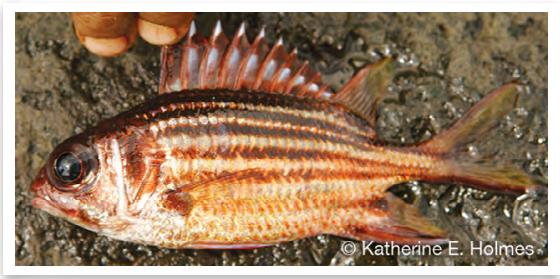

wēqitin̄ev $\sim$ Sargocentron ittodai

wērē n.com.suff. arse, buttocks as Syn: kērē 1

wērēk (n.poss) n.com.suff. my arse as blong mi

wirian (n.poss) n.com.suff. her/his/its arse as blong hem

wērēk $n$.com.suff. my arse as blong mi See: wērē

wērēreg $v$. fly flae wērērēn̄ëg n.com. necklace, something that is worn around one's neck nekles, samting we yu hangem long nek blong yu Syn: wetenge

wērēsōr n.com. place where the spirits of the dead go to rest when they are tired, a place on a point or hill wan ples we ol devel ol i go spel long hem, sapos ol i wokabaot go go ol i wantem spel. Hem i wan ples long wan poen o hil Söw wērēsōr nitiwial oko, nēr gē riv nitiwial oko, gē riv avē. And there is a resting place of spirits here, they planted one here, they planted in that place. Ale i gat wan ples blong ol devel long ples ia, ol i planem wan long ples ia, ol i planem long hem. (VVCMAA03Apr1306)

wērēt n.com. octopus nawita [POc *kuRita 'octopus']

wēsēm n.com. olive shell wan kaen sel blong solwota Family Olividae
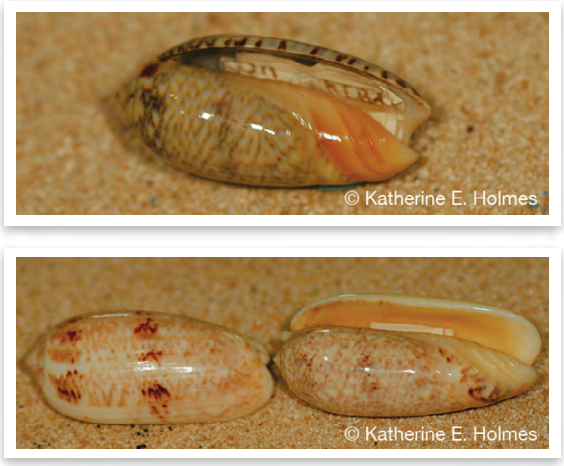

wēsēm olive shell

wēsēs n.com. generic name for sea snails, types of gastropod with snail type shells ol kaen nasese blong solwota we i fas long ston o rif wēsēs bē (comp.) n.com. kind of freshwater shellfish that is round and black, and red inside wan kaen sel blong wota we i raon mo baksaed blong hem i blak mo insaed long sel i red \{Lit. freshwater snail nasese blong wota\} 
wēsēs biēg (comp.) n.com. 1 • harp shell wan kaen sel blong solwota Family Harpidae $\mathbf{2} \cdot$ mitre shell wan kaen sel blong solwota Purpura sp. \{Lit. breadfruit snail bredfrut nasese\}

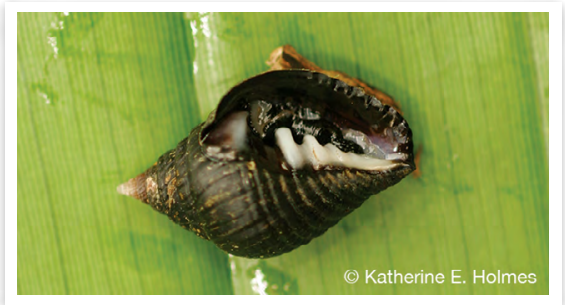

wēsēs biēg Purpura sp.

wēsēs malau (comp.) n.com. kind of turban shell wan kaen bigae blong solwota Turbo crassus \{Lit. snail that grows big bigae sel we i gru bigwan\}

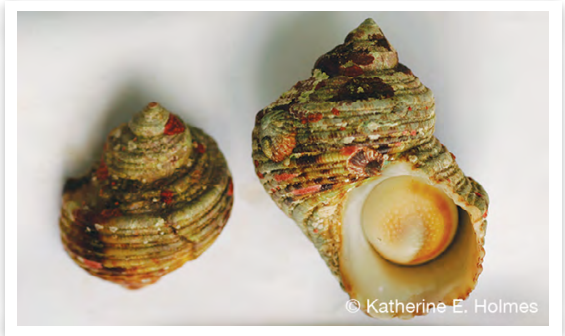

wēsēs malau Turbo crassus

wēsēs man (comp.) n.com. tapestry turban (gastropod shellfish) wan kaen sel blong solwota Turbo petholatus

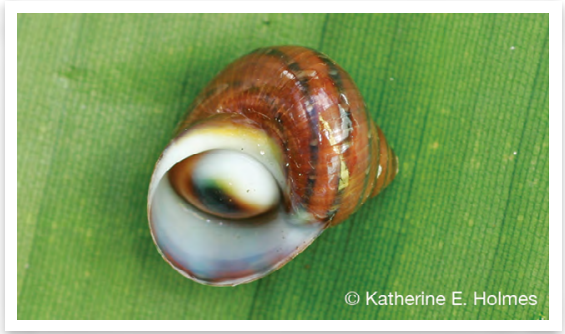

wēsēs man Turbo petholatus wēsēs nōnōm (comp.) n.com. kind of freshwater shellfish that can only be seen at night wan bigfala sel blong wota we i kamaot long naet nomo

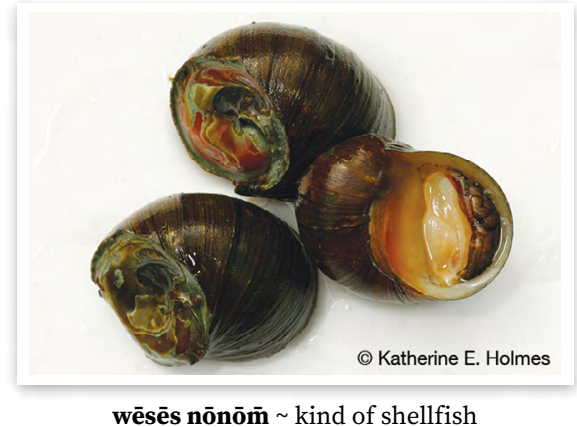

wēsēs tan̄ov (comp.) n.com. great green turban (gastropod shellfish) grinsnel Turbo marmoratus

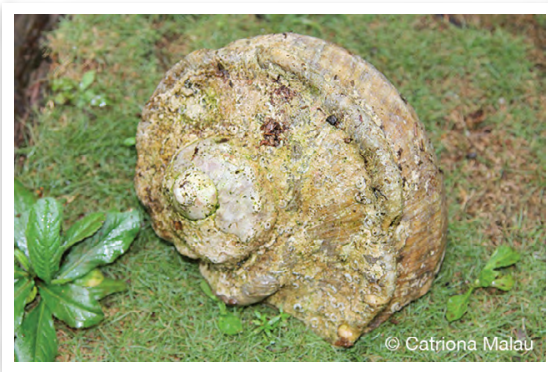

wēsēs tan̄ō̄ $\sim$ Turbo marmoratus

wēsēs vet (comp.) n.com. rough turban (gastropod shellfish) wan kaen bigae blong solwota Turbo setosus \{Lit. stone snail ston bigae\}

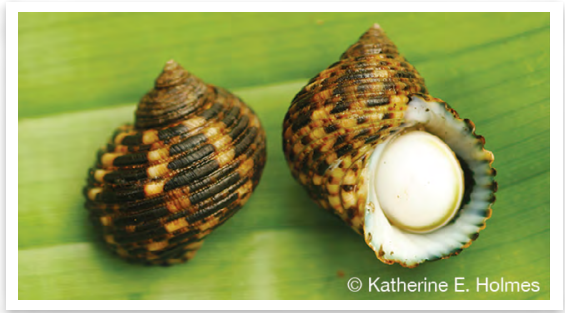

wēsēs vē̄ $\sim$ Turbo setosus 
wēsēs wōmalak (comp.) n.com. kind of turban shell wan kaen bigae blong solwota Turbo sparverius

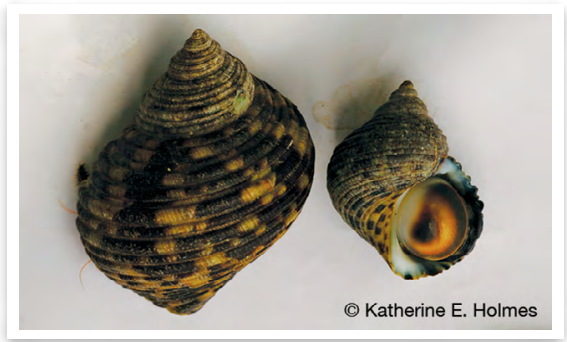

wēsēs wōmalak Turbo sparverius

wēsi inter. dunno, an interjection indicating that the speaker does not know what is being enquired about $\sim$ mi no save

Wēsilat n.loc. place name $\sim$ nem blong ples

wēsilivetel n.com. checkerboard wrasse (fish) wan kaen smolsmol fis Haliochoeres hortulanus

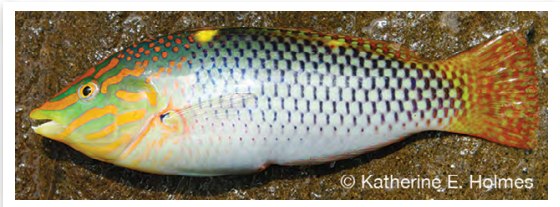

wēsilivetel Haliochoeres hortulanus

wēsin̄asin̄ n.com. white person, nonindigenous person $\sim$ waetman

wēsiri n.com. sharp needle-like piece from inside of base of sago palm rachis bun blong natanggura insaed long stamba See: wuduw

wēsustēgēr n.com. dry coconut that does not contain any liquid inside drae kokonas we i nomo gat wota i stap long hem

wēt n.com. carrying stick, length of wood used for carrying items over shoulder or between two people wud blong karem samting long hem wētēbisbis n.com. island thrush (bird) $\sim$ wan kaen pijin Turdus poliocephalus wētēltēliriaw n.com. snake sea cucumber wan samting olsem besdemea Synapta maculata

wètēq n.com. kind of arrow which has a blunt end so that it will bounce off a tree if the target is missed and not get stuck, used for shooting birds wan kaen aro we en blong hem i no sap blong hem i no save fas long wud taem yu sut wetem, i mas foldaon, ol i yusum blong sutum pijin See: tēq

wētēqēdur n.com. auger shell wan kaen sel blong solwota Terebra spp. \{Lit. dig hole digim hol\}

wètēsi gövur n.com. frame of house inside house that is used when building to sit on so that you can reach the roof to sew on the thatch $\sim$ smol brata blong haos, lada blong stanap long hem blong fasem haos \{Lit. small brother of house smol brata blong haos\}

wētēsisis n.com. cardinal honeyeater (bird, female - smaller and not as brightly coloured as male) redhed (pijin, woman) Myzomela cardinalis See: qötqötumen

wètēt 1 n.com. white-breasted woodswallow (bird) wan kaen pijin Artamus leucorhynchus

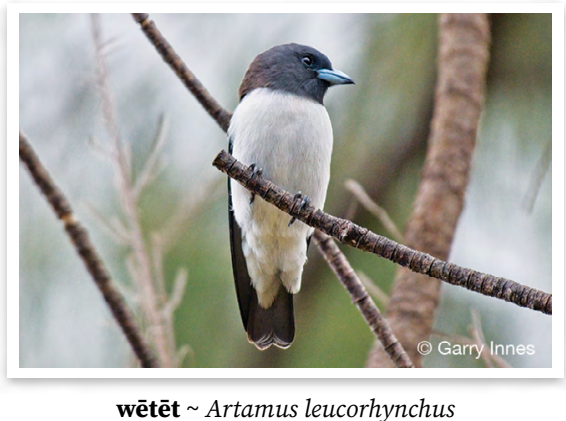

wētēt n.com. kind of tree wan kaen tri [The fruit is used as a glue. The wood is used for making spears and in house building. It grows in the forest, not close to villages. $\mathrm{Ol}$ i 
yusum kakae blong hem olsem glu. $\mathrm{Ol}$ i mekem spia long hem mo yusum blong bildim haos. Hem i gru long bigfala bus.]

wètētērē n.com. millipede kruked (bebet) Order Diplopoda

wētēv n.com. nautilus notilis, kaen sel blong solwota Nautilus pompilius

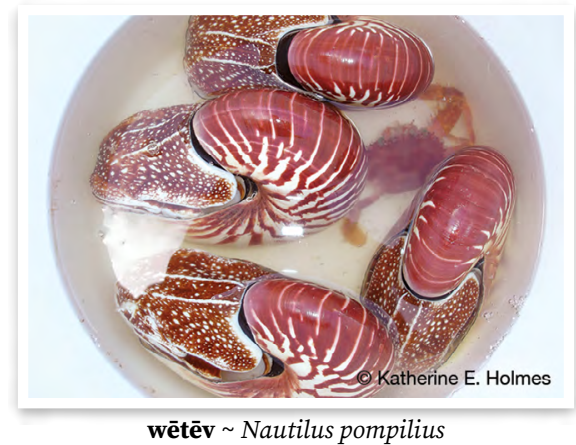

wètēvtēv n.com. kind of grass wan kaen gras Scleria polycarpa [It is believed that anyone who crunches up the seeds will have lots of children. If someone has lost their voice, they can eat the young shoots to make their voice come back. Bae yu graonem sid blong hem, bae yu karem fulap pikinini. Sapos voes blong yu i lus, bae yu kakae yangfala lif blong hem.] \{Lit. cutting thing, so named because the leaves are sharp and can cut the skin samting we i katkat\} wētēvut n.com. scorpionfish species kaen fis we $i$ gat nil Family scorpaenidae

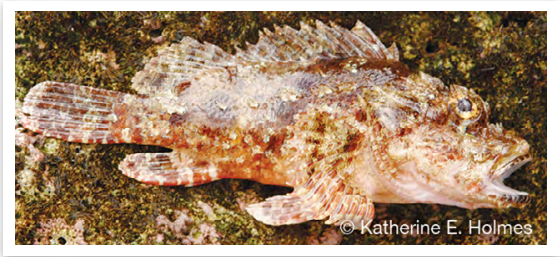

wētēvut $\sim$ Family scorpaenidae

wētēwil (fr.var. wētiwil) n.com. nerite (gastropod shellfish) nasese Family Neritidae wētēwil bēt (comp.) n.com. plicate nerite (gastropod shellfish) kaen nasese Nerita plicata

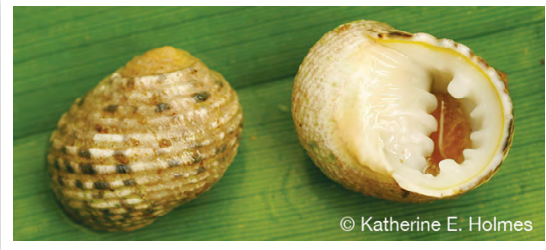

wētēwil bēt $~$ Nerita plicata

wētēwil kör (comp.) n.com. waved nerite (gastropod shellfish) kaen nasese Nerita undata

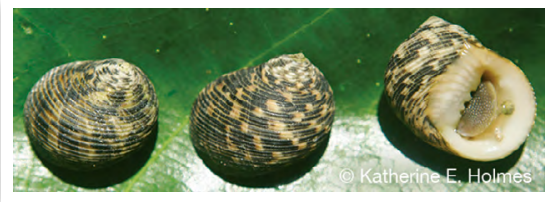

wētēwil kör Nerita undata

wētēwil mëv (comp.) n.com. maximum nerite (gastropod shellfish) kaen nasese we i stap long rif long ples we solwota i no stap kasem Nerita maxima

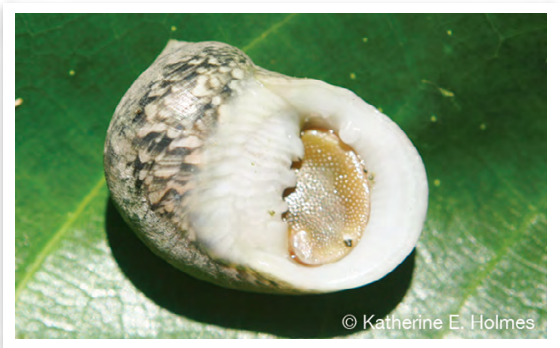

wētēwil mëv Nerita maxima

wētēwil serōn (comp.) n.com. kind of nerite (gastropod shellfish) kaen nasese Nerita polita

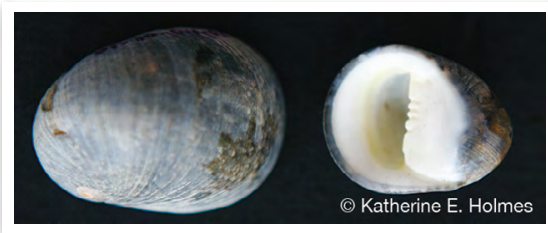

wētēwil serōn Nerita polita 
Wētika n.loc. place name nem blong ples

Wëtikō n.loc. place name nem blong ples

wētilmal n.com. part of house $\sim$ pat blong haos

wètilvarvar n.com. part of house pat blong haos

wētiwil (fr.var. of wētēwil)

wèvē $n$.com. variety of taro that has two types and is like wild taro. The corm is yellow and white inside. It is not strong and can fork kaen taro we i gat tu kaen blong hem. Hem i olsem waeltaro. Han blong hem i no dak tumas. Taem yu brekem top kakae blong hem i yelo mo i waet insaed. Hem i no strong mo i save karem fok wēvē lamkör (comp.) n.com. variety of weve taro that is very white inside but has a dark stem kaen wēvē taro we insaed blong hem i waet evriwan mo han blong hem i mo dak

wēvēl $n$.com. fantail warbler (bird) wan kaen pijin Gerygone flavolateralis wēvēs num. few fiu

wēvētuo n.com. smallmouth squirrelfish $\sim$ kaen redfis Sargocentron microstoma See: wörötuo wēvēvērēg n.com. vehicle, general term for car, utility vehicle, truck trak \{Lit. thing that runs samting we $i$ ron $\}$

wēviak n.com. grasshopper grashopa wēvinn̄e n.com. xiphoid process, cartilagenous extension of lower sternum of animal or human sofsof bun long ples we ol rib i joen, be daon

wēviñ $n . c o m$. piece of yam for planting $\sim$ pis yam blong planem [Taem yu wantem planem yam yu katem, afta yu katem blong i gat hol insaed, afta yu planem]

wēviriak $n . c o m$. rattle tree $\sim$ navangge Pangium edule wēviti $n$.com. variety of taro kaen taro wēwēn $n$.com. red beadtree, coralwood $\sim$ nabisa Adenanthera pavonina [Kind of tree with yellow flowers and identifiable by small hard red seeds in brown seed pod. Wan wud we flaoa blong hem i yelo mo i gat ol sid blong hem we i red we i red.]

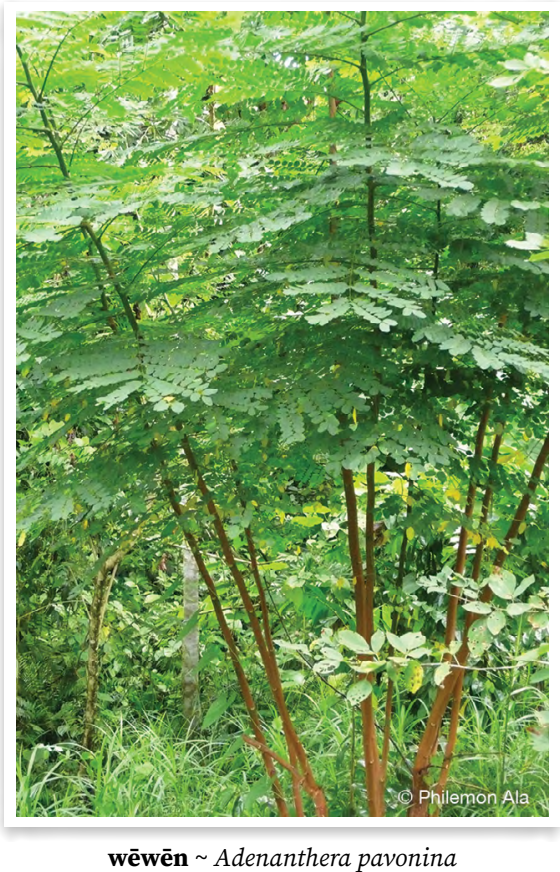

wēwēsēg $n$.com. variety of banana that has small, short fruit asis banana we hem i raon mo i sotfala

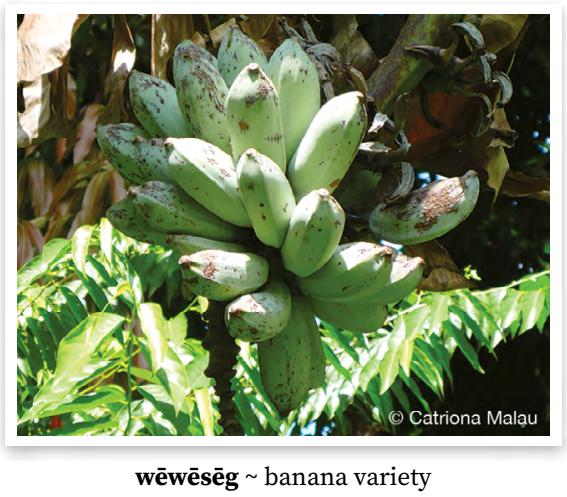




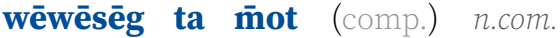
variety of banana kaen banana $\{$ Lit. wèwèsēg from Mota wèwèsēg blong Mota\}

wēwēsēg ta ōrbarbar n.com. variety of banana kaen banana \{Lit. wèwēsēg from Ureparapara wēwèsēg blong Ureparapara\}

wëbënbën $n . c o m$. spot spot

wëbëwbëwran n.com.suff. her/his/its ankle hinsis blong fut blong hem

wëlëklëk n.com. strap, thin piece of timber used in house construction to hold bamboo walling in place strap, wud blong holem taet wol blong haos See: lëk

wëmënël n.com. snack food (fruit, nuts) smol kakae olsem frut, nat

wërësrësak n.com. bailer, bailer for canoe samting blong belaot wota long kenu See: rës

wës $v i$. whistle $\sim$ wisel Nēn gëtë wëswës la masawre nēn a vēn o doot. We don't whistle when we are making sago palm thatch. Yumi no wisel taem yumi pinim lif natanggura.

wësëk n.com.suff. my egg eg blong mi See: wese

wësërsër n.com. clothes klos See: sër $_{3}$ \{Lit. thing to wear samting blong werem

wëtëvut n.com. kind of small insect which makes hole in the gound wan kaen smol bebet we hem i mekem hol long graon

wëvër (fr.var. of vër $r_{2}$ )

wëvër lo n.com. rosewood (tree) burao blong solwota Thespesia populnea

wiak $1 \cdot v t$. open $\sim$ openem Wiak ten sōw o tibiar e, ri èl ten, oso aē lē tibiar? Try to open up the basket, have a look, what is in the basket? Traem openem basket ia, traem luk, i gat wanem long basket? $(\mathrm{OQQ}) \mathbf{2} \cdot$ vi. open, be open open $\mathbf{O}$ mete gövur gē wiak. The door is open. Doa i open. wial $v t$. separate one part of something from another, open up or spread apart something which is made up of individual parts, like grass, hair or pieces of roofing openemaot o leftemap pat blong wan samting we $\mathrm{i}$ gat tu o moa pat blong hem, olsem yu lukluk insaed long gras o yu lukaotem laos long hea See: qias

wian $_{1}$ n.com. rain $\sim$ ren La masawre o wian ma mamas, o bē mē qēt vēvērēg. When the rain finishes, the creek stops flowing. Taem ren i finis, wota inomo ron. [POc *qusan 'rain']

löt wian (id.) vi. get rained on, not worry about getting wet in rain swim long ren Syn: suv wian

wian $_{2}$ n.com.suff. its fruit $\sim$ frut blong hem See: wiē

widiañ n.com. $\mathbf{1}$ - serpent's head cowrie (gastropod shellfish) wan kaen kaorisel Cypraea caputserpentis $\mathbf{2}$ • kind of children's game involving spinning a cowrie shell kaen pleplei blong ol pikinini

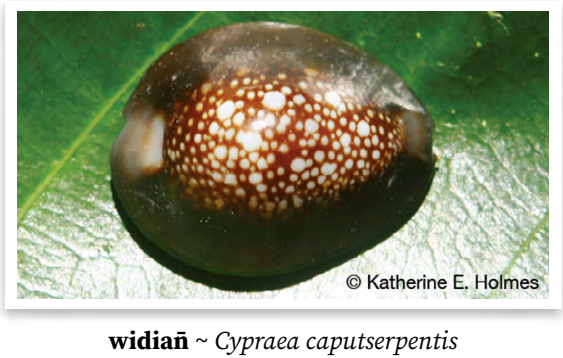

widiar n.com. 1 - kind of mushroom which glows in the dark kaen masrum we i stap saen long naet 2 - phosphorescence in the sea $\sim \mathrm{ol}$ smolsmol samting we i stap saen long solwota long naet

wido $n . c o m$. window $\sim$ windo

$\mathbf{w i e}$ n.com. fruit (of tree), generic term for fruit of plant frut (blong wud) Nana van sur o qētēgi wobalak ine, na bis o wiē balak. I'll go to the fig tree, and I'll pick the fig fruit. Mi go 
long stamba blong nambalanggo ia, mi karem frut blong nambalanggo. (GMM) $\left[P O c^{*}\right.$ puaq 'fruit: generic for fruit as a part of plants, the seed and its envelope'] wian $_{2}$ (n.poss) n.com.suff. its fruit frut blong hem

wik $n$.com. week wik [Bis]

wil $v t$. turn $\sim$ tanem

wilikseg (fr.var. wilikteg) v. look through something in order to find something lukluk tru blong faenem wan samting

wilikteg (fr.var. of wilikseg)

wiliqliaq n.com. fiveband flagtail (fish)

$\sim$ wan kaen fis Kuhlia mugil

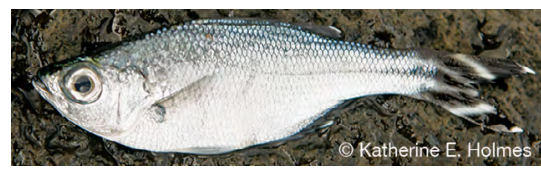

wiliqliaq Kuhlia mugil

Wimiar n.p.fr. term of endearment used by parent-in-law to child-inlaw nem we wan i yusum blong singaotem palika blong hem, we hem i woman blong boe blong hem o man blong gel blong hem

winiā̄ n.com. soursop (tree, fruit) karasol Annona muricata

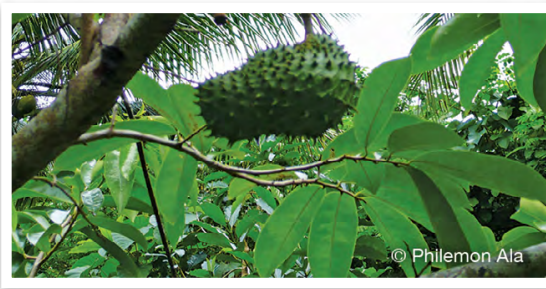

winiā̄ $~$ Annona muricata

winin bulëvu (fr.var. of wēnēn bulëvu) winir (fr.var. wēnir) n.com. house walling made from trunks of tree fern wol blong haos we ol i mekem long blakpam

winiwin $v i$. clean, be clean $\sim$ klin $\mathrm{Na}$ gëvrun nēn gē winiwin. Our house is clean. Haos blong yumi i klin. See: wurveg, win̄i n.com.suff. facial hair; beard, moustache mustas

wēn̄ēn (n.poss) n.com.suff. his facial hair mustas blong hem

win̄i tō (n.poss) n.com.suff. wattles of chicken, red flesh hanging at neck of chicken tufala samting we i hang andanit long maot blong man faol

win̄ik (n.poss) n.com.suff. my facial hair mustas blong mi

win̄i tō n.com.suff. wattles of chicken, red flesh hanging at neck of chicken tufala samting we $\mathrm{i}$ hang andanit long maot blong man faol See: win̄i win̄ik n.com.suff. my facial hair mustas blong mi See: win̄i

wiqian n.com. young coconut seedling with a small haustorium, the spongy embryo that grows inside when the coconut is germinating navara we insaed blong hem i smol nomo

wir n.com. kind of laplap that is rolled in island cabbage, cooked in saucepan, sliced, then has coconut milk squeezed on top laplap simboro we ol i kukum fastaem, afta ol i slaesem mo melekem

wiria n.com. red-bellied fruit dove (bird, immature(?), smaller and colour not as bright) grinpijin we hem i smol Ptilinopus greyii

wiria tabē (comp.) n.com. redbellied fruit dove (adult(?), larger) grinpijin we hem i bigwan Ptilinopus greyii

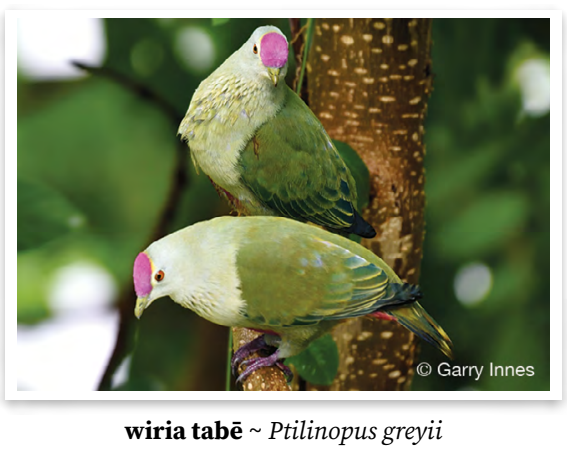


wirian n.com.suff. her/his/its arse $\sim$ as blong hem See: wērē

wiriatege n.com. variety of banana with very long, large fruit, it has a large trunk but does not bear lots of fruit kaen banana we kakae blong hem i longlongfala mo i bigwan, i gat bigfala stamba be hem i no karem fulap kakae

wirmamēgin n.com. kind of food dish wan kaen nalot we ol i kilim nalot finis, ol i melekem kokonas stret antap

wis n.com. barn owl (bird) hoknaet, wok naet (pijin) Tyto alba

wisiag $v i$. thicken, solidify kam tik wisil $v$. force $\sim$ fosem

wistaban n.com. variety of kava with yellow leaves wan kaen kava we lif blong hem i yelo

wiwi vi. hum (of mosquito) (moskito i) singsing $\mathbf{O}$ nem ni wiwi lö qörök. A mosquito is humming in my ear. Moskito i stap singsing long sorablong mi.

wiwieg $1 \cdot v i$. work wok Nē gē wiwieg vilēs e lö qöñ. She works all the time in the night. Hem i wok evritaem ia long naet. (DFF) 2 • n.com. work wok Nēr gē biriñ kēmi tala da o wiwieg. They will help you to do the work. Bae ol $i$ givhan long yufala blong mekem wok.

wo conj. and; conjunction that can join phrases and clauses mo Nēk kara gagneg min i Die wo i Mam. You just tell Mum and Dad. Bae yu jes talem long Mama mo Papa. (OQQ) O tōrtōr nē ga van qal tala gagneg min nēk o malmal wo min nēk o lōmegev. The preaching goes out to speak out to you, the young woman and to you, the young man. Toktok $i$ go kasem blong talemaot long yu yangfala gel mo long yu yangfala boe. (RKM) Ni mem o bred wo o biliag ni gen. He put down the bread and the buff-banded rail ate it. Hem i putum bred mo nambilak $i$ kakae. (RPP) wo ${ }_{2} v$ t. say talem Tēsi tan̄sar wo, 'Söw, nēk gō rōn̄teg?' The younger brother said, 'So, did you hear?' Smol brata i se, 'Ale, yu harem?'

wo $_{3}$ part. intensifying particle; absolutely, totally tumas, we

wo- pre. noun categorisation prefix, used with separate functions: before male names; before plant names, in reference to the fruit or the tree; and in the formation of new nouns maka we i go long fran blong nem blong wan man, long nem blong tri o frut blong tri mo long wan niufala wod we yu krietem (Allomorphs: wa-, we-, wē-, wë-, wō-, wö-)

wobalaklak n.com. sucker, suction cup on octopus arm, tentacle samting long han blong nawita we yu save fas long hem

wogarēr n.com. kind of hawkfish kaen fis

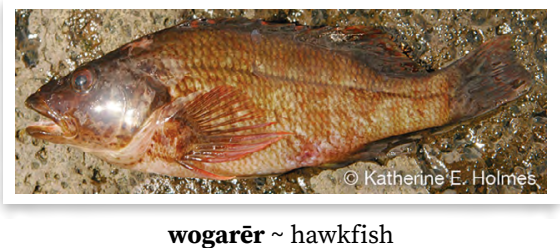

wok $\mathbf{1} \cdot$ n.com. work $\sim$ wok $\mathbf{2} \cdot v i$. work [Bis wok 'work']

wokē n.com. albino albino Syn: wulul wolkoko n.com. kind of plant that has small sharp hooks on the stem wan kaen plant we i gat huk long hem Caesalpinia crista

womalabōs n.com. lined cheek wrasse (fish) wan kaen fis Oxycheilinus diagrammus

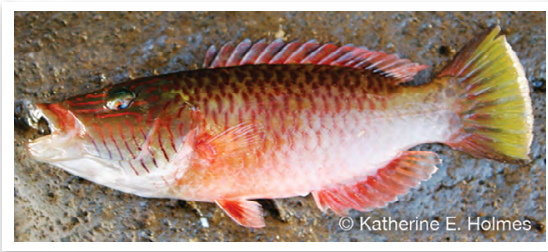

womalabōs $\sim$ Oxycheilinus diagrammus 
woman n.com. pawpaw, papaya popo Carica papaya

womal n.com. coral head korel, pat blong rif insaed long solwota

won̄ vi. light greyish, beige laet kala, olsem gre, laet braon Na tarbian mo won. His skin became a light greyish colour. Bodi blong hem i kam gre smol.

woo n.com. bamboo bambu Bambusa spp. Iñko nēn a tōt o tere woo ta nēn a butuñ o gövur. Then we cut some bamboo so that we can make the walling for the house. Nao ia yumi katem olbambu blong yumi mekem wol blong haos. (RBH.022)

woo mölum (comp.) n.com. kind of soft bamboo sofsof bambu Bambusa vulgaris \{Lit. soft bamboo sofsofbambu\}

woo vet (comp.) n.com. kind of strong bamboo which is thin, long and flexible and bends at the tip strong bambu we i smol be i longfala mo en blong hem i save benben Bambusa $\mathrm{sp}$. [This variety of bamboo is used for fishing poles. Kaen bambu ia ol i yusum blong hukum fis.] \{Lit. stone bamboo ston bambu\}

woo vet luwō (comp.) n.com. kind of strong, thick bamboo strong bambu we i tik mo i bigwan Bambusa sp. [This variety of bamboo is used for walling houses. Kaen bambu ia ol i yusum blong wivim wol blong haos.]

woo vet nötu (comp.) n.com. kind of strong bamboo that is thin strong bambu we i smol Bambusa sp. [This variety of bamboo is used for fishing poles and for narrow weaving for house walls. Kaen bambu ia ol i yusum blong hukum fis mo blong wivim wol blong haos i smolsmol.] \{Lit. bamboo for narrow weaving bambu blong wivim smolsmol\}

wooror n.com. toy pleplei \{Lit. thing for playing samting blong pleplei\} See: or woqat n.com. kind of wild ginger with white flowers and red fruit, which is white inside but black when ripe famle blong jinja we flaoa blong hem $\mathrm{i}$ waet mo frut i red, mo insaed long hem i waet, taem i raep i blak Alpinia sp.

wor $v t$. break, split open brekem $\mathbf{S i}$ odian̄, na tar wor na qōtōn tek o mataqē. If not, I will chop open your head with my axe. Sapos no, bae mi katem brekem hed blong yu wetem akis blong mi. (JRQ) See: mowor [POc *pora(k), *porak-i- 'break, damage'] woro n.com. dragon plum $\sim$ nakatambol Dracontomelon vitiense

worwor n.com. variety of banana that has long, large fruit and is yellow when ripe $\sim$ kaen banana we samtaem kakae blong hem i no kamaot long en be long medel. Hem i bigwan olsem vila, hem i grin mo taem i raep i yelo

wos $_{1} v t . \mathbf{1} \cdot$ bash, slap, pound; hammer, bash (nuts) to crack open $\sim$ hamarem No mō kōn̄ lēt o dem, ni van me, wos no, kik no, var no sōw lo qarörö dem wo nē ni mōl den no. I broke a yam, and so he came and slapped me, kicked me, and stomped on me down into the yam hole and he went home and left me there. Mi brekem yam, ale hem i kam, i kilim mi, kikim mi, stanap mo pusum mi i go daon long holblong yam mo hem i kam hom aot long mi. (DSR) 2 • build bildim $\left[P O c p^{w} a s a(r, R)\right.$ 'slap, hit']

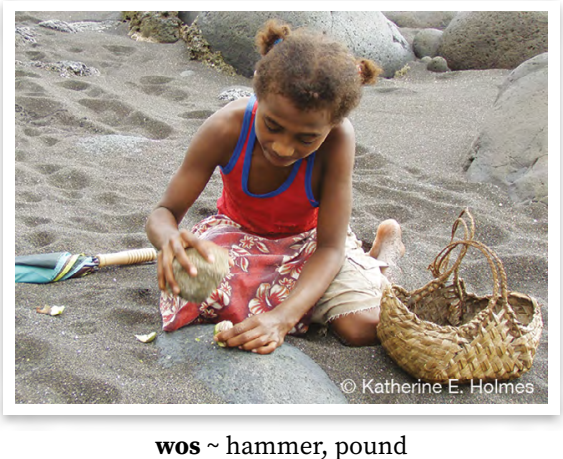


wos $_{2}$ n.com. boil, pus-filled swelling boela

wos matëdër (comp.) n.com. carbuncle, cluster of boils that are badly infected $\sim$ boela we i gat plante ae long wan ples

wosleqeñ $v$. method for cooking meat or fish by grilling a little, then wrapping up in leaves with salt and cooking again on coals $\sim$ fasin blong kukum mit o fis we yu putum long faea smol fastaem, afta paselem long lif wetem sol mo kukum long faea bakegen

wosvalqen $v$. cooking method $\sim$ kaen fasin blong kuk

wosvet n.com. Mossman mahogany, kind of hardwood tree with white flowers and red seeds wan kaen tri we flaoa blong hem i waet mo sid i red Dysoxylum arborescens See: qōmiat; walag

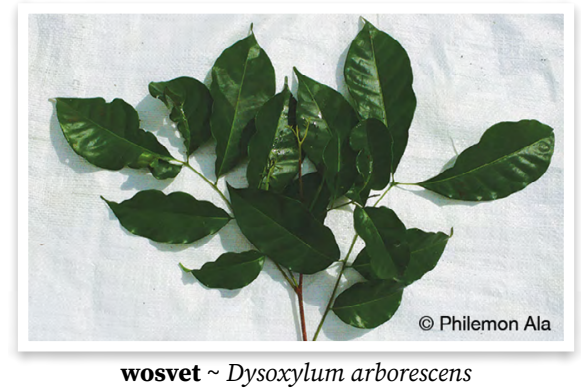

woswos n.com. kind of top shell famle blong troka Tectus pyramis

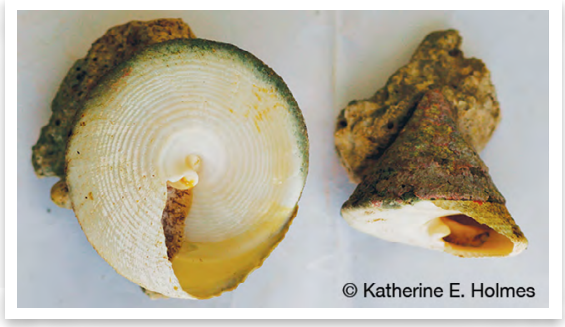

woswos Tectus pyramis

wot $_{1}$ vi. $\mathbf{1}$ - born, be born bon Ma qal wot o atmēn. The first to be born was a boy. Faswan blong hem i bon hem $i$ boe. (ESS) $2 \cdot$ materialise, come into being, happen bon long, kamaot $\mathrm{O}$ gēnē ga garaq mo wot min nēr. Something new had materialised for them. Wan niufala samting $i$ bin kamaot long olgeta. (ESP)

wot $_{2} v t$. castrate $\sim$ katem bol Tōlōw, nēk i van me, nēk i wot na bulëk o qō. Tomorrow, come and castrate my pig. Tumoro bae yu kam, yu katem bol blong pig blong mi.

wotag (fr.var. watag) n.com. cutnut, bush nut navele Barringtonia edulis wotag dere timiat (comp.) n.com. kind of cutnut that has dark red leaves and skin of fruit $\sim$ kaen navele we lif mo skin blong kakae blong hem i red olsem blad \{Lit. devil's blood cutnut navele blong blad blong devel\}

wotag dun (comp.) n.com. kind of cutnut $\sim$ kaen navele \{Lit. true cutnut trunavele\}

wotag sërsër (comp.) n.com. kind of cutnut $\sim$ kaen navele

wotag wos (comp.) n.com. kind of cutnut $\sim$ kaen navele \{Lit. cutnut that you can pound navele we yu kilim\}

wotag wōtō̄n (comp.) n.com. kind of cutnut $\sim$ kaen navele

wotag wöl (comp.) n.com. kind of cutnut kaen navele \{Lit. cutnut buy navele blong pem\}

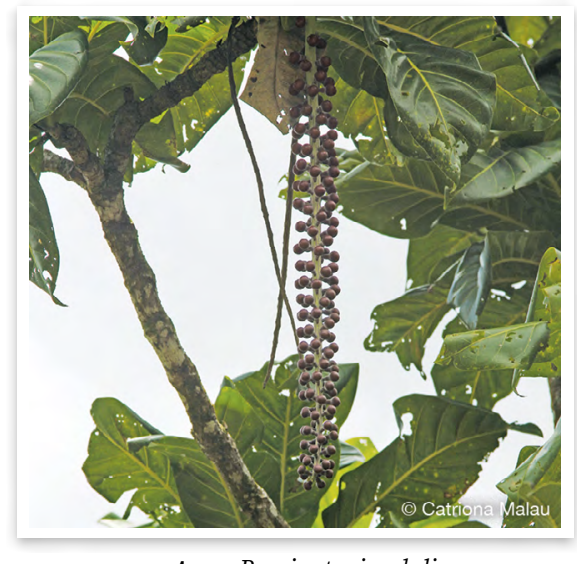

wotag Barringtonia edulis 
wotag bē n.com. kind of tree, of same genus as cutnut, but does not have edible nuts kaen wud we hem i famle blong navele, be yu no save kakae Barringtonia racemosa \{Lit. water cutnut navele blong wota\}

wotagavēl n.com. spotted boxfish fokona, fokonafis Ostracion meleagris wotalēbak $n$.com. variety of taro kaen taro

wotanaval n.com. variety of taro kaen taro

wotaqlislis n.com. unidentified variety of marine worm. The morphology of this variety is considerably different from that of those identified as un (Palola sp.) and it is likely that they are from a different family. They are short, black on top, red on the sides, and white on the underside. kaen wom blong solwota we hem i defren lelebet long palolo. Hem i sotsotfala, i blak antap, red long saed blong hem, mo bel blong hem $i$ waet. [At the time of the gathering of palolo, the wotaqlislis are the first to appear. These are gathered and thrown on the fire, the belief being that the 'true' palolo will be attracted by the scent of them burning. Taem ol man ol i go blong karem palolo, hemia nao hem i faswan blong kam. Hem i no rili stret wan we ol i wantem kakae, mekem se ol man ol i sakem long faea fastaem blong palolo ol i harem smel blong hem, ale ol i kam plante.] See: wömönmönösot

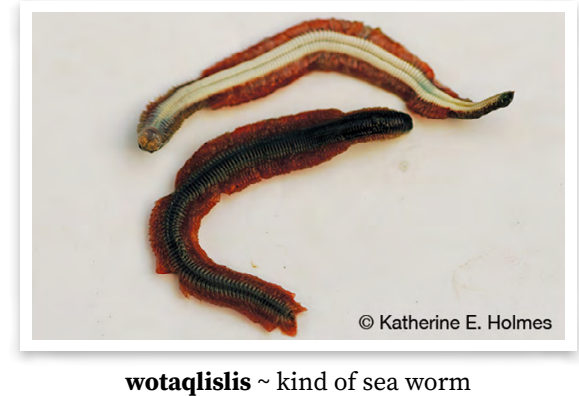

wotasiwōw n.com. variety of breadfruit that has elongated fruit kaen bredfrut we kakae blong hem i longwan

wotkērēvōr n.com. variety of taro kaen taro we han blong hem i laet grei mo kakae i waet. Taem yu planem wan nomo be hem i save karem plante kakae. Hem i wan gud taro blong nalot \{Lit. born at the base of volcano bon long as blong volkeno\}

wotlalabēvidavaqal n.com. variety of taro with dark blue stem and white corm. It does not fork kaen taro we han blong hem i dak blu mo kakae i waet. Hem i no save karem fok \{Lit. born on the frond of the davaqal fern bon long han blong davagal\}

wotliev n.com. variety of taro which is one of the largest varieties. There are two kinds, one with white stem, the other with a darker stem. The corm is white with a light green skin. It can fork kaen taro we hem i bigbigfala taro olgeta. I gat tu kaen blong hem, wan han blong hem i waet, wan han blong hem i dak lelebet. Kakae i waet mo skin blong kakae i laet grin. Samtaem i save karem fok \{Lit. born from fire bon long ples blong faea\}

wotlievgatgat n.com. variety of taro kaen taro

wotmēlēv n.com. variety of taro kaen taro

wotmindeli n.com. variety of taro kaen taro \{Lit. born to Deli bon long Deli\}

wotminmendala $n$.com. variety of taro $\sim$ kaen taro \{Lit. born to Mendala bon long Mendala\}

wotminsakna n.com. variety of taro kaen taro \{Lit. born to Jakna bon long Jakna\}

wotminviolette n.com. variety of taro $\sim$ kaen taro \{Lit. born to Violette bon long Violette\} 
wotminwērirtel n.com. variety of taro which has a stem that is red close to the leaf, but white near the corm. It is not large but grows long kaen taro we han blong hem antap, andanit long lif i red be klosap long kakae i waet. Hem i no save bigwan be i save longfala

wotoqog n.com. stocky hawkfish wan kaen fis Cirrhitus pinnulatus

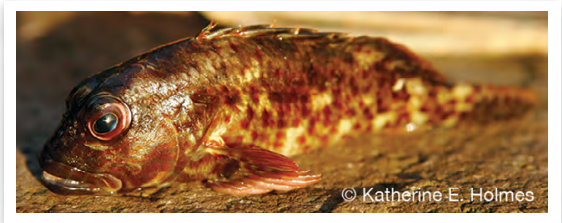

wotoqog Cirrhitus pinnulatus

wotoqtoq $_{1}$ n.com. $\mathbf{1}$ • be pregnant $\sim$ gat bel I ginon ni wotoqtoq kēl. His wife became pregnant again. Woman blong hem $i$ gat bel bakegen. (RPP.010) Syn: tek na taqan $\mathbf{2}$ - pregnant woman woman we i gat bel

wotoqtoq $_{2}$ n.com. hawkfish wan kaen fis Family Cirrhitidae

wotoqtoq $\mathbf{m a m} \overline{\mathbf{e}}$ n.com. scarlet robin (bird) wan kaen pijin Petroica multicolor $\{$ Lit. red pregnant belly red woman we igat bel\}

wotoqtoqolav n.com. stomach of cattle (cows only) bel blong buluk See: wöwöwut

wotvadadan̄ n.com. variety of taro which grows large and has a light green stem and white corm kaen taro we han blong hem i laet grin mo kakae i waet. Hem i save bigbigwan wotwot n.p.fr. kin relationship, one's father's mother's brother's children, reciprocally the same as one's father's sister's son's children, relationship between child of uncle and child of nephew (following male line only) $\sim$ famle memba we hem i pikinini blong angkel blong papa blong yu wovet n.com. spotfin squirrelfish kaen redfis Neoniphon sammara

wowolos n.com. Tahitian squirrelfish kaen redfis Sargocentron tiere

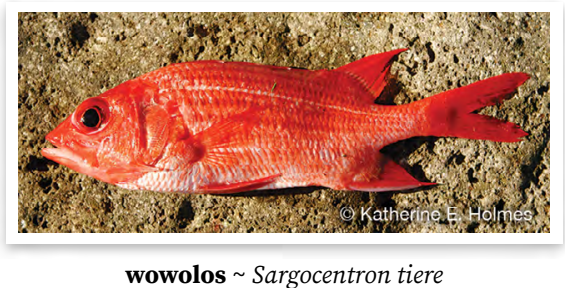

wōdōldōl n.com. trachea, windpipe pat blong bodi blong man mo animol we hem i paep blong pulum win long hem \{Lit. swallowing thing samting blong solem daon\}

wōg n.com. kind of freshwater crab wan kaen krab blong wota

wōgōlgōl n.com. cone shell wan kaen sel blong solwota, konsel Conus spp.

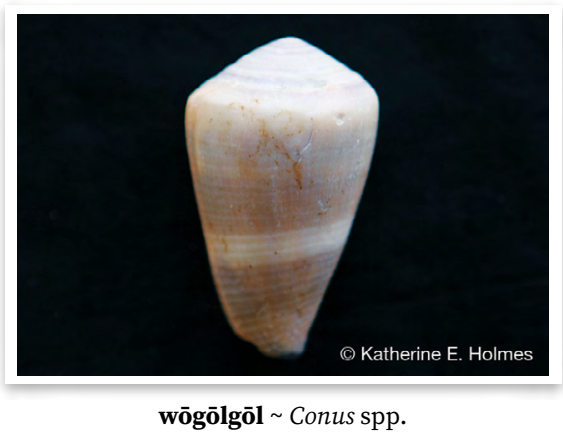

wōkōl n.com. Vanuatu mountain honeyeater (bird) longmaot blong hil (pijin) Phylidonyris notabilis

wōkōr n.com. slitgong that is made from bamboo or hollowed out wood tamtam we ol i mekem long bambu o wud Mam Tomas ge deñ o wōkōr lō vōrōg gēn. Papa Thomas will beat the slitgong this morning. Papa Tomas bae hem i kilim tamtam long moning ia. (VSJ) See: timiatwos [POc *kude 'hourglass drum'] 
WŌL

wōl 1 1 n.com. moon mun $\mathbf{2} \cdot$ n.com. month manis I ro vo ko go tog min nēn o wōl nivet. This woman is going to stay with us for four months. Woman ia bae hem i stap wetem yumi blong fo manis. (VSE) $\mathbf{3} \cdot$ n.com.suff. month of pregnancy $\sim$ manis (blong woman we i gat bel) Na wōlan̄ nivēs? How many months pregnant are you? Yu gat hamas manis? [POC *pulan 'moon, month']

wōlmeeñ (comp.) n.com. new moon fes saen blong mun i kam antap, taem $i$ gat mun be i no saen gud yet wōlmian (comp.) n.com. full moon ful mun \{ripe moon mun we $i$ raep\}

wōlōbōl (comp.) n.com. new moon niu mun \{unripe moon mun we $i$ no raep\}

wōlsial (comp.) n.com. moon that is visible during daylight $\sim$ mun we i stap antap yet taem i deilaet finis \{floating moon mun we iflot\}

wōlan (n.poss) n.com.suff. her Xth month (of pregnancy) manis blong hem

wulëk (n.poss) n.com.suff. my Xth month (of pregnancy) manis blong mi

wōl ${ }_{2}$ loc. to the left when facing the sea, in an anticlockwise direction around the island $\sim$ fes $i$ go daon long solwota, go long lefsaed Nēk i ukuëg den oko, nēk i van wōl me lo ere buluk. You leave from here, and you head in an anticlockwise direction going round the island to the cattle enclosure. Yu lego long ples ia, yu go long lefsaed olsem ia long fanis buluk. (RRS) See: Siag $_{2} \mathbf{2}$

wōl ${ }_{3}$ subord. because; introduces clause of reason from Van me, wōl nēk gōwē. Come, because you are nice. Kam, from yu naes. Syn: sur $_{2}$ wōl ${ }_{4}$ disc. discourse particle used when asking a question, indicating that one recognises that they are seeking information that they should already know, but want to be reminded $\sim$ wod we yu ademap long wan kwestin, blong soemaot se yu sud save ansa blong kwestin, be yu askem bakegen Na siañ wōl i sē? What's your name again? Wanem nem blong yu bakegen? (DSR)

wōlan n.com.suff. her Xth month (of pregnancy) manis blong hem See: wōl

wōlōg n.com. 1 • flexible wood for making trap wud blong bendem blong mekem trap 2 - handle of a $w o w$ trap handel blong fis trap wōw

wōlōg ${ }_{2} v$. bend benem

wōlōlōmeren n.com. kind of flowering plant with long shiny leaves and orange flowers kaen plant we hem i gat longfala lif we i saen mo flaoa blong hem i dak yelo Oxera vanuatuensis \{Lit. wisdom plant plant blong waes\}

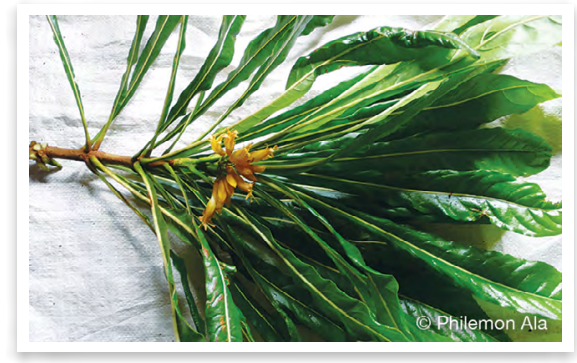

wōlōlōmeren Oxera vanuatuensis

wōlōm n.com. Indian mulberry noni, yalatri, yalawud Morinda citrifolia [An extract of the rotten fruit has a number of medicinal uses. Also will protect anyone who eats it before visiting a taboo place. Sapos yu kakae frut blong hem taem yu go long wan tabu ples, bae i no save 
spolem yu. Yu dring frut blong hem we yu bin livim i stap roten bae i save fiksim plante sik.]

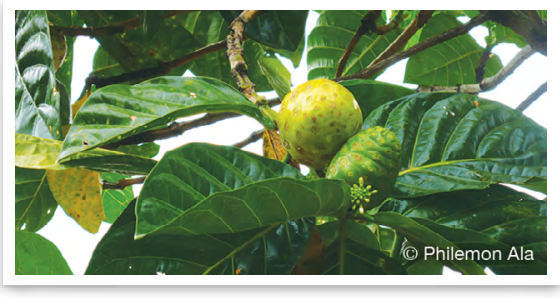

wōlōm $~$ Morinda citrifolia

wōlōrōr n.com. kind of small crab found on the sand, red with large claws wan kaen smol krab blong sanbij we kala blong hem i red mo tut blong hem i bigwan

wōlōwōl n.com. cross, cross beam kros, kroswud blong haos, kros blong Jisas

wōn 1 1 vi. join, close up, seal together $\sim$ tufala saed i mit, olsem fasem lif, tufala saed i mit antap, kaliko i brok, joenem mo somap bakegen La masawre nēn ma at van vavavan nē mō wōn, nēn a so o doot talë gëv o qötu. When we have tied the thatching on until it is joined, then we make the thatching to block the roof line where the thatching meets. Taem yumi somap go go hem i joen, yumi mekem natanggura blong kavremap top blong haos. (RBH) $\mathbf{2} \cdot v t$. seal (hole in), join together, close up joenem, silim I no tō wōnwōn in̄kē o qötu ak gēn. Here I am sealing up (a hole in) the front of the canoe here. Mi stap fiksimap (hollong) fored blong kenu ia. (VVKHVDP180ct0801.122)

wōnwōn (redup.) vi. whole, joined, complete fulwan (RBH.018)

wōn $v i$. blocked, be blocked fas Na qötuk mō wōnwōn. My head is blocked up (confused). Hed blong mi $i$ fas. See: qōrwōn wōnōnōg malgias n.com. kind of tree $\sim$ wan kaen tri [Wood used for axe handles and as firewood. Ol i yusum blong katem handel blong akis mo blong faea tu.]

wōnwōn n.com. flashflight fish wan kaen fis we ae blong hem i saen olsem toslaet

wōōr n.com. kind of woven basket which has a base and is made from rattan, woven with Freycinetia vines and lengths of Lygodium fern kaen basket we ol i wivim long rop mo fens See: gakarēs, gamērēr

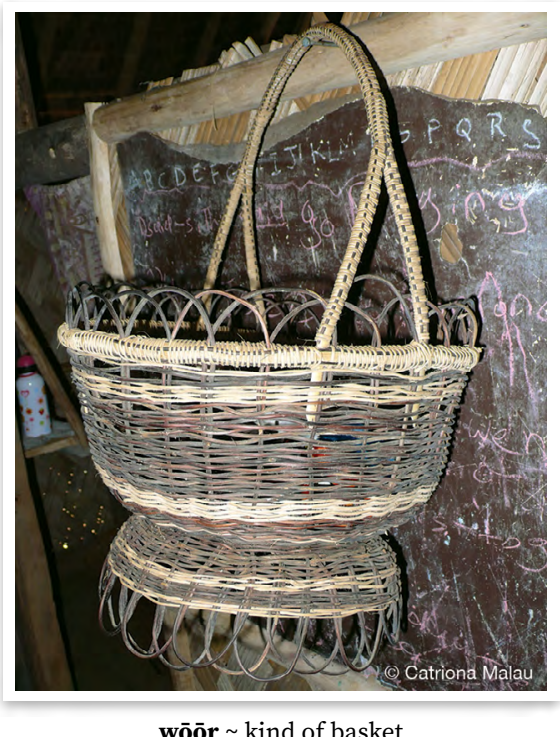

wōqōlmō $\overline{\mathbf{o}}_{1}$ n.com. kind of yam that is shaped like a crescent moon and can come out of the ground as it grows. There are two kinds, one with red skin, the other with white skin wan kaen yam we hem i sep olsem niu mun mo hem i gru i save kam antap long graon bakegen. I gat tu kaen blong hem, wan skin blong hem i red, wan i waet

wōqōlmō $\overline{\mathbf{o}}_{2}$ n.com. worm like creature wan kaen bebet we sep blong hem i olsem yam wōqōlmō 
WŌQŌLQQ̄L

wōqōlqōl n.com. back of neck bihaen long nek

wōqōqō n.com. beche de mer, sea cucumber $\sim$ besdemea, si kukamba Family Holothuriidae

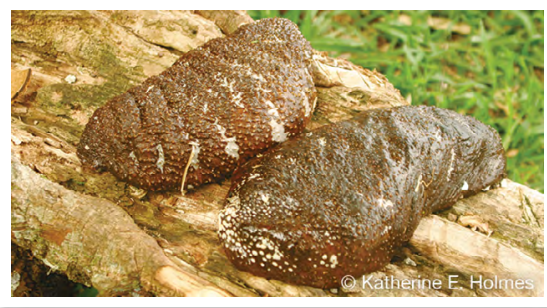

wōqōqōo Family Holothuriidae

wōr 1 vt. 1 - squeeze liquid out of (coconut, ground kava, etc.) melekem $\mathbf{2}$ • squeeze coconut milk onto food, either before or after cooking melekem (kakae) $\left[P O c{ }^{*} p o R o(s),{ }^{*} p o R o s i-i-\right.$ 'squeeze out, wring out (liquid)']

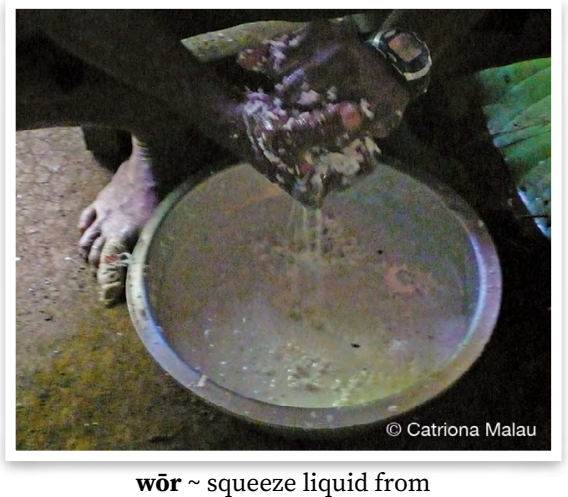

wōr logomonog (comp.) v. cooking style where food is cooked first then coconut milk is squeezed on top without being cooked again stael blong mekem kakae we yu mekem kakae i dan fastaem, afta yu melekem

wōr qarqar (comp.) v. cooking style where coconut milk is squeezed over uncooked food before being cooked, either in saucepan or parcel in oven or over fire stael blong kuk we yu melekem kakae fastaem, taem i no dan yet, afta yu kuk long sospen o pasolem mo bek long ston

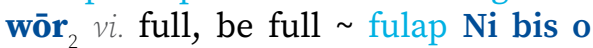
mìak, ni nësnës lö lölö tan̄, o tan̄ mō wōr wareg ren. He picked the Tahitian chestnuts, and put them in the basket, and the basket was really full. Hem i pikim ol namambe, i fulumap insaed long basket, basket i fulap gud nomo. Ant: $\bar{m}$ arö

wōrdes n.com. Polynesian triller (bird) wan kaen pijin Lalage maculosa

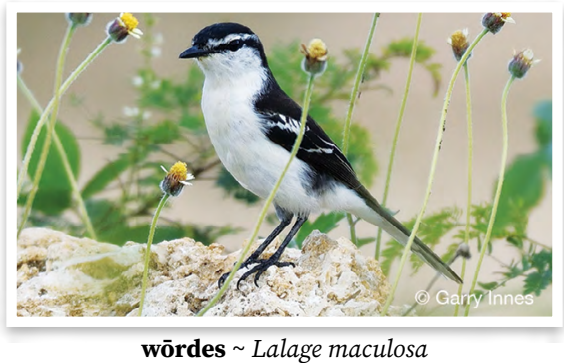

wōrkelkel n.com. kind of laplap that is made by mixing tuber or banana with coconut and wrapping in parcels to bake in oven $\sim$ wan kaen laplap we ol i salem maniok o wael yam o banana o kumala mo ol i miksim wetem melek kokonas bifo ol i bekem

wōrmar n.com. pumice ston we i kamaot long volcano we hem i no hevi [Used like sandpaper to make wood smooth. Ol i yusum blong mekem wud i smut.]

wōrō ${ }_{1}$ n.com. Kesteven's star-shell wan kaen sel olsem troka be i smol mo waet Astraea kesteveni

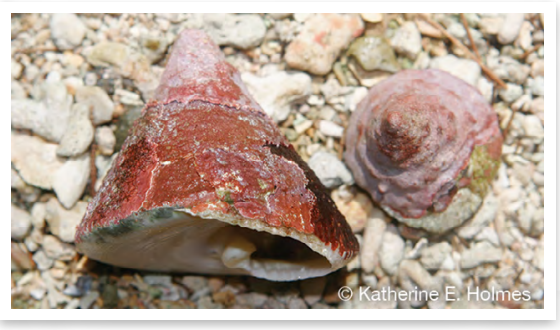

wōrō $~$ Astraea kesteveni 
wōrō ${ }_{2}$ n.com. dragonfly dragonflae wōrōqrōq n.com. bamboo rafter of house bambu blong somap lif long hem See: rōq

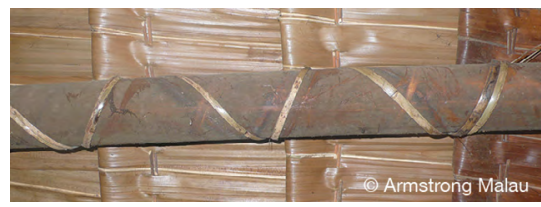

wōrōqrōq bamboo rafter

wōs n.com. paddle (for canoe) parel (blong kenu) Ni le o wōs, sō tēqēl, sō sō sō, sō dēñ rōw la maē. He took the paddle, and paddled out, paddled right out to the open sea. Hem i karem parel, parel $i$ go daon, parel go go i kasem daon olgeta long klia ples. (OHS) See: sō [POc * pose '(canoe) paddle']

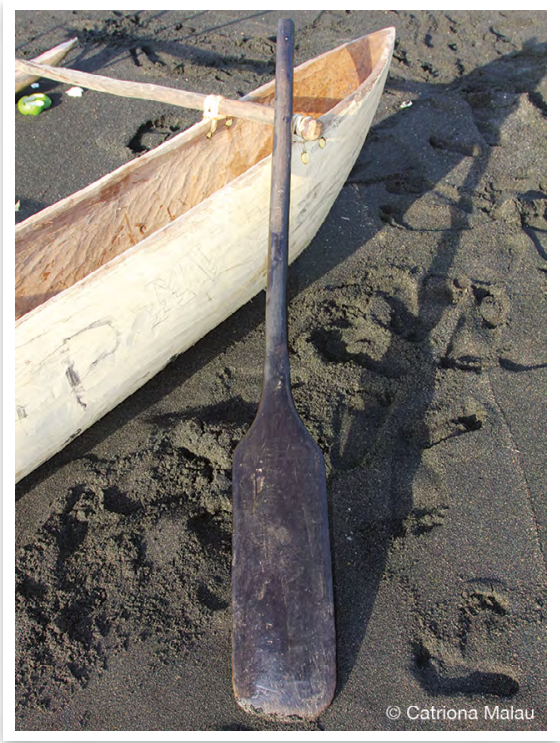

wōs paddle wōs 2 n.com. lungs lang, waetleva

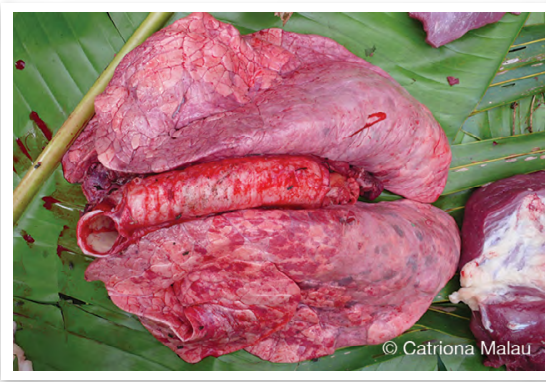

wōs lungs

wōsōk n.com. taro garden that is planted in dry ground and not irrigated garen taro we ol i planem long drae graon nomo

wōsōr n.com. cicada wan kaen bebet we hem i singaot long naet [There is a belief that if one hears a cicada in the night, it means the spirits will come. Long kastom ol i talem se sapos i kam long naet, i minim se ol devel ol i kam.] Syn: bōn̄

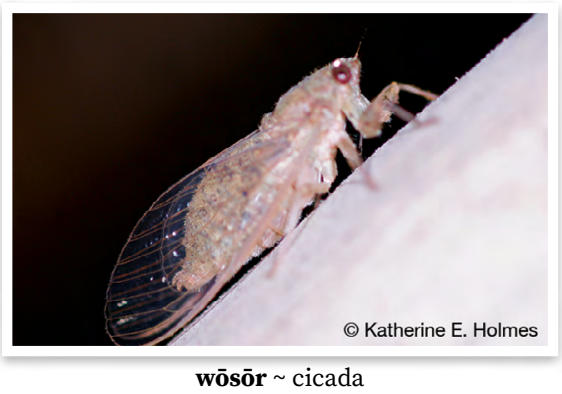

wōtōg n.com. reed that is used to sew sago onto, to make thatching rid we ol i yusum blong pinim natanggura long hem, mo tu wan pis blong taj wōtōgōrte n.com. kind of tree with very long fruit that are sticky like glue. The fruit is green when unripe and red when ripe. Used in house building wan kaen tri we frut blong hem i longlongwan mo i stiki olsem glu. Frut i grin be i kam red taem i raep. Ol i yusum wud blong wokem haos 
wōtōgsiar n.com. midrib of sago palm leaf which is used to make brooms bun blong lif natanggura we ol i yusum blong mekem brum Syn: döwurwur

wōtōkōl n.com. whimbrel (bird) wan kaen pijin Numenius phaeopus

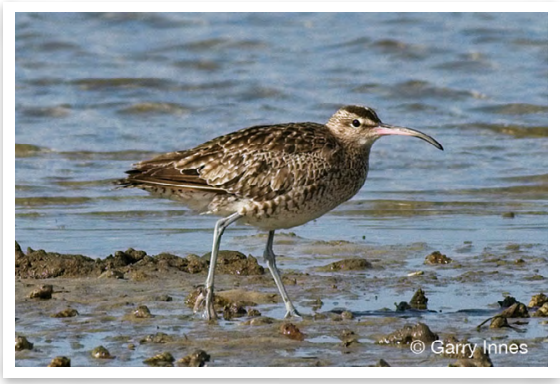

wōtōkōl Numenius phaeopus

wōtōn n.com. kind of fig with round fruits that are dark red when ripe, fruits are eaten by birds wan kaen tri we kakae blong hem i raon mo waet mo i dak red taem i raep mo ol pijin ol i kakae Ficus granatum [Yus blong hem i blong trapem pijin mo blong faeawud]

wōtōqagat n.com. seven-bar sergeant, banded sergeant (fish) wan kaen fis Abudefduf septemfasciatus

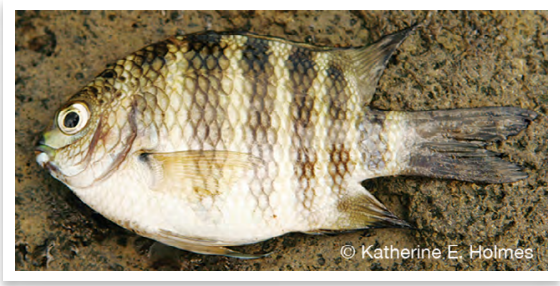

wōtōqagat Abudefduf septemfasciatus

wōtōw n.com. measurement unit for roof thatching pis natanggura we ol i mekem finis $\mathbf{O}$ wōtōw nitiwial, selen ni tevelēm. One slab of thatching is 50 vatu. Wan pis natanggura hem i 50 vatu. wōtōwōt n.com. pufferfish balongfis, bigbel Family Tetraodontidae [POc *[poto]poto 'pufferfish, porcupinefish'] wōvōnō n.com. kind of shrub, small tree kaen navenue Macaranga dioica [POc *pinu(q)an 'Macaranga spp. , perhaps $M$. involucrata']

wōvōt ${ }_{1}$ n.com. kind of shrub, with white flowers and large fruit that is red when ripe wan kaen tri we $i$ no bigwan be kakae blong hem i bigfala. Sep blong kakae i olsem nakavika mo i grin be taem i raep i red. Flaoa blong hem i waet Phaleria sp. [It is believed that if one throws the fruit at the abdomen of a pregnant pig, then the piglets will be red. Also, if one plays too much with the fruit then one's navel will become enlarged. I gat plante yus long kastom meresin, olsem tenge talō bōr, wan kaen soa. Sapos yu sakem frut blong hem long bel blong pig we i gat bel, bae ol pikinini blong hem bae ol i red.]

wōvōt ${ }_{2}$ n.com. bum as

wōw ${ }_{1}$ n.com. kind of fish trap used in the deep sea, which is like a lobster pot but used only for catching fish wan kaen net trap we yu yusum long medel si. Yu putum bet insaed mo $\mathrm{i}$ gat wan hol antap be taem ol fis ol $\mathrm{i}$ go insaed ol i no save kamaot bakegen

tul wōw (der.) $v$. throw wōw fish trap into the sea draonem wōw net long solwota

$\mathbf{w o ̄ w}_{2} v t$. tap lightly but firmly with something kilim smol wetem wan samting Nē mō wōw nēk tek oso? What did he tap you with? Hem i kilim yu long wanem

wōwaeg n.com. solid ball samting we $\mathrm{i}$ raon gud mo hem i no gat hol insaed 
wōwlan n.com. cowrie (gastropod shellfish) kaorisel Family Cypraeidae, Cypraea mauritiana

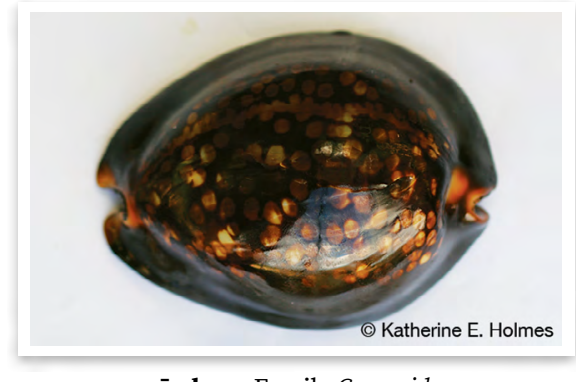

wōwlan Family Cypraeidae

wōwō n.com. ashes asis

wōwōsōl n.com. long-nosed emperor (fish) kaen redmaot (fis) Lethrinus olivaceus [POC *susul 'Lethrinus spp.']

wö n.com. kind of tree $\sim$ wan kaen tri Ixora asme [A hardwood, used to make digging stick for planting taro. Ol i yusum blong mekem stik blong planem taro.]

wöböw (abbrev. ga wöböw) n.com. aerial yam nabew, konkon yam Dioscorea bulbifera [Variety of yam that is not planted but grows wild and is only eaten in times of famine as it is bitter. A traditional medicine used for eye conditions. Kaen yam we ol i no planem, be hem i blong taem we i nogat kakae. Hem i kastom meresin taem ae i nogud.]

wöbulbulbōk n.com. glue berry tree glutri Cordia dichotoma

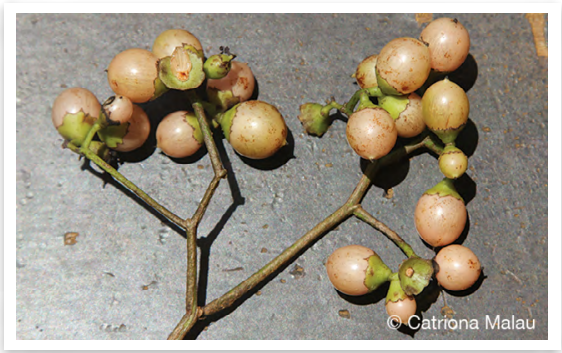

wöbulbulbōk Cordia dichotoma wödödö n.com. kind of tree, poison peach wan kaen tri Trema orientalis $[P O c * \operatorname{droRu}(\eta)$ 'Trema orientalis']

wödön $\mathbf{1} \cdot n$.com. method of baking in a stone oven, in which the stones are placed on the fire to heat, then when they are heated through and the fire has died down, the stones are removed, the food is placed on the bed of the oven, and the hot stones are placed on top of the food parcel wei blong bekem kakae we yu tekemaot ston, putum kakae long oven, afta putum hot ston antap long kakae [This method can be contrasted with the qarnis method, in which water is poured in a central hole to create steam, which contributes to the baking process. Fasin bek ia ol i no yusum wota blong kuk wetem stim olsem qarñis.] 2 - vt. method for setting wöwöwör qēsaqēs fish trap in sea, or waga prawn trap in river, in which stones are placed on top and around the trap to prevent it from being carried away by the current. putum trap blong fis long solwota o trap blong naora long wota mo putum ston raonem hem blong taed i no save muvum Na bien qēt o waga, na tek le qereñ bē, na wödön. I finish baiting the trap, I take it to the river, and I set it with stones around. Mi fidim trap finis, mi karem i go long wota, mi draonem mo putum ston long hem. (KBN)

wöduñduñ n.com. long, thick stick that is used for beating on wooden slab that is placed over hole in ground to make beat, especially for elñetiti dance longfala wud we ol i yusum blong kilim flat pis wud blong graon long taem blong danis, we i mekem bit blong elñetiti danis 
wögörtö n.com. spiny chiton, kind of mollusc strongbak Family Chitonidae

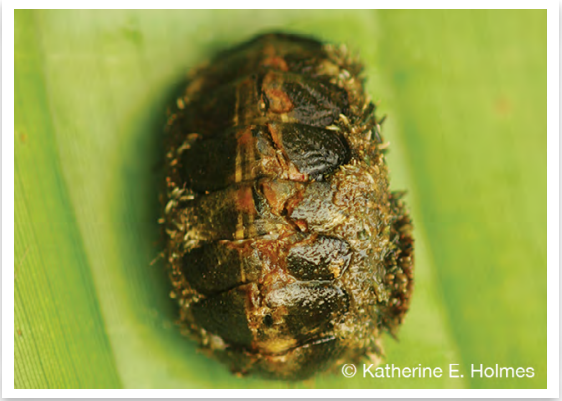

wögörtö Family Chitonidae

wökökö n.com. general name for caterpillars and other small wriggly creatures that don't have a specific name nem blong ol kaen smol samting olsem katapila

wökutökut n.com. kind of sea crab wan kaen smol krab blong solwota

wöl $v t .1$ - buy, purchase pem La masawre kemem mē dēñ siag e kemem a wöl o tiket. When we got up there, we bought the ticket. Taem mifala ikasem antap, mifala ipem tiket. (AAD) 2 - pay (someone), compensate pem No gö wöl nēk sur o wiwieg ine. I will pay you for the work. Bae mi pem yu from wok ia. $3 \cdot$ pay bride wealth for pem (woman) Nēk i vatvat min nēr vita nēr gö wöl i Roan lo sav wōl. You make an arrangement with them that they will pay the bride wealth for Roan in which month. Bae yu mekem promes wetem olgeta se bae ol i pem Roan long wanem manis. (CAA) wölwöl (redup.) n.com. shopping soping Qēt kemem a da o sönötu wölwöl. Then we did a bit of shopping. Afta mifala i mekem smol soping. (AAD) wölösömen $n$.com. testicles bol blong man o animol wölqareñi $v$. make payment to the people who dug a dead person's grave pem ol man we ol i digim gref blong ded man

wölubus n.com. corded turban (gastropod shellfish) wan kaen bigae blong solwota Turbo sparverius

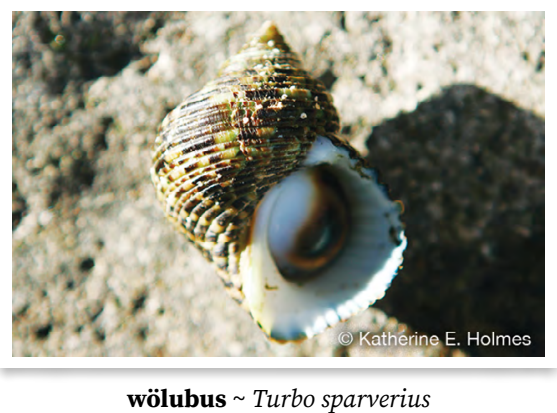

wölul (fr.var. wulul $)_{2}$ vi. make noise, as people shouting, singing and playing mekem noes, olsem ol man i singsingaot Nē gō rōñteg i rege mirmiar nēr gö wuwulul alo. He heard the children shouting and making lots of noise at the seaside. Hem $i$ harem olgeta pikinini i stap mekem noes long solwota. (DSR) See: qōrōtōt

Wölus n.p.fr. sister-in-law; husband's sister, brother's wife (for woman), reciprocal same generation in-law term for women sista blong man blong woman, woman blong brata blong woman

wōlōn (n.poss) n.p.bound her sisterin-law tawian blong hem

wöluk (n.poss) n.p.bound my sister-inlaw tawian blong mi

wömönmönösot n.com. unidentified variety of marine worm. The morphology of this variety is considerably different from that of those identified as Palola sp. and it is likely that they are from a different family. They are short in comparison with the palolo and light yelloworange in colour. kaen wom blong 
solwota we hem i defren lelebet long palolo. Hem i sotsotfala mo hem i laet red. [At the time of the gathering of palolo, the wömönmönösot are the last to appear. Their appearance is an indication that the swarming is nearly over. This variety is not eaten as they are not considered to taste good. Taem olgeta ol i stap kam i minim se ol tru palolo bae ol i finis nao. Ol i no stap kakae from we tes blong hem i no gud.] See: wotaqlislis

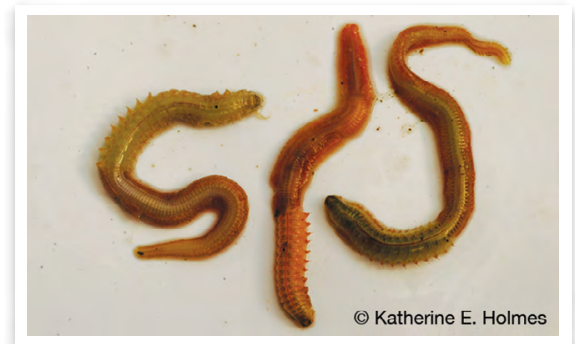

wömönmönösot kind of sea worm

wömön̄öln̄öl n.com. cartilage olsem bun be i sofsof

wömum n.com. quiet person $\sim$ man we i no stap toktok tumas

wömöötmöt n.com. area of bush, uninhabited area away from villages eria blong bus Rōrō a dörug nēr, vita a tek viteg nēr lö wömötmööt. The two of them tricked them in order to take them away into the deep bush.

Tufala $i$ trikim olgeta, olsem blong tekem olgeta $i$ go long bus. (RPP) See: $\overline{m o ̈ t}_{1} \mathbf{1}$

wönö crab or prawn that is carrying eggs that have not yet hatched krab o naora i gat eg be i no kamaot yet

wön̄ vi. be dry, become dried from heat kam drae from hot blong faea o san Nē ni mem siag le ev e, ni wön. He put it on top of the fire, and it became dry. Hem i putum antap long faea, hem i kam drae. (JRQ) Ner mö suvsuv qet, e in̄ko ner a wön̄wön gōr o 1ō. They've finished swimming, and now they are drying themselves in the sun. Ol i swim finis, ale nao ia ol i stap draemap olgeta long san.

wönsial n.com. driftwood wud we $i$ flot

wöqörö n.com. kind of freshwater gastropod shellfish that is found in muddy areas wan kaen sel blong wota we i stap long sofmad

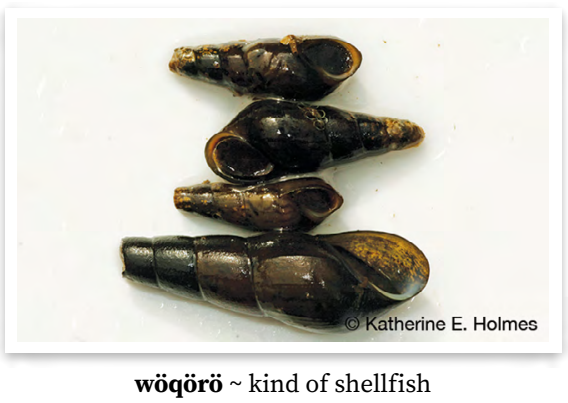

wöqut n.com. corner $\sim$ kona

wör $v t$. place in sunny position to dry putum long ples (olsem long san) blong mekem se i drae No mö wör o tere gogov. I hung the clothes up to dry. Mi hangem ol klos blong drae.

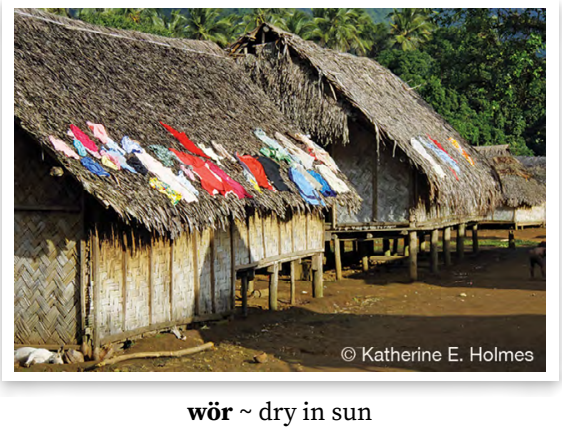

wörönö n.com. stake, length of wood inserted in ground for yam vine to grow up wud blong planem long graon blong yam i save gru long hem See: rönö 
wörön̄ötöwö n.com. aculeate rockshell wan kaen sel blong solwota Thais aculeata
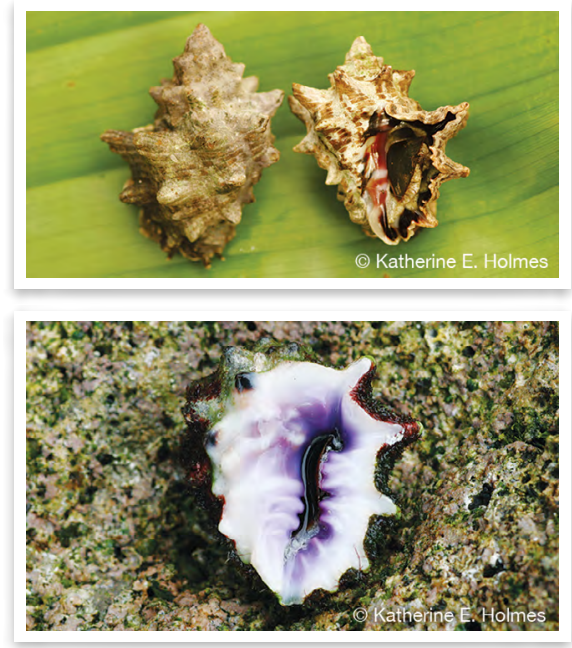

wörön̄ötöwö Thais aculeata

wörötuo n.com. smallmouth squirrelfish kaen redfis Sargocentron microstoma See: wēvētuo wörumrum n.com. fruit fly wan kaen smolsmol bebet we fulap i stap raon long frut we i raep gud. Ol pijin (swiflet) i stap kakae

wörurus tavav n.com. pancreas wan pat blong animol we i stap wetem gat

wösöm n.com. kind of gastropod shellfish wan kaen sel blong solwota [Shell that is used to make traditional shell money. Kaen sel we ol i yusum blong mekem sel mane.]

wösöwu n.com. seed $\sim$ sid I rege mirmiar o mēsi kur wösöwu biēg. Children love to eat breadfruit seeds all the time. Ol pikinini ol i stap kakae sid blong bredfrut oltaem.

wösöwunët n.com. bird wrasse (fish) kaen fis, famle blong blufis Gomphosus varius $\{$ Lit. red silkwood seed (due to shape) sidblong naduledule (from sep i semak)\}

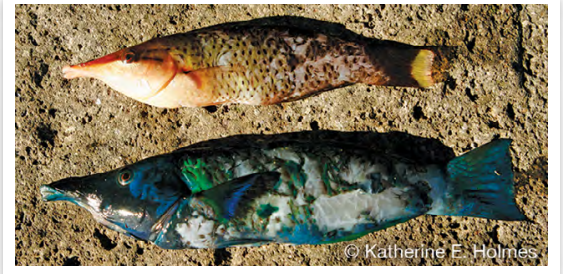

wösöwunët Gomphosus varius

wösurtege n.com. kind of pandanus basket which has two plaited straps for carrying, used for carrying cooked food and general items wan kaen basket blong pandanas we i gat tu rop blong karem, we ol i yusum blong karem kakae mo ol smol samting

wösusum̄almal (fr.var. susum̄almal) n.com. maculated top shell $\sim$ wan kaen selfis we i olsem troka be i raon mo sep blong hem i olsem titi blong wan gel Trochus maculatus \{Lit. young woman's breast titi blong yangfala gel\}

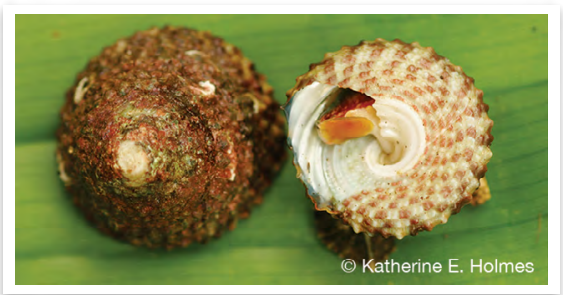

wösusum̄almāal Trochus maculatus

wösusurbē n.com. kind of shrub with round, bright orange fruit wan kaen tri we kakae blong hem i grin mo taem i raep $\mathrm{i}$ aranis-red Tabernaemontana sanguinea [Used for decoration by men in traditional dances. They are pierced on the end of a stick which is held while dancing. Women are not allowed to use them. I gat plante yus long kastom. Ol man ol i yusum frut blong hem long danis; ol i stikim long en blong brans blong wud mo danis wetem. Hem i blong man 
nomo i danis wetem. Most taem hem i gru long saed blong reva mo hem i wan smol tri nomo.]

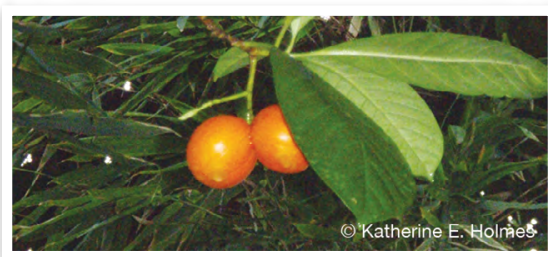

wösusurbē $\sim$ Tabernaemontana sanguinea

wösuwun n.com. disabled person who is unable to walk man we i no save wokbaot

wöt $v t$. $\mathbf{1} \cdot$ throw, throw away sakem Wöt ren me gēn, rivteg no. Throw it right here, close to me. Sakem stret long ples ia, klosap long mi. (DSR) $\mathbf{2}$ • contribute, give givim, sakem Nēk o memba talo asosiesen, nēk ga mas wöt na mōgōn o fi. If you are a member of the association, then you have to contribute your fee. Sapos yu memba blong asosiesen, bae yu mas sakem fi blong yu. (VSS)

wötörör n.com. caterpillar katapila wötörövarvar n.com. variety of yam which belongs to the grouping of 'male' yams wan kaen yam we i longlongfala mo insaed long hem i stat antap hem i red, i go daon long medel i miks red mo waet mo long en blong hem i waet

wötöv n.com. bottle botel

wötu n.com. top antap lö wötu sëkër at the top of the reef long antap long rif (AWW.027)

wöturturōrōōn n.com. bridled monocle bream (fish) wan kaen fis Scolopsis frenatus $\{$ Lit. thing that stands still samting we istanap kwaet\}

wöu n.com. route, way rod, wei blong go

wöul (fr.var. wuul) n.com. fivestripe wrasse (fish) kaen fis, famle blong blufis Thalassoma nigrofasciatum, T. quinquevittatum

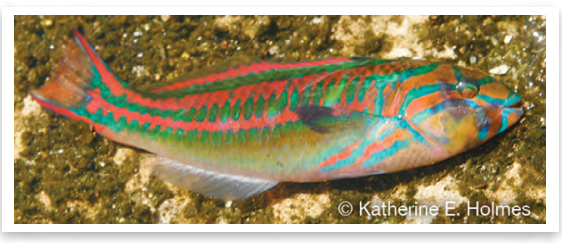

wöul Thalassoma spp.

wöuw n.com. Kind of yam that is very productive. It is one of the best tasting yams and is often grown in the taro garden $~$ wan kaen yam we hem i karem fulap kakae. Plante taem ol i planem long garen taro. Tes blong hem i gud, klosap i bitim ol narafala yam

wöwör $v$. weed $\sim$ widim [Poc *papo 'weed (a garden + )']

wöwös $\mathbf{1} \cdot v t$. wrap up, most commonly used to refer to making a cone shape from leaf, filling it, then sealing pasolem, speseli ol i talem taem we ol i foldem lif olsem kon, afta pasolem kakae insaed $\mathbf{2}$ - n.com. parcel, wrapped up goods, food pasol, kakae o wan samting we ol i bin pasolem Nēk tē gis ti e o wöwös so? What is in the parcel that you are holding? Yu stap holem ia wan pasol blong wanem? Syn: lölös $\mathbf{1}$, mon $\mathbf{1}$

wöwöwör qēsaqēs n.com. kind of trap used close to the shore for catching small fish and particularly lobster kaen trap we yu yusum klosap long so blong kasem ol smol fis mo speseli naora

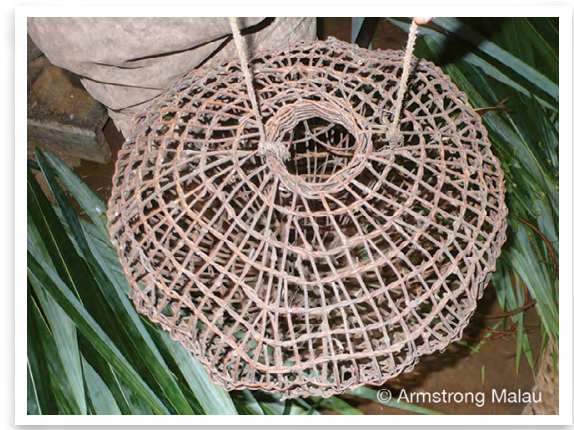

wöwöwör qēsaqēs woven trap 
wöwöwut n.com. smaller stomach of cattle, omasum handred rum (blong buluk) See: wotoqtoqolav

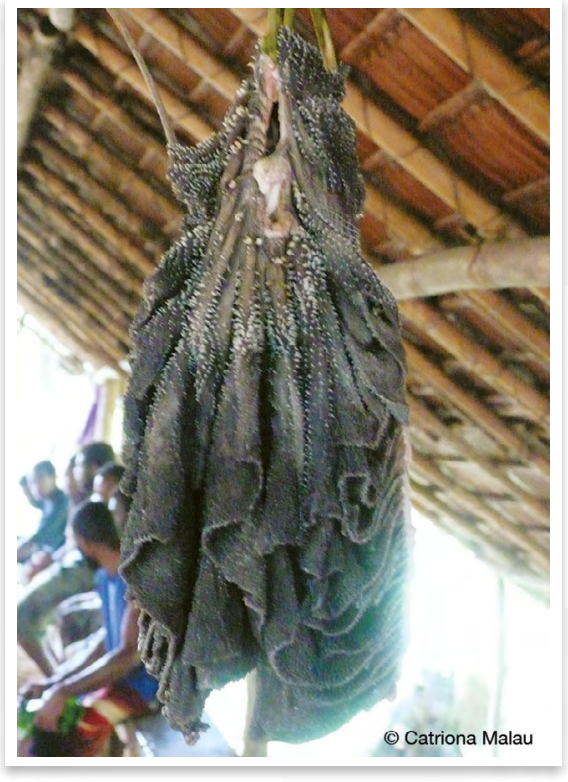

wöwöwut omasum

wöwut n.com. man of high rank who has studied the traditional rules and way of life in the men's house man we hem i bin go long nakamal blong lanem stret rod blong kastom

wu n.com. kind of small tree with hard wood wan kaen tri we hem i strong mo i no save gru bigwan [The wood is used as a taro planting stick. $\mathrm{Ol} \mathrm{i}$ katem blong planem taro]

wududuw (fr.var. of wēduduw)

wuduw n.com. sharp needle-like piece from inside of base of sago palm rachis (leaf stem) bun we $\mathrm{i}$ kamaot long insaed blong stamba blong lif natanggura [Used as tool for splitting pandanus leaves for weaving and for fastening thatch. $\mathrm{Ol}$ i yusum blong splitim pandanas mo pinim lif.] See: wēsiri

wul vt. peel, remove skin (from something that is soft and easy to peel, like a banana or vegetables that have been cooked) tekemaot skin (blong samting we $\mathrm{i}$ isi olsem banana o bredfrut we i dan) Na bērn̄ēñ ine, nē ni wulwul o gengen ine wo nēk i qōsqōs. Your partner peels the (cooked) food and you mash it. Patna blong yu $i$ karemaot skin blong kakae (we i dan) mo yu kilim. (JNN) See: sēm 1 [POc * ${ }^{*}\left({ }^{w}\right)$ ilit 'peel by hand (fruit, cooked food)']

wulewēsēston̄ov n.com. operculum (eye of green snail shell) ae blong grinsnel [Used like sandpaper for making wood smooth. Ol i save yusum olsem sanpepa blong mekem wud i smut.]

wulë busu n.com.suff. finger nail, toenail sel fingga

wulëk n.com.suff. my Xth month (of pregnancy) manis blong mi See: wōl

wulëwalal n.com. devil's horsewhip kaen plant Achyranthes aspera

wulul n.com. albino albino Syn: wokē wulul $_{2}$ (fr.var. of wölul)

wun $a d v$. probably, I think ating Kakaka na möguk wun mē qēt ti aē gēn. My tale, I think it is finished here. Storian blong mi ating i finis long ples ia. (DDP)

wun $_{2}$ v.part. high probability modality bae Syn: $\min _{2}$

wuqiat n.com. kind of tree whose wood is used as a digging stick for planting taro kaen wud we ol i yusum olsem wud blong planem taro

wur $v$. sweep brum See: döwurwur wurveg $v t$. clear, clean, prepare place for something klinim Am̄̄ō den nēn gö mörös ta nēn gē gis o gövur nitiwial, nēn a wurveg o tötönö gövur. Before we want to build a house, we clear the place for the house. Bifo yumi wantem bildim wan haos, bae yumi klinim ples blong haos. (RBH.001) See: winiwin 
wurveg, $a d v$. properly, correctly, according to one's aim or desire stret, gud Oo, kēti, kēti, nana vēn wurveg o mes. Oh, wait, wait, I've got to shoot enough fish. O, wet, wet, bae mi mas sutum gud ol fis. (JMT) Syn: wareg 1

wususrō n.com. kind of basket made from coconut leaves, used for carrying food and firewood kaen basket blong lif kokonas we ol i yusum blong kakae mo faeawud \{Lit. two breasted thing samting we $i$ gat tufala titi\}

wut n.com. louse laos [POc *kutu 'hair louse']

wutbōn n.com. bedbug stingfli

wutge $v$. mark spot at sea $\sim$ makem ples long solwota [This is a method used for marking the position at sea where one has placed a fish trap or caught a particular kind of fish. One notes the position at sea in relation to a tree or other landmark on the shore. Wei blong makem ples we yu bin draonem basket blong trapem fis o ples we yu save se yu bin karem fulap fis bifo.]

wutōt $v$. complain komplen Nèr a wuwutōt no. They are complaining to me. Ol i stap komplen long mi.

Wutrōw n.loc. $1 \cdot$ place name $\sim$ nem blong ples $\mathbf{2} \cdot$ tribe name $\sim$ nem blong traeb

wutu non n.com. Spanish mackerel (fish) kaen fis Scomberomorus commerson

wutu ōn n.com. spotted garden eel kaen namarae \{Lit. sand louse laos blong sanbij\}

wutumet n.com. drupe shell $\sim$ wan kaen sel blong solwota Drupa morum, Drupa grossularia

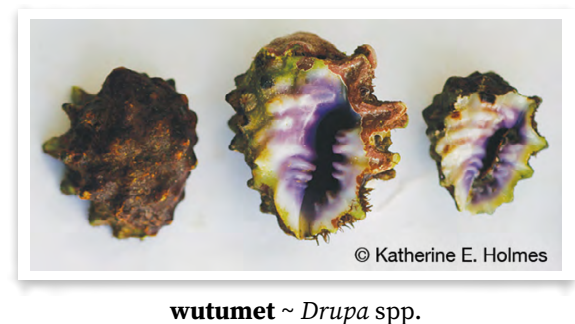

wutusērēt n.com. white-spotted grouper (fish) kaen los (fis) we i gat waet dot long hem Epinephelus caeruleopunctatus

wututan n.com. kind of insect like a cockroach but which lives in the ground and is small wan bebet we hem i olsem kakros be i smolsmol wan nomo we hem i stap long graon \{Lit. ground louse laos blong graon\}

wutwut $v$. stick out, bulge, rounded, of hill, forehead, stomach, bag kamaot, raon, olsem bigfala bel o top blong hil

wuul (fr.var. of wöul)

wuw $1 \cdot$ adj. many, plenty, lots plante, fulap Nē ni wölwöl o wuw savsav ine. He was buying lots of things. Hem $i$ stap pem fulap samting ia. $\mathbf{2}$ - vi. be many, plenty, become plentiful plante, fulap, kam plante $\mathbf{O}$ tō tö wuw ti a Tores o tō ta Vōnō Lav. The chickens that are becoming plentiful in the Torres Islands are chickens from Vanua Lava. Ol faol we ol i stap kam plante long Tores ol i faol blong Vanua Lava. (RTF) Syn: lōqtag, m̄ōrag, tutuqë 



\section{English - Vurës Finderlist}

The English finderlist presents English words with basic translation equivalents in Vurës. For the most part, no further information is provided. The purpose of the finderlist is simply to enable users to look up Vurës equivalents of English words. If a user wishes to know more about the meaning and use of the Vurës word, they should then search for that entry in the main part of the dictionary.

Some of the Vurës words listed are followed by a number in either subscript or bold font, or both types of numbers. A subscript number occurs if the word is a homonym, and thus directs the user to look up the appropriate homonym. A bold number occurs if the user is being directed to a specific sense of the Vurës word. For example, about sur 4 specifies that sur with the meaning 'about' is the fourth sense of the first listed homonym for sur.

Part of speech is not generally given, unless there is some level of ambiguity. That is, if the English word has a meaning associated with more than one part of speech. Thus, for example, English 'answer' is presented as answer vël, vt.; vëlvël n.com. to make it clear which Vurës word refers to the verb meaning of 'answer', and which the noun.

\section{$\mathbf{A} \sim \mathbf{a}$}

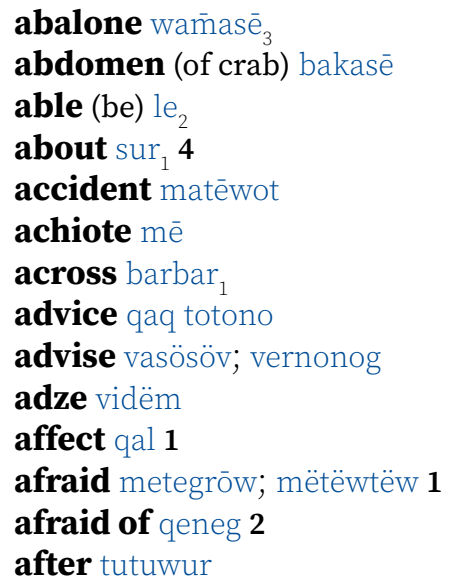

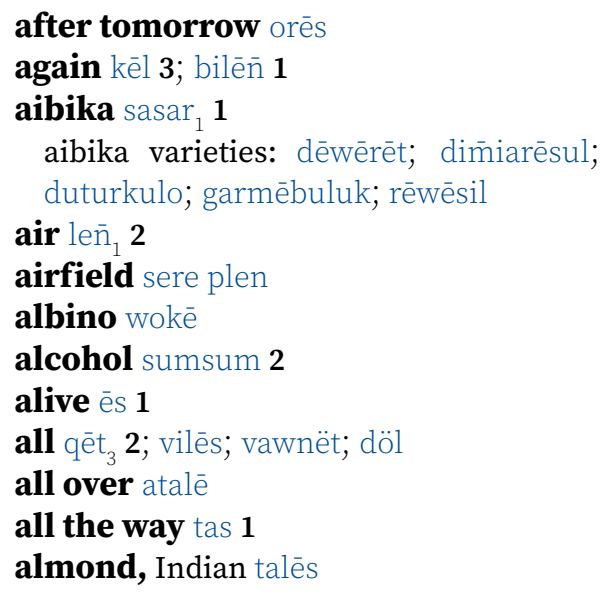


almond, native ñe

native almond varieties: ne dawel; ne dun; n̄e ta

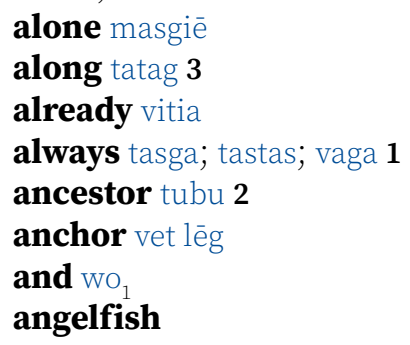

apart asaw

apple, Malay gavēg

argh! awi

argue kes; verliseg

arm bēni 1

her/his/its arm banēn

my arm bēnik

armpit gavivne

her/his/its armpit gavivnan

my armpit gavivnëk

arrive dēn $\mathbf{1}$; $\operatorname{rar}_{1}$

arrow qatē ${ }_{2}$; wētēq

kind of arrow meteër

poison arrow tōt ${ }_{2}$

arse kērē 1; wērē

her/his/its arse kirian; wirian

my arse kērēk; wērēk

ashes wōwō

ask baēr; vörus

at $\mathrm{lo}_{2} ; \mathrm{a}_{1}$

away rieg; viteg

axe mataqē; barbar $_{2}$; tele

\section{$\mathbf{B} \sim \mathbf{b}$}

baby tētē 1

\section{bachelor qilewer}

back tëwru; kērē $\mathbf{3}$; kulë $\mathbf{1}$

her/his/its back kōlan

my back kulëk

bad tisē

bait wēbē

bait hook $\mathrm{be}_{2}$

bake qan̄ris 2; wödön̄ $\mathbf{1}$

bald qas

bamboo woo

kinds of bamboo: woo mölum; woo vet nötu; woo vet luwō

banana vetel

banana varieties: bakrës; bē ${ }_{3}$; brisben; ëtëtlam; kukuög; lian manu; malēe; matmat malgias; matmat mamē; mianisi; malmalbak; retekraw; rēn̄rēn̄; rurun̄ög; samo; sēnē; sōbōt; siwit; tores; tulug; ulēulēi; vagvag; vetel mal; vetel talisōr; vietnam; vila; vinwōn; vinwōn ga qag; vinwōn gō tōtōrōg; wavagvag; wēwēsēg; wēwēsēg ta mot; wēwēsēg ta orbarbar; wiriatege; worwor bundle of bananas titigi

bang dun

bang (together) dorveg

banyan $\mathrm{bak}_{1}$

kinds of banyan: bak matqō; rēw

baptise gösuv rōñ

bark (dog) tōr 1 vt.

bark (tree) vinti 2 n.com.

barnacle wēbēbēs

barracuda, blackfin non

base kērē 2

bash lā̄seg; wos 1 1; qēs

bash (with stone) qos

basis qētēgi 2 


\section{basket tan̄; tibiar}

basket types: bor; garte wōl; gateteg; gavan̄; matqēt; qötuōw; tan̄; tibiar; wam̄asē $_{2}$; weteñe tō; wēgēbil ${ }_{2}$; wēgēt; wösurtege; wususrō corner of basket qörö nut basket toq

bass, red (fish) tenmē

bat tëqël

batfish dëvër

bathe suvsuv

be with bemrin̄ 2

beach $\overline{0} n_{2} 2$

beach mahogany bōgōr

beadtree, red wēwēn

beak ñere 2 2; ñusu $\mathbf{2}$

its beak n̄aran; n̄ōsōn

my beak n̄ërëk; n̄usuk

beam wēbēr

bear fruit $\bar{o} \mathrm{w}_{2}$

beard win̄i

beat deñ; dèn

beautiful mösövsöv

beauty leñas

because $\operatorname{sur}_{2} ;$ wōl $_{3}$

because of $\operatorname{sur}_{1} 3$

beche de mer wōqōqō

become van me $\mathbf{2}$

bedbug wutbōn

beetle

kinds of beetle: bōrōn̄; bustavēl

before am̄o $\mathbf{1}$; am̄ō den; götuë

before yesterday nonorēs

behind tuwur; kulë 2

behind her/him/it kōlan

behind me kulëk

behind them kulen nēr

belief nōmtuv 2

believe nōmtuv 1

belly teqe

her/his/its belly taqan

my belly tëqëk

belt gabelte

bend wōlōg ${ }_{2}$; kulum

betel nut wēmētigtig

big bëtutu; lav ; luwō $\mathbf{1}$ bigeye (fish) magalmevesoqötu

bird men

bird species: bagbaglō; biliag mamē;

bëlbëlvōlōn; mal biliag; malēe;

menmenlul; mensirsirqōōr; gōg;

gōg gabēt; gōg kör; mëkbē; mēlēg

wö; qon bulënqet; qon bulënrēlē;

qon tan̄sar; qon wēbēt; qötqötumen;

sōravar; tēgēr; tēwē; tēwē biliag; tēwē

ōlqön̄; tēwē rōwelteg; tërëw; tōrō; tōrō

butbut; tōwav ${ }_{2}$; tutrevak; tutwō; wasēe

wētēbisbis; wētēsisis; wētēt $t_{1}$;ēvēl;

wiria; wiria tabē; wotoqtoq mamē; wōrdes; wōkōl; wōtōkōl

kinds of shore bird: tēwē biliag; tēwē ōlqön; tēwē rōwelteg

bird's nest tötöu men

bird's nest fern dēqēt

Bislama qaq tala lam

bit, a mènē

bite $\mathrm{er}_{2}$; gar

bitter gōgōn 1

bivalve, species weger dun

black körkör 1

bleed dar 2

blenny (fish) liaw 2

bless vawē 1

blessing vawē 2

blind matwōn

block gëv; gōr ${ }_{2}$

blocked $\mathrm{wōn}_{2}$

blood dar 1

her/his/its blood daran

my blood dërëk

pig's blood dere qō

blow uv

blowfly lan̄ talōbō

blue tōtōrōg 3

blunt qosmötöl; löl

boat $a k$

body tarbiē

her/his/its body tarbian

my body tarbiēk

boil (sore) wos $_{2}$ n.com.

boil (water) gök $1 \mathrm{vi}$

bok choy raminwōlōnmögörviaomōtōsur 


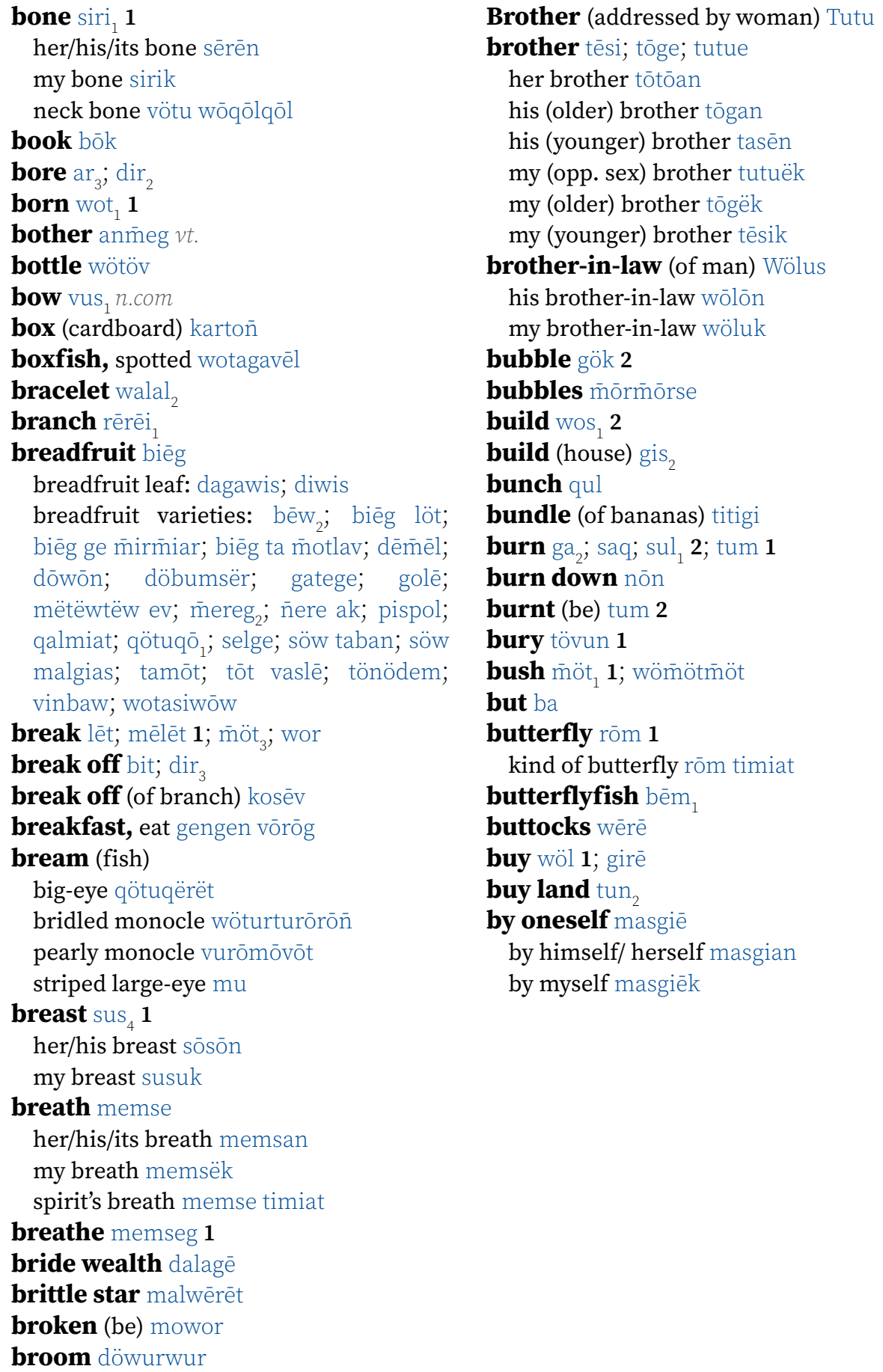


$\mathrm{C} \sim \mathrm{c}$

calf teqeliaw

call out $\bar{o} l_{1} 1$

canoe ak; ak sōsō; wēlwēl

sailing canoe ak geben

canoe tree $\bar{O} \mathrm{~V}$

cardinalfish matwiwi

careful (be) gitiag

carry sas; sesen̄; sōsōrvël; tatalēv

carry baby bēm 2

carry on shoulder sōltek

carry over shoulder bēmn̄eg

cartilage wömönöln̄öl

carve tes $_{2}$

carver $\bar{m} \ddot{g}$

cassava maniok

cassava groupings: maniok atatmēn;

maniok rereqe

cassava varieties: atina; maniok añañ;

raēs ga qag; raēs mamē; saemon; sus

rereqe; sus atatmēn; vasēr

castrate wot $_{2}$

casuarina ër

cat buskat; tivi

catch $\mathrm{SOkO}_{2}$; gis qal

caterpillar wötörör

cattle buluk

cause da 2

cave $l \bar{e}_{1}$

centipede tebedēmēēl

ceremonial ground $\mathrm{sar}_{1}$

change lies $\mathbf{1}$; lilies

charcoal watavren

charge vēvēg ${ }_{2}$

chase goro

chat kakaka 1

cheap masērig

cheek wadalñe

her/his/its cheek wadalñan

my cheek wadaln̄ëk

chest vere ${ }_{2} \mathbf{1}$

her/his/its chest varan 1

my chest vërëk 1

chestnut, Tahitian miak

chew $\overline{n a l}_{3}$; n̄ōr chick nötu tō

chicken $\mathrm{to}_{1}$

black chicken tō kör

chicken varieties: tō armiag; tō biliag;

tō menmenlul

female chicken tō reqe

red chicken tō mamē

white chicken tō bèt

chicken pox sislaqlaq

chief maranag; tövusmēēl

high ranking chief lan

child mirmiar; nötu

her/his/its child nōtōn

my child nötuk

chin balsiē

her/his/its chin balsian

my chin balsiēk

chiton, spiny wögörtö

choke (on) dōlgia

chop ger; $\operatorname{tar}_{1}$

church gövur gōrōn̄; gövur tatar

cicada wōsōr

clam tele $e_{2}$ giant clam tele tur

claw busu 2

clean winiwin

clean around vawseg

clear mānaē; tawseg; wurveg

cliff dō

climb rem

clinic gövur lisia

close (shut) bur 21

close (near) susus

close eyes gen mōvōt

close together verqiriñ; vōsōsōs

closed (be) bur $_{2} 2$

cloth gogov 2

clothes gogov 1; mölö; wësërsër

her/his clothes molon

my clothes mölök

cloud malēg

cloudy taqtaq

club qesekēr

coals matnōn 


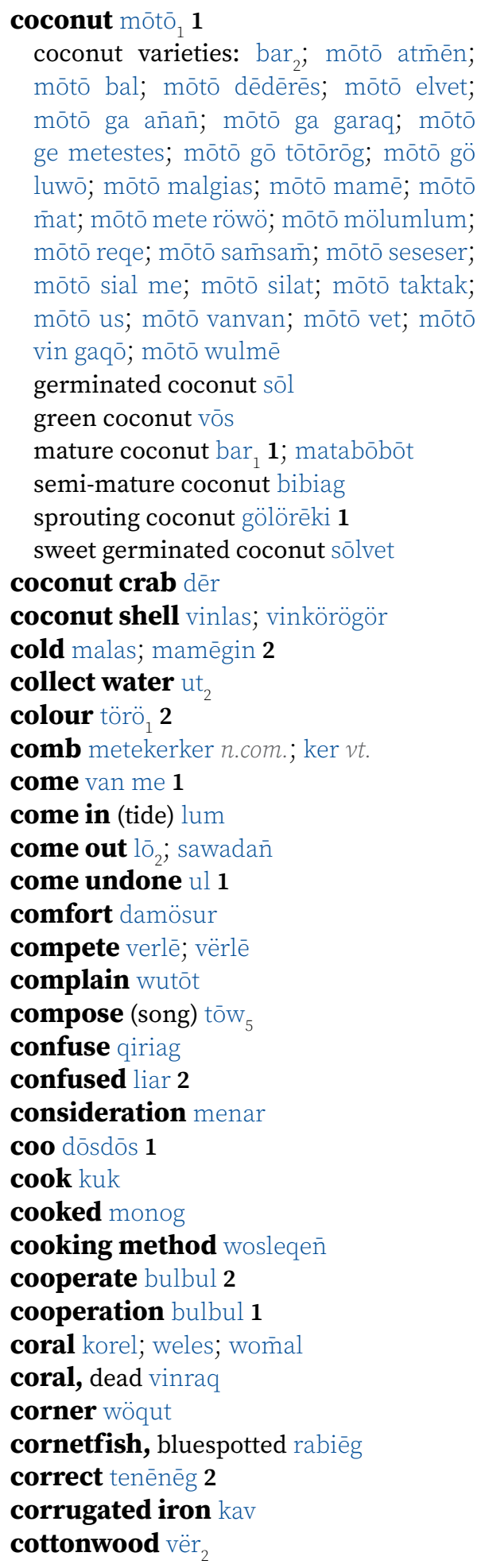

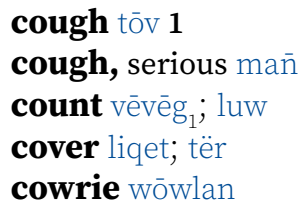




\section{$\mathbf{D} \sim \mathbf{d}$}

dab tōm
Dad Mam $\mathbf{1}$
damage da titisē
damselfish weñemërës; wēduduw
dance lak vi.; laklak n.com.
$\quad$ types of dance: eln̄etiti; liañ; mè; rësrës
$\quad$ den̄e; siwia
dark sil

day qön̄ $\mathbf{2}$; meren $\mathbf{1}$

become day meren 3

daylight meren $\mathbf{2}$

dead (be) miat

deaf qōrwōn

death matē 1

her/his/its death matian

my death matiēk

deep lam

depart ukuëg 1

descent group sögö

her/his descent group sogon

my descent group sögök

desire mörös 3

determined meneg 4

dew mëw

diarrhoea kirkir

die miat

different sisia

difficult mav 2

dig kev; gil 1

dig out gōqleg

digit busu 1

dinner, eat gengen revrev

dirty qōlō 1

discuss vövötös; vëtës

disobey liseg

distribute

ditch, small dōn

dive $q \bar{o} t$

dive down el

divide vēlēt; vēl ${ }_{3}$

do something vo 2

do what da gese dog genqirēg; tok

doll dole

dolphin $k \bar{e}_{1}$

don't kere; mawi; nitog

don't know wēsi

door mete gövur

dove

emerald dove mereg

fruit dove men dōsdōs; malqon

small Vanuatu fruit dove bëlbëlvōlōn

down $\operatorname{sur}_{3}$

downward sōw 2

dragonfly $\mathrm{wō}^{\mathrm{O}}{ }_{2}$

draw törö 1

dream qōrqōr

driftwood wön̄ial

drill wes

drink sum

drink kava wan 1

drip tir

drop gis tës

drown tul 1

drowsiness matmuturtur

drum timiatwos

drummer, long-finned rak

dry meren $_{2}$; rañ $\overline{1}_{1}$; mamas

dry (in sun) wör

dry up tërëkrëk

dugong qōlo

dull qōn̄ov

dust usu tan; tan vōvō 


\section{$\mathbf{E} \sim \mathbf{e}$}

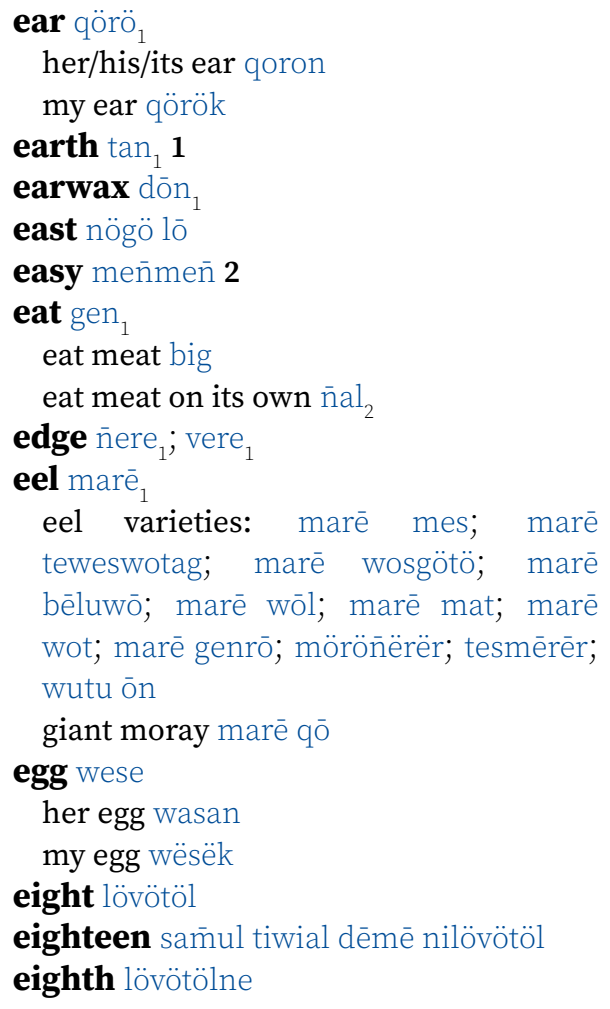

eyebrows burus mete

eyelashes burus mete

\section{$\mathbf{F} \sim \mathbf{f}$}

face nögö 1

her/his/its face nogon

my face nögök

face down vateqev

face upwards atelen

falcon, peregrine mal biliag

fall mēs

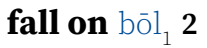

famine mar

fan riv $_{2} v t$.; dērivriv n.com.

fantail tēgēer

far aqit; arës

fart kir

fart, quietly vun

fascia tërtërgōr fast (no eating) gargarmamas

fast (quick) lelev

fat marak

father mam $\mathbf{1}$; teme her/his/its father taman my father tëmëk

fault bugë her/his/its fault bōgōn my fault bugëk

feast qetgengen

feather vul 2 chicken feather vulu tō

feed bien 1; bōgereg; ven̄en

feel rōñteg

female reqe $\mathbf{2}$

fence $\mathrm{ar}_{2}$ 


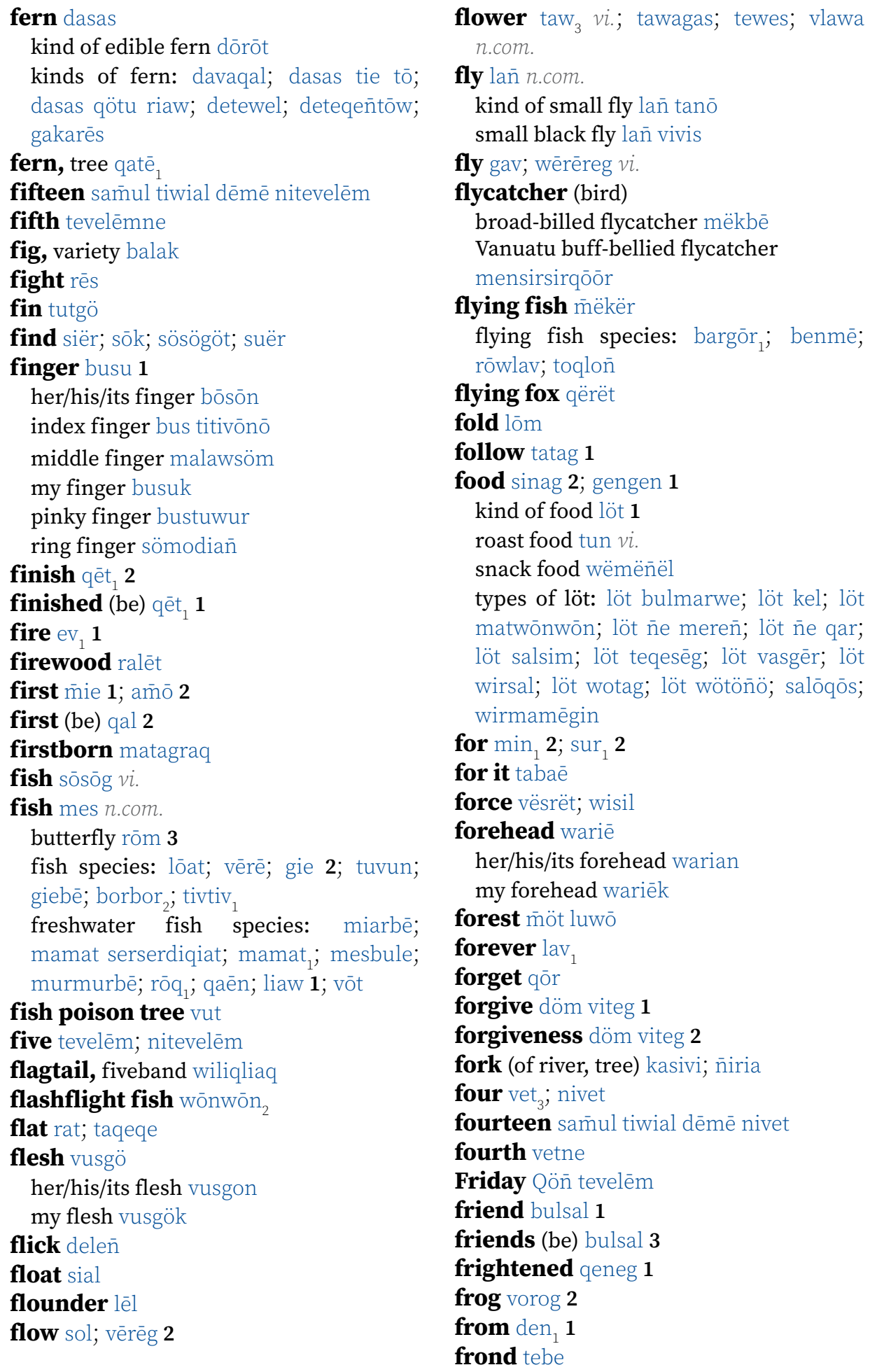


front nögö 2; qötu 3

fruit wiē

its fruit wian 2

fruit dove

fruit dove species wiria; wiria tabē

Vanuatu fruit dove sōravar fruit fly wörumrum

full wōr ${ }_{2}$

funeral matē $\mathbf{2}$

fusilier (fish) meswelwel

\section{$\mathbf{G} \sim \mathbf{g}$}

\section{game oror $\mathbf{2}$ \\ garden n̄en̄ere; tiqē \\ garden}

dry taro garden biriat

old garden wewestel

taro garden rot; vaslē; wōsōk

taro, planted in mud bōak $\mathbf{2}$

water taro garden mat

garfish qagar

gee! auw

generous mētigwē

generous (be) sōgsōg

genitals lösö

her/his genitals loson

my genitals lösök

get $l e_{1} 2$

get people sodēmēèl

get taro sigsig

gill (of fish) wawawn̄e

ginger sinsa

red flowering ginger wedeldel

ginger species gatavdor

give $l e_{1} \mathbf{3}$

give birth vösus; vawot

glass klas

glow mushroom widiar $\mathbf{1}$

gnaw n̄ōr

go $\operatorname{van}_{1} \mathbf{1} ; \operatorname{var}_{1} \mathbf{2}$

go around waliög $\mathbf{2}$

go ashore siōl

go down tēqēl $\mathbf{1}$; sōw $\mathbf{3}$

go downstream tēqēl $\mathbf{3}$

go home mōl 1

go inside $\mathrm{kal}_{2} \mathbf{5}$

go landward kal 22

go northwest tēēèl 4 go outside kalō

go seaward tēqēl 2

go southeast $\mathrm{kal}_{2} 4$

go through vesel

go up $\mathrm{kal}_{2} \mathbf{1}$

go upstream $\mathrm{kal}_{2} 3$

goatfish $\bar{m}$ as

dash-dot goatfish mas teñten

goldsaddle goatfish mas añan

yellowstripe goatfish mas sarsar

God Mam 2

good wē 1; gōwē

grab tiqër; sëwëw

grade-taking ceremony kōlkōl

grandchild Bum; Pōpō; tubu $\mathbf{1}$

her/his grandchild tōbōn

my grandchild tubuk

Grandma Diebum; Pōpō

Grandpa Mambum; Pōpō

grandparent tubu $\mathbf{1}$

her/his grandparent tōbōn

my grandparent tubuk

great-grandparent Bum tal

great-great-grandparent Bum vövörus

grass vēlis

grass species ditial ${ }_{2}$ mōōōt ; vēlis ōlōl;

wētēvtēv

grass skirt ber $_{1} \mathbf{1}$

wear grass skirt ber 2

grasshopper wēviak

grate ras 1

grate coconut geres; gör

grater via qatē; via

grave qër

great! dom

greedy survun 


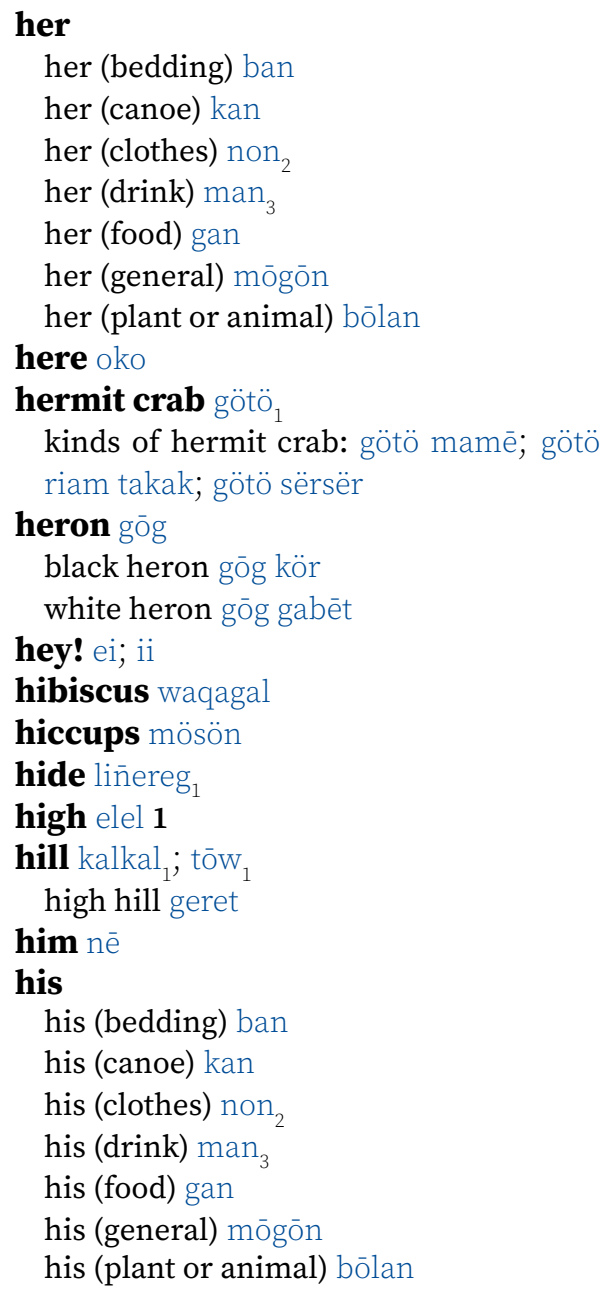

hit töwös; lā̄

hogfish mestēv

hold garveg; gis 1

hold up seben

hole lie; qaran̄; qarörö

hollow out ber 2

holothurian wētēltēliriaw

holy gōgōn

honeyeater

cardinal honeyeater (female) wētēsisis cardinal honeyeater (male) qötqötumen dark-brown honeyeater taw Vanuatu mountain honeyeater wōkōl

hook go $_{1}$

horn (of cattle) ser $_{2}$

hot tutun $\mathbf{1}$

hour $l \bar{o}_{1} 3$

house gövur

men's house gemel

how timiak avē $\mathbf{1}$

how many nivēs 1

hundred möldöl

hungry mēlin̄sal

hunt sōksōk; tēqatēq 1

hunt with light tut ${ }_{2}$

hurricane len̄vus

hurry gamlöt $\mathbf{2}$

hurt mëmës vi.

husk vinti $\mathbf{3}$

\section{$\mathbf{I} \sim \mathbf{i}$}

I $\mathrm{no}_{1}$
if $\mathrm{si}_{1}$
image tōtgial $\mathbf{1}$
important elel $\mathbf{2}$
in-laws
$\quad$ brother-in-law (of man) Wölus
$\quad$ child-in-law Wim̄iar
$\quad$ parent-in-law Qëlgël
sister-in-law (of woman) Wölus
incubator bird molo
individually masansan

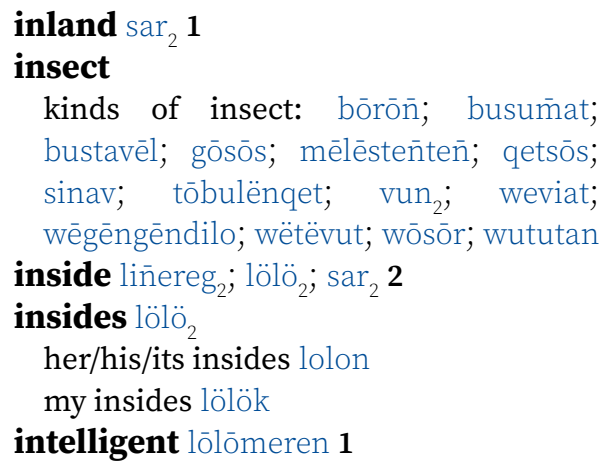




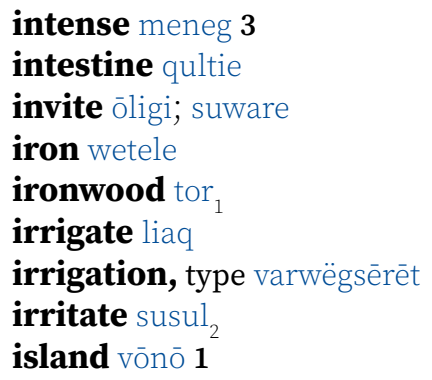

it nē

its

its (bedding) ban

its (canoe) kan

its (clothes) non 2 its (drink) man $_{3}$ its (food) gan its (general) mōgōn

its (plant or animal) bōlan

\section{$\mathbf{J} \sim \mathbf{j}$}

jaw balsiē

her/his/its jaw balsian

my jaw balsiēk

jealous gilal $\mathbf{3}$

jellyfish silat $\mathbf{2}$

join sö $\mathbf{1}$; wōn 1 1; bak $_{2}$ join side qeleg

joint $\mathrm{sö} \mathbf{2}$; mönug

joke, make sön̄sön

jump rōw

just gem $\mathbf{1}$; kara

\section{$\mathbf{K} \sim \mathbf{k}$}

\section{kava gē 1}

kava varieties: lakakēris ${ }_{2}$; wistaban

kava, wild dōmōtōtak

kill vus,

kind tarn̄i

kingfisher sēg

Pacific kingfisher sēg malgias

sacred kingfisher sēg mamē

kiss bōbōn

\author{
knee qöu \\ her/his/its knee qōōn \\ my knee qöuk \\ knife gasel \\ big knife gasel luwō \\ small knife gasel nötu \\ wooden knife meteges \\ knot, tie dutgōr vt. \\ know gilal $\mathbf{1}$ \\ knowledge gilal 2
}

\section{$\mathbf{L} \sim 1$}

lake tes

land $\tan _{1} 3$

language qaq 3

our language qaq ta minēn

lantern tree birbir

laplap laplap; lōk

kinds of laplap: biliagtat; lör; wewe;

wir; wōrkelkel

last qētqētne; basbasne laugh bor

laugh at borseg

laughter borbor

launch vasēlē

law $l \bar{e}_{2}$

lay flat tētēr

lazy meren $_{1}$; mōr

lead turmōō; gan̄veg

leader mie $\mathbf{3}$; qetegak 


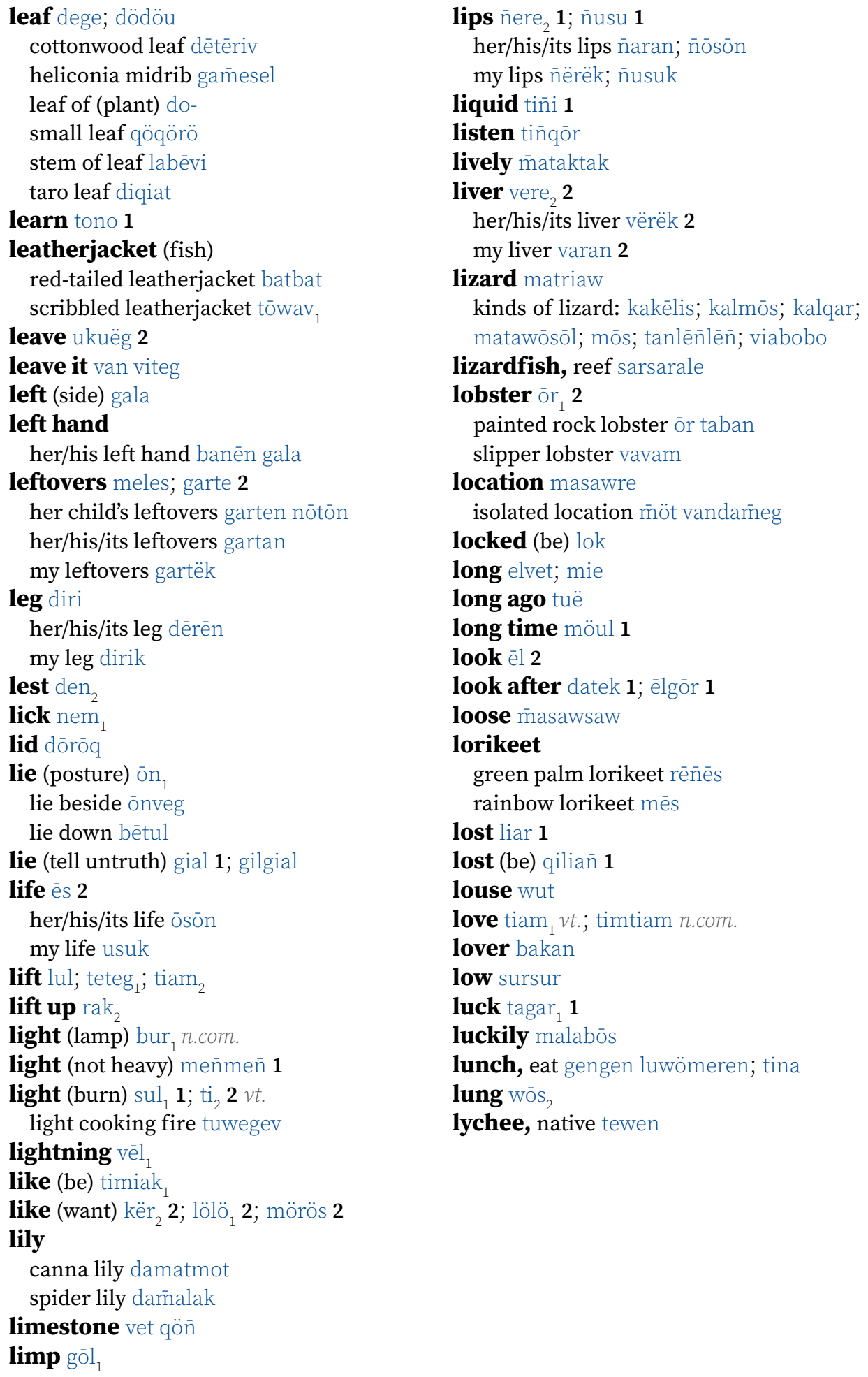




\section{$\mathbf{M} \sim \mathbf{m}$}

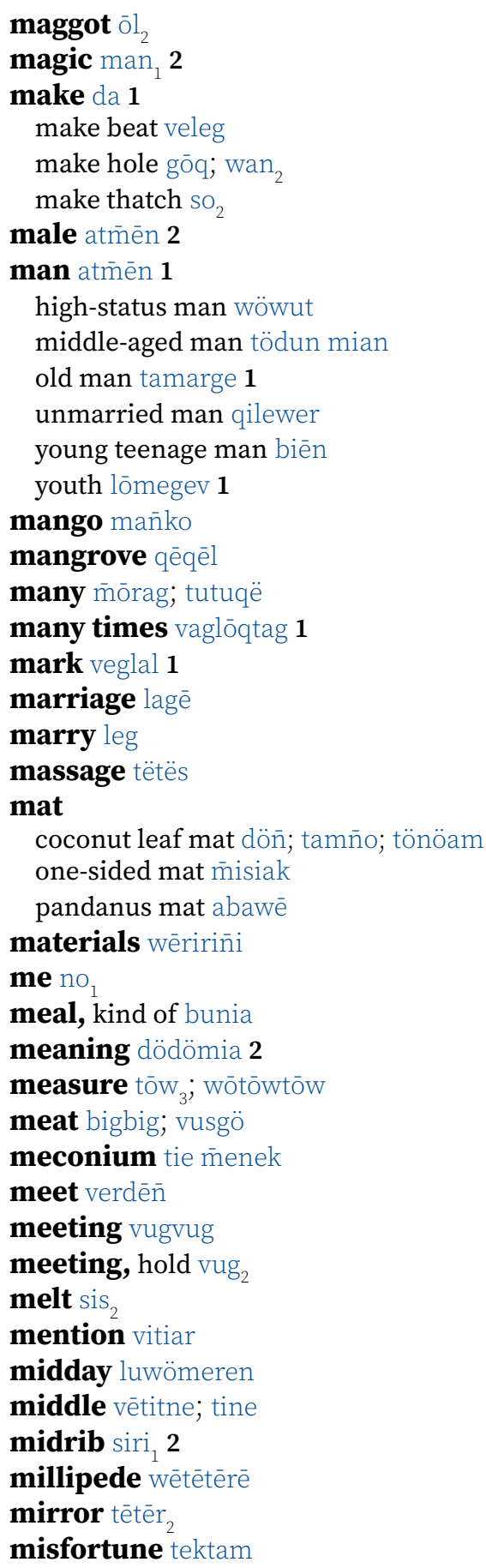

miss tokorës

missionary misonari

mist mërëv

mistake, make av

mistakenly tës

mix lër

moiety sögö

her/his/its sogon

my moiety sögök

Monday Made

money selen $\mathbf{2}$; wadē; söm $\mathbf{1}$; tiveg $\mathbf{1}$

money, shell söm $\mathbf{2}$

month wōl 1 2

month (of pregnancy), her Xth month wōlan my Xth month wulëk

\section{moon wōl 1 \\ moray}

black-blotched moray vusö

hookjaw moray val

painted moray vusö ōn

white-margined moray göwur

morinda wōlōm

morning vōrōg 1 n.com.

be morning vōrōg $\mathbf{2} v i$.

early morning lölön̄ö vōrōg

mosquito nem,

kinds of mosquito: nem biliag; nem

talöqön

moss lumlum 2

moth, kind of weverbōn

mother die $\mathbf{1}$; rētne her/his/its mother rētnan

my mother rētnëk

motorbike susuki

mouth vele

her/his/its mouth valan

my mouth vëlëk

move mögus

mud bōak 1

mulberry, native dimia

mullet (fish) gomot

Mum Die

mushroom, glowing widiar $\mathbf{1}$ 


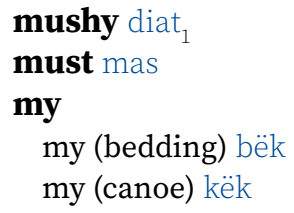

my (clothes) nök

my (drink) mëk

my (food) gëk

my (general) möguk

my (plant or animal) bulëk

\section{$\mathbf{N} \sim \mathbf{n}$}

nail (body part) wulë busu

nail (wooden) busmaraw

name (call) tōwereg

name sie n.com.

her/his/its name sian

my name siëk

narrow kokos 1

nausea lōlm̄usugsug

nautilus wētēv

navel butö

her/his/its navel buton

my navel butök

neck bibiē

her/his/its neck bibian

my neck bibiēk

neck, back of wōqōlqōl

necklace wetenge; wevet; wērērēn̄ëg

need mörös 1 ; lölö 1 ; kër ${ }_{2} 1$

needle wuduw

needle (plant) wēsiri

needlefish ut $_{1}$; til

nephew vann̄ö

her/his/its nephew vann̄on

my nephew vann̄ök

nerite (shellfish) wētēwil

nerite species: wētēwil bēt; wētēwil kör;

wētēwil mëv; wētēwil serōn

nest tötöu n.com. net giam $\mathbf{1}$

nettle tree silat 1

nettle tree variety silat gegeretō

new garaq

news rörö

nice lulum 2

nicolaia (plant) sör

niece vann̄ö

her/his/its niece vann̄on

my niece vann̄ök

night qön̄ 1 n.com.

be night qön $\mathbf{3} v i$.

nine lövövet

nineteen sam̄ul tiwial dēmē nilövövet

ninth lövövetne

nit (louse egg) lēs

no odiañ 1; ōōō

nod qet möwu

noise qōrōtōt 2; rarö

make noise wölul; dordor

pigeon noise tatörur

nose mödu

her/his/its nose mōdōn

my nose möduk

now in̄ko,

nut, bush wotag

nutmeg, wild darag

\section{O o}

octopus wērēt

kind of octopus on

oesophagus vötudōldōl

of den 4 4; taē; talo

offspring nötu often vaga 2

oh $\circ \circ$

oil, coconut bes $\mathbf{1}$

ok ale $_{2}$

old m̄usu 

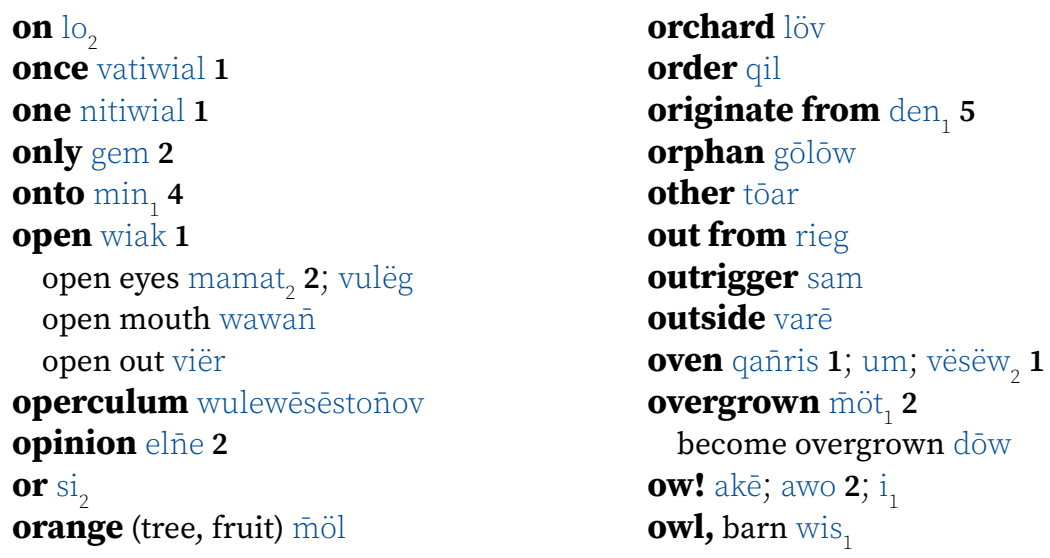

$\mathbf{P} \sim \mathbf{p}$

paddle sō vi.; wōs, n.com.

palm

coconut palm tur mōtō

palm varieties: bar $_{3}$; mōtōvōl

palolo (sea worm) un

palolo, female un lam

palolo, male un gal

pancreas wörurus tavav

pandanus vudege

dried pandanus dēmērir

dried pandanus leaf damarēn̄it

pandanus varieties: dērvag; $\operatorname{van}_{2}$

wild pandanus species: dootnönqet;

dökurut

papaya woman

parcel wöwös $\mathbf{2}$; mon $\mathbf{2}$

parent-in-law qelge

her/his parent-in-law qelgan

my parent-in-law qëlgëk

\section{parrotfish}

immature parrotfish demeles

large parrotfish sōm

parrotfish species: dēvēlavēl; tōr ${ }_{2}$

part taklē $\mathbf{2}$

partner bērn̄ē

her/his/its partner bērn̄ēn

my partner bērn̄ik

pass over levga

passionflower, stinking waga 2

\author{
passionfruit waga $_{2} 1$ \\ pat serev \\ path gersal; gersele \\ her/his/its path gersalan \\ my path gersëlëk
}

pawpaw woman

pay wöl 2; tiveg 2

pay back ser ${ }_{3}$

peace tamat

peekaboo qate $_{3}$

peel sēm, 1 ; wul

peel off er $r_{1}$; gag

peg bin

pelt vēvēr

penis qere

his/its penis qaran

my penis qërëk

people rege; $\mathrm{sul}_{2}$

person tañsar $\mathbf{1}$

old person tamarge $\mathbf{2}$

poor person masar

quiet person wömum

unfortunate person mōdō

unmarried person qilewer

unnameable person sian gōgōn

white person wēsin̄asin̄

personality metwu

her/his personality metwōn

my personality metwuk 
petiole qere

pheasant-dove, rufous-brown tōwav ${ }_{2}$

phosphorescence widiar $\mathbf{2}$

pick bis

pick up bet; gis rak; vil ${ }_{1}$

piece manrē; masrē; seve; vutrë

pierce es 2

pig $\mathrm{q} \overline{ }_{1}$

pig, intersex riaw $\mathbf{2}$

pigeon $q \circ n$

pigeon species: qon bulënqet; qon bulënrēlē; qon tan̄sar; qon wēbèt; tërëw; tutwō; tutrevak

white-throated pigeon tōrō

white-throated pigeon (male) tōrō butbut

pile up vëtur

pillow qetōlōn

pinch gin 2

pineapple $\operatorname{van}_{3}$

pitiful mamarseg $\mathbf{2}$

place sara; masawre 2; tönö; vene; vōnō

her/his/its place vanan

my place vënëk

place names: Bek; Bēut 1; Bōkrat; Koimaram; Laln̄etak; Laln̄evut; Lesa; Lēsē; Lion; Liwōn; Mere; Metir; Mēēēn; Namnog; Nam̄asmas; Nereqe; Neretan; Periōt; Qötuwis; Rekel; Rev; Sanleñ; Sasar; Selwō; Serewan; Sisiōl; Sol; Suvren; Tores; Tetgen; Vanlis; Vatov; Vetmōl; Vetrat; Vurës; Wasag; Wēsilat; Wētika; Wētikō

placenta be tētē $\mathbf{1}$; ben rōrō

plait vir

plan lugög

plane ak gavgav; plen

plant riv ${ }_{1} v t$.; rivriv n.com.

plant taro sōwereg; vas

plant species: busuqaēn; damataq; mēlēslēs ${ }_{2} ; \quad$ dunremēèlēg; dēnin; diwinwian; lēnötōo; wēnēn bulëvu; wesev; wulëwalal; dobormiak; dötöqötun; nisnias; wam̄ā̄as; waran̄ran̄ mamē; wolkoko; dövuln̄ö; dōmōtōtak mamē; do qōrgavgav ${ }_{2}$; lösönqō; wōlōlōmeren; mēvinvian dabalak; qōo; tartarawöw; waran̄rañ ga qag; do qōrgavgav

plantation plantesen

platform wēqē

platter, wooden tabē

play or; oror 1

plenty wuw 1

plenty (be) lōqtag

pluck sev 1

plum (tree, fruit)

dragon plum woro

great hog plum ur

plus dēmē

point ñusu 3 ; n̄ere 2

poisonous gōgōn ${ }_{1} 4$

poisonwood les

poke sus $_{1} \mathbf{1}$; sulör

pool qulös

porcupinefish terit

porcupinefish species: ganasēg; terit lam

posset lōt

post (house) sañ

central post sañ tine

poumuli (tree) mōmōo

pound $q \bar{o} \mathrm{~s}_{1} \mathbf{1}$

pour $\operatorname{lin}$

pour water in oven vun

prawn ${ }^{\circ} r_{1} 1$

freshwater prawn species: ōr bëlbël;

qaqet

pray tatar 1

prayer tatar 2

prayer hole qereñ ōlōl

preach tōrtōr $\mathbf{1}$

pregnant (be) tel ${ }_{1}$; wotoqtoq $_{1} \mathbf{1}$; tek na

taqan

prepare da taōrmat; taōrmat $\mathbf{2}$; vasgēr

present tabēva

pretend malmalē

prevent tiargōr

probably wun

progress tōtōtōw

prohibit vēlig

promise vatvat 1

properly wareg 2 ; wurveg $_{2}$ 


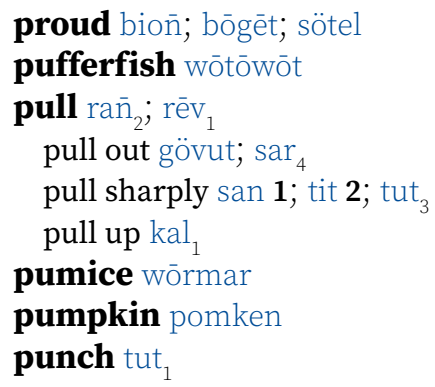

\section{$\mathbf{Q} \sim \mathbf{q}$}

queenfish barnaw

question vörus völu

quick (be) gamlöt 2 quickly gamlöt $\mathbf{1} ; \bar{m}^{\circ}{ }_{2}$ quiet (be) tarōn

quietly rōrōn 2

$\mathbf{R} \sim \mathbf{r}$

rabbitfish tömus

rabbitfish species: tömus kör; tömus añ;

tömus bul kör

rafter, bamboo wōrōqrōq

rail, buff-banded (bird) biliag

rain $\mathrm{re}_{2} ;$ ten $_{3}$; wian

very heavy rain deli

rainbow butöliaw

randomly valakreg

rank ronog

rat gōsōw

rattan gawol

raw qar 2

ray vër

reach dēñ 2

read vasōgō; rid

ready taōrmat $\mathbf{1}$

real turgi

reason manene

recognise $\mathrm{rak}_{3}$; èl rak

record bël 2

red mamē 1

red silkwood nët

reduce sōw 4

reed, kind of wōtōg

reef met; sëkër refuse gögörö

release ukuëg 3

remedy, plant tenge

remember döm kēl

remove le den; sev $\mathbf{2}$

remove insides bēs

remove midrib vidir $\mathbf{1}$

remove strip (pandanus) dērēs

repeat lulu

reply vëmlösal

respect döm mav

rest memseg 2

resuscitate da ês; vaēs

return kēl 1

revive van̄

rib(s) lele

her/his/its rib(s) lalan

my rib(s) lëlëk

right (entitlement) man $_{2}$

right (side) mōtō $_{2}$

right hand

her/his/its banēn mōtō

rigor mortis tētērē

ring qulön

ringworm vun $_{5}$ 

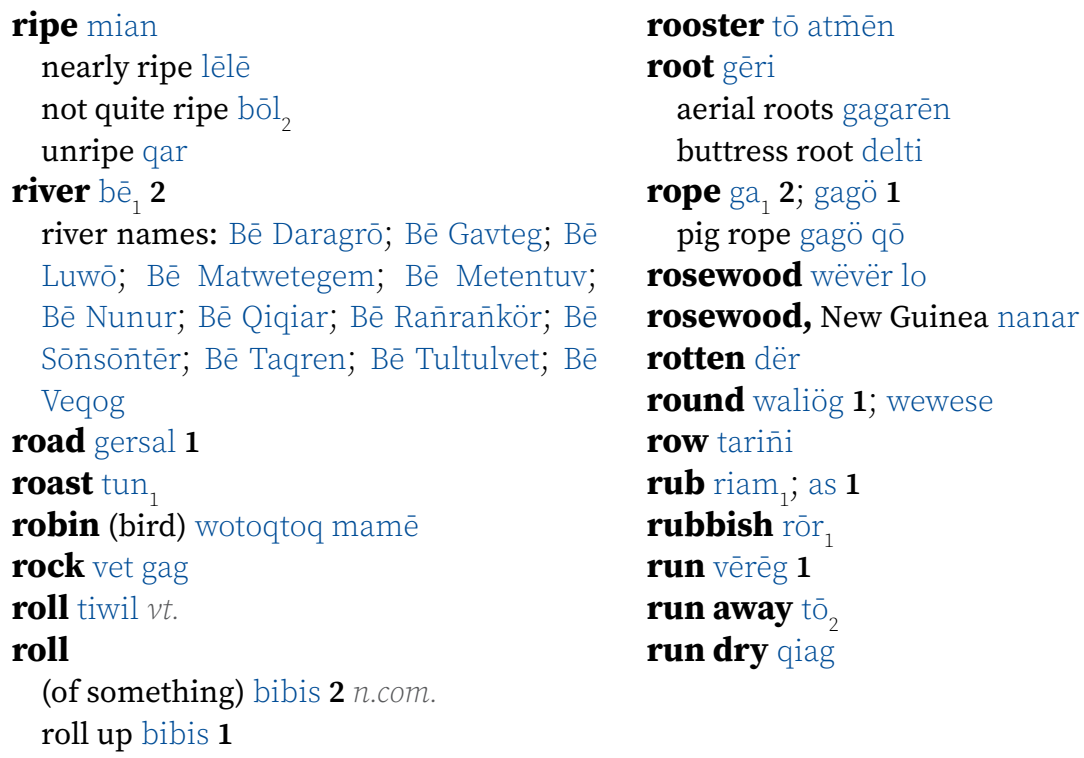

\section{$\mathbf{S} \sim \mathbf{S}$}

sad lōlwōn

sago palm doot 1

sail geben

sailfin tang (fish) tivtivnönqet

sailfish, Indo-Pacific mesgeben

saliva dinsöw $\mathbf{1}$

salt naw 4

salt water naw 2

salty gōgōn 2

same sasarēt 2

sand $\bar{o} n_{2} 1$

sandalwood sentawud

Santo Santo

sap tin̄i 2; ñalte

canarium sap bur ñe

sated (be) sis

Saturday Sarere

saucepan sospen

say $\mathrm{WO}_{2} ;$ qaq $\mathbf{1}$; vita $_{2}$

scabies gerger

scad, bigeye (fish) röwö

scar wēbēlēt

large scar wegemgem

school skul 1 scissors balbal 1

scorpionfish wētēvut

scrape $\operatorname{sir} \mathbf{1}$

scraps garat

scratch kër ${ }_{1}$; keres; rërës

scrub as 2

sea naw 1

calm sea $\operatorname{tar}_{2} 1$

deep sea lam

very deep sea butölam

sea fan ërlam

sea snake $\bar{m} a t$ tan tes; $\bar{m} e \mathbf{1}$

sea urchin wadas

sea urchin species sernönqet

seaside $l_{0}$

season to 2

seat tönö sigsiag

seaweed lumlum $\mathbf{1}$

seaweed variety lōw

seaworm

seaworm species: un; wotaqlislis;

wömönmönösot

secret maw $_{2}$

see $\mathrm{e} l 1$ 


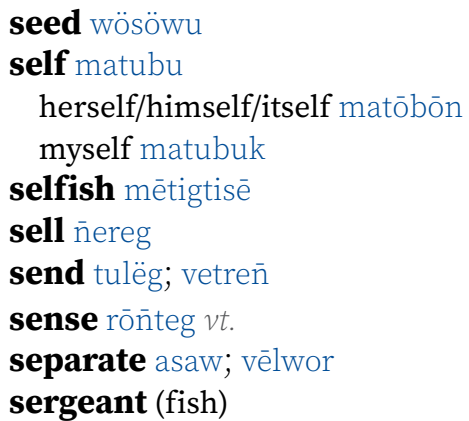

blackspot sergeant tōqagat

Indo-Pacific sergeant dananar seven-bar sergeant wōtōqagat

serve food lik

set (sun) tul 3

set trap wödön 2

seven lōvōrō

seventeen sam̄ul tiwial dēmē nilōvōrō

seventh lōvōrōne

sew susur

sew thatch vēn

sex, have bēl

shade mëlmël $\mathbf{1}$

shadow tamtiē

her/his/its shadow tamtian

my shadow tamtiēk

shake gōl ${ }_{2} v i$; kuëg vt.

shallow raras

share out gasēg

shark bōgō $_{1}$

blue shark bōgō dēm̄ēl

leopard shark bōgō n̄ōrn̄ōr

sandbar shark bōgō mes

sharp (blade) gegen

sharp (point) metestes

sharpen ras 2

shave $\operatorname{sir} \mathbf{3}$; sesev

she nē

shellfish weger

conch shell tōw cone shell wōgōlgōl

drupe shell wutumet

large freshwater shellfish gunögōsōw olive shell wēsēm shellfish species: matmamē; qōbetge; waakak; wamatwōn; watagōgōn; watawlētlēt; weger mel; weger sōl; weger ta; weger tur ; weqet; weswesak; weterōw; wēgim; wēsēs bē; wēsēs biēg; wēsēs man; wēsēs malau; wēsēs nōnōm; wēsēs tan̄ov; wēsēs vet; wēsēs wōmalak; wētēqēdur; woswos; wōrōo ${ }_{1}$ wölubus; wöqörö; wörönötöwö; wösöm

top shell wösusumalmal

shine sēlsēl

ship ak; bakalav

shirt sot

shit tia n.com.; titiav vi.

her/his/its shit tian

my shit tiēk

shock nian

shoot vēn ${ }_{1} v t$

shoot (with gun) tē $q_{2}$

shoot (new growth) ñili n.com.

coconut shoot n̄ēnēn $\mathbf{2}$

young shoot qöqö

shopping wölwöl

shore bōrōt

short qëtutu 1

shorts traosis 2

shout aw

show varēg

shower (rain) ninin

shrikebill, southern wase ${ }_{1}$

shrub

shrub species: bēkil; bormiak; busuqërët; damalalal; datā̄al; datam̄al añ; datamiar; datartarawöw; datawalwal; davarteker; deben tenēnēg; degetget; demen mav; dëmël; dimililial; ditial ; gōglo; qōmur; tutugēn tamarge; wösusurbē; vōnōdöwö; waran̄ran̄; wōvōnō; wōvōt

sibling, older same-sex tōge her/his tōgan my tōgëk

sibling, opposite sex tutue her/his tōtōan my tutuëk

sibling, younger same-sex tēsi her/his tasēn my tēsik 


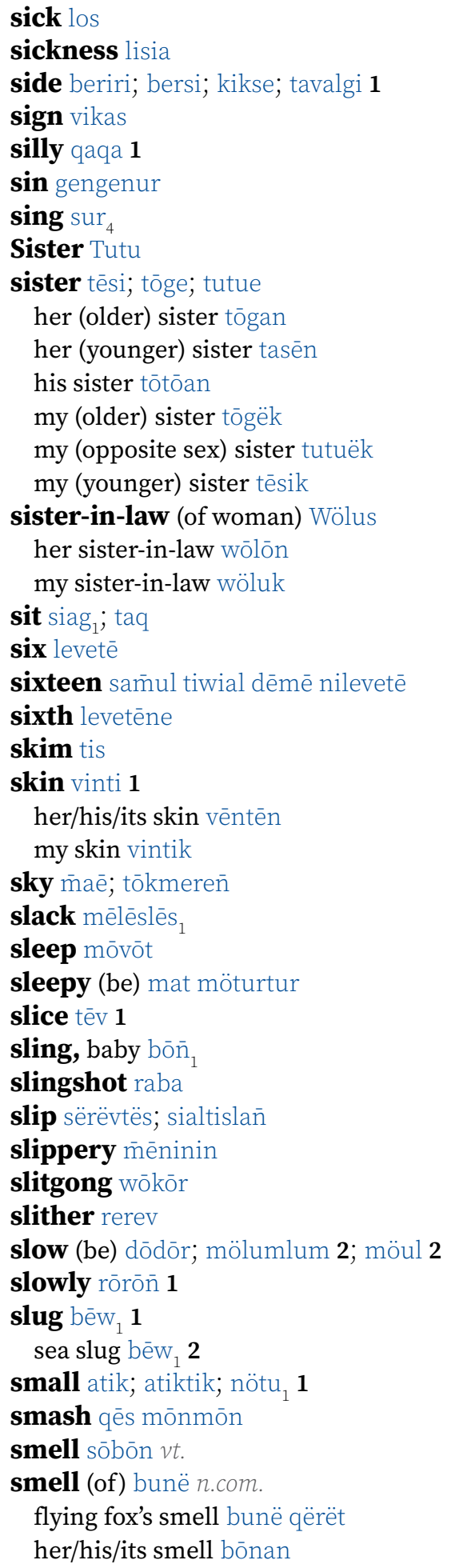

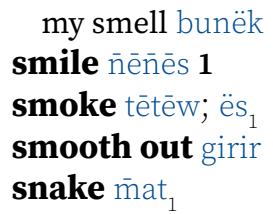




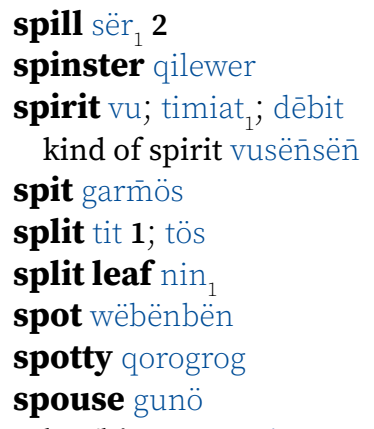

stick tutgëg $v t$.

carrying stick wēt

walking stick tutugon

stick out wutwut

stick to kat

still vēti

stingray vër

stingray species: vër dudut; vër mal; vër

matawasē; vër timiat

stink bōn vi.

stinkwood qōmiat

stir kel

stone vet $_{1}$ large stone mērēn

stone oven um

kind of stone oven um val

stonefish magte $_{1}$

story kakaka $\mathbf{3}$

traditional story eln̄evu

straight tenēnēg $\mathbf{1}$; wasren

straighten tun tenēnēg

strain sēn

strainer dinir; sēnsēn

stranger tëw

strap gagö 4; wëlëklëk

stream nötu bē

string gagö 2

strip out vetnia

strong lala; meneg $\mathbf{2}$; tor ${ }_{2}$

stubborn qetvet

stuck $\bar{m}$ ōō

stupidly qaqa 3

submerge tul 2

suck dim

sucker (banana) sili

sucker (octopus) wobalaklak

suckle sus $_{4} 2$

sugarcane töv

sugarcane variety dañdan̄

sun $\mathrm{lō}_{1} \mathbf{1}$

Sunday Sade

support vegsañ

sure dun 2

surf bēm naw

surface beret $_{2}$; n̄ēlida

surgeonfish

convict surgeonfish wēqiar

eyestripe surgeonfish vag mël 
larger surgeonfish vag orangeband surgeonfish törö smaller surgeonfish qōl striped surgeonfish beraw yellowfin surgeonfish vag mēel surprise $\bar{m}$ alan; $\bar{m}$ amal; vasas surprised (be) mamakē; malrōw surround kōk

swallow dōl

swamphen $\bar{m}$ atēk

swear $\mathrm{q}_{4}$

sweat memesgèt

sweep wur

sweep away gogoreg sweeper, copper (fish) mesteveltiwial; wetevel

sweet potato kōmar

sweet potato varieties: ga dōvōr ${ }_{2}$; pawa; spidbōt; tövutwen; tri manis; vorog $\mathbf{1}$; wese tō

sweetlips (fish)

black sweetlips benmomolo

oriental sweetlips gōrgōrbiliag

swell nen

swiftlet (bird) bagbaglō

swim gër $\mathbf{1}$; suvsuv

swing gakōkō n.com.; kōkō vi.

\section{$\mathbf{T} \sim \mathbf{t}$}

table tebol

tail gölö

her/his/its tail golon

my tail gölök

take tek $\mathbf{4}$; le 1

take notice nak

take off sër lō

take photo tōtgial 2

take place (of) lies $\mathbf{2}$

take rank kōl

talk about kakaka 2

tamanu, false wēgēbil 1

tame $\overline{m o} \bar{m} \mathrm{O}$

$\operatorname{tap}$ wōw $_{2} v t$.

taro sinag $\mathbf{1}$; qiat

giant taro ve $\bar{e}_{1}$

taro area tin̄

taro groupings: qiat atmēn; qiat reqe

taro paddy qēl

taro varieties: titiritōwvetam; wotmindeli; vinmötöl; ërmamē; rēgēt; wotliev; tewesqēqēl; mōvōl; burmatan; lakakēris; ${ }_{3}$ merlav; wotminmendala; qötuqō ${ }_{2}$; nōvōk; römöwuler; varvarsōm; wēviti; mesvōlōvōl; wasalav; qiat gōl; qiatlēlē; wotminwērirtel; lantar malgias; wederebiliag; lomonwer; wegeretqon; galaten̄tur; rēlēgtēl; taltal; siritimiat; biliag; wamal; wasanto; mensēkē; malmalei lantar; mōlkēl; qiat mingala; tan̄evsōs; tewesvër; wotkērēvōr; marēe qiat qet; mako; sēstan̄; wēvē; wakata qagqag; qiat rev; wotminviolette; manreva; wakata mamē; tewestamal; m̄ēw; ōrbarbar; wēbigqō; din̄venqiat; tana; wēvē lamkör; wotlievgatgat; mesmamēe ${ }_{2}$ wotmēlēv; qiat qön̄; rēsim; rēwurveg; mēvinvian ${ }_{2}$; wēlēbēōr; wotvadadan̄; akrikalja; len̄man; wasanto qagqag; wotlalabēvidavaqal; $\mathrm{rov}_{2} ;$ qiat minwog; marē wasalav mamē; wakata gatgat; wakata; nalumlum; masta; wotminsakna; wasēe $_{2} ;$ marē wasalav; wēmēèlēglēg; lantar lamkör; regeltëv; ten̄tur; busuōr; siag gēgēt ${ }_{2}$; tortor; bulalēv; wotanaval; matēkmëgërsurletes; lōkreg; lantar; waras; sarē; lōkreg mamē; rōvōl; wotalēbak; susdenlē; sēlēgtel; dogon; suwbē; böl; wēbigqō mamē; wasanto mamē; götö

taste tes

taste (of) lulumne

taste good dēdērēs; lulum $\mathbf{1}$

tattler, wandering (bird) tēwē biliag 


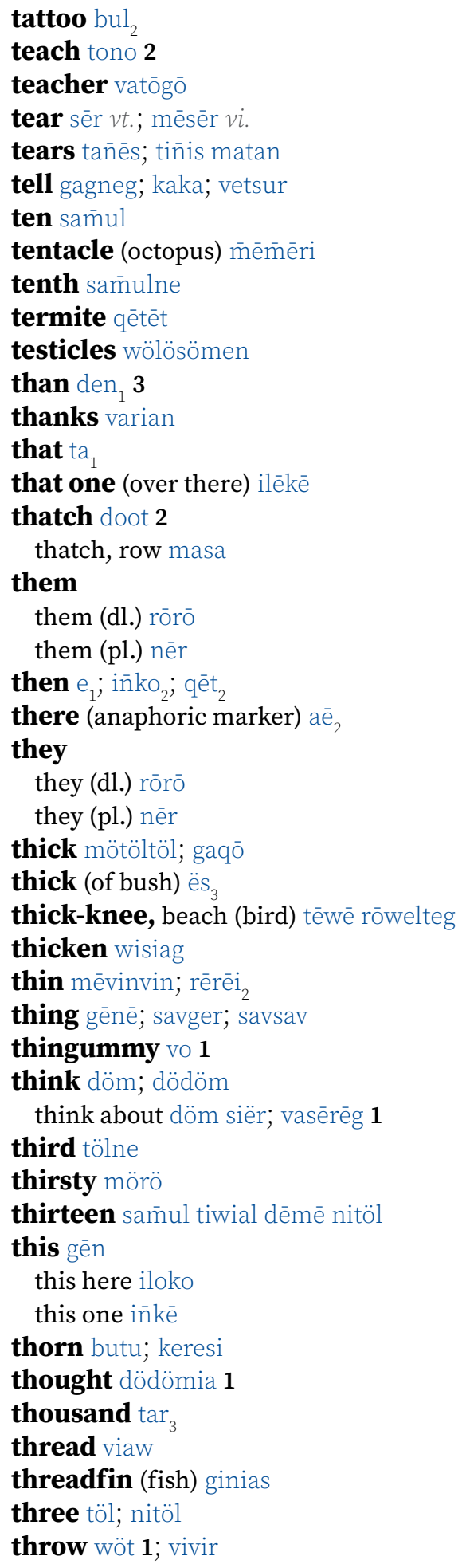

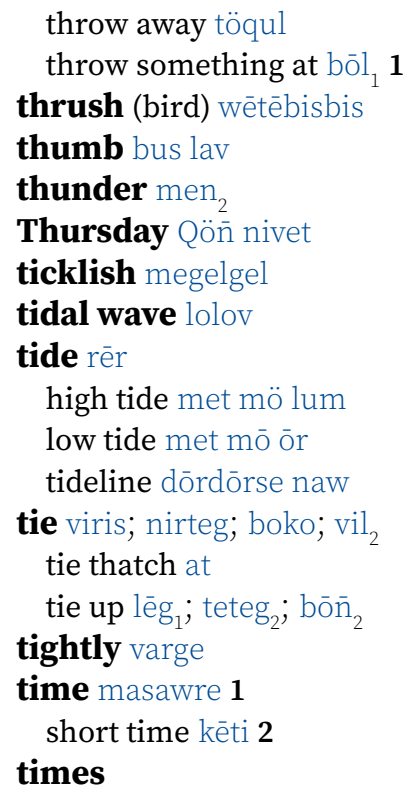

(do) eight times vaglövötöl

(do) five times vagtevelēm

(do) four times vagvet

(do) hundred times vagm̄öldöl

(do) many times vagwuw

(do) nine times vaglövövet

(do) seven times vaglōvōrō

(do) six times vaglevetē

(do) ten times vagsamul

(do) thousand times vagtar

(do) three times vagtöl

(do) two times vagrō

tingle malala

tired (be) n̄al ${ }_{1}$; iamron

to $\min _{1} \mathbf{1} ; \mathrm{lo}_{2} ; \mathrm{a}_{1}$

today garqe $\mathbf{1}$

toe busu 1

her/his/its toe bōsōn

my toe busuk

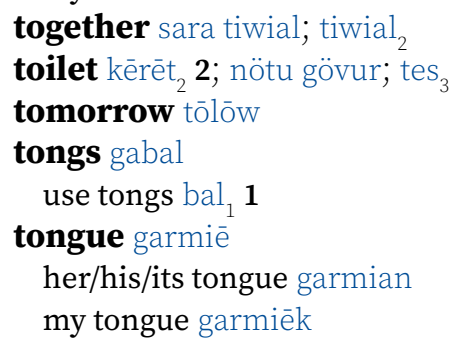

too bilēn̄ 2 
too much nimal; idian

tooth luwö 1

her/his/its tooth luwon

my tooth luwök

toothless luwö bas

top mēēē; qese; qötu 2; teqen̄; wötu

top shell walal

Torba province Torba

touch gis ${ }_{1} \mathbf{2}$; $\operatorname{san}_{2}$; tan̄qal

touch gently tēk

towards kēl 4; bo; sur 1

towline rērēv

toy wooror

trachea wōdōldōl

tradition kastom; sele ēs

trap

kinds of trap: gatitieg; wōw ${ }_{1}$ wöwöwör qēsaqēs;

prawn trap waga $_{1}$

rat trap walaqlaq gōsōw

tree rēntenge $\mathbf{1}$

cheese tree boboto

Indian coral tree rar $_{2}$

rattle tree wēviriak

tree fern dēmērmēr; qatē

tree fern leaf daqatē

tree species: $a_{1}$; ar ōl; ar tur; bēbētiqō;

bitbitiqō; boboros; bōgōrēmēs;

bul ; bustutun; dam̄āmas; darag

tōwlo; datam̄alqō; dēsēn; dōkōlōkōl;

dōmōvōtrev; dōōt; dōsōw; dōvōr nem;

dötöl; dunregōsōw; dun̄bönö; gan men;

gasēsēdignönqet; gasōg; gatötöqulqërët;

gawōlō; mala; malagavēg; malaqōōr;

malmalasē; malnët; markōn̄; marsam;

menmav; meteraram̄at; mēl; mēeriri;

mēririnek; nagnagsamul; nōnōn; qetge;

qēr ${ }_{1}$; qog; qō miat; rōqu; rōwō; sal ; sawa; sēqēg; sērsēr; sōgsōggenqet; tagar 2 ; talēs toko; tarak; tēq ; titiqō; totenanar; usugiav; varvar; vēkēmolo; vēlavēl 1 ; vēnvēn ${ }_{2}$; vētuboso; vōn̄ame; wakam; walag; ; wan̄arn̄ar; waran̄ran̄ marge; watamarge; wegerbak; weverbak; wēqiar $_{2}$; wēqiqiar; wētēt, ${ }_{2}$ wotag bē; wosvet; wōnōnōg malgias; wōtōgōrte; wōtōn̄; wōvōnō; wö; wöbulbulbōk; wödödö; wu; wuqiat

trevally, bluefin (fish) dötöv

tribe vēnēm̄

trick dörug

triggerfish bum

black triggerfish bum gōlōw

blue-finned triggerfish kurkurqōl

clown triggerfish ron̄ron̄oqar

orangelined triggerfish bum timiat

pallid triggerfish möörögötö

redtooth triggerfish bum qërët

starry triggerfish bum lam

triggerfish species bum lo

wedge-tail triggerfish sem

white-barred triggerfish sem

triller, Polynesian (bird) wōrdes

troll varwëw

trouble matēkatēk

trousers traosis $\mathbf{1}$

true dun $\mathbf{1}$

trunk qētēgi $\mathbf{1}$; turgēn $\mathbf{2}$

truth dun $\mathbf{3}$

try daēl; ten

Tuesday Qön nirō

turmeric waañ

turn tel ${ }_{2}$; wēlēs; wil

turn around kilo; taliwög

turn over le ris

turtle ${ }^{\circ} \mathrm{w}_{1}$

tusk luwö 2

twelve sam̄ul tiwial dēmē nirō

twenty sam̄ul rō

twice vagrō 1

twice, do vagrō 2

twin varam

twist gal

two rō; nirō 


\section{$\mathbf{U} \sim \mathbf{u}$}

\author{
umbilical cord gagö butö \\ umbrella abrela; vivi \\ umbrella leaf divivi \\ Uncle Tatat; Tat \\ uncle maröu \\ her/his uncle marōōn \\ my uncle maröuk \\ uncooked an \\ under tawle \\ underneath laln̄e \\ undo tuw 2 ; ul 2 \\ undried qar 3 \\ unicornfish \\ bluespine unicornfish meteërqōtōn \\ humpnose unicornfish ōm \\ orangespine unicornfish walaglē \\ spotted unicornfish mestalisōr
}

\author{
unity bultiwial \\ unripe qar 1 \\ unsuccessfully matē] \\ until gaidēn̄ \\ uproot mövut \\ upward $\operatorname{siag}_{2} 1$ \\ urinate $\operatorname{mim} 1$ \\ urine $\operatorname{mim} 2$ \\ her/his/its urine mimian \\ my urine mimiēk \\ us \\ us (dl. excl.) kōmōrōk \\ us (dl. incl.) dōrōk \\ us (pl. excl.) kemek; kemem \\ us (pl. incl.) nēn \\ uterus be tētē $\mathbf{2}$
}

\section{$\mathbf{V} \sim \mathbf{v}$}

\section{vagina vēsēn}

Vanua Lava Vōnō Lav

Vanuatu Vanuatu

vatu, 100 baon

vatu, $1,000 \mathrm{mil}$

vegetable cane visō

vehicle trak; wēvēvērēg

vein gabala

victory leaf dagarē

village vōnō 2

vine ga 1 vine species do; do man; dōsōwtöl; ga atat; ga del; ga dōvōr ; ga iaw; ga matawasē; ga mìien; ga ōl; ga sasēn̄ērn̄ēr; ga susul; ga taqav; ga vörur; gamalwuw; gamērēr dēmadēm; gamērēr qō; gatōw; gatöus; qörö gōsōw; röv; wetelwa

voice eln̄e $\mathbf{1}$

her/his/its voice elñan

my voice eln̄ëk

volcano vōr

vomit $\mathrm{lo}_{3}$ vi.; lōlō n.com.

\section{$\mathbf{W} \sim \mathbf{W}$}

wade gër 2

wait kēti 1; dērē 2; diar $\mathbf{2}$

wait for dērē $\mathbf{1}$; diar $\mathbf{1}$

wake up mamat $_{2} \mathbf{1}$; vi. vateber $v t$.

wall (of house) babēris; butuñ

types of walling: sasal; tereñ; winir

wander tetel want mörös $\mathbf{1}$; lölö 1 ; kër ${ }_{2} 1$

war vagal

warbler, fantail (bird) wēvē]

warm n̄ēsir

wart vōlō

wash qōs 2; gösuv; suv

watch out èlgōr 2 


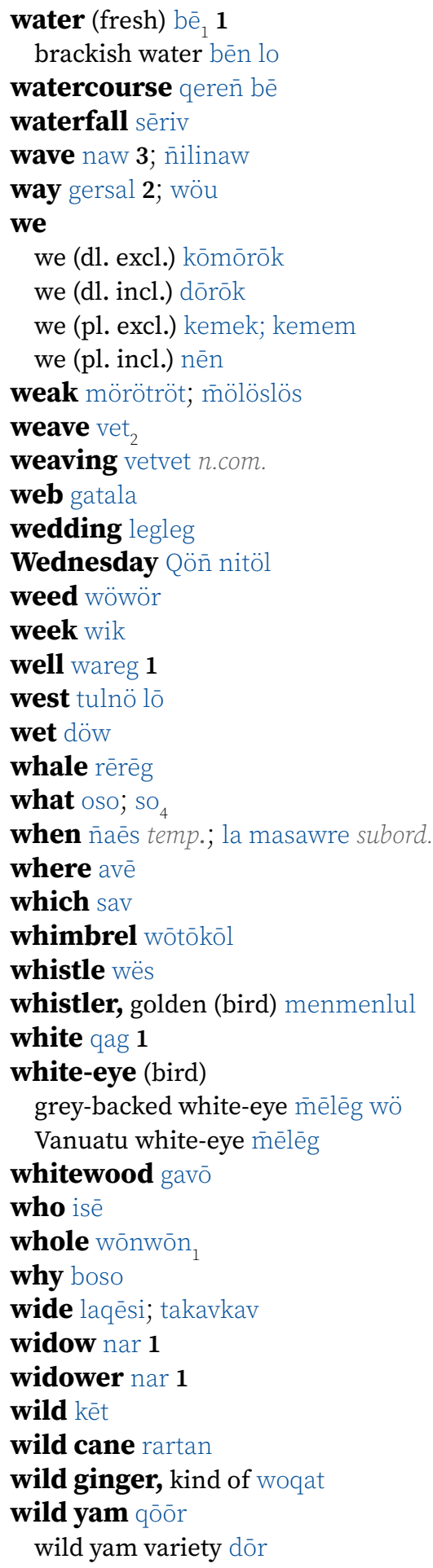

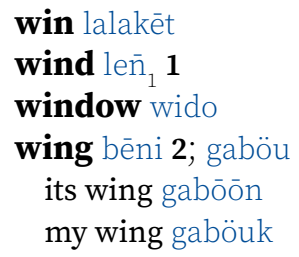




\begin{tabular}{|c|c|}
\hline \multicolumn{2}{|c|}{$\mathbf{Y} \sim \mathbf{y}$} \\
\hline 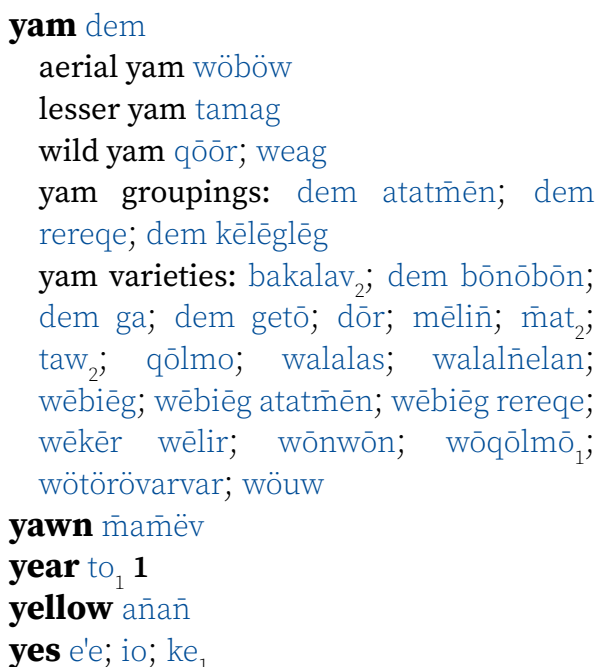 & 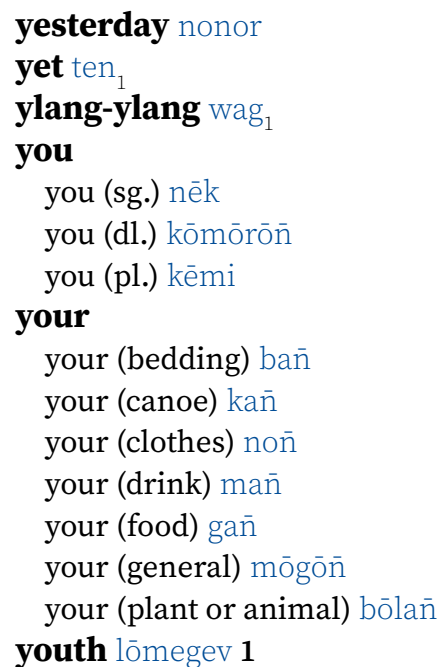 \\
\hline
\end{tabular}

\section{$\mathbf{Z} \sim \mathbf{Z}$}

zip metegongon 



\section{Bislama - Vurës Faendalis}

Pat blong diksonari ia hem i wan lis blong ol wod long Bislama mo i tanem ol wod ia i go long Vurës. Tingting bihaen long hem se sapos i gat wan wod long Bislama we yu wantem faenemaot mining blong hem long Vurës, bae yu jekem long ples ia. Faendalis ia i nogat fulap infomesen long hem olsem men pat blong diksonari. I gat ol wod long Bislama nomo wetem translesen blong olgeta wod ia i go long Vurës. I minim se sapos yu wantem moa infomesen long saed blong wan Vurës wod, bae yu faenemaot long men pat blong diksonari.

Sam long ol Vurës wod i gat namba we i kam afta long hem. Sapos wan smol namba stret afta long wod, i minim se i gat tu o moa wod we i saon semak long Vurës be i gat defren mining. Ale sapos yu wantem jekem wod long men diksonari, bae yu mas faenem wod wetem namba ia. Sapos namba hem i blak mo i bigwan, i minim se wod ia i gat sam smolsmol defrens long mining blong wod, mo defren mining i gat defren namba blong hem. Ale bae yu jekem mining we i go wetem stret namba. Eksampol, aoa lō 3 i talemaot se wod we i minim 'aoa' long Bislama, hem i lō long Vurës, be meksua se yu jekem lō from ${ } \bar{O}_{3}$ i minim 'traot'. Nogud yu jekem rongwan, bae i mekem se yu konfius smol! Ale taem yu jekem namba 3 mining blong $l_{1}$, bae yu luk se i gat wan eksampol blong lō we i minim 'aoa' insaed long wan sentens. (Namba 1 mining blong $l_{1}$ hem i 'san'.)

Wetem klosap evri wod long lis ia, bae yu luk se i no talemaot se wod ia hem i wan naon o veb o wanem long saed blong grama. Wetem wanwan wod nomo bae i talemaot, blong meksua se bae yu no konfius. Eksampol, bae yu luk brum wur vi.; döwurwur n.com. Long Bislama, yu save yusum 'brum' olsem veb (eksampol: 'Yu brum insaed long haos') mo tu olsem naon (eksampol: 'Yu karem brum blong mi'). Long lis ia i mekem i klia se wur hem i wan veb (vi.) olsem long fes eksampol, ale döwurwur hem i wan naon (n.com.) olsem long sekon eksampol. 


\section{$\mathbf{A} \sim \mathbf{a}$}

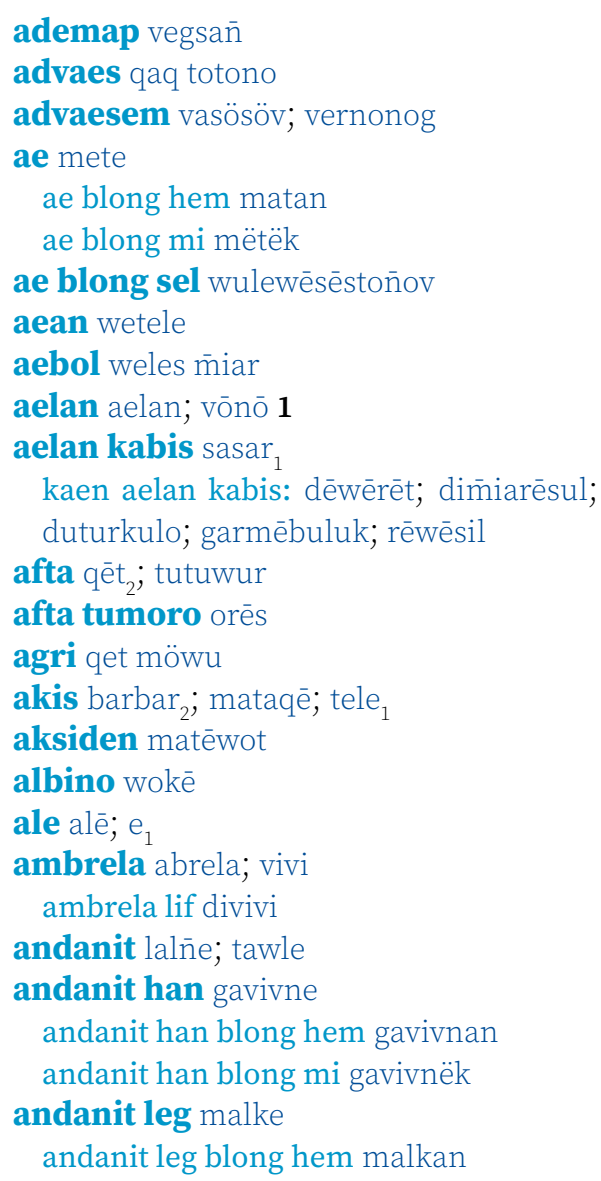

andanit leg blong mi malkëk

angka vet lēg

Angkel Tatat

angkel maröu

angkel blong hem marōōn

angkel blong mi maröuk

animol seleqō

anis magal

faea anis gen ${ }_{2}$;

kaen anis magal tutun

ansa vëmlösal $v$; vëlvël n.com.

ansarem vël ${ }_{2}$

antap mēēē; $\operatorname{sar}_{2} \mathbf{1}$; $\operatorname{siag}_{2} \mathbf{1}$; wötu

aoa $l_{1}, 3$

aot long den 3 ; rieg

aotsaed varē

aranis möl

aro qatē ${ }_{2}$; wētēq

kaen aro meteër

posen aro tōt

as wērē; kērē 1

as blong hem kirian; wirian

as blong mi kērēk; wērēk

asis wōwō

askem baēr; vörus

ating wun $_{1}$

awe! awi

awo! aaōō; akē; aqa; auw; awo 2; i

\section{$\mathbf{B} \sim \mathbf{b}$}

babol mōrmōōse

bakegen kēl 3; bilēñ 1

baksaed kulë 1

baksaed blong hem kōlan

baksaed blong mi kulëk

balongfis wōtōwōt

bambu woo

kaen bambu: woo mölum; woo vet nötu; woo vet luwō banana vetel

bandel blong banana titigi

kaen banana: bakrës; bē $\bar{j}_{3}$; brisben; ëtëtlam; kukuög; lian manu; malēe ${ }_{2}$; matmat malgias; matmat mamē; mianisi; malmalbak; retekraw; rēn̄rēn; rurun̄ög; samo; sēnē; sōbōt; siwit; tores; tulug; ulēulēi; vagvag; vetel mal; vetel talisōr; vietnam; vila; vinwōn; vinwōn ga qag; vinwōn gō tōtōrōg; wavagvag; wēwēsēg; wēwēsēg ta mot; wēwēsēg ta ōrbarbar; wiriatege; worwor 


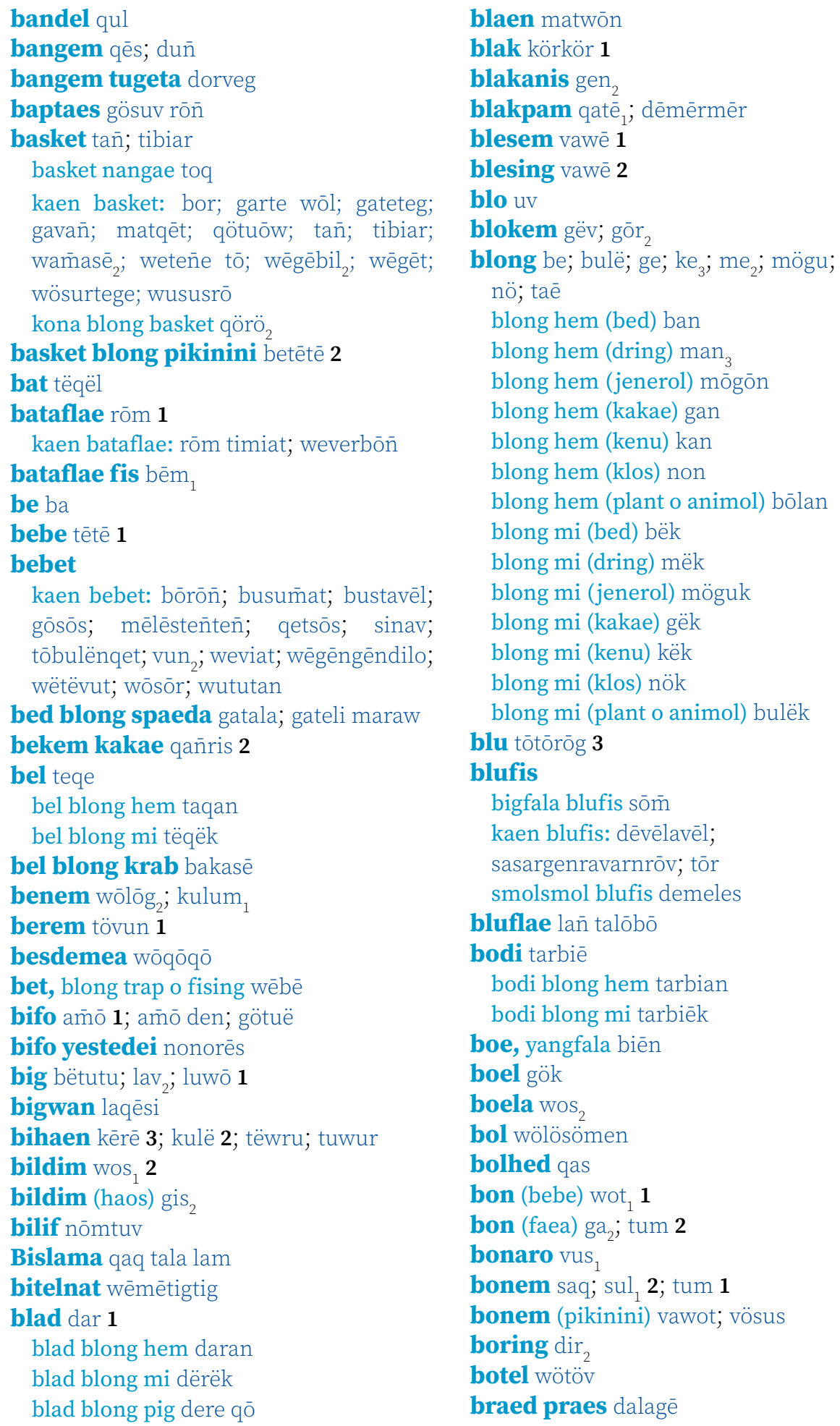




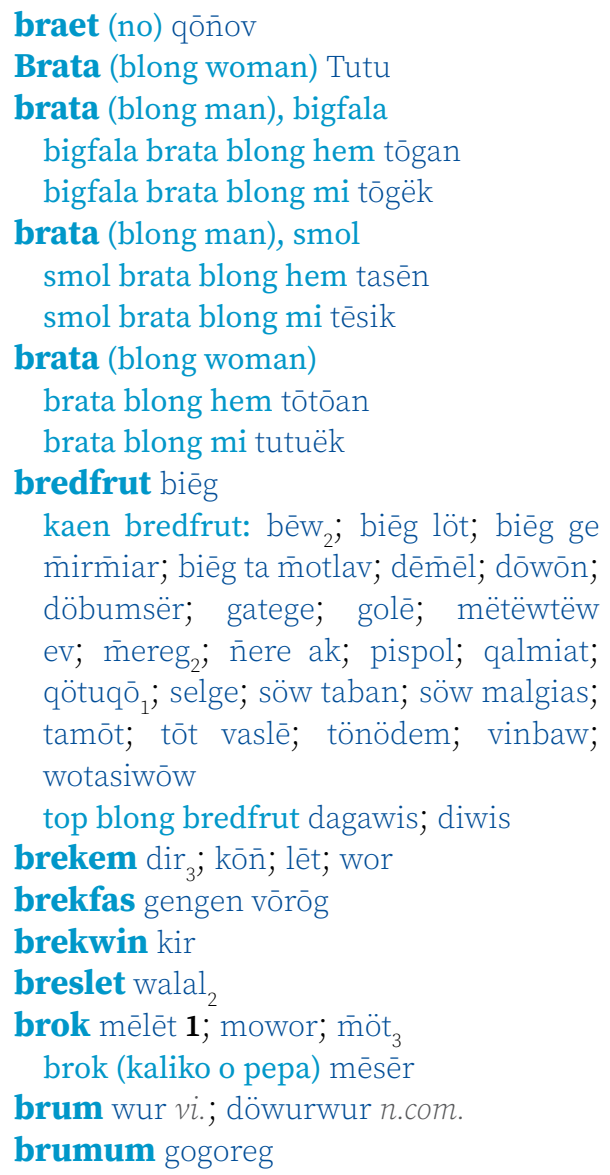

Bubu Bum; Pōpō

Bubu blong bubu Bum vövörus

Bubu man Mambum

Bubu woman Diebum

Mama o papa blong bubu Bum tal

\section{bubu tubu 1}

bubu blong hem tōbōn

bubu blong mi tubuk

smol bubu blong hem tōbōn

smol bubu blong mi tubuk

buk bōk

buluk buluk

bun siri $_{1} 1$

bun blong hem sērēn

bun blong mi sirik

bun blong leg daon siri wawalag

bun blong nek vötu wōqōlqōl

bunbun rērēi ${ }_{2}$

bunia bunia

burao vër ${ }_{2}$

bus möt eria blong bus wömōtmöt

\section{bus kabis}

kaen bus kabis: damalalal; datam̄al añ; davaqal; degetget; deteqeñtōw; detewel; dimililial; dōrōt

busnaef gasel luwō

\section{$\mathbf{D} \sim \mathbf{d}$}

daeva el; qōt
dan monog
dan (no) an; qar $\mathbf{2}$
danis lak vi.; laklak n.com.
kaen danis: eln̄etiti; lian̄; mēe; rësrës
den̄e; siwia
daon sursur; sōw $\mathbf{2}$
das tan vōvō
ded matē; miat
$\quad$ ded blong hem matian
ded blong mi matiēk
defren sisia
dei qöñ $\mathbf{2}$; meren $\mathbf{1}$
deilaet meren $\mathbf{2}$

\author{
devel timiat \\ digim gil 1 \\ digim graon kev \\ digimaot gōqleg \\ dina gengen luwömeren; tina \\ dipsi lam \\ disobei liseg \\ divaedem vēlwor \\ doa mete gövur \\ dog genqirēg; tok \\ dolfin $k \bar{e}_{1}$ \\ doli dole \\ dotdot biliag 4 \\ doti qōlō $\mathbf{1} v i$.
}


doti rōr n.com.

doti (blong sora) dōn

drae mamas; mereñ $\bar{n}_{2}$; rañ ${ }_{1}$; tërëkrëk

drae long san wör

no drae qar $\mathbf{3}$

solwota i drae met mōōr

drae (soa) maw $_{1}$ dragonflae wōrō

draon tul 1

draonem tul 2

drim qōrqōr

dring sum

dring kava wan 1

drop tir

\section{$\mathbf{E} \sim \mathbf{e}$}

eg wese

eg blong hem wasan

eg blong mi wësëk

eg blong laos lēs

ei! ei; ii

eit lövötöl

eitfala (man) bullövötöl

eitin sam̄ul tiwial dēmē nilövötöl eksampol tengeg

ekspektem döm dērē 1

emti marö

en ilsi; tavalgi 2

epot sere plen

evri vël ${ }_{1}$

evri taem tastas

evriwan vawnët; döl

\section{$\mathbf{F} \sim \mathbf{f}$}

faea $e_{1} 1$

faeawud ralēt

faef tevelēm; nitevelēm

faefala bultevelēm

faenem siër; sōk; sösögöt; suër

faerap töqöl

faet rēs

bigfala faet vagal

faetem tut

fak bēl

fan dērivriv

fanem riv $_{2}$

faol tō

blak faol tō kör

faol woman tō reqe

kaen faol: tō armiag; tō biliag; tō

menmenlul

pikinini blong faol nötu tō

red faol tō mamē

waet faol tō bèt

fas mōōō; vis

fasbon matagraq; mie $\mathbf{2}$

fasem lēg; ; nirteg; boko; bōn̄ fasfas ës ${ }_{3}$; verqirī̄

fasin metwu

fasin blong hem metwōn

fasin blong mi metwuk

fastaem am̄ō $\mathbf{2}$

faswan mie $\mathbf{1}$

faswan, kam qal 2

fenis $\mathrm{ar}_{2}$

fens dasas

fes nögö 1

fes blong hem nogon

fes blong mi nögök

feta vul 2

feta blong faol vulu tō

fidim ven̄en; bien 1; bōgereg

fiftin sam̄ul tiwial dēmē nitevelēm

fingga busu 1

fingga

fingga blong hem bōsōn

fingga blong mi busuk

las fingga bustuwur

medel fingga malawsöm

namba fo fingga sömodian 
nambatu fingga bus titivōnō

nambawan fingga bus lav

\section{finis vitia}

fis mes

kaen fis: barnaw; borbor ; bultarō; dananar; dëvër; gie; giebē; liaw; lōat; magalmevesoqötu; mamat; mamat serserdiqiat; mañde; matwiwi; meswelwel; mas an̄an̄; mas sarsar; mas ten̄teñ; mesbule; mesgeben; mesteveltiwial; mestēv; miarbē; mu; murmur; murmurbē; qaēn; qagar; qetmëv; qōtarō; qötuqërët; rabiēg; rak rōm 3; rōqu; rōwlav; röwö; sarsarale; sewerles; sōm; tenmē; terit lam; teqesqē; tewesgavēg; tivtiv ; tivtivnönqet; toqlon̄; tōqagat; tōr ${ }_{2}$; törö ; tuvun; vag mēēl; vag mël; vềrē; vōlōvōl; vōlōvōl sōm; vōt; vurōmōvōt; wen̄emërës; wetevel; weteltelvet; wēduduw; wēēwatē; wēgilgialev; wēqiar ${ }_{1} ; \quad$ wēqitin̄ev; wēsilivetel; wētēvut; wēvētuo; wiliqliaq; wogarēr; womalabōs; wotoqog; wotagavēl; wotoqtoq ${ }_{2}$; wovet; wowolos; wōnwōn ${ }_{2}$; wōtōqagat; wörötuo; wösöwunet; wöturturōrōn̄; wöul

fis, sop sasar,

fisposentri vut

fiu nivēs $\mathbf{2}$; wēvēs

flae lan̄ n.com. kaen flae, smolwan lan̄ tanō kaen flae, blakwan lan̄ vivis

flae gav; lil; wērēreg vi.

flaengfis mëkër kaen flaengfis: bargōr ${ }_{1}$; benmē; rōwlav; toqlon̄

\section{flaengfokis qërët}

flaoa tawagas; vlawa

flaoa blong (samting) tewes kaen flaoa: damalak; wēnēn bulëvu sakem flaoa $\operatorname{taw}_{3} v i$.

flas bōgēt

flat taqeqe

flat ples rat

flatfis lēl

flot sial

fo nivet; vet ${ }_{3}$

fofala (man) bulvet

fogetem qōr

fogivim döm viteg

fok (blong wota, wud) kasivi

fol

blong hem bōgōn

blong mi bugëk

blong papa blong mi bugën i tëmëk

foldaon mës

foldaon long $b o ̄ l_{1} 2$

foldem lōm

folem tatag 1

fored wariē

blong hem warian

blong mi wariēk

fosem vësrët; wisil

fotin samul tiwial dēmē nivet

foto tōtgial $\mathbf{1}$

Fraede Qön tevelēm

fraet qeneg $\mathbf{1}$; metegrōw; mëtëwtëw $\mathbf{1}$

fren bulsal n.com., vi.

frog vorog $\mathbf{2}$

from wanem boso

frut wiē

frut blong hem wian

fulap sis; ${ }_{1}$ wōr

fulap taem vaga $\mathbf{2}$; vaglōqtag $\mathbf{1}$

fullumap nës; sön̄ön

futbol, plei kikbol

futstep malke

\section{$\mathbf{G} \sim \mathbf{g}$}

garen n̄en̄ere; tiqē

garen, olfala wewestel

garen taro mat; wōsōk; rot; qēl; vaslē garen taro, drae biriat

gat qultie

gat bel tel ${ }_{1}$; wotoqtoq 1 ; tek na taqan 
gat kil mëvtia; mataq

gat klaod taqtaq

gel malmal $\mathbf{1}$

giaman malmalē; gial $\mathbf{1}$; gilgial giaman long dörug; gial $\mathbf{2}$

givim le $\mathbf{3}$

glad malaklak

gladhat mētigwè

gat gladhat sōgsōg 2

glas tētēr ${ }_{2}$; klas

glis mēeninin; sërëvtës; sialtislan̄

go $\operatorname{van}_{1} \mathbf{1} ; \operatorname{var}_{1} \mathbf{2}$

go antap kal

go aotsaed kalō

go daon sōw $\mathbf{3}$; tēqēl $\mathbf{1}$

go daon (san) tul $\mathbf{3}$

go hariap gamlöt $\mathbf{2}$

go hom mōl 1

go insaed kal $_{2} \mathbf{5}$ go long not tēqēl $\mathbf{4}$

go long saot $\mathrm{kal}_{2} \mathbf{4}$

go long solwota tēqēl $\mathbf{2}$

go tru vesel

graon $\tan _{1}$

graonem qirir; qisis

gras vēlis

kaen gras: ditial ${ }_{2}$; mōoōt ; vēlis ōlōl;

wētēvtēv

grashopa wēviak

grasket ber 1

werem grasket ber ${ }_{1} \mathbf{2}$

gref qër

grin tōtōrōg 1

grinpijin malqon; men dōsdōs

gris marak

gru malaw; tōw ${ }_{2}$; tōtōw

gud gōwē

\section{$\mathbf{H} \sim \mathbf{h}$}

had mav $\mathbf{2}$

hae elel $\mathbf{1}$

haebiskas waqagal

haed lin̄ereg

hafkakae garte $\mathbf{2}$

hafkakae blong hem gartan

hafkakae blong mi gartëk

halpem birin̄

hamak dimdiam

hamas nivēs $\mathbf{1}$

han bēni $\mathbf{1}$; rērēi

han blong hem banēn

han blong mi bēnik

han blong nawita mēēmēri

handel gagö 3

handred möldöl

hang diam

hangem lēg; ${ }_{2}$ simēdēg

hanggri mēlin̄sal

hant sōksōk; tēqatēq $\mathbf{1}$

haos gövur

smolhaos kērēt 2 2; nötu gövur; tes ${ }_{3}$

hapen n̄an̄aēr harem rōñteg

hariap gamlöt; lelev $\mathbf{1}$; möt $_{2}$

hariken len̄vus

hea vul

hea blong ae burus mete

hea blong hem vōlōn

hea blong mi vuluk

hed qötu 1

hed blong hem qōtōn

hed blong mi qötuk

heitem dasimreg

hem nē

hem wan masgian; matōbōn

hemia gēn; iloko; in̄kē hemia longwe e; ilēkē; ken

heven kētkēt 2

hevi mav 1

hil kalkal

hae hil geret

hip bal $_{2}$

hit (blong) sësëwu

hok mal

hoknaet wis ${ }_{1}$ 


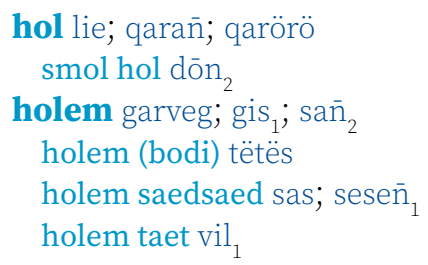

hon (blong buluk) ser hot tutun 1

hot smol m̄ēsir $\mathbf{1}$

hu isē

huk go n.com.; sōsōg vi.

\section{$\mathbf{I} \sim \mathbf{i}$}

i gat a $\bar{e}_{1}$

ia $\mathrm{ko}_{1}$; ine; ia

ilfis marē

impoten elel 2

inaf sasarēt $\mathbf{1}$

insaed lin̄ereg; $;$ 1ölö ${ }_{2}$; $\operatorname{sar}_{2} 2$

\section{insaed blong}

insaed blong hem lolon insaed blong mi lölök

invaetem ōligi; suware is (daerekson) mete lō $\mathbf{1}$ isi men̄men̄ 2

\section{$\mathbf{J} \sim \mathbf{j}$}

jajem vēvēg

jakol watavreñ

jalus gilal 3

jam rōw

jam antap leqer

jas kara

jea tönö sigsiag

jenis lilies

jenisim lies $\mathbf{1}$

jes (blong bodi) vere ${ }_{2} \mathbf{1}$

jes blong hem varan $\mathbf{1}$

jes blong mi vërëk $\mathbf{1}$

jif maranag; tövusm̄ēl

hae jif lan jikinpok sislaqlaq

jin balsiē

jin blong hem balsian

jin blong mi balsiēk

jinja sinsa

jo balsiē

jo blong hem balsian

jo blong mi balsiēk

joen sö 2; mönug

joenem sö 1 ; bak 2

jok dōlgia

jone (fis) datalēs

jos gövur gōrōn; gövur tatar; löglē

juiim (kakae) n̄ōr

\section{$\mathbf{K} \sim \mathbf{k}$}

kaen tarn̄i

kakae gengen $\mathbf{1}$; sinag $\mathbf{2}$ n.com.

bigfala kakae gengen luwō

smol kakae wëmën̄ël

kakae gen $;$; kur $\mathbf{1} v t$.; gengen vi.

kakae long medel dei gengen luwömeren

kakae long moning gengen vōrōg

kakae long sava gengen revrev kakae mit big

kakae wetem tut gar; n̄ōr

kala törö 2

kala lif kirkiar

kaen kala lif: galō; kirkiar m̄eter; kirkiar qet; kirkiar qere qō; kirkiar sas qön̄; wamamēgin

kalarem törö 1 
kaliko gogov $\mathbf{2}$

kaliko blong karem bebe bōn $\overline{1}_{1}$

kam me 1 ; van me $\mathbf{1}$

kam olfala tamarge $\mathbf{3}$

kamaot kalō; lōo ; sawadān

kamaot (brans blong wud) kosēv

kamaot long den $\mathbf{5}$

kamaot long eg dir

kamaot long graon mövut

kambak kēl 1

kamfotem damösur

kamso siōl

solwota i kamso met mö lum

kamtru dēn̄; rar ${ }_{1}$; rōw lō

kaofis qōlo

kaontem vēvēg; ; luw

kaorisel wōwlan kaen kaorisel widiañ $\mathbf{1}$

kapa kav

kapsaedem lin̄; sër ${ }_{1} 2$

kapsaedem wota vun 1

karasol winiam̄

karem bis; le $\mathbf{2}$; sōsōrvël

karem bebe bēm ${ }_{2}$

karem fok n̄iria

karem kakae ōw ${ }_{2}$

karem long solda sōltek

karem ova long solda bēmn̄eg

karem saedsaed sas; sesen̄

karem taro sigsig

karemaot er ${ }_{1}$; gag; le den

karemaot klos sër lō

karemaot skin (kakae) sēm 1

kari waan̄

karong, blu (fis) dötöv

kasem dēn̄; gaidēn̄; gis qal; qal $\mathbf{1}$; sëwëw; $\mathrm{sokO}_{2}$

kastom sele ès; kastom

kastom storian eln̄evu

katapila wötörör

katem bal 1 2; ger; gōr ${ }_{1}$; tēv $\mathbf{1}$; tōt

katem bol wot ${ }_{2}$

katon karton̄

kava gē 1

kaen kava wistaban

kavremap liqet; tër

kef $l \bar{e}_{1}$ kenu ak; ak sōsō; wēlwēl

kenu (wetem sel) ak geben

kenutri ōv

kilim lā̄; qōs 1 1; töwös; vus ${ }_{2}$

kilim (mekem bit) den̄

kilim pig kōl

kilim strong lam̄seg

kilim wetem ston qōs

kisim bōbōn

klaem rem

klamsel tele ${ }_{2}$

klaod malēg

klia tawseg; man̄aē

klif dō

klin winiwin

klinik gövur lisia

klinim vawseg

klinim insaed ber $_{2}$

klos gogov 1; mölö; wësërsër

klos blong hem molon; non ${ }_{2}$

klos blong mi mölök; nök

klos $\operatorname{bur}_{2} v$.

klosem bur $_{2} v$.

klosap susus

kof tōv 1

kof nogud man̄

kok qere ${ }_{2}$

kok blong hem qaran

kok blong mi qërëk

kokonas mōtō $_{1} 1$

drae kokonas matabōbōt

grin kokonas vōs

gru blong kokonas n̄ēn̄èn $\mathbf{2}$

haf drae kokonas qōtō

kaen kokonas: bar ; mōtō atm̄ēn; mōtō

bal; mōtō dēdērēs; mōtō elvet; mōtō ga

an̄añ; mōtō ga garaq; mōtō ge metestes; mōtō gō tōtōrōg; mōtō gö luwō; mōtō malgias; mōtō mamē; mōtō màt; mōtō mete röwö; mōtō mölumlum; mōtō reqe; mōtō sam̄sam̄; mōtō seseser; mōtō sial me; mōtō silat; mōtō taktak; mōtō us; mōtō vanvan; mōtō vet; mōtō vin gaqō; mōtō wulmē

sel kokonas vinlas; vinkörögör

stamba blong kokonas tur mōtō

wota blong kokonas tin̄i mōtō 
kolkol malas; mamēgin $\mathbf{2}$

kom metekerker n.com.

komem ker

kompit vërlē

komplen wutōt

komposem (singsing) tōw ${ }_{5}$

konfius liar $\mathbf{2}$

konfiusem qiriag

konkon gōgōn 12

kopereit bulbul 2

koperesen bulbul $\mathbf{1}$

korel korel; weles; womal

krab giav

kaen krab: gamar; giavēdun; giav kukut; giav matrōrōw; giav mërës; giav ōwōw; giav sem̄etöv; giav sēvsēv; giav sōsō; giav tam̄al; giav vaw; giav vet; gōdōw; görum; sursal; tēbar; tigian; wēgēriv; wēgilgialnaw; wōg; wōlōrōr; wökutökut krab kokonas dēr

krae ten̄; ten̄ten̄

krangke qaqa $\mathbf{1}$

kros en ; lōlenen; mēr kros tumas lōlgagar

krosem barbar ${ }_{1}$; sogolo

krulked kēlēglēg

kruked (bebet) wētētērē

kuk kuk

kumala kōmar

kaen kumala: ga dōvōr 2 ; pawa; spidbōt; tövutwen; tri manis; vorog $\mathbf{1}$; wese tō ${ }_{2}$

kwaet rōrōn $\mathbf{2}$

kwestin vörus völu

\section{$\mathbf{L} \sim \mathbf{1}$}

laef ès n.com., vi.

laef blong hem ōsōn

laef blong mi usuk

laekem kër 2 ; lölö 2 ; mörös 2

laen (famle) sögö

laen blong hem sogon

laen blong mi sögök

laen tarin̄i

laet bur $n$ n.com.

laet èlēl 2; sēlsēl vi.

laet (no hevi) men̄meñ $\mathbf{1}$

laetem $\mathrm{ti}_{2} 2$

laetem faea sul 1

laetning vēl

laf bor vi.; borbor n.com.

laf long borseg

lafet qetgengen

laki malabōs; $\operatorname{tagar}_{1} \mathbf{1}$

lanem tono 1

lang wōs

lanwis qaq 3

lanwis blong yumi qaq ta minēn

laos wut laplap laplap; lōk

kaen laplap: biliagtat; lör; wewe; wir; wōrkelkel

laswan basbasne; qētqētne

lavem tiam

lef han blong hem banēn gala

lefsaed gala

leftemap lul; rak ${ }_{2}$; sōltek; teteg ${ }_{1}$; tiam 2

leg diri

leg blong hem dērēn

leg blong mi dirik

lego dun̄ög; gis tës; ukuëg $\mathbf{2}$

lego kakae gargarmamas

leidaon bētul; ōn ; tētēr $_{1}$

lek (wota) tes

lelebet mēnē

les mereñ $;$; mōr

les long $\mathrm{ar}_{3}$; dir

lesen tin̄qōr

leva vere $\mathbf{2}$

leva blong hem varan $\mathbf{2}$

leva blong mi vërëk $\mathbf{2}$

leven sam̄ul tiwial dēmē nitiwial 
lida mie $\mathbf{3}$; qetegak

lidim gan̄veg; turm̄ō

lif dege; dödöu

ambrela lif divivi

bun blong lif labēvi; siri 2

drae lif pandanas damarēn̄it

lif blong (samting) do-

lif blong bredfrut dagawis; diwis

lif blong burao dētēriv

lif taro diqiat

yang lif qöqö; qöqörö

lif laplap damat

bun blong lif laplap gam̄esel

kaen lif laplap: damat dēnin; damat m̄öt

likim nem

lised matriaw

kaen lised: kakēlis; kalmōs; kalqar; matawōsōl; mōs; tanlēn̄lēñ; viabobo

loa $\bar{e}_{2}$

loeaken gawol lok lok

long a; bo ${ }_{1}$; den 1 1; $\min _{1} \mathbf{1}$

longfala elvet; mie

longmaot (fis) til; ut

longtaem tuë

longwe aqit; arës

los (fis)

kaen los: biliag; ; biliag lam; mewērwēr; mōmōo ${ }_{1}$ tiqialdaran̄ran̄; tiqialqō; tiria; tiriakör; wutusērēt

smol braon los wesrē

luk ēl 1

lukluk ēlēl $\mathbf{1}$

lukluk antap atelen̄

luk save ēl rak

lukaot gitiag; ēlgōr $\mathbf{2}$

lukaotem datek 1; ēlgōr $\mathbf{1}$

lukim ēl 2

lus liar 1; qilian̄ $\mathbf{1}$

$\mathbf{M} \sim \mathbf{m}$

maget $\bar{o} l_{2}$

majik $\operatorname{man}_{1} 2$

mak veglal $\mathbf{1}$

mak (long bel) gēri mōtō

makem tōm; tōw ${ }_{3}$; veglal 2

malet gomot

mama die $\mathbf{1}$; rētne

mama blong hem rētnan

mama blong mi rētnëk

Mama Die

man atm̄ēn; tan̄sar $\mathbf{1}$

fulap man rege

man sori mōdō

man we i no mared qilewer

medel man tödun mian

olfala man tamarge $\mathbf{1}$

pua man masar

man (blong) gunö

man blong hem ginon

man blong mi gunök

Mande Made

mane selen $\mathbf{2}$; söm $\mathbf{1}$; tiveg $\mathbf{1}$; wadē manfaol tō atm̄ēn

manggo man̄ko

maniok maniok

grup blong maniok: maniok atatmēen; maniok rereqe

kaen maniok: atina; maniok añan̄; raēs ga qag; raēs mamē; saemon; sus rereqe; sus atatm̄ēn; vasēr

manis wōl 2

manis (blong woman)

manis blong hem wōlan

manis blong mi wulëk

maot n̄ere $\mathbf{1}$; ñusu $\mathbf{1}$; vele

maot blong hem n̄aran; n̄ōsōn; valan

maot blong mi n̄ërëk; n̄usuk; vëlëk

maot blong pijin n̄usu 2; n̄ere 2

mared leg vi.; lagē n.com.; legleg n.com.

mas mas

masket tēqatēq 2

mat tönöam

lif kokonas mat dön

pandanas mat abawē 


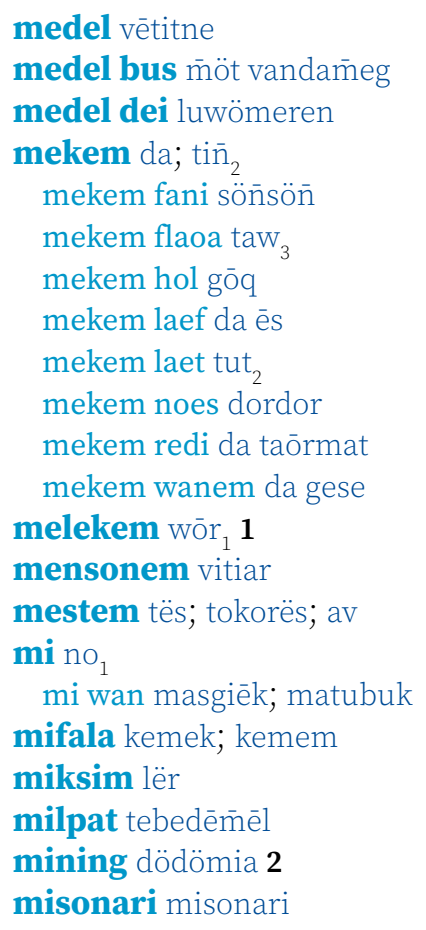

mit bigbig; vusgö n.com.

mit verdēñ vi.

miting vug 2 vi.; vugvug n.com.

mitufala kōmōrōk

mo wo $_{1}$

moa bilēn̄ 3

moning vōrōg 1

eli moning lölön̄ö vōrōg

kam moning vōrōg 2

morinda wōlōm

moskito nem

kaen moskito: nem biliag; nem talöqön

moto susuki

mun wōl 1

masrum (we i saen) widiar $\mathbf{1}$

mustas wini

mustas blong hem wēnēn

mustas blong mi win̄ik

mustasfis mas

muvmuv malaglag; m̄ōgōlgōl;

m̄ögus

\begin{tabular}{|c|c|}
\hline & \\
\hline $\begin{array}{l}\text { nabew wöböw } \\
\text { nabisa wēwēn } \\
\text { nandingori wag } \\
\text { naduledule nët } \\
\text { naef gasel } \\
\text { busnaef gasel luwō } \\
\text { smolnaef gasel nötu } \\
\text { wuden naef meteges } \\
\text { naen lövövet } \\
\text { naenfala (man) bullövövet } \\
\text { naentin sam̄ul tiwial dēmē nilövövet } \\
\text { naes lulum } \mathbf{2} \\
\text { naet qön̄ } \mathbf{1} \\
\text { nakamal gemel } \\
\text { nakatambol woro } \\
\text { nakato götö } \\
\text { kaen nakato: götö mamē; götö riam } \\
\text { takak; götö sërsër } \\
\text { nakavika gavēg }\end{array}$ & $\begin{array}{l}\text { nalaslas (wud, lif) dëm̄ël; dövuln̄ö; mölöl } \\
\text { kör } \\
\text { nalnal qesekēr } \\
\text { nalot löt } 1 \\
\text { kaen nalot: löt bulmarwe; löt kel; löt } \\
\text { matwōnwōn; löt n̄e meren̄; löt n̄e qar; } \\
\text { löt salsim; löt teqesēg; löt vasgēr; löt } \\
\text { wirsal; löt wotag; löt wötön̄ö; salōqōs; } \\
\text { wirmamēgin } \\
\text { nalumlum lumlum } \\
\text { kaen nalumlum lōw } \\
\text { namalao m̄olo } \\
\text { namalas meles } \\
\text { namambe miak } \\
\text { namarae marē } \\
\text { kaen namarae: göwur; marē bēluwō; } \\
\text { marē genrō; marē mat; marē mes; marē } \\
\text { qō; marē teweswotag; marē wosgötö; } \\
\text { marē wōl; marē wot; mörön̄ërër; } \\
\text { tesmērēr; val; vusö ōn; wutu ōn }\end{array}$ \\
\hline
\end{tabular}


nambaeit lövötölne

nambaeit (taem) vaglövötölne

nambafaef tevelēmne

nambafaef (taem) vagtevelēmne

nambafo vetne

nambafo (taem) vagvetne

nambanaen lövövetne

nambanaen (taem) vaglövövetne

nambangga $\mathrm{bak}_{1}$

kaen nambangga bak matqō

nambaseven lōvōrōne

nambaseven (taem) vaglōvōrōne

nambasikis levetēne

nambasikis (taem) vaglevetēne

nambaten samulne

nambaten (taem) vagsamulne

nambatri tölne

nambatri (taem) vagtölne

nambatu rōne

nambatu (taem) vagrōne

nambilak biliag,

nambirimbiri (tri) birbir

nambuton butö

nambuton blong hem buton

nambuton blong mi butök

namele mēèl

kaen namele m̄èl añañ

nandae (tri) darag

nandao (tri, frut) tewen

nangae (tri, nat) ñe

kaen nangae: n̄e dawel; n̄e dun; n̄e ta

nanggalat (tri) silat $\mathbf{1}$

kaen nanggalat silat gegeretō

nanggalat blong solwota silat $\mathbf{2}$

nanggaria (tri) dagarē

nao in̄ko

naora ōr

kaen naora blong wota: ōr bëlbël; qaqet

kaen naora blong solwota: ōr taban;

vavam

naos (tri, frut) ur

napiru (pijin) matēk

napoleong (fis) lēw

kaen napoleong: lēw biliag; lēw mal

narara (tri) rerev $_{1}$; rar $_{2}$

narasras via

narawan tōar nasama sam

nasara $\operatorname{sar}_{1}$

nasese wētēwil

kaen nasese: wētēwil bēt; wētēwil kör; wētēwil mëv; wētēwil serōn

nasiko (pijin) sēg

nasiviru (pijin) mēs; rēn̄ēs

natalae tele $e_{2}$

kaen natalae tele tur; wēgim

natambia tabē

natanggura doot $\mathbf{1}$

bun blong wēsiri; wuduw

mekem natanggura $\mathrm{SO}_{2}$

pis blong natanggura masa

natavoa (tri, nat) talēs

nating dā̄eg; nawōn

natongtong (tri) qēqēl

natora mianisi; tor

navara sōl

swit yangfala navara sōlvet

navele (tri, nat) wotag

kaen navele: wotag dere timiat; wotag dun; wotag sërsër; wotag wos; wotag wōtōn̄; wotag wöl

navenue (tri) gōglo; wōvōnō

naviso visō

nawimba (pijin) qon

nawita menek; on; wērēt

nek bibiē

bihaen long nek wōqōlqōl

nek blong hem bibian

nek blong mi bibiēk

nekles wetenge; wevet; wērērēn̄ēg

nem sie

nem blong hem sian

nem blong mi siëk

nemem tōwereg

nes blong pijin tötöu

net giam 1

ni (blong leg) qöu

ni blong hem qōōn

ni blong mi qöuk

nid mörös 3

nil butu; keresi

nilfis ganasēg; terit

niu garaq

nius rörö 
noes qōrōtōt 2; rarö

nogat odian̄ 1; ōōō

nogud tisē

nomo gem nus mödu

nus blong hem mōdōn

nus blong mi möduk

\section{$\mathbf{0} \sim \mathbf{0}$}

o $\mathrm{Si}_{2}$

oel kokonas bes 1

oktri ër

olbaot atalē; qaqa 3; valakreg

olfala musu

olfala man tamarge

olgeta nēr

olsem timiak

olsem wanem timiak avē $\mathbf{1}$

oltaem lav ${ }_{1}$; tasga; vaga $\mathbf{1}$ olwei tas 1

00 ००

open takavkav

openem wiak 1

openem ae vulëg; mamat 2

openem (han) viër

openem maot wawañ

ovarem levga

oven um

kaen oven vësëw 1

\section{$\mathbf{P} \sim \mathbf{p}$}

paenapol $\operatorname{van}_{3}$

Palika Wimiar

palika qelge

palika blong hem qelgan

palika blong mi qëlgëk

palolo un

pamken pomken

pamtri

kaen pamtri: bar ; mōtōvōl

lif blong pamtri tebe

pandanas vudege

drae lif pandanas damarēnit; dēmērir

kaen pandanas: dērvag; van $_{2}$

waelpandanas: dootnönqet; dökurut

Papa Mam 1

papa teme

papa blong hem taman

papa blong mi tëmëk

Papa God Mam 2

parel sō vi.; wōs n.com.

pasenfrut waga 1

wael pasenfrut waga 2

pasis metesa

pasol mon 2 pasolem lölös $\mathbf{1}$; m̄on $\mathbf{1}$

pat garte $\mathbf{1}$; taklē $\mathbf{2}$

patna bērn̄ē

patna blong hem bērn̄ēn

patna blong mi bērn̄ik

pem girē; tiveg 2; wöl 2

pem graon tun

pembak ser $_{3}$

pig $\mathrm{q}_{1}$

pig woman $\overline{\mathrm{mal}}$

pijin men

kaen pijin: bagbaglō; biliag mamē; bëlbëlvōlōn; mal biliag; malēe; menmenlul; mensirsirqōōr; gōg; gōg gabēt; gōg kör; mëkbē; mēēēg wö; qon bulënqet; qon bulënrēlē; qon tan̄sar; qon wēbēt; qötqötumen; sōravar; tēgēr; tēwē; tēwē biliag; tēwē ōlqön̄; tēwē rōwelteg; tërëw; tōrō; tōrō butbut; tōwav ${ }_{2}$; tutrevak; tutwō; wasēe; wētēbisbis; wētēsisis; wētēt, wēvēl; wiria; wiria tabē; wotoqtoq mamē; wōrdes; wōkōl; wōtōkōl

pilimap bet; gis rak 
pikinini mirmiar

pikinini blong hem nōtōn

pikinini blong mi nötuk

pikinini we i nogat mama mo papa gōlōw

smol pikinini (bebe) tētē $\mathbf{1}$

piko (fis) tömus

pilo qetōlōn

pin bin

pinim vēn

pinkfis qev

pinsim gin 2

pipol sul

pis (blong wan samting) manrē; masrē; seve; vutrë

pis (nogat faet) tamat

pisis seve

pispis mimiē n.com.

pispis blong hem mimian

pispis blong mi mimiēk

pispis mim $1 v i$.

plan lugög

planem riv

planem taro sōwereg; vas

plant

kaen plant: bēkil; bormiak; busuqaēn; busuqërët; dabalak; damatmot; damataq; daqatē; dasas qötu riaw; dasas tie tō; datamal; datamiar; datartarawöw; davarteker; deben tenēnēg; demen mav; dēnin; dēqēt; dëmël; dim̄ia; ditial ; diwinwian; do qōrgavgav; dōrōt; dökurut; dömöwön; dötöqötun; dunremēēēe;; gatavdor; lēnötō ${ }_{2}$; lösönqō; masēlasēl; mēlēslēs ${ }_{2} ; \quad$ mēvinvian ${ }_{1}$; nisnias; qōo; qōmur; tartarawöw; tutugēn tamarge; vōnōdöwö; wam̄amas $_{1}$; waran̄rañ; waran̄ran̄ ga qag; waran̄rañ mamē; wedeldel; wesev; wolkoko; wōvōt wōlōlōmeren; wösusurbē; wulëwalal

plante lōqtag; m̄ōrag; tutuqë

plantesen plantesen

plei or

pleplei oror vi.; wooror n.com.

plen ak gavgav ples masawre 2; sara; tönö

ples (blong) vene

nem blong ples Bek; Bēut 1; Bōkrat;

Koimaram; Laln̄etak; Laln̄evut; Lesa;

Lēsē; Lion; Liwōn; Mere; Metir; M̄ēsēn;

Namnog; Nam̄asmas; Nereqe; Neretan;

Periōt; Qötuwis; Rekel; Rev; Sanlen̄;

Sasar; Selwō; Serewan; Sisiōl; Sol;

Suvren; Tetgen; Tores; Vanlis; Vatov;

Vetmōl; Vetrat; Vurës; Wasag; Wēsilat;

Wētika; Wētikō

ples blong hem vanan

ples blong mi vënëk

ples blong tri löv

ples we san i kam antap nögö lō

ples ia oko

popel qöñön̄

popo woman

pos $\operatorname{san}_{1}$

medel pos sañ tine

posen gōgōn 4

praevet pat lösö

praevet pat blong hem loson

praevet pat blong mi lösök

praod bion̄; sötel

prea tatar $\mathbf{1}$ vi.; tatar $\mathbf{2}$ n.com.

presen tabēva

progres tōtōtōw

promes vatvat 1

prumbut $\operatorname{var}_{1} 1$

pul qulös

pule (fis)

kaen pule baw; martat; mestiagōsōw

pulum rañ ${ }_{2}$ rēv $_{1}$; tut ${ }_{3}$ pulumaot gövut; sar $_{4}$

pulum win memseg 1

pulum win (sno) n̄ōn̄ōr

pupu sel tōw ${ }_{4}$

puskat buskat; tivi

pusum qērēt; susn̄eg

putum mem; vasgēr

putum bet bēe putum ston bulës; bun 


\section{$\mathbf{R} \sim \mathbf{r}$}

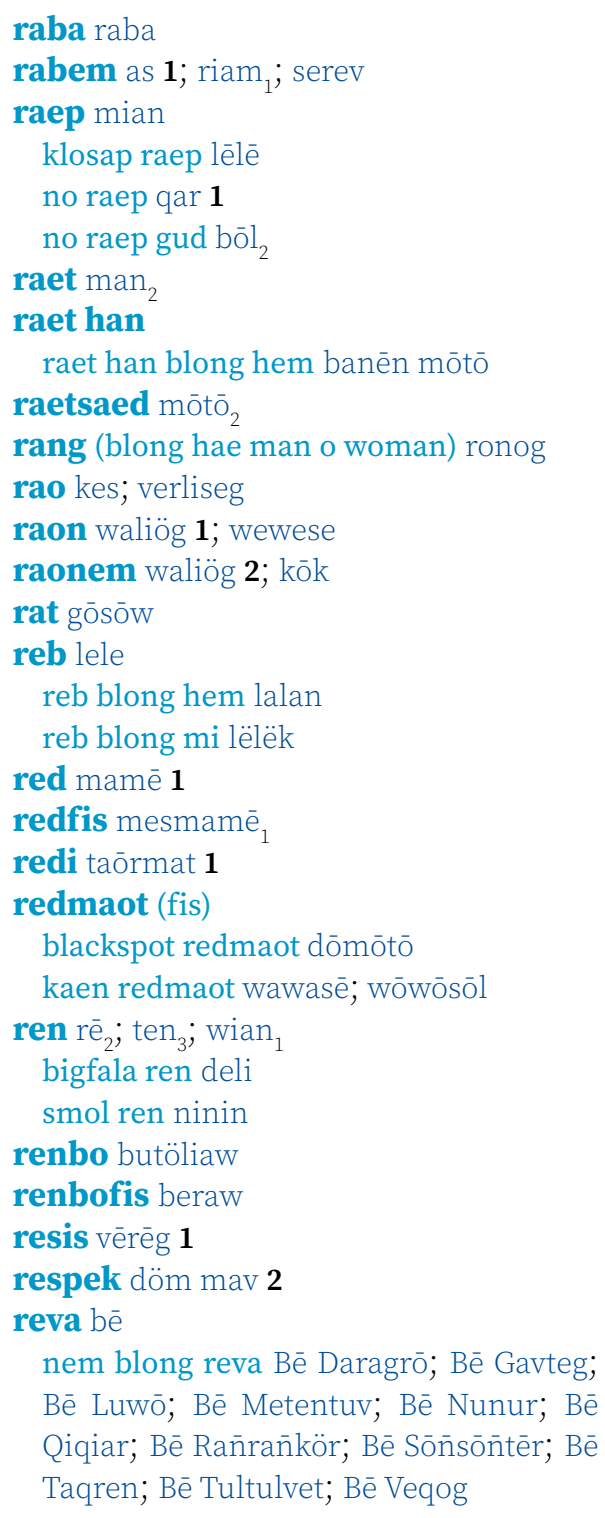

rid rid

ridim vasōgō

rif met; sëkër

rikodem bël 2

ring (long bambu) qulön

ringwom vun 5

ripitim lulu

risen manene

rod gersal 1; wöu

rod blong hem gersalan

rod blong mi gersëlëk

rod blong mared gersele legleg

rol (blong s.t.) bibis 2 n.com.

rolem tiwil

rolemap bibis $\mathbf{1}$

ron (wota) vērēg $\mathbf{1}$; sis ${ }_{2}$ ron bigwan sol ron drae qiag

ronem goro

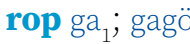

kaen rop: do; do man; dōsōwtöl; ga atat; ga del; ga dōvōr ${ }_{1}$; ga iaw; ga matawasē; ga mien; ga ōl; ga sasēn̄ērn̄ēr; ga susul; ga taqav; ga vörur gakalkalbōgō; gakarēs; gamalwuw; gamērēr; gamērēr dēmadēm; gamērēr qō; gatōw; gatöus; qörö gōsōw; röv; wetelwa rop blong pikinini gagö butö

roten dër

rus delti; gēri n.com.; tuntun vi.

rus antap gagarēn

rusum tun

\section{$\mathbf{S} \sim \mathbf{S}$}

sad lōlwōn

sado tamtiē

sado blong hem tamtian

sado blong mi tamtiēk saed bersi; beriri; kikse; vere $_{1}$ saed blong fes wadaln̄e saed blong fes blong hem wadaln̄an saed blong fes blong mi wadaln̄ëk 


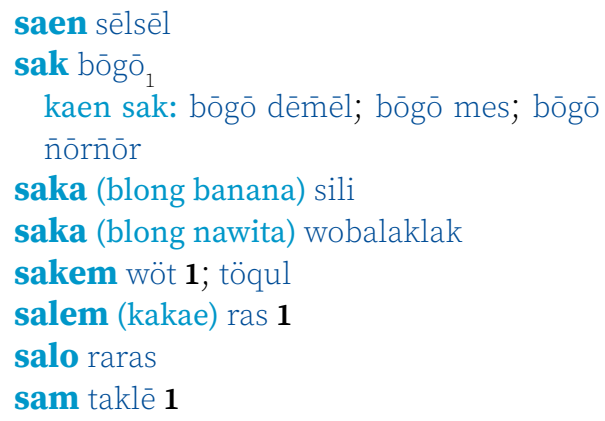
wamatwōn; watagōgōn; watawlētlēt; weger mel; weger sōl; weger ta; weger tur $_{2}$; weqet; weswesak; weterōw; wēgim; wēsēm; wēsēs bē; wēsēs biēg; wēsēs man; wēsēs malau; wēsēs nōnōm; wēsēs tan̄ov; wēsēs vet; wēsēs wōmalak; wētēqēdur; woswos; wōgōlgōl; wōrōa wölubus; wöqörö; wörön̄ötöwö; wösöm; wösusumalmàl; wutumet

sel fingga wulë busu

sel mane söm 2

selfis mētigtisē

semak sasarēt $\mathbf{2}$

sendem tulëg; vetren̄

sentawud sentawud

seraotem gasēg; vēl ${ }_{3}$

sevem (kava) sēn

sevem (kakae) lik

sevem (wud, mustas) sesev; sir $\mathbf{3}$

seven lōvōrō

sevenfala (man) bullōvōrō

seventin sam̄ul tiwial dēmē nilōvōrō

sid wösöwu

sidaon siag; ; taq

sik lisia n.com.; los vi.

sik (blong traot) lōlmususugsug

siks levetē

siksfala (man) bullevetē

sikstin sam̄ul tiwial dēmē nilevetē

sin gengenur

singaot aw; ōl 1

singaot (dog) tōr ${ }_{1}$

singim sur 4

singsing ës ${ }_{2}$ n.com

singsing sursur ës vi.

siok (tri) ër

sip ak; bakalav

sisis balbal 1

sisnek mat tan tes; me $\mathbf{1}$

Sista (blong man) Tutu

sista (blong man)

sista blong hem tōtōan

sista blong mi tutuëk

sista (blong woman), bigwan

bigfala sista blong hem tōgan

bigfala sista blong mi tōgëk

sista (blong woman), smol

smol sista blong hem tasēn

smol sista blong mi tēsik

sitsit tia n.com.

fes sitsit blong bebe tie menek

sitsit blong hem vēntēn

sitsit blong mi vintik 
sitsit titiav vi. sitsit wota kirkir

skae maē

skin vinti

skin blong hem vēntēn

skin blong mi vintik

skin blong maot n̄ere ${ }_{2} \mathbf{1}$; ñusu $\mathbf{1}$

skin blong maot blong hem n̄aran; n̄ōsōn

skin blong maot blong mi n̄ërëk; n̄usuk

skinim kok walas

skrabdak m̄olo

skras susul

skrasem keres; kër ${ }_{1}$; rërës; sir $\mathbf{1}$

skrasem kokonas geres; gör

skwisim bērseg; gēvir

slak gōl ${ }_{1}$; mēlēslēs ${ }_{1}$; mörötröt; masawsaw; mölöslös

slip mōvōt

slo dōdōr; mölumlum $\mathbf{2}$

sloslo rōrōñ 1 ; möul 2

smael n̄ēn̄ēs 1

smasem qēs mōnmōn

smat mataktak

smel bunë

smel blong hem bōnan

smel blong mi bunëk

smel nogud bōn

smelem sōbōn

smok ës

smokem tētēw

smol atik

smol (naro) kokos $\mathbf{1}$

smolsmol atiktik

smolwan nötu, $\mathbf{1}$

smolhaos kērēt ${ }_{2}$ 2; nötu gövur; tes ${ }_{3}$

smutum girir

snek mat

kaen snek: mat añ; mat biliag; tilē tan snis tarēn̄

so (solwota) bōrōt

soa mëmës vi.; mōnōg; qēsis n.com.

soemaot varēg

sofis non

sofmad bōak $\mathbf{1}$

sofsof mölumlum $\mathbf{1}$

sol (spirit) ete sol blong hem atan

sol blong mi ëtëk

sol (solwota) naw 4

solap nen

solda blong hem qötubanēn

solem daon dōl

solwota $\mathrm{l}_{1}$; naw $\mathbf{1}$

solwota, dip butölam

somap at; susur

soping wölwöl

sora qörö sora blong hem qoron sora blong mi qörök

sora blong fis wawawn̄e

sora i fas qōrwōn

sori mamarseg

sospen sospen

sot sot

sotfala qëtutu 1

sotleg mereg

spaeda maraw

kaen spaeda matētē

spel memseg 2

sperem es

speseli raksiag

spet dinsöw 1 n.com.; garmös

spia kar; ser

spirit vu; dēbit

kaen spirit vusën̄sën

spirit tamtiē

spirit blong hem tamtian

spirit blong mi tamtiēk

splitim tit 1; tös; weteg

splitim lif nin

spolem anmèeg; da titisē

spot wëbënbën

sta wam̄asē

stafis, kaen malwērēt

stamba qere $_{1}$; qētēgi; turgēn $\mathbf{2}$; vötu $\mathbf{1}$

stanap tur $\mathbf{1}$

stanap strong varseg

stanemap gilgeg; vetrug

stap tog 1

stap hem wan asaw

stap kwaet tarōn

stap wetem bemrin $\bar{n}_{1} 2$

statem qētēg $\mathbf{1} ; \mathrm{ti}_{2} \mathbf{1}$ 
statem faea sōs; tuwegev

stik (blong wokbaot) tutugon

stik faea tagrës

stikim dur $\mathbf{1}$; sus 1 1; sulör

stilim bël 1

stim sësëwu bē

stingfli wutbōn

stingre vër

kaen stingre: vër dudut; vër mal; vër

matawasē; vër timiat

ston vet

bigfala ston mērēn; vet gag

waet ston vet qön

ston oven um; um val

stonem bōl 1 ; vivir

stonfis magte

stopem tiargōr; vēlig

stori kaka

storian kakaka 2

strap gabelte; gagö 4; wëlëklëk

strenja tëw

stret tenēnēg; wareg $\mathbf{2}$; wasren stretem tun tenēnēg

string blong blad gabala

strong lala; meneg; tor $_{2}$

strongbak wögörtö

stronghed qetvet

strongskin bum

kaen strongskin: batbat; bum gōlōw;

bum lam; bum lo; bum timiat;

kurkurqōl; mestalisōr; meteërqōtōn; mörögötö; ōm; ron̄ron̄oqar; sem̄; tōwav yelo tel strongskin walaglē

sugaken töv

kaen sugaken dan̄dan̄

sukwe (kilim pig) kōlkōl

sunami lolov

sutum vēn

swea $\mathrm{qo}_{4}$

swet memesgēt

swim suvsuv

swimswim gër 1

swing gakōkō n.com.; kōkō vi.

swit dēdērēs; lulum $\mathbf{1}$

\section{$\mathbf{T} \sim \mathbf{t}$}

tabu gōgōn

tabu pat qösö

tabu pat blong hem qoson

tabu pat blong mi qösök

taed (man) iamron; mat möturtur

taed (blong solwota) rēr

taem to 2 ; la masawre; masawre $\mathbf{1}$

longfala taem möul

sot taem kēti $\mathbf{2}$

taem (mekem hamas taem)

mekem eit taem vaglövötöl

mekem faef taem vagtevelēm

mekem fo taem vagvet

mekem fulap taem vagwuw

mekem handred taem vagm̄öldöl

mekem naen taem vaglövövet

mekem seven taem vaglōvōrō

mekem sikis taem vaglevetē

mekem taosen taem vagtar

mekem ten taem vagsam̄ul mekem tri taem vagtöl

mekem tu taem vagrō

taet varge

taj (natanggura) doot $\mathbf{2}$

tajem gis $\mathbf{2}_{1}$; tañqal

talem gagneg; qaq $\mathbf{1}$; vetsur; vita $_{2} ; \mathrm{wO}_{2}$

tamanu bōgōr

fols tamanu wēgēbil tamanu blong bus bōgōrēmēs

tamtam wōkōr

tanda men

tanem wil; kilo; le ris; tel ${ }_{2}$ tanem wetem spun kel tanem raon taliwög

tang garmiē

tang blong hem garmian

tang blong mi garmiēk

tangkiu varian

taosen $\operatorname{tar}_{3}$ 
taro sinag 1; qiat

grup blong taro: qiat atmēn; qiat reqe kaen taro: titiritōwvetam; wotmindeli; vinmötöl; ërmamē; rēgēt; wotliev; tewesqēqēl; mōōōl; burmatan; lakakēris ${ }_{3} ; \quad$ merlav; wotminmendala; qötuqōo ${ }_{2}$ nōvōk; römöwuler; varvarsōm; wēviti; mesvōlōvōl; wasalav; qiat gōl; qiat lēlē; wotminwērirtel; lantar malgias; wederebiliag; lomonwer; wegeretqon; galaten̄tur; rēlēgtēl; taltal; siritimiat; biliag; wamal; wasanto; mensēkē; malmalei lantar; mōlkēl; qiat mingala; tan̄evsōs; tewesvër; wotkērēvōr; marēe qiat qet; mako; sēstan̄; wēvē; wakata qagqag; qiat rev; wotminviolette; manreva; wakata mamē; tewestamal; m̄ēw; ōrbarbar; wēbigqō; din̄venqiat; tana; wēvē lamkör; wotlievgatgat; mesmamēe $_{2}$; wotmēlēv; qiat qön̄; rēsim; rēwurveg; m̄ēvinvian ${ }_{2}$; wēlēbēōr; wotvadadan̄; akrikalja; len̄man; wasanto qagqag; wotlalabēvidavaqal; $\operatorname{rov}_{2}$; qiat minwog; marē wasalav mamē; wakata gatgat; wakata; nalumlum; masta; wotminsakna; wasēe $;$ marē wasalav; wēmēlēglēg; lantar lamkör; regeltëv; ten̄tur; busuōr; siag gēgēt ${ }_{2}$; tortor; bulalēv; wotanaval; matēkmëgërsurletes; lōkreg; lantar; waras; sarē; lōkreg mamē; rōvōl; wotalēbak; götö ${ }_{2}$; susdenlē; sēlēgtel; dogon; suwbē; böl; wēbigqō mamē; wasanto mamē top blong taro n̄ili qiat

Tasde Qön̄ nivet

tatu bul

tebol tebol

tede garqe 1

tek not nak

tekem tek $\mathbf{4}$; le 1 tekem foto tōtgial $\mathbf{2}$ tekem longtaem möul $\mathbf{1}$

tekemaot (gat) vetnia

tekemaot (lif) sev 2

tel gölö

tel blong hem golon

tel blong mi gölök ten samul

tenfala (man) bulsamul

terem sēr

tes tes tes blong lulumne

tija vatōgō

tijim tono 2

tik mötöltöl; gaqō

kam tik wisiag

tiklips (fis) benmomolo yelo tiklips gōrgōrbiliag

ting (tingting) dödöm vt. ting hevi döm mav $\mathbf{1}$ tingbaot döm siër; vasērēg $\mathbf{1}$; döm kēl tingting dödömia $\mathbf{1}$ n.com.

\section{ting blong slip}

ting blong slip blong hem ban

ting blong slip blong mi bëk

tintin mēvinvin

titi sus 4 n.com.

titi blong hem sōsōn

titi blong mi susuk

titi (long) dim; sus $_{4} 2 v t$.

toelet tes ${ }_{3}$; kērēt 2

toktok eln̄e $\mathbf{2}$ n.com. toktok blong hem eln̄an toktok blong mi eln̄ëk

toktok qaq $2 v$. tok kros long göl tokbaot vëtës strong toktok tōrtōr $\mathbf{2}$

tolaen rērēv

top qese; qötu $\mathbf{2}$; teqen̄ top blong wud n̄ēli

Torba provins Torba

tosta mörö

totel ōw

totin sam̄ul tiwial dēmē nitöl

trabol matēkatēk

traeb vēnēm̄

traem daēl

trak wēvēvērēg

traosis traosis 1

traot $l_{3} \bar{O}_{3}$ vi.; lōlō n.com. traot blong smol bebe lōt

trap gatitieg kaen trap waga; wōw ${ }_{1}$; wöwöwör qēsaqēs 
trap blong rat walaqlaq gōsōw

tri (namba) töl; nitöl

tri (wud) rēntenge $\mathbf{1}$

kaen tri: ar ${ }_{1}$; ar ōl; ar tur; bēbētiqō; bitbitiqō; boboros; bōgōrēmēs; bul ; bustutun; dam̄amas; darag tōwlo; datā̄alqō; dēsēn; dōkōlōkōl; dōmōvōtrev; dōōt; dōsōw; dōvōr nem; dötöl; dunregōsōw; dun̄bönö; gan men; gasēsēdignönqet; gasōg; gatötöqulqërët; gawōlō; mala; malagavēg; malaqōōr; malmalasē; malnët; markōñ; marsam; menmav; meterarā̄at; mēl; mēēiri; m̄ēririnek; nagnagsam̄ul; nōnōñ; qetge; qēr ${ }_{1}$; qog; qō miat; rōq ${ }_{3}$; rōwō; sal ; sawa; sēqēg; sērsēr; sōgsōggenqet; $\operatorname{tagar}_{2}$; talēs toko; tarak; tēq ; titiqō; totenanar; usugiav; varvar; vēkēmolo; vēlavēl vēnvēn ${ }_{2}$; vētuboso; vōn̄ame; wakam; walag; ; wan̄arn̄ar; waran̄ran̄ marge; watamarge; wegerbak; weverbak; wēqiar $;$; wēqiqiar; wētēt ${ }_{2}$; wotag bē; wosvet; wōnōnōg malgias; wōtōgōrte; wōtōn̄; wōvōnō; wö; wöbulbulbōk; wödödö; wu; wuqiat tri handred mööldöl vagtöl

trifala (man) bultöl

troka walal ${ }_{1}$; wösusum̄alm̄al

tru turgi; dun

tu bilēñ 2 mod.; rō; nirō num.

tu handred möldöl vagōrō

tudak lōlōqōr 2; sil

tufala rōrō

tufala (man) bulrō

tugeta tiwial $_{2}$

tumas idian; nimal;

tumora tōlōw

Tusde Qön̄ nirō

tut luwö 1

tut blong hem luwon

tut blong mi luwök

tut blong pig luwö (qō)

nogat tut luwö bas

twelf sam̄ul tiwial dēmē nirō

twenti samul rō

twin varam

twistim tugeta gal

\section{$\mathbf{V} \sim \mathbf{v}$}

Vanua Lava Vōnō Lav

Vanuatu Vanuatu

vatu, $1,000 \mathrm{mil}$

vatu, 100 baon

vilej vōnō 2 voes eln̄e $\mathbf{1}$

voes blong hem eln̄an

voes blong mi eln̄ëk

volkeno vōr

\section{$\mathbf{W} \sim \mathbf{W}$}

wael kēt

waelkava dōmōtōtak

kaen waelkava dōmōtōtak mamē

waelken rartan

waelpandanas dērvag; dootnönqet

waeltaro $\mathrm{ve}_{1}$

waepem meñ waes lōlōmeren; menar

waet qag 1

waetanis qētēt

waetbun (kabis)

raminwōlōnmögörviaomōtōsur

waetman tan̄sar gaqag

waetwud gavō 


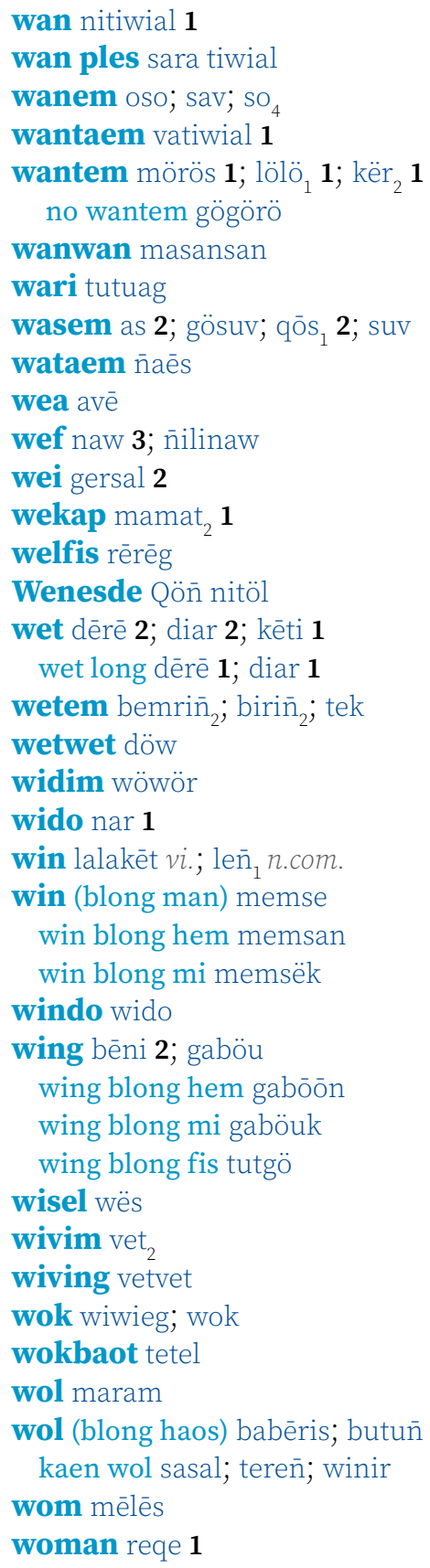

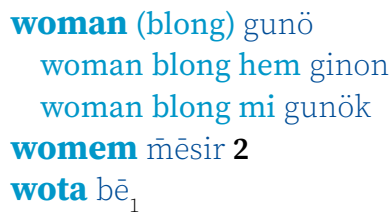
tarak; tēq ; titiqō; totenanar; usugiav; varvar; vēkēm̄olo; vēlavēl 1 ; vēnvēn vētuboso; vōn̄ame; wakam; walag ; wan̄arn̄ar; waran̄ran̄ marge; watamarge; wegerbak; weverbak; wēqiar ${ }_{2}$; wēqiqiar; wētēt ${ }_{2}$; wotag bē; wosvet; wōnōnōg malgias; wōtōgōrte; wōtōñ; wōvōnō; wö; wöbulbulbōk; wödödö; wu; wuqiat wud blong karemaot ston gabal wud we i flot wön̄sial 


\begin{tabular}{|c|c|}
\hline \multicolumn{2}{|c|}{$\mathbf{Y} \sim \mathbf{y}$} \\
\hline $\begin{array}{l}\text { yam dem } \\
\text { grup blong yam: dem atat̄̄ēn; dem } \\
\text { kēelēglēg; dem rereqe } \\
\text { kaen yam: bakalav } ; \text {; dem bōnōbōn; } \\
\text { dem ga; dem getō; dōr; mēlin̄; mat, } \\
\text { taw } 2 \text {; qōlmo; walalas; walaln̄elan; } \\
\text { wēbiēg; wēbiēg atat̄̄ēn; wēbiēg rereqe; } \\
\text { wēkēr wēlir; wōnwōn; wōqōlmōō; } \\
\text { wötörövarvar; wöuw } \\
\text { wael yam qōōr; weag } \\
\text { yangfala lōmegev } \mathbf{1}\end{array}$ & $\begin{array}{l}\text { yelo an̄an̄ } \mathbf{1} \\
\text { yes ke }{ }_{1} \text {; e'e } \\
\text { yestedei nonor } \\
\text { yet vēti } \\
\text { yia to } 1 \\
\text { yu nēk } \\
\text { yufala kēmi } \\
\text { yumi nēn } \\
\text { yumitu dōrōk } \\
\text { yuniti bultiwial } \\
\text { yutufala kōmōrōn̄ }\end{array}$ \\
\hline
\end{tabular}

\section{$\mathbf{Z} \sim \mathbf{Z}$}

zipa metegongon 



\section{Organisation of the thesaurus}

This thesaurus presents Vurës words organised according to semantic categories. This means that the words are grouped together according to their meaning, rather than alphabetically as in the main dictionary and finderlists. The structure of the thesaurus is based on that of Tonya Stebbins' Mali (Baining) Dictionary (2012). It is important to note that there are many different ways in which the words of a language could be grouped together on the basis of meaning, and the categories that are distinguished here reflect my interpretation based on my knowledge of Vurës language and culture. The categories do not reflect a particular Vurës interpretation of how words should be grouped together. There is no doubt that Vurës speakers will agree with some of my categorisations, but not all. Mali is a language spoken in Papua New Guinea, which like Vanuatu is a Melanesian country, and there are thus some sociocultural similarities between the two language communities. Both languages are spoken in similar tropical island environments by communities of subsistence farmers who place an emphasis on interaction with and use of the marine and rainforest environments. I therefore found that, for the most part, the categories distinguished by Stebbins for Mali reflect categories that are appropriate for Vurës. I added and removed some categories, partly on the basis of biases in the inclusions in the Vurës data, and partly on my interpretation of the data.

The primary motivation for including this type of thesaurus is so that it can be used to support teaching of literacy skills and cultural knowledge within the community. Teachers and parents are encouraged to use this section of the dictionary to focus on reinforcing traditional knowledge, particularly with young community members. It is for these users that many photos are included, so that a topic can be chosen, such as fish, plants or weaving, and Vurës people can practice learning the words, spelling and meanings of local concepts.

The categories that the thesaurus is organised into are listed below. Note that the thesaurus only includes the Vurës word, English and Bislama and meanings, and scientific name where relevant. Any user who would like more information for a particular word, such as part of speech or an example sentence, should check the word in the main dictionary for that information. Note also that if a word has more than one sense, then only the sense(s) relevant for the particular semantic category are included for an entry. Thus a word might be included under one heading for one sense, but under a different heading for a different sense. 


\section{Contents of Thesaurus}

\section{The natural world}

A Landscape, country and water

A0 General

A1 Topographical features (valley, hill, river, road)

A2 Celestial features (moon, stars)

A3 Water

A3.0 General (drown, splash, wet)

A3.1 Precipitation (hail, mist, dew)

A3.2 Water sources (springs, puddles, lakes)

A3.3 The ocean (waves, tides)

A4 Geological, other substances (sand, plastic, charcoal)

$B$ Weather and cyclic time

B1 Meteorological conditions (wind, cloud, thunder)

B2 Cyclical time

B2.1 Diurnal cycle (early morning, evening)

B2.2 Lunar cycle (new moon, high moon)

B2.3 Seasonal cycle (seasons, months, holidays)

B2.4 Weekly cycle (names of days)

B3 Geological events (volcanic eruption, earthquake)

C Spatial relations

C0 General

C1 Absolute direction (landward, seaward)

C2 Demonstratives (this, that over there)

C3 Pronouns

D Plants

D0 General (thorn, twig, leaf, bark)
D1 Trees and palms (rosewood, bush palm, orange tree)

D1.1 Coconuts

D1.2 Breadfruit

D1.3 Bananas

D2 Bushes, shrubs

D3 Grasses, bamboo, vines

D4 Tubers and roots

D4.1 Taro

D4.2 Yam

D4.3 Cassava

D4.4 Sweet potato

D5 Miscellaneous plants (seaweed, moss, mushrooms)

E Fauna

E0 General

E1 Mammals

E2 Birds

E3 Reptiles

E4 Water creatures

E4.0 Large sea creatures (sharks, dolphins)

E4.1 Fish

E4.2 Eels

E4.3 Prawns and crayfish

E4.4 Shell fish (oysters, shells)

E4.5 All other sea and shore life (sea cucumber, jellyfish)

E5 Crabs and hermit crabs

E6 Insects, other land arthropods, grubs

F $\quad$ Physical properties

F0 General

F1 Appearance

F1.1 Visibility and colour

F1.2 Size, shape, weight, pattern

F2 Temperature, texture 
F3 Sounds - their types and qualities

F4 Tastes and smells (sour, bitter, sweet, stink)

F5 Value (precious, worthless)
$G \quad$ Quantification

G1 Quantity (lots, another of the same type, nothing)

G2 Numerals

\section{Humans, human qualities}

H Human classification

H0 General (names, to name someone)

H1 Spirits and devils

H2 Peoples and language groups

H3 People by age, development, life cycle

H3.0 General (adolescent, infant)

H3.1 Male (young boy, adolescent male)

H3.2 Female (young girl, adolescent female)

H4 Roles and status terms

H5 Types of people not related to status/profession

H6 Address terms

I Human qualities and emotions

I1 Personality and moral evaluations (funny, talkative)

I2 Skill, ability, power (strong, courageous, skilled)

I3 Feelings and emotions (homesickness, jealous)

J Body parts and products (animal and human)

J0 General (head, body, egg)

J1 Internal organs (heart, stomach)

J2 Body products (urine, earwax, saliva)

J3 Extension of body (soul, voice, scar)

K Body states and functions

K1 Physiological reactions (breath, suck, cry, lay eggs)

K2 Body states
K2.1 Temporary (sleep, tired, thirsty, pregnant, bleed)

K2.2 Permanent (blind, deaf)

K3 Sickness, death, disease, injury (unconscious, vomit)

$L \quad$ Stance and motion

L1 Stance, position

L1.0 Stance, position - general (rest, crouch, wait)

L1.1 Stance, position - vertical axis (stand)

L1.2 Stance, position horizontal axis (sit, lie down)

L2 Movement

L2.0 Movement - general (arrive, fly, paddle, chase)

L2.1 Movement - vertical axis (walk, up, climb, dive)

L2.2 Movement - horizontal axis (roll, go across)

L3 Open and close

L3.1 Open (hole, gap, orifice, expose, empty)

L3.2 Close (block, obstruct, finish, switch off, fill)

L4 Exist (be, be lost)

L5 Do (attempt, succeed, make)

L6 Change of state (become)

$M$ Artefacts, material culture and manufactured things

M1 Tools, weapons, belongings

M2 Built environment

M2.0 General (build a house, housework, fence)

M2.1 Parts of a building (house posts, doorway)

M2.2 Furniture 
M3 Clothes, materials and decorations (coat, headdress)

M4 Weaving, woven goods (mat, basket, pandanus)

M5 Nautical (canoe, paddle, sail, anchor)
M6 Transfer of goods (sell, trade, steal)

M7 Vehicles (car, truck, motorcycle, aeroplane)

\section{Human interrelating, humans socially}

$N$ Kinship

N1 Kin terms

O Marriage, sex, nurturing

O1 Marriage and sexual relations

O2 Pregnancy and childbirth (birth, stages of pregnancy)

O3 Rearing babies and children (adoption, look after)

$P \quad$ Speaking, communication

and sounds

P1 Speaking (call out, ask, stutter, explain)

P2 Interjections, imperatives, function words

P2.1 Functional affixes and particles

P2.2 Interjections

P2.3 Conjunctions

P2.4 Subordinators

P2.5 Articles

P2.6 Discourse markers

P3 Negations, assertions, possibility (perhaps, true, no)

P4 Non-verbal communication (write, sign language)

P5 Sounds (dog growl, whistle, clapping, onomatopoeia)

Q Social interaction, relationships

and behaviour

Q0 General (behave, warn someone of trouble)

Q1 Fighting (enemy, get revenge)

Q2 Verbal quarrelling (provoke, ridicule, tease, annoy)

Q3 Making peace, maintaining social harmony
Q4 Games, entertainment, laughter, joking (play)

Q5 Being affected (being helped)

Q6 Permission and prohibition

Q7 Community 


\section{Human interaction with the natural world}

$R$ Religion, ceremony, law and the supernatural

R0 General

R1 Law, sacred places, legends

R2 Ceremony and rituals

R2.1 Ceremonial objects

R2.2 Ceremonies and rituals

R3 Death and mourning

R4 Taboos, strictures and secret knowledge

R5 Healing and medicine

R6 Singing and dancing

R7 Feasting

R8 Christianity

$S \quad$ Hunting, fishing, farming

S1 Hunting

S2 Fishing

S3 Farming and propagation (clearing land, planting)

$T \quad$ Fire and cooking

T1 Fire (cooking fire, matches, burn, smoke)

T2 Food preparation, cooking, distribution, eating (ripe)

$U$ Human uses of natural resources

U1 Food (meat, berries, bread)

U2 Narcotics (alcohol, tobacco)

$V \quad$ Thinking, perception and attention

V1 Sense and perception

V1.1 Seeing (look out, inspect, search)

V1.2 Hearing (listen)

V1.3 Tasting and smelling (try, taste)

V1.4 Physical sensations (feel)

V2 Cognition

V2.1 Mental states (be calm, be delirious)

V2.2 Mental processes and experiences (think, dream)

V2.3 Knowledge and learning (try, graduate)
W Handling and physical transfer

W0 General

W1 Taking, touching, obtaining (snatch, receive, put on)

W2 Holding, carrying, moving something around (lead)

W3 Letting go, giving, leaving be (pour, transfer, spread)

$X \quad$ Impact and surface contact

X0 General (split apart, throw, rip, break, dismantle)

X1 With an instrument (spear, mark, chop)

$\mathrm{X} 2$ Due to pressure (grind, snap, twist, burst)

X3 Sticking, tying, untying (tie up, cover)

X4 Surface contact (rub, peel, scrape, wipe)

$Y$ Speed, manner and relative time

Y0 General (how, like this, manner)

Y1 Speed and manner (fast, carefully, fiercely)

Y2 Relative time and duration (when, previously, new)

Y3 Serialisation (before doing something else, last)

Y4 Degree 


\section{A Landscape, country and water}

\section{AO General}

\section{maram world wol \\ masawre $2 \cdot$ place $~$ ples \\ $\tan _{1} \mathbf{1} \cdot$ earth, soil $\sim$ graon $\mathbf{2} \cdot$ ground $\sim$ graon $\mathbf{3} \cdot$ land $\sim$ graon \\ tönö place $\sim$ ples \\ vene place, owned area of land, place belonging to person or community ples, olsem ples blong man, ples we ol pipol ol i stap long hem \\ vanan her/his/its place $\sim$ ples blong hem \\ vënëk my place ples blong mi}

\section{A1 Topographical features}

bēn lo brackish water, spring water that comes up by the sea and thus is slightly salty but drinkable wota we i spring aot long solwota, yu save dring be i sol smol

bōak 1・ mud sofmad

bōrōt shore, sea shore, strip of sand or land close to the sea $\sim$ so, sanbij o eria we i klosap long solwota

dō cliff klif

gawuw valley $\sim$ vali

geret mountain, high hill maonten, tolfala hil

gersal $1 \cdot$ path, road $\sim \operatorname{rod}$

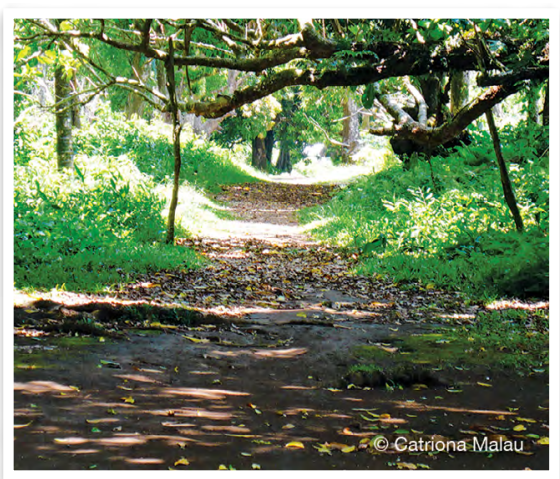

gersal $\sim$ path, road gersal liwō road, main road suitable for vehicles $\sim$ rod, men rod gersalan her/his/its path rod blong hem

gersëlëk my path rod blong mi

kalkal hill, slope of hill hil

lam deep water, deep freshwater pool, deep sea $\sim$ dip wota, dip solwota, dip ples

butölam very deep sea solwota $i$ dip olgeta

vētitnē lam deep sea between islands medel si bitwin tu aelan

le $\overline{\mathbf{e}}_{1}$ cave $\sim$ kef

lie hole hol

lie giav crab hole hol blong krab

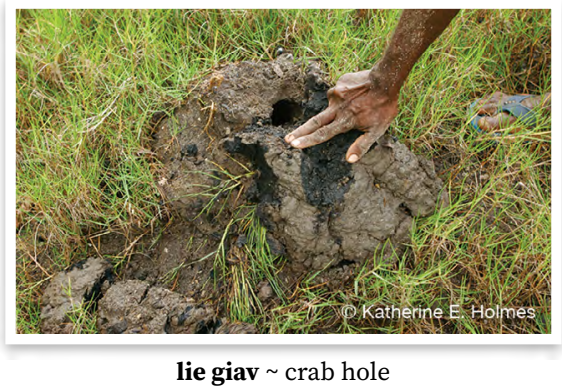

lo ${ }_{1}$ seashore, seaside, area by sea, but not including sea itself solwota, eria klosap long solwota

met area of reef close to shore that is covered during high tide but exposed during low tide $\sim$ rif, ples we solwota mo rif i stap tugeta mo man i save wokabaot long hem mo yusum blong fising

met siag tide is neither high nor low solwota i stap semak nomo, i no save drae mo i no save kam so.

met sörlav time when there is a very high high tide $\sim$ taem we solwota $i$ kam so we i bitim mak blong evri dei long manis 
mësu met ōrōr extreme low tide, which enables reef gleaning of species that would normally not be accessible $\sim$ taem solwota $i$ drae bigwan, mo i minim se yu save karem sam mit we mos taem i stap long dip ples

met mōo ōr low tide, tide going out solwota i drae

mete $1 \overline{0} \mathbf{1} \cdot$ east, place where sun rises $\sim$ ples we san i kam antap 2 • expression used to refer to Sola by people in Vurës, as Sola is towards the east, where sun rises $\sim$ wan wei blong talemaot Sola, from i stap long is, long ples we san i kam antap metesa anchorage, harbour, landing place $\sim$ pasis

möt 1 - bush bus 2 - overgrown, overgrown bushy area of land $\sim$ bus, eria we ol i no klinim gud

möt luwō forest fores, bigfala bus möt vandam̄eg isolated location, interior of island $\sim$ medel bus

nögö lō east, place where sun rises ples we san i kam antap

nenere garden $\sim$ garen

nere ${ }_{2} \mathbf{3}$ point of land $\sim$ poen

nere on stretch of beach where the sand meets the sea ples we sanbij i kasem solwota

nusu $3 \cdot$ point of land $\sim$ poen

ōn $\mathbf{1} \cdot$ sand $\sim$ sanbij $\mathbf{2}$ beach $\sim$ sanbij on wöt place where there is sand and surfable waves $~$ ples we $\mathrm{i}$ gat wef

plantesen plantation plantesen

qarörö hole hol

qereñ bè watercourse, creek gully, place where there is a creek, including creek and edge around it $\sim$ hol blong wota

qereñ ōlōl cave, hole in reef or pool that has special significance, where people go to pray, give money and ask the spirits for something that they want, or to appease and ask forgiveness from the spirits if they have done something wrong against them tabu hol long kef, long rif o long wota we ol man ol i go long hem blong prea long ol speret from wan samting o talem sori from wan samting we yu bin mekem

raras shallow, shallow place $\sim$ salo, ples we wota i no dip

rat flat place, area of land flat ples

rōwnölō east, place where sun rises

is, ples we san i kam antap

sere plen airfield $\sim$ epot

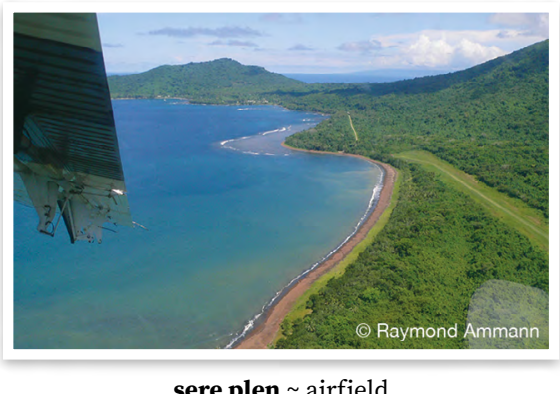

sëkër reef, coral, refers both to an area of reef and to coral heads that comprise the reef $\sim$ rif

$\tan _{1} \mathbf{1} \cdot$ earth, soil $\sim$ graon $2 \cdot$ ground graon $\mathbf{3} \cdot$ land $\sim$ graon

tes lake, large body of water $\sim$ bigfala wota, lek

tique garden $\sim$ garen

tōw hill, mountain hil, maonten

tulnö lō west, place where sun sets wes, ples we san i draon

velesgon plants growing closely together so one can't get through bus i fas

vene place, owned area of land, place belonging to person or community ples, olsem ples blong man, ples we ol pipol ol i stap long hem

vanan her/his/its place $\sim$ ples blong hem

vënëk my place ples blong mi 
vere wutwut mound, pile, rise of land from where one can see over lower land hip, hip blong graon o sanbij o smol top blong hil

vōnō $\mathbf{1} \bullet$ island aelan $\mathbf{2} \bullet$ village $\sim$ vilej nögö vōnō whole village, look or appearance of village $\sim$ eria blong vilej

vōr volcano volkeno

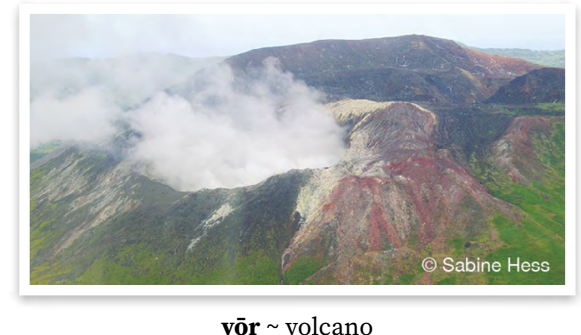

wömöötmöt area of bush, uninhabited area away from villages $\sim$ eria blong bus

\section{A2 Celestial features}

\section{$\mathbf{l o}_{1} \mathbf{1} \cdot \operatorname{sun} \sim \operatorname{san}$}

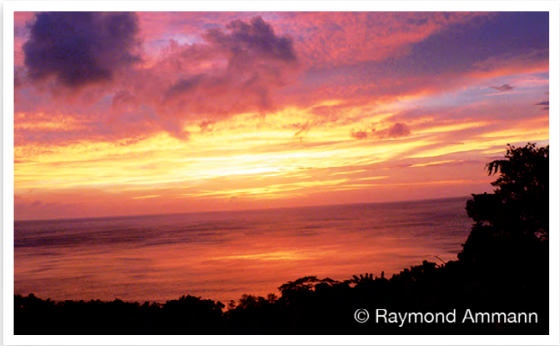

$1 \overline{\mathbf{o}} \sim \operatorname{sun}$

maē sky, open sky, high in air skae, antap long ea

wamas̄e $\overline{\mathbf{e}}_{1}$ star sta

wōl 1 1 moon mun

wōlmeeñ new moon fes saen blong mun i kam antap, taem i gat mun be i no saen gud yet

wōlmian full moon ful mun

wōlōbōl new moon niu mun

wōlsial moon that is visible during daylight $\sim$ mun we i stap antap yet taem i deilaet finis

\section{A3 Water}

\section{A3.0 General}

$\mathbf{b} \overline{\mathbf{e}}_{1} \mathbf{1} \cdot$ fresh water $\sim$ freswota $\mathbf{2} \cdot$ river $\sim$ wota

bē mamas dry creek bed drae wota, rod blong wota

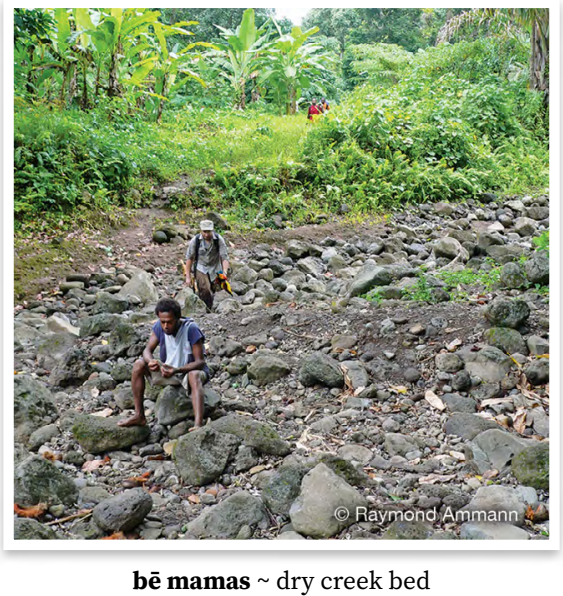

kērē bē mouth of river, place where river meets sea as blong wota, ples we wota i joenem solwota

mete bē source of river, creek ae blong wota, ples we wota i stat nögö bē place where the irrigation channel is directed into a taro paddy $\sim$ ples we ol i joenem wota i go insaed long garen taro

nötu bē stream smol reva

bēn lo brackish water, spring water that comes up by the sea and thus is slightly salty but drinkable wota we i spring aot long solwota, yu save dring be i sol smol

döw wet, be wet $\sim$ wetwet

lam deep water, deep freshwater pool, deep sea dip wota, dip solwota, dip ples

butölam very deep sea solwota $\mathrm{i}$ dip olgeta

vētitnē lam deep sea between islands medel si bitwin tu aelan '̄ōrmōōrse bubbles, foam babol 
mōōmōōsenaw sea foam spet blong solwota

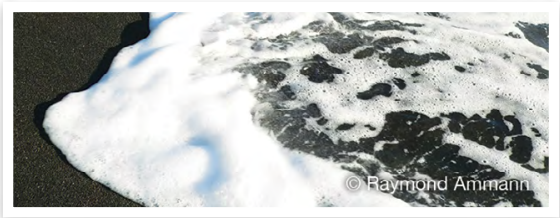

mōōrmōōrsenaw sea foam

$\mathbf{q} \overline{\mathbf{o}} \mathbf{S}_{2}$ run strongly, rapidly (of water) (wota i) ron strong qōsqōs large movement of water, as in wake of boat, water stirred up by propellers, etc. wota i muv bigwan, olsem from enjin blong bot

raras shallow, shallow place $\sim$ salo, ples we wota i no dip

sēriv waterfall wotafol

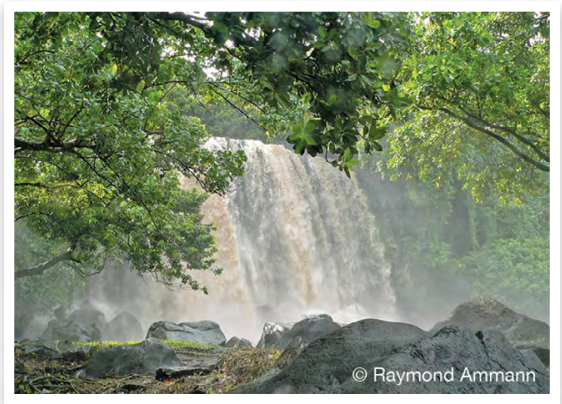

sēriv waterfall

taw $_{5}$ watercourse that starts after rain in a place where there was no water before wan wota we i stat niu long ples we i drae o i nogat wota bifo, be afta $i$ ren mo i gat wota

tin̄i $1 \cdot$ liquid, juice from something, as from fruit or sauce, liquid from cooked food wota (blong frut, kakae) $\mathbf{2}$ - sap of plant wota blong wud

tul $1 \cdot$ drown, fall down from height draon $\mathbf{2} \cdot$ submerge $\sim$ draonem

tul viteg draonem i go

ut ${ }_{2}$ collect water $\sim$ kasem wota
A3.1 Precipitation

deli very heavy rain $\sim$ ren we i strong we i strong

mërëv mist, fog sno, samting olsem smok we i kavremap ples taem i kolkol

miöw mist, fog samting olsem smok we i kavremap ples taem i kolkol ninin shower, light rain smol ren re $\overline{\mathbf{e}}_{2}$ rain $\sim$ ren

ten $_{3}$ rain $\sim$ ren

wian 1 rain $\sim$ ren

löt wian get rained on, not worry about getting wet in rain $\sim$ swim long ren

\section{A3.2 Water sources}

$\mathbf{b} \overline{\mathbf{e}}_{1} \mathbf{1} \cdot$ fresh water $\sim$ freswota $\mathbf{2}$ - river $\sim$ wota

bē mamas dry creek bed $\sim$ drae wota, rod blong wota

kērē bē mouth of river, place where river meets sea as blong wota, ples we wota i joenem solwota

mete bē source of river, creek ae blong wota, ples we wota i stat

nögö bē place where the irrigation channel is directed into a taro paddy ples we ol i joenem wota i go insaed long garen taro

nötu bē stream smol reva

mete qōsqōs source of spring water ples we spring wota i stat long hem mete vuvrës source of spring water ples we spring wota i stat long hem metewōs water source ples we wota i kamaot long hem

qulös pool pul

sol flow heavily (wota i) ron bigwan

A3.3 The ocean

beret $_{2}$ surface of sea antap blong solwota

dōrdōrse naw tideline ples we solwota i finis long hem

lum (tide) come in (solwota i) kamso 
met mö lum high tide, tide coming in solwota i kam so

met area of reef close to shore that is covered during high tide but exposed during low tide $\sim$ rif, ples we solwota mo rif i stap tugeta mo man i save wokabaot long hem mo yusum blong fising

met siag tide is neither high nor low solwota i stap semak nomo, i no save drae mo i no save kam so.

met sörlav time when there is a very high high tide $\sim$ taem we solwota $i$ kam so we i bitim mak blong evri dei long manis

mësu met ōrōr extreme low tide, which enables reef gleaning of species that would normally not be accessible $\sim$ taem solwota i drae bigwan, mo i minim se yu save karem sam mit we mos taem i stap long dip ples

met mō ōr low tide, tide going out solwota i drae

naw $\mathbf{1} \bullet$ sea $\sim$ solwota $2 \cdot$ sea water, salty water $\sim \operatorname{solwota} \mathbf{3} \cdot$ wave (of sea) $~$ wef (blong solwota)

dōrdōrsenaw tide mark mak we solwota i kam so, we yu harem noes blong solwota long ples ia nawēut rough sea solwota i raf nere on stretch of beach where the sand meets the sea ples we sanbij i kasem solwota

nilinaw wave $\sim$ wef

rēr tide $\sim$ taed

siōl go ashore kamso

$\operatorname{tar}_{2} \mathbf{1} \cdot$ calm sea $\sim$ solwota i kwaet $\mathbf{2}$ • (sea) become calm solwota i kam kwaet

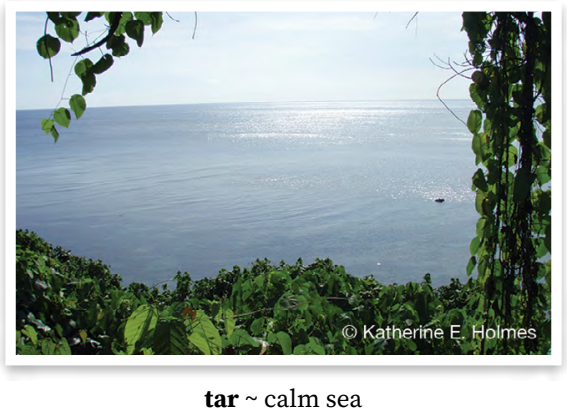

tiabasas go ashore kamso

viaw mes sea is neither calm nor rough solwota i no raf be i no kwaet

A4 Geological, other substances

lolov tsunami, tidal wave $~$ sunami, bigfala wef

rir earthquake etkwek

\section{B Weather and cyclic time}

\section{B1 Meteorological conditions}

butöliaw rainbow $\sim$ renbo

len $\overline{\mathbf{n}}_{1} \mathbf{1} \cdot$ wind $\sim$ win $\mathbf{2} \cdot$ air $\sim$ win

leñlimerlav southeast tradewind

$\sim$ tokelau, saotis win we i kam long Mere Lava

len̄limot northeasterly wind win i kam long notis long Mota

len̄lirō northerly wind win i kam long not long Reef Aelan

leñvus hurricane, cyclone $\sim$ hariken malēg cloud klaod

masalav southerly wind $\sim$ win we

i kam long saot

men $_{2}$ thunder $\sim$ tanda

mëw $\mathbf{w}_{2}$ dew $\sim$ drop wota long gras long

eli moning

rēe rain $\sim$ ren

sēlēg dark and cloudy, rain clouds tudak klaod, saen blong ren sēsēlēg dark, of sky, place (ples i) tudak 
taqtaq overcast, cloudy gat klaod, i no san be i no ren

ten $_{3}$ rain $\sim$ ren

tōk blue sky blu skae

tōkmereñ clear blue sky with no clouds skae we i blu nomo mo nogat klaod

tōwlo westerly wind $\sim$ win we i blo long wes

vēl lightning laetning

wian $_{1}$ rain $\sim$ ren

löt wian get rained on, not worry about getting wet in rain $\sim$ swim long ren

B2 Cyclical time

\section{B2.1 Diurnal cycle}

lölön̄ö vōrōg dawn, early morning eli moning

luwömeren midday, middle of day medel dei

meren $\mathbf{1} \cdot$ day, daytime $\sim$ dei $\mathbf{2} \cdot$ daylight $\sim$ deilaet $\mathbf{3} \cdot$ be daylight, become day $\sim$ kam deilaet

qön̄ $\mathbf{1}$ • night $\sim$ naet $\mathbf{2} \cdot$ day (as measurement of time) $\sim$ dei (olsem blong tokbaot hamas dei) $\mathbf{3}$ • become day kam dei

revrev late afternoon, evening; period preceding and following sunset aftenun, ivining

tinësqöñ middle of night $\sim$ medel naet vōrōg $\mathbf{1} \cdot$ morning moning $\mathbf{2}$ • be morning kam moning

\section{B2.2 Lunar cycle}

wōl 1 1 moon mun $\mathbf{2} \cdot$ month $\sim$ manis wōlmeeñ new moon fes saen blong mun i kam antap, taem i gat mun be i no saen gud yet

wōlmian full moon ful mun

wōlōbōl new moon niu mun

wōlsial moon that is visible during daylight $\sim$ mun we i stap antap yet taem i deilaet finis
B2.3 Seasonal cycle

mar famine taem we i nogat kakae $\overline{\mathbf{m}} \overline{\mathbf{o g}} \mathbf{\mathrm { t }} \mathrm{t}_{2}$ name of a season, when $\bar{m} \bar{o} g \bar{t} t$ flowers, just before dry season of rar, roughly February, March nem blong wan sisen blong yia, taem blong $\bar{m} \overline{o g} \bar{t}$ i gat flaoa, bifo long drae sisen

$\operatorname{rar}_{3}$ season when rar flowers, time of dry, cold weather, April to August sisen we narara hem i gat flaoa, hem i taem blong kolkol, stat long Eprel kasem long Ogis

to $\mathbf{1} \cdot$ year $\sim$ yia $\mathbf{2} \cdot$ season $\sim$ taem töu len hurricane season $\sim$ taem blong win, taem blong saeklon

\section{B2.4 Weekly cycle}

garqe $\mathbf{1} \cdot$ today $\sim$ tede $\mathbf{2} \cdot$ later on today, today in the afternoon or evening; earlier today, earlier the same day tede long aftenun, tede long moning finis $\mathbf{3} \cdot$ at the present time or age; these days $\sim$ long taem tede

in̄ko now $\sim$ nao

Made Monday Mande

nonor yesterday yestedei

nonorēs recent time period before yesterday $\sim$ bifo yestedei

naēs when $\sim$ wataem

orēs time period in the near future, after tomorrow afta tumoro

Qön̄ nirō Tuesday Tusde

Qön̄ nitöl Wednesday Wenesde

Qön̄ nivet Thursday Tasde

Qöñ tevelēm Friday Fraede

Sade Sunday $\sim$ Sande

Sarere Saturday $\sim$ Sarede

tōlōw tomorrow tumora

wik week wik

B3 Geological events

lolov tsunami, tidal wave $\sim$ sunami, bigfala wef

rir earthquake etkwek 


\section{Spatial relations}

CO General

$a_{1}$ locative, to, at, marking absolute location nouns long, maka we i go wetem ol nem blong ples

aqit (root qit) far longwe, farawe

arës (root rës ${ }_{2}$ ) distant, far, far away longwe, farawe

beriri side $\sim$ saed

bersi side, edge $\sim$ saed

$\mathbf{e}_{2} \mathbf{1}$ - that, distal modifier; as a modifier of locationals, indicates a greater distance $\sim$ ia longwe

gēn (fr.var. gēnak; igēn) this, this one, proximal demonstrative $\sim$ ia, hemia ilēkè (fr.var. lēkē) that one over there, distal presentative $\sim$ hemia longwe

iloko (fr.var. loko) this here, proximal presentative $\sim$ hemia, hemia ia

ilsi end $\sim$ en

ine (abbrev. ne) that, anaphoric demonstrative, referring back to a previously mentioned participant or event $\sim$ ia, wan samting we yu bin tokbaot finish

in̄ke this, this one, proximal demonstrative $\sim$ ia, hemia

$\mathbf{k a l}_{2} \mathbf{1} \cdot$ go up, motion or event directed upwards go antap $\mathbf{2} \cdot$ go landward, ashore, inland go antap long so, long bus $3 \cdot$ go upstream $\sim$ go antap (folem wota) $\mathbf{4} \cdot$ travel in a southeasterly direction (of travel beyond island to other islands) go long saot long narafala aelan $\mathbf{5} \cdot$ go inside, enter $\sim$ go insaed

kalō go outside $\sim$ go aotsaed

ken (fr.var. iken; ikene; kene) that one, that one there, distal demonstrative $\sim$ hemia

kikse side (of) saed (blong)

ko ${ }_{1}$ this, proximal modifier $\sim$ ia kulë 2 - behind, at the back, after (place or event) bihaen, afta kulen rōrō behind the two of them bihaen long tufala

laln̄e underneath andanit

linereg inside $\sim$ insaed

lo ${ }_{2}$ locative preposition, to, at, on, marking common nouns $\sim$ long

$\mathbf{l} \overline{\mathbf{o}}_{2}$ out, come out, move out $\sim$ aot, kamaot

lölö ${ }_{2}$ inside $\sim$ insaed

masawre $2 \cdot$ place $\sim$ ples

me 1 - towards speaker, in a direction towards the deictic centre $\sim \operatorname{kam} 2$. close by, proximal location, close to deictic centre $\sim$ ia, ples klosap long man we i toktok

mēēē top of, above, over antap

net towards addressee, in direction or facing towards person being spoken to $\sim$ i go long man we yu toktok long hem

nögö 2 - front, at the front, before fran

oko (abbrev. $\mathrm{ko}_{2}$ ) here, proximal location ples ia

qese top (of something tall, as person's head or hill) top (blong wan samting we i tolfala, olsem man o hil)

qese tōw top, peak of hill top blong hil

qötu $\mathbf{2}$ - top top $\mathbf{3} \cdot$ front, as in bow of ship, front of canoe, car $\sim$ fored, olsem blong kenu o trak

qötu vōnō area of village that is the section or edge towards the inland pat blong vilej we i stat long saed i go antap long bus

rivteg $\mathbf{1}$ - near, close by $\sim$ klosap 2 • near to, close to $\sim$ klosap long 
rōw 2 1 seaward, in a direction out to sea $\sim$ daon long solwota $2 \cdot$ outside $\sim$ aotsaed $\mathbf{3} \cdot$ go out, go outside $\sim$ go aotsaed, kamaot $4 \cdot$ come out and show oneself, appear to $\sim$ kamaot long

$\operatorname{sar}_{2} \mathbf{1} \cdot$ inland, landward $\sim$ antap (long bus) $\mathbf{2} \bullet$ inside $\sim$ insaed

$\operatorname{siag}_{2} \mathbf{1} \cdot$ upward $\sim \operatorname{antap} \mathbf{2} \cdot$ to the left when facing the sea, of places which are some distance away, not close by fes i go daon long solwota, go long lefsaed, be longwe lelebet

sōw $1 \cdot$ towards the right when facing the sea, in a clockwise direction around the island sapos yu fes $i$ go daon long solwota, go long raetsaed 2 - down, downwards, down low $\sim$ daon, stap daon $\mathbf{3} \cdot$ go down $\sim$ go daon

tavalgi $\mathbf{1} \bullet$ side $\mathbf{2} \cdot$ end $\sim$ en

tawle under, underneath $\sim$ andanit

teqeñ top top

tēqēl $1 \cdot$ go down, motion or event directed downwards $\sim$ go daon $\mathbf{2}$ • go seaward, go further out to sea go long solwota $\mathbf{3} \cdot$ go downstream $\sim$ go daon (folem wota) $4 \cdot$ travel in a northwesterly direction (of travel beyond island to other islands) $\sim$ go long not long narafala aelan

tëwru back bihaen

tuwur (fr.var. töwör) behind bihaen tutuwur after afta; bihaen

varē outside, open space outside of an enclosed space, as outside a house, in the open area of a village, on an open vehicle $\sim$ aotsaed

wōl ${ }_{2}$ to the left when facing the sea, in an anticlockwise direction around the island $\sim$ fes $i$ go daon long solwota, go long lefsaed
C1 Absolute direction

$\mathbf{k a l}_{2} \mathbf{1} \cdot$ go up, motion or event directed upwards go antap $2 \cdot$ go landward, ashore, inland go antap long so, long bus $3 \cdot$ go upstream go antap (folem wota) $\mathbf{4} \cdot$ travel in a southeasterly direction (of travel beyond island to other islands) $\sim$ go long saot long narafala aelan $\mathbf{5}$ - go inside, enter $\sim$ go insaed

rōw $\mathbf{w}_{2} \mathbf{1} \cdot$ seaward, in a direction out to sea $\sim$ daon long solwota $2 \cdot$ outside $\sim$ aotsaed $\mathbf{3} \cdot$ go out, go outside $\sim$ go aotsaed, kamaot $4 \cdot$ come out and show oneself, appear to $\sim$ kamaot long

$\operatorname{sar}_{2} \mathbf{1} \cdot$ inland, landward $\sim$ antap (long bus) $2 \cdot$ inside $\sim$ insaed

sōw $1 \cdot$ towards the right when facing the sea, in a clockwise direction around the island sapos yu fes $i$ go daon long solwota, go long raetsaed 2 - down, downwards, down low $\sim$ daon, stap daon $\mathbf{3} \cdot$ go down $\sim$ go daon

tēqēl 1 - go down, motion or event directed downwards $\sim$ go daon 2 • go seaward, go further out to sea go long solwota $\mathbf{3} \cdot$ go downstream $\sim$ go daon (folem wota) $\mathbf{4} \cdot$ travel in a northwesterly direction (of travel beyond island to other islands) $\sim$ go long not long narafala aelan

wōl ${ }_{2}$ to the left when facing the sea, in an anticlockwise direction around the island fes i go daon long solwota, go long lefsaed

\section{Demonstratives}

$\mathbf{e}_{2} \mathbf{1}$ that, distal modifier; as a modifier of locationals, indicates a greater distance $\sim$ ia longwe 2 - emphatic demonstrative, when modifying pronouns, specifies emphasis $\sim$ ia nao 
gēn (fr.var. gēnak; igēn) this, this one, proximal demonstrative $\sim$ ia, hemia ia here $\sim$ ia

ilēkē (fr.var. lēkē) that one over there, distal presentative hemia longwe

iloko (fr.var. loko) this here, proximal presentative $\sim$ hemia, hemia ia

ine (abbrev. ne) that, anaphoric demonstrative, referring back to a previously mentioned participant or event $\sim$ ia, wan samting we yu bin tokbaot finish

in̄kē this, this one, proximal demonstrative $\sim$ ia, hemia

ken (fr.var. iken; ikene; kene) that one, that one there, distal demonstrative $\sim$ hemia

ko this, proximal modifier $\sim$ ia

me 1 - towards speaker, in a direction towards the deictic centre $\sim \operatorname{kam} 2 \cdot$ close by, proximal location, close to deictic centre $~ i a$, ples klosap long man we i toktok

oko (abbrev. $\mathrm{ko}_{2}$ ) here, proximal location ples ia

soko emphatic marker, expression of surprise or emphasis regarding an item or event $\sim$ hemia ia

\section{C3 Pronouns}

dōrōk we two, us two; first person dual inclusive pronoun $\sim$ yumitu

kemem (fr.var. kemek) we, us; first person plural exclusive pronoun mifala

kēmi (fr.var. kimi) you, you all; second person plural pronoun yufala

kōmōrōk we two, us two; first person dual exclusive pronoun $\sim$ mitufala

kōmōrōñ you two; second person dual pronoun $\sim$ yutufala

nē he, she, it, him, her; third person singular pronoun $\sim$ hem

nēk you; second person singular pronoun $\sim$ yu

nēn (fr.var. $\operatorname{nin}_{2}$ ) we, us; first person plural inclusive pronoun $\sim$ yumi

nèr they, them; third person plural pronoun olgeta

no I, me; first person singular pronoun $\sim \mathrm{mi}$

rōrō they two, them two; third person dual pronoun $\sim$ tufala

\section{Plants}

\section{Do General}

butu thorn (of citrus fruits) nil (blong aranis)

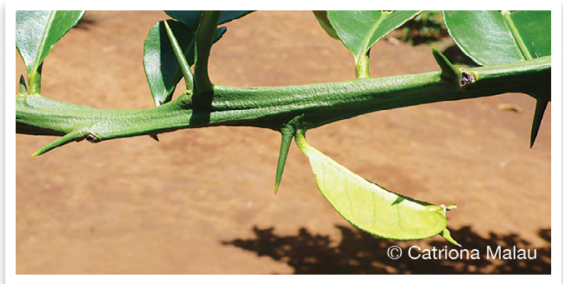

butu thorn (of citrus fruits) bōl ${ }_{2}$ unripe, ripening but not yet ripe $\sim$ klosap i raep be i no raep gud yet

bur ñe canarium sap wota blong nangae

damarēnit pandanus leaves that have dried on the branches $\sim$ lif pandanas we i drae nomo long stamba

dege leaf $\sim$ lif

delti buttress root, kind of tree root which starts high up on the trunk and is wide and long, reaching down to the ground $\sim$ kaen rus blong wud we hem i stat antap long stamba 
mo i kasem daon long graon, olsem blong nakatambol, bluwota, siok, natavoa o namambe

devga sago palm leaf that is dry and has brown mark on it lif natanggura we i gat braon mak olsem i drae

dödöu leaf, leaf of (a particular plant) $\sim$ lif, lif blong (wan tri)

gagarēn aerial roots $\sim$ rus we i gro antap

gagö $1 \cdot$ stem or runner of vine longfala gro blong rop

gamesel midrib, midvein of Heliconia indica leaf, when removed (and used for tying up food parcel, etc.) bun blong lif laplap

gèri root rus

keresi thorn (of wild yam, Chinese yam, umbrella leaf) nil blong wael yam, swityam, ambrela lif

labēvi stem or petiole and leaf of palm or non-branching plant, as taro, tree fern, coconut, sago palm; rachis, principal midrib han blong wud olsem kokonas o blakpam o taro

lēlē nearly ripe, of bananas which are ripe enough to eat, but still slightly unripe klosap raep, olsem banana we $\mathrm{i}$ gud blong kakae be $\mathrm{i}$ no raep gud yet

löv orchard, plantation, area where lots of useful plants and fruit and nut trees are planted for future generations $~$ ples we ol i planem fulap stamba blong frut o nat mo ol yusful plant long hem blong ol man long fiuja i save karem

mian ripe $\sim$ raep

nalte sap wota (blong wud)

nili (fr.var. n̄ēli) shoot, sprout, new growth of plant $\sim$ top blong wud, gru blong plant

nili pomken new growth of pumpkin plant, eaten as greens $\sim$ top blong pomken we hem i wan kabis nili qiat taro stems top blong taro

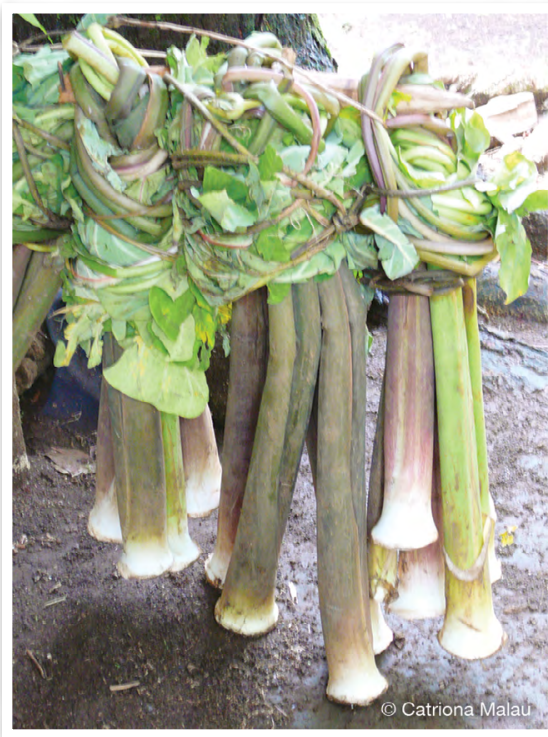

nili qiat $~$ taro stems

$\overline{\mathbf{o}} \mathbf{w}_{2}$ bear fruit karem kakae

qar $\mathbf{1}$ - unripe no raep $2 \cdot$ raw, uncooked $\sim$ no dan $\mathbf{3} \cdot$ undried, untreated, as of green leaves, rather than dried or heat treated leaves no drae, no dan, olsem lif we i grin nomo, we ol i no putum long faea blong mekem i strong.

qere petiole stamba

qētēgi (fr.var. qētigi; qitēgi; qitigi) $\mathbf{1}$ • trunk (of tree), body of tree stamba qöqö young shoot yang lif qöqörö small leaf smol leaf qulön ring-like mark around wood or plant stem, such as node of bamboo or sheath mark of sugarcane $\sim$ mak olsem ring we $i$ ronem samting olsem wud, bambu o sugaken

rērēi ${ }_{1}$ branch of tree or other plant han blong wud

sili sucker of banana, shoots from taro or other tubers, used to propagate new plants saka, olsem blong banana o narafala plant, blong planem 
siri $2 \cdot$ midrib, midvein of leaf, leaflet $\sim$ bun blong lif

siri qatē pieces from inside of trunk of tree fern, which is composed of layers of old branches that are brittle ol sap pisis blong insaed blong blakpam

$\mathbf{t a w}_{3}$ produce flower (plant) mekem flaoa

tawagas flower flaoa

tebe frond, leaf of palm tree or palmlike leaf $\sim$ lif blong pamtri

tewes flower of (a plant) flaoa blong (wan samting)

tinini $1 \cdot$ liquid, juice from something, as from fruit or sauce, liquid from cooked food wota (blong frut, kakae) $\mathbf{2}$ - sap of plant wota blong wud

tin̄i mōtō liquid from coconut (green or dry) wota blong kokonas (grin o drae)

turgēn $\mathbf{1} \cdot$ body bodi $\mathbf{2} \cdot$ trunk of tree $\sim$ stamba blong wud

vinti (fr.var. vēnti) $2 \cdot$ bark skin blong wud $3 \cdot$ husk (of nut, coconut), skin (of fruit, vegetable) skin blong nat o kokonas

vötu 1 - (of tree), trunk, above bole stamba, pat blong stamba daon

wēsiri sharp needle-like piece from inside of base of sago palm rachis bun blong natanggura insaed long stamba

wiē fruit (of tree), generic term for fruit of plant frut (blong wud) wian $_{2}$ its fruit $\sim$ frut blong hem

wōtōgsiar midrib of sago palm leaf which is used to make brooms $\sim$ bun blong lif natanggura we ol i yusum blong mekem brum

wösöwu seed $\sim \operatorname{sid}$
D1 Trees and palms

$\mathbf{a r}_{1}$ (fr.var. waar) kind of tree $\sim$ wan kaen tri Premna corymbosa ar ōl kind of ar tree that grows in the bush wan kaen ar tri blong bus ar tur kind of ar tree that grows near the sea and is a strong tree which does not bend wan kaen ar tri we i stap long solwota mo i strong mo i no save benben

$\mathbf{b a k}_{1}$ generic name for banyan trees, kind of banyan with fruit that are round and light red when ripe $\sim$ wan kaen nambangga we frut blong hem i raon mo i grin mo taem i raep i red waet Ficus spp.

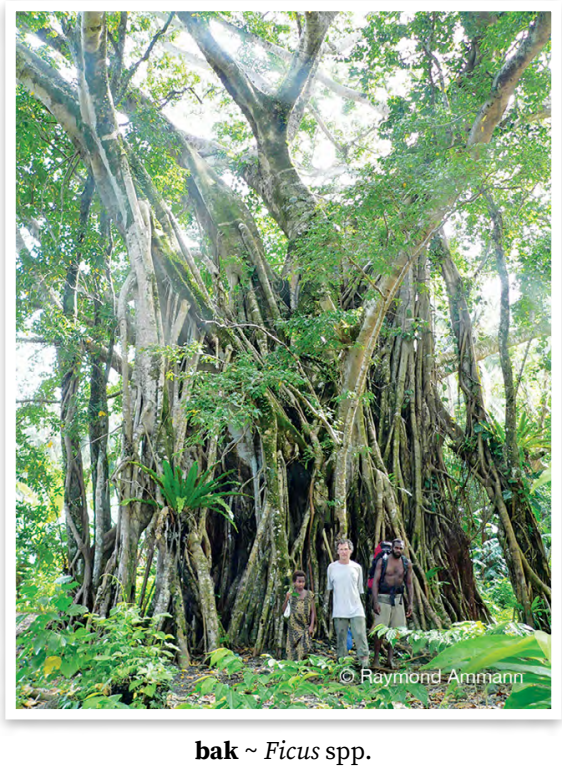

bak matqō kind of banyan with long fruit wan kaen nambangga we frut blong hem i longwan

balak variety of fig tree with edible fruit and leaves wan kaen tri we hem i famle blong nambangga mo yu save kakae frut mo lif blong hem Ficus wassa

dabalak leaf of balak that is eaten as wild greens $\sim$ lif blong balak we ol man ol i save kakae olsem bus kabis 
bar $_{3}$ kind of palm wan kaen pamtri Pelagodoxa henryana

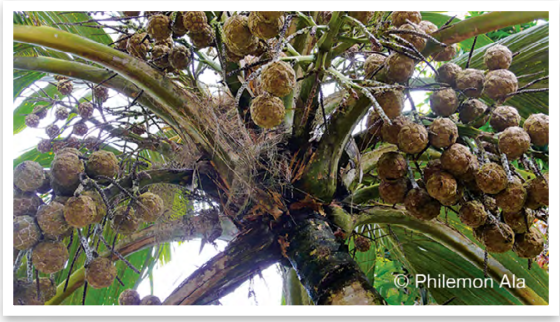

bar Pelagodoxa henryana

bēbètiq̄o kind of tree that has two varieties, one with white leaves, the other with red leaves. The wood is used for making arrows, and the leaves are used in traditional medicine wan kaen tri we $i$ gat tu kaen blong hem, wan lif i waet, narawan lif i red. Ol i yusum wud blong katem aro blong sutum pijin o faol. Ol i yusum blong lif meresin mo ol i melekem top blong hem blong smol pikinini

biēg breadfruit bredfrut Artocarpus altilis

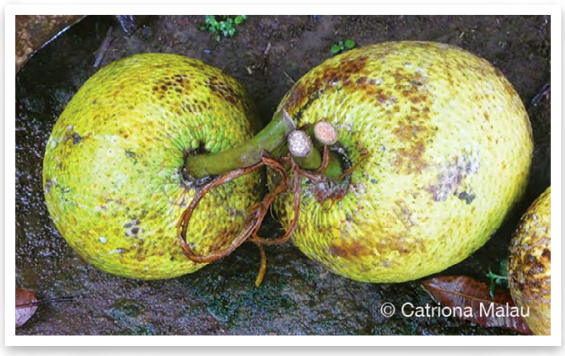

biēg Artocarpus altilis

birbir sea hearse, lantern tree $\sim$ nambirimbiri Hernandia nymphaeifolia

bitbitiq̄o kind of small tree with small red fruit and yellow flowers wan kaen tri we i smol mo frut blong hem i smol mo i red mo flaoa blong hem i yelo Psychotria trichostoma

boboros kind of tree related to balak, with small fruit that are white or pinkish when ripe $\sim$ wan kaen tri we hemi famle wetem balak. Kakae blong hem i smol mo taem i raep $\mathrm{i}$ waet o laet red

boboto cheese tree; kind of tree with small yellow flowers and small fruit that are black when ripe $\sim$ wael namamao, wan kaen tri we flaoa blong hem i smol mo i yelo mo frut blong hem i blak Glochidion sp.

bōgōr ballnut, Alexandrian laurel, beach mahogany, tamanu nambakura, tamanu blong solwota Calophyllum inophyllum

bōgōrēmēs kind of tree tamanu blong bus Calophyllum neo-ebudicum bul ${ }_{1}$ suicide tree, kind of tree with red or black fruit that has sticky sap wan kaen tri we frut blong hem i red o blak mo wota blong hem i olsem glu Cerbera manghas, C. odollam

bustutun kind of hardwood tree wan kaen wud Guettarda speciosa

dā̄amas kind of tree, one traditional use of which is to help crawling babies to be able to walk strongly wan kaen wud we kastom yus blong hem i blong ol pikinini we ol i wokabaot fo leg blong mekem se ol i save wokabaot strong

darag wild nutmeg nandae Myristica fatua

darag tōwlo kind of tree like wild nutmeg but which has larger fruit which are round wan kaen tri olsem nandae be kakae blong hem i bigwan mo i raon Myristica inutilis

datamalqō kind of tree wan kaen tri Pisonia grandis

datawalwal kind of small tree, grows to about 3 metres, with small yellow flowers and small fruit that are red when ripe $\sim$ wan kaen tri we kakae blong hem i smolsmol mo i grin be taem i raep i red, mo flaoa blong hem i yelo Phyllanthus ciccoides 
dēmērmēr tree fern blakpam Dicksonia spp.

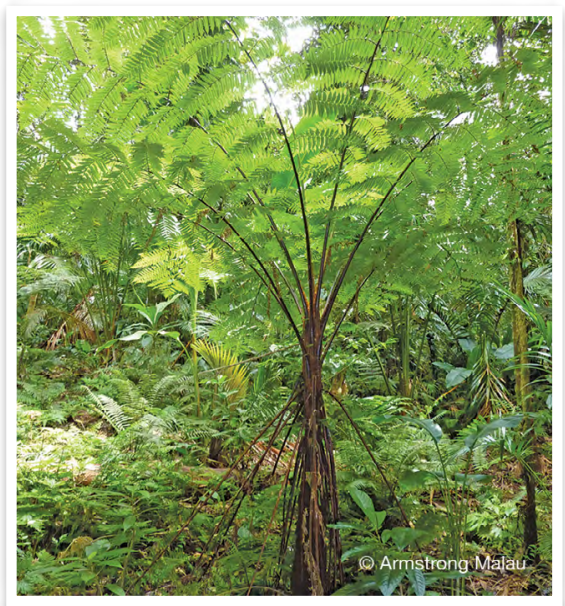

dēmērmēr Dicksonia spp.

dērvag beach pandanus

waelpandanas, wan kaen pandanas we $\mathrm{i}$ gro long solwota mo i save gro bigwan mo i gat longfala rus Pandanus sp.

dēsēn kind of tree wan kaen tri we i save gro eni ples

divivi umbrella leaf ambrela lif Licuala grandis

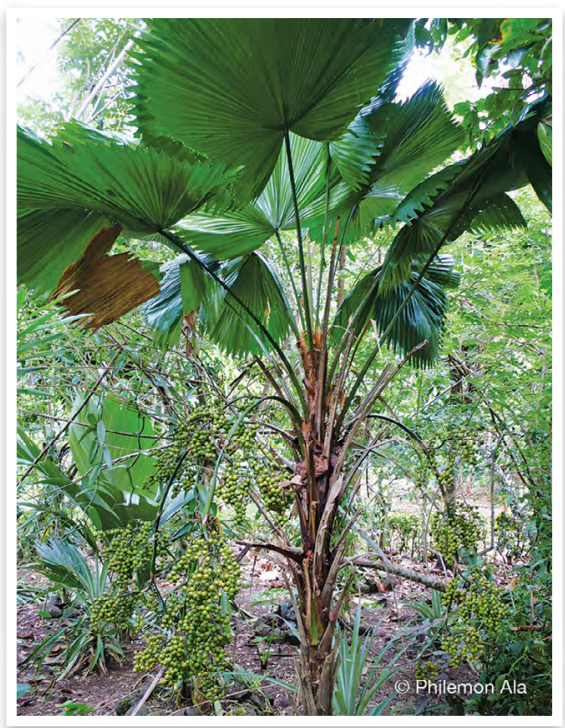

divivi Licuala grandis doot 1 - Vanuatu sago palm natanggura Metroxylon warburgii $\mathbf{2}$ • sago palm thatch $\sim$ lif natanggura, taj

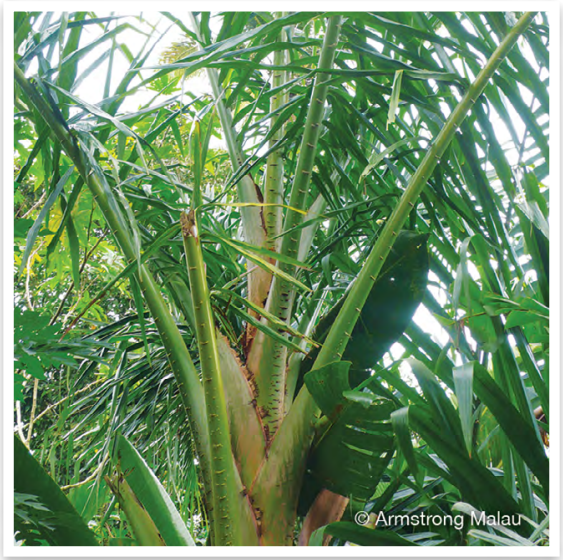

doot $\sim$ Metroxylon warburgii

dootnönqet wild bush pandanus that grows in moist environments. Leaves have very sharp edges waelpandanas blong bus we i laekem ples we i gat wota. Lif blong hem i sap mo i save katem yu Freycinetia sp.

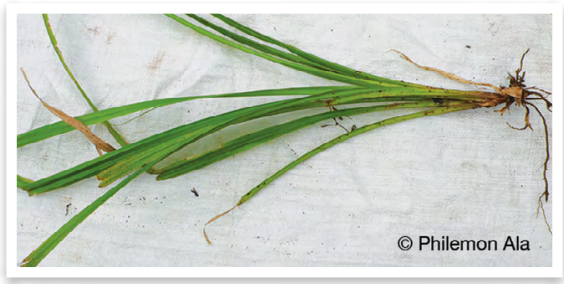

dootnönqet $\sim$ Freycinetia sp.

dōkōlōkōl kind of tree wan kaen tri Dillenia biflora

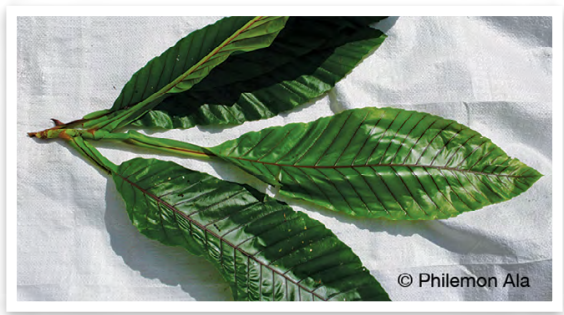

dōkōlōkōl Dillenia biflora 
dōmōvōtrev kind of small tree whose leaves close in the late afternoon wan kaen tri we lif blong hem i klos long aftenun Albizzia lebbeck

dōōt blind your eye, milky mangrove $\sim$ wan kaen tri Excoecaria agallocha

dōsōw kind of tree wan kaen tree Geniostoma rupestre

dōvōr nem kind of small tree wan kaen smol tri Vitex negundo

dötöl kind of tree with red flowers. Birds and flying fox eat the fruit wan kaen tri we i save gro bigwan mo i gat smel. Ol flaoa blong hem i red mo ol pijin mo flaengfokis i stap kakae

dunregōsōw kind of tree wan kaen tri

dun̄bönö Java cedar, bishop wood nakoka Bischofia javanica

ër casuarina, she-oak, beefwood, ironwood, whistling pine oktri, siok Casuarina equisetifolia

gan men species of fig tree which has fruit that is orange when ripe, growing close to the branch $\sim$ wan kaen tri we hem i famle blong nambangga, taem i raep, frut blong hem $i$ aranis Ficus tinctoria

gasēsēdignönqet kind of tree wan kaen tri Ficus aspera

gasōg tree with edible fruit wud we yumi save kakae frut blong hem Gnetum gnemon

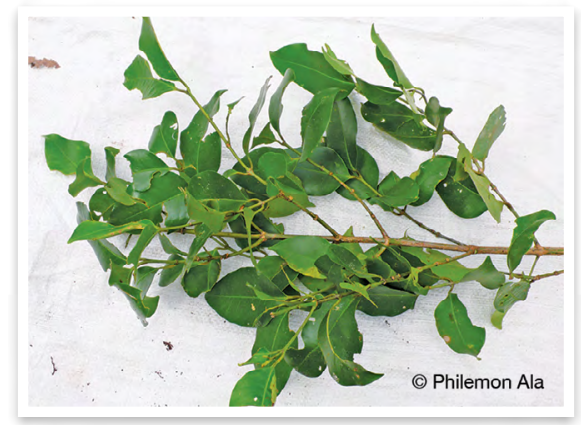

gasōg Gnetum gnemon gatötöqulqërët kind of dragon tree, shrubby plant wan kaen plant Dracaena sp.

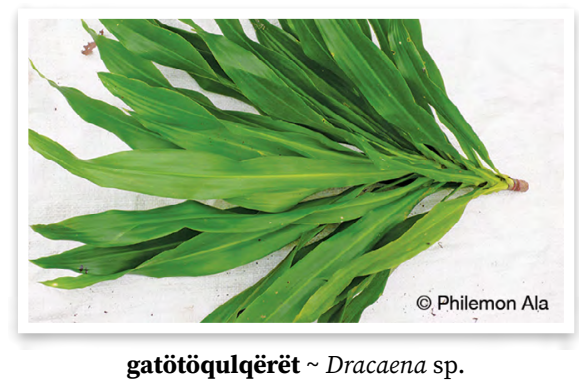

gavēg mountain apple, Malay apple, rose apple nakavika Syzygium malaccense

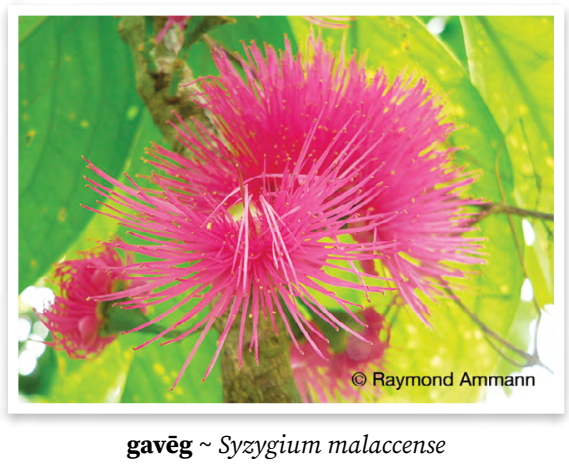

gavēgulul kind of tree like mountain apple but with white flowers wan kaen tri olsem nakavika be flaoa blong hem i waet

gavō whitewood waetwud Endospermum medullosum

gawōlo kind of tree that is used to make a strong rope $\sim$ wan kaen tri we $i$ gat rop blong hem insaed we $i$ strong tumas

gōglo kind of shrub, small tree kaen navenue Macaranga tanarius

les poisonwood posenwud Semecarpus neoebudica

mala kind of tree with fruit that is red on the outside and black inside $\sim$ wan kaen tri we frut blong hem i red long aotsaed, be insaed i blak Sterculia banksiana 
malagavēg kind of tree that is related to Malay apple, with pink flowers and small dark red to black fruit wan kaen tri we hem i famle blong nakavika, flaoa blong hem i pink mo frut i smolsmol mo i dak red o blak Syzygium buettnerianum

malaqōōr kind of tree with very long leaves that grows on the side of hills wan kaen tri we hem i gro long saed blong hil. Lif blong hem i longlongwan

malmalasē kind of tree wan kaen tri malnët kind of tree like red silkwood but the fruit are smaller and not eaten wan kaen tri olsem naduledule be kakae blong hem i smol mo man i no stap kakae

man̄ko mango manggo

markōn kind of tree wan kaen tri Metrosideros collina

marsam kind of tree, brown kurrajong wan kaen tri Commersonia bartramia

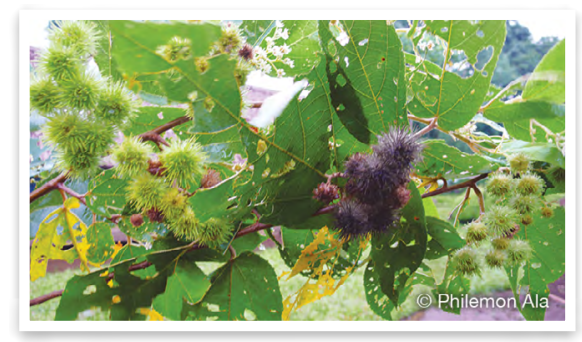

marsam Commersonia bartramia

matal puzzle tree namatal Kleinhovia hospita

menmav kind of tree, probably type of fig, with fruit that is green when unripe and white when ripe $\sim$ wan kaen tri we i olsem balak. Kakae blong hem $\mathrm{i}$ grin mo taem $\mathrm{i}$ raep $\mathrm{i}$ waet

meteraramat kind of tree kaen wud mē achiote, annatto; kind of shrub or small tree with bright red spiny fruits and red seeds wan kaen wud we $\mathrm{i}$ gat red frut blong hem wetem red sid insaed Bixa orellana

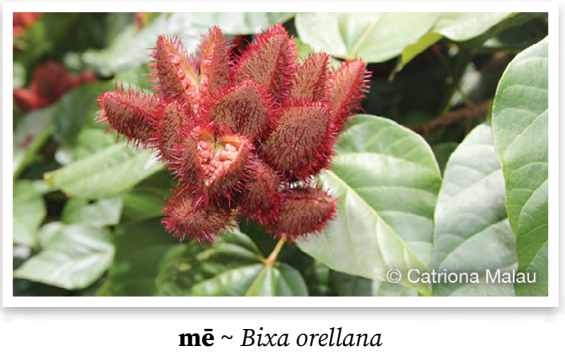

mēl kind of tree wan kaen wud Elaeocarpus floridanus

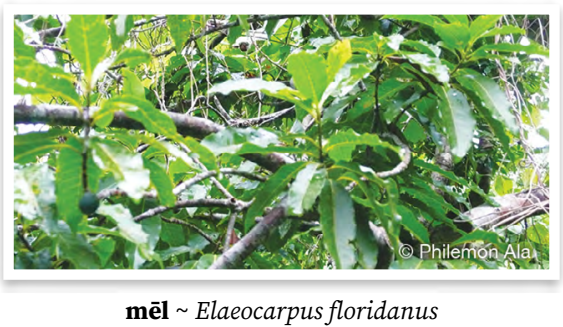

mōmō $\overline{\mathbf{m}}_{2}$ poumuli, tree species with small, black, inedible fruit namamao, namemiwa Flueggea flexuosa

mōtōvōl areca palm belonging to same family as betel nut kaen pamtri we i famle blong bitelnat Areca macrocalyx

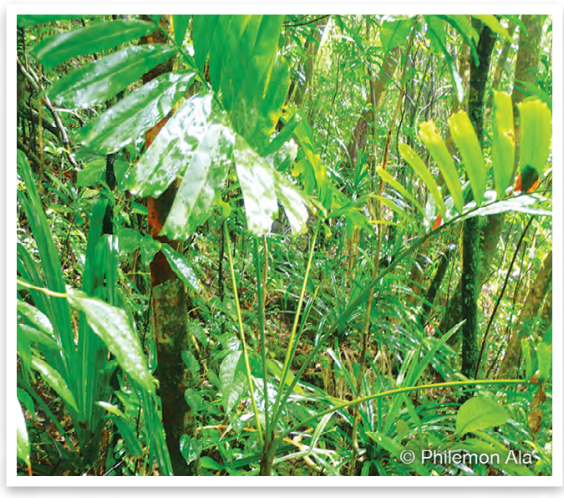

mōtōvōl Areca macrocalyx 
mēeriri kind of perennial climbing tree brata blong namariu; namariu blong solwota Acacia simplex

mēririnek kind of tree namariu blong solwota Acacia spirorbis

miak Tahitian chestnut $\sim$ namambe Inocarpus fagiferus

möll orange; generic term for citrus fruit, but mostly referring to orange $\sim$ aranis Citrus spp.

nagnagsamul kind of tree $\sim$ wan kaen tri

nanar New Guinea rosewood nananara, bluwota Pterocarpus indicus

nët red silkwood, large tree, growing up to 30 metres with large edible green fruit $\sim$ naduledule Burckella obovata

nōnōn kind of small tree wan kaen smol tri Ficus adenosperma

ne canarium nut, native almond nangae Canarium spp.

ne dawel kind of wild native almond which grows in the bush and is not eaten. It has white bark and can grow tall like a whitewood wan kaen wael nangae blong bus we ol man ol i no kakae. Skin blong hem i waet mo hem i save gro longwan olsem waetwud

ne dun kind of native almond which has flat nuts kaen nangae we i gat frut we i flat Canarium harveyi

ne ta kind of native almond which has round nuts kaen nangae we i gat frut we i raon Canarium indicum

òv canoe tree kenutri Gyrocarpus americanus

qate $\bar{e}_{1}$ tree fern blakpam Cyathea spp. qetge kind of tree $\sim$ wan kaen tri qēr ${ }_{1}$ kind of tree $\sim$ wan kaen tri Osmoxylon orientale

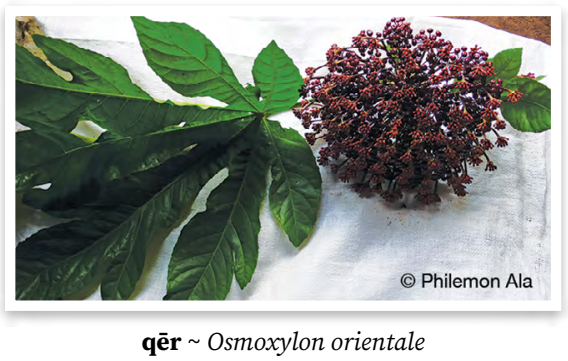

qog kind of tree wan kaen tri Neuburgia corynocarpa

qōmiat stinkwood; kind of tree with yellow fruit with red seeds inside stingwud; wan kaen tri we frut blong hem i yelo mo i gat red sid insaed Dysoxylum gaudichaudianum

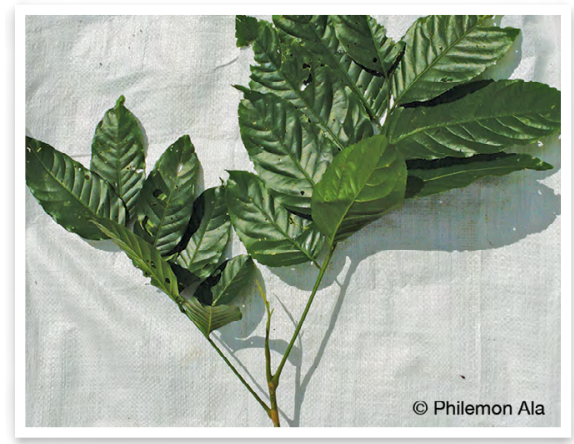

qōmiat Dysoxylum gaudichaudianum

$\mathbf{r a r}_{2}$ Indian coral tree $\sim$ narara Erythrina indica, E. variegata

rēntenge $1 \cdot$ tree, plant with woody trunk and branches, also covering palms, but not smaller shrubs tri, wud $\mathbf{2} \cdot$ wood, length of wood, stick, the wood of a tree $\sim$ wud

rēw kind of fig that does not have aerial roots. It has smaller fruits than a banyan, the fruits are red and yellow and eaten by birds wan kaen nambangga we rus blong hem i no kamaot antap. Frut blong hem i no bigbigwan tumas olsem nambangga. Ol frut ol i red mo yelo mo ol pijin ol i laek blong kakae 
rō $q_{3}$ kind of tree like a varvar which grows in the bush and has a thick double layer of bark wan kaen tri we $\mathrm{i}$ olsem varvar be $\mathrm{i}$ blong bus mo skin blong hem i strong dabol skin

rōwō kind of tree that has bark that is red on the underside $\sim$ wan kaen tri we insaed skin blong hem i red

$\mathbf{s a l}_{3}$ kind of tree $\sim$ wan kaen tri Homalanthus nutans

sawa mountain lantern tree bluwud Hernandia moerenhoutiana

sentawud sandalwood sentawud Santalum austrocaledonicum

sēqēg kind of tree wan kaen tri Elaeocarpus floridanus

sērsēr kind of tree like stinkwood but bigger and harder wood wan kaen tri olsem stingwud be i bigwan mo i strong

silat $1 \cdot$ nettle tree, fever nettle, devil nettle $\sim$ nanggalat Dendrocnide spp.

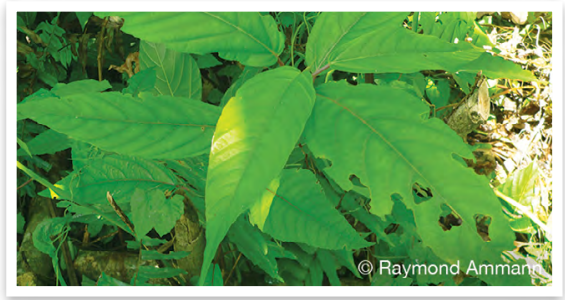

silat $\sim$ Dendrocnide spp.

silat gegeretō kind of nettle tree which has a powerful sting wan kaen nanggalat we hem i kakae man i strong

sōgsōggenqet kind of tree kaen wud Phaleria pentecostalis

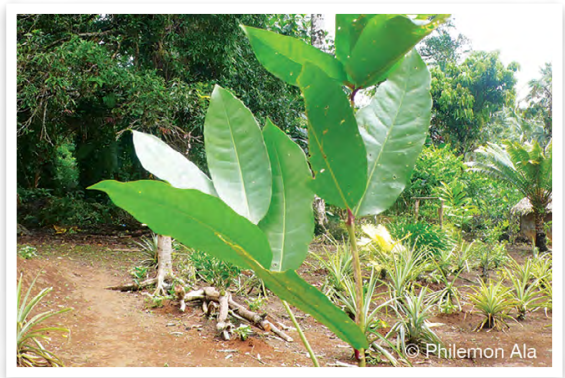

sōgsōggenqet $\sim$ Phaleria pentecostalis tagar $_{2}$ kind of tree $\sim$ wan kaen tri Melochia odorata

talēs sea almond, Indian almond natavoa Terminalia catappa

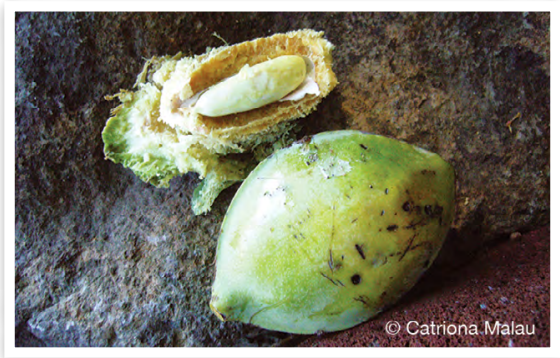

talēs Terminalia catappa

talēs toko kind of tree related to an Indian almond but which has small nuts wael natavoa Terminalia sepicana

tarak kind of tree wan kaen tri Ervatamia orientalis, E. pandacaqui, E. obtusciuscula

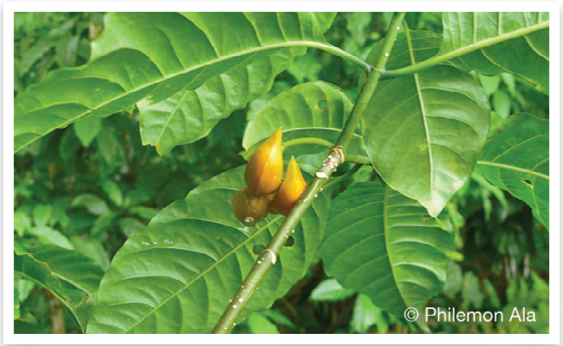

tarak Ervatamia orientalis

tewen native lychee $\sim$ nandao Pometia pinnata

tēq $q_{1}$ kind of tree with small black fruit $\sim$ wan kaen tri we kakae blong hem i smol mo taem i raep i blak Rhus taitensis

titiqō kind of tree kaen wud tor island teak, ironwood natora Intsia bijuga

totenanar kind of tree that has long white flowers with a strong perfume wan kaen tri we flaoa blong hem i waet mo i longlongfala mo i gat smel 
ur great hog plum naos Spondias dulcis

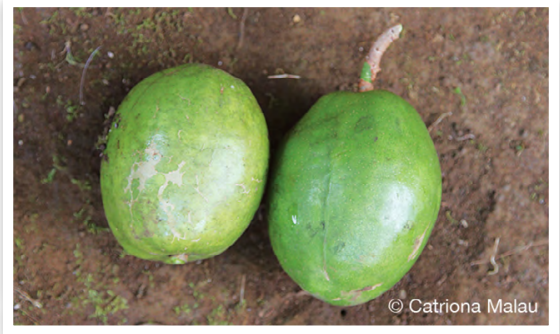

ur $\sim$ Spondias dulcis

usugiav kind of tree $\sim$ wan kaen tri we natora blong hem i blak Annona squamosa

$\operatorname{van}_{2}$ kind of pandanus that grows by the sea and has thorny leaves. The fruit is eaten by people and flying foxes $\sim$ wan kaen pandanas we i stap long solwota we $\mathrm{i}$ gat nil. Man mo flaengfokis ol i save kakae frut blong hem mo ol i juim mo afta spetemaot makas

varvar kind of tree wan kaen wud Neisosperma oppositifolia

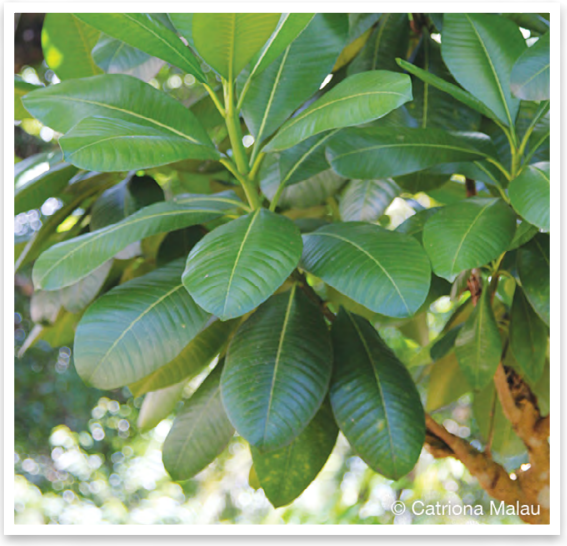

varvar Neisosperma oppositifolia

vēkēmolo kind of tree that grows to about 4 metres, with purple flower and brown fruit $\sim$ kaen tri we $i$ gat popel flaoa mo braon frut Psychotria aneityensis vēlavēl toi huremi, navasvas Alphitonia zizyphoides

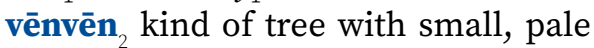
yellow flowers and small round fruit wan kaen tri we flaoa blong hem i smol mo i yelo mo kakae blong hem i raon Pavetta opulina

vètuboso kind of tree that has thick bark and roots similar to a mangrove wan kaen tri we skin blong hem i tik mo rus blong hem i olsem natongtong Crossostylis cominsii

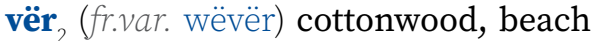
hibiscus burao Hibiscus tiliaceus

vōname kind of tree that has red flowers similar to a Malay apple. Flying foxes drink the nectar from the flowers wan kaen tri we flaoa blong hem i red mo i olsem nakavika be i strong. Flaengfokis i dring flaoa blong hem be ol i mas satem ae taem i dring o sapos no flaoa bae i stikim vudege (fr.var. vudëgë) pandanus, screw pine $\sim$ pandanas Pandanus spp.

vut fish poison tree, sea poison tree fisposentri Barringtonia asiatica

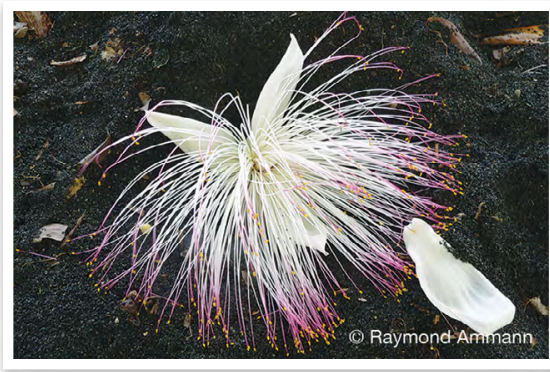

vut $\sim$ Barringtonia asiatica

wag $_{1}$ ylang-ylang, perfume tree nandingori Cananga odorata

wakam kind of tree with yellow flowers. It has nuts similar to native almonds which are yellow when ripe wan kaen tri we flaoa blong 
hem i yelo. Kakae blong hem i olsem nangae, i grin be taem i raep i yelo Finchia chloroxantha

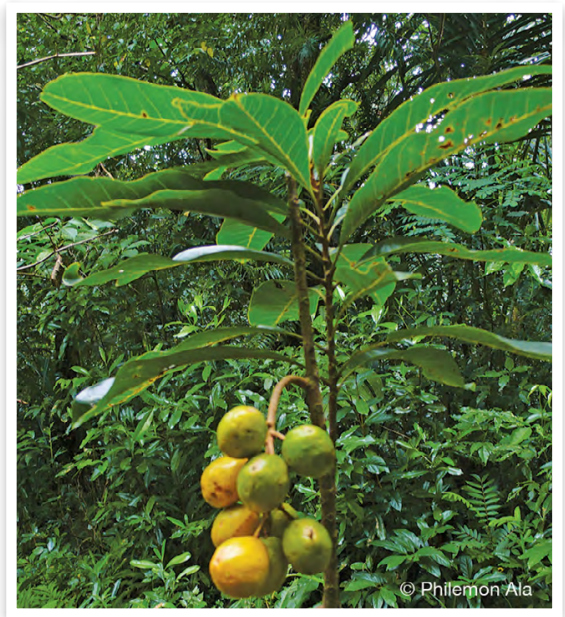

wakam Finchia chloroxantha

walag $_{1}$ kind of tree with large fruit with yellow skin and red seeds wan kaen tri we wud blong hem i strong mo frut blong hem i yelo mo insaed ol sid i red. Kakae blong hem i olsem kakae blong bintri Dysoxylum aneityensis

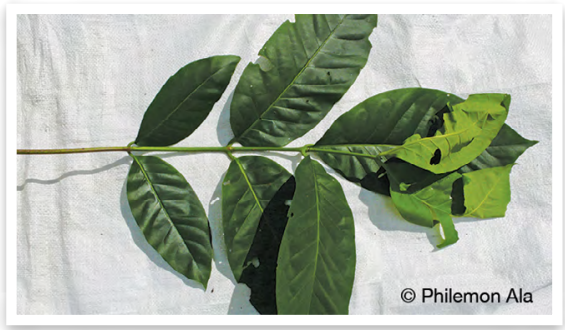

walag Dysoxylum aneityensis

wan̄arn̄ar kind of tree that has fruit similar to a mango which are red when ripe wan kaen tri we kakae blong hem i olsem manggo we i laet grin mo taem i raep i red Corynocarpus similis

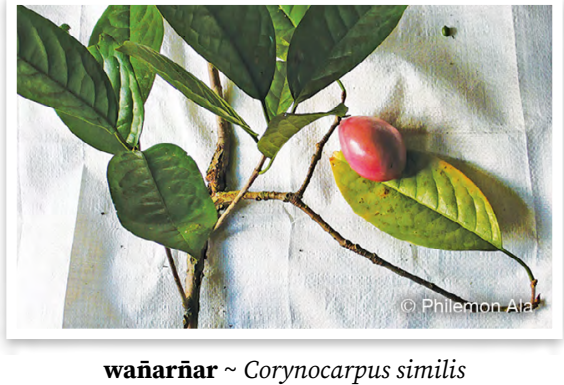

waran̄rañ marge kind of tree, fruit are red when ripe and similar to that of poumuli wan kaen tri we frut blong hem i red taem i raep mo hem i olsem kakae blong namamao Flacourtia rukam

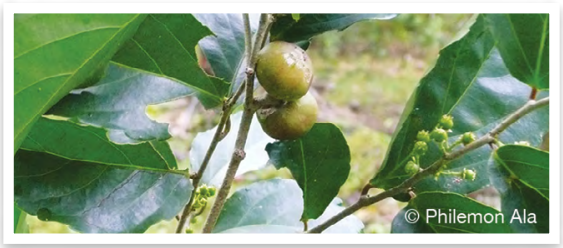

waran̄ran̄ marge Flacourtia rukam

watamarge kind of large tree with fruits which are similar to a Malay apple, but they are not commonly eaten as they are very sour. The fruit are red when ripe and long and oval shaped wan kaen tri we hem i bigwan lelebet. Kakae blong hem i olsem nakavika be man i no kakae tumas from i saoa. Taem i no raep i grin mo taem i raep i braet red. Hem i longfala lelebet Aceratium oppositifolium

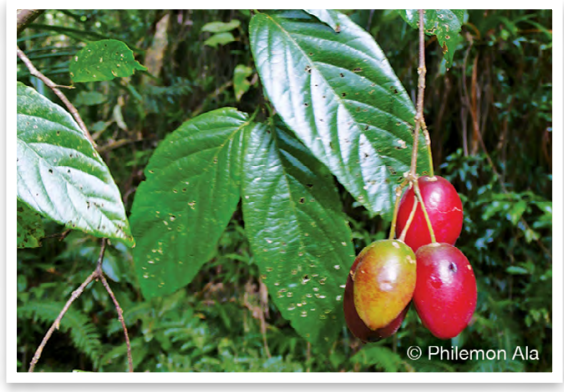

watamarge $\sim$ Aceratium oppositifolium 
wegerbak kind of tree wan kaen tri weverbak kind of tree $\sim$ wan kaen tri Olea paniculata

wēgēbil false tamanu; kind of tree with edible fruit that are dark red, black when ripe, red inside wan kaen tri we frut blong hem i dak red mo taem i raep i blak mo i red long insaed blong hem Garcinia pseudoguttifera

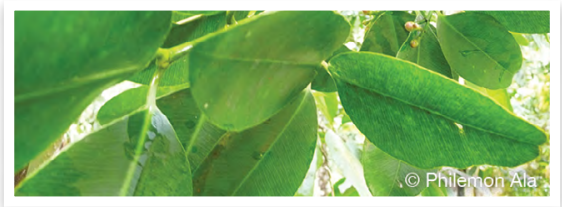

wēgēbil Garcinia pseudoguttifera

wēmētigtig betel nut, areca palm bitelnat, rabis kokonas, waelkokonas Areca catechu

wēqiar ${ }_{2}$ kind of small tree wan kaen smol tri Desmodium umbellatum

wequiqiar kind of tree that grows by the sea and has fruit that look like small beans wan kaen tri we i gru long solwota we kakae blong hem i olsem smolsmol bin

wètēt ${ }_{2}$ kind of tree wan kaen tri wēviriak rattle tree navangge Pangium edule

wēwēn red beadtree, coralwood nabisa Adenanthera pavonina

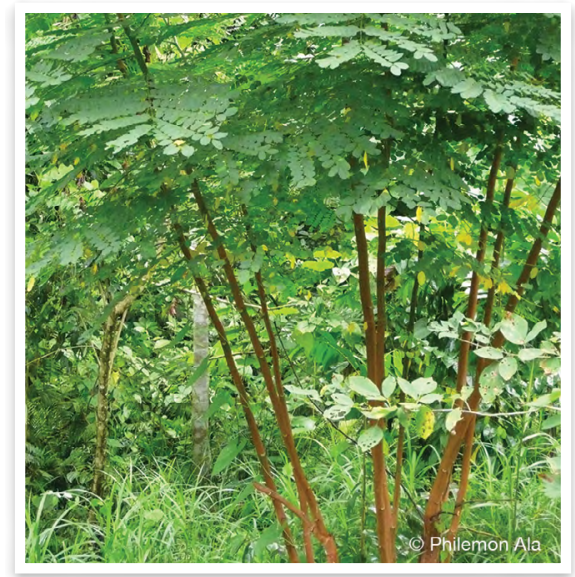

wēwēn Adenanthera pavonina wëvër lo rosewood burao blong solwota Thespesia populnea

winiā̄ soursop karasol Annona muricata

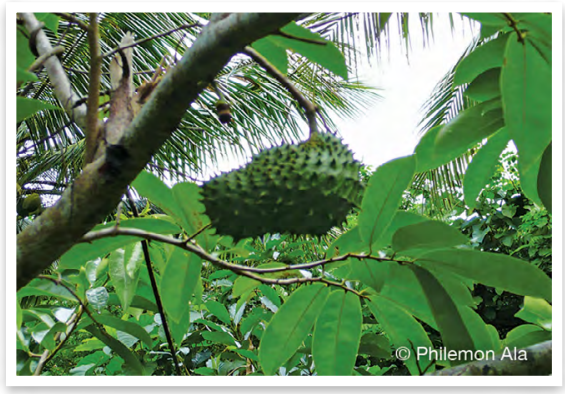

winiā̄ $~$ Annona muricata

woman pawpaw, papaya popo Carica papaya

woro dragon plum nakatambol

Dracontomelon vitiense

wosvet Mossman mahogany, kind of hardwood tree with white flowers and red seeds wan kaen tri we flaoa blong hem i waet mo sid i red Dysoxylum arborescens

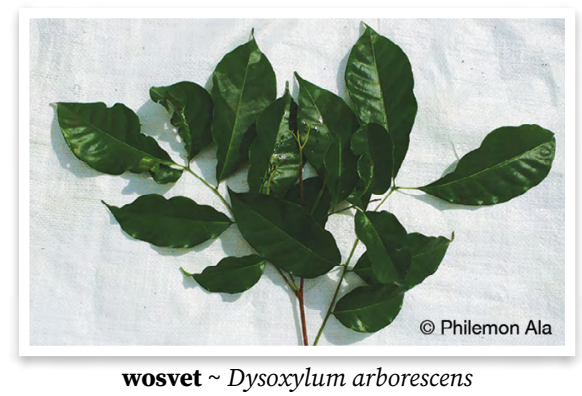

wotag (fr.var. watag) cutnut, bush nut $\sim$ navele Barringtonia edulis

wotag dere timiat kind of cutnut that has dark red leaves and skin of fruit kaen navele we lif mo skin blong kakae blong hem i red olsem blad

wotag dun kind of cutnut kaen navele

wotag sërsër kind of cutnut kaen navele

wotag wos kind of cutnut kaen navele 
wotag wōtōn kind of cutnut kaen navele

wotag wöl kind of cutnut kaen navele

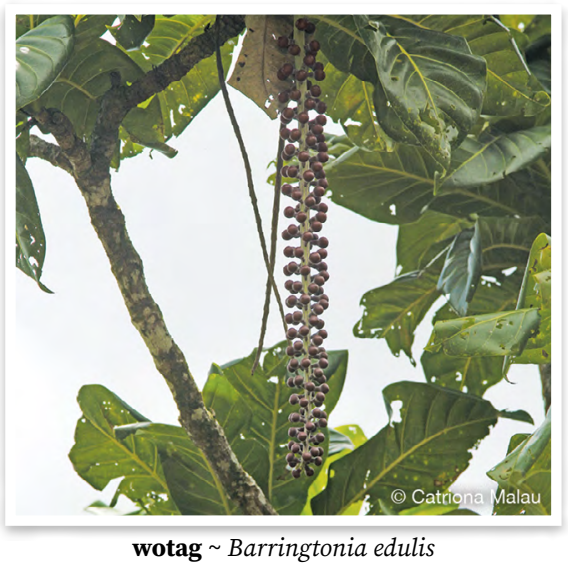

wotag bē kind of tree, of same genus as cutnut, but does not have edible nuts kaen wud we hem i famle blong navele, be yu no save kakae Barringtonia racemosa

wōlōm Indian mulberry noni, yalatri, yalawud Morinda citrifolia

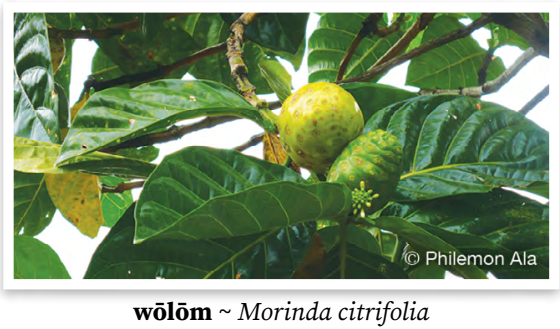

wōnōnōg malgias kind of tree wan kaen tri

wōtōgōrte kind of tree with very long fruit which are sticky like glue. The fruit is green when unripe and red when ripe. The wood is used in house building wan kaen tri we frut blong hem i longlongwan mo i stiki olsem glu. Frut i grin be i kam red taem i raep. Ol i yusum wud blong wokem haos wōtōn kind of fig with round fruits that are dark red when ripe, fruits are eaten by birds wan kaen tri we kakae blong hem i raon mo waet mo i dak red taem i raep mo ol pijin ol i kakae Ficus granatum

wōvōnō kind of shrub, small tree kaen navenue Macaranga dioica

wö kind of tree wan kaen tri Ixora asme

wöbulbulbōk glue berry tree glutri Cordia dichotoma

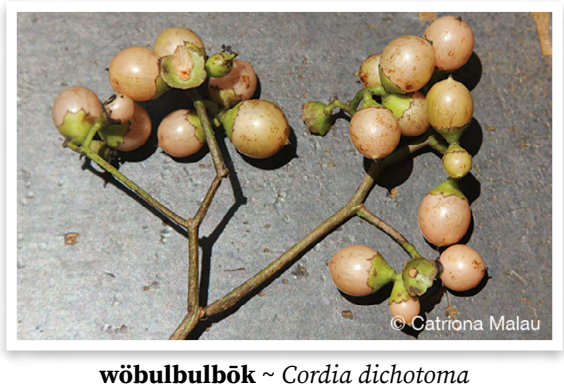

wödödö kind of tree, poison peach wan kaen tri Trema orientalis

wu kind of small tree with hard wood wan kaen tri we hem i strong mo i no save gro bigwan

wuqiat kind of tree whose wood is used as a digging stick for planting taro kaen wud we ol i yusum olsem wud blong planem taro

\section{D1.1 Coconuts}

bar $_{1} 1 \cdot$ mature coconut that has very thin flesh and so is not good for making copra and spongy growth inside is not good for eating kokonas we mit blong hem i tintin tumas, mekem se yu no save mekem kopra long hem $\mathbf{2}$ • (of coconut) has reached stage where the flesh has become thin and spongy growth inside is no longer good for eating (kokonas) i nomo gudfala navara blong kakae mo i nomo gud blong mekem kopra long hem 
bar 2 variety of coconut that has very small fruit that do not have much liquid in them but the liquid is very sweet kaen kokonas we hem i smol tumas be wota blong hem i swit

bes 1 - coconut oil, oily substance lining the flesh of germinated coconuts oel blong kokonas, krim we i stap wetem navara $\mathbf{2}$ - put coconut oil or grease on something, as to heal a sore or grease something rusty putum oel o krim blong kokonas long wan samting olsem long soa o long wan rosta aean

bibiag stage of coconut growth when the flesh is dry but the skin is still green $\sim$ mit blong kokonas i drae be skin blong hem i grin yet

gölörēki 1 - sprouting coconut that is just starting to germinate and has not yet formed spongy growth inside kokonas we i stat blong kam navara be wota i stap insaed yet $\mathbf{2}$ shoot of coconut begins to grow gro blong kokonas i stap kamaot be i no mekem gud navara yet

lovv grated coconut that has had the milk squeezed out of it makas blong kokonas

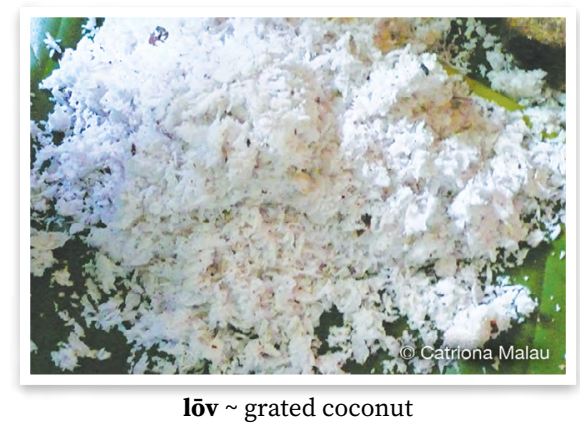

matabōbōt mature coconut $\sim$ drae kokonas

mōtō $1 \cdot$ coconut, generic term for coconut palm, and for fruit when ripe kokonas Cocos nucifera 2 coconut milk melek blong kokonas mōtō atm̄ēn variety of coconut that is characterised by rapid growth but poor productivity kaen kokonas we i gro kwiktaem be i no karem tumas kakae

mōtō bal variety of coconut where two palms grow from one coconut or two are planted side by side kaen kokonas we tu kokonas i gro i kamaot long wan kokonas o we ol i planem tu kokonas i stap klosap tumas

mōtō dēdērēs variety of coconut that has very sweet flesh and water $\sim$ kaen kokonas we kakae mo wota blong hem i swit tumas mōtō elvet variety of coconut which has long fruit kaen kokonas we frut blong hem i longfala

mōtō ga añan̄ variety of coconut that has yellow fruit and yellowish leaves kaen kokonas we frut blong hem i yelo mo lif i lelebet yelo

mōtō ga garaq variety of coconut that is a new hybrid kaen kokonas we i niuwan

mōtō ge metestes variety of coconut which has long fruit kaen kokonas we frut blong hem i longfala

mōtō gō tōtōrōg variety of coconut that has green fruit $\sim$ kaen kokonas we frut blong hem i grin

mōtō gö luwō large variety of coconut with thick meat kaen kokonas we i bigwan mo i gat bigfala kakae

mōtō malgias variety of coconut that has reddish greenish coloured fruit kaen kokonas we frut blong hem i grin red

mōtō mamē variety of coconut that has red fruit $\sim$ kaen kokonas we frut blong hem i red

mōtō mete röwö variety of coconut so called because the eyes on the shell stick out like those of the fish 
röwö kaen kokonas we ol i kolem olsem from ae blong hem i stik aot olsem ae blong wan fis, röwö

mōtō mölumlum variety of coconut that has soft flesh which is thus easy to remove from the shell kaen kokonas we kakae i sofsof mo i isi blong salem

mōtō mat variety of coconut that has stripes encircling the fruit kaen kokonas we $i$ gat ol straep i raonem frut

mōtō reqe variety of coconut that is characterised by slow growth yet high productivity kaen kokonas we i gro sloslo be i karem plante kakae mōtō sā̄sam variety of coconut which has long fruit kaen kokonas we frut blong hem i longfala

mōtō seseser variety of coconut that is easily husked kaen kokonas we $\mathrm{i}$ isi blong karemaot skin blong hem mōtō sial me variety of coconut that was introduced from another island kaen kokonas we i kam long narafala aelan

mōtō silat variety of coconut that has fragile leaves and inflorescences and is not highly productive $~$ kaen kokonas we lif mo flaoa blong hem i no strong mo hem i no karem tumas kakae

mōtō taktak variety of coconut kaen kokonas

mōtō us variety of coconut with flesh that is sweet and soft with lots of liquid kaen kokonas we i swit, i sofsof mo i gat plante wota

mōtō vanvan variety of coconut kaen kokonas

mōtō vet variety of coconut that has very hard flesh that is thus difficult to remove from shell kaen kokonas we $\mathrm{i}$ strong tumas mo i had blong selaot mōtō vin gaqō variety of coconut with a thick husk kaen kokonas we skin blong hem i tik

mōtō wulmē variety of coconut that has a reddish coloured ring surrounding where the peduncle is attached to the fruit $\sim$ kaen kokonas we $i$ gat red ring long frut long ples we i joen long han

mël green coconut that has no flesh on the shell, but has water inside, although the liquid is not sweet yet $\sim$ grin kokonas we hem i gat wota blong hem be i nogat mit mo yumi no stap dring from i no swit tumas

n̄ēnēn $\mathbf{1}$ • (shoot) start to emerge from coconut $\sim$ gro i stat blong kamaot long kokonas 2 • newly emerging coconut shoot $\sim$ gro blong kokonas

qōtō ${ }_{1}$ semi-mature coconut, which has fizzy liquid inside haf drae kokonas we wota insaed long hem i gat gas long hem

qōtōnögōsōw empty full shell of mature coconut that has not germinated and the husk and albumen inside have decomposed $\sim$ drae kokonas we hem i no kam navara mo skin mo kakae blong hem insaed i lus

sōgsōg ${ }_{1}$ variety of coconut that bears a large number of small fruit which have sweet liquid inside kaen kokonas we i karem fulap frut we ol i smolsmol mo wota blong hem i swit sōl young coconut seedling, general term for germinated coconut navara

sōlvet young coconut seedling that is firm and sweet navara we $i$ strong mo i swit

sōl kētkèt coconut that has germinated while still hanging to the tree, which has very sweet flesh $\sim$ kokonas we i kam navara taem i 
stap hang yet long stamba mo i no foldaon hem wan, navara blong hem i swit tumas

tur mōtō coconut palm stamba blong kokonas

vinkörögör coconut shell that has had flesh removed $\sim$ sel kokonas

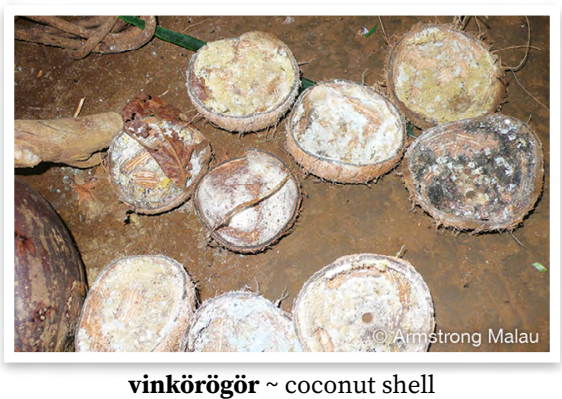

vinlas coconut shell with flesh removed, especially when used as a cup for drinking kava sel kokonas, olsem ol i dring kava long hem

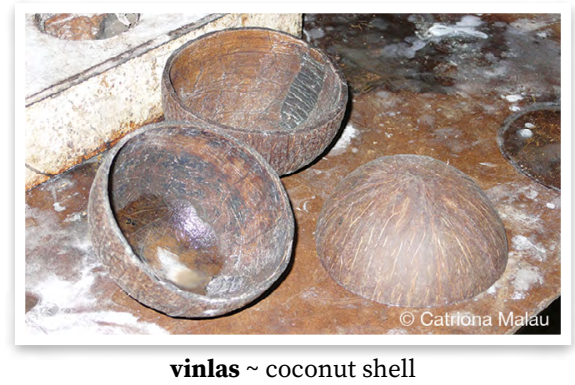

vōs green coconut grin kokonas

vōs gargarteqërët green coconut at beginning of stage appropriate for drinking, with soft shell, thin flesh and liquid inside $\sim$ grin kokonas we sel blong hem i sofsof, kakae blong hem $\mathrm{i}$ tintin mo i gat wota blong hem webeges coconut spathe (leafy sheath that encloses spadix (cluster of flowers)) samting olsem lif we $\mathrm{i}$ kavremap bandel blong flaoa blong kokonas wēlēwlēw coconut spadix (plant spike bearing flowers) samting blong kokonas we ol flaoa i stap gro long hem

wēsustēgēr dry coconut that does not contain any liquid inside $\sim$ drae kokonas we i nomo gat wota i stap long hem

wiqian young coconut seedling with a small haustorium (the spongy embryo that grows inside when the coconut is germinating) navara we insaed blong hem i smol nomo

\section{D1.2 Breadfruit}

bēw $_{2}$ variety of breadfruit kaen bredfrut

biēg ge mirmiar variety of breadfruit that has small fruit $\sim$ kaen bredfrut we kakae blong hem i smolwan

biēg löt variety of breadfruit that is good for making löt as it is neither too hard nor too soft kaen bredfrut we hem i gud blong nalot from hem i no sofsof tumas mo i no had tumas

biēg ta motlav variety of breadfruit that is good for boiling kaen bredfrut we hem i gud blong kuk

dēmēl variety of breadfruit that has elongated fruit $\sim$ kaen bredfrut we kakae blong hem i longwan

dōwōn variety of breadfruit that has small fruit kaen bredfrut we kakae blong hem i smolwan

döbumsër variety of breadfruit that has small fruit kaen bredfrut we kakae blong hem i smolwan

gatege variety of breadfruit that has small fruit kaen bredfrut we kakae blong hem i smolwan

gole variety of breadfruit that has elongated fruit $\sim$ kaen bredfrut we kakae blong hem i longwan

mëtëwtëw ev variety of breadfruit that cooks quickly kaen bredfrut we $i$ dan kwiktaem 
mereg variety of breadfruit that has small fruit kaen bredfrut we kakae blong hem i smolwan

nere ak variety of breadfruit kaen bredfrut

pispol variety of breadfruit that has large fruit kaen bredfrut we kakae blong hem i bigwan

qalmiat variety of breadfruit kaen bredfrut

qötuq $\overline{\mathbf{o}}_{1}$ variety of breadfruit that has large fruit kaen bredfrut we kakae blong hem i bigwan

selge variety of breadfruit that is good for making löt as it is neither too hard nor too soft $~$ kaen bredfrut we hem i gud blong nalot from hem i no sofsof tumas mo i no had tumas

söw malgias variety of breadfruit kaen bredfrut

söw taban variety of breadfruit $\sim$ kaen bredfrut

tamōt variety of breadfruit that is good for boiling kaen bredfrut we hem i gud blong kuk

tōt vaslēe variety of breadfruit kaen bredfrut

tönödem variety of breadfruit that has large fruit $\sim$ kaen bredfrut we kakae blong hem i bigwan

vinbaw variety of breadfruit $~$ kaen bredfrut

wotasiwōw variety of breadfruit that has elongated fruit $\sim$ kaen bredfrut we kakae blong hem i longwan

\section{D1.3 Bananas}

bakrës variety of banana kaen banana

$\mathbf{b} \overline{\mathbf{e}}_{3}$ variety of banana with very long, large fruit, and the fruit must be squeezed to enable peeling kaen banana we kakae blong hem i longlongfala mo i bigwan, mo yu mas skwisim bifo yu pilim

brisben variety of banana kaen banana ëtëtlam variety of banana that has a very tall trunk with small fruit growing in a long bunch kaen banana we stamba blong hem i gro hae bitim ol banana, kakae blong hem i smolsmol mo bandel i longfala

kukuög variety of banana that is only eaten ripe, not cooked when unripe, the fruit will fall off when you shake the trunk kaen banana we ol i kakae raepwan nomo mo taem yu sekem stamba ol raepwan i foldaon, mo hem i sotfala

lian manu variety of banana kaen banana

male $_{2}$ variety of banana that has roundish fruit, white flesh and does not have a strong flavour. The banana bundle grows upwards rather than hanging down $\sim$ kaen banana we kakae blong hem i raon mo i waet insaed be i nogat tumas tes. I sakem kakae blong hem i go antap

matmat malgias variety of banana that has whitish fruit, leaves and trunk, with long, large fruit $~$ kaen banana we skin mo stamba mo lif blong hem i waet mo hem i bigwan

matmat mamē variety of banana that has reddish fruit, leaves and trunk, with long, large fruit $\sim$ kaen banana we skin mo stamba mo lif blong hem i red

mianisi variety of banana with small, thin, bent fruit and short trunk kaen banana we hem i save gro hae. Tes blong kakae i defren lelebet be i olsem samo

malmalbak variety of banana that has long, large fruit $\sim$ kaen banana we stamba blong hem i bigfala mo hem i gro hae be hem i no karem fulap kakae. Taem ol i kukum i drae we i drae

retekraw variety of banana kaen banana 
rēn̄rēñ variety of banana that appears to be ripe when it is not completely ripe, and will be ripe approximately two days after first appearing to be ripe $~$ kaen banana we i save raep tu taem, hem i luk olsem i raep be yu mas wet, afta tumora bae i raep

rurun̄ög variety of banana that is only eaten ripe, not cooked and eaten when unripe. The fruit will fall from bundle when the trunk is shaken kaen banana we ol i kakae raepwan nomo mo taem yu sekem stamba ol raepwan i foldaon. Hem i sotfala

samo variety of banana with a short trunk and small, thin, bent fruit kaen banana we stamba blong hem i sotfala mo kakae blong hem i smol mo i benben

sēnē variety of banana kaen banana siwit variety of banana kaen banana

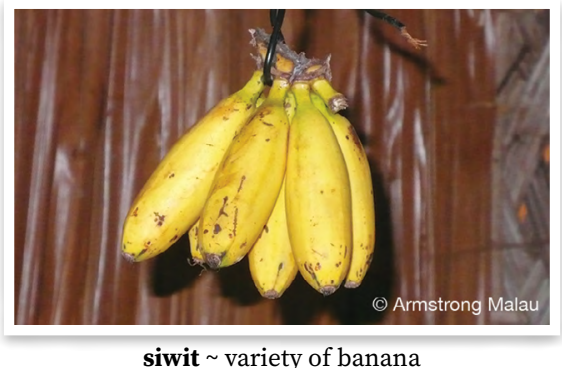

sōbōt variety of banana with small, thin, bent fruit and short trunk kaen banana we kakae blong hem i olsem samo be stamba i sotsotfala

tores variety of banana kaen banana tulug variety of banana, considered to be one of the original endemic varieties on Vanua Lava, with very long fruit, eaten only when ripe, not cooked when unripe kaen banana we kakae blong hem i longlongfala. Hem i blong kakae taem i raep mo yu mas skwisim fastaem

ulēulēi variety of banana kaen banana vagvag variety of banana kaen banana

vetel banana banana Musa spp. vetel mal variety of banana that has a large trunk but small fruit. The bundle can be quite long wan kaen banana we stamba blong hem i bigbigwan be kakae blong hem $i$ smol. Samtaem bandel blong hem i longfala

vetel talisōr variety of banana, traditionally considered one of the original endemic varieties, with red fruit, leaves and trunk wan kaen banana we hem i red, lif i red mo stamba blong hem i smol mo red

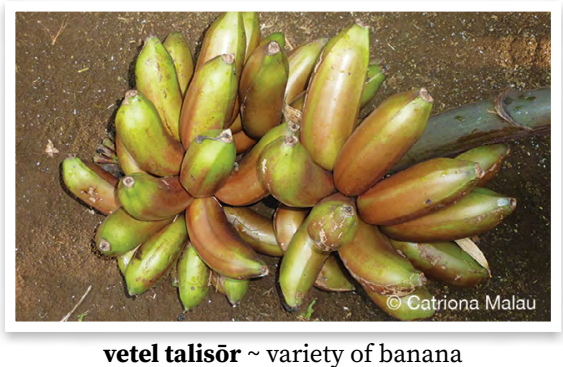

vietnam variety of banana kaen banana

vila variety of banana with long, large fruit kaen banana we kakae blong hem i longfala mo i bigwan

vinwōn variety of banana that is long and a flat shape kaen banana we hem i olsem asis banana be hem i longfala lelebet mo hem i save flat vinwōn ga qag variety of banana kaen banana

vinwōn gō tōtōrōg variety of banana kaen banana

wavagvag variety of banana that has a bundle that grows upwards instead of down. The fruit are roundish and yellow inside with red skin kaen banana we bandel blong hem i gro antap, i no hang daon. Kakae blong 
hem i raon mo taem i raep i red be insaed i yelo. Taem man i kakae i pispis yelo

wēwēsēg variety of banana that has small, short fruit asis banana we hem i raon mo i sotfala

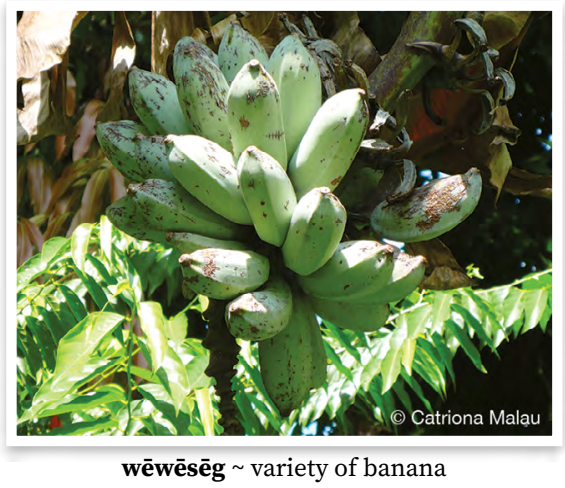

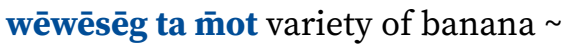
kaen banana

wēwēsēg ta ōrbarbar variety of banana kaen banana

wiriatege variety of banana with very long, large fruit, it has a large trunk but does not bear lots of fruit kaen banana we kakae blong hem i longlongfala mo i bigwan, i gat bigfala stamba be hem i no karem fulap kakae

worwor variety of banana that has long, large fruit and is yellow when ripe kaen banana we samtaem kakae blong hem i no kamaot long en be long medel. Hem i bigwan olsem vila, hem $\mathrm{i}$ grin mo taem $\mathrm{i}$ raep i yelo

\section{D2 Bushes, shrubs}

bēkil kind of shrub wan kaen plant Polyscias samoensis

bormiak evodia, shrub with distinctive, pleasant-smelling leaves, variety with smaller leaves wan kaen lif we i gat naes smel, lif blong hem i bigwan Evodia hortensis

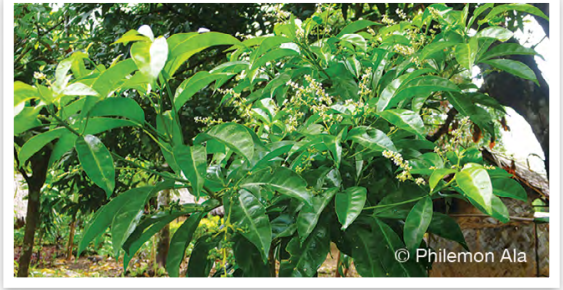

borm̄iak Evodia hortensis

busuqaēn kind of small flowering shrub with white flowers and small fruit that are red when ripe $\sim$ wan kaen wud we $i$ gat waet flaoa, frut blong hem i smolsmol mo i grin be taem i raep i red. Lif blong hem i smolsmol mo stamba i no save gro bigwan Psychotria aneityensis

busuqërët kind of shrub kaen smol wud Ascarina lanceolata

damalalal kind of shrub whose leaves are eaten as greens $\sim$ kaen bus kabis damat heliconia, plant with large leaves that are used to wrap laplap for cooking in stone oven $\sim$ lif laplap Heliconia indica

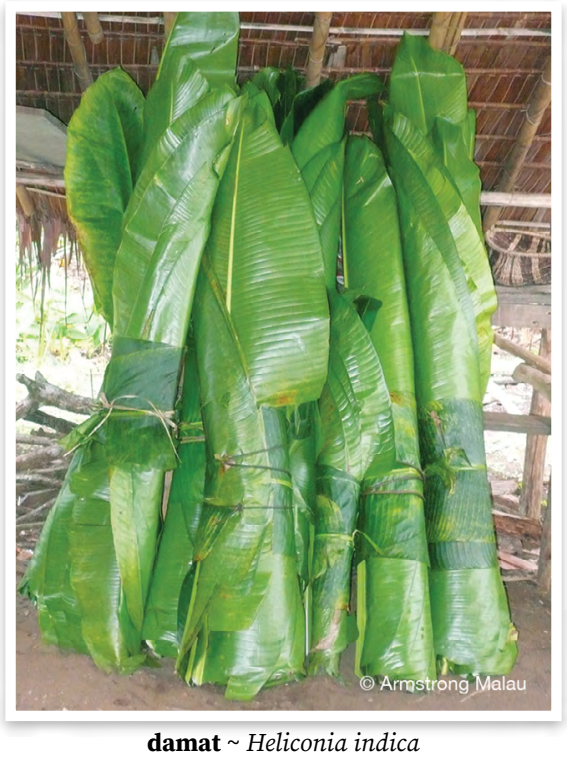

damat dēnin variety of heliconia that has small leaves kaen lif laplap we lif blong hem i smolsmol 
damat möt variety of heliconia which grows wild in the bush and is not cultivated kaen lif laplap we i gro long bus nomo

datamal caricature plant, kind of shrub with edible leaves $\sim$ wan kaen lif we yumi save kukum mo kakae Graptophyllum pictum

datamal an kind of shrub with edible leaves eaten as greens $\sim$ kaen bus kabis

datamiar small shrub with succulent stem and pale purple flowers $\sim$ wan kaen plant we flaoa blong hem i laet popel Coleus scutellarioides

datartarawöw bandicoot berry, kind of shrub with small round black fruit wan kaen plant we smol frut blong hem i raon mo i blak Leea indica

davaqal kind of edible fern that grows in the water or swamp wan kaen bus kabis we $\mathrm{i}$ olsem fens be $\mathrm{i}$ gro long wota o swam Pteris sp.

davarteker kind of shrub, grows to about 2 metres with tiny white flowers wan kaen plant we i gat smolsmol waet flaoa Maesa sp.

deben tenēnēg kind of small plant $\sim$ wan kaen smol plant Melicytus ramiflorus

degetget kind of shrub with leaves which are eaten as greens $\sim$ kaen bus kabis

demen mav kind of shrub wan kaen plant Ficus septica

deteqeñtōw kind of edible fern that grows on hill bus kabis we hem i gro long saed blong hil Pteris sp.

detewel kind of edible fern that grows in the water or swamp kaen bus kabis we i olsem fens be i gro long wota o swam Pteris sp.

dēwērēt aibika variety with reddish stems and veins of leaf $\sim$ kaen aelan kabis we i gat red han blong hem Abelmoschus manihot variety dëmēl ming aralia, kind of plant with edible leaves, that are yellowish green $~$ nalaslas, wan kaen tri we lif blong hem i gat ol poenpoen long saed blong hem Polyscias fruticosa, Polyscias guilfoylei

dimililial kind of shrub whose leaves are eaten as greens $\sim$ kaen bus kabis dimia kind of shrub, native mulberry $\sim$ wan kaen plant Pipturus argenteus

dimiarēsul aibika variety with lots of branches, eaten as greens $\sim$ kaen aelan kabis we i gat fulap han long hem Abelmoschus manihot variety

ditial ${ }_{1}$ kind of shrub wan kaen plant Selaginella durvillei

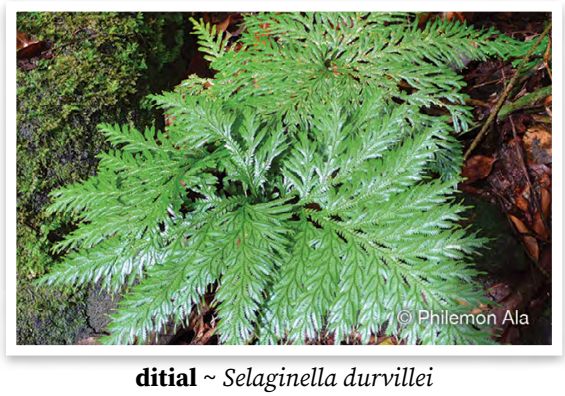

dōrōt type of edible fern bus kabis, kaen fens we yumi save kakae Diplazium harpeoides

dömöwön variety of caricature plant, kind of shrub with edible leaves kaen bus kabis

dövuln̄ö dinner plate aralia, kind of plant with edible leaves nalaslas Polyscias scutellaria

dunrē̄ēlēg kind of plant, shrub wan kaen wud Leucosyke australis

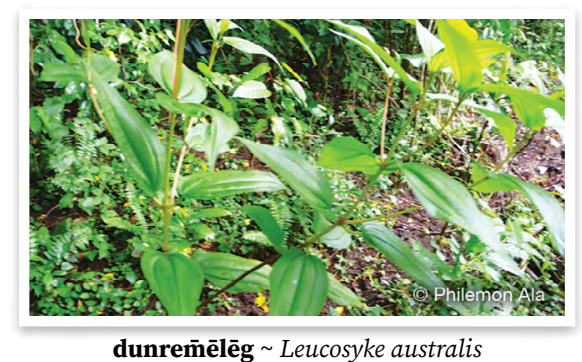


duturkulo (fr.var. döturkulo) variety of aibika, shrub with leaves that are commonly eaten as vegetable greens $\sim$ kaen aelan kabis Abelmoschus manihot variety

galō silver croton wan kaen tri Croton insularis

garmēbuluk variety of aibika, shrub with leaves which are commonly eaten as vegetable greens kaen aelan kabis Abelmoschus manihot variety

kirkiar croton kala lif Codiaeum variegatum

kirkiar mamēgin variety of croton that grows in the bush and is not planted near houses wan kaen kala lif we i stap long bus nomo

kirkiar meter variety of croton wan kaen kala lif

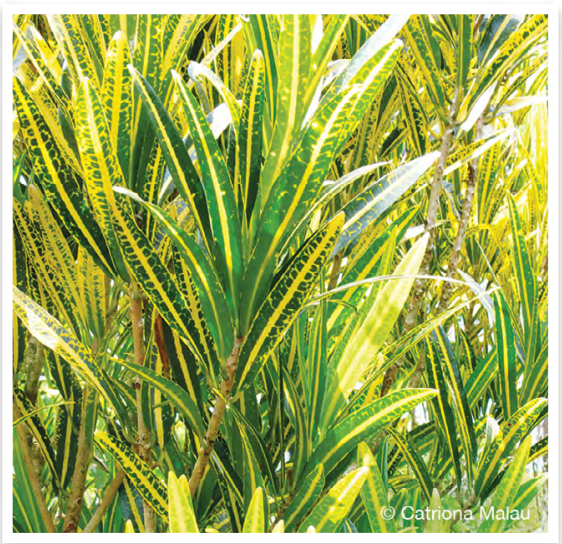

kirkiar meter variety of croton

kirkiar qere q⿳亠丷厂 variety of croton with curly leaves wan kaen kala lif we lif blong hem i tantanem

kirkiar qet variety of croton that is red and green $\sim$ wan kaen kala lif we i red mo grin

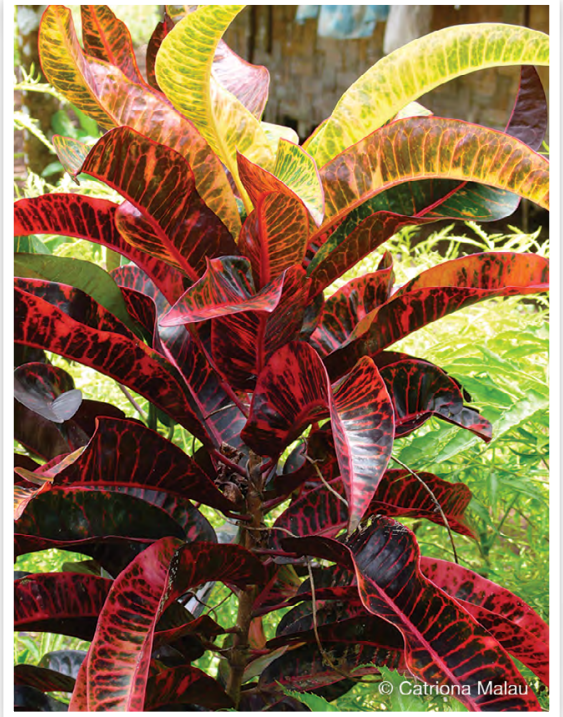

kirkiar qet $\sim$ variety of croton

kirkiar sas qön variety of croton wan kaen kala lif

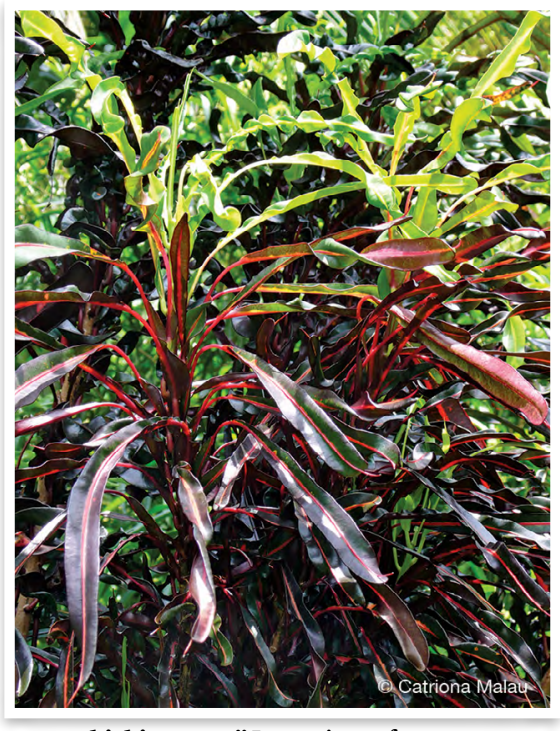

kirkiar sas qȫ̄ $~$ variety of croton

qēqūl mangrove natongtong Rhizophora spp.

qōmur (fr.var. dōqōmur) kind of shrub kaen plant 
rēwēsil variety of aibika, shrub with leaves which are commonly eaten as vegetable greens kaen aelan kabis Abelmoschus manihot variety

$\operatorname{sasar}_{1} \mathbf{1} \cdot$ aibika, kind of shrub that is planted for leaves that are eaten as greens aelan kabis Abelmoschus manihot $\mathbf{2}$ - greens, generic term for leaves that are eaten as greens, whether planted as a crop or gathered from wild plants aelan kabis, nem i kavremap ol defren kaen kabis

seg evodia, shrub with distinctive, pleasant-smelling leaves, variety with wider, larger leaves wan kaen lif we i gat naes smel, lif blong hem i bigwan Evodia hortensis

sör nicolaia; plant from the ginger family which has nice-smelling leaves, used as decoration for dancing and in the house kaen lif we hem i famle blong jinja, i gat naesfala smel blong hem mo ol i yusum long danis olsem dekoresen Hornstedtia sp.

tutugēn tamarge kind of small shrub with purple flowers wan kaen plant we i gat flaoa we i popel Blumea lacinuata

vōnōdöwö kind of shrub, with furry leaves and stem and small mauve flowers wan kaen smol plant Ageratum conyzioides

waqagal hibiscus haebiskas Hibiscus rosa-sinensis

waran̄rañ kind of shrub wan kaen tri Acalypha spp.

waran̄rañ ga qag plant with pale leaves kaen plant we lif blong hem i waet Acalypha forsteriana

waran̄rañ mamē plant with red leaves kaen plant we lif blong hem i red Acalypha hispida wōlōlōmeren kind of flowering plant with long shiny leaves and orange flowers kaen plant we hem i gat longfala lif we i saen mo flaoa blong hem i dak yelo Oxera vanuatuensis

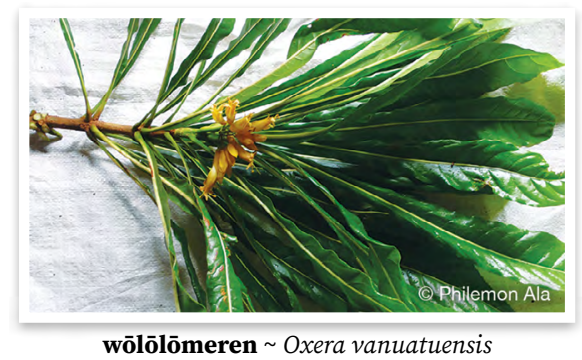

wōvōt kind of shrub, with fruit that is red when ripe wan kaen tri we i no bigwan be kakae blong hem i bigfala. Sep blong kakae i olsem nakavika mo $i$ grin be taem i raep $i$ red. Flaoa blong hem i waet Phaleria sp.

wösusurbē kind of shrub with round, bright orange fruit wan kaen tri we kakae blong hem i grin mo taem i raep $i$ aranis-red Tabernaemontana sanguinea

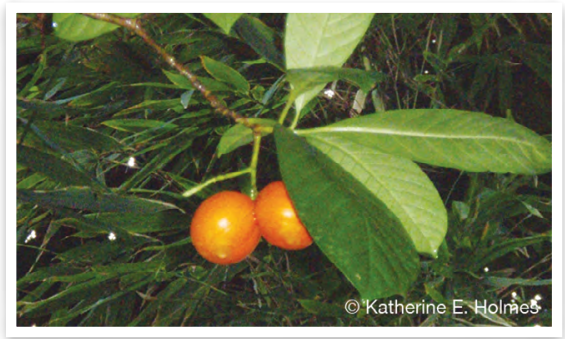

wösusurbē $\sim$ Tabernaemontana sanguinea

D3 Grasses, bamboo, vines

bō 1 - length of bamboo used for carrying water bambu blong fulumap wota 2 - length of bamboo used for making beat in dance bambu blong kilim long wud long taem blong danis

dañdan̄ sugarcane variety kaen sugaken 
ditial ${ }_{2}$ kind of grass with tiny white flowers wan kaen gras we i gat smolsmol waet flaoa

diwinwian kind of plant kaen lif Freycinetia tannaensis

do kind of vine $\sim$ wan kaen rop

do man kind of leaf from a vine that is used to poison fish wan kaen kastom lif blong wan rop we yu yusum blong posenem fish Derris elegans

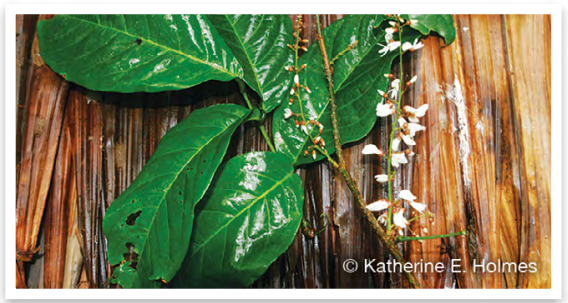

do man Derris elegans

do qōrgavgav leaf that looks like do man, which is used to poison fish, but do qörgavgav is not poisonous wan lif we i luk olsem do man be i nogat paoa blong posenem fis

dōsōwtöl kind of vine, ground creeper with purple flowers wan kaen rop we flaoa blong hem i popel Canavalia rosea

ga $_{1} \mathbf{1} \cdot$ vine $\sim$ rop

ga atat (fr.var. ga qir) climbing canelike vine $\sim$ wan rop we ol i yusum blong fasem haos Flagellaria indica

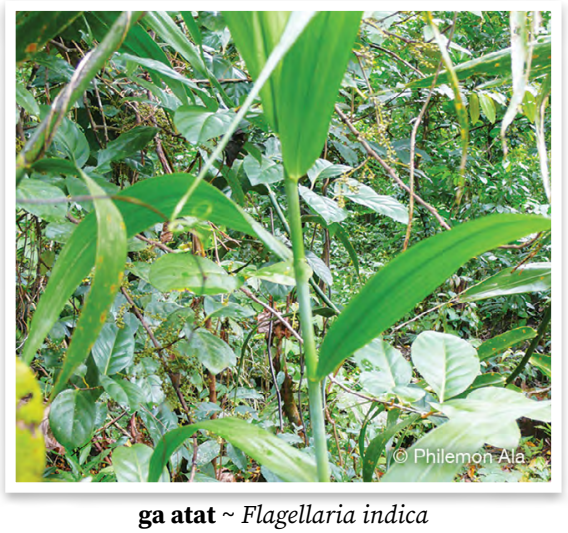

ga del kind of large leaved vine that grows up and wraps around trees $\sim$ wan kaen rop we i gro antap long wud we lif blong hem i bigwan Epipremnum pinnatum

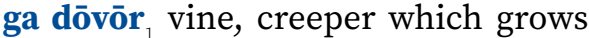
on beach, seashore wan kaen rop we i stap gro long sanbij mo klosap long solwota Ipomoea pes-caprae

ga iaw kind of vine wan kaen rop Anodendron paniculata

ga matawase kind of vine that grows straight and is white when immature and light brown when mature $\sim$ wan kaen rop we hem i smol nomo mo i save gro stret. Taem i yang i waet mo hem i kam laet braon Stephania forsteri

ga mien kind of vine $\sim$ wan kaen rop Epiprenum sp.

ga ōl kind of vine wan kaen rop

ga sasinirnir kind of vine wan kaen rop Cayratia trifolia

ga susul morning glory; kind of vine with blue flowers wan kaen smolsmol rop we i gro long garen mo long haos be no long bus Ipomoea acuminata

ga taqav kind of vine with large leaves wan kaen rop we lif blong hem i bigwan Merremia peltata

ga töus kind of vine which bears tubers similar to sweet potato $\sim$ wan kaen rop we i gat kakae blong hem olsem kumala

ga vörur kind of vine snek rop

gakalkalbōgō jackbean; kind of flowering vine $\sim$ wan kaen rop Canavalia cathartica

gakarēs kind of climbing fern wan kaen rope Lygodium reticulatum

gamalwuw kind of black vine wan kaen rop we i stap long bus mo hem i blak Smilax vitiensis

gamērēr kind of vine wan kaen rop Freycinetia spp. 
gamērēr dēmadēm kind of vine wan kaen rop Freycinetia flavida

gamērēr qō kind of vine wan kaen rop Freycinetia impavida

gatōw kind of strong vine that grows on hills and by the sea but not in the bush. It is hard but can be easily broken when immature wan kaen rop we i stap long hil mo long solwota be no long bus. I strong be yangwan i save brok isi Derris trifoliata

gatöus kind of vine that has fruit which is eaten in time of famine. It is very sour tasting wan kaen rop we ol i kakae frut blong hem long taem blong famin. Hem i konkon mo i saoa

gawol lawyer cane, rattan $\sim$ loeaken Calamus vanuatuensis

mēlēslēs ${ }_{2}$ kind of flowering plant in the nettle family $\sim$ kaen plant Elatostema macrophyllum

mēlēslēs lav kind of flowering plant in the nettle family kaen plant Elatostema beccadi

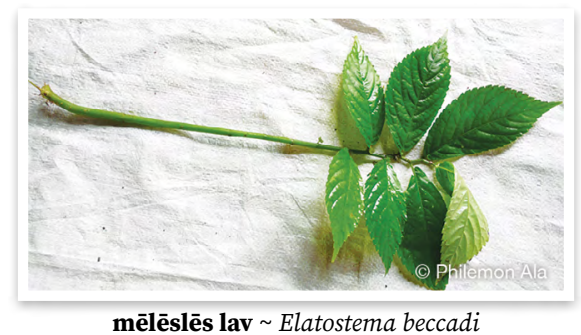

masēlasēl Job's tears; grain-bearing plant wan kaen gras we i longfala mo $i$ gat sid blong hem we i waet mo i strong mo ol i yusum olsem bid Coix lacryma

$\overline{\mathbf{m}} \mathbf{o} \mathbf{g} \mathbf{t}_{1}$ kind of grass wan kaen gras Oplismenus hirtellus

qörö gōsōw kind of vine that grows along the ground wan kaen rop $\mathrm{i}$ gro long graon nomo Geophyla repens rartan wild cane $\sim$ wael ken Miscanthus sp. röv vine used in weaving bird trap rop we ol i yusum blong wivim trap blong pijin

töv sugarcane $\sim$ sugaken Saccharum officinarum

vēlis grass, weeds gras

vēlis ōlōl false dodder, love vine, kind of slender rambling vine that grows on ground wan kaen rop we i gro long graon Cassytha filiformis

waga $_{2} \mathbf{1}$ - passionfruit pasenfrut Passiflora edulis

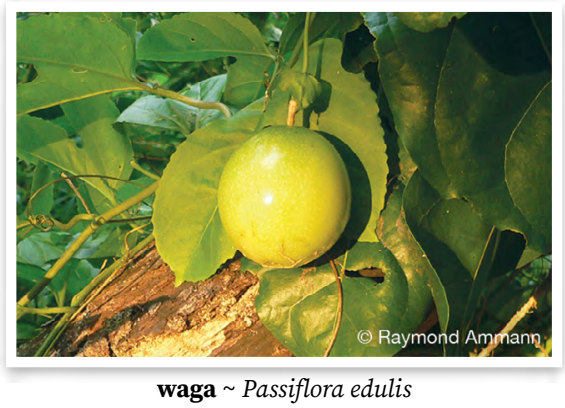

2 - stinking passionflower wael pasenfrut Passiflora foetida

wetelwa kind of vine, similar to a pumpkin with round fruit, the leaves are eaten as greens $~$ kaen rop we yumi save kakae top blong hem

wēnēn bulëvu (fr.var. winin bulëvu) perennial herb with branched cane-like stems kaen flaoa Donax cannaeformis

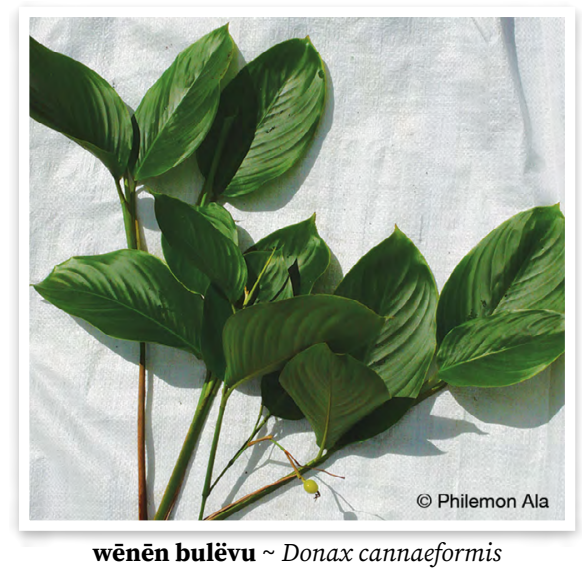


wētēvtēv kind of grass wan kaen gras Scleria polycarpa

woo bamboo bambu Bambusa spp. woo mölum kind of soft bamboo sofsof bambu Bambusa vulgaris woo vet kind of strong bamboo which is thin, long and flexible and bends at the tip strong bambu we i smol be i longfala mo en blong hem i save benben Bambusa sp.

woo vet luwō kind of strong bamboo that is thick and used for wide weaving for house walls strong bambu we i tik mo hem i bigwan blong wivim woling blong haos Bambusa sp.

woo vet nötu kind of strong bamboo that is thin and used for narrow weaving for house walls strong bambu we i smol blong wivim woling blong haos i smolsmol Bambusa sp.

\section{D4 Tubers and roots}

dem (fr.var. dëm) yam yam Dioscorea spp.

dem atat̄̄ēn primary grouping of varieties of yam, yams that bear long tubers ol kaen yam we kakae blong olgeta ol i longlongfala

dem kēlēglēg primary grouping of varieties of yam, yams that bear crooked tubers ol kaen yam we kakae blong olgeta ol i kruked

dem rereqe primary grouping of varieties of yam, yams that bear short tubers ol kaen yam we kakae blong olgeta ol i sotsotfala

kōmar sweet potato kumala Ipomoea batatas

maniok cassava, manioc maniok Manihot esculenta

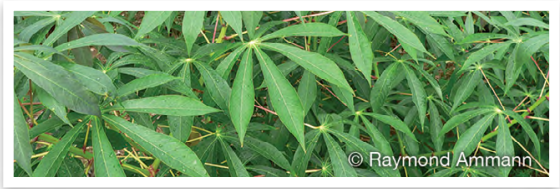

maniok Manihot esculenta qiat taro taro Araceae spp.

qiat atm̄ēn taro grouping, varieties of taro which are 'male' and don't fork grup blong ol taro, man taro we hem i no save karem fok

qiat reqe taro grouping, varieties of taro which can fork grup blong ol woman taro we ol i save karem fok

diqiat taro leaf, cooked and eaten as greens $\sim$ lif blong taro

sinsa ginger $\sim$ jinja

tamag lesser yam, Chinese yam wovile, swityam Dioscorea esculenta $\mathbf{v} \overline{\mathbf{e}}_{1}$ giant taro, elephant ear taro waeltaro Alocasia macrorrhiza

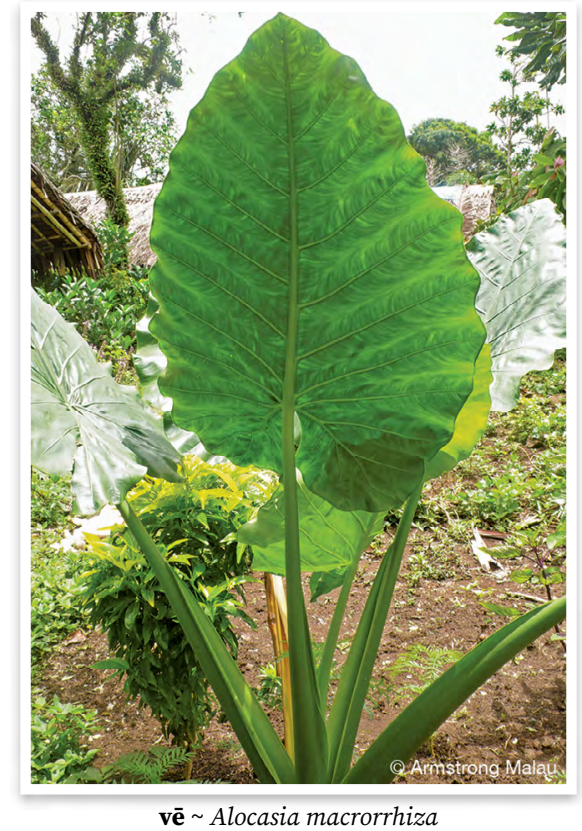

waan turmeric kari jinja Curcuma longa

\section{D4.1 Taro}

akrikalja variety of taro, 'male', which occurs in a variety of colours and shapes $\sim$ kaen taro

biliag $_{3}$ variety of taro, 'female', a type of marē, with a dark red stem kaen mare taro we hem i woman we han blong hem i dak red 
böl variety of taro, an old variety with a whitish stem that is no longer planted kaen taro blong bifo we ol i nomo planem we han blong hem i waet

bulalēv variety of taro, 'male', with a black stem kaen taro we hem i man we han blong hem i blak

burmatan variety of taro, 'male', with a dark green bottom, dark purple top and black petiole $~$ kaen taro we hem i man we han blong hem i dak grin daon mo antap i dak popel

busuōr variety of taro, 'female', with light red stem and top with light green stripes $\sim$ kaen taro we hem $i$ woman we han blong hem i laet red wetem grin straep long hem

din̄venqiat variety of taro, 'female', with a stem that is whitish at the bottom and light green at the top kaen taro we hem i woman we han blong hem i waet daon mo i grin antap

dogon variety of taro kaen taro

ërmamē variety of taro, with a light red stem with white stripes $\sim$ kaen taro we han blong hem $\mathrm{i}$ laet red mo i gat waet straep

galatentur variety of taro that has white tuber, skin and stem kaen taro we hem i waet evriwan, kakae wetem han mo skin tu i haf waet

götö variety of taro, 'male', with black stem, and white corm with red spots $\sim$ kaen taro we hem i man we han blong hem i blak mo kakae blong hem i waet wetem red spot

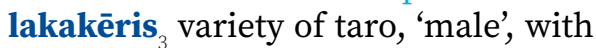
light green stem kaen taro we hem i man we han blong hem i laet grin

lantar variety of taro, 'female', with two types $\sim$ kaen taro we hem i woman mo hem i gat tu kaen blong hem lantar lamkör variety of taro, 'female', with black stem kaen taro we hem i wan kaen lantar. Han blong hem i dak mo kakae blong hem i waet

lantar malgias variety of taro, 'female', one of the largest tubers with a soft corm, stem is purple with light green at top kaen taro we hem i wan kaen blong lantar. Han blong hem i laet gre mo kakae blong hem i waet

len̄man variety of taro, 'female', with green stem with white or yellow stripes $\sim$ kaen taro we hem i woman we $\mathrm{i}$ gat grin han wetem waet o yelo straep long hem

lomonwer variety of taro with light green stem and yellow corm $\sim$ kaen taro we han blong hem i laet grin mo kakae blong hem i yelo

lōkreg variety of taro, 'female', with brown stem with light green stripes, and white corm kaen taro we han blong hem i laet braon mo kakae i waet. Hem i strong lelebet mo hem $i$ save karem fok

lōkreg mamē variety of taro, 'female', with red stem $\sim$ kaen taro we hem $i$ woman we han blong hem i red

mako variety of taro, 'male', with stem that is red at bottom and green at top $\sim$ kaen taro we hem i man we han blong hem i red daon mo grin antap

malmalei lantar variety of taro, 'male', family of lantar, with many stems that are dark green at bottom and light green at top, and small corms that are covered in roots and rot easily kaen taro we hem i man mo hem i famle lantar, we kakae blong hem i gat fulap rus blong hem mo $\mathrm{i}$ save roten isi. Han blong hem i dak grin daon mo i laet grin antap manreva variety of taro $\sim$ kaen taro 
mare $\bar{e}_{2}$ grouping of kinds of taro that don't fork but grow tall and crooked grup blong ol kaen taro we ol i no save karem fok be ol i save longfala mo benben (olsem namarae)

marē wasalav variety of taro, 'male' with stem that is white at bottom and light brown at top, with light black petiole kaen taro we hem i no save gro bigwan be i save longfala. Hem i sofsof nomo mo kakae i waet

marē wasalav mamē variety of taro, 'male', with red stem and white corm kaen taro we hem i man we han blong hem i red mo kakae blong hem i waet

masta variety of taro, with white stem that is red at bottom kaen taro we han blong hem i waet mo i red daon mensēkē variety of taro, 'male', family of lantar, with many stems that are dark green at bottom and light green at top, and small corms that are covered in roots and rot easily $\sim$ kaen taro we hem i man, we hem i famle lantar, we han blong hem i dak grin daon mo laet grin antap. Kakae blong hem i smol mo i gat fulap rus mo i save roten isi

mesmame $\bar{e}_{2}$ variety of taro, 'female', with red stem $\sim$ kaen taro we hem i woman mo stem blong hem i red

mesvōlōvōl variety of taro, 'female', with green stem kaen taro we hem i woman, we han blong hem i grin

mōlkēl variety of taro, 'female', stem with dark green bottom and dark purple top kaen taro we hem i woman mo stem blong hem i dak grin daon, popel antap

matēkmëgërsurletes variety of taro, 'female', with black stem and yellow corm kaen taro we hem i woman, we han blong hem i dak lelebet mo kakae blong hem i ful yelo merlav variety of taro, with tall dark red stem, and large light red corm $\sim$ kaen taro we stem blong hem i longfala mo i dak red, kakae blong hem i laet red

mēvinvian 2 variety of taro, 'female', with light green stem with light red stripes and light red corm kaen taro we hem i woman, we han blong hem i laet grin wetem laet red straep mo kakae blong hem i laet red

mēw variety of taro, 'female', with purple stem and wavy leaves with black veins on underside $\sim$ kaen taro we hem i woman we han blong hem i popel mo lif i gat blak laen long andanit blong hem

mōvōl variety of taro, 'female', with light purple stem, round leaves kaen taro, we hem i woman, stem i laet popel mo lif i raon

nalumlum variety of taro, 'male', with black stem with stripes $~$ kaen taro, we hem i man, wetem blak stem we i gat straep long hem

nōvōk variety of taro, 'male', with tall brown stem $\sim$ kaen taro, we hem i man, we stem blong hem i longfala mo i braon

ōrbarbar variety of taro, 'female' kaen taro we hem i woman

qiat taro taro Araceae spp.

diqiat taro leaf, cooked and eaten as greens $\sim$ lif blong taro

qiat atm̄ēn taro grouping, varieties of taro which are 'male' and don't fork grup blong ol taro, man taro we hem i no save karem fok

qiat gōl variety of marē taro, 'male', with light green stem and white corm kaen marē taro we hem i man mo i laet bitim evri taro. Hem i save bigwan smol mo hem i save longfala. Han blong hem i laet grin mo kakae i waet 
qiat lēlē variety of taro, 'female', that is considered to be the king of taros as it grows very tall, has a light green stem and white corm kaen taro we han blong hem i laet grin mo kakae i waet. Hem i save bigwan mo longlongfala, hem i king taro

qiat mingala variety of taro, 'female', with light green stem $\sim$ kaen taro we hem i woman, we han blong hem i laet grin

qiat minwog variety of taro, 'female', that is considered to be the king of taros as it grows very tall, has a light green stem and white corm kaen taro we hem i woman, han blong hem i laet grin mo kakae i waet. Hem i save bigwan mo longlongfala, hem i king taro

qiat qet variety of taro, 'female', with black stem and red corm $\sim$ kaen taro we hem i woman, han blong hem i blak mo kakae blong hem i red

qiat qön variety of taro, 'female', with black stem and red corm kaen taro we hem i woman, han blong hem i blak mo kakae blong hem i red

qiat reqe taro grouping, varieties of taro which can fork grup blong ol woman taro we ol i save karem fok

qiat rev variety of taro, a grouping that includes 'male' and 'female' varieties, all have many runners, there is variation in colour and shape grup taro we $\mathrm{i}$ gat plante kaen long hem, we sam i man, sam i woman, i gat defren kala mo sep be evriwan i gat fulap saka

qötuq $\overline{\mathbf{o}}_{2}$ variety of taro, 'male', with black stem with white stripes kaen taro we hem i man, we han blong hem i blak wetem waet straep

regeltëv variety of taro kaen taro rēgēt variety of taro, 'male', with light green stem and small red top $\sim$ kaen taro we hem i man we han blong hem i laet grin mo i red antap

rēlēgtēl variety of taro, 'female', with stem that is red at the bottom and purple at the top $\sim$ kaen taro we hem $\mathrm{i}$ woman mo han blong hem i dak lelebet mo kakae i waet. Skin blong hem i laet grin mo hem i save bigbigfala

rēsim variety of taro, with red stem and light red corm. It is not strong and cannot fork kaen taro we han blong hem i red mo kakae i miks red wetem waet. Hem i no strong mo hem i no save karem fok

rēwurveg variety of taro, 'female', with light brown stem and many roots on the corm $\sim$ kaen taro we hem i woman we han blong hem i laet braon mo kakae blong hem i gat fulap rus long hem

$\mathbf{r o v}_{2}$ variety of taro, 'female', with white stem and corm and red veins under the leaf, and can fork kaen taro we han blong hem $\mathrm{i}$ waet mo kakae blong hem i waet mo hem i save karem fok

rōvōl variety of taro, 'female' with stem that is white at bottom and red at top, with red veins under leaves $\sim$ kaen taro we hem i woman we han blong hem i waet daon mo red antap, mo i gat red mak long lif blong hem

römöwuler variety of taro, 'female', with very dark red stem $\sim$ kaen taro we hem i woman we han blong hem i dak red

sarē variety of taro, 'male', with stem that is white with a small amount of red $\sim$ kaen taro we han blong hem i waet wetem smol red, kakae blong hem i waet mo hem i no save karem fok

sēlēgtel variety of taro kaen taro 
sēstañ variety of taro, 'male', with a tall light green stem with red stripes $\sim$ kaen taro we hem i man, we han blong hem i laet grin mo i gat red straep long hem

siag gēgēt ${ }_{2}$ variety of taro, 'female', with light red stem with white stripes kaen taro we hem i woman we han blong hem i laet red wetem waet straep

sinag $\mathbf{1} \cdot$ taro $\sim \operatorname{taro} \mathbf{2} \cdot$ food $\sim$ kakae

siritimiat variety of taro, 'male', in mare grouping with dark stem, black petiole and red and white corm kaen mare taro we hem i no save bigwan be i save longfala mo i save benben. Han blong hem i dak popel mo kakae blong hem i miks laet red wetem waet. Hem i sofsof mo hem i taro blong rus we i save dan kwik

susdenlē variety of taro with red stem with white stripes kaen taro we han blong hem i red wetem waet straep

suwbē variety of taro, 'female', with white stem and corm. They are big and long and can be crooked like marē taro kaen taro we hem i woman mo han, skin mo kakae blong hem i waet. Hem i longfala mo bigfala mo hem i save ben olsem marē

taltal variety of taro, 'female', with black stem and light spots on leaves $\sim$ kaen taro we hem i woman, we han blong hem i haf dak mo han mo lif $\mathrm{i}$ gat waet spot. Kakae blong hem i waet mo hem i no stap karem fok

tana variety of taro, 'female', with dark red stem $\sim$ kaen taro we hem i woman, we han blong hem i dak red tan̄evsōs variety of taro, 'male' kaen taro we hem i man

teñtur variety of taro, 'female', with light green stem $\sim$ kaen taro we hem i woman, we han blong hem i laet grin tewesqēqēl variety of taro, 'female' with stem that is white at the bottom and red at top kaen taro we hem i woman, we han blong hem, daon i waet mo antap i red

tewestamal variety of taro, with green stem and black petiole $~$ kaen taro we han blong hem i grin mo i joen long lif hem i blak

tewesvër variety of taro, with red stem and light black petiole $\sim$ kaen taro we kala blong han mo kakae blong hem i miks red wetem waet, skin blong hem i red

titiritōwvetam variety of taro with green stem with brown stripes at base $\sim$ kaen taro we han blong hem i grin wetem braon straep daon

tortor variety of taro, 'female', with stem that is red at the bottom and green at the top kaen taro we hem i woman, we han blong hem i red daon mo grin antap

varvarsō̄m variety of taro with green stem and small black petiole, with white corm kaen taro we han blong hem i laet grin mo kakae i waet

vinmötöl variety of taro, 'female', with black stem and white corm $\sim$ kaen taro we hem i woman, we han blong hem i dak lelebet mo kakae i waet

wakata variety of taro kaen taro

wakata gatgat variety of taro, with white stem and white corm with red stripes kaen taro we han blong hem i waet mo kakae blong hem i waet wetem red straep

wakata mamē variety of taro, 'female', with tall red stem that has white stripes at the bottom, and white corm kaen taro we hem i woman, we han blong hem i longfala mo i red wetem waet straep daon, kakae blong hem i waet 
wakata qagqag variety of taro, 'male', with white stem and corm kaen taro we hem i man, we han mo kakae blong hem i waet

wamal variety of taro that has both 'male' and 'female' varieties, the 'male' with stem that is white at bottom and red at top, the 'female' with purple stem and black petiole kaen taro we $\mathrm{i}$ gat woman mo man wan blong hem, we woman, han blong hem i waet daon mo red antap, mo man, han blong hem $\mathrm{i}$ popel mo i blak long ples we lif i joes waras variety of taro, 'male', with white corm with red points kaen taro we hem i man, we kakae blong hem i waet wetem red long hem

wasalav variety of taro kaen taro

wasanto variety of taro that originated from Santo $\sim$ kaen taro we i kam long Santo

wasanto mamē variety of taro kaen taro

wasanto qagqag variety of taro originating in Santo that has white root $\sim$ kaen taro we i kam long Santo we kakae blong hem i waet

wase $_{2}$ variety of taro that has two types, one with light grey stem, the other with light green stem. They can have lots of forks, but are not good for eating in that case $\sim$ kaen taro we i gat tu kaen blong hem, wan han blong hem i laet grei, wan han blong hem i laet grin. Hem i strong lelebet. Hem i save karem plante fok be sapos olsem kakae i no gud

wederebiliag variety of marē taro that is not large but grows long and crooked, with light red stem with red stripes. The corm is white and is not strong but is good for roasting $\sim$ kaen mare taro we hem i no save bigwan be i save longfala mo i save benben. Han blong hem i laet red wetem ful red laen i go daon. Kakae blong hem i waet mo i no strong. Hem i spesel taro blong rusum

wegeretqon variety of taro $\sim$ kaen taro wēbigqō variety of taro $\sim$ kaen taro wēbigqō mamē variety of taro kaen taro

wēlēbēōr variety of taro, 'female', with light red stem and top with light green stripes $\sim$ kaen woman taro we han blong hem i laet red wetem laet grin straep

wēmēèèglèg variety of taro in marē grouping that has light red stem and white corm. It is stronger than other marē but not that strong kaen marē taro we han blong hem i laet red mo stamba klosap i waet. Kakae blong hem i waet mo hem i strong bitim ol narafala marè be i no strong tumas

wēvē variety of taro that has two types and is like wild taro. The corm is yellow and white inside. It is not strong and can fork kaen taro we $\mathrm{i}$ gat tu kaen blong hem. Hem i olsem waeltaro. Han blong hem i no dak tumas. Taem yu brekem top kakae blong hem i yelo mo i waet insaed. Hem i no strong mo i save karem fok wēvē lamkör variety of wèvē taro that is very white inside but has a dark stem kaen wèvē taro we insaed blong hem $\mathrm{i}$ waet evriwan mo han blong hem i mo dak

wēviti variety of taro kaen taro wotalēbak variety of taro $\sim$ kaen taro wotanaval variety of taro $\sim$ kaen taro wotkērēvōr variety of taro with a greyish stem and white corm. One stem can produce many corms. It is an ideal taro for making löt kaen taro we han blong hem i laet grei mo kakae i waet. Taem yu planem wan nomo be hem i save karem plante kakae. Hem i wan gud taro blong nalot 
wotlalabēvidavaqal variety of taro with dark blue stem and white corm. It does not fork kaen taro we han blong hem i dak blu mo kakae i waet. Hem i no save karem fok

wotliev variety of taro that is one of the largest varieties. There are two kinds, one with a white stem, the other with a darker stem. The corm is white with a light green skin. It can fork kaen taro we hem i bigbigfala taro olgeta. I gat tu kaen blong hem, wan han blong hem i waet, wan han blong hem i dak lelebet. Kakae i waet mo skin blong kakae i laet grin. Samtaem i save karem fok

wotlievgatgat variety of taro kaen taro

wotmēlēv variety of taro $\sim$ kaen taro

wotmindeli variety of taro $\sim$ kaen taro wotminmendala variety of taro kaen taro

wotminsakna variety of taro kaen taro

wotminviolette variety of taro kaen taro

wotminwērirtel variety of taro that has a stem that is red close to the leaf, but white near the corm. It is not large but grows long kaen taro we han blong hem antap, andanit long lif i red be klosap long kakae i waet. Hem i no save bigwan be i save longfala

wotvadadan variety of taro that grows large and has a light green stem and white corm kaen taro we han blong hem i laet grin mo kakae i waet. Hem i save bigbigwan

\section{D4.2 Yam}

bakalav $_{2}$ variety of yam that is between grouping of 'female' and 'male' yams. Unlike most yams, it is not easily recognisable on the basis of its leaf. It is very thorny and is red and white $\sim$ wan kaen yam we hem i no man mo hem i no woman. Hem $\mathrm{i}$ had blong luk save from lif blong hem nomo. I gat tumas nil blong hem mo insaed hem $i$ waet mo red

bargōr $\mathbf{r}_{2}$ yam that is unable to produce large tubers because it is planted with other large crops taem yu planem yam wetem bigfala kakae, yam i no save mekem bigfala kakae

dem (fr.var. dëm) yam yam Dioscorea spp.

dem atat̄̄ēn primary grouping of varieties of yam, yams that bear long tubers ol kaen yam we kakae blong olgeta ol i longlongfala

dem bōnōbōn variety of yam belonging to 'male' grouping, that has a distinctive smell wan kaen yam we i longfala mo i waet mo i gat smel blong hem

dem getō variety of yam that has red tuber, with varieties belonging to both 'male' and 'female' groupings $\sim$ wan kaen yam we insaed i red evriwan, i gat woman kaen mo man kaen tu

dem kēèeglēg primary grouping of varieties of yam, yams that bear crooked tubers ol kaen yam we kakae blong olgeta ol i kruked

dem rereqe primary grouping of varieties of yam, yams that bear short tubers ol kaen yam we kakae blong olgeta ol i sotsotfala

dem ga variety of yam that is not planted but grows wild in the bush kaen wael yam blong bus

dōr variety of wild yam strong wael yam

mēlin variety of yam that is grouped with the 'male' yams and is white inside wan kaen yam we $i$ longlongfala mo insaed i waet

mat variety of yam that belongs to the grouping of 'crooked' yams kaen yam we i stap long grup blong ol kruked yam 
qōlmo variety of yam that belongs to the grouping of 'crooked' yams kaen yam we i stap long grup blong ol kruked yam

qōōr wild yam wael yam Dioscorea nummularia

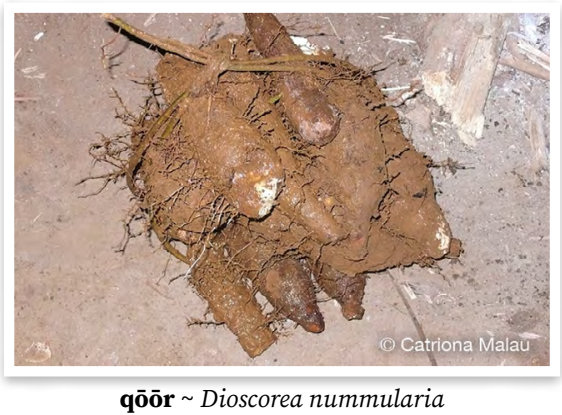

$\operatorname{taw}_{2}$ variety of yam that belongs to the grouping of 'female yams', which is white and soft wan kaen yam we $i$ sofsof mo i waet. Hem i save gro raon be samtaem i flat

walalas kind of wild yam wan kaen yam we hem i gru wael Dioscorea pentaphylla

walalnelan kind of yam that has a round shape, is white and can grow large wan kaen yam we hem i raon gud mo hem $i$ save bigbigwan mo hem i waet insaed

weag wild yam wael yam Dioscorea nummularia

wēbiēg variety of yam with a double layer of skin wan kaen yam we i gat dabol skin

wēbiēg atatīēèn variety of yam with a double layer of skin and white flesh wan kaen man yam we i gat dabol skin mo insaed i waet

wēbiēg rereqe variety of yam that can be red or white wan kaen woman yam we insaed i red o waet

wēkēr wōnwōn variety of yam belonging to the 'female' grouping $\sim$ wan kaen yam we i no save gro longfala tumas be i save gro bigfala. insaed blong hem i waet wēlir variety of yam that is not planted but grows wild in the bush $\sim$ wan kaen wael yam we i save longlongfala (olsem man) be i smol nomo antap we i mekem se $\mathrm{i}$ had blong digim

wōqōlmō kind of yam that is shaped like a crescent moon and can come out of the ground as it grows. There are two kinds, one with red skin, the other with white skin wan kaen yam we hem i sep olsem niu mun mo hem i gro i save kam antap long graon bakegen. I gat tu kaen blong hem, wan skin blong hem i red, wan i waet

wöböw (abbrev. ga wöböw) aerial yam nabew, konkon yam Dioscorea bulbifera

wötörövarvar variety of yam which belongs to the grouping of 'male' yams wan kaen yam we i longlongfala mo insaed long hem i stat antap hem i red, i go daon long medel i miks red mo waet mo long en blong hem i waet

wöuw kind of yam that is very productive. It is one of the best tasting yams and is often grown in the taro garden $\sim$ wan kaen yam we hem i karem fulap kakae. Plante taem ol i planem long garen taro. Tes blong hem i gud, klosap i bitim ol narafala yam

\section{D4.3 Cassava}

atina cassava variety $\sim$ kaen maniok

maniok cassava, manioc maniok Manihot esculenta

maniok añan variety of cassava that has yellow tubers kaen maniok we kakae blong hem i yelo

maniok atatīēn primary grouping of varieties of cassava, cassava that bear long, large tubers ol kaen maniok we kakae blong olgeta ol i longlongfala mo i bigwan 
maniok rereqe primary grouping of varieties of cassava, cassava that bear round, short tubers ol kaen maniok we kakae blong olgeta ol i raon mo i sotfala

raēs ga qag cassava variety $\sim$ kaen maniok

raēs mamē cassava variety kaen maniok

saemon cassava variety kaen maniok sus atat̄̄ēn variety of cassava belonging to 'male' grouping, with long, large tubers kaen maniok we kakae blong hem i longfala mo i bigwan

sus rereqe variety of cassava that has round, short tubers kaen maniok we kakae blong hem i raon mo i sotfala

vasēr cassava variety kaen maniok

\section{D4.4 Sweet potato}

ga dōvōr ${ }_{2}$ variety of sweet potato which is named for plant of same name as it has large leaves like that vine $~$ kaen kumala we i gat bigfala lif

pawa variety of sweet potato that has green vine $\sim$ kaen kumala we rop blong hem i grin

spidbōt variety of sweet potato that is named for the fact that it grows very quickly kaen kumala we ol i putum nem blong hem from hem i gro kwiktaem tumas

tövutwen variety of sweet potato that has a greenish vine $~$ kaen kumala we rop blong hem i grin smol

tri manis variety of sweet potato that is valued as it bears tubers only three months after planting kaen kumala we i stap tri manis nomo, afta i karem kakae

$\operatorname{vorog} 1 \cdot$ sweet potato variety $\sim$ kaen kumala

wese to $\overline{\mathbf{o}}_{2}$ variety of sweet potato that is so named as it is shaped like a chicken's egg kaen kumala we i tekem nem blong hem from i sep olsem eg blong faol
D5 Miscellaneous plants

dagarē (root garē) victory leaf, ti plant $\sim$ nanggaria Cordyline terminalis, $C$. fruticosa

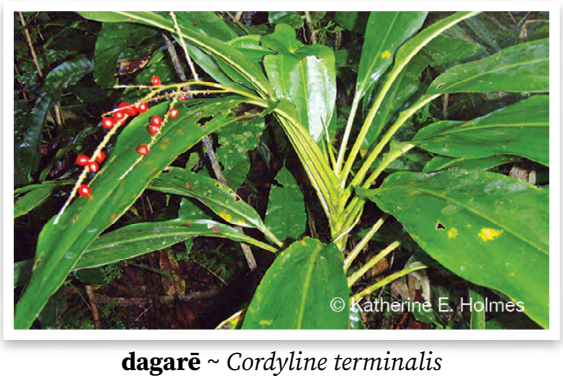

damatmot canna lily wan kaen flaoa Canna indica

damalak crinum lily, spider lily kaen waet flaoa Crinum asiaticum

damataq kind of small flowering plant with tiny mauve flowers wan kaen plant we i gat smolsmol popel flaoa Veronia cinnerea

dasas fern (generic) fens

dasas qötu riaw kind of fern wan kaen fens Angiospermum evecta

dasas tie tō kind of fern wan kaen fens Spherostephanos invisus

dēnin (fr.var. dinin) leaf like heliconia but smaller lif olsem lif laplap be i smolsmol Cominsia gigantea

dēqēt bird's nest fern wan kaen lif we i stap gro long stamba blong wud Asplenium nidus

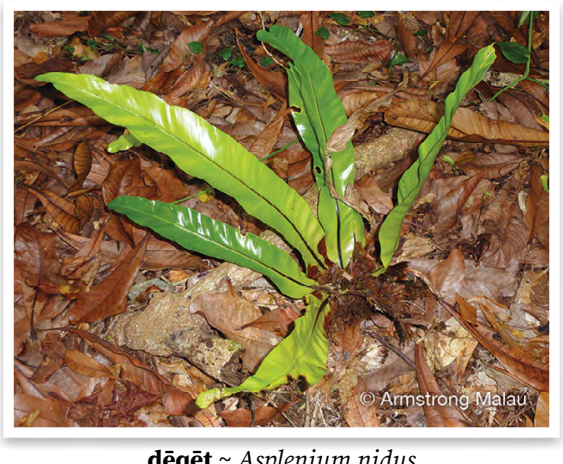

dēqēt Asplenium nidus 
dobormiak plant with strong scented yellowish, green leaves lif we $i$ gat smel blong hem we i yelo, grin Kopsia flavida

dōmōtōtak plant related to kava but which cannot be made into kava waelkava Macropiper latifolium

dōmōtōtak mamē plant related to kava with dark red leaves and branches waelkava we lif mo han blong hem i red

dökurut kind of wild pandanus, with yellow flower, fruit is red when ripe olsem wan kaen wael pandanas we i gat nil long lif blong hem Freycinetia tannaensis

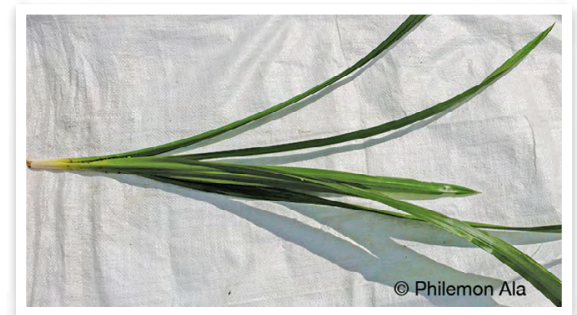

dökurut $\sim$ Freycinetia tannaensis

dötöqötun plant with stinging nettle like leaves with a serrated edge and tiny flowers wan kaen plant we hem i save kakae skin blong yu

gatavdor type of ginger plant kaen flaoa Alpinia sp.

gē $\mathbf{1}$ - kava plant kava Piper methysticum

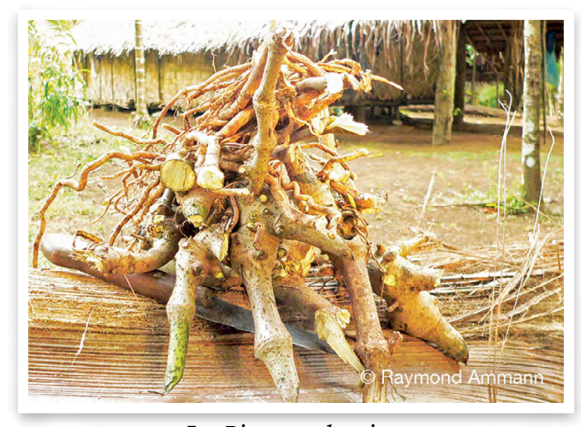

$\mathbf{g e ̄} \sim$ Piper methysticum lēnötō ${ }_{2}$ red firespike, kind of flowering plant with red flowers that look like rooster's comb wan kaen plant we i gat red flaoa blong hem we i luk olsem samting antap long hed blong man faol Odontonema stricta

lösönqō plant belonging to ginger family plant we hem i famle blong jinja Alpinia pacifica

lumlum 1 • seaweed nalumlum (blong solwota) $\mathbf{2} \cdot$ moss, algae nalumlum (long wud, long bus)

low kind of edible seaweed, which has two types, one red, one yellow $\sim$ kaen nalumlum blong solwota we yu save kakae

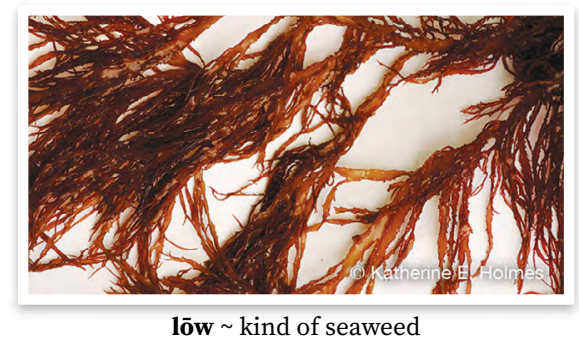

mèl cycad namele Cycas seemannii mēel añan̄ kind of cycad kaen namele

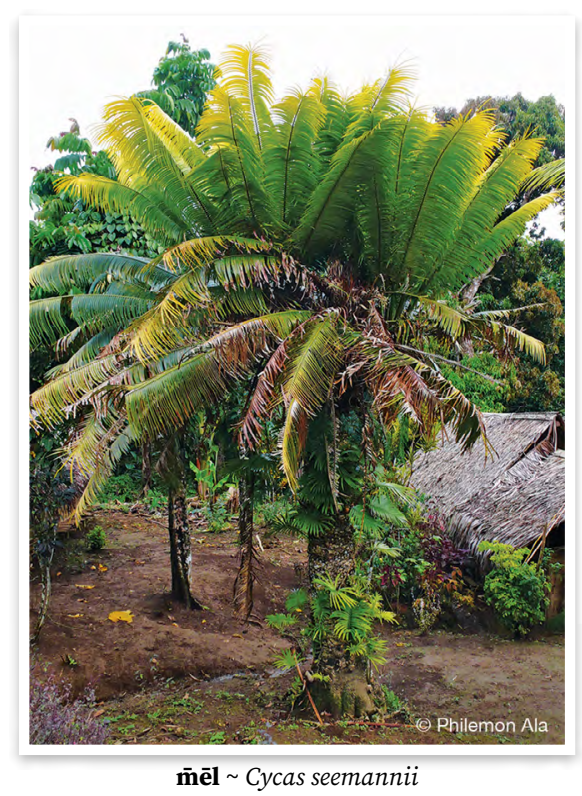


mēvinvian (fr.var. M̄ivian; mivinvian) kind of plant wan kaen plant Coprosma persicaefolia

nisnias kind of plant $\sim$ kaen plant Elatostagis falcata

pomken pumpkin pamken

$\mathbf{q} \overline{\mathbf{o}}_{3}$ kind of plant with thick succulent leaves, white flowers and red fruit with black seeds kaen plant we i gat waet flaoa, red frut mo blak sid Tapeinochilus sp.

raminwōlōnmögörviaomōtōsur bok choy, Chinese greens waetbun, jaenis kabis

tartarawöw kind of plant kaen plant Leea indica

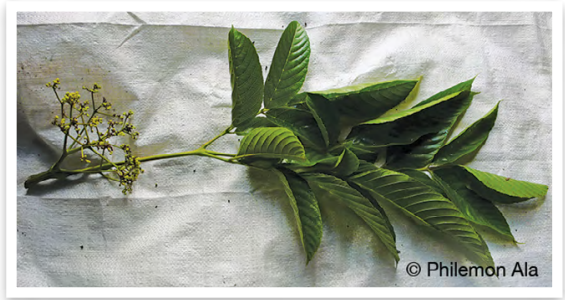

tartarawöw Leea indica

van $_{3}$ pineapple $\sim$ paenapol Ananas comosus

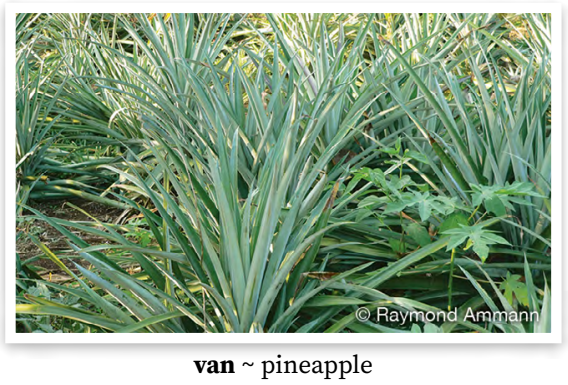

visō pitpit, duruka, vegetable cane naviso Saccharum edule

wamamēgin type of croton with green leaves famle blong kala lif be lif blong hem i grin nomo Codiaeum variegatum wamā̄as kind of plant wan kaen plant Evodia latifolia

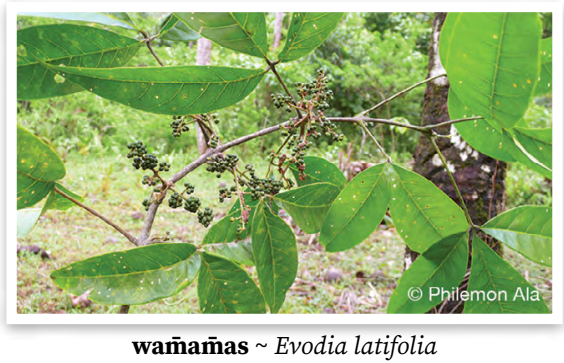

wedeldel red ginger, kind of flowering plant red flaoa we hem i famle blong jinja Alpinia purpurata

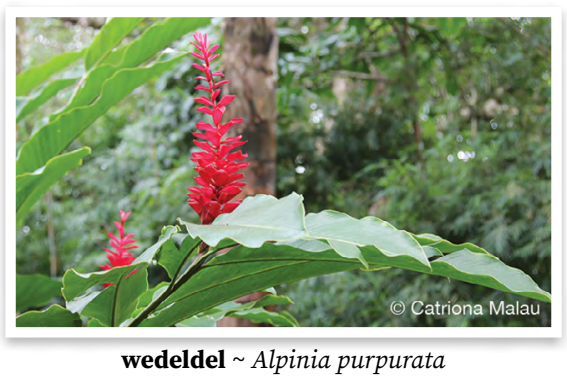

wesev plant with strong scented yellowish, green leaves lif we $\mathrm{i}$ gat smel blong hem we i yelo, grin Evodia hortensis

widiar 1 - kind of mushroom that glows in the dark kaen masrum we i stap saen long naet

wistaban variety of kava with yellow leaves wan kaen kava we lif blong hem i yelo

wolkoko kind of plant that has small sharp hooks on the stem $\sim$ wan kaen plant we i gat huk long hem Caesalpinia crista

wulëwalal devil's horsewhip kaen plant Achyranthes aspera 


\section{E Fauna}

\section{E0 General}

bakasē abdomen, under side of crabs and other crustaceans andanit, bel blong krab o naora

bēni $\mathbf{1} \cdot$ hand, arm han $\mathbf{2}$ - wing of bird wing blong pijin

banēn gala her/his/its left hand lef han blong hem

banēn mōtō her/his/its right hand raet han blong hem

banēn her/his/its hand han blong hem

bēnik my hand han blong mi

busu 1 - digit, finger, toe $\sim$ fingga 2 • claw fingga blong pijin, fingga blong krab, naora

bōsōn her/his/its finger, toe fingga blong hem

busu ōr prawn or lobster claw fingga blong naora

busuk my finger, toe fingga blong mi

dir ${ }_{1}$ hatch (eg i) brok

dōsdōs $1 \cdot \operatorname{coo}$, make noise like dove (grinpijin o sotleg) i singaot, mekem noes blong hem

gaböu wing wing

gabōōn its wing wing blong hem

gaböuk my wing wing blong mi

gargarke type of fatty looking substance, part of animal samting olsem gris we i stap wetem mit blong animol

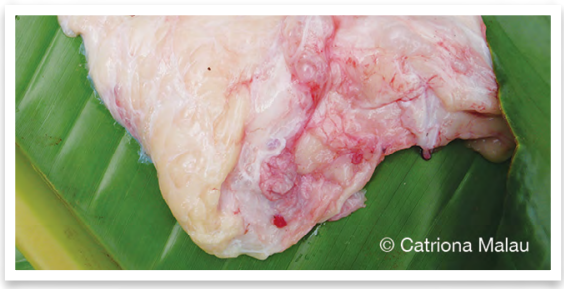

gargarke $\sim$ fatty substance

golon her/his/its tail tel blong hem gölö tail tel gölök my tail tel blong mi

lēnötō ${ }_{1}$ rooster's comb red samting we i stap antap long hed blong man faol

luwö $\mathbf{1} \cdot$ tooth $\sim$ tut $\mathbf{2} \cdot$ tusk $\sim$ tut (blong pig)

luwö bas toothless person, person who has lost all or most of their teeth $\sim$ man we i nogat tut

luwö qō pig's tusk tut blong pig

luwon her/his/its tooth, teeth tut blong hem

luwök my tooth, teeth tut blong mi marak fat (of animal) gris (blong animol)

men $_{1}$ bird $~$ pijin

mes fish $\sim$ fis

mō long feather of a rooster's tail longfala feta long tel blong man faol m̄ēmēri tentacle, arm of octopus han blong nawita

nere $\mathbf{1} \cdot$ lips $\sim$ lip, skin blong maot $2 \cdot$ beak $\sim$ maot blong pijin

naran her/his/its lips, beak skin blong maot blong hem

nërëk my lips skin blong maot blong mi

nusu $1 \cdot$ lips, snout (of animal such as pig) lip, skin blong maot $\mathbf{2} \cdot$ beak maot blong pijin

n̄ōsōn her/his/its lips, beak skin blong maot blong hem

nusuk my lips skin blong maot blong mi

seleqō animal animal

sōrsōr noise made by cicada noes we bebet we i kolem se bōn i mekem

taqtutun $1 \cdot$ sit on egg (of bird) pijin i slip long eg kasem taem eg i brok

tatörur noise made by pigeon $~$ noes blong nawimba

tikiar sound made by hen after laying an egg saon olsem woman faol i singaot afta hem i putum eg 


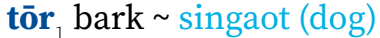

törtörök crow, sound made by rooster

$\sim$ man faol i singaot

tötöu nest nes

tötöu men bird's nest nes blong pijin

tutgö fin wing blong fis

vul $\mathbf{1}$ • hair $\sim$ hea $\mathbf{2}$ - feather $\sim$ feta

vōlōn her/his/its hair hea blong hem

vulu tō chicken feather $\sim$ feta blong faol

vuluk my hair hea blong mi

wawawne gill sora blong fis

wawleñ wing tip en blong wing

wēlēgian $\mathbf{1} \cdot$ her/his/its heel $\sim$ bihaen blong leg blong hem 2 - hoof (of animal) bihaen long leg blong animol

wobalaklak sucker, suction cup on octopus arm, tentacle samting long han blong nawita we yu save fas long hem

wotoqtoqolav stomach of cattle (cows only) bel blong buluk

wörurus tavav pancreas wan pat blong animol we i stap wetem gat

wöwöwut smaller stomach of cattle, omasum handred rum (blong buluk)

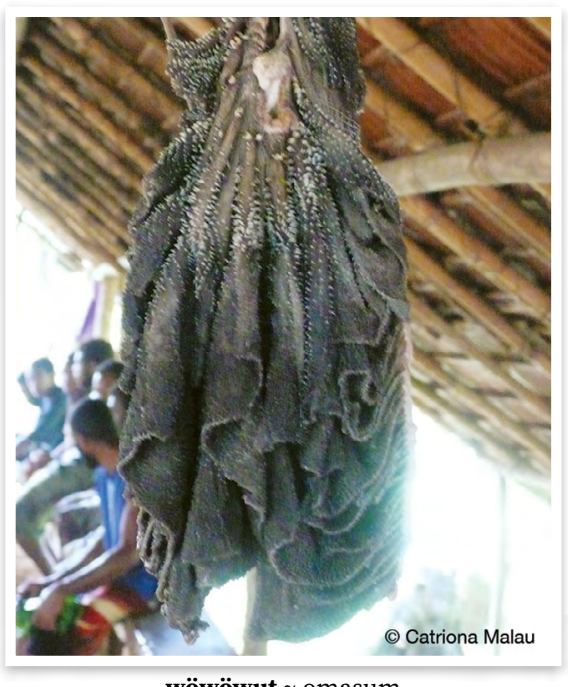

wöwöwut omasum wulewēsēstoñov operculum ae

blong grinsnel

E1 Mammals

buluk cow, cattle buluk

genqirēg $\operatorname{dog} \sim \operatorname{dog}$

gōsōw rat rat

mal sow pig woman

qërët flying fox $\sim$ flaengfokis

$\mathbf{q} \overline{\mathbf{o}}_{1}$ pig pig

riaw $\mathbf{1} \cdot$ hermaphrodite $\sim$ animol we hem i man mo woman wantaem 2 - intersex pig pig we hem i man mo woman wan taem

tëqël bat bat

tivi cat puskat

tok dog $\sim \operatorname{dog}$

E2 Birds

bagbaglō swiftlet wan kaen pijin Aerodramus spp.

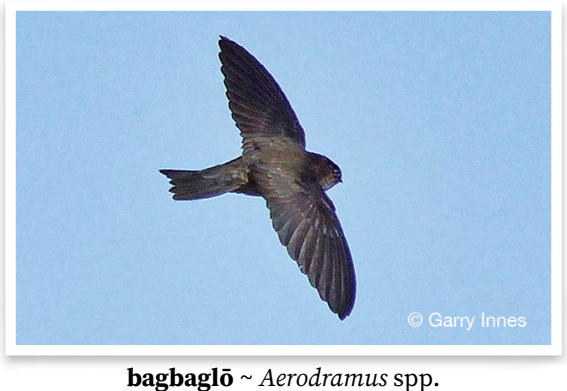

bëlbëlvōlōn small Vanuatu fruit dove $\sim$ grinpijin

biliag buff-banded rail nambilak Gallirallus philippensis

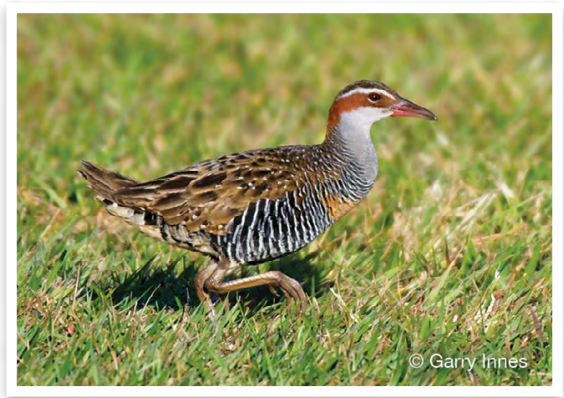

biliag Gallirallus philippensis 
biliag mamē white-browed crake wan kaen pijin Poliolimnas cinereus gōg reef heron longnek; naova Ardea sacra, Egretta sacra

gōg gabēt white heron waet longnek

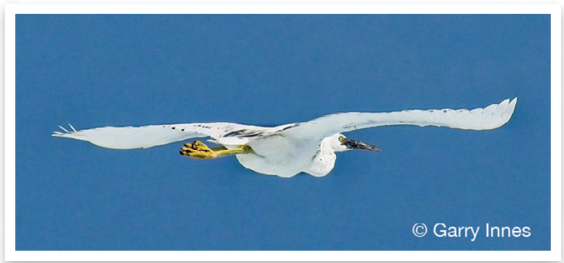

gōg gabēt white heron

gōg kör black heron blak longnek

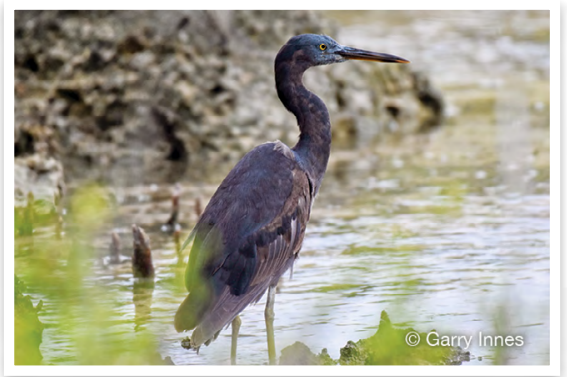

gōg kör black heron

mal common hawk, swamp harrier namala, igel, hok Circus approximans

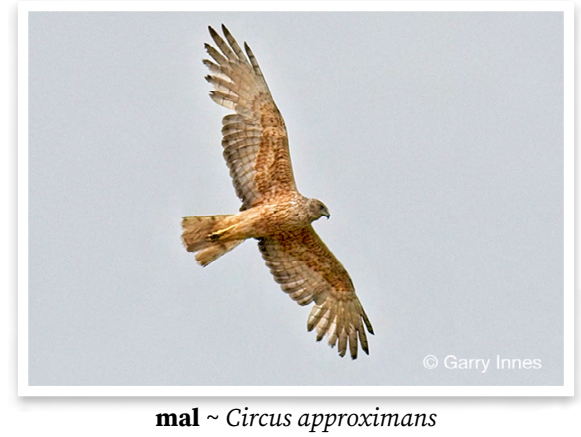

mal biliag peregrine falcon kaen pijin olsem hok be wing blong hem i longwan, bodi blong hem i moa smol Falco peregrinus

male $\overline{\mathbf{e}}_{1}$ bird species kaen pijin

malqon fruit dove $\sim$ grinpijin Ptilinopus spp. men $_{1}$ bird pijin

men dōsdōs fruit dove $\sim$ grinpijin

Ptilinopus spp.

menmenlul golden whistler $\sim$ wan kaen pijin

mensirsirqōōr Vanuatu buff-bellied flycatcher $\sim$ wan kaen pijin Neolalage banksiana

mēs rainbow lorikeet nasiviru Trichoglossus haematodus

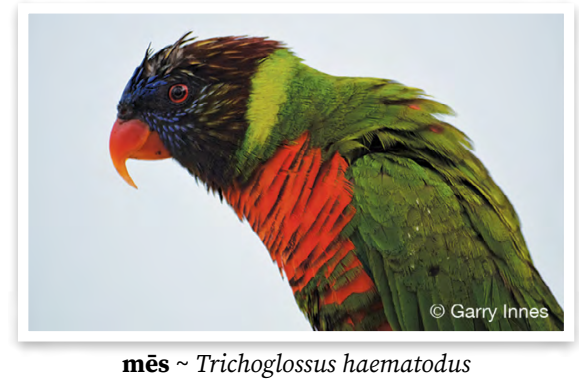

mëkbē broad-billed flycatcher wan kaen pijin Myiagra caledonica

matēk purple swamphen napiru, longnek Porphyrio porphyrio

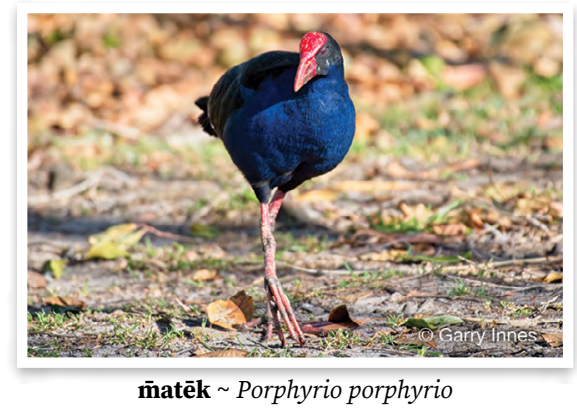

mereg 1 Pacific emerald dove $\sim$ sotleg Chalcophaps longirostris

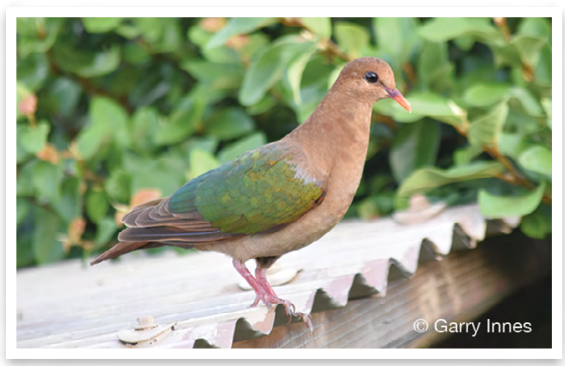

mereg Chalcophaps longirostris 
mēēlēg Vanuatu white-eye nalaklak wetem yelo bel Zosterops flavifrons mēēēg wö silvereye nalaklak Zosterops lateralis

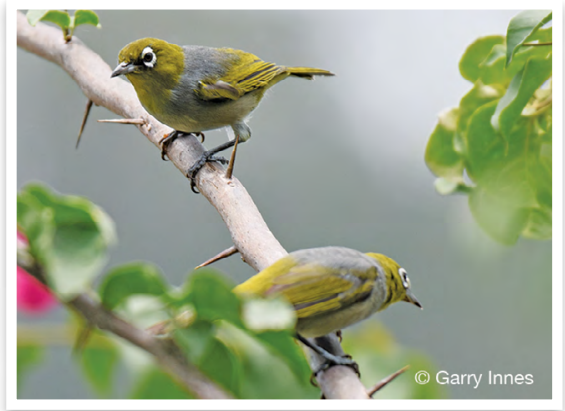

$\overline{\mathbf{m}} \mathbf{e} \mathbf{l e ̄ g}$ wö $~$ Zosterops lateralis

molo incubator bird, scrub fowl, megapode skrabdak, namalao Megapodius freycinet

qërët flying fox flaengfokis qon pigeon nawimba Ducula spp.

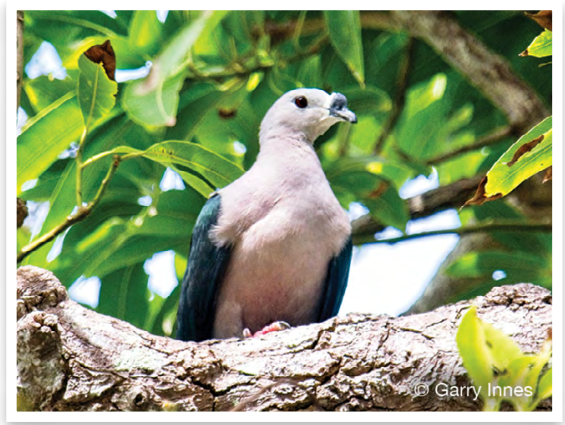

qon Ducula spp.

qon bulënqet kind of pigeon that has a ring on its leg kaen nawimba we i gat ring long leg blong hem

qon bulënrēlē kind of pigeon that has a lump on its beak (male) kaen nawimba wetem lam long maot blong hem (man)

qon tan̄sar kind of pigeon that has a straight beak (female) kaen nawimba we $i$ gat stret maot (woman)

qon wēbèt kind of pigeon that is white $\sim$ waet nawimba qötqötumen cardinal honeyeater (male) redhed (man) Myzomela cardinalis

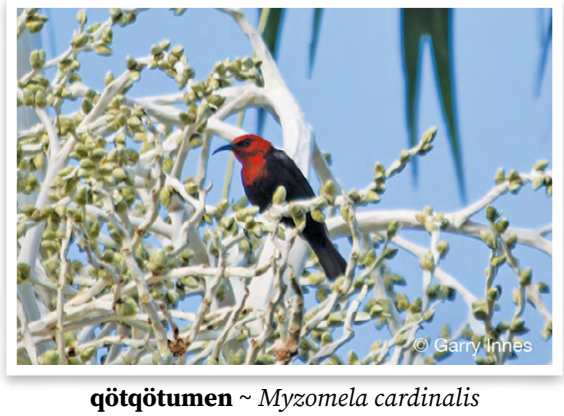

rēn̄ēs green palm lorikeet wan kaen pijin olsem nasiviru Charmosyna palmarum

sēg kingfisher nasiko, longmaot Todiramphus spp.

sēg malgias Pacific kingfisher nasiko Todiramphus sacer

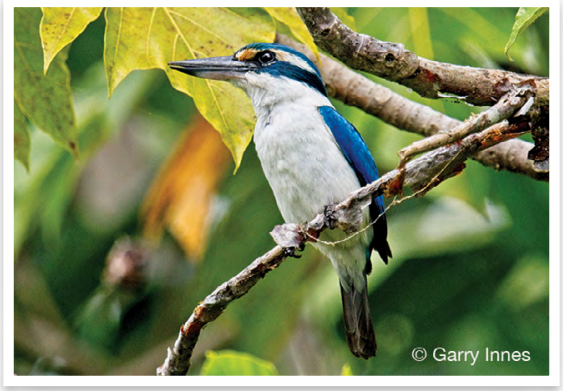

sēg malgias Todiramphus sacer

sēg mamē sacred kingfisher red nasiko Todiramphus sanctus

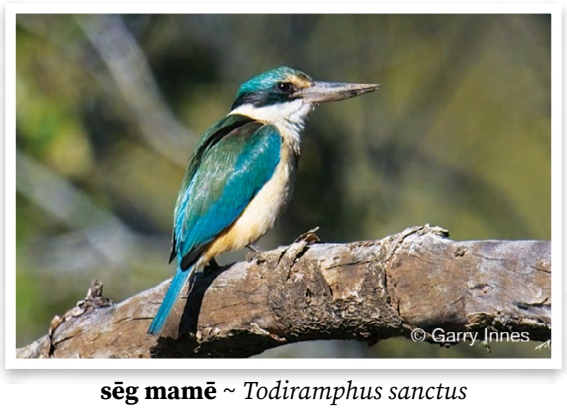

sōravar Vanuatu fruit dove bigfala grinpijin Ptilinopus tannensis 
tarere young chicken that is not a chick but not yet fully mature yang faol

taw $_{1}$ dark-brown honeyeater, greyeared honeyeater kaen pijin wetem longmaot, famle blong redhed we hem i braon Lichmera incana

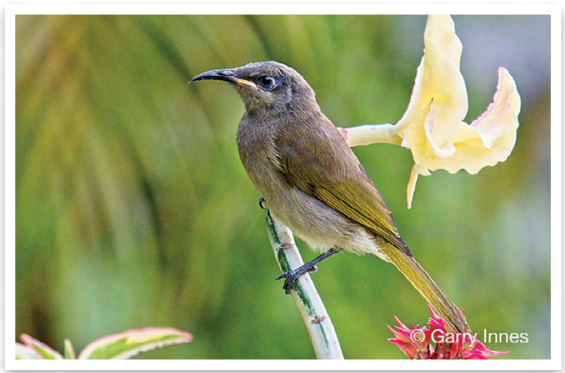

taw Lichmera incana

tēgēr fantail pijin blong trik Rhipidura spp.

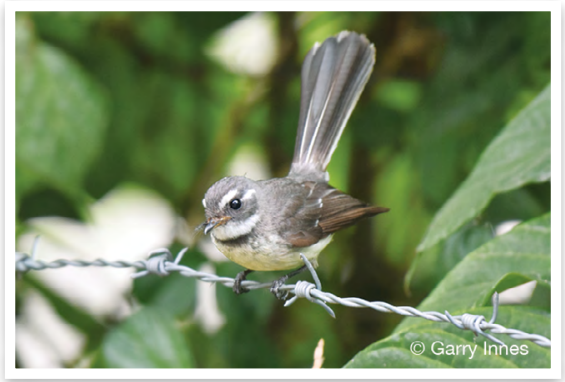

tēgēr Rhipidura spp.

tēwē shore birds pijin blong solwota tēwē biliag wandering tattler nasivi Heteroscelus incanus

tēwē ōlqön kind of shore bird smolsmol nasivi we hem i stap singaot oltaem

tēwē rōwelteg beach thick-knee kaen nasivi Esacus magnirostris

tërëw Vanuatu mountain pigeon nawimba blong hil Ducula bakeri

tōo ${ }_{1}$ chicken faol Gallus gallus nötu tō chick pikinini blong faol tō armiag chicken with light reddish brown feathers $\sim$ faol we kala blong hem i laet braon

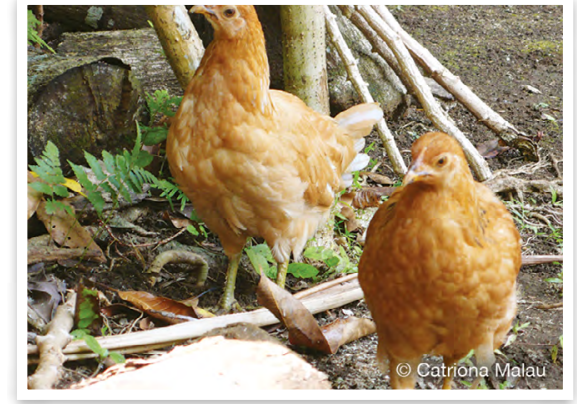

tō armiag brown chicken

tō atm̄ēn rooster $\sim$ man faol

tō bèt white chicken $\sim$ waet faol

tō biliag speckled chicken with colouring like buff-banded rail $\sim$ faol we kala blong hem i olsem nambilak

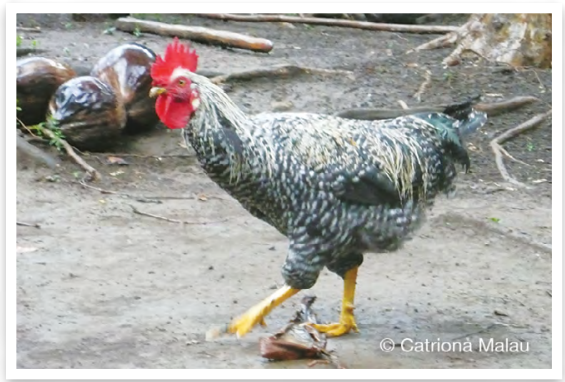

tō biliag speckled chicken

tō kör black chicken blak faol tō mamē red chicken $\sim$ red faol

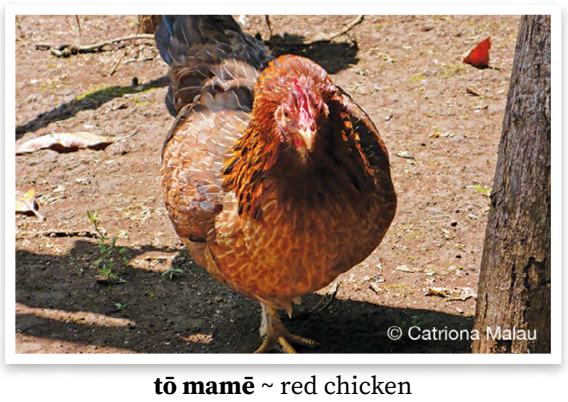

tō menmenlul red, white and black chicken faol we i gat tri kala long hem, red, waet mo blak

tō reqe hen $\sim$ woman faol 
tōrō white-throated pigeon nataroa Columba vitiensis

tōrō butbut male white-throated pigeon $\sim$ man nataroa

tōwav ${ }_{2}$ rufous-brown pheasant-dove longtel Macropygia mackinlayi

tutrevak kind of bird like a Vanuatu mountain pigeon but bigger wan kaen pijin olsem nawimba blong hil be bigwan

tutwō kind of bird like a Vanuatu mountain pigeon but smaller wan kaen pijin olsem nawimba blong hil be i smol lelebet

wase $_{1}$ southern shrikebill kaen pijin we hem i braon mo blak Clytorhynchus pachycephaloides

wètēbisbis island thrush wan kaen pijin Turdus poliocephalus

wētēsisis cardinal honeyeater (female - smaller and not as brightly coloured as male) redhed (woman) Myzomela cardinalis

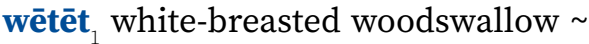
wan kaen pijin Artamusleucorhynchus

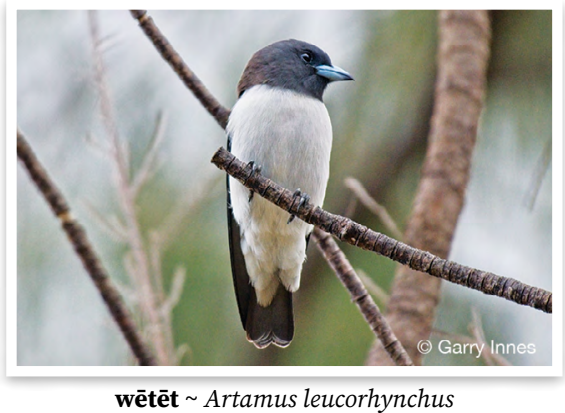

wēvēl fantail warbler wan kaen pijin Gerygone flavolateralis

wiria red-bellied fruit dove (immature(?), smaller and colour not as bright) grinpijin we hem $\mathrm{i}$ smol Ptilinopus greyii

wiria tabē red-bellied fruit dove (adult(?), larger) grinpijin we hem i bigwan Ptilinopus greyii

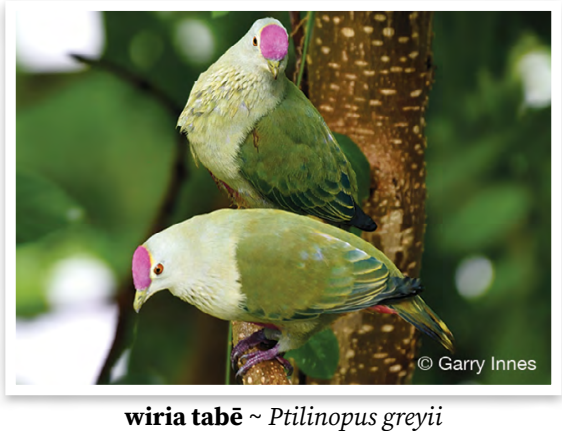

wis barn owl hoknaet, wok naet Tyto alba

wotoqtoq mamē scarlet robin wan kaen pijin Petroica multicolor wōkōl Vanuatu mountain honeyeater longmaot blong hil Phylidonyris notabilis

wōrdes Polynesian triller wan kaen pijin Lalage maculosa

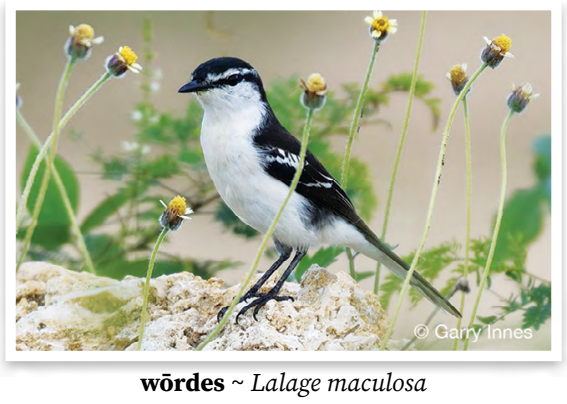

wōtōkōl whimbrel wan kaen pijin Numenius phaeopus

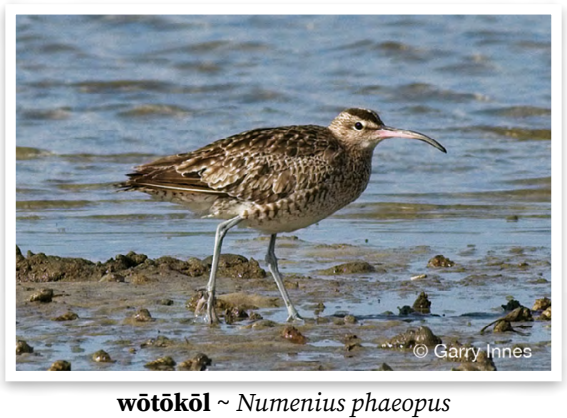




\section{E3 Reptiles}

kakēlis brown striped lizard braon lised wetem laen

kalmōs kind of lizard that is black with green-yellow belly wan kaen lised we hem i grin mo blak

kalqar green tree lizard $\sim$ grinlised Emoia sanfordii

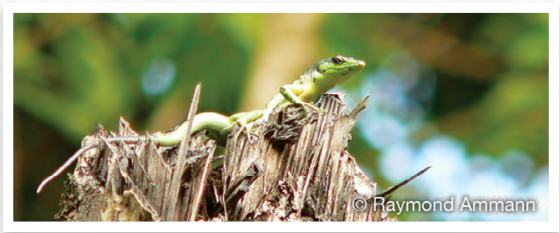

kalqar Emoia sanfordii

matawōsōl kind of lizard that is light brown with spots on its back and yellow belly. It sheds its skin when touched $\sim$ wan kaen lised we hem i laet braon wetem spot long baksaed mo yelo andanit. Taem yu holem skin blong hem i kamaot

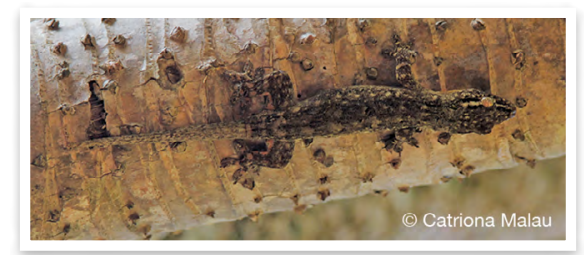

matawōsōl kind of lizard

matriaw lizard $\sim$ lised

mōs black lizard blak lised

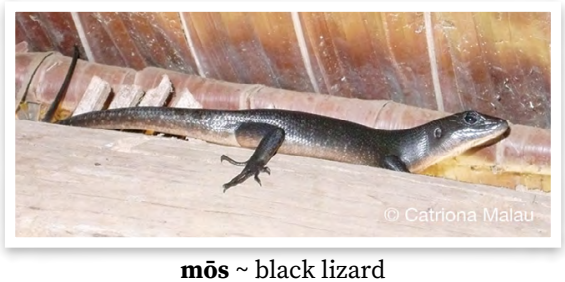

mat snake $~_{\text {snek }}$

mat an kind of snake that is yellow kaen snek we i yelo

mat biliag kind of snake that is spotted kaen snek we $\mathrm{i}$ gat dot long hem mat tan tes sea snake that is short and yellow and is found on surface wan snek blong solwota we i yelo mo i sotfala mo i stap swim antap long ol wef

tanlēn̄lēn kind of small black lizard that is found underneath logs and rocks wan kaen smol blak lised we hem i stap andanit long wud o ston

tilē tan kind of snake kaen snek

viabobo kind of dark brown lizard with large eyes. Its skin is rough and can scratch one's skin wan kaen lised we hem i dak braon mo hem i bigwan wetem bigbig ae. Skin blong hem i raf mo strong mo hem i save skrasem skin blong yu

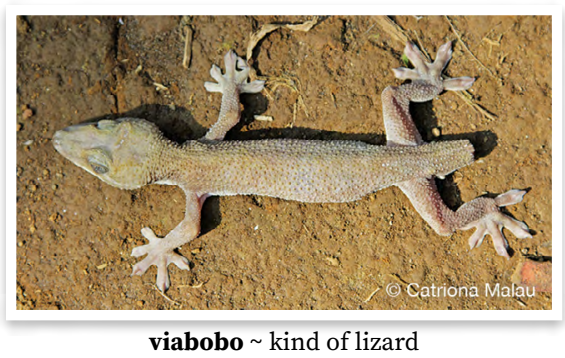

E4 Water creatures

\section{E4.0 Large sea creatures}

bōgō ${ }_{1}$ shark sak

bōgō dēmēèl blue shark wan kaen sak

bōgō mes sandbar shark wan kaen sak

bōgō n̄ōrn̄ōr leopard shark wan kaen sak

göwur white-margined moray wan

kaen namarae

$\mathbf{k} \overline{\mathbf{e}}_{1}$ dolphin dolfin

$\overline{\mathbf{o w}}$ turtle $\sim$ totel

qōlo dugong kaofis Dugong dugon

rērēg whale $\sim$ welfis

val hookjaw moray kaen namarae

vër ${ }_{1}$ stingray stingre 
vër dudut black-blotched stingray $\sim$ wan kaen stingre Taeniura melanospila

vër mal spotted eagle ray wan kaen stingre Aetobatus narinari vër matawasē Kuhl's stingray wan kaen stingre Neotrygon kuhlii

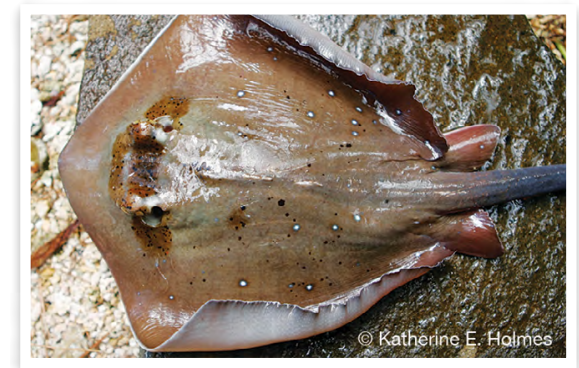

vër matawasē $~$ Neotrygon kuhlii

vër timiat manta ray wan kaen stingre Manta birostris

\section{E4.1 Fish}

bargōr 1 kind of flying fish that is found close to shore wan kaen smol flaengfis we i stap klosap long SO

barnaw doublespotted queenfish kaen fis Scomberoides lysan

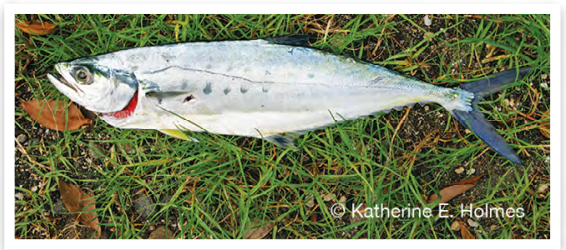

barnaw Scomberoides lysan

batbat red-tailed leatherjacket $\sim$ kaen strongskin

baw onespot snapper kaen pulefis we i gat yelo tel mo wan blak spot long hem Lutjanus monostigma

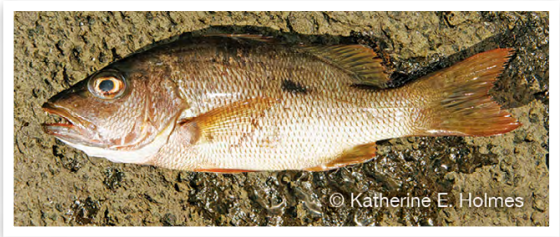

baw Lutjanus monostigma benmē kind of large flying fish with reddish pectoral fins $\sim$ wan kaen flaengfis we hem i bigwan mo wing blong hem i haf red

benmō̄olo black sweetlips tiklips Plectorhinchus gibbosus

beraw striped surgeonfish $\sim$ renbofis, blumak piko Acanthurus lineatus

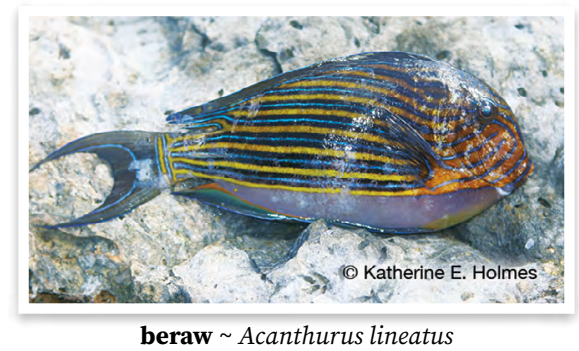

beretnaw common dart $\sim$ kaen fis Trachinotus botla

bēm butterflyfish bataflae fis Family Chaetodontidae

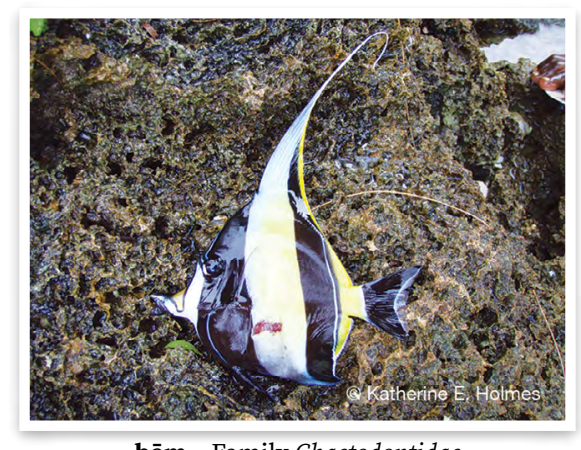

bēm Family Chaetodontidae

biliag $_{2}$ darkfin hind $\sim$ kaen los Cephalopholis urodeta

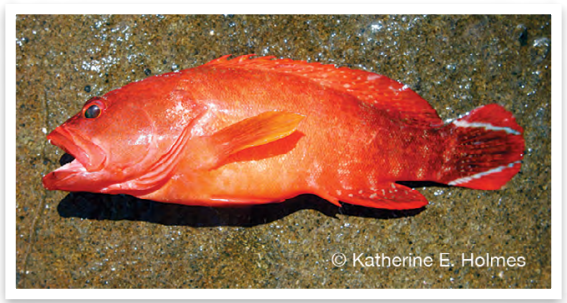

biliag Cephalopholis urodeta

biliag lam leopard grouper kaen los Cephalopholis leopardus borbor $_{2}$ kind of fish $\sim$ kaen fis 
bultarō yellowmask angelfish, regal angelfish papiong fis Pomacanthus xanthometopon, Pygoplites diacanthus bum triggerfish strongskin Family Balistidae

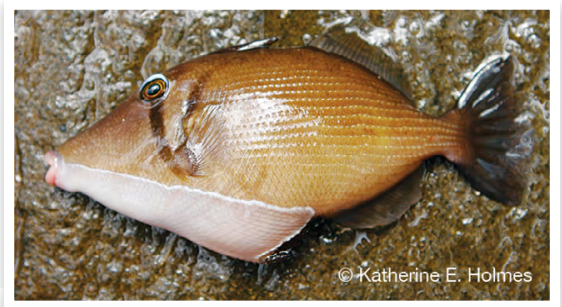

bum $\sim$ Family Balistidae

bum gōlōw black triggerfish kaen strongskin

bum lam starry triggerfish kaen strongskin Abalistes stellaris

bum lo kind of triggerfish kaen strongskin

bum qërët redtooth triggerfish kaen strongskin Odonus niger

bum timiat orangelined triggerfish kaen strongskin Balistapus undulatus

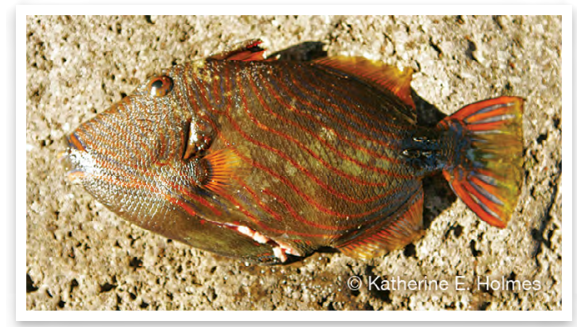

bum timiat $\sim$ Balistapus undulatus

dananar Indo-Pacific sergeant, scissortail sergeant $\sim$ wan kaen fis Abudefduf vaigiensis, Abudefduf sexfasciatus

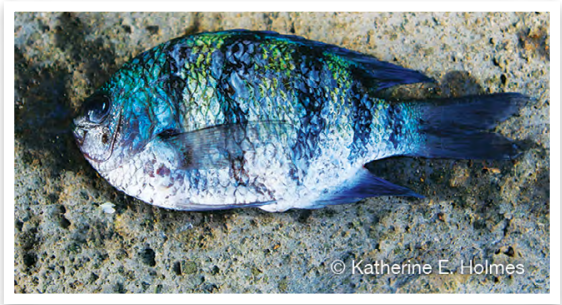

dananar Abudefduf spp. datalēs flametail snapper kaen pulefis Lutjanus fulvus

demeles general name for immature parrotfish species ol smol blufis we ol i no kam bigwan yet Chlorurus sordidus

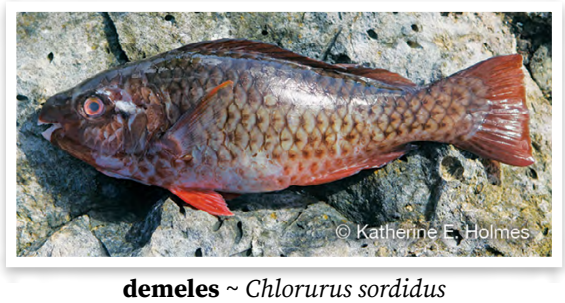

dēvēlavēl steephead parrotfish kaen

blufis Chlorus microrhinus

dëvër orbicular batfish wan kaen fis

Platax orbicularis

dōmōtō blackspot emperor blakspot redmaot Lethrinus harak

dötöv bluefin trevally blu karong Caranx melampygus

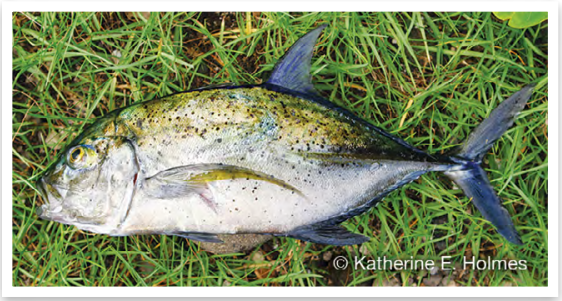

dötöv Caranx melampygus

ët tuna tuna

ganasēg freckled porcupinefish, black-blotched porcupinefish nilfis Diodon spp.

gie whitespotted surgeonfish $\sim$ wan kaen fis Acanthurus guttatus

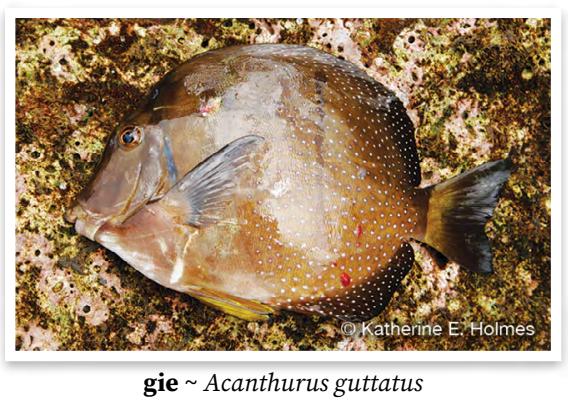


giebe kind of freshwater fish that is black with white stripes. It is flat, can be large, and is always found in pairs wan kaen fis blong wota we hem i blak wetem dak waet laen. Hem i flat mo hem i save bigwan. Ol taem ol i stap tu tugeta

ginias sixfinger threadfin $~$ wan kaen fis Polydactylus sexfilis

gomot striped mullet malet Mugil cephalus

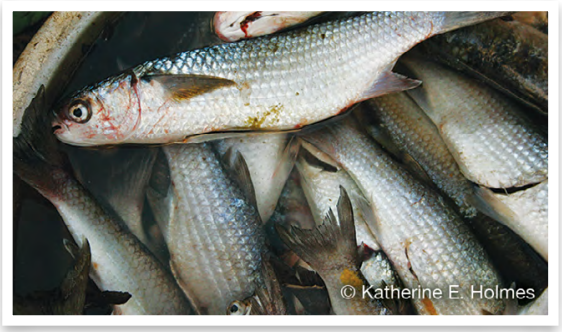

gomot $\sim$ Mugil cephalus

gōrgōrbiliag oriental sweetlips yelo tiklips Plectorhynchus orientalis

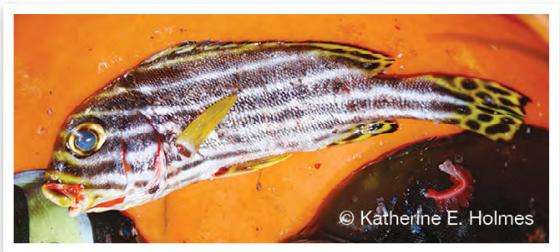

gōrgōrbiliag Plectorhynchus orientalis

kurkurqōl blue-finned triggerfish kaen strongskin

lèl flounder, flatfish flatfis, wansaed fis Family Bothidae, Family Samaridae

lèw (fr.var. löw) generic name for larger wrasses nem we i kavremap ol defren kaen bigfala napoleong Cheilinus spp.

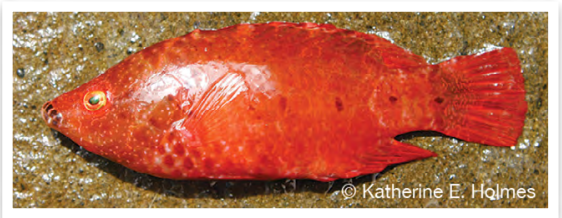

lēw Cheilinus spp. lēw biliag kind of large wrasse kaen bigfala napoleong

lēw mal humphead wrasse $~$ kaen napoleong fis Cheilinus undulatus

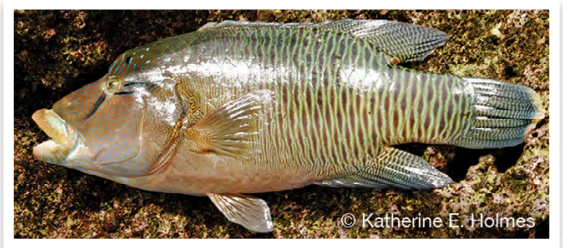

lēw mal Cheilinus undulatus

liaw $1 \cdot$ kind of freshwater fish $\sim$ wan kaen fis blong wota we i no save bigwan 2 - blenny, rockskipper species $\sim$ wan kaen smol fis we i stap jam long ston

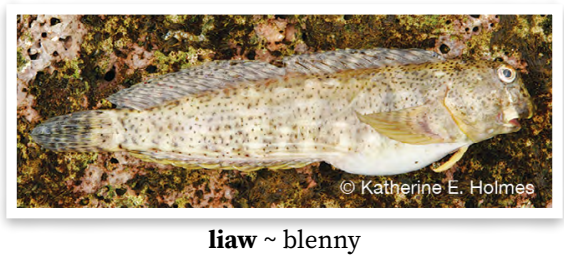

lōat kind of fish wan kaen fis

magalmevesoqötu bigeye $\sim$ wan kaen

fis Family Priacanthidae

magte $_{1}$ stonefish $\sim$ stonfis Family Scorpaenidae

mamat kind of freshwater fish that has large head and eyes. It is light grey and hides in holes in rocks. wan kaen fis blong wota we hem i save bigwan mo ae mo hed blong hem i bigwan. Hem i laet grei mo hem i stap long hol oltaem

mamat serserdiqiat kind of freshwater fish that is silver on its side with a black spot, and light grey on its back wan kaen fis blong wota we hem i silva long saed wetem blak spot. Hem i laet grei long baksaed blong hem. Hem i no save bigwan tumas 
man̄de sabre squirrelfish kaen redfis Sargocentron spiniferum

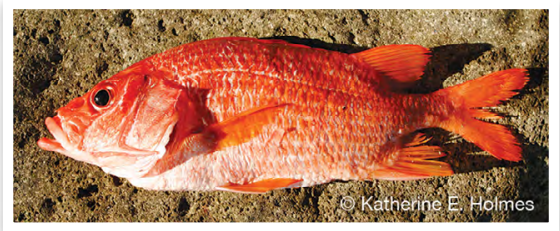

man̄de Sargocentron spiniferum

martat yellowstripe snapper, bluelined snapper $\sim$ kaen pulefis we hem i yelo wetem fo blu laen long hem Lutjanus kasmira

matwiwi cardinalfish wan kaen fis Family Apogonidae

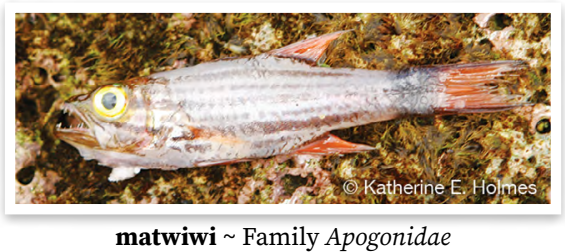

mes fish $\sim$ fis

mesbule kind of fish that lives in fresh water but moves to sea when mature. It is a dull silver and can be found in pairs wan kaen fis we $\mathrm{i}$ bon long wota be taem i bigwan $\mathrm{i}$ go stap long solwota. Hem i silva be i no braet mo i save bigwan. Ol i wokabaot tutu

mesgeben Indo-Pacific sailfish wan kaen fis Istiophorus platypterus

mesmame $\bar{e}_{1}$ pink squirrelfish $\sim$ redfis Sargocentron spp.

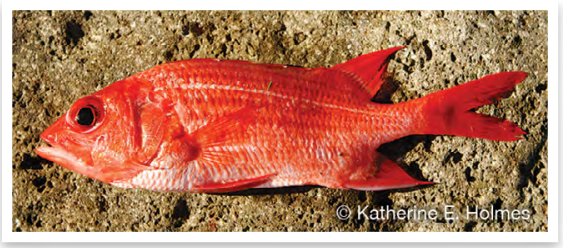

mesmamē $\sim$ Sargocentron spp.

mestalisōr spotted unicornfish kaen strongskin Naso brevirostris

mesteveltiwial copper sweeper $\sim$ wan kaen fis Pempheris oualensis mestēv hogfish kaen fis Bodianus axillaris

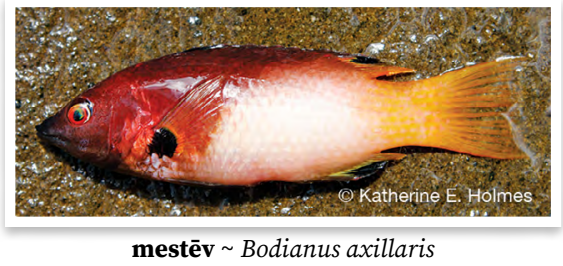

mestiagōsōw black and white snapper

kaen pulefis we hem i waet mo grei Macolor niger

meswelwel fusilier wan kaen fis Family Caesionidae

meteërqōtōn bluespine unicornfish kaen strongskin Naso unicornis

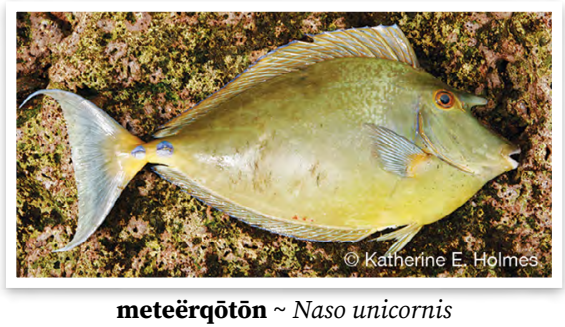

mewērwēr black-tipped grouper kaen los Epinephelus fasciatus

miarbē kind of freshwater fish that is red, and black on its back. It can live in the sea and grows quite large wan kaen fis blong wota we i red mo antap long hem i blak. Hem i save liv long solwota. Hem i save bigbigwan $\mathbf{m o ̄ m} \overline{\mathbf{o}}_{1}$ squaretail grouper $\sim$ kaen los Plectropomus areolatus

mu striped large-eye bream wan kaen fis Gnathodentex aurolineatus

murmur epaulet soldierfish wan kaen redfis wetem bigfala ae Myripristis kuntee

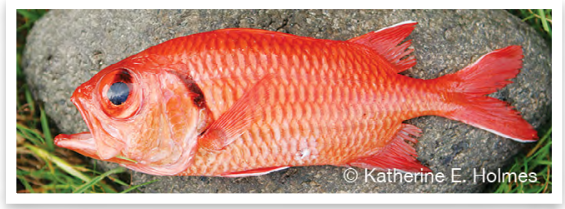

murmur Myripristis kuntee 
murmurbē kind of freshwater fish that is silver with a sharp mouth. It grunts like a pig. It lives in holes in rocks and can also move between fresh and salt water $\sim$ wan kaen fis blong wota we hem i silva mo fes blong hem i sap. Hem i mekem noes olsem pig. Hem i stap long hol mo hem i save stap long solwota

mas goatfish mustasfis Family Mullidae

mas añan goldsaddle goatfish wan kaen mustasfis Parupeneus cyclostomus

mas sarsar yellowfin goatfish, yellowstripe goatfish wan kaen mustasfis we ol i stap wokbaot long grup. Ol i longlongfala mo ol i waet wetem smol dak red spot long saed blong olgeta Mulloidichthys sp.

mas teñtē̄ dash-dot goatfish wan kaen mustasfis Parupeneus barberinus

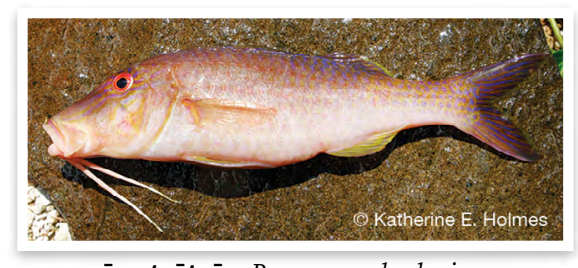

mas ten̄teñ $\sim$ Parupeneus barberinus

mëkër flying fish flaengfis

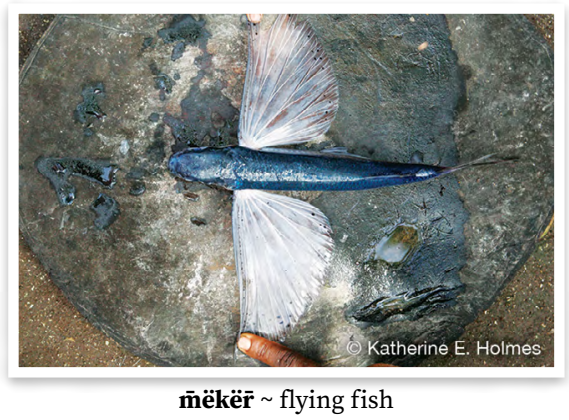

möörögötö pallid triggerfish kaen strongskin Sufflamen bursa non blackfin barracuda $\sim$ sofis Sphyraena genie

ōm humpnose unicornfish kaen strongskin Naso tuberosus

qaēn kind of small freshwater fish. Lays its eggs in the sea in a nest like a spider's nest. When the eggs hatch, they move upstream. They have suckers so they can cling to the rocks to move upstream. A popular eating fish. wan kaen smolsmol fis blong wota we hem i gud blong kakae. Hem i putum eg long solwota wan taem long wan yia mo basket blong eg i olsem blong spaeda. Taem ol pikinini i kamaot ol i klaem folem wota i go antap. Ol i gat samting olsem nawita blong ol i save fas long ston blong klaem i go antap long wota

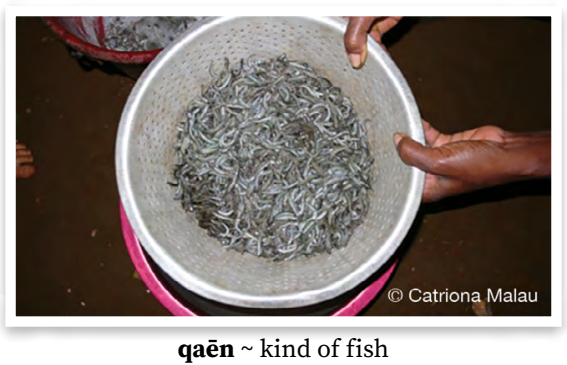

qagar garfish wan kaen fis Family Hemiramphidae

qetmëv surge wrasse wan kaen fis Thalassoma purpureum

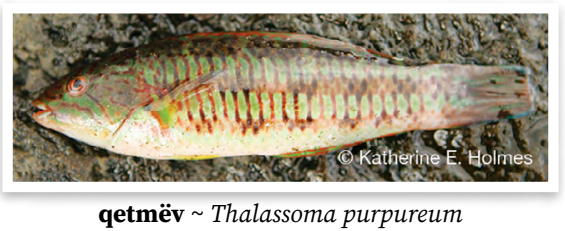

qev humpback snapper $\sim$ kaen pulefis Lutjanus gibbus 
qōl surgeonfish, name covers smaller species of surgeonfish $\sim$ fis we i gat naef, ol smolsmol wan blong hem Acanthurus spp.

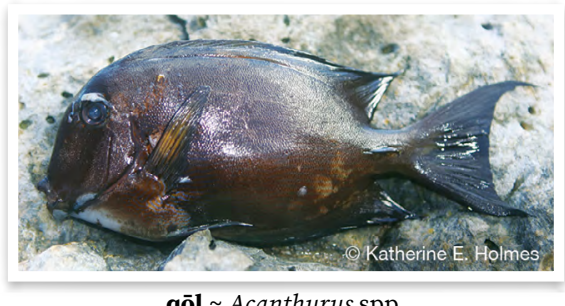

qōl Acanthurus spp.

qōtarō angelfish papiong fis Family Pomacanthidae

qötuqërët big-eye bream wan kaen fis Monotaxis grandoculis

rabiēg bluespotted cornetfish, smooth flutemouth wan kaen longfala fis Fistularia commersonii

$\mathbf{r a k}_{1}$ long-finned drummer wan kaen fis Kyphosus vaigiensis

rēki topsail drummer, Pacific chub, drummer bigbel Kyphosus cinerascens, K. pacificus

ron̄ron̄oqar clown triggerfish $\sim$ kaen strongskin we i gat bigfala waet spot long hem Balistoides conspicillum

rōm 3 - butterflyfish bataflae fis Chaetodon spp.

rō $\mathbf{q}_{1}$ kind of freshwater fish malet blong wota we i save bigbigwan

rōwlav kind of flying fish, larger than other kinds, white with black spots, from deep sea $\sim$ wan kaen flaengfis we hem i bigwan olgeta. Hem i waet be i gat blak spot blong hem. Hem i blong big si blong aotsaed olgeta

röwö bigeye scad kaen bigfala fis blong dipsi Selar crumenophthalmus sarsarale reef lizardfish wan kaen fis Synodus variegatus

sasar $_{2}$ wrasse sop Family Labridae sasargenravarnrōv bluespotted wrasse wan kaen fis Anampses caeruleopunctatus

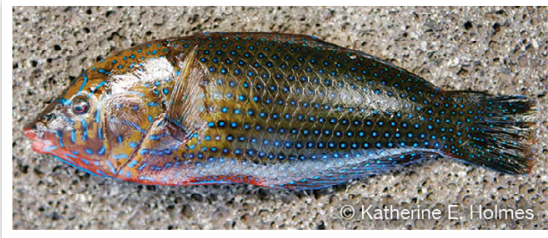

sasargenravarnrōv Anampses caeruleopunctatus

se⿳亠丷厂 white-barred triggerfish, wedgetail triggerfish $\sim$ kaen strongskin Rhinecanthus aculeatus, $R$. rectangulus sewerles several species of hawkfish wan kaen fis Neocirrhites sp., Paracirrhites spp.

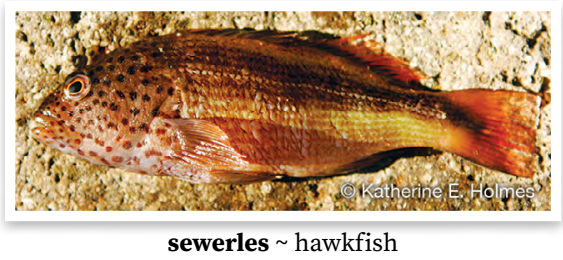

sōm parrotfish, refers to all large species, when they are blue $\sim$ blufis, ol bigwan blong hem Family Scaridae

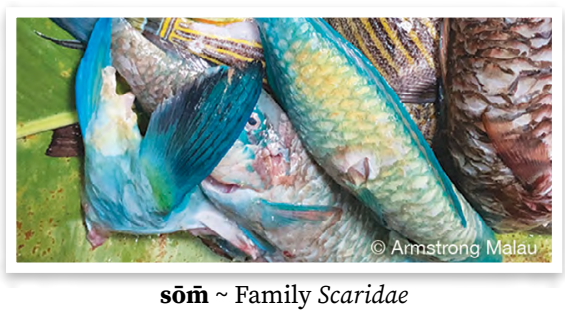

tenmē red bass wan kaen snapa, pulefis Lutjanus bohar

teqesqē spiny squirrelfish $\sim$ kaen redfis Sargocentron spiniferum

terit porcupinefish $\sim$ nilfis Family Diodontidae

terit lam spotted porcupinefish wan kaen nilfis Diodon hystrix

tewesgavēg tailspotsquirrelfish kaen redfis Sargocentron caudimaculatum

til reef needlefish, keeltail needlefish, juvenile needlefish longmaot, ol pikinini o smolsmol kaen blong hem Strongylura incisa, Platybelone argalus 
tiqialdaran̄ran̄ peacock grouper kaen los Cephalopholis argus

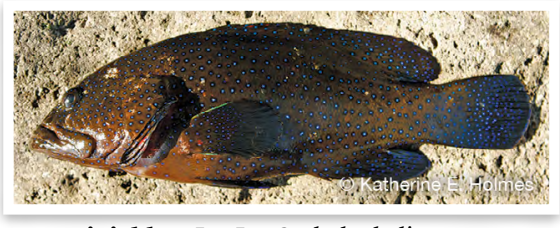

tiqialdarā̄rañ $\sim$ Cephalopholis argus

tiqialq̄ō potato grouper kaen los Epinephelus tukula

tiria lyretail grouper $\sim$ red los Variola louti

tiriakör redmouth grouper kaen los Aethaloperca rogaa

tivtiv ${ }_{1}$ kind of fish wan kaen fish

tivtivnönqet sailfin tang kaen kalakala fis we i gat naef Zebrasoma desjardinii

toqlon kind of flying fish wan kaen flaengfis we hed mo tel blong hem i smol be bel blong hem i bigwan. Hem i stap aotsaed be no tumas

tōqagat blackspot sergeant wan kaen fis Abudefduf sordidus

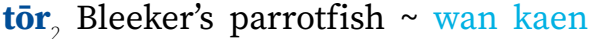
blufis Chlorus bleekeri

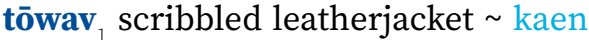
strongskin Aluterus scriptus

tömus rabbitfish piko Family Siganidae

tömus añ kind of rabbitfish kaen piko Siganus sp.

tömus bul kör kind of rabbitfish kaen piko Siganus puellus

tömus kör kind of rabbitfish kaen piko Siganus stellatus

törö ${ }_{2}$ orangeband surgeonfish $\sim$ kaen fis we $i$ gat naef, wetem orenj spot long hem Acanthurus olivaceus

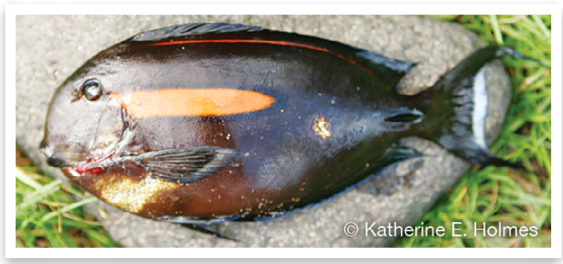

törö Acanthurus olivaceus ut reef needlefish, keeltail needlefish, mature needlefish longmaot, ol bigwan blong hem Strongylura incisa, Platybelone argalus

ut lam kind of needlefish kaen longmaot

ut timiat kind of needlefish kaen longmaot

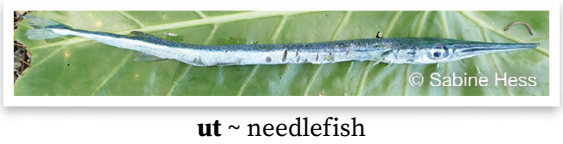

vag surgeonfish, name covers larger species of surgeonfish $\sim$ fis we i gat naef, ol bigwan blong hem Family Acanthuridae, Acanthurus maculiceps A. albipectoralis

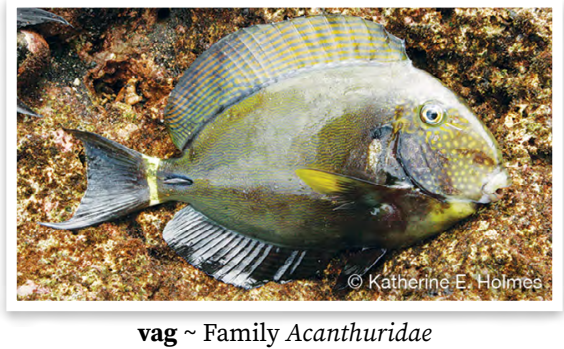

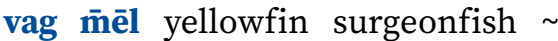
wan kaen fis we i gat naef Acanthurus xanthopterus

vag $\overline{\text { mël }}$ eyestripe surgeonfish wan kaen fis we $\mathrm{i}$ gat naef Acanthurus dussumieri

vērē oyena mojarra, common mojarra silva fis Family Gerreidae

vōlōvōl Klunzinger's wrasse wan kaen smolsmol fis Thalassoma klunzingeri

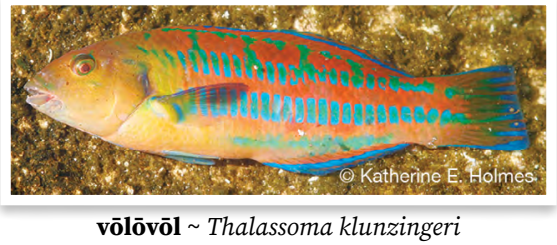

vōlōvōl sōmē Christmas wrasse wan kaen smolsmol fis Thalassoma trilobatum 


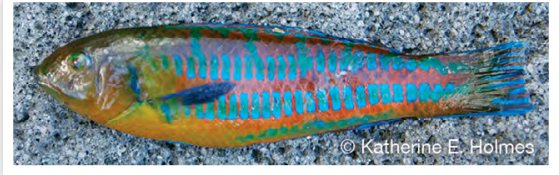

vōlōvōl sō̄̄̄ Thalassoma trilobatum

vōt kind of freshwater fish wan kaen

fis blong wota we hem i no save agensem karen

vurōmōvōt pearly monocle bream wan kaen fis Scolopsis margaritifer walaglē orangespine unicornfish yelo tel strongskin Naso lituratus

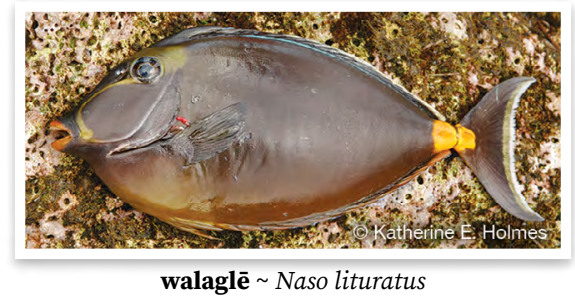

wawasē sweetlip emperor kaen redmaot Lethrinus miniatus

wen̄emërës damselfish species wan kaen fis Family Pomacentridae

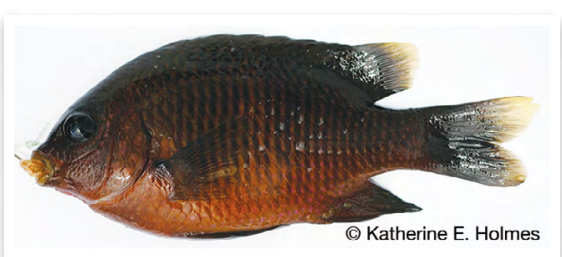

wen̄emërës Family Pomacentridae

wesrē honeycomb grouper smol braon los Epinephelus merra

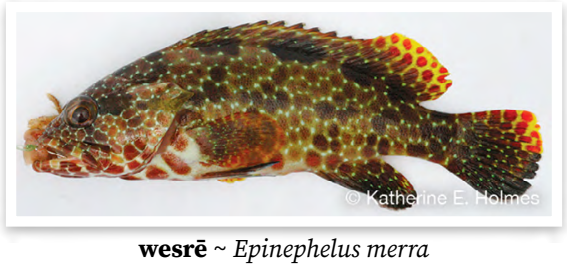

weteltelvet blackside, freckled hawkfish $\sim$ wan kaen fis Paracirrhites forsteri

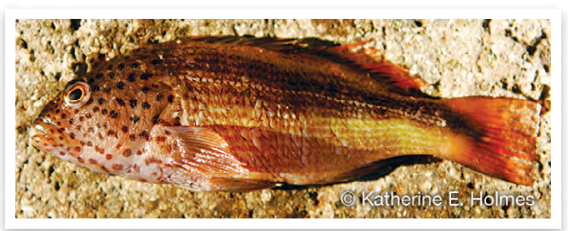

weteltelvet $\sim$ Paracirrhites forsteri

wetevel copper sweeper $\sim$ kaen fis Pempheris oualensis

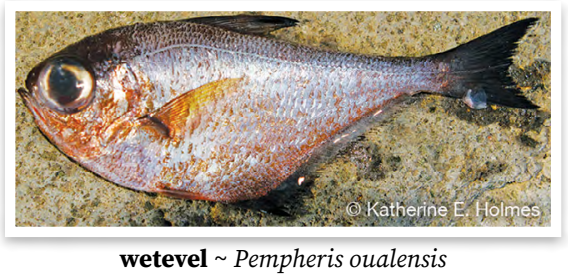

wēduduw (fr.var. wududuw) damselfish $\sim$ wan kaen fis Amblyglyphidodon spp.

wēēwatē blackfin squirrelfish, spotfin squirrelfish $\sim$ kaen redfis Neoniphon spp.

wēgilgialev smallmouth squirrelfish kaen redfis Sargocentron microstoma

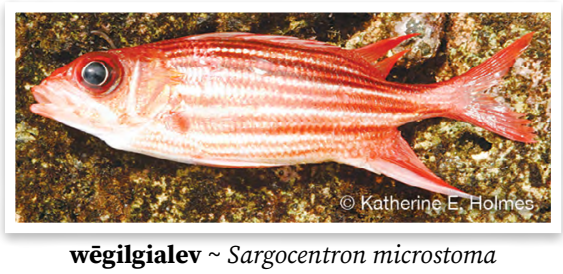

wēlēm immature trevally, jacks and kingfishes smolwan blong karong, taem i bigwan i gat defren nem wēqiar $_{1}$ convict surgeonfish, tang wan kaen fis Acanthurus triostegus

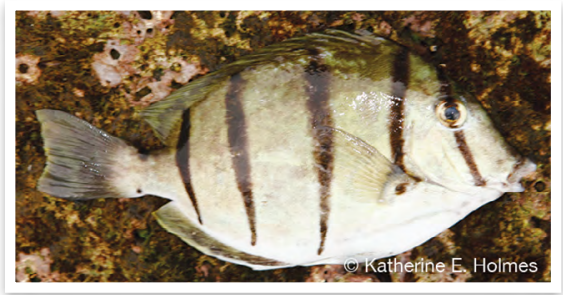

wēqiar Acanthurus triostegus 
wēqitin̄ev samurai squirrelfish kaen redfis Sargocentron ittodai

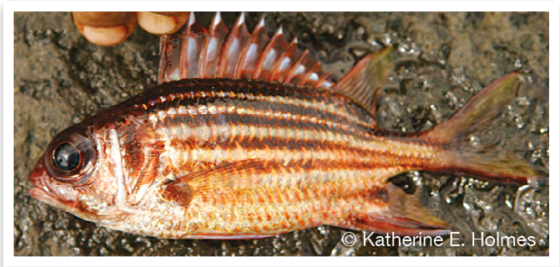

wēqitin̄ev $\sim$ Sargocentron ittodai

wēsilivetel checkerboard wrasse wan kaen smolsmol fis Haliochoeres hortulanus

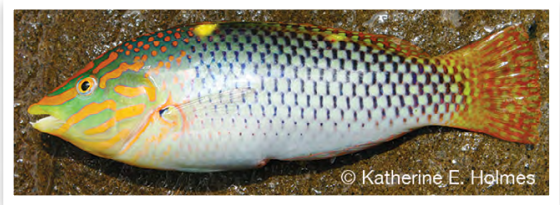

wēsilivetel $\sim$ Haliochoeres hortulanus

wētēvut scorpionfish species kaen fis we i gat nil Family scorpaenidae

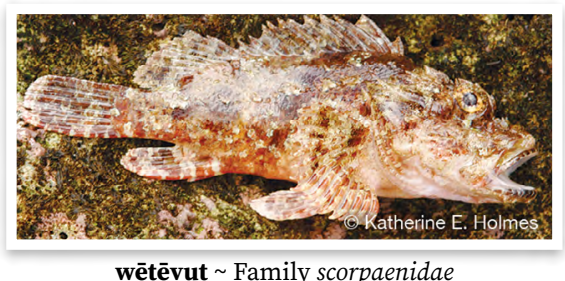

wēvètuo smallmouth squirrelfish

kaen redfis Sargocentron microstoma wiliqliaq fiveband flagtail wan kaen fis Kuhlia mugil

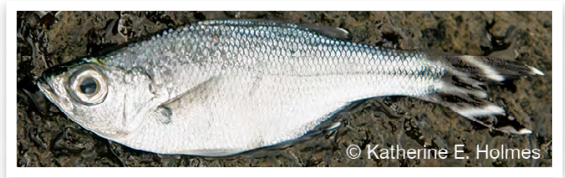

wiliqliaq Kuhlia mugil

wogarēr kind of hawkfish kaen fis

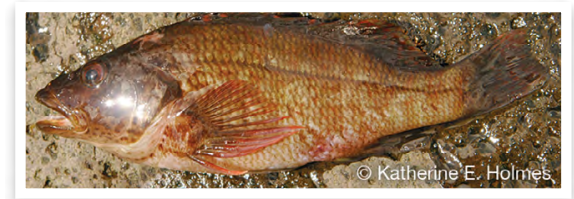

wogarēr $\sim$ hawkfish womalabōs linedcheek wrasse wan kaen fis Oxycheilinus diagrammus

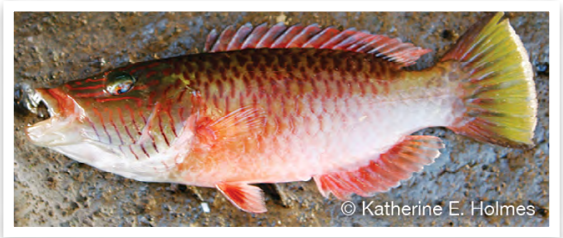

womalabōs Oxycheilinus diagrammus

wotagavēl spotted boxfish fokona, fokonafis Ostracion meleagris

wotoqog stocky hawkfish wan kaen fis Cirrhitus pinnulatus

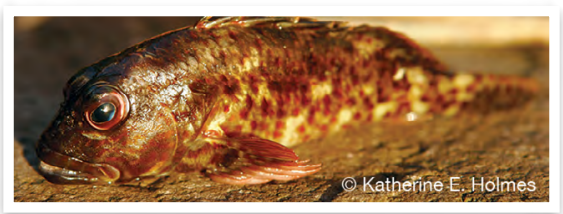

wotoqog Cirrhitus pinnulatus

wotoqtoq $_{2}$ hawkfish $\sim$ wan kaen fis Family Cirrhitidae

wovet spotfin squirrelfish $\sim$ kaen redfis Neoniphon sammara

wowolos Tahitian squirrelfish kaen redfis Sargocentron tiere

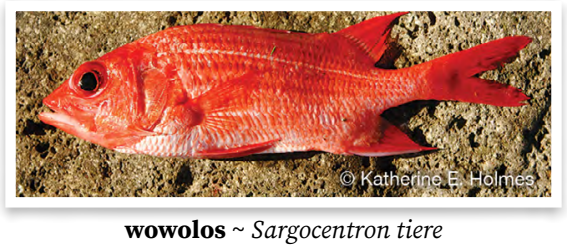

wōnwōn flashflight fish wan kaen fis we ae blong hem i saen olsem toslaet

wōtōqagat seven-bar sergeant, banded sergeant wan kaen fis Abudefduf septemfasciatus

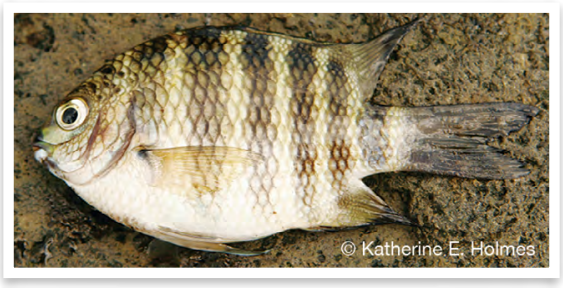

wōtōqagat Abudefduf septemfasciatus 
wōtōwōt pufferfish balongfis, bigbel

Family Tetraodontidae

wōwōsōl long-nosed emperor kaen redmaot Lethrinus olivaceus

wörötuo smallmouth squirrelfish kaen redfis Sargocentron microstoma wösöwunët bird wrasse kaen fis, famle blong blufis Gomphosus varius

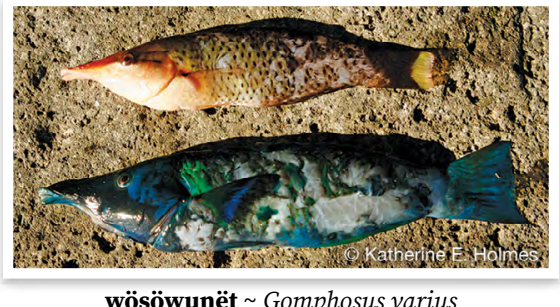

wöturturōrōn̄ bridled monocle bream

$\sim$ wan kaen fis Scolopsis frenatus

wöul (fr.var. wuul) fivestripe wrasse kaen fis, famle blong blufis Thalassoma nigrofasciatum, T. quinquevittatum

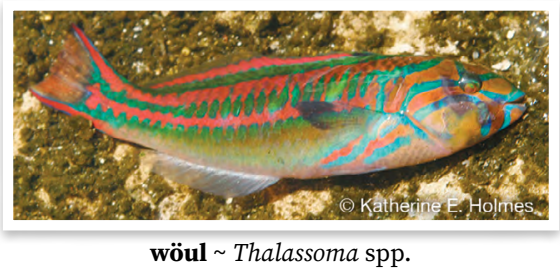

wutu non Spanish mackerel kaen fis Scomberomorus commerson

wutusērēt white-spotted grouper kaen los we i gat waet dot long hem Epinephelus caeruleopunctatus

\section{E4.2 Eels}

\section{marē eel namarae, ilfis}

marē bēluwō kind of freshwater eel that lives in rivers, with a large head, light grey with small spots wan kaen namarae blong wota we hed blong hem i bigwan mo i laet grei wetem ol smolsmol spot. Hem i liv long bigfala wota marē genrō kind of eel that dwells where water meets the sea. It is flat and yellow with black spots wan kaen namarae we i stap klosap long solwota long ples we solwota mo wota i miks. Hem i kakae long tu ples, long wota mo solwota. Hem i flat we i flat mo i yelo wetem blak spot long hem

marē mat kind of freshwater eel, light grey with small spots wan kaen namarae blong wota we hem $i$ laet grei wetem ol smolsmol spot.

marē mes mottled moray wan kaen namarae blong solwota marē qō giant moray wan kaen namarae blong solwota

marē teweswotag starry eel, moray $\sim$ wan kaen namarae blong solwota marē wosgötö girdled reef eel wan kaen namarae blong solwota marē wot kind of freshwater eel, black with white belly, has large eyes. It dwells in swamps and muddy waters wan kaen namarae we baksaed blong hem i blak gud mo bel blong hem i waet. Ae blong hem i bigwan. I stap oltaem long swam mo ples we i gat sofmad

marē wōl kind of freshwater eel, short and silver wan kaen namarae we kala blong hem i silva mo hem i sotwan

mörönërër kind of eel that is thin but long $(150 \mathrm{~cm})$ and speckled brown wan kaen namarae we hemi tintin be longfala mo kala blong hem i braon

tesmērēr kind of freshwater eel, very long, grey with yellow belly and light red tail. It is quite aggressive wan kaen namarae blong wota we hem i save kakae man mo hem i save kamaot hanting long drae ples. Hem i longlongfala mo kala blong hem i grei, andanit i yelo mo fin mo tel i laet red 
vusö black-blotched moray wan kaen namarae Gymnothorax sp.

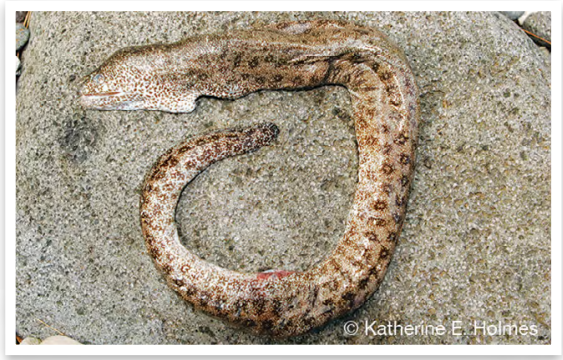

vusö Gymnothorax sp.

vusö ōn painted moray kaen namarae

wutu ōn spotted garden eel kaen namarae

\section{E4.3 Prawns and crayfish}

$\overline{\mathbf{o r}} \mathbf{1}_{1} \mathbf{1}$ - freshwater prawn naora Macrobrachium sp. $\mathbf{2} \cdot$ lobster $\sim$ naora blong solwota Panulirus spp.

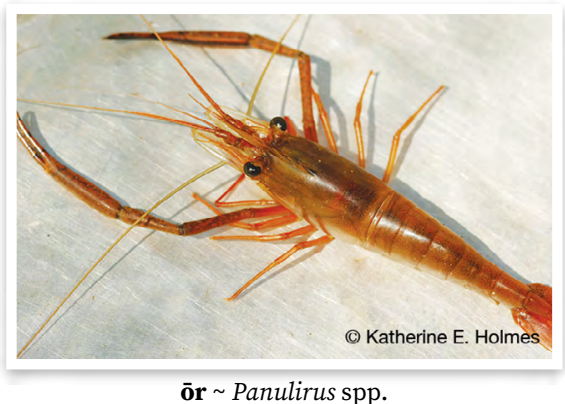

ōr bëlbël kind of freshwater prawn, medium-sized that does not grow large, whitish with spots $\sim$ wan kaen naora we hem i medel saes mo i no save gro bigwan. Hem i waet smol wetem ol spot long hem

òr taban painted rock lobster wan kaen naora blong solwota Panulirus versicolor

qaqet kind of small freshwater prawn wan kaen smolsmol naora

vavam slipper lobster flat naora Parribacus caledonicus
E4.4 Shell fish

gunögōsōw kind of large freshwater shellfish wan bigfala sel blong wota we i stap long saed blong wota matmamē rock shell wan kaen sel blong solwota Thais sp.

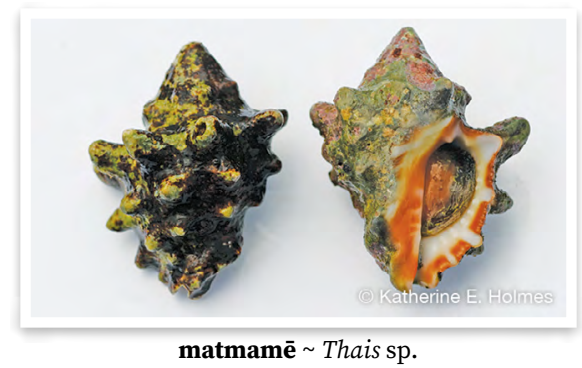

qōbetge common Pacific vase wan kaen sel blong solwota Vasum turbinellus

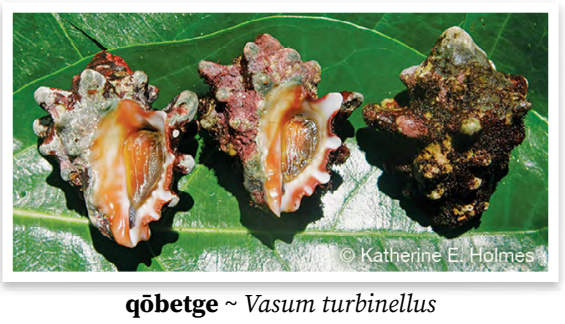

tele clam $\sim$ natalae

tele tur giant clam bigfala natalae, klamsel Tridacna sp.

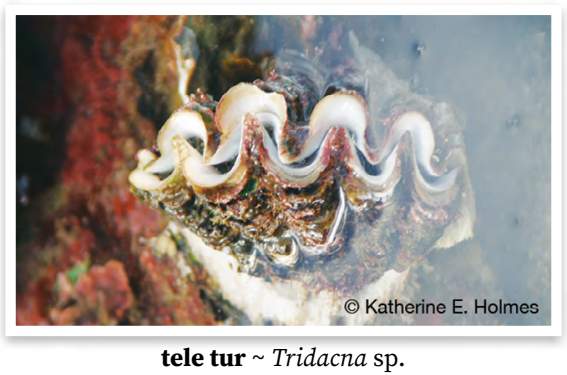

tōw 4 (fr.var. tōw mölum) triton trumpet, conch shell pupu Charonia tritonis 
waakak kind of freshwater mollusc, which can be found in places where the water runs fast $\sim$ wan kaen flat sel blong wota we i stap long ples we wota i save ron strong

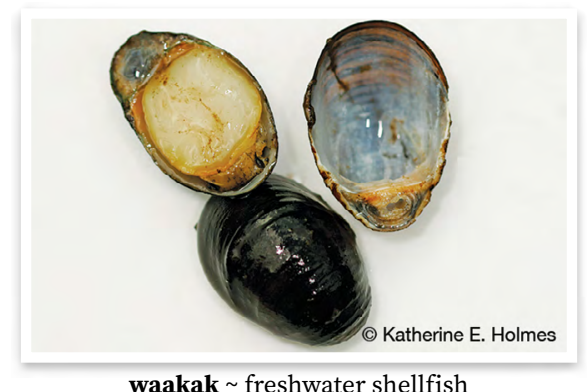

walal top shell troka Trochus niloticus

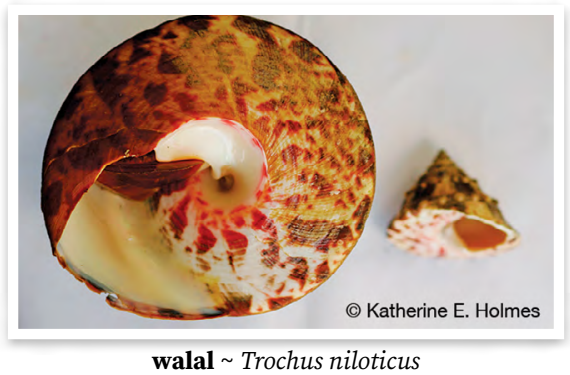

wamatwōn monodont wan kaen sel blong solwota Monodonta sp.

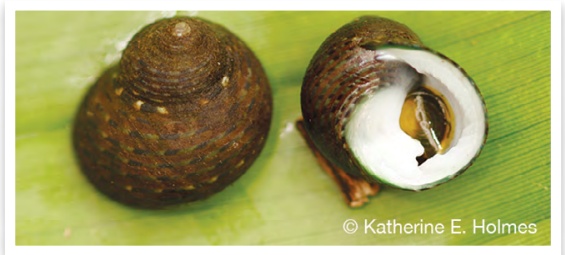

wamatwōn Monodonta sp.

wamase $\overline{\mathbf{e}}_{3}$ abalone $\sim$ kaen sel Haliotis asinina

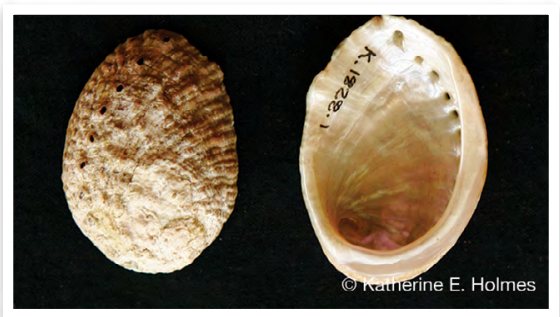

wā̄asē $\sim$ Haliotis asinina watagōgōn kind of gastropod shellfish

wan kaen nasese we i stap long ston

watawlētlēt Pacific star shell wan kaen sel blong solwota Astraea haematraga

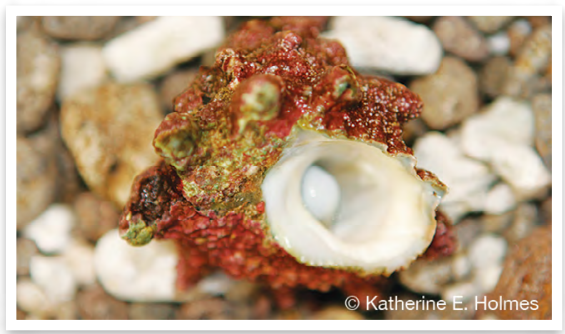

watawlētlēt Astraea haematraga

weger kind of gastropod shellfish, generic term which covers a number of bivalve and limpet species grup blong sel blong solwota, kaen sel we ol i yusum blong skrasem kakae mo smutum pandanas

weger dun sunset shell wan kaen sel blong solwota

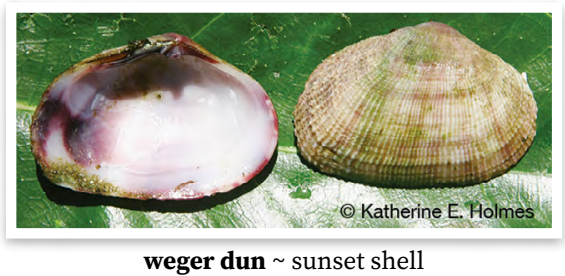

weger mel common turtle limpet paua Cellana testudinaria

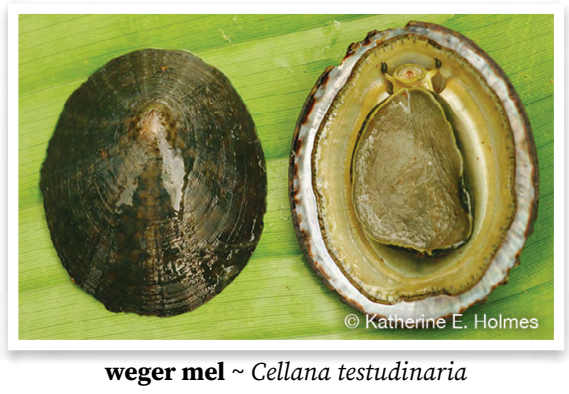

weger sōl kind of small shellfish which is found on the sand $\sim$ kaen smolsmol selfis we i stap long sanbij 
weger ta Pacific sugar limpet wan kaen sel blong solwota Patelloida saccharina

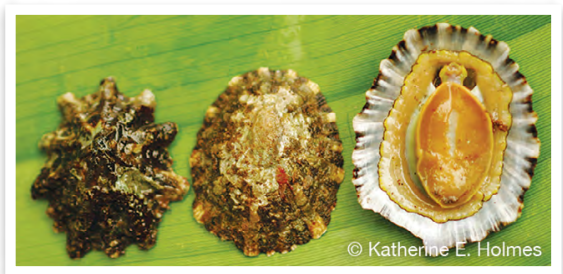

weger ta $\sim$ Patelloida saccharina

weger tur ${ }_{1}$ pen shell wan kaen sel blong solwota Pinna bicolor

weger tur 2 Pacific asaphis, sunset shell wan kaen sel blong solwota Asaphis violascens

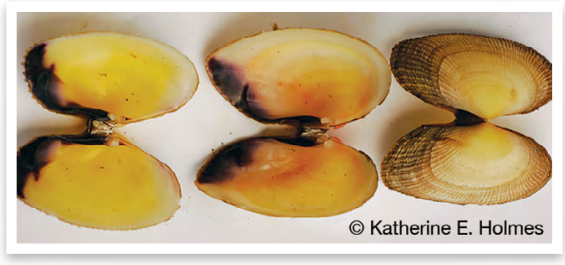

weger tur $\sim$ Asaphis violascens

weqet kind of freshwater shellfish that has spines that can stick into one's feet and break off if trodden on wan kaen nasese we i stap long wota mo i save stikim man

weswesak filamentous horse conch, used in the past to drill holes in canoe for binding canoe wan kaen sel blong solwota, sel we yu save yusum olsem dril, bifo ol i yusum blong mekem hol long kenu Pleuroplaca filamentosa

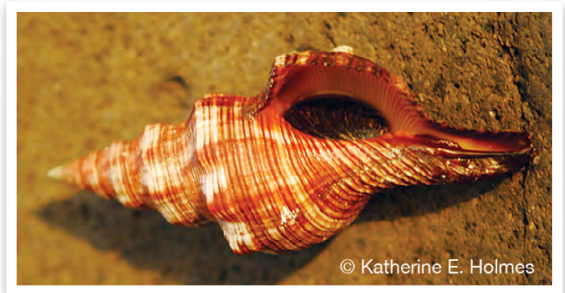

weswesak Pleuroplaca filamentosa weterōw smooth moon turban wan kaen sel blong solwota Lunella cinerea

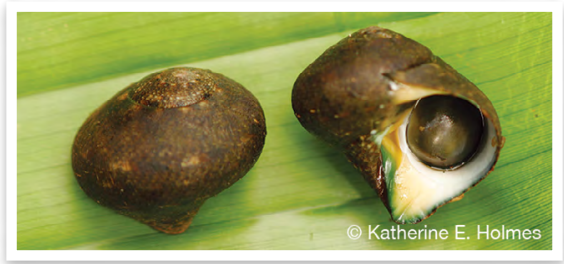

weterōw $\sim$ Lunella cinerea

wēgim giant clam that can move natalae we i save wokabaot Tridacna sp.

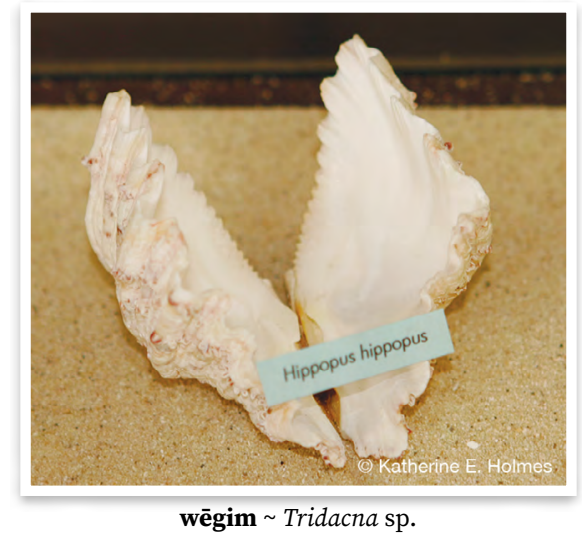

wēsēm olive shell wan kaen sel blong solwota Family Olividae
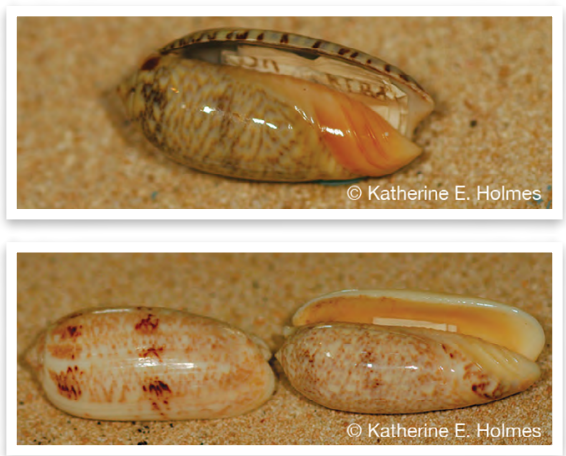

wēsēm Family Olividae

wēsēs generic name for sea snails, types of gastropod with snail type shells ol kaen nasese blong solwota we i fas long ston o rif 
wēsēs bē kind of freshwater shellfish that is round and black and red inside wan kaen sel blong wota we i raon mo baksaed blong hem i blak mo insaed long sel i red

wēsēs biēg $\mathbf{1} \cdot$ harp shell wan kaen sel blong solwota Family Harpidae 2 - mitre shell wan kaen sel blong solwota Purpura sp.

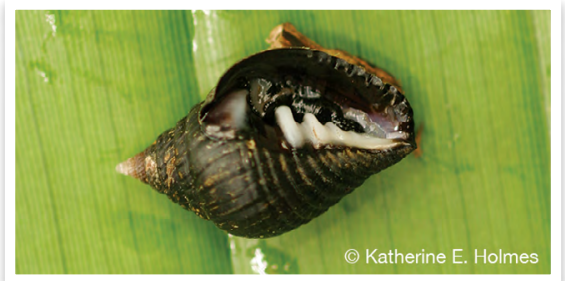

wēsēs biēg Purpura sp.

wēsēs malau kind of turban shell wan kaen bigae blong solwota Turbo crassus

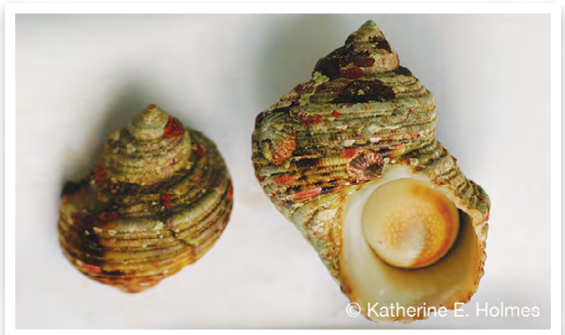

wēsēs malau Turbo crassus

wēsēs man tapestry turban wan kaen sel blong solwota Turbo petholatus

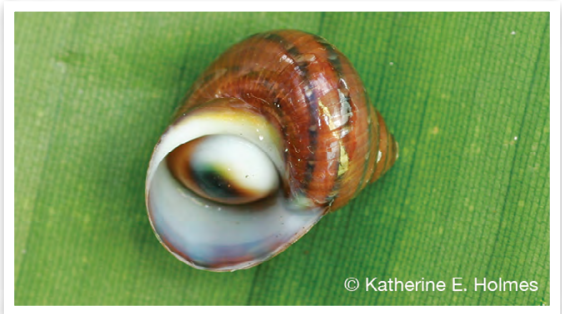

wēsēs man Turbo petholatus

wēsēs nōnōm kind of freshwater shellfish that can only be seen at night wan bigfala sel blong wota we i kamaot long naet nomo

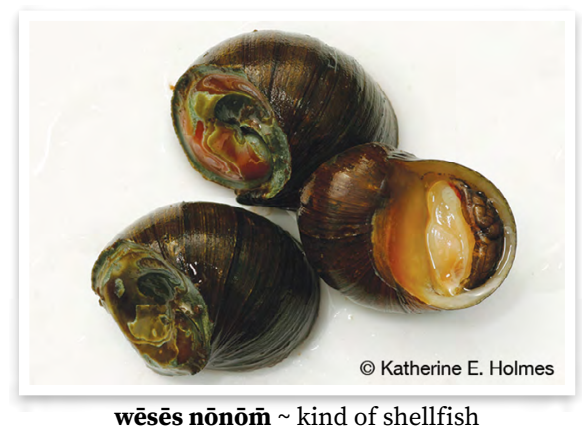

wēsēs tan̄ov great green turban grinsnel Turbo marmoratus

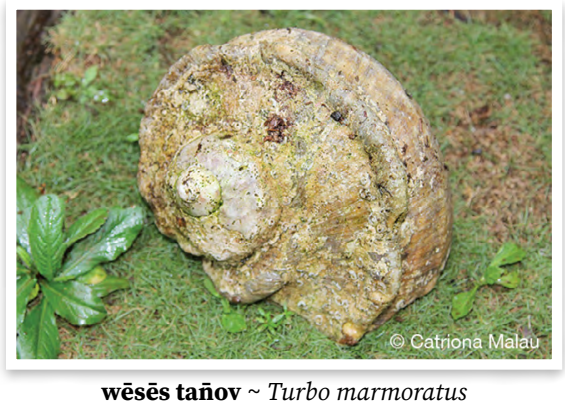

wēsēs vet rough turban wan kaen bigae blong solwota Turbo setosus

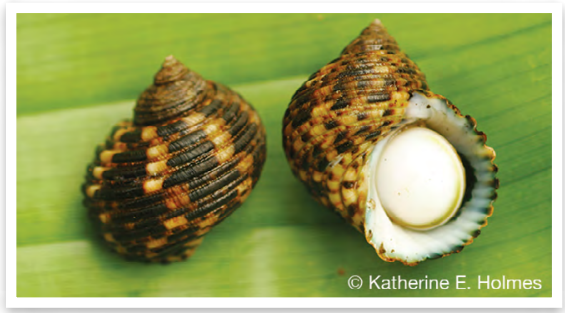

wēsēs vet $\sim$ Turbo setosus

wēsēs wōmalak kind of turban shell wan kaen bigae blong solwota Turbo sparverius

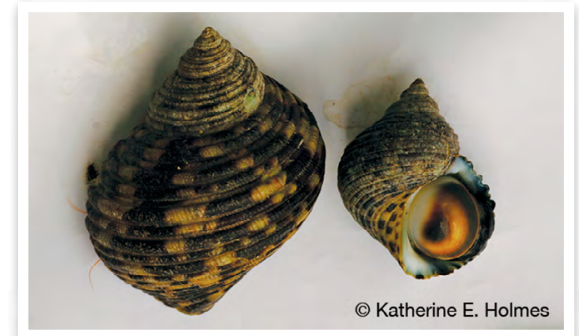

wēsēs wōmalak Turbo sparverius 
wētēqēdur auger shell wan kaen sel blong solwota Terebra spp.

wētēwil (fr.var. wētiwil) nerite nasese Family Neritidae

wētēwil bēt plicate nerite kaen nasese Nerita plicata

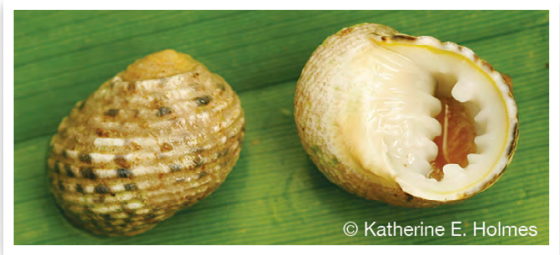

wētēwil bēt $~$ Nerita plicata

wētēwil kör waved nerite kaen nasese Nerita undata

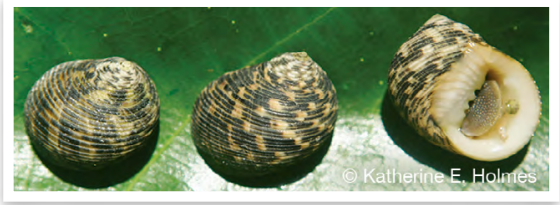

wētēwil kör Nerita undata

wētēwil mëv maximum nerite kaen nasese we i stap long rif long ples we solwota i no stap kasem Nerita maxima

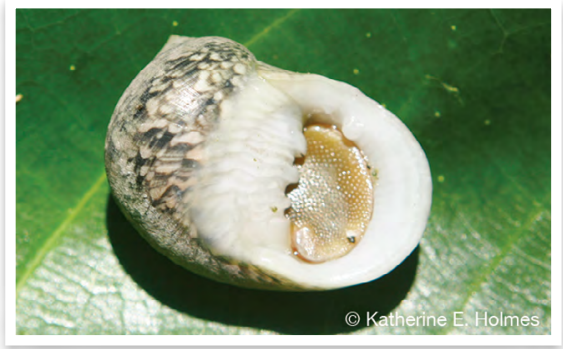

wētēwil mëv Nerita maxima

wètēwil serōn kind of nerite kaen nasese Nerita polita

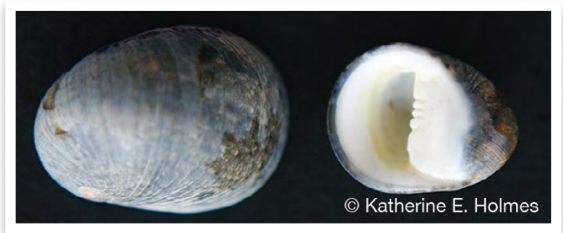

wētēwil serōn $~$ Nerita polita widiañ $1 \bullet$ serpent's head cowrie wan kaen kaorisel Cypraea caputserpentis

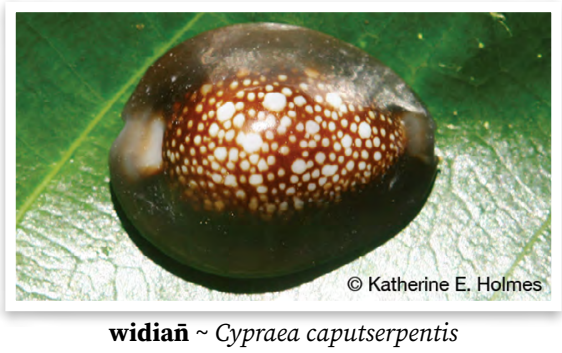

woswos kind of top shell famle blong troka Tectus pyramis

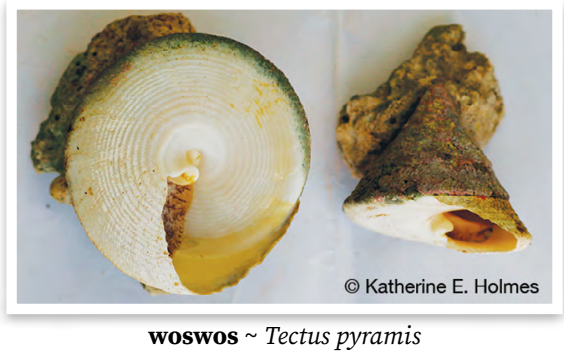

wōgōlgōl cone shell wan kaen sel blong solwota, konsel Conus spp.

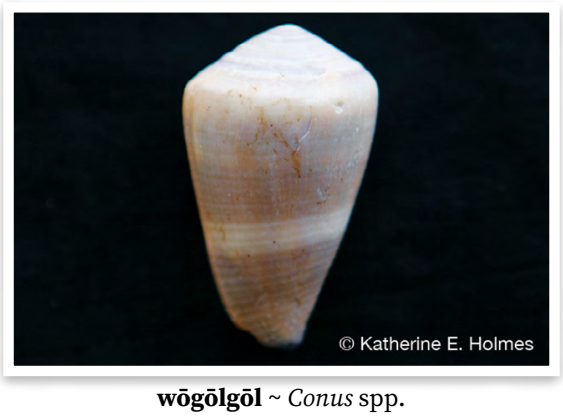

wōrō sel olsem troka be i smol mo waet Astraea kesteveni

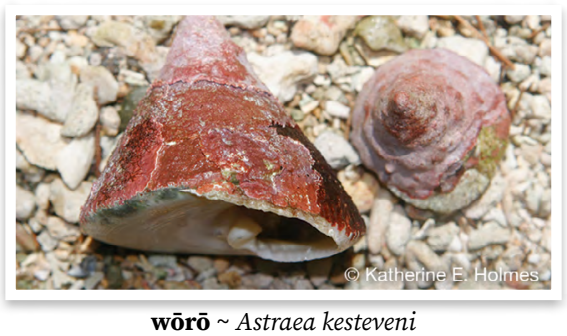


wōwlan cowrie kaorisel Family Cypraeidae; Cypraea mauritiana

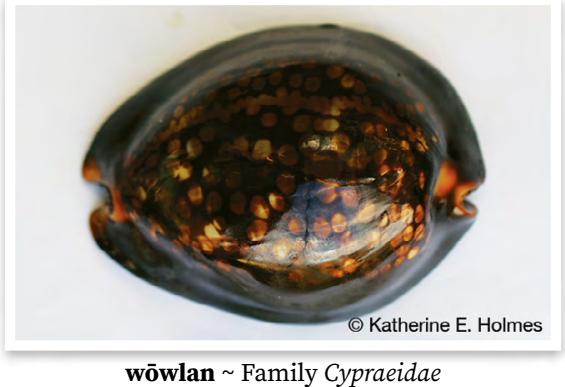

wölubus corded turban wan kaen bigae blong solwota Turbo sparverius (?)

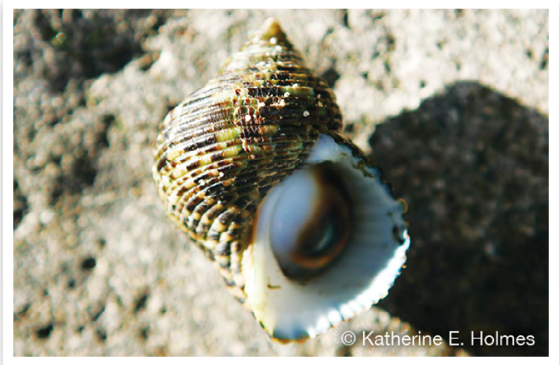

wölubus Turbo sparverius (?)

wöqörö kind of freshwater shellfish which is found in muddy areas wan kaen sel blong wota we i stap long sofmad

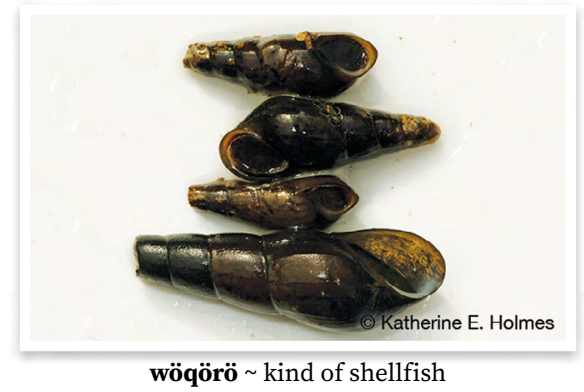

wörön̄ötöwö aculeate rock-shell wan kaen sel blong solwota Thais aculeata
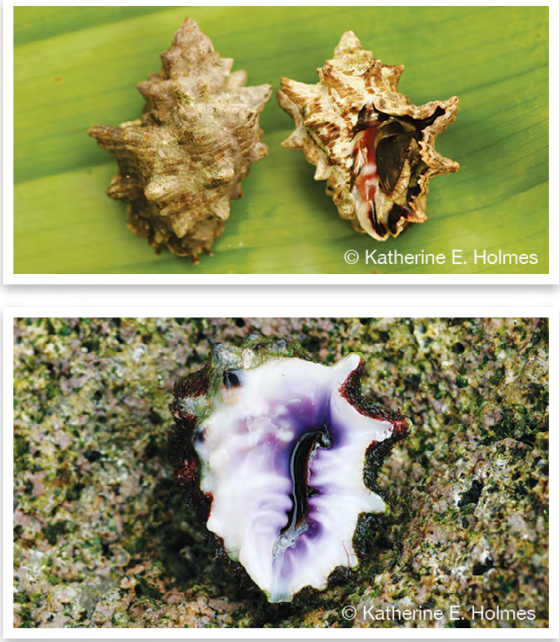

wörön̄ötöwö Thais aculeata

wösöm kind of gastropod shellfish wan kaen sel blong solwota

wösusumàmà (fr.var. susum̄almal) maculated top shell wan kaen selfis we i olsem troka be i raon mo sep blong hem i olsem titi blong wan gel Trochus maculatus

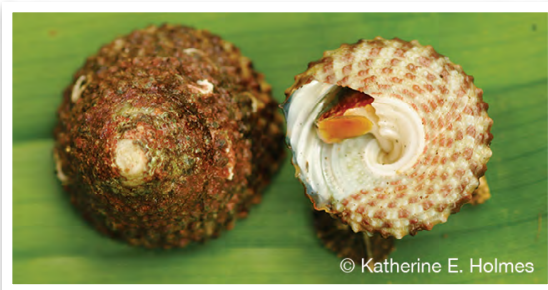

wösusū̄almāal Trochus maculatus

wutumet drupe shell wan kaen sel blong solwota Drupa morum, Drupa grossularia

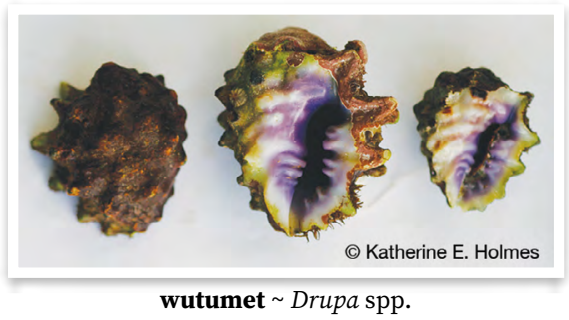




\section{E4.5 All other sea and shore life}

bēw 1 1 kind of slug that is brown slag, samting olsem snel be i nogat sel blong hem $\mathbf{2}$ - kind of sea slug samting olsem besdemea we i stap long solwota

ërlam sea fan samting blong solwota we i luk olsem wan fan Plexauridae spp.

malwērēt brittle star kaen stafis we bodi i smol mo ol leg i longwan, mo i gat ol smolsmol nil long hem

me 1 - banded sea snake, banded sea krait sisnek we hem i blak mo waet Laticauda colubrina

menek $\mathbf{1} \cdot$ squid $\sim$ nawita $\mathbf{2} \cdot$ cuttlefish $\sim$ nawita, katelfis

on kind of large octopus, can be taller than man, with a big head bigfala nawita we i gat bigfala hed

sernönqet kind of sea urchin with sharp spines samting blong solwota we i olsem sel mo i gat ol sap nil long hem Family Diaematidae

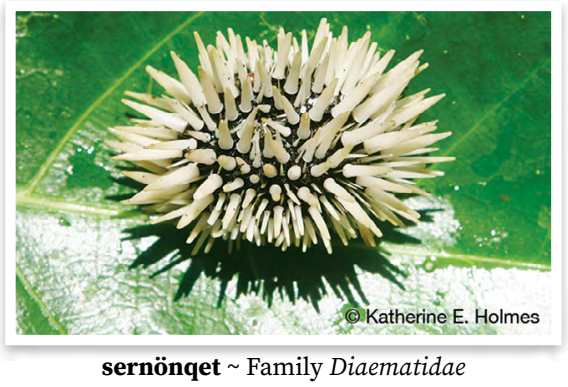

sëkër reef, coral, refers both to an area of reef and to coral heads that comprise the reef $\sim$ rif

un palolo, sea worm, marine annelid $\sim$ palolo, siwom Palola viridis

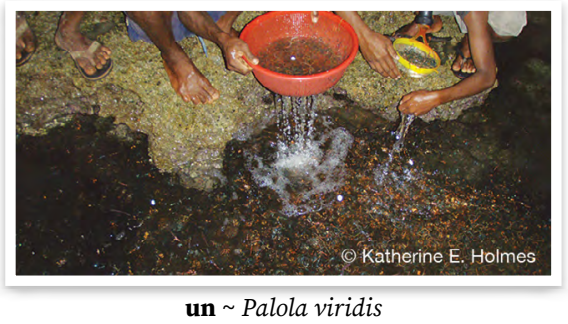

un gal male of the species Palola viridis, distinguished by its light brown colouring palolo wom we hem i man wan, we hem i laet braon

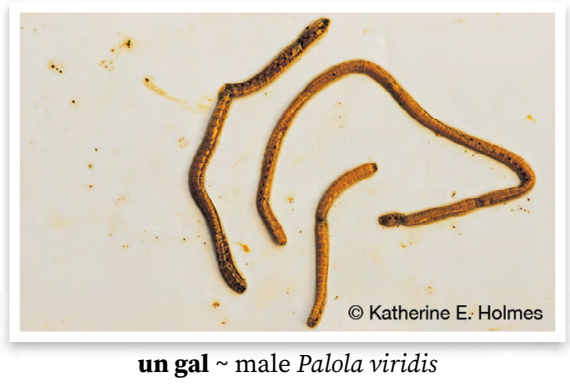

un lam female of the species Palola viridis, distinguished by its deep blue-green colouring palolo wom we hem i woman wan, hem i dak mo i blu o grin

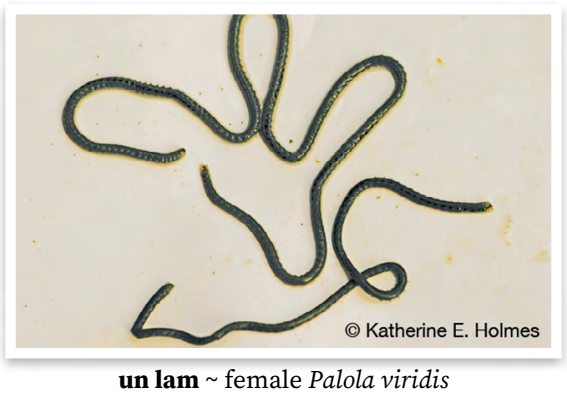

vinraq dead coral rif we i ded finis wadas kind of sea urchin with thick blunt spines $\sim$ kaen sel blong solwota we i gat nil long hem be hem i no sap Family Cidaridae

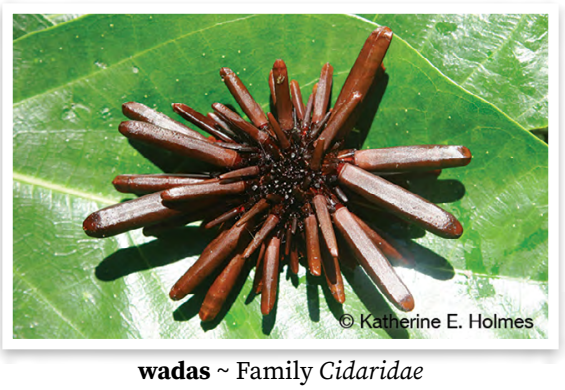

weles coral, both live and dead rubble $\sim$ korel, olsem pat blong rif o we i ded finis 
wēbēbēs barnacle kokias we i fas long ston o botom blong sip

wērēt octopus nawita

wētēltēliriaw snake sea cucumber

wan samting olsem besdemea Synapta maculata

wètēv nautilus notilis, kaen sel blong solwota Nautilus pompilius

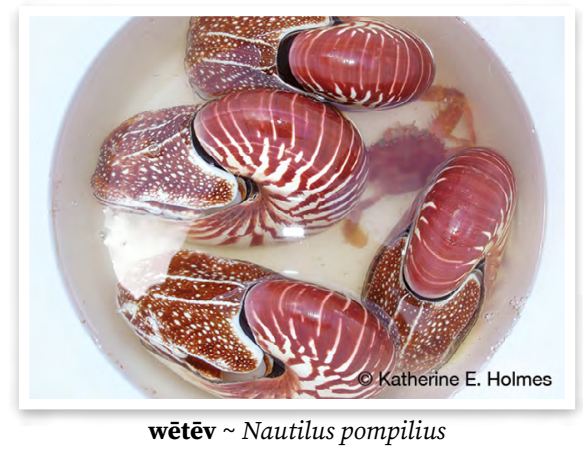

widiar 2 - phosphorescence in the sea $\sim$ ol smolsmol samting we i stap saen long solwota long naet

womal coral head korel, pat blong rif insaed long solwota

wotaqlislis unidentified variety of marine worm. The morphology of this variety is considerably different from that of those identified as Palola sp. and it is likely that they are from a different family. They are short, black on top, red on the sides, and white on the underside. kaen wom blong solwota we hem i defren lelebet long palolo. Hem i sotsotfala, i blak antap, red long saed blong hem, mo bel blong hem i waet.

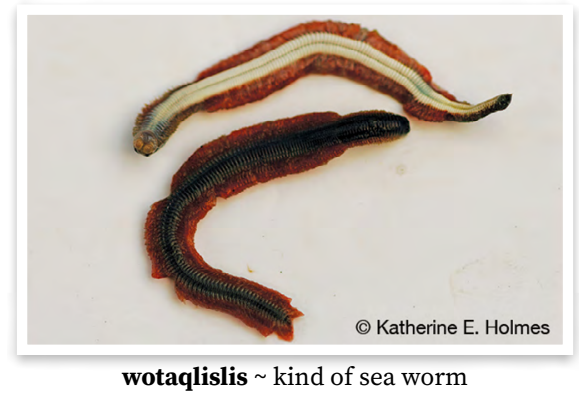

wōqōqō beche de mer, sea cucumber besdemea, si kukamba Family Holothuriidae

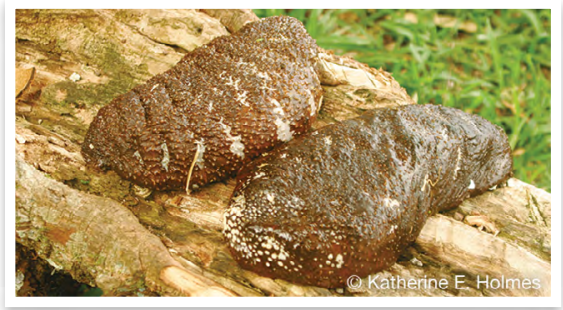

wōqōqō Family Holothuriidae

wögörtö spiny chiton strongbak Family Chitonidae

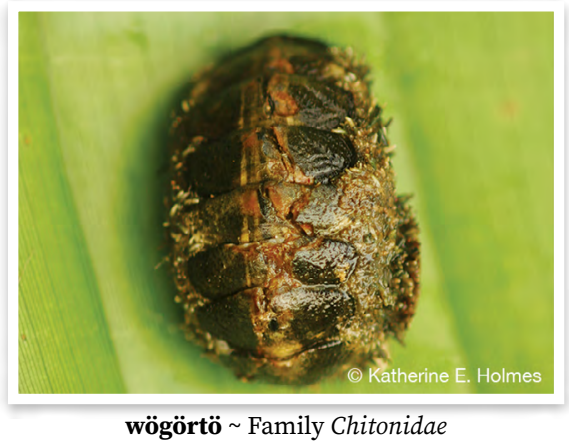

wömönmönösot unidentified variety of marine worm. The morphology of this variety is considerably different from that of those identified as Palola sp. and it is likely that they are from a different family. They are short in comparison with the palolo and light yellow-orange in colour. kaen wom blong solwota we hem i defren lelebet long palolo. Hem i sotsotfala mo hem i laet red.

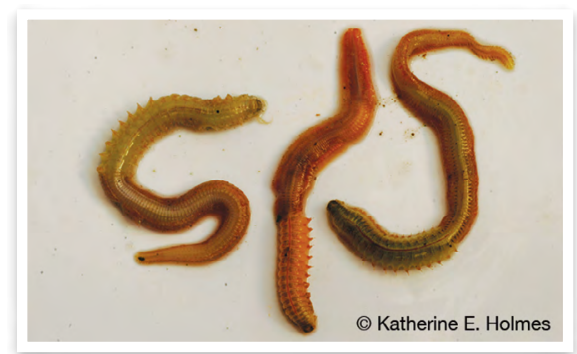

wömönmönösot kind of sea worm 
E5 Crabs and hermit crabs

dēr coconut crab krab kokonas Birgus latro

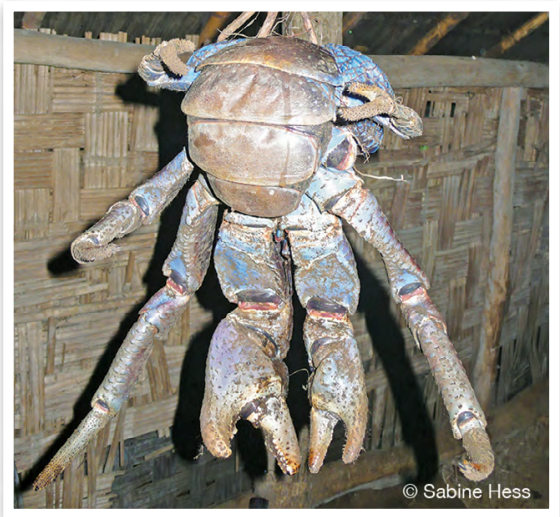

dēr Birgus latro

gamar mottled lightfoot, rocky shore crab wan kaen krab we i stap long so Grapsus albolineatus

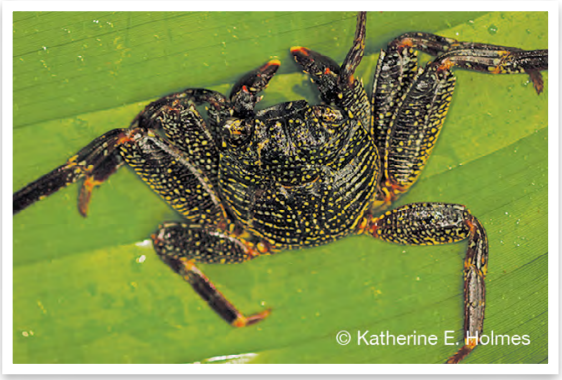

gamar Grapsus albolineatus

giav crab krab

giav kukut sargassum crab wan kaen krab blong solwota Family Grapsidae

giav matrōrōw kind of crab with big eyes, blue back and white underside. Found in the bush or shore, close to the sea wan kaen krab we ae blong hem i bigbigwan mo baksaed blong hem i blu, front i waet. Hem i stap long bus o so be klosap long solwota giav mërës mottled lightfoot crab wan kaen krab we i stap long so Grapsus albolineatus giav ōwōw spanner crab wan kaen krab blong solwota Ranina ranina giav semetöv kind of crab that dwells in holes in rotten trees in the water. It is white and light brown wan kaen krab we i stap insaed long wota insaed long hol blong wud. Hemi lelebet waet mo laet braon giav sēvsēv kind of crab wan kaen krab giav sōsō kind of freshwater crab which lives in swamps $\sim \mathrm{krab}$ Kaledoni

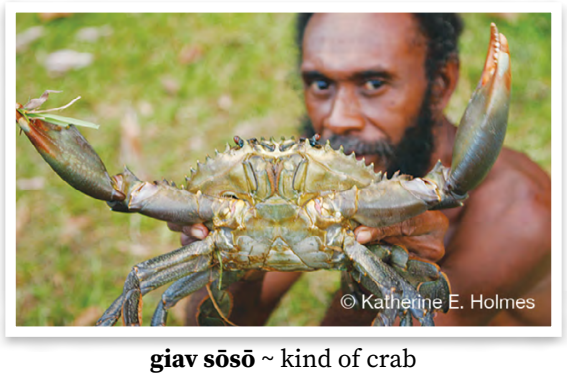

giav tamal mottled lightfoot crab wan kaen krab we i stap long so Grapsus albolineatus

giav vaw kind of reef crab with red spots on shell wan kaen krab we i stap long rif we i gat red dot long baksaed Carpilius maculatus

giav vet kind of shore crab wan kaen krab we i stap long so Plagusia dentipes

giavēdun land crab (with hairy legs) wan kaen krab we hem i blak mo hem i gat tumas hea blong hem. Hem i stap long drae ples be hem i go long solwota taem $i$ gat eg

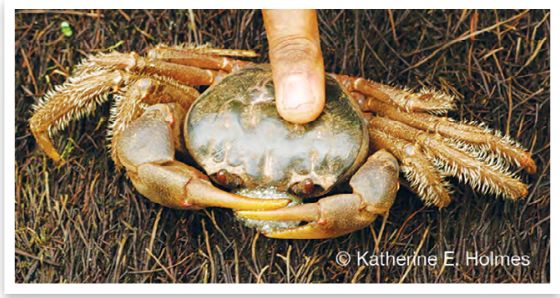

giavēdun kind of crab 
gōdōw red-eyed crab wan kaen krab blong solwota Eriphia sebana

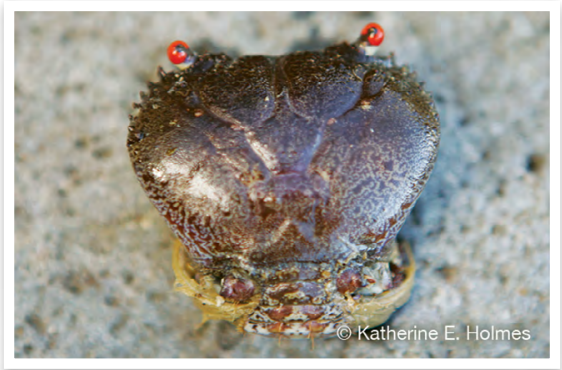

gōdōw Eriphia sebana

görum black land crab that is red when cooked, lives in holes in trees or in the ground wan blak krab we i stap long bus insaed long wud o hol long graon. Taem yu kukum hem i red Gecarcinus quadratus

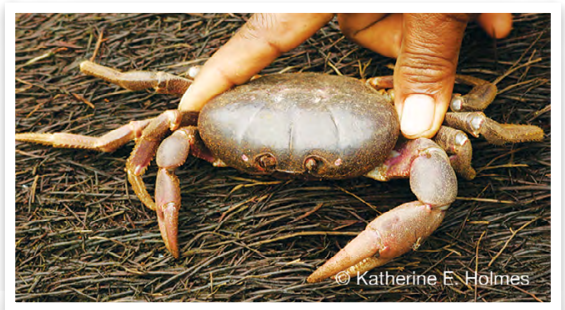

görum Gecarcinus quadratus

götö ${ }_{1}$ hermit crab nakato

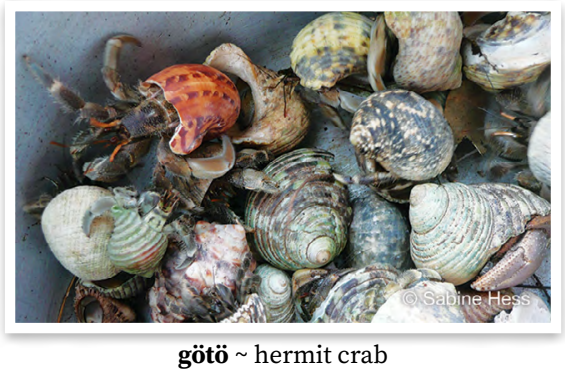

götö mamē kind of land hermit crab $\sim$ wan kaen nakato we i stap nomo long drae ples

götö riam takak kind of black hermit crab found in the bush wan kaen blak nakato blong bus götö sërsër kind of small hermit crab that lives in the sea wan kaen smol nakato we i stap insaed long solwota

sursal land crab (with hairy legs) wan kaen krab we hem i blak mo hem i gat tumas hea blong hem. Hem i stap long drae ples be hem i go long solwota taem i gat eg Gecarcinidae sp.

tēbar kind of crab, white, which dwells by the sea where there is sand and mud $\sim$ wan kaen waet krab we i stap long solwota long ples we i gat sanbij mo sofmad

tigian kind of sea crab that is black with claws that are white underneath $\sim$ krab blong solwota we hem i laet blak mo ol fingga blong hem ol i waet andanit

wēgēriv ghost crab wan kaen krab blong solwota Ocypode ceratophthalma

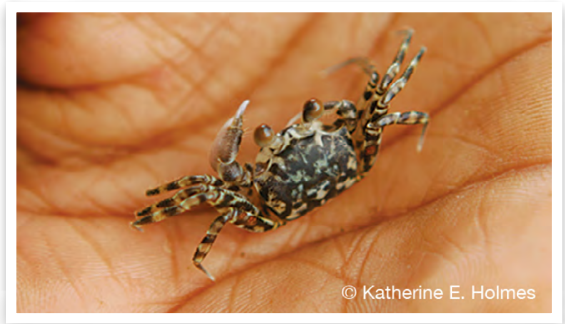

wēgēriv Ocypode ceratophthalma

wēgilgialnaw fiddler crab kaen krab Uca sp.

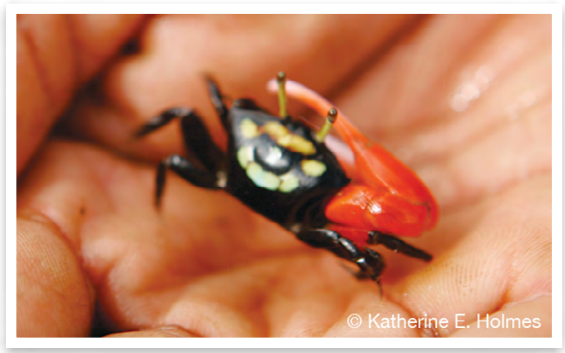

wēgilgialnaw Uca sp.

wōg kind of freshwater crab wan kaen krab blong wota 
wōlōrōr kind of small crab found on the sand, red with large claws wan kaen smol krab blong sanbij we kala blong hem i red mo tut blong hem i bigwan

wökutökut kind of sea crab wan kaen smol krab blong solwota

E6 Insects, other land arthropods, grubs

$\mathbf{b}_{\mathbf{e}} \mathbf{w}_{1} \mathbf{1} \cdot$ kind of slug that is brown slag, samting olsem snel be i nogat sel blong hem $\mathbf{2}$ - kind of sea slug samting olsem besdemea we i stap long solwota

bōñ cicada wan kaen bebet we hem i singaot long naet

bōrō̄n kind of beetle shaped like a turtle with hard wings and a strong smell wan kaen bebet we wing blong hem i strong mo sep blong hem i olsem totel. Hem i sting

bustavēl kind of colourful, shiny beetle wan kaen bebet we wing blong hem i strong mo i kalakala mo i saen

busumat kind of insect kaen bebet

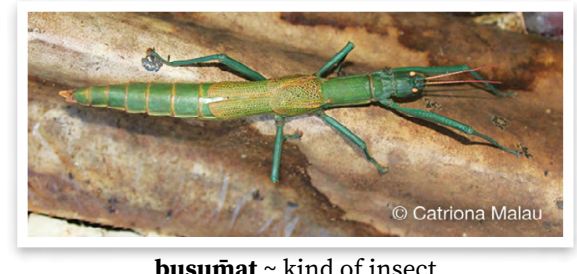

gatala web bed blong spaeda gateli maraw spider web bed blong spaeda

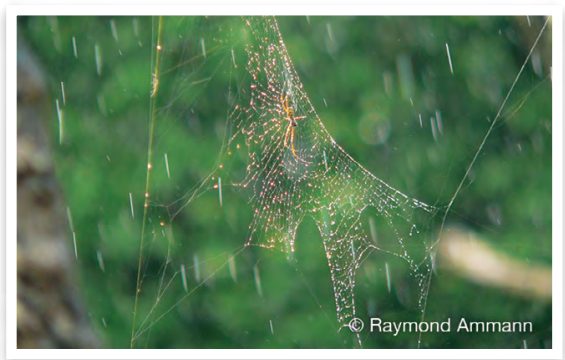

gateli maraw spider web gen $_{2}$ fire ant $\sim$ blakanis Solenopsis geminata

gōsōs insect that damages yam and taro and Hong Kong taro bebet we i stap kakae yam mo taro mo Fiji taro Papuana uninodis

lan̄ fly flae

lan̄ talōbō blowfly bluflae

lañ tanō kind of small fly that is found by freshly dug ground, they cause damage to fruits like oranges wan kaen smol flae we i kamaot taem yu digim niufala graon; ol i spolem ol frut olsem aranis

lan̄ vivis small black fly smol blak flae

lēs nit, louse egg eg blong laos

magal ant $\sim$ anis

magal tutun kind of large, reddish, biting ant, produces painful bite wan kaen bigfala anis we hem i hafred. Taem i kakae man i soa mo i hot wantaem

maraw spider spaeda

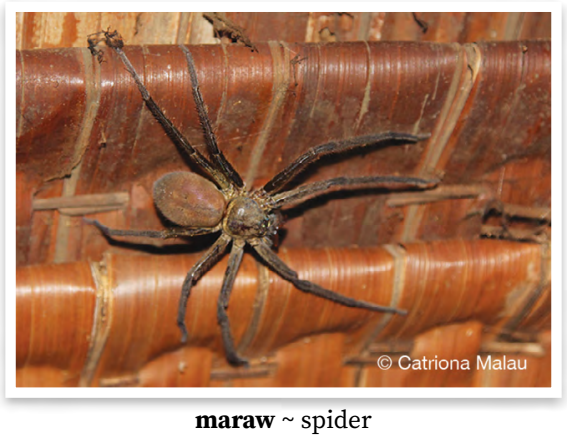

mèlēs worm wom

mēlēstenten kind of insect that is similar to a cicada, both in the way it looks and noise that it makes wan kaen bebet we hem i wokabaot long graon mo hem i save jam. Hem i singaot oltaem

matètē kind of small spider. When you touch its web, the spider shakes $\sim$ wan kaen smol spaeda. Kaen we taem yu tajem web blong hem i seksek 
nem 2 mosquito moskito

nem biliag kind of mosquito that is black with grey spots wan kaen moskito we hem i blak wetem grei spot

nem talöqön kind of mosquito that is brown and comes out at night wan kaen moskito long naet we hem i braon

$\overline{\mathbf{o}} \mathbf{1}_{2}$ maggot $\sim$ maget

qetsōs kind of insect like a termite but larger wan kaen bebet we hem i olsem waetanis be i bigwan lelebet

qētēt termite, white ant waetanis

$\mathbf{q} \overline{\mathbf{o}}_{2}$ type of insect, large and black, that bites people and fights with cockroaches $\sim$ bebet we i save kakae man. Hem i blak mo bigfala mo hem i save faetem kakros

rōm $\mathbf{1}$ - butterfly bataflae

rōm timiat kind of big, black butterfly with white dots wan kaen bataflae we hem i bigbigfala mo hem i blak wetem waet dot

sinav kind of insect that is like a cockroach but small wan kaen bebet we hem i olsem kakros be i smolsmol. Ol i laek blong stap antap long faea from we ol i laekem hit mo smok blong faea

tebedēmēēl centipede $\sim$ milpat Order Chilopoda

tōbulënqet kind of insect like a dragonfly wan kaen bebet olsem dragonflae be i smolsmol mo i waet. Taem yu tajem i save jam longwe

vun $_{2}$ kind of insect that destroys yam kaen bebet we i kakae yam

weswes piles of fecal pellets, probably from a type of termite, appearance is like dried yeast, but slightly larger, pest unidentified and not seen, only the deposit $\sim$ hip blong ol smolsmol raon samting we i kamaot long wud taem wan kaen bebet i kakae weverbōn kind of moth that comes out in the early evening, at dusk, and makes a loud buzzing noise with the beating of its wings kaen bataflae blong naet we hem i kamaot taem tudak i kam, mo i mekem noes wetem wing blong hem

weviat type of insect that lives in wood, edible bebet we i stap long wud we man i save kakae

wēgēngēndilo kind of insect like a grasshopper but smaller, found around stones by the sea kaen bebet we hem i olsem grashopa be i smol mo i stap long ol ston long saed blong solwota long ples we solwota $\mathrm{i}$ finis long hem

wētētērē millipede kruked (bebet) Order Diplopoda

wēviak grasshopper grashopa

wëtëvut kind of small insect that makes hole in the gound wan kaen smol bebet we hem i mekem hol long graon

wōqōlmōo ${ }_{2}$ worm-like creature that has shape like yam called wōqōlmō wan kaen bebet we sep blong hem i olsem yam wōqōlmō

wōrō dragonfly dragonflae

wōsōr cicada wan kaen bebet we hem i singaot long naet

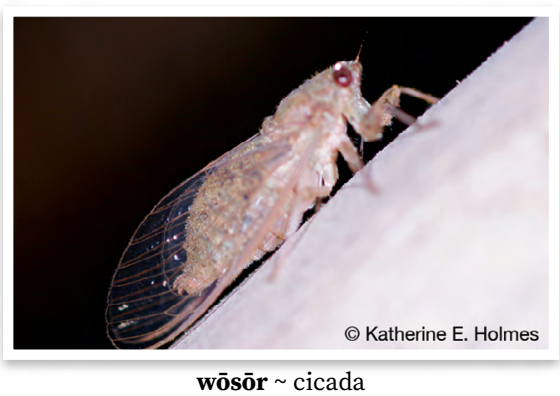

wökökö general name for caterpillars and other small wriggly creatures that don't have a specific name nem blong ol kaen smol samting olsem katapila 
wörumrum fruit fly wan kaen smolsmol bebet we fulap i stap raon long frut we i raep gud. Ol pijin (swiflet) i stap kakae

wötörör caterpillar katapila

wut louse laos wutbōn bedbug stingfli

wututan kind of insect like a cockroach but which lives in the ground and is small wan bebet we hem i olsem kakros be i smolsmol wan nomo we hem i stap long graon

\section{F Physical properties}

\section{Fo General}

garaq new $\sim$ niu
musu old $\sim$ olfala, wan samting (olsem
naef, haos) we hem i olfala

qar $\mathbf{1}$ - unripe $\sim$ no raep $2 \cdot$ raw, uncooked $\sim$ no dan $\mathbf{3} \cdot$ undried, untreated, as of green leaves, rather than dried or heat treated leaves no drae, no dan, olsem lif we i grin nomo, we ol i no putum long faea blong mekem i strong.

wōn 1 1 join, close up, seal together $\sim$ tufala saed i mit, olsem fasem lif, tufala saed i mit antap, kaliko i brok, joenem mo somap bakegen $\mathbf{2}$ - seal (hole in), join together, close up joenem, silim

wōnwōn ${ }_{1}$ whole, joined, complete fulwan

\section{F1 Appearance}

\section{F1.1 Visibility and colour}

añañ $1 \cdot$ yellow, be yellow yelo 2 • yellowness, yellow (one) yelowan

biliag $_{4}$ speckled, modifier derived from name for buff-banded rail, to indicate that the referent has speckled colouring dotdot, olsem kala blong nambilak

körkör 1 - black, be black blak 2 • blackness, black (one) blakwan $3 \cdot$ dark (colour) blak, tudak

mamē $\mathbf{1} \cdot$ red, be red $\sim$ red $\mathbf{2} \cdot$ redness, red (one) $\sim$ redwan mëlmël $1 \cdot$ shade, cool place sed, ples i kolkol 2 - become cool kam kolkol

qag $\mathbf{1} \cdot$ white $\sim$ waet $\mathbf{2} \cdot$ whiteness, white (one) $\sim$ waetwan $\mathbf{3} \cdot$ light (in colour), pale $\sim$ laet kala

qōlō $\mathbf{1} \cdot$ dirty, be dirty doti $\mathbf{2} \cdot$ become dirty, be dirty kam doti

qōn̄ov dull, be dull no braet

qöñöön purple $\sim$ popel

sēlsēl shine, emit light laet, saen

sil darkness, pitch black tudak

tōtōrōg $\mathbf{1} \cdot$ green, be green $\sim$ grin $\mathbf{2}$ • greenness, green (one) grinwan $\mathbf{3} \cdot$ blue, be blue $~$ blu $4 \cdot$ blueness, blue (one) bluwan

törö 1 1 make marking, draw, paint, dye $\sim$ kalarem, putum kala long wan samting $\mathbf{2} \cdot$ colour, markings $\sim$ kala

wamalak white with black splotches, referring to colour of pig or sometimes dog kala blong pig (o dog) we i waet mo blak smol

winiwin clean $\sim$ klin

won̄ light greyish, beige laet kala, olsem grei, laet braon

F1.2 Size, shape, weight, pattern

atik small, be small smol atiktik small smolsmol bëtutu big, large bigfala

bibis 1 - roll up, as pandanus, hair, mat $\sim$ rolemap wan longfala samting 


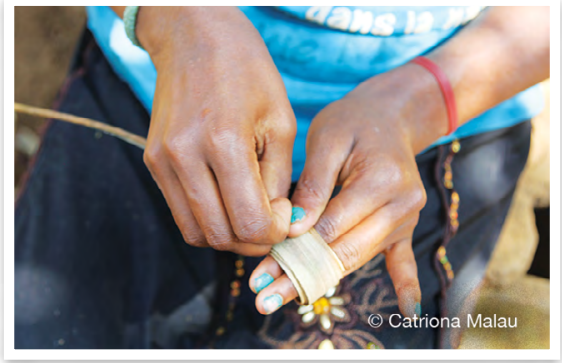

bibis roll up

2 roll (of something) rol (blong wan samting)

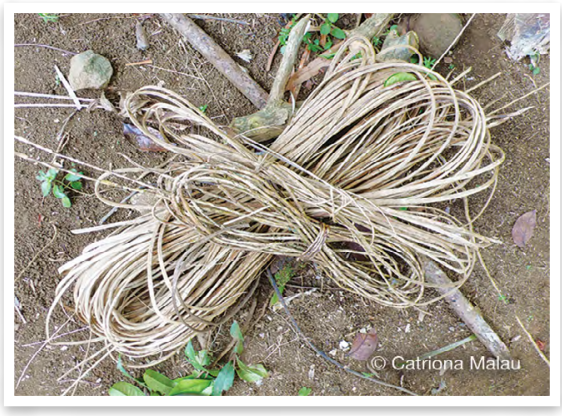

bibis roll

elel $1 \cdot$ high, high up, tall $\sim$ hae, antap $\mathbf{2} \cdot$ important $\sim$ impoten

elvet long, be long long, longfala

kēlēglēg crooked, bent, not straight kruked

$\mathbf{l a v}_{2}$ big big

luwō (fr.var. liwō) $\mathbf{1}$ - big, be big, sizable, become big, expand bigfala, kam bigwan $\mathbf{2}$ - important, high ranking hae, impoten

mav $1 \cdot$ heavy $\sim$ hevi, no laet

men̄meñ $1 \cdot$ light (weight), not heavy

laet, no hevi

mēvinvin thin tintin

mie long longfala

mötöltöl thick tik

nötu (fr.var. sönötu; sunötu) $\mathbf{1} \cdot$ small, be small, become small $\sim \operatorname{smol} 2$ • narrow, be narrow, become narrow $\sim$ smol, naro

qëtutu (fr.var. sëqëtutu) $\mathbf{1}$ - short, be short $\sim$ sotfala $2 \cdot$ be short, become short $\sim$ sotfala, kam sotwan rat flat place, area of land $\sim$ flat ples

sēsēvēl slanted, not flat no stret, no

lei flat

sial m̄ōtōtō level, even level

sursur down low $\sim$ daon

taqeqe flat $\sim$ flat

tenēnēg 1 - straight, not bent or curved stret, no kruked

tōw ${ }_{3}$ measure, mark out $\sim$ makem, mesarem

wōtōwtōw length of wood used to measure something out wud blong makem

waliög 1 - round, be round, spherical $\sim$ raon

wewete elongated, long and narrow longfala mo bunbun

\section{F2 Temperature, texture}

döw wet, be wet $\sim$ wetwet

gegen sharp (edge, as in knife) sap (long saed, olsem naef)

malas cold kolkol

mamas dry drae

mamēgin $1 \cdot$ be cold, cold $\sim$ kolkol 2 • cold $\sim$ kolkol

mere $\overline{\mathbf{n}}_{2}$ dry, dry up, dry out (wood, leaf, fruit, etc.), become withered (vegetation) drae (olsem wud o lif) metestes sharp (point, as in end of spear, knife) sap (poen, olsem en blong spia o naef)

mölumlum 1 • soft sofsof $2 \cdot$ slow, slowly slo, sloslo

qosmötöl blunt no sap

tutun $\mathbf{1} \cdot$ hot $\sim$ hot $\mathbf{2}$ ' have fever $\sim$ gat fiva

F3 Sounds - their types and qualities

aw shout, cry out singaot

dēn beat, tap lightly, bang, beating just to make a noise, rather than to make music or signal to people kilim (bambu, wud) blong mekem noes nomo 
qōrōtōt 1 - make a lot of noise, be noisy $\sim$ mekem tumas noes $\mathbf{2} \cdot$ loud sounds, too much noise $\sim$ tumas noes

rarö noise $\sim$ noes

tarōn be quiet, be calm, be peaceful stap kwaet

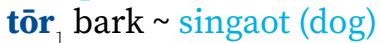

töqöl boom, bang, make a loud booming noise mekem bigfala noes olsem saon blong tanda

F4 Tastes and smells

bōn smell bad, as rotten food smel nogud

bunë smell smel

bōnan her/his/its smell smel blong hem

bunë qërët flying fox's smell smel blong flaengfokis

bunëk my smell smel blong mi

dēdērēs taste good swit

gagönus taste very good $\sim$ i swit we i swit gōgōn 1 • bitter konkon $\mathbf{2} \cdot$ salty, be salty konkon, sol $\mathbf{3} \bullet$ sour, be sour konkon $4 \cdot$ poisonous, be poisonous $\sim$ posen

lulum $1 \cdot$ have a good taste (of food) (kakae) i swit

lulumne taste (of something) tes blong wan samting

rōn̄ōbōn smell, passive sense of smell $\sim$ harem smel

sōbōn smell, sniff scent, active sensing of smell smelem

F5 Value

gōwē good gud

mav $2 \cdot$ difficult, hard, not easy $\sim$ had, no isi

men̄meñ $2 \cdot$ easy isi, no had

tisē bad $\sim$ nogud

turgi real, proper, true stret, barava, tru

wē $\mathbf{1} \cdot \operatorname{good}$, be good $\sim \operatorname{gud} \mathbf{2} \cdot$ be well, healthy stap gud, kam gud

\section{G Quantification}

\section{G1 Quantity}

bilēñ (fr.var. bilēn) $\mathbf{1} \cdot$ again, modifies verb in the verb complex $\sim$ bakegen 2 - as well, too, modifies noun in the noun phrase $\sim$ tu $3 \cdot$ more, other, another, modifier in the noun phrase $\sim$ moa, bakegen

döl all evriwan

garte 1 - leftovers, part remaining from something pat we i stap yet 2 - leftovers, food that someone has left uneaten $\sim$ hafkakae

gartan her/his/its leftovers hafkakae blong hem

garten nōtōn her child's leftovers hafkakae blong pikinini blong hem gartëk my leftovers hafkakae blong mi

gem (fr.var. gëm) $\mathbf{1}$ • just nomo $\mathbf{2}$ • only, just nomo

lōqtag plenty, many, be many plante, fulap

luw count (something) kaontem marö empty emti mōorag many, be many plante nivēs (fr.var. nives) $\mathbf{1} \cdot$ how many hamas $\mathbf{2}$ - few, several $\sim$ fiu, hamas nomo

sasarēt $\mathbf{1} \cdot$ enough $\sim$ inaf $\mathbf{2} \cdot$ same, equal semak, stret

taklē 1 - some, part of samfala, pat $2 \cdot$ part, partial $\sim$ haf, pat 
terē (fr.var. tere) plural marker, occuring before common nouns ol, olgeta

tōar (fr.var. tuar) other, another, the other, one of (a group) narawan

tutuqë many, plenty, be many fulap, plante

vaglōqtag $\mathbf{1} \cdot$ many times $\sim$ fulap taem $\mathbf{2}$ - do many times $\sim$ mekem fulap taem

vēvēg count (something) kaontem

vilēs all, all over evriwan

wōr $\mathbf{r}_{2}$ full, be full fulap

wuw $1 \cdot$ many, plenty, lots plante, fulap 2 - be many, plenty, become plentiful plante, fulap, kam plante

\section{G2 Numerals}

basbasne last laswan

bullevetē six together, group of six (of people) sikisfala (man)

bullōvōrō seven together, group of seven (of people) sevenfala (man)

bullövötöl eight together, group of eight (of people) eitfala (man)

bullövövet nine together, group of nine (of people) naenfala (man)

bulrō two together, group of two (of people) tufala (man)

bulsamul ten together, group of ten (of people) tenfala (man)

bultevelēm five together, group of five (of people) faefala (man)

bultöl three together, group of three (of people) trifala (man)

bulvet four together, group of four (of people) fofala (man)

levetē six siks

levetēne sixth, sixth one $\sim$ nambasikis

lōvōrō seven seven

lōvōrōne seventh, seventh one nambaseven

lövötöl eight eit

lövötölne eighth, eighth one nambaeit

lövövet nine naen lövövetne ninth, ninth one nambanaen

mölldöl hundred, reach, become a hundred $\sim$ handred, kasem handred möldöl vagōrō two hundred tu handred

möldöl vagtöl three hundred tri handred

-ne ordinal suffix ending long namba we i talemaot oda blong namba, olsem namba tu, namba tri, etc

ni- numeral marker maka we i go long fored blong ol namba

nirō (root rō) two tu

nitevelēm (root tevelēm) five $\sim$ faef

nitöl (root töl) three tri

nivet $(\text { root vet })_{3}$ ) four $\sim$ fo

qētqētne last laswan

rōne second, second one $~$ nambatu

sam̄ul ten ten

sam̄ul rō twenty twenti

samul tiwial dēmē nilevetē sixteen $\sim$ sikstin

samul tiwial dēmē nilövövet nineteen $\sim$ naentin

sam̄ul tiwial dēmē nilōvōrō seventeen $\sim$ seventin

sam̄ul tiwial dēmē nilövötöl eighteen eitin

sam̄ul tiwial dēmē nirō twelve twelf

sam̄ul tiwial dēmē nitevelēm fifteen $\sim$ fiftin

sam̄ul tiwial dēmē nitiwial eleven leven

sam̄ul tiwial dēmē nitöl thirteen totin

sam̄ul tiwial dēmē nivet fourteen fotin

samulne tenth, tenth one $\sim$ nambaten $\operatorname{tar}_{3}$ become, reach a thousand kasem taosen

tar vagōrō two thousand tu taosen tar vatiwial one thousand $\sim$ wan taosen

tevelēmne fifth nambafaef 
tölne third, third one nambatri vag- multiplicative prefix, added to numeral, indicates that the action is carried out specified number of times maka we i go long ol namba mo i talem se mekem hamas taem we namba i talemaot

vaglōvōrō 1 • seven times seven taem $\mathbf{2} \cdot$ do seven times $\sim$ mekem seven taem

vaglövötöl 1 • eight times eit taem $\mathbf{2} \cdot$ do eight times $\sim$ mekem eit taem vaglövövet $\mathbf{1} \cdot$ nine times $\sim$ naen taem $2 \cdot$ do nine times $\sim$ mekem naen taem

vagmöldöl 1 - hundred times handred taem $\mathbf{2} \cdot$ do a hundred times $\sim$ mekem handred taem

vagrō $\mathbf{1} \cdot$ twice $\sim$ tu taem $\mathbf{2} \cdot$ do twice $~$ mekem tu taem

vagrōne second time nambatu taem vagsamul $1 \cdot$ ten times $\sim$ ten taem $\mathbf{2} \cdot$ do ten times $\sim$ mekem ten taem vagtar $1 \cdot$ thousand times $\sim$ taosen taem $\mathbf{2} \cdot$ do thousand times $\sim$ mekem taosen taem

vagtevelēm 1 - five times $\sim$ faef taem 2 - do five times $\sim$ mekem faef taem vagtevelēmne fifth time $\sim$ nambafaef taem

vagtöl $\mathbf{1} \cdot$ three times $\sim$ tri taem $\mathbf{2} \cdot$ do three times $\sim$ mekem tri taem vagtölne third time $\sim$ nambatri taem vagvet $\mathbf{1}$ - four times $\sim$ fo taem $\mathbf{2}$ do four times $\sim$ mekem fo taem

vagvetne fourth time $\sim$ nambafo taem vagwuw $1 \cdot$ many times $\sim$ fulap taem $2 \cdot$ do many times $\sim$ mekem fulap taem

vetne fourth, fourth one $\sim$ nambafo wēvēs few fiu

\section{H Human classification}

\section{HO General}

masar poor person, who has no valuables, such as pigs and other valuable goods, money and land pua man we hem i nogat ol samting olsem pig mo narafala samting we $\mathrm{i}$ gat valiu blong hem

$\mathbf{0} \mathbf{l}_{1} \mathbf{2} \cdot$ call, give name to place or person $\sim$ singaotem (nem)

rege people, group of two or more people ol man, olgeta

sie name $\sim$ nem

sian her/his/its name $\sim$ nem blong hem

siëk my name nem blong mi

sul $_{2}$ people, general population, unified group of people pipol, ol man tan̄sar $\mathbf{1} \cdot$ person $\sim \operatorname{man} \mathbf{2} \cdot$ become a person, feel like you have become human again $~$ kam man

H1 Spirits and devils

dēbit good spirit (can be of dead or live person) who gives people good things gudfala speret, blong man we $i$ ded o laef yet, we i save givim gudfala samting long yu

Qet name of mythical creation figure, God God blong kastom

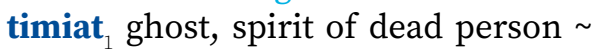
devel

tubu $2 \cdot$ ancestor $\sim$ bubu blong bifo tōbōn her/his ancestor bubu blong hem

tubuk my ancestor bubu blong mi 
vēlavēl ${ }_{2}$ person who knows how to poison people $\sim$ man we hem i save posenem man

vu magical spirit, a supernatural being that appears to people $\sim$ wan speret we i save kam helpem man

vusën̄sën̄ good spirit gudfala speret

\section{H2 Peoples and language groups}

tan̄sar $\mathbf{1} \cdot$ person $\sim \operatorname{man} 2 \cdot$ become a person, feel like you have become human again $~$ kam man

tan̄sar gaqag caucasian, white person $\sim$ waetman

tegvōnō local person man ples

wēsin̄asin̄ white person, nonindigenous person $\sim$ waetman

H3 People by age, development, life cycle

\section{H3.0 General}

masar poor person, who has no valuables, such as pigs and other valuable goods, money and land pua man we hem i nogat ol samting olsem pig mo narafala samting we i gat valiu blong hem

rege people, group of two or more people ol man, olgeta

sie name $\sim$ nem

sian her/his/its name $\sim$ nem blong hem

siëk my name nem blong mi

sul people, general population, unified group of people $\sim$ pipol, ol man

tan̄sar $\mathbf{1} \cdot$ person $\sim \operatorname{man} \mathbf{2} \cdot$ become a person, feel like you have become human again $~$ kam man

\section{H3.1 Male}

atm̄ēn $\mathbf{1} \cdot \operatorname{man} \sim \operatorname{man} \mathbf{2} \cdot$ male $\sim$ man biēn young unmarried male, in early stages of puberty, no facial growth as yet (younger than lomegev) yangfala boe, olsem se hem i nomo pikinini be i nogat mustas yet lōmegev $1 \cdot$ youth, young unmarried man $\sim$ yangfala $\mathbf{2}$ be youth, become youth $\sim$ kam yangfala

tamarge 1 old man olfala man $\mathbf{2}$ - old person $\sim$ olfala $\mathbf{3}$ grow old, become old $\sim$ kam olfala

\section{H3.2 Female}

dēm childless woman, woman who has never given birth woman we i neva karem pikinini

magte $_{2}$ old woman $\sim$ olfala woman

malmal $1 \cdot$ young unmarried woman, teenager $\sim$ yangfala gel $\mathbf{2}$ be young woman, become young woman kam yangfala gel

reqe $\mathbf{1} \cdot$ woman $\sim$ woman $\mathbf{2}$ - female woman

H4 Roles and status terms

bōg $\overline{\mathbf{o}}_{2}$ man who has not learned the traditional ways in the men's house to gain rank $\sim$ man we hem i no bin go long nakamal blong lanem stret rod blong kastom

maranag chief jif

meter woman of high rank who has studied to learn the true traditional way of life $\sim$ woman we hem i bin go long nakamal blong lanem stret rod blong kastom

m̄ëg carver, carpenter, handyman, man who has skill for making things $\sim$ man we hem i save katem wud, o gat skil blong mekem samting

mëg simsim carver who is very slow and careful about making good work $\sim$ man we hem i katem wud sloslo be hem i mekem gudwan

mëg sōbōr person who carves randomly with no skill $\sim$ man we $i$ katem wud olbaot nomo

mie 2 - first-born child, first thing, first one $\sim$ fasbon, fas samting $\mathbf{3} \cdot$ leader $\sim$ lida

qetegak captain, leader kapten, lida ronog rank rang 
sarsar woman who has not gained rank and learned the true traditional ways woman we hem i no bin go long nakamal

tövusm̄ēl chief who has gained rank in traditional way by kiling pig stret kastom jif we hem i bin kilim pig

vatōgō teacher $\sim$ tija

wöwut man of high rank who has studied the traditional rules and way of life in the men's house $\sim$ man we hem $\mathrm{i}$ bin go long nakamal blong lanem stret rod blong kastom

H5 Types of people not related to status/ profession

bulsal 1 - friend, mate, can be sexual partner or platonic friend $\sim$ fren $2 \cdot$ friend, used as an address term fren $\mathbf{3}$ - be friends, become friends $\sim$ fren $\mathbf{4}$ - become sexual partners fren (olsem gelfren mo boefren)

gōlōw orphan, child whose parents have died $\sim$ pikinini we $i$ nogat mama mo papa

mōdō unfortunate person, who people feel sorry for because they have no family, partner, or some other bad things are affecting their life $~$ man sori we laef blong hem i nogud tumas from i nogat famle, nogat man o woman blong hem, mo sam samting nogud long laef blong hem i mekem se ol man i sori long hem

nar 1 - widow, widower wido, woman we man blong hem i ded, man we woman blong hem i ded 2 - give up one specific favourite food for 1,000 days out of respect after a close relative has died $\sim$ taem mama o papa i ded, ol pikinini ol i mas givap wan kaen kakae we papa o mama $\mathrm{i}$ bin fidim olgeta wetem taem ol i smol. Ol i no save kakae kasem wan taosen dei. Tu ol i mas givim smol mane long hem we i ded mo hangem rop (blong burao) long nek blong hem blong givim saen se hem i lego wan kakae

tavalalē enemy badfren, enemi

varam twin $\sim$ twin

wömum quiet person $\sim$ man we i no stap toktok tumas

\section{H6 Address terms}

Bulsal 2 - Friend, used as an address term $\sim$ Fren

Bum Grandma, Grandpa, Grandson/ daughter; reciprocal address term used by grandparent to grandchild or grandchild to grandparent $\sim \mathrm{Bubu}$

Die Mum Mama

Diebum Grandma Bubu woman

Ika 1 - Wife, used as an address term $\sim$ Waef, olsem yu singaot long hem $\mathbf{2}$-address term, to someone who is close to you $\sim$ Dia

Mam 1 $\operatorname{Dad} \sim$ Papa 2 • God Papa God Mambum Grandad, Grandpa Bubu man

Qalēg reciprocal term used to address parents of spouse or spouse of child, father-in-law, mother-in-law, daughter-in-law, son-in-law palika

Rëwël brother-in-law; wife's brother, sister's husband (for man), reciprocal same generation in-law address term for men brata blong woman blong man, man blong sista blong man

Tatat (abbrev. Tat) Uncle, address term used towards uncle Angkel, nem we yu singaot long angkel

Tētē $\mathbf{2} \cdot$ My child, term of endearment used by parent to child $\sim$ Bebe, nem we mama o papa i yusum blong singaotem smol pikinini blong hem

Tutu address term used towards opposite sex sibling, brother (of a girl), sister (of a boy) brata blong wan gel o sista blong wan boe 
Vann̄ö niece, nephew, child of sister (of a man) smol angkel, pikinini blong sista (blong wan man)

Wimiar term of endearment used by parent-in-law to child-in-law nem we wan i yusum blong singaotem palika blong hem, we hem i woman blong boe blong hem o man blong gel blong hem
Wölus sister-in-law; husband's sister, brother's wife (for woman), reciprocal same generation in-law term for women sista blong man blong woman, woman blong brata blong woman

\section{Human qualities and emotions}

I1 Personality and moral evaluations

bioñ proud praod

bōgēt feel proud, act proudly, show off flas, mekem flas

bulbul $1 \cdot$ cooperation, togetherness, friendship $\sim$ kopereisen $\mathbf{2} \cdot$ cooperate $\sim$ kopereit

döm mav $\mathbf{1} \cdot$ respect $\sim$ ting hevi, respek $\mathbf{2} \cdot$ respect $\sim$ respek

lōlōqōr $\mathbf{1}$ - forgetful save fogetem tumas samting $\mathbf{2} \cdot$ heathen, lacking Christian enlightenment $\sim$ taem $\mathrm{O}$ man blong tudak

lōlwōn sad harem nogud, filing insaed long hat i no stret

meren $\overline{\mathbf{n}}_{1}$ lazy les

metwu personality, behaviour, manner, habit $\sim$ fasin metwōn her/his/its personality fasin blong hem

metwuk my personality fasin blong mi

mētigtisē selfish, not kind hearted selfis, fasin i nogud

mētigwē generous, kind hearted, well mannered gladhat, gudfala fasin

mōr lazy les

qaqa 1 - silly, ridiculous behaviour, ridiculous behaving person krangke, krangke fasin 2 - crazy person, behave in a ridiculous way $\sim$ mekem ol samting we i no stret olsem i hafmad; man we i krangke 3 - stupidly, not properly, randomly

olbaot, long kragke fasin

sen lie, tell untruth $\sim$ giaman

sōgsōg ${ }_{2}$ be generous $\sim$ gat gladhat

sötel proud, pride $\sim$ praod, wan blong mekem flas blong hem

survun greedy, putting oneself first before others, thinking highly of oneself $\sim$ kriti, putum yu wan mo daonem narawan

I2 Skill, ability, power

lōlōmeren $\mathbf{1}$ • wise, intelligent $\sim$ waes

2 -wisdom, knowledge $\sim$ waes, save tor $_{2}$ strong strong, taf

I3 Feelings and emotions

dasimreg hate, reject $\sim$ heitem, no wantem nating

en be angry, cross with $~$ kros long

göl speak angrily to (someone) tok kros (long)

liar $\mathbf{1} \cdot$ lost, be lost lus $\mathbf{2} \cdot$ confused, be confused $\sim$ konfius

lōlenen angry at $\sim$ kros long

lōlgagar very angry kros bigwan, kros tumas

lölö $\mathbf{1}$ ・ want $\sim$ wantem $\mathbf{2} \cdot$ like laekem 
malaklak $\mathbf{1} \cdot$ happy $\sim \operatorname{glad} \mathbf{2} \cdot$ happiness $\sim \operatorname{glad}$

mamakē be surprised at, about sapraes long

mamarseg (abbrev. marseg) 1 • pity, feel sorry (for) $\sim$ sori (long) $\mathbf{2}$ • unfortunate, pitiful sori

mēr $\mathbf{1} \cdot$ angry, be angry $\sim \operatorname{kros} \mathbf{2} \cdot$ anger $\sim$ kros

mëtëwtëw 1 - afraid, frightened fraet $\mathbf{2}$ - afraid of, frightened of $\sim$ fraet long mörös $\mathbf{1} \cdot$ want, need $\sim$ wantem $\mathbf{2} \cdot$ like

$\sim$ laekem $\mathbf{3} \cdot$ desire $\sim$ nid

malrōw be surprised $\sim$ sapraes

mataklav very happy glad tumas

qeneg 1 - afraid, frightened $\sim$ fraet

2 - fear, be afraid of $\sim$ fraet long

tēbēg express love for fasin blong soemaot lav long

tiam ${ }_{1}$ love lavem

timtiam love $\sim$ laf

\section{J Body parts and products}

\section{Jo General}

bakasē abdomen, under side of crabs and other crustaceans $\sim$ andanit, bel blong krab o naora

balsiē chin, jaw jin, jo

balsian her/his/its chin, jaw jin, jo blong hem

balsiēk my chin, jaw jin, jo blong mi

bēni $\mathbf{1} \cdot$ hand, arm han $2 \cdot$ wing of bird wing blong pijin

banēn gala her/his/its left hand lef han blong hem

banēn mōtō her/his/its right hand raet han blong hem

banēn her/his/its hand han blong hem

bēnik my hand han blong mi

bibiē neck nek

bibian her/his/its neck nek blong hem

bibiēk my neck nek blong mi

burus mete eyelashes, eyebrows hea blong ae

bus lav thumb nambawan fingga bus titivōnō index finger, pointer finger nambatu fingga long han

bustuwur pinky, baby finger las fingga long han busu 1 - digit, finger, toe $\sim$ fingga 2 • claw fingga blong pijin, fingga blong krab, naora

bōsōn her/his/its finger, toe fingga blong hem

busu ōr prawn or lobster claw fingga blong naora

busuk my finger, toe fingga blong mi

butö navel nambuton

buton her/his/its navel nambuton blong hem

butök my navel nambuton blong mi

diri leg, foot $\sim$ leg

dērēn her/his/its leg leg blong hem dirik my leg leg blong mi

gaböu wing wing

gabōōn its wing wing blong hem gaböuk my wing wing blong mi gaböu tō chicken wing jikinwing, wing blong faol

gagö butö umbilical cord rop we i joenem nambuton i go long mama gala left lefsaed

gargarke type of fatty looking substance, part of animal samting olsem gris we $i$ stap wetem mit blong animol 
garmiē tongue $\sim$ tang garmian her/his/its tongue tang blong hem

garmiēk my tongue $\sim$ tang blong $\mathrm{mi}$ gavivne armpit andanit blong han gavivnan her/his/its armpit andanit han blong hem gavivnëk my armpit andanit han blong mi

gölö tail tel golon her/his/its tail tel blong hem gölök my tail tel blong mi

kulë $\mathbf{1}$ • back (body part) baksaed $\mathbf{2}$ • behind, at the back, after (place or event) bihaen, afta

kōlan her/his/its back baksaed blong hem

kulëk my back baksaed blong mi lele rib(s) reb

lalan his/her/its rib(s) reb blong hem

lëlëk my rib(s) reb blong mi

lēnötō rooster's comb red samting we $i$ stap antap long hed blong man faol

lösö genitals, covers full genitals for male or female $\sim$ praevet pat blong hem

loson her/his genitals praevet pat blong hem

lösök my genitals praevet pat blong mi

luwö $\mathbf{1} \cdot$ tooth $\sim$ tut $\mathbf{2} \cdot$ tusk $\sim$ tut (blong pig)

luwö bas toothless person, person who has lost all or most of their teeth $\sim$ man we i nogat tut

luwö qō pig's tusk tut blong pig

luwon her/his/its tooth, teeth $\sim$ tut blong hem

luwök my tooth, teeth tut blong mi malawsöm middle finger medel fingga

malke sole (of foot), footstep andanit fut, futstep

malkan her/his/its sole andanit leg blong hem malkëk my sole andanit leg blong mi

marak fat (of animal) gris (blong animol)

mete eye $\sim$ ae

matan his/her/its eye $\sim$ ae blong hem

mëtëk my eye ae blong mi

mō long feather of a rooster's tail longfala feta long tel blong man faol

mōtō ${ }_{2}$ right $\sim$ raetsaed

mödu nose $~$ nus

mōdōn her/his/its nose nus blong hem

möduk my nose nus blong mi

mēèmèri tentacle, arm of octopus han blong nawita

nögö 1 • face fes

nogon her/his/its face $\sim$ fes blong

hem

nögök my face fes blong mi

nere 1 • lips $\sim$ lip, skin blong maot 2 • beak maot blong pijin

naran her/his/its lips, beak skin blong maot blong hem

n̄ërëk my lips skin blong maot blong mi

n̄usu $1 \cdot$ lips, snout (of animal such as pig) lip, skin blong maot $\mathbf{2} \cdot$ beak maot blong pijin

n̄ōsōn her/his/its lips, beak skin blong maot blong hem

nusuk my lips skin blong maot blong mi

qere ${ }_{2}$ penis $\sim \mathrm{kok}$

qaran his/its penis kok blong hem qërëk my penis kok blong mi

qörö ${ }_{1}$ ear sora

qoron her/his/its ear sora blong hem

qörök my ear sora blong mi

qösö groin, area of body around genitals pat blong bodi raon long ol tabu pat

qoson her/his groin tabu eria blong hem

qösök my groin tabu eria blong mi 
qötu $1 \cdot$ head hed

qōtōn her/his/its head hed blong hem

qötuk my head hed blong mi

qötubanēn her/his/its shoulder solda blong hem

qöu knee ni

qōōn her/his/its knee(s) ni blong hem

qöuk my knee ni blong mi

siri $1 \cdot$ bone $\sim$ bun

sērēn her/his/its bone(s) bun

blong hem

sirik my bone(s) bun blong mi

siri wawalag shin bone $\sim$ bun blong leg daon

sö $\mathbf{1} \cdot$ join joenem $\mathbf{2} \cdot$ joint, as in joint where two bones meet, joint in crafted wooden item joen, olsem joen blong tufala bun o joen long wud

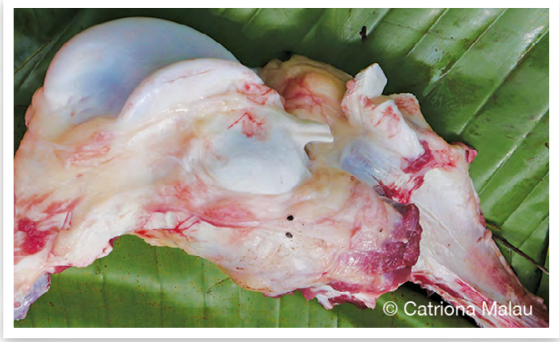

sö $\sim$ joint

sömodian̄ ring finger namba fo fingga long han

sus $_{4} \mathbf{1} \cdot$ breast $\sim$ titi

sōsōn her breasts titi blong hem

susuk my breasts titi blong mi

tarbiē body bodi

tarbian her/his/its body bodi blong hem

tarbiēk my body bodi blong mi

teqe belly bel

taqan her/his/its belly bel blong hem

tëqëk my belly bel blong mi

teqeliaw calf (of leg) mit we i stap bihaen long leg daon teqelsan back of head and back of neck bihaen hed, bitwin solda, stat antap long hed

turgēn $\mathbf{1} \cdot$ body bodi

tutgö fin wing blong fis

vele mouth $\sim$ maot valan her/his/its mouth maot blong hem

vërëk my mouth maot blong mi

vere $_{2} \mathbf{1} \cdot$ chest (of body) $\sim$ jes $\mathbf{2} \cdot$ liver $\sim$ leva

varan $1 \cdot$ her/his/its chest $\sim$ jes blong hem $\mathbf{2} \bullet$ her/his/its liver $\sim$ leva blong hem;

vërëk $\mathbf{1} \cdot$ my chest jes blong mi $\mathbf{2} \cdot$ my liver $\sim$ leva blong mi

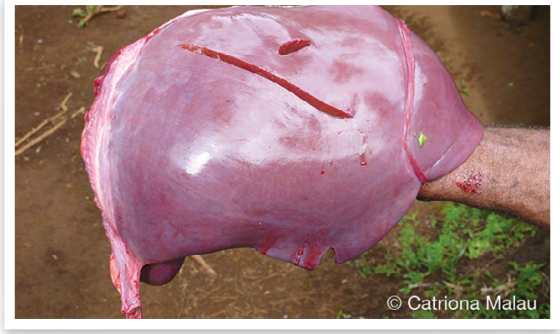

vere liver

vēsēn her/its vagina kan blong hem vikian her/his/its thigh top blong leg blong hem

vinti (fr.var. vēnti) $\mathbf{1}$ • skin skin vēntēn her/his/its skin skin blong hem

vintik my skin skin blong mi

vötu wōqōlqōl neck bone bun blong nek

vötubanēn her/his/its arm han (am) blong hem

vul 1 - hair hea $2 \cdot$ feather $\sim$ feta vōlōn her/his/its hair hea blong hem

vulu tō chicken feather feta blong faol

vuluk my hair hea blong mi

vusgö meat, flesh mit vusgon her/his/its meat $\sim$ mit blong hem 
wadalne cheek saed blong fes wadaln̄an her/his/its cheek saed blong fes blong hem

wadalnëk my cheek saed blong fes blong mi

walag, lower leg, between knee and ankle $\sim$ pat blong leg daon, bitwin ni mo angkol

wariē forehead $\sim$ fored

warian her/his/its forehead fored blong hem

wariēk my forehead fored blong mi

wawawne gill sora blong fis

wawleñ wing tip en blong wing

weles miar eyeball aebol, raon pat blong ae

wēlēgian $1 \cdot$ her/his/its heel bihaen blong leg blong hem $\mathbf{2}$ - hoof (of animal) bihaen long leg blong animol

wērē arse, buttocks as wērēk my arse as blong mi wirian her/his/its arse as blong hem

wëbëwbëwran her/his/its ankle hinsis blong fut blong hem

wobalaklak sucker, suction cup on octopus arm, tentacle samting long han blong nawita we yu save fas long hem

wotoqtoqolav stomach of cattle (cows only) bel blong buluk

wōqōlqōl back of neck bihaen long nek

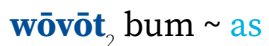

wölösömen testicles bol blong man o animol

wörurus tavav pancreas wan pat blong animol we i stap wetem gat

wöwöwut smaller stomach of cattle, omasum handred rum (blong buluk)

wulewēsēston̄ov operculum $\sim$ ae blong grinsnel
J1 Internal organs

gabala vein string blong blad gabala mōgōn her/his/its vein string blong blad blong hem

gëwtu stomach $\sim$ bel

giam 2 - net covering stomach $\sim$ net we i kavremap gat

mönug joint, where two parts of skeleton are joined together $\sim$ joen blong bodi

qultie intestine $~$ gat qultian her/his/its intestines, guts gat blong hem

tërtërgōr fascia, covering over meat waet samting we i stap bitwin long skin mo mit

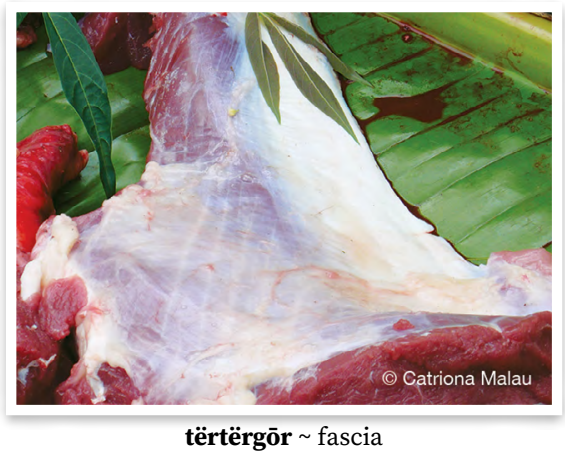

varwōs mōgōn her/his/its lungs lang blong hem

vere $\mathbf{1} \cdot$ chest (of body) jes $\mathbf{2} \cdot$ liver $\sim$ leva

varan $\mathbf{1} \cdot$ her/his/its chest $\sim$ jes blong hem $\mathbf{2} \bullet$ her/his/its liver $\sim$ leva blong hem;

vërëk $\mathbf{1}$ • my chest jes blong mi $\mathbf{2}$ • my liver $\sim$ leva blong mi

vötudōldōl oesophagus rod blong kakae

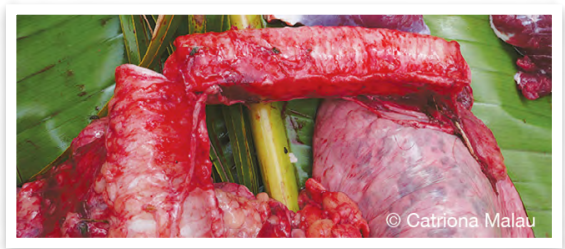

vötudōldōl oesophagus 
wēmiedaran (abbrev. miedar) her/his/ its heart hat blong hem

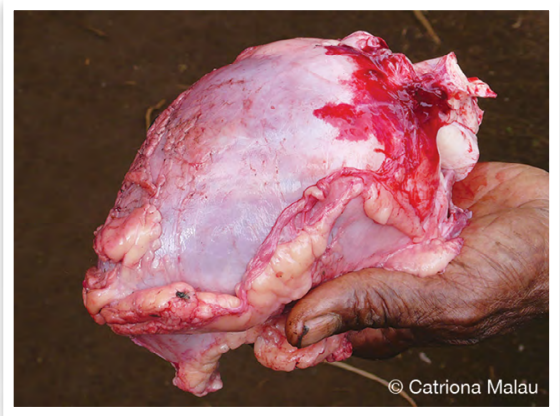

wēm̄iedaran $\sim$ heart

wēm̄ikm̄iak kidney kidni

wēvinn̄e xiphoid process, cartilaginous extension of lower sternum sofsof bun long ples we ol rib i joen, be daon

wōdōldōl trachea, windpipe pat blong bodi blong man mo animol we hem i paep blong pulum win long hem

wōs $\mathbf{s}_{2}$ lung lang, waetleva

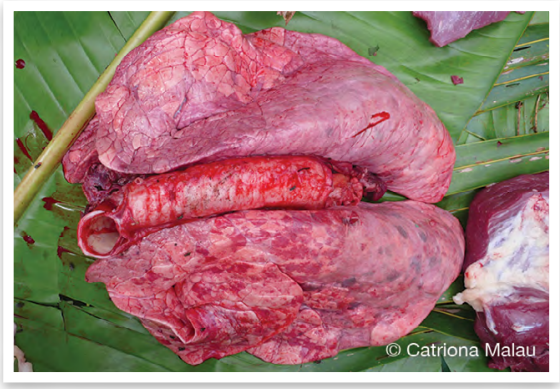

wōs lung

\section{J2 Body products}

dar $\mathbf{1} \cdot$ blood $\sim$ blad $\mathbf{2} \cdot$ bleed $\sim($ blad i) ron

daran her/his/its blood blad blong hem

dërëk my blood blad blong mi

dinsöw $1 \cdot$ saliva, dribble coming out of mouth, especially as with baby spet we i kamaot long maot (olsem blong bebe) dōn earwax $\sim$ doti blong sora

garmöös (fr.var. garmēs) $\mathbf{1} \cdot$ spit $\sim$ spet

$2 \cdot$ spit, spit on $\sim$ spet, spet long

kērēt ${ }_{2} \mathbf{1}$ - poo, excrement $\sim$ sitsit $\mathbf{2}$ • toilet $\sim$ toelet

lōt posset, baby milk vomit traot blong smol bebe

marak fat (of animal) gris (blong animol)

memesgēt sweat swet

menn̄ērēs dried mucus, snot wota blong nus we i drae

mim $1 \cdot$ urinate $\sim$ pispis $2 \cdot$ urine pispis

mimiē urine, wee pispis

mimian her/his/its urine $~$ pispis blong hem

mimiēk my urine pispis blong mi

nan $1 \cdot$ pus $\sim$ doti wota blong soa $o$ boela 2 - rheum, gound, mucus discharge from eyes during sleep or from conjunctivitis doti wota blong ae o blong sik red ae

nör snot wota blong nus

tan̄ēs tears wota blong ae

tia shit, excrement $\sim$ sitsit

tian her/his/its faeces $\sim$ sitsit blong hem

tie tō chicken faeces sitsit blong faol

tiēk my faeces sitsit blong mi

tie menek meconium; baby's first faeces which is almost black in colour $\sim$ fes sitsit blong bebe after hem ibon

tin̄is matan his/her tears wota blong ae blong hem

vul 1 • hair $\sim$ hea $2 \cdot$ feather $\sim$ feta vōlōn her/his/its hair hea blong hem

vuluk my hair hea blong mi

wese egg eg

wasan her egg eg blong hem

wësëk my egg eg blong mi

win̄i facial hair; beard, moustache mustas 


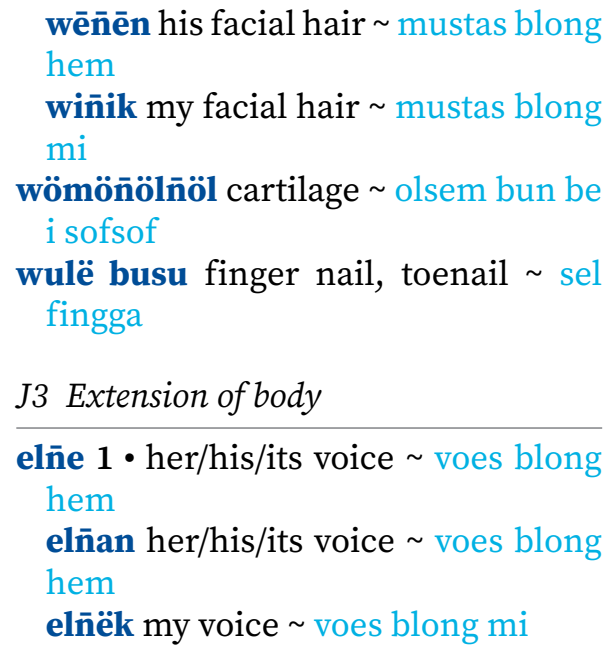

ete soul sol

atan her/his/its soul sol blong hem

ëtëk my soul sol blong mi

masgiē alone, by oneself $\sim$ hem wan masgian by himself, herself, itself hem wan

masgiēk by myself mi wan

qas be bald, bald person $\sim$ bolhed

tamtiē $1 \cdot$ shadow, reflection $\sim$ sado 2 - spirit, soul of a person $\sim$ speret blong wan man

tamtian her/his/its shadow, soul sado, speret blong hem

tamtiēk my shadow, soul sado, speret blong mi

wēbēlēt scar trak blong soa

\section{$K$ Body states and functions}

\section{K1 Physiological reactions}

bor laugh laf

borbor laughter $\sim$ laf

borseg laugh at $\sim$ laf long

burmat flutter eyelashes, make seductive looks to someone mekem ae, lukluk long wan man o woman we yu laekem hem

dar $\mathbf{1} \cdot \operatorname{blood} \sim \operatorname{blad} \mathbf{2} \cdot$ bleed $\sim($ blad i) ron

dim suck titi long

dōl swallow solem (daon)

dōlgia $\mathbf{1} \cdot$ choke $\sim$ no solem daon gud kakae o wota mo i fas long trot 2 • choke on jok long

garmös (fr.var. garmēs) $\mathbf{1} \cdot$ spit spet $\mathbf{2} \cdot$ spit, spit on $\sim$ spet, spet long

kir fart $\sim$ brekwin

kirkir have diarrhoea sitsit wota

$\mathbf{1} \overline{\mathbf{o}}_{3}$ vomit $\sim$ traot

lōlō vomit traot

lōlgagar very angry kros bigwan, kros tumas mamat $_{2} \mathbf{1} \cdot$ wake up $\sim$ wekap $\mathbf{2} \cdot$ open eyes, have one's eyes open $\sim$ openem ae, ae i open

memse breath $\sim$ win (blong hem) memsan her/his/its breath win blong hem

memse timiat spirit's breath $\sim$ win blong devel

memsëk my breath win blong mi

memseg $\mathbf{1}$ • breathe $\sim$ pulum win 2 • rest $\sim$ spel

memesgēt sweat swet

mim $\mathbf{1} \cdot$ urinate $\sim$ pispis $\mathbf{2} \cdot$ urine pispis

mösön hiccups hikap

mamēèv yawn yon, openem waed maot, olsem taem yu taed

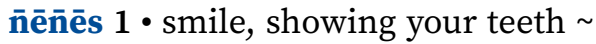
smael, soem tut blong yu 2 - show teeth $\sim$ soemaot tut

$\overline{\mathbf{n}} \mathbf{o} \bar{n} \mathbf{r}$ snore $\sim$ pulum win

sus $_{4} \mathbf{2} \cdot$ suckle, suck on $\sim$ titi, titi long

tarēñ sneeze $\sim$ snis

titiav shit, defecate $\sim$ sitsit 
tōv $\mathbf{1} \cdot$ cough $\sim$ kof $\mathbf{2} \cdot$ have a cold $\sim$ kof, kasem kof

vun $_{4}$ fart inaudibly $\sim$ man $i$ fat we i kwaet nomo

\section{K2 Body states}

\section{K2.1 Temporary}

gargarmamas fast, refrain from eating certain foods for a particular reason lego kakae mo dring

iamron tired $\sim$ taed

malala tingle, have pins and needles ded, filing long bodi taem yu sidaon long leg o han blong yu

mamēgin $1 \cdot$ be cold, cold kolkol $2 \cdot$ cold $\sim$ kolkol

mataq fresh wound, cut $\sim$ gat kil

mav 1 - heavy $~$ hevi, no laet 2 • difficult, hard, not easy $\sim$ had, no isi meneg 1 - hard, be hard strong, olsem i no sofsof $\mathbf{2}$ - be strong, powerful strong, olsem i gat paoa 3 - intense $\sim$ strong, olsem i gat paoa blong hem $\mathbf{4} \cdot$ be determined strong, olsem fasin i strong

mēlin̄sal be hungry, hunger hanggri mëmës hurt, be sore, ache soa

mëvtia wound gat kil

mörö thirst, be thirsty tosta

mörötröt weak, faint, as from hunger slak olbaot, nogat wan samting $i$ pulum tugeta paoa blong olgeta

möölöslös weak slak

nal tired, slack taed, slak

qaqa 1 - silly, ridiculous behaviour, ridiculous behaving person krangke, krangke fasin 2 - crazy person, behave in a ridiculous way mekem ol samting we i no stret olsem i hafmad; man we i krangke 3 - stupidly, not properly, randomly olbaot, long krangke fasin

qorogrog have tiny lumps, spots, as on baby's skin gat spot long bodi (blong bebe) rēg $1 \cdot$ swell, become swollen $\sim$ solap $\mathbf{2}$ - fat, be fat $\sim$ fatfat

sak swell $\sim$ solap

wamasal kind of unidentified lump on skin that becomes a sore $~$ kaen soa long skin

wawarēs lump on skin (unknown skin condition, like pimple but with no pus inside) spot long skin

wē $\mathbf{1} \cdot \operatorname{good}$, be good $\sim \operatorname{gud} \mathbf{2} \cdot$ be well, healthy stap gud, kam gud

wēqig swelling, lump on person or tree that swells but does not hurt ples long bodi o wud we i solap be i no soa

\section{K2.2 Permanent}

atm̄ēn $\mathbf{1} \cdot \operatorname{man} \sim \operatorname{man} \mathbf{2} \cdot$ male $\sim$ man

matwōn blind blaen

qōrwōn deaf person, be deaf $~$ sora $i$ fas

reqe $1 \cdot$ woman $\sim$ woman $2 \cdot$ female $\sim$ woman

wösuwun cripple, person who is unable to walk man we i no save wokbaot

K3 Sickness, death, disease, injury

bakō skin condition like ringworm where the skin becomes scaly and isn't strong, peeling off easily wan sik blong skin we i olsem ringwom mo skin i luk olsem skel blong fis mo i save kamkamaot

ēlgōr $1 \cdot$ look after, care for $\sim$ lukaotem $\mathbf{2}$ - watch out, be careful lukaot gerger scabies sik blong skras görgör kind of skin condition in which skin has lots of tiny, rough bumps, like sandpaper, but is not too itchy kaen sik blong skin we i gat ol smolsmol lam long hem, be i no skras tumas

kirkir have diarrhoea sitsit wota

lēs nit, louse egg eg blong laos 
lisia sickness, illness, disease sik, siknes

los sick, be sick sik

$\mathbf{1} \overline{\mathbf{o}}_{3}$ vomit $\sim$ traot

lōlō vomit traot

lōlmusugsug nausea, feeling that one is going to vomit sik olsem yu harem se bae yu traot

mañ serious cough, with wheezing, asthma kof nogud

mataq fresh wound, cut gat kil maw $_{1}$ heal (soa i) drae, kam gud

mete mōtō (fr.var. wemete mōtō) pimple spot long skin, espesli we i stap long fes blong ol yangfala, mo $i$ gat wota blong hem

mëvtia wound gat kil

mōnōg sore on body soa long bodi

qet mëmës headache, have headache

$\sim$ hed i soa

qēsis sore, similar to a boil, caused by a small scratch which then becomes infected, swells up and fills with pus $\sim$ kaen soa we hem i gat doti wota insaed olsem boela

qorogrog have tiny lumps, spots, as on baby's skin gat spot long bodi (blong bebe)

sislaqlaq chicken pox jikinpok

sosov skin condition caused by getting too wet and cold in rain, resulting in swollen, itchy, spotty skin skin i skras, i solap, i tik, from yu stap long taem tumas long ren mo kolkol

taqgilgil lie down motionless, with no energy, as when someone is very sick taem we siknes i kasem wan man mo hemi no save muv, i leidaon nomo

tenge leaf remedy, plant used for traditional medicine, fertilising crops, aiding fishing and other traditional remedies and purposes lif meresin o lif we ol i yusum long saed blong kastom olsem blong fis o long garen tōv $\mathbf{1} \cdot$ cough $\sim \operatorname{kof} \mathbf{2} \cdot$ have a cold $\sim$ kof, kasem kof

tutun $\mathbf{1} \cdot$ hot $\sim$ hot $\mathbf{2}$. have fever $\sim$ gat fiva

vaēs resuscitate $\sim$ mekem i laef

van̄ revive $\sim$ mekem i kam laef bakegen

vësëw treat illness by taking leaves of certain medicinal plants, steeping them in boiling water, then covering the person with the treatment so that they can breath in the steam $\sim$ tritim sik wetem lif meresin we yu kukum ol lif long wota, afta yu mekem man we i sik i smelem stim

vōn sore on scalp, caused by lice soa long hed we laos i mekem

vun $_{5}$ ringworm $\sim$ ringwom vun meren type of ringworm where the skin is dry with no liquid coming out unless it is scratched kaen ringwom we wota i no stap kamaot long skin oltaem

vun qar $1 \cdot$ type of ringworm where the liquid is coming out all the time kaen ringwom we wota blong hem i stap ron oltaem 2 - type of disease that affects trees and other plants, where liquid comes out of the affected part kaen sik blong wud we wota i stap kamaot long skin blong hem oltaem

wamasal kind of unidentified lump on skin that becomes a sore $\sim$ kaen soa long skin

wawarēs lump on skin (unknown skin condition, like pimple but with no pus inside) spot long skin

wos $_{2}$ boil, pus-filled swelling boela wos matëdër carbuncle, cluster of boils that are badly infected boela we i gat plante ae long wan ples

wösuwun cripple, person who is unable to walk man we i no save wokbaot 


\section{Stance and motion}

\section{L1 Stance, position}

\section{L1.0 Stance, position - general}

dèrē $\mathbf{1} \bullet$ wait for $\sim$ wet long $\mathbf{2}$ • wait wet

diam hang hang

dimdiam hammock, something one can hang in $\sim$ hamak

diar $\mathbf{1} \cdot$ wait for $\sim$ wet long $\mathbf{2} \bullet$ wait wet

kat stick to fas long

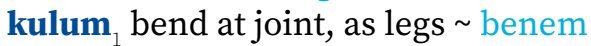
memseg $2 \cdot$ rest $\sim$ spel

rivteg $1 \cdot$ near, close by $\sim$ klosap 2 • near to, close to klosap long

tenēnēg 1 - straight, not bent or curved stret, no kruked

tog $1 \cdot$ stay, be in a place, remain in a place or way, live in a place $\sim$ stap turm̄̄o go first, be in front, lead stanap fastaem, go fastaem, lidim

L1.1 Stance, position - vertical axis

gēgēt be on balls of feet or tippy toes, not full, flat feet $~$ stanap long en $\mathrm{o}$ fingga blong leg

$\operatorname{siag}_{1}$ sit, sit down $\sim$ sidaon

siag gēgēt ${ }_{1}$ squat sidaon, olsem yu bendem leg mo as blong yu i no tajem graon

taq sit, be in sitting position $\sim$ sidaon tur 1 - stand, stand up standap 2 • have a stance, maintain a position on a particular thinking or argument stanap long wan tingting

\section{L1.2 Stance, position - horizontal axis}

atelen face upwards, for items that have division into top side and underside, such as person lying, bowl, plate, cup fes o luk i go antap, olsem man i leidaon, kap o plet

bētul lie down $~$ leidaon

ōn lie, lie down leidaon ōnveg lie beside, lie next to leidaon klosap long

vateqev face downwards, for items that have division into top side and underside, such as person lying, bowl, plate, cup fes o luk i go daon, olsem man i leidaon, kap o plet

\section{L2 Movement}

\section{L2.0 Movement-general}

dēn̄ $\mathbf{1} \bullet$ arrive $\sim$ kasem $\mathbf{2} \bullet$ reach, arrive at $\sim$ kasem

gav fly flae

gōl ${ }_{2}$ shake, shudder $\sim$ seksek

$\mathbf{k a l}_{2} \mathbf{1} \cdot$ go up, motion or event directed upwards go antap $\mathbf{2} \cdot$ go landward, ashore, inland go antap long so, long bus $3 \cdot$ go upstream $\sim$ go antap (folem wota) $\mathbf{4}$ - travel in a southeasterly direction (of travel beyond island to other islands) $\sim$ go long saot long narafala aelan $\mathbf{5} \cdot$ go inside, enter $\sim$ go insaed

kēl $\mathbf{1}$ - return kambak 2 - face towards, be directed towards $\sim$ fes $\mathrm{i}$ go long

kuëg shake, move, push on swing sekem, muvum, pusum

lak dance $\sim$ danis

le ris turn, turn over $\sim$ tanem

lil run, move very quickly resis, ron kwiktaem tumas

malaglag wobbly, be wobbly muvmuv

mōōōlgōl wobbly, be wobbly muvmuv

qēt 1 - finish, be finished, end, cease $\sim$ finis 2 - finish, complete, stop, cease $\sim$ finisim

rerev $_{2}$ slither, creep, movement low to the ground and generally as something that does not have legs 
kalkal, wokbaot, muv olsem wan samting we i nogat leg, olsem snek, snel, nawita, slug

sial float $\sim$ flot

sialtislan̄ slip glis

siōl go ashore kamso

sō paddle $\sim$ parelem

tatag $\mathbf{1}$ - follow $\sim$ folem $\mathbf{2} \cdot$ do according to, in accordance with $\sim$ mekem folem

tetel walk around, go somewhere just for enjoyment, with no particular purpose $\sim$ wokbaot, wokbaot blong enjoem nomo

$\mathbf{t} \overline{\mathbf{o}}_{2}$ run away $\sim$ ronwe

ukuëg $\mathbf{1}$ • depart, leave go, lego 2 • leave (place, person) lego

$\operatorname{van}_{1} 1 \cdot$ go (unmarked for direction) go

van me $\mathbf{1} \cdot$ come $\sim \operatorname{kam} 2 \cdot$ become $~$ kam

$\operatorname{var}_{1} \mathbf{1} \cdot$ stomp, tread heavily on prumbut $\mathbf{2} \cdot$ go in upwards direction $\sim$ go antap $\mathbf{3} \cdot$ stand on, kick something with stanap long, kik samting wetem

verdēn̄ meet, join $\sim$ mit

vērēg $\mathbf{1} \cdot$ run $\sim$ ron $\mathbf{2}$ - flow (of water, liquid, etc.) (wota i) ron 3 - run (tap), cause to flow $\sim$ mekem i ron wērēreg fly flae

\section{L2.1 Movement-vertical axis}

el dive down $\sim$ daeva i go daon, stap antap long sefes mo go daon

$\mathbf{k a l}_{2} \mathbf{1} \cdot$ go up, motion or event directed upwards go antap $\mathbf{2} \cdot$ go landward, ashore, inland go antap long so, long bus $3 \cdot$ go upstream $~$ go antap (folem wota) $\mathbf{4} \cdot$ travel in a southeasterly direction (of travel beyond island to other islands) $\sim$ go long saot long narafala aelan $\mathbf{5} \cdot$ go inside, enter go insaed

mës fall foldaon

rem climb klaem rōr ${ }_{2}$ go deep inside, either down or lengthwise, fall into hole, sink into something go dip insaed long wan samting, i go daon o go longwe, draon insaed long hol

rōw jump jam

sōw $3 \cdot$ go down $\sim$ go daon

tēqēl 1 - go down, motion or event directed downwards $\sim$ go daon 2 • go seaward, go further out to sea go long solwota $\mathbf{3} \cdot$ go downstream $\sim$ go daon (folem wota) 4 • travel in a northwesterly direction (of travel beyond island to other islands) $\sim$ go long not long narafala aelan

tōrak stand up, get up stanap, girap tul $1 \cdot$ drown, fall down from height $\sim$ draon $\mathbf{2}$ - submerge $\sim$ draonem $\mathbf{3}$ • set (of sun) $\sim$ go daon, draon (san) tul viteg draonem $i$ go

\section{L2.2 Movement - horizontal axis}

gër $\mathbf{1} \cdot$ swim $\sim \operatorname{swim~} \mathbf{2}$ - wade through water $\sim$ wokbaot krosem wota

kalkal (fr.var. kakal) crawl wokbaot fo leg

rēv pull pulum

waliög $1 \cdot$ round, be round, spherical raon $\mathbf{2} \cdot$ go around something, cover all areas $\sim$ raonem wan samting, no mata wanem sep

L3 Open and close

\section{L3.1 Open}

dur $1 \cdot$ stab, poke hole in something $\sim$ stikim $2 \cdot \operatorname{dig}$ hole $\sim$ digim hol 3 - hollow out inside of canoe digimaot insaed blong kenu

lie hole $\sim$ hol

mamat $_{2} \mathbf{2}$ - open eyes, have one's eyes open $\sim$ openem ae, ae i open

mëw open and close i open, i klos

mamaē clear, be open, not enclosed (of area) klia, open ples

marö empty emti

$\overline{\mathbf{m}} \mathbf{o} \mathbf{l} \overline{\mathrm{o}}$ form hole, have hole, cavity brok, gat hol 
qarañ hole $\sim$ hol

qarörö hole hol

qètēg $\mathbf{1}$ - start stat, statem $\mathbf{2}$ • inceptive, first verb in serial verb construction, indicating the inception of the action of the verb that follows $\sim$ statem

viër open out flat openem i kam flat vulëg open (eye) openem (ae)

wawañ open mouth openem maot

wiak 1 • open openem 2 - open, be open $\sim$ open

\section{L3.2 Close}

bur $_{2} \mathbf{1} \cdot$ close, shut $\sim$ sarem 2 - be closed $\sim$ klos

tövun (fr.var. tuvun) $\mathbf{1}$ • bury berem $\mathbf{2} \cdot$ fill in (hole), cover over something in hole $\sim$ berem

wōn 1 - join, close up, seal together $\sim$ tufala saed i mit, olsem fasem lif, tufala saed i mit antap, kaliko i brok, joenem mo somap bakegen $\mathbf{2}$ - seal (hole in), join together, close up joenem, silim

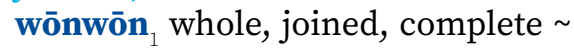
fulwan

wōn blocked, be blocked fas

wōr ${ }_{2}$ full, be full fulap

\section{L4 Exist}

$\mathbf{a} \overline{\mathbf{e}}_{1}$ have, existential predicate marker $\sim$ i gat

dun $1 \cdot$ true, be true, real $\sim$ tru $2 \cdot$ sure, tell truth $\sim$ sua, tru $\mathbf{3} \bullet$ truth $\sim$ tru

ès $\mathbf{1} \cdot$ alive, be alive $\sim$ laef $\mathbf{2} \cdot$ life $\sim$ laef (blong)

timiak 1 - like, marks manner or similative role $\sim$ olsem $2 \cdot$ be like $\sim$ olsem

\section{L5 Do}

da $1 \cdot$ make, do mekem $2 \cdot$ cause; occurs as initial verb in causative serial verb construction $\sim$ mekem 3 - do something mean or bad to someone; mistreat, tease, hassle mekem daēl try traem

gis build (house) bildim (haos)

qētēg $\mathbf{1}$ - start $\sim$ stat, statem $\mathbf{2}$ • inceptive, first verb in serial verb construction, indicating the inception of the action of the verb that follows $\sim$ statem

taōrmat $1 \cdot$ be ready, be prepared redi $2 \cdot$ prepare, make something ready $\sim$ mekem redi

da taōrmat prepare $\sim$ mekem redi

wiwieg $1 \bullet$ work $\sim$ wok $2 \bullet$ work $\sim$ wok

\section{L6 Change of state}

lies $1 \cdot$ change, exhange, replace one thing with another $\sim$ jenisim $\mathbf{2} \cdot$ take someone's place, do something in place of another tekem ples blong narafala man

lilies change (clothes) jenis, jenisim klos

meren̄ dry, dry up, dry out (wood, leaf, fruit, etc.), become withered (vegetation) drae (olsem wud o lif) qar $\mathbf{1}$ - unripe $~$ no raep 2 - raw, uncooked $\sim$ no dan $\mathbf{3} \cdot$ undried, untreated, as of green leaves, rather than dried or heat treated leaves no drae, no dan, olsem lif we i grin nomo, we ol i no putum long faea blong mekem i strong.

qètēg $\mathbf{1}$ - start stat, statem $\mathbf{2}$ • inceptive, first verb in serial verb construction, indicating the inception of the action of the verb that follows $\sim$ statem

$\mathbf{r a n}_{1}$ dry (yam, wild yam, taro, etc.) drae (olsem yam, wael yam, taro)

ris $\mathbf{1} \cdot$ turn $\sim$ tanem $2 \cdot$ change into tanem i kam

ti 1 1 start $\sim$ statem $\mathbf{2} \bullet$ light $\sim$ laetem

tōw $\mathbf{w}_{2}$ grow $\sim$ gro

van me $\mathbf{1} \cdot$ come $\sim \operatorname{kam~} 2 \cdot$ become $~$ kam

wör place in sunny position to dry putum long ples (olsem long san) blong mekem se i drae 


\section{Artefacts, material culture and manufactured things}

M1 Tools, weapons, belongings

balbal $\mathbf{1} \cdot$ scissors $\sim$ sisis $2 \cdot$ cut (with scissors) $\sim$ katem (wetem sisis)

barbar $_{2}$ axe akis

bele handle, hard handle as of knife, cup, kettle handel, olsem blong naef, kap, ketel

bur light laet

busmaraw nail, wooden stake $\sim$ nil

dinir strainer for kava sev blong kava

döwurwur broom brum

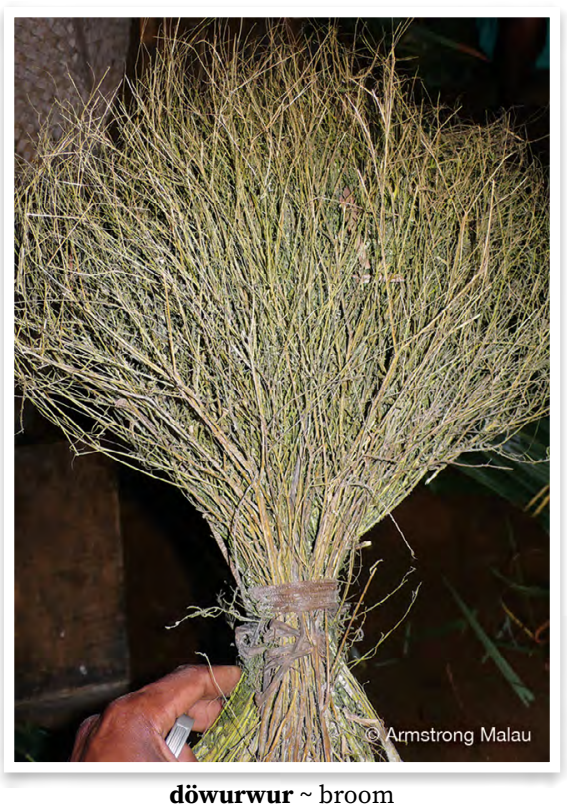

ga $_{1} \mathbf{2}$ - rope, string, other rope-like items used for tying rop

gabal long wooden tongs used for picking up hot stones or charcoal from stone oven wud blong karemaot ston long oven

gakōkō swing made from rope swing galēm long wooden pole used for evenly spreading charcoal and heat of stones in stone oven $\sim$ wud blong miksimap jakol mo ston long oven

gasel knife $\sim$ naef

gasel luwō machete, large knife, used mostly for garden work and chopping wood busnaef, big naef gasel nötu small knife, kitchen knife used mainly for cooking, simple daily use $\sim$ smolnaef

gasim length of bamboo used for removing breadfruit from the tree bambu we ol i yusum blong karemaot bredfrut long stamba

gatitieg bird or pig trap made from rope $\sim$ trap blong pijin o pig

giam $1 \cdot$ net $\sim$ net

giam vus net with a handle $\sim$ kaen net wetem handel blong hem

gil 2 - digging stick $\sim$ stik blong digim graon 3 - stick used for removing husk from coconut wud blong karemaot skin long kokonas

kar spear with four prongs and rubber spia we hem i gat raba blong hem mataqē stone or clamshell axe $\sim$ ston akis o akis wetem sel blong natalae metekerker comb kom

qesekēr club, length of wood used as weapon $\sim$ nalnal

qōrser kind of spear with several prongs on the end $\sim$ wan kaen spia we $\mathrm{i}$ gat plante poen long en blong hem

ririn̄i belongings, possessions, things $\sim$ ting (blong wan man)

wēririn̄i materials needed for making a particular item, such as house, canoe, basket ol samting we yu nidim blong mekem wan samting ser $_{1}$ spear spia 
tabē carved wooden platter, used for pounding nalot and making kava natambia, wuden plet

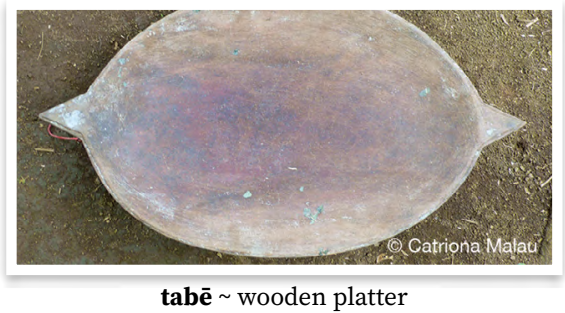

tele axe $\sim$ akis

tēqès tool for cutting wood to make it flat tul blong katem wud i flat

timiatèq gun masket

tutugon walking stick wokingstik

via grater for grating nuts and tubers

to make laplap and nalot samting

blong salem nangae o ol kakae

via qatē grater made from stem of tree fern samting blong salem laplap, nat o samting olsem we ol i mekem long blakpam

via tin grater made from old tin that has holes punched into it samting blong salem laplap, nat o samting olsem we ol i mekem long tin

vidëm adze, tool for hollowing out canoe aj, tul blong katem kenu vus bow $~_{1}$ bonaro

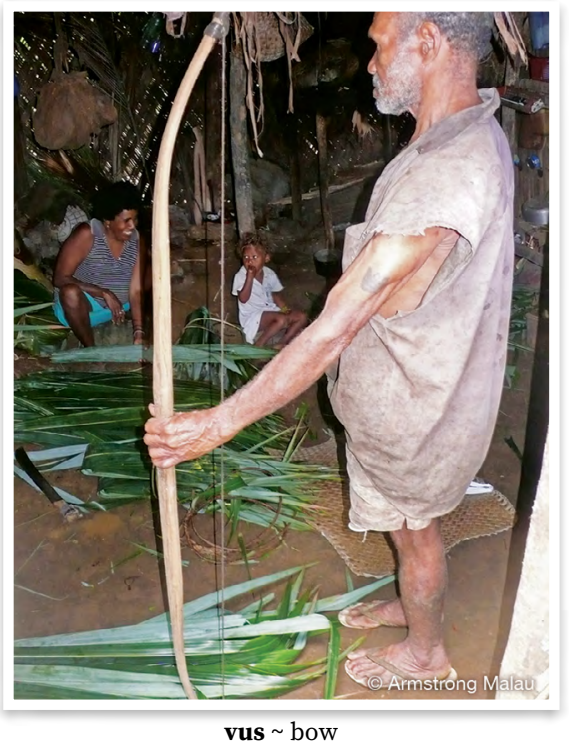

walaqlaq gōsōw rat trap trap blong rat

wetele iron aean

wetele lan̄lan̄ metal rods used as support for saucepan when cooking over fire aean blong ples blong kuk

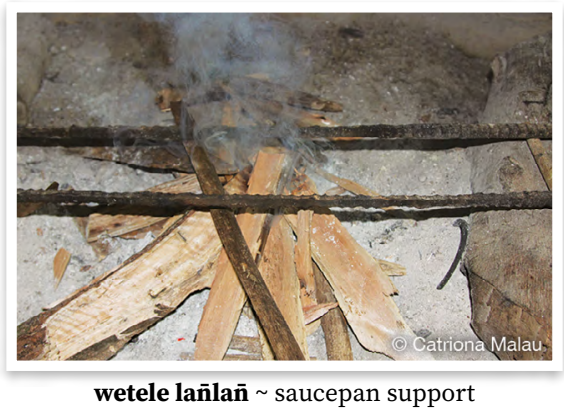

wèt carrying stick, length of wood used for carrying items over shoulder or between two people wud blong karem samting long hem wētēq kind of arrow which has a blunt end so that it will bounce off a tree if the target is missed and not get stuck, used for shooting birds wan kaen aro we en blong hem i no sap blong hem i no save fas long wud taem yu sut wetem, i mas foldaon, ol i yusum blong sutum pijin

wow ${ }_{1}$ kind of fish trap used in the deep sea, which is like a lobster pot but used only for catching fish wan kaen net trap we yu yusum long medel si. Yu putum bet insaed mo i gat wan hol antap be taem ol fis ol i go insaed ol i no save kamaot bakegen

tul wōw throw wōw fish trap into the sea draonem wōw net long solwota wötöv bottle botel

wöwöwör qèsaqēs kind of trap used close to the shore for catching small fish and particularly lobster kaen trap we yu yusum klosap long so blong kasem ol smol fis mo speseli naora 


\section{M2 Built environment}

M2.0 General

$\mathbf{a r}_{2}$ fenced in area, pen, compound, enclosure $\sim$ fenis

doot 2 - sago palm thatch $\sim$ lif natanggura, taj

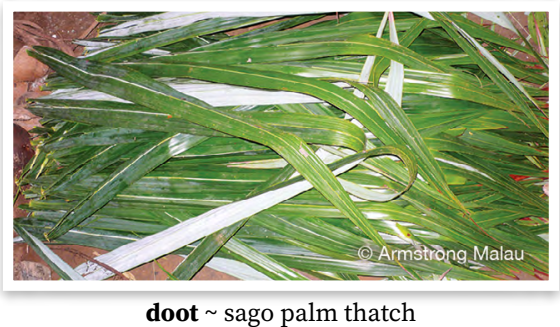

gis ${ }_{2}$ build (house) bildim (haos)

gövur house, generic name for building haos

gövur gōrōn̄ church jos

gövur lisia hospital, clinic klinik

gövur tatar church jos

kulë gövur back, sides and roof of house, sections of house behind the front $~$ baksaed blong haos, we $\mathrm{i}$ kavremap saed mo ruf

nögö gövur front of house fored blong haos

qötu gövur roof beam of house top blong haos

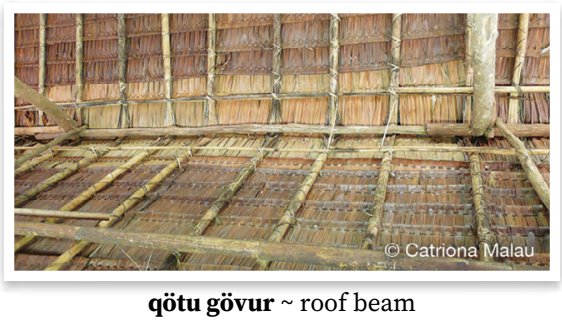

nötu gövur toilet, out house smolhaos

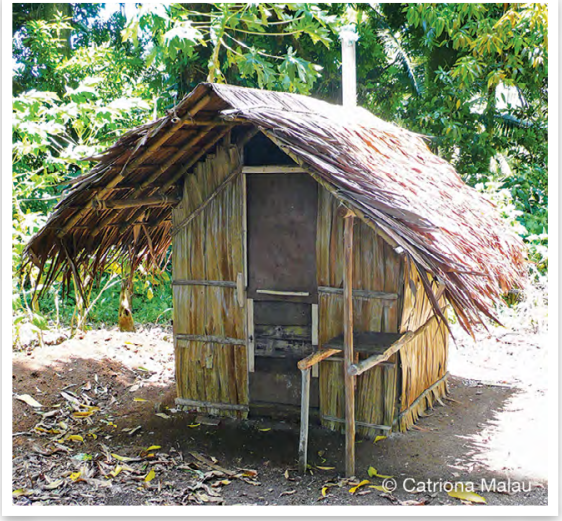

nötu gövur toilet house

tes $_{3}$ toilet $\sim$ toelet

vōnō $2 \cdot$ village $\sim$ vilej

nögö vōnō whole village, look or appearance of village $\sim$ eria blong vilej

\section{M2.1 Parts of a building}

at tie thatch slabs onto bamboo rafters

$\sim$ fasem lif natanggura $i$ go antap long bambu

at rēv tie sago roof thatching loosely, not close together $\sim$ fasem lif natanggura we i no fasfas tumas at tit tie sago roof thatching with lots of leaves close together $\sim$ fasem lif natanggura we i fasfas gud wetem plante lif klosap

babēris $1 \cdot$ wall of house $\sim$ wol blong haos $\mathbf{2} \cdot$ weave wall of house $\sim$ wivim wol blong haos

bēmtawal piece of sago palm thatch which covers over the top of the roof natanggura we ol i somap blong blokem top blong haos

bēr tie bamboo rafters to beam of roof before tying thatch onto rafters $\sim$ fasem bambu long ol wud (bifo somap taj i go long hem)

butun 1 - front wall of house $\sim$ fron wol blong haos $\mathbf{2} \cdot$ make front wall of house $\sim$ wivim fran blong haos 
butū lav front walling of house made by tying together large upright lengths of bamboo woling long fored blong haos we ol i fasem tugeta ol bigfala bambu

butun̄ rēg front walling of house made by tying together small upright lengths of bamboo woling long fored blong haos we ol i fasem tugela ol smolsmol bambu

gas make roof of house by making two lengths of bamboo join to make cross $\sim$ krosem tufala bambu i mit long antap blong haos

gemel traditional building used only by men of rank for ceremonial purposes and meetings $\sim$ nakamal gëv cover the ridge of a roof, where the two sides meet, make ridge flashing blokem top blong haos

gëv bulës make the ridge flashing for house roof using method of bending sago palm thatch over join wei blong blokem top blong haos we yu benem wan natanggura i go antap blong blokem top blong haos gëv gonsōgōrō make the ridge flashing for house roof by sewing together two slabs of sago palm thatch and placing on join wei blong blokem top blong haos wetem tu natanggura we yu somap tugeta mo benem antap long haos

gilgeg stand up post stanemap pos gonsōgōrō two pieces of thatch joined together to make one piece to cover over top of roof $\sim$ tu natanggura we ol i somap tugeta blong kavremap top blong haos

gövruqō thin length of bamboo tied onto edge of thatching on roof to hold the thatching tight $\sim$ longfala bambu we $\mathrm{i}$ tintin we ol $\mathrm{i}$ fasem long en blong natanggura blong i holem taet

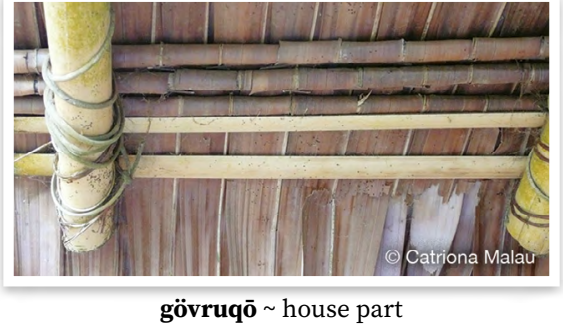

gövur house, generic name for building haos

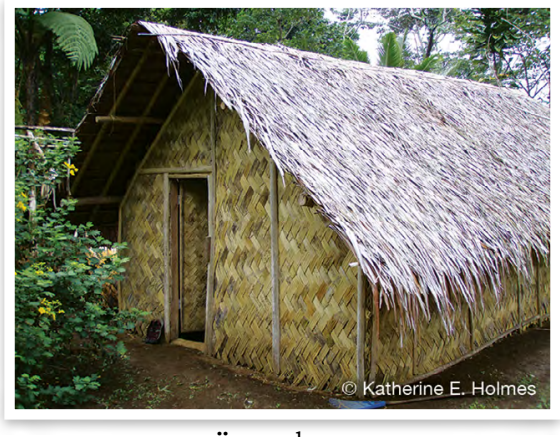

gövur gōrōn̄ church jos

gövur lisia hospital, clinic klinik

gövur tatar church jos

kulë gövur back, sides and roof of house, sections of house behind the front $~$ baksaed blong haos, we $\mathrm{i}$ kavremap saed mo ruf

nögö gövur front of house fored blong haos

lëk nail piece of timber on outside of bamboo walling to hold it in place putum strap long haos

lōlō count how many rows of thatching are needed to make roofing $\sim$ faenemaot hamas ro blong lif (masa) i nidim, sapos yu no makem fastaem

masa row of slabs of thatch that make up roofing ro blong pis natanggura we ol i bin fasem blong mekem rufing blong haos

masa dirdir short slab of thatch that is made if there is a short row left at the end when you are thatching 
a roof $\sim$ pis natanggura blong ruf we ol i mekem i sotwan blong filimap ples long en

masa taq side of roof that has more slabs saed blong ruf blong haos we hem i gat moa natanggura long hem masa tur side of roof with less slabs $\sim$ saed blong ruf blong haos we natanggura i no fulap

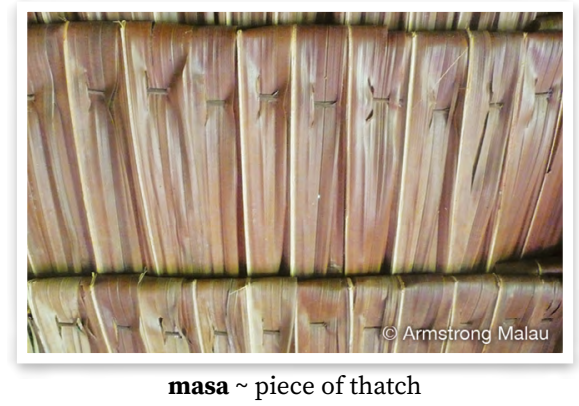

mete gövur door doa

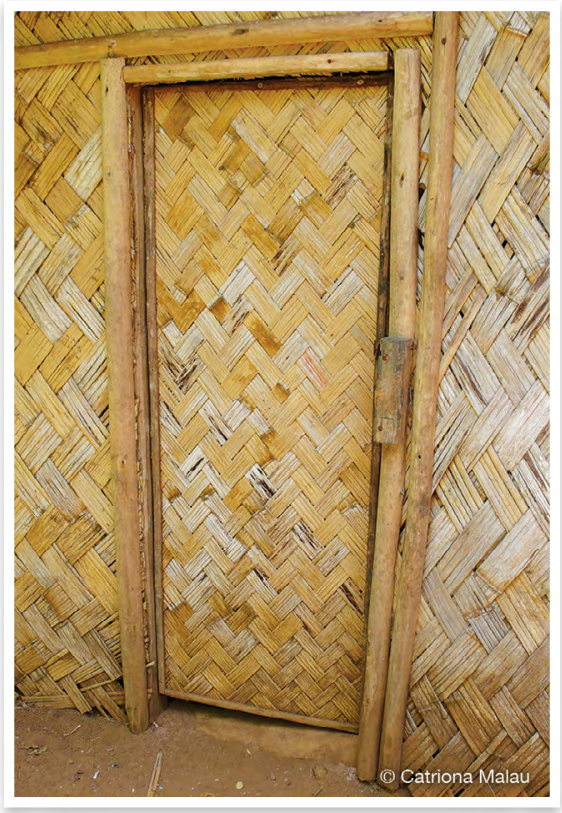

mete gövur door

mun mend roof of house by changing individual pieces of thatch $\sim$ jenesim wanwan lif natanggura insaed long haos mēēē tele weaving pattern, for which two strands are woven over, two strands under, alternating the smooth side facing out, then the rough side facing out, can refer to bamboo walling, coconut, pandanus stael blong wiv we tu pis i go ova, tu pis i go andanit, mo yu jenisim, wan antap blong lif o bambu, narawan andanit blong hem

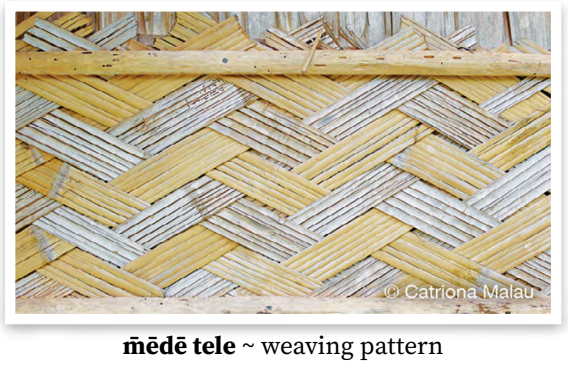

$\mathbf{r o ̄} \mathbf{q}_{2}$ bend length of bamboo over the top of the house in making roof putum bambu i go ova long top blong mekem haos

sagar remove damaged piece of thatch from roof $\sim$ tekemaot olfala lif natanggura bifo yu mekem niu wan bakegen

sañ ${ }_{1}$ house post pos blong haos sañ tine central post of house medel pos blong haos

san̄möt part of house pat blong haos sasal tie lengths of unsplit bamboo together to make walling for house wei blong mekem wol blong haos we yu dabolem ol pis bambu

so cut sago leaves, bring and prepare thatch $\sim$ mekem natanggura

so gamēlēs remove midrib from sago palm but don't remove it all, and therefore the leaf will snap when you try to bend it to make thatch karemaot bun blong natanggura be yu no karemaot gud

sus $_{3}$ place marker to indicate position of house posts stikim mak, wud folem mak blong haos 
teren make walling of house by lining up and tying together lengths of bamboo laenemap mo fasem ol bambu blong mekem wol blong haos

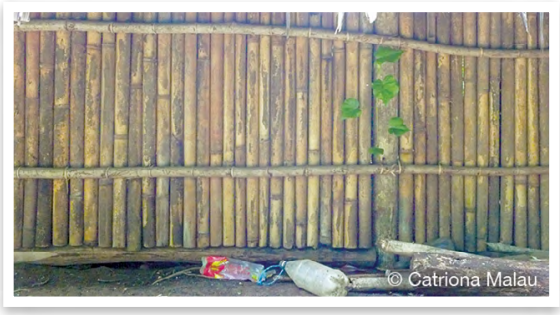

tereñ $\sim$ make walling

varar weaving pattern where two strips are woven under, two over; term used for weaving pandanus, coconut leaves, bamboo walling stael blong wiv we tu pis i go ova, tu pis i go anda; stael ia hem i blong pandanas, kokonas o bambu blong haos

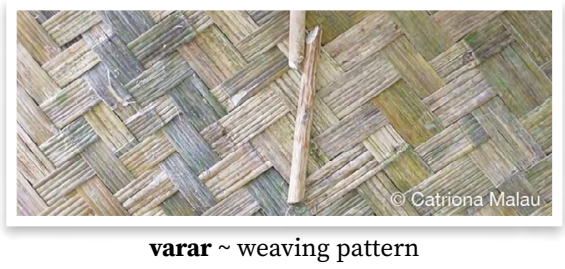

varar tur two over, two under weaving pattern, straight up and down stael blong wiv we tu pis i go ova, tu pis i go anda, i stanap stret

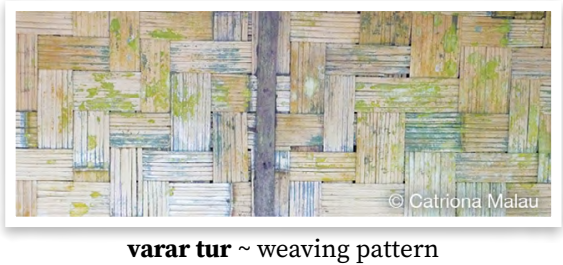

vēn make thatch slabs for roofing

by pinning sago palm leaves over a length of reed $\sim$ pinim lif natanggura waatlëk part of house pat blong haos weselegōsōw part of house pat blong haos weseñebēr part of house pat blong haos

wēbēr beam rel

wētēsi gövur frame of house inside house that is used when building to sit on so that you can reach the roof to sew on the thatch $\sim$ smol brata blong haos, lada blong stanap long hem blong fasem haos

wētilmal part of house pat blong haos

wètilvarvar part of house pat blong haos

wëlëklëk strap, thin piece of timber used in house construction to hold bamboo walling in place strap, wud blong holem taet woling blong haos

winir (fr.var. wēnir) house walling made from trunks of tree fern woling blong haos we ol i mekem long blakpam

wōlōwōl cross, cross beam kros, kroswud blong haos, kros blong Jisas

wōrōqrōq bamboo rafter of house bambu blong somap lif long hem

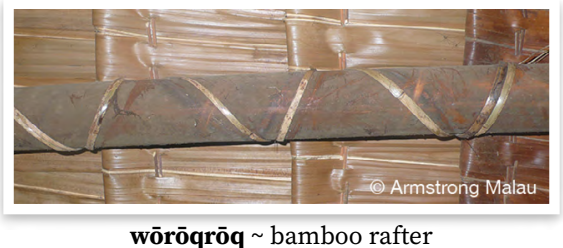

wōtōg reed that is used to sew sago onto to make thatching rid we ol i yusum blong pinim natanggura long hem, mo tu wan pis blong taj wōtōw measurement unit for roof thatching pis natanggura we ol $\mathrm{i}$ mekem finis

wöqut corner kona

wuduw sharp needle-like piece from inside of base of sago palm rachis bun we i kamaot long insaed blong stamba blong lif natanggura 


\section{M2.2 Furniture}

abawē pandanus mat pandanas mat be relational classifier used to mark possession of items that are used for bedding by the possessor $\sim$ blong (yusum blong tokbaot ona blong bed o samting blong slip)

ban her/his/its (things for bedding) $\sim$ ting blong slip blong hem

ben i nötuk my child's (bedding) ting blong slip blong pikinini blong mi

bëk my (things for bedding) ting blong slip blong mi

döñ coconut leaf mat $\sim$ lif kokonas mat

qetōlōñ pillow pilo

tētēr ${ }_{2}$ mirror glas, miro

tönö sigsiag seat, chair, stool, bench ples blong sidaon, jea

tönöam bed or sleeping mat bed o mat we yu leidaon long hem

wēqē shelf, bed, storage platform bed

wēqē n̄e shelf built over fireplace for smoking canarium nuts $\sim$ bed we ol i bildim ova long faea blong smokem nangae

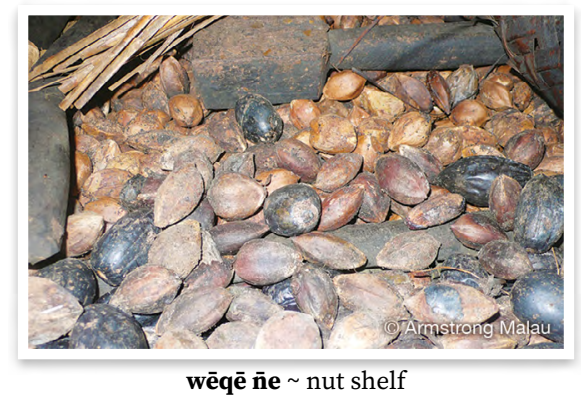

M3 Clothes, materials and decorations

ber $1 \cdot 1 \cdot$ grass skirt, skirt made from beach pandanus $\sim$ grasket

bōñ baby sling, cloth used for carrying baby kaliko blong karem pikinini bul tattoo tatu

gabelte belt $\sim$ strap

gogov $\mathbf{1} \cdot$ clothes $\sim$ klos $2 \cdot$ cloth $~$ kaliko

metegongon zip, opening of trousers

sipa, samting blong blokem fran blong traosis

mölö clothes klos

molon her/his clothes $\sim$ klos blong hem

mölök my clothes klos blong mi

rēn̄ëg wear around neck hangem long nek

sagēl decorate, as with flowers and colourful leaves, or planting colourful plants on boundary dekoreitem, olsem yu putum flaoa long haos o planem kalakala flaoa long eria

sër ${ }_{3}$ wear, put on (clothes, accessories) $\sim$ werem, havem (klos, savat)

sër $1 \overline{\mathbf{o}}$ take off, remove (clothes, accessories) karemaot (klos)

sot shirt $\sim$ sot

timiat headdress worn by men for particular dances hedres we ol man i werem long taem blong danis walal $_{2}$ bracelet $\sim$ breslet wetenge necklace $\sim$ nekles

wevet necklace $\sim$ nekles

wērērēnēeg necklace, something that is worn around one's neck nekles, samting we yu hangem long nek blong yu

wësërsër clothes klos

M4 Weaving, woven goods

abawē pandanus mat pandanas mat bès ${ }_{1}$ prepare cane or vine for weaving by stripping woody insides from outer bark karemaot insaed blong loeaken o rop, redi blong wivim

bor 2 1 kind of large coconut leaf basket which is used for transporting food wan kaen basket blong lif kokonas we ol i yusum blong 
paselem plante kakae insaed olsem blong sanem $\mathbf{2} \cdot$ small basket that is filled with food and hung up outside someone's house to indicate that another person has failed to meet an appointment, such as for a meal or drinking kava, with that person. The community will then know that this person has been let down by another basket we man i fulumap wetem kakae mo hangem aotsaed long haos blong ol man ol i save se wan narafala man i mestem mo i no kam taem hem i bin mekem promes wetem man we i hangem basket ia

dēmērir pandanus that has dried on the plant pandanas we i drae long stamba

dērēs tear strip from edge of coconut or pandanus leaf to make narrower for use in weaving teremaot pat blong lif kokonas o pandanas sapos i bigwan tumas taem yu wantem wiv
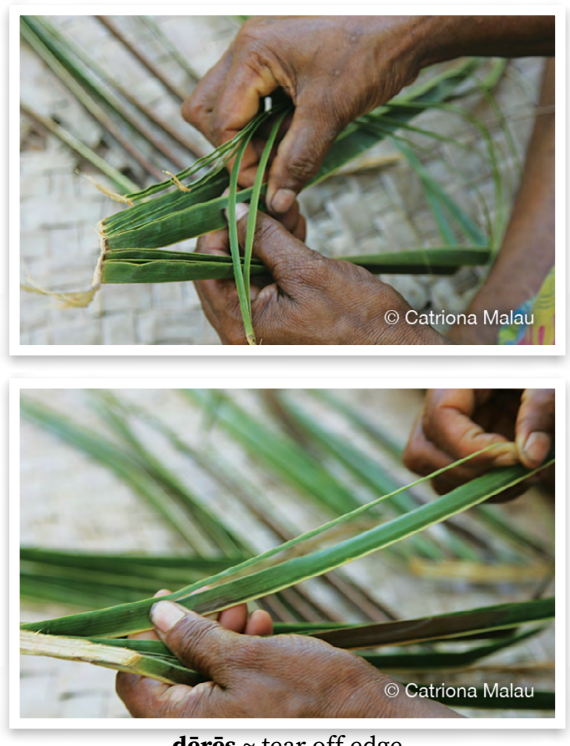

dērēs tear off edge dērivriv (fr.var. dirivriv) fan $\sim$ fan

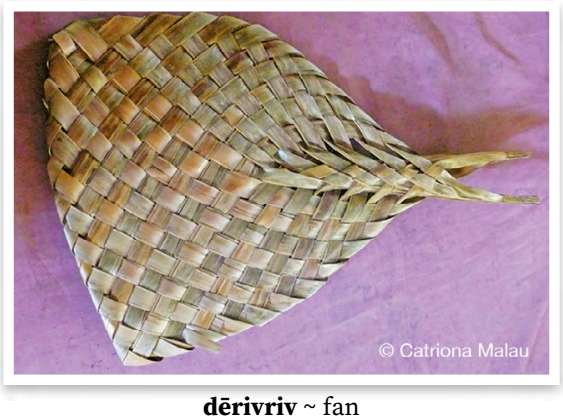

dön coconut leaf mat lif kokonas mat

dötösqar green (undried) pandanus leaf grin lif blong pandanas

gakarēs kind of climbing fern wan kaen rope Lygodium reticulatum

gamalwuw kind of black vine wan kaen rop we i stap long bus mo hem i blak Smilax vitiensis

gamērēr kind of vine wan kaen rop Freycinetia spp.

gamērēr dēmadēm kind of vine wan kaen rop Freycinetia flavida

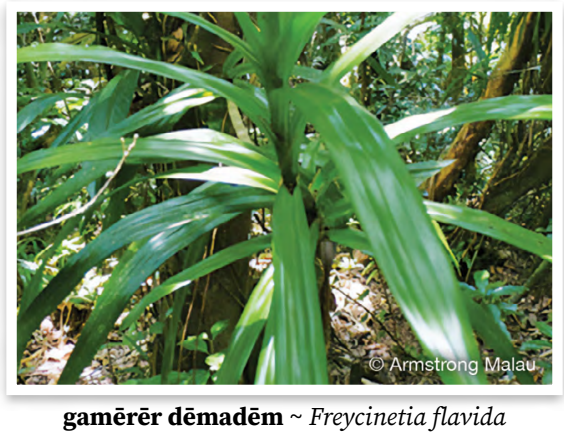

gamērēr qō kind of vine wan kaen rop Freycinetia impavida

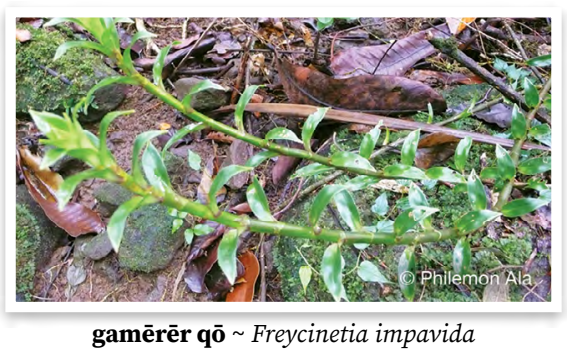


garte wōl kind of basket, with curved base wan kaen basket we hem i sep olsem hafmun

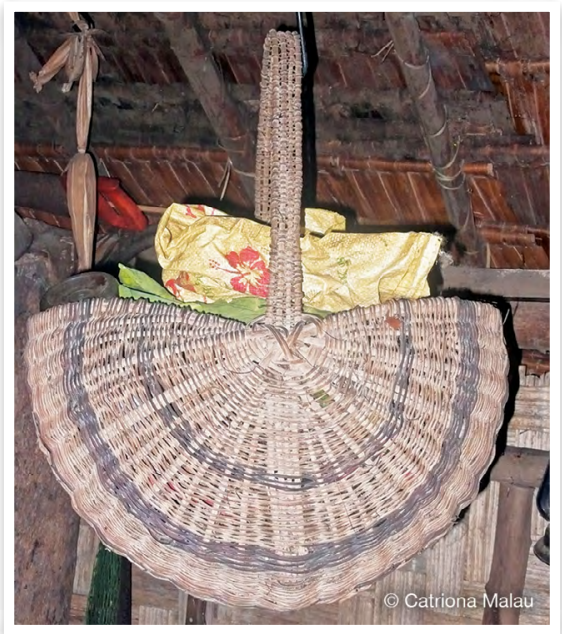

garte wōl kind of basket

gateteg kind of basket made from coconut leaves and used for carrying firewood wan kaen basket blong faeawud we ol i wivim long lif kokonas

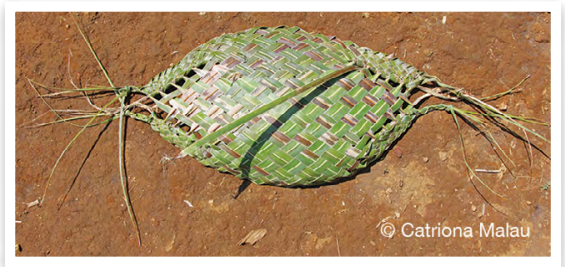

gateteg kind of basket

gavan̄ kind of food basket woven with a black vine, gamalwuw wan kaen basket blong kakae we ol i wivim wetem blak rop

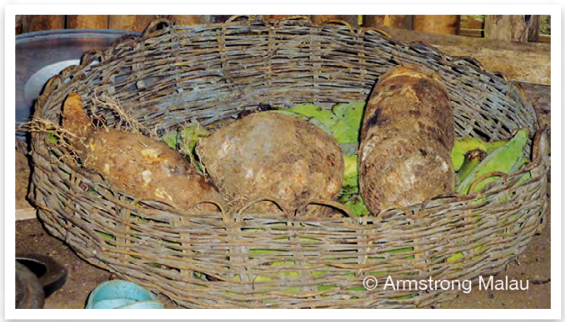

gavañ $~$ kind of food basket girir smooth out pandanus leaves using limpet shell, in preparation for weaving smutum pandanas wetem sel, redi blong wivim
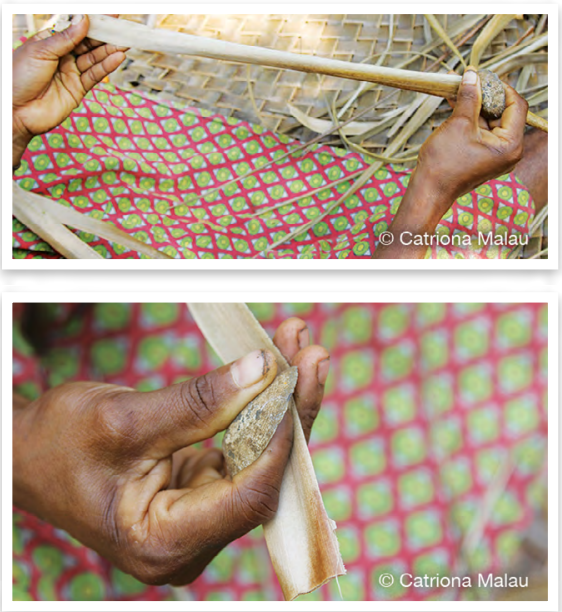

girir smooth pandanus

matqēt flat coconut leaf basket which is used especially in cooking, as a scraps basket and also for placing parcels of hot cooked food inside basket blong lif kokonas we hem i flat mo ol i yusum long saed blong kuk blong putum kakae o doti blong kakae insaed

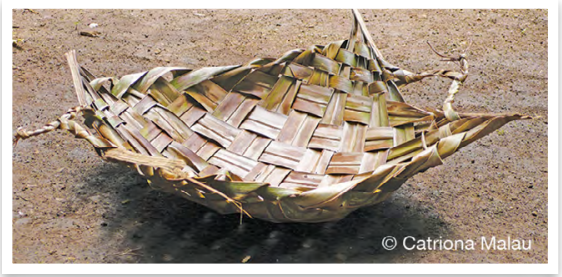

matqēt flat coconut leaf basket

mēèdē tele weaving pattern, for which two strands are woven over, two strands under, alternating the smooth side facing out, then the rough side facing out, can refer to bamboo walling, coconut, pandanus $\sim$ stael blong wiv we tu pis i go ova, tu pis i go andanit, mo yu jenisim, wan antap blong lif o bambu, narawan andanit blong hem 
misiak coconut leaf mat with only one side lif kokonas wetem wan saed nomo

$\operatorname{nin}_{1}$ split leaf or leaflet in two along midrib, split length of vine or cane into strips splitim lif, karemaot bun blong lif

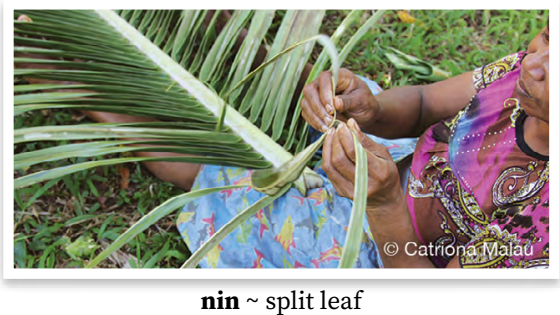

qeleg plait together two strips of coconut leaflets or pandanus leaves as first stage in making basket or mat joenem tugeta tu saed blong lif kokonas o pandanas blong wivim
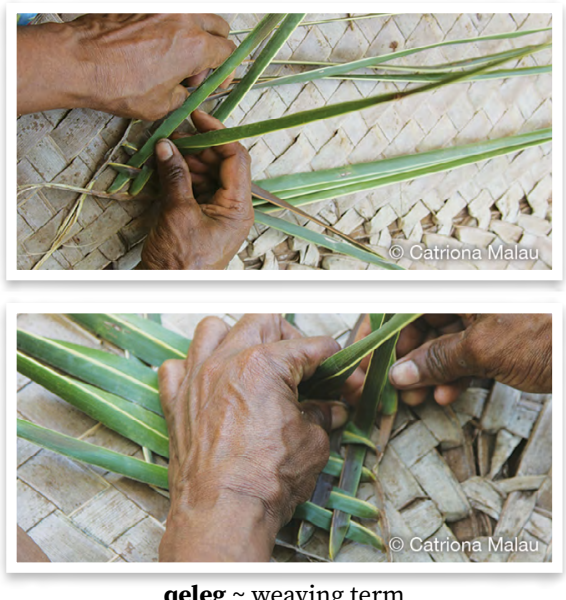

qeleg weaving term

qörö ${ }_{2}$ strip of coconut leaflets incorporated into basket to form corner pis lif kokonas we ol i ademap long basket blong mekem kona blong hem

qötuōw kind of basket made from coconut leaves and hung in the kitchen, used especially for putting bivalve shells in, which are used for scraping food and coconuts wan kaen basket we ol i mekem long lif kokonas mo yusum blong putum plasta sel insaed long kijin

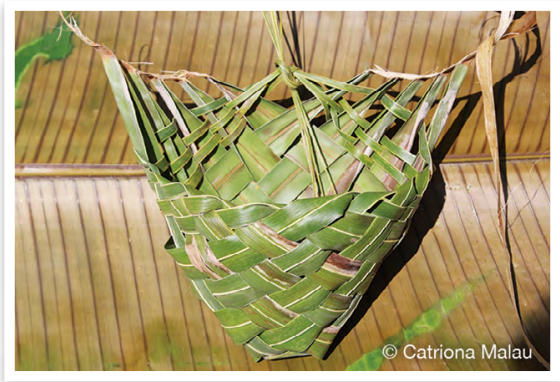

qötuōw kind of basket

ran̄ōrō plait, weave twill pattern; plait strips over two crossing elements wivim tutu, wivim pis pandanas o bambu i go ova long tu narafala pis, i no wan nomo

rēvōrō weave two strands over and two strands under $\sim$ wivim tu i go antap, tu i go andanit

ro soak, immerse in water $\sim$ draonem long wota

sergeg (after plaiting together sections composed of two pandanus half leaves) lay sections down, overlapping, ready to plait together $\sim$ tekem ol pis pandanas we yu bin wivim tu lif tugeta, joenem plante tugeta blong statem mat

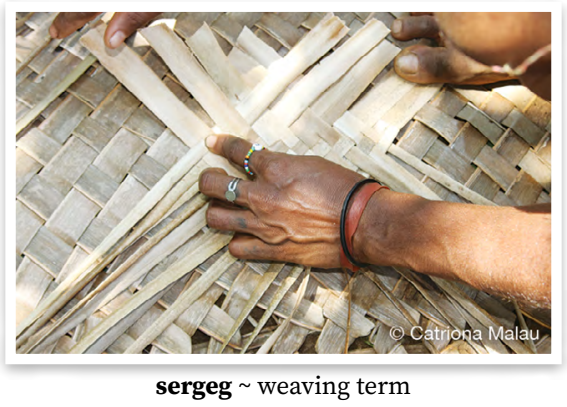

susuñu make fringe (on mat or cloth) $\sim$ katem pisisim en (blong mat o kaliko) 
tamn̄o coconut leaf mat lif kokonas mat

tan̄ pandanus basket, usually small, especially one used for carrying personal effects, also for collecting nuts or small amounts of food basket pandanas, blong yusum blong ol smolsmol ting blong yu

tiam $_{2}$ upsett (basket), prepare to weave, plait sides (of basket) leftemap basket, redi blong wivim saed blong hem

tibiar basket, generic term used to refer to coconut leaf, pandanus and cane or reed baskets basket, nem ia i kavremap ol kaen basket blong kokonas, pandanas mo narafala kaen

tibiar gamalwuw kind of food basket woven with a black vine wan kaen basket blong kakae we ol i wivim wetem blak rop

tig finish (basket or mat) by plaiting or threading in ends to prevent from coming undone $\sim$ finisim ol sotsot pis blong pandanas o rop blong mat o basket blong mekem se ol i no save kamkamaot

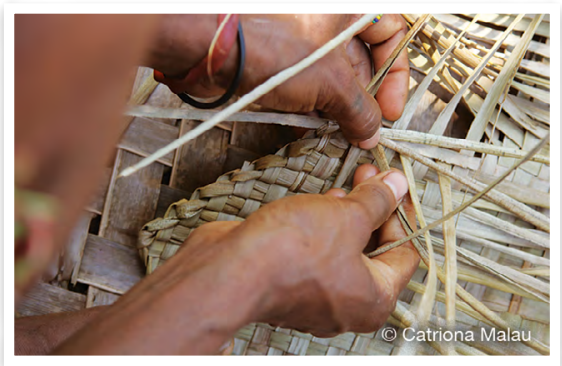

tig weaving term

toq bottle shaped basket which is used for storing dry canarium nuts basket blong fulumap drae nangae insaed we hem i sep olsem wan bigfala botel

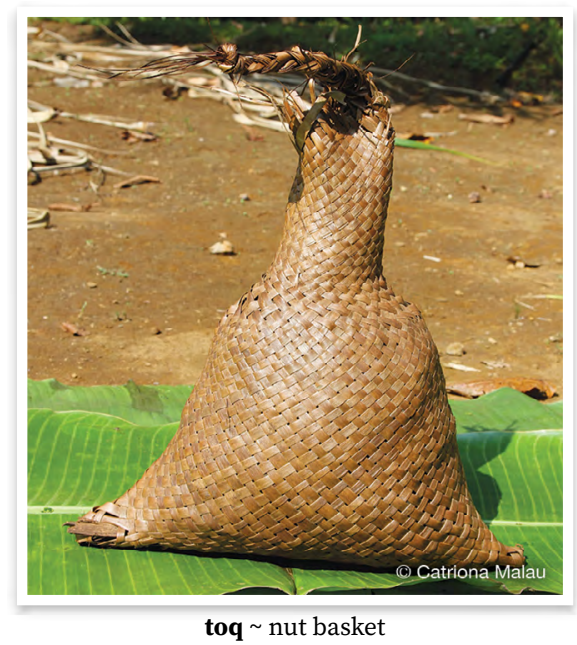

tönöam bed or sleeping mat bed o mat we yu leidaon long hem

tös strip thorny edges (of pandanus) with needle-like instrument and split into strips ready for weaving $\sim$ splitim pandanas smolsmol mo tekemaot bun o nil

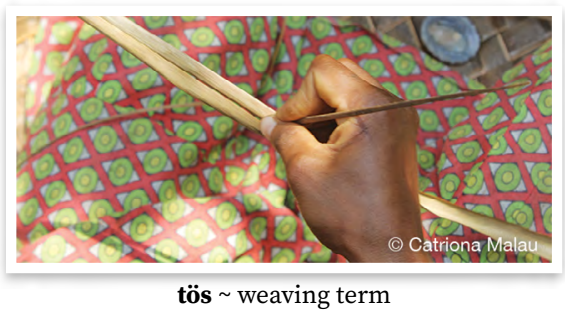

varar weaving pattern where two strips are woven under, two over; term used for weaving pandanus, coconut leaves, bamboo walling stael blong wiv we tu pis i go ova, tu pis i go anda; stael ia hem i blong pandanas, kokonas o bambu blong haos

varar tur two over, two under weaving pattern, straight up and down stael blong wiv we tu pis i go ova, tu pis i go anda, i stanap stret 
vet ${ }_{2}$ plait, weave [refers both generally to interlacing of sets of elements (as plaiting of coconut leaves, pandanus) and specifically to interlacing of a set of weft elements across fixed warp elements] wiv, wivim

vetvet weaving, in process or completed items $\sim$ wiving we i stap mekem o i mekem finis
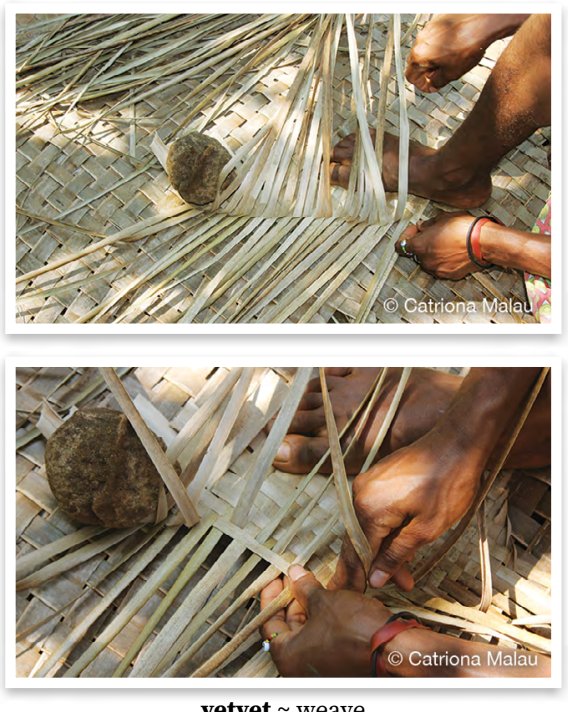

vinit plait in additional pandanus leaf strips to create second side of pandanus mat joenem lif pandanas blong statem narafala saed blong mat

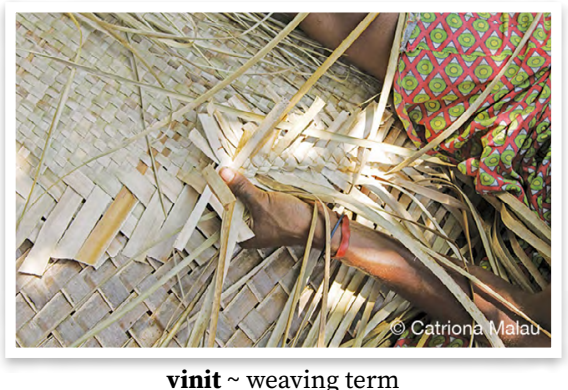

vir plait, braid, French braid, plait a cord $\sim$ tanem (hea o rop o saed blong mat)

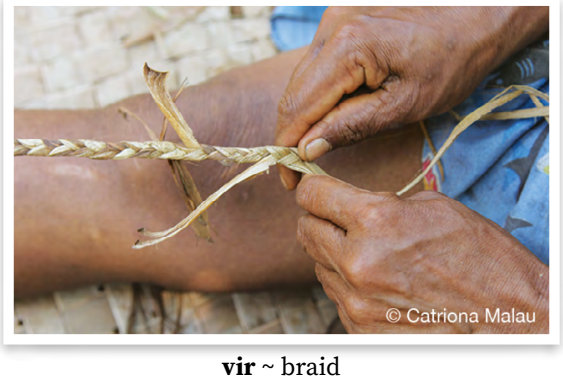

gavirvir braid, French braid rop we ol i tanem

vudege (fr.var. vudëgë) pandanus, screw pine pandanas Pandanus spp.

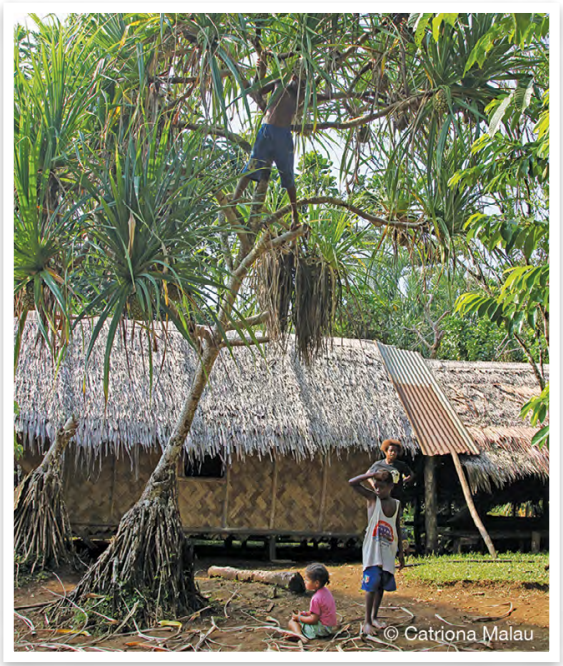

vudege $\sim$ pandanus

waga $_{1}$ basket trap for catching freshwater prawns $\sim$ basket blong kasem naora

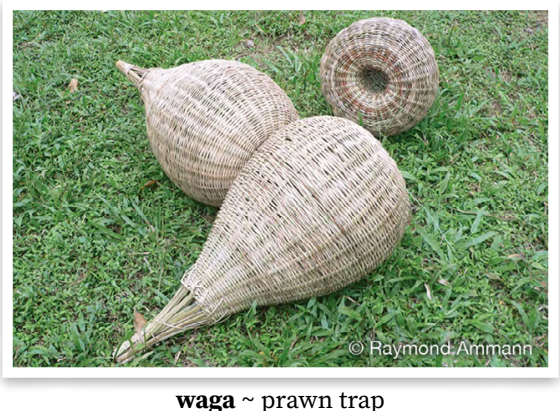

waga $\sim$ prawn trap 
wamase $\overline{\mathbf{e}}_{2}$ kind of basket, shaped like a star wan kaen basket we i gat sep olsem sta

weteñe tō basket traditionally used for keeping chicks in to feed them, which now has other uses, made from Donax canniformis and woven in lattice style $\sim$ basket blong kipim smol faol insaed blong fidim

wēgèbil ${ }_{2}$ kind of small coconut leaf basket that is used especially when reef gleaning, for putting shellfish in kaen basket blong lif kokonas we ol i yusum taem ol i go karem mit long solwota, blong fulumap ol sel insaed long hem

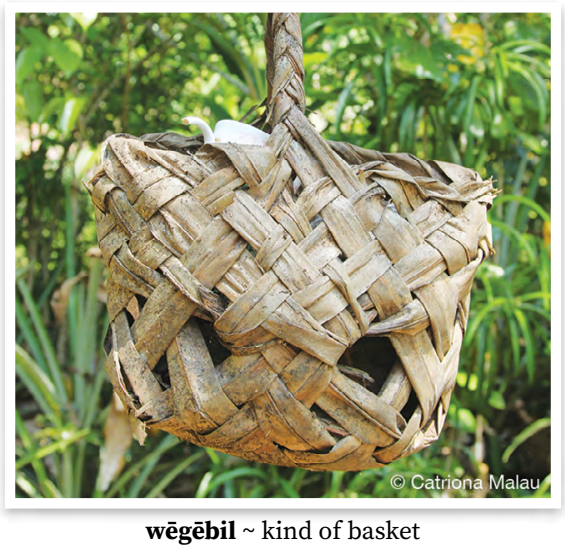

wēgèt kind of coconut leaf basket used for holding and transporting food wan kaen basket blong lif kokonas we ol i yusum blong karem kakae long garen o hangem kakae long hem

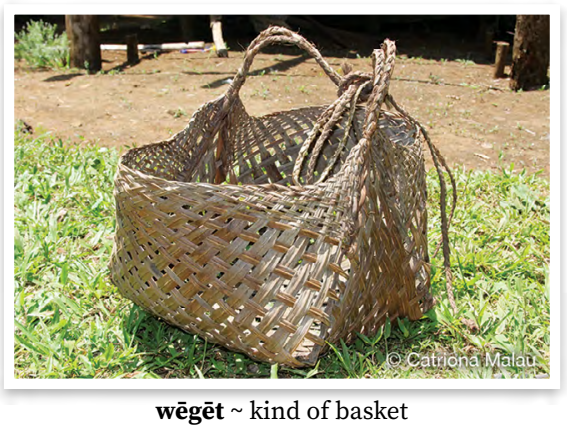

wōōr kind of woven basket that has a base and is made from rattan, woven with Freycinetia vines and lengths of Lygodium fern kaen basket we ol i wivim long rop mo fens

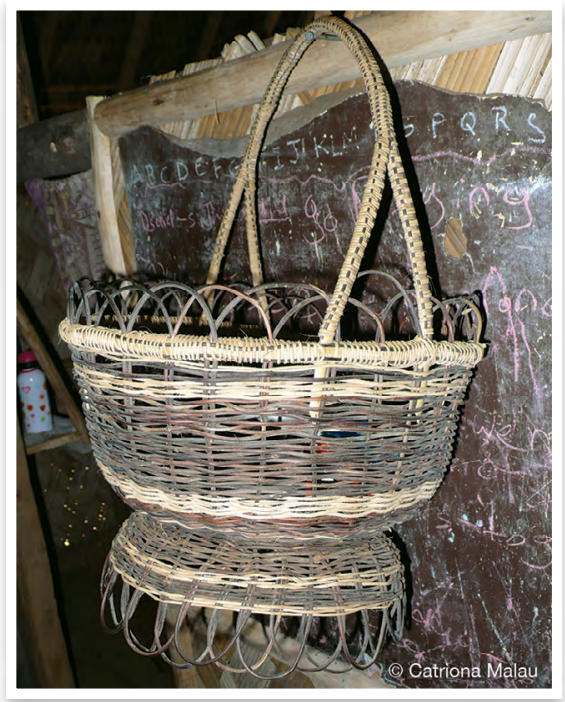

wōōr $\sim$ kind of basket

wösurtege kind of pandanus basket that has two plaited straps for carrying, used for carrying cooked food and general items wan kaen basket blong pandanas we i gat tu rop blong karem, we ol i yusum blong karem kakae mo ol smol samting

wöwöwör qēsaqēs kind of trap used close to the shore for catching small fish and particularly lobster kaen trap we yu yusum klosap long so blong kasem ol smol fis mo speseli naora

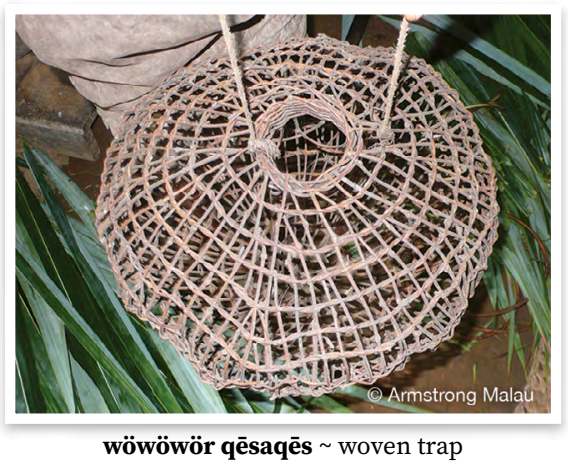


wuduw sharp needle-like piece from inside of base of sago palm rachis bun we i kamaot long insaed blong stamba blong lif natanggura

wususrō kind of basket made from coconut leaves, used for carrying food and firewood kaen basket blong lif kokonas we ol i yusum blong kakae mo faeawud

\section{M5 Nautical}

ak canoe, boat, ship kenu, sip ak geben sailing canoe $\sim$ sel kenu ak sōsō canoe kenu

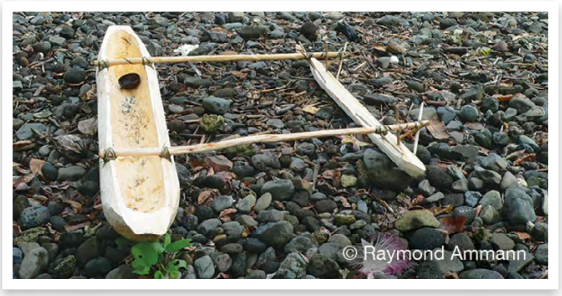

ak sōsō $\sim$ canoe

bakalav $_{1}$ ship sip

bertañtan feel body of canoe to test whether it is thick or not, to see if it needs to be hollowed out more holem bodi blong kenu blong testem se i tik o no

buswōtōt wooden stakes hammered into float to join float to cross booms of outrigger $\sim$ smol wud we i holem taet rel $\mathrm{i}$ joen long nasama blong kenu

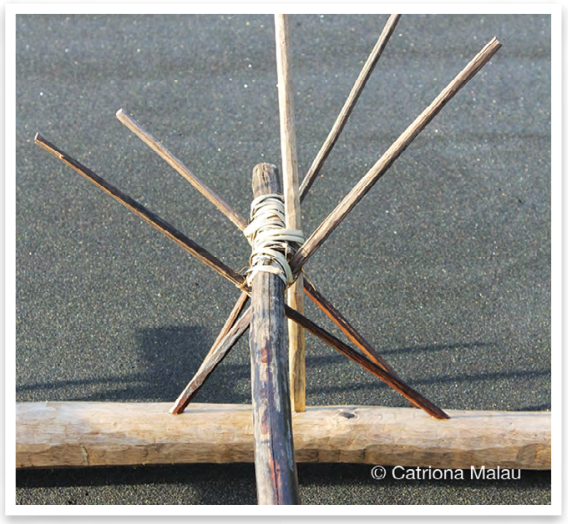

buswōtōt outrigger stakes ēwatē wooden sticks used to join crossboom to outrigger boom of canoe and hold it in place $\sim$ smol wud we i nilim wud i go long nasama blong kenu blong holem taet

geben sail sel

kewō large canoe bigfala kenu

qetegak captain, leader $\sim$ kapten, lida rës bail (water) belaot

sam outrigger float $\sim$ nasama

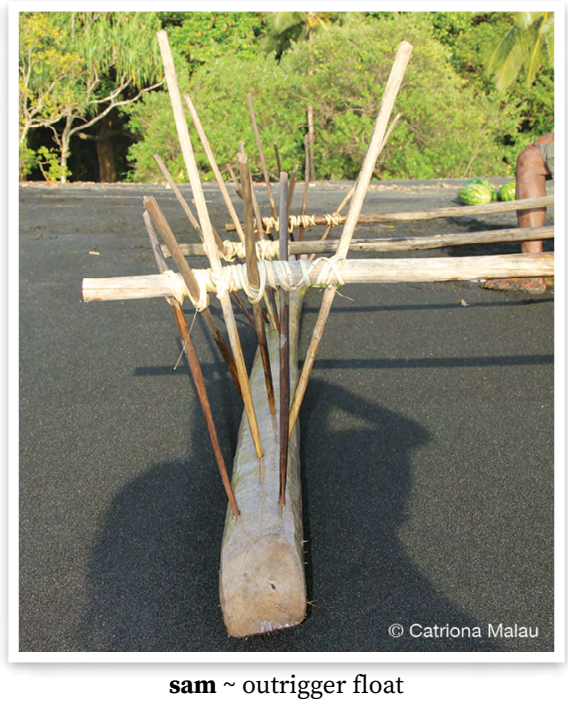

sō paddle parel, pul long kenu

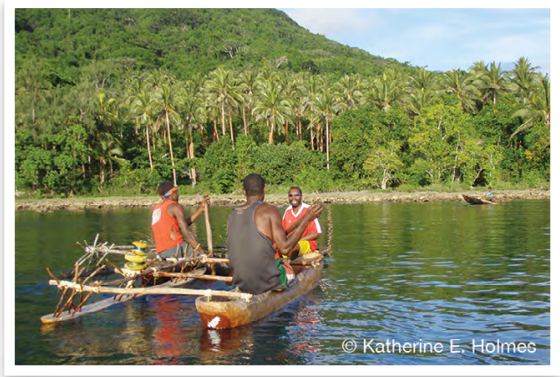

$\mathbf{s o ̄} \sim$ paddle

vasēlē launch (canoe) lonjem, putum (kenu) i go long solwota vidëm adze, tool for hollowing out canoe aj, tul blong katem kenu wēlwēl canoe kenu wërësrësak bailer, bailer for canoe samting blong belaot wota long kenu 
wōs 1 paddle (for canoe) parel (blong kenu)

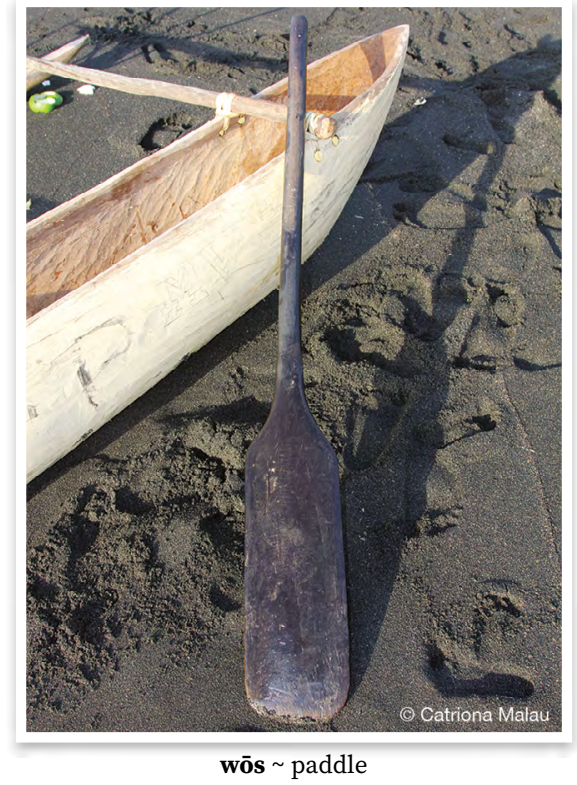

M6 Transfer of goods

baon 100 vatu, monetary unit used to refer to multiples of 100 vatu 100 vatu

bël $1 \cdot$ steal stilim

bit break off length of shell money $\sim$ karemaot pis sel mane

dalagē payment of bride wealth braed praes

girēe buy pem

girē qalēg (at marriage ceremony) give money to parents of husband, to confirm their relationship status as in-laws long taem blong mared woman i givim mane long ol papa mo mama blong boe

girē wël (at marriage ceremony) give money to sisters of husband (for woman) to confirm their relationship status as in-laws $\sim$ long taem blong mared woman wetem ol famle blong hem ol i givim smol mane long brata mo sista blong boe mil 1,000 vatu, monetary unit used to refer to multiples of 1,000 vatu 1,000 vatu

masar poor person, who has no valuables, such as pigs and other valuable goods, money and land pua man we hem i nogat ol samting olsem pig mo narafala samting we $i$ gat valiu blong hem

nereg sell salem

rēq pay fine for saying the name of your parent-in-law or child-in-law, or otherwise not showing respect to them pem smol faen sapos yu no bin respektem gud palika blong yu, olsem yu singaotem nem blong palika o yu ovarem hem

rēqrēq fine paid for saying your parent or child-in-law's name or otherwise not showing respect to them $\sim$ faen blong pem sapos yu no bin respektem gud palika blong yu, olsem yu singaotem nem blong palika o yu ovarem hem

selen 1 - 10 vatu, 10 vatu coin, monetary unit used to refer to multiples of 10 vatu, up to 100 vatu 10 vatu, koen we i gat valiu blong 10 vatu mo yu save ademap kasem long 100 vatu $\mathbf{2}$ • money, general term for money mane

ser ${ }_{3}$ pay back, return money in exchange for something given pembak

söm $\mathbf{1} \cdot$ money mane $\mathbf{2} \cdot$ shell money, traditional money, used as a value item for trading and payments in cultural exchanges sel mane we ol i bin yusum bifo blong pem ol samting long kastom

tabēva present $\sim$ presen

tiveg 1 - money, payment for something mane, amaon blong pem from wan samting 2 - pay (someone, something) pem

tun $_{2}$ buy land from another tribe pemaot graon long narafala traeb long kastom 
wadē money mane

wöl $\mathbf{1}$ • buy, purchase pem $\mathbf{2} \cdot$ pay (someone), compensate $\sim$ pem 3 . pay bride wealth for $\sim$ pem (woman) wölwöl shopping soping

\section{M7 Vehicles}

ak canoe, boat, ship kenu, sip ak gavgav plane $\sim$ plen

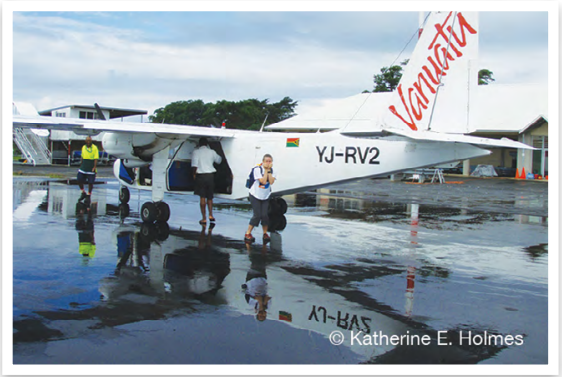

ak gavgav $\sim$ plane ak geben sailing canoe $\sim$ sel kenu

ak sōsō canoe kenu

$\mathbf{k e}_{3}$ relational classifier used to mark ownership of forms of transport, such as a canoe blong (yusum blong tokbaot ona blong kenu o trak) kan her/his/its (canoe) blong hem (kenu)

ken tëmëk belonging to my father (canoe) blong papa blong mi (kenu)

këk my (canoe) blong mi (kenu) plen plane $\sim$ plen susuki motorbike $\sim$ moto trak vehicle, car, utility vehicle $\sim$ trak wēvēvērēg vehicle, general term for car, utility vehicle, truck trak

\section{N Kinship}

\section{N1 Kin terms}

bērn̄ē partner, friend, person one associates with patna, fren

bērn̄ēn her/his/its partner patna blong hem

bērn̄ik my partner patna blong mi

Bum Grandma, Grandpa, Grandchild; reciprocal address term used by grandparent to grandchild or grandchild to grandparent $\sim \mathrm{Bubu}$

Bum tal Great-Grandma, GreatGrandpa mama o papa blong bubu

Bum vövörus Great-great-grandparent Bubu blong bubu

Die Mum Mama

Diebum Grandma Bubu woman

die 1 - mother (generic) mama 2 • call (someone) mother $\sim$ singaotem mama long

gunö spouse man blong woman; woman blong man ginon her/his spouse $\sim$ man/woman blong hem

gunök my spouse woman/man blong mi

Ika $1 \cdot$ wife, used as an address term $\sim$ waef, olsem yu singaot long hem

malaen ex-wife, ex-husband olfala woman o man blong yu blong bifo we yu nomo stap wetem hem

Mam $1 \cdot$ Dad Papa

Mambum Grandad, Grandpa Bubu man

maröu maternal uncle; brother of mother, husband of sister of father angkel

marōōn her/his uncle angkel blong hem

maröuk my uncle angkel blong mi nötu 2 child (of), offspring pikinini (blong hem) 
nōtōn her/his/its child pikinini blong hem

nötuk my child pikinini blong mi Qalēg reciprocal term used to address parents of spouse or spouse of child, father-in-law, mother-in-law, daughter-in-law, son-in-law palika qelge reciprocal term used to refer to parents of spouse or spouse of child, father-in-law, mother-inlaw, daughter-in-law, son-in-law palika, papa o mama blong man o woman blong yu, man o woman blong pikinini blong yu

qelgan her/his parent-in-law palika blong hem

qëlgëk my parent-in-law palika blong mi

rētne mother (of someone) mama (blong)

rētnan her/his/its mother mama blong hem

rētnëk my mother mama blong mi

Rëwël brother-in-law; wife's brother, sister's husband (for man), reciprocal same generation in-law address term for men $\sim$ brata blong woman blong man, man blong sista blong man

savan which relative of him/her, or which part of his/her body? wanem famle blong hem, o wanem pat blong bodi blong hem?

sian gōgōn name that one uses to refer to or address someone who has the same name as an in-law whose name you are unable to say, due to cultural restrictions of the relationship nem we yu yusum blong talemaot wan man o woman we hem i gat semak nem olsem wan tawian o palika blong yu, from we nem i semak, i tabu blong singaotem nem

sögö moiety, descent group laen sogon her/his descent group laen blong hem sögök my descent group laen blong mi

Tatat (abbrev. Tat) Uncle, address term used towards uncle Angkel, nem we yu singaot long angkel

tawian brother-in-law, sister-in-law tawi, tawian

teme father, father's brother papa blong hem

taman her/his father papa blong hem

tëmëk my father papa blong mi

tèsi younger same sex sibling smol brata blong wan boe o smol sista blong wan gel

tasēn her/his/its younger sibling smol brata o sista blong hem

tēsi rësrës youngest sibling lasbon brata, sista

tēsik my younger sibling smol brata o sista blong mi

tētē $\mathbf{1} \cdot$ baby bebe, smol pikinini 2 - My child, term of endearment used by parent to child $\sim$ Bebe, nem we mama o papa i yusum blong singaotem smol pikinini blong hem

tōgan her/his/its older same sex sibling big brata blong wan boe o big sista blong wan gel

tubu 1 - grandparent, grandchild, reciprocal term used between grandparent and grandchild $\sim$ bubu, smol bubu $\mathbf{2} \cdot$ ancestor $\sim$ bubu blong bifo

tōbōn her/his grandparent/ grandchild bubu blong hem tubuk my grandparent/grandchild bubu blong mi

Tutu address term used towards opposite sex sibling, brother (of a girl), sister (of a boy) brata blong wan gel o sista blong wan boe

tutue opposite sex sibling, brother (of a girl), sister (of a boy) brata blong wan gel o sista blong wan boe 
tōtōan her/his/its opposite sex sibling brata blong hem (blong gel) o sista blong hem (blong man)

tutuen Hilton Hilton's sister sista blong Hilton

tutuëk my brother (for girl), my sister (for boy) brata blong mi (gel i toktok) o sista blong mi (boe i toktok) vann̄on niece, nephew, child of sister (of a man) smol angkel, pikinini blong sista blong wan man

Vann̄ö niece, nephew, child of sister (of a man) smol angkel, pikinini blong sista (blong wan man)

Wimiar term of endearment used by parent-in-law to child-in-law nem we wan $i$ yusum blong singaotem palika blong hem, we hem i woman blong boe blong hem o man blong gel blong hem

wotwot kin relationship, one's father's mother's brother's children, reciprocally the same as one's father's sister's son's children, relationship between child of uncle and child of nephew (following male line only) $\sim$ famle memba we hem i pikinini blong angkel blong papa blong yu

Wölus sister-in-law; husband's sister, brother's wife (for woman), reciprocal same generation in-law term for women sista blong man blong woman, woman blong brata blong woman

\section{O Marriage, sex, nurturing}

\section{O1 Marriage and sexual relations}

bat go secretly in search of sexual partner krip from fren

bēl have sexual intercourse fak

bōbōn kiss kis, kisim

dalagē payment of bride wealth braed praes

irir reject spouse because you believe that they have been unfaithful (man o woman) i no wantem stap gud wetem woman o man blong hem from hem $i$ ting se hem $i$ gat narafala fren

lagē marriage mared

lagē gē tisē bad marriage, in that it is not correct according to the rules of tradition dictating whom one should marry mared we i no stret long kastom, olsem we wan i mared wetem famle

lagē gōwē good marriage, in that it is correct according to the rules of tradition dictating whom one should marry mared we i folem stret rod blong kastom leg marry (in church), be married mared (long jos)

legleg marriage ceremony (in church) mared (long jos)

leg verser a son marries daughter of an uncle and daughter marries son of the same uncle, so daughter is exchanged for son and there is no need to pay a bride price $\sim$ pikinini gel blong angkel i maredem boe blong nara angkel, mo narafala boe i maredem gel blong sem angkel bakegen, mekem se ol i eksjenisim pikinini mo i no nid blong pem woman, jas mekem kakae

legser man who promises to marry but then changes his mind $\sim$ no save mared gud

mökölage food and presents for the house given with bride price $\sim$ ol samting we ol i givim wetem braed praes olsem kakae mo ol samting blong haos 


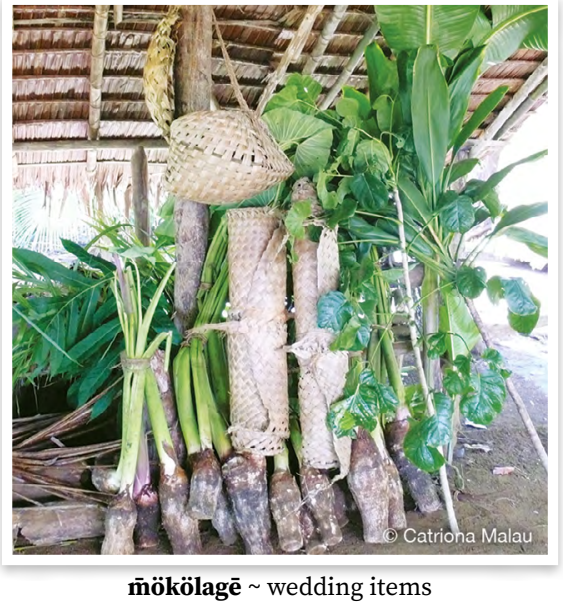

qalēg gōr make an agreement between the parents that a particular girl or woman will be married to a man blokem, putum tabu long wan gel mo givim mane se bae tufala i mared sersöm return some of bride price if a couple divorce, the woman's family must return money to the man's family who paid the bride price givim bak mane sapos mared i finis mo man mo woman i divos, famle blong woman i mas mekem long famle blong man we ol i bin pem woman ia

\section{O2 Pregnancy and childbirth}

ben rōrō placenta samting we $\mathrm{i}$ kamaot long mama, taem bebe i bon, we i bin fidim pikinini

be tētē $1 \cdot$ placenta samting we $i$ kamaot long mama, taem bebe i bon, we i bin fidim pikinini 2 uterus basket blong pikinini

dēm childless woman, woman who has never given birth woman we $\mathrm{i}$ neva karem pikinini

gösuv miar bathe baby in a traditional ceremony that occurs 5-10 days after the baby is born, the aunts of the child bathe the child and the father pays the aunts for this contribution $\sim$ faef o ten dei afta fes pikinini i bon, ol sista blong papa ol i kam wasem pikinini mo flasem hem mo afta papa i mas pem olgeta miarmōvōl stillbirth pikinini we hem i ded taem hem i bon

miarōbōl new mother, mother who has recently given birth mama blong smol bebe

tel (abbrev. of telsiër) be pregnant, become pregnant $\sim$ gat bel

vawot give birth bonem

völöt traditional practice in which when the first child of a couple is born, the family of the mother come and try to throw stones at the father of the child and his family taem fes bon pikinini i bon, famle blong mama ol i traem stonem papa blong pikinini wetem ol famle

vösus give birth, lay egg bonem, karem pikinini, putum eg

wot 1 - born, be born bon

wotoqtoq $_{1} \mathbf{1} \cdot$ be pregnant $\sim$ gat bel $\mathbf{2} \bullet$ pregnant woman $\sim$ woman we $i$ gat bel

wōl $1_{1} \mathbf{3} \cdot$ month of pregnancy $\sim$ manis (blong woman we i gat bel) wōlan her Xth month (of pregnancy) $\sim$ manis blong hem

wulëk my Xth month (of pregnancy)

$\sim$ manis blong mi

\section{O3 Rearing babies and children}

datek $1 \cdot$ look after $\sim$ lukaotem

ēlgōr $1 \bullet$ look after, care for $\sim$ lukaotem 2 - watch out, be careful lukaot gōlōw orphan, child whose parents have died $\sim$ pikinini we $i$ nogat mama mo papa

$\overline{\mathbf{n}} \mathbf{a} \overline{\mathbf{n}}$ feed baby by chewing their food first to make it smooth, then feeding them fidim bebe, olsem yu juim kakae blong hem fastaem, afta fidim long hem taem i sofsosf

sus $_{4} \mathbf{2} \cdot$ suckle, suck on $\sim$ titi, titi long 


\section{P Speaking, communicaton and sounds}

\section{P1 Speaking}

aw shout, cry out $\sim$ singaot

baēr ask (someone) for something, ask (something) from someone askem wan samting long wan man

eln̄e $1 \cdot$ her/his/its voice $\sim$ voes blong hem 2 - words, opinion presented, what someone has said toktok folem tingting blong wan man

elñan her/his/its voice voes blong hem

elnëk my voice voes blong mi

elneul explanation $\sim$ toktok we i mekem i klia

eln̄evu traditional story kastom storian

gadödö qaqaq original, old-fashioned way of speaking olfala fasin blong toktok

gagneg tell, say to $\sim$ talem

gial $1 \cdot$ lie, tell untruth $\sim$ giaman $2 \cdot$ lie, tell untruth to $\sim$ giaman long gilgial lie giaman

kaka tell story, narrate $\sim$ stori

kakaka 1 - chat, speak storian, toktok $\mathbf{2} \cdot$ talk about, tell story about $\sim$ storian (long) $\mathbf{3} \bullet$ story, tale, speech $\sim$ storian

mamar ask of, plead, implore askem bigwan

òl 1 1 call, call out to $\sim$ singaotem ōlōl shout, call out $\sim$ singaot

qaq $\mathbf{1} \cdot$ say $\sim$ talem se $\mathbf{2} \cdot$ speak, talk $\sim$ toktok $3 \cdot$ language of a place lanwis

qaq ta minēn language of Vurës lanwis blong Vurës

qaq tala lam Bislama Bislama

sal 1 - message $\sim$ mesej $\mathbf{2} \cdot$ send message $\sim$ sendem mesej

sen lie, tell untruth giaman

suware invite $\sim$ invaetem tatar $1 \cdot$ pray $\sim$ prea $2 \cdot$ prayer $\sim$ prea

tono $\mathbf{1}$ - learn lanem $\mathbf{2}$ - teach, instruct $\sim$ lanem, tijim

vatvat 1 - promise, arrange, make agreement $\sim$ promes 2 - promise, agreement, arrangement $\sim$ promes

vetsur tell $\sim$ talem

vël ${ }_{2}$ answer, reply to ansarem, riplae long

vëlvël answer ansa

vörus ask (someone); ask (about) askem (man)

vörus völu question kwestin

P2 Interjections, imperatives, function words

\section{P2.1 Functional affixes and particles}

$\mathbf{a}_{2}$ nonsingular default aspect marker $\sim \mathrm{i}$

$\mathbf{a} \overline{\mathbf{e}}_{2}$ anaphoric marker, referring to previously mentioned place or topic, there, about (it) long hem, long ples ia (wan samting o ples we i bin tokbaot finis)

do- leaf of; derivational prefix which occurs both on nouns referring to plants, to specify a leaf of that plant, and also with a limited use to produce nouns referring to useful products made with leaves, such as a fan (dérivriv) or broom (döwurwur) lif blong (wan samting), wan maka we i talemaot se lif blong wan tri o plant o wan samting we i mekem long lif, olsem fan (dêrivriv) o brum (döwurwur) (Allomorphs: da-, de-, dē-, dë-, dō-, dö-)

go ${ }_{2}$ imperfective aspect marker $\sim$ maka we $\mathrm{i}$ go wetem veb we i talemaot se aksen i tek ples long fiuja o i refe 
long wan situesen we i stap olsem nomo (Allomorphs: ga, ge, gē, gë, gi, gō, gö)

goto negative particle, not $\sim$ no (Allomorphs: gata, gete, gētē, gëtë, giti, gōtō, götö)

$\mathbf{i}_{3}$ second person singular default aspect marker wod we i folem nēk 'yu', bifo long veb long sentens

kara just, of an event that has happened very recently or will happen very shortly after an upcoming event $\sim$ jas

$\mathbf{k e}_{2}$ emphatic particle $\sim$ we, tumas

kere dehortative marker; don't no; wod we i go bifo long veb long sentens, taem yu talemaot long wan man se bae i no mekem wan samting mas must $~$ mas

mawi dehortative; don't no; yusum blong talemaot se no mekem wan samting

mēsi emphatic marker, premodifying nouns, indicating that the subject has the characteristic indicated to a large degree $\sim$ tumas

$\min _{2}$ (abbrev. mi) high probability modality bae

mito future negative proclitic $\sim$ bae $i$ no; wod we i go bifo long veb we i talemaot se aksen bae i no tekples (Allomorphs: mita, mite, mitē, mitë, miti, mitō, mitö)

mo perfect aspect marker bin (Allomorphs: ma, me, mē, më, mi, mō, mö)

mönö (fr.var. mēnē) a bit smol

na first person singular default aspect marker mi (Allomorphs: nana)

ni (fr.var. nini) third person singular nonspecific aspect marker $\sim \mathrm{i}$

nitog prohibitive mood particle, dehortative marker; don't no; wod we $\mathrm{i}$ go bifo long veb long sentens, taem yu talemaot long wan man se bae i no mekem wan samting ren emphatic marker; right, absolutely, totally, definitely tumas, tru, stret; i makem strong toktok we yu talemaot

ri second person non-singular imperative marker yusum taem yu talem wan grup blong mekem wan samting

tabaē (abbrev. taē) of it, for it, anaphoric form blong hem, toktok we $\mathrm{i}$ gobak long narafala toktok we yu talem finis

ten $_{1}$ yet $\sim$ yet

ten $_{2}$ connative particle, try $\sim$ traem

terè (fr.var. tere) plural marker, occuring before common nouns ol, olgeta

ti ${ }_{1}$ eventive aspect marker

to ${ }_{2}$ progressive aspect marker, indicates an event that is in progress stap, go go, yusum taem yu tokbaot wan aksen we i stap gohed

towo immediate progressive aspect marker, marks an event currently in progress $\sim$ go go, yusum blong tokbaot wan samting we i stap hapen nao ia nomo (Allomorphs: tawa, tewe, tēwē, tëwë, tōwō, töwö)

va- causative prefix $\sim$ maka we $\mathrm{i}$ go long fored blong veb we i minim se mekem

$\operatorname{van}_{1} \mathbf{1} \cdot$ go (unmarked for direction) go $\mathbf{2}$ - on and on, postverbal particle indicating continuous aspect, continuation of an activity $~$ go go

ver collective subject, preverbal marker that indicates either that the subjects are performing the action of the verb collectively or that the action is reciprocal tugeta

vēti (fr.var. viti) still yet

vitia already $\sim$ finis

wo ${ }_{3}$ intensifying particle; absolutely, totally tumas, we

wo- noun categorisation prefix, used with separate functions: before male names; in plant names, in 
reference to the fruit or the tree; and in the formation of new nouns maka we i go long fran blong nem blong wan man, long nem blong tri o frut blong tri mo long wan niufala wod we yu krietem (Allomorphs: wa-, we-, wē-, wë-, wō-, wö-)

wun $_{2}$ high probability modality bae

\section{P2.2 Interjections}

aaōō expression of surprise, wow, gee $\sim$ awo

ake ow! expression used to indicate that you are hurt awo! toktok we i talemaot se yu kasem kil

ale $\overline{\mathbf{e}}_{2}$ ok, alright ale

aqa expression of surprise, disbelief, horror; gee, gosh awo, toktok blong singaot blong talem se yu sapraes long wan samting

auw gee! awo!

awi argh! awe!

awo $1 \cdot$ general interjection, used to express positive emotion, wow! woah! awo! 2 •general interjection, used to express negative emotion, hey! ow! awo!

dom great, word used by and with deaf people, from Bislama 'top' top, long lanwis blong ol def we i kamaot long 'top'

e'e yes $\sim$ yes

ei hey ei

i ow! awo!

ii hey! ei!

io yes $\sim$ yes

$\mathbf{k e}_{1}$ yes $\sim$ yes

kērēt ${ }_{1}$ yeah right! Interjection used teasingly to indicate that you know that what you or another interlocutor said is an exaggeration or inaccurate ei no!

kēti (fr.var. kiti) $\mathbf{1} \cdot$ wait, hold on $\sim$ wet, wet smol $\mathbf{2} \cdot$ short time $\sim$ sot taem

madagese (abbrev. medese) what's happening? what's going on? olsem wanem? mamarseg (abbrev. marseg) 1 - pity, feel sorry (for) $\sim$ sori (long) $\mathbf{2}$ • unfortunate, pitiful $\sim$ sori $\mathbf{3} \cdot$ sorry $\sim$ sori

odian̄ (abbrev. dian̄) 1 • no nogat 2 - negative nonverbal predicate, be not $\sim$ nogat

oo oh oo

$\overline{\mathbf{o}} \overline{\mathbf{o}} \mathbf{\text { no}}$; interjection used as negative response to a question, or to indicate a negative attitude to a proposition nogat

qaēl hey! awo!

qate $_{3}$ peekaboo! Interjection used in playing game of surprise with small children

timiak avē $1 \cdot$ how olsem wanem $2 \cdot$ what's going on? olsem wanem? varian thanks $\sim$ tangkyu

varwöt serves you right $\sim \mathrm{i}$ gud ia (talemaot long wan man taem wan nogud samting i hapen long hem be yu ting se i stret from wanem we hem i bin mekem)

wèsi dunno, an interjection indicating that the speaker does not know what is being enquired about $\sim$ mi no save

\section{P2.3 Conjunctions}

$\mathbf{a l e}_{1}$ so, then, conjunction used to link coordinate clauses $\sim$ ale

ba but be

dēmē plus, used to conjoin numerals, thousands and hundreds and tens and units wod we $\mathrm{i}$ joenem tugeta tu pat blong hae namba, olsem tu handred mo twenti

$\mathbf{e}_{1}$ then, so; conjunction used to join coordinate clauses $~$ ale

in̄ko then $\sim$ nao

qēt ${ }_{2}$ then, clause initial consecutive marker finis, afta

$\mathbf{s i}_{2}$ or $\sim 0$

söwlē (abbrev. söw) then, so ale

wo and; conjunction that can join phrases and clauses $\sim$ mo 


\section{P2.4 Subordinators}

a⿳亠̄̄o den before, introduces temporal adverbial clause $\sim$ bifo long

den $_{2}$ lest, in case, introduces adversative clause $\sim$ nogud

la masawre when, introduces temporal adverbial clause $\sim$ taem

$\mathbf{s i}_{1}$ if, introduces conditional clause sapos

so relativiser, that $\sim$ ia

sur $_{2}$ because, introduces adverbial clause of reason $\sim$ from

tabo in order to, so that, introduces adverbial purposive clause $\sim$ blong

talo ${ }_{2}$ in order to, so that, introduces adverbial purposive clause $\sim$ blong

timiak $_{2}$ (abbrev. tiak) $\mathbf{1} \cdot$ like, introduces adverbial clause of manner $\sim$ olsem $\mathbf{2} \cdot$ like olsem

vita $(\text { abbrev. ta })_{1}$ that, complementiser introducing complement clause $\sim$ se

wōl ${ }_{3}$ because, introduces clause of reason $\sim$ from

\section{P2.5 Articles}

$\mathbf{a}_{1}$ locative, to, at, marking absolute location nouns long, maka we i go wetem ol nem blong ples

$\mathbf{i}_{2}$ personal article denoting nouns in personal noun class maka we $\mathrm{i}$ go wetem naon we i talemaot nem blong man o famle

ira plural personal article ol; wod we $i$ go bifo long naon long sentens

lo ${ }_{2}$ locative preposition, to, at, on, marking common nouns $\sim$ long

na the, a, article denoting noun that is in possessive construction $\sim$ wan, maka blong naon

o the, a, article denoting noun in class of common nouns wan, maka blong ol naon

ra plural marker denoting personal nouns ol, maka we i go wetem nem blong man we i soemaot se i gat plante man long grup

\section{P2.6 Discourse markers}

$\mathbf{k} \overline{\mathbf{e}}_{2}$ emphatic particle $\sim$ tumas

mina discourse particle $\sim$ we

timiak $_{2}$ (abbrev. tiak) $\mathbf{1} \cdot$ like, introduces adverbial clause of manner $\sim$ olsem

$\mathbf{2} \cdot$ like olsem

wana discourse particle $\sim$ we

wōl discourse particle used when asking a question, indicating that you recognise that you are seeking information that you should already know, but want to be reminded wod we yu ademap long wan kwestin, blong soemaot se yu sud save ansa blong kwestin, be yu askem bakegen

P3 Negations, assertions, possibility

odian̄ (abbrev. dian̄) 1 • no nogat 2 - negative nonverbal predicate, be not nogat

$\overline{\mathbf{o}} \overline{\mathbf{o}} \mathbf{\overline { n }}$ no; interjection used as negative response to a question, or to indicate a negative attitude to a proposition nogat

rivteg 1 - near, close by klosap $\mathbf{2} \cdot$ near to, close to $\sim$ klosap long $\mathbf{3} \bullet$ nearly, soon klosap

wēsi dunno, an interjection indicating that the speaker does not know what is being enquired about $\sim$ mi no save wun probably, I think ating

wun $_{2}$ high probability modality $\sim$ bae

P4 Non-verbal communication

bōk book buk

kilmat blink eyes to make a sign to a woman that you want her $\sim$ mekem ae long woman blong talemaot long hem se yu wantem hem

n̄ēnēs $1 \cdot$ smile, showing your teeth smael, soem tut blong yu $\mathbf{2} \cdot$ show teeth soemaot tut

qet möwu nod head, show agreement $\sim$ sakem hed se i oraet 
$\mathbf{r e ̄ v}$, write raetem

vasōgō read ridim

vikas communicate in sign language

toktok wetem han

P5 Sounds

aw shout, cry out singaot

dēn beat, tap lightly, bang, beating just to make a noise, rather than to make music or signal to people kilim (bambu, wud) blong mekem noes nomo qōrōtōt 1 - make a lot of noise, be noisy $\sim$ mekem tumas noes $\mathbf{2} \cdot$ loud sounds, too much noise $\sim$ tumas noes

rarö noise noes

tarōñ be quiet, be calm, be peaceful stap kwaet

tōr $\mathbf{r}_{1}$ bark singaot (dog)

töqöl boom, bang, make a loud booming noise mekem bigfala noes olsem saon blong tanda

\section{Q Social interaction, relationships and behaviour}

\section{QO General}

metwu personality, behaviour, manner, habit fasin

metwōn her/his/its personality fasin blong hem

metwuk my personality fasin blong mi

qetgengen feast, planned celebratory feast lafet, wan kakae we yumi ekspektem blong holem, plan blong mekem kakae

sal $_{1} \mathbf{1} \cdot$ message $\sim$ mesej $\mathbf{2} \cdot$ send message $\sim$ sendem mesej

tōrtōr 1 - preach, give important speech, as in at wedding givim hevi toktok $\mathbf{2} \cdot$ speech $\sim$ strong toktok

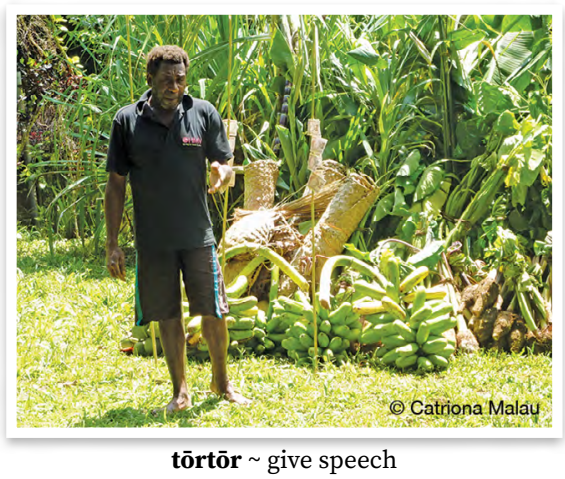

Q1 Fighting

dar $\mathbf{1} \cdot$ blood $\sim$ blad $\mathbf{2} \cdot$ bleed $\sim($ blad i) ron daran her/his/its blood blad blong hem

dere qō pig's blood blad blong pig dërëk my blood blad blong mi

lā̄ bash, hit, beat strongly kilim strong

mëmës hurt, be sore, ache soa

rēs 1 - fight, wrestle, struggle $\sim$ faet

2 - struggle, fight an urge $\sim$ traem blong winim

tavalalē enemy badfren, enemi

tēq, shoot (with gun) sutum (wetem masket)

tēqatēq $1 \cdot$ hunt with bow and arrow in the bush (birds and flying fox) hant wetem bonaro long bus (blong sutum pijin o flaengfokis) $\mathbf{2}$ • gun masket

timiatēq gun $\sim$ masket

töwös hit kilim

tut ${ }_{1}$ punch $\sim$ faetem

vagal war, large scale fighting wo, bigfala faet 
vēn shoot (with bow and arrow or spear) sutum (wetem bonaro o spia)

vēnvēn go shooting go blong sut

wos $_{1} \mathbf{1}$ - bash, slap, pound; hammer, bash (nuts) to crack open $\sim$ hamarem $\mathbf{2}$ - build bildim

\section{Q2 Verbal quarrelling}

dörug trick, play a trick on, deceive trikim, giaman long

gial $1 \cdot$ lie, tell untruth $\sim$ giaman $2 \cdot$ lie, tell untruth to $\sim$ giaman long $3 \cdot$ trick, pretend to $\sim$ giaman long gilgial lie $\sim$ giaman

kes argue, row with $\sim$ rao

liseg disobey, argue with disobei, no folem tingting blong

vergöl argue, row rao

verliseg argue $\sim$ rao

Q3 Making peace, maintaining social harmony

birin̄ help givhan long, halpem

bulsal $1 \cdot$ friend, mate, can be sexual partner or platonic friend $\sim$ fren $2 \cdot$ friend, used as an address term fren $3 \cdot$ be friends, become friends $\sim$ fren $\mathbf{4}$ - become sexual partners fren (olsem gelfren mo boefren)

döm mav 1 • respect $\sim$ ting hevi, respek

$2 \cdot$ respect $\sim$ respek

qet möwu nod head, show agreement $\sim$ sakem hed se i oraet

tamat peace $\sim$ pis

Q4 Games, entertainment, laughter, joking

bēm naw surf, go surfing karem wef long solwota

bor laugh laf

borbor laughter $\sim$ laf

borseg laugh at laf long

kikbol play soccer plei futbol

or play plei oror 1 - play pleplei $2 \cdot$ game $\sim$ pleplei

vërlē (fr.var. verlē) compete, race kompit, resis

\section{Q5 Being affected}

birin̄ help givhan long, halpem

datek $\mathbf{1}$ •look after lukaotem $\mathbf{2}$ • have knowledge of, possess and use a certain skill or knowledge $\sim$ gat save long wan samting, holem wan save blong yusum

ēlgōr 1 • look after, care for lukaotem 2 - watch out, be careful lukaot

gasēg share out, separate, divide seraotem

mamarseg (abbrev. marseg) 1 - pity, feel sorry (for) sori (long) $\mathbf{2}$ • unfortunate, pitiful $\sim$ sori $3 \cdot$ sorry $\sim$ sori

ōligi invite invaetem

Q6 Permission and prohibition

baēr ask (someone) for something, ask (something) from someone askem wan samting long wan man

$\mathbf{l} \overline{\mathbf{e}}_{2}$ law loa

liseg disobey, argue with disobei, no folem tingting blong

qil order, tell someone that they must do something talem long wan man se i mas mekem wan samting

tiargōr prevent, stop someone from doing something stopem

Q7 Community

bultiwial unity yuniti

sal 1 - message $\sim$ mesej $2 \cdot$ send message $\sim$ sendem mesej

vug ${ }_{2}$ have meeting $\sim$ miting vugvug meeting miting 


\section{$R$ Religion, ceremony, law and the supernatural}

\section{RO General}

dun $1 \cdot$ true, be true, real $\sim \operatorname{tru} 2 \cdot$ sure, tell truth $\sim$ sua, tru $\mathbf{3} \cdot$ truth $\sim$ tru

löglē religion, church $\sim$ jos, skul

nōmtuv $\mathbf{1} \cdot$ believe biliv $2 \cdot$ belief bilif

tatar $\mathbf{1} \cdot$ pray $\sim$ prea $2 \cdot$ prayer $\sim$ prea

R1 Law, sacred places, legends

bōgō man who has not learned the traditional ways in the men's house to gain rank man we hem i no bin go long nakamal blong lanem stret rod blong kastom

Dōl name of mythical creation figure, enemy of the main creation figure Qet nem blong man long kastom storian

$\mathbf{e v}_{1} \mathbf{1} \cdot$ fire $\sim$ faea $\mathbf{2} \cdot$ hell $\sim$ hel

gōrōn̄ holy, sacred, spiritually meaningful tabu

kētkēt 1 • in air, up high above ground $\sim$ antap, long ea, i no long graon $\mathbf{2}$ - heaven $\sim$ heven

$\mathbf{l} \overline{\mathbf{e}}_{2}$ law $~ 1$ oa

maranag chief $\sim$ jif

meter woman of high rank who has studied to learn the true traditional way of life woman we hem i bin go long nakamal blong lanem stret rod blong kastom

miar veser call someone father when they also call you father (of man) singaotem papa long man we hem i singaotem papa long yu

qeren̄ ōlōl cave, hole in reef or pool that has special significance, where people go to pray, give money and ask the spirits for something that you want, or to appease and ask forgiveness from the spirits if you have done something wrong against them tabu hol long kef, long rif o long wota we ol man ol i go long hem blong prea long ol speret from wan samting o talem sori from wan samting we yu bin mekem

sar ${ }_{1}$ ceremonial ground $\sim$ nasara

seretimiat area associated with the spirits of the dead, on the top of a hill nasara blong ol devel. Hem $i$ wan ples antap long hil, wan ples we ol spirit ol i go stap long hem

serevugvug meeting place for spirits of the dead, underneath a banyan tree ples we ol devel ol i mit long hem. Hem i wan ples andanit long wan nambangga we i klin we i klin sölö put taboo, place marker to indicate restriction, usually a cycad leaf is placed to indicate, for example, that people should not fish or should not take the fruit from a tree putum namele lif, olsem tabu blong soemaot se ol man ol i no sud mekem wan samting, olsem tekem kakae long wan wud

sösölö a cycad leaf placed to indicate a restriction on use of plant or area lif namele we yu putum blong makem tabu

tövusm̄ēl chief who has gained rank in traditional way by kiling pig stret kastom jif we hem i bin kilim pig

wèrēsōr place where the spirits of the dead go to rest when they are tired, a place on a point or hill wan ples we ol devel ol i go spel long hem, sapos ol i wokabaot go go ol i wantem spel. Hem i wan ples long wan poen o hil 
wöwut man of high rank who has studied the traditional rules and way of life in the men's house $\sim$ man we hem $\mathrm{i}$ bin go long nakamal blong lanem stret rod blong kastom

\section{$R 2$ Ceremony and rituals}

\section{R2.1 Ceremonial objects}

ganar rope made from cottonwood which one hangs around the neck as a sign that one has given up a particular type of food out of respect when a close relative has died $\sim$ rop we man i hangem long nek blong hem blong soemaot se hem i givap wan kaen kakae taem mama o papa blong hem i ded

gon ganar tie a rope around ones neck as a symbol to indicate that you are mourning the death of ones father or mother, the rope also indicates that one is giving up a particular food for the mourning period taem we man i fasem rop long nek blong hem blong soemaot se hem i givap wan kaen kakae taem mama o papa blong hem i ded

möökölagē food and presents for the house given with bride price $\sim \mathrm{ol}$ samting we ol i givim wetem braed praes olsem kakae mo ol samting blong haos

timiatwos traditional wooden upright drum tamtam

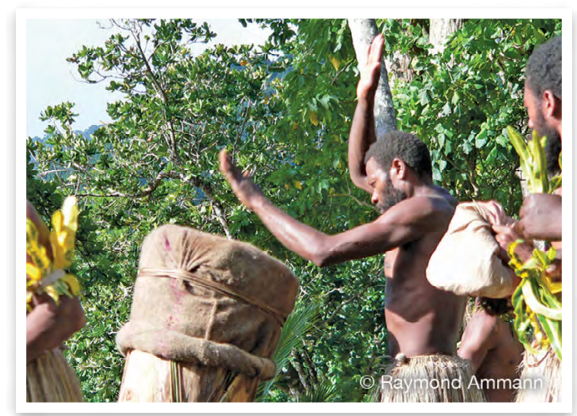

timiatwos $\sim$ wooden drum tōw ${ }_{4}$ (fr.var. tōw mölum) triton trumpet, conch shell pupu Charonia tritonis

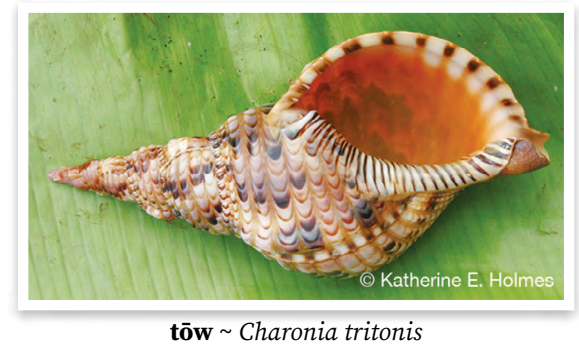

wōkōr slitgong that is made from bamboo or hollowed out wood tamtam we ol i mekem long bambu o wud

\section{R2.2 Ceremonies and rituals}

da lagē pay bride price pem woman genqinimate last feast that is made to celebrate someone's death, 1,000 days after the death $\sim$ kakae las dei long wan taosen dei blong ded man. Hem i kakae blong finisim tingbaot man we i ded mo blong stat blong fogetem. Hem i no dei blong sore, i dei blong glad. Hem i talem se i naf blong tingbaot, nao ol i save fogetem gëvgëv perform ceremony when someone has killed another person, to pay the person's family for their life $\sim$ pem laef blong wan man we yu bin kilim

gon wōmōotō make an agreement that two small children will marry when they come of age. The boy's family gives money and coconuts to the girl's father. Two germinating coconuts are tied together and must be planted together as a symbol of the bond mekem agrimen se bae tufala pikinini i mared taem tufala pikinini i smol nomo. Famle blong boe ol i fasem tu kokonas tugeta mo givim long papa blong gel wetem mane. Ol i mas planem tu kokonas 
tugeta mo hem i wan saen se bae tufala i mared. Hem i wan strong agrimen we bae ol i no save brekem gōrga cut the mourning rope from the neck of a man who has fasted during period of mourning for father or mother. The ceremony is performed in the morning of the 999th day after person has died. The same family who tied the rope around the person's neck must cut the rope, then they give the person the food that he has not eaten during the mourning period, and he must pay them. seremoni we ol i mekem long eli moning long naen handred naenti naen dei blong wan ded man. $\mathrm{Ol}$ man we ol i bin hangem rop long man we hem i fas long kakae, ol i kam katem rop. Afta ol i givim kakae long man we hem i no kakae kakae ia mo man ia i mas pem hem.

gösuv miar bathe baby in a traditional ceremony that occurs 5-10 days after the baby is born, the aunts of the child bathe the child and the father pays the aunts for this contribution $\sim$ faef o ten dei afta fes pikinini i bon, ol sista blong papa ol i kam wasem pikinini mo flasem hem mo afta papa i mas pem olgeta

kōl perform grade-taking ceremony, that a man performs in order to increase in rank as a chief, which involves killing of pigs kilim pig long seremoni blong leftemap rang

kōlkōl grade-taking ceremony, ceremony that a man performs in order to increase in rank as a chief $\sim$ seremoni we man o woman i save mekem blong tekem rang. Woman i mekem long haos, man i mekem long nakamal

leleverser ceremony in which the family of a man who has died give money to other members of the family wan seremoni we ol famle blong wan man we hem i ded ol i givim mane long olgeta insaed long famle

matē $\mathbf{1} \cdot$ death $\sim \operatorname{ded} \mathbf{2} \cdot$ funeral, death ceremony $\sim$ seremoni blong ded matian her/his/its death $\sim$ ded blong hem

matiēk my death ded blong mi

miarten noises that are made with bamboo flutes and other things when people are making a vōnōmōōo ceremony ol noes we ol man ol i mekem wetem flut o wanem, taem ol i mekem vōnōin̄ōtō

qalēg gōr make an agreement between the parents that a particular girl or woman will be married to a man blokem, putum tabu long wan gel mo givim mane se bae tufala i mared qin̄imatē measurement of days since someone's death $\sim$ dei blong ded we yu kaontem kasem wan taosen dei

sersöm return some of bride price if a couple divorce, the woman's family must return money to the man's family who paid the bride price givim bak mane sapos mared i finis mo man mo woman i divos, famle blong woman i mas mekem long famle blong man we ol i bin pem woman ia

siganar remove rope from the neck of a man who has not met the goal of giving up a certain food for 1,000 days during the mourning period after the death of a close relation tekemaot rop (ganar) long wan man we hem i no save lego kakae we hem i bin talem se bae hem i no kakae kasem wan taosen dei blong wan man long famle blong hem i ded

sōq perform grade-taking ceremony, by killing of pig kilim pig long seremoni blong tekem rang

tuleg act of children of a dead man making payment to their father's family to claim their right to his 
land and property taem wan man i ded, ol pikinini blong ded man ol i mas givim mane long famle blong papa blong pemaot graon mo ol propeti long ol famle blong mekem se i graon blong olgeta nao

veltiwial time when the family of a couple who are to be married come together in order to settle the couple together long taem blong mared ol famle blong man mo woman i kam tugeta blong putum tugeta boe mo gel

vōnō̄mōōō tradition that is performed in the afternoon of the fourth day after someone has died, in which the heads of the families of the man who has died do such things as remove the dead man's house or cut wood in his garden. The children of the dead man must then pay the people who have done this $\sim$ long aftenun long namba fo dei blong wan man i ded, ol man (lida blong olgeta hem i famle blong man we hem i ded) ol i kam katem wud long garen o tekemaot haos blong ded man mo ol samting olsem. Afta pikinini blong ded man i mas givim mane long ol man we ol i mekem

völöt traditional practice in which when the first child of a couple is born, the family of the mother come and try to throw stones at the father of the child and his family taem fes bon pikinini i bon, famle blong mama ol i traem stonem papa blong pikinini wetem ol famle

vusarōr kill pig in order to release the spirit of someone who has died. The dead person is quiet but the pig squeals to represent the person crying as their spirit is released. $\sim$ kilim pig blong lego spirit blong man we i ded. Ded man i kwaet be pig i singaot blong representem ded man i singaot mo hem i lego spirit. vusveslivmat kill a pig or chicken to close the eyes of someone who has died, a ceremony performed by one of the children of person who has died kilim pig o faol blong klosem ae blong man we i ded, wan pikinini blong man we i ded i mekem

wölqareñi make payment to the people who dug a dead person's grave pem ol man we ol i digim gref blong ded man

\section{R3 Death and mourning}

matē $\mathbf{1} \cdot$ death $\sim \operatorname{ded} \mathbf{2} \cdot$ funeral, death ceremony $\sim$ seremoni blong ded matian her/his/its death $\sim$ ded blong hem

matiēk my death ded blong mi

miat die, be dead $\sim$ ded

miarten noises that are made with bamboo flutes and other things when people are making a vōnōmōto ceremony ol noes we ol man ol i mekem wetem flut o wanem, taem ol i mekem vōnōinōtō

onvegtëm lay in the same house with the body of someone who has died after sunset, until the next day when they can remove the body in daylight $\sim$ sapos wan man hem i ded long sapa, ol i mas slip wetem hem kasem neks dei

qin̄imatē measurement of days since someone's death, counted up until 1,000 days dei blong ded we yu kaontem kasem wan taosen dei

siganar remove rope from the neck of a man who has not met the goal of giving up a certain food for 1,000 days during the mourning period after the death of a close relation tekemaot rop (ganar) long wan man we hem i no save lego kakae we hem i bin talem se bae hem i no kakae kasem wan taosen dei blong wan man long famle blong hem i ded 
tövun (fr.var. tuvun) $\mathbf{1}$ bury berem $\mathbf{2}$ - fill in (hole), cover over something in hole berem

turgēn $\mathbf{1} \cdot$ body $\sim$ bodi $\mathbf{2} \cdot$ trunk of tree $\sim$ stamba blong wud

vōnōmōōlō tradition that is performed in the afternoon of the fourth day after someone has died, in which the heads of the families of the man who has died do such things as remove the dead man's house or cut wood in his garden. The children of the dead man must then pay the people who have done this long aftenun long namba fo dei blong wan man i ded, ol man (lida blong olgeta hem i famle blong man we hem i ded) ol i kam katem wud long garen o tekemaot haos blong ded man mo ol samting olsem. Afta pikinini blong ded man i mas givim mane long ol man we ol i mekem

vusarōr kill pig in order to release the spirit of someone who has died. The dead person is quiet but the pig squeals to represent the person crying as their spirit is released. $\sim$ kilim pig blong lego spirit blong man we i ded. Ded man i kwaet be pig i singaot blong representem ded man i singaot mo hem i lego spirit.

vusveslivmat kill a pig or chicken to close the eyes of someone who has died, a ceremony performed by one of the children of person who has died kilim pig o faol blong klosem ae blong man we i ded, wan pikinini blong man we i ded i mekem

wölqareñi make payment to the people who dug a dead person's grave pem ol man we ol i digim gref blong ded man
R4 Taboos, strictures and secret knowledge

$\operatorname{man}_{1} \mathbf{1} \cdot$ intoxicate, stun, poison, as in kava, alcohol or poison making you feel drunk or delirious kilim, mekem i drong, olsem man $\mathrm{i}$ posenem fis o kava $\mathrm{i}$ kilim man $2 \cdot$ magic, words used when performing magic or to support use of traditional healing and other purposes majik, toktok blong talemaot long taem blong yusum kastom meresin mo sam narafala samting

maw $_{2}$ secret $\sim$ wan save we bae yu yusum be yu no save lego

$\overline{\mathbf{o}} \mathbf{l} \mathbf{o} \mathbf{l}_{2}$ traditional secret prayer made at a sacred place to ask for something desired kastom prea long tabu ples blong askem wan samting

salgōr sacred and restricted act or thing samting we i tabu

sian gōgōn name that one uses to refer to or address someone who has the same name as an in-law whose name you are unable to say, due to cultural restrictions of the relationship nem we yu yusum blong talemaot wan man o woman we hem i gat semak nem olsem wan tawian o palika blong yu, from we nem i semak, i tabu blong singaotem nem

vēl ${ }_{2}$ use magic to kill a person yusum majik blong kilim wan man i ded

\section{R5 Healing and medicine}

sus 1 - poke, prod, pierce stikim 2 • point (with finger) $\sim$ poen (wetem fingga) $3 \cdot$ inject, give injection to stikim (wetem stik meresin)

tenge leaf remedy, plant used for traditional medicine, fertilising crops, aiding fishing and other traditional remedies and purposes 
lif meresin o lif we ol i yusum long saed blong kastom olsem blong fis o long garen

\section{$R 6$ Singing and dancing}

ber $_{1} \mathbf{1} \cdot$ grass skirt, skirt made from beach pandanus $\sim$ grasket $\mathbf{2}$-wear grass skirt $~$ havem grasket

bō 1 - length of bamboo used for carrying water bambu blong fulumap wota $\mathbf{2}$ - length of bamboo used for making beat in dance bambu blong kilim long wud long taem blong danis

diat $_{2}$ make a mistake and not reach the end when performing a dance mestem mo no kasem stret en blong danis

elnetiti style of traditional dance that is performed at the ceremony marking 1,000 days since someone's death wan danis we ol man ol i danis long wan taosen dei blong wan ded man. Ol man ol i danis blong soemaot se ol i glad mo ol i stat blong fogetem

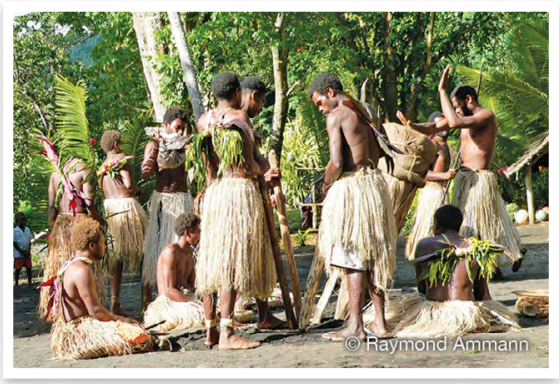

elnetiti style of dance

ës 2 song singsing

lak dance $\sim$ danis

laklak dance $\sim$ danis

lalēbur thick slab of wood that is placed over hole in ground and used to make the sound and rhythm, especially for elnetiti dance $\sim$ bigfala pis wud we ol i putum ova long wan hol long graon mo ol i kilim wetem wud blong mekem gudfala saon blong danis, speseli blong elñetiti

liañ style of traditional dance $\sim$ kaen kastom danis

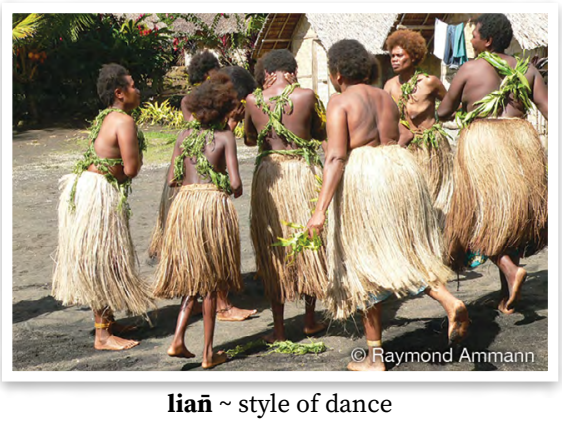

me 2 - name of a traditional dance nem blong wan kaen kastom danis

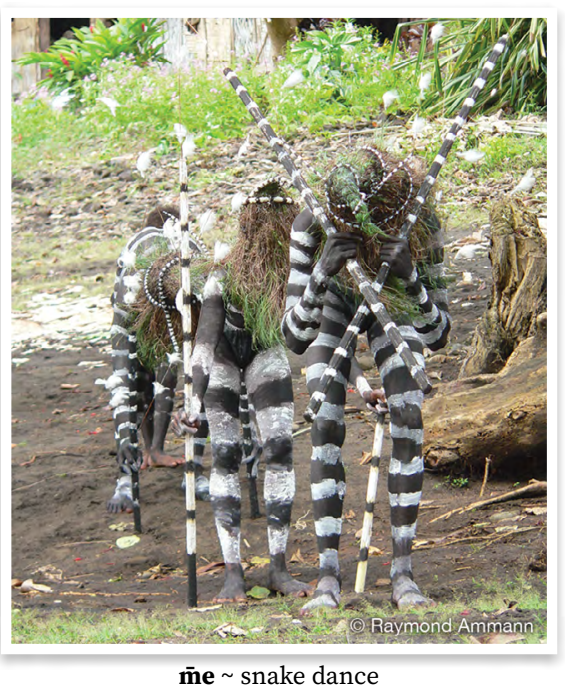

rësrës deñe style of dance that is made at a ceremony held five days after someone's death, when their spirit leaves the earth to go to the place of the spirits (lisōr) wan kaen danis we ol i mekem long faef dei blong ded blong wan man, taem spirit blong hem i go long ples blong ol ded (lisōr) 
rëw sing singim, singsing

sēsēlit water music wota miusik

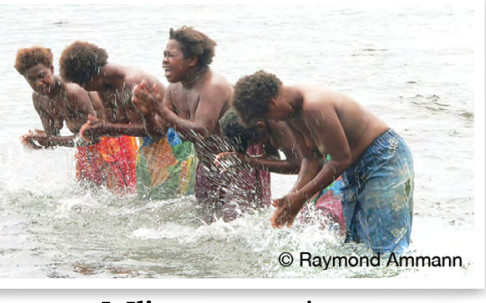

sēsēlit water music

siwia kind of public dance kaen pablik danis

sur ${ }_{4}$ sing singim

timiat $_{2}$ headdress worn by men for particular dances hedres we ol man i werem long taem blong danis

timiatwos traditional wooden upright drum tamtam

tōw ${ }_{5}$ compose (song) komposem (singsing)

veleg beat (usually bamboo or wood) with one stick in each hand to make music or dance beat kilim tamtam, bambu blong mekem miusik, o man i danis long hem

weveveleg sticks used for beating slitgong wud we ol i yusum blong kilim tamtam

wōkōr slitgong that is made from bamboo or hollowed out wood tamtam we ol i mekem long bambu o wud

wöduñdun long, thick stick that is used for beating on wooden slab that is placed over hole in ground to make beat, especially for elnetiti dance longfala wud we ol i yusum blong kilim flat pis wud long graon long taem blong elñetiti danis, we $\mathrm{i}$ mekem bit blong danis
R7 Feasting

gis salmiat make preparations for a feast priper blong mekem bigfala kakae, speseli long wan taosen dei blong wan ded man

qetgengen feast, planned celebratory feast lafet, wan kakae we yumi ekspektem blong holem, plan blong mekem kakae

\section{R8 Christianity}

\section{gengenur $\sin \sim \sin$}

gōrōn holy, sacred, spiritually meaningful tabu

gösuv rōn̄ baptise baptaes

gövur house, generic name for building haos gövur gōrōn̄ church jos gövur tatar church jos

leg marry (in church), be married mared (long jos)

legleg marriage ceremony (in church) mared (long jos)

lōlōqōr $\mathbf{1}$ • forgetful save fogetem tumas samting $\mathbf{2} \cdot$ heathen, lacking Christian enlightenment $\sim$ taem $\mathrm{O}$ man blong tudak

Mam 1 1 Dad $\sim$ Papa $2 \cdot$ God $\sim$ Papa God tatar $1 \cdot$ pray $\sim$ prea $2 \cdot$ prayer $\sim$ prea vawē $\mathbf{1} \cdot$ bless blesem $\mathbf{2} \cdot$ blessing blesing 


\section{S Hunting, fishing, farming}

\section{S1 Hunting}

bien $\mathbf{1} \cdot$ feed $\sim$ fidim $\mathbf{2}$ put bait in trap $\sim$ putum bet long basket naora

gatitieg bird or pig trap made from rope $\sim$ trap blong pijin o pig

san 1 - pull with quick movement, as in pulling in a fish pulum, jokem 2 - kind of trap for catching chickens and birds on the ground. A rope is tied to a piece of wood and a loop made in the rope, then food is scattered around on the ground. Then the rope is pulled tightly when a bird walks inside the loop wan kaen trap blong kasem faol o pijin long graon we yu fasem rop long wud long graon, putum kakae raon long rop mo jokem kwiktaem taem pijin i stanap insaed long rop

san via try to pull in a fish but it gets away pulum fis be yu mestem

san qal pull fish successfully pulum fis

sēsēdig method of trapping birds wei blong kasem pijin wetem trap we yu fasem rop long brans blong tri mo yusum frut blong atraktem pijin ga sēsēdig rope for trapping birds rop blong trap blong pijin

tēqatēq $1 \cdot$ hunt with bow and arrow in the bush (birds and flying fox) hant wetem bonaro long bus (blong sutum pijin o flaengfokis) $\mathbf{2}$-gun masket

tieg lay trap putum trap

timiatēq gun $\sim$ masket

tit $\mathbf{1} \cdot$ split $\sim$ splitim $2 \cdot$ pull rope down quickly and sharply when trying to catch bird with a sēsēdig bird trap jokem string blong kasem pijin long sēsēdig tut ${ }_{2}$ hunt at night, using light to shine on prey, such as flying fox, coconut crab, crab, prawns mekem laet, olsem blong kasem krab, flaengfokis, naora long naet

vus bow bonaro

wètèq kind of arrow that has a blunt end so that it will bounce off a tree if the target is missed and not get stuck, used for shooting birds wan kaen aro we en blong hem i no sap blong hem i no save fas long wud taem yu sut wetem, i mas foldaon, ol i yusum blong sutum pijin

\section{S2 Fishing}

beret $_{1}$ try to shoot something (e.g. fish with bow and arrow, bird with shanghai or person with stone) but be unsure if it can be hit as it is far away or can't be seen properly traem sut (olsem fis wetem bonaro, pijin wetem lastik o stonem man we i stap long bus) be yu no sua se yu save kasem from maet i stap longwe tumas o yu no save luk gud

beberet training to shoot a target with a bow and arrow trening blong sut wetem bonaro

b $\overline{\mathbf{e}}_{2}$ bait hook putum bet long huk

gasalē right size place for setting net where the fish won't be able to get away stret ples blong putum net we ol fis bae ol i no save lus

giam $\mathbf{1} \cdot$ net $\sim$ net $\mathbf{2} \cdot$ net covering stomach $\sim$ net we i kavremap gat giam vus net with a handle $\sim$ kaen net wetem handel blong hem tē giam place net putum net go hook, fish hook huk blong fis kal pull in fishing line, pull up fish pulum string 
kar spear with four prongs and rubber $\sim$ spia we hem i gat raba blong hem meteër arrow made from wood of casuarina, with sharp point, used for shooting fish aro we ol i mekem long wud blong oktri, we i gat sap en blong hem mo ol i yusum blong sutum fis

qēsqēs fishing technique where one pounds hermit crabs so that they smell and then throws them in the sea to attract fish wan fasin blong kasem fis we yu kilim hed blong nakato blong mekem se i smel, mo afta yu sakem long solwota blong atraktem fis

qōrser kind of spear with several prongs on the end $\sim$ wan kaen spia we $\mathrm{i}$ gat plante poen long en blong hem

qōt dive (for) daeva (long)

qōtqōt go diving daeva

rērēv towline $\sim$ tolaen

ser $_{1}$ spear spia

so $_{3}$ gather, harvest palolo worm karem palolo, wom blong solwota

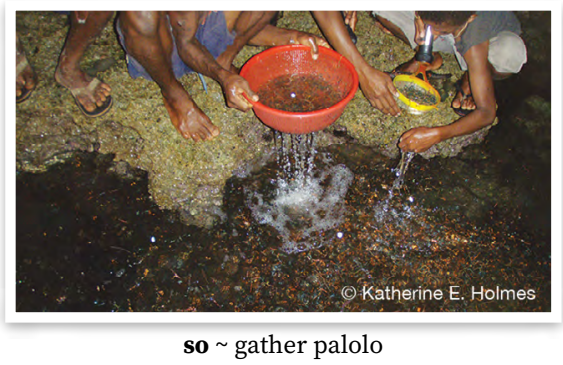

sōsōg fish, go fishing go huk

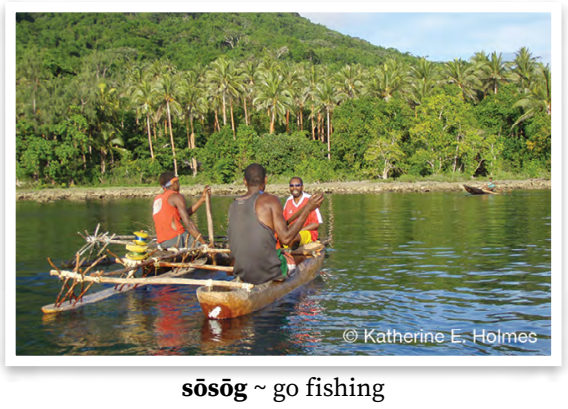

varwëw troll (fishing line) fasin blong fising long bot we yu lego string we i stap long rol

vetbōñ rope used for picking up a wōw fish trap from the bottom of the sea. It has a stone on the end that has a hole in it for tying the rope to it, and a special hook rop wetem ston mo huk long en blong hem blong pulum wōw net we i stap long solwota

vëkrës scale, remove scales from fish $\sim$ tekemaot skel blong fis

vidir (fr.var. vēdir) $\mathbf{1} \cdot$ remove midrib from sago palm leaf $\sim$ tekemaot bun blong lif natanggura 2 - shoot liaw fish sutum liaw fis

vovon̄on go fishing, looking for shellfish or other sea or freshwater creatures $\sim$ lukaotem mit long wota o solwota

vötuga reel, fishing reel wud blong rolem rop i stap long hem

vötuga sēsēdig reel for rope for catching birds wud blong rolem rop i stap long hem blong kasem ol pijin

vötuga sōsōsōg fishing reel wud blong rolem string blong fising $i$ stap long hem

vukrē scale skel blong fis

vunvun poison fish $\sim$ posenem fis vun viteg method of poisoning fish in deep sea $\sim$ fasin blong posenem fis long dip si

waraēl fishing bamboo pole $\sim$ bambu blong hukum fis

wēbē bait bet

$\mathbf{w o ̄ l o g}_{1} \mathbf{1} \cdot$ flexible wood for making trap wud blong bendem blong mekem trap $\mathbf{2} \cdot$ handle of a $w \bar{w} w$ trap $\sim$ handel blong fis trap wōw

wōw 1 kind of fish trap used in the deep sea, which is like a lobster pot but used only for catching fish wan kaen net trap we yu yusum long medel si. Yu putum bet insaed 
mo i gat wan hol antap be taem ol fis ol $\mathrm{i}$ go insaed ol i no save kamaot bakegen

tul wōw throw wōw fish trap into the sea $\sim$ draonem wow net long solwota wödön $\mathbf{1} \cdot$ method of baking in a stone oven, in which the stones are placed on the fire to heat, then when they are heated through and the fire has died down, the stones are removed, the food is placed on the bed of the oven, and the hot stones are placed on top of the food parcel wei blong bekem kakae we yu tekemaot ston, putum kakae long oven, afta putum hot ston antap long kakae $\mathbf{2} \cdot$ method for setting wöwöwör qēsaqēs fish trap in sea, or waga prawn trap in river, in which stones are placed on top and around the trap to prevent it from being carried away by the current. putum trap blong fis long solwota o trap blong naora long wota mo putum ston raonem hem blong taed i no save muvum

wöwöwör qēsaqēs kind of trap used close to the shore for catching small fish and particularly lobster kaen trap we yu yusum klosap long so blong kasem ol smol fis mo speseli naora

wutge mark spot at sea makem ples long solwota

\section{S3 Farming and propagation}

biriat garden where taro is planted in dry ground on hill $\sim$ drae garen taro antap long hil

bōak $\mathbf{1} \cdot \operatorname{mud} \sim \operatorname{sofmad} \mathbf{2} \cdot$ taro garden planted in mud $\sim$ garen taro we ol $\mathrm{i}$ planem long sofmad

dōw become overgrown (garden) bus i kavremap garen $\mathbf{e v}_{2}$ use digging stick to loosen taro, after pull out of ground yusum wud blong mekem graon i sofsof, mo pulumaot taro long graon

gil $1 \cdot$ dig, dig up food plants, etc. digim $2 \cdot$ digging stick stik blong digim graon $\mathbf{3}$ - Stick used for removing husk from coconut $\sim$ wud blong karemaot skin long kokonas

gōq make hole with digging stick to plant taro $\sim$ digim graon wetem wud blong mekem hol blong taro

kor make kindling, cut trees and branches which you have cut down into smaller pieces so that they will dry and you can burn them katkatem ol brans mo wud we yu bin katem blong livim i drae redi blong bonem

liaq irrigate $\sim$ putum wota i go insaed long garen taro

löñ $\mathbf{1}$. place leaf over fire in order to block and reduce the heat $\sim$ putum lif long faea fastaem blong blokem sapos i bigwan tumas 2 - block off water to change watercourse so it flows into taro paddy blokem wota blong mekem i go long garen taro

mat taro garden planted in running water or mud, where the water is never drained off, taro paddy garen taro long wota o sofmad we wota i stap long hem oltaem

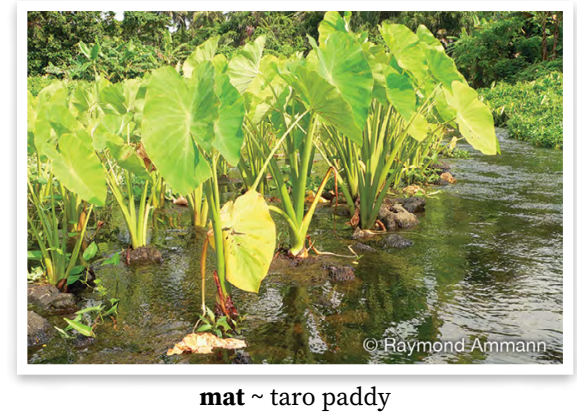

mat bōak taro garden planted in mud with no stones, where water wells up when the ground is dug 
garen taro we ol i planem long sofmad, we i nogat ston mo wota i kam antap taem ol i digim graon

mat vetvet taro garden planted only in stones with running water garen taro we ol i planem long ples we wota i ron long ol ston

mat wöwörös taro garden planted in small stones and thin soil with running water $\sim$ garen taro we ol i planem long ples we $\mathrm{i}$ gat ol smolsmol ston mo wota i ron long hem

n̄en̄ere garden garen

qèel (pl. qēlaqēl) taro paddy; garden for planting taro which is sometimes irrigated and sometimes not during the period of growth. The taro are planted in dry ground, then irrigated, then drained for weeding, then irrigated again kaen garen taro we ol i planem long drae graon, afta ol i putum wota, afta lego wota long taem blong widim

qël grow and spread out (of vine or yam) rop i gro mo spred aot

ran̄rañ clean garden $\sim$ klinim, katem garen

riv ${ }_{1}$ plant $\sim$ planem

rivriv plant $\sim$ samting we yu planem

rot taro garden $\sim$ garen taro

rönö plant stake in ground for yam to grow up planem wud long graon blong yam i save gro long hem

sar $_{4}$ pull out, referring to pulling taro from ground pulumaot, olsem pulumaot taro long graon

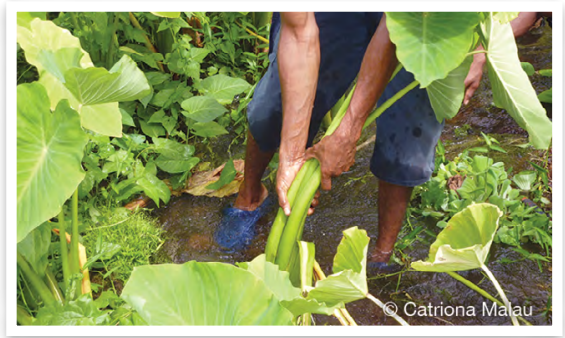

sar pull out taro sartan first sign that yam is starting to dry out $\sim$ fes saen blong yam i stap go drae (lif i yelo o rop i drae)

sigsig harvest taro from paddy gardens $\sim$ karem taro long garen

sōwereg plant yam or water taro in the same place where it was dug up planem yam o wota taro i gobak long sem ples we yu bin tekemaot

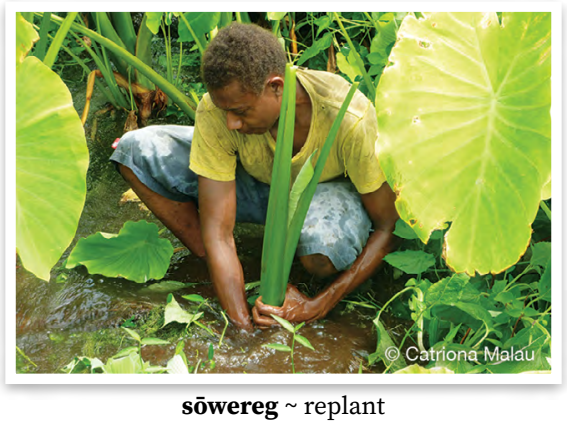

tin̄ taro paddy unit within a qēl, mat or bōak separated by mud or stone walls step insaed long garen taro tiqū garden $~$ garen

tōw ${ }_{2}$ grow $\sim$ gro

$\operatorname{var}_{2}$ plant (island cabbage) planem (aelan kabis)

varwëgsērēt first flooding in process of irrigating of taro paddy. In this step the water remains in the paddy for five days before it is drained fas taem we yu putum wota i go long garen taro blong i stap faef dei vas plant taro $\sim$ planem garen taro

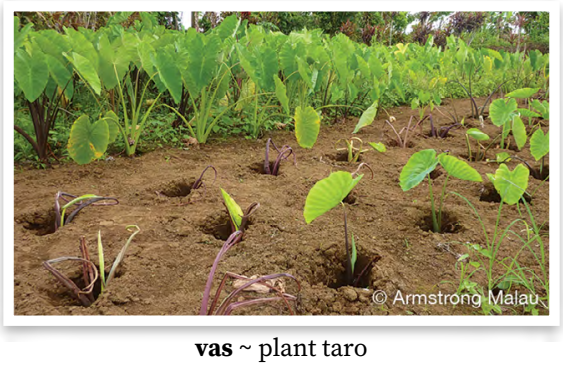

vaslē area where lots of taro gardens $(q \bar{e} l)$ are all planted together $\sim$ ples blong fulap garen wota taro $(q \bar{e} l)$ 
vug $_{1}$ row of yams in garden ro blong yam long garen

vun section of yam garden $~$ eria blong garen yam

vuteg plant (yam) planem (yam)

wewestel area that used to be a vegetable garden, which is not currently being tended, but still produces produce, so people can go there to harvest crops $\sim$ ples blong olfala garen we ol man ol i nomo planem kakae long hem, be ol kakae i stap gro yet, mekem se ol man ol i save go karem kakae long ples ia wēviñ piece of yam for planting pis yam blong planem

wōsōk taro garden that is planted in dry ground and not irrigated $~$ garen taro we ol i planem long drae graon nomo

wörönö stake, length of wood inserted in ground for yam vine to grow up wud blong planem long graon blong yam i save gro long hem

wöwör weed widim

\section{T Fire and cooking}

T1 Fire

$\mathbf{e v}_{1} \mathbf{1} \cdot$ fire $\sim$ faea $\mathbf{2} \cdot$ hell $\sim$ hel

ga burn up, burn out of control bon nogud

nōn (of fire) burn down to coals, ready for cooking go daon (faea) redi blong kuk

ralēt firewood $\sim$ faeawud

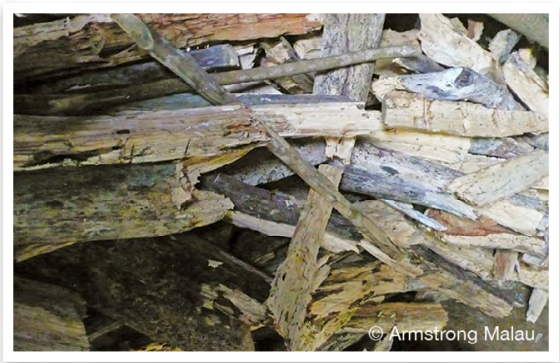

ralēt $\sim$ firewood

rēe draw leaf, such as coconut, pandanus, heliconia, over fire to make more hardy and supple for use in weaving and cooking bonem lif long faea, olsem kokonas, pandanas, lif laplap, blong mekem se i sofsof mo i save stap longtaem sul 1 • light (fire) laetem faea $\mathbf{2} \cdot$ burn (something) bonem (samting) susul 1 - burn a bird after plucking $\sim$ bonem pijin taem yu tekemaot feta finis $\mathbf{2}$ - irritate mouth (said of taro and wild yam) $\sim$ taro o wael yam i kakae man

tum $1 \cdot$ burn bonem 2 - burn, be burnt $\sim$ bon

tuwegev (fr.var. tuwëgev) make fire in stone oven $\sim$ mekem faea insaed long oven

watavren charcoal jakol

wōwō ashes asis

T2 Food preparation, cooking,

distribution, eating

an be uncooked, not properly cooked $\sim$ no dan gud

bal $_{1} \mathbf{1} \cdot$ use wooden tongs to pick up hot stones or charcoal from stone oven $\sim$ tekemaot ston o jakol long oven wetem wud $\mathbf{2}$ - cut (with scissors) katem (wetem sisis)

big eat (meat, greens, protein with main meal) kakae (mit o kabis wetem kakae) 
bigbig meat, greens, protein component of meal mit, pat blong kakae we i no men kakae, olsem kabis o kokonas

big töw eat grated coconut flesh, as you scrape it out of the shell, either on its own or with a staple food kakae kokonas, taem yu skrasem kokonas, yu kakae hem wan o tugeta wetem wan kakae

bōnësnës fill length of bamboo with food (e.g. taro, yam, meat, prawns) and roast over fire $\sim$ fulumap kakae insaed long bambu mo rusum long faea

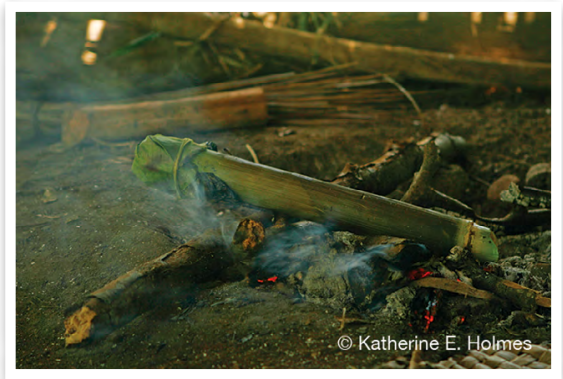

bōnësnës cook in bamboo

bun place stones on fire of oven putum ston antap long faea

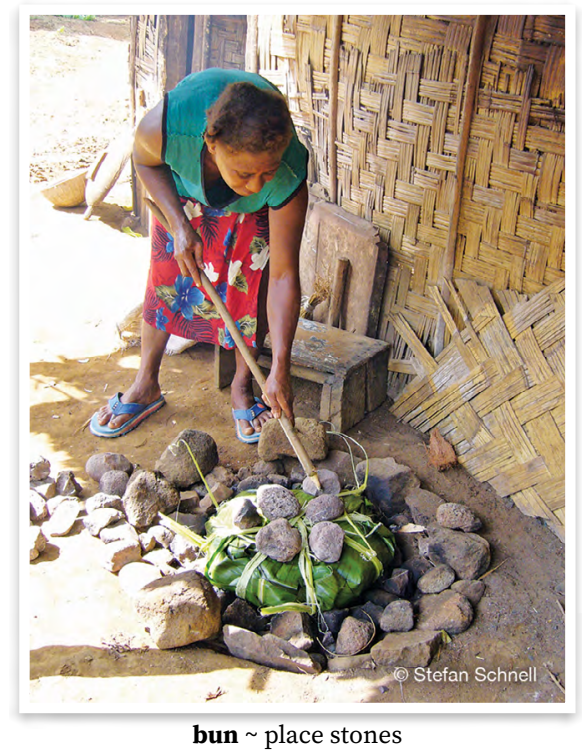

demete leaves fastened together and used to cover opening of stone oven ol lif we ol i fasem tugeta blong blokem ae blong oven

dēdērēs taste good swit

dētēriv leaves of cottonwood tree that have been pinned together to produce a large leaf mat that is used to cover over food when it is baking in stone oven $\sim$ stikim tugeta lif blong burao o navenue blong mekem i bigwan blong kavremap kakae taem i bek long oven

dër rot, be rotten, as in food deteriorating or other items disintegrating roten, olsem kakae, klos, haos

diat mushy, soft (of food that has been overcooked) sofsof kakae we i dan tumas, olsem raes i kam olsem laplap, o yam i tumas wota

dōleg mix löt food dish after it has been pounded $\sim$ miksim nalot wetem wud afta yu bin kilim finis

dōrōq cottonwood leaves fastened together to form a lid for covering the opening of a steam oven lid blong blokem ae blong oven we ol i mekem long lif blong burao o navenue

en flatten out löt ñe mereñ food dish flatem kaen nalot ia we ol i kolem se löt ñe mereñ

gabal long wooden tongs used for picking up hot stones or charcoal from stone oven wud blong karemaot ston long oven 


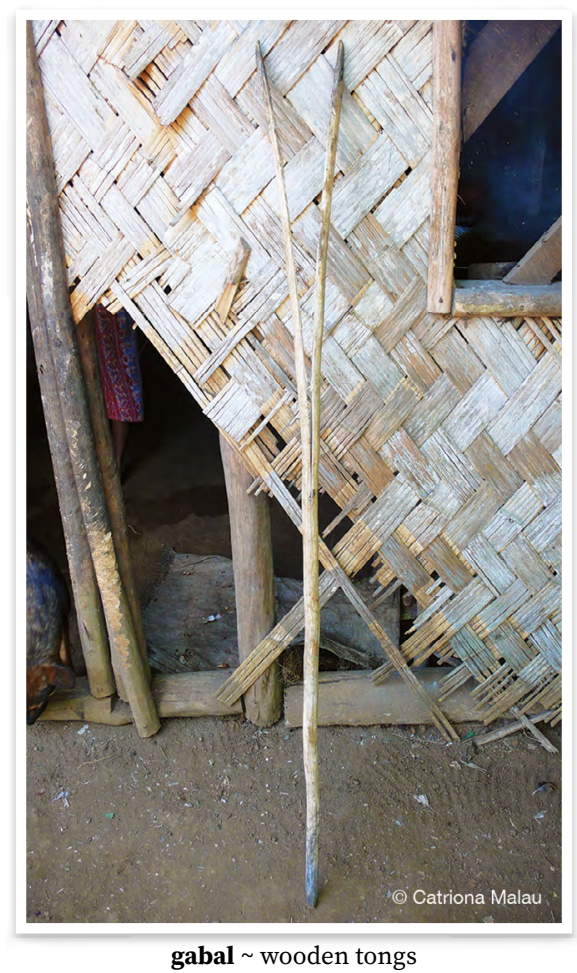

galēm long wooden pole used for evenly spreading charcoal and heat of stones in stone oven wud blong miksimap jakol mo ston long oven

gatitimeles piece of wood with hooks on it for hanging food in the kitchen $\sim$ wud we i gat huk long hem we ol i hangem antap blong hangem kakae long hem

gen $_{1}$ eat, eat staple part of meal kakae, kakae men kakae

gengen $\mathbf{1}$ - food, meal, staple part of meal, either cooked or uncooked kakae, men kakae, we i dan o i no dan $\mathbf{2}$ - food crops, staple food plants $\sim$ kakae long garen

gengen luwō feast $~$ bigfala kakae, lafet

gengen luwömeren eat lunch, meal in middle of day kakae dina gengen revrev eat dinner, evening meal kakae long sapa gengen vōrōg eat breakfast dring

ti long moning

genvēl something that you don't want to eat but you must force yourself to eat samting we yu no wantem kakae be yu mas kakae mo yu fosem nomo blong kakae

genwiwiris fussy eater, someone who is choosy about what they want to eat and won't eat if you cook something other than what they want man we hem i jusum kakae we hem i wantem nomo mo sapos yu kukum nara kaen kakae bae hem i no wantem kakae

geres grate coconut skrasem kokonas

gëvtun make meal using method where the food is wrapped in leaves, then place the parcel of food on top of the coals of the fire mekem kakae folem fasin we yu paselem kakae fastaem, afta yu putum long faea. Samtaem yu save rusum kakae fastaem

gil 1 - dig, dig up food plants, etc. digim 2 - digging stick $\sim$ stik blong digim graon $\mathbf{3}$ - Stick used for removing husk from coconut $\sim$ wud blong karemaot skin long kokonas

gök $\mathbf{1}$ • boil boel $\mathbf{2}$ - bubble, dissolve $\sim$ boel (meresin)

gör grate (coconut), scrape out insides of something firm $\sim$ skrasem kokonas, skrasemaot insaed long wan samting we i strong

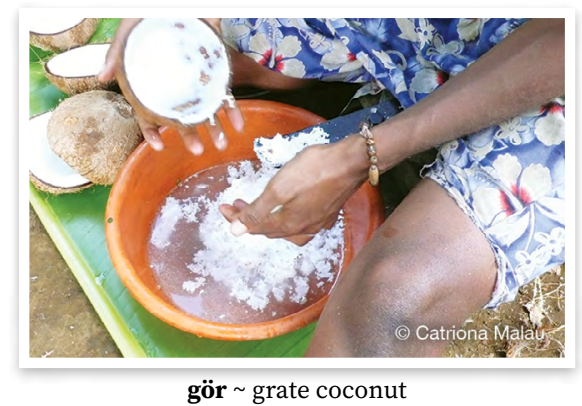


kuk cook, used occasionally as generic cooking term, but more commonly with specific reference to cooking food in a saucepan, rather than over fire or in stone oven kuk, kuk long sospen

kurqar not cooked properly hafdan, kakae we i no dan gud

lèm stir up and spread charcoal and hot stones in stone oven with long stick (galèm); use lever action yusum wud (gatēm) blong tekemaot bigfala pis wud o jakol long bigfala oven blong vësëw bifo i redi blong putum kakae

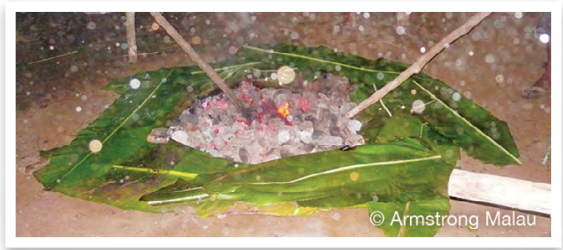

lēm lever with stick

lik serve food, remove food from oven

sevem kakae, karemaot kakae long oven

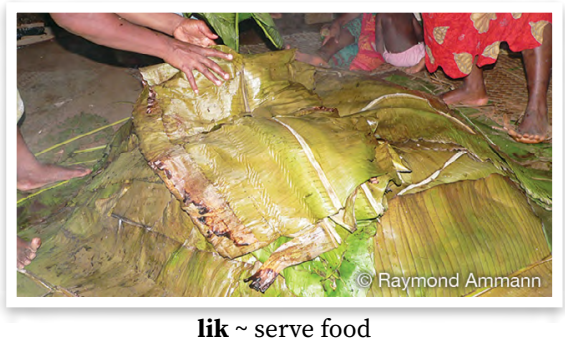

liqet cover food with large leaves for cooking in stone oven $\sim$ kavremap kakae wetem lif insaed long oven

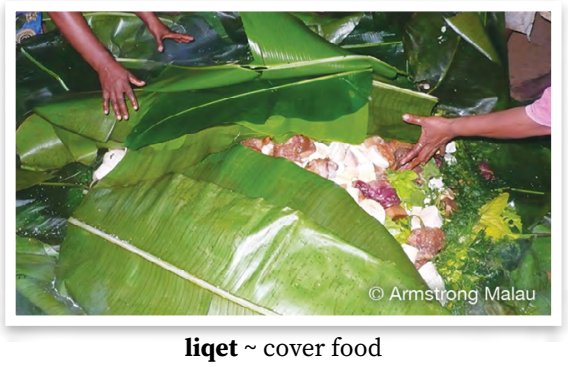

meteges wooden knife for cutting löt food dish wuden naef blong katem nalot

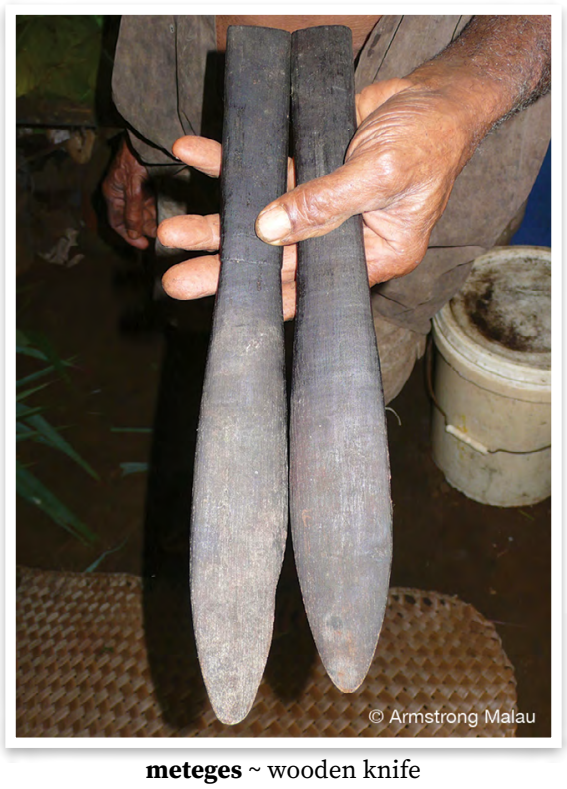

monog cooked $\sim$ dan

$\bar{n}_{2}$ eat only meat without the staple part of a meal kakae mit witaot kakae

nal ${ }_{3}$ chew, as something chewy like toffee, bubble gum juim, kakae samting we i taf

qan̄ris (fr.var. qarn̄is) $\mathbf{1}$ - method of baking in stone oven $\sim$ fasin blong bek long oven $\mathbf{2}$ bake food in stone oven bek, bekem kakae long oven qar $\mathbf{1}$ - unripe $\sim$ no raep $2 \cdot$ raw, uncooked $\sim$ no dan $\mathbf{3} \cdot$ undried, untreated, as of green leaves, rather than dried or heat treated leaves no drae, no dan, olsem lif we i grin nomo, we ol i no putum long faea blong mekem i strong

q $\overline{\mathbf{o}} \mathbf{t} \overline{\mathbf{o}}_{2}$ cupboard that is built over the fire in kitchen to store canarium nuts or breadfruit, so that they are dried and kept safe from rats $\sim$ haos blong putum drae nangae o bredfrut 
insaed long hem. Hem i go ova long faea mo ol i somap doa blong rat i no save kakae ol nangae o bredfrut

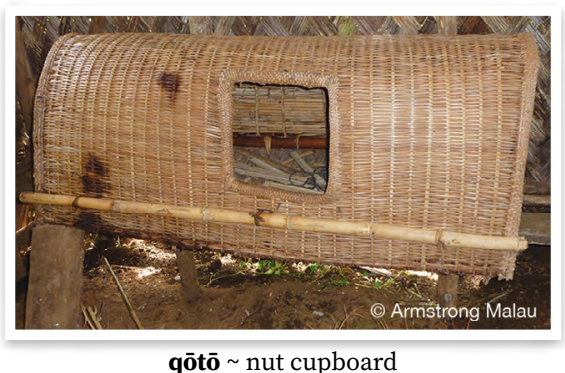

qusmamas eat a meal without any meat kakae drae, kakae kakae we i no gat mit blong hem

ras 1 - grate nuts or tubers for nalot or laplap rasras, salem kakae $\mathbf{2}$ - sharpen $\sim$ sapenem

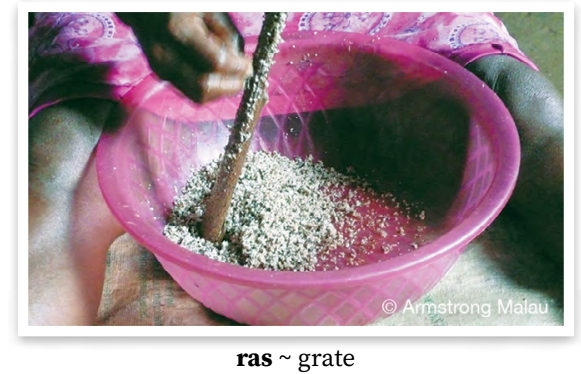

rèn̄ē roast meat over fire or charcoal rusum mit long faea

rötö heat leftover food, reheat next to fire or on coals, to prevent from going off $\sim$ hitim namalas blong mekem se i no sting

sal mamēgin style of making meal by which staple foods are wrapped with meat and greens in heliconia leaves with hot stones inside to help the food cook stael blong mekem kakae we yu pasolem ol hot ston insaed long lif laplap wetem kakae, mit mo kabis

salsal make meal by wrapping staple food with meat and greens in heliconia leaves with hot stones inside to help the food cook, the food is then placed on hot stones or coals so that it is cooking from inside and outside the parcel mekem kaen kakae we yu paselem kakae wetem mit, putum hot ston insaed bitwin lif laplap, mo afta yu putum long faea

sēm 1 - peel, remove skin from (something hard, as uncooked vegetables, copra) tekemaot skin (blong samting we i strong olsem taro o kopra) 2 - remove coconut flesh from shell and husk, to produce copra $\sim$ selaotem

sir $1 \cdot$ scrape, scrape dirt from the outside of taro and other root vegetables skrasem doti (blong taro) $\mathbf{2}$ - remove skin, bark tekemaot skin

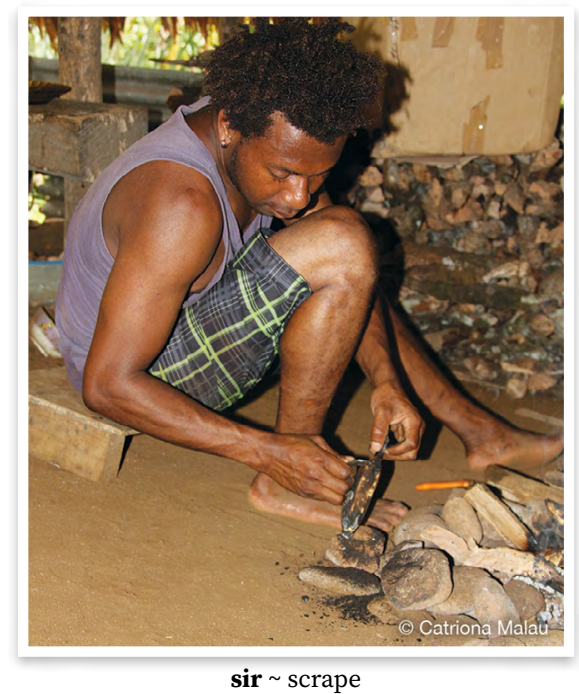

sum drink dring

sumsum 1 • drink $\sim$ dring $2 \cdot$ alcohol, alcoholic beverage $\sim$ dring, alkohol

tabē carved wooden platter, used for pounding nalot and making kava natambia, wuden plet

tētēw smoke, dry over smoke smokem

wètētēw wood for smoking wud blong smokem 
tumsölönev food is burnt on the outside but raw in the middle after roasting in a fire that is too hot kakae $\mathrm{i}$ bon long afsaed be i no dan long insaed, taem yu rus be faea i hot tumas

tun roast staple foods, as taro, banana, cassava, etc. (not meat) over fire or embers fasin blong rusum kakae we yu putum ova long faea o jakol

tuntun roast food $\sim$ rus

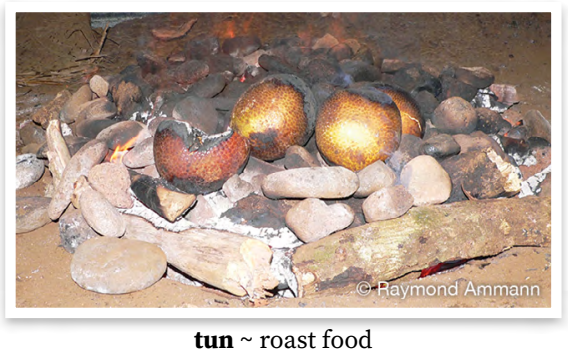

tuwegev (fr.var. tuwëgev) make fire in stone oven $\sim$ mekem faea insaed long oven

um oven made by digging hole in the ground and baking food with hot stones $\sim$ graon oven

um val kind of stone oven for which a big hole is dug in the ground, which is then filled with big stones kaen oven we ol i digim hol, afta fulumap wetem bigfala ston

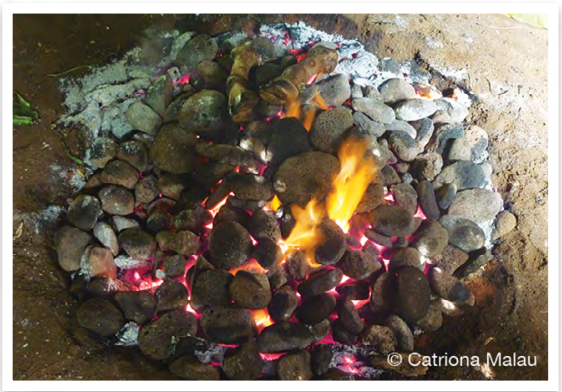

um $\sim$ stone oven

vësëw 2 • stone oven $\sim$ hol blong oven 2 - bake food in stone oven $\sim$ bekem kakae long oven via grater for grating nuts and tubers to make laplap and nalot samting blong salem nangae o ol kakae via qatē grater made from stem of tree fern samting blong salem laplap, nat o samting olsem we ol i mekem long blakpam

via tin grater made from old tin that has holes punched into it samting blong salem laplap, nat o samting olsem we ol i mekem long tin

vun 1 pour water into oven to produce steam by which food will cook kapsaedem wota antap long kakae long oven

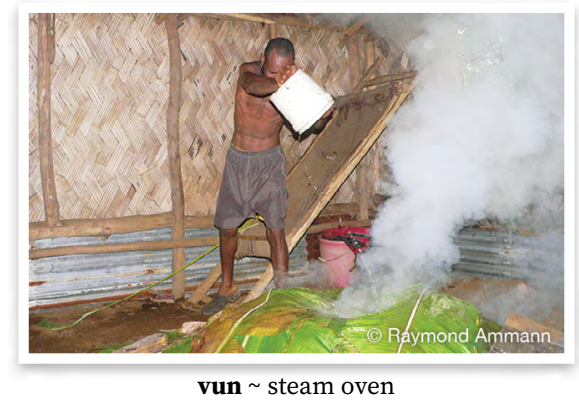

vutulöt (fr.var. vötulöt) large wooden pestle used for pounding löt food dish wud blong kilim nalot

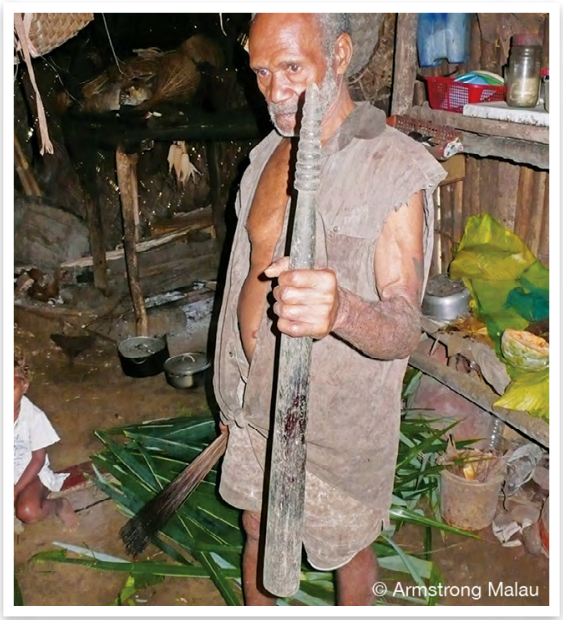

vutulöt wooden pestle 
weger tele metal coconut grater aean blong skrasem kokonas

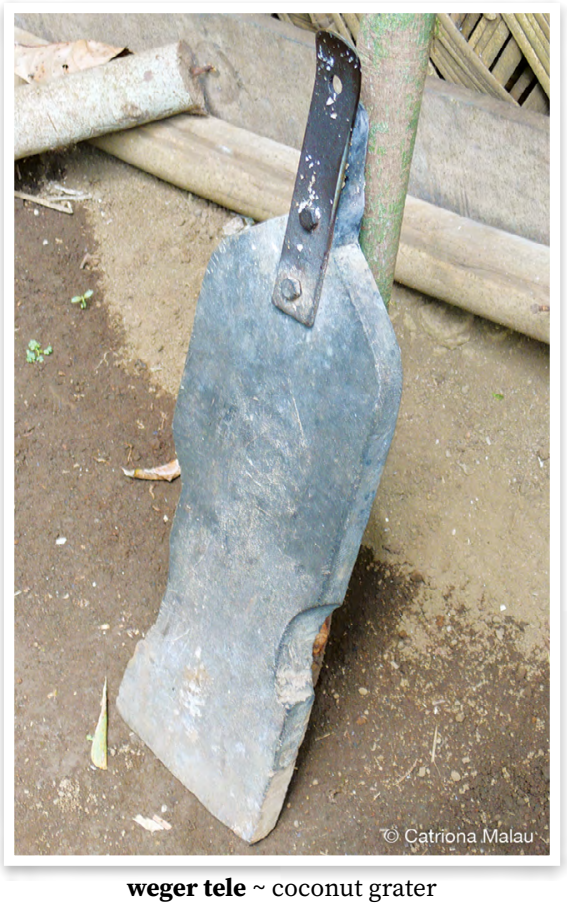

wetele lan̄lan̄ metal rods used as support for saucepan when cooking over fire aean blong ples blong kuk

wosleqen method for cooking meat or fish by grilling a little, then wrapping up in leaves with salt and cooking again on coals $\sim$ fasin blong kukum mit o fis we yu putum long faea smol fastaem, afta paselem long lif wetem sol mo kukum long faea bakegen

wosvalqeñ cooking method kaen fasin blong kuk

wōr 1 1 squeeze liquid out of (coconut, ground kava, etc.) melekem 2 • squeeze coconut milk onto food, either before or after cooking melekem (kakae)

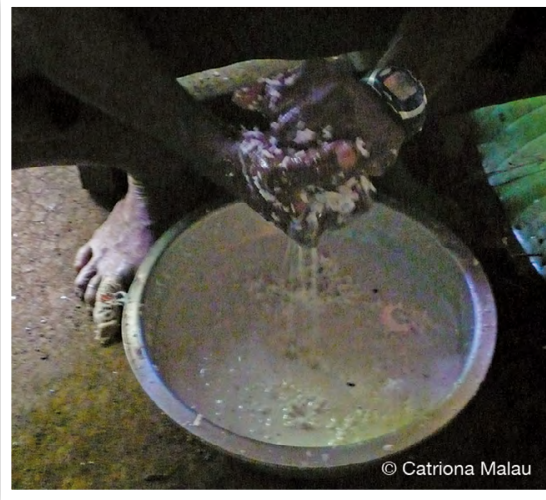

wōr squeeze liquid from

wōr logomonog cooking style where food is cooked first then coconut milk is squeezed on top without being cooked again stael blong mekem kakae we yu mekem kakae i dan fastaem, afta yu melekem

wōr qarqar cooking style where coconut milk is squeezed over uncooked food before being cooked, either in saucepan or parcel in oven or over fire stael blong kuk we yu melekem kakae fastaem, taem i no dan yet, afta yu kuk long sospen o pasolem mo bek long ston

wul peel, remove skin (from something which is soft and easy to peel, like a banana or vegetables which have been cooked) tekemaot skin (blong samting we $\mathrm{i}$ isi olsem banana o bredfrut we i dan) 


\section{U Human uses of natural resources}

\section{U1 Food}

biliagtat kind of laplap food dish that has two layers of laplap with a layer of grated canarium nuts between, either roasted or raw wan kaen laplap we $\mathrm{i}$ gat tu laen wetem grin o drae nangae long medel

bunia food dish, where different kinds of root vegetables are baked in leaves in stone oven with coconut milk bunia

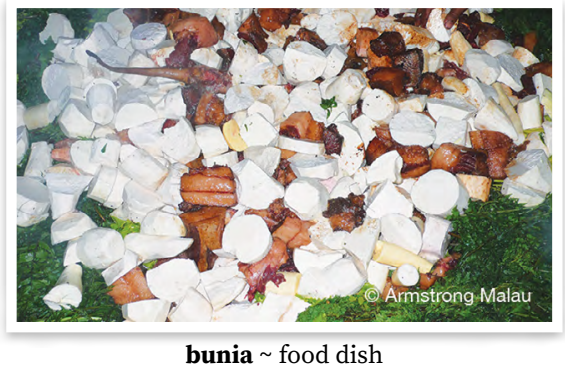

dagawis (fr.var. diwis) young leaf of breadfruit, which is cooked and eaten as greens $\sim$ top blong bredfrut, blong kakae olsem kabis

döwis soft edible tips of breadfruit leaves top blong bredfrut

garat food scraps (e.g. taro skin, fish bones) doti blong kakae (olsem bun blong fis, skin blong taro)

gargarmamas fast, refrain from eating certain foods for a particular reason $\sim$ lego kakae mo dring

garte 1 - leftovers, part remaining from something pat we i stap yet 2 - leftovers, food that someone has left uneaten $\sim$ hafkakae gartan her/his/its leftovers hafkakae blong hem

garten nōtōn her child's leftovers hafkakae blong pikinini blong hem gartëk my leftovers hafkakae blong mi kör dried breadfruit biscuit, used as famine food after hurricane bredfrut biskit

lōk laplap, food dish made from grated tubers or banana, generally cooked in stone oven laplap

wōr lōk simboro, food dish made from grated banana or tubers, wrapped in leaves of Abelmoschus manihot and cooked in coconut milk $\sim$ simboro

lōlōs food dish that is made from grated canarium nuts and datamal leaves, wrapped up and cooked on hot stones wan kaen kakae we ol i mekem smolsmol pasel blong datamal lif wetem nangae we ol i bin rasras mo ol i paselem mo putum antap long hot ston

lör kind of laplap that has grated canarium nuts mixed in before being spread out and wrapped laplap we yu miksim wetem nangae, afta jas paselem

löt 1 - kind of food dish made from breadfruit, taro or cassava, which is boiled or roasted, pounded to make a thick paste, then mixed or covered with coconut cream or nuts $\sim$ nalot 2 . make löt mekem nalot

löt bulmarwe kind of food dish made from pounded root vegetables or breadfruit, which has grated roasted cutnuts on top wan kaen nalot we i gat drae navele antap long hem we ol i bin rasras

löt kel kind of food dish made from pounded root vegetables or breadfruit, which has roasted cutnuts mixed with sugarcane juice on top wan kaen nalot we $\mathrm{i}$ gat drae navele antap we ol i bin miksim wetem wota blong sugaken 
löt matwōnwōn kind of food dish made from pounded breadfruit, with a hole in the centre that is filled with grated cutnuts, cooked with hot volcanic stones $\sim$ wan kaen bredfrut nalot we ol i kilim finis mo afta rasras grin navele, putum insaed long hol long medel wetem hot ston, kavremap mo livim i dan (10-15 minit)

löt n̄e mereñ kind of food dish made from pounded root vegetables or breadfruit, with grated roasted canarium nuts on top wan kaen nalot we $i$ gat drae nangae antap long hem

löt ne qar kind of food dish made from pounded root vegetables or breadfruit, with grated raw canarium nuts on top wan kaen nalot we i gat grin nangae antap

löt salsim kind of food dish made from pounded root vegetables or breadfruit, topped with coconut milk that has been cooked until the oil separates from the caramelised coconut wan kaen nalot we ol i putum melek kokonas antap we ol i bin kukum go go i braon mo oel blong hem i kamaot

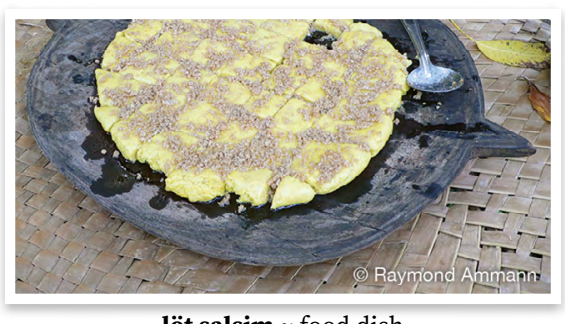

löt teqesēg kind of food dish made from pounded taro, with coconut milk poured in a hole in the centre, then the whole dish is wrapped in heliconia leaves and cooked over hot coals wan kaen taro nalot we ol i mekem hol long medel, melekem kokonas insaed, paselem mo putum antap long faea löt vasgēr kind of food dish made from pounded root vegetables or breadfruit, topped with caramelised coconut cream and oil that has been cooked in a coconut shell over the fire wan kaen nalot wetem kokonas antap. Ol i melekem kokonas long sel kokonas mo putum antap long faea blong mekem se oel i kamaot mo kokonas i braon

löt wirsal kind of food dish made from pounded root vegetables or breadfruit, topped with coconut cream that has been cooked by the heat of a hot stone inside a coconut shell wan kaen nalot we ol i putum melek kokonas antap. Ol i melekem insaed long sel kokonas we i gat hot ston insaed

löt wotag kind of food dish made from pounded root vegetables or breadfruit, topped with grated roasted cutnuts $\sim$ wan kaen nalot we $i$ gat drae navele antap

löt wötön̄ö kind of food dish made from pounded root vegetables or breadfruit, with a hole in the centre filled with grated green coconut flesh, which then has hot volcanic stones added, and is wrapped in heliconia leaves so that the coconut cooks wan kaen nalot we ol i rasras frut blong kokonas we i no grin be i no drae yet. Ol i kilim nalot finis, afta ol i mekem hol long medel mo putum kokonas wetem hot ston insaed. Afta ol i kavremap wetem lif laplap mo livim i dan (5 o 10 minit).

meles leftover food, food from previous day, which may need to be reheated namalas, kol kakae, hafkakae

mamas grated canarium nuts wrapped in datamal leaves and baked in a stone oven $\sim$ nangae we ol i pasolem long lif wetem lif blong datañal mo bekem long oven 
naw $\mathbf{1} \cdot$ sea $\sim$ solwota $\mathbf{2} \cdot$ sea water, salty water $\sim$ solwota $\mathbf{3} \cdot$ wave (of sea) $\sim$ wef (blong solwota) $4 \cdot$ salt $\sim$ sol

dōrdōrsenaw tide mark mak we solwota i kam so, we yu harem noes blong solwota long ples ia

nawēut rough sea solwota i raf

rirve if one only has a small amount of meat, soak small pieces of it in salty water, then when it is eaten with a meal, one will feel that it is sufficient sapos yu gat smol mit nomo blong kakae wetem kakae, sapos yu tekem smolsmol pis mit ia mo yu putum long wota wetem sol, bae i mekem se yu fulap long smol mit

rov $_{1}$ food dish made by mixing various leafy greens with grated raw canarium nuts, wrapping them and baking in stone oven wan kaen kakae we ol i rasras grin nangae mo paselem wetem defren kaen kabis, afta ol i bekem

salōqōs kind of food dish wan kaen nalot wetem melek blong kokonas antap. Ol i kukum kokonas blong mekem se i tik be i no braon

siaw $_{1}$ food dish in which sliced ripe breadfruit is layered with canarium nuts, wrapped and baked in stone oven wan kaen kakae we ol i slaesem raep bredfrut mo lerem wetem nangae we ol $\mathrm{i}$ bin rasras

sinag $\mathbf{1} \cdot$ taro $\sim$ taro $\mathbf{2} \cdot$ food, staple food kakae

tivtiv $_{2}$ cover made from sago palm leaves, used to cover breadfruit when baking in stone oven in order to keep heat in wan samting we ol i somap lif natanggura mo yusum blong kavremap bredfrut long oven blong kipim hit i stap insaed

wam̄amas $_{2}$ food dish in which grated canarium nuts are rolled up in datamal leaves, then wrapped in heliconia leaves and baked in stone oven kaen kakae we ol i rasras nangae, rolem long lif dataīal, pasolem long lif laplap, afta bekem

wewe kind of laplap that is rolled in island cabbage and then cooked in saucepan rather than baked laplap simboro

wëmën̄ël snack food (fruit, nuts) smol kakae olsem frut, nat

wir kind of laplap that is rolled in island cabbage, cooked in saucepan, sliced, then has coconut milk squeezed on top laplap simboro we ol i kukum fastaem, afta ol i slaesem mo melekem

wirmamēgin kind of food dish wan kaen nalot we ol i kilim nalot finis, ol i melekem kokonas stret antap

wōrkelkel kind of laplap that is made by mixing tuber or banana with coconut and wrapping in parcels to bake in oven wan kaen laplap we ol i salem maniok o wael yam o banana o kumala mo ol i miksim wetem melek kokonas bifo ol i bekem

\section{U2 Narcotics}

gē $\mathbf{1}$ - kava plant $\sim$ kava Piper methysticum $\mathbf{2} \cdot$ drink prepared from the roots of the kava plant $\sim$ kava we ol i mekem dring aot long rus blong hem

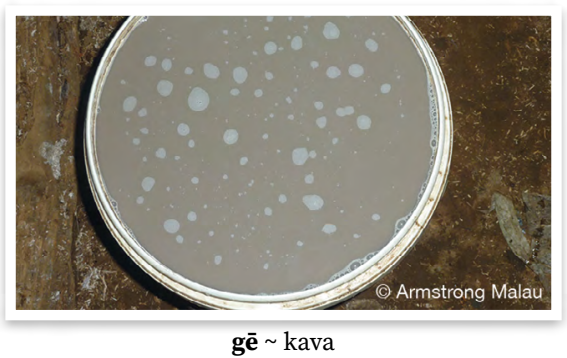

is homebrew made from yeast $\sim$ dring we ol i mekem long yis

sum drink dring

sumsum $\mathbf{1} \cdot$ drink $\sim$ dring $\mathbf{2} \cdot$ alcohol, alcoholic beverage $\sim$ dring, alkohol 


\section{Thinking, perception and attention}

V1 Sense and perception

\section{V1.1 Seeing}

èl $\mathbf{1} \cdot$ see $\sim$ luk $2 \cdot$ look lukim èlēl $1 \cdot$ view, vision, insight, opinion $\sim$ lukluk 2・ light laet

èl rak recognise, realise (by seeing) luk save

rērēt peep, peek, look secretively or with particular intent $\sim$ pusum hed blong luk haed

sēk look for lukaotem

V1.2 Hearing

rōn̄teg $\mathbf{1}$ ' hear harem $\mathbf{2} \cdot$ feel, sense $\sim$ harem, filim

tinqōōr listen (to) lesen

V1.3 Tasting and smelling

rōn̄ōbōn smell, passive sense of smell $\sim$ harem smel

sōbōn smell, sniff scent, active sensing of smell smelem

\section{V1.4 Physical sensations}

gis $1 \cdot$ hold, carry, hold onto $\sim$ holem, karem $\mathbf{2} \cdot$ touch $\sim$ tajem $\mathbf{3} \cdot$ host, hold celebration, ceremony $\sim$ hostem lafet, seremoni

rōn̄teg $\mathbf{1}$ • hear harem $\mathbf{2}$ • feel, sense $\sim$ harem, filim

V2 Cognition

\section{V2.1 Mental states}

malaklak $\mathbf{1} \cdot$ happy $\sim \operatorname{glad} \mathbf{2} \cdot$ happiness $\sim \operatorname{glad}$

metegrōw afraid, frightened $\sim$ fraet mēr $\mathbf{1} \cdot$ angry, be angry $\sim \operatorname{kros} \mathbf{2} \cdot$ anger $\sim$ kros

mëtëwtëw 1 - afraid, frightened fraet $\mathbf{2} \cdot$ afraid of, frightened of $\sim$ fraet long

mā̄al surprise $\sim$ sapraes qaqa 1 - silly, ridiculous behaviour, ridiculous behaving person krangke, krangke fasin 2 - crazy person, behave in a ridiculous way $\sim$ mekem ol samting we i no stret olsem i hafmad; man we i krangke 3 - stupidly, not properly, randomly olbaot, long krangke fasin

qeneg $\mathbf{1}$-afraid, frightened $\sim$ fraet $\mathbf{2}$ • fear, be afraid of $\sim$ fraet long qiriag confuse $\sim$ konfiusem rōrōn̄ $\mathbf{1} \cdot$ slowly sloslo $\mathbf{2} \cdot$ quietly kwaet $\mathbf{3} \cdot$ calmly, still $\sim$ kwaet tutuag worry, suffer $\sim$ wari

\section{V2.2 Mental processes and experiences}

dödöm think, think that, think about $\sim$ ting, ting se

dödöm tutun think strongly, be determined $\sim$ tingting strong blong mekem wan samting

dödömia 1 - thought, thinking, idea $\sim$ tingting $\mathbf{2} \cdot$ meaning $\sim$ mining

döm kēl think about, remember tingbaot

döm mav $\mathbf{1} \cdot$ respect $\sim$ ting hevi, respek $2 \cdot$ respect $\sim$ respek

döm siër think about, think of tingbaot

döm viteg 1 - forgive fogivim 2 • forgiveness $\sim$ fogivnes

ēl rak recognise, realise (by seeing) luk save

gilal $\mathbf{1} \cdot$ know $\sim$ save $\mathbf{2} \cdot$ knowledge, wisdom $\sim$ save, waes $\mathbf{3} \cdot$ jealous, express jealousy jalus

lugög plan plan

luw count (something) kaontem

matgagar anger ae i kros

nōmtuv 1 • believe biliv $2 \cdot$ belief $~$ bilif

qōr forget $\sim$ foget, fogetem 
qōrqōr $\mathbf{1} \cdot$ dream $\sim$ drim $\mathbf{2} \cdot$ dream about $\sim$ drim long $\mathbf{3} \cdot$ dream $\sim$ drim $\mathbf{r e ̄ v _ { 2 }}$ write $\sim$ raetem

rōñteg rak understand, sense, comprehend, realise $\sim$ harem save vasērēg 1 - think about, consider tingbaot $\mathbf{2} \cdot$ idea, thought $\sim$ tingting vasōgō read ridim

vēvēg ${ }_{1}$ count (something) kaontem

V2.3 Knowledge and learning

daēl try traem

dun $\mathbf{1} \cdot$ true, be true, real $\sim$ tru $2 \cdot$ sure, tell truth $\sim$ sua, tru $3 \cdot$ truth $\sim$ tru gilal $1 \cdot$ know save $2 \cdot$ knowledge, wisdom save, waes $\mathbf{3} \cdot$ jealous, express jealousy jalus

lōlōmeren $\mathbf{1} \cdot$ wise, intelligent $\sim$ waes $2 \cdot$ wisdom, knowledge $\sim$ waes, save mav $1 \cdot$ heavy hevi, no laet $2 \cdot$ difficult, hard, not easy $\sim$ had, no isi qaq totono advice $\sim$ advaes

skul 1 • school skul 2 - go to school, carry out schooling skul, go long skul

tono $\mathbf{1}$ - learn lanem 2 - teach, instruct $\sim$ lanem, tijim

vasösöv advise, instruct givim advaes long

\section{W Handling and physical transfer}

\section{Wo General}

salmiat be ready, be prepared $\sim$ redi taōrmat 1 - be ready, be prepared redi $2 \cdot$ prepare, make something ready $\sim$ mekem redi

da taōrmat prepare $\sim$ mekem redi

W1 Taking, touching, obtaining

bet pick up pikimap

bël $1 \cdot$ steal $\sim$ stilim $2 \cdot$ record, with audio or video recorder $\sim$ rikodem voes o video

dēñ $\mathbf{1} \bullet$ arrive $\sim$ kasem $\mathbf{2} \bullet$ reach, arrive at $\sim$ kasem

gövut pull out something that is tightly held within something, as in ground, uproot pulumaot wan samting we $i$ fas gud bitwin o insaed long wan samting, olsem pulumaot wud long graon

kal ${ }_{1}$ pull in fishing line, pull up fish pulum string

kulum $_{3}$ gather together in bundle and tie up mekem bandel mo fasem taet le 1 • take tekem $\mathbf{2} \cdot$ get, bring karem, karem i kam $\mathbf{3} \cdot$ give $\sim$ givim le den remove, take away, take out karemaot

mem put putum

nës put into (container) fulumap insaed wan samting

qal 1 • affect, attain, reach $\sim$ kasem 2 • be first kam faswan

$\mathbf{s a n}$ touch, hold just with fingertips $\sim$ holem, tajem be wetem fingga nomo, i no wetem hol hand

sar $_{4}$ pull out, referring to pulling taro from ground pulumaot, olsem pulumaot taro long graon

sër ${ }_{3}$ wear, put on (clothes, accessories)

$\sim$ werem, havem (klos, savat)

sër lō take off, remove (clothes, accessories) karemaot (klos)

sëwëw grab, catch something with quick movement kasem kwiktaem nomo

soko catch $\sim$ kasem

sōk find faenem

sön̄ön put inside (container) fulumap insaed

sösögöt find faenem 
tañqal touch (with hand) tajem (wetem han)

tēk touch gently tajem sloslo, tajem smol

tiqër grab with two hands, tackle, wrap arms around kasem, holem taet

tut ${ }_{3}$ pull using quick sharp motion pulum taet hariap

ut collect water $\sim$ kasem wota

wö̀wör weed $\sim$ widim

W2 Holding, carrying, moving something around

bēm ${ }_{2}$ carry (baby) with piece of cloth, carry person or thing on back karem pikinini o man long baksaed

bēmn̄eg (fr.var. bimn̄eg) carry on shoulder, hang over shoulder, as carrying basket with strap over shoulder and basket hanging down karem long solda, hangem long solda, olsem basket

gan̄veg lead lidim

kuëg shake, move, push on swing sekem muvum, pusum

lik serve food, remove food from oven sevem kakae, karemaot kakae long oven

rak $_{2}$ lift, lift up leftemap

$\mathbf{r e ̄ v}_{1}$ pull pulum

rêv vasese pull canoe out of the water to the tideline, so it is half in and half out, in case a quick getaway is needed pulum kenu i kam antap smol nomo, haf long solwota, haf aotsaed, blong mekem se yu save pulum i go daon hariap sapos yu wantem go kwiktaem

rieg away from, out, out of the way aot long

sas carry something heavy, holding with one hand on either side, or two people carrying, one on each side $\sim$ karem, wan i holem wan saed, narawan i holem narasaed, o wan man i yusum tu han blong leftemap

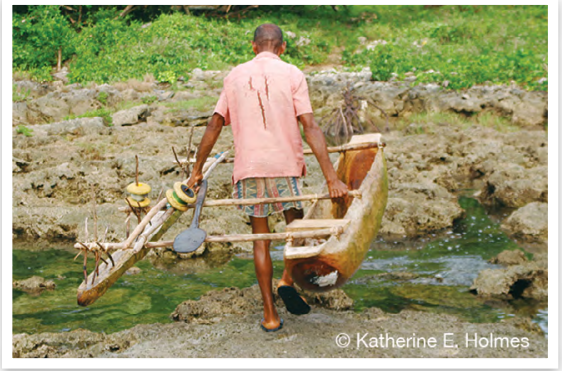

sas carry

sesen carry something heavy, holding with one hand on either side, or two people carrying, one on each side $\sim$ karem, wan i holem wan saed, narawan i holem narasaed, o wan man i yusum tu han blong leftemap sër ${ }_{1} \mathbf{1} \cdot$ spill, be spilt $\sim$ kapsaed $\mathbf{2} \cdot$ spill, pour, tip out (anything, either liquid or solid) kapsaedem

sōltek carry on one shoulder, especially heavy items $\sim$ karem (wan hevi samting) long wan solda

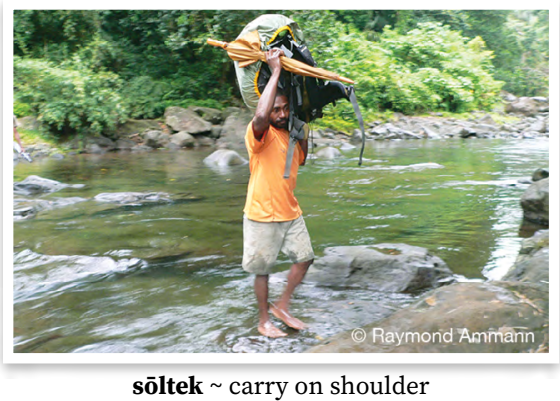

sōsōrvël carry using length of wood over shoulder, with half the load in front and half behind $\sim$ karem long wud long solda

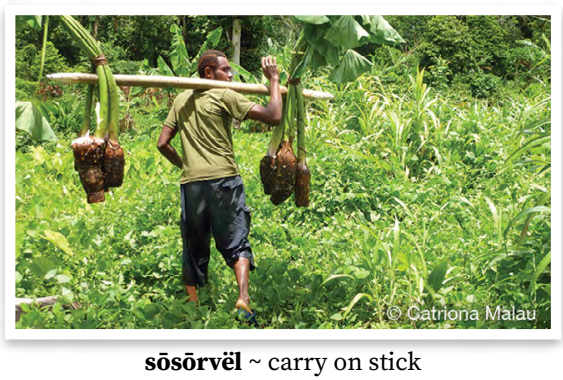


tatalēv carry load hanging on a stick held over shoulder karem wetem stik ova long wan solda

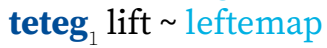

tiwil roll, toss around $\sim$ rolem

turm̄̄ō go first, be in front, lead stanap fastaem, go fastaem, lidim

vetrug stand up, erect stanemap

vëtur place in piles, pile up hipimap

W3 Letting go, giving, leaving be

bōl 1 - throw (something roundish and short) at $\sim$ stonem (wetem raon, sotfala samting) $2 \cdot$ fall down onto (of something heavy) (wan samting hevi) foldaon i go antap long

dun̄ög leave something with someone, leave something behind putum wan samting, livim i stap (olsem se bae yu jas kambak from o man i karem i kambak o wan i kam from)

gasēg share out, separate, divide seraotem

gatöqul throw (something long) at stonem (wetem longfala samting)

le 1 •take tekem 2 - get, bring karem, karem i kam $\mathbf{3} \cdot$ give $\sim$ givim

lër mix miksim

lin pour into something kapsaedem i go long wan samting qērēt push, push in button, push something down, press $\sim$ pusum

sër ${ }_{1} \mathbf{1} \cdot$ spill, be spilt $\sim$ kapsaed $\mathbf{2} \cdot$ spill, pour, tip out (anything, either liquid or solid) kapsaedem

susgeg push (stake or length of other material) into tight narrow space pusum (stik o samting olsem) i go fas long wan hol

susn̄eg push pusum

töqul throw away sakem

tövun (fr.var. tuvun) $\mathbf{1}$ • bury berem $2 \cdot$ fill in (hole), cover over something in hole $\sim$ berem

ukuëg 1 - depart, leave go, lego $\mathbf{2}$ - leave (place, person) lego $\mathbf{3}$ • release, let go of, allow to happen, let $\sim$ lego wan samting we yu holem 4 - leave something to continue, especially continue unaided for a time lego i stap

vēl ${ }_{3}$ divide, distribute, separate seraotem

vun 1 pour water into oven to produce steam by which food will cook kapsaedem wota antap long kakae long oven

wöt $1 \cdot$ throw, throw away $\sim$ sakem $2 \cdot$ contribute, give $\sim$ givim, sakem

\section{$X$ Impact and surface contact}

\section{Xo General}

da titisē damage, affect something in a negative way $\sim$ spolem

kōn snap, bend and break brekem

lam bash, hit, beat strongly kilim strong

lam̄eg bash against something hard, like a stone $\sim$ kilim long ston o long wan samting we i strong

qēs bang against something bangem $\mathbf{q} \overline{\mathbf{o}} \mathbf{s}_{1} \mathbf{1} \cdot$ pound, as in pounding löt with pestle, pounding clothes against rock $\sim$ kilim, olsem blong mekem nalot 2 - wash (clothes) $\sim$ wasem (klos)

qōs do pound leaf used for poisoning fish and mix with sand $\sim$ stonem lif blong posenem fis mo miksim wetem sanbij

sēr tear, rip terem

töwös hit kilim 
tut ${ }_{1}$ punch faetem

weteg crack, break open, split splitim

wor break, split open $\sim$ brekem

wos $_{1} \mathbf{1}$ - bash, slap, pound; hammer, bash (nuts) to crack open $\sim$ hamarem 2 build bildim

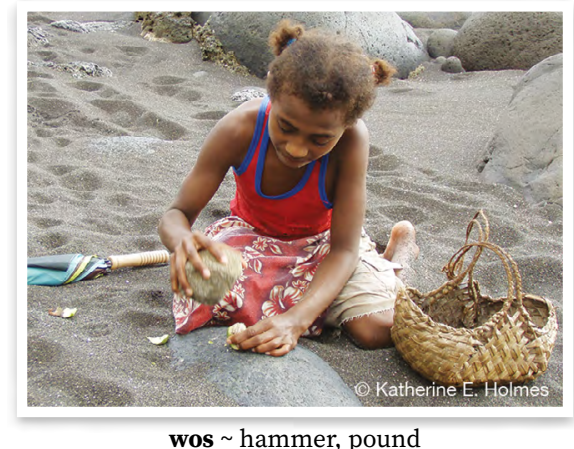

\section{X1 With an instrument}

bal $_{1} \mathbf{1} \cdot$ use wooden tongs to pick up hot stones or charcoal from stone oven $\sim$ tekemaot ston o jakol long oven wetem wud $\mathbf{2}$ - cut (with scissors) katem (wetem sisis)

es $1 \cdot$ spear sperem $2 \cdot$ pierce, poke, use sharp pointed object to pierce something stikim, yusum wan samting we i sap long en blong hem blong stikim wan samting

es do pound leaves for making fish poison mekem posen lif, sperem lif wetem wud blong mekem plante es mōtō remove husk from coconut sperem kokonas blong karemaot skin

ger chop, cut using chopping action with considerable effort required for effect, as object is solid, hard katem, olsem yu katem wan samting we i strong mo yu mas hadwok long hem

ras $1 \cdot$ grate nuts or tubers for nalot or laplap rasras, salem kakae $\mathbf{2}$ • sharpen $\sim$ sapenem $\mathbf{r e ̄ v _ { 2 }}$ write $\sim$ raetem

rōt cut with shell or small knife, placing finger on edge of knife or shell to guide and support the cutting action katem long naef o sel mo yu yusum fingga blong gaedem

sus 1 - poke, prod, pierce stikim $2 \cdot$ point (with finger) $\sim$ poen (wetem fingga) $\mathbf{3} \cdot$ inject, give injection to stikim (wetem stik meresin)

$\operatorname{tar}_{1}$ chop, cut using chopping action with considerable effort required for effect, as object is solid, hard katem, olsem long akis we i hadwok blong mekem

tes $_{2}$ carve, use knife or chisel to cut and shape something from wood katem wud

tes ak carve canoe $\sim$ katem kenu

tè-q $\mathbf{q}_{2}$ shoot (with gun) sutum (wetem masket)

tēv $\mathbf{1}$ • cut, slice $\sim$ katem, slaesem $\mathbf{2}$ • operate on $\sim$ katem (long hospitel)

tōt cut using chopping action, with minimal effort required for effect katem, jopem, be i no kadwok tumas

tutgëg stick, poke something into something stikim wan samting

vēn shoot (with bow and arrow or spear) sutum (wetem bonaro o spia)

vēnvēn go shooting go blong sut

\section{X2 Due to pressure}

bis pinch and twist to break off, as in picking (fruit) from tree $\sim$ karem (frut) long tri

qērēt push, push in button, push something down, press $\sim$ pusum

qirir grind kava graonem

wag $_{2}$ squeeze with hand, like massaging skwisim long han, olsem yu holem man 
X3 Sticking, tying, untying

lölös $\mathbf{1} \cdot$ wrap up pasolem $\mathbf{2} \cdot$ parcel $\sim$ pasol

mon $\mathbf{1} \cdot$ wrap up $\sim$ pasolem $\mathbf{2} \cdot$ parcel $\sim$ pasol

sö 1 • join joenem $2 \cdot$ joint, as in joint where two bones meet, joint in crafted wooden item joen, olsem joen blong tufala bun o joen long wud

ul 1 - come undone, unfastened kamaot (olsem samting we ol i bin fasem) 2 - undo, untie, unwrap, unfasten slakem, openemaot, karemaot (rop)

wōn 1 1 join, close up, seal together tufala saed i mit, olsem fasem lif, tufala saed i mit antap, kaliko i brok, joenem mo somap bakegen 2 • seal (hole in), join together, close up joenem, silim

wōnwōn ${ }_{1}$ whole, joined, complete fulwan

wöwös 1 - wrap up, most commonly used to refer to making a cone shape from leaf, filling it, then sealing pasolem, speseli ol i talem taem we ol i foldem lif olsem kon, afta pasolem kakae insaed 2 - parcel, wrapped up goods, food pasol, kakae o wan samting we ol i bin pasolem

\section{X4 Surface contact}

as $\mathbf{1} \cdot$ rub $\sim$ rabem $\mathbf{2}$ - wash, scrub (plates) wasem

er 1 peel off, remove pandanus leaves from trunk, cut around and peel off layer of wood from tree trunk karemaot pandanas long stamba

gör grate (coconut), scrape out insides of something firm skrasem kokonas, skrasemaot insaed long wan samting we i strong keres scratch, use scratching motion to dig skrasem, digim

kër ${ }_{1}$ scratch with fingernails skrasem, wetem sel fingga

men̄ wipe $\sim$ waepem

nem lick likim

rërës scratch skrasem

riam $_{1}$ rub rabem

$\mathbf{s} \overline{\mathbf{e}}_{1}$ peel bark from wood $\sim$ karemaot skin long wud

sēm 1 • peel, remove skin from (something hard, as uncooked vegetables, copra) tekemaot skin (blong samting we i strong olsem taro o kopra) 2 - remove coconut flesh from shell and husk, to produce copra selaotem

sërëvtës slip glis

sialtislañ slip glis

sir 1 - scrape, scrape dirt from the outside of taro and other root vegetables skrasem doti (blong taro) $\mathbf{2}$ - remove skin, bark tekemaot skin

siriv peel dried pandanus leaves from the plant $\sim$ karemaot lif pandanas we i drae finis long stamba

tëtës massage holem (bodi)

$\operatorname{var}_{1} \mathbf{1} \cdot$ stomp, tread heavily on prumbut $\mathbf{2}$ go in upwards direction $\sim$ go antap $\mathbf{3}$ - stand on, kick something with stanap long, kik samting wetem

vidir (fr.var. vēdir) $\mathbf{1} \cdot$ remove midrib from sago palm leaf $~$ tekemaot bun blong lif natanggura 2 - shoot liaw fish sutum liaw fis

wul peel, remove skin (from something that is soft and easy to peel, like a banana or vegetables that have been cooked) tekemaot skin (blong samting we $\mathrm{i}$ isi olsem banana o bredfrut we i dan)

wur sweep brum

wurveg $_{1}$ clear, clean, prepare for something klinim 


\section{Y Speed, manner and relative time}

YO General

gem (fr.var. gëm) $\mathbf{1} \cdot$ just $\sim$ nomo $\mathbf{2}$ • only, just nomo

kara just, of an event that has happened very recently or will happen very shortly after an upcoming event $\sim$ jas

manene reason $\sim$ risen

sisia different $\sim$ defren

\section{Y1 Speed and manner}

gamlöt $\mathbf{1} \cdot$ quickly hariap, kwiktaem

$2 \cdot$ be quick, hurry up go hariap

gem (fr.var. gëm) $\mathbf{1} \cdot$ just $~$ nomo $\mathbf{2}$ • only, just nomo

kara just, of an event that has happened very recently or will happen very shortly after an upcoming event $\sim$ jas

lelev fast, be fast $\sim$ hariap

manene reason $\sim$ risen

mav 1 - heavy hevi, no laet 2 • difficult, hard, not easy had, no isi meneg $1 \cdot$ hard, be hard strong, olsem i no sofsof $\mathbf{2}$ - be strong, powerful strong, olsem i gat paoa 3 - intense $\sim$ strong, olsem i gat paoa blong hem $\mathbf{4} \cdot$ be determined strong, olsem fasin i strong

mölumlum $\mathbf{1} \cdot$ soft $\sim$ sofsof $\mathbf{2} \cdot$ slow, slowly slo, sloslo

möul 1 - for a long time, take a long time $\sim$ tekem longtaem $2 \cdot$ slow, be slow sloslo

sisia different $\sim$ defren

tenēnēg 1 - straight, not bent or curved stret, no kruked $\mathbf{2} \cdot$ correct, right, correctly $\sim$ stret, gud

valakreg randomly, not in ordered way olbaot

wareg $1 \cdot$ well, completely, totally gud $\mathbf{2} \cdot$ properly $\sim$ stret

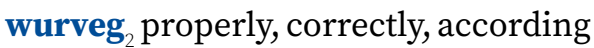
to one's aim or desire stret, gud

Y2 Relative time and duration

garqe $\mathbf{1} \cdot$ today $\sim$ tede $\mathbf{2} \cdot$ later on today, today in the afternoon or evening; earlier today, earlier the same day tede long aftenun, tede long moning finis $3 \cdot$ at the present time or age; these days $\sim$ long taem tede

la masawre when, introduces temporal adverbial clause $\sim$ taem

$\mathbf{l a v}_{1}$ forever, without ceasing oltaem, wantaem

möul 1 - for a long time, take a long time $\sim$ tekem longtaem $\mathbf{2} \cdot$ slow, be slow sloslo

orēs time period in the near future, after tomorrow afta tumoro

tastas always $\sim$ evri taem

tōlōw tomorrow $\sim$ tumoro

vaga $1 \cdot$ always, all the time $\sim$ oltaem, evritaem $\mathbf{2}$ often, regularly $\sim$ fulap taem

$\operatorname{van}_{1} \mathbf{1} \cdot$ go (unmarked for direction) go $\mathbf{2}$ - on and on, postverbal particle indicating continuous aspect, continuation of an activity $\sim$ go go

vatiwial $\mathbf{1} \cdot$ once $\sim$ wantaem $\mathbf{2}$ - at once, straight away wantaem

vèti (fr.var. viti) still yet

\section{Y3 Serialisation}

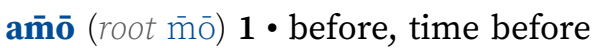
now $\sim$ bifo, fastaem $\mathbf{2}$ - first, in front $\sim$ fastaem

basbasne last laswan

bilēñ (fr.var. bilēn) $\mathbf{1} \cdot$ again, modifies verb in the verb complex $\sim$ bakegen $2 \cdot$ as well, too, modifies noun in the noun phrase $\sim$ tu $3 \cdot$ more, other, another, modifier in the noun phrase moa, bakegen 


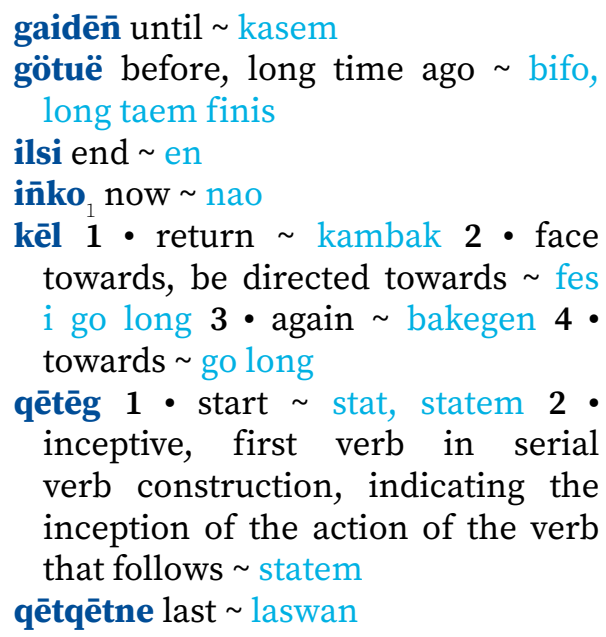

Y4 Degree

döl all evriwan

gem (fr.var. gëm) $\mathbf{1} \cdot$ just $\sim$ nomo $\mathbf{2} \bullet$ only, just $\sim$ nomo

idian very, really, too, too much, degree adverb tumas

kara just, of an event that has happened very recently or will happen very shortly after an upcoming event $\sim$ jas

nimal very, really, too, too much, degree adverb tumas

nötu (fr.var. sönötu; sunötu) $\mathbf{1} \cdot$ small, be small, become small smol

ren emphatic marker; right, absolutely, totally, definitely tumas, tru, stret; $i$ makem strong toktok we yu talemaot

rivteg 1 - near, close by $\sim$ klosap $\mathbf{2} \cdot$ near to, close to $\sim$ klosap long $\mathbf{3} \cdot$ nearly, soon $\sim$ klosap

vilēs all, all over evriwan 


\section{Appendix}

This appendix contains two tables that list all of the recorded Vurës texts that together form the corpus from which this dictionary has been created. Many of the entries in the dictionary include an example sentence to illustrate the meaning of the headword. Most of these examples have been taken from recorded texts, which are publically available in digital archives. If this is the case, the example is followed by a reference, which looks like this: (CAA.061). The reference inside the brackets identifies the text from which the example was taken, and in some cases also indicates a sentence number. This list of texts is included for any interested dictionary users who wish to access the original recordings and hear examples being spoken.

Table 1 includes a list of all texts which are stored in the PARADISEC and ELAR archives. Column 1 in the table presents the abbreviated code for the text I have used in the dictionary references. Column 2 gives the longer file name, which is used in the PARADISEC archive, found at: catalog.paradisec.org.au/collections/ $\mathrm{CH} 1 /$. My collection is all stored under the collection ID CH1. Column 3 then identifies the names of the Vurës speakers who are in the recordings.

Table 1: Texts stored in PARADISEC and ELAR archives

\begin{tabular}{|l|l|l|}
\hline Code & Tape & Speaker(s) \\
\hline AAD & (Written) & Armstrong M̄ëglemete Malau \\
\hline ASS & CH1-200202-AASS & Andrew Bō \\
\hline DFF & CH1-200202-ADFF & Dudley Lōlōwia \\
\hline ESS & CH1-200202-AESS & Emely Rēlin̄veg Qiat \\
\hline EWB & CH1-200202-AEWB & Emely Rēlin̄veg Qiat \\
\hline JTF & CH1-200202-AJTF & Jesse Rēlin̄ Gōrbē \\
\hline RBH & CH1-200202-BRBH & Roy Wutōt Lōmegev Kipe \\
\hline RPT & CH1-200202-BRPT & Roy Wutōt Lōmegev Kipe \\
\hline RRS & CH1-200202-BRRS & Roy Wutōt Lōmegev Kipe \\
\hline RTF & CH1-200202-BRTF & Roy Wutōt Lōmegev Kipe \\
\hline WRN & CH1-200202-BWRN & Welsam Segir Malau Qiat \\
\hline DNS & CH1-200203-ADNS & Doran Rörösōq \\
\hline NBQ & CH1-200203-ANBQ & Noris Menegqiat \\
\hline
\end{tabular}


A DICTIONARY OF VURËS, VANUATU

\begin{tabular}{|c|c|c|}
\hline Code & Tape & Speaker(s) \\
\hline RKM & CH1-200203-ARKM & Roy Wutōt Lōmegev Kipe \\
\hline DDL & CH1-200203-BDDL & Drusila Winnie \\
\hline JMT & CH1-200203-BJMT & Jimmy Brown \\
\hline HWM & CH1-200203-HWM & Hosea Waras \\
\hline DHN & CH1-200204-ADHN & Kathy Doris \\
\hline ESP & CH1-200204-AESP & Eli Field Malau Vutvut \\
\hline JWM & CH1-200204-AJWM & Joana Leo \\
\hline $\mathrm{KBN}$ & CH1-200204-AKBN & Kali Rēgōrlē Malau \\
\hline CAA & CH1-200204-BCAA & $\begin{array}{l}\text { Doran Rörösōq, Joana Leo, Noris Meneg Qiat, } \\
\text { Emely Rēlin̄veg Qiat }\end{array}$ \\
\hline DDP & CH1-200204-BDDP & Doran Rörösōq \\
\hline VSB & CH1-200205-VSB & Brian Manliwōs \\
\hline VSE & CH1-200205-VSE & Eli Field Malau \\
\hline VSG & CH1-200205-VSG & Jerry Gilbert \\
\hline VSJ & CH1-200205-VSJ & Seveniah \\
\hline VSM & CH1-200205-VSM & Jeremiah Malau \\
\hline VSS & CH1-200205-VSS & Samson Blondel \\
\hline VST & CH1-200205-VST & Tomas Sakalmes \\
\hline VSV & CH1-200205-VSV & John Vira \\
\hline ATB & CH1-200206-АATB & Armstrong M̄ëglemete Malau \\
\hline AWW & CH1-200206-BAWW & Armstrong M̄ëglemete Malau \\
\hline BQD & CH1-200206-BBQD & Barnabas Manar \\
\hline SBB & CH1-200206-BSBB & Steven Bet \\
\hline $\mathrm{KM} \overline{\mathrm{MT}}$ & CH1-200206-KMT & Kate Ruth \\
\hline M2D & CH1-200207-M2D & Kathy Doris \& Kate Ruth \\
\hline M2E & CH1-200207-M2E & Eli Field Malau \& Roy Kipe \\
\hline M3E & CH1-200207-M3E & Eli Field Malau \& Roy Kipe \\
\hline M4D & CH1-200207-M4D & Kathy Doris \& Kate Ruth \\
\hline M4E & CH1-200207-M4E & Eli Field Malau \& Roy Kipe \\
\hline TT2 & CH1-200207-TT2 & Kathy Doris \& Kate Ruth \\
\hline TT3 & CH1-200207-TT3 & Kathy Doris \& Kate Ruth \\
\hline JRQ & CH1-200208-AJRQ & John Elizabeth Kökör \\
\hline JNN & CH1-200208-BJNN & Jokoped Leo \\
\hline MTM & CH1-200208-BMTM & Miriam \\
\hline SSW & CH1-200208-BSSW & Paul Sēkrot \\
\hline EDM & CH1-200208-EDM & Emely Rēlin̄veg Qiat \\
\hline ATW & CH1-200209-AATW & Arthur Malvanvan \\
\hline FRN & CH1-200209-BFRN & Freda Lona Malau \\
\hline WNW & CH1-200209-BWNW & Walter Lekēl \\
\hline
\end{tabular}




\begin{tabular}{|c|c|c|}
\hline Code & Tape & Speaker(s) \\
\hline $\mathrm{DPD}$ & CH1-200209-DPD & Dērēs Laklaqiat \\
\hline DTB & CH1-200209-DTB & Dērēs Laklaqiat \\
\hline DWW & CH1-200209-DWW & Derek Wilton \\
\hline WWM̄ & CH1-200210-AWWM̄ & Nightingale Webster Liar \\
\hline BNR & CH1-200210-BBNR & Brenden Malau \\
\hline DPR & CH1-200210-BDPR & Kathy Doris \\
\hline DSR & CH1-200210-BDSR & Alice Dina Raymond \\
\hline GNR & CH1-200210-BGNR & Gōsōw Atchfield \\
\hline JSD & CH1-200210-BJSD & Jonas Sakalmes \\
\hline NRL & CH1-200210-BNRL & Nanet Jack \\
\hline OHS & CH1-200210-BOHS & Owen Qalgislē \\
\hline OSK & CH1-200210-BOSK & Obed Rereman \\
\hline RPP & CH1-200210-RPP & Ray Wanwott \\
\hline ADT & CH1-200211-AADT & Albert Mason Vörus Lōmegev \\
\hline TSR & CH1-200211-ATSR & Tam̄at Togor \\
\hline WDR & CH1-200211-AWDR & Walter Lekēl \\
\hline GEM & CH1-200211-BGEM & Father Gregory Manliwōs \\
\hline GMM & CH1-200211-BGMM & Father Gregory Manliwōs \\
\hline LLF & CH1-200211-BLLF & Arthur Linka \\
\hline OQQ & CH1-200211-BOQQ & Owen Qalgislē \\
\hline ASA & CH1-200212-ASA & Angas \\
\hline EKK & CH1-200301EKK-01 & Eli Field Malau Vutvut \\
\hline HSK & CH1-200301HSK-01 & Hosea Waras \\
\hline AGG & CH1-200401AGG-01 & Amelton Levi \\
\hline ASW & CH1-200401ASW-01 & Amelton Levi \\
\hline BCB & CH1-200401BCB-01 & Bōkrat choir \\
\hline $\mathrm{BDC}$ & CH1-200401BDC-01 & Basil Gideon \\
\hline $\mathrm{BDP}$ & CH1-200401BDP-01 & Basil Gideon \\
\hline BGF & CH1-200401BGF-01 & Bōkrat choir \\
\hline BTB & CH1-200401BTB-01 & Basil Gideon \\
\hline DMM & CH1-200401DMM-01 & Dick Cheney \\
\hline DRV & CH1-200401DRV-01 & Dimas \\
\hline DWB & CH1-200401DWB-01 & Delila Liges John \\
\hline EQG & CH1-200401EQG-01 & Emely Rēlin̄veg Qiat \\
\hline FKS & CH1-200401FKS-01 & Frankie Dickinson Silas \\
\hline HRV & CH1-200401HRV-01 & Hosea Waras \\
\hline JFH & CH1-200401JFH-01 & Joyce Edwin \\
\hline JTS & CH1-200401JTS-01 & John Elizabeth Kökör \\
\hline
\end{tabular}


A DICTIONARY OF VURËS, VANUATU

\begin{tabular}{|l|l|l|}
\hline Code & Tape & Speaker(s) \\
\hline LDP & CH1-200401LDP-01 & Livae Gōrōs \\
\hline MTM & CH1-200401MTM-01 & Miriam \\
\hline NDP & CH1-200401NDP-01 & Nellie \\
\hline SS1 & CH1-200401SS1-01 & Sabela Röuldena \\
\hline SS2 & CH1-200401SS2-01 & Sabela Röuldena \\
\hline SWG & CH1-200401SWG-01 & Sabela Röuldena \\
\hline AFH & CH1-200402AFH-01 & Albet Clemen \\
\hline AQA & CH1-200402AQA-01 & Albet Clemen \\
\hline AQD & CH1-200402AQD-01 & Albet Clemen \\
\hline EFS & CH1-200402EFS-01 & Elton John Sakalmes \\
\hline GGM & CH1-200402GGM-01 & Gladys Retelvegsöm \\
\hline GLH & CH1-200402GLH-01 & Gladys Retelvegsöm \\
\hline CAW & CH1-200403CAW-01 & $\begin{array}{l}\text { Eli Field Malau, Catriona Hyslop, Armstrong Malau, } \\
\text { Doran Rörösōq }\end{array}$ \\
\hline
\end{tabular}

Table 2 includes a list of all texts stored in the DoBeS archive. Column 1 gives the reference used in the dictionary, which is the file name for the recordings stored in the archive at: archive.mpi.nl/tla/islandora/object/tla\%3A1839_00_000 0_0000_000A_24A7_1. Column 2 then identifies the names of the Vurës people who speak in the recordings.

Table 2: Texts stored in DoBeS archive

\begin{tabular}{|l|l|}
\hline Recording name & Speaker(s) \\
\hline VVAMVDP04Jun0801-JN & Joana Leo, Nesta Riliar \\
\hline VVAMVDP04Jun0802-JN & Joana Leo, Nesta Riliar \\
\hline VVAMVDP04Jun0801-KP & Children from Laln̄evut \\
\hline VVAMVDP04Jun0802-EF & Eli Field Malau \\
\hline VVAMVDP04Jun0802-LN & Members of the Doran family \\
\hline VVAMVDP05Jun0801-LN & Members of the Doran family \\
\hline VVAMVDP05Jun0801-KB & Kali Malau, Barrington \\
\hline VVAMVDP05Jun0801-SF & Sēkrot \\
\hline VVAMVDP05Jun0801-NQ & Members of the Doran family \\
\hline VVAMVDP05Jun0801-TT & Kali Malau, Barrington \\
\hline VVAMVDP05Jun0802-DW & Dudley Lōlōwia \\
\hline VVAMVDP05Jun0802-LN & Members of the Doran family \\
\hline VVCMVDP21Nov0701-AR & Angeline Rösuvwian \\
\hline VVCMVDP21Nov0701-AS & Abigail Sōrōrbum \\
\hline VVCMVDP21Nov0701-BN & Brilliant Nomtuv \\
\hline VVCMVDP21Nov0701-GM & Godfrey Manar \\
\hline VVCMVDP21Nov0701-JL & Joana Leo \\
\hline
\end{tabular}




\begin{tabular}{|c|c|}
\hline Recording name & Speaker(s) \\
\hline VVCMVDP21Nov0701-JR & Juliana Revug \\
\hline VVCMVDP21Nov0701-VD & Brilliant Nomtuv \\
\hline VVCMVDP22Nov0701-JR & Juliana Revug \\
\hline VVCMVDP22Nov0701-KD & Kathy Doris \\
\hline VVCMVDP22Nov0701-MC & Mothy Claudia Ōlveliesbum \\
\hline VVCMVDP22Nov0702-KD & Kathy Doris \\
\hline VVCMVDP22Nov0702-OM & Olive Mōlvitegmam \\
\hline VVCMVDP22Nov0702-CO & Doris, Revug \& Olive \\
\hline VVCMVDP22Nov0703-CO & Doris, Revug \& Olive \\
\hline VVCMVDP22Nov0703-KR & Kate Ruth \\
\hline VVCMVDP22Nov0703-NR & Nesta Riliar \\
\hline VVCMVDP22Nov0704-AG & Armstrong Malau, Godden Wesley Felix, Eli Field Malau \\
\hline VVCMVDP22Nov0704-BJ & Brilliant Nomtuv, Joana Leo \\
\hline VVCMVDP22Nov0704-BN & Brilliant Nomtuv \\
\hline VVCMVDP22Nov0704-JL & Joana Leo \\
\hline VVCMVDP22Nov0704-VW & Brilliant Nomtuv \\
\hline VVCMVDP24Nov0701-NM & Noris Menegqiat \\
\hline VVCMVDP24Nov0701-RK & Roy Kipe \\
\hline VVCMVDP24Nov0701-SL & Victoria, Tewes, Armstrong, Doran, Noris \\
\hline VVCMVDP24Nov0701-VW & Brilliant Nomtuv \\
\hline VVCMVDP24Nov0702-N1 & Noris Menegqiat \\
\hline VVCMVDP24Nov0702-N2 & Noris Menegqiat \\
\hline VVCMVDP24Nov0702-N3 & Noris Menegqiat \\
\hline VVCMVDP24Nov0702-SL & Johnny Wege, Noris \\
\hline VVCMVDP24Nov0702-T1 & Helen Tewes \\
\hline VVCMVDP24Nov0702-T2 & Helen Tewes \\
\hline VVCMVDP24Nov0702-Q1 & Eli Field Malau \\
\hline VVCMVDP25Nov0701-MK & Abraham Vetreñ, Eli Field Malau \\
\hline VVCMVDP25Nov0701-Q2 & Armstrong Malau, Nesta Riliar, Joana Leo \\
\hline VVCMVDP25Nov0701-Q3 & Joana Leo \\
\hline VVCMAA30Mar13 & Eli Field Malau \\
\hline VVCMAA03Apr1301 & Eli Field Malau \\
\hline VVCMAA03Apr1302 & Eli Field Malau \\
\hline VVCMAA03Apr1303 & Eli Field Malau \\
\hline VVCMAA03Apr1304 & Eli Field Malau \\
\hline VVCMAA03Apr1305 & Eli Field Malau \\
\hline VVCMAA03Apr1306 & Eli Field Malau \\
\hline VVCMAA03Apr1307 & Eli Field Malau \\
\hline
\end{tabular}


A DICTIONARY OF VURËS, VANUATU

\begin{tabular}{|c|c|}
\hline Recording name & Speaker(s) \\
\hline VVCMAA03Apr1308 & Eli Field Malau \\
\hline VVCMAA03Apr1309 & Eli Field Malau \\
\hline VVKHVDP21Jul0701-MC & Mothy Claudia Ōlveliesbum \\
\hline VVKHVDP21Jul0701-NL & Nelson Leo \\
\hline VVKHVDP21Jul0702-NL & Nelson Leo \\
\hline VVKHVDP21Jul0702-CS & Mahi Rose Rōtōvitegbum, Nesta Riliar \\
\hline VVKHVDP21Jul0702-SW & $\begin{array}{l}\text { Mahi Rose Rōtōvitegbum, Nesta Riliar, Donna } \\
\text { Isabel Risiveglōmegev, Mothy Claudia Ōlvelesbum, } \\
\text { Angeline Wēlēñ }\end{array}$ \\
\hline VVKHVDP23Jul0701-CC & Graham Mumeg \\
\hline VVKHVDP23Jul0701-CF & Nesta Kathleen, Minnie Ēllavbum \\
\hline VVKHVDP23Jul0701-EF & Eli Field Malau \\
\hline VVKHVDP23Jul0701-FH & Nesta Kathleen, Minnie Ēllavbum \\
\hline VVKHVDP29Jul0701-CC & Graham Mumeg \\
\hline VVKHVDP29Jul0701-EF & Eli Field Malau \\
\hline VVKHVDP29Jul0701-JR & Joana Leo \\
\hline VVKHVDP29Jul0701-NT & Nicegella Tiqu \\
\hline VVKHVDP01Aug0701-CF & Cecilia Fay Serig \\
\hline VVKHVDP01Aug0701-CR & Cliff Richard \\
\hline VVKHVDP01Aug0701-DS & Dickinson Silas \\
\hline VVKHVDP01Aug0701-EK & Eunice Kensnet \\
\hline VVKHVDP01Aug0701-IK & Isabel Kenedy Virseglōmegev \\
\hline VVKHVDP01Aug0701-JP & Jonis Paul \\
\hline VVKHVDP01Aug0701-MI & Majorie Ivet \\
\hline VVKHVDP01Aug0701-RA & Robert Andison \\
\hline VVKHVDP01Aug0701-SU & Isabel Kenedy Virseglōmegev \\
\hline VVKHVDP01Aug0701-WR & Websta Nightingale Riliar \\
\hline VVKHVDP11Aug0701-WT & Watason Tin̄in̄ \\
\hline VVKHVDP11Aug0701-PF & $\begin{array}{l}\text { Watason Tin̄in, Hilton Malau, Eli Field Malau, } \\
\text { Jimmy Brown, Johnstar, Kevin Mark, Christian, Stewart, } \\
\text { Graham Mumeg, Barrington }\end{array}$ \\
\hline VVKHVDP12Aug0701-PF & $\begin{array}{l}\text { Watason Tin̄in̄, Hilton Malau, Eli Field Malau, Jimmy } \\
\text { Brown, Johnstar, Kevin Mark, Christian, Stewart, } \\
\text { Graham Mumeg, Barrington }\end{array}$ \\
\hline VVKHVDP13Oct0801 & $\begin{array}{l}\text { Eli Field Malau, Frank Bolin, Cliff Richard Sirig, } \\
\text { Robert Anderson, Chrisben Dereseum Kukut } \\
\text { Juliette Han Reteñsurbum, Chrisy Etna Tuntelqiat, Marta } \\
\text { Gartesöm, Fanny Rigiskilo, Angela Doreen Roquasa, } \\
\text { Isabel Kennedy Virserlōmegev }\end{array}$ \\
\hline
\end{tabular}




\begin{tabular}{|l|l|}
\hline Recording name & Speaker(s) \\
\hline VVKHVDP15Oct0801 & $\begin{array}{l}\text { Robert Anderson Kukut, Michael Rowson, Jim Borsa, } \\
\text { Goliak, Jovil, Andre, Simon, } \\
\text { Jesse Riwianlav, Merilyn Webas, Janet Reteorigengen } \\
\text { Marta Gartesöm, Juliette Han Reten̄surbum, Chrisy Etna } \\
\text { Tuntelqiat, Margret Broton Raman̄serlē }\end{array}$ \\
\hline VVKHVDP18Oct0801 & Dickinson Silas, Cliff Richard Sirig, Arman Sirig \\
\hline VVKHVDP18Oct0802 & $\begin{array}{l}\text { Websta Nightingale, Bridely Whitely, Silvian Mako, } \\
\text { Simian Wēbas, Dina Alice Raymond, Marta Gartesöm, } \\
\text { others from Wasaga }\end{array}$ \\
\hline VVKHVDP20Oct0801 & Celestial Alforce, Ann Fredy Welin, Natalie Agnes \\
\hline VVKHVDP20Oct0802 & $\begin{array}{l}\text { Merilyn Wēbas and family, Robert Anderson, } \\
\text { Dickinson Silas }\end{array}$ \\
\hline
\end{tabular}


